Prepared for the U.S. Department of Energy under Contract DE-AC05-76RL01830

\title{
Characterization Data Package for Containerized Sludge Samples Collected from Engineered Container SCS-CON-210
}

\author{
MS Fountain \\ SK Fiskum \\ DL Baldwin \\ SJ Bos \\ CA Burns \\ CD Carlson
}

September 2013

DS Coffey
RC Daniel
CH Delegard
MK Edwards
LR Greenwood
D Neiner

BM Oliver

KN Pool

AJ Schmidt RW Shimskey

SI Sinkov

D Neiner
LA Snow
CZ Soderquist
CJ Thompson
T Trang-Le
MW Urie 


\title{
DISCLAIMER
}

This report was prepared as an account of work sponsored by an agency of the United States Government. Neither the United States Government nor any agency thereof, nor Battelle Memorial Institute, nor any of their employees, makes any warranty, express or implied, or assumes any legal liability or responsibility for the accuracy, completeness, or usefulness of any information, apparatus, product, or process disclosed, or represents that its use would not infringe privately owned rights. Reference herein to any specific commercial product, process, or service by trade name, trademark, manufacturer, or otherwise does not necessarily constitute or imply its endorsement, recommendation, or favoring by the United States Government or any agency thereof, or Battelle Memorial Institute. The views and opinions of authors expressed herein do not necessarily state or reflect those of the United States Government or any agency thereof.

\author{
PACIFIC NORTHWEST NATIONAL LABORATORY \\ operated by \\ BATTELLE \\ for the \\ UNITED STATES DEPARTMENT OF ENERGY \\ under Contract DE-AC05-76RL01830
}

Printed in the United States of America

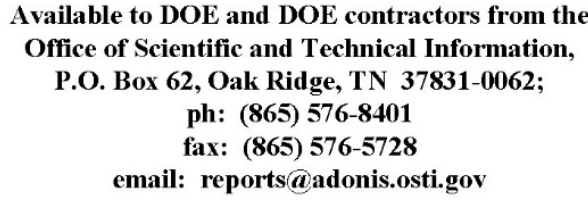

Available to the public from the National Technical Information Service

5301 Shawnee Rd., Alexandria, VA 22312 ph: (800) 553-NTIS (6847)

email: orders@ntis.gov $<$ http://www.ntis.gov/about/form.aspx $>$

Online ordering: http://www.ntis.gov 


\section{Characterization Data Package for Containerized Sludge Samples Collected from Engineered Container SCS-CON-210}

\author{
MS Fountain \\ SK Fiskum \\ DL Baldwin \\ SJ Bos \\ CA Burns \\ CD Carlson
}

\author{
DS Coffey \\ $\mathrm{RC}$ Daniel \\ $\mathrm{CH}$ Delegard \\ MK Edwards \\ LR Greenwood \\ D Neiner
}

\author{
BM Oliver \\ KN Pool \\ AJ Schmidt \\ RW Shimskey \\ SI Sinkov
}

\author{
LA Snow \\ CZ Soderquist \\ CJ Thompson \\ T Trang-Le \\ MW Urie
}

September 2013

Prepared for

the U.S. Department of Energy

under Contract DE-AC05-76RL01830

Pacific Northwest National Laboratory

Richland, Washington 99352 
PNNL-20650, Rev. 2

\section{Revision History}

\begin{tabular}{|c|c|c|}
\hline $\begin{array}{l}\text { Revision } \\
\text { Number }\end{array}$ & Description & Date \\
\hline 0 & First issue & August 2011 \\
\hline 1 & $\begin{array}{l}\text { Results for ASR } 8878 \text { and ASR } 8919 \text { were inserted and discussed in Sections } 4.0 \text { and } \\
5.0 \text { and primarily address results from undissolved solids analyses. Appendix D was } \\
\text { updated to include both ASR reports. Two surveillance reports and one Occurrence } \\
\text { Report were inserted into Table J.6 and Table J.4 (Appendix J), respectively. Minor } \\
\text { editorial changes were incorporated throughout. }\end{array}$ & October 2011 \\
\hline 2 & $\begin{array}{l}\text { According to the CH2MHill Plateau Remediation Company, Sludge Treatment Project } \\
\text { (STP) Quality Assurance Project Plan/Sampling and Analysis Plan (QAPjP/SAP KBC- } \\
\text { 33786), Revision } 1 \text { of this characterization data package was validated and assessed by } \\
\text { STP staff. The resulting STP validation report (PRC-STP-00560) } \\
\text { reviews included detailed } \\
\text { QAPjP/SAP documents) for the sludge in engineered container SCS-CON-210. As a } \\
\text { consequence of the validation reviews of the laboratory data package, in addition to the } \\
\text { baseline laboratory data qualifiers and data summaries provided in the validation report, } \\
\text { a set of general corrections were also identified (e.g., typographical errors, general } \\
\text { technical inconsistences, sample identification issues, etc.). While the STP validation } \\
\text { report should be used as the primary source for STP sludge characterization and the } \\
\text { corresponding STP Databook (HNF-SD-SNF-TI-015, Vol. 2, Rev. 24) }{ }^{\text {(b) }} \text { for design } \\
\text { data, there are instances where certain detailed background information is only } \\
\text { available in the Pacific Northwest National Laboratory (PNNL) report and data } \\
\text { package. To assist future data users that have such background needs (e.g., for STP } \\
\text { Phase 2), Revision } 2 \text { of the original report and data package (PNNL-20650) is being } \\
\text { issued to correct for the general errors found during the STP validation that were } \\
\text { subsequently confirmed by PNNL. Appendix J provides a detailed description of the } \\
\text { changes for this revision. } \\
\text { Figure } 1 \text { shows the hierarchy of SCS-CON-210 characterization data documentation } \\
\text { and data authorization, and specifically how PNNL-20650 Revision } 1 \text { and Revision } 2 \\
\text { reports are used relative to the STP-approved data. }\end{array}$ & September 2013 \\
\hline
\end{tabular}

(a) Makenas BJ, RB Baker, JA Pottmeyer and TJ Welsh. 2013. Data Validation and Assessment by the Sludge Treatment Project for Characterization Data from Engineered Container SCS-CON-210, PRC-STP-00560, Revision 0, 0A, and 0B, CH2M Hill Plateau Remediation Company, Richland Washington.

(b) Johnson ME. 2013. Spent Nuclear Fuel Project Technical Databook, Volume 2, Sludge. HNF-SD-SNF-TI015, Volume 2, Revision 24, CH2M Hill Plateau Remediation Company, Richland Washington. 
PNNL-20650, Rev. 2

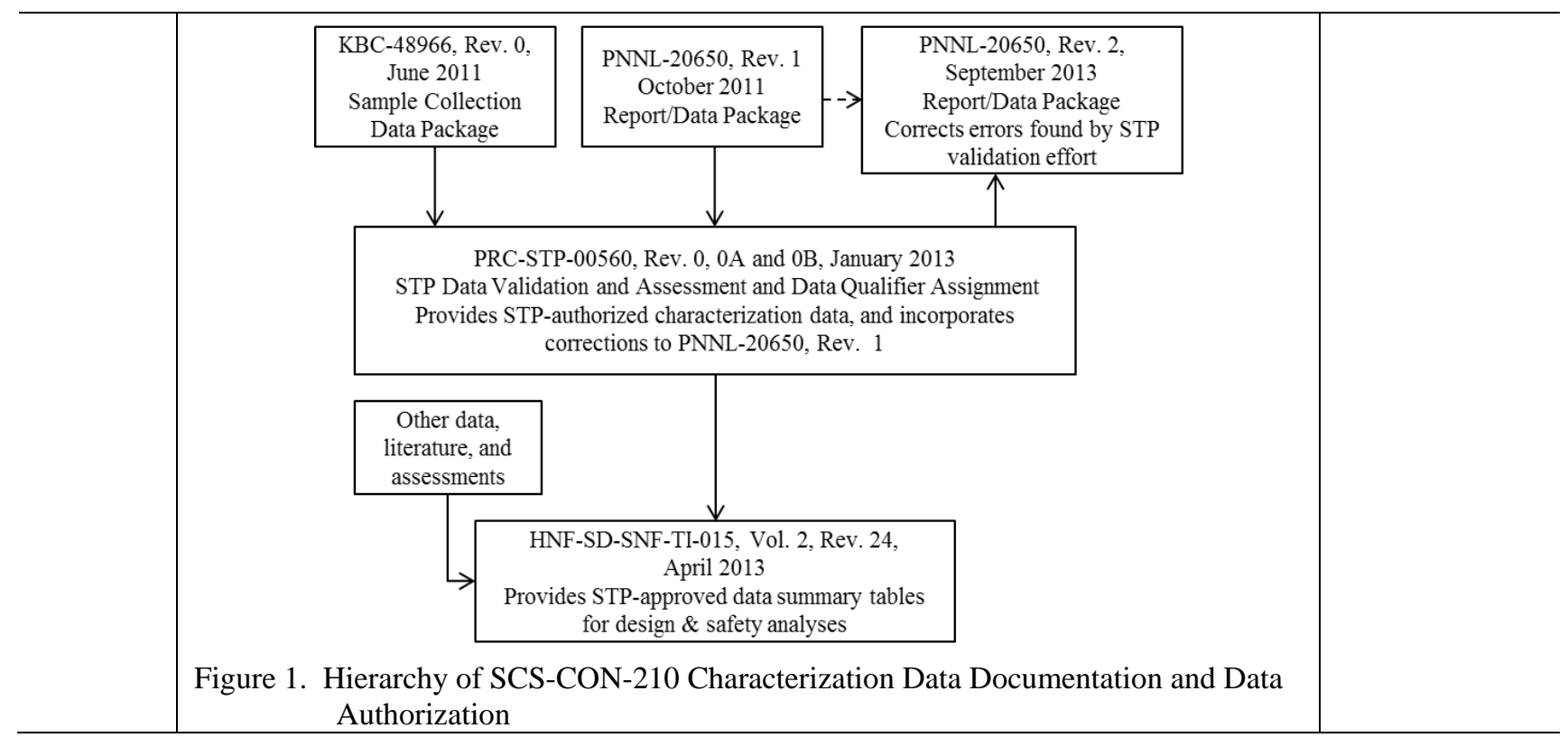




\section{Certification Statement for Revsion 2}

We certify that the data presented in PNNL-20650 Revision 2 describing the characterization analysis of K Basin containerized sludge samples collected from Engineered Containers SCS-CON-210 conform to the requirements in the following documents:

- Quality Assurance Project Plan/Sampling and Analysis Plan (QAPjP/SAP): Quality Assurance Project Plan/Sampling and Analysis Plan for Sludge in the KW Engineered Containers; KBC-33786, Rev. 2, December 2009

- DOE/RL-96-68 HASQARD, Revision 3, Volumes 1 and 4, Hanford Analytical Services Quality Assurance Requirements Document, Administrative and Laboratory Technical Requirements

- Analytical Support Operations (ASO) Quality Assurance Plan, ASO-QAP-001, Rev. 7

- Laboratory procedures and test instructions

- CH2M Hill Plateau Remediation, Contract 36402, Release 40, Statement of Work, Technical Support for Planning, Sampling and Analyzing KW Container Sludges (October 3, 2008)

Any deviations from procedures or test instructions are addressed in the completed test instructions (DVD attachment) and analysis report narratives (appendices) via references to Occurrence Reports. Throughout the project, Occurrence Reports were issued to inform participants and our client when analytical issues of concern were identified. When required, Occurrence Reports are elevated to Deficiency Reports to document conditions when data quality objective issues were not satisfied and procedure or plan deviations occurred or to Nonconformance Reports when an item did not meet specifications. A summary of the Occurrence Reports are summarized in Appendix J. There were no Deficiency Reports for this work activity and one Nonconformance Report was generated.

Multiple levels of data verification activities were performed as discussed in Appendix J.

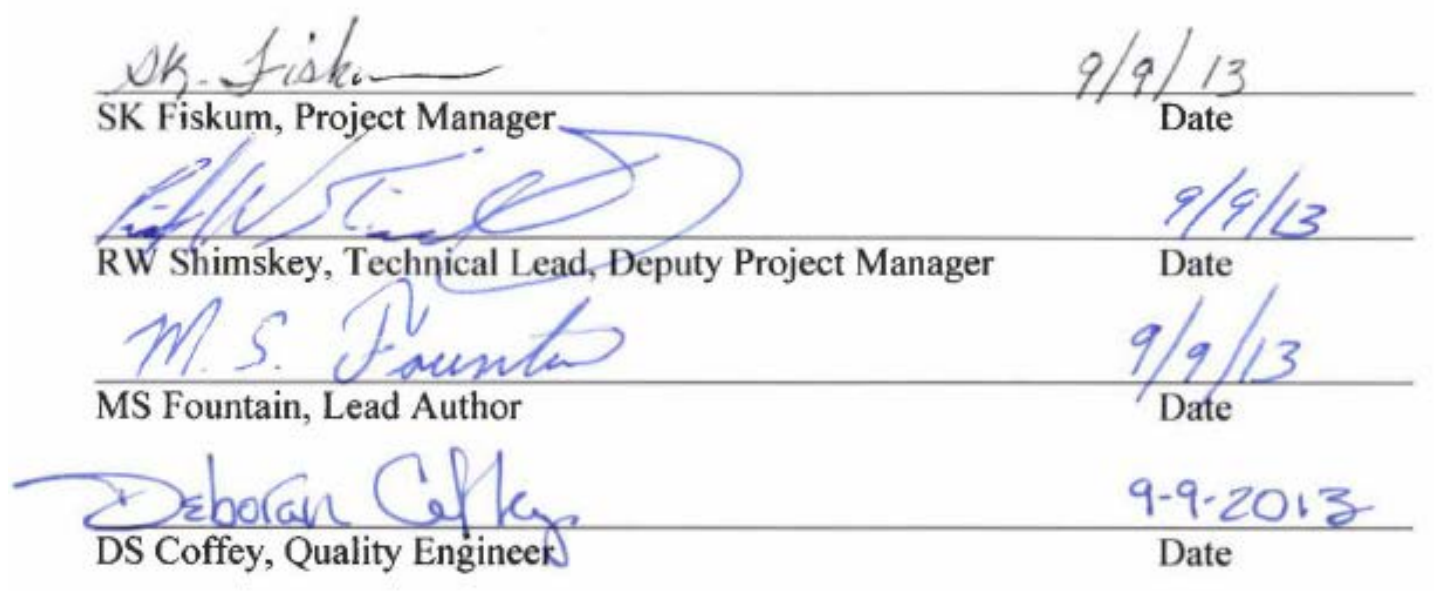


PNNL-20650, Rev. 2

\section{Certification Statement}

We certify that the data presented in this report for the characterization analysis of $\mathrm{K}$ Basin containerized sludge samples collected from Engineered Containers SCS-CON-210 conform to the requirements in the following documents:

- Quality Assurance Project Plan/Sampling and Analysis Plan (QAPjP/SAP): Quality Assurance Project Plan/Sampling and Analysis Plan for Sludge in the KW Engineered Containers; KBC-33786, Rev. 2, December 2009

- DOE/RL-96-68 HASQARD, Revision 3, Volumes 1 and 4, Hanford Analytical Services Quality Assurance Requirements Document, Administrative and Laboratory Technical Requirements

- Analytical Support Operations (ASO) Quality Assurance Plan, ASO-QAP-001, Rev. 7

- Laboratory procedures and test instructions

- CH2M Hill Plateau Remediation, Contract 36402, Release 40, Statement of Work, Technical Support for Planning, Sampling and Analyzing KW Container Sludges (October 3, 2008)

Any deviations from procedures or test instructions are addressed in the completed test instructions (DVD attachment) and analysis report narratives (appendices) via references to Occurrence Reports. Throughout the project, Occurrence Reports were issued to inform participants and our client when analytical issues of concern were identified. When required, Occurrence Reports are elevated to Deficiency Reports to document conditions when data quality objective issues were not satisfied and procedure or plan deviations occurred. A summary of the Occurrence Reports are summarized in Appendix J. There were no Deficiency Reports for this work activity and one Non-Conformance Report was generated.

Multiple levels of data verification activities were performed as discussed in Appendix J.

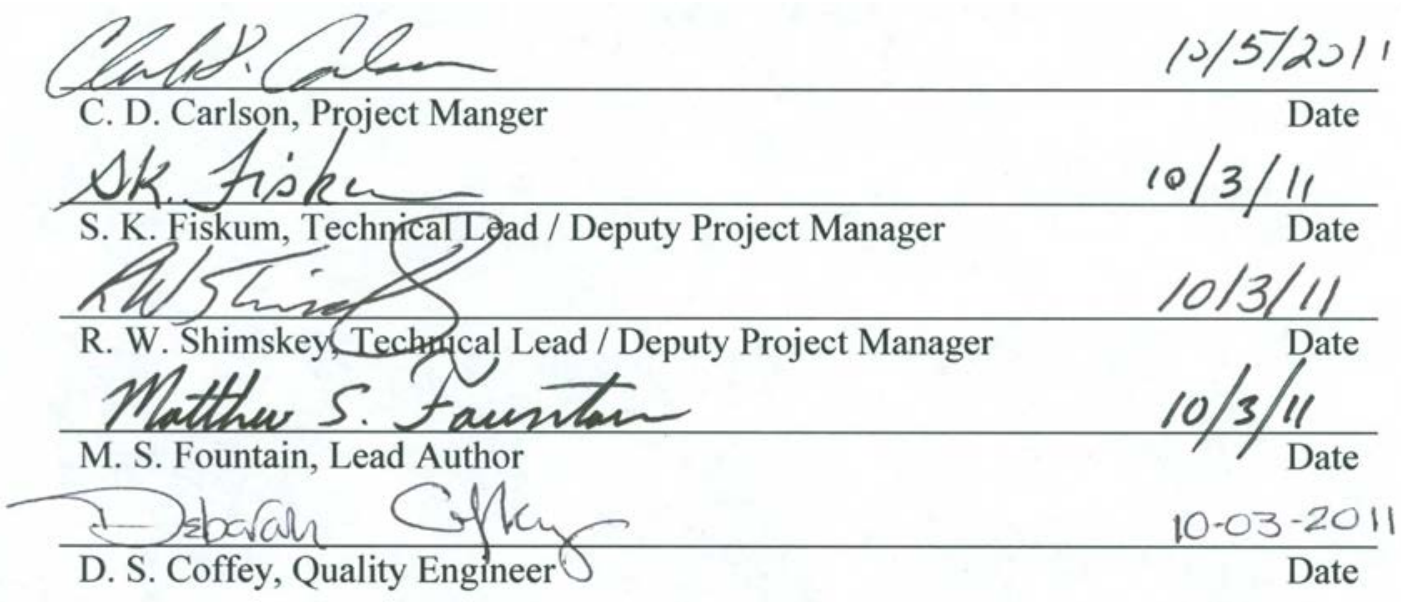



PNNL-20650, Rev. 2

\section{Summary}

This data package contains the K Basin sludge characterization results obtained by Pacific Northwest National Laboratory during processing and analysis of four sludge core samples collected from Engineered Container SCS-CON-210 in 2010 as requested by CH2M Hill Plateau Remediation Company. Sample processing requirements, analytes of interest, detection limits, and quality control sample requirements are defined in the KBC-33786, Rev. 2, Quality Assurance Project Plan/Sampling and Analysis Plan for Sludge in the KW Engineered Containers (Baker et al. 2009).

The core processing scope included reconstitution of a sludge core sample distributed among four to six 4-L polypropylene bottles into a single container. The reconstituted core sample was then mixed and subsampled to support a variety of characterization activities. Additional core sludge subsamples were combined to prepare two container composites. Only one container composite was fractionated by wet sieving through 2,000- $\mu \mathrm{m}$ and $500-\mu \mathrm{m}$ mesh sieves. Each sieve fraction was sampled to support a suite of analyses. The unsieved container composite supported rheology testing.

The core composite analysis scope included sludge density determination, radioisotope analysis, and metals analysis, including the Waste Isolation Pilot Plant Hazardous Waste Facility Permit metals (with the exception of mercury). The container composite analysis included most of the core composite analysis scope plus particle size distribution, particle density, rheology, and crystalline phase identification.

A summary of the received samples, core sample reconstitution and subsampling activities, container composite preparation and subsampling activities, physical properties, and analytical results are

presented. Supporting data and documentation are provided in the appendices. There were no cases of sample or data loss and all of the available samples and data are reported as required by the Quality Assurance Project Plan/Sampling and Analysis Plan; 100\% completeness was attained. 



\section{Acknowledgements}

The authors wish to thank the following staff who conducted much of the hands-on preparation and analytical work: Jake Bolke, Dustin Blundon, Brittany Carman, Jason Cartwright, Jeffery Chenault, Nicole Green, Ben Palma, Victor Sauceda, Tim Smith, and Jamin Trevino for sample receipt and hot cell work; Jennifer Carter for inductively coupled plasma optical emission spectrometry analyses; Katharine Carson and Lori Darnell for radiochemical separations and kinetic phosphorescence analysis (KPA) of uranium; Michael Cantaloub for his help in radioisotope counting; David Kallsen and Crystal Rutherford for total organic and total inorganic carbon analyses; Margaret Smoot for sample container preparations. We wish to also thank Jenifer Braley for her help in reviewing isotopic and KPA reports; Tracy Kelley for her help in project records; Mike Parker for his help with report format and technical editing; Lisa Middleton for her help in quality engineer reviews; and the many radiological control technicians and health physics staff whose efforts further enhanced operational safety.

The authors would also like to gratefully acknowledge the technical and programmatic support received from Wally Rutherford, Ron Baker, and Mike E Johnson of CHPRC and Terri Welsh of MSA. Their insights and reviews significantly enhanced the value of this characterization effort. 

PNNL-20650, Rev. 2

\section{Acronyms and Abbreviations}

AEA

ALE

AOI

ASO

ASTM

ASR

At\%

BTR

BS

CCB

CCV

CEC

CHPRC

COC

DI

DL

DOE

DQO

DVD

EQL

GEA

HASQARD

HLRF

ICB

ICDD

ICP-OES

ICV

ID

IDL

IWTS

$\mathrm{KBC}$

$\mathrm{KE}$

KOP

KPA

KW alpha energy analysis

Arid Land Ecology (Reserve)

analyte of interest

Analytical Support Operations

American Society for Testing and Materials

analytical service request

atom percent

Buyer's Technical Representative

blank spike

continuing calibration blank

continuing calibration verification

Consolidated Electrodynamics Corp.

CH2M Hill Plateau Remediation Company

chain of custody

deionized

detection limit

Department of Energy

data quality objectives

digital versatile disc

estimated quantitation limit

gamma energy analysis

Hanford Analytical Services Quality Assurance Requirements Documents

High Level Radiochemistry Facility

initial calibration blank

International Centre for Diffraction Data

inductively coupled plasma optical emission spectroscopy

initial calibration verification

identification

instrument detection limit

Integrated Water Treatment System

K-Basin Closure (Project)

K-East (Basin)

knockout pot

kinetic phosphorescence analysis

K-West (Basin) 
LCC

LCS

LCTS

LRB

LSC

MCC

MDA

MDC

MDL

M\&TE

MS

NARA

NBS

NIST

NM

NR

OIER

OTRS

PB

PDF

PNNL

PQL

PS

PSD

PUREX

QA

QAP

QAPjP/SAP

QC

RPD

RPL

RSD

SAL

SCC

SFO large container composite, particles greater than 2,000 microns (or $+2,000 \mu \mathrm{m}$ ) sieve fraction

laboratory control sample

laboratory-created test sample

laboratory record book

liquid scintillation counter

middle container composite, particles between 2,000 and 500 microns

(or $-2,000 /+500 \mu \mathrm{m}$ ) sieve fraction

minimum detectable activity

minimum detectible concentration

minimum detection limit

measuring and test equipment

matrix spike

National Archives and Records Administration

National Bureau of Standards

National Institute of Standards and Technology

nuclear material or not measured, see context

not recovered or not reported, see context

organic ion exchange resin

one time request for shipment

preparation blank

powder diffraction file (XRD analyses) or portable document format

Pacific Northwest National Laboratory

process quantification limit

post-digestion spike

particle size distribution

Plutonium and Uranium Extraction

quality assurance

quality assurance plan

Quality Assurance Project Plan/Sampling and Analysis Plan

quality control

relative percent difference

Radiochemical Processing Laboratory

relative standard deviation

Shielded Analytical Laboratory

small container composite, particles less than 500 microns (or $-500 \mu \mathrm{m}$ ) sieve fraction

Shielded Facility Operations 


$\begin{array}{ll}\text { SNF } & \text { spent nuclear fuel } \\ \text { SPC } & \text { statistical process control } \\ \text { SRM } & \text { standard reference material } \\ \text { SSC } & \text { shielded sample container } \\ \text { STP } & \text { Sludge Treatment Project, CHPRC } \\ \text { SWB } & \text { standard waste box } \\ \text { TC } & \text { total carbon } \\ \text { TI } & \text { test instruction } \\ \text { TIC } & \text { total inorganic carbon } \\ \text { TIMS } & \text { thermal ionization mass spectrometry } \\ \text { TOC } & \text { total organic carbon } \\ \text { TRIM } & \text { Total Records Information Management } \\ \text { TS } & \text { total solids } \\ \text { UDS } & \text { undissolved solids } \\ \text { UHP } & \text { ultra-high purity } \\ \text { WIPP } & \text { Waste Isolation Pilot Plant } \\ \text { XRD } & \text { x-ray diffraction (spectrometry) }\end{array}$





\section{Definitions}

Settled sludge $\quad$ Sludge that has been fully suspended and then settled $\sim 24 \mathrm{~h}$ from a relatively large fluidic headspace (i.e., batch settled). Typically, this batch settling leads to sample stratification, with the denser/larger particles at the bottom of the sample and the lighter/smaller particles at the top of the "settled sample".

Note 1: based on testing performed with simulants, the "settled" density was found to be more reproducible than "dewatered" (or "as-sampled") density from one sampling session to the next. This trend was observed during actual waste testing. Note 2: in accordance with the Quality Assurance Project Plan/Sampling and Analysis Plan (QAPjP/SAP), analyte concentrations are to be reported on a settled sludge basis.

Dewatered sludge Sludge that has been thickened by "batch settling” followed by mixing/ homogenizing the settled sludge and removing any resulting displaced interstitial water. Based on past experience with sludge, in the dewatered state, sludge possesses sufficient shear strength to suspend/uniformly disperse the dense uranium metal particles. Consequently, it is in this dewatered state that subsamples are collected from the parent sample. Once sampled, cover-water was added back to the dewatered sludge to avoid sample drying and maintain sludge integrity.

Note: the "dewatered" state is an arbitrary measure since the water entrainment condition in the solids will range somewhere between a free-flowing condition with free-standing water on the surface (settled sludge) and mixed and centrifuged, where the maximum water is squeezed out of solids interstices. The time it takes to reach this state can be highly variable, as it is affected by the sludge composition and settling rates. In testing with simulants, the dewatered condition exhibited significantly more variability in density relative to settled sludge. This trend was observed during actual waste testing.

As-sampled sludge The actual state of the sludge at the point of sampling; in most instances this is the "dewatered" sludge state, but in the case of sieved sample material retained on a sieve it is taken as "air-dried" (dried at ambient conditions in the hot cell).

Dry sludge $\quad$ Sludge that has been dried to a constant mass at $105^{\circ} \mathrm{C}$.

Slurry volume The combined sludge volume (settled or dewatered) and fluid volume above the solids.

Estimated volume The uncertainty in measured volume is dependent on a plurality of factors. These uncertainty include the spread in volume graduation marks on a container, the interpolation applied to the volume when the meniscus/level is between graduation marks, the inner diameter of the container, the evenness of the surface being measured, the amount of material that may be coating the upper interior container walls, and obscuration from other factors.

Opportunistic A variety of opportunistic data were collected (e.g., volume, mass, inductively measurements coupled plasma optical emission spectrometry [ICP-OES] analytes, radioisotope analytes, etc.) with little or no additional cost to the project. The QAPjP/SAP did not specifically require these data be collected, and quality control (QC) sample acceptance limits requirements were not established for these analyses. The opportunistic data are presented to provide a broader understanding of the data set as a whole. 



\section{Contents(a)}

Certification Statement for Revsion 2 2.............................................................................. iv

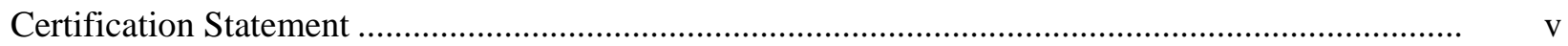

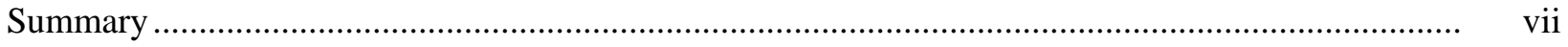

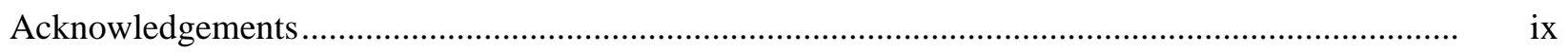

Acronyms and Abbreviations ................................................................................................... xi

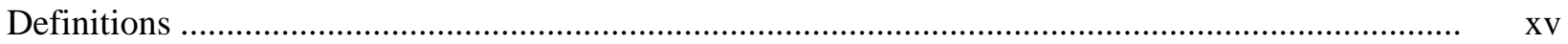

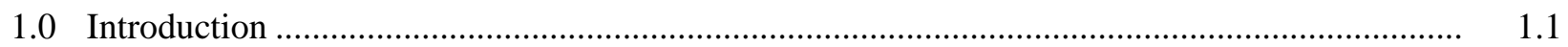

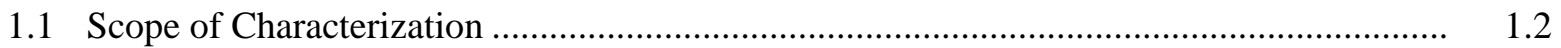

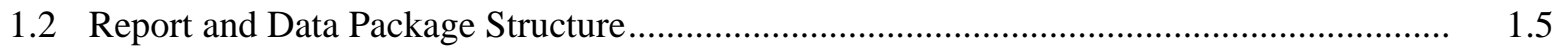

1.3 Brief History/Overview of K Basin Sludge .................................................................. 1.7

1.4 Sample Collection/Sample Plan ................................................................................ 1.10

2.0 Sample Receipt and Processing ................................................................................. 2.1

2.1 Core Reconstitution and Subsampling Overview ......................................................... 2.4

2.2 Container Compositing and Subsampling Overview ..................................................... 2.15

2.3 Physical Properties: Wet Sludge Densities and Mass Conversion Factors ......................... 2.21

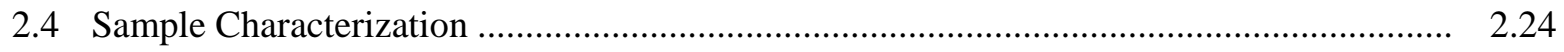

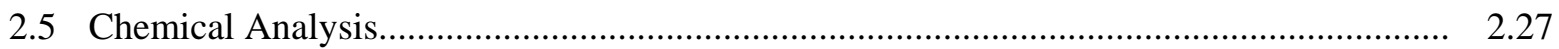

2.5.1 Acid Digestion for ICP-OES and Radionuclides............................................. 2.27

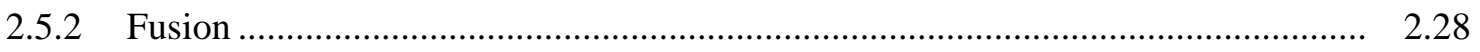

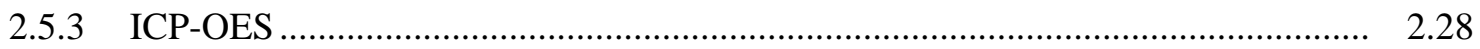

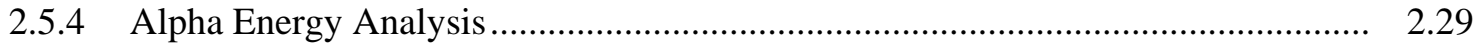

2.5.5 Gamma Energy Analysis ............................................................................... 2.31

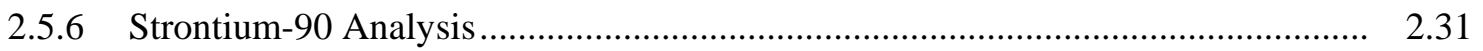

2.5.7 Total Uranium (Kinetic Phosphorescence Analysis).......................................... 2.32

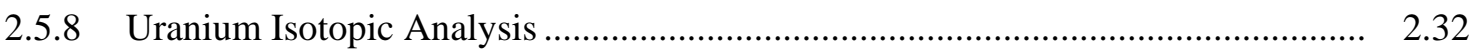

2.5.9 Plutonium Isotopic Analysis...................................................................... 2.32

2.5.10 Uranium Speciation-Metal and Oxidation State Ratio....................................... 2.33

2.5.11 Total Organic Carbon and Total Inorganic Carbon ............................................... 2.34

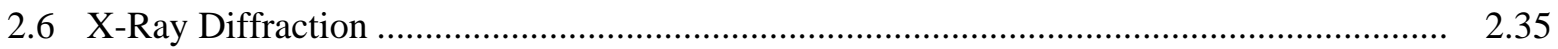

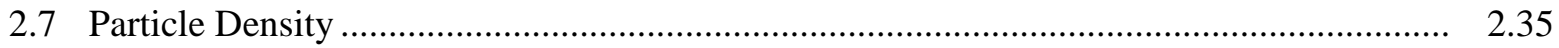

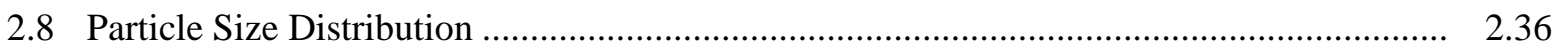

2.9 Rheology/Shear Strength Measurement ......................................................................... 2.38

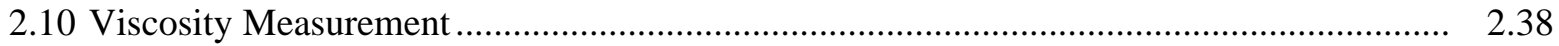

(a) The report and appendices are also published on DVD. 
3.0 Physical Property Characterization Samples: Sludge Densities and Conversion Factors to Dry and Wet Mass Bases

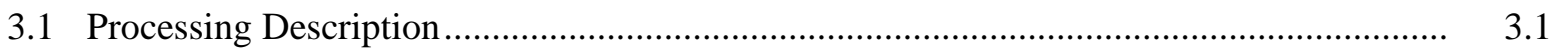

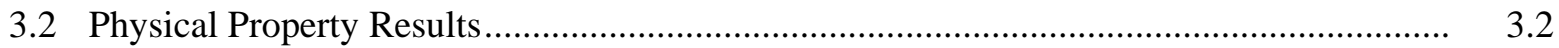

3.3 Volume Measurements and Morphology Assessments of Dried Physical Property Samples

4.0 Engineered Container SCS-CON-210 Core Samples .......................................................

4.1 Core Sample Observations …...........................................................................................

4.2 Holding Time Limits .................................................................................................. 4.11

4.3 Acid Dissolution for Chemical and Radiochemical Analysis ........................................... 4.11

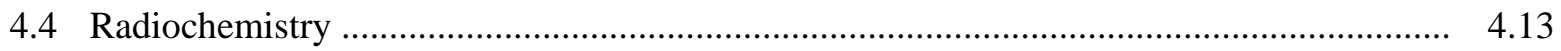

4.4.1 Neptunium (Alpha Energy Analysis) …............................................................. 4.13

4.4.2 Plutonium (Alpha Energy Analysis).............................................................. 4.21

4.4.3 Americium (Alpha Energy Analysis) …............................................................... 4.21

4.4.4 Gamma Energy Analysis ................................................................................ 4.21

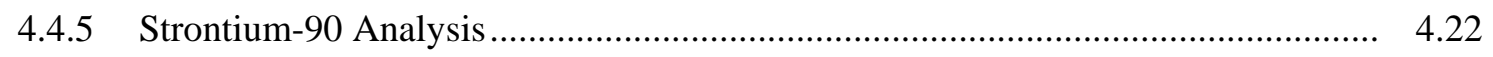

4.4.6 Total Uranium (Kinetic Phosphorescence Analysis)........................................... 4.22

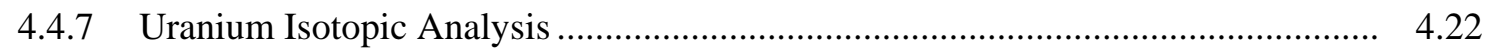

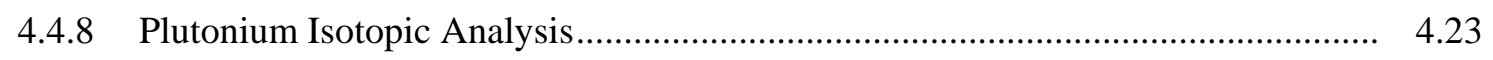

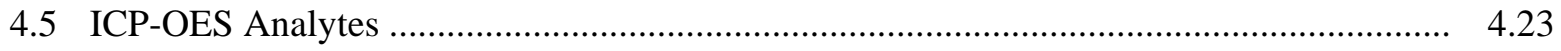

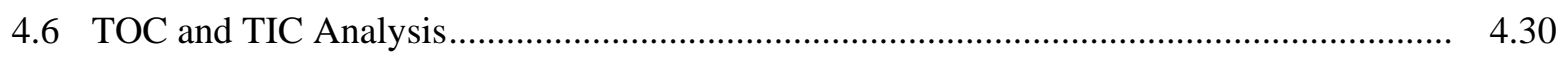

4.7 Uranium Speciation Results ....................................................................................... 4.34

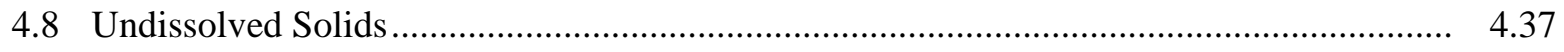

5.0 SCS-CON-210 Container Composite Samples .................................................................... 5.1

5.1 Container Composite Preparations and Observations .................................................. 5.1

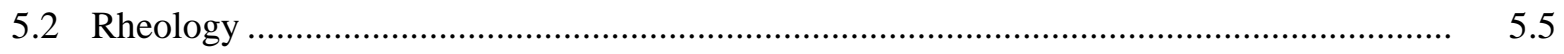

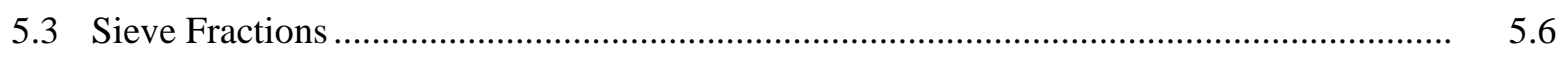

5.4 Particle Densities and Bulk Volume ….......................................................................... 5.10

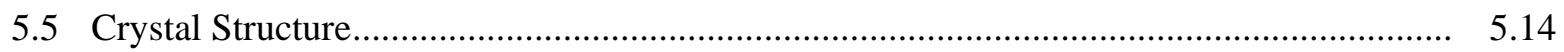

5.6 Particle Size Distribution .................................................................................... 5.17

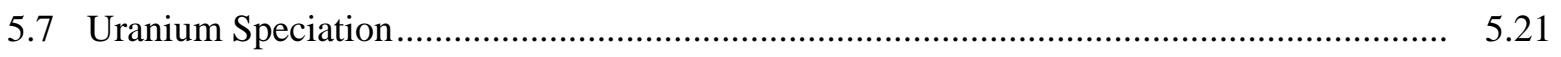

5.8 Chemical and Radiochemical Analysis .......................................................................... 5.22

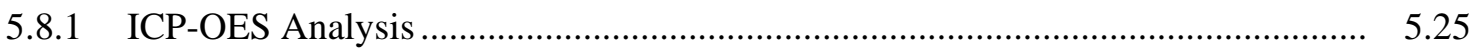

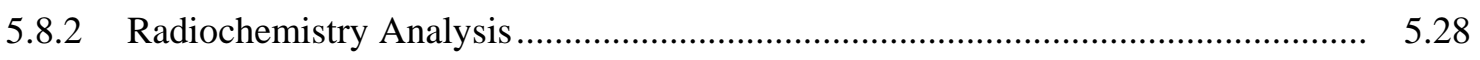

5.8 .3 Undissolved Solids ....................................................................................... 5.31

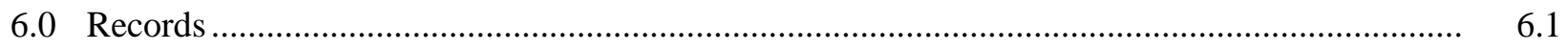

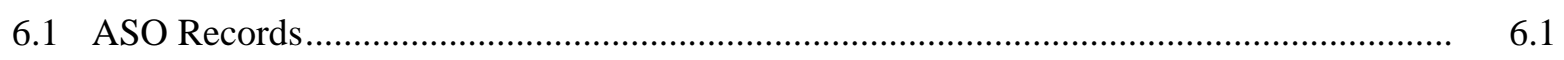

6.2 Isotopic Distribution Mass Spectrometry Records ......................................................... 6.1

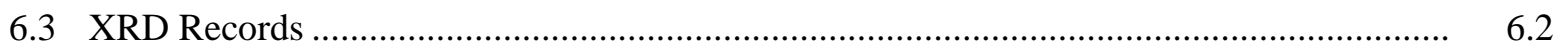




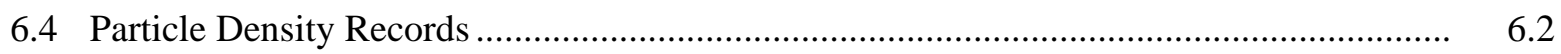

6.5 Rheology Records ................................................................................................... 6.2

6.6 Particle Size Distribution Records ................................................................................ 6.2

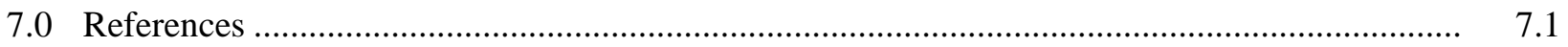

Appendix A - Completed Chain of Custody Forms .............................................................. A.1

Appendix B - ASR 8798 and Results ..................................................................................... B.1

Appendix C - ASR 8824 and Results .............................................................................. C.1

Appendix D - ASR 8878 and ASR 8919 Results .................................................................. D.1

Appendix E - Isotopic Analysis Request and Results .................................................................. E.1

Appendix F - X-Ray Diffraction Analysis ............................................................................ F.

Appendix G - Particle Density .................................................................................................. G.1

Appendix H - Rheology …....................................................................................................... H.1

Appendix I - Particle Size Distribution .................................................................................... I. I

Appendix J - Verification Processes and Revision 2 Correction Summary .................................... J.. 


\section{Figures}

1.1 Project Scope with PNNL Characterization Scope Shown in the Red Dotted Box ................... 1.2

1.2 Generalized Core Sample Analysis Flowchart.................................................................. 1.3

1.3 Generalized Container Sludge Composite Sample Analysis Flowchart ................................... 1.4

1.4 Cut-Away View of Engineered Containers SCS-CON-210 Through 260 ….............................11

1.5 Sample Locations in Engineered Containers …................................................................. 1.12

2.1 Depiction of K Basin Sludge Core and Equipment Blank Sampling Effort with Identification of Scope Associated with Specific Test Instructions ........................................... 2.3

2.2 System Schematic for Fluid and Sludge Removal ................................................................. 2.4

2.3 Prototypic Image of Sludge Core Reconstitution Step into the 960-mL Jar with Modified

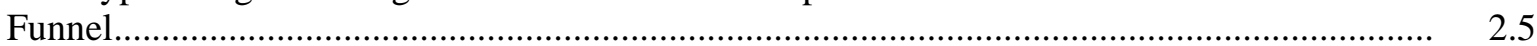

2.4 Schematic of a Core Sample Reconstitution ......................................................................... 2.5

2.5 Examples of Polybottles with Starting Concentrated Slurry and Emptied Polybottles

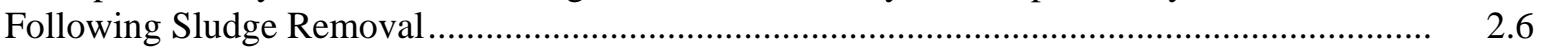

2.6 Sample Preparation for Subsampling ......................................................................... 2.7

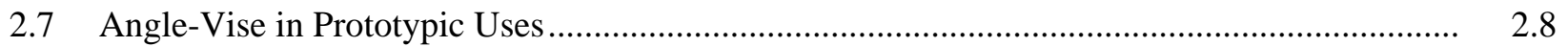

2.8 Overall Processing and Subsampling Scheme for the SCS-CON-210 Core Samples ............... 2.9

2.9 Overall Processing Schematic for the KW210-CC-A Container Composite Supporting

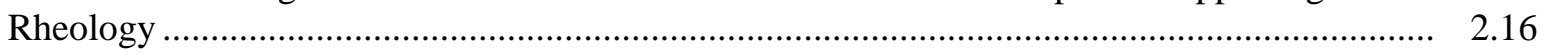

2.10 Initial Processing and Sieving Schematic of the KW210-CC-N Container Composite............. 2.16

2.11 Fines-Collection Assembly Supporting Wet Sieving Activity................................................ 2.17

2.12 Prototypic Subsampling of the Dry Sludge Solids Collected on the 500- $\mu m$ Mesh Sieve ........ 2.18

2.13 Processing Schematic of the KW210-CC-N Container Composite, $-500-\mu$ m Fraction ............. 2.19

2.14 Sample Processing for Physical Property Measurements ......................................................... 2.22

2.15 MacBeth ColorChecker ${ }^{\circledR}$ Rendition Chart ...................................................................... 2.24

3.1 Sample Preparation for Subsampling ................................................................................

3.2 Sample Processing for Physical Property Measurements ...................................................... 3.2

3.3 Settling Behavior of Physical Property Samples Collected from the KW210-A1 Core ............ 3.8

3.4 Settling Behavior of Physical Property Samples Collected from the KW210-A4 Core ............ 3.8

3.5 Settling Behavior of Physical Property Samples Collected from the KW210-B1 Core ............. 3.9

3.6 Settling Behavior of Physical Property Samples Collected from the KW210-B3 Core ............. 3.9

3.7 Select Post-Test Examination Images of TI001-A1-K ....................................................... 3.11

3.8 Select Post-Test Examination Images of TI002-A4-K …..................................................... 3.12

3.9 Select Post-Test Examination Images of TI002-B1-L ......................................................... 3.13

3.10 Select Dried Solids Examination Images of TI001-B3-K .................................................... 3.14

3.11 Select Dried Solids Examination Images of SCS-CON-210 Container Composite Samples ..... 3.15

4.1 MacBeth Color Checker Chart Images in HLRF During Reconstitution and Subsampling of the SCS-CON-210 Core Samples 
4.2 Color Chart Images Before and After Camera Failure.

4.3 Images of Reconstituted Core KW210-A1, Associated Subsamples, and a Dried Solids Examination

4.4 Sludge Profile Images for All Four Reconstituted Core Samples After Initial Remix and Settle Test.

4.5 Images of Magnetic Material Adhering to Telfon-Coated Spinbar Magnet in U Metal Subsamples.

4.6 Images of Dried Residues and Particles on Qorpak Jar Walls .....

4.7 Images of Scum Layer and Suspended Particles at Air/Liquid Interface.

4.8 Images of Gel-Like Material on Mouth of 4-L Core Sample Polybottles...

4.9 Images of Resin Beads on Top of Fines Prior to Mixing.....

4.10 Images of Vertical Fluid Channels at Jar Walls and Solids Mounds at Sludge Surface for B1 and B3 Cores

4.11 Time-Lapse Images of Fluid Ejecting Solids From the Sludge Surface and Mound Formation at the Wall in KW210-B3 Reconstituted Core Sample

4.12 Photographs of UDS from the KW210-A Core Subsamples

4.13 Photographs of UDS from the KW210-B Core Subsamples

4.14 Percent of Analyte Remaining in the UDS Measured by GEA Following Acid Digestion for SCS-CON-210 Reconstituted Core Samples

4.15 Raw X-Ray Powder Diffraction Patterns for the KW210 UDS Samples

4.16 Percent of Analyte Remaining in the UDS Measured by ICP-OES and AEA Screen in the SCS-CON-210 Reconstituted Core Samples

4.17 Relationship of Al, Fe, S, U, and Pu to OIER in the Parent Analytical Subsample.....

4.18 Total ${ }^{239+240} \mathrm{Pu}, \mathrm{U}$, and Fe Concentrations and Partitions Between Acid Digestate and UDS Phases for Each Core Subsample

5.1 Relative Settled Sludge Volume and Dry Mass Contributions of Core Samples to the Container Composite Samples with the Propagated 1- $\sigma$ Error

5.2 MacBeth Color Chart, In-Cell Image , and Scanned Image

5.3 KW210-CC-A After Mixing and Settling.....

5.4 KW210-CC-N After Mixing and Settling

5.5 KW210-LCC Solids Captured on the 2,000- $\mu \mathrm{m}$ Mesh Sieve......

5.6 KW210-LCC Transferred to Jar.

5.7 KW210-MCC Solids Captured on the 500- $\mu \mathrm{m}$ Sieve...

5.8 KW210-SCC After Mixing and Settling.....

5.9 Images of TI008-L, $+2,000-\mu \mathrm{m}$ Sieve Fraction of the KW210-CC-N Container Composite.....

5.10 Images of TI008-MK and TI008-ML , $-2,000-\mu \mathrm{m} /+500-\mu \mathrm{m}$ Sieve Fraction of the KW210-CC-N Container Composite .

5.11 Images of TI008-SK and TI008-SL in the Gas Pycnometry Analysis Cup, $-500-\mu \mathrm{m}$ Sieve Fraction of the KW210-CC-N Container Composite.

5.12 Sample Vials Containing As-Received TI008-MK and TI008-ML After Placement in the Glovebox 
5.13 XRD Powder Diffraction Patterns for the KW210-MCC and KW210-SCC Samples ............ 5.16

5.14 Average Cumulative Volume \% Undersize Plot of KW210-SCC .......................................... 5.19

5.15 Average Volume \% PSD Plot of KW210-SCC ..................................................................... 5.19

5.16 Analyte Content in KW210-CC-N Sieve Fractions ............................................................ 5.30

5.17 KW210-CC-N UDS Phase Split Fractions …................................................................... 5.32

5.18 XRD Patterns of KW210-CC-N Container Composite Sieve Fraction Sample UDS................ 5.33

5.19 Relationship of Al, Fe, S, U, and Pu to OIER Content in the Parent Analytical Sample........... 5.36

5.20 Total ${ }^{239+240} \mathrm{Pu}, \mathrm{U}, \mathrm{Fe}$ and ${ }^{137} \mathrm{Cs}$ Content and Partitions Between Acid Digestate and UDS Phases for the MCC and SCC Sieve Fractions ..................................................................... 5.37

5.21 Undigested KW210-MCC Sample Split for GEA …....................................................... 5.38 


\section{Tables}

2.1 Samples Received from Engineered Containers SCS-CON-210 ............................................ 2.2

2.2 Cross-Reference of Core Sample ID to Test Instruction ID and Title ...................................... 2.3

2.3 Cross-Reference of SCS-CON-210 A1 Core Sample to TI Subsample ID, Subsample Mass, RPL/ASO ID, ASR ID, and General Analysis Scope................................................... 2.10

2.4 Cross-Reference of SCS-CON-210 A4 Core Sample to TI Subsample ID, Subsample Mass, RPL/ASO ID, ASR ID, and General Analysis Scope................................................... 2.11

2.5 Cross-Reference of SCS-CON-210 B1 Core Sample to TI Subsample ID, Subsample Mass, RPL/ASO ID, ASR ID, and General Analysis Scope................................................... 2.12

2.6 Cross-Reference of SCS-CON-210 B3 Core Sample to TI Subsample ID, Subsample Mass, RPL/ASO ID, ASR ID, and General Analysis Scope.

2.7 Cross-reference of Core Sample IDs, TI Subsample IDs, and Original RPL/ASO IDs to TIMS and UDS Sample ID Assignments.

2.8 Sieve Parameters

2.9 Cross-Reference of SCS-CON-210 Container Composite Samples, TI Subsample ID, AsSampled Mass, Split Date, RPL/ASO Subsample ID, and General Analysis Scope.....

2.10 Cross-reference of Container Composite Sample Description, TI Subsample IDs, and Original RPL/ASO IDs to UDS Sample ID Assignments

2.11 Digestion, Chemical, and Physical Property Analytical Procedures and QC Sample Criteria ... 2.25

2.12 Radioisotopic Analytical Procedures and QC Sample Criteria .............................................. 2.26

2.13 Summary of Alpha Emitters and Corresponding Energies .................................................. 2.29

2.14 Condition Matrix for PSD Determinations ................................................................ 2.37

3.1 Sludge Densities and Mass Conversion Factors for Samples from SCS-CON-210 ................. 3.3

3.2 SCS-CON-210 Sludge Density Changes for Subsamples K and L with Movement ................. 3.10

3.3 Dried Physical Property Sample Observations ..................................................................... 3.16

4.1 Sample Holding Times Supporting WIPP Hazardous Waste Permit Metals Analysis .............. 4.11

4.2 SCS-CON-210 Chemical Analysis Characterization Samples for Acid Dissolution, Net UDS Masses, and UDS Weight Fraction .

4.3 Radionuclide Results in Acid Digestate, Dry Sludge Mass Basis ........................................ 4.14

4.4 Radionuclide QC Sample Results in Acid Digestate ......................................................... 4.15

4.5 Radionuclide Results in Acid Digestate, Wet Settled Sludge Mass Basis ................................ 4.16

4.6 Uranium and Plutonium TIMS Isotopic Ratios in Acid Digestate........................................... 4.17

4.7 Uranium and Plutonium TIMS QC Sample Results in Acid Digestate................................... 4.18

4.8 Uranium and Plutonium Isotope Mass and Activity Distributions in Acid Digestate, Dry

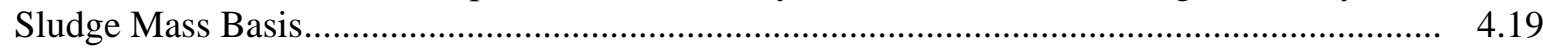

4.9 Uranium and Plutonium Isotope Mass and Activity Distributions in Acid Digestate, Wet Settled Sludge Mass Basis ............................................................................................ 4.20

4.10 ICP-OES Analytes of Interest in Acid Digestate, Dry Sludge Mass Basis .............................. 4.25

4.11 ICP-OES Opportunistic Analytes in Acid Digestate, Dry Sludge Mass Basis ....................... 4.26 
4.12 ICP-OES QC Sample Results for Analytes of Interest in Acid Digestate ............................. 4.27

4.13 ICP-OES Analytes of Interest in Acid Digestate, Wet Settled Sludge Mass Basis ................. 4.28

4.14 ICP-OES Opportunistic Analytes in Acid Digestate, Wet Settled Sludge Mass Basis ............. 4.29

4.15 TOC of SCS-CON-210 Core Samples, Dry Sludge Mass Basis ............................................... 4.32

4.16 TIC of SCS-CON-210 Core Samples, Dry Sludge Mass Basis .............................................. 4.33

4.17 TOC and TIC of SCS-CON-210 Core Samples, Wet Settled Sludge Mass Basis .................... 4.34

4.18 TOC and TIC QC Summary Table for the LCTS, Dry Sludge Mass Basis .............................. 4.34

4.19 Uranium Metal in SCS-CON-210 Core Samples .................................................................. 4.35

4.20 Uranium Oxidation State Measurements in SCS-CON-210 Core Samples .............................. 4.36

4.21 GEA Analysis of UDS and Analyte Fractionation to the UDS ...............................................4.40

4.22 Dry Mass Fraction of OIER in the Analytical Core Samples ................................................... 4.42

4.23 Summary of Phases Found via X-Ray Powder Diffraction on UDS Following Acid Digestion and Bulk OIER Removal from SCS-CON-210 Core Samples ................................ 4.45

4.24 Analytes in UDS Fusion, Dry UDS Mass Basis ........................................................... 4.46

5.1 SCS-CON-210 Core Subsamples for Container Composite KW210-CC-A.............................. 5.1

5.2 SCS-CON-210 Core Subsamples for Container Composite KW210-CC-N.............................. 5.2

5.3 Shear Strength Summary for KW210-CC-A ................................................................. 5.5

5.4 K West Basin Simulant Shear Strength Summary and Implied Safety Basis ......................... 5.6

5.5 Dry Mass Distribution of KW210-CC-N Container Composite Sieve Fractions ...................... 5.10

5.6 Particle Density Results ................................................................................................. 5.12

5.7 Approximate Bulk Density and Volume of Dried Solids....................................................... 5.13

5.8 Volume Comparisons of the KW210-CC-N Container Composite to the SCC and Combined Fractions ....................................................................................................... 5.14

5.9 Crystalline Phases Identified in KW210-SCC ..................................................................... 5.14

5.10 Crystalline Phases Identified in KW210-MCC .................................................................. 5.15

5.11 Default Optical Properties Used for PSD Analysis of KW210-SCC Samples ........................ 5.17

5.12 Test Condition Matrix for PSD Determinations ................................................................. 5.17

5.13 Cumulative Percent Undersize at 1-min Recirculation Time and Post-Sonication.................... 5.18

5.14 Summary Table of Selected Percentile Values for KW210-SCC and K Basin Simulants......... 5.20

5.15 Uranium Speciation in KW210-CC Sieve Fractions MCC and SCC: Metal Concentration and Oxide Fractionation ............................................................................................. 5.21

5.16 Uranium Speciation QC Sample Results Summary .......................................................... 5.22

5.17 ASR 8824 Batch Processing by PNL-ALO-129 Acid Digestion ........................................... 5.23

5.18 KW210-CC Sieve Fraction Sample Holding Times Supporting WIPP Hazardous Waste

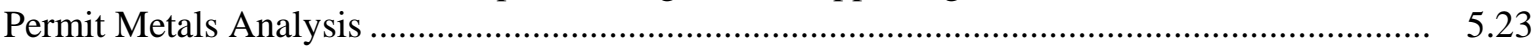

5.19 Chemical Analysis Characterization Samples for Acid Dissolution, Net Residue Masses, and UDS Weight Fractions ....................................................................................... 5.24

5.20 KW210-CC Sieve Fractions ICP-OES AOI Results, Dry Mass Basis .................................... 5.26

5.21 KW210-CC Sieve Fractions ICP-OES Opportunistic Analyte Results, Dry Mass Basis .......... 5.27 
5.22 Acid Soluble Analyte Distribution Between MCC and SCC Sieve Fractions Relative to the Calculated Input Composition

5.23 KW210-CC Sieve Fraction Radionuclide Analysis, Dry Mass Basis

5.24 Radionuclide Results in KW210-CC-N Sieve Fraction UDS, Dry Mass Basis

5.25 Dry Mass Fractionation of OIER in the KW210-CC-N Sieve Fractions

5.26 Summary of Crystalline Phases Found via X-Ray Powder Diffraction on UDS Following Acid Digestion and Bulk OIER Removal from KW210-CC-N Sieve Fraction Samples

5.27 Analytes in UDS Fusion, Dry UDS Mass Basis 5.35

5.28 Sieve Fraction Samples Mass Basis used to Create Figure 5.20..... 5.36

5.29 Radionuclide Results in KW210-MCC Sieve Fraction Separated Solids , Dry Mass Basis ...... 5.39

$5.30{ }^{137}$ Cs Distribution in Components of the MCC Fraction.......................................................... 5.39

6.1 Records Summary and Contacts for Retrieval ................................................................ 6.2

The complete summary report and data package (procedures, completed test instructions, analysis reports, calculations, and images) are provided on DVD-52578-PNNL-20650 Rev. 2, Sections I-IV, September 2013. The DVD package contents are:

Section I: Electronic Version (Adobe PDF) of Characterization Data Package for Containerized Sludge Samples Collected from Engineered Container SCS-CON-210, PNNL-20650, Rev. 2, September 2013.

Section II: Standard Laboratory Operating and Analytical Procedures

\begin{tabular}{|c|c|c|c|}
\hline Analysis Objective & Procedure Number & Rev. & Procedure Title \\
\hline TI Implementation & RPL-OP-001 & 9 & Routine Research Operations \\
\hline $\begin{array}{l}\text { Chemical Analysis } \\
\text { Acid Digestion }\end{array}$ & PNL-ALO-129 & 0 & $\mathrm{HNO}_{3}-\mathrm{HCl}$ Acid Extraction of Solids Using a Dry-Block Heater \\
\hline $\begin{array}{l}\text { Chemical Analysis } \\
\text { Fusion }\end{array}$ & PNL-ALO-115 & 1.1 & Solubilization of Metals from Solids Using a $\mathrm{KOH}-\mathrm{KNO}_{3}$ Fusion \\
\hline $\begin{array}{l}\text { U Metal and ICP- } \\
\text { OES Measurable } \\
\text { Analytes }\end{array}$ & RPG-CMC-211 & 3 & $\begin{array}{l}\text { Determination of Elemental Composition by Inductively Coupled } \\
\text { Argon Plasma Optical Emission Spectrometry (ICP-OES) }\end{array}$ \\
\hline $\mathrm{U} / \mathrm{KPA}$ & RPG-CMC-4014 & 1 & Uranium by Kinetic Phosphorescence Analysis \\
\hline $\begin{array}{l}\text { U Metal and U } \\
\text { Speciation Acid } \\
\text { Digestion }\end{array}$ & RPG-CMC-107 & 1 & $\begin{array}{l}\text { Sample Preparation for Determination of Uranium Metal } \\
\text { Concentrations in Sludge }\end{array}$ \\
\hline $\mathrm{U}(\mathrm{IV})$ : U(VI) Ratio & RPG-CMC-255 & 0 & $\begin{array}{l}\text { Sample Preparation and Analysis for Determining Uranium Oxide } \\
\text { Oxidation States in K Basin Sludges }\end{array}$ \\
\hline $\begin{array}{l}\text { Solution Density } \\
\text { Determination }\end{array}$ & PNL-ALO-501 & 0 & $\begin{array}{l}\text { Laboratory Procedure for Measurement of Physical and Rheological } \\
\text { Properties of Solutions, Slurries, and Sludges }\end{array}$ \\
\hline
\end{tabular}


PNNL-20650, Rev. 2

Section II: Standard Laboratory Operating and Analytical Procedures (contd)

\begin{tabular}{|c|c|c|c|}
\hline Analysis Objective & Procedure Number & Rev. & Procedure Title \\
\hline TOC/TIC & RPG-CMC-385 & 2 & Carbon Measured in Solids, Sludge, and Liquid Matrices \\
\hline XRD & PNNL-RPG-268 & 4 & Solids Analysis by X-Ray Diffraction \\
\hline Particle Density & APEL-PIP-4 & 2 & $\begin{array}{l}\text { Gas Pycnometry Method for Apparent Specific Gravity } \\
\text { Determinations of Consolidated Solids }\end{array}$ \\
\hline $\begin{array}{l}\text { Particle Size } \\
\text { Distribution }\end{array}$ & RPL-COLLOID-01 & 1 & Particle Size Analysis Using Malvern MS2000 \\
\hline $\begin{array}{l}\text { Viscosity Shear } \\
\text { Strength and Flow } \\
\text { Curve }\end{array}$ & RPL-COLLOID-02 & 2 & $\begin{array}{l}\text { Measurement of Physical and Rheological Properties of Solutions, } \\
\text { Slurries, and Sludges }\end{array}$ \\
\hline \multirow[t]{2}{*}{ U-Isotopic Analysis } & RPG-CMC-4017 & 0 & $\begin{array}{l}\text { Analysis of Environmental Water Samples for Actinides and } \\
\text { Strontium-90 }\end{array}$ \\
\hline & PNNL-98523-264 & 2 & Mass Spectrometer Isotopic Analysis (TIMS) \\
\hline \multirow{3}{*}{$\begin{array}{l}{ }^{238} \mathrm{Pu},{ }^{239+240} \mathrm{Pu}, \\
{ }^{241} \mathrm{Am} \text {, and }{ }^{237} \mathrm{~Np}- \\
\mathrm{AEA}\end{array}$} & RPG-CMC-4017 & 0 & $\begin{array}{l}\text { Analysis of Environmental Water Samples for Actinides and } \\
\text { Strontium-90 }\end{array}$ \\
\hline & RPG-CMC-496 & 0 & Coprecipitation Mounting of Actinides for Alpha Spectroscopy \\
\hline & RPG-CMC-422 & 2 & Solutions Analysis: Alpha Spectrometry \\
\hline \multirow[t]{2}{*}{ Pu-Isotopic Analysis } & RPG-CMC-455 & 0 & $\begin{array}{l}\text { Separation of Uranium and Plutonium for Isotopic Analysis by Mass } \\
\text { Spectrometry }\end{array}$ \\
\hline & PNNL-98523-264 & 2 & Mass Spectrometer Isotopic Analysis (TIMS) \\
\hline $\begin{array}{l}\text { Gamma-emitters } \\
\text { - GEA }\end{array}$ & RPG-CMC-450 & 1 & $\begin{array}{l}\text { Gamma Energy Analysis (GEA) and Low-Energy Photon } \\
\text { Spectrometry (LEPS) }\end{array}$ \\
\hline \multirow{2}{*}{$\begin{array}{l}\text { Gross Alpha } \\
\text { and Beta }\end{array}$} & RPG-CMC-4001 & 1 & Source Preparation for Gross Alpha and Gross Beta Analyses \\
\hline & RPG-CMC-408 & 2 & Total Alpha and Beta Analysis \\
\hline \multirow[t]{2}{*}{ AEA Screen } & RPG-CMC-496 & 0 & Coprecipitation Mounting of Actinides for Alpha Spectroscopy \\
\hline & RPG-CMC-422 & 2 & Solutions Analysis: Alpha Spectrometry \\
\hline \multirow[t]{2}{*}{${ }^{90} \mathrm{Sr}$} & RPG-CMC-476 & 0 & Strontium Separation Using Eichrom Strontium Resin \\
\hline & RPG-CMC-474 & 1 & $\begin{array}{l}\text { Measurement of Alpha and Beta Activity by Liquid Scintillation } \\
\text { Spectrometry }\end{array}$ \\
\hline \multirow[t]{3}{*}{ General } & 52578-ADM-001 & 0 & $\begin{array}{l}\text { K Basin Requests for Analysis and Guidelines for Data Reporting } \\
\text { and Technical, QE, and Spreadsheet Reviews }\end{array}$ \\
\hline & $\begin{array}{l}\text { ADM-RSEG- } \\
\text { BALANCES }\end{array}$ & 0 & Balance Calibration Verification \\
\hline & SOP-SFO-512 & 0 & Receive K Basin Sludge via Standard Waste Box (SWB) \\
\hline
\end{tabular}


Section III: Test Instructions, Laboratory Record Books, and Data Analysis Files

\begin{tabular}{|c|c|}
\hline Folder ID & Contents Description \\
\hline 52578-TI001 & $\begin{array}{l}\text { K West Basin Sample Receipt and Preparation of Consolidated Core } \\
\text { Composites: SCS-CON-210-A1 and SCS-CON-210-B3 }\end{array}$ \\
\hline 52578-TI002 & $\begin{array}{l}\text { K West Basin Sample Receipt and Preparation of Consolidated Core } \\
\text { Composites: SCS-CON-210-A4 and SCS-CON-210-B1 }\end{array}$ \\
\hline 52578-TI008 & $\begin{array}{l}\text { Preparation of K West Basin Consolidated Container Composite for SCS-CON- } \\
210\end{array}$ \\
\hline 52578-TI037 & $\begin{array}{l}\text { Particle Size Distribution Measurements for K West Basin Consolidated } \\
\text { Container Composite SCS-CON-210, Using Minus } 500 \text { micron Particle Size } \\
\text { Fraction Samples }\end{array}$ \\
\hline 52578-TI039 & $\begin{array}{l}\text { Rheological Measurements for K West Basin Consolidated Container } \\
\text { Composite SCS-CON-210 }\end{array}$ \\
\hline \multirow[t]{2}{*}{ 52578-TI040 } & $\begin{array}{l}\text { Processing of the Undissolved Solids from the K West Basin Core Samples } \\
\text { Collected from SCS-CON-210 for Additional Analysis }\end{array}$ \\
\hline & $\begin{array}{l}\text { This folder also contains the data calculation spreadsheet underpinned by this } \\
\text { test instruction: Sample Split Masses TI040 Rev. 1.xlsx. }\end{array}$ \\
\hline ASR Data Reductions & $\begin{array}{l}\text { Contains ASR } 8798 \text { Data Reduction.xlsx, ASR } 8798 \text { Data Reduction } \\
\text { Rev1.xlsx, ASR } 8798 \text { Data Reduction Rev2.xlsx, ASR 8824 Data } \\
\text { Reduction.xlsx, ASR } 8824 \text { Data Reduction Rev1.xlsx, and ASR } 8824 \text { Data } \\
\text { Reduction Rev2.xlsx. Spreadsheets combine physical properties information } \\
\text { with analytical data and produces summarized results. QE surveillance of ASR } \\
\text { 8824 Data Reduction Rev1.xlsx and ASR } 8798 \text { Data Reduction Rev2.xlsx also } \\
\text { included. }\end{array}$ \\
\hline KW210 Bottle Volumes & $\begin{array}{l}\text { Contains KW210_Bottle_Volumes.xls. Compares measurements of sludge } \\
\text { volume of received core samples in 4-L polybottles to the measured volume of } \\
\text { the reconstituted core after addition of each core sample. Individual core } \\
\text { sample bottle information and reconstituted core settling behavior over time is } \\
\text { also captured. }\end{array}$ \\
\hline LRBs & Applicable pages of LRB 60605 that specifically relate to KW210 work. \\
\hline TI001 Physical Properties & $\begin{array}{l}\text { Contains KW210-A1 Rev2.xlsx and KW210-B3 Rev2.xlsx files. Spreadsheets } \\
\text { calculate and summarize the physical properties and settling of the reconstituted } \\
\text { core subsamples. }\end{array}$ \\
\hline TI002 Physical Properties & $\begin{array}{l}\text { Contains KW210-A4 Rev2.xlsx and KW210-B1 Rev2.xlsx files. Spreadsheets } \\
\text { calculate and summarize the physical properties and settling of the reconstituted } \\
\text { core subsamples. }\end{array}$ \\
\hline TI008 Physical Properties & $\begin{array}{l}\text { Contains KW210CC Rev. 1.xlsx Spreadsheet calculates and summarizes the } \\
\text { physical properties and settling of the container composite samples. }\end{array}$ \\
\hline TIMS Data Analysis & $\begin{array}{l}\text { Contains the spreadsheet used to normalize the raw TIMS data to atom\% for } \\
\text { reporting, KW210 TIMS data July } 19 \text { 2011.xlsx. }\end{array}$ \\
\hline
\end{tabular}


PNNL-20650, Rev. 2

Section IV: Photographic Images

\begin{tabular}{|c|c|}
\hline Folder ID & Contents Description \\
\hline Color Charts & $\begin{array}{l}\text { A color chart image was collected each day core reconstitution and subsampling work } \\
\text { was conducted. Other work activities have a color chart view in the background of the } \\
\text { image. }\end{array}$ \\
\hline KW210 A1 Core & $\begin{array}{l}\text { This folder contains } 6 \text { subfolders entitled: 4-L Polybottle Inspections, Core } \\
\text { Reconstitution Stages, Core Subsample Settling, Reconstituted Core Inspections, } \\
\text { Reconstituted Core Settling, and Reconstituted Core Subsampling. }\end{array}$ \\
\hline KW210 A4 Core & $\begin{array}{l}\text { This folder contains } 6 \text { subfolders entitled: 4-L Polybottle Inspections, Core } \\
\text { Reconstitution Stages, Core Subsample Settling, Reconstituted Core Inspections, } \\
\text { Reconstituted Core Settling, and Reconstituted Core Subsampling. }\end{array}$ \\
\hline KW210 B1 Core & $\begin{array}{l}\text { This folder contains } 6 \text { subfolders entitled: 4-L Polybottle Inspections, Core } \\
\text { Reconstitution Stages, Core Subsample Settling, Reconstituted Core Inspections, } \\
\text { Reconstituted Core Settling, and Reconstituted Core Subsampling. }\end{array}$ \\
\hline KW210 B3 Core & $\begin{array}{l}\text { This folder contains } 6 \text { subfolders entitled: 4-L Polybottle Inspections, Core } \\
\text { Reconstitution Stages, Core Subsample Settling, Reconstituted Core Inspections, } \\
\text { Reconstituted Core Settling, and Reconstituted Core Subsampling. }\end{array}$ \\
\hline $\begin{array}{l}\text { KW210 Container } \\
\text { Composite }\end{array}$ & $\begin{array}{l}\text { This folder contains } 6 \text { subfolders entitled: KW210-CC poke test, KW210-LCC, } \\
\text { KW210-MCC, KW210-SCC poke test, Pre and Post comp, Settling test KW210-SCC }\end{array}$ \\
\hline $\begin{array}{l}\text { KW210 Particle Density } \\
\text { Measurement }\end{array}$ & This folder contains the images collected from the particle density subsamples. \\
\hline $\begin{array}{l}\text { KW210 Dry Solids } \\
\text { Inspections }\end{array}$ & $\begin{array}{l}\text { The primary physical properties samples }(\mathrm{K} \text { and } \mathrm{L}) \text { for each core were dried at } 105^{\circ} \mathrm{C} \text {. } \\
\text { Images documenting the inspection of the vials and dry materials are included. }\end{array}$ \\
\hline KW210 UDS & $\begin{array}{l}\text { This folder contains images of the undissolved solids (UDS) after removal from the hot } \\
\text { cell from the core samples (subfolder: Cores) and the container composite samples } \\
\text { (subfolder: Cont Comp). }\end{array}$ \\
\hline $\begin{array}{l}\text { KW210MK-ML Resin } \\
\text { and Non-Resin }\end{array}$ & $\begin{array}{l}\text { This folder contains images of the resin bead fraction and non-resin bead fraction after } \\
\text { first combining samples TI008-MK and TI008-ML and then separting the resin beads } \\
\text { from the combined UDS sample. }\end{array}$ \\
\hline SAL 24hr Settle Tests & $\begin{array}{l}\text { Archive and } \mathrm{M} \text { samples from each core were remixed and 24-hr settling tests completed } \\
\text { in SAL. Images are captured of slurry and sludge levels at the start and end of the } \\
\text { settling tests. }\end{array}$ \\
\hline
\end{tabular}




\subsection{Introduction}

The Sludge Treatment Project (STP), managed for the U.S. Department of Energy (DOE) by CH2M Hill Plateau Remediation Company (CHRPC), is responsible for operating the K West (KW) Basin fuel storage pool and managing the sludge (and other materials) that it contains. The STP mission includes characterization, containment, safe removal, treatment, and transportation of all sludge (treated or untreated) from the KW Basin. Sludge from the floor and pits of the KW and K East (KE) Basins has been consolidated into five large engineered containers: SCS-CON-210, 220, 240, 250, and 260. Settler sludge was retrieved and transferred into Engineered Container SCS-CON-230.

Pacific Northwest National Laboratory (PNNL) was contracted by CHPRC to conduct detailed physical, chemical, and radioisotopic characterization on K Basin sludge samples collected by CHPRC in 2010 from Engineered Container SCS-CON-210. The characterization effort was required to establish

- the nuclear material (NM) accountability values for the KW Basin floor and pit sludge inventory and

- to provide specific characterization data to support the final process design to retrieve, treat, store, and package the sludge for disposal at the Waste Isolation Pilot Plant (WIPP).

All characterization testing was conducted at the PNNL Radiochemical Processing Laboratory (RPL). This summary report and data package presents the verified characterization results, as well as the supporting characterization documentation. These verified data will undergo further validation and assessment for use by the STP and others. The final/validated data for general use by the various endusers will be documented elsewhere (e.g., STP validation and assessment reports [“brown covers”], supporting documents [Plys and Schmidt 2006], and STP engineering databook [Schmidt 2010]).

This report is the third in a series of $\mathrm{K}$ Basin engineered container characterization reports and data packages. Fiskum et al. (2011a, 2011b) reported the results from Engineered Containers SCS-CON-220, 240, 250, and 260; Shimskey et al. (2011) reported the results from Engineered Container SCS-CON-230. Much of the previous reports' structure, verbiage, and diagram descriptions are repeated in this report, allowing it to stand alone as a complete report and data package. The text and diagrams have been updated as appropriate.

The overall scope of the characterization effort supported by PNNL is provided in Figure 1.1 (see highlighted text in the red dotted box). In this case, PNNL is shown as receiving, processing, and analyzing samples, followed by project-driven data verification. Data validation is conducted separately, outside of the PNNL scope.

The sample handling and characterization requirements for this work are defined in KBC-33786, Quality Assurance Project Plan/Sampling and Analysis Plan (QAPJP/SAP) for Sludge in the KW Engineered Containers, Revision 2 (Baker et al. 2009). The work was performed by PNNL under CHPRC Contract 36402-40 and executed under PNNL Project Number 52578. 


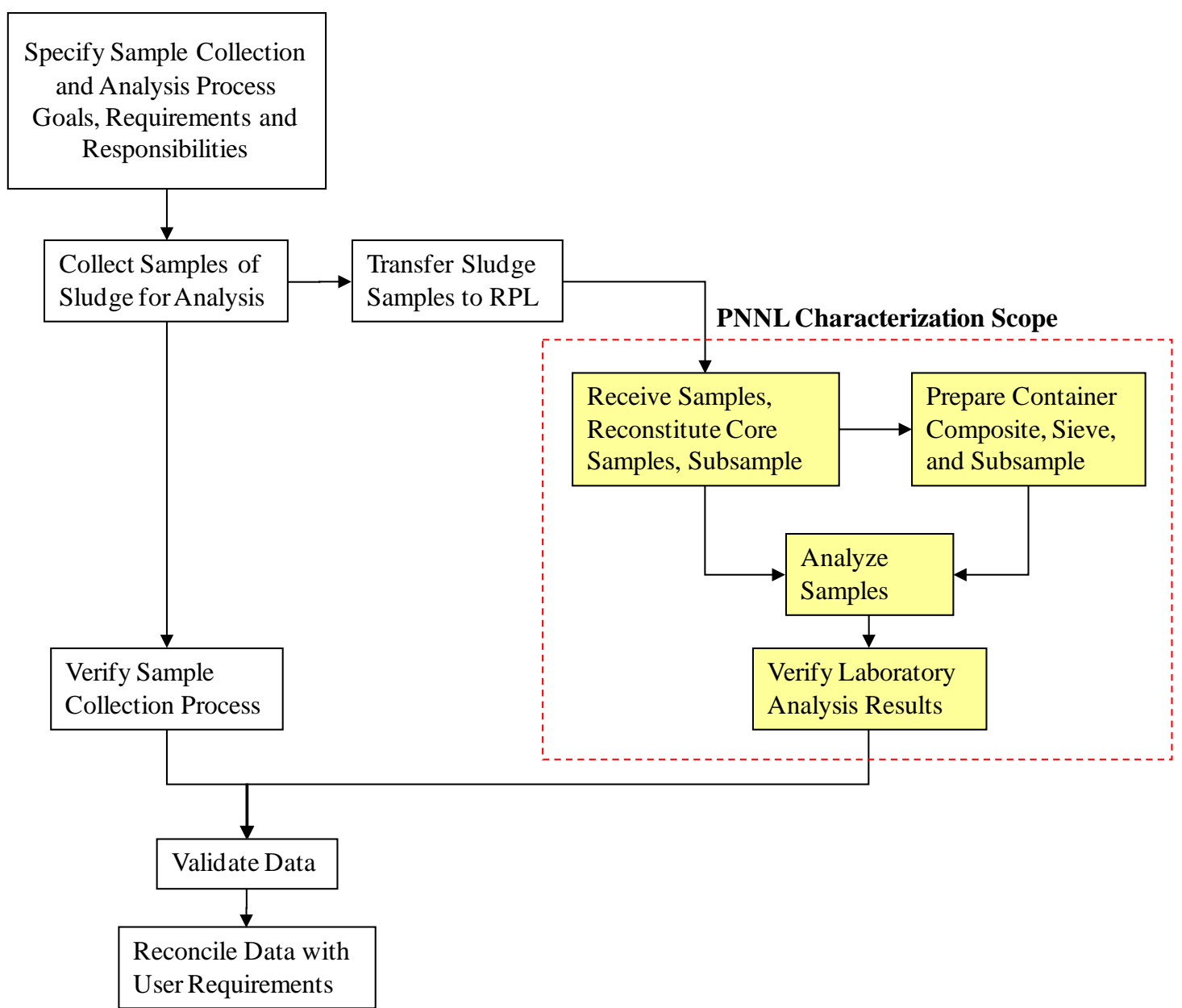

Figure 1.1. Project Scope with PNNL Characterization Scope Shown in the Red Dotted Box

\subsection{Scope of Characterization}

Four sludge core samples were collected from SCS-CON-210 (located at the KW Basin) in 4-L sample bottles. Each of the core samples was distributed among four to six sample bottles. The sample bottles were shipped to the RPL in a heavily shielded sample container (SSC) (i.e., lead-lined "Super Pig”) within a standard Hanford waste box (SWB) with chain of custody (COC) documentation. Each shipment consisted of two to four sample bottles; nine shipments were received at RPL between November 18, 2010 and January 4, 2011.

Upon receipt at RPL, the sample bottles were transferred from the SWB into a shielded hot cell at the High Level Radiochemistry Facility (HLRF). After initial observations and volume measurements, the core samples were reconstituted by combining the sample materials from the appropriate sample bottles. Each core sample was then mixed and subsampled to support a variety of analytical activities. A portion of the core samples was combined to prepare a container composite sample. The container composite sample was size-fractionated by wet sieving through a 2,000- $\mu \mathrm{m}$ mesh sieve and a 500- $\mu \mathrm{m}$ mesh sieve to generate three distinct particle-size fractions. Each sieve fraction was sampled to support a suite of analyses. A second duplicate container composite was created but was not sieved; it was analyzed for shear strength. 
Figure 1.2 and Figure 1.3 are generalized flow charts adapted from the QAPjP/SAP KBC-33786, Rev. 2 (not intended to show all operational steps) that summarize the characterization scope associated with the core samples and the container composite samples. Some additional sample analyses were conducted that were not explicitly addressed in the QAPjP/SAP. These analyses are discussed in Sections 4.0 and 5.0.

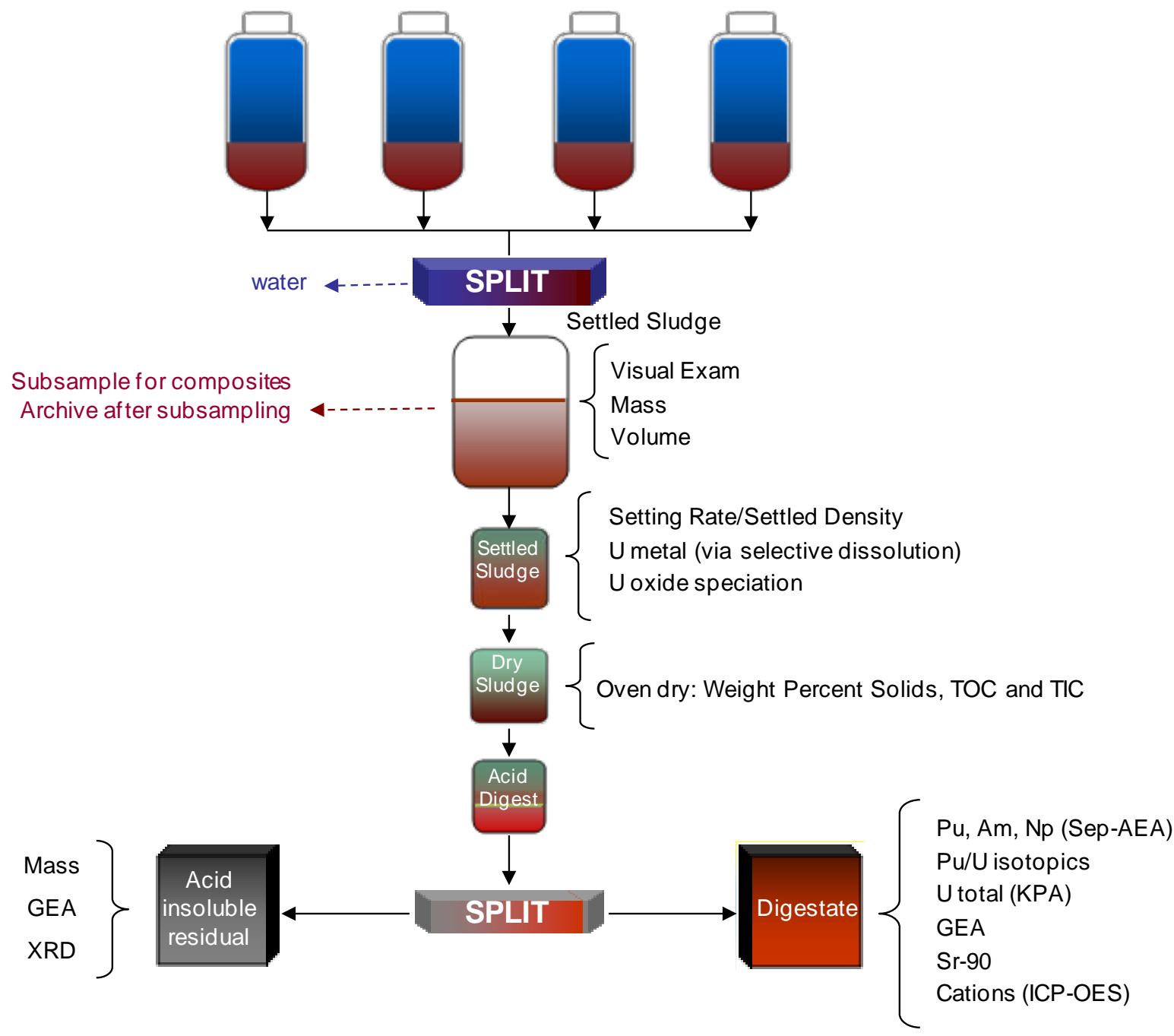

Figure 1.2. Generalized Core Sample Analysis Flowchart

The activities associated with sample consolidation, subsampling, and physical characterization were controlled and documented in detailed work documents (e.g., test instructions [TIs] or technical procedures). The sample handling and characterization requirements were complex, and before implementation, each TI underwent extensive internal reviews. Also, in accordance with the QAPjP/SAP and the Statement of Work (SOW) for Contract 36402-40, the TIs were reviewed and approved by the CHPRC Contract 36402-40 Buyer's Technical Representative (BTR) who was also the Manager of the CHPRC/STP Sampling and Characterization group. Approval and concurrence were documented by the BTR's signature on the TI. 


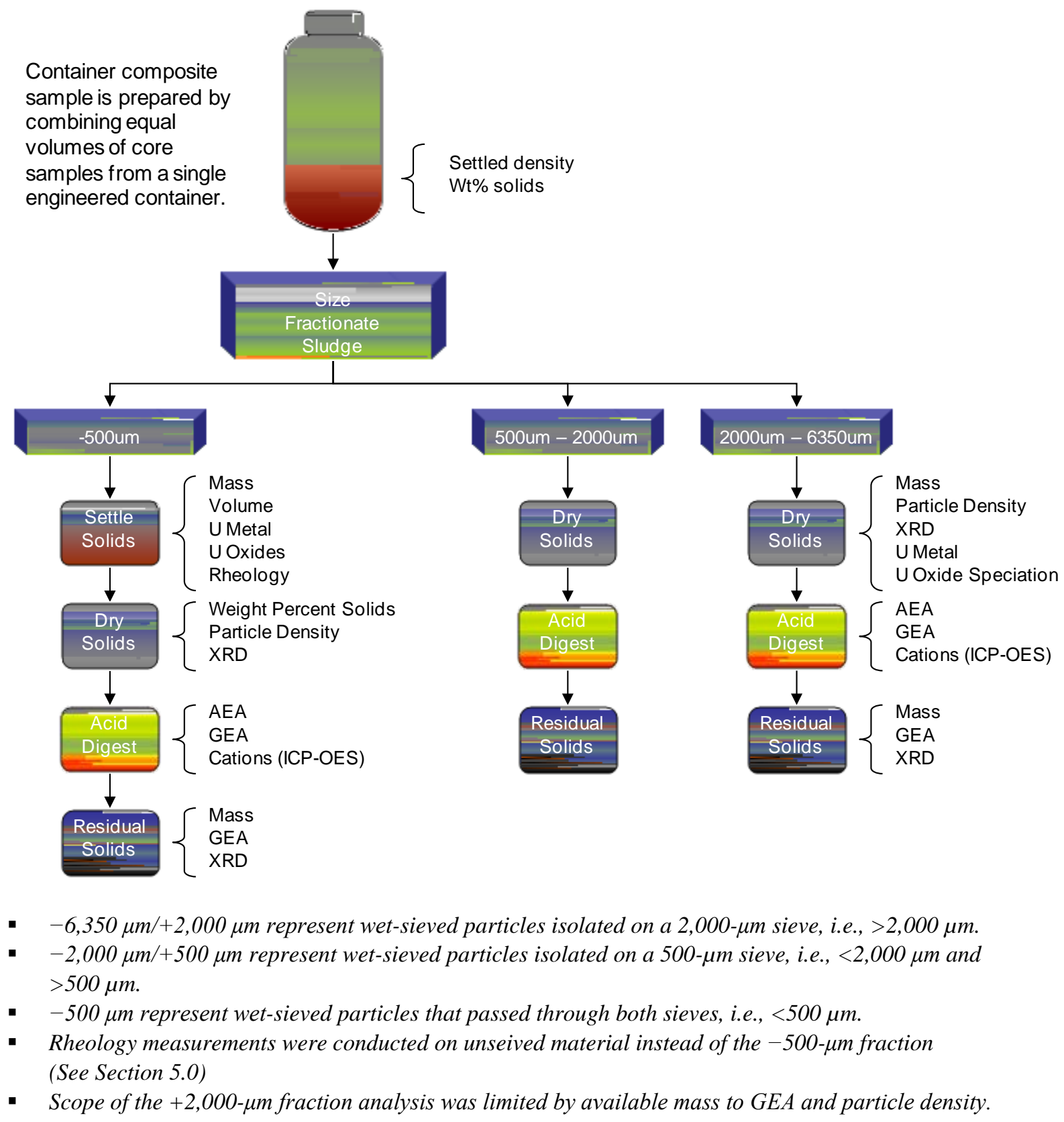

Figure 1.3. Generalized Container Sludge Composite Sample Analysis Flowchart

Analyses and associated quality control (QC) sample requirements in the QAPjP/SAP were provided to the analytical laboratory (referred to as the RPL Analytical Support Operations [ASO] laboratory) in the form of Special Instructions appended to the Analytical Service Request (ASR). Because the analytical requirements were complex, these Special Instructions were reviewed and approved both internally by PNNL staff and by the BTR to help make sure all requirements were properly conveyed to the ASO staff. As with the TIs, approval and concurrence were documented by signature on the Special Instructions. 
All physical, chemical, and radioisotope analyses reported in this document were performed between November 2010 and August 2011.

\subsection{Report and Data Package Structure}

The contents and structure of the summary report and data package are described below.

\section{Section 2.0. Sample Receipt and Processing}

This section provides an overview of PNNL sample receipt, processing, subsampling, and characterization activities.

The physical and chemical characterization scope for the sludge samples is defined in the KBC-33786, Rev. 2 QAPjP/SAP (Baker et al. 2009). The approach taken by PNNL to execute the specific scope is detailed in Section 2.0 of this report. Section 2.0 documents sample receipt at the RPL and provides an overview of the sample characterization approach, including detailed descriptions of sample flow for the following activities:

- reconstitution of core samples by combining sludge material retrieved from the 4-L sample bottles

- sludge subsampling and subsample processing

- disposition of the water from the samples

- preparation of two container composites

- container composite sieving, subsampling, and sample processing.

In addition, test approaches and procedures used for physical and chemical (inclusive of radioisotopes) characterization are identified and described and quality control (QC) sample requirements and acceptance criteria for each analysis are provided.

Sample identification (ID) nomenclature used in this characterization effort included engineered container ID (SCS-CON-210), core IDs (e.g., A1), TI subsample IDs (e.g., TI001-A1-B), ASR numbers (e.g., 8798), and RPL analytical laboratory IDs (e.g., 11-0419). Section 2.0 includes sample ID cross reference tables to assist in the navigation of the document and appendices.

\section{Section 3.0. Physical Property Characterization Samples: Sludge Densities and Conversion Factors to Dry and Wet Mass Bases}

KBC-33786, Rev. 2 QAPjP/SAP identifies wet settled-sludge density as a key property that must be determined for core (and other) samples. Chemical/radioisotope analysis results were determined and reported by ASO on a dry-mass basis and, for U metal, an as-sampled mass basis. Section 3.0 provides results from density determinations, wt $\%$ solids measurements, and resulting conversion factors that allow the user to convert analyte concentrations from a dry mass to a settled sludge mass basis and from an as-sampled mass to a settled sludge mass basis. These factors can also be used to convert analyte concentration from a mass to a volume basis. Primary physical property samples were used for these measurements. Physical property data were also collected from the parent sample (the reconstituted core) and other core subsamples collected for additional processing (e.g., characterization subsamples and 
container composite subsamples). These data were collected with the same rigor as the primary physical property sample data, and allow the data user to assess the general representativeness of the physical properties and subsamples. ${ }^{\text {(a) }}$

The primary physical property samples, typically 6 to $20 \mathrm{~mL}$, were dried at $105^{\circ} \mathrm{C}$. Before discarding, the dried physical property samples were evaluated for apparent bulk volume and bulk morphology (i.e., powder vs. agglomerated monoliths) for insights into sludge behavior if it were allowed to dry out. ${ }^{\text {(b) }}$ Section 3.0 includes a summary of these evaluations.

\section{Section 4.0. Engineered Container SCS-CON-210 Core Samples}

Four core samples were collected from Engineered Container SCS-CON-210 (i.e., floor and pit sludge originating from the KW Basin). Section 4.0 discusses the processing, reconstitution, observations (including photographs), and characterization results from each of these four core samples (see Figure 1.2). Consistent with KBC-33786, Rev. 2 QAPjP/SAP reporting requirements, characterization results are presented on a dry-solids mass basis and a settled-sludge mass basis. Also, for each type of analysis, a table is provided summarizing the process batch QC sample results for assessment of precision and accuracy (i.e., relative percent difference [RPD], laboratory control sample [LCS]/blank spike [BS] recoveries, matrix spike [MS] recoveries, and post-spike recoveries).

In accordance with the QAPjP/SAP, residual solids from the acid digestion preparation were evaluated by gamma energy analysis (GEA). Sufficient mass remained in these residual solids that further analyses were deemed necessary per direction from the BTR to better characterize the chemical composition of this material. Section 4.0 includes detailed discussion of the residual solids characterization.

A variety of data were collected on analytes not specifically listed in the QAPjP/SAP (e.g., 27 analytes measured by inductively coupled plasma-optical emission spectrometry [ICP-OES], radioisotope analytes [e.g., $\mathrm{Cm}$ ].) with little or no additional cost to the project, which this report defines as opportunistic. The QAPjP/SAP did not specify QC sample acceptance limits requirements for these opportunistic analytes and the results may not be supported by the data quality evaluation rigor applied to the specified analytes of interest. Therefore, the quality of these opportunistic data may be considered indeterminate.

\section{Section 5.0. SCS-CON-210 Container Composite Sample}

Subsamples of each of the four core samples were collected and combined to create two container composite samples of equivalent composition. After measuring the physical properties, one of the container composites was sieved into three size fractions. Each sieve fraction was characterized in accordance with KBC-33786, Rev. 2 QAPjP/SAP, based on sample availability. In addition to chemical/ radiochemical analyses (ICP-OES, GEA, alpha energy analysis [AEA] screen), characterization included crystalline phase identification by $\mathrm{x}$-ray diffraction (XRD) and particle density measurement. The

(a) Depending on the sample type, mass and/or volume measurements in the settled, as-sampled, and/or dried condition were collected. The uncertainties of these measurements were assessed and the propagated error of density and mass ratios were calculated.

(b) Consistent with the QAPjP/SAP, the characterization scope beyond the QAPjP/SAP was approved by the BTR in response to "in-field" observations/opportunities and unforeseen situations. 
smallest sieve fraction (i.e., containing particles $<500 \mu \mathrm{m}$ ) was characterized by particle-size distribution (PSD). The second container composite was not sieved and was used for shear strength testing. This section details the processing, observations, and characterization results from the container composite and the sieve fractions.

\section{Section 6.0. Records}

Records supporting the analytical results are maintained by the ASO records system, and copies of non-ASO records are provided in this report and data package. Section 6.0 details the types of ASO records that are stored and describes how they can be retrieved.

\section{Section 7.0. References}

Section 7.0 lists the references cited in this report.

\section{Appendices}

Copies of the COC records are provided in Appendix A. Appendices B through D provide all ASO-generated reports in accordance with each ASR request. Each appendix is specific to one ASR. Appendices E, F, G, H, and I provide additional sample characterization results and supporting data (thermal ionization mass spectrometry [TIMS], XRD, particle density, rheology, and PSD, respectively). Appendix $\mathrm{J}$ addresses the processes used to verify the data and provides a summary of Revision 2 corrections in this report.

\subsection{Brief History/Overview of K Basin Sludge}

Nuclear reactors were operated to produce weapons-grade plutonium from 1944 to 1990 at the U.S. Department of Energy's (DOE's) Hanford Site in Eastern Washington. The KE and KW reactors were built in the 1950s and operated into the early 1970s. Each reactor had a large water-filled concrete basin used for temporary storage of its irradiated fuel (approximately 125 feet by 67 feet and 21 feet deep, holding approximately 1.2 million gallons of water). One of the other newer reactors, N-Reactor, operated from 1964 through 1987. During N-Reactor operations from 1972 until 1983, fuel reprocessing at Hanford's Plutonium and Uranium Extraction (PUREX) facility was suspended because of safety and other concerns. With fuel processing suspended, spent nuclear fuel (SNF) accumulated to a point that exceeded the capacity of the N-Reactor storage basin, requiring utilization of the basins at the KE and KW Reactors for additional storage. Reprocessing at PUREX resumed from 1983 until 1989, but approximately 2,100 metric tons of Zircaloy-clad (with a small portion of aluminum-clad single pass), metallic uranium SNF remained in the K Basins when PUREX was permanently shut down in 1990. Some of the SNF was damaged when it was discharged from the N-Reactor. The damage was primarily to the cladding and occurred as a result of collisions among the fuel assemblies discharged and handled after irradiation at the reactors. Subsequent corrosion of the exposed uranium metal fuel resulted in the generation of radioactive sludge components. K Basin sludge is a mix of spent fuel products (including metallic uranium, zircaloy-2 cladding, plutonium, and fission and activation products), small fuel fragments, iron and aluminum oxides, concrete grit, sand, dirt, particulates of operational processes (e.g., ion exchange material), and material of biological origin (e.g., insects, plant matter). For the purpose of differentiating SNF and debris (e.g., nuts, bolts, wires) from the sludge, any material that will pass through a screen with $0.64 \mathrm{~cm}(0.25 \mathrm{in}$.) openings is defined by the STP as sludge. 
The KE Basin was not cleaned out before being used to store N-Reactor fuel; the first N-Reactor fuel was received at KE Basin in 1975. Therefore, some fraction of the sludge material originated while the basin was used to support the KE Reactor operations. The basin pool was located inside a steel framed building, but the building was not air tight, and windblown dust and other environmental debris were able to enter the pool. The pool walls and floor were constructed of concrete (uncoated), and with time, degradation of the concrete (i.e., grit and small aggregates) contributed to the sludge volume.

Corrosion products from carbon steel and aluminum structures and components are significant sludge constituents (Johnson and Burke 1995). Material from water quality control operations, including sand (from sand filters) and organic and inorganic ion exchange materials (from the ion exchange systems), are known to be in the sludge. At the KE Basin, N-Reactor SNF was stored in open-top fuel storage canisters (constructed of aluminum and stainless steel), and 83\% of these fuel canisters had open-screened bottoms. Significant quantities of fuel corrosion products were generated within these canisters. The open tops and screened-bottoms provided a pathway for this uranium-rich "canister sludge” (Makenas et al. 1997) to migrate to the basin floor. However, the fraction of this canister sludge that ended up on the KE basin floor (vs. the quantity that remained with the SNF through fuel cleaning operations) is not precisely known (Pearce and Klimper 2001). After SNF was removed from the KE Basin, sludge on the basin floors and pits was vacuumed into large containers in the KE Basin (2004/2005). Subsequently, this sludge was transferred via hose-in-hose and multiple pumps to the engineered containers (SCS-CON-240, 250, and 260) located in the KW Basin (sludge transfer from KE to KW was completed in 2007). Some composition variability is to be expected between the containers because precise control of which KE Basin sludge sources went into which of the three specific engineered containers was not a criterion for the transfer. Characterization of the core samples collected from Engineered Containers SCS-CON-240, 250, and 260 has been previously reported (Fiskum et al. 2011b).

At the KW Basin, before its use for N-Reactor fuel storage (1976), the basin pool was cleaned (i.e., any sludge from operation of the KW reactor was removed), and the concrete surface was coated. The N-Reactor fuel stored in the KW Basin was placed into closed fuel storage containers (each canister's lid was sealed with a Grafoil ${ }^{\circledR}$ gasket), and most fuel corrosion products were retained in the canisters during storage. As a result of the SNF pool management practices employed at the KW Basin, the quantity of sludge that accumulated in the KW Basin pit and floors was less than that in the KE Basin. Before commencement of fuel cleaning ${ }^{(a)}$ and fuel transfer operations in the KW Basin, the small quantity of sludge on the basin floor was assumed to consist primarily of dust and sediment with a relatively low concentration of fuel corrosion products (fuel was in closed canisters). Similar to the KE Basin operations, sandfilters and ion exchange systems were used to maintain basin pool water quality, and materials from these operations (sand and possibly ion exchange material) are present in the sludge. After start-up of fuel cleaning and packaging operations in the KW Basin in 2000, some "fuel wash sludge," containing higher concentrations of uranium metal and fuel corrosion products (and likely ion exchange materials from KE Basin) was introduced to certain areas of the KW Floor. This fuel wash sludge is expected to make up the majority of the radionuclide inventory in the KW floor and pit sludge. Also, when recovering the fuel from the KW storage canisters, Grafoil ${ }^{\circledR}$ pieces and particles (from disintegration of the gasket material when lids were removed from the fuel storage canisters) were introduced to the KW Basin pool and floor. Sludge originating from the floor and pits in the KW Basin

(a) All fuel formerly stored in the KE Basin was transferred to the KW Basin where it was washed/cleaned, packaged, and removed (2000 to 2004). 
has been consolidated into Engineered Containers SCS-CON-210 and 220. Characterization of the core samples collected from SCS-CON-220 has been previously reported (Fiskum et al. 2011b).

The particulate sludge generated from fuel packaging operations in the KW Basin was captured in equipment including Knock-Out Pots (KOPs), strainers and settler tubes that are part of the KW Integrated Water Treatment System (IWTS). Some of this fuel wash sludge also escaped to the KW Basin floor and pits. KOP sludge (which includes the associated strainers) is functionally represented by sludge that did not pass through $\leq 600^{(a)} \mu \mathrm{m}$ mesh screens. The sludge captured within the 10 settler tanks passed through these screens and is referred to as the Settler sludge. The Settler sludge was retrieved and transferred into Engineered Container SCS-CON-230. Characterization of the core samples collected from SCS-CON-230 has been previously reported (Shimskey et al. 2011).

As of November 2010, all sludge additions to SCS-CON-210 from the KW floor cleaning activities were completed. The objective of the sampling and analysis campaign is the characterization of the KW sludge after being consolidated in the Engineered Container SCS-CON-210.

Significant challenges exist in characterizing, handling, treating, and packaging the K Basin sludge:

- Presence of uranium metal-Uranium metal is present in the sludge as determined by scientific investigations and observations of $\mathrm{K}$ Basin spent fuel handling activities. Uranium metal is pyrophoric and generates flammable hydrogen gas when reacted with anoxic water. While the sludge is safely stored in the water-filled basin, future handling and removal from the basin and transport must account for this issue during process steps.

- Remote handling — Sludge is highly radioactive, and ion exchange material in the sludge has increased the quantity of ${ }^{137} \mathrm{Cs}$ retained within the sludge.

- Difficult to handle - Sludge is a heterogeneous mixture of materials that consists primarily of very fine particles $(<50 \mu \mathrm{m})$ but also contains up to $1 / 4$-in. pieces of debris and uranium metal particles. These materials range in density from about 1 (e.g., Grafoil ${ }^{\circledR}$ ) to about $19 \mathrm{~g} / \mathrm{cm}^{3}$ (uranium metal). Experience has shown that mobilization and transport of sludge (within the basins) is a tedious and time-intensive undertaking. Due to the very fine particulate nature of much of the sludge, the recapture of mobilized sludge is also challenging. High transfer velocities are needed to address the density and upper particle size of the uranium metal. Existing characterization data within a given sludge type are highly variable because of its heterogeneous nature and variable sources.

A number of major sludge characterization campaigns (Makenas et al. 1996, 1997, 1998, 1999; and Baker and Welsh $2001^{(\mathrm{b})}$ ) have been conducted in the past. These campaigns were conducted before sludge consolidation/containerization efforts (i.e., the samples were collected from original sludge locations in the basins). The goals of the past characterization efforts were to provide baseline characterization data and specific associated data to support the sludge disposition alternative being pursued at the time of sampling. Results from these past efforts are summarized in the data quality objectives (DQO) document (Westcott et. al 2009) and KBC-33786, Rev. 2 QAPjP/SAP (Baker et al. 2009).

(a) The sludge was screened with either a 500 or $600 \mu \mathrm{m}$ screen; $\leq 600 \mu \mathrm{m}$ is used for convenience.

(b) Baker and Welsh. 2001. "Summary of Initial Laboratory Data from the Consolidated and Single Pull Core Sludge Sampling Campaigns.” Memo 01-SNF/RBB004, To: KL Pearce, from RB Baker and TL Welsh, Fluor Hanford, May 10, 2001. 
Based on stakeholder decisions as documented in the DQO (Westcott et. al 2009), a new sampling and characterization analysis campaign, starting in 2009, was deemed necessary because:

1. Transfers of floor and pit sludge into containers may have affected the sludge since it was previously characterized.

2. The fraction of KE canister sludge that migrated to the KE floor vs. the fraction that followed the SNF through the fuel washing process is uncertain.

3. There has been no direct characterization of some types of sludge (including sludge from the KW Basin floor, pits, and settler tubes).

4. A new design concept to treat the $\mathrm{K}$ Basin sludges is being developed, and updated characterization information and special process testing ${ }^{(a)}$ will allow refinement of design concepts and flowsheets.

The DQO document provides in-depth specific problem statements, background, and identified required characterization data for the campaign.

\subsection{Sample Collection/Sample Plan}

Sludge that originally resided on the KE Basin floors and pits is now consolidated in Engineered Containers SCS-CON-240, 250, and 260. Floor and pit sludge originating in the KW Basin is now consolidated into Engineered Containers SCS-CON-210 and 220. Sludge in the KW Settler tanks has been retrieved and is now consolidated in SCS-CON-230. Engineered containers are $11 \mathrm{ft} 10 \mathrm{in}$. long, $5 \mathrm{ft}$ wide, and $13 \mathrm{ft}$ tall, and each contains between 1 and $8.1 \mathrm{~m}^{3}$ of sludge. Engineered Container SCS-CON-210 contains about $4 \mathrm{~m}^{3}$ of sludge. ${ }^{\text {(b) }}$ Figure 1.4 shows a cut-away view of an engineered container. (Note: Further details and background on engineered containers are provided in the QAPjP/SAP [Baker et al. 2009].)

During sludge retrieval and consolidation into the engineered containers, sludge was introduced from two inlet distribution heads positioned above the sludge bed (Figure 1.4). With this mode of sludge introduction, it is expected that the sludge was generally deposited in a series of horizontal layers or strata. The composition of the sludge in a given layer of the KW engineered container is expected to vary based on the distance from the two inlet distribution heads (see Westcott 2009, DQO Section 8.0). Larger and denser particles likely settled closer to the inlet distribution heads. Lighter/finer particles are more likely to be uniformly distributed throughout the given layer.

While some mixing occurred during containerization, it is possible that some compositional identity (based on original sludge locations) was maintained. As such, there are potential layers in containers that may correspond to the location from which the sludge was collected and retrieved. Additionally, there may also be some compositional variation or stratification due to settling of sludge during the transfer

(a) Results from settling tests defined in the DQO and QAPjP/SAP for Engineered Containers SCS-CON-220, 240, 250, and 260 have been previously reported (Fiskum et al. 2010). Settler sludge settling tests have been conducted and reporting is pending as of August 2011. As of June 2011, settling tests have been deleted from processing scope; instead the samples of SCS-CON-210 sludge will be processed in a prototypic treat-andtransfer system with added flocculating agent (this process testing sequence includes settling steps).

(b) Interoffice Memorandum CHPRC-1004013, from RK Nissen to JF Thompson, November 17, 2010. 
process. Therefore, sludge within the containers may be stratified because of two primary factors: 1) the original location where the sludge was retrieved and 2) variability in sludge particle size and density during settling. This potential stratification was taken into account while developing the sampling plan (Westcott 2009, Section 8.0).

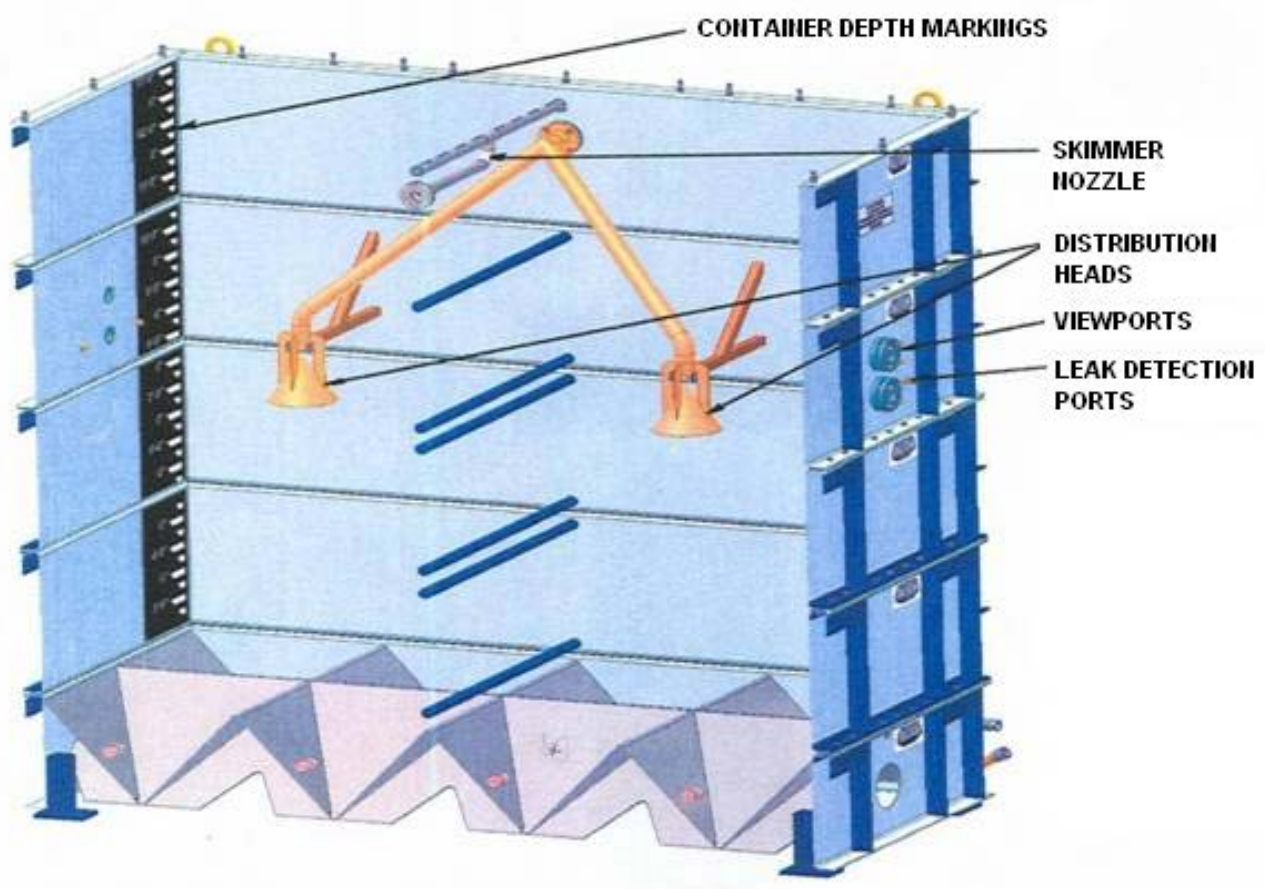

Figure 1.4. Cut-Away View of Engineered Containers SCS-CON-210 Through 260

As described in Baker et al. (2009), a probabilistic sample design was used, consistent with SW-846 and WIPP requirements for waste characterization. Full vertical cores of sludge were collected from each randomly selected sample location identified in each of the engineered containers. The sample locations available in each engineered container were constrained to the eight locations shown in Figure 1.5. The same eight locations are available for each of the engineered containers because all of the engineered containers are of the same design. Four of the sample locations are identified where sludge is more likely to contain larger and denser sludge particles (near distribution heads). These locations are identified with an "A" on Figure 1.5. Four of the sample locations are identified where sludge is less likely to contain larger and denser sludge particles (away from distribution heads). These locations are identified with a "B" on Figure 1.5. The "A" and "B" sample location identification has been maintained throughout the sampling and characterization campaign.

The DQO (Westcott 2009) specified that two representative vertical core samples (one "A" and one "B" sample) of the sludge in each of the three containers with KE-originating sludge (SCS-CON-240, 250, and 260) were required for supporting STP process design tasks. Four representative vertical core samples of the sludge in each of two containers with KW-originating sludge (SCS-CON-210 and 220) were required to provide the basis for determining the required NM accountability values and sludge characterization in support of STP process design tasks. Note, this KW-originating sludge was not sampled during the past characterization campaigns; therefore, more samples per container were collected. 


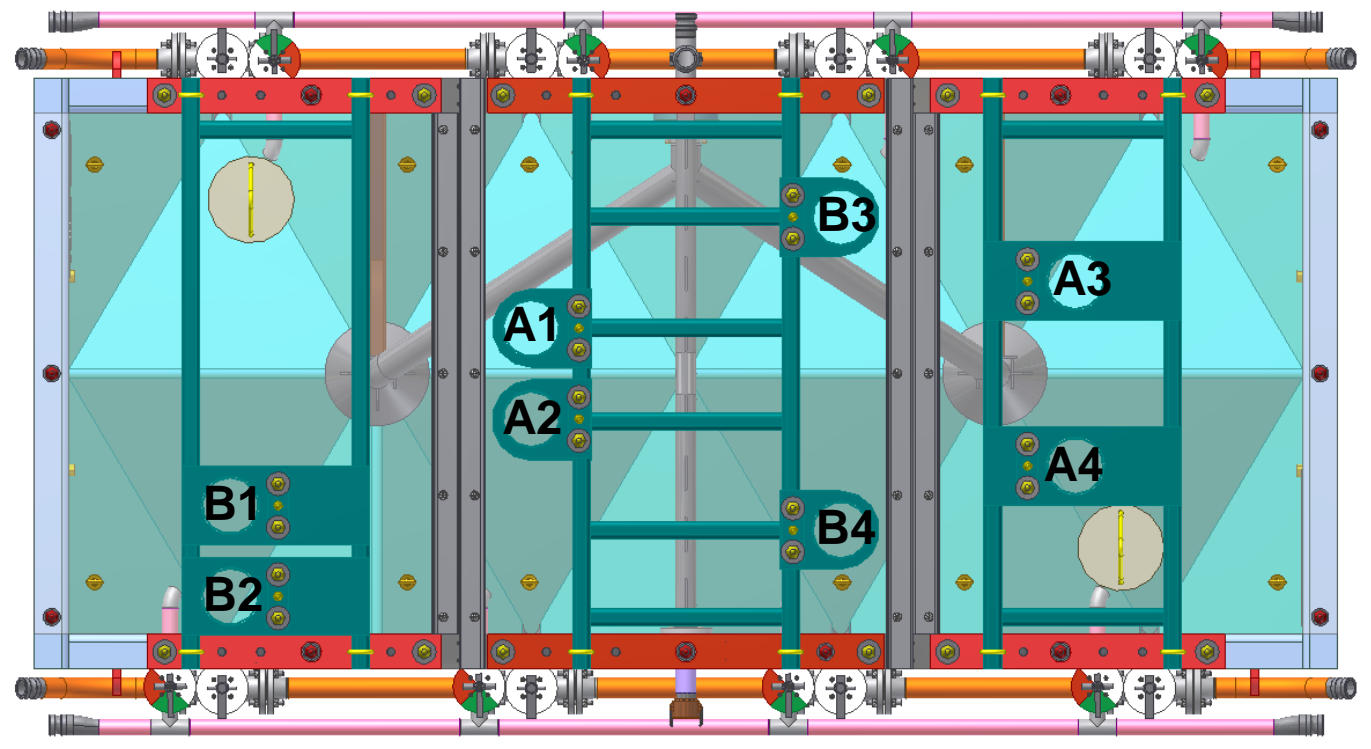

Potential Core Sample Locations

A = Near Distribution Heads

B = Away from Distribution Heads

Figure 1.5. Sample Locations in Engineered Containers (top view looking down)

A design feature that is not apparent in Figure 1.5 is the clear polycarbonate cover bolted over the open top of the container to help control sludge overflow during filling and prevent the introduction of foreign material (dropped from above). K Basin operations require that these covers stay in place for process control of this material. Therefore, for sampling of the sludge, a series of holes were drilled through the polycarbonate covers. This drilling potentially introduced a small quantity of polycarbonate turnings to the sludge.

The sample-collection apparatus consists of an isolation tube (1 or 2 in. diameter) that is inserted through a hole in the top cover of the engineered container all the way down through the sludge to the bottom of the container (Baker et al. 2009, Section 3.1). For the sampling of SCS-CON-210, only 1-in. diameter isolation tubes were used. The sample system design incorporates a feature to provide a good seal between the container egg-crate bottom surface (Figure 1.4) and the end of the isolation tube. For sample collection, an extraction tube is slid inside the isolation tube, and sludge is suctioned 20 to 25 vertical feet into one of several sample polybottles configured in series. The sample polybottles are staged in a shielded cart located on the deck above the basin. Three heavy-walled 4-L sample polybottles are in the cart at one time (however, the third bottle is used only as a safety backup bottle and is not normally filled).

After samples were collected at the KW Basin into the 4-L sample polybottles, they were loaded into SSCs, which were then packaged into one or two SWBs. Each shipment consisted of two to four sample polybottles. Sample shipment was conducted in accordance with the shipping safety document (i.e., DOE 2009).

A separate data package documenting the sample collection and shipment activities conducted at the KW Basin has been prepared (Thompson and Ross 2011). 


\subsection{Sample Receipt and Processing}

Four top-to-bottom core samples were collected from November to December 2010 from Engineered Container SCS-CON-210 located in the KW Basin. Each core sample was packaged in multiple 4-L polypropylene bottles (polybottles) and shipped in heavily shielded sample containers (i.e., lead-lined "Super Pigs") within a series of SWBs to the HLRF located at PNNL's RPL. Samples were received under COC (COC copies are available in Appendix A). Table 2.1 provides a summary of the polybottle ID, COC number, sampling date at the K Basin, sample receipt date at the HLRF, opportunistically measured gamma dose rates, and nominal measured volumes. Note that samples with the prefix "KW210" represent core samples from Engineered Container SCS-CON-210. The second alphanumeric designation (i.e., A1, A4, B1, and B3) represents the core ID (as shown in Figure 1.5). The final letter (e.g., -A, -B, -C) designates the sequential polybottle container of the core sample material. A single core sample was collected in four to six polybottles.

The gamma dose rates listed in Table 2.1 were opportunistically determined from each sludge sample container. Measurements were collected from the bottom of the container during the transfer process into the HLRF using a handheld extension dosimeter (Telescan XEXTI-0017) or standard dosimeter (Eberline RO-20). Every sample polybottle was enclosed in a plastic shipping bag. Because the sludge had settled to the container bottom, each dose rate should represent the highest rate for the sample.

The core sample volumes provided in Table 2.1 were determined following sludge consolidation processing into safety-coated, volume-graduated, 960-mL Qorpak jars; re-agitation, and both a 24-hr and $>112$-hr settling period. Each sample volume was correlated to a height determined from a decal ruler affixed to the jar. The sludge height uncertainty was estimated to be $\pm 0.2 \mathrm{~cm}$, corresponding to a volume uncertainty of $\pm 12 \mathrm{~mL}$ (specific to the 960 -mL Qorpak jar). ${ }^{\text {(a) }}$

Sludge from individual cores was reconstituted, subsampled, and processed in accordance with directions given in detailed TIs. Sludge was subsampled from each reconstituted core sample to be used for preparation of two container composites. The container composite sludge processing and subsampling was also detailed in a TI. Before implementation, each TI underwent extensive internal reviews and was then submitted for review and approval by the BTR. Table 2.2 provides a cross-reference of core sample and container composite sample to TI identification.

Figure 2.1 depicts the project scope and its correlation to TIs. Sludge characterization results from other engineered containers have been previously determined and reported (Fiskum et al. 2011a, b, Shimskey et al. 2011) and the associated TIs are shaded in gray. The SCS-CON-210 sludge characterization TIs (current scope discussed herein) are not shaded. Completed TI packages are provided to the BTR as Portable Document Format (PDF) documents in digital format. ${ }^{\text {(b) }}$ A TI package includes the completed and reviewed TI, reviewed calculation spreadsheets (inclusive of tabulated TI data, data calculations supporting the physical property testing, and opportunistic settling rate observations), photograph contact sheets, measuring and test equipment (MT\&E) identification and calibration records, key communications between the project and BTR, a surveillance performed by the PNNL project Quality Engineer (QE), and occurrence reports (as applicable). The QE reviewed the TIs

(a) Although the linear dimension uncertainty of $\pm 0.2 \mathrm{~cm}$ was constant, the volume uncertainty changes with changing jar shapes.

(b) DVD-52578-PNNL-20650 Rev. 2, Section III, September 2013. 
Table 2.1. Samples Received from Engineered Containers SCS-CON-210

\begin{tabular}{|c|c|c|c|c|c|c|c|c|}
\hline \multirow[b]{2}{*}{ Client Sample ID } & \multirow[b]{2}{*}{$\begin{array}{l}\text { COC } \\
\text { No. }\end{array}$} & \multirow[b]{2}{*}{$\begin{array}{l}\text { Sample } \\
\text { Date }\end{array}$} & \multirow[b]{2}{*}{$\begin{array}{l}\text { Receipt } \\
\text { Date }\end{array}$} & \multicolumn{2}{|c|}{ Dose Rate, R/h } & \multicolumn{3}{|c|}{$\begin{array}{l}\text { Reconstituted Sample } \\
\text { Volume, } \mathrm{mL}^{(\mathrm{c})}\end{array}$} \\
\hline & & & & (a) & (b) & $\begin{array}{l}24 \mathrm{hr} \\
\text { Settled }\end{array}$ & $\begin{array}{l}>112 \mathrm{hr} \\
\text { Settled }\end{array}$ & $\begin{array}{c}\text { As- } \\
\text { Sampled }\end{array}$ \\
\hline KW210-A1-A ${ }^{(\mathrm{d})}$ & 6695 & $12 / 29 / 10$ & $1 / 4 / 11$ & 3 & 2 & \multirow{4}{*}{$\begin{array}{c}224 \\
(23 \mathrm{hr})\end{array}$} & \multirow{4}{*}{$\begin{array}{c}212 \\
(136 \mathrm{hr})\end{array}$} & \multirow[t]{4}{*}{168} \\
\hline KW210-A1-B & 6695 & $12 / 29 / 10$ & $1 / 4 / 11$ & - & 0.45 & & & \\
\hline KW210-A1-C & 6697 & $12 / 30 / 10$ & $1 / 4 / 11$ & - & 0.045 & & & \\
\hline KW210-A1-D & 6697 & $12 / 30 / 10$ & $1 / 4 / 11$ & - & 0.015 & & & \\
\hline KW210-A4-A (d) & 6662 & $11 / 17 / 10$ & $11 / 18 / 10$ & 2 & - & \multirow{6}{*}{$\begin{array}{c}199 \\
(23 \mathrm{hr})\end{array}$} & \multirow{6}{*}{$\begin{array}{c}193 \\
(281 \mathrm{hr})\end{array}$} & \multirow[t]{6}{*}{174} \\
\hline KW210-A4-B & 6662 & $11 / 17 / 10$ & $11 / 18 / 10$ & 0.35 & - & & & \\
\hline KW210-A4-C & 6664 & $11 / 18 / 10$ & $12 / 2 / 10$ & 4 & - & & & \\
\hline KW210-A4-D & 6664 & $11 / 18 / 10$ & $12 / 2 / 10$ & 0.3 & - & & & \\
\hline KW210-A4-E & 6668 & $11 / 19 / 10$ & $12 / 6 / 10$ & 0.06 & - & & & \\
\hline KW210-A4-F & 6668 & $11 / 19 / 10$ & $12 / 6 / 10$ & 0.008 & - & & & \\
\hline KW210-B1-A ${ }^{(\mathrm{d})}$ & 6674 & $12 / 1 / 10$ & $12 / 8 / 10$ & - & 0.12 & \multirow{6}{*}{$\begin{array}{c}616 \\
(24.5 \mathrm{hr})\end{array}$} & \multirow{6}{*}{$\begin{array}{c}560 \\
(113 \mathrm{hr})\end{array}$} & \multirow[t]{6}{*}{523} \\
\hline KW210-B1-B & 6674 & $12 / 1 / 10$ & $12 / 8 / 10$ & - & 0.25 & & & \\
\hline KW210-B1-C & 6678 & $12 / 7 / 10$ & $12 / 14 / 10$ & - & 0.27 & & & \\
\hline KW210-B1-D(e) & 6678 & $12 / 7 / 10$ & $12 / 14 / 10$ & - & 0.07 & & & \\
\hline KW210-B1-F & 6682 & $12 / 9 / 10$ & $12 / 16 / 10$ & - & 0.22 & & & \\
\hline KW210-B1-G & 6682 & $12 / 9 / 10$ & $12 / 16 / 10$ & - & 0.08 & & & \\
\hline KW210-B3-A (d) & 6686 & $12 / 15 / 10$ & $12 / 27 / 10$ & 1 & - & \multirow{6}{*}{$\begin{array}{c}585 \\
(23 \mathrm{hr})\end{array}$} & \multirow{6}{*}{$\begin{array}{c}554 \\
(136 \mathrm{hr})\end{array}$} & \multirow[t]{6}{*}{485} \\
\hline KW210-B3-B & 6686 & $12 / 15 / 10$ & $12 / 27 / 10$ & 0.5 & - & & & \\
\hline KW210-B3-C & 6689 & $12 / 17 / 10$ & $12 / 27 / 10$ & 0.8 & - & & & \\
\hline KW210-B3-D ${ }^{(f)}$ & 6689 & $12 / 17 / 10$ & $12 / 27 / 10$ & 0.3 & - & & & \\
\hline KW210-B3-E & 6692 & $12 / 27 / 10$ & $12 / 29 / 10$ & 0.7 & - & & & \\
\hline KW210-B3-F & 6692 & $12 / 27 / 10$ & $12 / 29 / 10$ & 0.045 & - & & & \\
\hline
\end{tabular}

(a)/(b) Dose rates were taken at contact and uncorrected from the bottom of the sample container while it was still encased in the plastic (shipping) bag. The dosimeter was a Telescan XEXTI-0017 (a) or the Eberline RO-20 (b). Historically, the Telescan XEXTI-0017 has provided slightly higer dose rates than the Eberline RO-20. This is likely due to the different radiation sensing area geometry. The Telescan has a cylindrical, 360 degree, sensing geometry and the Eberline has a flat, one-directional sensing area.

(c) The volumes are accurate to only two significant figures; the third significant figure is shown for information only. The settled volume represents the volume of the core sample after re-agitation and settling for $\sim 24 \mathrm{hr}$. The $>112 \mathrm{hr}$ settling volume represents the core composites after a second re-agitation and settling for $112 \mathrm{hr}$ or more. The as-sampled volume represents the core sample volume after excess water was removed and and sludge was mixed prior to subsampling.

(d) One subsample collected from each reconstituted core sample was inadvertently given the client sample ID. The client sample IDs (e.g., KW210-A1-A, KW210-A4-A) designated in this table are different samples from the samples identically identified in Tables 2.3, 2.4, 2.5 and 2.6.

(e) Polybottle ID KW210-B1-E was not provided to PNNL because of a sampling equipment problem at KW Basin. A sample bottle was initially assigned this identification but found to contain no significant sludge material, hence it was not shipped from KW Basin to PNNL (Thompson and Ross 2011).

(f) Polybottle ID KW210-B3-D contained a flow deflector tube when received into HLRF (Thompson and Ross 2011). 
Table 2.2. Cross-Reference of Core Sample ID to Test Instruction ID and Title

\begin{tabular}{lcl}
\hline Core ID & TI ID & \multicolumn{1}{c}{ TI Title } \\
\hline KW210-A1 & 52578-TI001 & $\begin{array}{l}\text { K West Basin Sample Receipt and Preparation of Consolidated Core } \\
\text { Composites: SCS-CON-210-A1 and SCS-CON-210-B3 }\end{array}$ \\
\hline KW210-B3 & & $\begin{array}{l}\text { K West Basin Sample Receipt and Preparation of Consolidated Core } \\
\text { Composites: SCS-CON-210-A4 and SCS-CON-210-B1 }\end{array}$ \\
KW210-A4 & 52578-TI002 & $\begin{array}{l}\text { Preparation of K West Basin Consolidated Container Composite for } \\
\text { SCS-CON-210 }\end{array}$ \\
\hline $\begin{array}{l}\text { Container } \\
\text { Composites }\end{array}$ & 52578-TI008 & \\
KW210-CC-A \& & & \\
\hline
\end{tabular}

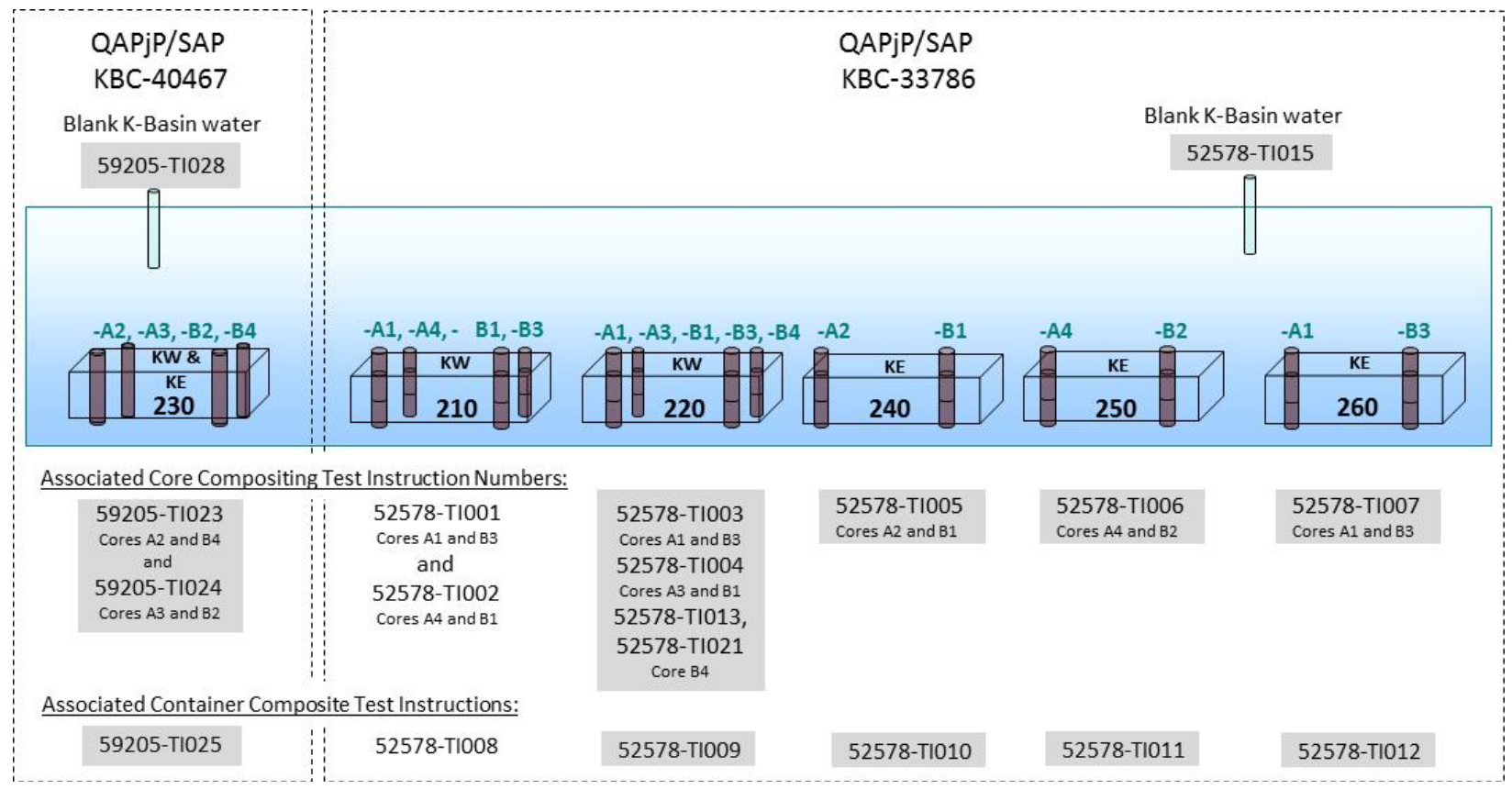

Note: The K Basin indicators (i.e., KW and KE) refer to the source of the sludge in the containers. The three-digit numbers (e.g., "210") are abbreviations of the full engineered container designations (e.g., SCS-CON-210). The gray shading indicates scope that was previously completed and reported. The focus of this report is the SCS-CON210 container material.

Figure 2.1. Depiction of K Basin Sludge Core and Equipment Blank Sampling Effort with Identification of Scope Associated with Specific Test Instructions

to evaluate completeness, that all entries were recorded properly, the errors were corrected appropriately and when needed, that justification was present to describe data corrections, that any issues affecting data quality were documented as Occurrence Reports, and that the attachments to the TI were at the stage to become a project record. Additionally, TIs were reviewed to assure that technical reviews were performed, issues were dispostioned, and that data were traceable to point of use, generally Excel spreadsheets, which also required technical review and comment/issue resolution. 


\subsection{Core Reconstitution and Subsampling Overview}

Samples were transferred into the HLRF and allowed to settle for at least one day in the as-received 4-L polybottle. Sludge height measurements for the polybottles were collected; however, for most cases the sludge volumes were so low that meaningful volume measures could not be determined from the settled sludge height (see DVD-52578-PNNL-20650 Rev. 2, Section III, September 2013). Most of the clarified supernatant water (or liquid) was removed by vacuum-assisted siphoning. Figure 2.2 shows a schematic of the fluid removal system. Components (i.e., wand and rods) in the system were made of stainless steel, and Tygon ${ }^{\circledR}$ pressure tubing. Some water $(\sim 200 \mathrm{~mL})$ was left with the solids. This concentrated sludge slurry was agitated, suspending solids in the remaining solution, and then poured into a 960-mL, wide-mouth, safety-coated, glass jar. A stainless steel canning jar funnel equipped for stability (welded pipe) and manipulator use (ring clamp) was positioned over the compositing jar to aid quantitative sludge transfer (see Figure 2.3). Figure 2.4 shows a flow diagram of the overall core reconsitituion process and targeted data collection.

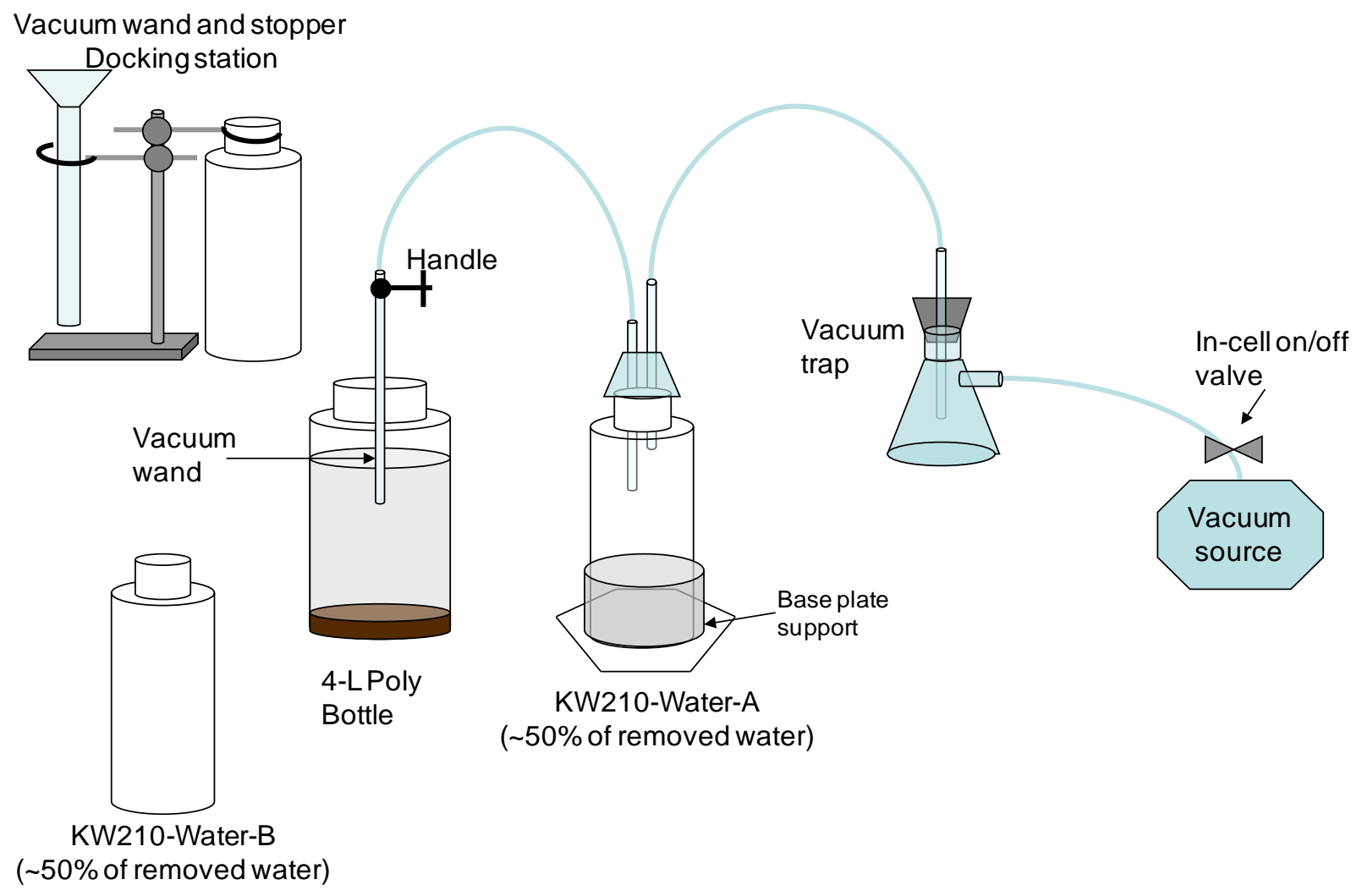

Figure 2.2. System Schematic for Fluid and Sludge Removal 


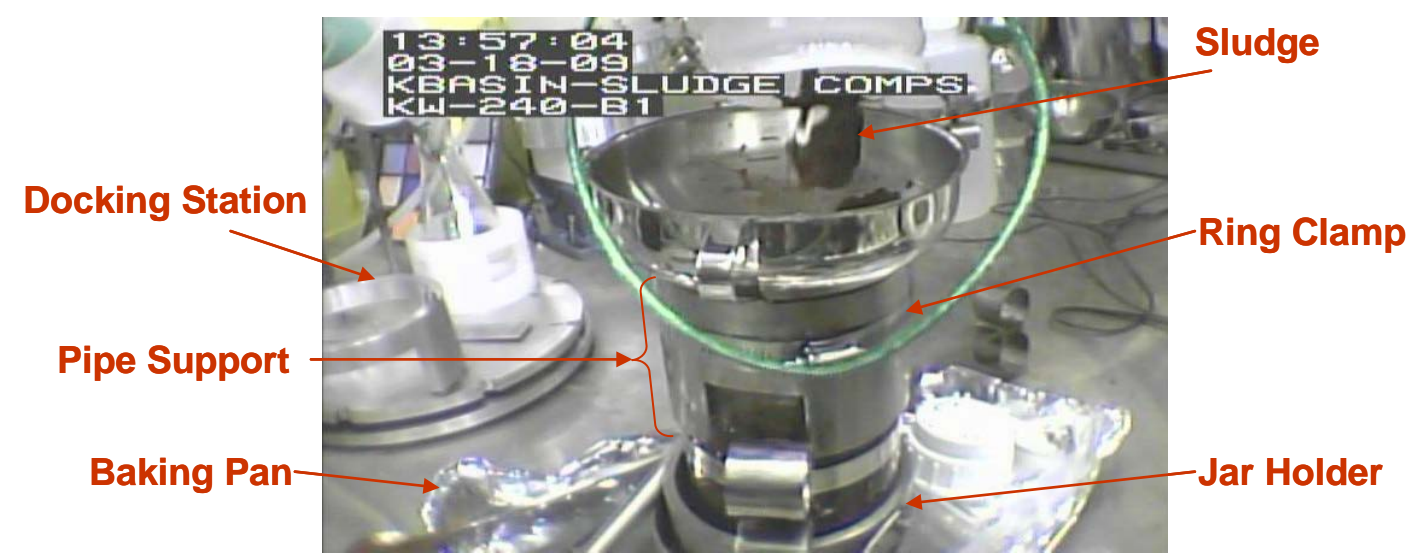

Notes:

- The pipe welded to the stainless steel funnel is provided for funnel stability in the bottle. The ring clamp on the pipe supported manipulator finger rings (opposite side in image).

- $\quad$ The jar was set into a special holder to minimize chances for tipping.

- An aluminum baking pan was used to provide a clean work surface for handling equipment.

- Part of the docking station is visible at the left.

Figure 2.3. Prototypic Image of Sludge Core Reconstitution Step into the 960-mL Jar with Modified Funnel

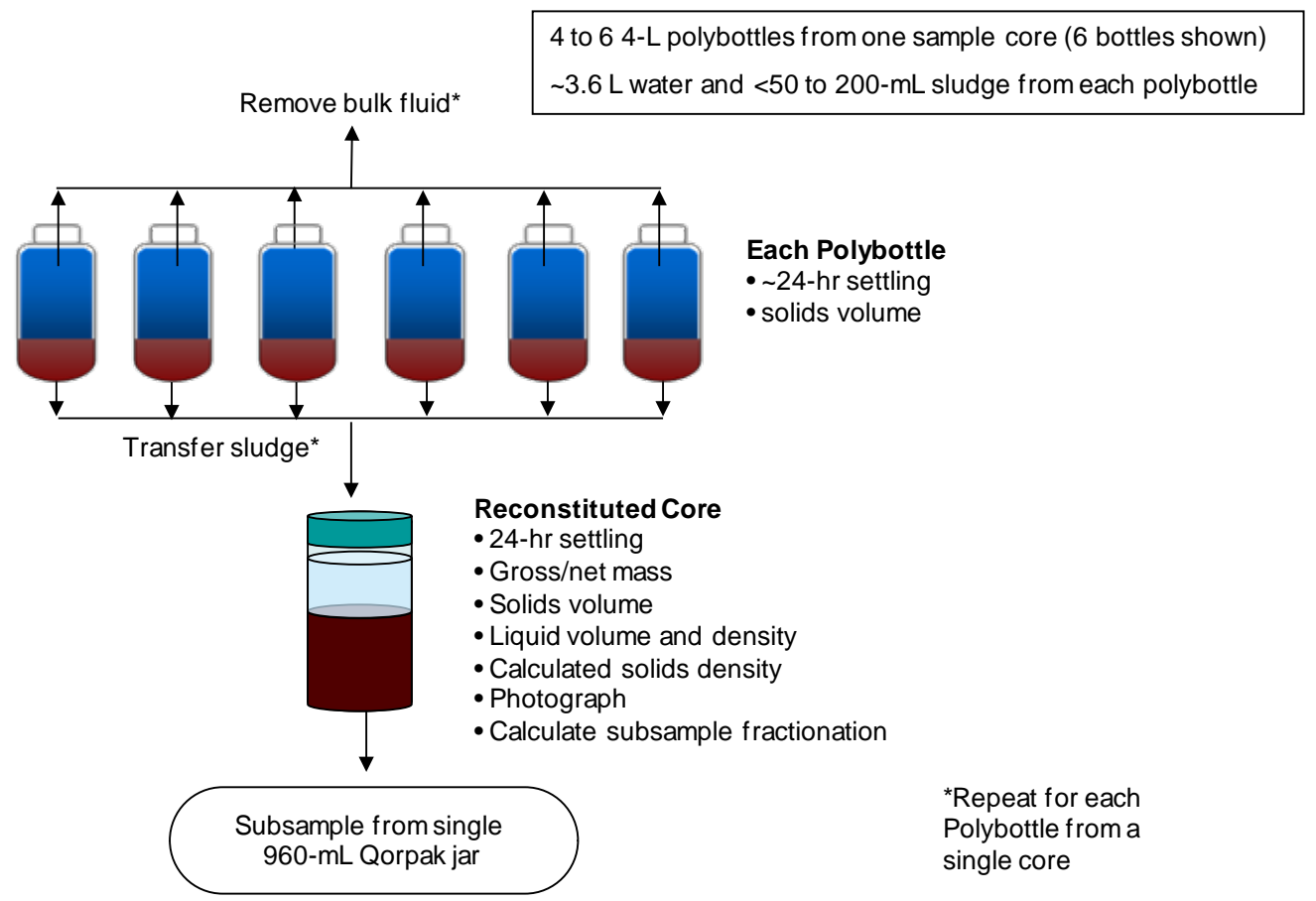

Figure 2.4. Schematic of a Core Sample Reconstitution

$\mathrm{K}$ Basin water was added to the residual solids in the polybottle for entrainment and removal by vacuum transfer. The entrained residual solids were collected in a separate glass bottle with a tapered neck; this design feature allowed for efficient solids transfer after collection and settling. The process was repeated until all solids were effectively removed from the 4-L polybottle (i.e., effectively, a quantitative transfer, as verified by visual inspection using an in-cell camera). Examples of full and emptied polybottles depicting the typically achieved quantitative transfers are shown in Figure 2.5. 


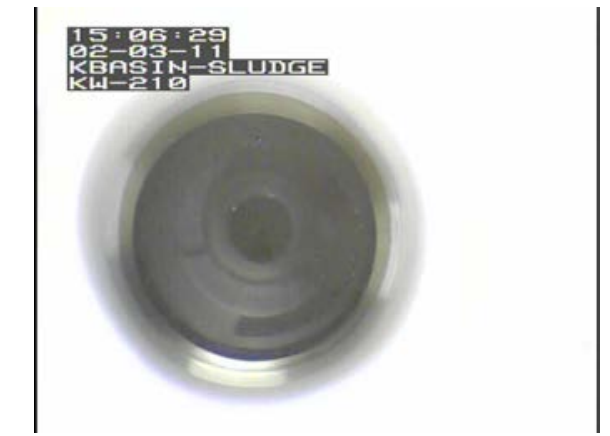

Bottle KW210-A1-B Prior to Bulk Transfer

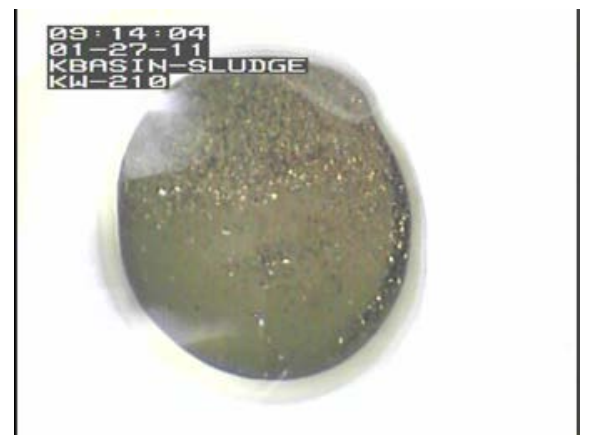

Bottle KW210-A4-C Prior to Bulk Transfer

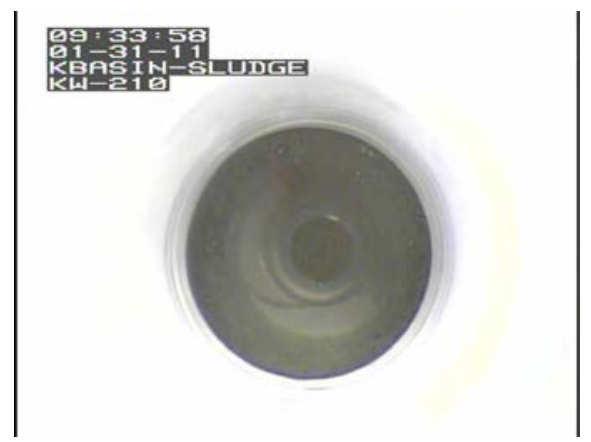

Bottle KW210-B1-C Prior to Bulk Transfer

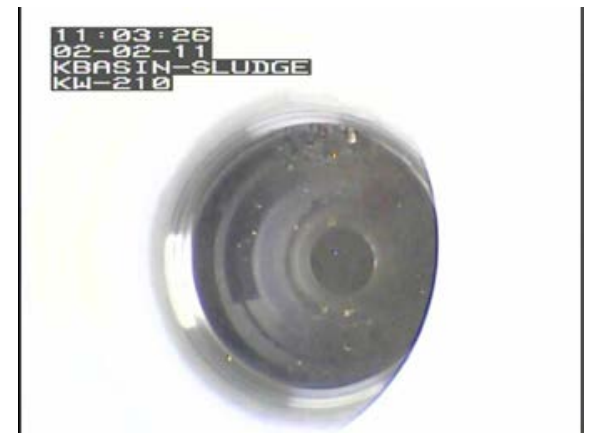

Bottle KW210-B3-C Prior to Bulk Transfer

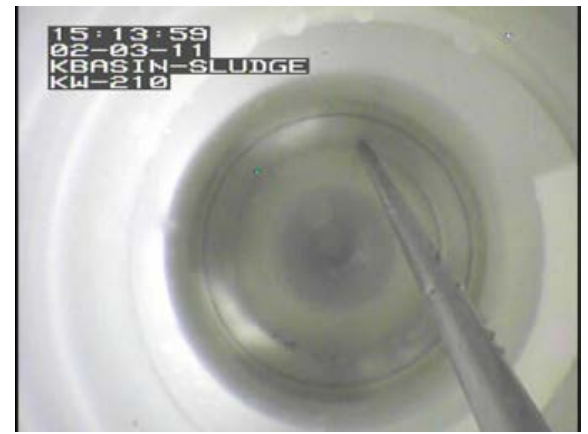

Bottle KW210-A1-B After Final Rinse

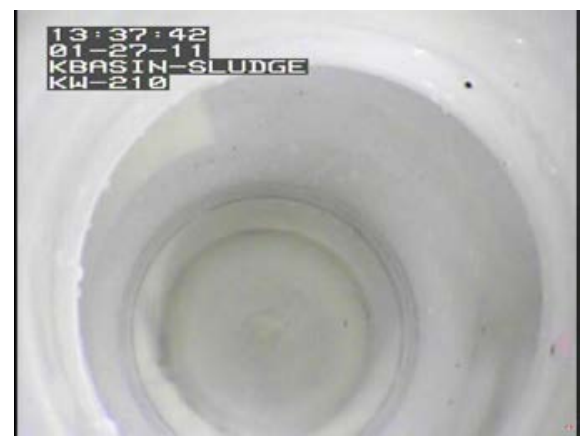

Bottle KW210-A4-C After Final Rinse

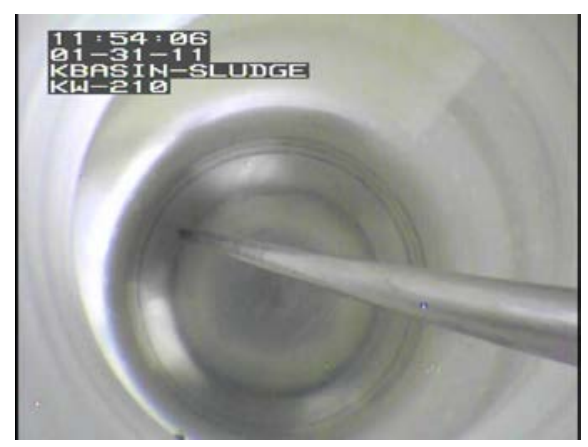

Bottle KW210-B1-C After Final Rinse

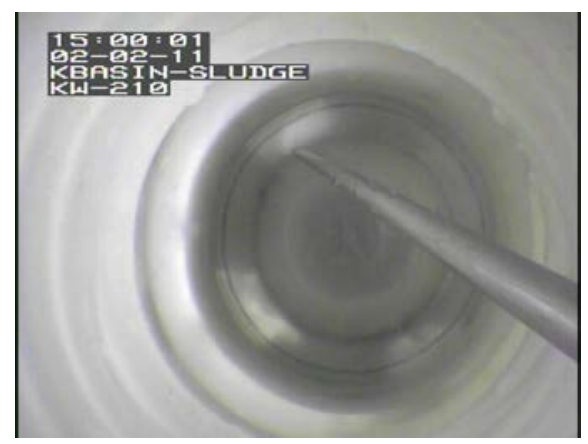

Bottle KW210-B3-C After Final Rinse

Note: Images are looking down into the polybottle with in-cell camera.

Figure 2.5. Examples of Polybottles with Starting Concentrated Slurry and Emptied Polybottles Following Sludge Removal 
The sludge removal and collection process was repeated for each core sample component, combining the sludge into a single jar and thus reconstituting the core sample (originally distributed across four to six 4-L polybottles) into a single jar (Figure 2.4). In all cases, the core sample volume was sufficiently small that the entire core sample could be reconstituted into a single 960-mL Qorpak jar and still allow sufficient headspace for efficient mixing. Once the core sample was reconstituted into the jar, the sludge was suspended by swirling and turning the jar. Then, the sludge was allowed to settle for $\sim 24 \mathrm{hr}$. The $24-$ hr settled sludge volume was determined based on the sludge height. Opportunistic characterization data and photographs were collected on each core sample: settling behavior during the 24-hr settling period, the gross mass, total and settled sludge volumes, and fluid head volume were determined. The settled sludge mass and density were calculated from these parameters.

The reconstituted sludge samples were thickened before final mixing and subsampling by removing the fluid head from the sludge, mixing the wet sludge with a stainless steel spoon, allowing the sludge to settle, and removing the fluid head again (Figure 2.6). The process was repeated until the sludge appeared to have sufficient strength to suspend dense particles. Final dewatering was conducted by carefully tipping the sample jar to create a pool of fluid with enough depth to be retrieved with a transfer pipet. To accomplish this, an angle-vise, fitted with a custom-made mounting block, was used to hold the 960-mL jars at a given angle (Figure 2.7). ${ }^{\text {(a) }}$ A transfer pipet was then used to retrieve the pooled water above the sludge.

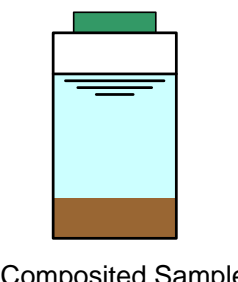

Composited Sample

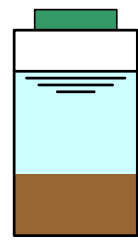

Settled Sample
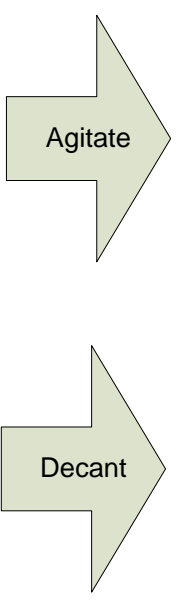
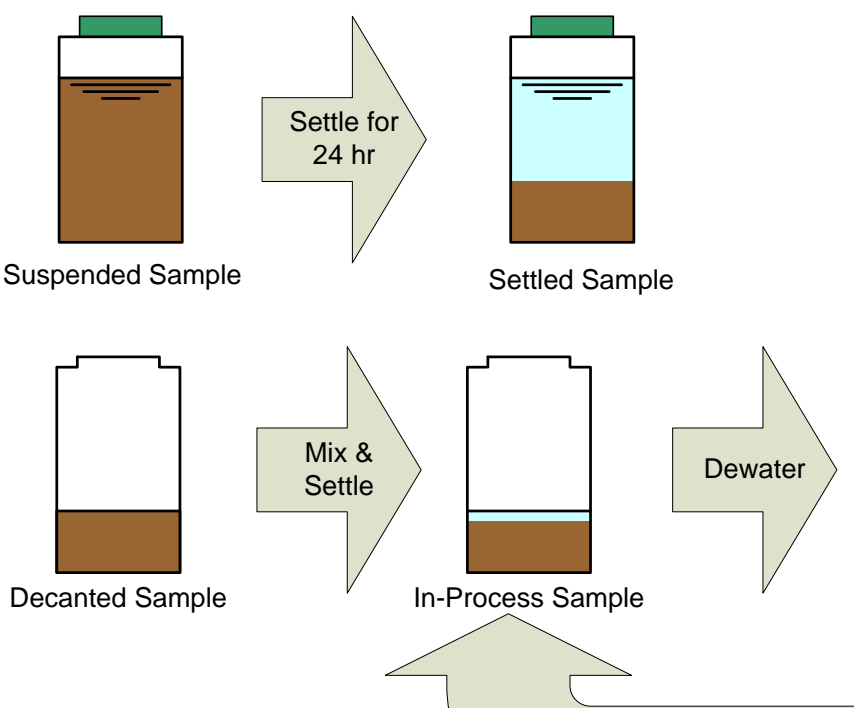

Dewatered Sample

Figure 2.6. Sample Preparation for Subsampling

(a) The angle-vise was also used to hold the polybottle still for uncapping and for initial gross water removal. 


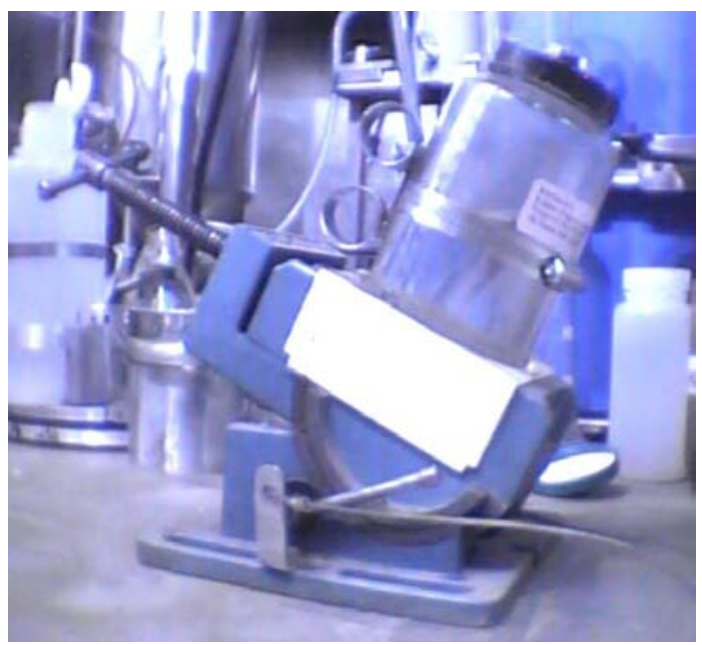

Vise is holding the compositing jar at angle for continued sludge dewatering activity.

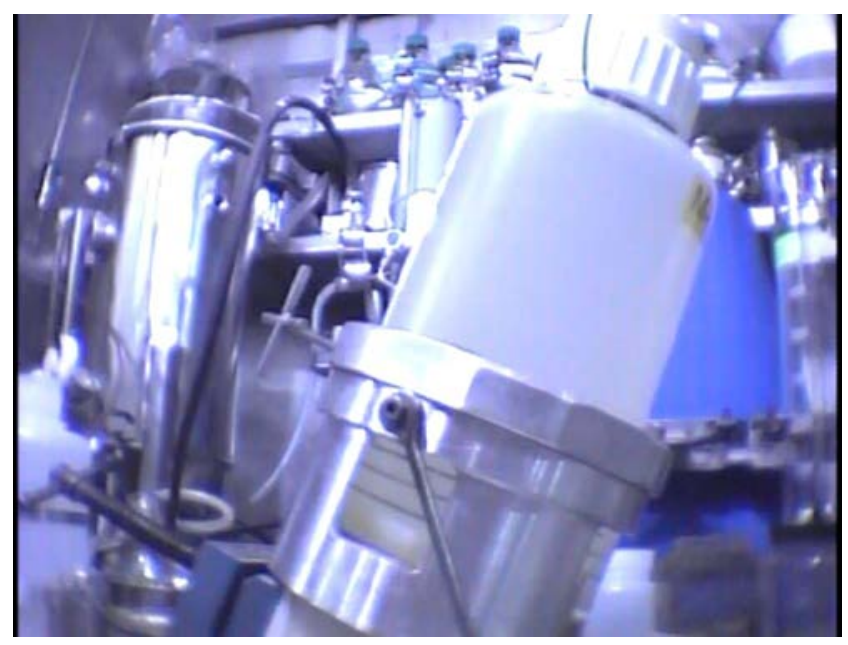

Vise holding the 4-L polybottle still for uncapping and water removal.

Figure 2.7. Angle-Vise in Prototypic Uses

The sludge was thickened to provide more uniform sludge subsamples that would be less prone to particle segregation based on particle size or density. After achieving a thickened state, and before subsampling, the sludge was homogenized by mixing with a stainless steel spoon for 5 min. For subsampling, all subsample containers were staged, and aliquots of mixed/thickened sludge were transferred to the containers. Each subsampling operation was conducted in a single uninterrupted session at the hot cell window to minimize differences in the wt\% solids being transferred into each subsample container.

Multiple subsamples were pulled from the mixed and thickened sludge to satisfy a multiplicity of analytical objectives (Figure 2.8). Additional characterization samples were collected in case of disagreement between the primary sample and duplicate, observed analytical problems, or required reanalysis. Test instructions 52578-TI001 and 52578-TI002 defined a specific subsampling strategy that required at least $140 \mathrm{~mL}$ of sludge be available per reconstituted core sample (as-sampled basis). The majority of this volume $(80 \mathrm{~mL})$ was to create a container composite. However the volumes of the "A" core sludge samples (i.e., A1 and A4) were about half of the anticipated volumes and a large population of particles were observed that would likely not pass through the $500-\mu \mathrm{m}$ pore size sieve. Reducing the volume of $<500-\mu \mathrm{m}$ (referred to as “ $-500-\mu \mathrm{m}$ ”) sieve fraction material would likely confound the ability to collect shear strength and flow curve measurements on this fraction (a situation previously observed on the SCS-CON-220 sample [Fiskum et al. 2011b] which precluded the ability to obtain reliable rheological data). Therefore the approach to the container composite creation and testing was modified with the BTR's concurrence. Two samples (30 mL and $40 \mathrm{~mL}$ ) were collected, instead of one $80-\mathrm{mL}$ sample, from each core sample to create two independent container composites. One container composite was destined to support the analytical characterization and the other was to support the rheological characterization (shear strength only, the flow curve analysis was cancelled per direction/concurrance of the BTR). The container composite created specifically for shear strength testing was assured to be of sufficient volume to support the test. Engineered Container SCS-CON-210 composite testing is described in Section 2.2. The original subsampling strategy was also modified to eliminate one contingency physical property subsample from each core sample. 
PNNL-20650, Rev. 2

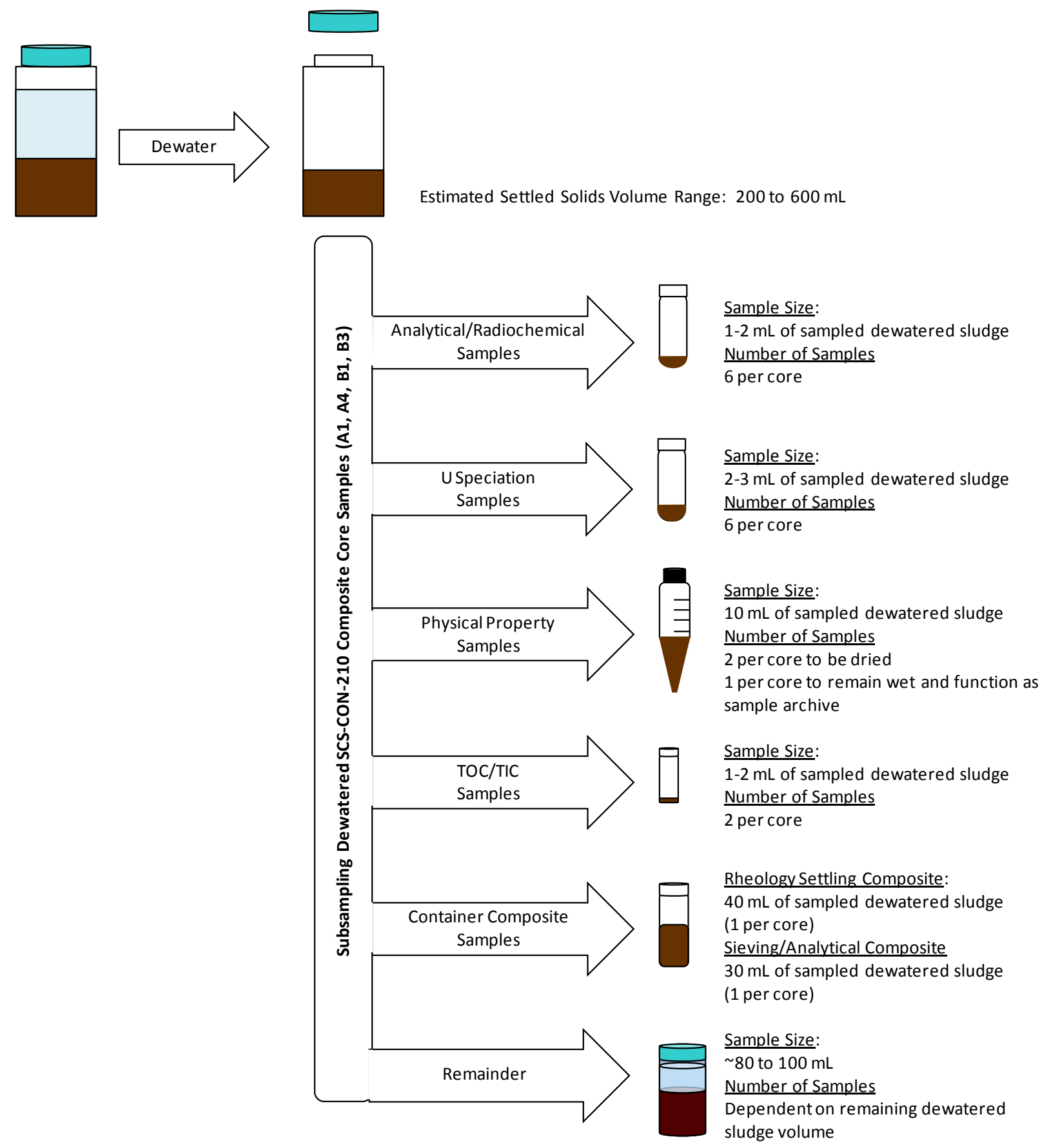

Note: Subsamples collected to create the container composite were adjusted to $30 \mathrm{~mL}$ and $40 \mathrm{~mL}$ from each core sample. This was done to allow creation of an independent composite for rheology testing, removing the strictures of obtaining enough -500- $\mu$ m sieve size material to support rheology as well as characterization.

Figure 2.8. Overall Processing and Subsampling Scheme for the SCS-CON-210 Core Samples

The "B" core sludge samples (i.e., B1 and B3) had more than sufficient volume to support all testing. The remaining " $\mathrm{B}$ ” core sludge was distributed among three to four 120-mL Qorpak jars in readiness for long term storage tests, as directed by the BTR. The "A" core sludge samples (i.e., A1 and A4) were essentially consumed from subsampling. 
Cross-references between core sample IDs, subsample IDs (from TIs), as-sampled (wet) mass, ASO sample IDs, and ASR IDs are provided in Table 2.3 through Table 2.6. These IDs will be referenced throughout this report. Note that the subsample collection date is provided in the table annotations.

Table 2.3. Cross-Reference of SCS-CON-210 A1 Core Sample (KW210-A1) to TI Subsample ID, Subsample Mass, RPL/ASO ID, ASR ID, and General Analysis Scope

\begin{tabular}{lcccl}
\hline & $\begin{array}{c}\text { As-Sampled } \\
\text { Mass (g) }\end{array}$ & RPL/ASO ID & ASR & \multicolumn{1}{c}{ Analysis } \\
\hline Kubsample ID & 245.83 & --- & --- & Parent core sample \\
KW210-A1-1 & 62.61 & --- & --- & Container composite \\
TI001-A1-B & 2.05 & $11-0419-129-S$ & 8798 & Chemical analysis \\
TI001-A1-C & 2.28 & $11-0419-129-D$ & 8798 & Chemical analysis duplicate \\
TI001-A1-D & 2.49 & --- & --- & Chemical analysis reserve \\
TI001-A1-E & 2.08 & --- & --- & Chemical analysis reserve \\
TI001-A1-F & 2.27 & --- & --- & Chemical analysis reserve \\
TI001-A1-G & 2.50 & --- & --- & Chemical analysis reserve \\
TI001-A1-H & 1.3503 & $11-0419-C-S$ & 8798 & TOC/TIC \\
TI001-A1-J & 1.0210 & $11-0419-C-D$ & 8798 & TOC/TIC duplicate \\
TI001-A1-K & 14.74 & --- & --- & Primary physical properties \\
TI001-A1-L & 18.50 & --- & --- & Primary physical properties \\
TI001-A1-M & 20.07 & --- & --- & Analytical archive; supplemental \\
& & & & physical properties \\
TI001-A1-N & 44.62 & --- & --- & Container composite \\
TI001-A1-P & 3.89 & $11-0419-107-S$ & 8798 & Uranium speciation \\
TI001-A1-Q & 4.55 & $11-0419-107-D$ & 8798 & Uranium speciation duplicate \\
TI001-A1-R & 4.09 & --- & --- & Uranium speciation reserve \\
TI001-A1-S & 4.79 & --- & --- & Uranium speciation reserve \\
TI001-A1-T & 4.14 & --- & --- & Uranium speciation reserve \\
TI001-A1-U & 4.79 & --- & --- & Uranium speciation reserve \\
KW210-A1-Archive-A & 16.76 & --- & --- & Archive sample \\
KW210-A1-Archive-B & NM & --- & Archive sample \\
KW210-A1-Archive-C & NM & --- & -- & Archive sample \\
\hline
\end{tabular}

(a) Subsampling date: February 22, 2011.

(b) Inadvertently one sample was given an ID identical to a client sample ID. The sample designated "KW210-A1-A" here is a different sample from the sample identically identified in Table 2.1.

(c) Two subsamples from each reconstituted core sample were collected to create two separate container composites according to TI 52578-TI008. Before compositing, the physical properties and settling behavior of these samples were evaluated.

(d) Samples were obtained through composite jar washes and as-sampled masses were not measured.

NM: Not Measured.

The net sample masses were calculated from data collected in TI 52578-TI001.

Gray shading indicates samples that were collected but not used. 
Table 2.4. Cross-Reference of SCS-CON-210 A4 Core Sample (KW210-A4) to TI Subsample ID, Subsample Mass, RPL/ASO ID, ASR ID, and General Analysis Scope

\begin{tabular}{lcccl}
\hline Subsample ID & $\begin{array}{c}\text { As-Sampled } \\
\text { Mass (g) }\end{array}$ & RPL/ASO ID & ASR & \multicolumn{1}{c}{ Analysis } \\
\hline KW210-A4-1 & 281.64 & --- & --- & Parent core sample \\
KW210-A4-A & (b) & --- & --- & Container composite ${ }^{(\mathrm{c})}$ \\
TI002-A4-B & 24.51 & $11-0420-129-S$ & 8798 & Chemical analysis \\
TI002-A4-C & 2.30 & $11-0420-129-D$ & 8798 & Chemical analysis duplicate \\
TI002-A4-D & 2.61 & --- & --- & Chemical analysis reserve \\
TI002-A4-E & 2.18 & --- & --- & Chemical analysis reserve \\
TI002-A4-F & 2.55 & --- & --- & Chemical analysis reserve \\
TI002-A4-G & 2.03 & --- & --- & Chemical analysis reserve \\
TI002-A4-H & 1.4289 & $11-0420-C-S$ & 8798 & TOC/TIC \\
TI002-A4-J & 2.0583 & $11-0420-C-D$ & 8798 & TOC/TIC duplicate \\
TI002-A4-K & 14.67 & --- & --- & Primary physical properties \\
TI002-A4-L & 16.76 & --- & --- & Primary physical properties \\
TI002-A4-M & 23.34 & --- & --- & Analytical archive; supplemental \\
& & & & physical properties \\
TI002-A4-N & 49.38 & --- & --- & Container composite \\
TI002-A4-P & 4.80 & $11-0420-107-S$ & 8798 & Uranium speciation \\
TI002-A4-Q & 3.99 & $11-0420-107-D$ & 8798 & Uranium speciation duplicate \\
TI002-A4-R & 4.37 & $11-0967-107-S$ & 8824 & Uranium speciation \\
TI002-A4-S & 4.36 & $11-0967-107-D$ & 8824 & Uranium speciation duplicate \\
TI002-A4-T & 4.30 & --- & --- & Uranium speciation reserve \\
TI002-A4-U & 4.02 & --- & --- & Uranium speciation reserve \\
KW210-A4-Archive-A & 37.22 & --- & --- & Archive sample \\
KW210-A4-Archive-B & NM & --- & --- & Archive sample \\
KW210-A4-Archive-D & (d) & NM & --- & Archive sample \\
\hline
\end{tabular}

(a) Subsampling date: February 21, 2011.

(b) Inadvertently one sample was given an ID identical to a client sample ID. The sample designated "KW210A4-A" here is a different sample from the sample identically identified in Table 2.1.

(c) Two subsamples from each reconstituted core sample were collected to create two separate container composites according to TI 52578-TI008. Before compositing, the physical properties and settling behavior of these samples were evaluated.

(d) KW210-A4-Archive-C was not created.

(e) Samples were obtained through composite jar washes and as-sampled masses were not measured.

NM: Not Measured.

The net sample masses were calculated from data collected in TI 52578-TI002.

Gray shading indicates samples that were collected but not used. 
Table 2.5. Cross-Reference of SCS-CON-210 B1 Core Sample (KW210-B1) to TI Subsample ID, Subsample Mass, RPL/ASO ID, ASR ID, and General Analysis Scope

\begin{tabular}{|c|c|c|c|c|}
\hline Subsample ID & $\begin{array}{l}\text { As-Sampled } \\
\text { Mass (g) }\end{array}$ & RPL/ASO ID & ASR & Analysis \\
\hline KW210-B1-1 & 612.10 & --- & --- & Parent core sample \\
\hline KW210-B1-A ${ }^{(b)}$ & 45.45 & --- & --- & Container composite ${ }^{(\mathrm{c})}$ \\
\hline TI002-B1-B & 1.84 & 11-0421-129-S & 8798 & Chemical analysis \\
\hline TI002-B1-C & 1.98 & 11-0421-129-D & 8798 & Chemical analysis duplicate \\
\hline TI002-B1-D & 2.15 & 11-0421-129-MS & 8798 & Chemical analysis matrix spike \\
\hline TI002-B1-E & 2.00 & --- & --- & Chemical analysis reserve \\
\hline TI002-B1-F & 2.51 & --- & --- & Chemical analysis reserve \\
\hline TI002-B1-G & 2.38 & --- & --- & Chemical analysis reserve \\
\hline TI002-B1-H & 1.7868 & 11-0421-C-S & 8798 & TOC/TIC \\
\hline TI002-B1-J & 2.0942 & 11-0421-C-D & 8798 & TOC/TIC duplicate \\
\hline TI002-B1-K & 14.85 & --- & --- & Primary physical properties \\
\hline TI002-B1-L & 12.51 & --- & --- & Primary physical properties \\
\hline TI002-B1-M & 20.52 & --- & --- & $\begin{array}{l}\text { Analytical archive; supplemental } \\
\text { physical properties }\end{array}$ \\
\hline TI002-B1-N & 37.28 & --- & --- & Container composite $^{(\mathrm{c})}$ \\
\hline TI002-B1-P & 4.18 & 11-0421-107-S & 8798 & Uranium speciation \\
\hline TI002-B1-Q & 4.62 & 11-0421-107-D & 8798 & Uranium speciation duplicate \\
\hline TI002-B1-R & 3.97 & 11-0421-107-MS & 8798 & Uranium (metal-only) matrix spike \\
\hline TI002-B1-S & 4.41 & --- & --- & Uranium speciation reserve \\
\hline TI002-B1-T & 4.15 & --- & --- & Uranium speciation reserve \\
\hline TI002-B1-U & 4.08 & --- & --- & Uranium speciation reserve \\
\hline KW210-B1-Archive-1 ${ }^{(\mathrm{d})}$ & 102.57 & --- & --- & Archive sample \\
\hline KW210-B1-Archive-2 ${ }^{\text {(d) }}$ & 89.69 & --- & --- & Archive sample \\
\hline KW210-B1-Archive-3(d) & 108.75 & --- & --- & Archive sample \\
\hline KW210-B1-Archive-4 $4^{(\mathrm{d})}$ & 104.13 & --- & --- & Archive sample \\
\hline KW210-B1-Archive-5 & $\mathrm{NM}^{(\mathrm{e})}$ & --- & --- & Archive sample \\
\hline KW210-B1-Archive-6 & $\mathrm{NM}^{(\mathrm{e})}$ & --- & --- & Archive sample \\
\hline
\end{tabular}

(a) Subsampling date: February 17, 2011.

(b) Inadvertently one sample was given an ID identical to a client sample ID. The sample designated "KW210-B1-A" here is a different sample from the sample identically identified in Table 2.1.

(c) Two subsamples from each reconstituted core sample were collected to create two separate container composites according to TI 52578-TI008. Before compositing, the physical properties and settling behavior of these samples were evaluated.

(d) Samples were aliquoted into 120-mL Qorpak jars to support the long-term storage testing or as directed by the BTR.

(e) Samples were obtained through composite jar washes and as-sampled masses were not measured.

NM: Not Measured.

The net sample masses were calculated from data collected in TI 52578-TI002.

Gray shading indicates samples that were collected but not used. 
Table 2.6. Cross-Reference of SCS-CON-210 B3 Core Sample (KW210-B3) to TI Subsample ID, Subsample Mass, RPL/ASO ID, ASR ID, and General Analysis Scope

\begin{tabular}{|c|c|c|c|c|}
\hline Subsample ID & $\begin{array}{l}\text { As-Sampled } \\
\text { Mass (g) }\end{array}$ & RPL/ASO ID & ASR & Analysis \\
\hline KW210-B3-1 & 602.56 & --- & --- & Parent core sample \\
\hline KW210-B3-A ${ }^{(b)}$ & 54.24 & --- & --- & Container composite $e^{(c)}$ \\
\hline TI001-B3-B & 2.26 & 11-0422-129-S & 8798 & Chemical analysis \\
\hline TI001-B3-C & 2.24 & 11-0422-129-D & 8798 & Chemical analysis duplicate \\
\hline TI001-B3-D & 2.64 & --- & --- & Chemical analysis reserve \\
\hline TI001-B3-E & 2.09 & --- & --- & Chemical analysis reserve \\
\hline TI001-B3-F & 2.23 & --- & --- & Chemical analysis reserve \\
\hline TI001-B3-G & 2.48 & --- & --- & Chemical analysis reserve \\
\hline TI001-B3-H & 1.8483 & 11-0422-C-S & 8798 & TOC/TIC \\
\hline TI001-B3-J & 1.5905 & 11-0422-C-D & 8798 & TOC/TIC duplicate \\
\hline TI001-B3-K & 12.58 & --- & --- & Primary physical properties \\
\hline TI001-B3-L & 12.97 & --- & --- & Primary physical properties \\
\hline TI001-B3-M & 20.57 & --- & --- & $\begin{array}{l}\text { Analytical archive; supplemental } \\
\text { physical properties }\end{array}$ \\
\hline TI001-B3-N & 38.27 & --- & --- & Container composite $e^{(\mathrm{c})}$ \\
\hline TI001-B3-P & 4.33 & 11-0422-107-S & 8798 & Uranium speciation \\
\hline TI001-B3-Q & 3.93 & 11-0422-107-D & 8798 & Uranium speciation duplicate \\
\hline TI001-B3-R & 4.23 & --- & --- & Uranium speciation reserve \\
\hline TI001-B3-S & 4.59 & --- & --- & Uranium speciation reserve \\
\hline TI001-B3-T & 4.13 & --- & --- & Uranium speciation reserve \\
\hline TI001-B3-U & 4.40 & --- & --- & Uranium speciation reserve \\
\hline KW210-B3-Archive-1 ${ }^{\text {(d) }}$ & 92.84 & --- & --- & Archive sample \\
\hline KW210-B3-Archive-2 ${ }^{\text {(d) }}$ & 94.11 & -- & --- & Archive sample \\
\hline KW210-B3-Archive-3 ${ }^{(\mathrm{d})}$ & 96.70 & --- & --- & Archive sample \\
\hline KW210-B3-Archive-4 & $\mathrm{NM}^{(\mathrm{e})}$ & --- & --- & Archive sample \\
\hline
\end{tabular}

(a) Subsampling date: February 18, 2011.

(b) Inadvertently one sample was given an ID identical to a client sample ID. The sample designated "KW210B3-A" here is a different sample from the sample identically identified in Table 2.1.

(c) Two subsamples from each reconstituted core sample were collected to create two separate container composites according to TI 52578-TI008. Before compositing, the physical properties and settling behavior of these samples were evaluated.

(d) Samples were aliquoted into 120-mL Qorpak jars to support the long-term storage testing or as directed by the BTR.

(e) Sample was obtained through composite jar washes and as-sampled masses were not measured.

NM: Not Measured.

The net sample masses were calculated from data collected in TI 52578-TI001.

Gray shading indicates samples that were collected but not used. 
Samples processed with the PNL-ALO-129 acid digestion procedure, $\mathrm{HNO}_{3}-\mathrm{HCl}$ Acid Extraction of Solids Using a Dry-Block Heater, for chemical and radioisotope analyses (e.g., TI001-A1-B) were further processed after much of the initial analytical scope was completed. Aliquots of the acid digestate were prepared for $\mathrm{U}$ and $\mathrm{Pu}$ isotopic measurement by TIMS once the total $\mathrm{U}$ and $\mathrm{Pu}$ concentrations were determined. The undissolved solids (UDS) were processed according to TI 52578-TI040 ${ }^{(a)}$ to obtain subsamples of 0.1 to $0.2 \mathrm{~g}$ for fusion and analysis. Additional aliquots of the UDS were processed according to TI 52578-TI040 to remove the bulk of the resin beads and subsequently the bead and nonbead fractions were weighed. One sample from each core that was depleted in resin beads was analyzed for crystalline structures by XRD. In all cases, the "daughter" samples were assigned modified IDs to delineate them from the parent samples. Table 2.7 cross-references the parent sample IDs to the daughter sample IDs for each of these analyses.

Table 2.7. Cross-reference of Core Sample IDs, TI Subsample IDs, and Original RPL/ASO IDs to TIMS and UDS Sample ID Assignments

\begin{tabular}{|c|c|c|c|c|}
\hline \multirow[b]{2}{*}{ Core Sample ID } & \multirow[b]{2}{*}{ Subsample ID } & \multirow{2}{*}{$\begin{array}{c}\text { Original } \\
\text { RPL/ASO ID } \\
\text { ASR } 8798 \\
\end{array}$} & \multicolumn{2}{|c|}{ Daughter Sample Designation } \\
\hline & & & $\begin{array}{l}\text { TIMS U Preparation } \\
\text { (Acid Digestate) }\end{array}$ & $\begin{array}{l}\text { TIMS Pu Preparation } \\
\text { (Acid Digestate) }\end{array}$ \\
\hline \multirow[t]{2}{*}{ KW210-A1 } & TI001-A1-B & 11-0419-129-S & 11-0419-129-S-U & 11-0419-129-S-Pu \\
\hline & TI001-A1-C & 11-0419-129-D & 11-0419-129-D-U & 11-0419-129-D-Pu \\
\hline \multirow[t]{2}{*}{ KW210-A4 } & TI002-A4-B & $11-0420-129-S$ & 11-0420-129-S-U & 11-0420-129-S-Pu \\
\hline & TI002-A4-C & 11-0420-129-D & 11-0420-129-D-U & 11-0420-129-D-Pu \\
\hline \multirow[t]{2}{*}{ KW210-B1 } & TI002-B1-B & 11-0421-129-S & 11-0421-129-S-U & 11-0421-129-S-Pu \\
\hline & TI002-B1-C & 11-0421-129-D & 11-0421-129-D-U & 11-0421-129-D-Pu \\
\hline \multirow[t]{3}{*}{ KW210-B3 } & TI001-B3-B & 11-0422-129-S & 11-0422-129-S-U & 11-0422-129-S-Pu \\
\hline & TI001-B3-C & 11-0422-129-D & 11-0422-129-D-U & 11-0422-129-D-Pu \\
\hline & & & ASR 8878; RPL/ASO ID (UDS) & XRD (UDS) \\
\hline \multirow[t]{2}{*}{ KW210-A1 } & TI001-A1-B & 11-0419-129-S & TI040-0419S-1b; 11-1279 & -- \\
\hline & TI001-A1-C & 11-0419-129-D & TI040-0419D-2b; 11-1280 & TI040-419D-2x \\
\hline \multirow[t]{3}{*}{ KW210-A4 } & TI002-A4-B & $11-0420-129-S$ & TI040-0420S-2c; 11-1281 & -- \\
\hline & TI002-A4-C & 11-0420-129-D & TI040-0420D-1c; 11-1282 & TI040-420D-2x \\
\hline & & & TI040-0420D-2c ${ }^{(\mathrm{a})} ; 11-1282 \mathrm{D}$ & -- \\
\hline \multirow[t]{2}{*}{ KW210-B1 } & TI002-B1-B & 11-0421-129-S & TI040-0421S-2b; 11-1283 & -- \\
\hline & TI002-B1-C & 11-0421-129-D & TI040-0421D-1b; 11-1284 & TI040-421D-2x \\
\hline \multirow[t]{2}{*}{ KW210-B3 } & TI001-B3-B & $11-0422-129-S$ & TI040-0422S-1c; 11-1285 & -- \\
\hline & TI001-B3-C & 11-0422-129-D & TI040-0422D-1c; 11-1286 & TI040-422D-2x \\
\hline
\end{tabular}

(a) Processed as batch duplicate.

Note: The TIMS analysis was not conducted by the ASO; the ASO prepared purified Pu and U fraction samples for TIMS analysis.

(a) Processing of the Undissolved Solids Samples from the K West Basin Core Samples Collected from SCS-CON210 for Additional Analysis, SK Fiskum, May 2011. 


\subsection{Container Compositing and Subsampling Overview}

Container composite samples were intended to be created from the combination of equal settled sludge volumes from each core sample in accordance with the QAPJP/SAP. Subsamples collected for container composite preparation from each core sample were transferred from the HLRF to the Shielded Analytical Laboratory (SAL). Two independent container composites were prepared, one to support rheology testing and one to support sieving and characterization according to TI 52578-TI008. ${ }^{\text {(a) }}$ Initial preparations of each container composite were identical. The "-A" samples (e.g., Table 2.3, KW210-A1A) that had been collected from each core sample were combined to create a container composite (KW210-CC-A) in a 480-mL, safety-coated, Qorpak glass jar. Similarly, the “-N” samples (e.g., Table 2.3, TI001-A1-N), collected from each core sample, were combined to create a container composite (KW210-CC-N) in a 240-mL, safety-coated, Qorpak glass jar. An adhesive decal millimeter scale was affixed to each jar to facilitate determination of volume as a function of height. The contents were mixed by shaking the bottles and fully suspending the solids in a fluid head of deionized (DI) water. The solids were allowed to settle $24 \mathrm{hr}$. The settled solids volumes were tracked as a function of time (opportunistically) to create a settling curve. Physical-property information (i.e., settled solids volume, total slurry volume, and mass) were collected on each container composite.

Primary physical property subsamples were collected from each container composite sample. The headspace fluid was removed and set aside, and the sludge was mixed. The process was repeated until the sludge consistency had thickened enough to suspend dense particles. This thickening was performed to aid in providing more uniform sludge subsamples that would be less prone to particle segregation based on particle size or density. Two physical-property samples of thickened sludge were then collected from each container composite into 40-mL heavy-walled glass centrifuge tubes and then weighed. After subsampling, the reserved water was added back to the solids.

Preparation and processing of the KW210-CC-A container composite is summarized in Figure 2.9. The KW210-CC-A material was transferred directly to shear strength testing (see Section 2.9) without sieving. Preparation and processing of the KW210-CC-N container composite is summarized in Figure 2.10.

The KW210-CC-N container composite supporting analytical characterization was required to be fractionated into three sieve sizes: $>2,000 \mu \mathrm{m},<2,000$ and $>500 \mu \mathrm{m}$, and $<500 \mu \mathrm{m}$. Large-diameter sieves were used to process the wet sludge to best accommodate the large ( $120 \mathrm{~mL})$ sample volume. The sieves were 8-in. diameter $\times 2$-in. deep and composed of stainless steel woven wire cloth (Advantech Manufacturing, New Berlin, WI and distributed by VWR). The sieves were manufactured in compliance with American Society for Testing and Materials (ASTM) Specification E-11. The sieve product summary is provided in Table 2.8 .

(a) Preparation of K West Basin Consolidated Container Composite for SCS-CON-210. SK Fiskum, March 2011. 
PNNL-20650, Rev. 2

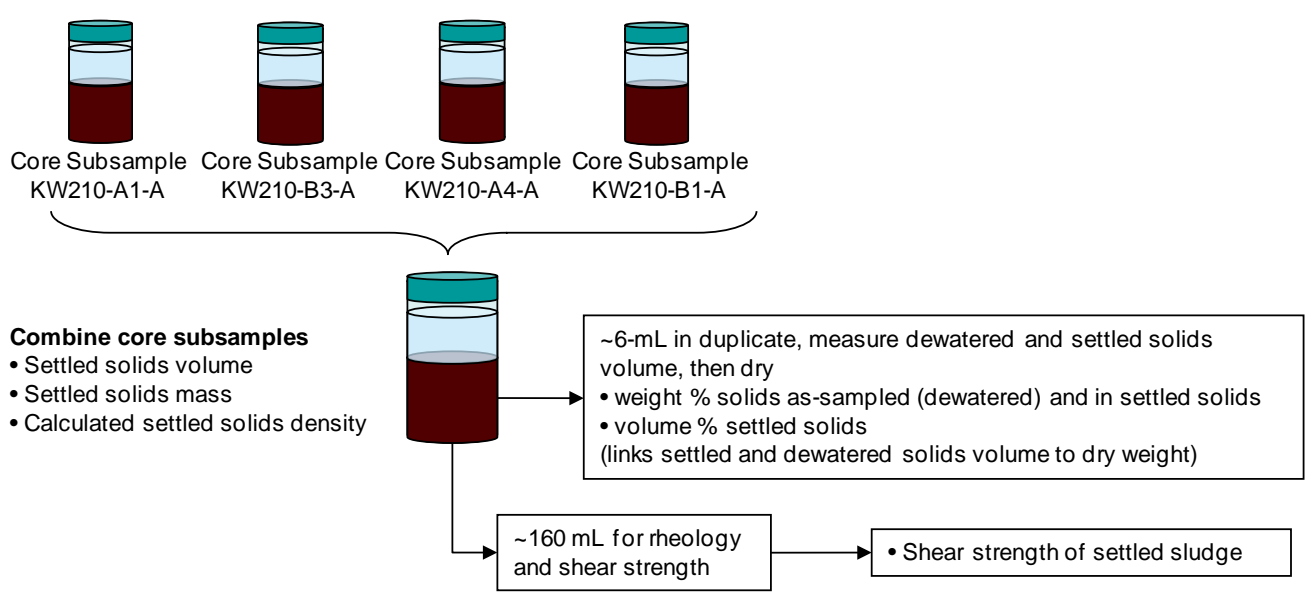

Figure 2.9. Overall Processing Schematic for the KW210-CC-A Container Composite Supporting Rheology

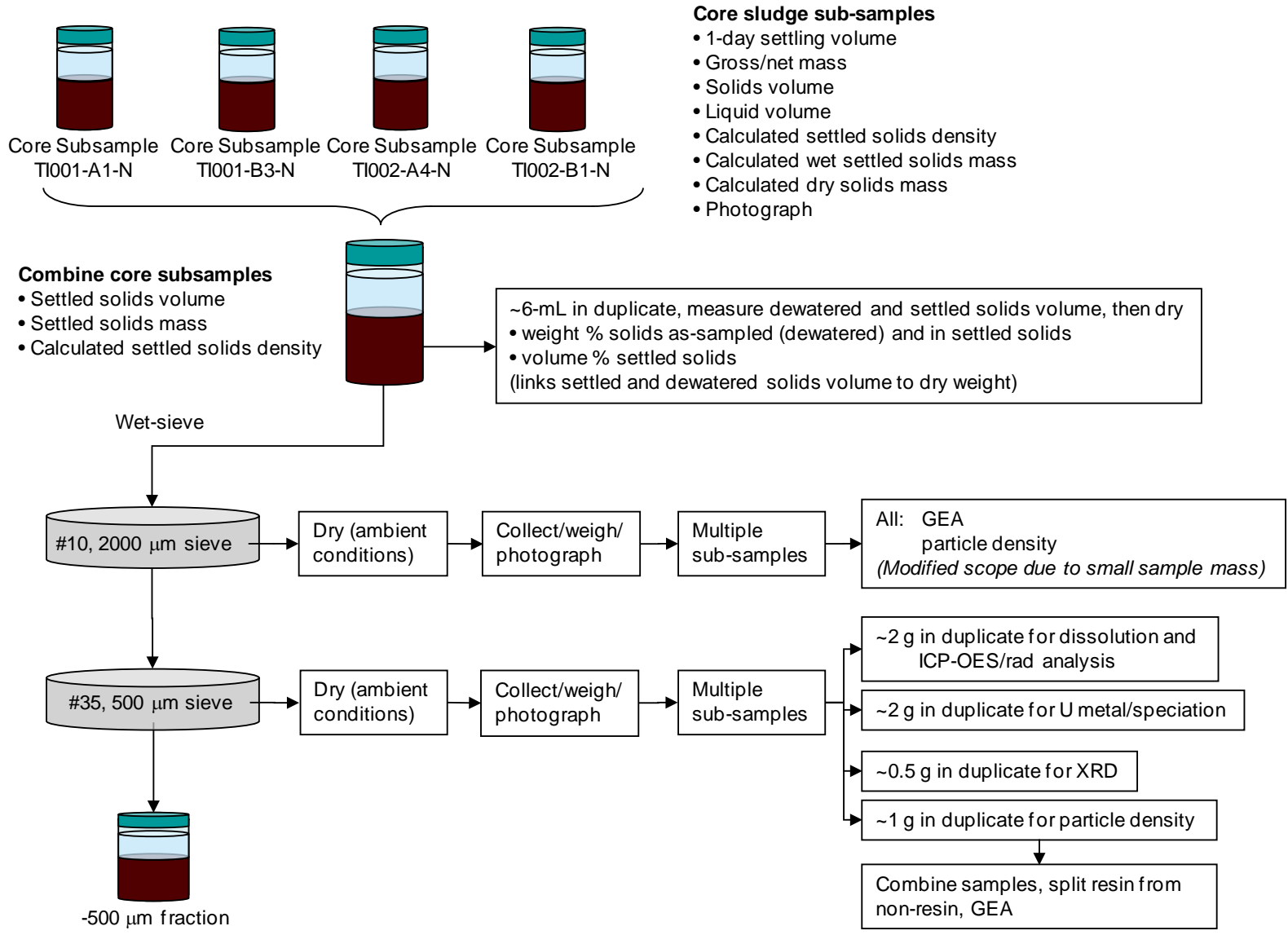

- The $>2,000-\mu m$ (referred to as " $+2,000-\mu m$ ") sieve fraction was too small to support the full analytical suite (targeted to equal the scope of the $-2,000-\mu \mathrm{m} /+500-\mu \mathrm{m}$ sieve fraction; only GEA analysis followed by particle density measurement was conducted on the whole collected sample.

- Processing of the -500- $\mu$ m container composite fines is defined in Figure 2.13.

Figure 2.10. Initial Processing and Sieving Schematic of the KW210-CC-N Container Composite 
Table 2.8. Sieve Parameters

\begin{tabular}{ccc}
\hline $\begin{array}{c}\text { US Series Sieve } \\
\text { Designation }\end{array}$ & $\begin{array}{c}\text { Standard } \\
\text { Designation }\end{array}$ & $\begin{array}{c}\text { Sieve Serial } \\
\text { Number }\end{array}$ \\
\hline$\# 10$ & $2.0 \mathrm{~mm}$ & 10SS8F417114 \\
$\# 35$ & $500 \mu \mathrm{m}$ & 35SS8F440521 \\
\hline
\end{tabular}

The two sieves were stacked with the 2.0 -mm mesh sieve on top of the $500-\mu \mathrm{m}$ mesh sieve. These were placed onto a custom-made support stand welded to a stainless steel funnel (see Figure 2.11) such that the fines could be directed into a collection bottle after exiting the sieve. This configuration minimized the chance of losing solids. The sieves and sieve holder had been previously used on other K Basin sludge samples (Fiskum et al. 2011b). They were rinsed and wiped in-cell before use.

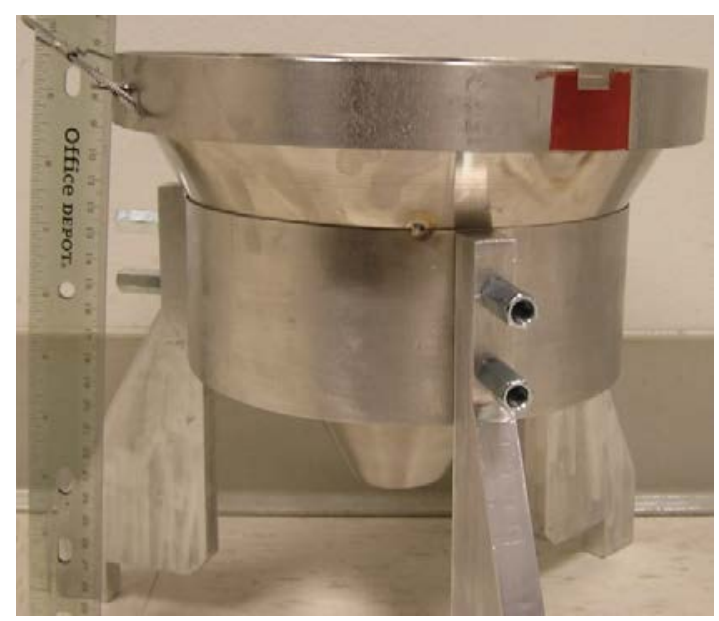

Side view

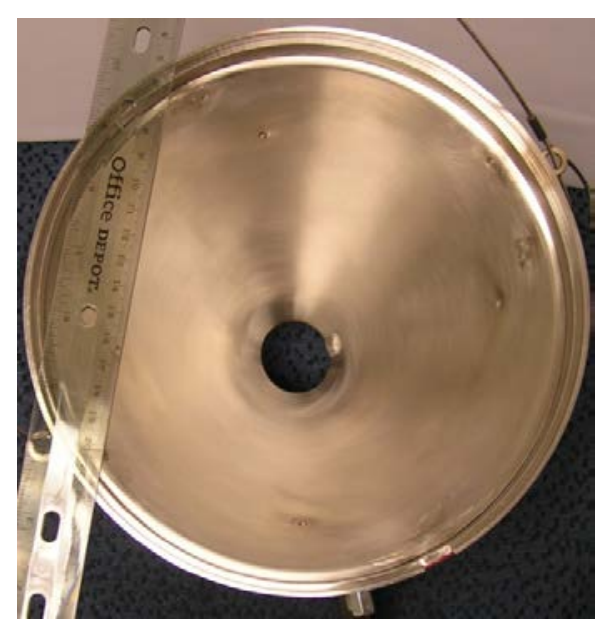

Looking down

Figure 2.11. Fines-Collection Assembly Supporting Wet Sieving Activity

The solids were re-suspended and transferred onto the stacked sieve array. Water was used to facilitate quantitative transfer and to wash the solids through the sieve openings. The collection bottle was replaced as it was filled. Washing continued until solids visually appeared well-rinsed. The top sieve $(2,000 \mu \mathrm{m})$ was removed. The solids continued to be washed on the $500-\mu \mathrm{m}$ sieve until solids visually appeared well-rinsed, and no additional fines were falling into the collection bottle. The fines were collected in a rotation of four to five glass bottles; after the fines settled, the aqueous phase was retrieved and used in additional solids washing. The solids fractions collected on the sieve fabric were allowed to air dry overnight under ambient temperature conditions (typically 25 to $30^{\circ} \mathrm{C}$ ) and air flow conditions. Solids were then retrieved from each sieve by inverting them over a duplicate sieve support assembly and then collecting the solids in a jar. The dry solids were weighed. The $>2,000-\mu \mathrm{m}$ (referred to as " $+2,000-\mu \mathrm{m}$ ") fraction mass was small and the sample was not split but analyzed in its entirety. The $-2,000-\mu \mathrm{m} /+500-\mu \mathrm{m}$ fraction was split using an inverted Y-connector attached to a funnel. Splits were repeated in a cascade fashion until appropriate analytical sizes were obtained (see example, Figure 2.12). This splitting effort was performed to produce representative subsamples.

The $-500-\mu \mathrm{m}$ wet sludge collected in the four to five bottles was composited into a single $480-\mathrm{mL}$ glass jar. The combined solids were processed in a similar manner to the core composite material (see Figure 2.13). An opportunistic settling curve was collected on the bulk solids then multiple subsamples were pulled from the mixed and thickened sludge to satisfy a multiplicity of analytical objectives. 
PNNL-20650, Rev. 2

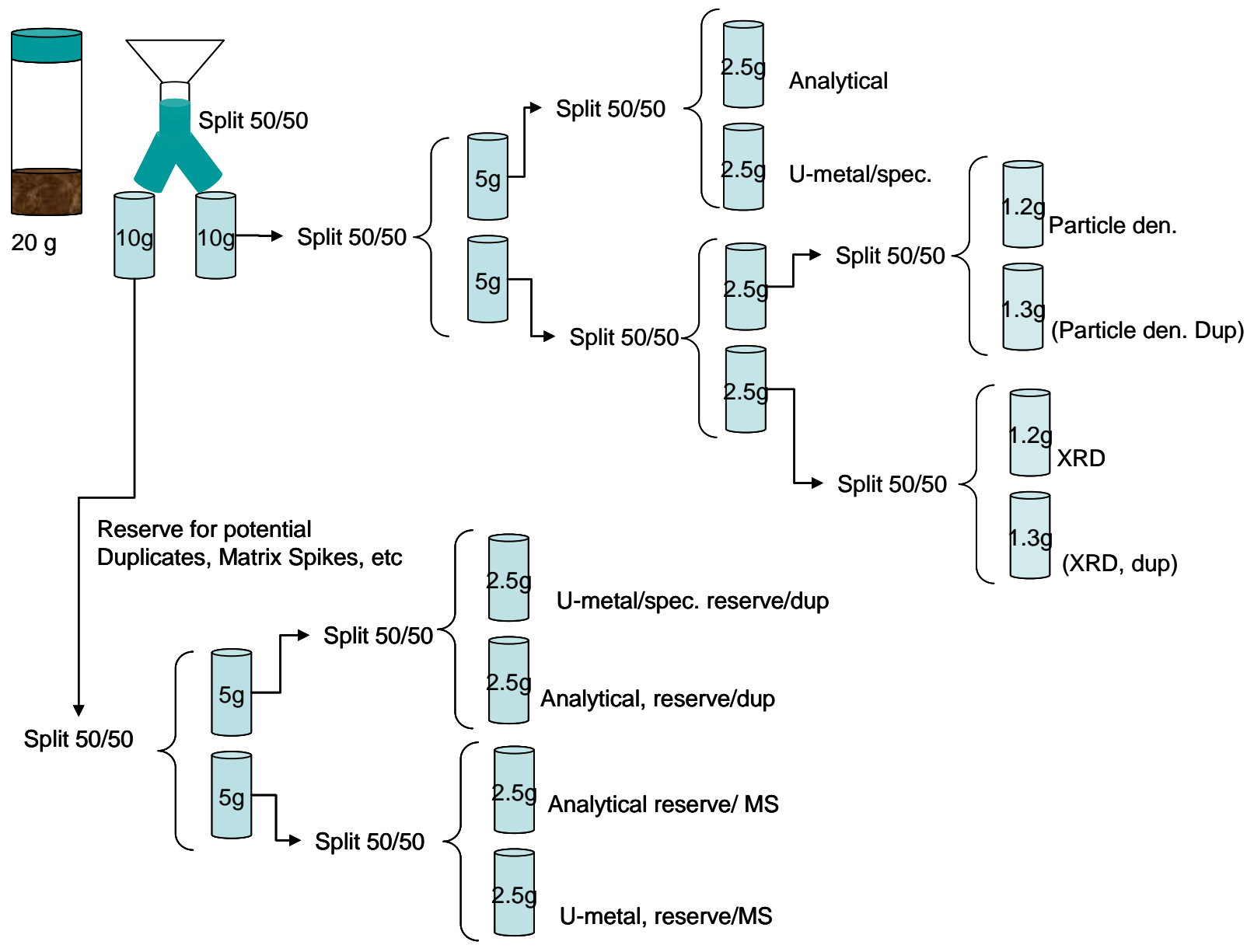

Figure 2.12. Prototypic Subsampling of the Dry Sludge Solids Collected on the $500-\mu m$ Mesh Sieve 
PNNL-20650, Rev. 2

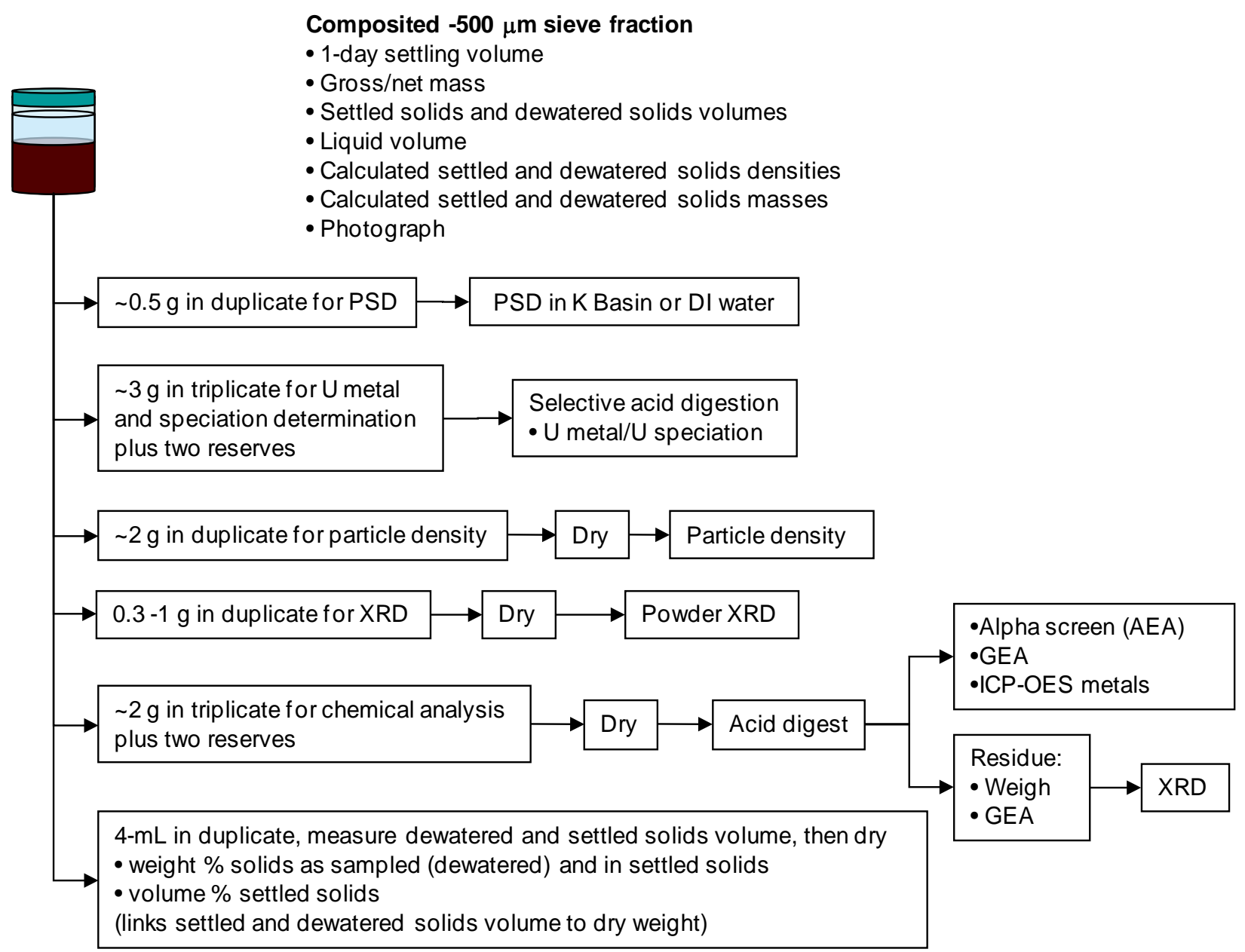

Figure 2.13. Processing Schematic of the KW210-CC-N Container Composite, $-500-\mu \mathrm{m}$ Fraction

A crosswalk among container composite IDs, subsample IDs (from TIs), as-sampled mass, sample split date, ASO sample IDs (RPL/ASO ID), ASR IDs, and analyses requested is provided in Table 2.9. More than the minimum required characterization samples were collected as a contingency in case of disagreement between duplicates, and/or when analytical problems were observed, and reanalysis was required.

The UDS collected following acid digestion were processed to support fusion analysis and XRD evaluation. These daughter samples are cross-referenced to the original material as shown in Table 2.10. 
Table 2.9. Cross-Reference of SCS-CON-210 Container Composite Samples, TI Subsample ID, AsSampled Mass, Split Date, RPL/ASO Subsample ID, and General Analysis Scope

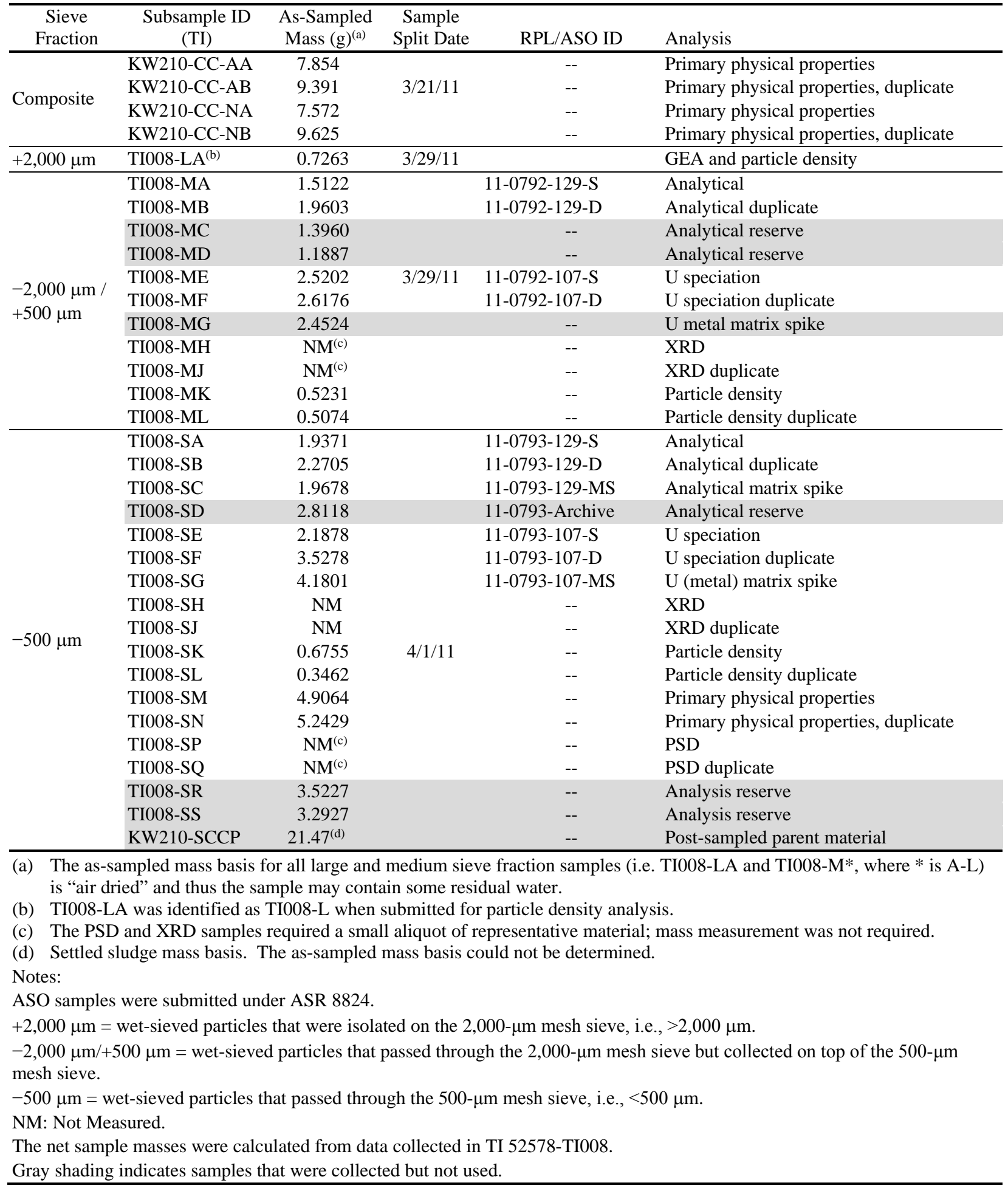


Table 2.10. Cross-reference of Container Composite Sample Description, TI Subsample IDs, and Original RPL/ASO IDs to UDS Sample ID Assignments

\begin{tabular}{|c|c|c|c|c|c|}
\hline \multirow{2}{*}{$\begin{array}{l}\text { Container } \\
\text { Sample } \\
\text { Description }\end{array}$} & \multirow[b]{2}{*}{$\begin{array}{l}\text { Subsample } \\
\text { ID }\end{array}$} & \multirow{2}{*}{$\begin{array}{c}\text { Original } \\
\text { RPL/ASO ID } \\
\text { ASR } 8824\end{array}$} & \multicolumn{3}{|c|}{ UDS Daughter Sample Designation } \\
\hline & & & $\begin{array}{c}\text { ASR } 8878 \\
\text { (RPL/ASO ID) }\end{array}$ & XRD & $\begin{array}{c}\text { ASR } 8919 \\
\text { (RPL/ASO ID) }\end{array}$ \\
\hline \multirow[t]{4}{*}{$\begin{array}{l}-2,000 \mu \mathrm{m} / \\
+500 \mu \mathrm{m}\end{array}$} & TI008-MA & 11-0792-129-S & $\begin{array}{l}\text { TI040-0792S-1c } \\
(11-1289)\end{array}$ & & \\
\hline & TI008-MB & 11-0792-129-D & $\begin{array}{l}\text { TI040-0792D-1c } \\
\quad(11-1290)\end{array}$ & TI040-792D-2x & \\
\hline & $\begin{array}{l}\text { TI008-MK \& } \\
\text { TI008-ML } \\
\text { Combined }\end{array}$ & & & & $\begin{array}{l}\text { KW210 MK \& } \\
\text { ML Resin Beads } \\
\text { (11-1555) }\end{array}$ \\
\hline & & & & & $\begin{array}{c}\text { KW210 MK \& ML } \\
\text { Residual Solids } \\
(11-1556)\end{array}$ \\
\hline \multirow[t]{3}{*}{$-500 \mu \mathrm{m}$} & TI008-SA & 11-0793-129-S & $\begin{array}{l}\text { TI040-0793S-1a } \\
\quad(11-1287)\end{array}$ & & \\
\hline & TI008-SB & 11-0793-129-D & $\begin{array}{c}\text { TI040-0793D-1b } \\
(11-1288)\end{array}$ & TI040-793D-2x & \\
\hline & & & $\begin{array}{c}\text { TI040-0793D-2b(a) } \\
(11-1288 \mathrm{D})\end{array}$ & & \\
\hline
\end{tabular}

(a) Processed as batch duplicate.

\subsection{Physical Properties: Wet Sludge Densities and Mass Conversion Factors}

Samples collected for primary physical property measurements were processed in accordance with the specific TI. Figure 2.14 depicts the overall primary physical property sample handling process. After measuring the as-sampled mass and volume, $\mathrm{K}$ Basin water was added to each sample. The ratio of fluid to as-sampled solids varied somewhat from sample to sample, but for the primary physical property characterization samples, the volume of fluid added was approximately equal to the volume of as-sampled sludge. Solids were then re-suspended by shaking (with manipulator hand and the aid of a vortex mixer) and allowed to settle undisturbed for up to $24 \mathrm{hr}$ at which time constant volume (undisturbed) had been attained. Settling curves were generated opportunistically from the primary physical property samples and samples collected for the container composites. The settled solids volume and the total (settled solids plus the headspace water) volume were recorded or later determined from the video documentation, and the gross mass was measured. Most of the fluid headspace was removed (without disturbing the settled solids) and placed into a separate tared vial. The wet solids and removed fluid were dried to constant mass at $105^{\circ} \mathrm{C}$. 


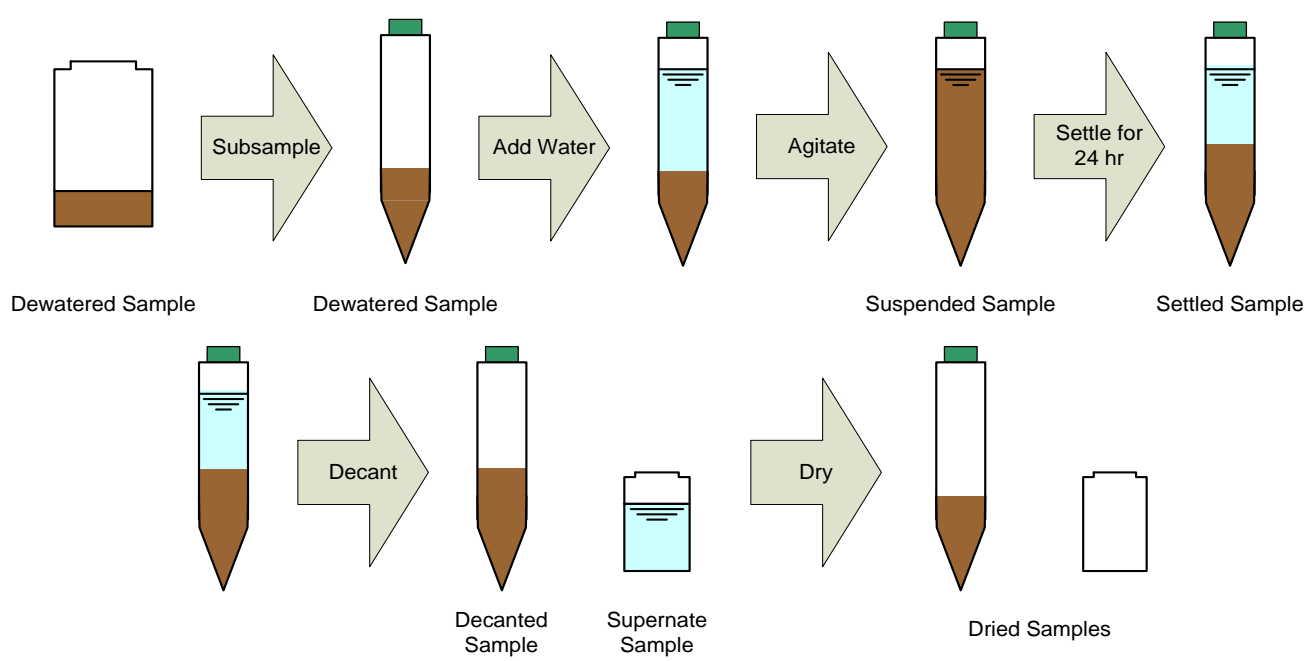

- Top row shows collection of a dewatered sample and how it is returned to a settled sludge state.

- Bottom row shows processing of a sample from a settled sludge state to a dry state.

Figure 2.14. Sample Processing for Physical Property Measurements

The physical properties of the sludge (i.e., density, wt\% solids) were assessed from duplicate "primary" subsamples collected from each core sample, container composite sample, and the $-500-\mu \mathrm{m}$ sieve fraction. Additional physical property data were collected from other samples where possible for sample-to-sample assessment of the general representativeness of the primary physical properties and subsamples.

- The primary physical property subsamples were used to determine 1) the as-sampled sludge mass, volume, and density, 2) the settled sludge mass, volume, and density after dispersion in water and settling for $24 \mathrm{hr}$, and 3) the wt\% solids (i.e., from measurement of the dry sludge mass). These samples were the primary linkage source for all physical properties (i.e., used to convert from as-sampled to settled to dry mass basis) associated with the reconstituted core samples, the container composite samples, and $-500-\mu \mathrm{m}$ sieve size fraction of the container composite. Primary physical property samples were collected from each of the test materials in heavy-walled, 40-mL glass centrifuge tubes (Kimble-Kontes, Vineland, NJ, part number 45200-40). The lower tapered portion tube height is $5 \mathrm{~cm}$ and the total tube height (to the bottom of the neck) is $12 \mathrm{~cm}$; the inside diameter increases from $\sim 0.5 \mathrm{~cm}$ to $\sim 2.5 \mathrm{~cm}$. Volume measures of the as-sampled sludge were sometimes confounded by sludge hold-up on the sample vessel sides.

- The reconstituted core samples and the container composite samples in the parent glass Qorpak jars were evaluated for as-sampled sludge mass, volume, and density and settled sludge mass, volume, and density. In these cases, the ratio of fluid to settled sludge prior to mixing was not as carefully controlled and depended in part on available space in the sample jar. Volume measures of the assampled sludge were sometimes confounded by sludge hold-up on the sample vessel sides.

- Subsamples collected from the reconstituted core samples and the $-500-\mu \mathrm{m}$ sieve size fraction of the container composite sample were evaluated for as-sampled sludge mass, volume, and density and settled sludge mass, volume, and density. In these cases, the sample containers varied in material of construction (glass and plastic) and inside diameter. As with the parent samples, the ratio of fluid volume to settled sludge volume was not carefully controlled and depended in part on available space in the sample container. Volume measures of the as-sampled sludge were sometimes confounded by sludge hold-up on the sample vessel sides. 
- For the core samples, chemical analysis subsamples were dried by the ASO, and the dried sludge mass was reported such that the g dry solids/g sampled sludge could be determined. These physical property data were generated using the same conditions and processes as the primary physical property samples.

- The density for all contact water was considered equivalent to DI water, and assumed to have a density of $1.000 \pm 0.005 \mathrm{~g} / \mathrm{cm}^{3}$. (a)

The data collected from samples of various sizes, showed that the physical properties were generally consistent within the range of sample sizes used in this characterization effort (i.e., several grams to several hundred grams). In the KW Basin, the sludge depth in the engineered containers varied from $\sim 10$ to $183 \mathrm{~cm}$; in the laboratory, the sludge depth in sample jars ranged from $<2.5$ to $10 \mathrm{~cm}$. The settling rate and extent of sludge compaction or density may be influenced by sludge morphology, composition, concentration (sludge depth), and container dimension.

The collected data supported calculations of a variety of physical property attributes as defined in the equations below.

$$
\begin{aligned}
& \text { as-sampled sludge mass (M) } \quad \mathrm{M}=\mathrm{M}_{\mathrm{G}}-\mathrm{m}_{\mathrm{CT}} \\
& \begin{array}{r}
\text { dry sludge mass per } \\
\frac{\mathrm{g}_{\text {dry }}}{\mathrm{g}_{\text {as-sampled }}}
\end{array}=\frac{\mathrm{m}_{\mathrm{DS}}}{\mathrm{M}} \\
& \text { settled sludge mass }\left(\mathrm{m}_{\mathrm{s}}\right) \quad \mathrm{m}_{\mathrm{S}}=\mathrm{m}_{\mathrm{G}}-\mathrm{m}_{\mathrm{CT}}-\left[\left(\mathrm{v}_{T}-\mathrm{v}_{\mathrm{S}}\right) \times \mathrm{D}_{\mathrm{L}}\right] \\
& \begin{array}{l}
\text { dry sludge mass per } \\
\text { settled sludge mass }
\end{array} \quad \frac{\mathrm{g}_{\text {dry }}}{\mathrm{g}_{\text {settled }}}=\frac{\mathrm{m}_{\mathrm{DS}}}{\mathrm{m}_{\mathrm{S}}} \\
& \begin{array}{r}
\text { as-sampled sludge mass per } \\
\text { settled sludge mass }
\end{array} \quad \frac{\mathrm{g}_{\text {as-sampled }}}{\mathrm{g}_{\text {settled }}}=\frac{\mathrm{M}}{\mathrm{m}_{\mathrm{S}}} \\
& \text { settled sludge density } \quad \mathrm{D}_{\mathrm{S}}=\frac{\mathrm{m}_{\mathrm{S}}}{\mathrm{v}_{\mathrm{S}}}
\end{aligned}
$$

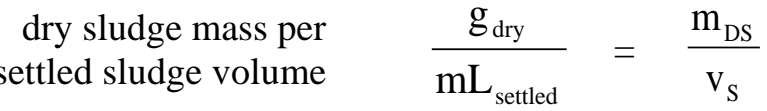

where $\quad \mathrm{M}_{\mathrm{G}}=$ gross mass of as-sampled sludge plus container tare (g)

$\mathrm{m}_{\mathrm{CT}}=$ tare mass of container $(\mathrm{g})$

$\mathrm{m}_{\mathrm{G}}=$ mass of container tare + wet settled sludge + liquid above sludge (g)

$\mathrm{v}_{\mathrm{T}}=$ total slurry volume (liquid plus settled sludge) $(\mathrm{mL})$

$\mathrm{v}_{\mathrm{S}}=$ volume of settled sludge $(\mathrm{mL})$

$\mathrm{D}_{\mathrm{L}}=$ liquid density $(\mathrm{g} / \mathrm{mL})$

$\mathrm{m}_{\mathrm{DS}}=$ mass of dried sludge $(\mathrm{g})$.

Note: Dissolved solids in liquid were found to be insignificant.

(a) The water density was assigned $0.005 \mathrm{~g} / \mathrm{cm}^{3}$ uncertainty which was propagated into the overall uncertainties as appropriate. 
Before disposal, and following drying to constant mass, the primary physical property samples were subjected to volume measurement and morphology assessments. The volume was measured directly without attempting to further settle solids. Before drying, interstitial spaces around settled particles were filled with water. After drying, interstitial spaces around solids were filled with air. Some retraction of the solids away from the vessel walls was observed; this increased void space was ignored. Morphology was determined in two stages: 1) the centrifuge tube was inverted to assess solids flow, and 2) the agglomerated material was prodded to assess resistance (this was a subjective measure through the constraint of a manipulator).

Images were intermittently collected with an in-cell video camera during all phases of the sample handling, subsampling, and sieving. Still images were collected from the video DVD. ${ }^{(a)}$ To the extent practical, a MacBeth ColorChecker ${ }^{\circledR}$ Rendition Chart (MacBeth Color Chart) was included in the field-ofview in an effort to better define the sludge sample coloration. Figure 2.15 shows a scanned image of the MacBeth Color Chart.

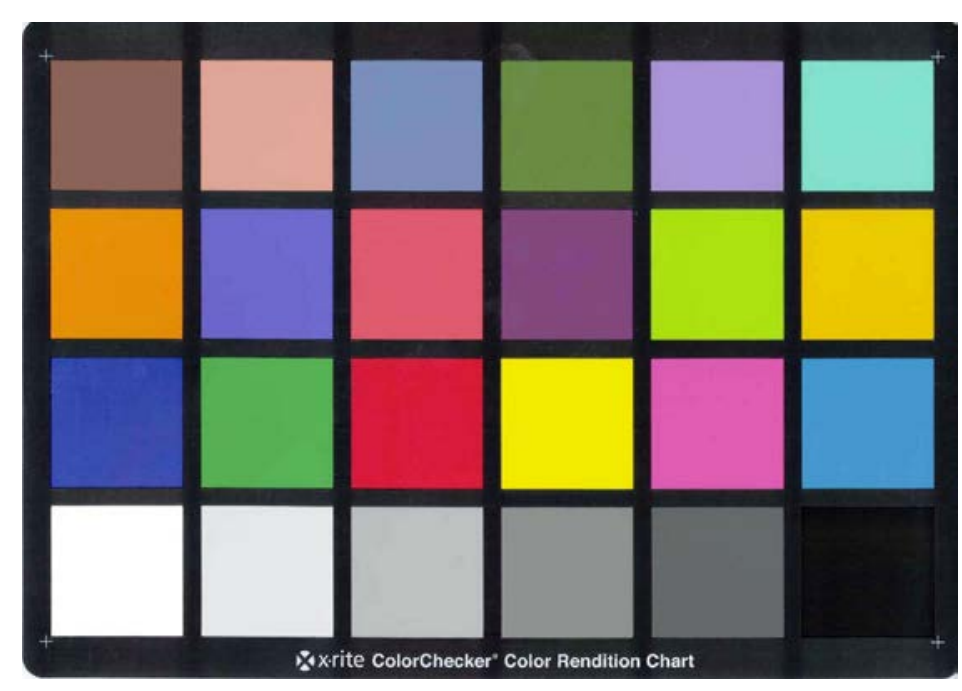

Figure 2.15. MacBeth ColorChecker ${ }^{\circledR}$ Rendition Chart

\subsection{Sample Characterization}

All chemical, radiochemical, U speciation, and total organic carbon (TOC)/ total inorganic carbon (TIC) characterization samples were submitted to the ASO under various ASRs (as previously identified). Detailed Special Instructions were provided to the ASO, defining project-specific DQOs and requirements (see Appendices B-D). Each Special Instruction was reviewed internally and then provided to the BTR for review and concurrence before finalizing. The multiple reviews confirmed that the complexity of the required analyses was accurately conveyed to the ASO. Similarly, to assure that the client DQOs were properly delineated, the TIMS analysis requests were developed with a series of instructions that were first reviewed internally and then by the BTR before being issued for analysis.

Table 2.11 and Table 2.12 provide crosswalks of the analysis procedures, related QC sample acceptance criteria, and required duplicate frequency. Full copy procedures are provided as part of the

(a) Video DVDs will not be maintained as project records. Relevant still images are documented in the TIs and this report. The inventory of DVDs will be provided to STP CHPRC as non-record, information copies. 
Table 2.11. Digestion, Chemical, and Physical Property Analytical Procedures and QC Sample Criteria

\begin{tabular}{|c|c|c|c|c|c|c|c|}
\hline Analysis Objective & Procedure Number & Procedure Title & $\begin{array}{l}\text { Duplicate } \\
\text { (Precision) }\end{array}$ & $\begin{array}{l}\text { Blank Spike } \\
\text { (Accuracy) }\end{array}$ & $\begin{array}{l}\text { Matrix Spike } \\
\text { (Accuracy) }\end{array}$ & $\begin{array}{l}\text { Calibration } \\
\text { Verification }\end{array}$ & $\begin{array}{l}\text { Duplicate }^{(a)} \\
\text { Frequency }\end{array}$ \\
\hline TI implementation & RPL-OP-001 & Routine Research Operations & NA & NA & NA & NA & NA \\
\hline $\begin{array}{l}\text { Acid digestion for } \\
\text { chemical analysis }\end{array}$ & PNL-ALO-129 & $\begin{array}{l}\mathrm{HNO}_{3}-\mathrm{HCl} \text { Acid Extraction of Solids Using a } \\
\text { Dry-Block Heater }\end{array}$ & NA & NA & NA & NA & $\begin{array}{c}\text { 100\% Cores } \\
\text { 1/Container Batch }\end{array}$ \\
\hline $\begin{array}{l}\text { Fusion for UDS } \\
\text { dissolution }\end{array}$ & PNL-ALO-115 & 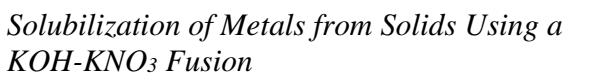 & NA & NA & NA & NA & $1 / \mathrm{Batch}^{(\mathrm{b})}$ \\
\hline $\begin{array}{l}\text { ICP-OES for acid } \\
\text { digestates and U } \\
\text { metal analysis }\end{array}$ & RPG-CMC-211 & $\begin{array}{l}\text { Determination of Elemental Composition by } \\
\text { Inductively Coupled Argon Plasma Optical } \\
\text { Emission Spectrometry (ICP-OES) }\end{array}$ & $\begin{array}{c} \pm 20 \% / \\
( \pm 20 \% \text { U metal } \\
>0.1 \text { wt } \%)\end{array}$ & $\begin{array}{c} \pm 20 \% \\
( \pm 25 \% \\
\text { U metal })\end{array}$ & $\begin{array}{l} \pm 20 \% \\
\text { (NA U metal) }\end{array}$ & $\begin{array}{l}\mathrm{LLS} \pm 25 \% \mathrm{ICV} \\
\text { and } \\
\mathrm{CCV} \pm 10 \%^{(\mathrm{c})}\end{array}$ & $\begin{array}{c}\text { 100\% Cores } \\
\text { 1/Container Batch }\end{array}$ \\
\hline $\mathrm{U} / \mathrm{KPA}$ for total $\mathrm{U}$ & RPG-CMC-4014 & Uranium by Kinetic Phosphorescence Analysis & $\pm 20 \%$ & $\pm 20 \%$ & $\pm 25 \%$ & $\pm 7.5 \%$ & $100 \%$ Cores \\
\hline $\begin{array}{l}\text { Acid Digestion for } \\
\text { U Speciation }\end{array}$ & RPG-CMC-107 & $\begin{array}{l}\text { Sample Preparation for Determination } \\
\text { Uranium Metal Concentrations in Sludge }\end{array}$ & NA & NA & NA & NA & $\begin{array}{c}\text { 100\% Cores } \\
\text { 1/Container Batch }\end{array}$ \\
\hline $\mathrm{U}(\mathrm{IV})$ : U(VI) Ratio & RPG-CMC-255 & $\begin{array}{l}\text { Sample Preparation and Analysis for } \\
\text { Determining Uranium Oxide Oxidation States } \\
\text { in K Basin Sludges }\end{array}$ & NA & $\pm 25 \%$ & NA & NA & 1/Container Batch \\
\hline TOC/TIC analysis & RPG-CMC-385 & $\begin{array}{l}\text { Carbon Measured in Solids, Sludge, and } \\
\text { Liquid Matrices }\end{array}$ & $\pm 20 \%$ & $\pm 25 \%$ & $\pm 25 \%$ & NA & $100 \%$ Cores \\
\hline $\begin{array}{l}\text { XRD preparation } \\
\text { and analysis }\end{array}$ & PNNL-RPG-268 & Solids Analysis by X-Ray Diffraction & NA & NA & NA & NA & NA \\
\hline $\begin{array}{l}\text { Particle density } \\
\text { analysis }\end{array}$ & APEL-PIP-4 & $\begin{array}{l}\text { Gas Pycnometry Method for Apparent Specific } \\
\text { Gravity Determinations of Consolidated Solids }\end{array}$ & $\pm 5 \%$ & NA & NA & $\pm 1 \%$ & 1/Container Batch \\
\hline $\begin{array}{l}\text { Particle size } \\
\text { distribution analysis }\end{array}$ & RPL-COLLOID-01 & Particle Size Analysis Using Malvern MS2000 & NA & NA & NA & NA & 1/Container Batch \\
\hline $\begin{array}{l}\text { Shear strength } \\
\text { analysis }\end{array}$ & RPL-COLLOID-02 & $\begin{array}{l}\text { Measurement of Physical and Rheological } \\
\text { Properties of Solutions, Slurries, and Sludges }\end{array}$ & NA & NA & NA & NA & NA \\
\hline \multicolumn{8}{|c|}{$\begin{array}{l}\text { (a) A laboratory duplicate sample is an aliquot of one of the original samples processed in duplicate through entire the preparative processes. Subsamples from each core were processed in duplicate; } \\
\text { hence, } 100 \% \text { of the core samples were analyzed as true duplicates. Only one duplicate per batch was required for the container composite analysis. }\end{array}$} \\
\hline \multirow{2}{*}{\multicolumn{8}{|c|}{$\begin{array}{l}\text { (b) The fusion preparation (applied to undissolved solids) was not specified in the QAPjP/SAP; therefore, co } \\
\text { (c) Calibration verification criteria for the ICP-OFS analysis was established in procedure RPG-CMC-211. }\end{array}$}} \\
\hline & & & $\mathrm{C}-211$. & & & & \\
\hline
\end{tabular}


Table 2.12. Radioisotopic Analytical Procedures and QC Sample Criteria

\begin{tabular}{|c|c|c|c|c|c|c|c|}
\hline Analysis Objective & Procedure Number & Procedure Title & $\begin{array}{l}\text { Duplicate } \\
\text { (Precision) }\end{array}$ & $\begin{array}{c}\text { Blank Spike } \\
\text { (Accuracy) }\end{array}$ & $\begin{array}{c}\text { Matrix } \\
\text { Spike } \\
\text { (Accuracy) }\end{array}$ & $\begin{array}{l}\text { Calib. Verif./ } \\
\text { Counter } \\
\text { Control }^{(a)} \\
\end{array}$ & $\begin{array}{l}\text { Duplicate }(\mathrm{b}) \\
\text { Frequency }\end{array}$ \\
\hline \multirow[t]{2}{*}{$\begin{array}{l}\mathrm{U}-\text { Isotopic } \\
\text { analysis }\end{array}$} & RPG-CMC-4017 & $\begin{array}{l}\text { Analysis of Environmental Water Samples for Actinides and } \\
\text { Strontium-90 }\end{array}$ & $\pm 20 \%$ & NA & NA & $\pm 2 \%^{(\mathrm{d})}$ & $100 \%$ Cores \\
\hline & PNNL-98523-264 & Mass Spectrometer Isotopic Analysis (TIMS) & & & & & \\
\hline \multirow[t]{2}{*}{$\begin{array}{l}\mathrm{Pu}-\text { Isotopic } \\
\text { analysis }\end{array}$} & RPG-CMC-455 & $\begin{array}{l}\text { Separation of Uranium and Plutonium for Isotopic Analysis by } \\
\text { Mass Spectrometry }\end{array}$ & $\pm 20 \%$ & NA & NA & $\pm 6 \%^{(\mathrm{d})}$ & $100 \%$ Cores \\
\hline & PNNL-98523-264 & Mass Spectrometer Isotopic Analysis (TIMS) & & & & & \\
\hline \multirow[t]{3}{*}{$\begin{array}{l}{ }^{238} \mathrm{Pu},{ }^{239+240} \mathrm{Pu} \\
\text {-AEA }\end{array}$} & RPG-CMC-4017 & $\begin{array}{l}\text { Analysis of Environmental Water Samples for Actinides and } \\
\text { Strontium-90 }\end{array}$ & $\pm 20 \%$ & $\pm 20 \%$ & $\pm 25 \%$ (c) & NA & $100 \%$ Cores \\
\hline & RPG-CMC-496 & Coprecipitation Mounting of Actinides for Alpha Spectroscopy & & & & & \\
\hline & RPG-CMC-422 & Solutions Analysis: Alpha Spectrometry & & & & & \\
\hline \multirow[t]{3}{*}{${ }^{241} \mathrm{Am}-\mathrm{AEA}$} & RPG-CMC-4017 & $\begin{array}{l}\text { Analysis of Environmental Water Samples for Actinides and } \\
\text { Strontium-90 }\end{array}$ & $\pm 20 \%$ & $\pm 20 \%$ & $\pm 25 \%$ (c) & NA & $100 \%$ Cores \\
\hline & RPG-CMC-496 & Coprecipitation Mounting of Actinides for Alpha Spectroscopy & & & & & \\
\hline & RPG-CMC-422 & Solutions Analysis: Alpha Spectrometry & & & & & \\
\hline $\begin{array}{l}\text { Gamma-emitters } \\
\text {-GEA }\end{array}$ & RPG-CMC-450 & $\begin{array}{l}\text { Gamma Energy Analysis (GEA) and Low-Energy Photon } \\
\text { Spectrometry (LEPS) }\end{array}$ & $\pm 20 \%$ & NA & NA & SPC & $\begin{array}{c}100 \% \text { Cores } \\
\text { 1/Container Batch }\end{array}$ \\
\hline \multirow[t]{3}{*}{${ }^{237} \mathrm{~Np}-\mathrm{AEA}$} & RPG-CMC-4017 & $\begin{array}{l}\text { Analysis of Environmental Water Samples for Actinides and } \\
\text { Strontium-90 }\end{array}$ & $\pm 20 \%$ & $\pm 20 \%$ & $\pm 25 \%$ (c) & NA & $100 \%$ Cores \\
\hline & RPG-CMC-496 & Coprecipitation Mounting of Actinides for Alpha Spectroscopy & & & & & \\
\hline & RPG-CMC-422 & Solutions Analysis: Alpha Spectrometry & & & & & \\
\hline AEA screen & $\begin{array}{l}\text { RPG-CMC-496 } \\
\text { RPG-CMC-422 }\end{array}$ & $\begin{array}{l}\text { Coprecipitation Mounting of Actinides for Alpha Spectroscopy } \\
\text { Solutions Analysis: Alpha Spectrometry }\end{array}$ & $\pm 25 \%$ & $\pm 20 \%$ & $\pm 25 \%$ (c) & NA & 1/Container Batch \\
\hline${ }^{90} \mathrm{Sr}$ analysis & $\begin{array}{l}\text { RPG-CMC-476 } \\
\text { RPG-CMC-474 }\end{array}$ & $\begin{array}{l}\text { Strontium Separation Using Eichrom Strontium Resin } \\
\text { Measurement of Alpha and Beta Activity by Liquid Scintillation } \\
\text { Spectrometry }\end{array}$ & $\pm 20 \%$ & $\pm 25 \%$ & $\pm 25 \%$ (c) & SPC & $50 \%$ Cores \\
\hline $\begin{array}{l}\text { Total alpha and total } \\
\text { beta screen analysis }\end{array}$ & $\begin{array}{l}\text { RPG-CMC-4001 } \\
\text { RPG-CMC-408 }\end{array}$ & $\begin{array}{l}\text { Source Preparation for Gross Alpha and Gross Beta Analysis } \\
\text { Total Alpha and Beta Analysis }\end{array}$ & NA & NA & NA & NA & NA \\
\hline
\end{tabular}

(a) Counting instruments were checked daily with counter control samples to confirm that performance was within statistical process control (SPC).

(b) A laboratory duplicate sample is an aliquot of one of the original samples processed in duplicate through the entire preparative processes. Each core was processed in duplicate; hence, $100 \%$ of the core samples were analyzed as true duplicates. Only one duplicate per batch was required for the container composite analysis.

(c) In accordance with the ASO QA Plan, post- preparative blank and matrix spikes were performed for Pu-AEA, Am-AEA, Np-AEA, AEA screen, and ${ }^{90}$ Sr.

(d) The isotopic analysis calibration verification standard criteria apply to ${ }^{235} \mathrm{U},{ }^{238} \mathrm{U}$, ${ }^{239} \mathrm{Pu}$, and ${ }^{240} \mathrm{Pu}$ isotopes and are based on the historical TIMS performance established through control charts. Control charts were not prepared for the other $U$ and Pu isotopes. The Analysis Request approved by the BTR passed down the accuracy requirement for isotope concentrations exceeding 0.1 atom\%. See Occurrence Report 52578-05-09-11 in Appendix E for details.

TIMS: thermal ionization mass spectrometry; AEA: alpha energy analysis; GEA: gamma energy analysis; LEPS: low energy photon spectroscopy; NA: not applicable. 
complete data package. All reconstituted core samples were analyzed in duplicate. Duplicate frequency for the container composite samples was specified as one per analytical batch. The matrix spike (MS) was prepared from a triplicate sample that was spiked with the analytes of interest (AOIs). The laboratory control samples (LCSs) or blank spike (BS) samples were spiked as appropriate for the analysis. The LCS/BS and MS samples for radionuclides and U kinetic phosphorescence analysis (KPA) analyses were prepared post-digestion at the analytical workstation. In the case of TIC/TOC analysis, each duplicate sample was analyzed in small-mass triplicate sub-aliquots; the MS was created from another replicate sub-aliquot.

Project staff conducted physical property characterization activities outside of the ASO (XRD, particle density, PSD, and rheology) directly on samples. The particle density and XRD samples were submitted under analysis requests that incorporated relevant elements of the QAPJP/SAP data quality requirements. The PSD and rheology characterizations were conducted per client-approved TIs implementing the indicated operational procedure.

All laboratory procedures used are provided as part of the complete data package on DVD-52578PNNL-20650 Rev. 2, Section II, September 2013.

\subsection{Chemical Analysis}

Chemical analyses (ICP-OES and radionuclide analysis) were conducted on the duplicate core subsamples and the container composite sieve fraction samples following acid digestion or fusions. The following sections provide brief descriptions. Cited procedures and reports found in Appendices B through D contain additional details for sample processing, QC sample processing, and instrument calibration. Analytes not listed as AOIs in the QAPJP/SAP were measured opportunistically as the procedures and detection systems allowed (with little or no additional cost to the project) and were not necessarily subjected to the same evaluation rigor applied to AOIs. Therefore, the quality of these opportunistic data is indeterminate.

\subsubsection{Acid Digestion for ICP-OES and Radionuclides}

Process QC samples prepared for the acid digestion included sample duplicates (all core and sieve fraction samples), a BS, and MS sample (the BS and MS sample were spiked with ICP-OES AOIs), a BS (spiked only with $U$ to support KPA), and one preparation blank (PB). The samples processed for chemical analysis were dried to constant mass at $105^{\circ} \mathrm{C}$. The dried residue was then acid-digested per PNL-ALO-129, Rev. 0, $\mathrm{HNO}_{3}$-HCl Acid Extraction of Solids Using a Dry-Block Heater, modified to double the reagent acid volumes and concentrations ( $8 \mathrm{M}$ each $\mathrm{HNO}_{3}$ and $\mathrm{HCl}$ ). The acid volumes used were proportioned to the mass of dried sample, with $8 \mathrm{~mL}$ of each acid for samples $1 \mathrm{~g}$ or less, $12 \mathrm{~mL}$ of each acid for samples between 1 and $1.5 \mathrm{~g}$, and $16 \mathrm{~mL}$ of each acid for samples between 1.5 and $2 \mathrm{~g}$.

The post-digested UDS were separated from the aqueous phase and thoroughly rinsed with acidic solution. The aqueous phase was brought to a $100-\mathrm{mL}$ volume in a volumetric flask, and aliquots were distributed for ICP-OES and radiochemical analyses. The solution distributed to the workstations is termed the digestate. The UDS was dried to constant mass at $105^{\circ} \mathrm{C}$. The UDS was removed from the SAL and counted by GEA. Sample digestion descriptions and mass measurements are provided in Appendices B and C. 
The UDS samples were split into smaller sizes using the splitting technique previously identified (Figure 2.12) to support fusion processing. One sample split from each sample was fractionated to remove resin beads from the non-resin bead fraction. This was done on a best effort basis according to TI 52578-TI040 ${ }^{(a)}$. Material was placed on folded paper; rolling particles (resin beads) were collected into a vial and the non-rolling fraction was collected in a separate vial. Each fraction was weighed to estimate the mass associated with the resin and non-resin material. One of the duplicate samples of non-resin material was evaluated by XRD (Table 2.7 and Table 2.10). Core and container composite UDS subsamples were selected and submitted for fusion processing and ICP-OES and AEA screen (Table 2.7 and Table 2.10).

\subsubsection{Fusion}

Selected UDS subsamples from ASR 8798 (Table 2.7) and ASR 8824 (Table 2.10) were processed according to ASR 8878 Special Instructions and were dissolved with the aid of fusion. Fusion was conducted in accordance with procedure PNL-ALO-115, Rev. 1.1, Solubilization of Metals from Solids Using a $\mathrm{KOH}-\mathrm{KNO}_{3}$ Fusion using a $\mathrm{KOH} / \mathrm{KNO}_{3}$ flux in a nickel crucible. The sample size for fusion processing ranged from 0.1 to $0.2 \mathrm{~g}$, therefore large process dilution factors apply. The LCS used in fusion preparations was National Institute of Standards and Technology (NIST) standard reference material (SRM) 2710 Montana Soil. The fused material was dissolved and brought to a $100-\mathrm{mL}$ volume with DI water. The solution was then analyzed by ICP-OES for Al, Ca, Fe, S, Si, Zr, and U, for gross alpha, and by AEA screen for ${ }^{238} \mathrm{Pu} /{ }^{241} \mathrm{Am}$ and ${ }^{239+240} \mathrm{Pu}$. Process $\mathrm{QC}$ for the fusion preparations included a sample duplicate, an LCS, and one PB.

\subsubsection{ICP-OES}

Analysis was conducted using a PerkinElmer Model 5300DV ICP-OES equipped to handle radioactive samples in accordance with procedure RPG-CMC-211, Rev. 3, Determination of Elemental Composition by Inductively Coupled Argon Plasma Optical Emission Spectrometry (ICP-OES). Instrument calibration was conducted per the manufacturer-recommended procedure using multi-analyte custom standard solutions traceable to the NIST. Detailed calibration descriptions are located in the ICP-OES reports provided in Appendices B, C, and D.

Typically, the acid digestates were diluted $10 \times$ at the workstation to reduce the acid concentration and solution density at the instrument. However, the digestates also were run undiluted in an effort to meet the detection limits required for several elements (i.e., As, Sb, Se, Tl). The direct analysis run introduces potential matrix issues related to increased solution density and acidity. Samples prepared by fusion required larger dilution $(\sim 100 \times)$ to reduce the flux salt concentration. All reported results were corrected for the sample-specific process and dilution factors.

(a) Processing of the Undissolved Solids Samples from the K West Basin Core Samples Collected from SCS-CON210 for Additional Analysis, SK Fiskum, May 2011. 
Analytes are reported in two tiers. AOIs were specifically designated in the QAPjP/SAP for the core samples and the container composite samples and have been fully evaluated relative to QC sample performance. The AOIs for the fusion preparations were designated in the ASR (8878) with BTR concurrence. Other analytes were opportunistically measured but have not been fully evaluated for QC performance.

The ICP-OES report lists analyte concentrations that are less than the method detection limit (MDL) as "--"; the MDLs for these analytes were estimated by multiplying the instrument detection limit (IDL) of the analyte by the process factor or "multiplier". Sample results shown in brackets "[ ]" were less than the estimated quantitation limit (EQL) but greater than the MDL. Both the IDL and EQL are based on acidified water and/or fusion flux matrices (as applicable). Routine precision and bias is typically $\pm 15 \%$ or less for samples in dilute, acidified water at analyte concentrations >EQL up to the established linear range. Bracketed values have potential uncertainties $>15 \%$. The maximum number of significant figures for all ICP-OES measurements is two. The third significant figure provided for analytes detected greater than the EQL is provided for additional information.

\subsubsection{Alpha Energy Analysis}

Radioisotopes measured by alpha spectrometry require radiochemical separations to provide a pure chemically separated solution containing the radioisotopes of interest. This allows the preparation of a mount that is free of residue that would otherwise attenuate the alpha emission, resulting in peak broadening and absorption. Separation also allows for elemental purification such that other alphaemitting radionuclides with equivalent energies are stripped from the test fraction. A typical AEA system has an energy resolution of $\sim 0.03 \mathrm{MeV}$. Table 2.13 shows the significant alpha emitters expected in the test samples, their corresponding primary alpha-emission energies, the primary interfering radioisotopes, and a method to resolve interference. Some isotopes have essentially equivalent energies and must be chemically separated to provide accurate analysis (e.g., ${ }^{238} \mathrm{Pu}$ and ${ }^{241} \mathrm{Am}$ ). Other isotopes require radiochemical separation followed by TIMS to differentiate (e.g., ${ }^{239} \mathrm{Pu}$ and ${ }^{240} \mathrm{Pu}$ ).

Table 2.13. Summary of Alpha Emitters and Corresponding Energies

\begin{tabular}{llll}
\hline & & $\begin{array}{c}\text { Potential } \\
\text { Interfering } \\
\text { Isotope }\end{array}$ & Resolution to Interference \\
\hline${ }^{238} \mathrm{Pu}$ & Alpha Energy, $\mathrm{MeV}^{(\mathrm{a})}$ & ${ }^{241} \mathrm{Am}$ & radiochemical separation \\
${ }^{239} \mathrm{Pu}$ & $5.46,5.50$ & ${ }^{240} \mathrm{Pu}$ & TIMS \\
${ }^{240} \mathrm{Pu}$ & $5.14,5.16$ & ${ }^{239} \mathrm{Pu}$ & TIMS \\
${ }^{242} \mathrm{Pu}$ tracer & $5.12,5.17$ & None & NA \\
${ }^{237} \mathrm{~Np}$ & $4.86,4.90$ & ${ }^{234} \mathrm{U}$ & radiochemical separation \\
${ }^{234} \mathrm{U}$ & $4.77,4.79$ & ${ }^{237} \mathrm{~Np}$ & radiochemical separation \\
${ }^{235} \mathrm{U}$ & $4.72,4.78$ & ${ }^{236} \mathrm{U}$ & TIMS \\
${ }^{236} \mathrm{U}$ & $4.32,4.36,4.40,4.56,4.60$ & ${ }^{235} \mathrm{U}$ & TIMS \\
${ }^{238} \mathrm{U}$ & $4.44,4.49$ & None & NA \\
${ }^{241} \mathrm{Am}$ & $4.15,4.20$ & ${ }^{238} \mathrm{Pu}$ & radiochemical separation \\
${ }^{243} \mathrm{Am}$ tracer & $5.44,5.49$ & None & NA \\
\hline
\end{tabular}

(a) Browne and Firestone 1986. 
All alpha spectra were collected on Ortec silicon charged-particle detectors mounted in model 576A Dual Alpha Spectrometers operated with Canberra GENIE2000 Alpha Acquisition and Analysis software. Detectors were energy- and efficiency-calibrated with NIST-traceable standards.

\subsubsection{Neptunium (Alpha Energy Analysis)}

An aliquot of each core sample and duplicate acid digestate was passed through a strong-base anion exchange resin bed in a hydrochloric acid matrix in accordance with procedure RPG-CMC-4017, Rev. 0, Analysis of Environmental Water Samples for Actinides and Strontium-90. The Np fraction collected from the anion exchange column was processed through a second anion exchange separation to more completely remove interference from $\mathrm{U}$. The ${ }^{234} \mathrm{U}$ (4.72 and $4.78 \mathrm{MeV}$ alpha energies) and ${ }^{237} \mathrm{~Np}$ (4.77 and $4.79 \mathrm{MeV}$ alpha energies) cannot be resolved by alpha spectrometry. The purified $\mathrm{Np}$ fraction was then co-precipitated and mounted for alpha counting in accordance with procedure RPG-CMC-496, Rev. 0, Coprecipitation Mounting of Actinides for Alpha Spectroscopy, and counted by alpha spectrometry in accordance with procedure RPG-CMC-422, Rev. 2, Solutions Analysis: Alpha Spectrometry. Each alpha spectrum was assessed for ${ }^{237} \mathrm{~Np}$. An internal tracer was not used, and the results are reported uncorrected. Each alpha spectrum was also examined for the presence of ${ }^{238} \mathrm{U}$ to assess the degree of separation from the $U$ and potential ${ }^{234} \mathrm{U}$ interference. A ${ }^{237} \mathrm{~Np}$ BS and MS (spiked into the acid digestate at the workstation) were analyzed with each analytical batch. The recovery of the BS and MS provide information on batch performance. See the analytical report in Appendix B for additional detail.

\subsubsection{Plutonium and Americium (Alpha Energy Analysis)}

Core samples were analyzed for ${ }^{238} \mathrm{Pu},{ }^{239+240} \mathrm{Pu}$, and ${ }^{241} \mathrm{Am}$ concentrations. An initial gross alpha screen analysis was conducted on the acid digestate to optimize the test sample size and tracer addition (gross alpha results are included in the radiochemistry reports provided in Appendices B and C). The acid digestate aliquots were traced with ${ }^{242} \mathrm{Pu}$ and ${ }^{243} \mathrm{Am}$; these were used as internal standards correcting for radiochemical yields and counter efficiencies. The BS and MS were also spiked (post-digestion) with ${ }^{239} \mathrm{Pu}$ and ${ }^{241} \mathrm{Am}$. Each aliquot was then processed through a strong-base anion exchange resin bed in a hydrochloric acid matrix in accordance with procedure RPG-CMC-4017. The Pu and Am fractions were collected separately. The purified Pu and Am fractions were co-precipitated and mounted for alpha counting in accordance with procedure RPG-CMC-496 and counted by alpha spectrometry in accordance with procedure RPG-CMC-422.

The Pu alpha spectra were evaluated for ${ }^{238} \mathrm{Pu}$, ${ }^{239+240} \mathrm{Pu}$, and tracer ${ }^{242} \mathrm{Pu}$. Note that the ${ }^{239} \mathrm{Pu}$ and ${ }^{240} \mathrm{Pu}$ have nearly identical alpha energies and cannot be resolved; these isotope activities are reported as a sum. Each spectrum was also examined for the presence of ${ }^{243} \mathrm{Am}$ tracer to assess the degree of separation from Am. The $\mathrm{U}$ isotopes alpha emission energies do not interfere with those of Pu isotopes.

The Am alpha spectra were evaluated for ${ }^{241} \mathrm{Am}$ and tracer ${ }^{243} \mathrm{Am}$. Each spectrum was also examined for the presence of ${ }^{239+240} \mathrm{Pu}$ to assess the degree of separation from $\mathrm{Pu}$. Alpha emission energies from $\mathrm{U}$ isotopes do not interfere with those of Am isotopes. 


\subsubsection{Alpha Screen (Alpha Energy Analysis)}

Aliquots from acid digestion of container composite samples and fusion processing of UDS from both core samples and container composite samples were measured for alpha emitters from their AEA spectra. An initial gross alpha screen analysis was conducted on the acid digestate to optimize the test sample size (gross alpha results are included in the radiochemistry reports provided in Appendices $\mathrm{C}$ and $\mathrm{D}$ ). The acid digestate aliquots were processed directly by co-precipitation with $\mathrm{NdF}_{3}$ and mounted for alpha counting in accordance with procedure RPG-CMC-496. The mounts were counted by alpha spectrometry in accordance with procedure RPG-CMC-422. The alpha spectra were evaluated for ${ }^{238} \mathrm{Pu} /{ }^{241} \mathrm{Am}$ (unresolved spectral peaks), ${ }^{239+240} \mathrm{Pu}$, and ${ }^{243+244} \mathrm{Cm}$ (opportunistically) for spectral peaks. Spectral peaks from $U$ isotopes were not present. From an activity concentration basis, ${ }^{234} U$ and ${ }^{238} U$ were at least $400 \times$ less than that of ${ }^{238} \mathrm{Pu} /{ }^{241} \mathrm{Am}$ and ${ }^{239+240} \mathrm{Pu}$ in the original core samples. Batch QC for the alpha screen included a process sample duplicate, process blank, BS, and MS. The BS and MS samples were postdigestion spiked with ${ }^{239} \mathrm{Pu}$ at the workstation. See Appendices $\mathrm{C}$ and $\mathrm{D}$ for additional information.

\subsubsection{Gamma Energy Analysis}

Core and container composite sample acid digestate aliquots $(2 \mathrm{~mL}$ and/or $10 \mathrm{~mL}$ in glass liquid scintillation counter [LSC] vials) were counted directly on high-purity germanium detectors operated with Canberra GENIE2000 Gamma Analysis Software (VMS and PC versions). Analysis was conducted according to RPG-CMC-450, Rev. 1, Gamma Energy Analysis (GEA) and Low-Energy Photon Spectrometry (LEPS). Because this was a direct analysis (i.e., no chemical separations were applied), no LCS, BS, or MS samples were required or prepared. Energy and efficiency calibrations were checked through statistical process control of the counter calibration verification standards. Sample count times were established such that the required MDLs for all AOIs could be reached. Interference from Compton scattering of high ${ }^{137} \mathrm{Cs}$ concentrations may have affected achievable detection limits of other (opportunistic) gamma-emitting isotopes at energies $<661 \mathrm{keV}$.

Solids (e.g., UDS) were similarly placed in glass LSC vials for direct GEA measurement. Unlike liquid samples, their geometries could not be carefully controlled to match the calibration standards. The solids samples were counted at $\sim 10 \mathrm{~cm}$ from the detector face to minimize the differences between the sample geometry and the calibration geometry. (Calibrations are performed using aqueous samples and reproducible geometries.) The UDS samples consisted of mostly resin beads (based on observation) and silica (based on XRD analyses, however, the exact sample mineralogical composition and associated density were not known). The small sample size ( $1 \mathrm{~g})$ minimized the absorption effects; therefore, density corrections were not applied. See Appendices B, C, and D for additional information.

\subsubsection{Strontium-90 Analysis}

An initial gross beta screen analysis was conducted on each core sample acid digestate to optimize the test sample size (results are included in the radiochemistry report provided in Appendix B). Aliquots of the acid digestate were processed through Sr Resin (Eichrom Technologies, Lisle, IL) to purify the Sr fraction in accordance with procedure RPG-CMC-476, Rev. 0, Strontium Separation Using Eichrom Strontium Resin, (tracer- and carrier-free). The purified Sr fraction was then analyzed by LSC in accordance with procedure RPG-CMC-474, Rev. 1, Measurement of Alpha and Beta Activity by Liquid Scintillation Spectrometry. See the ASO report in Appendix B for additional information on instrument calibration and control. The gross beta screen results were compared with the sum of beta emitters $\left({ }^{137} \mathrm{Cs}\right.$ 
and ${ }^{90} \mathrm{Sr} /{ }^{90} \mathrm{Y}$ ) for indication of agreement. BS and MS samples were prepared at the analytical workstation by spiking with a ${ }^{90} \mathrm{Sr}$ standard into the acid digestate.

\subsubsection{Total Uranium (Kinetic Phosphorescence Analysis)}

Aliquots of the core sample acid digestates were diluted $(\sim 10,000 \times)$ and analyzed directly by KPA (Chem Chek Instruments, Richland, WA) in accordance with procedure RPG-CMC-4014, Rev. 1, Uranium by Kinetic Phosphorescence Analysis. A hot cell BS prepared with only added U was provided with the samples. The MS sample (U spiked into the acid digestate) was prepared at the analytical workstation (post-digestion spike). Calibration verification standards were also analyzed during the analytical run to assess calibration stability. See the analytical report for additional detail in instrument calibration and calibration verification (Appendix B).

\subsubsection{Uranium Isotopic Analysis}

Aliquots of each core sample acid digestate were processed in accordance with procedure RPG-CMC4017 to purify the U component. Subsample aliquot sizes ( $0.1 \mathrm{~mL}$ of the acid digestate) were determined after analyzing the digestate total $\mathrm{U}$ concentrations by ICP-OES. The PB (1 mL acid digestate) was also prepared for TIMS analysis.

A portion of the isolated $U$ fraction was then mounted on a Re filament and analyzed by TIMS on a CEC (Consolidated Electrodynamics Corp.) 21-703 single stage mass spectrometer in accordance with procedure PNNL-98523-264, Rev. 2, Mass Spectrometer Isotopic Analysis (TIMS). The calibration of the instrument was assessed with U standard reference material NIST U-030. Details of the sample analysis and instrument calibrations are located in Appendix E.

\subsubsection{Plutonium Isotopic Analysis}

Aliquots of each of the core sample acid digestates were processed in accordance with procedure RPG-CMC-455, Rev. 0, Separation of Uranium and Plutonium for Isotopic Analysis by Mass Spectrometry to isolate the Pu fraction using strong base anion exchanger. Subsample aliquot size ( $0.1 \mathrm{~mL}$ of undiluted digestate) was determined after analyzing the digestate Pu concentrations by AEA. The PB ( $1 \mathrm{~mL}$ acid digestate) was also prepared for TIMS analysis. No tracer was added because $\mathrm{Pu}$ atom ratios were needed.

A portion of the isolated Pu fraction was then mounted on a Re filament and analyzed by TIMS in accordance with procedure PNNL-98523-264. The calibration of the instrument was assessed with $\mathrm{Pu}$ standard reference material NIST Pu-946. Details of the sample analysis and instrument calibrations are located in Appendix E.

The ${ }^{238} \mathrm{U}$ is a major component of the sample matrix. Despite care in the separation, a very small carry-over of $U$ into the Pu fraction is expected to interfere with the analysis of ${ }^{238} \mathrm{Pu}$ (from the ${ }^{238} \mathrm{U}$ isobar). Therefore, the ${ }^{238} \mathrm{Pu}$ analysis by AEA is expected to provide more accurate results for this radioisotope. The correct contribution of ${ }^{238} \mathrm{Pu}$ with respect to ${ }^{239} \mathrm{Pu}$ was determined by the following steps: 
- The AEA provides relative amounts of ${ }^{238} \mathrm{Pu}$ and combined ${ }^{239,240} \mathrm{Pu}$ in terms of activities $(\mu \mathrm{Ci})$ per gram of sludge. In the AEA, the ${ }^{238} \mathrm{U}$ interference does not contribute to the ${ }^{238} \mathrm{Pu}$ or to the ${ }^{239,240} \mathrm{Pu}$ AEA peaks.

- The relative contributions of ${ }^{239} \mathrm{Pu}$ and ${ }^{240} \mathrm{Pu}$ to the ${ }^{239,240} \mathrm{Pu}$ AEA peak are apportioned based on the ${ }^{239} \mathrm{Pu}$ and ${ }^{240} \mathrm{Pu}$ TIMS values and the specific activities of the individual ${ }^{239} \mathrm{Pu}$ and ${ }^{240} \mathrm{Pu}$ isotopes. This provides activities of the individual ${ }^{239} \mathrm{Pu}$ and ${ }^{240} \mathrm{Pu}$ isotopes per gram of sludge.

- The activities of the ${ }^{238} \mathrm{Pu}$ and the ${ }^{239} \mathrm{Pu}$ per gram of sludge then are known.

- The atom percent (At\%) of ${ }^{238} \mathrm{Pu}$, with respect to the At\% of ${ }^{239} \mathrm{Pu}$ reported by TIMS is calculated using the following equation:

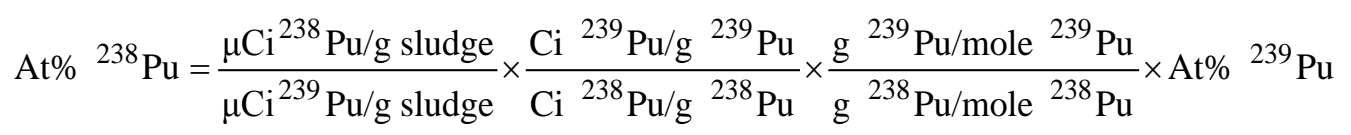

in which the first term includes the specific activities of ${ }^{238} \mathrm{Pu}$ and ${ }^{239} \mathrm{Pu}$ in the sludge, the second term includes the specific activities of ${ }^{238} \mathrm{Pu}$ and ${ }^{239} \mathrm{Pu}$ with respect to their isotopically pure element (from National Bureau of Standards [NBS] values), the third term includes the atomic weights of the ${ }^{238} \mathrm{Pu}$ and ${ }^{239} \mathrm{Pu}$ isotopes (from NBS values), and the fourth term is the At $\%$ of ${ }^{239} \mathrm{Pu}$ found by TIMS.

- Because the At $\%{ }^{238} \mathrm{Pu}$, with respect to the At $\%{ }^{239} \mathrm{Pu}$ (TIMS), as determined by this method, is less than the ${ }^{238} \mathrm{Pu}$ value reported by TIMS, the individual plutonium isotope percentages, by the above calculation for ${ }^{238} \mathrm{Pu}$ and by TIMS for the other Pu isotopes, are summed and renormalized. This calculation approach provided the correct At\% of all of the plutonium isotopes.

\subsubsection{Uranium Speciation-Metal and Oxidation State Ratio}

Uranium speciation was determined on the core and container composite samples following a stepwise acid dissolution, spectrophotometry, and U analysis by ICP-OES. The U speciation included analysis for the $\mathrm{U}$ oxidation state (U[IV] and $\mathrm{U}[\mathrm{VI}]$ ) distribution for the uranium oxides and analysis of the $U$ metal concentration. Most of the samples taken for $U$ speciation were not dried; a small amount of $\mathrm{K}$ Basin water had been added to the as-sampled wet solids shortly after the sample was taken to protect them from drying during the interim storage period before analysis. However, the container composite middle-sieve fraction samples (i.e., $-2,000 \mu \mathrm{m} /+500 \mu \mathrm{m}$ ) had been air-dried in the SAL.

The samples were processed in accordance with RPG-CMC-107, Rev. 1, Sample Preparation for Determining Uranium Metal Concentrations in Sludge. The ASO reports (Appendices B and C) provide details of sample digestion processing and analysis. The post-digested phosphoric acid solution was provided for spectrophotometric analysis to determine the distribution to the U(IV) and U(VI) oxidation states in accordance with procedure RPG-CMC-255, Rev. 0, Sample Preparation and Analysis for Determining Uranium Oxide Oxidation States in K Basin Sludges. The detection limit for both U(IV) and $\mathrm{U}(\mathrm{VI})$ is $0.001 \mathrm{M}$. The post-digested nitric acid solution was provided for $\mathrm{U}$ analysis by ICP-OES in accordance with procedure RPG-CMC-211 to determine U metal concentration. The process quantitation limit (PQL) is $0.03 \mathrm{wt} \% \mathrm{U}$ metal in the sludge matrix; the detection limit is $0.011 \mathrm{wt} \% \mathrm{U}$ metal, both on a settled sludge basis. Unlike other analyte concentrations reported by the ASO (reported on a dry mass basis), the $U$ metal results were reported on an as-sampled mass basis. 
Batch QC samples included sample duplicates, one process blank, and one LCS. Each LCS contained $\sim 0.5 \mathrm{~g} \mathrm{U}_{3} \mathrm{O}_{8}, 0.6 \mathrm{~g}$ Hanford sand, and $\sim 0.01 \mathrm{~g} \mathrm{U}$ metal (weighed to the nearest $0.00001 \mathrm{~g}$ ). The LCS served to determine the method performance for $\mathrm{U}$ oxide oxidation state distribution $\left[\mathrm{U}_{3} \mathrm{O}_{8}\right.$ is $33.3 \%$ $\mathrm{U}(\mathrm{IV})]$ and for U metal recovery. A MS sample was also analyzed but was not required by the QAPJP/SAP; it was included to provide additional information on method performance. The MS sample was prepared by adding $\sim 0.01 \mathrm{~g} \mathrm{U}$ metal to a triplicate sample of sludge.

Previous U oxidation state measurements of K Basin sludge have shown that a known interference in the sludge matrix is present and that U(IV) and U(VI) oxidation state results should be used with caution. Ferrous iron (Fe[II]), present as magnetite, has been observed in K Basin sludge (Fiskum et al. 2011a, b) and has been shown to chemically reduce $\mathrm{U}(\mathrm{VI})$ to $\mathrm{U}(\mathrm{IV})$ in $\mathrm{Na}_{2} \mathrm{SO}_{4} / \mathrm{H}_{3} \mathrm{PO}_{4}$ solutions. While the amount of Fe(II) is not known, the concentration of Fe in sludge samples is high enough (at 3 to $10 \mathrm{wt} \%$ on a dry basis) to cause concern. The U(VI) fractions can be considered lower bounding values and the U(IV) fractions the upper bounding values.

\subsubsection{Total Organic Carbon and Total Inorganic Carbon}

Samples collected for TOC and TIC measurements were distributed directly to the workstation for analysis in accordance with RPG-CMC-385, Rev. 2, Carbon Measured in Solids, Sludge, and Liquid Matrices. This method was revised specifically for $\mathrm{K}$ Basin work to accommodate measurements of organic carbon that potentially include organic ion exchange resin (OIER) and Grafoil ${ }^{\circledR}$ via sequential temperature combustion at 600 and $1,000^{\circ} \mathrm{C}$, respectively (Fiskum et al. 2011a). The combustion method was required to convert OIER to $\mathrm{CO}_{2}$. (Oxidation of OIER in solution using the Ag[I] catalyzed persulfate oxidation method at $\sim 95^{\circ} \mathrm{C}$ was not effective.) Analytical sample size is generally limited so that the product $\mathrm{CO}_{2}$ does not exceed 5,000 $\mu \mathrm{g}$ (as $\mathrm{C}$ ). Special care was further required of the sludge samples because of their high activity and requisite drying, which resulted in a dispersible powder.

The TIC/TOC subsamples collected from each core sample were dried to constant weight at $\sim 105^{\circ} \mathrm{C}$ such that the sample mass basis could be directly related to the settled sludge mass basis using the conversion factors from physical property samples. Small subsamples from each dried sample were transferred to quartz reaction vessels and weighed. Each subsample was then transferred to the tube furnace set at $600^{\circ} \mathrm{C}$ and allowed to react with the carrier oxygen sweep gas. The gas was processed through barium chromate catalyst to ensure complete oxidation of carbon to $\mathrm{CO}_{2}$. The $\mathrm{CO}_{2}$ was absorbed in a solution and its reaction product titrated from an electrolytically generated titrant; the titrant was measured in terms of coulombs. All $\mathrm{CO}_{2}$ collected from the $600^{\circ} \mathrm{C}$ oxidation was attributed to TOC. Once the combustion at $600^{\circ} \mathrm{C}$ was completed, the same analytical subsample was then subjected to the same oxidation process except at $1,000^{\circ} \mathrm{C}$, a process that was shown to oxidize carbonate compounds and Grafoil ${ }^{\circledR}$ to $\mathrm{CO}_{2}$. All $\mathrm{CO}_{2}$ collected from the $1,000^{\circ} \mathrm{C}$ oxidation temperature was attributed to TIC.

The Coulometrics coulometer is calibrated at the factory; instrument calibration is not performed as part of this procedure. However, a minimum of two initial calibration verification samples (ICVs) $\alpha-D$ glucose, and two initial blanks are analyzed before sample analysis, after analysis of every 15 samples, and at the end of the day. The results for all samples are corrected for the average contribution from the blanks and average recovery of the ICVs.

Method performance is evaluated from the LCS and MS sample each spiked with $\alpha$-D-glucose and calcium carbonate. For the ASR 8798 analyses sequential temperature oxidation method performance 
was further evaluated with a mixture of Purolite ${ }^{\circledR}$ NRW37 OIER and Grafoil ${ }^{\circledR}$ that was processed both before and after the sludge samples were processed. Triplicate measurements were made on small subsamples from each duplicate sludge sample providing a total of six measurements per core sample.

\subsection{X-Ray Diffraction}

Samples were prepared for XRD analysis in accordance with procedure PNNL-RPG-268, Rev. 4, Solids Analysis by X-Ray Diffraction. Samples TI008-SH and TI008-SJ were provided as slurries and required an initial drying step. Each vial containing slurry material was covered with filter paper such that water (vapor) could pass through, yet contain particulate. To enhance the drying process, each vial was placed in a bed of Drierite and evaporation continued at room temperature until complete (based on visual examination). In general, the process took several days. All other samples were provided as dry material and were used as received.

All the samples were mixed with amyl acetate to produce slurries, transferred into a boron carbide mortar, and ground to a fine powder. After grinding, a small fraction of each sample was pipetted onto individual Krylon-covered glass slides (one sample per slide) and allowed to dry. The dried samples were then covered with Kapton ${ }^{\circledR}$. Selected sample preparations were mixed with the rutile $\left(\mathrm{TiO}_{2}\right)$ internal standard from SRM 674b.

Each sample was analyzed using a Rigaku Ultima IV diffractometer XRD unit equipped with a high speed position sensitive detector system D/teX and a copper X-ray tube (Cu K $\alpha$ radiation, $\lambda=1.5418 \AA$ ). Data were collected in a step scan mode between 5 and $65^{\circ}$ 2-theta with a step size of $0.02^{\circ}$, at a speed of $2 \%$ min. The X-ray tube operating conditions were $40 \mathrm{kV}$ and $44 \mathrm{~mA}$.

Crystalline phase identification was conducted using JADE software, Version 9.0 (Materials Data Inc., Livermore, CA), search match routines with comparison to the International Centre for Diffraction Data (ICDD) database Powder Diffraction File (PDF)-2 (2009). The ICDD database included the Inorganic Crystal Structure Database maintained by Fachinformationszentrum, Karlsruhe, Germany. Phase identification incorporated chemistry restrictions based on the elements determined from chemical analysis. In this case, the search included the following elements: $\mathrm{C}, \mathrm{Na}, \mathrm{K}, \mathrm{Mg}, \mathrm{Ca}, \mathrm{B}, \mathrm{Al}, \mathrm{Sr}, \mathrm{Si}, \mathrm{O}, \mathrm{H}$, $\mathrm{Cd}, \mathrm{Cr}, \mathrm{Ba}, \mathrm{Cu}, \mathrm{Ni}, \mathrm{Pb}, \mathrm{Mn}, \mathrm{Fe}, \mathrm{Zn}, \mathrm{Zr}$, Ti, and U. Selection of these elements addresses the evaluation of hydrate compounds, Al hydrates, U hydrides, U oxides, and $\mathrm{Zr}$ metal plus any other identifiable compounds.

\subsection{Particle Density}

Sample particle density was determined by gas pycnometry using an AccuPyc 1330 (Micromeritics, Norcross, GA) in accordance with procedure APEL-PIP-4, Rev 2, Gas Pycnometry Method for Apparent Specific Gravity Determinations of Consolidated Solids. This method measures the amount of displaced ultra-high purity (UHP) He gas in each sample as determined by pressure. The two pressures observed upon 1) filling the sample chamber and then 2) discharging it into a second empty chamber allowed computation of the sample solid phase volume using Boyle's Law. In this method, gas molecules rapidly fill the tiniest pores of the sample; thus, only the truly solid phase of the sample displaced the gas. Ultimately, the sample volume was assessed according to Equation 2.9 (Lowell 2004): 
PNNL-20650, Rev. 2

$$
\mathrm{V}_{\mathrm{S}}=\mathrm{V}_{\mathrm{C}}+\left(\frac{\mathrm{V}_{\mathrm{R}}}{1-\frac{\mathrm{P}_{1}}{\mathrm{P}_{2}}}\right)
$$

where $V_{\mathrm{S}}=$ sample volume

$\mathrm{V}_{\mathrm{C}}=$ volume of the empty sample chamber

$\mathrm{V}_{\mathrm{R}}=$ reference volume

$\mathrm{P}_{1}=$ first pressure measured (sample chamber only)

$\mathrm{P}_{2}=$ second pressure measured (after expansion of the gas into the combined volumes of sample chamber and reference chamber).

Once the sample volume was determined, the density was calculated based on the sample mass introduced into the pycnometer. The pycnometer was calibrated per the manufacturer's instructions using a certified calibration standard and confirmed with a secondary certified standard at the beginning of each series of analyses.

Duplicate sample slurries were collected from the $-500-\mu \mathrm{m}$ sieve fraction of the container composite sample and required an initial drying step. The samples were dried at ambient conditions within the glovebox to provide proper containment of the highly radioactive materials. The $+2,000-\mu \mathrm{m}$ and the $-2,000-\mu \mathrm{m} /+500-\mu \mathrm{m}$ sieve fraction samples were submitted for analysis already air-dried (in the SAL).

Analytical samples sizes were volume-limited to fit within the 1- $\mathrm{cm}^{3}$ pycnometer sample cup. The subsampling was conducted such that the provided sample could be nearly quantitatively transferred to the analysis cup thus eliminating additional subsampling error. Each sample was placed in the cup, weighed, and analyzed five sequential times; the results were averaged and the density, with standard deviation of the average, was reported. Details of the sample analysis are provided in Appendix G.

\subsection{Particle Size Distribution}

PSD was determined on duplicate sample slurries collected from the $-500-\mu \mathrm{m}$ sieve fraction of the container composite sample in accordance with procedure RPL-COLLOID-01 Rev. 1, Particle Size Analysis Using Malvern MS2000. Specific processing parameters were delineated in TI 52578-TI037, Particle Size Distribution Measurements for K West Basin Consolidated Container Composite SCS-CON210, Using Minus 500 Micron Particle Size Fraction Samples.

A Mastersizer 2000 (Malvern Instruments Ltd., Worcestershire, UK) equipped with a Hydro $\mu \mathrm{P}$ dispersion unit was used to analyze the samples. The instrument software (Mastersizer 2000 Version 5.60, Malvern Instruments, Ltd., Copyright 2009) was used to calculate the PSD (i.e., fractional volume contribution versus particle diameter) from the laser diffraction patterns using Mie scattering theory (see instrument manual and Malvern product literature for additional information). Based on the equipment specifications, particles with diameters between 0.02 and 2,000 $\mu \mathrm{m}$ can be analyzed by the Malvern Mastersizer 2000; however, it is a challenge for the Hydro $\mu \mathrm{P}$ dispersion unit to suspend and detect greater than $\sim 150 \mu \mathrm{m}$ diameter components (and/or high density materials). 
The QAPJP/SAP does not provide specific precision or accuracy requirements for PSD measurements, but defers to procedural controls. Therefore, per procedure, the Malvern analyzer was to be checked with size distribution standards once at the beginning of a series of analyses that may span several days not exceeding a calendar quarter ( 90 days). The median value $\left(\mathrm{D}_{50}\right)$ of three standard measurements should deviate less than a target value of $10 \%$ from the certified value together with its standard deviation unless other criteria are provided by the manufacturer of the standard material. Before conducting particle-size measurements with sludge samples, the PSD of a particle size standard, Malvern Quality Audit Standard QAS3004, traceable to NIST was measured to verify instrument performance. The standard consisted of polydispersed silica particles with diameters falling primarily between 13 and $130 \mu \mathrm{m}$. The results from this measurement were compared to the standard's certificate of analysis provided by the supplier, and the acceptable performance of the size analyzer was confirmed before running sludge samples in accordance with procedure RPL-COLLOID-01.

An in-depth study (initial parameter evaluation) was conducted using K Basin simulants (Burns et al. 2011) and on actual K Basin sludge samples (Fiskum et al. 2011b). The parameters evaluated included: refractive index, particle adsorption index, shape factor (spherical/non-spherical), recirculation time, recirculator rpm (mixing/resuspension), and sonication (power and duration). From these studies, a standard set of operational and analysis parameters were developed and recommended for use when measuring other K Basin sludge samples to provide a consistent PSD measurement method for all samples. For the current study, the same instrument configuration and operational/analysis parameters were used for the $-500-\mu \mathrm{m}$ sieve fraction samples collected from the SCS-CON-210 container composites. Appendix I provides detailed information on the selected run parameters.

Sample slurries received from the SAL were subsampled using a transfer pipet. The slurry was mixed and solids suspended by rapid transfer in and out of the transfer pipet. Then, a sample was collected and transferred dropwise into the Hydro $\mu \mathrm{P}$ dispersion unit until obscuration was satisfactory. The sampled material was analyzed for PSD in accordance with Table 2.14 and then removed from the system.

Table 2.14. Condition Matrix for PSD Determinations

\begin{tabular}{ccc}
\hline Recirculation Rate, $\mathrm{RPM}^{(\mathrm{a})}$ & Recirculation Time, min $^{(\mathrm{a})}$ & Sonication $^{(\mathrm{a}, \mathrm{b})}$ Power, Time \\
\hline 2,000 & 1 & Pre-sonication \\
2,000 & 3 & $100 \% / 120$ sec \\
2,000 & 5 & Post-sonication \\
\hline
\end{tabular}

(a) The two initial measurement conditions for recirculation rpm, recirculation time and sonication power and time are based on results of the parameter evaluation in TI 52578TI017 (Fiskum et al. 2011b). A third measurement condition (at 5 min recirculation) was added to further evaluate the impacts of sonication on the measured PSD.

(b) The Hydro $\mu \mathrm{P}$ dispersion cell employed for particle size analysis is equipped with variable power sonicator with an ultrasound power of up to 20W (at an instrument sonicator setting of $100 \%)$.

Other Measures/Conditions

Number of PSD measurements/conditions: each measurement consists of three separate observations (with each observation consisting of a10 s red light measurement and a $10 \mathrm{~s}$ blue light measurement). 


\subsection{Rheology/Shear Strength Measurement}

Rheology testing (shear strength) was conducted on the whole PSD (i.e., unseived) of the container composite (KW210-CC-A) in accordance with procedure RPL-COLLOID-02 Rev. 1, "Measurement of Physical and Rheological Properties of Solutions Slurries and Sludges”. Specific processing parameters were delineated in TI 52578-TI039, Rheological Measurements for K West Basin Consolidated Container Composite SCS-CON-210.

The QAPjP/SAP does not provide specific accuracy or precision requirements for these tests, but defers to procedural controls. Viscometer performance checks were conducted before and after a series of measurements (series time window of up to 30 days) with NIST-traceable viscosity standards. For testing with SCS-CON-210 materials, a $96.0 \mathrm{cP}$ viscosity standard purchased from Brookfield Engineering Laboratories was employed. The acceptance criterion of $\pm 10 \%$ applies for this standard and translates to an acceptable viscosity range of 86.4 to $105.6 \mathrm{cP}$

Shear strength was measured on settled sludge samples. The measurements were performed in the SAL with a Haake RV20-M5 viscometer. The RV20-M5 viscometer is capable of evaluating fluid rheological properties using Couette flow devices or shear strength using shear vanes.

For shear strength measurements, two different shear vane tools were used: 1 ) a $6 \times 6 \mathrm{~mm}$ (vane diameter by height) four-paddle vane that can measure strengths up to $110,000 \mathrm{~Pa}$ and 2) a $16 \times 16 \mathrm{~mm}$ (vane diameter by height) four-paddle vane that can measure strengths up to $\sim 5,700 \mathrm{~Pa}$. Vane tool selection is dependent on expected sample shear strength and sample container geometries. The $6 \times 6 \mathrm{~mm}$ vane tool was employed for the initial shear strength characterization of the as-received material to accommodate the potential for higher-than-expected shear strengths. The $16 \times 16 \mathrm{~mm}$ vane was used on all other shear strength characterizations.

RPL-COLLOID-02 outlines geometrical spacing requirements for vane tool and solids containers when testing for shear strength. For all of the test cases reported herein, the combination of container and sludge volume yielded a settled solids configuration that met these test geometry requirements.

\subsection{Viscosity Measurement}

Apparent viscosity was not determined on the SCS-CON-210 container composite. The unseived material contained a significant amount of large particles $(>500 \mu \mathrm{m})$ which would not meet the minimum requirements for the geometry of the flow curve measurement apparatus in SAL. A minimum geometry gap of 3 times the largest particle diameter is required for flow curve measurements to ensure that the material does not get caught inside the geometry; a factor of 10 is desirable. The maximum gap available to us in SAL is the MV2 geometry, this geometry has a gap of $2.6 \mathrm{~mm}$ which does not permit the analysis of particles with one linear dimension greater than $\sim 850 \mu \mathrm{m}$. The $-500-\mu \mathrm{m}$ sieve fraction material did not contain enough sample volume to support the flow curve measurement. This scope deletion was directed/concurred by the BTR. 


\subsection{Physical Property Characterization Samples: Sludge Densities and Conversion Factors to Dry and Wet Mass Bases}

This section provides a summary of the results generated from the physical property characterization subsamples obtained from the core and container composite samples from Engineered Container SCS-CON-210. These include wet sludge densities (settled and as-sampled mass bases), weight percent (wt\%) solids, and mass conversion factors as follows:

- dry mass: sampled sludge mass

- as-sampled sludge mass: settled sludge mass

- dry mass: settled sludge mass.

\subsection{Processing Description}

Two wet-density bases are given for each sample: settled density and as-sampled density. As-sampled density refers to the thickened state of sludge at the point at which subsamples were collected from the parent sample. Before subsampling was conducted, the sludge was batch-settled, excess water was removed, and the sludge was mixed and allowed to re-settle. This process of dewatering, mixing, and settling was continued until a thickened sludge state was obtained (i.e., a state at which the sludge possessed sufficient shear strength to suspend/uniformly disperse any dense uranium metal particles) (Figure 3.1). Attainment of the thickened state was a subjective measure based on visual observation of the rheology (i.e., sludge flow from the mixing spoon). Once sufficiently thickened, the sludge was mixed and then subsampled (i.e., as-sampled sludge). The mass and volume of the as-sampled sludge in the various subsample containers was measured. Processing details are provided in Section 2.3.

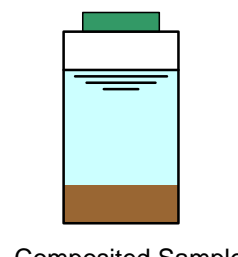

Composited Sample

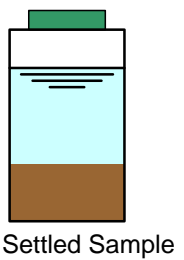

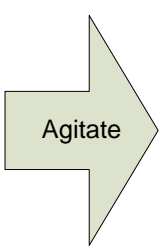

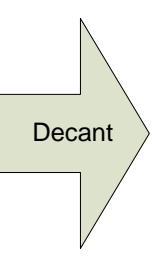

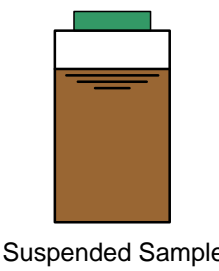
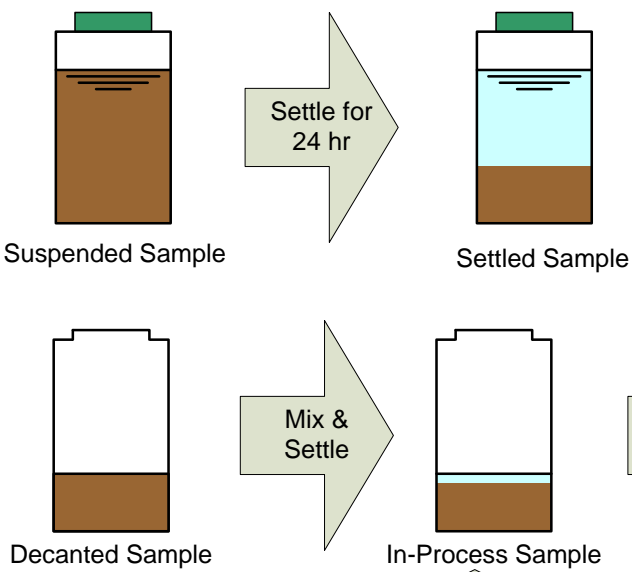

Settled Sample
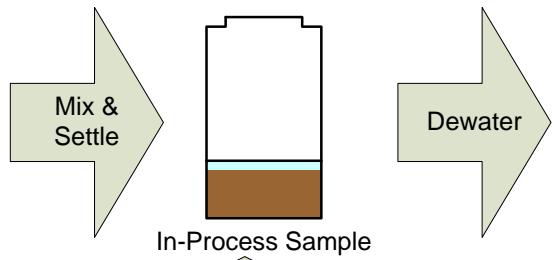

Dewatered Sample

Repeat Mix and Settle Until Sludge Strength Sufficient

to Suspend Dense Particles

Figure 3.1. Sample Preparation for Subsampling 
After the as-sampled sludge mass and volume were measured, select samples, including the physical property samples, were returned to a settled sludge state. K Basin water was added to the as-sampled sludge, and the water and sludge were mixed to create a suspension (typically 20 to $80 \mathrm{vol} \%$ settled solids and more narrowly $~ 50$ vol\% settled solids for the primary physical property subsamples), and then the suspension was allowed to settle undisturbed. The mass and volume of the settled sludge were measured to provide settled sludge densities in accordance with the QAPJP/SAP (see Section 2.3). Figure 3.2 depicts the sample manipulation processes. The settled sludge density values in Table 3.1 represent the undisturbed, settled sludge volume ( 24-h).

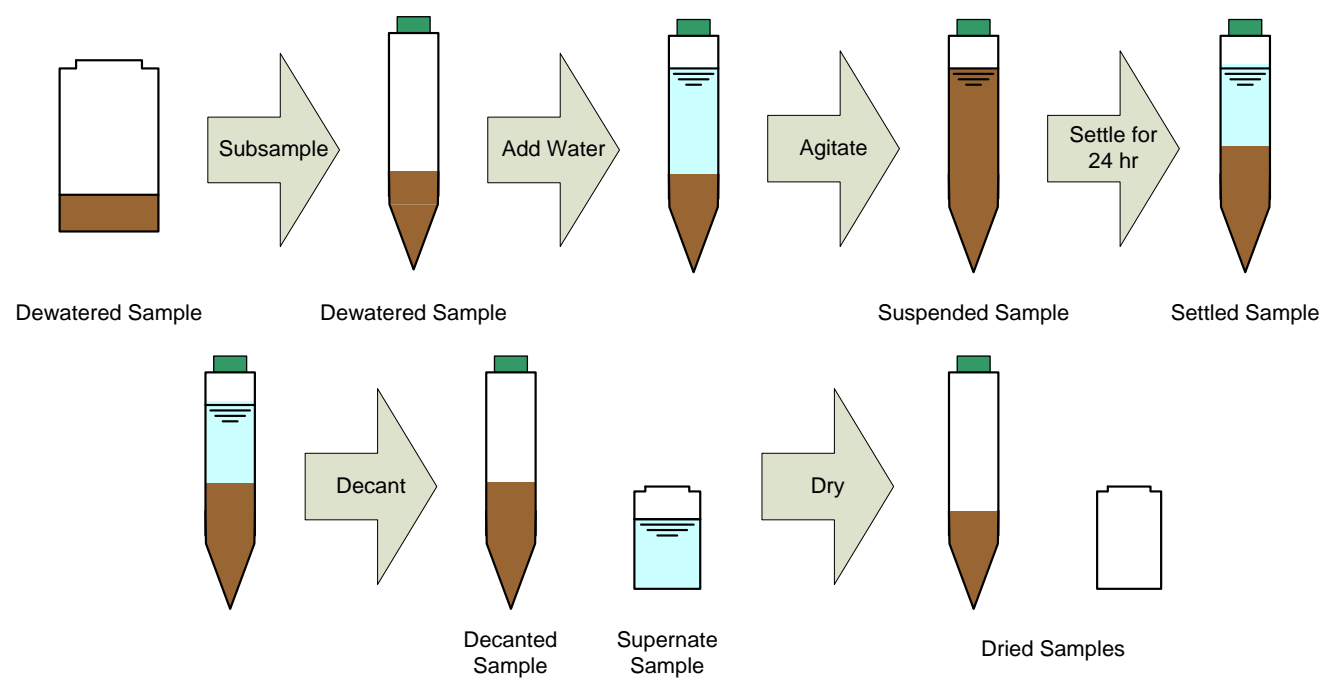

- Top row shows collection of a dewatered sample and how the dewatered sample is returned to a settled sludge state.

- Bottom row shows the processing of a sample from a settled sludge state to a dry state.

Figure 3.2. Sample Processing for Physical Property Measurements

\subsection{Physical Property Results}

Table 3.1 provides the sludge densities and conversion factors for all samples from SCS-CON-210. The values are relevant for understanding analyte concentration data presented later in this report. Details on how the density and wt\% solids measurements were performed are provided in Section 2.3. Opportunistic data obtained from the reconstituted core samples and other characterization subsamples are also presented in Table 3.1 for comparison purposes.

The average physical properties of the primary sample pairs that were specifically collected for physical property measurements are shown in bold text in Table 3.1 - along with related uncertainties. These average values were used to convert analyte concentration data from a dry solids mass basis to a wet settled sludge mass basis (chemical characterization results) or from an as-sampled sludge mass basis to a settled sludge mass basis ( $\mathrm{U}$ metal results) in subsequent sections of this report.

The opportunistic data gathered from the core reconstitution and container composite vessels (large sample masses) and other analytical subsamples (small sample masses) are shown in italics as supplemental information. These data provide an estimate of the overall representativeness and reproducibility of the average values and demonstrate that the density values were generally consistent at a range of sample sizes. 
Table 3.1. Sludge Densities and Mass Conversion Factors for Samples from SCS-CON-210

\begin{tabular}{|c|c|c|c|c|c|c|c|c|c|c|c|}
\hline \multirow[b]{2}{*}{$\begin{array}{l}\text { Core and Sample } \\
\text { Identification }\end{array}$} & \multirow{2}{*}{$\begin{array}{l}\text { As-Sampled } \\
\text { Sludge } \\
\text { Mass, }^{(a)} \mathrm{g}\end{array}$} & \multicolumn{2}{|c|}{ Settled Density ${ }^{(a)}$} & \multicolumn{2}{|c|}{ As-Sampled Density ${ }^{(\mathrm{a}, \mathrm{b})}$} & \multicolumn{6}{|c|}{ Mass Ratios ${ }^{(\mathrm{a})}$} \\
\hline & & $\mathrm{g} / \mathrm{cm}^{3}$ & $\begin{array}{l}\text { Relative \% } \\
\text { Uncertainty }\end{array}$ & $\mathrm{g} / \mathrm{cm}^{3}$ & $\begin{array}{l}\text { Relative \% } \\
\text { Uncertainty }\end{array}$ & $\begin{array}{l}\text { g dry / g } \\
\text { sampled }\end{array}$ & $\begin{array}{l}\text { Relative \% } \\
\text { Uncertainty }\end{array}$ & $\begin{array}{l}\text { g sampled / } \\
\text { g settled }\end{array}$ & $\begin{array}{l}\text { Relative \% } \\
\text { Uncertainty }\end{array}$ & $\begin{array}{l}\text { g dry / } \\
\text { g settled }\end{array}$ & $\begin{array}{l}\text { Relative \% } \\
\text { Uncertainty }\end{array}$ \\
\hline \multicolumn{12}{|l|}{ Core A1 ${ }^{(\mathrm{c})}$} \\
\hline TI001-A1-K & 14.74 & 1.33 & $10.1 \%$ & 1.40 & $9.5 \%$ & 0.5671 & $0.5 \%$ & 0.7649 & $7.4 \%$ & 0.4338 & $7.4 \%$ \\
\hline TI001-A1-L & 18.50 & 1.31 & $8.4 \%$ & 1.42 & $7.7 \%$ & 0.5724 & $0.4 \%$ & 0.8040 & $6.2 \%$ & 0.4602 & $6.2 \%$ \\
\hline $\begin{array}{l}\text { KW210-A1 } \\
\text { Average (K \& L) }\end{array}$ & --- & 1.32 & $6.6 \%$ & 1.41 & $6.1 \%$ & 0.5697 & $0.3 \%$ & 0.7845 & $4.8 \%$ & 0.4470 & $4.8 \%$ \\
\hline RPD & --- & $1.06 \%$ & --- & $1.37 \%$ & ---- & $0.93 \%$ & --- & $4.98 \%$ & --- & $5.91 \%$ & --- \\
\hline KW210-A1-1 & 245.83 & 1.38 & $8.2 \%$ & 1.46 & $7.1 \%$ & --- & --- & 0.8433 & $5.9 \%$ & --- & --- \\
\hline KW210-A1-A & 62.61 & 1.49 & $10.0 \%$ & 1.57 & $8.8 \%$ & --- & --- & 0.8686 & $6.9 \%$ & --- & --- \\
\hline TIO01-A1-B & 2.05 & --- & --- & --- & --- & 0.5476 & $3.9 \%$ & ---- & ---- & ---- & ---- \\
\hline TI001-A1-C & 2.28 & ---- & ---- & ---- & ---- & 0.5605 & $3.5 \%$ & ---- & ---- & ---- & ---- \\
\hline TI001-A1-M & 20.07 & 1.40 & $5.7 \%$ & 1.43 & $7.2 \%$ & ---- & ---- & 0.7977 & $1.2 \%$ & ---- & ---- \\
\hline TIO01-A1-N & 44.62 & 1.46 & $3.9 \%$ & 1.49 & $3.3 \%$ & ---- & ---- & 0.8491 & $2.7 \%$ & ---- & ---- \\
\hline KW210-A1-Archive-A & 16.76 & 1.23 & $40.1 \%$ & $\mathrm{NR}^{(\mathrm{d})}$ & ---- & --- & ---- & 1.020 & $30.5 \%$ & --- & --- \\
\hline \multicolumn{12}{|l|}{ Core A4 ${ }^{(\mathrm{c})}$} \\
\hline TI002-A4-K & 14.67 & 1.34 & $12.1 \%$ & 1.47 & $5.0 \%$ & 0.6272 & $3.5 \%$ & 0.9106 & $8.8 \%$ & 0.5711 & $8.8 \%$ \\
\hline TI002-A4-L & 16.76 & 1.44 & $10.8 \%$ & 1.52 & $9.1 \%$ & 0.6277 & $3.1 \%$ & 0.8958 & $7.6 \%$ & 0.5623 & $7.6 \%$ \\
\hline $\begin{array}{l}\text { KW210-A4 } \\
\text { Average (K \& L) }\end{array}$ & --- & 1.39 & $8.1 \%$ & 1.50 & $5.3 \%$ & 0.6274 & $2.3 \%$ & 0.9032 & $5.8 \%$ & 0.5667 & $5.8 \%$ \\
\hline RPD & ---- & $6.92 \%$ & ---- & $3.75 \%$ & ---- & $0.08 \%$ & ---- & $1.65 \%$ & ---- & $1.56 \%$ & ---- \\
\hline KW210-A4-1 & 281.64 & 1.60 & $8.3 \%$ & 1.61 & $6.9 \%$ & --- & --- & 0.9137 & $5.5 \%$ & ---- & --- \\
\hline KW210-A4-A & 74.51 & 1.50 & $8.5 \%$ & 1.49 & $7.0 \%$ & ---- & ---- & 0.8754 & $5.9 \%$ & ---- & --- \\
\hline TIO02-A4-B & 2.30 & ---- & ---- & ---- & ---- & 0.6189 & $3.5 \%$ & ---- & ---- & ---- & ---- \\
\hline TIO02-A4-C & 2.61 & ---- & ---- & ---- & ---- & 0.6117 & $3.1 \%$ & ---- & ---- & ---- & ---- \\
\hline TI002-A4-M & 23.34 & 1.54 & $5.7 \%$ & 1.56 & $6.7 \%$ & ---- & ---- & 0.8436 & $1.1 \%$ & ---- & ---- \\
\hline TI002-A4-N & 49.38 & $\mathrm{NR}^{(\mathrm{f})}$ & & 1.62 & $3.3 \%$ & ---- & ---- & $\mathrm{NR}^{(\mathrm{e})}$ & & --- & ---- \\
\hline KW210-A4-Archive-A & 37.22 & 1.54 & $9.8 \%$ & 1.72 & $16.2 \%$ & --- & --- & 0.9068 & $9.1 \%$ & --- & --- \\
\hline \multicolumn{12}{|l|}{ Core B1 } \\
\hline TI002-B1-K & 14.85 & 1.12 & $9.5 \%$ & $\mathrm{NR}^{(\mathrm{f})}$ & ---- & 0.4204 & $0.5 \%$ & 0.7812 & $7.5 \%$ & 0.3284 & $7.5 \%$ \\
\hline TI002-B1-L & 12.51 & 1.07 & $11.1 \%$ & 1.14 & $9.1 \%$ & 0.4197 & $0.6 \%$ & 0.7809 & $8.9 \%$ & 0.3277 & $8.9 \%$ \\
\hline $\begin{array}{l}\text { KW210-B1 } \\
\text { Average (K \& L) }\end{array}$ & --- & 1.09 & $7.3 \%$ & NA & NA & 0.4201 & $0.4 \%$ & 0.7810 & $5.8 \%$ & 0.3281 & $5.8 \%$ \\
\hline RPD & ---- & $4.62 \%$ & ---- & NA & ---- & $0.17 \%$ & ---- & $0.04 \%$ & ---- & $0.21 \%$ & ---- \\
\hline
\end{tabular}


Table 3.1. (contd)

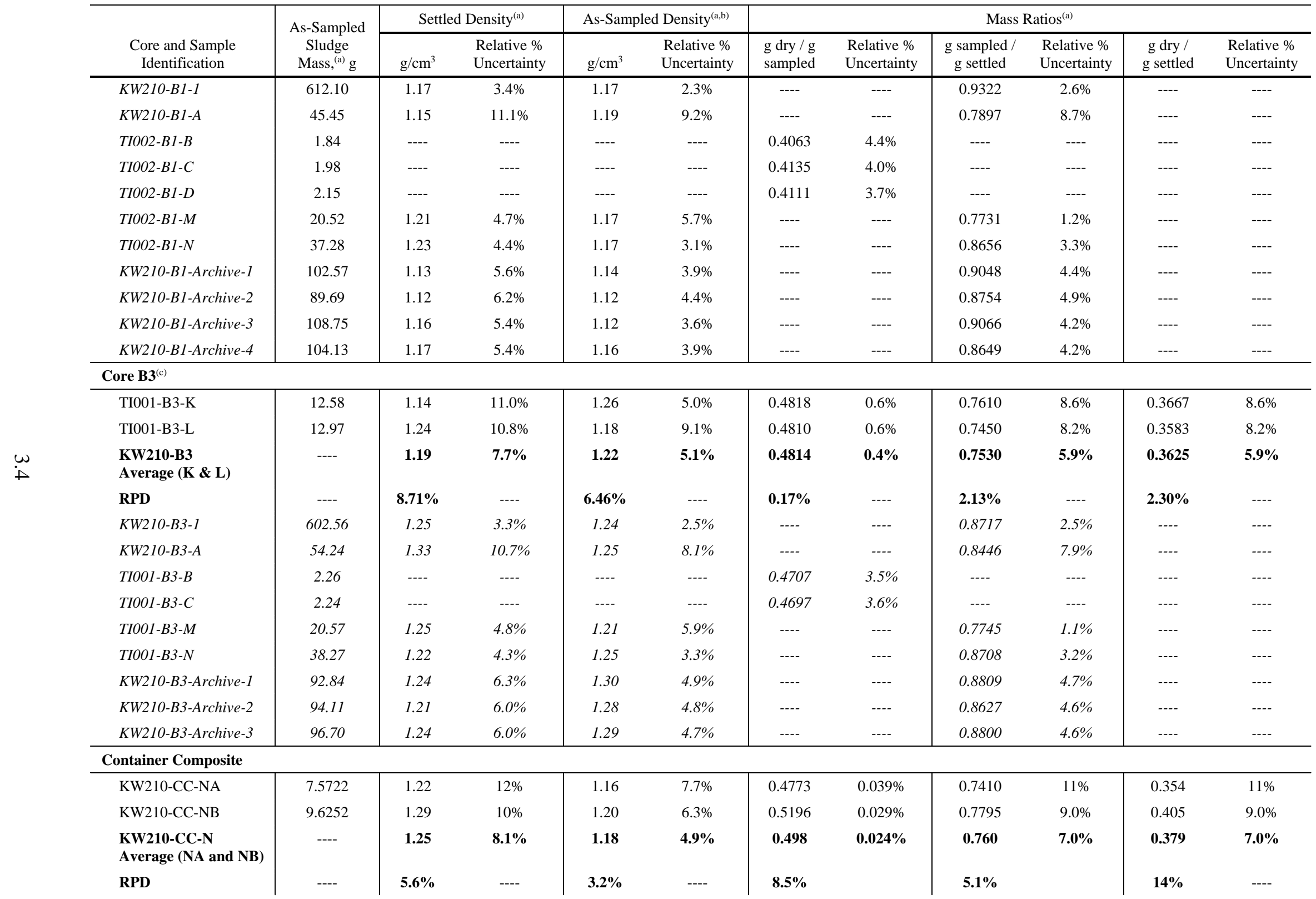


Table 3.1. (contd)

\begin{tabular}{|c|c|c|c|c|c|c|c|c|c|c|c|}
\hline \multirow[b]{2}{*}{$\begin{array}{l}\text { Core and Sample } \\
\text { Identification }\end{array}$} & \multirow{2}{*}{$\begin{array}{l}\text { As-Sampled } \\
\text { Sludge } \\
\text { Mass, }{ }^{(a)} \mathrm{g}\end{array}$} & \multicolumn{2}{|c|}{ Settled Density ${ }^{(\mathrm{a})}$} & \multicolumn{2}{|c|}{ As-Sampled Density ${ }^{(\mathrm{a}, \mathrm{b})}$} & \multicolumn{6}{|c|}{ Mass Ratios ${ }^{(a)}$} \\
\hline & & $\mathrm{g} / \mathrm{cm}^{3}$ & $\begin{array}{l}\text { Relative \% } \\
\text { Uncertainty }\end{array}$ & $\mathrm{g} / \mathrm{cm}^{3}$ & $\begin{array}{l}\text { Relative \% } \\
\text { Uncertainty }\end{array}$ & $\begin{array}{l}\text { g dry / g } \\
\text { sampled }\end{array}$ & $\begin{array}{l}\text { Relative \% } \\
\text { Uncertainty }\end{array}$ & $\begin{array}{l}\text { g sampled / } \\
\text { g settled }\end{array}$ & $\begin{array}{l}\text { Relative \% } \\
\text { Uncertainty }\end{array}$ & $\begin{array}{l}\text { g dry / } \\
\text { g settled }\end{array}$ & $\begin{array}{l}\text { Relative \% } \\
\text { Uncertainty }\end{array}$ \\
\hline KW210-CC-AA & 7.8543 & 1.22 & $12 \%$ & 1.12 & $7.1 \%$ & 0.5037 & $0.036 \%$ & 0.7556 & $11 \%$ & 0.381 & $11 \%$ \\
\hline KW210-CC-AB & 9.3908 & 1.31 & $11 \%$ & 1.17 & $6.3 \%$ & 0.5208 & $0.029 \%$ & 0.7953 & $9.4 \%$ & 0.414 & $9.4 \%$ \\
\hline $\begin{array}{l}\text { KW210-CC-A } \\
\text { Average (AA and AB) }\end{array}$ & ---- & 1.27 & $8.2 \%$ & 1.15 & $4.7 \%$ & 0.512 & $0.023 \%$ & 0.775 & $7.1 \%$ & 0.397 & $7.1 \%$ \\
\hline RPD & ---- & $7.0 \%$ & ---- & $4.5 \%$ & ---- & $3.3 \%$ & & $5.1 \%$ & & $8.5 \%$ & ---- \\
\hline KW210-CCA & 250.554 & 1.29 & $5.3 \%$ & 1.25 & $4.0 \%$ & NA & NA & 0.8828 & $3.9 \%$ & NA & NA \\
\hline KW210-CCN & 178.768 & 1.23 & $4.9 \%$ & 1.23 & $3.5 \%$ & NA & NA & 0.9402 & $3.7 \%$ & NA & NA \\
\hline \multicolumn{12}{|l|}{ Fines, $-500 \mu \mathrm{m}$} \\
\hline TI008-SM & 4.9064 & 1.27 & $15 \%$ & 1.23 & $7.5 \%$ & 0.4700 & $0.061 \%$ & 0.7555 & $11 \%$ & 0.355 & $11 \%$ \\
\hline TI008-SN & 5.2429 & 1.30 & $13 \%$ & 1.31 & $7.5 \%$ & 0.4777 & $0.056 \%$ & 0.7211 & $9.7 \%$ & 0.344 & $9.7 \%$ \\
\hline $\begin{array}{l}\text { KW210-SCC } \\
\text { Average (SM and SN) }\end{array}$ & ---- & 1.29 & $9.8 \%$ & 1.27 & $5.3 \%$ & 0.474 & $0.041 \%$ & 0.738 & $7.3 \%$ & 0.350 & $7.3 \%$ \\
\hline RPD & ---- & $1.9 \%$ & ---- & $6.6 \%$ & ---- & $1.6 \%$ & & $4.7 \%$ & & $3.0 \%$ & ---- \\
\hline KW210-SCC & 52.283 & 1.08 & $14 \%$ & 1.38 & $13 \%$ & NA & NA & 0.8525 & $12 \%$ & NA & NA \\
\hline TIO08-SA & 1.9371 & ---- & --- & ---- & --- & 0.4581 & $0.16 \%$ & --- & --- & ---- & ---- \\
\hline TIO08-SB & 2.2705 & ---- & ---- & ---- & ---- & 0.4791 & $0.13 \%$ & --- & ---- & ---- & ---- \\
\hline TIO08-SC & 1.9678 & ---- & ---- & ---- & --- & 0.4573 & $0.16 \%$ & --- & --- & --- & ---- \\
\hline
\end{tabular}

(a) Individual mass measurements are presented in appropriate significant figures. Individual mass ratios and averages are presented to three and four significant digits in this table. Volume measurements (and thus density) are accurate to two significant figures. The third significant figure of the density values are shown for information only. The additional digits are provided for information and to help the reader re-create computed values without introducing the rounding error.

(b) Volume measurements of the dewatered or as-sampled sludge were challenging due to sludge smearing and uneven surfaces. Consequently, as-sampled sludge density values should be used with caution.

(c) Additional archive subsamples were collected by sequentially rinsing residual solids coating the interior composite containers with K Basin water for cores A1, A4, and B3. The settled sludge volumes were typically $<5 \mathrm{~mL}$ and relative uncertainties of the settled density measurements exceeded $90 \%$ for cores A1 and A4. The sequential rinses were collected in different bottles and may not be representative of the reconstituted core without initial recombination.

(d) KW210-A1-Archive-A as-sampled sludge density was not reported because of a severely sloped sludge level and resulting uncertainty of the measured value.

(e) TI002-A4-N settled sludge density and g sampled/g settled conversion factor are not reported because the re-suspension of the as-sampled sludge was not successful. The phase ratio of suspending solution to sludge was small and there appeared to be an unknown compound in the liquid phase that clung to the side walls and cap of the sample vial after agitation. This resulted in a settled sludge volume and total slurry volume that was not credible. See OR 52578-08-19-11 in TI 52578-TI002 for details.

(f) TI002-B1-K as-sampled sludge density was not reported because the sludge level was severely sloped. This resulted in an as-sampled density greater than three times the standard deviation of the B1 subsamples.

Notes:

Refer to Definitions (page xiii) for descriptions of as-sampled, settled, and dried sludge conditions.

Calculations of averages, relative percent differences (RPDs), and relative uncertainty were performed using untruncated values (Microsoft Excel calculation spreadsheet). Input data and Excel spreadsheet calculations used to generate data for this table are provided in DVD-52578-PNNL-20650 Rev. 2, Section III, September 2013.

The italicized values are provided as supplemental, opportunistic data.

NR: Not Reported. NA: Not Applicable. 
The relative uncertainty of each measurement was calculated using variance propagation. The relative uncertainties of the net as-sampled sludge masses ranged from $0.01 \%$ to $4.4 \%$ and are not shown in Table 3.1. ${ }^{\text {(a, b) }}$ The density uncertainties were primarily driven by the volume uncertainty component. Volume measurements were made by visually reading the decal millimeter scale affixed to the sample jars (e.g., $\pm 2 \mathrm{~mm}=12 \mathrm{~mL}$ in the 960 -mL jar) or from factory-calibrated and marked centrifuge tubes. Volume measurements of as-sampled sludge were sometimes affected by sludge smears on the inside walls and uneven sludge levels (i.e., the thickened as-sampled sludge was difficult to level). Smearing was not an issue for settled sludge and the sludge settled in a level manner. In all cases, the volume could only be measured to two significant figures.

For all cores, the 24-hr settled sludge volume was considerably different from the as-sampled sludge volume. The sludge mass difference between the as-sampled and settled states can be significant as it is driven primarily by the difference in sludge volumes. The average mass ratios of the as-sampled to settled sludge ranged from 0.74 to 0.90 (Table 3.1). However, the as-sampled and settled sludge densities were not much different.

The relative uncertainties for average values of the primary characterization pairs given in Table 3.1 were propagated in accordance with standard practices and ranged from 0.02 to $9.8 \%$. ${ }^{\text {(c) }}$ Another measure of precision, RPD, was also determined for each primary physical property characterization pair and ranged from 0.04 to $14 \%$.

The mass of dry solids per gram of settled sludge (g dry/g settled) is provided as a ratio. Multiplying this value by 100 will provide the wt\% dry solids in settled sludge, as requested in the QAPJP/SAP.

All sludge-sample aliquots were weighed in the wet dewatered or as-sampled form. Data initially normalized to the as-sampled mass (e.g., U metal) convert to a settled solids mass basis by multiplying by the conversion factor "g sampled/g settled" (Equation 3.1).

$$
\frac{U_{\text {metal }}}{\mathrm{g}_{\text {sampled }}} \times \frac{\mathrm{g}_{\text {sampled }}}{\mathrm{g}_{\text {settled }}}=\frac{\mathrm{U}_{\text {metal }}}{\mathrm{g}_{\text {settled }}}
$$

(a) Relative \% Uncertainty = (absolute uncertainty / measured value) $\mathrm{x} 100$.

(b) The relative uncertainty of the as-sampled sludge mass measurements varied as a function of sample mass, balance type, and balance tolerance control limits. For example, using the HLRF balance, a 2.05-g analytical sample (collected in the $40-\mathrm{mL}$ Oak Ridge centrifuge tube) has a 3.9\% relative uncertainty. A 14.74-g physical property sample (collected in the $40-\mathrm{mL}$ glass centrifuge tubes) and weighed on the HLRF balance has a $0.54 \%$ relative uncertainty. Individual sample mass uncertainties are provided in the physical properties spreadsheets contained in DVD-52578-PNNL-20650 Rev. 2, Section III, September 2013.

(c) Relative uncertainties for average values were calculated as follows:

Step 1-Calculate the uncertainty of each measurement in absolute units (standard deviation).

Step 2-Calculate the variance associated with the mean function $(\mathrm{X}+\mathrm{Y}) / 2)$. The variance of this function is the constant squared $\left[(1 / 2)^{2}=1 / 4\right]$ times the sum of $\operatorname{Var}(X)$ and $\operatorname{Var}(Y)$ where $\operatorname{Var}(\mathrm{X})$ is the answer in step 1 for the first measurement squared, and $\operatorname{Var}(\mathrm{Y})$ is the answer in step 1 for the second measurement squared.

Step 3-Add the $\operatorname{Var}(\mathrm{X})$ and $\operatorname{Var}(\mathrm{Y})$, divide by 4, and take the square root of the answer.

Step 4-Put the answer back into relative terms (RSD) by dividing the answer in step 3 by the overall average of the two measurements. 
Data initially normalized to the dry sample mass (e.g., chemistry and radionuclide results) convert to the settled solids mass basis by multiplying by the conversion factor "g dry/g settled" (Equation 3.2).

$$
\frac{A_{X}}{g_{\text {dry }}} \times \frac{g_{\text {dry }}}{g_{\text {settled }}}=\frac{A_{X}}{g_{\text {settled }}}
$$

All physical properties samples, reconstituted core samples, and container composite samples for SCS-CON-210 were re-agitated to acquire a settled sludge volume. Constant settled sludge volume was generally achieved within $1.5 \mathrm{hr}$ of undisturbed settling after mixing/suspension. Plots of settled sludge volume as a function of time were opportunistically collected on the primary physical property sample pairs (Figure 3.3 through Figure 3.6) and many of the other collected samples (see DVD-52578-PNNL-20650 Rev. 2, Section III, September 2013). In all cases, the samples rapidly settled in the first ten minutes, then slowly approached the final measured settled volume within $1.5 \mathrm{hr}$. Because no changes in settled sludge volumes were seen after $12 \mathrm{hr}$, final settled sludge volumes were recorded prior to the 24-hr mark.

Settled sludge volumes were re-measured after transferring the samples from HLRF to SAL to assess compaction as a function of the sample handling. SCS-CON-210 material appeared to be heterogeneous in nature (i.e., with respect to particle morphology and size distributions). The previously characterized physical properties sludge subsamples collected from SCS-CON-220 containerized sludge core samples appeared to have similar heterogeneous characteristics and significantly compacted from handling activities (Fiskum et al. 2011b). Table 3.2 shows the change in settled sludge densities for the SCS-CON-210 primary physical properties samples and compares them to the as-sampled densities. 


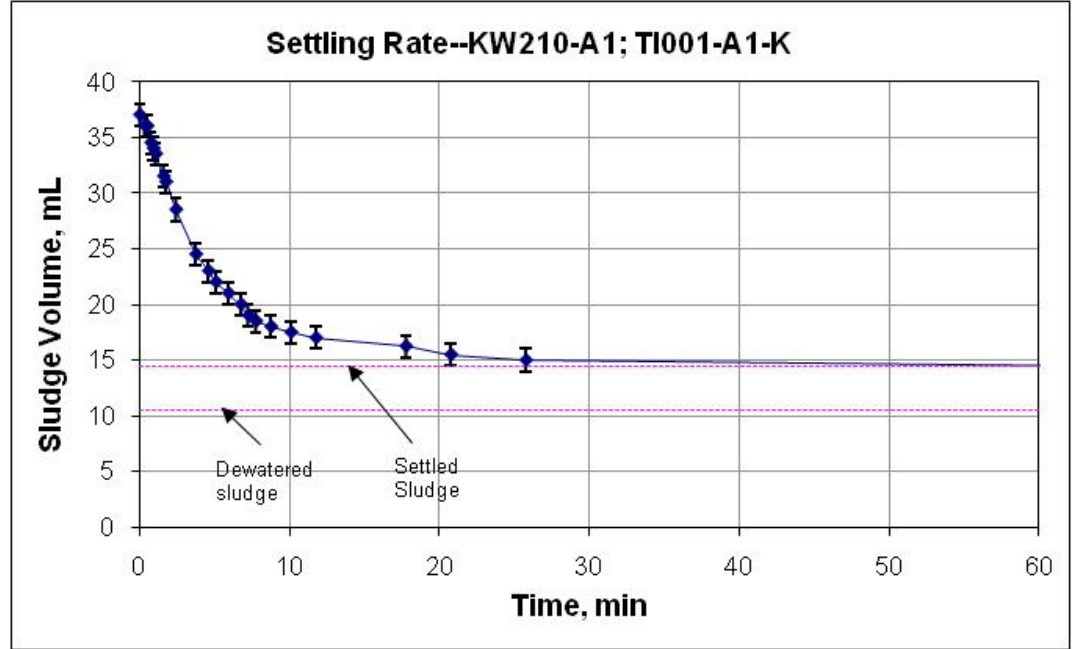

A. Settling Behavior of Subsample TI001-A1-K

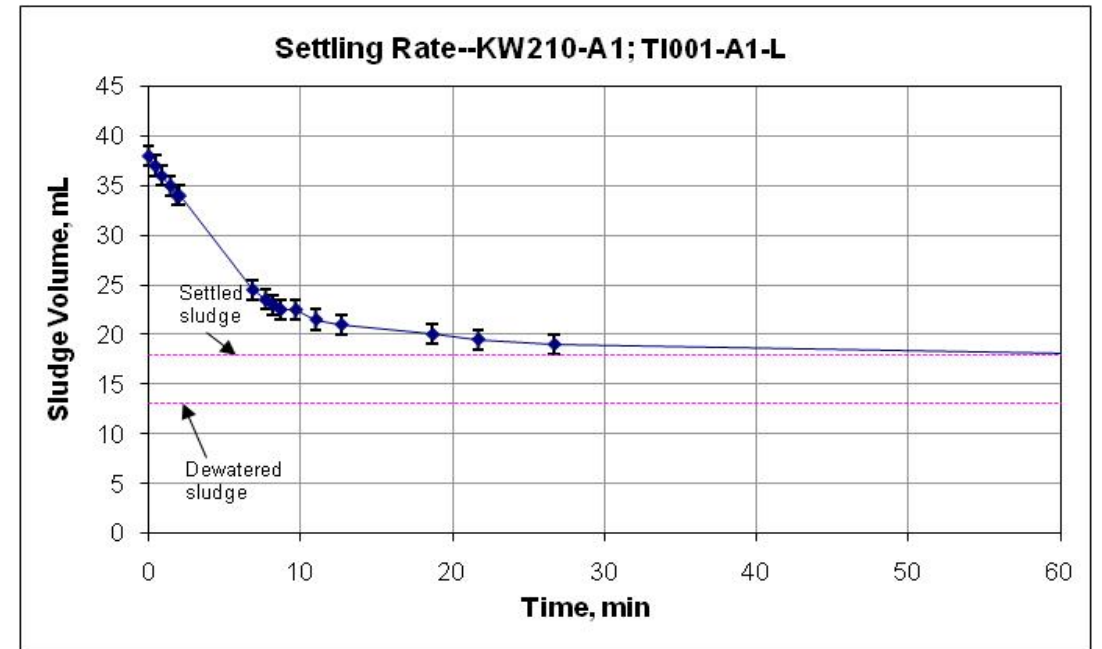

B. Settling Behavior of Subsample TI001-A1-L

Figure 3.3. Settling Behavior of Physical Property Samples Collected from the KW210-A1 Core

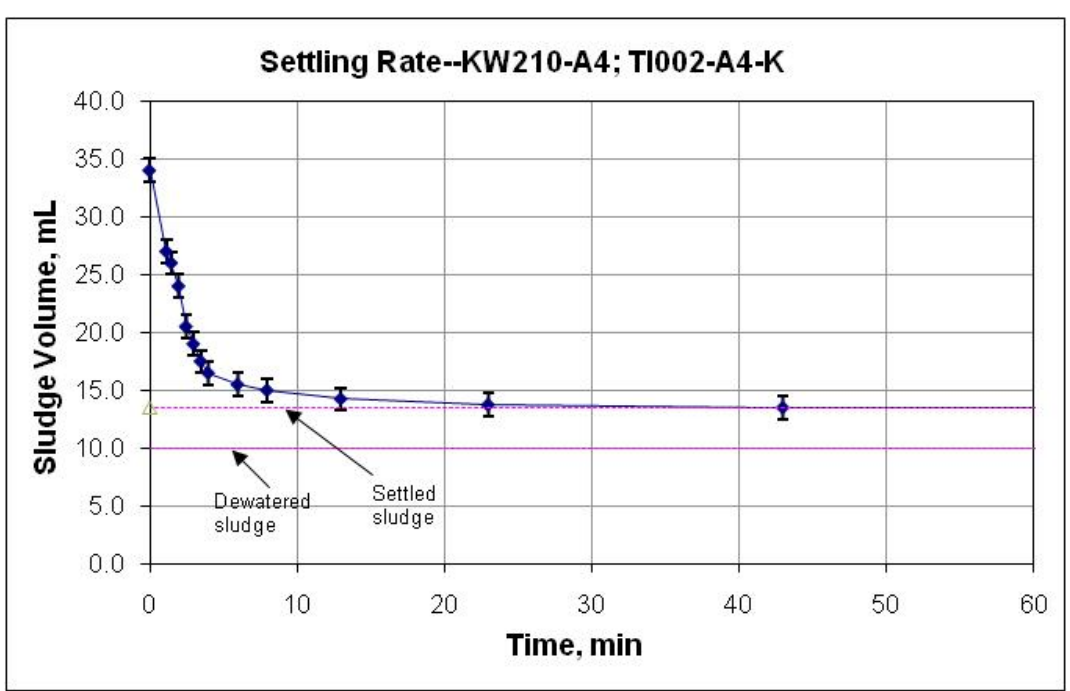

A. Settling Behavior of Subsample TI002-A4-K

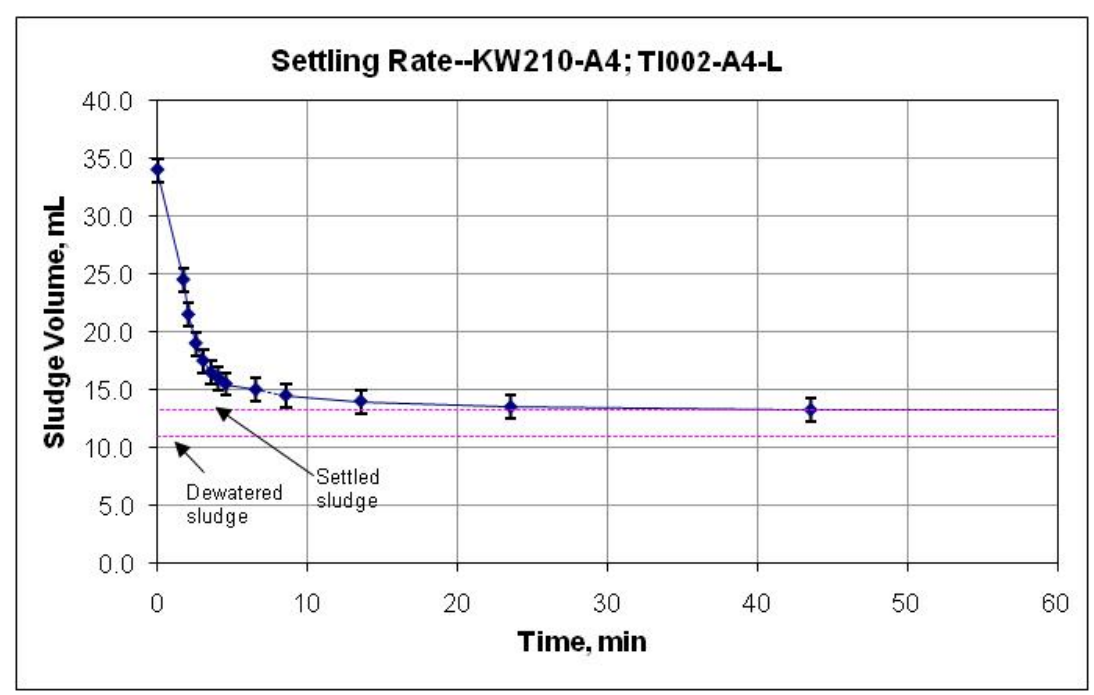

B. Settling Behavior of Subsample TI002-A4-L

Figure 3.4. Settling Behavior of Physical Property Samples Collected from the KW210-A4 Core 


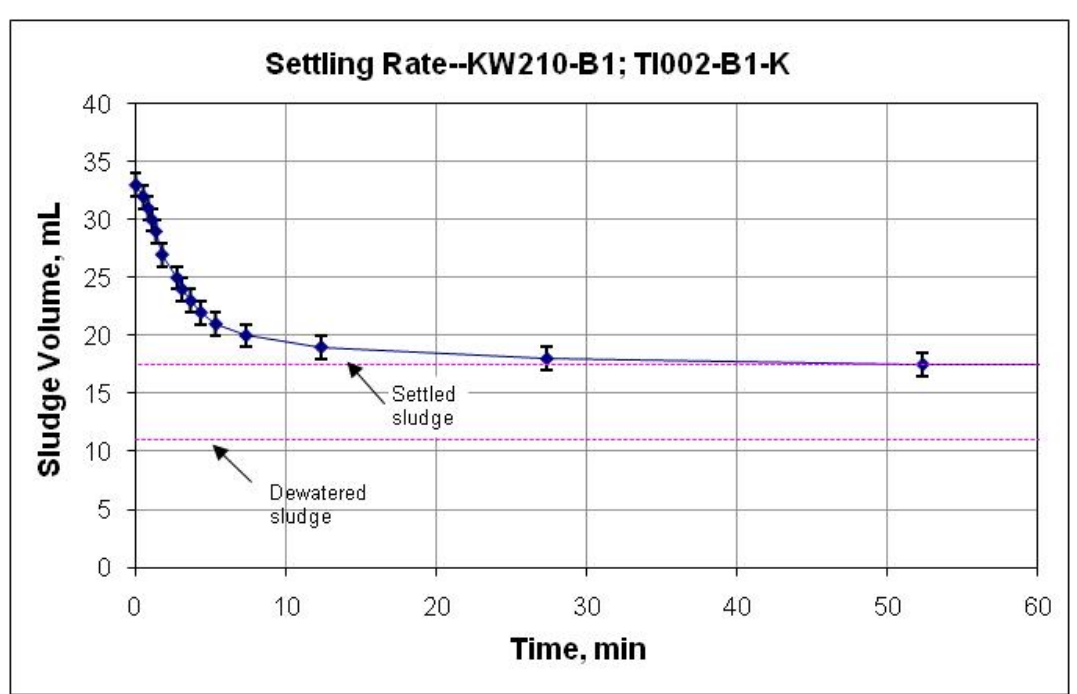

A. Settling Behavior of Subsample TI002-B1-K

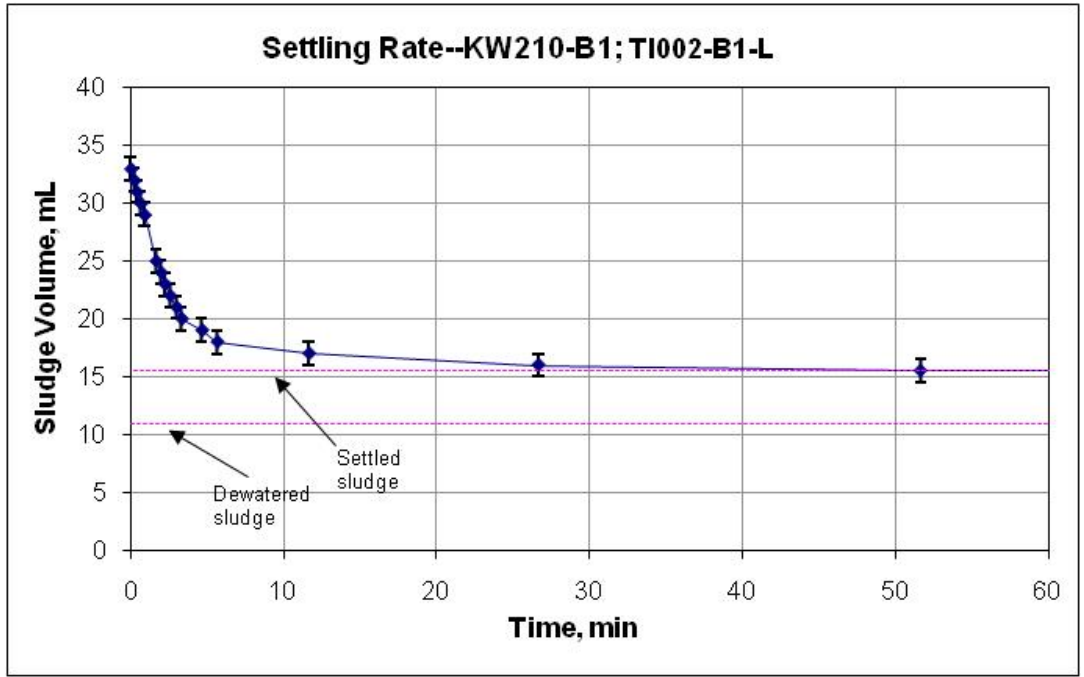

B. Settling Behavior of Subsample TI002-B1-L

Figure 3.5. Settling Behavior of Physical Property Samples Collected from the KW210-B1 Core

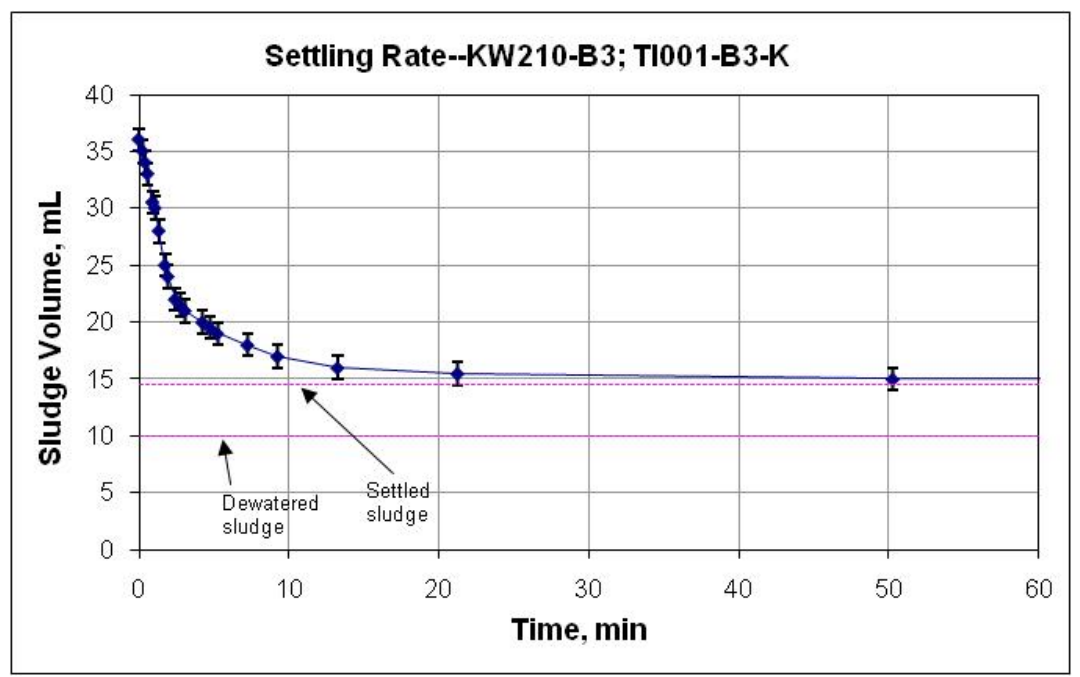

A. Settling Behavior of Subsample TI001-B3-K

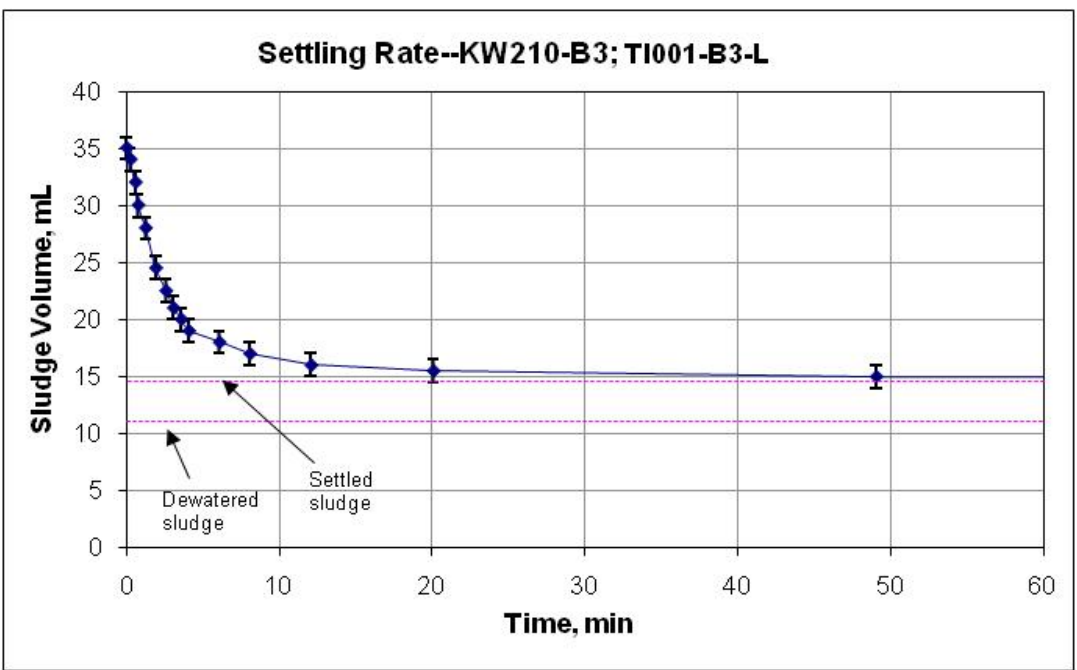

B. Settling Behavior of Subsample TI001-B3-L

Figure 3.6. Settling Behavior of Physical Property Samples Collected from the KW210-B3 Core 
PNNL-20650, Rev. 2

Table 3.2. SCS-CON-210 Sludge Density Changes for Subsamples K and L with Movement

\begin{tabular}{|c|c|c|c|c|c|c|c|c|}
\hline \multirow[b]{4}{*}{ Condition } & \multicolumn{8}{|c|}{ Sludge Density } \\
\hline & \multicolumn{4}{|c|}{ Core A1 } & \multicolumn{4}{|c|}{ Core A4 } \\
\hline & \multicolumn{2}{|c|}{ TI001-A1-K } & \multicolumn{2}{|c|}{ TI001-A1-L } & \multicolumn{2}{|c|}{ TI002-A4-K } & \multicolumn{2}{|c|}{ TI002-A4-L } \\
\hline & $\mathrm{g} / \mathrm{cm}^{3}$ & Uncertainty & $\mathrm{g} / \mathrm{cm}^{3}$ & Uncertainty & $\mathrm{g} / \mathrm{cm}^{3}$ & Uncertainty & $\mathrm{g} / \mathrm{cm}^{3}$ & Uncertainty \\
\hline 24-h Settled & 1.33 & $10.1 \%$ & 1.31 & $8.4 \%$ & 1.34 & $12.1 \%$ & 1.44 & $10.8 \%$ \\
\hline Moved to SAL ${ }^{(a)}$ & 1.37 & $11.5 \%$ & 1.38 & $9.3 \%$ & 1.43 & $12.3 \%$ & 1.54 & $11.3 \%$ \\
\hline \multirow[t]{4}{*}{ As-sampled } & 1.40 & $9.5 \%$ & 1.42 & $7.7 \%$ & 1.47 & $5.0 \%$ & 1.52 & $9.1 \%$ \\
\hline & \multicolumn{4}{|c|}{ Core B1 } & \multicolumn{4}{|c|}{ Core B3 } \\
\hline & \multicolumn{2}{|c|}{ TI002-B1-K } & \multicolumn{2}{|c|}{ TI002-B1-L } & \multicolumn{2}{|c|}{ TI001-B3-K } & \multicolumn{2}{|c|}{ TI001-B3-L } \\
\hline & $\mathrm{g} / \mathrm{cm}^{3}$ & Uncertainty & $\mathrm{g} / \mathrm{cm}^{3}$ & Uncertainty & $\mathrm{g} / \mathrm{cm}^{3}$ & Uncertainty & $\mathrm{g} / \mathrm{cm}^{3}$ & Uncertainty \\
\hline 24-h Settled & 1.12 & $9.5 \%$ & 1.07 & $11.1 \%$ & 1.14 & $11.0 \%$ & 1.24 & $10.8 \%$ \\
\hline Moved to SAL ${ }^{(a)}$ & 1.13 & $13.4 \%$ & 1.11 & $11.6 \%$ & 1.22 & $11.4 \%$ & 1.22 & $16.7 \%$ \\
\hline As-sampled & $\mathrm{NR}^{(\mathrm{b})}$ & ---- & 1.14 & $9.1 \%$ & 1.26 & $5.0 \%$ & 1.18 & $9.1 \%$ \\
\hline $\begin{array}{l}\text { (a) Containers were } \\
\text { (b) TI002-B1-K as-s } \\
\text { measurement of } \\
\text { deviation of the } \\
\end{array}$ & $\begin{array}{l}\text { oved fro } \\
\text { pled sl } \\
\text { sludge } \\
\text { er B1 st } \\
\end{array}$ & $\begin{array}{l}\text { he HLRF to } t \\
\text { e density was } \\
\text { lume. This re } \\
\text { amples. }\end{array}$ & $\begin{array}{l}\text { SAL } \\
\text { trepo }\end{array}$ & $\begin{array}{l}\text { NR) becal } \\
\text { ulated, as }\end{array}$ & slud & $\begin{array}{l}\text { gel was seve } \\
\text { greater tha }\end{array}$ & slope & $\begin{array}{l}\text { founding } \\
\text { e standard }\end{array}$ \\
\hline
\end{tabular}

\subsection{Volume Measurements and Morphology Assessments of Dried Physical Property Samples}

Before discarding the $105^{\circ} \mathrm{C}$-dried physical property characterization samples, they were opportunistically evaluated for apparent bulk volume and evidence of agglomeration. The simple examinations included prodding the agglomerate with a spatula to qualitatively assess strength. General observations included:

- Much of the dry material was free flowing and granular.

- Fines formed friable and relatively weak agglomerates. Fibers that were found mixed in the fines helped hold the agglomerates together. These fiber-reinforced agglomerates were broken apart with relatively little force using a 1-cm wide stainless steel spatula.

- No evidence of the formation of strong agglomerates was observed for the core subsamples.

- Strong agglomerates were observed on each of the $-500-\mu \mathrm{m}$ sieve fraction container composite samples.

- Generally, the dried sludge did not cling to the glass centrifuge tube walls.

- Sludge typically contracted 18 to 51\% from a settled to a dried volume basis.

- Significant quantities of OIER beads were observed in all samples; they visually appeared to be in a greater abundance in the " $\mathrm{B}$ " core samples.

Figures 3.7 through 3.10 show select images of SCS-CON-210 core primary physical properties samples post-drying, Figure 3.11 shows select images of SCS-CON-210 container composite and $-500-\mu \mathrm{m}$ sieved fraction samples post-drying. Table 3.3 summarizes observations collected from selected core and container composite samples. [Note: The inside diameter of the centrifuge tubes shown in Figures 3.7 through 3.11 increases from $\sim 0.5 \mathrm{~cm}$ at the cone tip to $\sim 2.5 \mathrm{~cm}$ at the top of the tapered cone region.] 


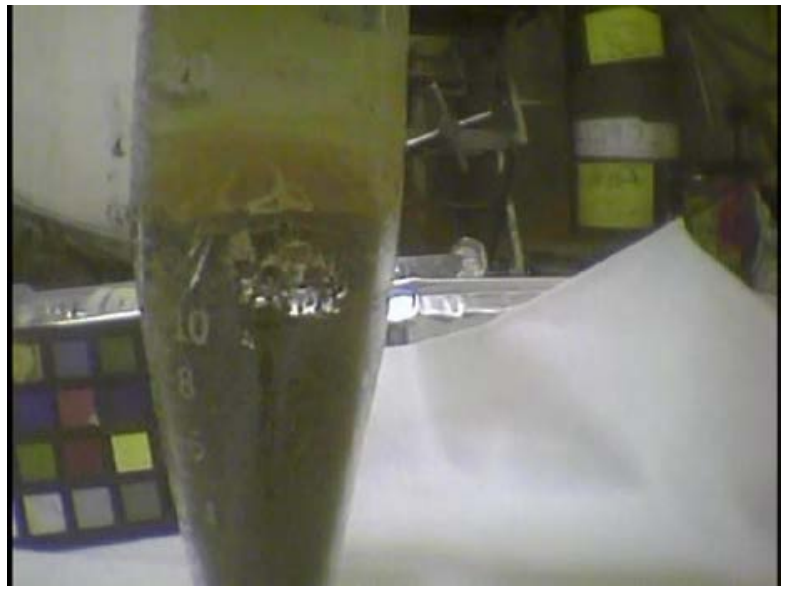

a) TI001-A1-K initial look at dried solids levels, some material adhered to centrifuge tube wall.

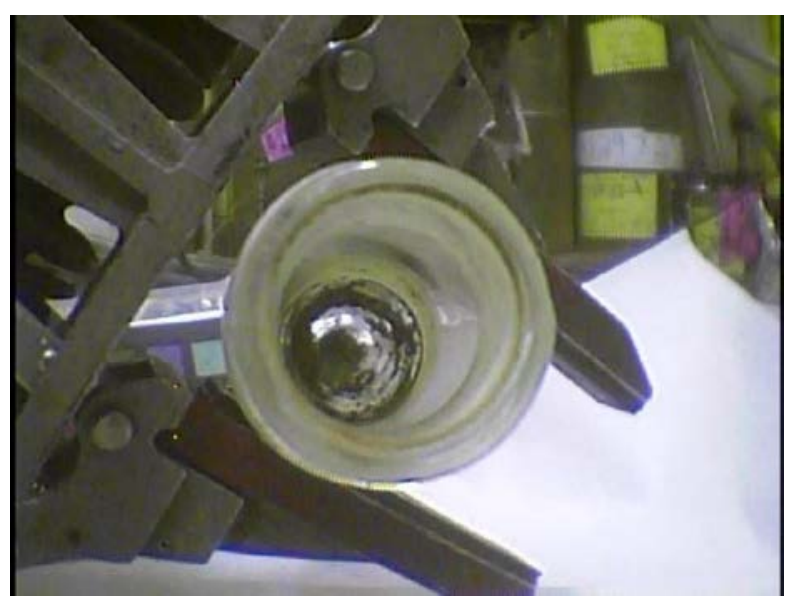

c) TI001-A1-K vial after pouring out solids. Small removable heel and some material adhered to centrifuge tube wall at sludge/water interface.

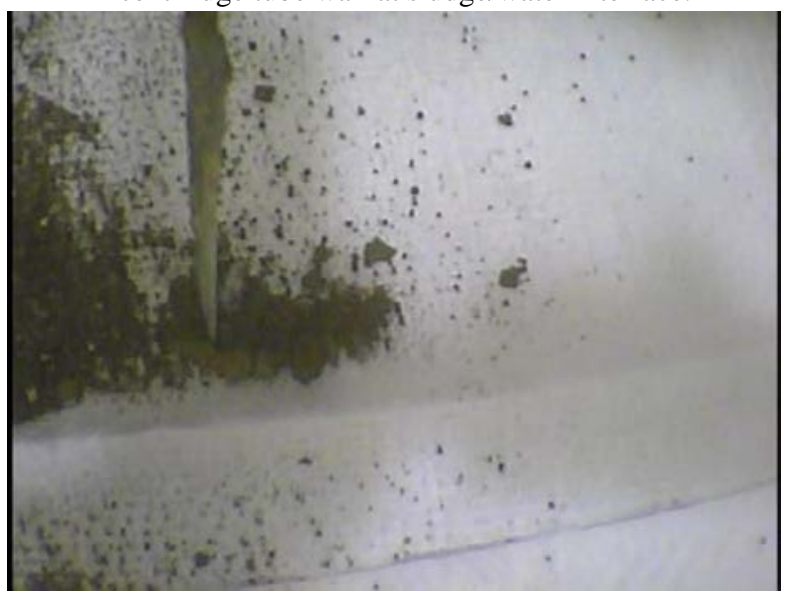

e) TI001-A1-K agglomerate broken into pieces with little force as indicated by material resting point in immediate sectioning area.

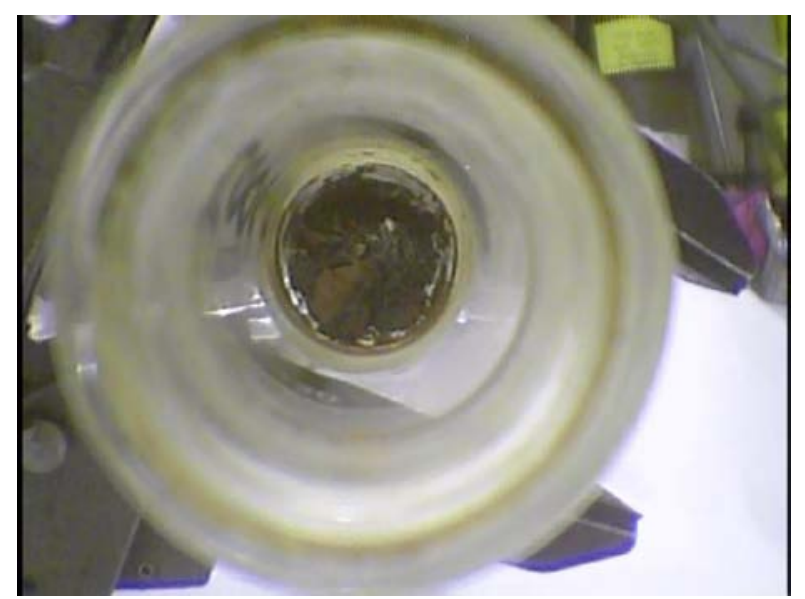

b) TI001-A1-K prior to pouring out solids. Large agglomerate pieces on top of underlying granular particles.

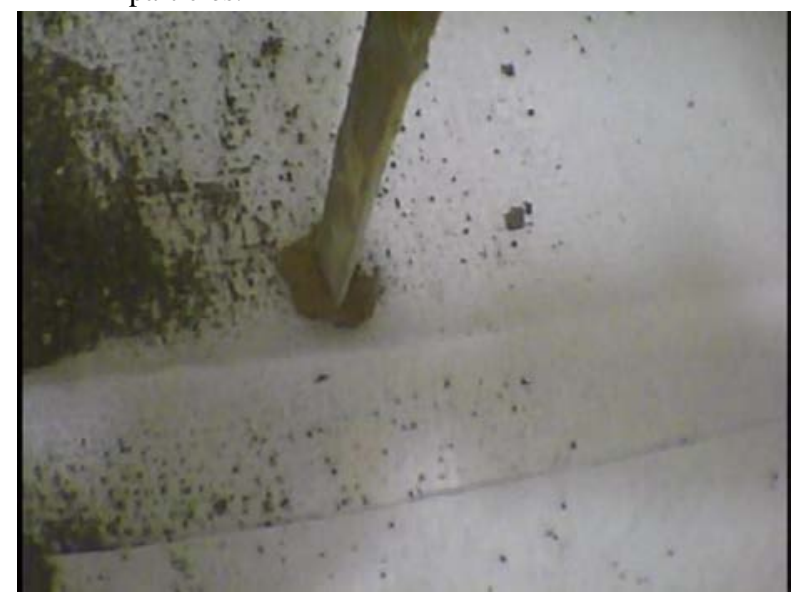

d) TI001-A1-K agglomerate particle with 1-cm wide stainless spatula. Little effort required to break.

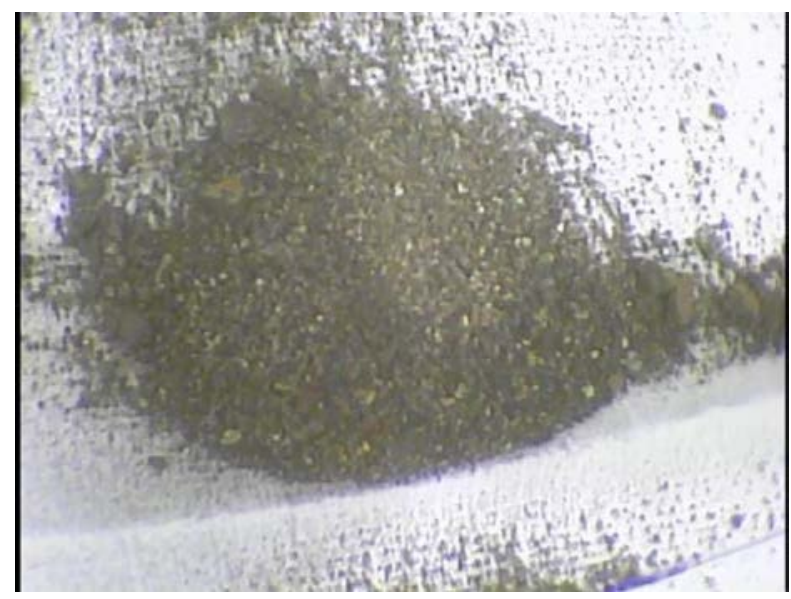

f) TI001-A1-K bulk materials image capturing general particle size, color, and morphology.

Figure 3.7. Select Post-Test Examination Images of TI001-A1-K (KW210-A1) 


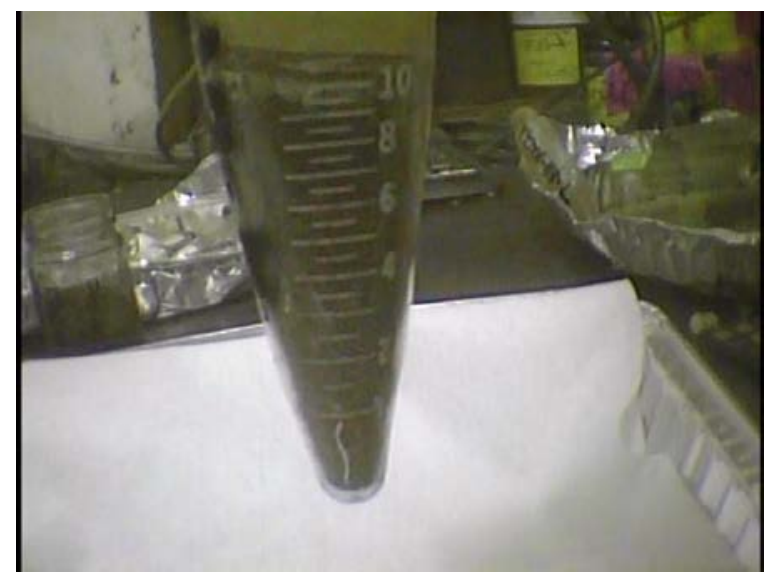

a) TI002-A4-K initial look at dried solids levels, some material adhered to centrifuge tube wall.

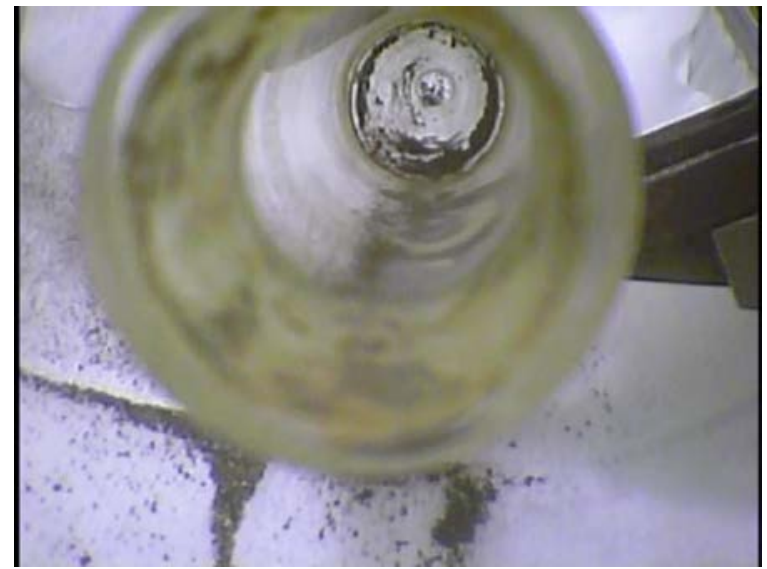

c) TI002-A4-K vial after pouring out solids. Small removable heel and some material adhered to centrifuge tube wall at sludge/water interface.

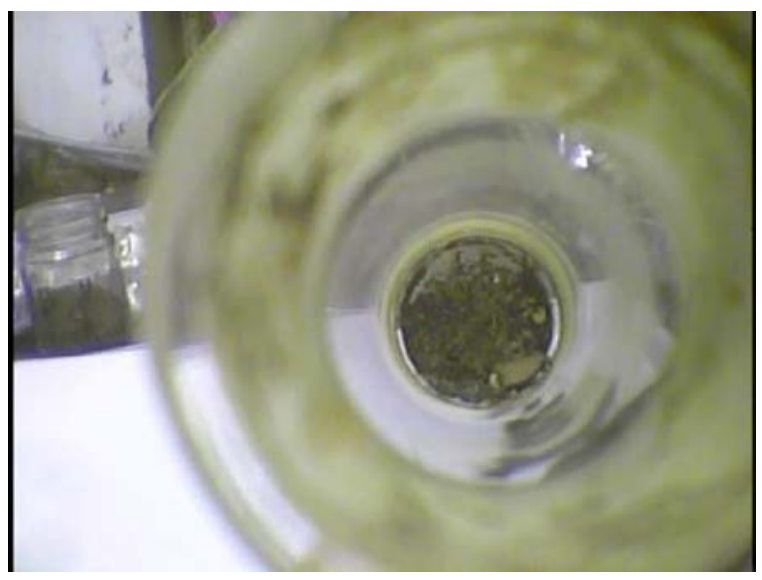

b) TI002-A4-K prior to pouring out solids. Large agglomerate pieces on top of underlying granular particles.

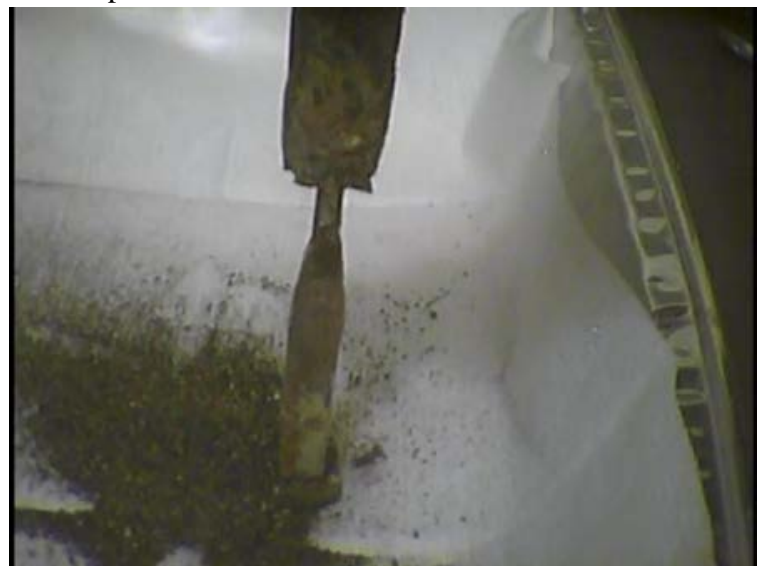

d) TI002-A4-K agglomerate particle with 1-cm wide stainless spatula. Little effort required to break.

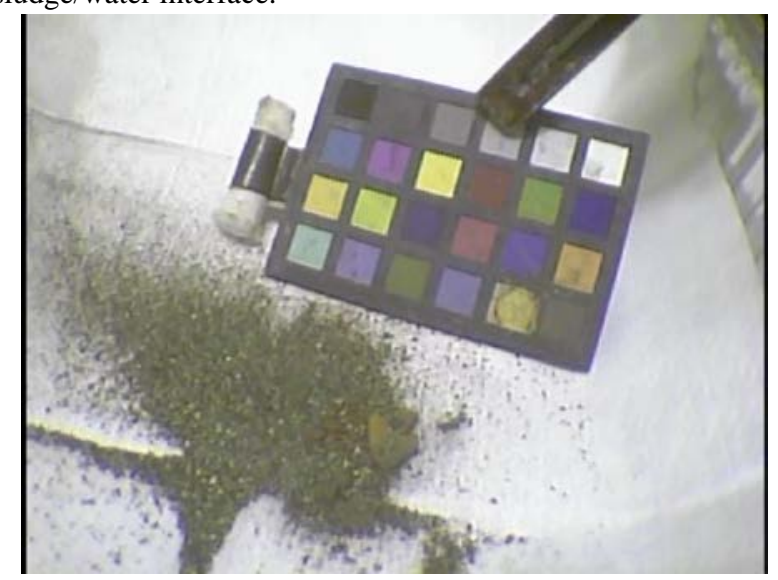

e) TI002-A4-K bulk materials image capturing general particle size, color, and morphology.

Figure 3.8. Select Post-Test Examination Images of TI002-A4-K (KW210-A4) 


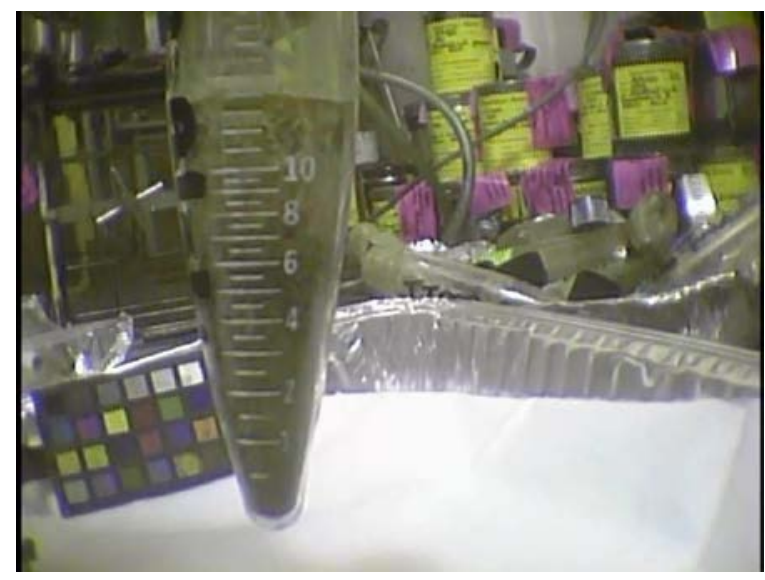

a) TI002-B1-L initial look at dried solids levels, some material adhered to centrifuge tube wall.

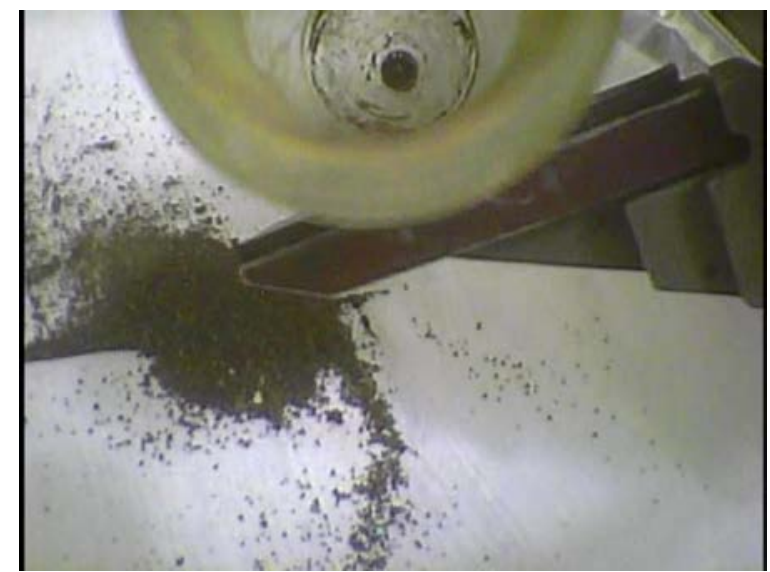

c) TI002-B1-L vial after pouring out solids. Small removable heel and some material adhered to centrifuge tube wall at sludge/water interface.

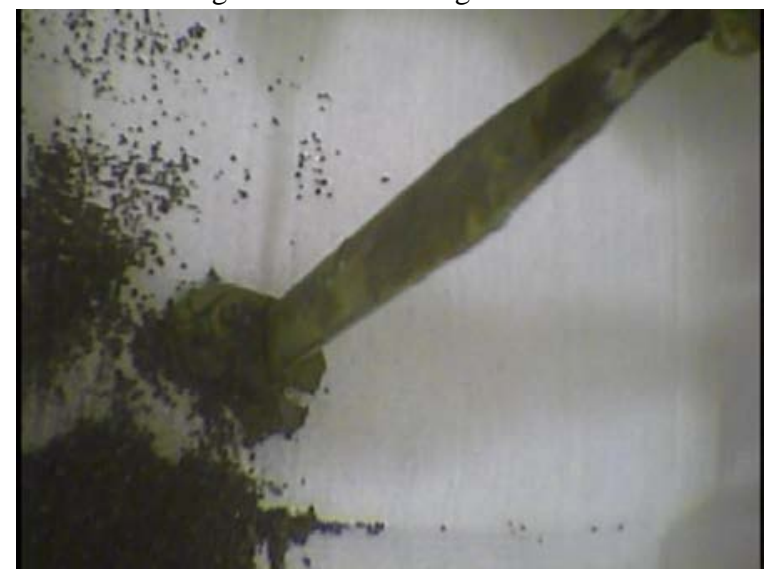

e) TI002-B1-L agglomerate broken into pieces with little force as indicated by material resting point in immediate sectioning area.

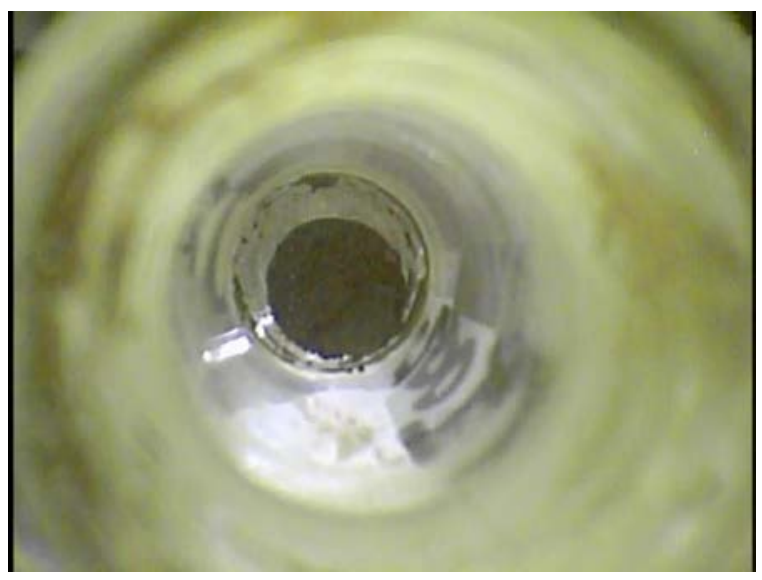

b) TI002-B1-L prior to pouring out solids. Large agglomerate pieces on top of underlying granular particles.

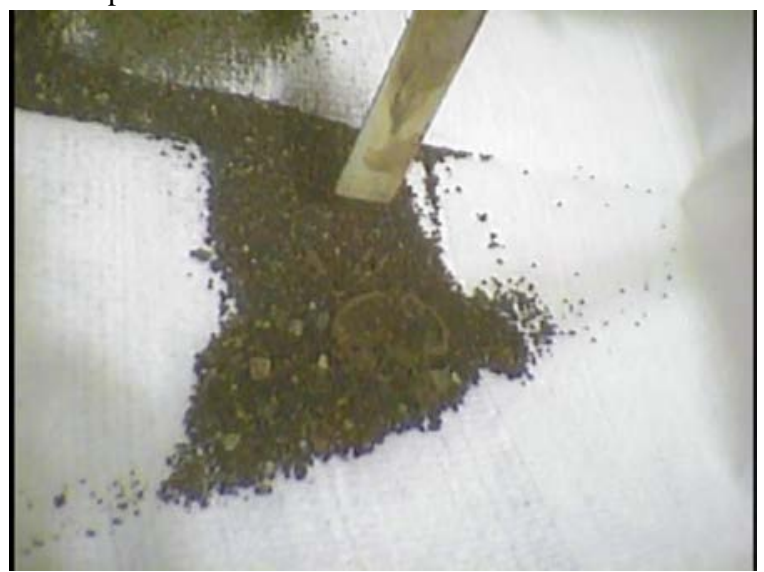

d) TI002-B1-L agglomerate particle with 1-cm wide stainless spatula. Little effort required to break.

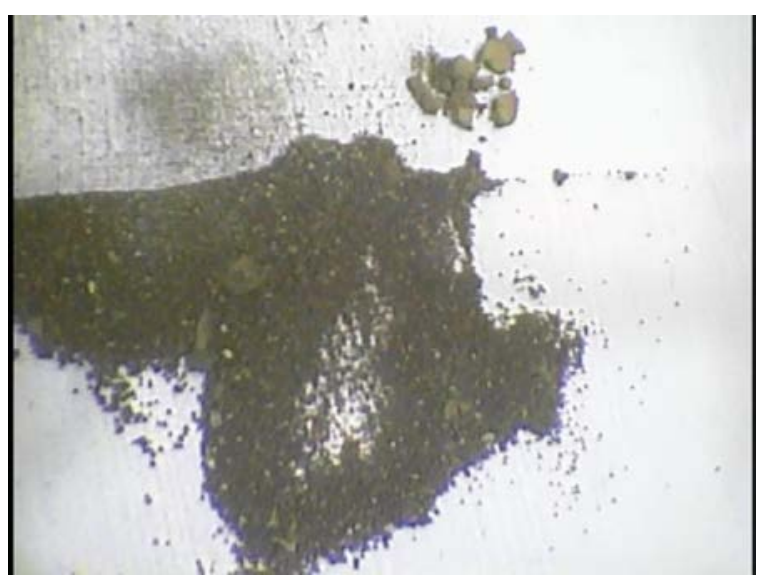

f) TI002-B1-L bulk materials image capturing general particle size, color, and morphology.

Figure 3.9. Select Post-Test Examination Images of TI002-B1-L (KW210-B1) 


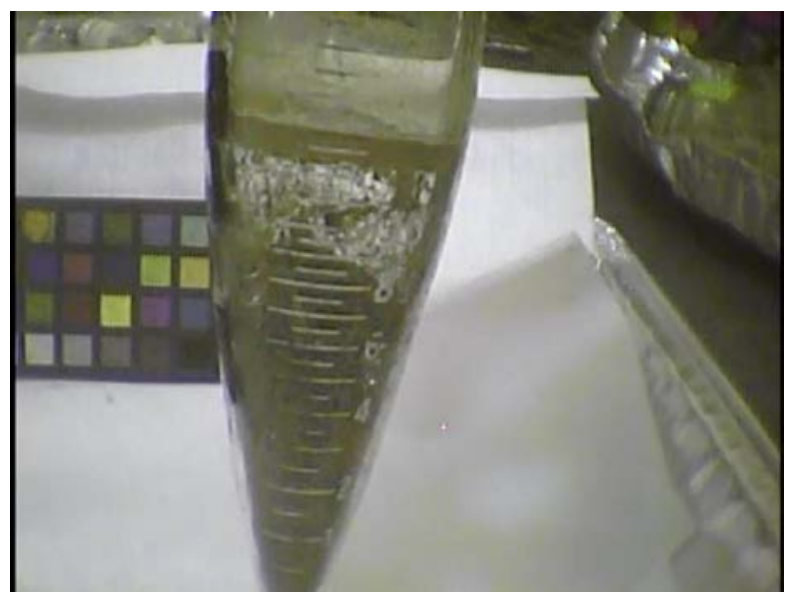

a) TI001-B3-K initial look at dried solids levels, some material adhered to centrifuge tube wall.

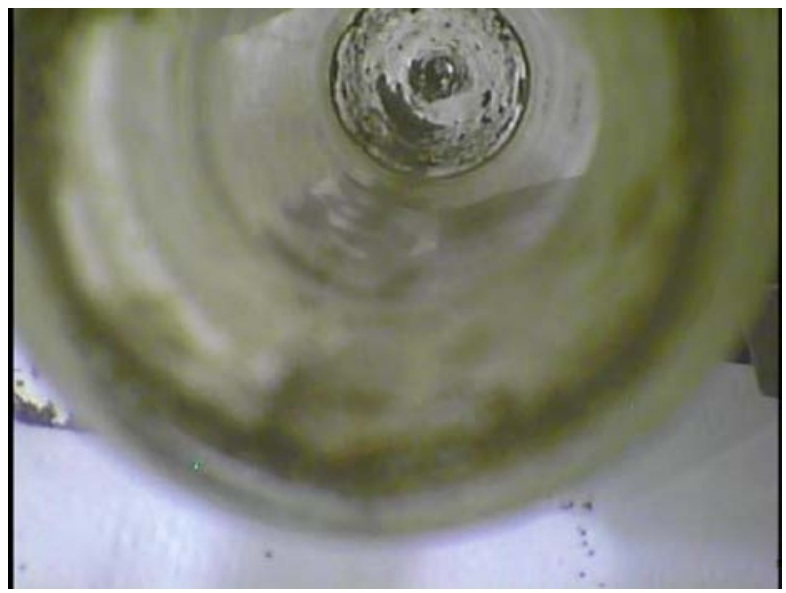

c) TI001-B3-K vial after pouring out solids. Small removable heel and some material adhered to centrifuge tube wall at sludge/water interface.

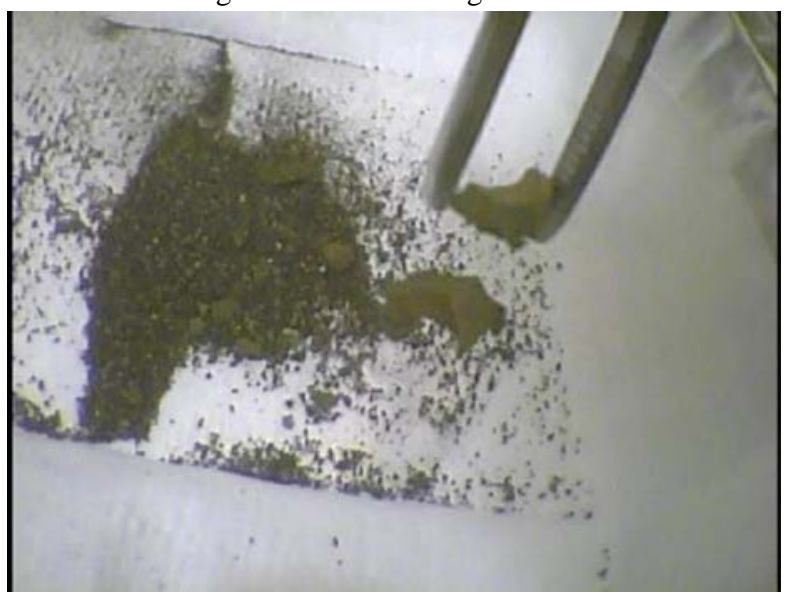

e) TI001-B3-K agglomerate pieces held together by fiber. One piece held by tweezers, other piece suspended by fiber strand.

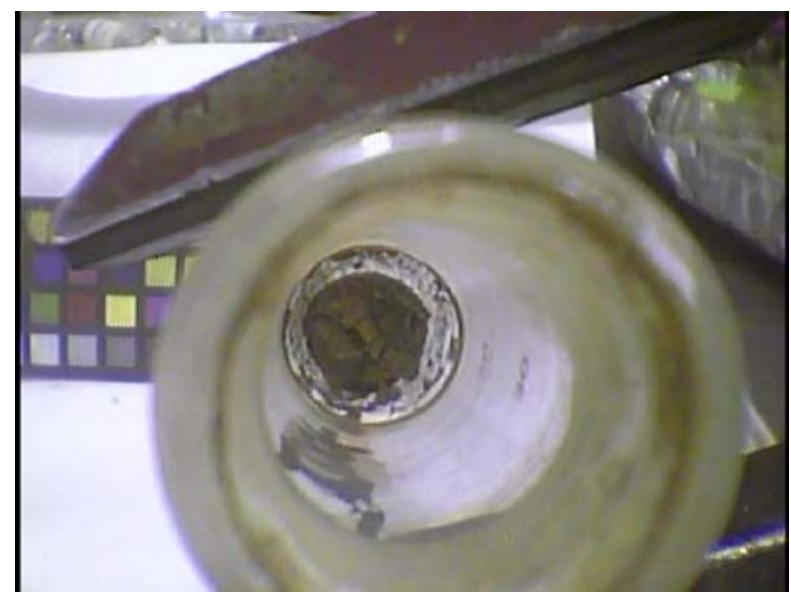

b) TI001-B3-K prior to pouring out solids. Large agglomerate pieces on top of underlying granular particles.

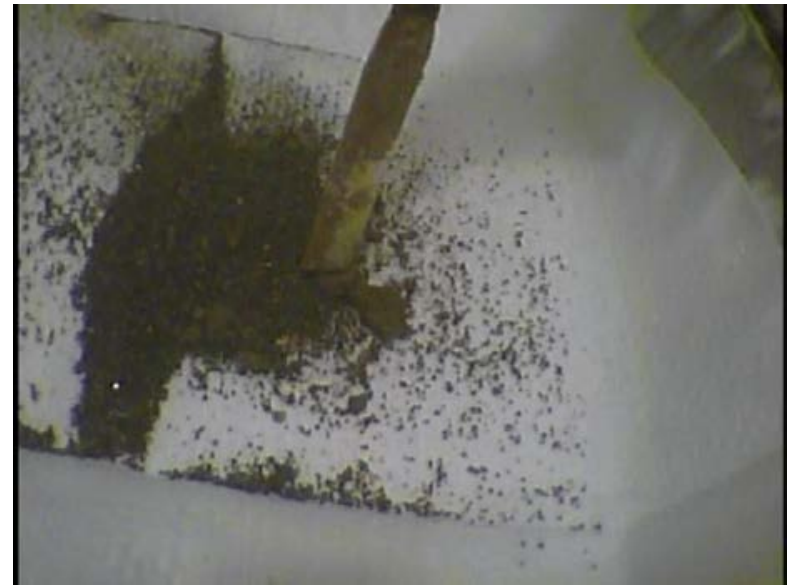

d) TI001-B3-K agglomerate particle with 1-cm wide stainless spatula. Little effort required to break.

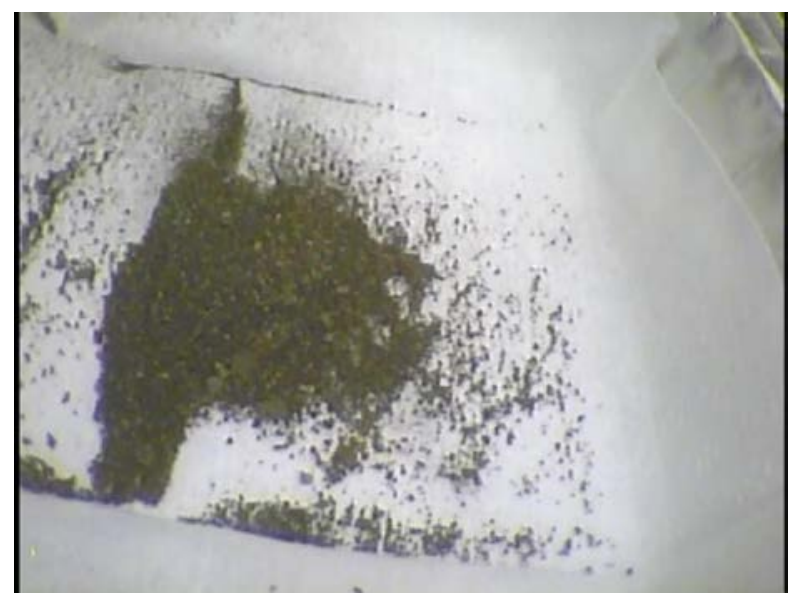

f) TI001-B3-K bulk materials image capturing general particle size, color, and morphology.

Figure 3.10. Select Dried Solids Examination Images of TI001-B3-K (KW210-B3) 


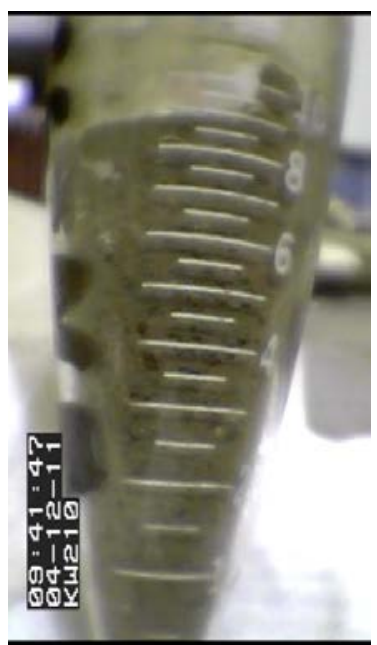

a) TI008-NB, KW210 container composite (un-sieved).

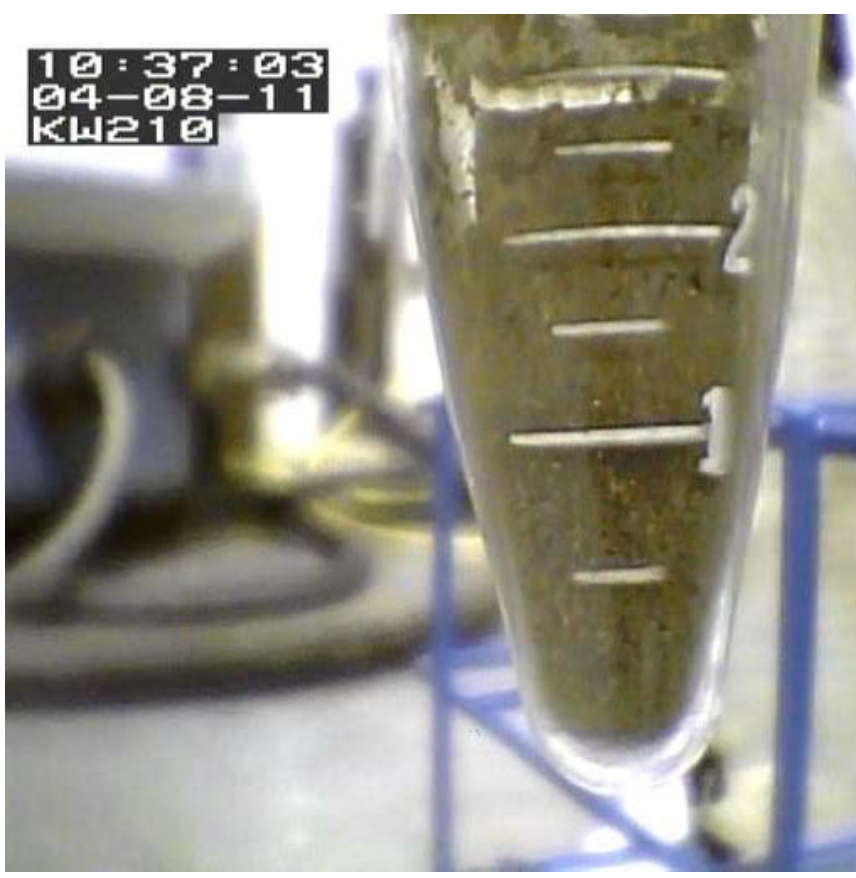

c) TI008-SN (TI008-SM was identical), $-500-\mu \mathrm{m}$ sieve fraction. Note the monolithic structure between 2 and 3 mL mark.

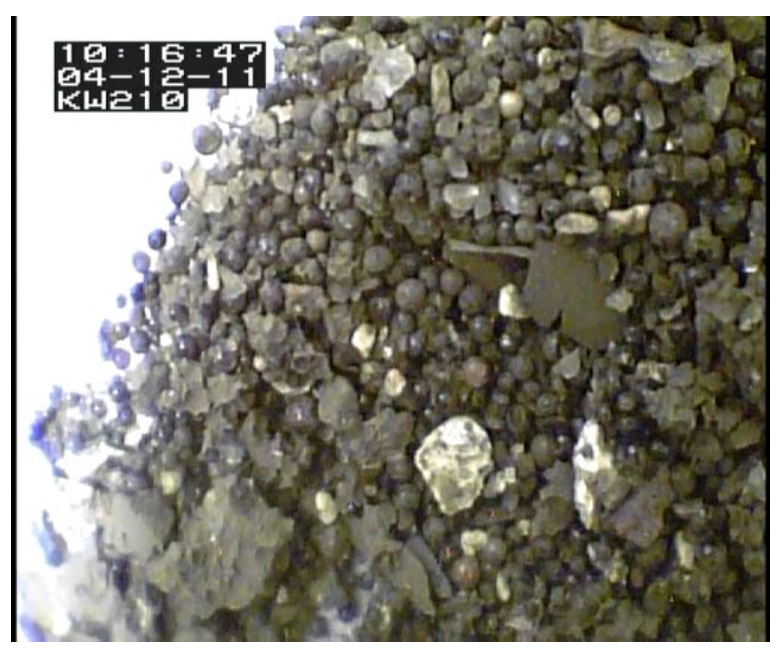

b) TI008-NB poured from centrifuge tube.

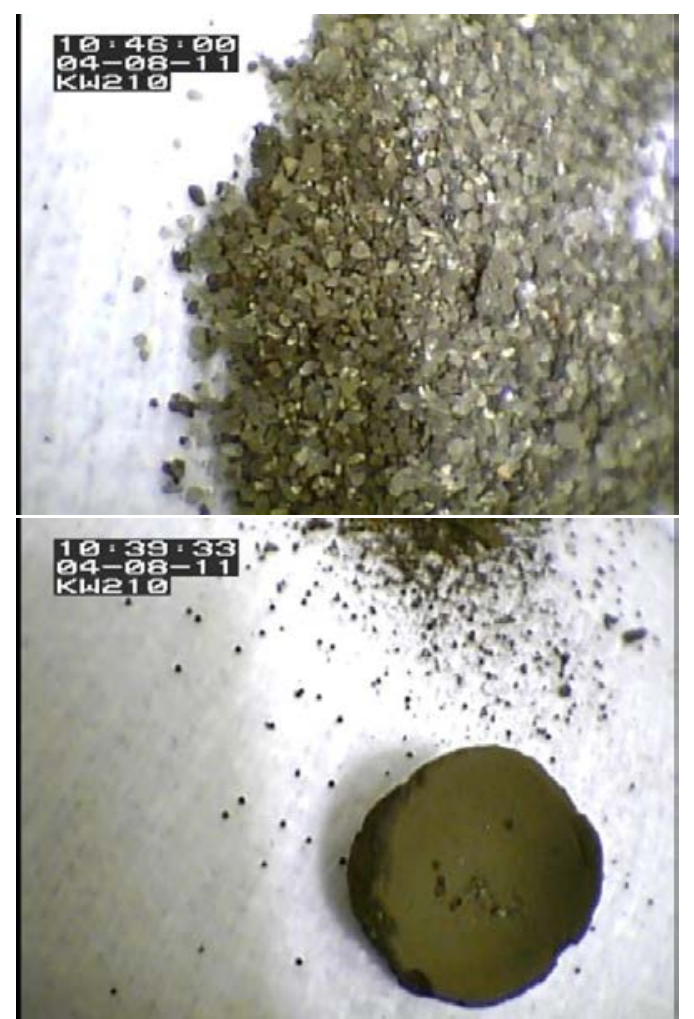

d) TI008-SM, the monolithic structure remained cohesive after pouring from the glass centrifuge tube. The structure was hard, requiring significant effort to break.

Figure 3.11. Select Dried Solids Examination Images of SCS-CON-210 Container Composite Samples 
PNNL-20650, Rev. 2

Table 3.3. Dried Physical Property Sample Observations

\begin{tabular}{|c|c|c|c|c|}
\hline $\begin{array}{l}\text { Core and Sample } \\
\text { Identification }\end{array}$ & $\begin{array}{l}\text { Settled } \\
\text { Solids } \\
\text { Volume, } \\
\text { mL }\end{array}$ & $\begin{array}{l}\text { Dried } \\
\text { Solids } \\
\text { Volume, } \\
\text { mL }\end{array}$ & $\begin{array}{l}\text { Volume } \\
\text { Reduction } \\
\quad(\%)\end{array}$ & Observations \\
\hline \multicolumn{5}{|l|}{ Core A1 } \\
\hline TI001-A1-K & 12 & 8.5 & 29 & $\begin{array}{l}\text { Very light, flaky, easily removed from glass centrifuge tube wall. } \\
\text { Powdery. Breaks in clumps. At sludge/water interface, dried } \\
\text { material on glass difficult to remove. Small heel remained in } \\
\text { centrifuge tube (vial) after pour. }\end{array}$ \\
\hline TI001-A1-L & 14.5 & 9 & 38 & $\begin{array}{l}\text { Poured well and granular. Flaky. Large particulates and fines. } \\
\text { Large chunks with porosity. Small heel remained in centrifuge tube } \\
\text { after pour. }\end{array}$ \\
\hline \multicolumn{5}{|l|}{ Core A4 } \\
\hline TI002-A4-K & 10.5 & 8.5 & 19 & $\begin{array}{l}\text { Material loosely adhered to centrifuge tube walls. Large } \\
\text { agglomerate formed on top of the bed of fines. Material composed } \\
\text { of fines, granules, agglomerates, and some flakes. Long, hair-like } \\
\text { fibers holding several large agglomerates together. }\end{array}$ \\
\hline TI002-A4-L & 11 & 9 & 18 & $\begin{array}{l}\text { Material composed of fines, granules, agglomerates, and some } \\
\text { flakes. Agglomerates broke apart with little force. Small heel } \\
\text { remained in centrifuge tube after pour. }\end{array}$ \\
\hline \multicolumn{5}{|l|}{ Core B1 } \\
\hline TI002-B1-K & 11.5 & 8 & 30 & $\begin{array}{l}\text { Poured well and little solids adhered to centrifuge tube. Large } \\
\text { agglomerate particles. More force required to break large } \\
\text { agglomerates. Material composed of fines, granules, large } \\
\text { agglomerates, and some flakes. }\end{array}$ \\
\hline TI002-B1-L & 13.25 & 6.5 & 51 & $\begin{array}{l}\text { Same as TI002-B1-K. In addition, a long, hair-like fiber holding } \\
\text { several large agglomerates together was observed. Small heel } \\
\text { remained in centrifuge tube after pour. }\end{array}$ \\
\hline \multicolumn{5}{|l|}{ Core B3 } \\
\hline TI001-B3-K & 12.5 & 7 & 44 & $\begin{array}{l}\text { Granular, fines, and some large agglomerates. Poured well and } \\
\text { little solids adhered to centrifuge tube. More difficult to break large } \\
\text { agglomerates than A1 material. Long, hair-like fiber holding } \\
\text { several large agglomerates together. }\end{array}$ \\
\hline TI001-B3-L & 13 & 7 & 46 & $\begin{array}{l}\text { Large agglomerates formed on top of dry solids. Many fines. } \\
\text { Material did not adhere to centrifuge tube. Large agglomerates on } \\
\text { top of fines, embedded fibers, and porous. Large chunks difficult to } \\
\text { break. Small heel remained in centrifuge tube after pour. }\end{array}$ \\
\hline \multicolumn{5}{|c|}{ Container Composite } \\
\hline KW210-CC-AA & 8.5 & 4.5 & 47 & $\begin{array}{l}\text { Granular particles poured easily with only minor adherence to the } \\
\text { glass walls. }\end{array}$ \\
\hline KW210-CC-AB & 9.0 & 5.7 & 37 & $\begin{array}{l}\text { Granular particles that poured easily. A large whitish chunk was } \\
\text { observed and it was brittle. }\end{array}$ \\
\hline KW210-CC-NA & 8.4 & 4.5 & 46 & $\begin{array}{l}\text { Granular particles poured easily with only minor adherence to the } \\
\text { glass walls. }\end{array}$ \\
\hline KW210-CC-NB & 9.6 & 5.7 & 41 & $\begin{array}{l}\text { Granular particles poured easily with only minor adherence to the } \\
\text { glass walls. }\end{array}$ \\
\hline \multicolumn{5}{|l|}{ Fines, $-500 \mu \mathrm{m}$} \\
\hline TI008-SM & 5.1 & 2.5 & 51 & $\begin{array}{l}\text { A distinct formation was observed as a cohesive hockey-puck shape } \\
\text { on top of granular material ( } \sim 20 \% \text { of the material volume). All } \\
\text { items poured out of the centrifuge tube easily. The puck-shaped } \\
\text { monolith was hard and required effort to crack. The rest of the } \\
\text { material was granular in appearance. }\end{array}$ \\
\hline TI008-SN & 5.6 & 2.9 & 48 & Same as TI008-SM. \\
\hline
\end{tabular}




\subsection{Engineered Container SCS-CON-210 Core Samples}

Four core samples were taken from Engineered Container SCS-CON-210. This section discusses the processing and characterization results from each core sample. Refer to Section 2.0 for discussions of sample receipt and preparation processes.

\subsection{Core Sample Observations}

As discussed in Section 2.3, color representation relative to the MacBeth ColorChecker ${ }^{\circledR}$ Rendition Chart (MacBeth Color Chart or equivalent) was required per the QAPjP/SAP. Example images of the MacBeth Color Chart taken with the in-cell camera in the HLRF (where polybottle receipt and core reconstitution activities occurred) are shown in Figure 4.1. As in previous compositing campaigns (Fiskum et al. 2011b), most green and blue shades reproduced to some degree, but red hues appeared muted or missing. These color reproduction issues need to be considered when viewing digital images captured during processing activities for the SCS-CON-210 core samples. In addition, varying lighting conditions in the hot cell tended to cause shades of sludge layers between video stills to differ. For this reason, attempts were made to capture images with the MacBeth Color Chart present.

The in-cell video camera failed on February 7, 2011 during an overnight composite jar settling test for core A1 and had to be replaced. No progressive degradation was observed leading up to the failure, which has been directly attributed to extended camera exposure to the high-activity samples and high dose rates in cell. Figure 4.2 compares video stills of the MacBeth Color Chart before and after the camera was replaced. Comparison of the video stills shows no detectable color loss. The camera failure occurred after all core compositing was completed and prior to any subsampling activities.

All SCS-CON-210 core samples required only one jar for sludge collection because the total volume collected from each core sample was less than $650 \mathrm{~mL}$. Material in each of the four reconstituted core samples was visually heterogeneous, varied in color, and contained observable stratification layers. No unusually large particulate was visible in the core sample (see Section 5.0 for sieved material images).

Dewatering the reconstituted core samples took between one and three days (as described in Section 2.1); they were subsampled once the material consistency exhibited sufficient strength to suspend dense particles. KW210-B1 and B3 cores required four dewatering steps prior to the final five-minute core mixing step just before the start of subsampling. KW210-A1 and A4 cores required three dewatering steps and two dewatering steps, respectively. The desired consistency for "A" cores was achieved more quickly and with less effort than the "B" cores.

Subsamples $\mathrm{H}$ and $\mathrm{J}$ were collected first into clean vials and delivered directly to analytical workstations for subsequent analytical work (i.e., TIC/TOC). Analytical samples were collected next into 40-mL Oak Ridge digestion centrifuge tubes, followed by collection of physical property and container composite samples. When sufficient sludge volume remained in the reconstituted core sample jar, archive samples were collected (using the sample spoon) into 120-mL Qorpak jars. In addition, residual solids were rinsed from the reconstituted core sample jars and collected as archive samples to minimize sample loss. 


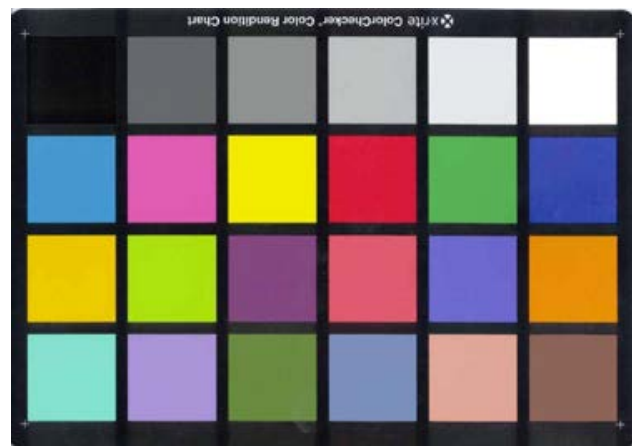

MacBeth Checker Color Chart Standard Note: Image is aligned to match 01/28/11 in-cell video still.

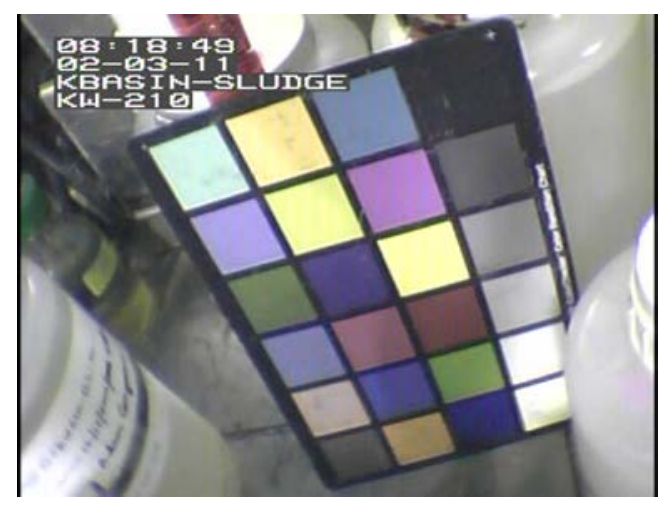

HLRF Color Chart: 02/03/11

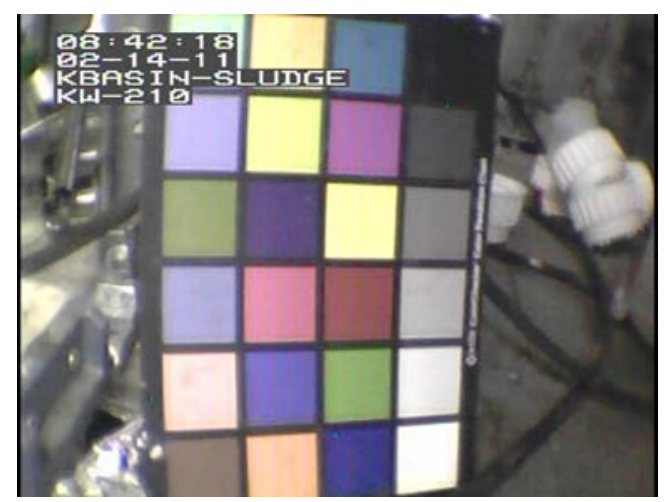

HLRF Color Chart: 02/14/11

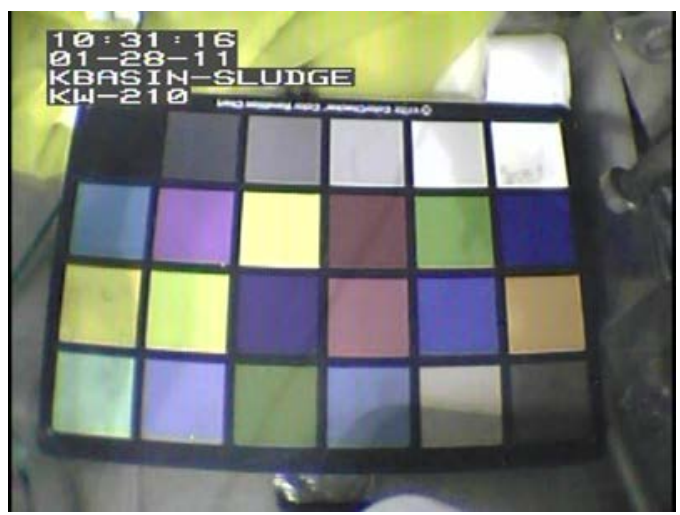

HLRF Color Chart: 01/28/11

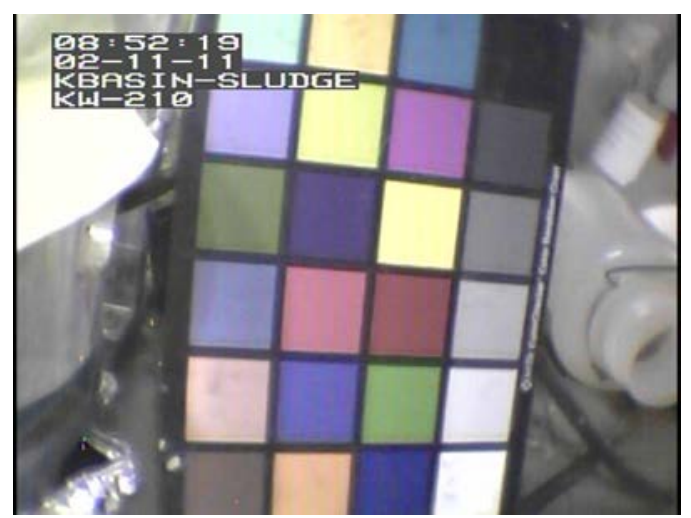

HLRF Color Chart: 02/11/11

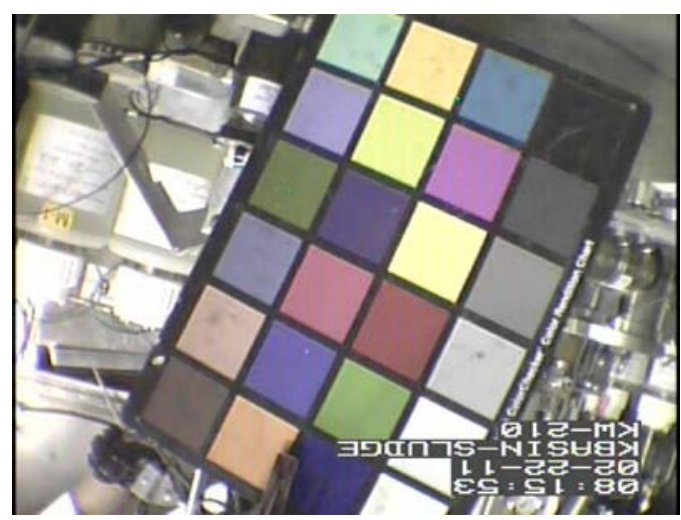

HLRF Color Chart: 02/22/11

Figure 4.1. MacBeth Color Checker Chart Images in HLRF During Reconstitution and Subsampling of the SCS-CON-210 Core Samples

Images capturing the KW210-A1 core processing, from the reconstituted core to the examination of the dried primary physical properties samples, are shown in Figure 4.3. The images provided in Figure 4.3 are representative of the other three cores in terms of processing. Similar images for all four cores are provided in data package DVD-52578-PNNL-20650 Rev. 2, Section IV, September 2013. 


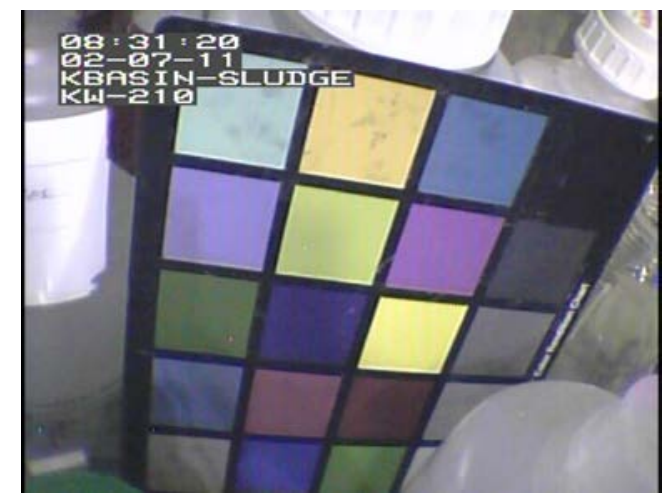

HLRF Color Chart Image on 02/07/11

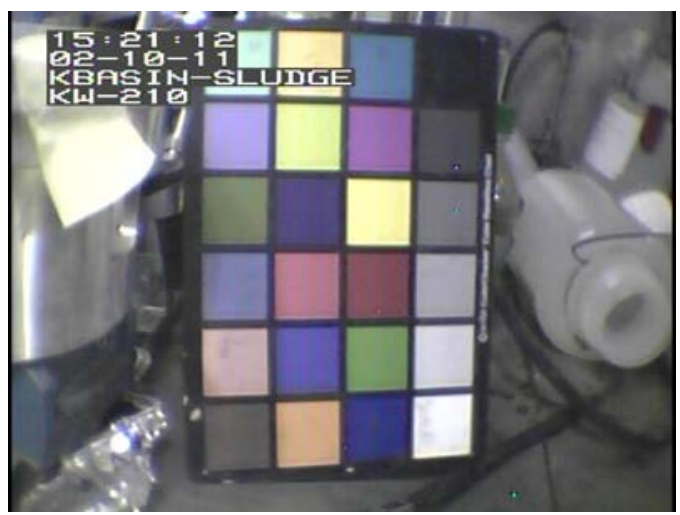

HLRF Color Chart Image on 02/10/11

Figure 4.2. Color Chart Images Before and After Camera Failure

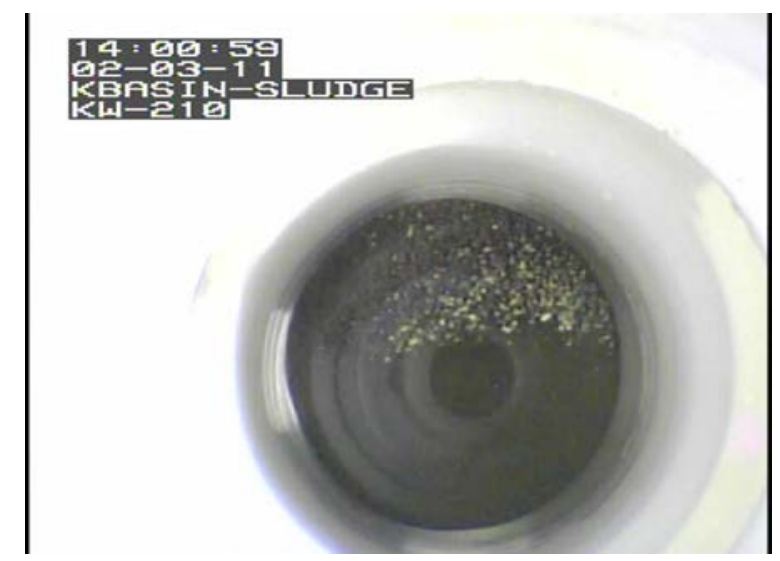

KW210-A1-A Initial 4-L Polybottle, Core Sample

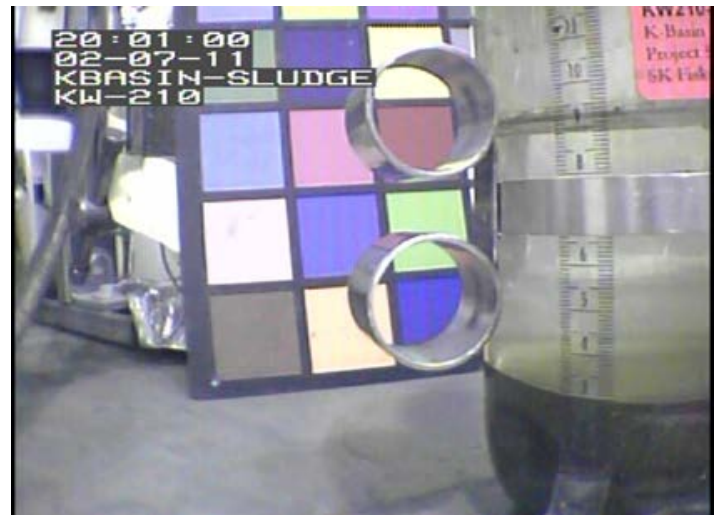

KW210-A1 Reconstituted Core Sample, Mixed and Settled

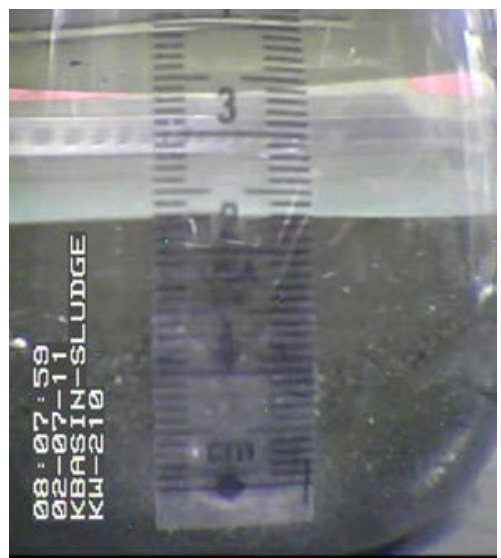

KW210-A1 Reconstituted Core Sample, Prior to Mixing

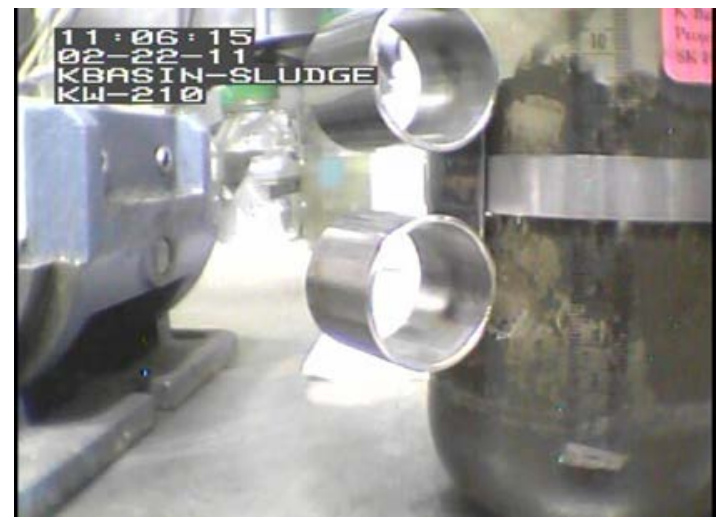

KW210-A1 Reconstituted Core Sample, Dewatered

Figure 4.3. Images of Reconstituted Core KW210-A1, Associated Subsamples, and a Dried Solids Examination 


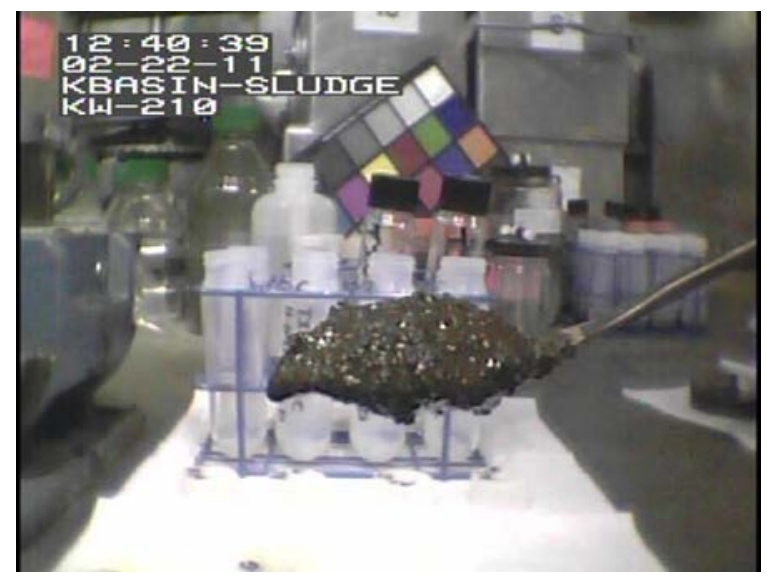

Subsampling Analytical

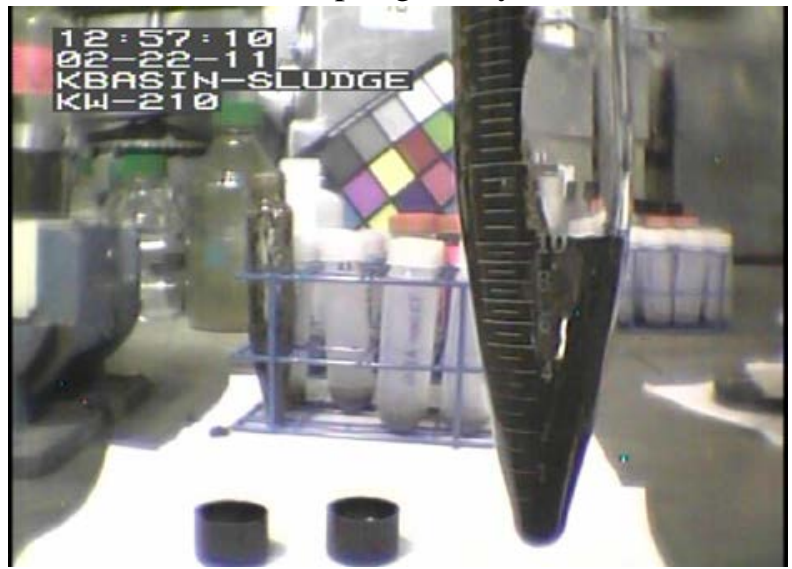

As-Sampled TI001-A1-K

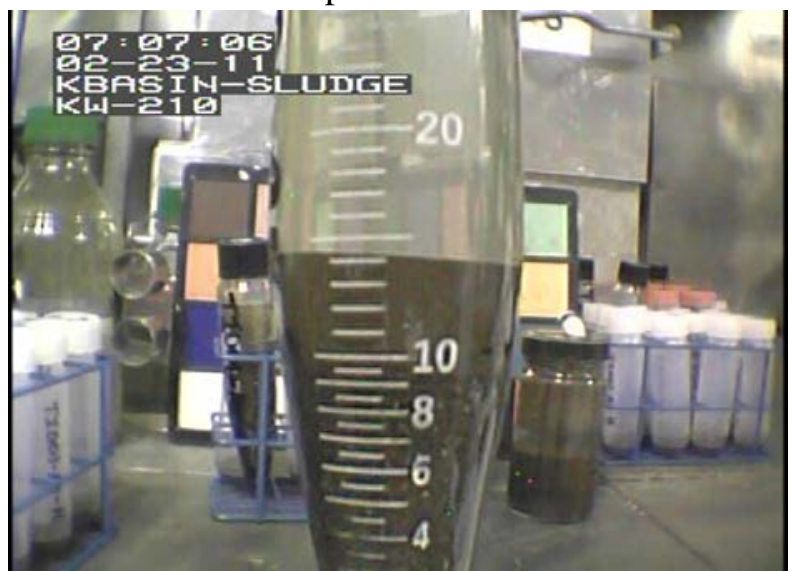

Rewatered and Settled TI001-A1-K

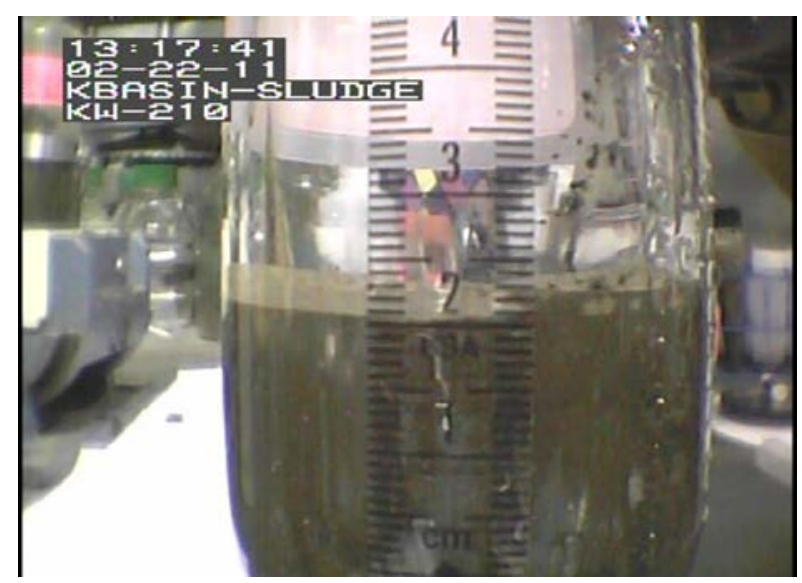

KW210-A1-A Sludge Level As-Sampled

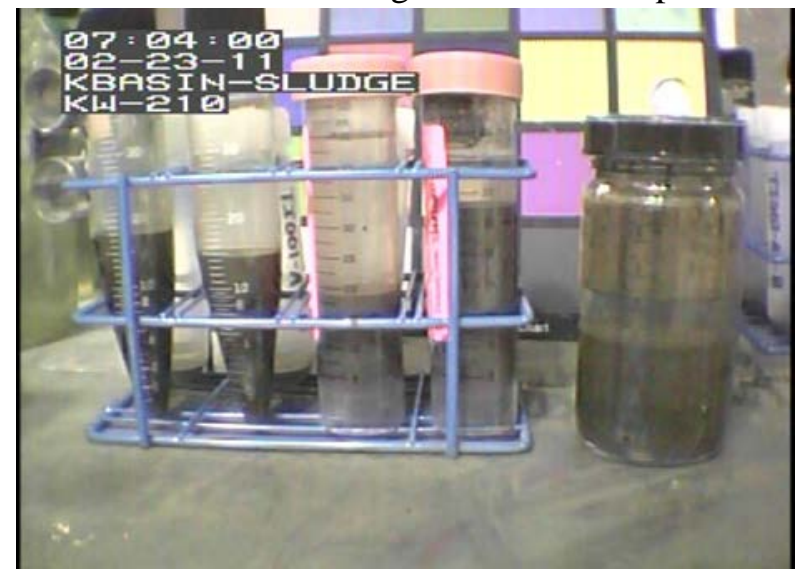

KW210-A1 Subsample Settling Test

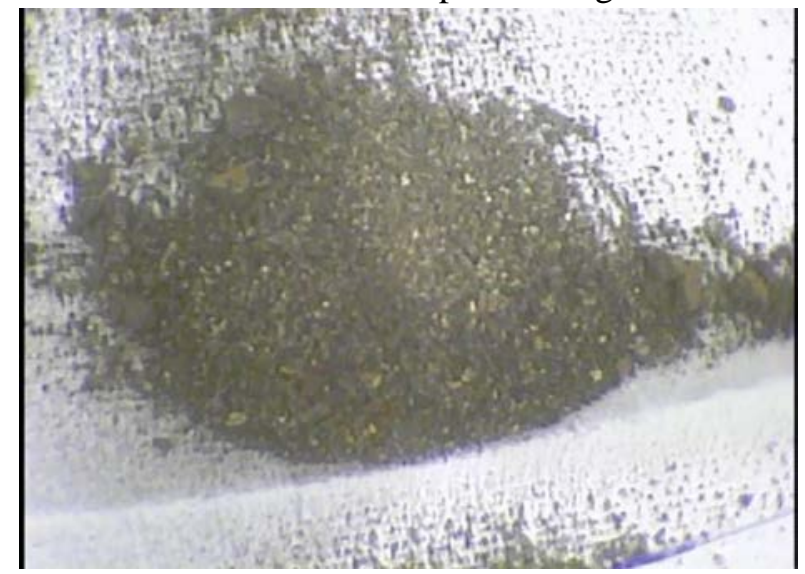

TI001-A1-K Dried Solids Inspection (“Poke Test”)

Figure 4.3. (contd)

Figure 4.4 provides sludge profile pictures of each of the four cores for a visual comparison of sludge morphology. The settled A1 and A4 core samples had similar material distributions with three distinct layers; first layer (bottom) a mixture of fines and agglomerates, second layer (middle) of primary granular agglomerates, and third (top) layer of grayish fines. The settled B1 and B3 core samples were also similar in appearance and settling behavior, but visually different from " $\mathrm{A}$ " cores. The " $\mathrm{B}$ " core samples had more dispersed fines distributed among a high number of resin beads. 


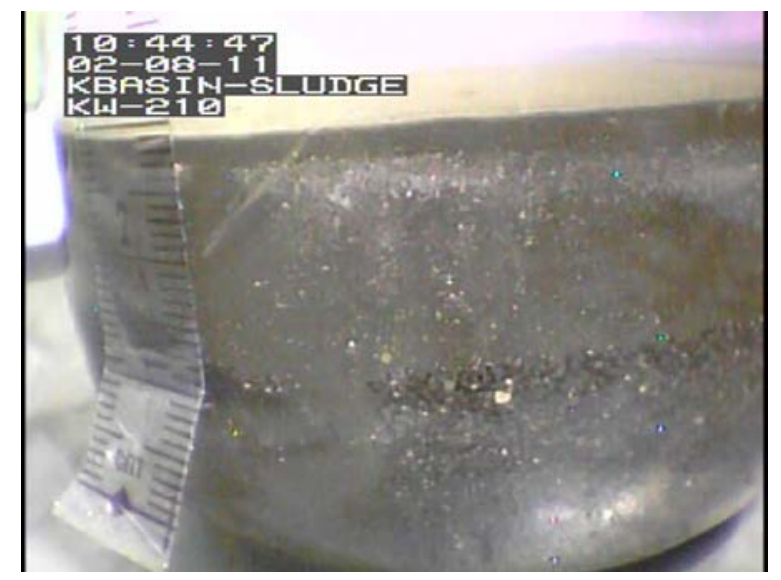

KW210-A1

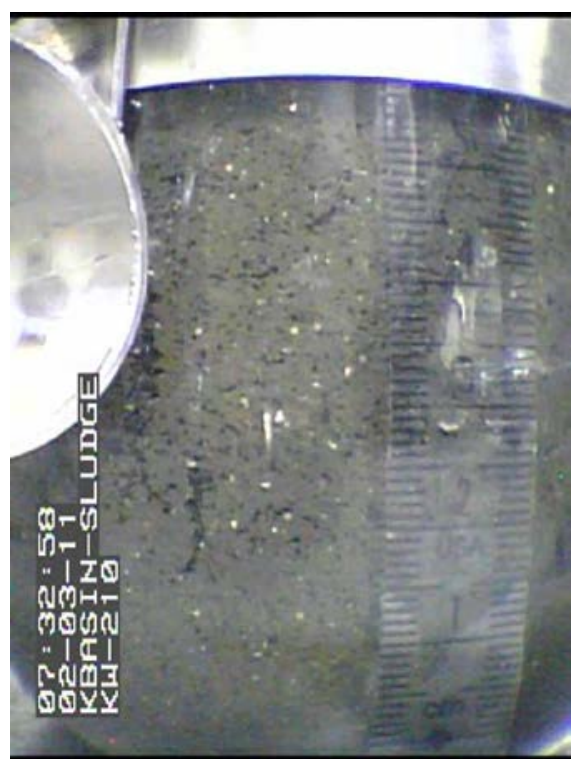

KW210-B1

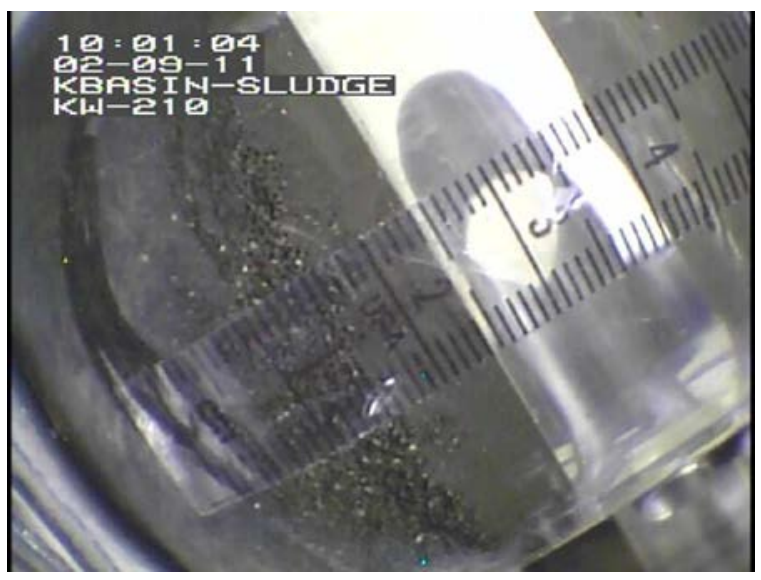

KW210-A4

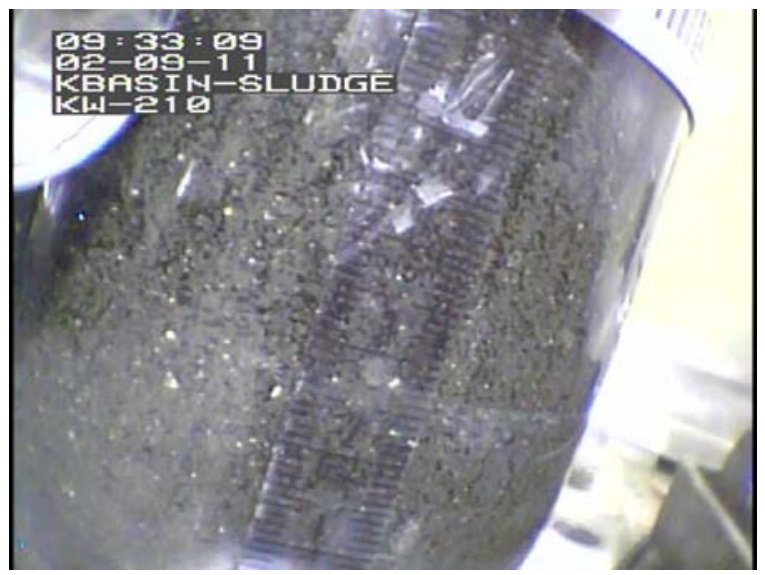

KW210-B3

Figure 4.4. Sludge Profile Images for All Four Reconstituted Core Samples After Initial Remix and Settle Test

Select images of unique observations made for the reconstituted cores are provided in Figures 4.5 through 4.10. The images document noteworthy and unique physical observations such as:

- material adhering to Teflon ${ }^{\mathrm{TM}}$-coated, magnetic spinbar in Oak Ridge tube

- solids materials and residues drying to jar walls

- scum layer and suspended particles at air/liquid interface

- gel-like material on mouth of 4-L core sample polybottles

- resin beads on top of fines prior to mixing

- vertical fluid channeling and solids mounds formation at sludge surface

- sludge surface mound formation and solids-ejecting plume captured in time lapsed photos. 
Teflon-coated spinbar magnets were introduced into the Oak Ridge tubes for $\mathrm{U}$ metal analysis subsamples $\mathrm{P}$ and Q. Two example images, provided in Figure 4.5, captured the magnetic material adhering to the Teflon-coated magnets in samples TI002-B1-P and TI001-A1-Q. These images were captured after the subsamples were remixed and settled. All four cores produced this observation.

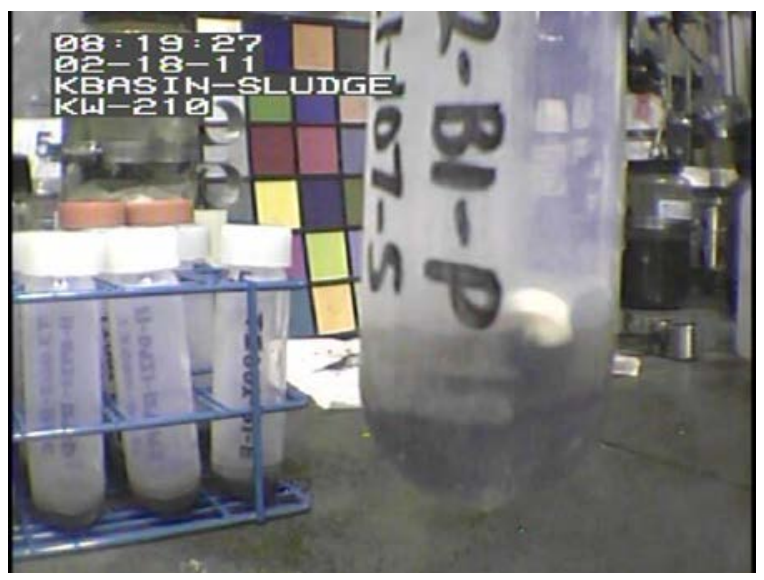

TI002-B1-P

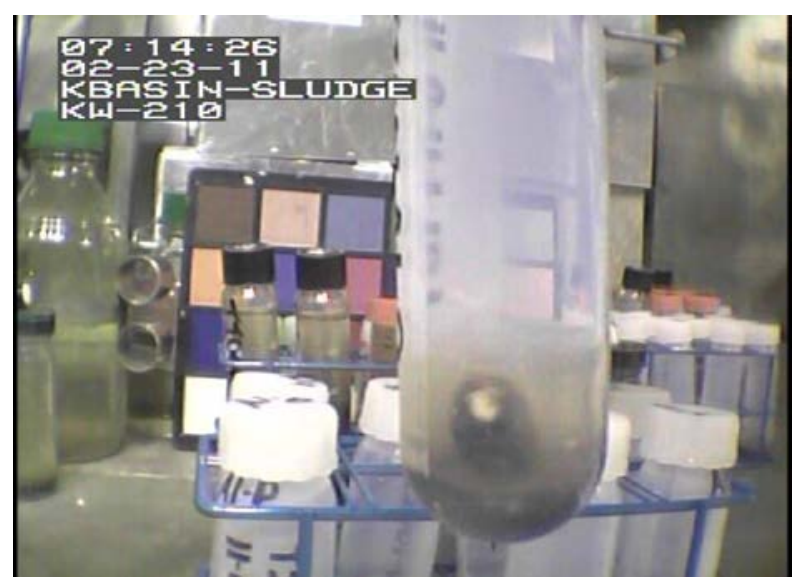

TI001-A1-Q

Figure 4.5. Images of Magnetic Material Adhering to Telfon-Coated Spinbar Magnet in U Metal Subsamples

Solids residues and particles were observed to adhere to the jar walls for all four cores. After mixing and settling, Qorpak jar walls also became coated with what appeared to be a residue with many agglomerated solids distributed on the walls. Figure 4.6 provides select images for cores A1 and A4.

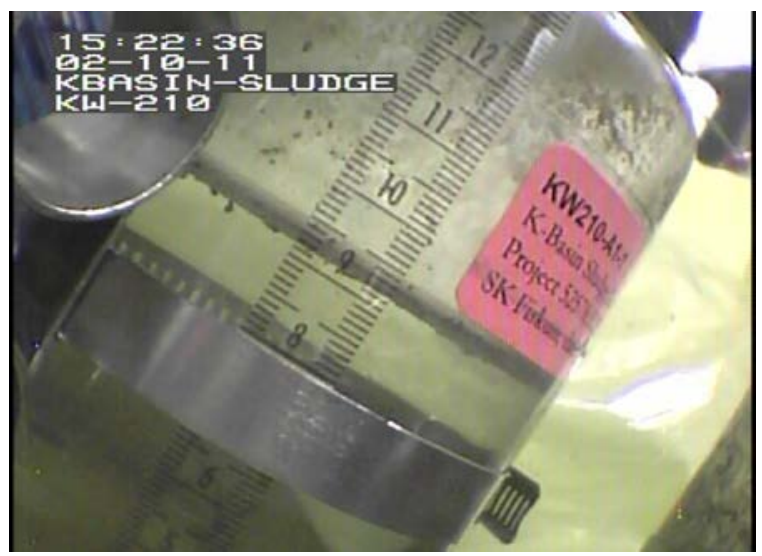

KW210-A1 Reconstituted Core, Surface Scum, Suspended Particles at Surface, and Dried Residue on Qorpak Jar Wall

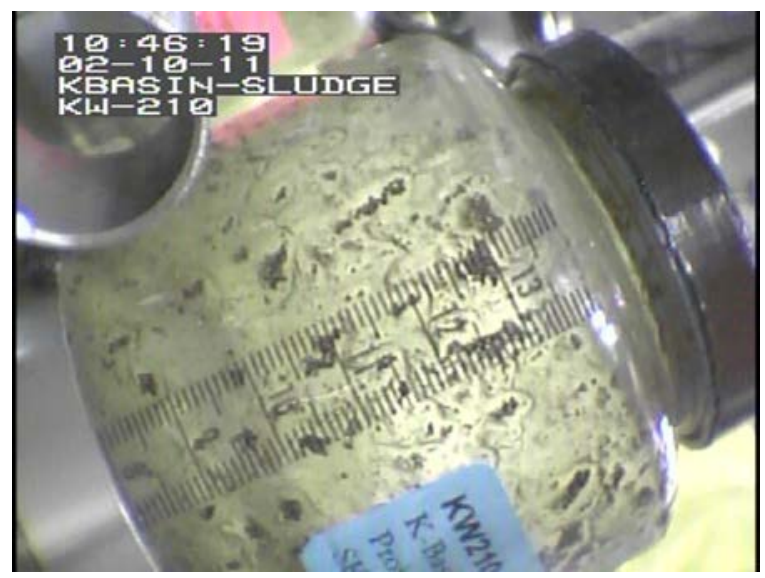

KW210-A4 Dried Solids Adhered to Qorpak Jar After Remixing and Settling Sludge

Figure 4.6. Images of Dried Residues and Particles on Qorpak Jar Walls

All four cores exhibited similar scum layer formation at the air/liquid interface in the reconstituted core sample jars. This observation was also made during subsample settling tests. Figure 4.7 provides two select images capturing the scum layer. The scum frequently retained large particulates at the surface, apparently by surface tension, and often released the large particulates to the sludge layer below when the liquid surface was lightly disturbed. 


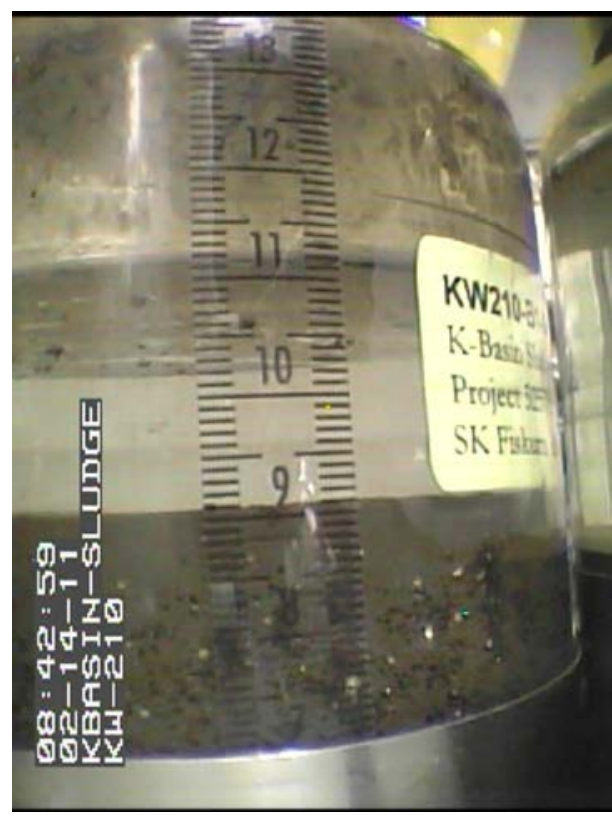

KW210-B1 Reconstituted Core (Bottom Up View), Surface Scum and Suspended Particles at Liquid/Air Interface

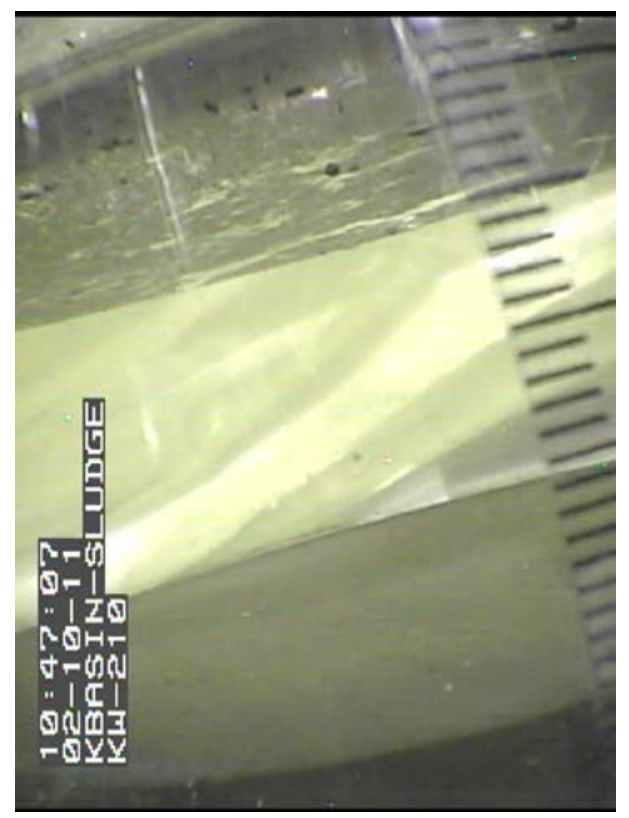

KW210-A4 Reconstituted Core (Bottom Up View), Surface Scum and Suspended Particles at Liquid/Air Interface

Figure 4.7. Images of Scum Layer and Suspended Particles at Air/Liquid Interface

The observed scum material may be related to the gel-like material observed on the mouth of some 4-L polybottles while reconstituting the core samples. Sample images of this gel-like material are provided in Figure 4.8. This material was observed while bulk transferring the core sample to the composite jar. As with all four cores, the gel-like material was combined with the bulk sludge sample after rinsing the residual solids and vacuum transferring from the polybottle and transferring to the Qorpak composite jar. All 4-L polybottles were completely clean after the sludge transfer.

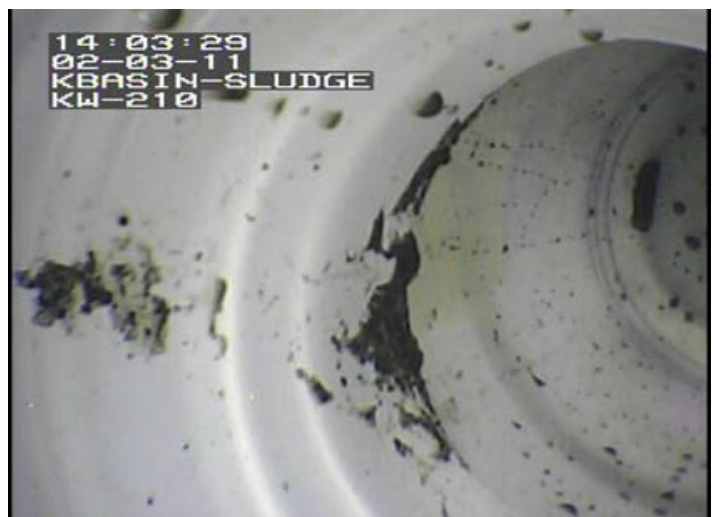

KW210-A1-A Gel-Like Material Adhesion to Polybottle Neck

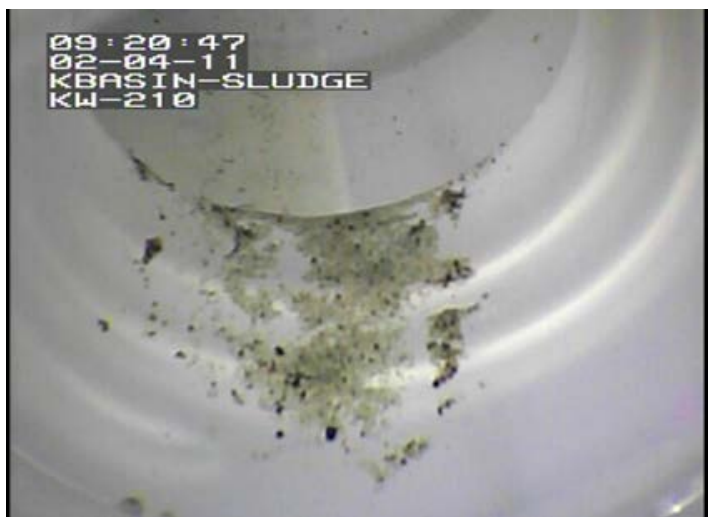

KW210-A1-B Gel-Like Material Adhesion to Polybottle Neck

Figure 4.8. Images of Gel-Like Material on Mouth of 4-L Core Sample Polybottles 
A notable observation on the behavior of the " $A$ " cores was the compression of the sludge and movement of the fines as water and resin beads were displaced. Figure 4.9 presents two images of the KW210-A4 reconstituted core prior to mixing and settling. The black particles in the images appear to be resin beads suspended or mounded above the bulk fines level. When this core was mixed and settled this behavior was not observed again.

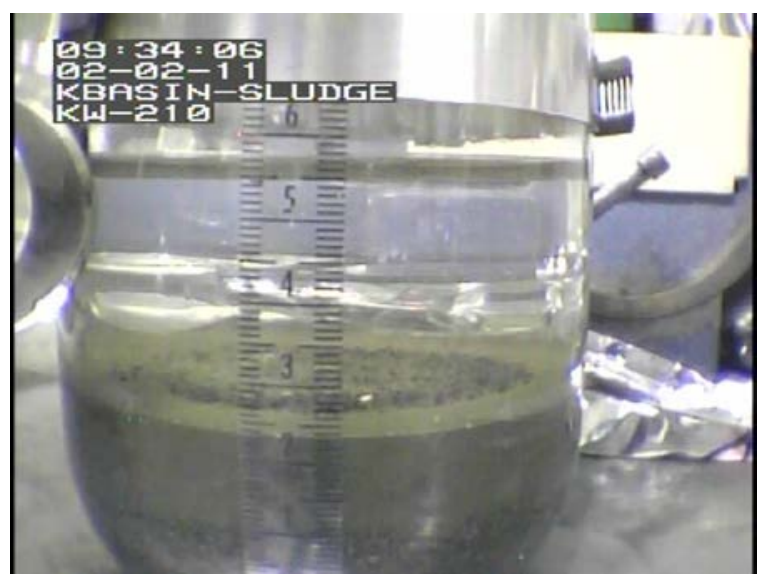

KW210-A4 Particles on Top of

Fines Prior to Mixing

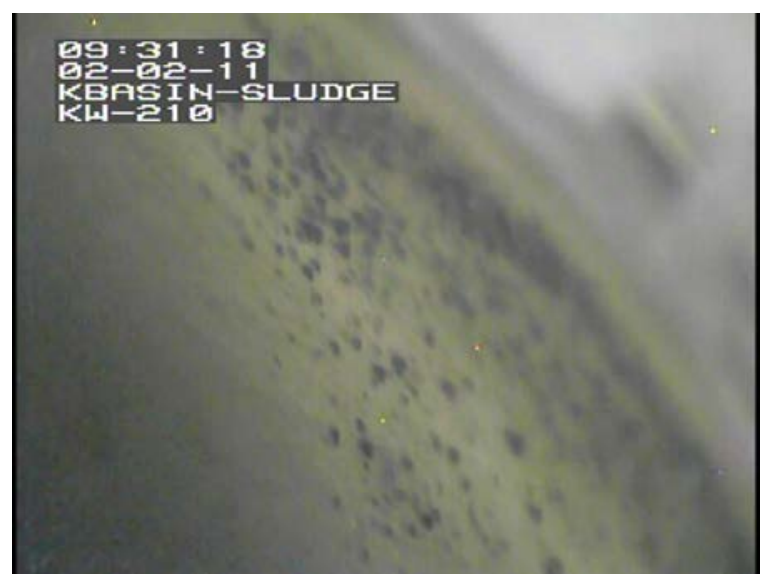

KW210-A4 Particles on Top of

Fines Prior to Mixing

Figure 4.9. Images of Resin Beads on Top of Fines Prior to Mixing

Vertical fluid channeling and the formation of solids mounds at the sludge surface were observed uniquely in the "B" cores. After mixing the reconstituted core samples and allowing them to settle, vertical channels and voids appeared in the sludge (see Figure 4.10). If the jar was disturbed, these channels would frequently link to the sludge surface and fluid flow was observed that resulted in mound formations. The sediment mounds were commonly created at the jar walls, but also distributed on the sludge surface. The cause of this behavior is believed to be interstitial water trapped below a sludge fines layer cap. When a fissure was created or broadened by the jar disturbance, the fluid head pressure drove the material to the sludge/water interface. Fines and smaller granular particles were carried to the surface. This analogous behavior is described by the geologic term "clastic dike." Figure 4.11 shows the development of the "clastic dike" habit in KW210-B3. 


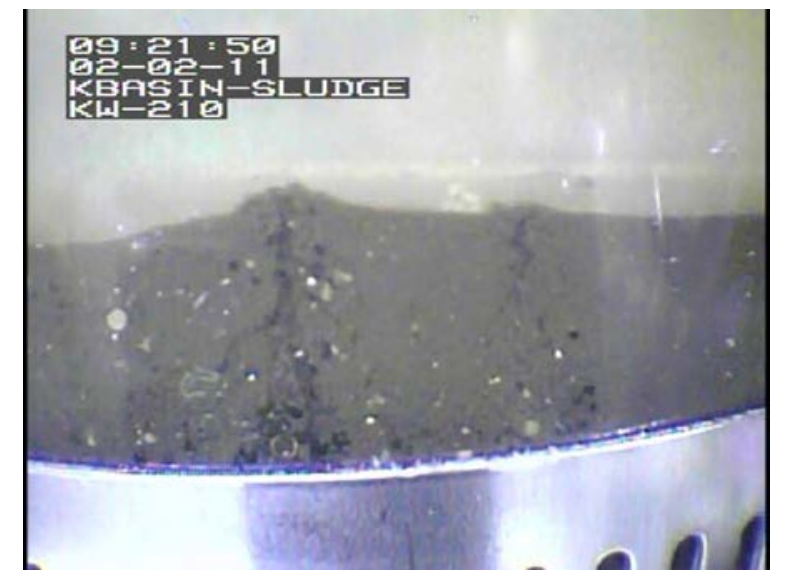

KW210-B1 Vertical Fluid Channels and Mounds

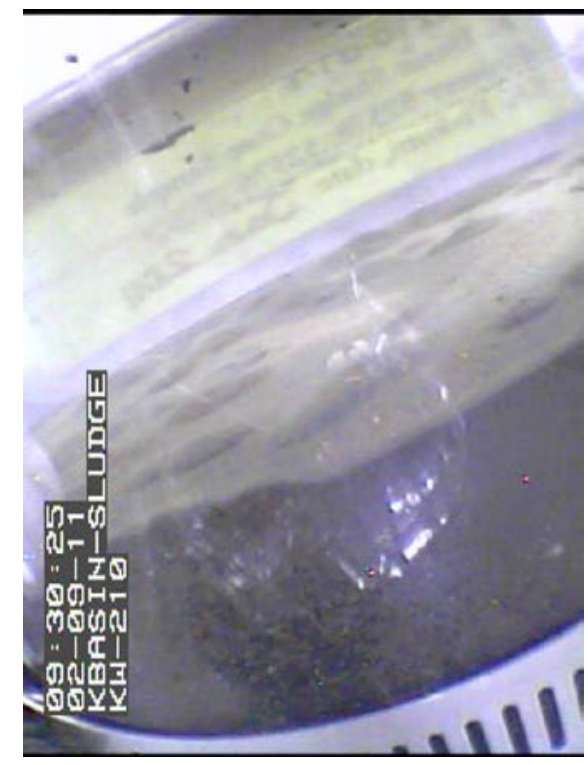

KW210-B3 Surface Mounds

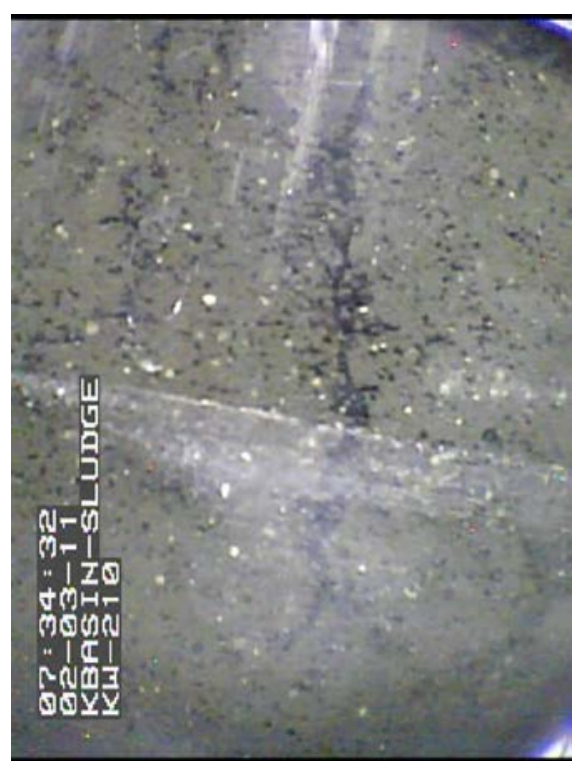

KW210-B1 Vertical Fluid Channels

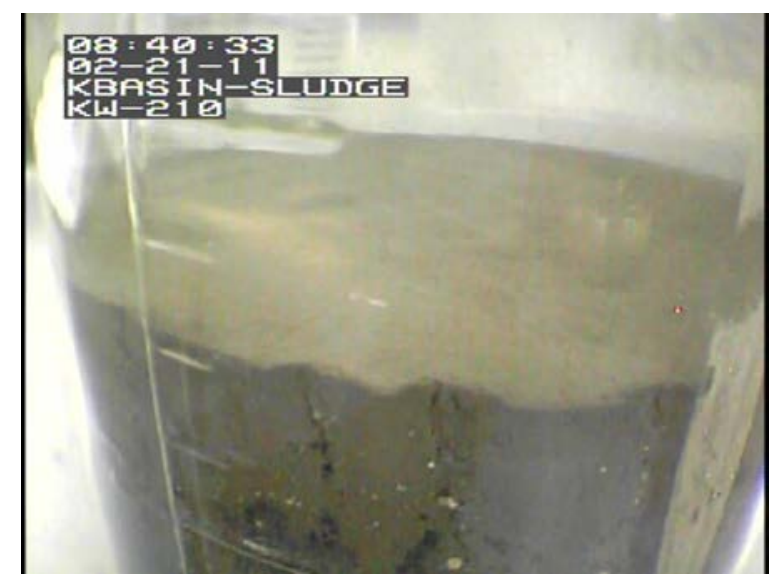

KW210-B3-Archive-1

Vertical Fluid Channels and Mounds

Figure 4.10. Images of Vertical Fluid Channels at Jar Walls and Solids Mounds at Sludge Surface for B1 and B3 Cores 


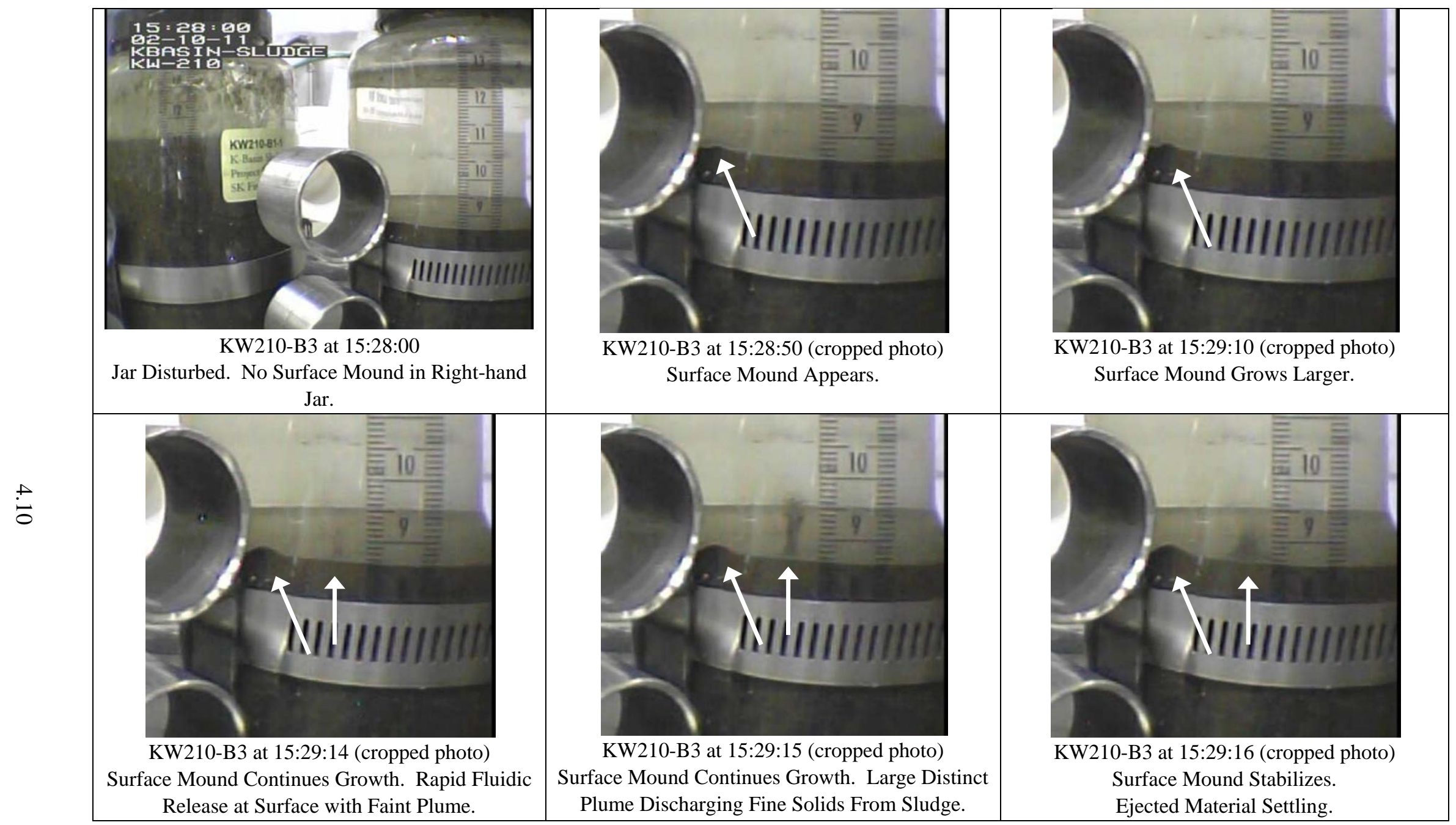

Figure 4.11. Time-Lapse Images of Fluid Ejecting Solids From the Sludge Surface and Mound Formation at the Wall in KW210-B3 Reconstituted Core Sample 


\subsection{Holding Time Limits}

As stated in the data quality objectives document (Westcott et al. 2009), characterization of the reconstituted core samples may be used to assign hazardous waste codes (WIPP permit metals) in accordance with the WIPP Hazardous Waste Permit. To this end, a six-month holding time limit, defined from sample collection date to the metals analysis date, was applied to the samples. The samples were typically collected over a period of several days; ICP-OES analyses were conducted in a two-day span. The effective holding time was calculated from the most conservative standpoint (first sample date and last analysis date). The attained holding times for each core sample are provided in Table 4.1. Each core sample was processed within the six-month limit as defined above.

Table 4.1. Sample Holding Times Supporting WIPP Hazardous Waste Permit Metals Analysis

\begin{tabular}{c|c|c|c|c|c|c}
\hline $\begin{array}{c}\text { Core Sample } \\
\text { ID }\end{array}$ & $\begin{array}{c}\text { RPL/ASO } \\
\text { ID }^{(\mathrm{a})}\end{array}$ & $\begin{array}{c}\text { Sample } \\
\text { Date } \\
\left(\mathrm{T}_{0}\right)\end{array}$ & $\begin{array}{c}\text { End of } \\
\text { 6-Month } \\
\text { Holding Time }\end{array}$ & $\begin{array}{c}\text { Acid } \\
\text { Digestion } \\
\text { Date }\end{array}$ & $\begin{array}{c}\text { ICP-OES } \\
\text { Analysis Date }\end{array}$ & $\begin{array}{c}\text { Effective } \\
\text { Holding Time, } \\
\text { Months }^{(\mathrm{c})}\end{array}$ \\
\hline KW210-A4 & $11-0420$ & $11 / 17 / 10$ & $5 / 17 / 11$ & & & 4.0 \\
KW210-B1 & $11-0421$ & $12 / 1 / 10$ & $6 / 1 / 11$ & & & 3.5 \\
KW210-B3 & $11-0422$ & $12 / 15 / 10$ & $6 / 15 / 11$ & $3 / 7 / 11$ & $3 / 15 / 11-3 / 16 / 11$ & 3.0 \\
KW210-A1 & $11-0419$ & $12 / 29 / 10$ & $6 / 29 / 11$ & & & 2.6 \\
\hline
\end{tabular}

(a) RPL/ASO ID extensions included -129-S and -129-D.

(b) Sample date is conservatively applied to the date material was first collected from the isolation tube.

(c) The holding time was calculated from the most conservative application of dates, i.e., the first sample date and the last ICP-OES analysis date. For calculation purposes, a month was assumed to be 30 days.

\subsection{Acid Dissolution for Chemical and Radiochemical Analysis}

The reconstituted core samples were processed as one analytical batch (in accordance with ASR 8798) as described in Section 2.5.1. The pre-digestion dry mass, settled sludge mass, and UDS dry mass following the acid digestion procedure are shown in Table 4.2. The wt\% of UDS (residual solids) was calculated on both the dried sample mass basis and the settled sludge mass basis. The wt\% UDS varied between samples, ranging from 64 to $88 \mathrm{wt} \%$ on a dry sample mass basis, and from 27 to $40 \mathrm{wt} \%$ wet settled solids sample mass basis.

All samples exceeded the decision criterion of $5 \mathrm{wt} \%$ UDS (settled sludge mass) and thus triggered the need for GEA and XRD analyses (see Table 3-8 of the QAPjP/SAP [KBC-33786, Rev. 2]). The UDS samples were subsampled to support the $\mathrm{KOH} / \mathrm{KNO}_{3}$ fusion (PNL-ALO-115) dissolutions to measure ICP-OES analytes and alpha emitting isotopes by AEA in accordance with ASR 8878. The UDS composition is discussed in Section 4.8. 
Table 4.2. SCS-CON-210 Chemical Analysis Characterization Samples for Acid Dissolution, Net UDS Masses, and UDS Weight Fraction

\begin{tabular}{|c|c|c|c|c|c|c|c|c|c|}
\hline \multirow[b]{2}{*}{$\begin{array}{c}\text { Container } \\
\text { Core ID }\end{array}$} & \multirow[b]{2}{*}{ Sample ID } & \multirow[b]{2}{*}{ RPL/ASO ID } & \multirow[b]{2}{*}{$\begin{array}{l}\text { Sample Pre- } \\
\text { digest Dry } \\
\text { Mass }^{(a)} \text {, gdry }\end{array}$} & \multirow{2}{*}{$\begin{array}{l}\text { Average } \\
\text { Conversion } \\
\text { Factor }^{(\mathrm{b})} \text {, } \\
\text { gdry } / \mathrm{g}_{\text {settled }}\end{array}$} & \multirow{2}{*}{$\begin{array}{c}\text { Pre-digest } \\
\text { Settled Sample } \\
\text { Mass }^{(\mathrm{c})} \text {, } \\
\text { gsettled }^{\text {sed }}\end{array}$} & \multirow{2}{*}{$\begin{array}{l}\text { UDS Post } \\
\text { Digest Dry } \\
\text { Mass }^{(a)}, \\
\text { gresidual }^{2}\end{array}$} & \multicolumn{3}{|c|}{ Weight Fraction UDS (Residual) } \\
\hline & & & & & & & $\begin{array}{l}\text { Dry Mass } \\
\text { Basis }^{(\mathrm{d})}, \\
\text { gresidual / gdry }\end{array}$ & $\begin{array}{l}\text { RPD or } \\
\text { RSD }\end{array}$ & $\begin{array}{c}\text { Settled Mass } \\
\text { Basis }^{(\mathrm{e})} \text {, } \\
\text { gresidual / gsettled }\end{array}$ \\
\hline \multirow[t]{2}{*}{ KW210-A1 } & TI001-A1-B & 11-0419-129-S & 1.1233 & 0.4470 & 2.51 & 0.7262 & 0.6465 & $1.43 \%$ & 0.289 \\
\hline & TI001-A1-C & 11-0419-129-D & 1.2773 & 0.4470 & 2.86 & 0.8140 & 0.6373 & & 0.285 \\
\hline \multirow[t]{2}{*}{ KW210-A4 } & TI002-A4-B & $11-0420-129-S$ & 1.4258 & 0.5667 & 2.52 & 1.0004 & 0.7016 & $0.09 \%$ & 0.398 \\
\hline & TI002-A4-C & 11-0420-129-D & 1.5994 & 0.5667 & 2.82 & 1.1212 & 0.7010 & & 0.397 \\
\hline \multirow[t]{3}{*}{ KW210-B1 } & TI002-B1-B & 11-0421-129-S & 0.7459 & 0.3281 & 2.27 & 0.6192 & 0.8301 & $2.95 \%$ & 0.272 \\
\hline & TI002-B1-C & 11-0421-129-D & 0.8192 & 0.3281 & 2.50 & 0.7206 & 0.8796 & & 0.289 \\
\hline & TI002-B1-D & 11-0421-129-MS & 0.8818 & 0.3281 & 2.69 & 0.7621 & 0.8643 & & 0.284 \\
\hline \multirow[t]{2}{*}{ KW210-B3 } & TI001-B3-B & $11-0422-129-S$ & 1.0626 & 0.3625 & 2.93 & 0.9163 & 0.8623 & $0.98 \%$ & 0.313 \\
\hline & TI001-B3-C & 11-0422-129-D & 1.0515 & 0.3625 & 2.90 & 0.9157 & 0.8709 & & 0.316 \\
\hline
\end{tabular}

(a) Mass measurements were made using four-place analytical balances. Results are found in analytical report "Mass Measurements” under ASR 8798 (see Appendix B).

(b) Conversion factors for $\mathrm{g}_{\mathrm{dry}} / \mathrm{g}_{\text {settled }}$ are calculated from the average values of the primary physical property samples from each core as reported in Table 3.1. Individual mass ratios and averages are presented to four significant figures in this table. However, the accuracies of the mass ratios are only valid to the third significant digit; the fourth digit is provided for information and to help the reader re-create computed values without introducing the rounding error.

(c) The pre-digested settled sample mass is calculated from dividing the pre-digest dry mass by the average conversion factor (gdry/gsettled) for the core. Data are presented to three significant figures to better represent the estimated accuracy.

(d) The weight fraction for UDS on a dry mass basis is calculated by dividing the dry mass of the UDS by the dry mass of the pre-digested solids. Data are presented to the appropriate significant figures.

(e) The weight fraction for UDS on a settled mass basis is calculated by multiplying the weight fraction for UDS on a dry mass basis by the average conversion factor (gdry/gsettled) for the core. Data are presented to three significant figures to better represent the estimated accuracy.

Calculations of data presented above were performed using un-truncated values using a Microsoft Excel calculation spreadsheet. Calculation spreadsheets in Microsoft Excel are provided in DVD-52578-PNNL-20650 Rev. 2, Section III, September 2013. 


\subsection{Radiochemistry}

Table 4.3 through Table 4.5 show the radioisotope analytical results for samples analyzed under ASR 8798. Results are presented on a dry mass basis (Table 4.3) with a companion summary of the QC sample results (precision and post-digestion BS and MS recoveries). The PB results are shown with the companion samples. The PB represents a blank prepared during the acid dissolution step and serves as an indicator of potential sources of contamination. The concentration basis of the PB was normalized to the average sample mass. Table 4.4 indicates the specific sample used for the MS evaluation. The QAPjP/SAP delineated a precision of $\pm 20 \%$ between sample and duplicate results and accuracy to be within \pm 20 to $\pm 25 \%$ as respectively measured from the BS and MS recoveries (see Table 2.12). All of the $\mathrm{QAPjP} / \mathrm{SAP}$ requirements for precision (RPD) and accuracy (BS and MS percent recoveries) for the analytes listed in Table 4.4 were met. Results are also expressed in terms of wet settled sludge mass basis. The factors used to convert dry sludge mass to wet settled sludge mass were calculated from the average physical properties (Section 3.0) and are summarized in Table 4.5. The conversion to the wet settled mass basis was calculated according to Equation 3.2. The reference dates for the reported radioisotopes are provided in the corresponding data table. Analytical reports for ASR 8798 are found in Appendix B.

Table 4.6 through Table 4.9 provide the TIMS analytical results of the isotopic ratios for $\mathrm{U}$ and $\mathrm{Pu}$. Results are presented in terms of At\% and wt\% in Table 4.6. As discussed in Section 2.5.9, Pu isotopic results were normalized to the ${ }^{238} \mathrm{Pu}$ AEA results. The QC sample results (RPD, results from the analysis of standard calibration verification check samples) are presented in Table 4.7 and discussed in detail in the sections below. The wt\% results were converted to units of $\mu \mathrm{g} / \mathrm{g}$ and $\mu \mathrm{Ci} / \mathrm{g}$ on a dry mass and settled mass basis in Table 4.8 and Table 4.9 respectively. Supporting documents for TIMS analytical results are found in Appendix E.

Specific radiochemistry results discussions are provided in the following sections.

\subsubsection{Neptunium (Alpha Energy Analysis)}

The alpha spectra were assessed for ${ }^{237} \mathrm{~Np}$ and ${ }^{238} \mathrm{U}$. Low levels of ${ }^{238} \mathrm{U}$ were observed in all of the samples. Three sample preparations contained ${ }^{238} \mathrm{U}$ equivalent to $\sim 9 \%$ of the ${ }^{237} \mathrm{~Np}$ activity detected in the sample (TI001-A1-C, TI002-B1-B, TI001-B3-B). The results from these samples were higher than their associated duplicate sample. The ${ }^{234} \mathrm{U}$ activity is $\sim 1.4 \times$ the ${ }^{238} \mathrm{U}$ activity for this sample set (based on the isotopic distribution). Therefore a $9 \%{ }^{238} \mathrm{U}$ impurity would be expected to increase the ${ }^{237} \mathrm{~Np}$ alpha emission signal by an additional $\sim 13 \%$ from the ${ }^{234} \mathrm{U}$ component $\left({ }^{237} \mathrm{~Np}\right.$ and ${ }^{234} \mathrm{U}$ have equivalent alpha emission energies).

The ${ }^{237} \mathrm{~Np}$ results were nearly equivalent to the requested detection limit of $1 \mathrm{E}-3 \mu \mathrm{Ci} / \mathrm{g}$. The RPDs for each duplicate pair ranged from 2 to 82\%; duplicate sample results from the A1 and B1 cores exceeded the $\pm 20 \%$ acceptance criterion. Relative $1-\sigma$ uncertainties for each measurement ranged from 10 to 29\% (actual uncertainties assigned to each sample are provided in Appendix B). These uncertainties represent the total propagated error associated with processing and counting operations, and include weighing errors, volume uncertainties, and counting error; they do not incorporate sub-sampling uncertainty (i.e., duplicate precision). The higher uncertainties were dominated by counting error, indicating that the ${ }^{237} \mathrm{~Np}$ activity was nearing the detection limit. 
Table 4.3. Radionuclide Results in Acid Digestate, Dry Sludge Mass Basis (ASR 8798)

\begin{tabular}{|c|c|c|c|c|c|c|c|c|c|c|}
\hline \multirow[b]{3}{*}{ Sample ID: } & \multicolumn{5}{|l|}{ Container: } & \multicolumn{5}{|c|}{ SCS-CON-210 } \\
\hline & Core: & \multicolumn{2}{|c|}{$\mathrm{A} 1$} & \multicolumn{2}{|c|}{ A4 } & \multicolumn{3}{|c|}{ B1 } & \multicolumn{2}{|c|}{ B3 } \\
\hline & $\mathrm{PB}$ & TI001-A1-B & TI001-A1-C & TI002-A4-B & TI002-A4-C & TI002-B1-B & TI002-B1-C & TI002-B1-D ${ }^{(b)}$ & TI001-B3-B & TI001-B3-C \\
\hline $\begin{array}{c}\mathrm{RPL} / \mathrm{ASO} \\
\mathrm{ID}> \\
\end{array}$ & $\begin{array}{c}11-0419- \\
129-\mathrm{PB}\end{array}$ & $\begin{array}{c}11-0419- \\
129-S\end{array}$ & $\begin{array}{c}11-0419- \\
129-D\end{array}$ & $\begin{array}{c}11-0420- \\
129-S\end{array}$ & $\begin{array}{c}11-0420- \\
129-D\end{array}$ & $\begin{array}{c}11-0421- \\
129-S\end{array}$ & $\begin{array}{c}11-0421- \\
129-D \\
\end{array}$ & $\begin{array}{l}11-0421- \\
129-M S\end{array}$ & $\begin{array}{c}11-0422- \\
129-S \\
\end{array}$ & $\begin{array}{c}11-0422- \\
129-D\end{array}$ \\
\hline AEA & \multicolumn{10}{|c|}{ MCi/g, Reference Dates: ${ }^{241} \mathrm{Am}$ April 11-12, 2011; ${ }^{238,239+240} \mathrm{Pu}$ April 8-12, 2011; ${ }^{237} \mathrm{~Np}$ April 14-19, 2011 (reference dates same as counting dates) } \\
\hline${ }^{237} \mathrm{~Np}$ & $<2 \mathrm{E}-6$ & $1.74 \mathrm{E}-3$ & $2.16 \mathrm{E}-3^{(\mathrm{a})}$ & $1.30 \mathrm{E}-3$ & 1.19E-3 & $1.78 \mathrm{E}-3^{(\mathrm{a})}$ & 7.49E-4 & -- & $1.14 \mathrm{E}-3^{(\mathrm{a})}$ & $1.12 \mathrm{E}-3$ \\
\hline${ }^{238} \mathrm{Pu}$ & $<3 E-6$ & $2.27 \mathrm{E}+0$ & $2.56 \mathrm{E}+0$ & $2.30 \mathrm{E}+0$ & $2.01 \mathrm{E}+0$ & $1.59 \mathrm{E}+0$ & $1.65 \mathrm{E}+0$ & -- & $1.85 \mathrm{E}+0$ & $1.77 \mathrm{E}+0$ \\
\hline${ }^{239+240} \mathrm{Pu}$ & $<3 E-6$ & $1.78 \mathrm{E}+1$ & $1.92 \mathrm{E}+1$ & $1.66 \mathrm{E}+1$ & $1.47 \mathrm{E}+1$ & $1.23 \mathrm{E}+1$ & $1.26 \mathrm{E}+1$ & -- & $1.36 \mathrm{E}+1$ & $1.26 \mathrm{E}+1$ \\
\hline${ }^{241} \mathrm{Am}$ & $5.67 \mathrm{E}-6$ & $1.82 \mathrm{E}+1$ & $1.97 \mathrm{E}+1$ & $1.71 \mathrm{E}+1$ & $1.43 \mathrm{E}+1$ & $1.51 \mathrm{E}+1$ & $1.48 \mathrm{E}+1$ & -- & $1.54 \mathrm{E}+1$ & $1.47 \mathrm{E}+1$ \\
\hline AEA & \multicolumn{10}{|l|}{$\mu \mathrm{g} / \mathrm{g}$} \\
\hline${ }^{237} \mathrm{~Np}$ & $<3 \mathrm{E}-3$ & $2.47 \mathrm{E}+0$ & $3.07 \mathrm{E}+0$ & $1.85 \mathrm{E}+0$ & $1.70 \mathrm{E}+0$ & $2.54 \mathrm{E}+0$ & $1.06 \mathrm{E}+0$ & -- & $1.63 \mathrm{E}+0$ & $1.59 \mathrm{E}+0$ \\
\hline${ }^{241} \mathrm{Am}$ & 1.65E-6 & $5.31 \mathrm{E}+0$ & $5.75 \mathrm{E}+0$ & $4.98 \mathrm{E}+0$ & $4.16 \mathrm{E}+0$ & $4.39 \mathrm{E}+0$ & $4.32 \mathrm{E}+0$ & -- & $4.49 \mathrm{E}+0$ & $4.28 \mathrm{E}+0$ \\
\hline Total U & \multicolumn{10}{|l|}{$\mu \mathrm{g} / \mathrm{g}$} \\
\hline U/KPA & 0.02 & 98,000 & 108,000 & 87,900 & 77,100 & 55,500 & 48,800 & -- & 56,200 & 53,100 \\
\hline U/ICP-OES & $<11$ & 93,800 & 103,000 & 82,800 & 73,600 & 51,900 & 45,600 & -- & 55,700 & 51,000 \\
\hline GEA & \multicolumn{3}{|c|}{$\mu \mathrm{Ci} / \mathrm{g}$, Reference Date: January 11, 2011} & & & & & & & \\
\hline${ }^{60} \mathrm{Co}$ & $<4 \mathrm{E}-5$ & $2.30 \mathrm{E}-1$ & $1.41 \mathrm{E}-1$ & $1.34 \mathrm{E}-1$ & $1.21 \mathrm{E}-1$ & $7.34 \mathrm{E}-2$ & 7.31E-2 & $6.73 E-2$ & $6.86 \mathrm{E}-2$ & $6.39 \mathrm{E}-2$ \\
\hline${ }^{134} \mathrm{Cs}$ & $<4 \mathrm{E}-5$ & $<3 \mathrm{E}-3$ & $<6 \mathrm{E}-3$ & $<3 \mathrm{E}-3$ & $<6 \mathrm{E}-3$ & $<4 \mathrm{E}-3$ & $<3 \mathrm{E}-3$ & $<5 \mathrm{E}-3$ & $<5 \mathrm{E}-3$ & $<5 \mathrm{E}-3$ \\
\hline${ }^{137} \mathrm{Cs}$ & $2.19 \mathrm{E}-4$ & $3.68 \mathrm{E}+2$ & $3.93 \mathrm{E}+2$ & $3.38 \mathrm{E}+2$ & $3.81 \mathrm{E}+2$ & $1.43 \mathrm{E}+2$ & $1.68 \mathrm{E}+2$ & $1.50 \mathrm{E}+2$ & $1.13 \mathrm{E}+2$ & $1.02 \mathrm{E}+2$ \\
\hline${ }^{152} \mathrm{Eu}$ & $<2 \mathrm{E}-4$ & $<5 \mathrm{E}-3$ & $<8 \mathrm{E}-3$ & $<5 \mathrm{E}-3$ & $<9 \mathrm{E}-3$ & $<4 \mathrm{E}-3$ & $<7 \mathrm{E}-3$ & $<1 \mathrm{E}-2$ & $<1 \mathrm{E}-2$ & $<1 \mathrm{E}-2$ \\
\hline${ }^{154} \mathrm{Eu}$ & $<1 \mathrm{E}-4$ & 7.30E-1 & 7.59E-1 & $5.84 \mathrm{E}-1$ & $5.41 \mathrm{E}-1$ & $4.71 \mathrm{E}-1$ & $5.16 \mathrm{E}-1$ & $4.76 \mathrm{E}-1$ & $5.28 \mathrm{E}-1$ & $5.14 \mathrm{E}-1$ \\
\hline${ }^{155} \mathrm{Eu}$ & $<2 \mathrm{E}-4$ & $1.18 \mathrm{E}-1$ & $1.26 \mathrm{E}-1$ & $<6 \mathrm{E}-2$ & $<1 \mathrm{E}-1$ & $8.71 \mathrm{E}-2$ & $1.20 \mathrm{E}-1$ & $<7 \mathrm{E}-2$ & $8.04 \mathrm{E}-2$ & 7.35E-2 \\
\hline${ }^{241} \mathrm{Am}$ & $<3 \mathrm{E}-4$ & $1.96 \mathrm{E}+1$ & $2.13 \mathrm{E}+1$ & $1.64 \mathrm{E}+1$ & $1.39 \mathrm{E}+1$ & $1.41 \mathrm{E}+1$ & $1.68 \mathrm{E}+1$ & $1.38 \mathrm{E}+1$ & $1.48 \mathrm{E}+1$ & $1.39 \mathrm{E}+1$ \\
\hline \multicolumn{11}{|l|}{ Beta/LSC } \\
\hline${ }^{90} \mathrm{Sr}$ & $<1 \mathrm{E}-4$ & $3.44 \mathrm{E}+2$ & $3.37 \mathrm{E}+2$ & $2.71 E+2$ & $2.72 \mathrm{E}+2$ & $1.39 \mathrm{E}+2$ & $1.55 \mathrm{E}+2$ & -- & $1.44 \mathrm{E}+2$ & $1.34 \mathrm{E}+2$ \\
\hline
\end{tabular}

(a) The sample Np fraction contained a slight quantity of $\mathrm{U} ;{ }^{234} \mathrm{U}$ has an equivalent alpha emission energy as ${ }^{237} \mathrm{~Np}$ and would cause slight high bias. See Section 4.4 .1 .

(b) Sample TI002-B1-D was the batch MS sample that was spiked with analytes of interest for ICP-OES. From a GEA perspective, it can be used as a confirmatory sample for KW210 Core B1.

Relative uncertainties were typically between 2 and $6 \%$ except for ${ }^{237} \mathrm{~Np}$ where 10 to $29 \%$ uncertainties were reported. Analytical results including 1 -sigma uncertainties from ASR 8798 are found in Appendix B.

RPD calculations reported in Table 4.4 for KW210 Core B1 are based on samples TI002-B1-B and TI002-B1-C.

Shaded analyte results indicate the QAPjP/SAP precision criterion of $\pm 20 \%$ was exceeded. 
Table 4.4. Radionuclide QC Sample Results in Acid Digestate (ASR 8798)

\begin{tabular}{|c|c|c|c|c|c|c|c|}
\hline \multirow[b]{3}{*}{ Analyte } & \multicolumn{4}{|c|}{ Container SCS-CON-210 Core Samples } & \multirow{2}{*}{$\begin{array}{c}\text { LCS/BS } \\
\text { Recovery, }\end{array}$} & \multirow{2}{*}{$\frac{\text { MS }}{\text { Recovery, }}$} & \multirow{2}{*}{$\begin{array}{c}\text { MS } \\
\text { Sample }\end{array}$} \\
\hline & A1 & A4 & $\mathrm{B} 1^{\text {(a) }}$ & B3 & & & \\
\hline & RPD (\%) & RPD (\%) & RPD (\%) & RPD (\%) & $\%$ & $\%$ & ID \\
\hline${ }^{237} \mathrm{~Np}$ & 22 & 9 & 82 & 2 & 102 & 103 & TI001-B3-B \\
\hline${ }^{238} \mathrm{Pu}$ & 12 & 13 & 3 & 5 & -- & -- & -- \\
\hline${ }^{241} \mathrm{Am}$ & 8 & 18 & 2 & 5 & 102 & 97 & TI001-B3-B \\
\hline \multicolumn{8}{|l|}{ Total U } \\
\hline $\mathrm{U} / \mathrm{KPA}$ & 10 & 13 & 13 & 6 & 103 & 103 & TI001-B3-C \\
\hline U/ICP-OES & 9.6 & 12 & 13 & 8.9 & 100 & NR & -- \\
\hline \multicolumn{8}{|l|}{ GEA } \\
\hline${ }^{152} \mathrm{Eu}$ & --- & --- & --- & --- & -- & -- & -- \\
\hline${ }^{154} \mathrm{Eu}$ & 4 & 8 & 9 & 3 & -- & -- & -- \\
\hline${ }^{155} \mathrm{Eu}$ & 7 & --- & 32 & 9 & -- & -- & -- \\
\hline${ }^{241} \mathrm{Am}$ & 8 & 17 & 17 & 6 & -- & -- & -- \\
\hline \multicolumn{8}{|l|}{ Beta/LSC } \\
\hline${ }^{90} \mathrm{Sr}$ & 2 & 0.5 & 11 & 8 & 99 & 98 & TI001-B3-B \\
\hline
\end{tabular}

(a) Sample TI002-B1-D was the batch MS sample spiked with analytes of interest for ICP-OES. From a GEA perspective, it can be used as a confirmatory sample for KW210 Core B1. Relative percent deviation (RPD) calculations for the KW210 Core B1 are based on samples TI002-B1-B and TI002-B1-C.

NR: not recovered; spike was too low relative to the uranium present in the sample matrix.

Dashed lines indicate the property was not applicable. The RPD could not be determined because the analyte concentration was less than the detection limit; the spike recovery did not apply.

Analytical results and uncertainties from ASR 8798 samples are found in Appendix B.

Shaded analyte results indicate the QAPjP/SAP precision criterion of $\pm 20 \%$ was exceeded. 
Table 4.5. Radionuclide Results in Acid Digestate, Wet Settled Sludge Mass Basis (ASR 8798)

\begin{tabular}{|c|c|c|c|c|c|c|c|c|c|c|}
\hline \multirow[b]{3}{*}{ Sample ID: } & \multirow{2}{*}{$\begin{array}{r}\text { Container: } \\
\text { Core: } \\
\end{array}$} & \multicolumn{9}{|c|}{ SCS-CON-210 } \\
\hline & & \multicolumn{2}{|c|}{ A1 } & \multicolumn{2}{|c|}{ A4 } & \multicolumn{3}{|c|}{$\mathrm{B} 1$} & \multicolumn{2}{|c|}{ B3 } \\
\hline & PB & TI001-A1-B & TI001-A1-C & TI002-A4-B & TI002-A4-C & TI002-B1-B & TI002-B1-C & TI002-B1-D(b) & TI001-B3-B & TI001-B3-C \\
\hline $\begin{array}{c}\text { RPL/ASO } \\
\text { ID }>\end{array}$ & $\begin{array}{c}11-0419- \\
129-\mathrm{PB}\end{array}$ & $\begin{array}{c}11-0419- \\
129-S \\
\end{array}$ & $\begin{array}{c}11-0419- \\
129-D\end{array}$ & $\begin{array}{c}11-0420- \\
129-S \\
\end{array}$ & $\begin{array}{c}11-0420- \\
129-\mathrm{D} \\
\end{array}$ & $\begin{array}{c}11-0421- \\
129-S\end{array}$ & $\begin{array}{c}11-0421- \\
129-D\end{array}$ & $\begin{array}{l}11-0421- \\
129-M S\end{array}$ & $\begin{array}{c}11-0422- \\
129-S\end{array}$ & $\begin{array}{c}11-0422- \\
129-\mathrm{D}\end{array}$ \\
\hline $\begin{array}{c}\text { Conversion } \\
\text { Factor }^{(a)} \\
\end{array}$ & 0.426 & 0.447 & 0.447 & 0.567 & 0.567 & 0.328 & 0.328 & 0.328 & 0.363 & 0.363 \\
\hline AEA & \multicolumn{10}{|c|}{ $\mu \mathrm{Ci} / \mathrm{g}$, Reference Date: ${ }^{241} \mathrm{Am}$ April 11-12, 2011; ${ }^{238,239+240} \mathrm{Pu}$ April 8-12, 2011; ${ }^{237} \mathrm{~Np}$ April 14-19, 2011 (reference dates same as counting dates) } \\
\hline${ }^{237} \mathrm{~Np}$ & $9.84 \mathrm{E}-7$ & 7.77E-4 & $9.66 \mathrm{E}-4^{(\mathrm{c})}$ & 7.39E-4 & $6.76 \mathrm{E}-4$ & $5.85 \mathrm{E}-4^{(\mathrm{c})}$ & $2.46 \mathrm{E}-4$ & -- & 4.15E-4 ${ }^{(\mathrm{c})}$ & 4.05E-4 \\
\hline${ }^{238} \mathrm{Pu}$ & $1.44 \mathrm{E}-6$ & $1.02 \mathrm{E}+0$ & $1.15 \mathrm{E}+0$ & $1.30 \mathrm{E}+0$ & $1.14 \mathrm{E}+0$ & $5.22 \mathrm{E}-1$ & $5.40 \mathrm{E}-1$ & -- & $6.72 \mathrm{E}-1$ & $6.42 \mathrm{E}-1$ \\
\hline${ }^{239+240} \mathrm{Pu}$ & $1.38 \mathrm{E}-6$ & $7.97 \mathrm{E}+0$ & $8.56 \mathrm{E}+0$ & $9.40 \mathrm{E}+0$ & $8.34 \mathrm{E}+0$ & $4.02 \mathrm{E}+0$ & $4.12 \mathrm{E}+0$ & -- & $4.92 \mathrm{E}+0$ & $4.58 \mathrm{E}+0$ \\
\hline${ }^{241} \mathrm{Am}$ & $2.42 \mathrm{E}-6$ & $8.15 \mathrm{E}+0$ & $8.82 \mathrm{E}+0$ & $9.68 \mathrm{E}+0$ & $8.08 \mathrm{E}+0$ & $4.95 \mathrm{E}+0$ & $4.86 \mathrm{E}+0$ & -- & $5.59 \mathrm{E}+0$ & $5.32 \mathrm{E}+0$ \\
\hline AEA & \multicolumn{10}{|l|}{$\mu \mathrm{g} / \mathrm{g}$} \\
\hline${ }^{237} \mathrm{~Np}$ & $1.40 \mathrm{E}-3$ & $1.11 \mathrm{E}+0$ & $1.37 \mathrm{E}+0$ & $1.05 \mathrm{E}+0$ & $9.62 \mathrm{E}-1$ & 8.32E-1 & $3.49 \mathrm{E}-1$ & -- & $5.89 \mathrm{E}-1$ & $5.75 \mathrm{E}-1$ \\
\hline${ }^{241} \mathrm{Am}$ & $7.04 \mathrm{E}-7$ & $2.37 \mathrm{E}+0$ & $2.57 \mathrm{E}+0$ & $2.82 \mathrm{E}+0$ & $2.36 \mathrm{E}+0$ & $1.44 \mathrm{E}+0$ & $1.42 \mathrm{E}+0$ & -- & $1.63 \mathrm{E}+0$ & $1.55 \mathrm{E}+0$ \\
\hline Total U & \multicolumn{10}{|l|}{$\mu \mathrm{g} / \mathrm{g}$} \\
\hline $\mathrm{U} / \mathrm{KPA}$ & 0 & 43,800 & 48,300 & 49,800 & 43,700 & 18,200 & 16,000 & -- & 20,400 & 19,200 \\
\hline U/ICP-OES & $<4.5$ & 41,900 & 46,000 & 46,900 & 41,700 & 17,000 & 15,000 & -- & 20,200 & 18,500 \\
\hline GEA & \multicolumn{10}{|c|}{$\mu \mathrm{Ci} / \mathrm{g}$, Reference Date: January 11, 2011} \\
\hline${ }^{60} \mathrm{Co}$ & $<2 \mathrm{E}-5$ & $1.03 \mathrm{E}-1$ & $6.30 \mathrm{E}-2$ & 7.59E-2 & $6.86 \mathrm{E}-2$ & $2.41 \mathrm{E}-2$ & $2.40 \mathrm{E}-2$ & $2.21 \mathrm{E}-2$ & 2.49E-2 & $2.32 \mathrm{E}-2$ \\
\hline${ }^{134} \mathrm{Cs}$ & $<2 \mathrm{E}-5$ & $<1 \mathrm{E}-3$ & $<3 \mathrm{E}-3$ & $<2 \mathrm{E}-3$ & $<3 \mathrm{E}-3$ & $<1 \mathrm{E}-3$ & $<1 \mathrm{E}-3$ & $<2 \mathrm{E}-3$ & $<2 \mathrm{E}-3$ & $<2 \mathrm{E}-3$ \\
\hline${ }^{137} \mathrm{Cs}$ & 9.33E-5 & $1.64 \mathrm{E}+2$ & $1.76 \mathrm{E}+2$ & $1.92 \mathrm{E}+2$ & $2.16 \mathrm{E}+2$ & $4.69 \mathrm{E}+1$ & $5.51 \mathrm{E}+1$ & $4.92 \mathrm{E}+1$ & $4.10 \mathrm{E}+1$ & $3.70 \mathrm{E}+1$ \\
\hline${ }^{152} \mathrm{Eu}$ & $<9 \mathrm{E}-5$ & $<2 \mathrm{E}-3$ & $<4 \mathrm{E}-3$ & $<3 \mathrm{E}-3$ & $<5 \mathrm{E}-3$ & $<1 \mathrm{E}-3$ & $<2 \mathrm{E}-3$ & $<4 \mathrm{E}-3$ & $<4 \mathrm{E}-3$ & $<4 \mathrm{E}-3$ \\
\hline${ }^{154} \mathrm{Eu}$ & $<4 \mathrm{E}-5$ & $3.26 \mathrm{E}-1$ & 3.39E-1 & 3.31E-1 & 3.07E-1 & $1.55 \mathrm{E}-1$ & $1.69 \mathrm{E}-1$ & $1.56 \mathrm{E}-1$ & $1.91 \mathrm{E}-1$ & $1.86 \mathrm{E}-1$ \\
\hline${ }^{155} \mathrm{Eu}$ & $<9 \mathrm{E}-5$ & $5.27 \mathrm{E}-2$ & 5.63E-2 & $<3 \mathrm{E}-2$ & $<6 \mathrm{E}-2$ & $2.86 \mathrm{E}-2$ & $3.94 \mathrm{E}-2$ & $<2 \mathrm{E}-2$ & $2.91 \mathrm{E}-2$ & $2.66 \mathrm{E}-2$ \\
\hline${ }^{241} \mathrm{Am}$ & $<1 \mathrm{E}-4$ & $8.76 \mathrm{E}+0$ & $9.52 \mathrm{E}+0$ & $9.29 \mathrm{E}+0$ & $7.88 \mathrm{E}+0$ & $4.63 \mathrm{E}+0$ & $5.51 \mathrm{E}+0$ & $4.53 \mathrm{E}+0$ & $5.37 \mathrm{E}+0$ & $5.04 \mathrm{E}+0$ \\
\hline \multicolumn{11}{|c|}{ Beta/LSC $\quad \mu \mathrm{CH} / \mathrm{g}$, Reference Date: April 1, 2011 (reference date same as separation date) } \\
\hline${ }^{90} \mathrm{Sr}$ & $6.21 \mathrm{E}-5$ & $1.54 \mathrm{E}+2$ & $1.51 \mathrm{E}+2$ & $1.53 \mathrm{E}+2$ & $1.54 \mathrm{E}+2$ & $4.57 \mathrm{E}+1$ & $5.08 \mathrm{E}+1$ & -- & $5.22 \mathrm{E}+1$ & $4.84 \mathrm{E}+1$ \\
\hline
\end{tabular}

(a) The conversion factor, gdry/gsettled, was determined from the primary physical property samples (see Table 3.1).

(b) Sample TI002-B1-D was the batch MS sample spiked with analyte of interest for ICP-OES. From a GEA perspective, it can be used as a confirmatory sample for KW210 Core B1.

(c) The sample Np fraction contained a slight quantity of $\mathrm{U} ;{ }^{234} \mathrm{U}$ has an equivalent alpha emission energy as ${ }^{237} \mathrm{~Np}$ and would cause slight high bias. See Section 4.4 .1 .

RPD calculations reported in Table 4.4 for KW210 Core B1 are based on samples TI002-B1-B and TI002-B1-C.

Shaded analyte results indicate the QAPjP/SAP precision criterion of $\pm 20 \%$ was exceeded. 
PNNL-20650, Rev. 2

Table 4.6. Uranium and Plutonium TIMS Isotopic Ratios in Acid Digestate

\begin{tabular}{|c|c|c|c|c|c|c|c|c|}
\hline \multirow{3}{*}{$\begin{array}{r}\text { Container: } \\
\text { Core: } \\
\text { Sample ID: } \\
\end{array}$} & \multicolumn{8}{|c|}{ SCS-CON-210 } \\
\hline & \multicolumn{2}{|c|}{ A1 } & \multicolumn{2}{|c|}{ A4 } & \multicolumn{2}{|c|}{ B1 } & \multicolumn{2}{|c|}{ B3 } \\
\hline & TI001-A1-B & TI001-A1-C & TI002-A4-B & TI002-A4-C & TI002-B1-B & TI002-B1-C & TI001-B3-B & TI001-B3-C \\
\hline $\begin{array}{c}\text { RPL/ } \\
\text { ASO ID > }\end{array}$ & $\begin{array}{c}\text { 11-0419- } \\
129-S\end{array}$ & $\begin{array}{c}\text { 11-0419- } \\
129-D\end{array}$ & $\begin{array}{c}11-0420- \\
129-S\end{array}$ & $\begin{array}{c}\text { 11-0420- } \\
129-D\end{array}$ & $\begin{array}{c}\text { 11-0421- } \\
129-S\end{array}$ & $\begin{array}{c}\text { 11-0421- } \\
129-D\end{array}$ & $\begin{array}{c}11-0422- \\
129-S\end{array}$ & $\begin{array}{c}11-0422- \\
129-D\end{array}$ \\
\hline \multicolumn{9}{|c|}{ TIMS Data, Reference Date: May 3-9, 2011} \\
\hline Analyte & Atom\% & & & & & & & \\
\hline${ }^{233} \mathrm{U}$ & 0 & 0 & 0 & 0 & 0 & 0 & 0 & 0 \\
\hline${ }^{234} U^{(a)}$ & 0.008 & 0.007 & 0.007 & 0.007 & 0.007 & 0.007 & 0.008 & 0.011 \\
\hline${ }^{235} \mathrm{U}$ & 0.711 & 0.709 & 0.702 & 0.705 & 0.706 & 0.704 & 0.710 & 0.714 \\
\hline${ }^{236} U^{(a)}$ & 0.073 & 0.076 & 0.072 & 0.074 & 0.071 & 0.072 & 0.071 & 0.077 \\
\hline${ }^{238} \mathrm{U}$ & 99.208 & 99.208 & 99.220 & 99.214 & 99.216 & 99.217 & 99.211 & 99.198 \\
\hline Analyte & Mass\% & & & & & & & \\
\hline${ }^{233} \mathrm{U}$ & 0 & 0 & 0 & 0 & 0 & 0 & 0 & 0 \\
\hline${ }^{234} U^{(a)}$ & 0.0078 & 0.0066 & 0.0067 & 0.0073 & 0.0065 & 0.0069 & 0.0076 & 0.0107 \\
\hline${ }^{235} \mathrm{U}$ & 0.702 & 0.700 & 0.693 & 0.696 & 0.697 & 0.695 & 0.701 & 0.705 \\
\hline${ }^{236} U^{(a)}$ & 0.073 & 0.075 & 0.071 & 0.073 & 0.071 & 0.072 & 0.070 & 0.077 \\
\hline${ }^{238} \mathrm{U}$ & 99.217 & 99.218 & 99.229 & 99.224 & 99.226 & 99.227 & 99.221 & 99.208 \\
\hline \multicolumn{9}{|l|}{ Isotopic $\mathrm{Pu}$} \\
\hline Analyte & \multicolumn{8}{|c|}{ Atom\% (Normalized) } \\
\hline${ }^{238} \mathrm{Pu}$ & 0.0619 & 0.0651 & 0.0678 & 0.0667 & 0.0630 & 0.0639 & 0.0663 & 0.0685 \\
\hline${ }^{239} \mathrm{Pu}$ & 86.115 & 86.250 & 85.926 & 86.203 & 86.431 & 86.235 & 86.251 & 86.016 \\
\hline${ }^{240} \mathrm{Pu}$ & 12.918 & 12.920 & 13.219 & 12.995 & 12.794 & 12.982 & 12.918 & 13.183 \\
\hline${ }^{241} \mathrm{Pu}$ & 0.674 & 0.516 & 0.533 & 0.508 & 0.486 & 0.485 & 0.535 & 0.522 \\
\hline${ }^{242} \mathrm{Pu}$ & 0.231 & 0.250 & 0.254 & 0.228 & 0.227 & 0.234 & 0.230 & 0.209 \\
\hline Analyte & \multicolumn{8}{|c|}{ Mass\% (Normalized) } \\
\hline${ }^{238} \mathrm{Pu}$ & 0.0617 & 0.0648 & 0.0675 & 0.0664 & 0.0627 & 0.0635 & 0.0660 & 0.0682 \\
\hline${ }^{239} \mathrm{Pu}$ & 86.061 & 86.197 & 85.872 & 86.150 & 86.378 & 86.182 & 86.198 & 85.963 \\
\hline${ }^{240} \mathrm{Pu}$ & 12.964 & 12.966 & 13.266 & 13.041 & 12.839 & 13.029 & 12.964 & 13.230 \\
\hline${ }^{241} \mathrm{Pu}$ & 0.680 & 0.520 & 0.537 & 0.512 & 0.490 & 0.489 & 0.539 & 0.526 \\
\hline${ }^{242} \mathrm{Pu}$ & 0.233 & 0.253 & 0.257 & 0.231 & 0.230 & 0.237 & 0.232 & 0.212 \\
\hline
\end{tabular}

(a) Most of the measured ${ }^{234} U$ and ${ }^{236} U$ ratios of NIST standard U-030 exceeded the allowed $\pm 2 \%$ difference from the certified values for the five performance checks of the system (see Appendix E for the individual results). The 1- $\sigma$ uncertainties of the ${ }^{234} \mathrm{U}$ and ${ }^{236} \mathrm{U}$ values were large (much greater than $2 \%$, relative) and generally bracketed the $\pm 2 \%$ criterion range. The Analysis Request approved by the BTR only passed down the requirement for isotopes where the concentration exceeded an atom\% of 0.1 (i.e., specifically applicable to ${ }^{235} U$ and ${ }^{238} U$ ). See Occurrence Report 52578-05-09-11 in Appendix E for details.

The samples prepared by the ASO for U TIMS analysis included suffix “-U”; the samples provided for Pu TIMS analysis had a suffix "-Pu". These are not shown in the sample ID at the top for brevity and clarity.

Shaded values indicate RPD exceeded 20\%; however, the $\pm 20 \%$ QAPjP/SAP criterion only applies to isotopes where the atomic abundance is greater than $1 \%$.

Additional significant figures (obtained from the supporting analytical data) are shown for additional information. See TIMS report in Appendix E. Calculation spreadsheets in Microsoft Excel are provided in DVD-52578-PNNL-20650 Rev. 2, Section III, September 2013. 
PNNL-20650, Rev. 2

Table 4.7. Uranium and Plutonium TIMS QC Sample Results in Acid Digestate

\begin{tabular}{cccccc}
\hline Container: & \multicolumn{5}{c}{ SCS-CON-210 } \\
\hline Core: & A1 & A4 & B1 & B3 & $\begin{array}{c}\text { Calibration Verification Standard } \\
\text { \%ifference of Pooled Average } \\
\text { from Certificate Value }\end{array}$ \\
\hline Analyte & RPD (\%) & RPD (\%) & RPD (\%) & RPD (\%) & SRM U-030 \\
\hline${ }^{233} \mathrm{U}$ & --- & --- & --- & --- & --- \\
${ }^{234} \mathrm{U}$ & 17 & 8.4 & 5.6 & 33 & $0.61^{(\mathrm{b})}$ \\
${ }^{235} \mathrm{U}$ & 0.26 & 0.43 & 0.33 & 0.58 & -0.88 \\
${ }^{236} \mathrm{U}$ & 3.1 & 2.7 & 1.2 & 8.3 & $-2.66^{(\mathrm{b})}$ \\
${ }^{238} \mathrm{U}$ & 0.001 & 0.006 & 0.001 & 0.013 & 0.03 \\
\hline Normalized & & & & & SRM Pu-946 \\
${ }^{238} \mathrm{Pu}$ & 5.0 & 1.7 & 1.4 & 3.3 & 0.29 \\
${ }^{239} \mathrm{Pu}$ & 0.16 & 0.32 & 0.23 & 0.27 & 0.05 \\
${ }^{240} \mathrm{Pu}$ & 0.013 & 1.7 & 1.5 & 2.0 & -0.35 \\
${ }^{241} \mathrm{Pu}$ & 27 & 4.7 & 0.22 & 2.5 & 0.69 \\
${ }^{242} \mathrm{Pu}$ & 8.0 & 11 & 2.9 & 9.2 & -0.36 \\
\hline
\end{tabular}

(a) The RPD values were calculated from the unrounded sample and the duplicate atom\% values (TIMS values obtained from the TIMS calculation spreadsheet [Appendix E]). The Excel calculation spreadsheet file is provided in Appendix E (ASR 8798 Data Reduction Rev2.xlsx). Excel files can also be found in DVD-52578-PNNL-20650 Rev. 2, Section III, September 2013. Shaded blocks indicate that the RPD of the duplicate pair exceeded $\pm 20 \%$. However, the isotopic abundances of ${ }^{240} \mathrm{Pu},{ }^{241} \mathrm{Pu},{ }^{234} \mathrm{U}$ and ${ }^{236} \mathrm{U}$ were $<1$ atom\%; therefore the $\pm 20 \%$ RPD precision criterion was not applicable.

(b) Most of the measured ${ }^{234} \mathrm{U}$ and ${ }^{236} \mathrm{U}$ ratios of NIST standard $\mathrm{U}-030$ exceeded the allowed $\pm 2 \%$ difference from the certified values for the five performance checks of the system (see Appendix E for the individual results). The 1- $\sigma$ uncertainties of the ${ }^{234} \mathrm{U}$ and ${ }^{236} \mathrm{U}$ values were large (much greater than $2 \%$, relative) and generally bracketed the $\pm 2 \%$ criterion range. The Analysis Request approved by the BTR only passed down the requirement for isotopes where the concentration exceeded an atom\% of 0.1 (i.e., specifically applicable to ${ }^{235} U$ and ${ }^{238} U$ ). See Occurrence Report 52578-05-09-11 in Appendix E for details.

SRM: Standard Reference Material. 
PNNL-20650, Rev. 2

Table 4.8. Uranium and Plutonium Isotope Mass and Activity Distributions in Acid Digestate, Dry Sludge Mass Basis

\begin{tabular}{|c|c|c|c|c|c|c|c|c|}
\hline \multirow{3}{*}{$\begin{array}{r}\text { Container: } \\
\text { Core: } \\
\text { Sample ID: } \\
\end{array}$} & \multicolumn{8}{|c|}{ SCS-CON-210 } \\
\hline & \multicolumn{2}{|c|}{ A1 } & \multicolumn{2}{|c|}{ A4 } & \multicolumn{2}{|c|}{ B1 } & \multicolumn{2}{|c|}{ B3 } \\
\hline & TI001-A1-B & TI001-A1-C & TI002-A4-B & TI002-A4-C & TI002-B1-B & TI002-B1-C & TI001-B3-B & TI001-В3-С \\
\hline $\begin{array}{c}\text { RPL/ } \\
\text { ASO ID > }\end{array}$ & $\begin{array}{c}11-0419- \\
129-S\end{array}$ & $\begin{array}{c}\text { 11-0419- } \\
129-D\end{array}$ & $\begin{array}{c}11-0420- \\
129-S\end{array}$ & $\begin{array}{c}11-0420- \\
129-D\end{array}$ & $\begin{array}{c}11-0421- \\
129-S\end{array}$ & $\begin{array}{c}11-0421- \\
129-D\end{array}$ & $\begin{array}{c}11-0422- \\
129-S\end{array}$ & $\begin{array}{c}\text { 11-0422- } \\
129-\mathrm{D}\end{array}$ \\
\hline Isotopic U & \multicolumn{8}{|c|}{ TIMS Data, Reference Date: May 3-9, 2011} \\
\hline Analyte & \multicolumn{8}{|l|}{$\mu \mathrm{g} / \mathrm{g}$} \\
\hline${ }^{233} \mathrm{U}$ & $0 \mathrm{E}+0$ & $0 \mathrm{E}+0$ & $0 \mathrm{E}+0$ & $0 \mathrm{E}+0$ & $0 \mathrm{E}+0$ & $0 \mathrm{E}+0$ & $0 \mathrm{E}+0$ & $0 \mathrm{E}+0$ \\
\hline${ }^{234} U^{(a)}$ & $7.6 \mathrm{E}+0$ & $7.1 \mathrm{E}+0$ & $5.9 \mathrm{E}+0$ & $5.6 \mathrm{E}+0$ & $3.6 \mathrm{E}+0$ & $3.4 \mathrm{E}+0$ & $4.3 \mathrm{E}+0$ & $5.7 \mathrm{E}+0$ \\
\hline${ }^{235} \mathrm{U}$ & $6.88 \mathrm{E}+2$ & $7.56 \mathrm{E}+2$ & $6.09 \mathrm{E}+2$ & $5.36 \mathrm{E}+2$ & $3.87 \mathrm{E}+2$ & $3.39 \mathrm{E}+2$ & $3.94 \mathrm{E}+2$ & $3.74 \mathrm{E}+2$ \\
\hline${ }^{236} \mathrm{U}^{(\mathrm{a})}$ & $7.1 \mathrm{E}+1$ & $8.1 \mathrm{E}+1$ & $6.3 \mathrm{E}+1$ & $5.7 \mathrm{E}+1$ & $3.9 E+1$ & $3.5 E+1$ & $4.0 \mathrm{E}+1$ & $4.1 \mathrm{E}+1$ \\
\hline${ }^{238} \mathrm{U}$ & $9.723 E+4$ & $1.072 \mathrm{E}+5$ & $8.722 E+4$ & $7.650 \mathrm{E}+4$ & $5.507 \mathrm{E}+4$ & $4.842 \mathrm{E}+4$ & $5.576 \mathrm{E}+4$ & $5.268 \mathrm{E}+4$ \\
\hline Analyte & \multicolumn{8}{|l|}{$\mu \mathrm{Ci} / \mathrm{g}$} \\
\hline${ }^{233} \mathrm{U}$ & $0 \mathrm{E}+0$ & $0 \mathrm{E}+0$ & $0 \mathrm{E}+0$ & $0 \mathrm{E}+0$ & $0 \mathrm{E}+0$ & $0 \mathrm{E}+0$ & $0 \mathrm{E}+0$ & $0 \mathrm{E}+0$ \\
\hline${ }^{234} \mathrm{U}^{(\mathrm{a})}$ & 4.7E-2 & 4.4E-2 & 3.7E-2 & 3.5E-2 & 2.3E-2 & $2.1 \mathrm{E}-2$ & 2.7E-2 & $3.5 \mathrm{E}-2$ \\
\hline${ }^{235} \mathrm{U}$ & $1.49 \mathrm{E}-3$ & $1.63 \mathrm{E}-3$ & $1.32 \mathrm{E}-3$ & $1.16 \mathrm{E}-3$ & 8.36E-4 & 7.33E-4 & $8.51 \mathrm{E}-4$ & 8.09E-4 \\
\hline${ }^{236} \mathrm{U}^{(\mathrm{a})}$ & 4.6E-3 & $5.2 \mathrm{E}-3$ & $4.1 \mathrm{E}-3$ & 3.7E-3 & $2.5 \mathrm{E}-3$ & 2.3E-3 & $2.6 \mathrm{E}-3$ & $2.6 \mathrm{E}-3$ \\
\hline${ }^{238} \mathrm{U}$ & $3.268 \mathrm{E}-2$ & 3.602E-2 & 2.932E-2 & $2.571 \mathrm{E}-2$ & $1.851 \mathrm{E}-2$ & $1.628 \mathrm{E}-2$ & $1.874 \mathrm{E}-2$ & $1.771 \mathrm{E}-2$ \\
\hline Isotopic $\mathrm{Pu}$ & \multicolumn{8}{|c|}{ TIMS Data, Reference Date: May 18 and 25, 2011} \\
\hline Analyte & \multicolumn{8}{|c|}{$\mu \mathrm{g} / \mathrm{g}$ (Normalized) } \\
\hline${ }^{238} \mathrm{Pu}$ & 1.33E-1 & $1.50 \mathrm{E}-1$ & $1.34 \mathrm{E}-1$ & $1.18 \mathrm{E}-1$ & $9.29 \mathrm{E}-2$ & $9.61 \mathrm{E}-2$ & $1.08 \mathrm{E}-1$ & $1.03 \mathrm{E}-1$ \\
\hline${ }^{239} \mathrm{Pu}$ & $1.854 \mathrm{E}+2$ & $1.992 \mathrm{E}+2$ & $1.708 \mathrm{E}+2$ & $1.528 \mathrm{E}+2$ & $1.281 \mathrm{E}+2$ & $1.303 \mathrm{E}+2$ & $1.413 E+2$ & $1.303 E+2$ \\
\hline${ }^{240} \mathrm{Pu}$ & $2.793 \mathrm{E}+1$ & $2.996 \mathrm{E}+1$ & $2.639 \mathrm{E}+1$ & $2.312 \mathrm{E}+1$ & $1.904 \mathrm{E}+1$ & $1.970 \mathrm{E}+1$ & $2.125 \mathrm{E}+1$ & $2.006 \mathrm{E}+1$ \\
\hline${ }^{241} \mathrm{Pu}$ & $1.46 \mathrm{E}+0$ & $1.20 \mathrm{E}+0$ & $1.07 \mathrm{E}+0$ & $9.08 \mathrm{E}-1$ & $7.26 \mathrm{E}-1$ & 7.39E-1 & 8.84E-1 & $7.98 \mathrm{E}-1$ \\
\hline${ }^{242} \mathrm{Pu}$ & $5.03 \mathrm{E}-1$ & $5.84 \mathrm{E}-1$ & $5.11 \mathrm{E}-1$ & 4.09E-1 & $3.41 \mathrm{E}-1$ & $3.58 \mathrm{E}-1$ & $3.81 \mathrm{E}-1$ & $3.21 \mathrm{E}-1$ \\
\hline Analyte & \multicolumn{8}{|c|}{$\mu \mathrm{Ci} / \mathrm{g}$ (Normalized) } \\
\hline${ }^{238} \mathrm{Pu}$ & $2.27 \mathrm{E}+0$ & $2.56 \mathrm{E}+0$ & $2.30 \mathrm{E}+0$ & $2.01 \mathrm{E}+0$ & $1.59 \mathrm{E}+0$ & $1.65 \mathrm{E}+0$ & $1.85 \mathrm{E}+0$ & $1.77 \mathrm{E}+0$ \\
\hline${ }^{239} \mathrm{Pu}$ & $1.150 \mathrm{E}+1$ & $1.235 E+1$ & $1.060 \mathrm{E}+1$ & $9.475 E+0$ & $7.946 \mathrm{E}+0$ & $8.085 \mathrm{E}+0$ & $8.763 E+0$ & $8.084 \mathrm{E}+0$ \\
\hline${ }^{240} \mathrm{Pu}$ & $6.336 \mathrm{E}+0$ & $6.797 \mathrm{E}+0$ & $5.988 \mathrm{E}+0$ & $5.246 \mathrm{E}+0$ & 4.320E +0 & $4.471 \mathrm{E}+0$ & $4.821 \mathrm{E}+0$ & $4.551 \mathrm{E}+0$ \\
\hline${ }^{241} \mathrm{Pu}$ & $1.52 E+2$ & $1.25 \mathrm{E}+2$ & $1.11 \mathrm{E}+2$ & $9.42 \mathrm{E}+1$ & $7.54 \mathrm{E}+1$ & 7.67E+1 & $9.18 \mathrm{E}+1$ & $8.28 \mathrm{E}+1$ \\
\hline${ }^{242} \mathrm{Pu}$ & $1.99 \mathrm{E}-3$ & $2.31 \mathrm{E}-3$ & $2.02 \mathrm{E}-3$ & $1.62 \mathrm{E}-3$ & $1.35 \mathrm{E}-3$ & $1.42 \mathrm{E}-3$ & $1.51 \mathrm{E}-3$ & $1.27 \mathrm{E}-3$ \\
\hline
\end{tabular}

(a) Most of the measured ${ }^{234} \mathrm{U}$ and ${ }^{236} \mathrm{U}$ ratios of NIST standard $\mathrm{U}-030$ exceeded the allowed $\pm 2 \%$ difference from the certified values for the five performance checks of the system (see Appendix E for the individual results). The 1- $\sigma$ uncertainties of the ${ }^{234} \mathrm{U}$ and ${ }^{236} \mathrm{U}$ values were large (much greater than $2 \%$, relative) and generally bracketed the $\pm 2 \%$ criterion range. The Analysis Request approved by the BTR only passed down the requirement for isotopes where the concentration exceeded an atom\% of 0.1 (i.e., specifically applicable to ${ }^{235} \mathrm{U}$ and ${ }^{238} \mathrm{U}$ ). See Occurrence Report 52578-05-09-11 in Appendix E for details.

Shaded values indicate RPD exceeded 20\%; however, the $\pm 20 \%$ QAPjP/SAP criterion only applies to isotopes where the atom abundance is greater than $1 \%$.

See TIMS report in Appendix E. Calculation spreadsheets in Microsoft Excel are provided in DVD-52578-PNNL-20650 Rev. 2, Section III, September 2013. 
PNNL-20650, Rev. 2

Table 4.9. Uranium and Plutonium Isotope Mass and Activity Distributions in Acid Digestate, Wet Settled Sludge Mass Basis

\begin{tabular}{|c|c|c|c|c|c|c|c|c|}
\hline \multirow{3}{*}{$\begin{array}{r}\text { Container: } \\
\text { Core: } \\
\text { Sample ID: }\end{array}$} & \multicolumn{8}{|c|}{ SCS-CON-210 } \\
\hline & \multicolumn{2}{|c|}{ A1 } & \multicolumn{2}{|c|}{ A4 } & \multicolumn{2}{|c|}{ B1 } & \multicolumn{2}{|c|}{ B3 } \\
\hline & TI001-A1-B & TI001-A1-C & TI002-A4-B & TI002-A4-C & TI002-B1-B & TI002-B1-C & TI001-B3-B & TI001-B3-C \\
\hline $\begin{array}{c}\text { RPL/ } \\
\text { ASO ID > }\end{array}$ & $\begin{array}{c}\text { 11-0419- } \\
129-S\end{array}$ & $\begin{array}{c}\text { 11-0419- } \\
129-D\end{array}$ & $\begin{array}{c}11-0420- \\
129-S\end{array}$ & $\begin{array}{c}11-0420- \\
129-D\end{array}$ & $\begin{array}{c}11-0421- \\
129-S\end{array}$ & $\begin{array}{c}11-0421- \\
129-D\end{array}$ & $\begin{array}{c}11-0422- \\
129-S\end{array}$ & $\begin{array}{c}\text { 11-0422- } \\
129-\mathrm{D}\end{array}$ \\
\hline Isotopic U & \multicolumn{8}{|c|}{ TIMS Data, Reference Date: May 3-9, 2011} \\
\hline Analyte & \multicolumn{8}{|l|}{$\mu \mathrm{g} / \mathrm{g}$} \\
\hline${ }^{233} \mathrm{U}$ & $0 \mathrm{E}+0$ & $0 \mathrm{E}+0$ & $0 \mathrm{E}+0$ & $0 \mathrm{E}+0$ & $0 \mathrm{E}+0$ & $0 \mathrm{E}+0$ & $0 \mathrm{E}+0$ & $0 \mathrm{E}+0$ \\
\hline${ }^{234} U^{(a)}$ & $3.4 \mathrm{E}+0$ & $3.2 \mathrm{E}+0$ & $3.3 \mathrm{E}+0$ & $3.2 \mathrm{E}+0$ & $1.2 \mathrm{E}+0$ & $1.1 \mathrm{E}+0$ & $1.6 \mathrm{E}+0$ & $2.1 \mathrm{E}+0$ \\
\hline${ }^{235} \mathrm{U}$ & $3.08 \mathrm{E}+2$ & $3.38 \mathrm{E}+2$ & $3.45 \mathrm{E}+2$ & $3.04 \mathrm{E}+2$ & $1.27 \mathrm{E}+2$ & $1.11 \mathrm{E}+2$ & $1.43 \mathrm{E}+2$ & $1.36 \mathrm{E}+2$ \\
\hline${ }^{236} U^{(a)}$ & $3.2 \mathrm{E}+1$ & $3.6 \mathrm{E}+1$ & $3.6 \mathrm{E}+1$ & $3.2 \mathrm{E}+1$ & $1.3 \mathrm{E}+1$ & $1.1 \mathrm{E}+1$ & $1.4 \mathrm{E}+1$ & $1.5 \mathrm{E}+1$ \\
\hline${ }^{238} \mathrm{U}$ & $4.35 \mathrm{E}+4$ & $4.79 \mathrm{E}+4$ & $4.94 \mathrm{E}+4$ & $4.34 \mathrm{E}+4$ & $1.81 \mathrm{E}+4$ & $1.59 \mathrm{E}+4$ & $2.02 E+4$ & $1.91 \mathrm{E}+4$ \\
\hline Analyte & \multicolumn{8}{|l|}{$\mu \mathrm{Ci} / \mathrm{g}$} \\
\hline${ }^{233} \mathrm{U}$ & $0 \mathrm{E}+0$ & $0 \mathrm{E}+0$ & $0 \mathrm{E}+0$ & $0 \mathrm{E}+0$ & $0 \mathrm{E}+0$ & $0 \mathrm{E}+0$ & $0 \mathrm{E}+0$ & $0 \mathrm{E}+0$ \\
\hline${ }^{234} U^{(a)}$ & $2.1 \mathrm{E}-2$ & $2.0 \mathrm{E}-2$ & $2.1 \mathrm{E}-2$ & $2.0 \mathrm{E}-2$ & $7.4 \mathrm{E}-3$ & $6.9 \mathrm{E}-3$ & $9.7 \mathrm{E}-3$ & $1.3 \mathrm{E}-2$ \\
\hline${ }^{235} \mathrm{U}$ & $6.65 \mathrm{E}-4$ & 7.31E-4 & $7.46 \mathrm{E}-4$ & $6.57 \mathrm{E}-4$ & $2.74 \mathrm{E}-4$ & $2.40 \mathrm{E}-4$ & 3.09E-4 & 2.93E-4 \\
\hline${ }^{236} U^{(a)}$ & $2.1 \mathrm{E}-3$ & 2.3E-3 & 2.3E-3 & $2.1 \mathrm{E}-3$ & 8.3E-4 & 7.4E-4 & 9.3E-4 & $9.5 \mathrm{E}-4$ \\
\hline${ }^{238} \mathrm{U}$ & $1.46 \mathrm{E}-2$ & $1.61 \mathrm{E}-2$ & $1.66 \mathrm{E}-2$ & $1.46 \mathrm{E}-2$ & $6.07 \mathrm{E}-3$ & $5.34 \mathrm{E}-3$ & $6.79 \mathrm{E}-3$ & $6.42 \mathrm{E}-3$ \\
\hline Isotopic $\mathrm{Pu}$ & \multicolumn{8}{|c|}{ TIMS Data, Reference Date: May 18 and 25, 2011} \\
\hline Analyte & \multicolumn{8}{|c|}{ 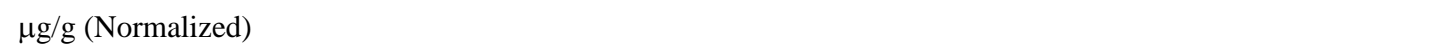 } \\
\hline${ }^{238} \mathrm{Pu}$ & $5.94 \mathrm{E}-2$ & $6.69 \mathrm{E}-2$ & 7.61E-2 & $6.67 \mathrm{E}-2$ & $3.05 \mathrm{E}-2$ & $3.15 \mathrm{E}-2$ & $3.92 \mathrm{E}-2$ & $3.75 \mathrm{E}-2$ \\
\hline${ }^{239} \mathrm{Pu}$ & $8.29 \mathrm{E}+1$ & $8.90 \mathrm{E}+1$ & $9.68 \mathrm{E}+1$ & $8.66 \mathrm{E}+1$ & $4.20 \mathrm{E}+1$ & $4.28 \mathrm{E}+1$ & $5.12 \mathrm{E}+1$ & $4.72 \mathrm{E}+1$ \\
\hline${ }^{240} \mathrm{Pu}$ & $1.25 \mathrm{E}+1$ & $1.34 \mathrm{E}+1$ & $1.50 \mathrm{E}+1$ & $1.31 \mathrm{E}+1$ & $6.25 \mathrm{E}+0$ & $6.46 \mathrm{E}+0$ & $7.70 \mathrm{E}+0$ & $7.27 \mathrm{E}+0$ \\
\hline${ }^{241} \mathrm{Pu}$ & $6.54 \mathrm{E}-1$ & 5.37E-1 & $6.05 \mathrm{E}-1$ & 5.15E-1 & $2.38 \mathrm{E}-1$ & $2.42 \mathrm{E}-1$ & $3.20 \mathrm{E}-1$ & $2.89 \mathrm{E}-1$ \\
\hline${ }^{242} \mathrm{Pu}$ & $2.25 \mathrm{E}-1$ & $2.61 \mathrm{E}-1$ & $2.90 \mathrm{E}-1$ & $2.32 \mathrm{E}-1$ & $1.12 \mathrm{E}-1$ & $1.17 \mathrm{E}-1$ & $1.38 \mathrm{E}-1$ & $1.16 \mathrm{E}-1$ \\
\hline Analyte & \multicolumn{8}{|c|}{$\mu \mathrm{Ci} / \mathrm{g}$ (Normalized) } \\
\hline${ }^{238} \mathrm{Pu}$ & $1.02 \mathrm{E}+0$ & $1.15 \mathrm{E}+0$ & $1.30 \mathrm{E}+0$ & $1.14 \mathrm{E}+0$ & $5.22 \mathrm{E}-1$ & $5.40 \mathrm{E}-1$ & $6.72 \mathrm{E}-1$ & $6.42 \mathrm{E}-1$ \\
\hline${ }^{239} \mathrm{Pu}$ & $5.14 \mathrm{E}+0$ & $5.52 \mathrm{E}+0$ & $6.00 \mathrm{E}+0$ & $5.37 \mathrm{E}+0$ & $2.61 \mathrm{E}+0$ & $2.65 \mathrm{E}+0$ & $3.18 \mathrm{E}+0$ & $2.93 \mathrm{E}+0$ \\
\hline${ }^{240} \mathrm{Pu}$ & $2.83 \mathrm{E}+0$ & $3.04 \mathrm{E}+0$ & $3.39 \mathrm{E}+0$ & $2.97 \mathrm{E}+0$ & $1.42 \mathrm{E}+0$ & $1.47 \mathrm{E}+0$ & $1.75 \mathrm{E}+0$ & $1.65 \mathrm{E}+0$ \\
\hline${ }^{241} \mathrm{Pu}$ & $6.79 \mathrm{E}+1$ & $5.57 \mathrm{E}+1$ & $6.28 \mathrm{E}+1$ & $5.34 \mathrm{E}+1$ & $2.47 \mathrm{E}+1$ & $2.52 \mathrm{E}+1$ & $3.33 \mathrm{E}+1$ & $3.00 \mathrm{E}+1$ \\
\hline${ }^{242} \mathrm{Pu}$ & $8.89 \mathrm{E}-4$ & $1.03 \mathrm{E}-3$ & 1.15E-3 & $9.17 \mathrm{E}-4$ & $4.42 \mathrm{E}-4$ & 4.64E-4 & $5.46 \mathrm{E}-4$ & $4.61 \mathrm{E}-4$ \\
\hline
\end{tabular}

(a) Most of the measured ${ }^{234} U$ and ${ }^{236} U$ ratios of NIST standard $U-030$ exceeded the allowed $\pm 2 \%$ difference from the certified values for the five performance checks of the system (see Appendix E for the individual results). The 1- $\sigma$ uncertainties of the ${ }^{234} \mathrm{U}$ and ${ }^{236} \mathrm{U}$ values were large (much greater than $2 \%$, relative) and generally bracketed the $\pm 2 \%$ criterion range. The Analysis Request approved by the BTR only passed down the requirement for isotopes where the concentration exceeded an atom\% of 0.1 (i.e., specifically applicable to ${ }^{235} \mathrm{U}$ and ${ }^{238} \mathrm{U}$ ). See Occurrence Report 52578-05-09-11 in Appendix E for details.

Shaded values indicate RPD exceeded 20\%; however, the $\pm 20 \%$ QAPjP/SAP criterion only applies to isotopes where the atomic concentration is greater than $1 \%$.

See TIMS report in Appendix E. Calculation spreadsheets in Microsoft Excel are provided in DVD-52578-PNNL-20650 Rev. 2, Section III, September 2013. 
The accuracy, expressed as post-digestion BS and MS recoveries (102 and 103\%), was within the acceptance limits identified in the QAPjP/SAP (BS: 80 to 120\%; MS: 75 to 125\%). The hot cell acid digestion PB showed no detectable ${ }^{237} \mathrm{~Np}$ activity (<2E-6) and indicated that contamination from hot cell processing activities was unlikely.

\subsubsection{Plutonium (Alpha Energy Analysis)}

The duplicate pair results for ${ }^{238} \mathrm{Pu}$ and ${ }^{239+240} \mathrm{Pu}$ showed RPDs ranging between 3 and $12 \%$ for ${ }^{238} \mathrm{Pu}$ and 2 to $12 \%$ for ${ }^{239+240} \mathrm{Pu}$ - each meeting the QAPjP/SAP requirements. Relative 1- $\sigma$ uncertainties ranged from 3 to $6 \%$ for ${ }^{238} \mathrm{Pu}$ measurements from 2 to $3 \%$ for ${ }^{239+240} \mathrm{Pu}$ measurements (actual uncertainties assigned to each sample and analyte are provided in Appendix B). These uncertainties represent the total propagated error associated with processing and count operations and include weighing errors, volume uncertainties, and counting error; they do not incorporate sub-sampling uncertainty. The accuracy, expressed as post-digestion BS and MS recoveries of the ${ }^{239} \mathrm{Pu}$ analytical spike (100 and $101 \%$ ), was within the acceptance limits identified in the QAPjP/SAP. The hot cell acid digestion PB resulted in no detectable activity for ${ }^{238} \mathrm{Pu}$ or ${ }^{239+240} \mathrm{Pu}$. A ${ }^{242} \mathrm{Pu}$ and ${ }^{243} \mathrm{Am}$ tracer was added to every sample before the radiochemical separations. There was no evidence of ${ }^{243} \mathrm{Am}$ in the alpha spectra, indicating that the ${ }^{238} \mathrm{Pu}$ analyte peak was not confounded by the presence of ${ }^{241} \mathrm{Am}$. Radiochemical yields based on recoveries of the ${ }^{242} \mathrm{Pu}$ internal standard ranged from 94 to $108 \%$.

\subsubsection{Americium (Alpha Energy Analysis)}

The duplicate pair results for ${ }^{241} \mathrm{Am}$ by AEA showed RPDs ranging from 2 to $18 \%$. Relative 1- $\sigma$ measurement uncertainties (inclusive of all radiochemistry sources of laboratory errors-volumetric, mass, and counting uncertainties) for ${ }^{241} \mathrm{Am}$ were $2 \%$ (see ASO analysis report in Appendix B). The accuracy, expressed as post-digestion BS and MS recoveries of the ${ }^{241} \mathrm{Am}$ analytical spike (102 and 97\%, respectively), was within the acceptance limits identified in the QAPjP/SAP. The hot cell acid digestion PB showed detectable activity for ${ }^{241} \mathrm{Am}$. However, the blank activity concentration was six orders of magnitude less than the observed lowest sample activity concentration. $\mathrm{A}{ }^{242} \mathrm{Pu}$ and ${ }^{243} \mathrm{Am}$ tracer was added to every sample before the radiochemical separations. The alpha spectra were free of ${ }^{239+240} \mathrm{Pu}$ indicating that ${ }^{238} \mathrm{Pu}$ was not present and thus not confounding the ${ }^{241} \mathrm{Am}$ peak. Radiochemical yields based on recoveries of ${ }^{243} \mathrm{Am}$ internal standard ranged from 92 to $114 \%$.

The ${ }^{241}$ Am activity concentrations from GEA (where no radiochemical separations were applied) were also compared to the concentrations determined by AEA (where chemical separations were applied). The ratio of AEA/GEA results ranged from 0.88 to 1.07 with an average ratio of 1.00 with a standard deviation of 0.07 . The close agreement between the two techniques provides another measure of the overall measurement accuracy for this analyte.

\subsubsection{Gamma Energy Analysis}

Five of the seven gamma-emitting radionuclides of interest were detected in the sample aliquots: ${ }^{60} \mathrm{Co},{ }^{137} \mathrm{Cs}$, ${ }^{154} \mathrm{Eu}$, and ${ }^{241} \mathrm{Am}$ were detected in all samples; ${ }^{155} \mathrm{Eu}$ was detected in three sample duplicate pairs (not detected in KW210-A4) in quantities below the requested detection limit. Neither ${ }^{134} \mathrm{Cs}$ nor ${ }^{152} \mathrm{Eu}$ were detected, and their minimum detectible concentrations (MDCs) are reported. In most cases, the RPDs were within $\pm 20 \%$; RPDs exceeding the QAPjP/SAP criteria include ${ }^{60}$ Co in KW210-A1 (RPD 
of 48\%) and ${ }^{155} \mathrm{Eu}$ in KW210-B1 (RPD 32\%). Relative 1- $\sigma$ measurement uncertainties (inclusive of all radiochemistry lab errors-volumetric, mass, and counting uncertainties) for ${ }^{60} \mathrm{Co},{ }^{137} \mathrm{Cs},{ }^{154} \mathrm{Eu}$ and ${ }^{241} \mathrm{Am}$ ranged from 2 to 5\% (with the exception of TI002-B1-C where 20\% 1- $\sigma$ error was reported for ${ }^{241} \mathrm{Am}$ ). The $1-\sigma$ error for ${ }^{155} \mathrm{Eu}$ results ranged from 7 to $19 \%$. Specific uncertainties assigned to each sample are provided in Appendix B. The hot cell PB resulted in no detectable analyte activity except for ${ }^{137} \mathrm{Cs}$, which was five orders of magnitude less than the lowest activity in the samples. The GEA reference date is January 11, 2011.

\subsubsection{Strontium-90 Analysis}

The duplicate pair results for ${ }^{90} \mathrm{Sr}$ showed RPDs ranging from 0.5 to $11 \%$, meeting the QAPjP/SAP criterion. The reported $1-\sigma$ uncertainty (inclusive of all sources of radiochemistry laboratory errorvolumetric, mass, and counting uncertainties) for each ${ }^{90} \mathrm{Sr}$ sample concentration was $\pm 2 \%$. The accuracy, expressed as BS and MS recoveries of the ${ }^{90} \mathrm{Sr}$ analytical spike (99 and 98\%, respectively), was within the acceptance limits identified in the QAPjP/SAP. No ${ }^{90} \mathrm{Sr}$ activity was detected in the hot cell $\mathrm{PB}$, indicating cross-contamination was not likely to have occurred. (The radiochemistry report is provided in Appendix B.) The reference date for the reported ${ }^{90} \mathrm{Sr}$ concentration is the separation date (April 1, 2011).

\subsubsection{Total Uranium (Kinetic Phosphorescence Analysis)}

The measured precision of the duplicate pairs ranged from 6 to 13\% RPD and met the QAPjP/SAP requirement of $\pm 20 \% \mathrm{RPD}$. The reported measurement uncertainty for each U sample concentration was $\pm 4 \%(1-\sigma)$. The BS, prepared as part of the sample digestion activity, recovered $103 \%$, meeting the QAPjP/SAP requirement. Recovery of the post-digestion MS was also 103\%. The calibration check sample results ranged from 102 to $103 \%$ of true value, which was within the $\pm 7.5 \%$ limits defined in the QAPJP/SAP.

The KPA U results demonstrated agreement with the ICP-OES U results. The U KPA/U ICP-OES ratio averaged 1.05 with a standard deviation of 0.02 . The close agreement between the two techniques provides another measure of the overall accuracy for this analyte.

\subsubsection{Uranium Isotopic Analysis}

Table 4.6 shows the results of the TIMS uranium isotopic analysis conducted on May 3-9, 2011. All $\mathrm{U}$ isotopes of interest were detected except ${ }^{233} \mathrm{U}$. The duplicate pairs resulted in atom\% precision measures of 6 to $33 \%$ for ${ }^{234} U$ (measured values had high uncertainties 13 to $45 \%$ ), $\leq 1 \%$ RPD for ${ }^{235} U$, $\leq 8 \%$ RPD for ${ }^{236} \mathrm{U}$, and $\leq 0.01 \%{ }^{238} \mathrm{U}$ (specific measurement uncertainties for each isotope are provided in Appendix E). The TIMS accuracy was assessed with one standard reference material (SRM) U-030. The QAPjP/SAP-delineated accuracy for U TIMS was $\pm 2 \%$. Measures of the major $U$ isotopes, ${ }^{235} U$ and ${ }^{238} U$, from all five analysis runs easily met this requirement (see Appendix E). However, the measured concentrations of for ${ }^{234} \mathrm{U}$ and ${ }^{236} \mathrm{U}$ exceeded $\pm 2 \%$ during some of the performance checks of the system. The high spread of the SRM ${ }^{234} \mathrm{U}$ and ${ }^{236} \mathrm{U}$ atom\% values was not unexpected considering their isotopic concentrations are low at 0.019 and 0.020 atom\%, respectively. This issue is discussed in detail in Occurrence Report 52578-05-09-11 located in Appendix E with the TIMS final report. The relative 
difference of the certified values from the pooled averages for this analysis set were less than $3 \%$ ( $-0.88 \%$ for ${ }^{235} \mathrm{U}$ and $0.03 \%$ for ${ }^{238} \mathrm{U}$ ) as shown in Table 4.7 .

The wt\% of each $\mathrm{U}$ isotope in each core subsample was determined by multiplying the atom\% (Table 4.6) by the atomic mass of the specific $U$ isotope and then dividing by the atomic weight of $U$ (238.03). The $\mathrm{U}$ isotope concentration $(\mu \mathrm{g} / \mathrm{g})$ was determined by multiplying the $\mathrm{wt} \%$ of each $\mathrm{U}$ isotope by the $\mathrm{U}$ KPA concentration in Table 4.3 . The $\mathrm{U}$ isotope concentration $(\mu \mathrm{Ci} / \mathrm{g})$ was determined by multiplying the mass concentration of each isotope by the specific activity (Ci/g) of the isotope.

\subsubsection{Plutonium Isotopic Analysis}

Plutonium TIMS analysis was conducted May 18 and 25, 2011. All Pu isotopes of interest were detected. The TIMS results shown in Table 4.6, Table 4.8, and Table 4.9 were normalized to ${ }^{238} \mathrm{Pu}$ determined from Pu AEA analysis (see calculation defined in Section 2.5.9). The normalization was considered necessary because there was a significant probability that $U$ could contaminate the Pu fraction confounding the ${ }^{238} \mathrm{Pu}$ with isobaric ${ }^{238} \mathrm{U}$.

All duplicate pairs met the QAPjP/SAP precision criteria of $\pm 20 \%$ RPD (applicable to isotopes with atom abundance is greater than $1 \%$, i.e., ${ }^{239} \mathrm{Pu}$ and ${ }^{240} \mathrm{Pu}$ ). The minor isotopes of the duplicate pairs also were within $\pm 20 \%$ RPD with one exception; ${ }^{241} \mathrm{Pu}$ in KW210-A1 resulted in 27\% RPD. Accuracy for Pu isotopic distribution analysis by TIMS was assessed with SRM Pu-946; all measured isotopes for each of the nine standard runs were measured within $\pm 6 \%$ of the certified value allowed by the QAPjP/SAP. The relative difference of the certified and decay-corrected values from the pooled averages for this analysis set were less than $1 \%\left(0.05 \%\right.$ for ${ }^{239} \mathrm{Pu}$ and $0.35 \%$ for $\left.{ }^{240} \mathrm{Pu}\right)$. These values are shown in Table 4.7 and further details are provided in the TIMS final report located in Appendix E.

The wt\% and isotope concentrations for each Pu isotope were calculated analogously to the $\mathrm{U}$ concentrations after normalizing to the ${ }^{238} \mathrm{Pu}$ (AEA) concentration (see Sections 2.5.9 and 4.4.7).

\subsection{ICP-OES Analytes}

The ICP-OES-measured AOI concentrations for ASR 8798 are shown in Table 4.10. Table 4.11 presents opportunistically measured analytes. The analytes are reported on a dry mass basis in both tables. The ICP-OES measurements have a general overall uncertainty of $\pm 15 \%$ or better where analytes are greater than estimated quantitation limit (EQL) and in dilute, acidified water. Bracketed values indicate that concentrations were measured at less than the EQL but greater than the method detection limit; uncertainty for these values will likely be higher than $15 \%$. The less-than concentrations were determined by multiplying the IDL by the sample-specific process factor; process factor components include the sample size and final volume of digestate.

Overall QC results for the AOIs are summarized in Table 4.12. Additional details (also the opportunistic analytes) are provided in the ICP-OES analytical report for ASR 8798 located in Appendix B. The RPD was calculated only where both duplicate pair values exceeded the EQL. A majority of the RPD measurements met the QAPjP/SAP criterion of $\pm 20 \%$. However, several RPD failures (highlighted with gray shading in the tables) were found in three of the core samples. 
Accuracy was assessed through the LCS/BS and MS recoveries (where the spike was added before digestion), as well as from the post-digestion spikes (PSs) and serial dilution. All LCS/BS recoveries met the acceptance criterion of 80 to $120 \%$. While a majority of the MS recoveries also met the acceptance criteria as well, four analytes did not: Sb (45\%), Tl (30\%), Zn (74\%), and Zr (244\%). The MS recoveries for $\mathrm{Sb}$ and $\mathrm{Tl}$ were significantly lower than had been obtained previously (Fiskum et al. 2011a, b, and Shimskey et al. 2011). ${ }^{\text {(a) }}$ The low MS recoveries point to significant matrix-related signal depression issues with respect to these two analytes. The high Zr MS recovery indicates inhomogeneity in the subsamples for this analyte; the duplicate pair associated with the MS sample had a high Zr RPD of $135 \%$, also indicative that the subsamples collected for analysis varied in $\mathrm{Zr}$ concentration. In selected cases (for analytes Al, Ca, Fe, and U), MS recoveries were not able to be calculated and thus not reported (NR). This occurred when the sample analyte concentration greatly exceeded the spiked amount. All PS recovery results met the acceptance criterion (80 to 120\%); a further indication that a matrix effect exists. The serial dilution compared analyte concentrations above EQL after an additional $5 \times$ dilution at the workstation and is an additional measure of accuracy. The calculated percent differences were well below the $10 \%$ criterion established within the ASO procedure for those analytes where MS recoveries were not possible.

Detection limit requirements defined in the QAPjP/SAP were met for all AOIs with the exception of Se in the KW210-B1 duplicate pair. The duplicate pair (nominal MDL $=22 \mu \mathrm{g} / \mathrm{g}$ on a dry mass basis) exceeded the targeted $20 \mu \mathrm{g} / \mathrm{g}$ limit delineated in the ASR. However, the nominal detection limit was $7 \mu \mathrm{g} / \mathrm{g}$ on a wet settled sludge mass basis, which met the objective of the QAPjP/SAP (KBC-33786, Rev.2; Table 3-8). The PB showed analyte concentrations above the MDL but less than EQL for Ba, $\mathrm{Cr}$, $\mathrm{Na}, \mathrm{B}, \mathrm{Bi}, \mathrm{K}, \mathrm{P}, \mathrm{Sn}$, and Sr.

Table 4.13 and Table 4.14 show the AOIs and opportunistic analytes, respectively, on a wet settled sludge mass basis. As in Table 4.10 through Table 4.11, analyte concentrations are shaded where RPDs exceeded $\pm 20 \%$. However, DQO requirements were not applicable for opportunistic analytes.

(a) The historically low Sb, Se, and Tl MS recoveries in the K Basin sludge sample matrices were communicated to the BTR in PNNL correspondence 52578-2011-L06. The issues concerning 100\% attainment of the various QAPjP/SAP requirements on all QC samples were discussed in detail in PNNL correspondence 59205-2011L04 (K Basin Settler sludge). Because the same issues apply to the KW210 core sample results, the letters are provided as part of this data package in Appendix B. 
Table 4.10. ICP-OES Analytes of Interest in Acid Digestate, Dry Sludge Mass Basis (ASR 8798)

\begin{tabular}{|c|c|c|c|c|c|c|c|c|c|}
\hline $\begin{array}{l}\text { Sample } \\
\text { ID: }\end{array}$ & $\begin{array}{r}\text { Container: } \\
\text { Core: } \\
\text { PB }\end{array}$ & \multicolumn{8}{|c|}{ SCS-CON-210 } \\
\hline Analyte & $(\mu \mathrm{g} / \mathrm{g})$ & $(\mu \mathrm{g} / \mathrm{g})$ & $(\mu g / g)$ & $(\mu g / g)$ & $(\mu \mathrm{g} / \mathrm{g})$ & $(\mu g / g)$ & $(\mu \mathrm{g} / \mathrm{g})$ & $(\mu \mathrm{g} / \mathrm{g})$ & $(\mu \mathrm{g} / \mathrm{g})$ \\
\hline $\mathrm{Ag}$ & $<1.7$ & [5.5] & [3.8] & {$[2.8]$} & $\begin{array}{l}{[2.8]} \\
\end{array}$ & [4.2] & $\begin{array}{l}{[3.5]} \\
\end{array}$ & $\begin{array}{l}{[2.0]} \\
\end{array}$ & $\begin{array}{l}{[3.9]} \\
\end{array}$ \\
\hline $\mathrm{Al}$ & $<4.0$ & 32,500 & 32,600 & 20,700 & 22,400 & 15,600 & 15,500 & 12,400 & 12,100 \\
\hline As & $<8.4$ & 89.2 & 98.4 & 72.3 & 72.7 & [46] & [41] & [54] & [50] \\
\hline $\mathrm{Ba}$ & {$[0.24]$} & 192 & 199 & 135 & 143 & 75.4 & 78.0 & 66.8 & 62.4 \\
\hline $\mathrm{Be}$ & $<0.059$ & 6.99 & 10.2 & 12.2 & 6.70 & 3.03 & 18.6 & 2.76 & 2.51 \\
\hline $\mathrm{Ca}$ & $<1.9$ & 7,640 & 6,750 & 6,690 & 7,740 & 7,410 & 6,590 & 8,350 & 7,510 \\
\hline $\mathrm{Cd}$ & $<1.3$ & 35.9 & 33.5 & 29.8 & 34.6 & 65.5 & 57.4 & 48.8 & 48.8 \\
\hline $\mathrm{Cr}$ & [3.4] & 354 & 277 & 569 & 337 & 287 & 274 & 225 & 218 \\
\hline $\mathrm{Fe}$ & $<4.0$ & 86,800 & 88,600 & 96,400 & 93,700 & 35,100 & 27,500 & 31,800 & 27,800 \\
\hline $\mathrm{Mg}$ & $<0.54$ & 1140 & 1010 & 730 & 943 & 582 & 648 & 588 & 582 \\
\hline $\mathrm{Na}$ & [14] & 708 & 655 & 1,420 & 1650 & 1,710 & 1,610 & 1,460 & 1,340 \\
\hline $\mathrm{Ni}$ & $<2.3$ & 185 & 128 & 334 & 206 & 223 & 178 & 157 & 156 \\
\hline $\mathrm{Pb}$ & $<18$ & 345 & 345 & 238 & 264 & 319 & 322 & 313 & 287 \\
\hline $\mathrm{Sb}^{(\mathrm{a})}$ & $<1.9$ & [8.6] & [8.0] & [9.4] & [8.5] & $<2.9$ & [4.6] & [5.1] & [3.9] \\
\hline Se & $<15$ & $<15$ & $<13$ & $<12$ & $<11$ & $<23$ & $<21$ & $<16$ & $<16$ \\
\hline $\mathrm{Tl}^{(\mathrm{a})}$ & $<7.6$ & [9.2] & [8.7] & [6.6] & [6.8] & $<12$ & $<10$ & $<8.1$ & $<8.2$ \\
\hline $\mathrm{U}$ & $<11$ & 93,800 & 103,000 & 82,800 & 73,600 & 51,900 & 45,600 & 55,700 & 51,000 \\
\hline V & $<1.0$ & [15] & [15] & [13] & 14.2 & [9.8] & [11] & [9.8] & [8.4] \\
\hline $\mathrm{Zn}^{(\mathrm{a})}$ & $<4.9$ & 469 & 398 & 291 & 383 & 394 & 243 & 211 & 200 \\
\hline $\mathrm{Zr}^{(\mathrm{a})}$ & $<0.74$ & 334 & 369 & 373 & 148 & 77.6 & 400 & 91.8 & 97.2 \\
\hline
\end{tabular}

(a) The batch MS recovery exceeded the QAPjP/SAP criteria, see Table 4.12 .

Shaded analyte results indicate the QAPjP/SAP precision criterion of $\pm 20 \%$ was exceeded.

Results shown in brackets indicate that the analyte concentrations were greater than the MDL but less than the EQL.

Less-than values represent the instrument detection limit multiplied by the process factors. 
Table 4.11. ICP-OES Opportunistic Analytes in Acid Digestate, Dry Sludge Mass Basis (ASR 8798)

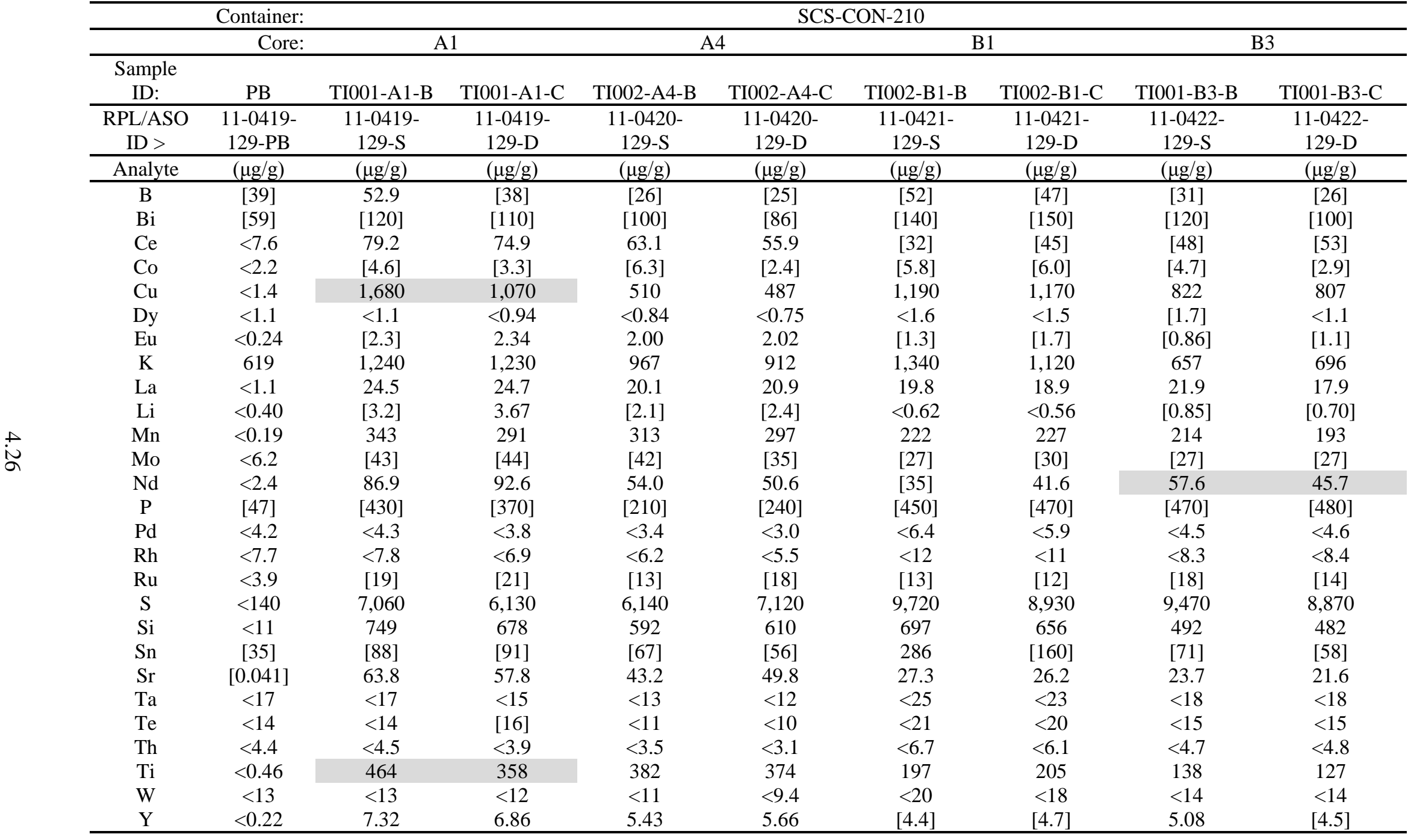

Results shown in brackets indicate that the analyte concentrations were greater than the MDL but less than the EQL. Opportunistic analytes are analytes not identified within the (QAPjP/SAP) and are shown for information-only; QAPjP/SAP QC sample requirements do not apply to these analytes. Less-than values represent the instrument detection limit multiplied by the process factors.

Shaded analyte results indicate the sample and duplicate sample RPD exceeded $\pm 20 \%$. 
Table 4.12. ICP-OES QC Sample Results for Analytes of Interest in Acid Digestate (ASR 8798)

\begin{tabular}{|c|c|c|c|c|c|c|c|c|}
\hline \multirow{2}{*}{$\begin{array}{r}\text { Container: } \\
\text { Core: }\end{array}$} & \multicolumn{4}{|c|}{ SCS-CON-210 (a) } & \multirow{2}{*}{$\begin{array}{l}\mathrm{LCS} / \mathrm{BS}^{(\mathrm{b})} \\
\text { Recovery, }\end{array}$} & \multirow{2}{*}{$\begin{array}{c}\mathrm{MS}^{(\mathrm{b})} \\
\text { Recovery, }\end{array}$} & \multirow{2}{*}{$\begin{array}{l}\text { Post Spike } \\
\text { Recovery, }\end{array}$} & \multirow{2}{*}{$\begin{array}{c}\text { Serial } \\
\text { Dilution, }{ }^{(\mathrm{c})}\end{array}$} \\
\hline & A1 & A4 & B1 & B3 & & & & \\
\hline Analyte & RPD & RPD & RPD & RPD & $\%$ & $\%$ & $\%$ & \% Diff \\
\hline Ag & -- & -- & -- & -- & 96 & 100 & 99 & -- \\
\hline $\mathrm{Al}$ & 0.4 & 7.6 & 0.8 & 2.1 & 101 & NR & 102 & 1.6 \\
\hline As & 9.9 & 0.6 & -- & -- & 85 & 90 & 97 & -- \\
\hline $\mathrm{Ba}$ & 3.8 & 5.5 & 3.3 & 6.9 & 97 & 104 & 101 & 3.1 \\
\hline $\mathrm{Ca}$ & 12.4 & 14.6 & 11.6 & 10.6 & 99 & NR & 101 & 1.8 \\
\hline $\mathrm{Cd}$ & 7.0 & 15.0 & 13.2 & 0.1 & 100 & 90 & 104 & -- \\
\hline $\mathrm{Cr}$ & 24.5 & 51.0 & 4.6 & 3.0 & 95 & 102 & 101 & 0.2 \\
\hline $\mathrm{Fe}$ & 2.1 & 2.9 & 24.4 & 13.7 & 105 & NR & 99 & 2.1 \\
\hline $\mathrm{Mg}$ & 12.1 & 25.4 & 10.8 & 1.1 & 102 & 118 & 101 & 8.3 \\
\hline $\mathrm{Na}$ & 7.8 & 14.9 & 6.4 & 8.5 & 103 & 119 & 100 & 0.3 \\
\hline $\mathrm{Pb}$ & 0.0 & 10.2 & 0.8 & 8.7 & 100 & 105 & 100 & -- \\
\hline $\mathrm{Sb}$ & -- & -- & -- & -- & 89 & 45 & 95 & -- \\
\hline Se & -- & -- & -- & -- & 89 & 96 & 99 & -- \\
\hline $\mathrm{Tl}$ & -- & -- & -- & -- & 84 & 30 & 92 & -- \\
\hline $\mathrm{U}$ & 9.6 & 11.7 & 13.0 & 8.9 & 100 & NR & 98 & 1.7 \\
\hline $\mathrm{V}$ & -- & -- & -- & -- & 94 & 99 & 99 & -- \\
\hline $\mathrm{Zn}$ & 16.3 & 27.3 & 47.3 & 5.3 & 93 & 74 & 99 & 6.8 \\
\hline $\mathrm{Zr}$ & 9.8 & 86.4 & 135.0 & 5.7 & 104 & 244 & 104 & 3.5 \\
\hline
\end{tabular}

(a) Shaded RPD values indicate the QAPjP/SAP precision criterion of $\pm 20 \%$ was exceeded. A dashed line indicates that the analyte concentration was below EQL and RPD could not be determined.

(b) The LCS and MS targeted recoveries were 80 to $120 \%$. Shaded MS recoveries indicate QAPjP/SAP accuracy criterion of $\pm 20 \%$ ( 80 to $120 \%$ analyte recovery) was exceeded.

(c) The dashed line indicates the analyte in the diluted sample was $<$ EQL and the percent difference could not be determined.

NR: Not Recovered; analyte concentration in the sample far exceeded the added spike amount. 
Table 4.13. ICP-OES Analytes of Interest in Acid Digestate, Wet Settled Sludge Mass Basis (ASR 8798)

\begin{tabular}{|c|c|c|c|c|c|c|c|c|c|}
\hline \multirow[b]{3}{*}{ Sample ID: } & \multirow{2}{*}{$\begin{array}{r}\text { Container: } \\
\text { Core: } \\
\end{array}$} & \multicolumn{8}{|c|}{ SCS-CON-210 } \\
\hline & & \multicolumn{2}{|c|}{ A1 } & \multicolumn{2}{|c|}{ A4 } & \multicolumn{2}{|c|}{$\mathrm{B} 1$} & \multicolumn{2}{|c|}{ B3 } \\
\hline & PB & TI001-A1-B & TI001-A1-C & TI002-A4-B & TI002-A4-C & TI002-B1-B & TI002-B1-C & TI001-B3-B & TI001-B3-C \\
\hline $\begin{array}{c}\mathrm{RPL} / \mathrm{ASO} \\
\mathrm{ID}>\end{array}$ & $\begin{array}{c}11-0419- \\
129-\mathrm{PB} \\
\end{array}$ & $\begin{array}{c}11-0419- \\
129-S \\
\end{array}$ & $\begin{array}{c}11-0419- \\
129-\mathrm{D}\end{array}$ & $\begin{array}{c}11-0420- \\
129-S \\
\end{array}$ & $\begin{array}{c}11-0420- \\
129-\mathrm{D}\end{array}$ & $\begin{array}{c}11-0421- \\
129-S \\
\end{array}$ & $\begin{array}{c}11-0421- \\
129-\mathrm{D} \\
\end{array}$ & $\begin{array}{c}11-0422- \\
129-S \\
\end{array}$ & $\begin{array}{c}\text { 11-0422- } \\
129-\mathrm{D}\end{array}$ \\
\hline Analyte & $(\mu \mathrm{g} / \mathrm{g})$ & $(\mu \mathrm{g} / \mathrm{g})$ & $(\mu \mathrm{g} / \mathrm{g})$ & $(\mu \mathrm{g} / \mathrm{g})$ & $(\mu \mathrm{g} / \mathrm{g})$ & $(\mu g / g)$ & $(\mu \mathrm{g} / \mathrm{g})$ & $(\mu \mathrm{g} / \mathrm{g})$ & $(\mu \mathrm{g} / \mathrm{g})$ \\
\hline $\mathrm{Ag}$ & $<0.71$ & [2.5] & [1.7] & {$[1.6]$} & [1.6] & [1.4] & {$[1.1]$} & {$[0.73]$} & {$[1.4]$} \\
\hline $\mathrm{Al}$ & $<1.7$ & 14,500 & 14,600 & 11,700 & 12,700 & 5,120 & 5,090 & 4,500 & 4,390 \\
\hline As & $<3.6$ & 39.9 & 44.0 & 41.0 & 41.2 & [15] & [13] & [20] & [18] \\
\hline $\mathrm{Ba}$ & {$[0.10]$} & 85.8 & 89.0 & 76.5 & 81.0 & 24.7 & 25.6 & 24.2 & 22.6 \\
\hline $\mathrm{Be}$ & $<0.025$ & 3.12 & 4.56 & 6.91 & 3.80 & 0.994 & 6.10 & 1.00 & 0.910 \\
\hline $\mathrm{Ca}$ & $<0.82$ & 3,420 & 3,020 & 3,790 & 4,390 & 2,430 & 2,160 & 3,030 & 2,720 \\
\hline $\mathrm{Cd}$ & $<0.56$ & 16.0 & 15.0 & 16.9 & 19.6 & 21.5 & 18.8 & 17.7 & 17.7 \\
\hline $\mathrm{Cr}$ & [1.4] & 158 & 124 & 322 & 191 & 94.2 & 89.9 & 81.6 & 79.0 \\
\hline $\mathrm{Fe}$ & $<1.7$ & 38,800 & 39,600 & 54,600 & 53,100 & 11,500 & 9,020 & 11,500 & 10,100 \\
\hline $\mathrm{Mg}$ & $<0.23$ & 510 & 451 & 414 & 534 & 191 & 213 & 213 & 211 \\
\hline $\mathrm{Na}$ & {$[6.0]$} & 316 & 293 & 805 & 935 & 561 & 528 & 529 & 486 \\
\hline $\mathrm{Ni}$ & $<1.0$ & 82.7 & 57.2 & 189 & 117 & 73.2 & 58.4 & 56.9 & 56.6 \\
\hline $\mathrm{Pb}$ & $<7.9$ & 154 & 154 & 135 & 150 & 105 & 106 & 113 & 104 \\
\hline $\mathrm{Sb}^{(\mathrm{a})}$ & $<0.82$ & [3.8] & [3.6] & [5.3] & [4.8] & $<1.0$ & [1.5] & [1.8] & [1.4] \\
\hline Se & $<6.4$ & $<6.8$ & $<5.9$ & $<6.8$ & $<6.0$ & $<7.5$ & $<6.8$ & $<5.8$ & $<5.9$ \\
\hline $\mathrm{Tl}^{(\mathrm{a})}$ & $<3.2$ & [4.1] & [3.9] & [3.7] & [3.9] & $<3.8$ & $<3.4$ & $<2.9$ & $<3.0$ \\
\hline $\mathrm{U}$ & $<4.5$ & 41,900 & 46,000 & 46,900 & 41,700 & 17,000 & 15,000 & 20,200 & 18,500 \\
\hline $\mathrm{V}$ & $<0.41$ & [6.7] & [6.7] & {$[7.4]$} & 8.05 & [3.2] & [3.6] & [3.6] & [3.0] \\
\hline $\mathrm{Zn}^{(\mathrm{a})}$ & $<2.1$ & 210 & 178 & 165 & 217 & 129 & 79.7 & 76.5 & 72.5 \\
\hline $\mathrm{Zr}^{(\mathrm{a})}$ & $<0.31$ & 149 & 165 & 211 & 83.9 & 25.5 & 131 & 33.3 & 35.2 \\
\hline
\end{tabular}

(a) The batch MS recovery exceeded the QAPjP/SAP criteria, see Table 4.12.

Shaded analyte results indicate the QAPjP/SAP precision criterion of $\pm 20 \%$ was exceeded.

Results shown in brackets indicate that the analyte concentrations were greater than the MDL but less than the EQL. Less-than values represent the instrument detection limit multiplied by the process factors. 
Table 4.14. ICP-OES Opportunistic Analytes in Acid Digestate, Wet Settled Sludge Mass Basis (ASR 8798)

\begin{tabular}{|c|c|c|c|c|c|c|c|c|c|}
\hline & Container: & & & & SCS-C & $\mathrm{N}-210$ & & & \\
\hline & Core: & & & & & & & & \\
\hline $\begin{array}{l}\text { Sample } \\
\text { ID: }\end{array}$ & PB & TI001-A1-B & TI001-A1-C & TI002-A4-B & TI002-A4-C & TI002-B1-B & TI002-B1-C & TI001-B3-B & TI001-B3-C \\
\hline $\begin{array}{c}\text { RPL/ASO } \\
\text { ID }> \\
\end{array}$ & $\begin{array}{c}11-0419- \\
129-\mathrm{PB} \\
\end{array}$ & $\begin{array}{c}11-0419- \\
129-S\end{array}$ & $\begin{array}{c}\text { 11-0419- } \\
\text { 129-D }\end{array}$ & $\begin{array}{c}11-0420- \\
129-S \\
\end{array}$ & $\begin{array}{c}\text { 11-0420- } \\
129-\mathrm{D}\end{array}$ & $\begin{array}{c}11-0421- \\
129-S \\
\end{array}$ & $\begin{array}{c}\text { 11-0421- } \\
129-\mathrm{D}\end{array}$ & $\begin{array}{c}11-0422- \\
129-S\end{array}$ & $\begin{array}{c}\text { 11-0422- } \\
129-D\end{array}$ \\
\hline Analyte & $(\mu \mathrm{g} / \mathrm{g})$ & $(\mu \mathrm{g} / \mathrm{g})$ & $(\mu \mathrm{g} / \mathrm{g})$ & $(\mu \mathrm{g} / \mathrm{g})$ & $(\mu \mathrm{g} / \mathrm{g})$ & $(\mu \mathrm{g} / \mathrm{g})$ & $(\mu \mathrm{g} / \mathrm{g})$ & $(\mu \mathrm{g} / \mathrm{g})$ & $(\mu \mathrm{g} / \mathrm{g})$ \\
\hline $\mathrm{B}$ & [17] & 23.6 & [17] & [15] & [14] & [17] & [15] & [11] & [9.4] \\
\hline $\mathrm{Bi}$ & [25] & [54] & [49] & [57] & [49] & [46] & [49] & [44] & [36] \\
\hline $\mathrm{Ce}$ & $<3.3$ & 35.4 & 33.5 & 35.8 & 31.7 & [10] & [15] & [17] & [19] \\
\hline Co & $<0.94$ & {$[2.1]$} & [1.5] & [3.6] & [1.4] & [1.9] & {$[2.0]$} & [1.7] & [1.1] \\
\hline $\mathrm{Cu}$ & $<0.60$ & 751 & 478 & 289 & 276 & 390 & 384 & 298 & 293 \\
\hline Dy & $<0.45$ & $<0.48$ & $<0.42$ & $<0.48$ & $<0.43$ & $<0.53$ & $<0.48$ & [0.62] & $<0.41$ \\
\hline $\mathrm{Eu}$ & $<0.10$ & {$[1.0]$} & 1.05 & 1.13 & 1.14 & {$[0.43]$} & {$[0.56]$} & {$[0.31]$} & {$[0.40]$} \\
\hline $\mathrm{K}$ & 264 & 554 & 550 & 548 & 517 & 440 & 367 & 238 & 252 \\
\hline $\mathrm{La}$ & $<0.45$ & 11.0 & 11.0 & 11.4 & 11.8 & 6.50 & 6.20 & 7.94 & 6.49 \\
\hline $\mathrm{Li}$ & $<0.17$ & [1.4] & 1.64 & [1.2] & [1.4] & $<0.20$ & $<0.18$ & {$[0.31]$} & [0.25] \\
\hline Mn & $<0.082$ & 153 & 130 & 177 & 168 & 72.8 & 74.5 & 77.6 & 70.0 \\
\hline Mo & $<2.6$ & [19] & [20] & [24] & [20] & [8.9] & [9.8] & [9.8] & [9.8] \\
\hline $\mathrm{Nd}$ & $<1.0$ & 38.8 & 41.4 & 30.6 & 28.7 & [11] & 13.6 & 20.9 & 16.6 \\
\hline $\mathrm{P}$ & [20] & [190] & [170] & [120] & [140] & [150] & [150] & [170] & [170] \\
\hline $\mathrm{Pd}$ & $<1.8$ & $<1.9$ & $<1.7$ & $<1.9$ & $<1.7$ & $<2.1$ & $<1.9$ & $<1.6$ & $<1.7$ \\
\hline $\mathrm{Rh}$ & $<3.3$ & $<3.5$ & $<3.1$ & $<3.5$ & $<3.1$ & $<3.9$ & $<3.5$ & $<3.0$ & $<3.0$ \\
\hline $\mathrm{Ru}$ & $<1.6$ & [8.5] & [9.4] & [7.4] & [10] & [4.3] & [3.9] & [6.5] & [5.1] \\
\hline $\mathrm{S}$ & $<60$ & 3,160 & 2,740 & 3,480 & 4,030 & 3,190 & 2,930 & 3,430 & 3,220 \\
\hline $\mathrm{Si}$ & $<4.9$ & 335 & 303 & 335 & 346 & 229 & 215 & 178 & 175 \\
\hline Sn & [15] & [39] & [41] & [38] & [32] & 93.8 & [52] & [26] & [21] \\
\hline $\mathrm{Sr}$ & [0.017] & 28.5 & 25.8 & 24.5 & 28.2 & 8.96 & 8.60 & 8.59 & 7.83 \\
\hline $\mathrm{Ta}$ & $<7.1$ & $<7.6$ & $<6.6$ & $<7.6$ & $<6.7$ & $<8.4$ & $<7.6$ & $<6.5$ & $<6.6$ \\
\hline $\mathrm{Te}$ & $<6.0$ & $<6.4$ & [7.2] & $<6.4$ & $<5.7$ & $<7.0$ & $<6.4$ & $<5.5$ & $<5.5$ \\
\hline Th & $<1.9$ & $<2.0$ & $<1.7$ & $<2.0$ & $<1.8$ & $<2.2$ & $<2.0$ & $<1.7$ & $<1.7$ \\
\hline $\mathrm{Ti}$ & $<0.19$ & 207 & 160 & 216 & 212 & 64.6 & 67.3 & 50.0 & 46.0 \\
\hline W & $<5.6$ & $<6.0$ & $<5.2$ & $<6.0$ & $<5.3$ & $<6.6$ & $<6.0$ & $<5.1$ & $<5.2$ \\
\hline $\mathrm{Y}$ & $<0.094$ & 3.27 & 3.07 & 3.08 & 3.21 & [1.4] & [1.5] & 1.84 & [1.6] \\
\hline
\end{tabular}

Results shown in brackets indicate that the analyte concentrations were greater than the MDL but less than the EQL.

Opportunistic analytes are analytes not identified within the QAPJP/SAP and are shown for information only; QAPjP/SAP QC sample requirements did not apply to these analytes. Shaded analyte results indicate the sample and duplicate sample RPD exceeded $\pm 20 \%$.

Less-than values represent the instrument detection limit multiplied by the process factors. 


\subsection{TOC and TIC Analysis}

Table 3-4a and Figure 3-4 of the QAPJP/SAP specified the analysis for TOC and indicated that the samples are to be analyzed on a wet (settled sludge mass basis); Table 3-8 indicated TOC/TC ${ }^{(a)}$ be performed. As discussed in the following paragraphs, and in accordance with the BTR-approved ASR 8798 Special Instructions, the samples were dried at $105^{\circ} \mathrm{C}$ to constant weight before TOC and TIC analyses were performed.

Grafoil $^{\circledR}$ and OIER were found to be present in some sludge samples based on observations of the sludge make-up during previous containerized sludge characterization activities (Fiskum et al. 2011b). Consequently, the BTR provided additional direction to include the analysis of Grafoil ${ }^{\circledR}$ and the carbon contribution from OIER in K Basin sludge samples. These observations and requirements drove the need to use sequential temperature combustion processing $\left(600\right.$ and $\left.1,000^{\circ} \mathrm{C}\right)$ as previously described (Fiskum et al. 2011a) to oxidize the carbon in these materials (procedure RPG-CMC-385, Rev. 2). Using this method, the TOC results from OIER Purolite NRW37 samples were shown to recover 77.7 to $107.8 \%$ (average of $90 \pm 8 \%$ ), and the TIC from Grafoil ${ }^{\circledR}$ were shown to recover 66.6 to $111 \%$ (average of $103 \pm 8 \%$ ) (Fiskum et al. 2011a). The OIER material is considered an upper limit in difficulty for organic compound combustion; therefore, any other organic species present will also be effectively combusted at $600^{\circ} \mathrm{C}$. Similar rationale applies to the Grafoil ${ }^{\circledR}$ combustion in that all inorganic carbon forms will oxidize at $1,000^{\circ} \mathrm{C}$ (e.g., calcium carbonate). Therefore, results are not exclusive to these materials in the sample, but will be inclusive of them.

Small, representative subsamples collected for TOC and TIC analyses from the dewatered parent material (i.e., core samples) were transferred to 30 -mL glass vials and removed from the hot cell. The transferred subsample masses varied sample to sample and needed to be $<1 \mathrm{~g}$ (for ALARA purposes). Each subsample contained significant headspace and no additional water was added to maintain them in a wetted condition prior to analysis. The TOC and TIC analyses were performed at the workstation several months after collection (June 2011).

The subsamples collected from the parent material were dried at $105^{\circ} \mathrm{C}$ to constant mass. This resulted in a normalized mass basis for each subsample and allowed the dry-mass/wet-settled mass conversion factor (Table 3.1) to be applied such that the TOC and TIC concentrations could be reported on a wet-settled sludge mass basis. (Note that an attempt to subsample wetted sludge at the analytical workstation would be confounded because the water content, and thus the mass basis, would be different from known conditions such as dried, as-sampled, and as-settled sample material.) Three small replicate subsamples were collected from the dried sample material for sequential TOC and TIC analyses. The small replicate subsamples were collected on a best-effort basis to be as representative as possible of the supplied material. The replicate subsample masses ranged from 3.5 to $64.8 \mathrm{mg}$. The analytical samples were driven to small sizes by the nature of the material (i.e., high organic carbon content, high dose rate, high actinide concentrations, and small PSD leading to highly dispersible sample characteristics) and the processing location (i.e., fume hood) where the carbon analyzer was located. Many of the samples analyzed for the K Basin project have high carbon content (typically from OIER and Grafoil ${ }^{\mathbb{R}}$ ), so small sample sizes are required to prevent overwhelming the detector system with product $\mathrm{CO}_{2}$ (upper working limit is generally limited to $\sim 5,000 \mu \mathrm{g} \mathrm{C}$ ).

(a) The TC is the sum of TIC and TOC. 
It is understood that the sample collection, storage, and drying process could result in loss of volatile organic compounds, if present. Any component that volatilizes around $105^{\circ} \mathrm{C}$ will be lost in this method; typically this condition applies to organic solvents (benzene boiling point is $80^{\circ} \mathrm{C}$ and octane $\sim 125^{\circ} \mathrm{C}$ [Weast and Astle 1979]). Organic salts tend to volatilize or decompose at higher temperatures (for example, the melting point of sodium formate is $253^{\circ} \mathrm{C}$, the melting point of glucose is $146^{\circ} \mathrm{C}$ [Weast and Astle 1979]).

Table 4.15 and Table 4.16 summarize the TOC and TIC concentrations, respectively, in terms of $\mu \mathrm{g}$ carbon per g dry sample for each replicate subsample, along with the associated dry mass sample size. The EQL (considered 5× the MDL) was exceeded for all reported sample results except for the TIC on the duplicate B1 core subsamples and one of the B3 core subsamples. The precision of the three replicate samples from each analysis is shown in the data tables. Although significant variability existed in the " $A$ " core sample TOC results, the RPD between the weighted average of the sample and duplicate (each were measured in triplicate) met the QAPjP/SAP precision criterion for each core.

The TIC results showed a lot more variability within the replicate sample set and the weighted average of the duplicate pairs than was observed with the TOC results. All TIC results were close to the MDL, a consequence of the small sample aliquot taken for analysis to accommodate the high TOC content. Table 4.17 summarizes the TOC and TIC concentrations on a wet settled mass basis from the weighted average sample results and the conversion factor (g dry/g settled) found in Table 3.1.

Each sample was processed on a different day because of the long duration of an analysis run. A LCS was processed each day samples were processed; two samples were prepared with analytical spike $\alpha$-D-glucose and calcium carbonate (normal spike materials to assess system performance). The TOC LCS (a-D-glucose) recovered between 93 and 115\%; the TIC LCS (calcium carbonate) recovered between 80 and 97\%. One analytical spike sample recovery (132\% TOC on sample 11-419-C-S) exceeded the acceptance limit ${ }^{(\mathrm{a})}$; all others met acceptance criteria (see Appendix B).

Additionally, staff were directed to prepare a laboratory-created test sample (LCTS) consisting of combined Purolite NRW37 and Grafoil ${ }^{\circledR}$ (RPL/ASO ID 11-0423-C-S and -D). The LCTS was processed at the beginning and at the conclusion of the sludge sample processing. Recoveries of the LCTS TOC and TIC were assessed and are summarized in Table 4.18. All TOC and TIC recoveries were within $\pm 10 \%$ of the known $\mathrm{C}$ concentration indicating system performance was acceptable.

(a) The high analytical spike recovery was most likely an artifact of the subsampling processing and associated TOC variability between sub-samples. 
Table 4.15. TOC of SCS-CON-210 Core Samples, Dry Sludge Mass Basis (ASR 8798)

\begin{tabular}{|c|c|c|c|c|c|c|}
\hline Core: & \multicolumn{6}{|c|}{ KW210-A1 } \\
\hline TI ID: & \multicolumn{3}{|c|}{ TI001-A1-H } & \multicolumn{3}{|c|}{ TI001-A1-J } \\
\hline \multirow[t]{2}{*}{ RPL/ASO Sample ID: } & \multicolumn{3}{|c|}{ 11-0419-C-S } & \multicolumn{3}{|c|}{ 11-0419-C-D } \\
\hline & $\begin{array}{c}\text { Sample } \\
\text { size, g }\end{array}$ & $\begin{array}{l}\text { TOC, } \\
\mu \mathrm{g} / \mathrm{g}\end{array}$ & $\begin{array}{c}\text { TOC, } \\
\mu \mathrm{g}\end{array}$ & $\begin{array}{c}\text { Sample } \\
\text { size, g }\end{array}$ & $\begin{array}{l}\text { TOC, } \\
\mu \mathrm{g} / \mathrm{g}\end{array}$ & $\begin{array}{c}\text { TOC, } \\
\mu \mathrm{g}\end{array}$ \\
\hline Replicate 1 & 0.0326 & 131,000 & 4,271 & 0.0184 & 130,000 & 2,392 \\
\hline Replicate 2 & 0.0165 & 178,000 & 2,937 & 0.0241 & 193,000 & 4,651 \\
\hline Replicate 3 & 0.0058 & 104,000 & 603 & 0.0097 & 97,400 & 945 \\
\hline RSD & \multicolumn{3}{|c|}{$27 \%$} & \multicolumn{3}{|c|}{$35 \%$} \\
\hline Total & 0.0549 & & 7,811 & 0.0522 & & 7,988 \\
\hline \multicolumn{6}{|l|}{ Weighted Average } & \\
\hline RPD of Weighted Averages & \multicolumn{6}{|c|}{$7.2 \%$} \\
\hline Core: & \multicolumn{6}{|c|}{ KW210-A4 } \\
\hline TI ID & \multicolumn{3}{|c|}{ TI002-A4-H } & \multicolumn{3}{|c|}{ TI002-A4-J } \\
\hline \multirow[t]{2}{*}{ RPL/ASO Sample ID: } & \multicolumn{3}{|c|}{ 11-0420-C-S } & \multicolumn{3}{|c|}{ 11-0420-C-D } \\
\hline & $\begin{array}{c}\text { Sample } \\
\text { size, g }\end{array}$ & $\begin{array}{l}\text { TOC, } \\
\mu \mathrm{g} / \mathrm{g}\end{array}$ & $\begin{array}{c}\text { TOC, } \\
\mu \mathrm{g}\end{array}$ & $\begin{array}{c}\text { Sample } \\
\text { size, g }\end{array}$ & $\begin{array}{l}\text { TOC, } \\
\mu \mathrm{g} / \mathrm{g}\end{array}$ & $\begin{array}{c}\text { TOC, } \\
\mu \mathrm{g}\end{array}$ \\
\hline Replicate 1 & 0.0189 & 40,900 & 773 & 0.0200 & 51,500 & 1,030 \\
\hline Replicate 2 & 0.0340 & 72,300 & 2,458 & 0.0648 & 93,900 & 6,085 \\
\hline Replicate 3 & 0.0577 & 94,700 & 5,464 & 0.0362 & 118,000 & 4,272 \\
\hline RSD & \multicolumn{3}{|c|}{$39 \%$} & \multicolumn{3}{|c|}{$38 \%$} \\
\hline Total & 0.1106 & & 8,695 & 0.1210 & & 11,386 \\
\hline \multicolumn{7}{|l|}{ Weighted Average } \\
\hline RPD of Weighted Averages & \multicolumn{6}{|c|}{$18 \%$} \\
\hline Core: & \multicolumn{6}{|c|}{ KW210-B1 } \\
\hline TI ID & \multicolumn{3}{|c|}{ TI002-B1-H } & \multicolumn{3}{|c|}{ TI002-B1-J } \\
\hline \multirow{2}{*}{ RPL/ASO Sample ID: } & & 1-0421-C & & & $1-0421-C$ & \\
\hline & $\begin{array}{c}\text { Sample } \\
\text { size, g }\end{array}$ & $\begin{array}{l}\text { TOC, } \\
\mu \mathrm{g} / \mathrm{g}\end{array}$ & $\begin{array}{c}\text { TOC, } \\
\mu \mathrm{g}\end{array}$ & $\begin{array}{c}\text { Sample } \\
\text { size, g }\end{array}$ & $\begin{array}{l}\text { TOC, } \\
\mu \mathrm{g} / \mathrm{g}\end{array}$ & $\begin{array}{c}\text { TOC, } \\
\mu \mathrm{g}\end{array}$ \\
\hline Replicate 1 & 0.0132 & 518,000 & 6,838 & 0.0046 & 439,000 & 2,019 \\
\hline Replicate 2 & 0.0035 & 516,000 & 1,806 & 0.0101 & 505,000 & 5,101 \\
\hline Replicate 3 & 0.0188 & 510,000 & 9,588 & 0.0148 & 499,000 & 7,385 \\
\hline RSD & & $0.8 \%$ & & & $7.6 \%$ & \\
\hline Total & 0.0355 & & 18,232 & 0.0295 & & 14,505 \\
\hline 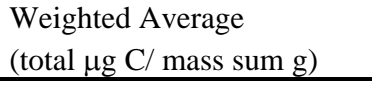 & & 513,600 & & & 491,700 & \\
\hline RPD of Weighted Averages & & & & & & \\
\hline Core: & & & $\mathrm{KH}$ & $-\mathrm{B} 3$ & & \\
\hline TI ID & & I001-B3- & & & I001-B3 & \\
\hline RPL/ASO Sample ID: & & 1-0422-C & & & L-0422-C & \\
\hline & $\begin{array}{c}\text { Sample } \\
\text { size, g }\end{array}$ & $\begin{array}{l}\text { TOC, } \\
\mu \mathrm{g} / \mathrm{g}\end{array}$ & $\begin{array}{c}\text { TOC, } \\
\mu \mathrm{g}\end{array}$ & $\begin{array}{c}\text { Sample } \\
\text { size, g }\end{array}$ & $\begin{array}{l}\text { TOC, } \\
\mu \mathrm{g} / \mathrm{g}\end{array}$ & $\begin{array}{c}\text { TOC, } \\
\mu \mathrm{g}\end{array}$ \\
\hline Replicate 1 & 0.0096 & 356,000 & 3,418 & 0.0106 & 353,000 & 3,742 \\
\hline Replicate 2 & 0.0261 & 366,000 & 9,553 & 0.0219 & 378,000 & 8,278 \\
\hline Replicate 3 & 0.0053 & 464,000 & 2,459 & 0.0141 & 371,000 & 5,231 \\
\hline RSD & & $15 \%$ & & & $4 \%$ & \\
\hline Total & 0.041 & & 15,429 & 0.0466 & & 17,251 \\
\hline $\begin{array}{l}\text { Weighted Average } \\
\text { (total } \mu \mathrm{g} \mathrm{C} \text { / mass sum g) }\end{array}$ & & 376,330 & & & 370,200 & \\
\hline RPD of Weighted Averages & & & & & & \\
\hline
\end{tabular}


Table 4.16. TIC of SCS-CON-210 Core Samples, Dry Sludge Mass Basis (ASR 8798)

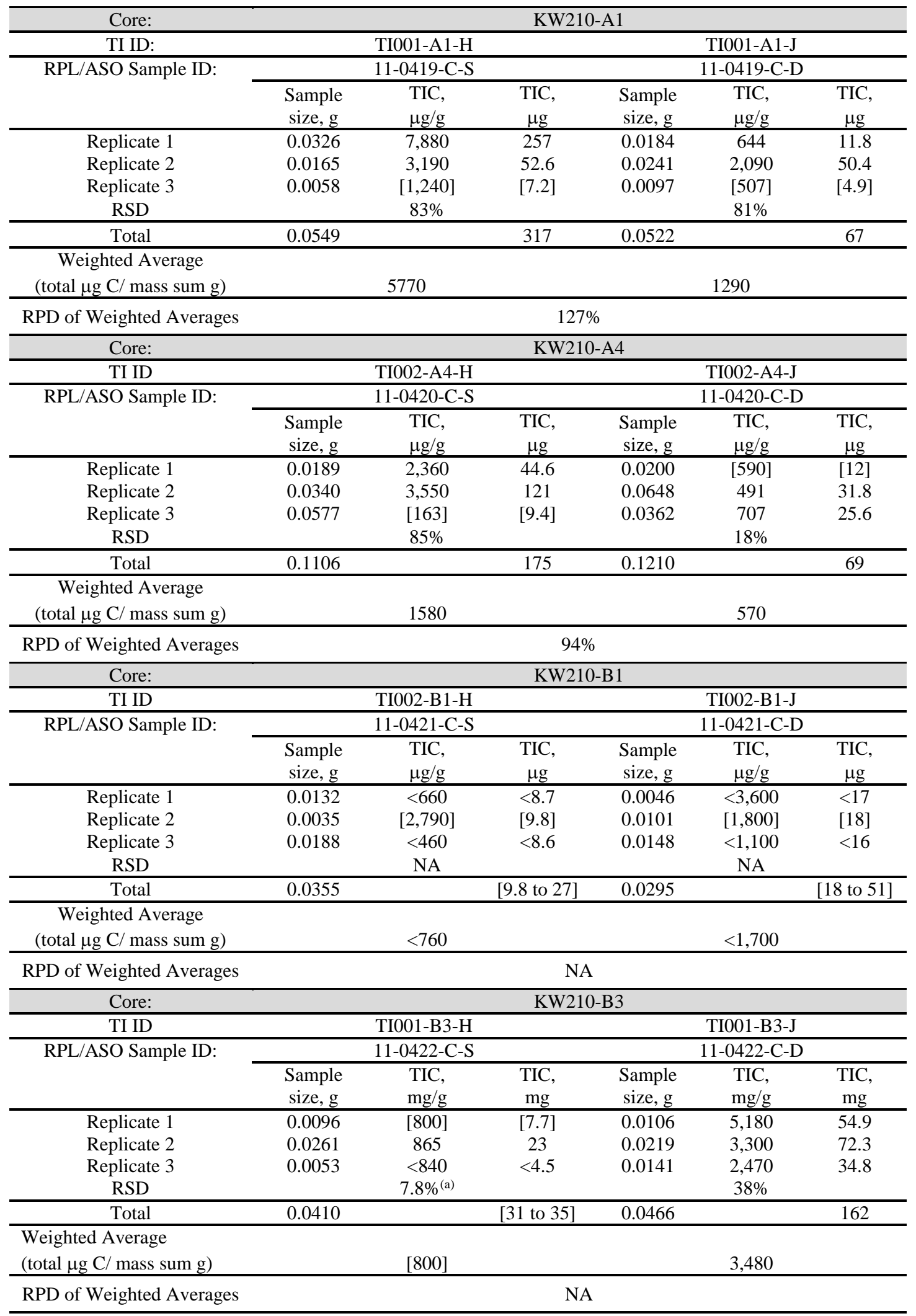

(a) The precision (RPD) was calculated from the two measurements above the MDL. 
PNNL-20650, Rev. 2

Table 4.17. TOC and TIC of SCS-CON-210 Core Samples, Wet Settled Sludge Mass Basis (ASR 8798)

\begin{tabular}{|c|c|c|c|c|}
\hline \multirow[b]{2}{*}{ TI ID > } & \multicolumn{2}{|c|}{ KW210-A1 } & \multicolumn{2}{|c|}{ KW210-A4 } \\
\hline & TI001-A1-H & TI001-A1-J & TI002-A4-H & TI002-A4-J \\
\hline RPL/ASO ID > & 11-0419-C-S & 11-0419-C-D & 11-0420-C-S & 11-0420-C-D \\
\hline TOC & 63,600 & 68,400 & 44,600 & 53,300 \\
\hline \multirow[t]{2}{*}{ TIC } & 2,580 & 577 & 895 & 323 \\
\hline & \multicolumn{2}{|c|}{ KW210-B1 } & \multicolumn{2}{|c|}{ KW210-B3 } \\
\hline TI ID > & TI002-B1-H & TI002-B1-J & TI001-B3-H & TI001-B3-J \\
\hline RPL/ASO ID > & 11-0421-C-S & 11-0421-C-D & 11-0422-C-S & 11-0422-C-D \\
\hline TOC & 169,000 & 161,000 & 136,000 & 134,000 \\
\hline TIC & $<250^{(\mathrm{a})}$ & $<560^{(\mathrm{a})}$ & {$[290]^{(b)}$} & 1,260 \\
\hline \multicolumn{4}{|c|}{$\begin{array}{l}\text { (a) Two of the three replicate TIC analysis results were }<\text { MDL and one result was }<\text { EQL; the reported less-than } \\
\text { value provides a bounding assessment of the TIC concentration in the sample. }\end{array}$} & $\begin{array}{l}\text { (b) One of the three replicate TIC analysis results was }<\text { MDL, one result was }<\text { EQL, and one result was }>\text { EQL; the } \\
\text { brackets indicate the sample result has significant uncertainty. }\end{array}$ \\
\hline \multicolumn{5}{|c|}{ Shading indicates RPD of the sample and duplicate exceeded $\pm 20 \%$. } \\
\hline \multicolumn{5}{|c|}{$\begin{array}{l}\text { Calculations of data presented above were performed using untruncated values in Table } 3.1 \text { to convert weighted } \\
\text { average results in Table } 4.15 \text { and Table } 4.16 \text { from as-dried mass basis to a wet as-settled sludge mass basis. } \\
\text { Calculation spreadsheets using Microsoft Excel are provided in data package DVD-52578-PNNL-20650 Rev. 2, } \\
\text { Section III, September } 2013 \text {. }\end{array}$} \\
\hline
\end{tabular}

Table 4.18. TOC and TIC QC Summary Table for the LCTS, Dry Sludge Mass Basis (ASR 8798)

\begin{tabular}{|c|c|c|c|c|c|c|}
\hline & \multicolumn{3}{|c|}{ Purolite NRW37at $600^{\circ} \mathrm{C}$ (TOC) } & \multicolumn{3}{|c|}{ Grafoil $^{\circledR}$ at $1,000^{\circ} \mathrm{C}$ (TIC) } \\
\hline & Sample size, $\mathrm{g}$ & $\begin{array}{l}\text { Result, } \\
\mu \mathrm{gC} / \mathrm{g}\end{array}$ & $\begin{array}{c}\text { Recovery, } \\
\%\end{array}$ & Sample size, g & $\begin{array}{l}\text { Result, } \\
\mu \mathrm{gC} / \mathrm{g}\end{array}$ & $\begin{array}{c}\text { Recovery, } \\
\%\end{array}$ \\
\hline \multicolumn{7}{|l|}{ Before Series } \\
\hline 11-0423-C-S & 0.0029 & 612,000 & 91.0 & 0.0046 & $1,060,000$ & 106.9 \\
\hline 11-0423-C-D & 0.0053 & 636,000 & 94.7 & 0.0044 & $1,050,000$ & 105.6 \\
\hline \multicolumn{7}{|l|}{ After Series } \\
\hline 11-0423-C-S & 0.0044 & 726,000 & 108 & 0.0080 & 953,000 & 95.7 \\
\hline 11-0423-C-D & 0.0047 & 687,000 & 102 & 0.0044 & 981,000 & 98.6 \\
\hline
\end{tabular}

\subsection{Uranium Speciation Results}

The sample digestion (RPG-CMC-107) for all core samples (ASR 8798) was completed on March 2, 2011, while the sample digestion for KW210-A4 re-analysis (ASR 8824) was completed on April 13, 2011. The PB samples were prepared with $0.6 \mathrm{~g}$ of Hanford sand from the Arid Land Ecology Reserve (ALE); LCS samples were prepared using the ALE sand with measured quantities of $\mathrm{U}_{3} \mathrm{O}_{8}$ powder and $\mathrm{U}$ metal beads. Reagent blank samples were also analyzed for $\mathrm{U}$. Duplicate samples from each core were prepared for evaluation of method precision. A third core subsample was spiked with U metal and processed as the batch MS sample (the MS sample was supplemental to the QAPjP/SAP-defined requirements). Table 4.19 and Table 4.20 summarize the $U$ metal and oxidation state results for each core sample. 
PNNL-20650, Rev. 2

Table 4.19. Uranium Metal in SCS-CON-210 Core Samples (ASR 8798 and 8824)

\begin{tabular}{|c|c|c|c|c|c|c|c|}
\hline $\begin{array}{c}\text { Sample } \\
\text { Description }\end{array}$ & Sample ID & RPL/ASO ID & $\begin{array}{c}\mathrm{U} \text { in } \\
7^{\text {th }} \text { rinse } \\
(\mu \mathrm{g} / \mathrm{mL})\end{array}$ & $\begin{array}{c}\text { U Metal, } \\
\text { As-Sampled } \\
\text { (wt\%) }\end{array}$ & $\begin{array}{l}\text { U Metal } \\
\text { Settled } \\
(\mathrm{wt} \%)^{(\mathrm{a})}\end{array}$ & $\begin{array}{l}\text { U Metal } \\
\text { Recovery } \\
(\%)\end{array}$ & $\begin{array}{l}\text { RPD } \\
\text { (\%) }\end{array}$ \\
\hline \multicolumn{8}{|l|}{ ASR 8798} \\
\hline LCS & LCS & 11-0419-107-LCS & 0.405 & --- & --- & 102 & --- \\
\hline Hanford Sand & PB 2 & 11-0419-107-B2 & 0.023 & $<0.0006$ & --- & --- & --- \\
\hline \multirow[t]{2}{*}{ KW210-A1 } & TI001-A1-P & 11-0419-107-S & 0.362 & 0.0685 & 0.0537 & --- & NA \\
\hline & TI001-A1-Q & 11-0419-107-D & 0.546 & 0.116 & 0.0910 & --- & \\
\hline \multirow[t]{2}{*}{ KW210-A4 } & TI002-A4-P & $11-0420-107-S$ & 0.814 & 1.19 & 1.07 & -- & 54 \\
\hline & TI002-A4-Q & 11-0420-107-D & 0.583 & 0.685 & 0.619 & -- & \\
\hline \multirow[t]{2}{*}{ KW210-B1 } & TI002-B1-P & $11-0421-107-S$ & 0.080 & $<0.011$ & $<0.0086$ & --- & NA \\
\hline & TI002-B1-Q & 11-0421-107-D & 0.186 & 0.0191 & 0.0149 & --- & \\
\hline Matrix spike & TI002-B1-R & 11-0421-107-MS & 0.092 & $0.0102^{\text {(b) }}$ & --- & 99 & \\
\hline \multirow[t]{2}{*}{ KW210-B3 } & TI001-B3-P & $11-0422-107-S$ & 0.519 & 0.0176 & 0.0133 & --- & NA \\
\hline & TI001-B3-Q & 11-0422-107-D & 0.335 & 0.0130 & 0.0098 & --- & \\
\hline \multicolumn{8}{|l|}{ ASR 8824} \\
\hline LCS & LCS & 11-0792-107-LCS & 0.146 & --- & --- & 103 & --- \\
\hline Hanford Sand & PB 2 & 11-0792-107-B2 & $<0.012$ & 0.00065 & --- & --- & --- \\
\hline \multirow[t]{2}{*}{ KW210-A4 } & TI002-A4-R & 11-0967-107-S & 0.475 & 0.825 & 0.745 & --- & 4 \\
\hline & TI002-A4-S & 11-0967-107-D & 0.937 & 0.795 & 0.718 & --- & \\
\hline
\end{tabular}

(a) The conversion factors ( $\mathrm{g}_{\text {sampled }} / \mathrm{g}_{\text {settled}}$ ) are provided in Table 3.1.

(b) The MS sample from ASR 8798 was taken from the B1 core (TI002-B1-R). The U metal concentration of MS sample before the U metal spike was added is back-calculated to be $0.0102 \mathrm{wt} \%$ on an as-sampled mass basis. This is lower than the method detection limit of $0.011 \mathrm{wt} \%$.

Shaded blocks indicate that the RPD of the duplicate pair exceeded $\pm 20 \%$.

NA: Not Applicable. The RPD calculation of duplicates is not performed when uranium concentration is $<0.1$ wt $\%$ of the assampled sludge.

Analytical results from ASR 8798 are found in Appendix B and the results for ASR 8824 are found in Appendix C. 
PNNL-20650, Rev. 2

Table 4.20. Uranium Oxidation State Measurements in SCS-CON-210 Core Samples

(ASR 8798 and 8824)

\begin{tabular}{|c|c|c|c|c|c|c|c|}
\hline $\begin{array}{c}\text { Sample } \\
\text { Description }\end{array}$ & Sample ID & RPL/ASO ID & $\begin{array}{l}\mathrm{U}(\mathrm{IV}) \\
(\mathrm{mol} \%)\end{array}$ & $\begin{array}{l}\mathrm{U}(\mathrm{VI}) \\
(\mathrm{mol} \%)\end{array}$ & $\begin{array}{l}\text { RPD U(IV) } \\
(\%)\end{array}$ & $\begin{array}{l}\text { RPD } \\
\text { U(VI) } \\
(\%)\end{array}$ & $\begin{array}{l}\text { Quality } \\
\text { Checks }\end{array}$ \\
\hline \multicolumn{8}{|l|}{ ASR 8798} \\
\hline LCS & LCS & 11-0419-107-LCS & 33.7 & 66.3 & --- & --- & Pass $^{(a)}$ \\
\hline \multirow[t]{2}{*}{ KW210-A1 } & TI001-A1-P & $11-0419-107-S$ & 88.4 & 11.6 & 1.7 & 14 & Pass $^{(b)}$ \\
\hline & TI001-A1-Q & 11-0419-107-D & 90.0 & 10.0 & --- & --- & \\
\hline \multirow[t]{2}{*}{ KW210-A4 } & TI002-A4-P & $11-0420-107-S$ & $>94.1$ & $<5.9^{(\mathrm{c})}$ & --- & --- & NA \\
\hline & TI002-A4-Q & 11-0420-107-D & $>91.2$ & $<8.8^{(\mathrm{c})}$ & --- & --- & \\
\hline \multirow[t]{2}{*}{ KW210-B1 } & TI002-B1-P & $11-0421-107-S$ & $>87.4$ & $<12.6^{(\mathrm{c})}$ & --- & --- & \\
\hline & TI002-B1-Q & 11-0421-107-D & $>88.4$ & $<11.6^{(\mathrm{c})}$ & --- & --- & NA \\
\hline Matrix spike & TI002-B1-R & 11-0421-107-MS & $>85.9$ & $<14.1^{(\mathrm{c})}$ & --- & --- & \\
\hline \multirow[t]{2}{*}{ KW210-B3 } & TI001-B3-P & $11-0422-107-S$ & $>89.5$ & $<10.5^{(\mathrm{c})}$ & --- & --- & NA \\
\hline & TI001-B3-Q & 11-0422-107-D & $>90.5$ & $<9.5^{(\mathrm{c})}$ & --- & --- & \\
\hline \multicolumn{8}{|l|}{ ASR 8824} \\
\hline LCS & LCS & 11-0792-107-LCS & 34.2 & 65.8 & --- & --- & Pass $^{(a)}$ \\
\hline \multirow[t]{2}{*}{ KW210-A4 } & TI002-A4-R & 11-0967-107-S & $>91.8$ & $<8.2$ & --- & --- & NA \\
\hline & TI002-A4-S & 11-0967-107-D & $>92.1$ & $<7.9$ & --- & --- & \\
\hline \multicolumn{8}{|c|}{$\begin{array}{l}\text { (a) The LCS requirement per procedure is } \pm 5 \% \text { (absolute) of the nominal from the standard to pass. The standard value for } \\
\mathrm{U}(\mathrm{IV}) \text { is } 33.3 \% \text {, allowing for the LCS to fall between } 28.3 \% \text { and } 38.3 \% \text {. The standard value for } \mathrm{U}(\mathrm{VI}) \text { is } 66.7 \% \text {, allowing } \\
\text { the LCS to fall between } 61.7 \% \text { and } 71.7 \% \text {. }\end{array}$} \\
\hline \multicolumn{8}{|c|}{$\begin{array}{l}\text { (b) The QAPjP/SAP requires that the sample and all duplicates shall agree within } \pm 5 \% \text { of the average when the } \mathrm{U} \text { concentration } \\
\text { of each oxidation state is greater than } 0.001 \mathrm{M} \text {. }\end{array}$} \\
\hline \multicolumn{8}{|c|}{ (c) The U(VI) concentration measured by spectrophotometry were $<0.001 \mathrm{M}$. } \\
\hline \multicolumn{8}{|c|}{$\begin{array}{l}\text { The U oxidation state ratios for } \mathrm{K} \text { Basin sludge samples should be used with caution. Ferrous iron (Fe[II]) present as magnetite } \\
\text { has been observed in } \mathrm{K} \text { Basin sludge, and has been shown to reduce } \mathrm{U}(\mathrm{VI}) \text { to } \mathrm{U}(\mathrm{IV}) \text { in } \mathrm{Na}_{2} \mathrm{SO}_{4} / \mathrm{H}_{3} \mathrm{PO}_{4} \text { solutions. The U(VI) can } \\
\text { be considered a lower bounding case and the U(IV) fraction the upper bounding case. }\end{array}$} \\
\hline \multicolumn{8}{|c|}{$\begin{array}{l}\text { NA: Not Applicable. The RPD calculation of duplicate samples is not performed when the U concentration of one oxidation } \\
\text { state is below detection. }\end{array}$} \\
\hline \multicolumn{8}{|c|}{ Analytical results from ASR 8798 are found in Appendix B and the results for ASR 8824 are found in Appendix C. } \\
\hline
\end{tabular}

Table 4.19 contains the $\mathrm{U}$ metal concentration results and QC sample results for assessment of precision (RPD) and accuracy (LCS, MS sample, PB samples). Because the A4 core duplicate pair sample results varied significantly (RPD $=54 \%$ ), two reserve A4 core subsamples were processed for $\mathrm{U}$ metal according to ASR 8824. In the second analysis, the precision met acceptance criterion (4\% RPD) and the duplicate results were between the results of the duplicate pair of the initial analyses. The uncertainty for $\mathrm{U}$ metal concentrations $>0.03 \mathrm{wt} \%$ is estimated to be $\pm 25 \%(2-\sigma)$ or less.

The $\mathrm{U}$ measured in the solids $7^{\text {th }}$ rinse solution (before nitric acid dissolution of $U$ metal) ranged from 0.08 to $0.81 \mu \mathrm{g} / \mathrm{mL}$, well below the $100-\mu \mathrm{g} / \mathrm{mL}$ acceptance threshold, indicating the measured $\mathrm{U}$ metal was not confounded by carryover of non-metallic U. The PBs contained no or minute amounts of $\mathrm{U}(\leq 0.00065 \mathrm{wt} \%)$ indicating that results were not confounded by contamination during processing. The U metal LCS for both batches recovered 102 and 103\%, respectively. The U metal MS sample was not 
required by the QAPjP/SAP and was processed for additional information; sample TI002-B1-R (ASR 8798) was spiked with a U metal bead that recovered 99\%. The quantity of U metal in the sample before the $U$ metal bead was added was back-calculated / estimated by subtracting the quantity of the known U metal spike. Its result was between the results of the duplicate pair but below the $0.011 \mathrm{wt} \%$ MDL.

The A4 core samples contained 4.2 to $4.7 \mathrm{wt} \%$ total $\mathrm{U}$ on a settled mass basis (ICP-OES, Table 4.13). With the A4 core showing an average of $0.79 \mathrm{wt} \% \mathrm{U}$ metal on a settled mass basis (Table 4.19) as determined by results from all four samples, $18 \%$ of the total U exists as U metal (0.79 wt\%/4.5 wt\%).

Table 4.20 summarizes the $\mathrm{U}$ oxidation state measurements performed along with the QC samples. The Hanford sand blanks and the reagent blanks from both batches showed the U(IV) and U(VI) concentrations to be less than $0.001 \mathrm{M}$. The LCS measurements were also within $\pm 5 \%$ of the theoretical oxidation state ratio for $\mathrm{U}_{3} \mathrm{O}_{8}(33.3 \% \mathrm{U}[\mathrm{IV}], 66.7 \% \mathrm{U}[\mathrm{VI}])$, and therefore within the $\pm 25 \%$ allowed deviation in the QAPjP/SAP. All the core samples were found to be overwhelmingly $(>86 \%)$ in the $\mathrm{U}(\mathrm{IV})$ oxidation state. This is supported by the XRD measurements of the $-500-\mu \mathrm{m}$ sieve fraction where crystalline $\mathrm{U}$ phases were present as $\mathrm{UO}_{2}$ and $\mathrm{U}_{4} \mathrm{O}_{9}$ (see Section 5.4). However, these $\mathrm{U}(\mathrm{IV})$ and $\mathrm{U}(\mathrm{VI})$ oxidation state ratios should be used with caution. Ferrous iron (Fe[II]), present as magnetite, has been observed in K Basin sludge (Fiskum et al. 2011b), and has been shown to reduce U(VI) to U(IV) in $\mathrm{Na}_{2} \mathrm{SO}_{4} / \mathrm{H}_{3} \mathrm{PO}_{4}$ solutions (Fiskum et al. 2011a). While the amount of $\mathrm{Fe}(\mathrm{II})$ is not known, the concentration of $\mathrm{Fe}$ in sludge samples is significant enough ( 0.9 to $5 \mathrm{wt} \%$ on a settled sludge mass basis) to cause concern (the possibility of the presence of magnetite is indicated by the qualitative observations of magnetic material in the subject samples as shown in Figure 4.5). The U(VI) can be considered to be the lower bounding case and the U(IV) fraction the upper bounding case.

\subsection{Undissolved Solids}

As discussed in Section 4.3, the UDS (or residual solids) fractions of the analytical core samples following the acid digestion (PNL-ALO-129) were well over $5 \mathrm{wt} \%$ (wet settled mass basis). Per the QAPjP/SAP, UDS $>5 \mathrm{wt} \%$ require GEA and XRD analysis. Figure 4.12 and Figure 4.13 show photographs of the residual solids after being removed from the hot cells. The material contains a variety of particle types: resin beads, sand, and gray platy pieces. The acid digestion procedure (PNL-ALO-129) is not expected to dissolve OIER or sand (quartz). 


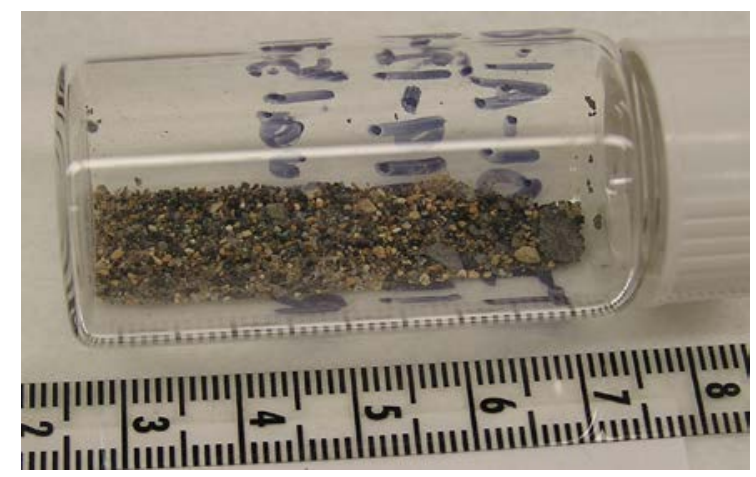

TI001-A1-B

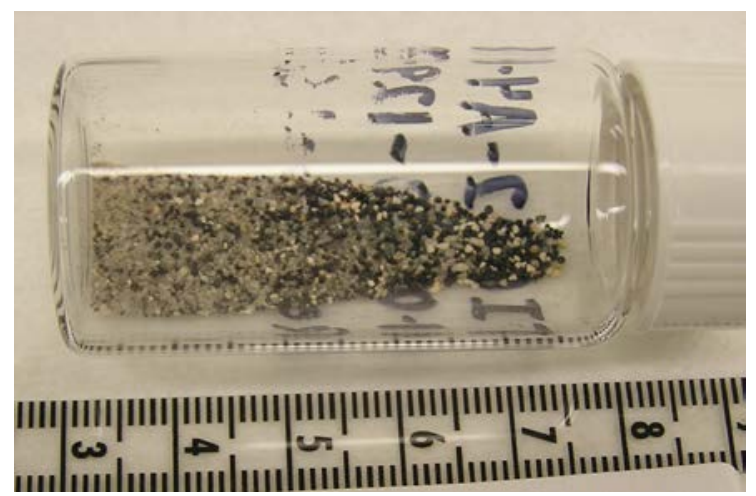

TI002-A4-B

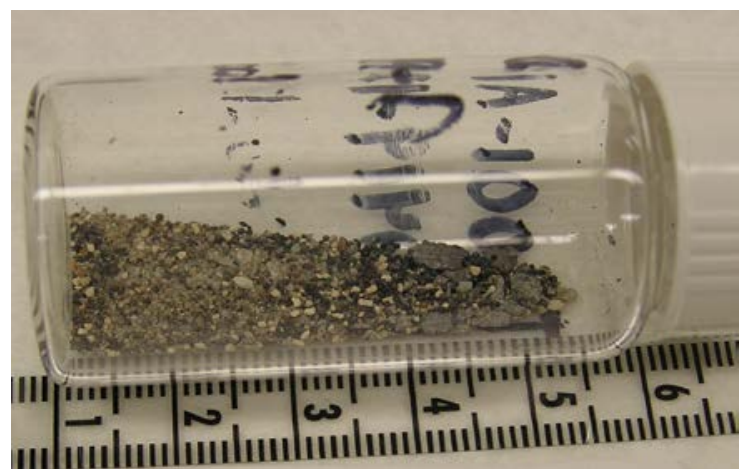

TI001-A1-C

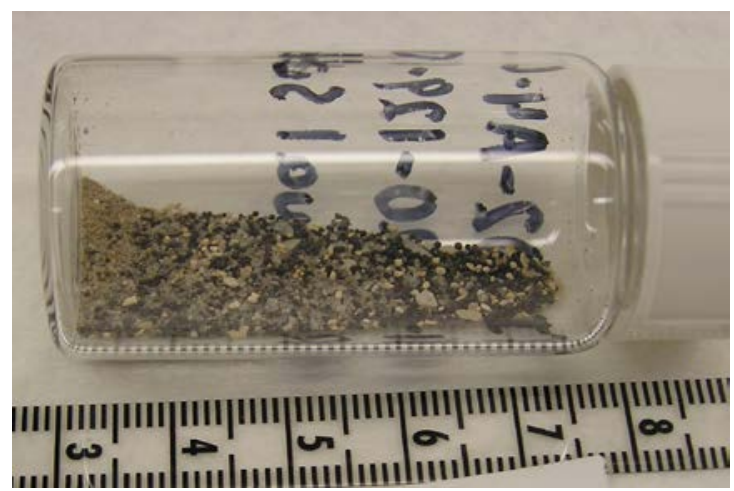

TI002-A4-C

Note: The ruler scale is a centimeter scale with millimeter subdivisions.

Figure 4.12. Photographs of UDS from the KW210-A Core Subsamples (Core A1 on top; Core A4 on bottom) 


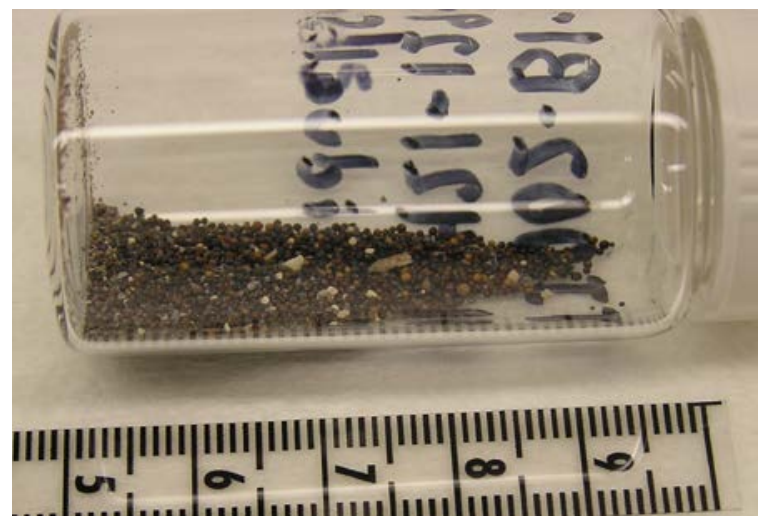

TI002-B1-B

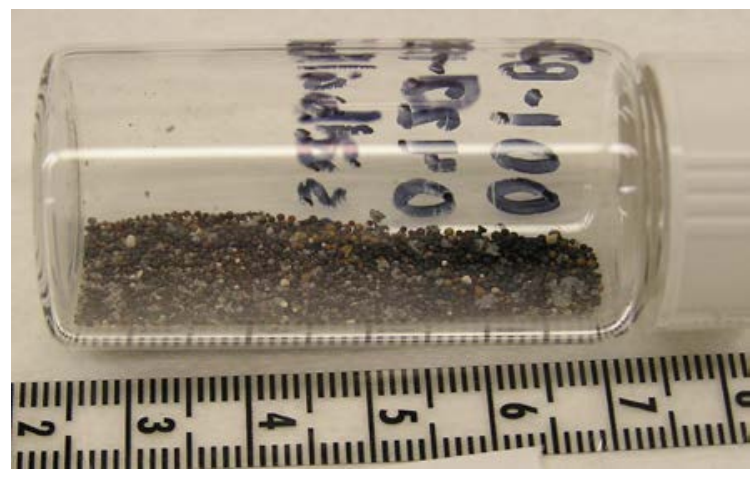

TI001-B3-B

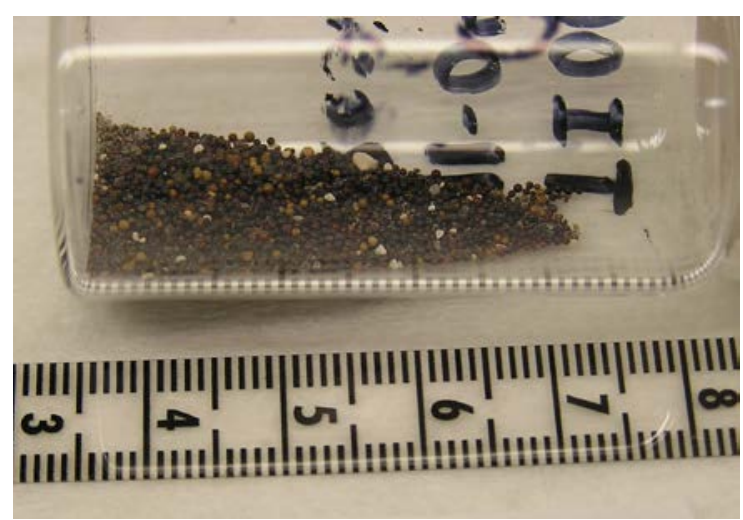

TI002-B1-C

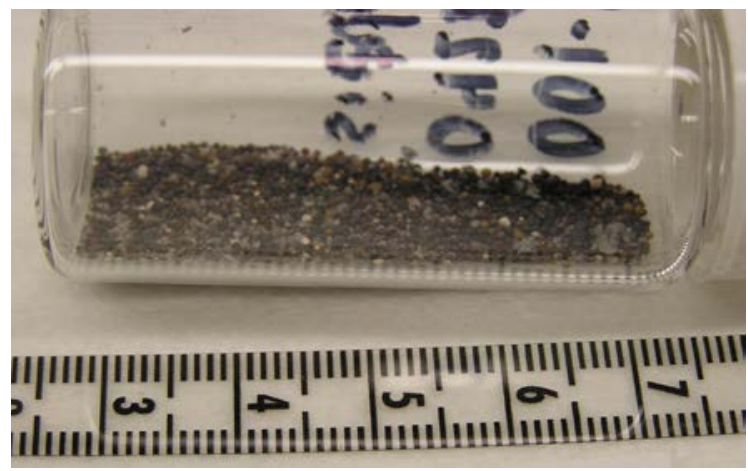

TI001-B3-C

Note: The ruler scale is a centimeter scale with millimeter subdivisions.

Figure 4.13. Photographs of UDS from the KW210-B Core Subsamples (Core B1 on top; Core B3 on bottom)

The UDS were measured directly by GEA according to ASR 8798 and Special Instructions to assess fractionation of ${ }^{137} \mathrm{Cs},{ }^{154} \mathrm{Eu}$, and ${ }^{241} \mathrm{Am}$ between digestate and solids. Table 4.21 shows the analyte concentrations. The UDS ${ }^{134} \mathrm{Cs},{ }^{152} \mathrm{Eu}$, and ${ }^{155} \mathrm{Eu}$ concentrations were near or below the MDL and were not further evaluated. Table 4.21 also shows the fractionation of detected analytes between the aqueous digestates and UDS. Analyte fractionation to the UDS was calculated according to Equation 4.1.

$$
X_{U D S}=\frac{\left(C_{U D S} \times g_{U D S}\right)}{\left[\left(C_{U D S} \times g_{U D S}\right)+\left(C_{S} \times g_{S}\right)\right]}
$$

where $\quad \mathrm{X}_{\mathrm{UDS}}=$ fraction of analyte not dissolved from acid digestion

$\mathrm{C}_{\mathrm{s}}=$ concentration of analyte in the acid digestate

$\mathrm{C}_{\text {UDS }}=$ concentration of analyte in UDS fraction (post-acid digestion)

guDS $=$ mass of UDS (post-acid digestion)

$\mathrm{g}_{\mathrm{s}}=$ mass of dry solids in aliquot before digestion (pre-digest dry mass). 
Table 4.21. GEA Analysis of UDS and Analyte Fractionation to the UDS (ASR 8798)

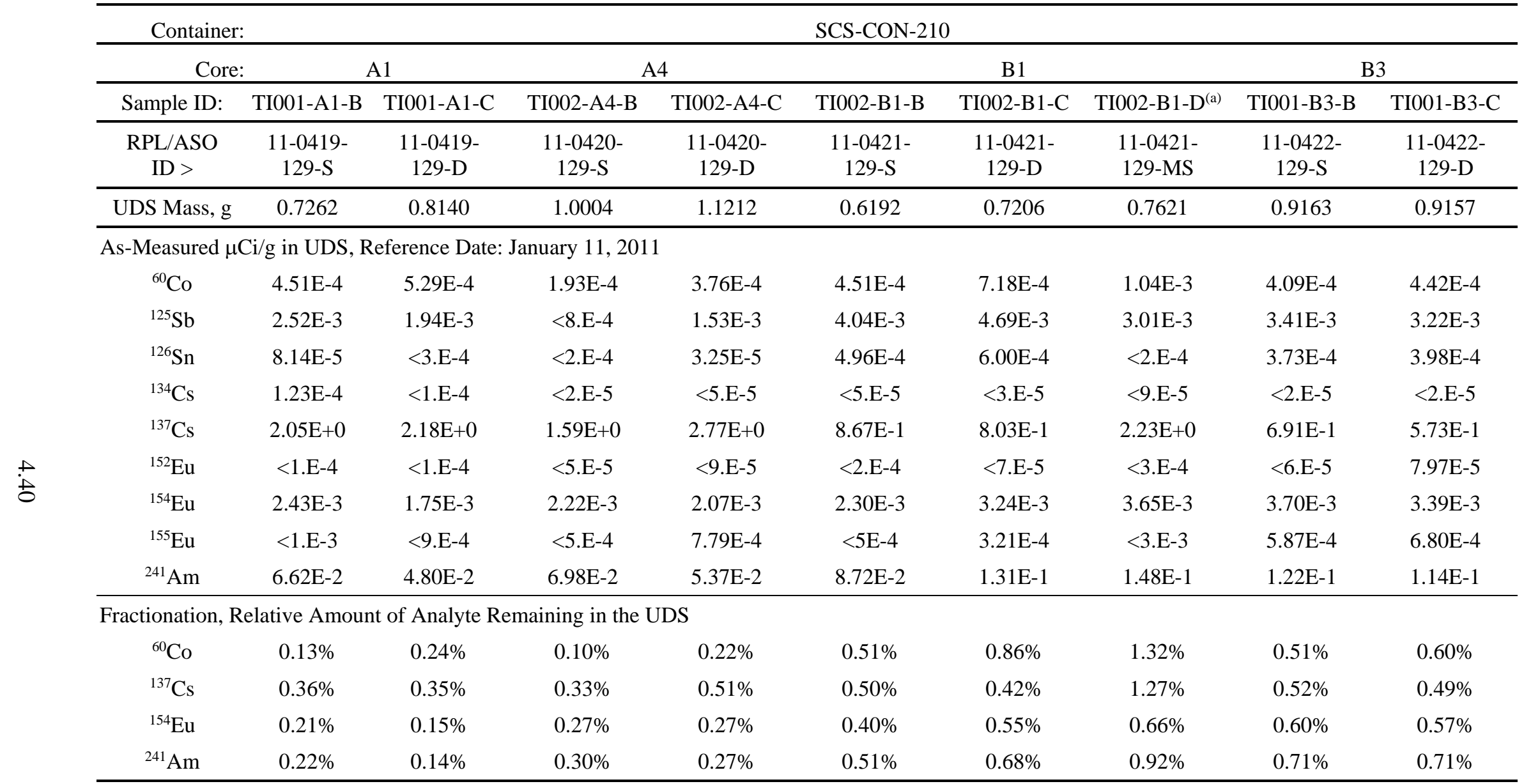

(a) Sample TI002-B1-D was prepared as a MS for ICP-OES analysis only; the GEA results are provided for additional information.

Calculation spreadsheets using Microsoft Excel are provided in data package DVD-52578-PNNL-20650 Rev. 2, Section III, September 2013. Analytical results and the 1- $\sigma$ uncertainty from ASR 8798 are found in Appendix B. 
Overall, the GEA measurements showed that the UDS in the sample and duplicate sample contained less than $1 \%$ of the total activity when compared to the dissolved fraction of the parent samples. Each analyte $\left({ }^{60} \mathrm{Co},{ }^{137} \mathrm{Cs},{ }^{154} \mathrm{Eu}\right.$, and $\left.{ }^{241} \mathrm{Am}\right)$ averaged $0.4 \%$ fractionation to the UDS. The ICP-OES MS sample (TI002-B1-D) results are shown for additional information; the ${ }^{60} \mathrm{Co}$ and ${ }^{137} \mathrm{Cs}$ analyte fractionation to the UDS slightly exceeded $1 \%$ in this sample.

Figure 4.14 compares the GEA-measured analyte fractionation (Table 4.21) in the UDS. The chart demonstrates that the initial analytical digestion succeeded in dissolving the significant fraction of the ${ }^{137} \mathrm{Cs}$, ${ }^{154} \mathrm{Eu}$, and ${ }^{241} \mathrm{Am}$ and that slightly higher fractions of ${ }^{154} \mathrm{Eu}$ and ${ }^{241} \mathrm{Am}$ were retained in the "B" cores UDS samples relative to the "A" core UDS samples. The slightly higher fractions of ${ }^{137} \mathrm{Cs}$ and ${ }^{241} \mathrm{Am}$ in sample TI002-B1-D may result from slight differences in the acid digestate chemistry associated with the addition of the ICP-OES MS analytes. The altered acid digestate matrix may have slightly altered the OIER affinity for specific (cationic and anionic) analytes. This behavior is discussed in more detail for the remainder of this section.

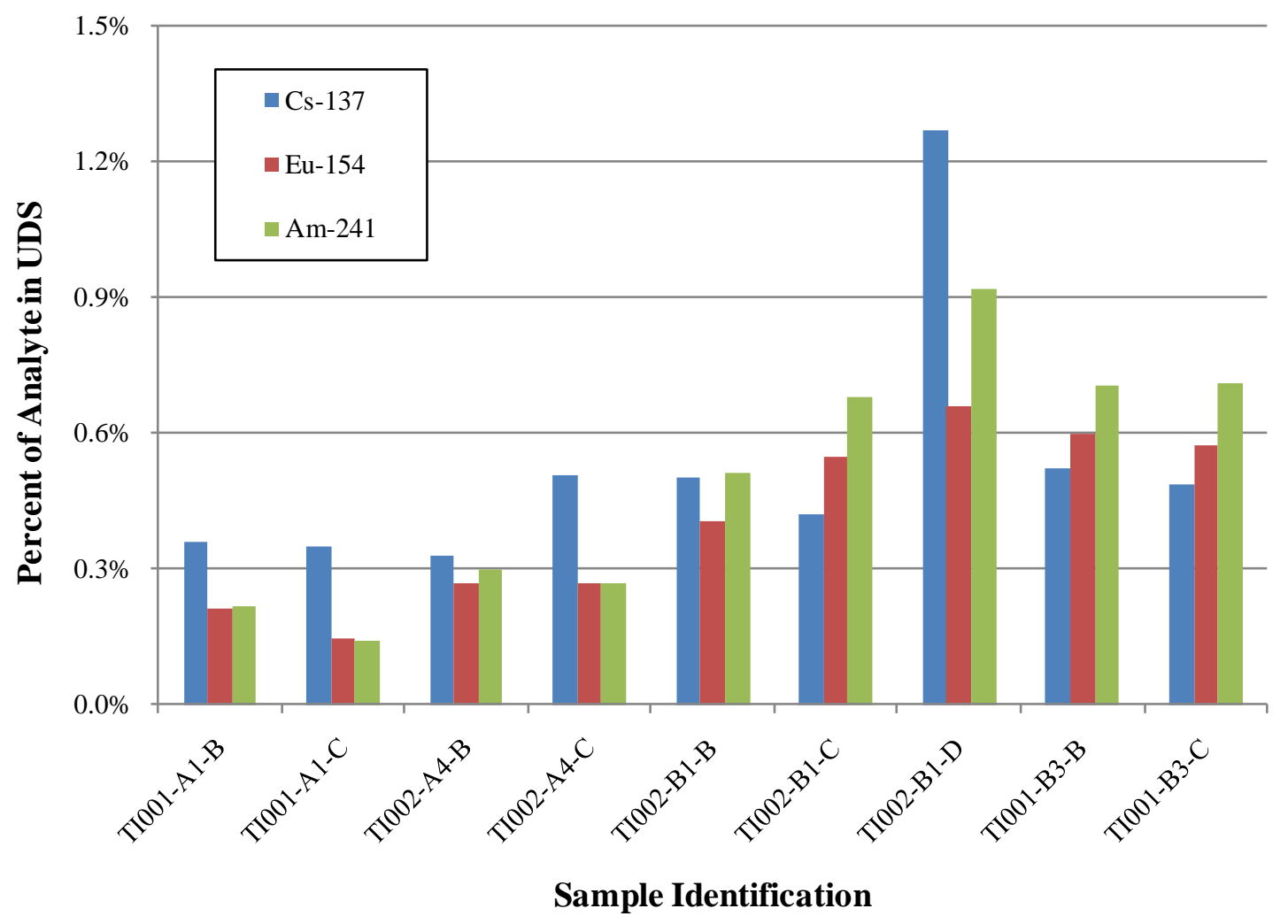

Figure 4.14. Percent of Analyte Remaining in the UDS Measured by GEA Following Acid Digestion for SCS-CON-210 Reconstituted Core Samples

In addition to XRD analysis, the BTR directed fusion dissolution of the UDS followed by ICP-OES, gross alpha, and AEA screen. To prepare for these analyses, the UDS were subsampled using the Y-splitter in a cascade (see Figure 2.12) to create small subsamples appropriate for fusion and samples appropriate for XRD.

The sample splits collected for XRD were phase-separated by panning off the rolling resin beads into a vial separate from the non-rolling/non-resin material. The rolling particles were the spherical-shaped 
OIER. The intention of removing the OIER was two-fold: reduce the confounding effects of an amorphous background in the powder diffraction pattern and obtain mass fraction data from the resin and non-resin components splits. Both the OIER and the non-OIER fractions were weighed. The split was not perfect; some cross-contamination between material types was observed. However general trends in the approximate mass distribution were recorded. The approximate OIER mass fractions in the UDS samples are provided in Table 4.22. The calculated OIER mass fraction in the dry sample (before acid digestion) was calculated according to Equation 4.2 and is also provided in Table 4.22. This calculation approach assumes no resin mass was lost during the acid digestion processing.

$$
\frac{\mathrm{M}_{\mathrm{UDS}} \times\left[\frac{\mathrm{m}_{\mathrm{OIER}}}{\mathrm{m}_{\mathrm{UDS}}}\right]}{\mathrm{M}_{\mathrm{S}}}=\mathrm{SF}_{\mathrm{OIER}}
$$

$$
\begin{aligned}
\text { Where } \mathrm{M}_{\text {UDS }} & =\text { Mass of UDS in the analytical sample } \\
\mathrm{m}_{\text {OIER }} & =\text { Mass of OIER in the fractionated subsample } \\
\mathrm{m}_{\text {UDS }} & =\text { Mass of UDS in the fractionated subsample (OIER plus non-OIER) } \\
\mathrm{M}_{\mathrm{S}} & =\text { Mass of dry parent sample before acid digestion } \\
\text { SF }_{\text {OIER }} & =\text { Mass fraction of the OIER in the dry parent sample before acid digestion }
\end{aligned}
$$

Table 4.22. Dry Mass Fraction of OIER in the Analytical Core Samples

\begin{tabular}{cccccccc}
\hline & & \multicolumn{2}{c}{$\begin{array}{c}\text { CIER in UDS } \\
\text { Core }\end{array}$} & \multicolumn{2}{c}{$\begin{array}{c}\text { Calculated OIER in } \\
\text { Dry Parent Sample }\end{array}$ (a) } & \multicolumn{2}{c}{$\begin{array}{c}\text { Calculated OIER Based on } \\
\text { Measured TOC }\end{array}$} \\
\cline { 3 - 8 } Sample & RPL/ASO ID & wt \% & RPD & wt \% & RPD & RPL/ASO ID & wt\% \\
\hline A1 & $11-419-129-S$ & 33.7 & & 22 & & $11-0419-C-S$ & 21 \\
& $11-419-129-D$ & 31.8 & 5.9 & 20 & 7.4 & $11-0419-C-D$ & 23 \\
\hline A4 & $11-420-129-S$ & 26.3 & & 18 & & $11-0420-C-S$ & 12 \\
& $11-420-129-D$ & 24.7 & 6.0 & 17 & 6.1 & $11-0420-C-D$ & 14 \\
\hline B1 & $11-421-129-S$ & 87.1 & & 72 & & $11-0421-C-S$ & 76 \\
& $11-421-129-D$ & 84.9 & 2.5 & 75 & 3.3 & $11-0421-C-D$ & 73 \\
\hline B3 & $11-422-129-S$ & 56.4 & & 49 & & $11-0422-C-S$ & 56 \\
& $11-422-129-D$ & 57.0 & 1.0 & 50 & 2.0 & $11-0422-C-D$ & 55 \\
\hline
\end{tabular}

(a) Mass fractions should be used with caution because the fractionation of OIER from non-OIER was not perfect. See text for discussion.

The OIER mass fraction results were compared to the TOC concentrations measured using furnace combustion. The weight percent of OIER was calculated from the TOC results according to Equation 4.3:

$$
\frac{\mathrm{g}_{\text {TOC }}}{\mathrm{g}_{\text {sample }}} \times \frac{\mathrm{g}_{\text {Purolite }}}{0.672 \mathrm{~g}_{\text {TOC }}}=\mathrm{wt} \% \text { OIER }
$$

where: $\mathrm{g}_{\mathrm{TOC}} / \mathrm{g}_{\text {sample }}=$ ASO-reported TOC concentration

g $_{\text {Purolite }} / 0.672 \mathrm{~g}_{\text {TOC }}=$ Reciprocal of the TOC content in Purolite NRW37 ion exchange resin 
The calculation assumes all TOC is from OIER and consistent with the Purolite NRW37 carbon content of $67.2 \mathrm{wt} \%$ (Fiskum et al. 2011a). The result summary is provided in Table 4.22. Generally the wt\% OIER measured from separation of the resin from the non-resin material agreed well with the wt $\%$ OIER calculated from TOC. The sample sizes for the direct mass measures ranged from 0.23 to $0.53 \mathrm{~g}$ and were much greater than the sample sizes for TOC, which ranged from 0.0035 to $0.065 \mathrm{~g}$.

One non-OIER solid sample from each core was submitted for XRD analysis. Figure 4.15 presents a summary of the powder diffractions for all samples along with the stick-figure standard crystalline phases. Table 4.23 summarizes the crystalline phases identified in the UDS along with the sample progenitor IDs, ICDD card numbers, and relative intensities. The UDS samples primarily consisted of quartz and anorthite as major crystalline phases. Some of these samples also contained as minor phases aegerine and calcium sulfate. No other crystalline components were identified; all reflections were identified. The UDS did not contain oxyhydroxides, hydrates and hydroxides, aluminum oxides, oxyhydroxides and hydrates, or zirconium metal and zirconia (from cladding materials) as crystalline phases.

The UDS subsample splits (containing OIER) from all eight core subsamples were further split into small representative aliquots applicable for $\mathrm{KOH} / \mathrm{KNO}_{3}$ fusion (0.1 to $0.2 \mathrm{~g}$ ). After fusion the digestates were split. One split was analyzed by ICP-OES for AOIs Al, Ca, Fe, S, Si, U, and Zr; the other split was analyzed for gross alpha and AEA screen for ${ }^{239+240} \mathrm{Pu}$ measurement. The fusion and analyses were conducted according to ASR 8878 and Special Instructions (Appendix D). ${ }^{\text {(a) }}$ This analysis was supplemental scope to that defined in the QAPjP/SAP. All process QC samples met the ASR-delineated requirements. The LCS was prepared using NIST SRM 2710 Montana Soil and recovery values ranged from 95 to 107\% for Al, Ca, Fe, and Si. The core sample/sample duplicate analyte results were within $18 \%$ RPD, and analytes in the PB were $<$ MDL. See Appendix D for details and measurement uncertainties.

Table 4.24 provides the AOI results and their relative mass and activity fractionations to the UDS (results of ICP-OES opportunistic analytes and gross alpha can be found in Appendix D). Analyte fractionation to the UDS was calculated according to Equation 4.1 where CuDs was determined from the analysis of the fused sample. The pre-digest dry mass (gs) and UDS mass (guDs) values are reported in Table 4.2 and the concentrations of analytes in the acid digestate $\left(\mathrm{C}_{S}\right)$ are reported in Table 4.10. Figure 4.16 compares Fe, U, and Pu fractionations in the UDS for each core sample. The chart vividly shows the large step change in retention for these analytes in the "B" core sample UDS, which also had the highest calculated dry wt\% OIER as shown in Table 4.22.

(a) The UDS associated with the container composite $-2,000-\mu \mathrm{m} /+500-\mu \mathrm{m}$ and $-500-\mu \mathrm{m}$ sieve fraction samples were processed as part of ASR 8878. Core sample 11-0420-129-D and container composite SCC sample 11-793-129-D were split and run as batch duplicates. All process QC samples were shared between the core and container composite samples. 


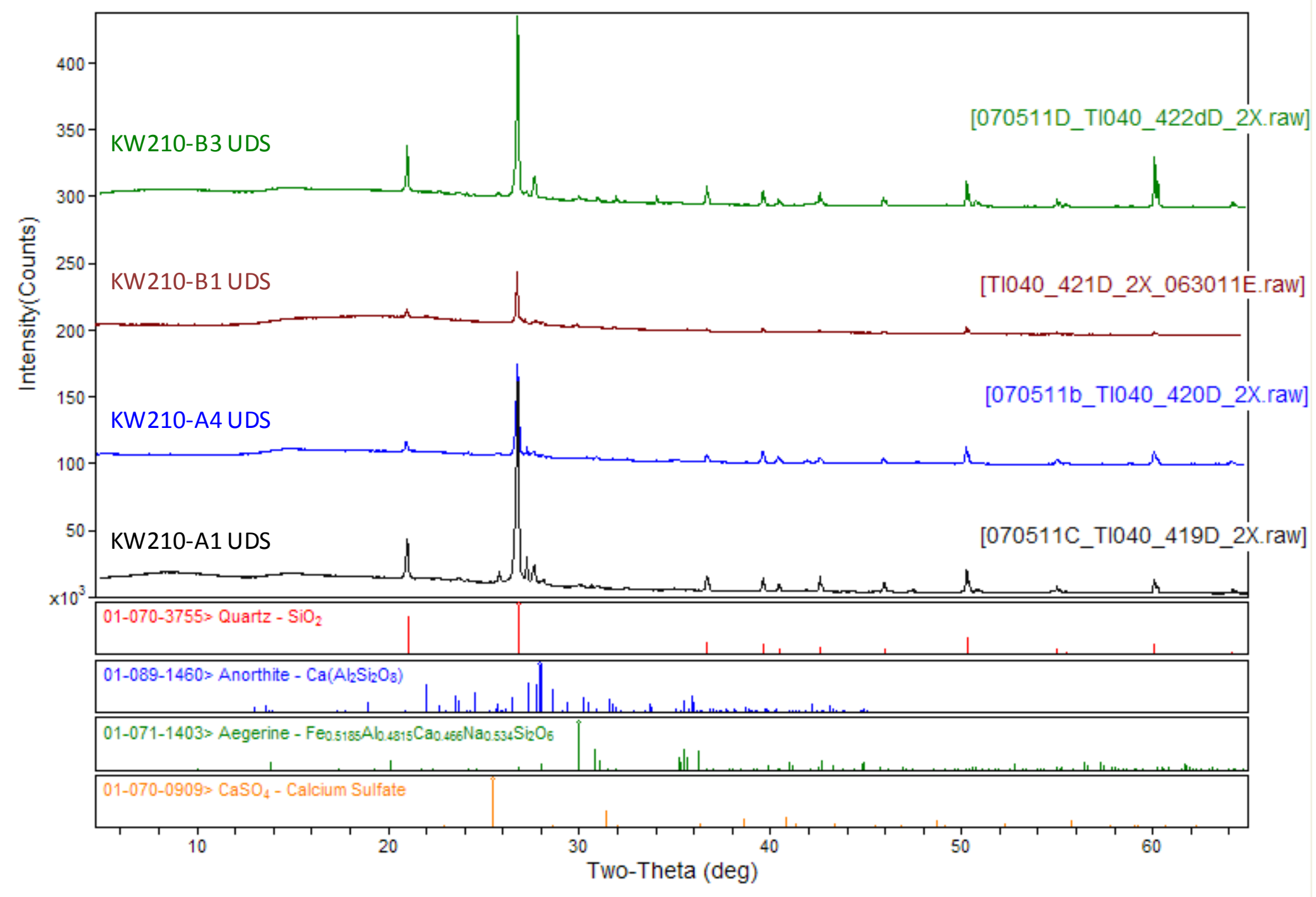

Note: Patterns were not background-subtracted.

Figure 4.15. Raw X-Ray Powder Diffraction Patterns for the KW210 UDS Samples 
Table 4.23. Summary of Phases Found via X-Ray Powder Diffraction on UDS Following Acid Digestion and Bulk OIER Removal from SCS-CON-210 Core Samples

\begin{tabular}{|c|c|c|c|c|c|}
\hline $\begin{array}{c}\text { XRD } \\
\text { Sample ID }\end{array}$ & $\begin{array}{l}\text { RPL/ASO Parent ID } \\
\text { (TI Parent ID, } \\
\text { Core ID) }\end{array}$ & $\begin{array}{c}\text { ICDD } \\
\text { Card Number }\end{array}$ & $\begin{array}{c}\text { Relative } \\
\text { Intensity }^{(a)}\end{array}$ & Formula & $\begin{array}{c}\text { Common } \\
\text { Phase Name }\end{array}$ \\
\hline \multirow[t]{2}{*}{ TI040-419D-2x } & $11-0419-129-D$ & 01-070-3755 & Major & $\mathrm{SiO}_{2}$ & Quartz \\
\hline & $\begin{array}{c}\text { (TI001-A1-C, } \\
\text { KW210-A1) }\end{array}$ & $01-089-1460$ & Major & $\mathrm{Ca}\left(\mathrm{Al}_{2} \mathrm{Si}_{2} \mathrm{O}_{8}\right)$ & Anorthite \\
\hline \multirow[t]{4}{*}{ TI040-420D-2x } & \multirow{4}{*}{$\begin{array}{c}\text { 11-0420-129-D } \\
\text { (TI002-A4-C, } \\
\text { KW210-A4) }\end{array}$} & 01-070-3755 & Major & $\mathrm{SiO}_{2}$ & Quartz \\
\hline & & 01-089-1460 & Major & $\mathrm{Ca}\left(\mathrm{Al}_{2} \mathrm{Si}_{2} \mathrm{O}_{8}\right)$ & Anorthite \\
\hline & & 01-071-1403 & Minor & $\mathrm{Ca}_{0.466} \mathrm{Fe}_{0.5185} \mathrm{Al}_{0.815} \mathrm{Na}_{0.534} \mathrm{O}_{6} \mathrm{Si}_{2}$ & Aegerine \\
\hline & & 01-070-0909 & Minor & $\mathrm{CaSO}_{4}$ & Calcium sulfate \\
\hline \multirow[t]{2}{*}{ TI040-421D-2x } & \multirow{2}{*}{$\begin{array}{c}\text { 11-0421-129-D } \\
\text { (TI002-B1-C, } \\
\text { KW210-B1) }\end{array}$} & 01-070-3755 & Major & $\mathrm{SiO}_{2}$ & Quartz \\
\hline & & 01-089-1460 & Major & $\mathrm{Ca}\left(\mathrm{Al}_{2} \mathrm{Si}_{2} \mathrm{O}_{8}\right)$ & Anorthite \\
\hline \multirow[t]{3}{*}{ TI040-422D-2x } & \multirow{3}{*}{$\begin{array}{l}\text { 11-0422-129-D } \\
\text { (TI001-B3-C, } \\
\text { KW210-B3) }\end{array}$} & 01-070-3755 & Major & $\mathrm{SiO}_{2}$ & Quartz \\
\hline & & 01-089-1460 & Major & $\mathrm{Ca}\left(\mathrm{Al}_{2} \mathrm{Si}_{2} \mathrm{O}_{8}\right)$ & Anorthite \\
\hline & & 01-071-1403 & Minor & $\mathrm{Ca}_{0.466} \mathrm{Fe}_{0.5185} \mathrm{Al}_{0.815} \mathrm{Na}_{0.534} \mathrm{O}_{6} \mathrm{Si}_{2}$ & Aegerine \\
\hline
\end{tabular}


Table 4.24. Analytes in UDS Fusion, Dry UDS Mass Basis (ASR 8878)

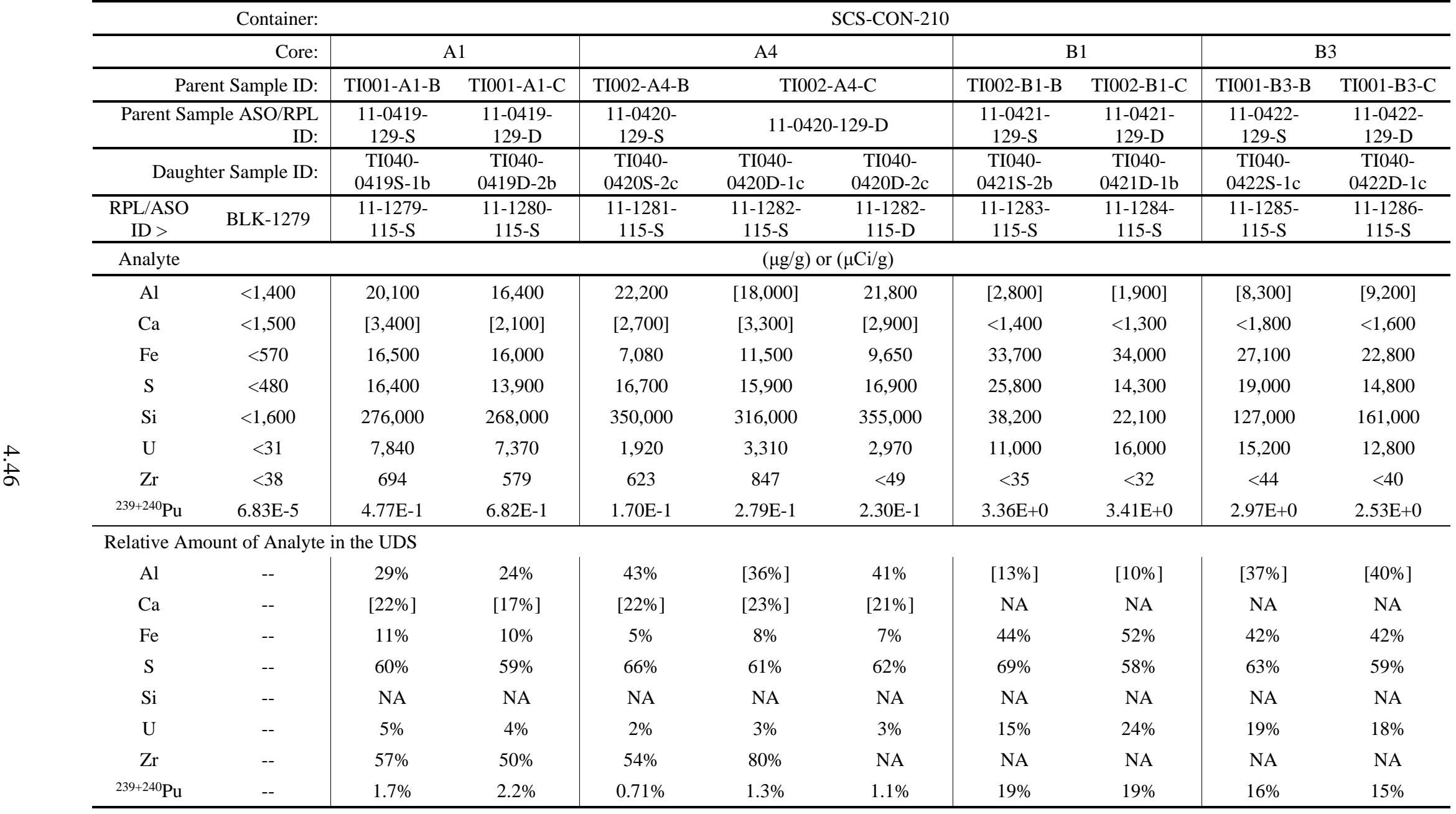

Analyte results shown in brackets indicate that the analyte concentrations were greater than the MDL but less than the EQL. Less-than values represent the instrument detection limit multiplied by the process factors. See Appendix D for measurement uncertainties.

Percent values in brackets indicate one of the input parameters was $<$ EQL but $>$ MDL.

NA: Not Applicable; an input factor was $<$ MDL or, in the case of Si, the analyte was not expected to dissolve in the PNL-ALO-129 acid digestion. 
PNNL-20650, Rev. 2

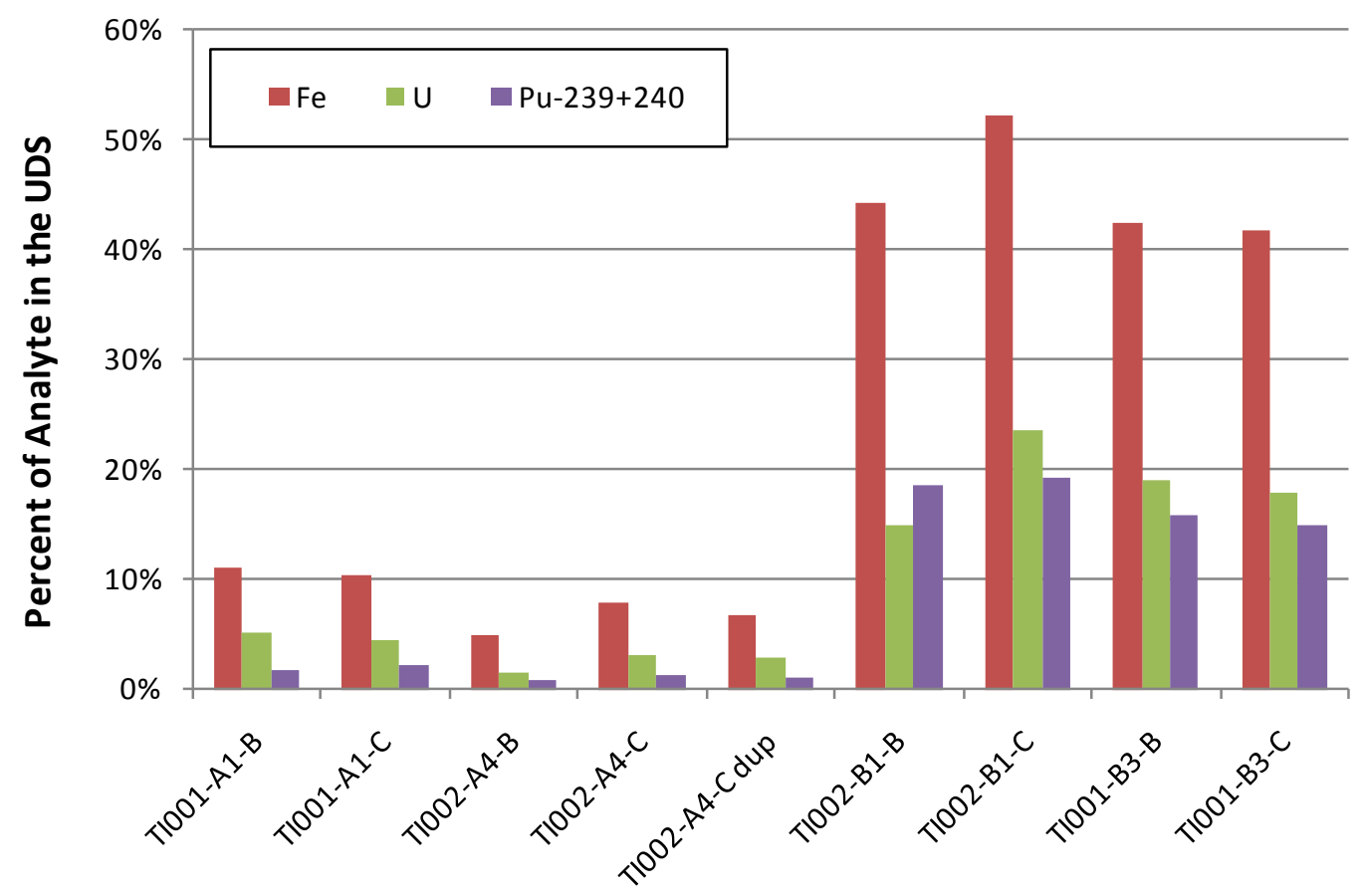

Sample Identification

Figure 4.16. Percent of Analyte Remaining in the UDS Measured by ICP-OES and AEA Screen in the SCS-CON-210 Reconstituted Core Samples

Figure 4.17 graphically presents the $\mathrm{Al}, \mathrm{Fe}, \mathrm{S}, \mathrm{U}$ and Pu retention in the UDS as a function of the OIER content in the dry parent sample (before subjection to PNL-ALO-129 acid digestion, see Table 4.22). The UDS contained $\sim 50$ to $60 \%$ of the total S content; no partitioning trend was observed as a function of OIER fraction in the sample. The $S$ is postulated to come from the sulfonic acid functional group in the cation resin where only a percentage of the $S$ was amendable to acid dissolution. Aluminum did not show a relationship to the OIER content; it appears to be a "spectator" species with respect to OIER content and exchange. The UDS aluminum is probably associated with anorthite and aegerine (as identified by XRD, see Table 4.23). Iron is present in aegerine, which was found as a minor crystalline phase in A4 and B3 core samples. However, a direct relationship appears to exist between Fe in the UDS and OIER content. Uranium phases in the UDS were not identified by XRD; however, up to $24 \%$ of the U content was found in the "B" core sample UDS. The Pu was similarly higher in the "B" core sample UDS, which contained up to $19 \%$ of the Pu content.

The direct relationship between the OIER content in the parent sample material to Fe, $\mathrm{U}$, and $\mathrm{Pu}$ retention in the UDS suggests that analyte anionic or cationic species exchanged onto the OIER during the PNL-ALO-129 acid digestion and/or solids rinse steps. Similarly, analytes exchanging onto OIER were reported previously with increases in Fe and ${ }^{239+240} \mathrm{Pu}$ concentrations by factors of 17 and 290, respectively, when a $\mathrm{K}$ Basin OIER (NRW-37) was exposed to a $6 \mathrm{M} \mathrm{HNO}_{3}$ dissolver solution at $95^{\circ} \mathrm{C}$ for $7 \mathrm{hr}$ (Schmidt et al 1999). In this same 1999 study, the U concentration increased by only a factor of 1.07. In contrast, the present work (where $8 \mathrm{M} \mathrm{HNO}_{3}$ combined with equal volume of $8 \mathrm{M} \mathrm{HCl}$ was used) $\mathrm{U}$ retention in UDS tracked well with the ${ }^{239+240} \mathrm{Pu}$ retention and Fe retention was greater than $\mathrm{Pu}$ retention (see Figure 4.17). Acid type and concentration are known to affect the affinity of specific (cationic and anionic) analytes onto OIER (Coleman 1965). The fraction of Fe, U, and ${ }^{239+240} \mathrm{Pu}$ in the 
OIER presented in Table 4.24 likely does not represent the analyte fractions in the sludge OIER currently contained in SCS-CON-210 because these analytes were not dissolved in-situ and the contacting medium is essentially $\mathrm{pH} 7$ water (not acidic). Additional analyses of undigested OIER would be required to confirm this assessment.

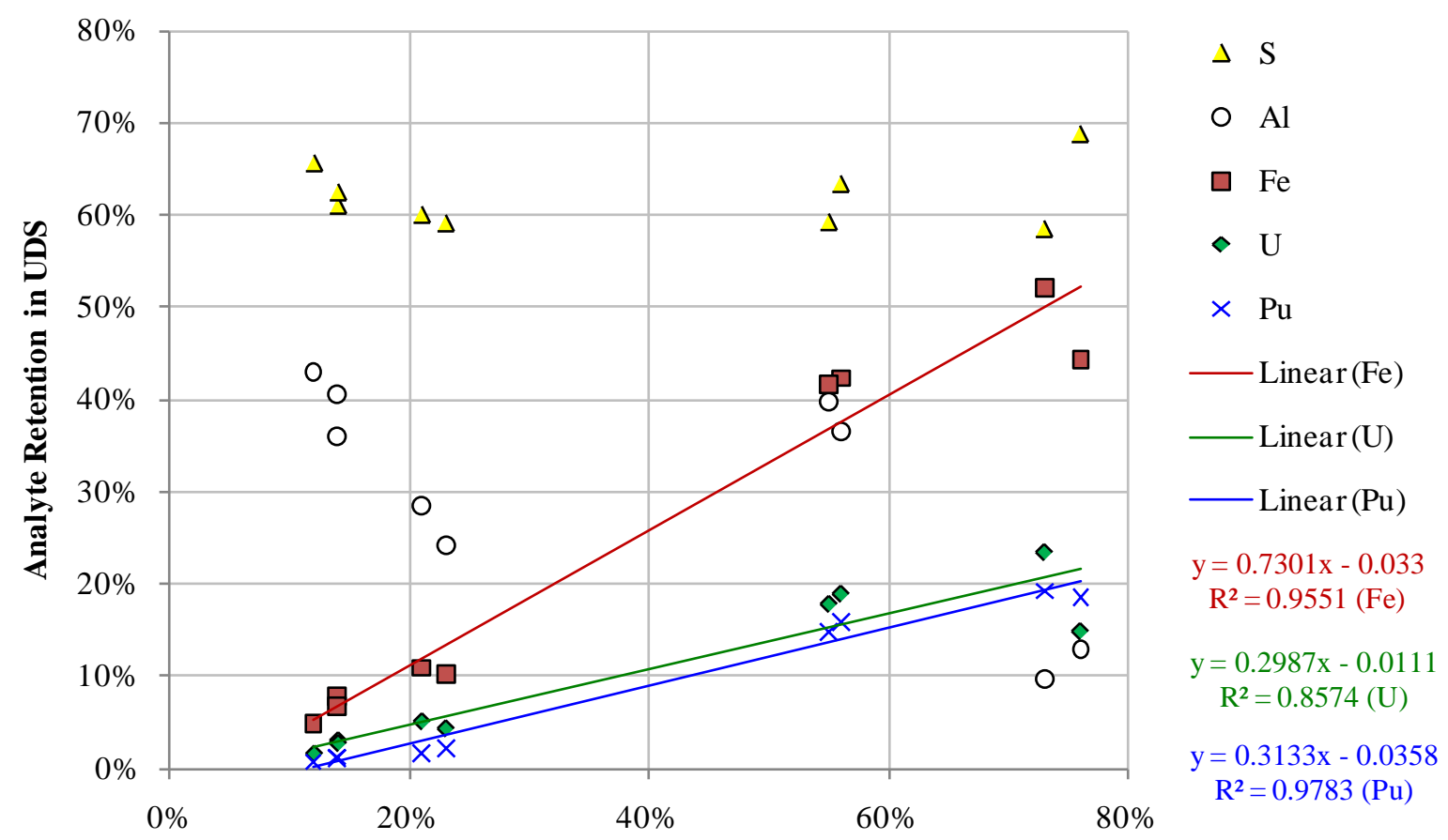

Weight Fraction OIER in the Analytical Subsample

- $\quad P u, U$, and Fe increased in the UDS as OIER content increased in the parent analytical sample.

- Al and $S$ in the UDS showed no relationship to increasing OIER content.

- Linear trend lines are provided for illustrative purposes and do not attempt to provide a model fit.

Figure 4.17. Relationship of Al, Fe, S, U, and Pu to OIER in the Parent Analytical Subsample

The relative mass fractions of $\mathrm{Pu}, \mathrm{U}$, and Fe in the UDS versus the acid digestate were compared. This required that the $\mathrm{Pu}, \mathrm{U}$, and Fe concentrations in the UDS be re-normalized to the initial dry mass basis of each core sample and duplicate. The re-normalization was conducted according to Equation 4.4

$$
\mathrm{Ci}=\mathrm{Ci} i_{\mathrm{UDS}} \times\left(\frac{\mathrm{g}_{\text {residual }}}{\mathrm{g}_{\mathrm{dry}}}\right)
$$

where $\mathrm{Ci}=$ Concentration of UDS analyte $i$ in the original dry analytical sample $\left(\mu \mathrm{Ci} / \mathrm{g}_{\mathrm{dry}}\right)$

$\mathrm{Ci}_{\mathrm{UDS}}=$ Concentration of analyte $i$ in the UDS ( $\left.\mu \mathrm{Ci} / \mathrm{g}_{\text {UDS }}\right)$

$\frac{\mathrm{g}_{\text {residual }}}{\mathrm{g}_{\text {dry }}}=$ UDS weight fraction, see Table 4.2.

Figure 4.18 compares the core sample $\mathrm{Pu}, \mathrm{U}$, and Fe total analyte concentrations in each sample (combined red and blue bars) relative to the analyte concentration that dissolved in the PNL-ALO-129 acid digestion (blue bars) and the normalized analyte concentration that was associated with the UDS (red bars). The concentration is presented on a dry mass basis. The total Pu concentrations did not differ greatly between core samples. The total $U$ and Fe concentrations were higher in the "A" cores than the "B" cores. 
PNNL-20650, Rev. 2
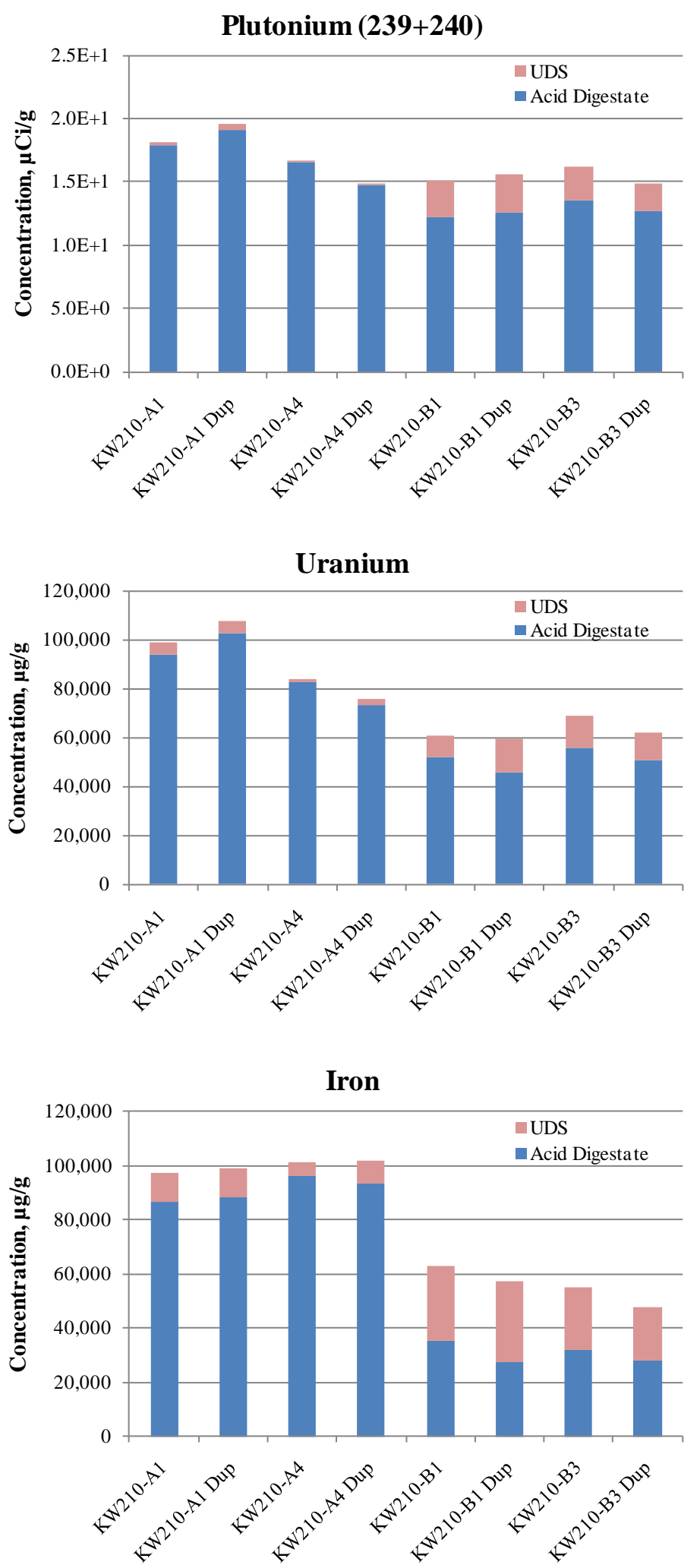

Figure 4.18. Total ${ }^{239+240} \mathrm{Pu}$, U, and Fe Concentrations and Partitions Between Acid Digestate and UDS Phases for Each Core Subsample 


\subsection{SCS-CON-210 Container Composite Samples}

The four reconstituted core samples from Engineered Container SCS-CON-210 were subsampled to support the creation of two independent container composite samples of similar composition. After measuring gross physical properties, the container composite samples were subsampled to collect primary physical property samples. One of the container composite samples was then wet-sieved into three size fractions. Each sieve fraction was characterized to the extent possible, given limitations on sample size. The other container composite was used to measure shear strength. This section details the processing and characterization results from each container composite and sieve fraction. Refer to Section 2.2 for a detailed discussion of the container composite preparation processes.

\subsection{Container Composite Preparations and Observations}

The goal defined in the QAPjP/SAP (KBC-33786, Rev. 2) was to combine equal volumes (within $\pm 10 \%$ per TI direction) of settled sludge from each core sample to prepare the SCS-CON-210 container composites. The settled sludge volume after a 24-hr settling time was used as the comparative volume basis. (It was noted that additional sludge settling occurred after subsamples were transferred to the SAL with three to four weeks of additional settling time. The volume compaction associated with sample movement and extended settling time ranged from $\sim 6$ to $22 \%$.)

Table 5.1 and Table 5.2 summarize the core sample masses (as-sampled) and settled sludge volumes used to create the two container composites. The relative fractions of each core sample in the container composites on both a settled sludge volume basis and a dried sludge mass basis are also provided. After combination of the component core subsamples, the 24-hr settled sludge volumes and the as-sampled sludge masses were measured on each container composite. The measured 24-hr settled volumes and dewatered sludge masses of each container composite show reasonable agreement with the summed settled volumes and masses of the component subsamples.

Table 5.1. SCS-CON-210 Core Subsamples for Container Composite KW210-CC-A

\begin{tabular}{|c|c|c|c|c|c|c|}
\hline Description & $\begin{array}{l}\text { Constituent } \\
\text { Core ID }\end{array}$ & $\begin{array}{l}\text { TI Sample } \\
\text { Tracking ID }\end{array}$ & $\begin{array}{l}\text { As-Sampled } \\
\text { Mass, } \text { g }^{\text {(a) }}\end{array}$ & $\begin{array}{c}\text { Settled } \\
\text { Volume, } \\
\text { mL }^{(a)}\end{array}$ & $\begin{array}{c}\text { Settled } \\
\text { Volume } \\
\text { Fraction, \% }\end{array}$ & $\begin{array}{c}\text { Core Dry } \\
\text { Mass }^{(b)} \\
\text { Fraction, \% }^{2}\end{array}$ \\
\hline \multirow{5}{*}{$\begin{array}{l}\text { Component } \\
\text { Subsamples }\end{array}$} & KW210-A1 & KW210-A1-A (c) & 62.61 & 48 & 24 & 28.0 \\
\hline & KW210-A4 & KW210-A4-A ${ }^{(\mathrm{c})}$ & 74.51 & 57 & 28 & 36.6 \\
\hline & KW210-B1 & KW210-B1-A ${ }^{(\mathrm{c})}$ & 45.45 & 50 & 25 & 15.0 \\
\hline & KW210-B3 & KW210-B3-A ${ }^{(\mathrm{c})}$ & 54.24 & 48 & 24 & 20.5 \\
\hline & --- & Sum & 236.81 & 203 & & \\
\hline $\begin{array}{l}\text { Container } \\
\text { Composite }\end{array}$ & --- & KW210-CC-A & $250.55^{(\mathrm{d})}$ & $220^{(\mathrm{d})}$ & --- & --- \\
\hline
\end{tabular}

(a) The masses and 24-hr settled sludge volumes are from component core composite subsamples before combining them to create the container composite.

(b) The dry mass was calculated from the as-sampled mass and the conversion factors $\mathrm{g}_{\mathrm{dry}} / \mathrm{g}_{\text {sampled }}$ (See Table 3.1).

(c) Inadvertently core subsample IDs were identical to client sample IDs. These samples are different from the samples identically identified in Table 2.1.

(d) Measured volume and mass after combining the core subsamples. 
Table 5.2. SCS-CON-210 Core Subsamples for Container Composite KW210-CC-N

\begin{tabular}{|c|c|c|c|c|c|c|}
\hline Description & $\begin{array}{c}\text { Constituent } \\
\text { Core ID }\end{array}$ & $\begin{array}{c}\text { TI Sample } \\
\text { Tracking ID }\end{array}$ & $\begin{array}{c}\text { As-Sampled } \\
\text { Mass, g }\end{array}$ & $\begin{array}{c}\text { Settled } \\
\text { Volume, } \\
\mathrm{mL}^{(\mathrm{a})}\end{array}$ & $\begin{array}{c}\text { Settled } \\
\text { Volume } \\
\text { Fraction, \% }\end{array}$ & $\begin{array}{c}\text { Core Dry } \\
\text { Mass }^{(b)} \\
\text { Fraction, \% }\end{array}$ \\
\hline \multirow{5}{*}{$\begin{array}{l}\text { Component } \\
\text { Subsamples }\end{array}$} & KW210-A1 & TI001-A1-N & 44.62 & 36 & 26 & 28.1 \\
\hline & KW210-A4 & TI002-A4-N & 49.38 & $29^{(\mathrm{c})}$ & 21 & 34.2 \\
\hline & KW210-B1 & TI002-B1-N & 37.28 & 35 & 26 & 17.3 \\
\hline & KW210-B3 & TI001-B3-N & 38.27 & 36 & 26 & 20.4 \\
\hline & -- & Sum & 169.55 & 136 & & \\
\hline $\begin{array}{l}\text { Container } \\
\text { Composite }\end{array}$ & -- & KW210-CC-N & $178.77^{(\mathrm{d})}$ & $155^{\text {(d) }}$ & -- & --- \\
\hline
\end{tabular}

(a) The masses and 24-hr settled sludge volumes are from component core composite subsamples before combining them to create the container composite.

(b) The dry mass was calculated from the as-sampled mass and the conversion factors $\mathrm{g}_{\mathrm{dry}} / \mathrm{g}_{\text {sampled }}$ (See Table 3.1).

(c) The settled volume is suspected to be too low because the as-sampled volume was recorded at $30.5 \mathrm{~mL}$. Using the primary physical property sample results, the settled volume should be closer to $39 \mathrm{~mL}$ :

$$
49.38 \mathrm{~g}_{\text {sampled }} \times\left(\frac{1 \mathrm{~g}_{\text {settled }}}{0.9032 \mathrm{~g}_{\text {sampled }}}\right) \times\left(\frac{1 \mathrm{~mL}_{\text {settled }}}{1.39 \mathrm{~g}_{\text {settled }}}\right)=39 \mathrm{~mL}
$$

(d) Measured volume and mass after combining the core subsamples.

Ideally the settled sludge volume contributions from the component core subsamples would be $25 \% \pm$ $2.5 \%$. Only one core sample was outside of this range: TI002-A4-N at $21 \%$ volume contribution. The volume basis for this sample is suspected to be significantly too low; applying correction factors from physical property samples to the as-sampled mass results in an estimated 39-mL volume for TI002-A4-N. ${ }^{\text {(a) }}$ If the volume of TI002-A4-N is corrected to $39 \mathrm{~mL}$, the relative volume contribution increases to $27 \%$ with the other cores contributing 24 to 25 vol\%; this better matches the KW210-CC-A composition.

On a dry mass basis, the relative component core subsample contributions showed large variation. The A4 core dry mass contribution to the container composites was the highest followed by A1 core. Both "B" core samples contributed substantially less dry mass to the container composites than the "A" cores. These dry mass variations were consistently reflected in each of the container composites. Figure 5.1 shows graphically how the volume percent settled sludge and mass percent dry sludge for the two container composites compare. The relative dry mass contribution of the KW210-A4 core subsamples showed close agreement (34.2 vs 36.6\%) whereas the relative settled volume contribution showed significant discrepancy (21 vs $28 \%$ ).

A variety of images were collected while processing the container composite in the SAL. Color representation relative to the MacBeth ColorChecker ${ }^{\circledR}$ Rendition Chart (MacBeth Color Chart) was required per the QAPjP/SAP. Figure 5.2 shows the MacBeth Color Chart taken with the in-cell camera during the KW210-CC processing interval with direct comparison to the truer color image (out-of-cell scanned image). As indicated in Section 4.0, the red hues were largely washed out of the in-cell pictures.

(a) The observations for this sample and anomalous results are documented in Occurrence Report 52578-8-19-11, located with TI 52578-TI002 (DVD-52578-PNNL-20650 Rev. 2, Section III, September 2013). 


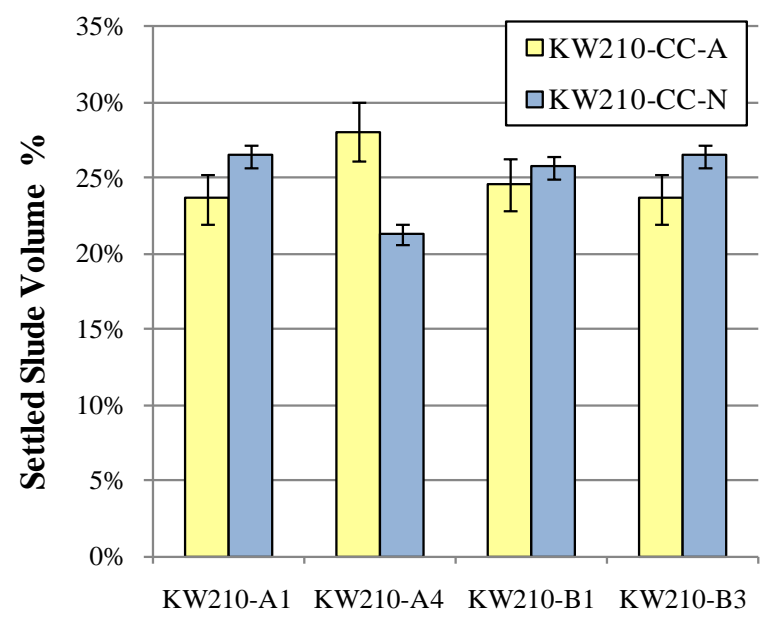

Core Sample

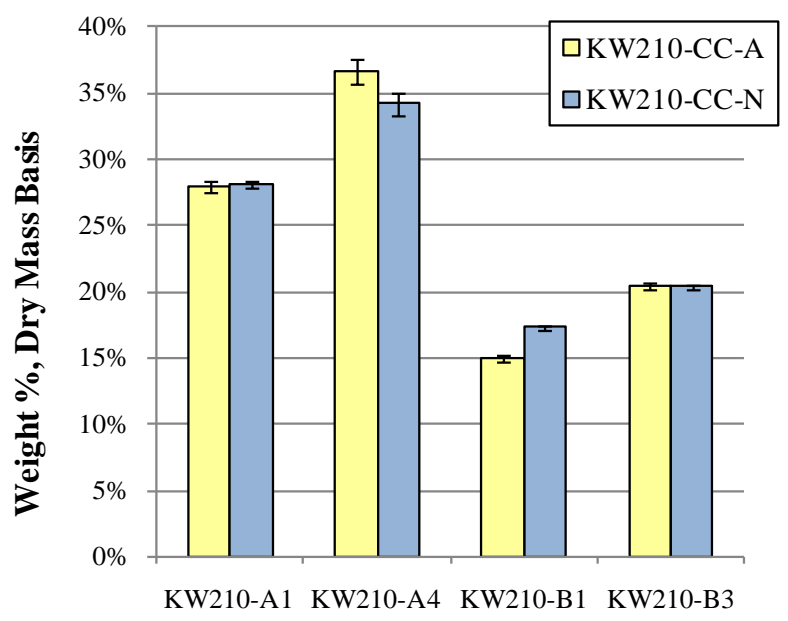

Core Sample

Figure 5.1. Relative Settled Sludge Volume and Dry Mass Contributions of Core Samples to the Container Composite Samples with the Propagated 1- $\sigma$ Error

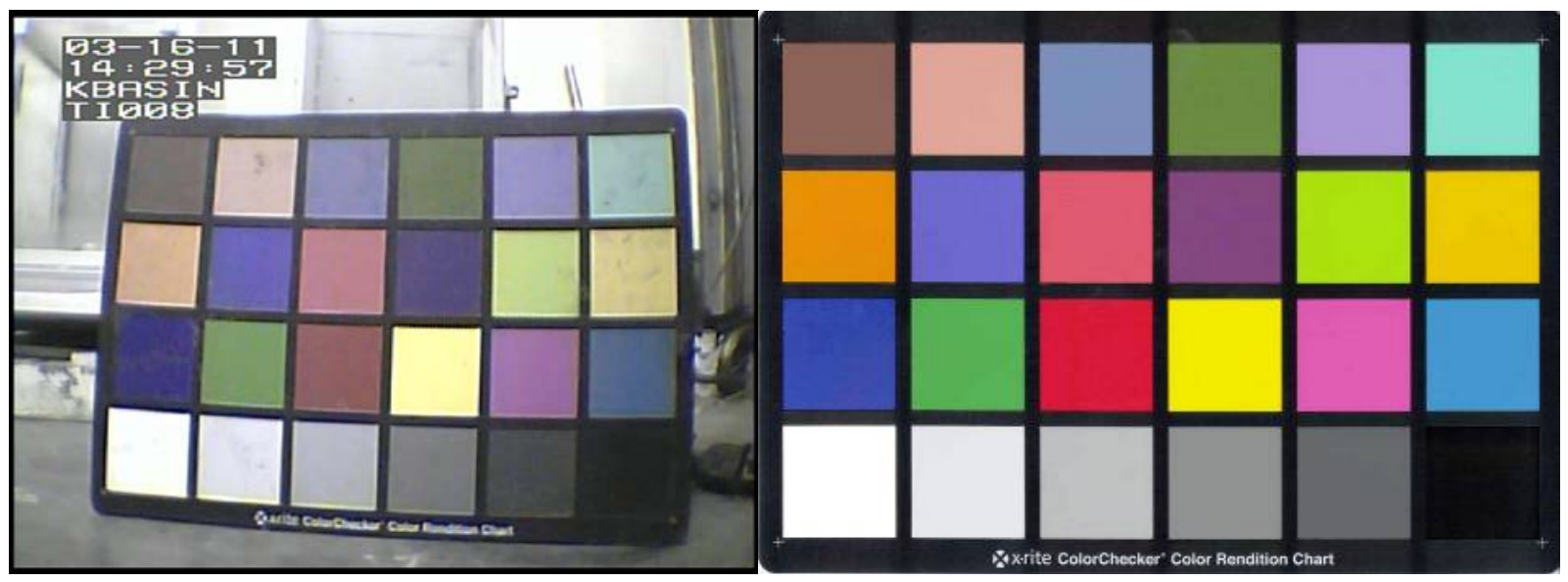

Figure 5.2. MacBeth Color Chart, In-Cell Image (Left), and Scanned Image (Right)

Images of the container composite samples are shown in Figure 5.3 and Figure 5.4. The parent material is shown in the 480-mL (for KW210-CC-A) and 240-mL (for KW210-CC-N) Qorpak jars; the physical property samples are shown in the $40-\mathrm{mL}$ centrifuge tubes. The images were collected after the sludge in the jars was mixed and allowed 24-hr settling time. The volume of settled sludge was approximately equal to the mobilizing fluid (water), and good mixing and batch settling were obtained. Gas bubbles associated with this material were not apparent. 


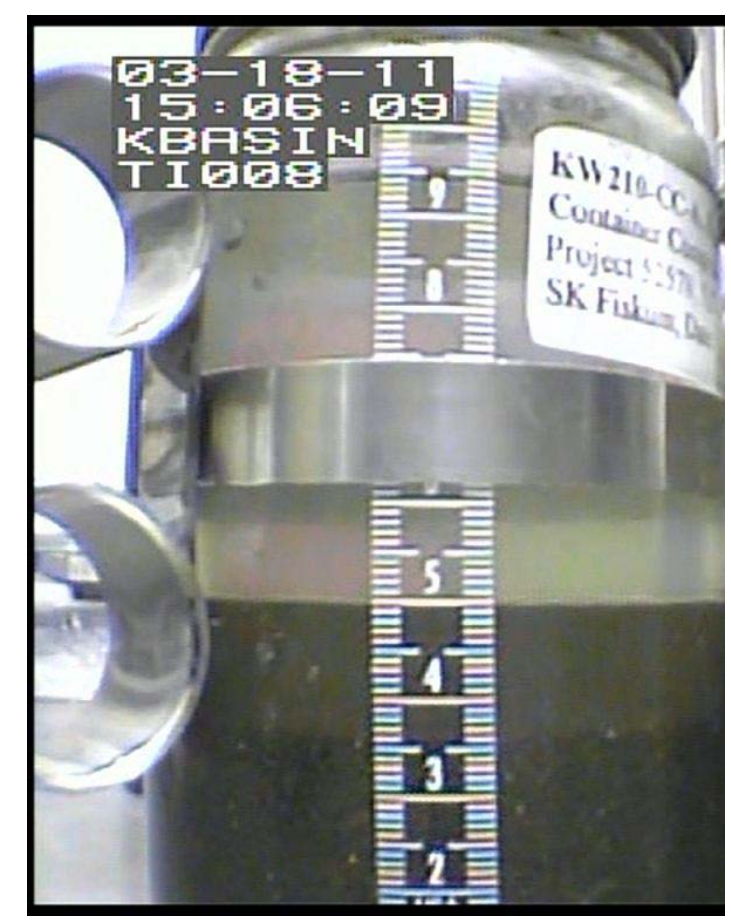

Parent Material (KW210-CC-A)

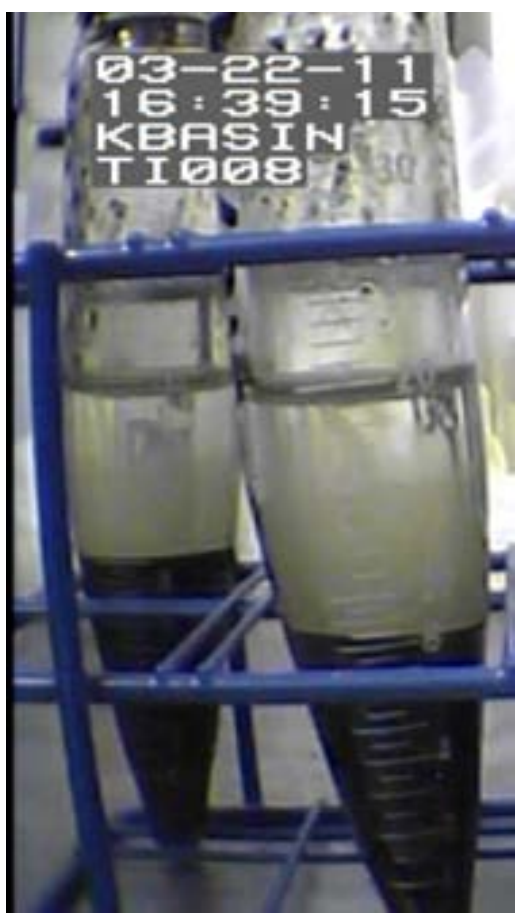

Physical Property Samples

Figure 5.3. KW210-CC-A After Mixing and Settling

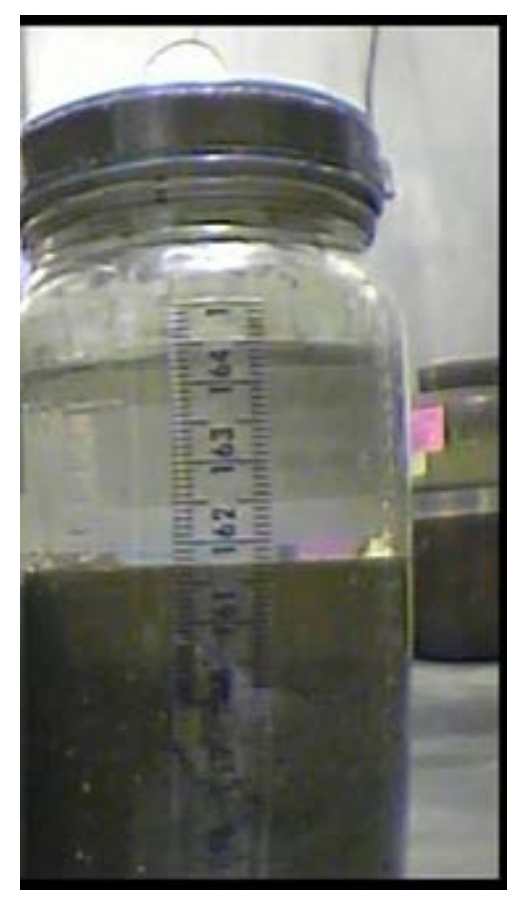

Parent Material (KW210-CC-N)

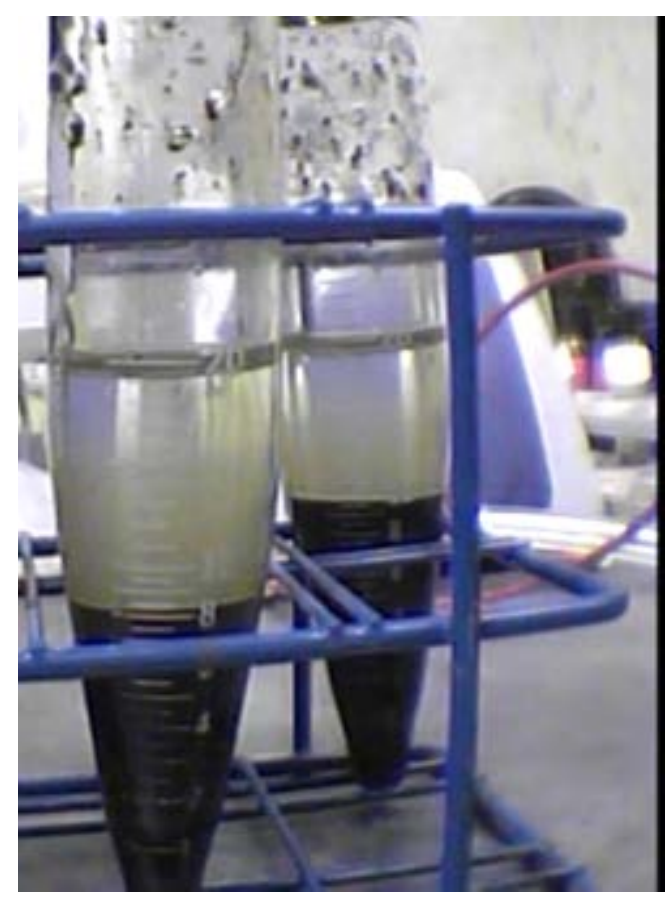

Physical Property Samples

Figure 5.4. KW210-CC-N After Mixing and Settling 


\subsection{Rheology}

Shear strength measurements were performed on only one KW210 unsieved container composite sample: KW210-CC-A. Appendix H contains the detailed rheology report. A total of four shear strength measurements were performed: one measurement of the as-received settled solids strength, two measurements of the settled solids strength after transfer to a small container and approximately $48 \mathrm{hr}$ after solids resuspension, and one opportunistic measurement at an immersion depth of $26 \mathrm{~mm}$ (to evaluate if any significant differences in shear strength resulted as function of depth).

The as-received sample, with a settling time of approximately 11 weeks, was measured using a $6 \times 6 \mathrm{~mm}$ vane tool to avoid exceeding the maximum operating torque of the RV20-M5 measuring system. This is consistent with October 11, 2010 guidance from CHPRC directing PNNL to use a $6 \times 6 \mathrm{~mm}$ vane tool for initial shear strength measurements. The initial strength of the sample was established to be within the working stress range of the $16 \times 16 \mathrm{~mm}$ vane and all subsequent measurements were made with this larger vane tool. The sample was transferred to a smaller container so that the $16 \times 16 \mathrm{~mm}$ vane tool could be used, as there was insufficient settled solids height to use this vane in the original container. Table 5.3 provides a summary of all shear strength measurements. Table 5.3 also presents the average shear strength for sample KW210-CC-A, which was determined from the first and second settle-and-mix operations. For each test, the vane was immersed into the center of the jar at the indicated depth. The uncertainty for shear strength measurement average was calculated as twice the standard error of mean ${ }^{\text {(a) }}$ of the two $\sim 48$ hr settled solids shear strengths. This provides an estimate of the lower bound of $95 \%$ confidence limits for the mean. Shear strength variation with vane depth had been previously observed with granular, heterogeneous simulant samples (Burns et al. 2011). Therefore, an opportunistic measurement at an immersion depth of $26 \mathrm{~mm}$ (which is greater than the standard immersion depth of one vane height) was taken to probe changes in shear strength with immersion depth.

Table 5.3. Shear Strength Summary for KW210-CC-A (all measurements were taken at ambient cell temperature $\left.\left[\sim 29^{\circ} \mathrm{C}\right]\right)$

\begin{tabular}{ccccc}
\hline Sample ID & Measurement & $\begin{array}{c}\text { Settling } \\
\text { Time }(\mathrm{hr})\end{array}$ & $\begin{array}{c}\text { Immersion } \\
\text { Depth }(\mathrm{mm})\end{array}$ & $\begin{array}{c}\text { Shear Strength } \\
(\mathrm{Pa})\end{array}$ \\
\hline KW210-CC-A & As-Received $^{(\mathrm{a})}$ & 1,824 & 6 & $\sim 281$ \\
[Note: Sample was & $1^{\text {st }}$ Mix & 50 & 16 & $63^{\text {(b) }}$ \\
not sieved.] & $2^{\text {nd }}$ Mix & 47 & 16 & $58^{\text {(b) }}$ \\
& $2^{\text {nd }}$ Mix & 47 & 26 & 113 \\
& Average & & & $60 \pm 5^{\text {(c) }}$ \\
\hline
\end{tabular}

(a) Initial, as-received, shear strength measurement using a $6 \times 6 \mathrm{~mm}$ vane. Subsequent measurements were taken with a $16 \times 16 \mathrm{~mm}$ vane after the sample was transferred to a smaller container.

(b) Used in average calculation.

(c) Reported uncertainty is twice the standard error of the mean (which approximated the lower bound of the $95 \%$ confidence limits).

(a) The standard error of a mean is calculated as the sample standard deviation divided by the square root of the sample size, $\mathrm{N}$ ( $\mathrm{N}=2$ for the shear strength measurements reported herein). 
It should be noted that the as-received measurement indicated a shear strength of $\sim 281 \mathrm{~Pa}$; however, given that this measurement was subject to significant noise and that the measured value represents only $\sim 0.2 \%$ full-scale reading with the $6 \times 6 \mathrm{~mm}$ vane, this measurement is subject to large uncertainty.

Vane depth dependence was observed for the KW210 container composite sample used in this study under the mixing/settling regime employed. Typically, such increases in shear strength with depth are indicative of stratification of solids or lithostatic effects.

After mixing and settling for $\sim 47$ to $50 \mathrm{hr}$, the shear strength of the material was reduced considerably from the as-received condition. Subsequent mixing and settling operations yielded settled materials with similar shear strength; thus, the initial mixing operation appeared to have fully disrupted any sample structure.

For comparison against the results of actual KW Basin unseived sludge fraction shear strength, the shear strength results of the KW Container sludge simulant (KW-B, $<500-\mu \mathrm{m}$ fraction) are provided in Table 5.4. The KW Container sludge simulant results show shear strength ranging from 800 to 2,400 $\mathrm{Pa}$ (Burns et al. 2011), with an implied design/safety basis value for size-fractionated samples containing particles $<250 \mu \mathrm{m}$ of $\sim 500 \mathrm{~Pa}$ (Plys and Schmidt 2006). The shear strength results for sample KW210-CC-A are bounded by those listed in Table 5.4. Similar to KW210-CC-A, the KW-B simulant evidenced shear strength variation with immersion depth, with a high measurement of $\sim 6,000 \mathrm{~Pa}$. Variation of the KW-B shear strength with immersion depth was attributed to "particle settling and continued dewatering of the simulant" (Burns et al. 2011). Similar mechanisms may contribute to the vertical stress variations observed in KW210-CC-A.

Table 5.4. K West Basin Simulant ( $-500-\mu \mathrm{m}$ Sieve Fraction) Shear Strength Summary and Implied Safety Basis

\begin{tabular}{ccc}
\hline Basis & Shear Strength [Pa] & Reference \\
\hline $\begin{array}{c}\text { Measurements of KW-B }(<500-\mu \mathrm{m}) \text { Simulant } \\
\text { Implied Design/Safety; based on samples } \\
\text { containing only particles }<250 \mu \mathrm{m}\end{array}$ & 800 to 2,400 & Burns et al. 2011 \\
\hline
\end{tabular}

\subsection{Sieve Fractions}

The KW210-CC-N container composite material was sieved through the 2,000- $\mu \mathrm{m}$ and $500-\mu \mathrm{m}$ mesh sieve assembly. The materials that collected on the $2,000-\mu \mathrm{m}$ mesh sieve were designated large container composite (LCC) or $+2,000 \mu \mathrm{m}$; materials that collected on the $500-\mu \mathrm{m}$ mesh sieve were designated middle container composite (MCC) or $-2,000 \mu \mathrm{m} /+500 \mu \mathrm{m}$; and materials that passed through all sieves were designated small container composite (SCC) or $-500-\mu \mathrm{m}$.

Images of LCC material are shown in Figure 5.5 and Figure 5.6. Some of the LCC particles were reflective and plate-shaped, which is consistent in appearance with Grafoil ${ }^{\circledR}$ particles. The LCC fraction also included light and dark rock-like materials, resin beads, and thread-like material. Although most particles appeared to exist in shades of gray, some particles appeared red to yellow in coloration. 


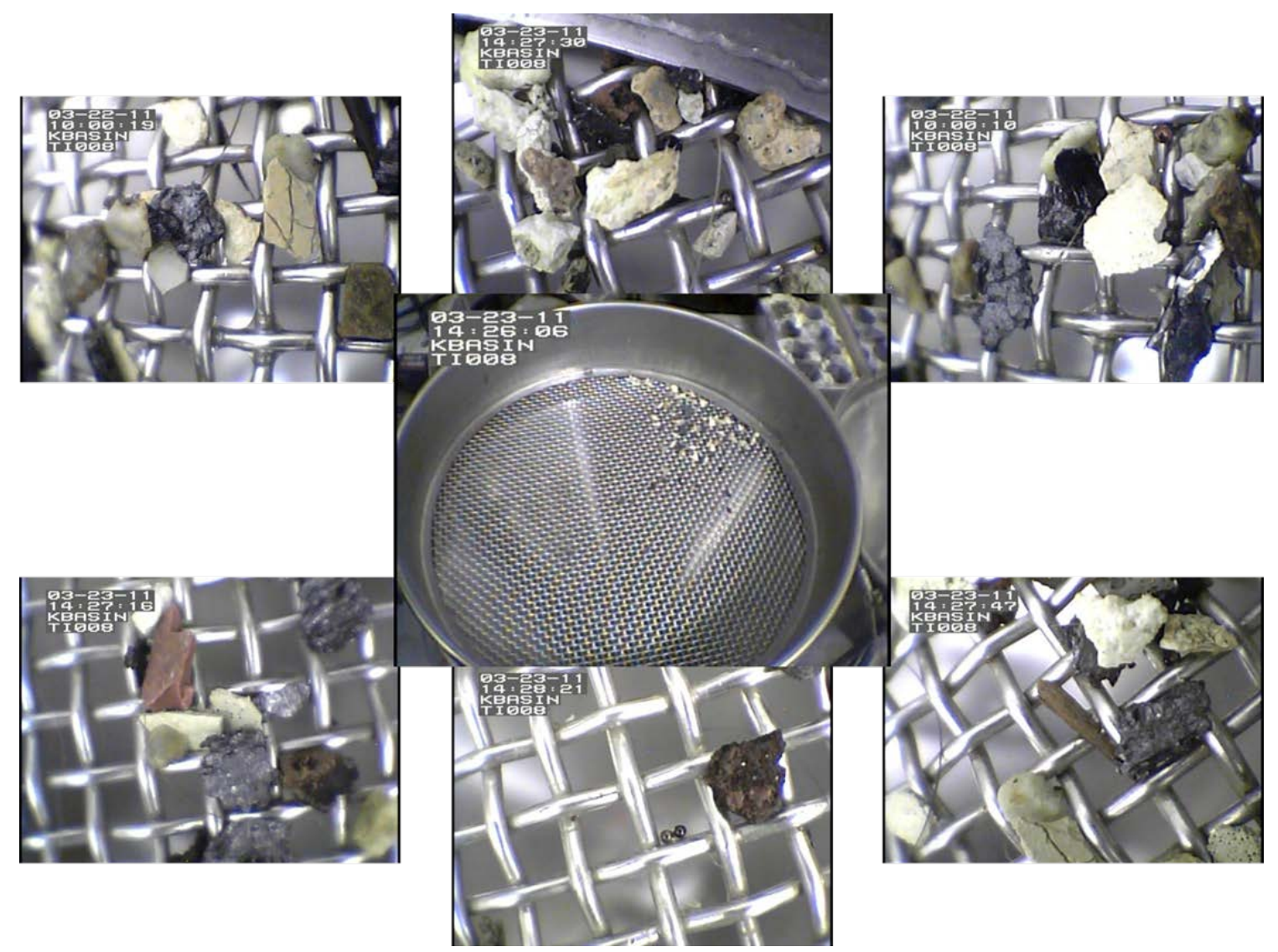

Note: There was a wide variety of particle morphologies; some particles appeared to have reflective surfaces.

Figure 5.5. KW210-LCC Solids Captured on the 2,000- $\mu \mathrm{m}$ Mesh Sieve 

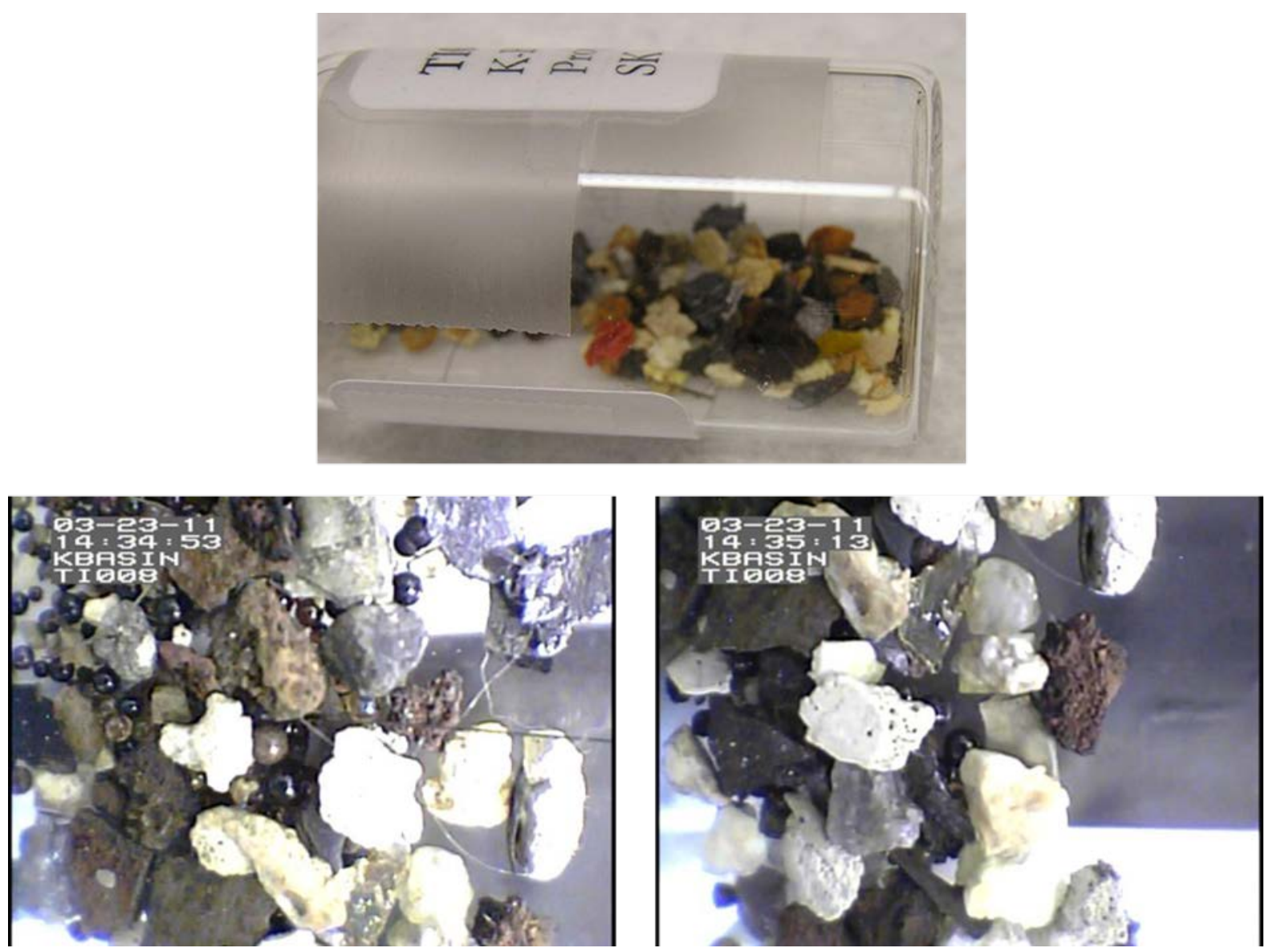

Note the reflective surfaces, the thread-like material, and the resin beads. The upper photograph was taken outside of the hot cell, so the colors better represent the "true" colors (sample is in a 20-mL glass scintillation vial).

Figure 5.6. KW210-LCC Transferred to Jar

The MCC consisted mostly of resin beads, almost transparent irregularly shaped particles, and a few colored (green and red) particles (see Figure 5.7). 


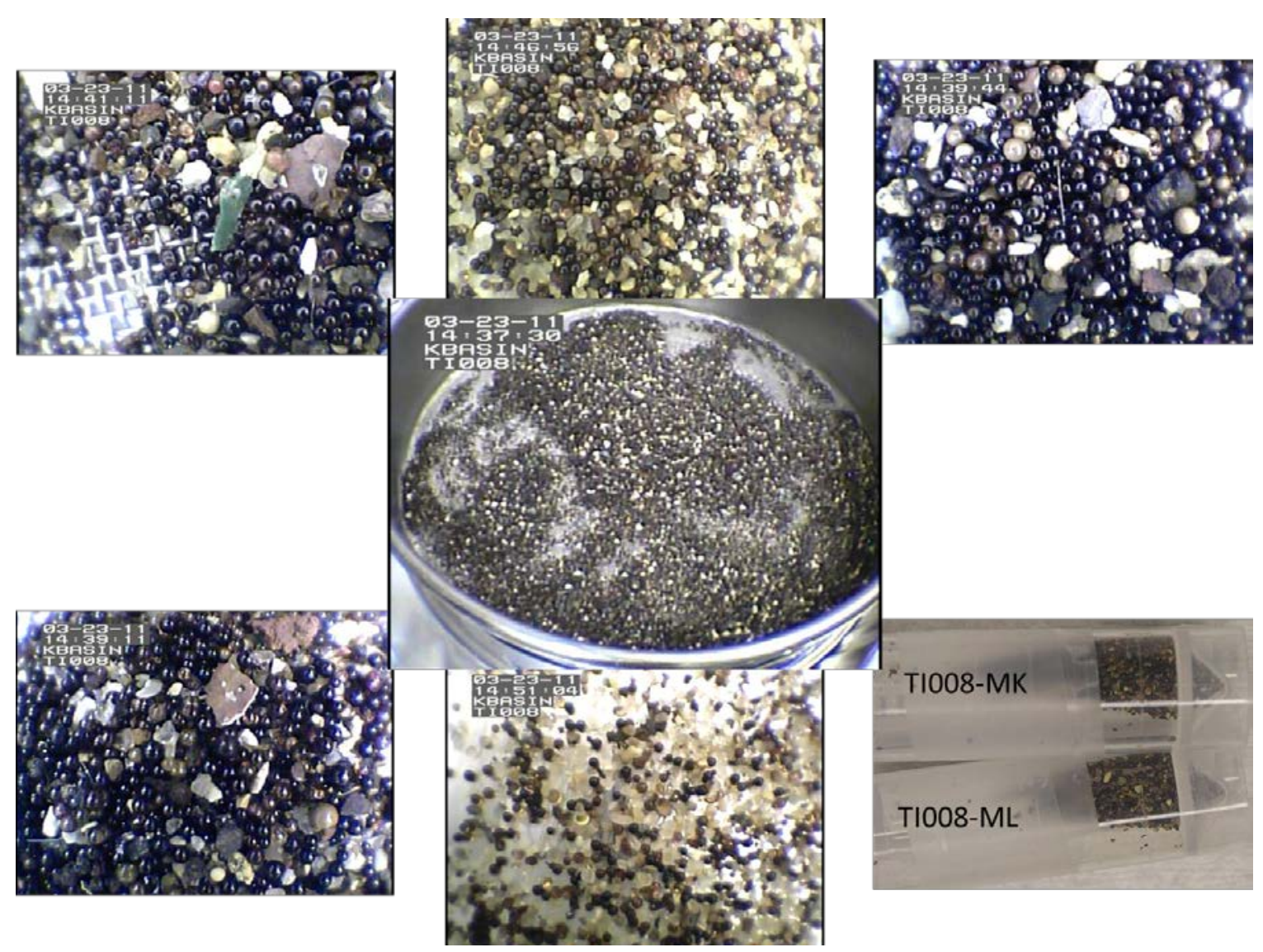

Note: The lower right shows aliquots of the sample that were removed from the hot cell for particle density measurement (TIO08-ML and -MK in 5-mL plastic vials).

Figure 5.7. KW210-MCC Solids Captured on the 500- $\mu \mathrm{m}$ Sieve

Figure 5.8 shows the SCC fraction after 23-hr and 142-hr settling. It appeared to be homogenously muddy-brown. No apparent stratification was observed.
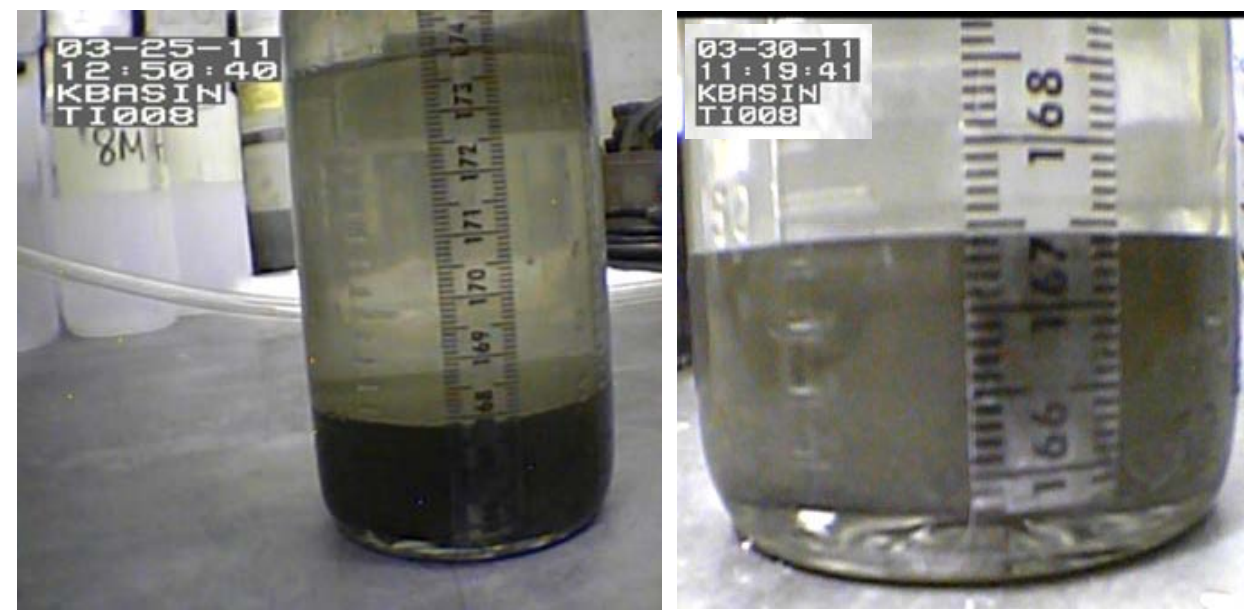

Note: The sludge layer is the dark brown material; clarified water is above. The total (water plus sludge) volume was $\sim 214 \mathrm{~mL}$. The 23-hr settled sludge volume was $~ 57 \mathrm{~mL}$ (left); the 142-hr settled sludge volume was $\sim 44 \mathrm{~mL}$ (right).

Figure 5.8. KW210-SCC After Mixing and Settling 
Table 5.5 summarizes the mass distributions on a dry mass basis for each sieve fraction. The calculated dry masses of the initial composite and the $-500-\mu \mathrm{m}$ fraction were determined from the total settled solids volume and the physical property characterization samples (see Section 3.0). The dry masses of the wet sludge therefore corresponded to higher uncertainty. The dry mass fraction of the MCC fraction was calculated by multiplying the net fraction mass by the calculated wt\% solids $(90.4 \%$, which was determined from drying the duplicate ASO samples [TI008-MA and TI008-MB] at $105^{\circ} \mathrm{C}$ to constant mass). The mass fractionation was calculated from the sample fraction dry mass divided by the sum of the dry fraction masses (77 g). The calculated input dry fraction mass (65 $\pm 6 \mathrm{~g}[1-\sigma]$ ) was lower than the calculated dry mass sum of fractions $(77 \pm 3 \mathrm{~g}[1-\sigma])$. The difference could be associated with experimental error or the resin beads in the MCC fraction may contain additional water.

Table 5.5. Dry Mass Distribution of KW210-CC-N Container Composite Sieve Fractions

\begin{tabular}{|c|c|c|c|c|c|}
\hline & KW210-CC & & KW210-LCC & KW210-MCC & KW210-SCC \\
\hline Parameter & $\begin{array}{c}\text { Calculated Initial } \\
\text { Composite }^{(a)}\end{array}$ & $\begin{array}{c}\text { Sum of Dry } \\
\text { Fractions }\end{array}$ & $+2,000 \mu \mathrm{m}$ & $-2,000 \mu \mathrm{m} /+500 \mu \mathrm{m}$ & $-500 \mu \mathrm{m}$ \\
\hline Mass (g) & $65 \pm 6$ & $77 \pm 3$ & 0.7263 & 55.2 & 21.5 \\
\hline $\begin{array}{l}\text { Dry mass } \\
\text { fraction }\end{array}$ & -- & 1.00 & 0.0094 & 0.71 & 0.28 \\
\hline
\end{tabular}

(a) Mass of fraction of composite that was sieved. A portion of the composite had been subsampled for physical property measurements; the calculated initial composite mass accounts for this subsampling activity.

The MCC fraction contained approximately 2.5 times the mass of the SCC fraction. There was just sufficient volume and mass of the SCC fraction to accommodate all targeted subsample collections. The LCC fraction constituted $<1 \mathrm{wt} \%$ of the container composite. The small mass collected for the LCC fraction limited the targeted analysis suite to direct GEA followed by particle density—no dissolution processing.

\subsection{Particle Densities and Bulk Volume}

Five samples were submitted for analysis, one LCC, two MCC, and two SCC fraction samples. The full particle density analysis report is provided in Appendix G. Figure 5.9 through Figure 5.11 present images of the sample materials. The TI008-L, TI008-MK, and TI008-ML samples had particles of varying shapes, sizes, and colors. The TI008-MK and TI008-ML samples had visible resin beads and appeared to have a significant charge, as evidenced by the particles clinging to the plastic container walls (see Figure 5.12). The TI008-SK and TI008-SL samples appeared as a dark brown powder. 

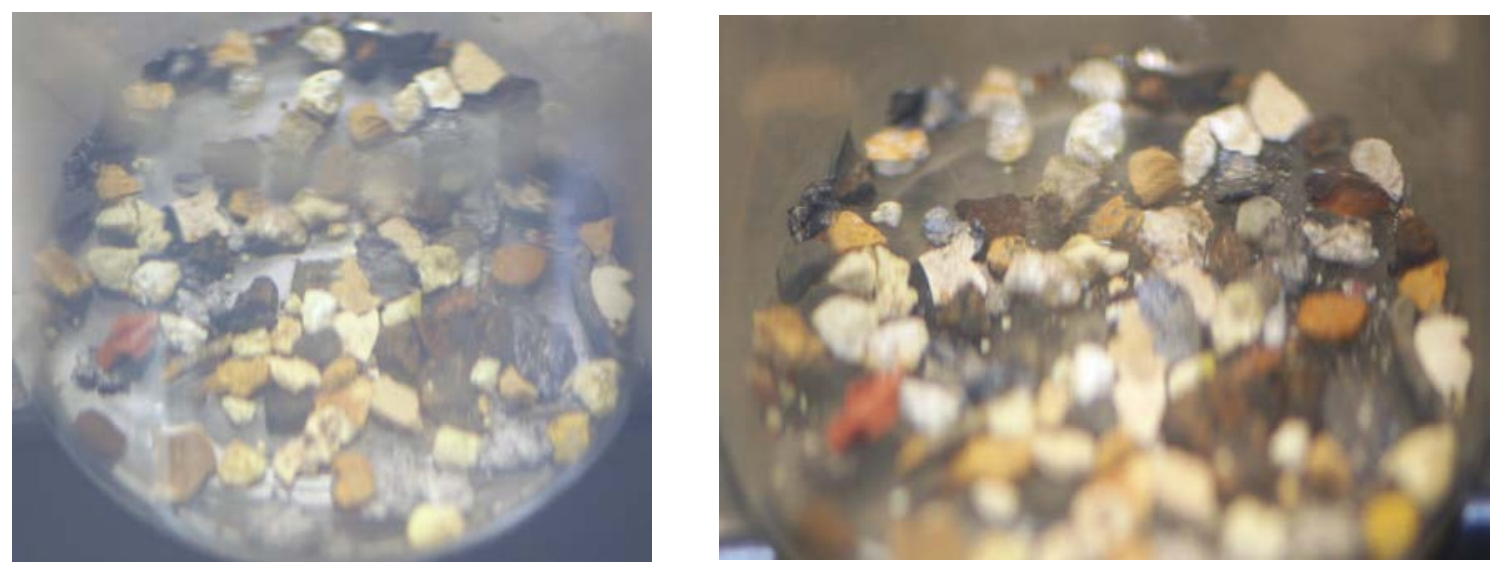

Note: Focus issues are an artifact of the glovebox window.

Figure 5.9. Images of TI008-L, $+2,000-\mu \mathrm{m}$ Sieve Fraction of the KW210-CC-N Container Composite
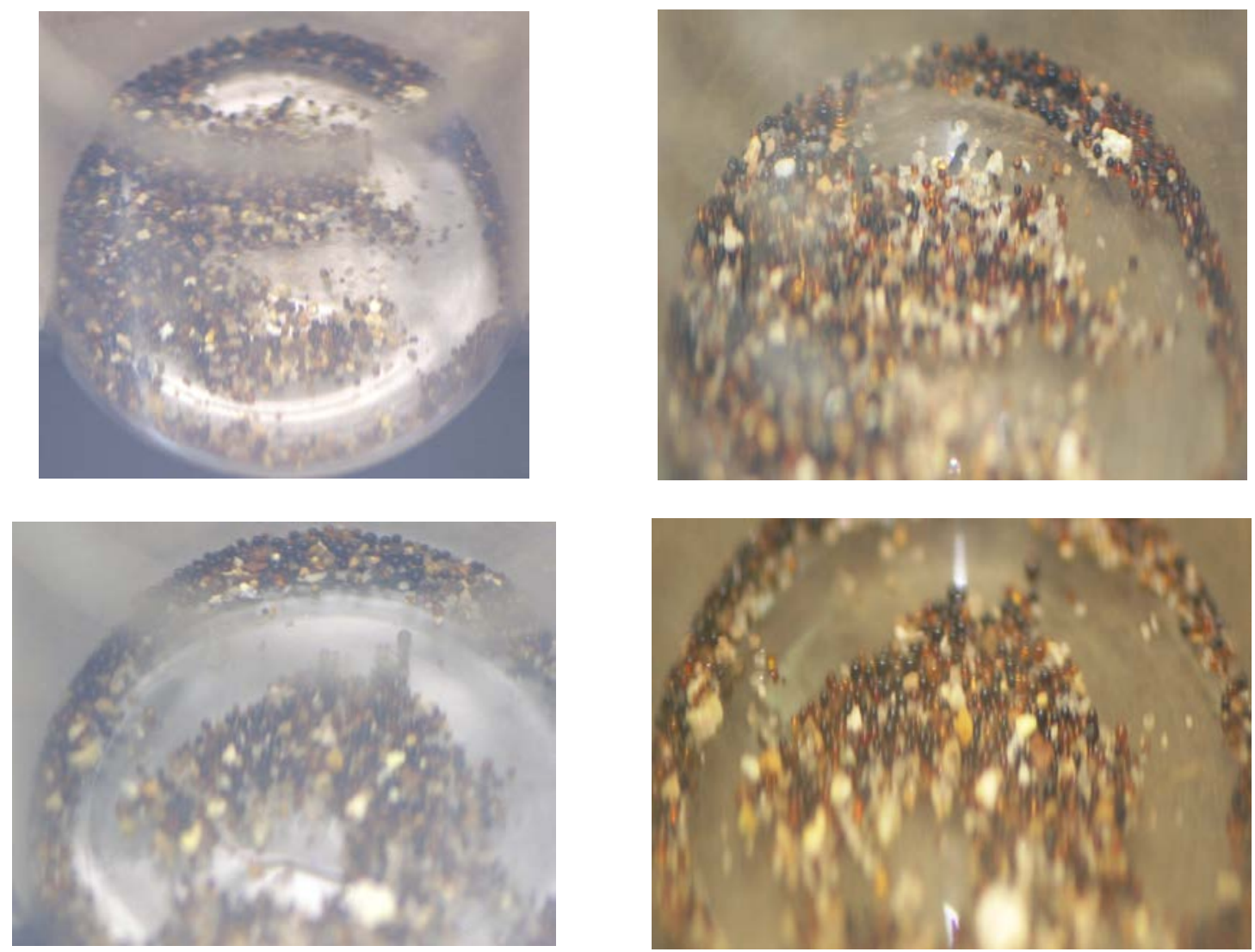

Note: Focus issues are an artifact of the glovebox window.

Figure 5.10. Images of TI008-MK (top row) and TI008-ML (bottom row), $-2,000-\mu \mathrm{m} /+500-\mu \mathrm{m}$ Sieve Fraction of the KW210-CC-N Container Composite 


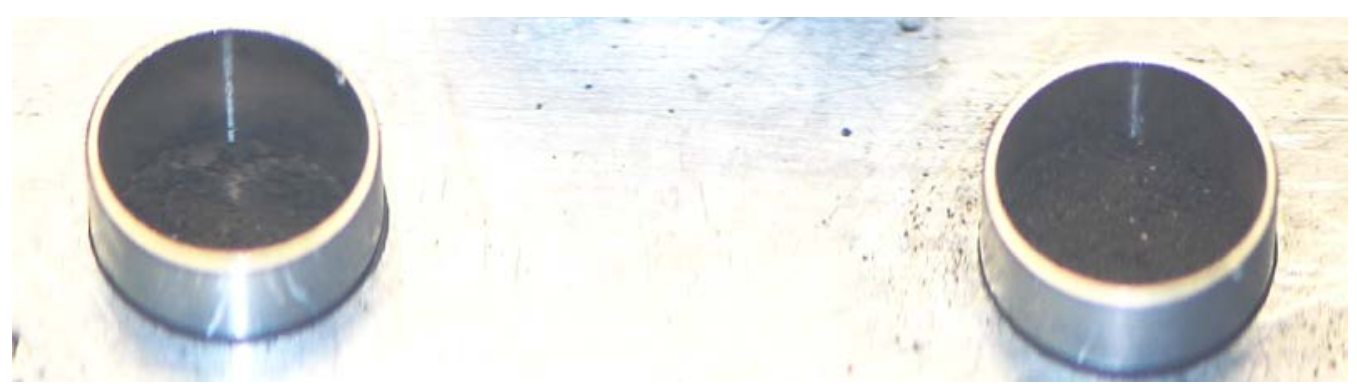

Figure 5.11. Images of TI008-SK and TI008-SL in the Gas Pycnometry Analysis Cup, $-500-\mu \mathrm{m}$ Sieve Fraction of the KW210-CC-N Container Composite

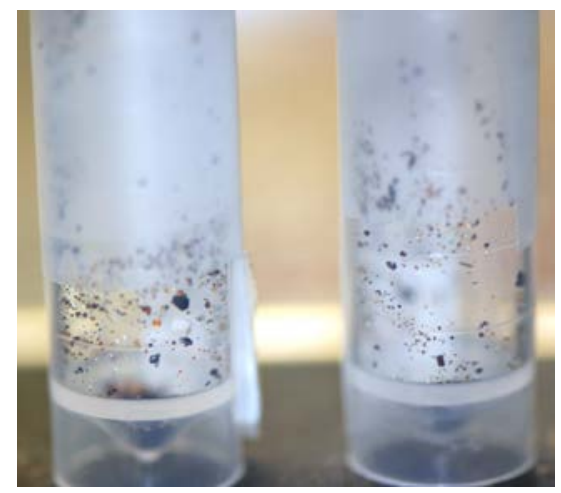

Figure 5.12. Sample Vials Containing As-Received TI008-MK and TI008-ML After Placement in the Glovebox

The sample volumes were small enough to allow most if not all material transfer into a tared 1- $\mathrm{cm}^{3}$ pycnometer sample cup for analysis. The pycnometer cup holding the sample was placed into the sample chamber and analyzed five sequential times. The results were averaged and the density with standard deviation was recorded. Table 5.6 presents the density results in $\mathrm{g} / \mathrm{cm}^{3}$ along with \%RSD for the five replicate sample runs. The averaged sample result and the averaged duplicate sample result were used to determine the RPD. Data are provided in Appendix G.

Table 5.6. Particle Density Results

\begin{tabular}{|c|c|c|c|c|c|c|c|}
\hline $\begin{array}{l}\text { Sieve } \\
\text { Fraction }\end{array}$ & Sample ID & Physical Description & $\begin{array}{l}\text { Air-Dried } \\
\text { Sample } \\
\text { Mass (g) })^{(a)}\end{array}$ & $\begin{array}{c}\text { Mass } \\
\text { Analyzed } \\
(\mathrm{g})^{(\mathrm{b})}\end{array}$ & $\begin{array}{c}\text { Average } \\
\text { Density, } \\
\text { g/cm }{ }^{3}\end{array}$ & $\begin{array}{l}\text { Sample } \\
\text { \% RSD }\end{array}$ & $\begin{array}{c}\% \\
\mathrm{RPD}\end{array}$ \\
\hline$+2,000 \mu \mathrm{m}$ & TI008-L ${ }^{(c)}$ & $\begin{array}{l}\text { Particles of varying shapes, sizes } \\
\text { and colors }\end{array}$ & 0.7588 & 0.6780 & 2.60 & 0.15 & NA \\
\hline \multirow{2}{*}{$\begin{array}{l}-2,000 \mu \mathrm{m} / \\
+500 \mu \mathrm{m}\end{array}$} & TI008-MK & \multirow{2}{*}{$\begin{array}{l}\text { Particles of varying shapes, sizes } \\
\text { and colors. Resin beads evident. }\end{array}$} & 0.5231 & 0.4142 & 1.62 & 0.04 & \multirow[t]{2}{*}{0} \\
\hline & TI008-ML & & 0.5074 & 0.3420 & 1.62 & 0.03 & \\
\hline \multirow[t]{2}{*}{$-500 \mu \mathrm{m}$} & TI008-SK & \multirow[t]{2}{*}{ Dark brown powder } & 0.6755 & 0.6355 & 2.57 & 0.05 & \multirow[t]{2}{*}{3} \\
\hline & TI008-SL & & 0.3462 & 0.2962 & 2.49 & 0.07 & \\
\hline
\end{tabular}

(a) The tare masses of the vials were recorded in TI 52578-TI008. The samples were air-dried in the glovebox and a gross weight was obtained. Mass of the subdivision is the difference between the gross and tare masses.

(b) Mass of the sample removed from vial and placed in analysis sample cup.

(c) Sample TI008-L was labeled TI008-LA when analyzed by GEA per ASR 8824.

NA: Not Applicable. 
The QAPjP/SAP delineated a particle density precision of $\pm 5 \%$. Over $50 \%$ of each subsample was analyzed, minimizing possible variations from subsampling at the workstation. Because the LCC sample was not split for analysis, the RPD could not be calculated. The duplicate sample precision for the $-2,000-\mu \mathrm{m} /+500-\mu \mathrm{m}$ (TI008-MK and TI008-LK) and -500- $\mu \mathrm{m}$ (TI008-SK and TI008-SK) sieve fractions met the QAPjP/SAP criterion.

Table 5.7 summarizes the bulk dry density, dry mass, bulk dry volume, and absolute dry volume for the LCC and MCC sieve fractions (as applicable). These measures were determined opportunistically and acceptance criteria do not apply. The bulk density should be used with caution because it includes void space. The dry mass was taken from Table 5.5 and is shown again for information.

Table 5.7. Approximate Bulk Density and Volume of Dried Solids

\begin{tabular}{lcccc}
\hline & $\begin{array}{c}\text { Dry Bulk } \\
\text { Density, g/mL } \\
\text { Sample ID }\end{array}$ & $\begin{array}{c}\text { Bry Mass, } g \\
\left(\mathrm{D}_{\mathrm{B}}\right)\end{array}$ & $\begin{array}{c}\text { Bolk } \\
\text { Volume, } \mathrm{mL} \\
\left(\mathrm{V}_{\mathrm{B}}\right)\end{array}$ & $\begin{array}{c}\text { Absolute } \\
\text { Volume, mL } \\
\left(\mathrm{V}_{\mathrm{A}}\right)\end{array}$ \\
\hline KW210-LCC & -- & 0.7263 & -- & 0.279 \\
KW210-MCC & 1.0 & 55.18 & 55 & 34.1 \\
\hline The bulk density value should be used with caution since this measure includes the void fraction. \\
Dry masses were copied from Table 5.5 and are supplied here for reference.
\end{tabular}

The bulk volume refers to the total volume of solids (inclusive of void space) in the sieve fraction and was calculated according to Equation 5.1.

$$
\mathrm{V}_{\mathrm{B}}=\frac{\mathrm{M}}{\mathrm{D}_{\mathrm{B}}}
$$

where $V_{B}$ is the bulk volume, $M$ is the total mass of sieve fraction, and $D_{B}$ is the bulk density (determined from a sample aliquot). The bulk volume of the LCC solids was not determined because of the difficulty of measuring a small volume of large granular materials in a vial. The MCC bulk density was determined opportunistically from the volume of a sample split measured in a 50-mL, volume-calibrated centrifuge tube and its corresponding net mass. In this case, the MCC solids were poured into the centrifuge tubeno other method was employed to further pack the particles in the tube.

The absolute volumes of the MCC and LCC samples were calculated from the total sieve fraction mass, and the particle density (determined by gas pycnometry) according to Equation 5.2.

$$
\mathrm{V}_{\mathrm{A}}=\frac{\mathrm{M}}{\mathrm{D}_{\mathrm{A}}}
$$

where $\mathrm{V}_{\mathrm{A}}$ is the absolute volume, $\mathrm{M}$ is the total mass of sieve fraction, and $\mathrm{D}_{\mathrm{A}}$ is the absolute density determined by gas pycnometry. The absolute volume represents the total particle volume of the sieve fraction free of void space. The absolute volume for the MCC fraction was $\sim 62 \%$ of the bulk volume.

Table 5.8 compares the 24-hr settled sludge volumes of the initial container composite before sieving to the SCC fraction. The SCC fraction represented $42 \mathrm{vol} \%$ of the total container composite. The sum of combined wet settled SCC sludge volume and the absolute volumes of the MCC and LCC fractions is 
also provided in Table 5.8. The summed volumes represented only $67 \%$ of the total container composite. The drying process of the MCC and LCC fractions could have resulted in a significant contraction of resin beads and other components and/or the OIER volume measured from gas pycnometry did not include the resin void spaces (pore spaces). The observations for the KW210 container composite material are consistent with those reported for the KW220 container composite (Fiskum et al. 2011b).

Table 5.8. Volume Comparisons of the KW210-CC-N Container Composite to the SCC and Combined Fractions

\begin{tabular}{|c|c|c|c|c|}
\hline \multirow{2}{*}{$\begin{array}{l}\text { Initial Settled } \\
\text { Wet Sludge } \\
\text { Composite } \\
\text { Volume, mL } \\
\text { (a) }\end{array}$} & \multicolumn{2}{|c|}{ Wet SCC Volume Fraction } & \multicolumn{2}{|c|}{$\begin{array}{c}\text { Combined Wet SCC and Dry MCC } \\
\text { and LCC Volume Fractions }\end{array}$} \\
\hline & $\begin{array}{l}\text { Settled Wet } \\
\text { Sludge, } \mathrm{mL}^{\text {(a) }}\end{array}$ & $\%$ of Composite & $\begin{array}{l}\text { Total, } \\
\mathrm{mL}^{(\mathrm{b})}\end{array}$ & $\%$ of Composite \\
\hline 137 & 57 & $42 \%$ & 91.3 & $67 \%$ \\
\hline
\end{tabular}

(a) Measured after $\sim 24 \mathrm{hr}$ of settling. The volume uncertainty was estimated to be $\pm 5 \mathrm{~mL}$.

(b) The volume sum combined the wet settled SCC volume plus the absolute dry volumes of the LCC and MCC fractions.

\subsection{Crystal Structure}

Two sample aliquots from each of the MCC and the SCC solids were characterized for crystalline phases. The full XRD analysis reports with XRD patterns can be found in Appendix F.

Table 5.9 summarizes the crystalline phases identified in the two subsamples collected for analysis from each of the SCC solids $(-500 \mu \mathrm{m})$. These solids were dominated by quartz $\left(\mathrm{SiO}_{2}\right)$ and uranium oxide $\left(\mathrm{UO}_{2}\right.$ and $\left.\mathrm{U}_{4} \mathrm{O}_{9}\right)$, present as major phases. Unassigned peaks are also shown. In all cases the unassigned peaks were very low intensity and exhibited broadening over $\sim 0.3^{\circ} 2$-theta with a non-descript peak center.

Table 5.9. Crystalline Phases Identified in KW210-SCC (-500- $\mu$ m Fraction)

\begin{tabular}{|c|c|c|c|}
\hline \multirow[b]{2}{*}{ Phase Present } & \multirow{2}{*}{$\begin{array}{c}\text { Chemical } \\
\text { Formula }\end{array}$} & \multicolumn{2}{|c|}{ Relative Intensities (Rietveld Analysis) } \\
\hline & & TI008-SH & TI008-SJ \\
\hline Quartz & $\mathrm{SiO}_{2}$ & Major & Major \\
\hline Uraninite & $\mathrm{UO}_{2}$ & Major & Major \\
\hline Uranium oxide & $\mathrm{U}_{4} \mathrm{O}_{9}$ & Major & Major \\
\hline Unassigned peaks & & $23.6^{\circ}, 34.5^{\circ}, 35.6^{\circ(\mathrm{b})}$ & $24^{\circ}$, (a) $^{\circ} 35^{\circ}, 35.8^{\circ}$, (b) $37^{\circ}, 38^{\circ}, 51.5^{\circ}$ \\
\hline
\end{tabular}

(a) The peak at $24^{\circ} 2$-theta was found only on the subsample that included the internal $\mathrm{TiO}_{2}$ tracer. It was consistent with the peak located at $23.6^{\circ} 2$-theta in TI008-SH.

(b) The peaks at 35.6 and $35.8^{\circ}$ 2-theta for TI008-SH and TI008-SJ, respectively, represent the same peak. See text for discussion of peak center identification.

Table 5.10 summarizes the crystalline phases identified in the two subsamples collected for analysis from each of the MCC solids $(-2,000 \mu \mathrm{m} /+500 \mu \mathrm{m})$. Only quartz was identified in the MCC fraction. 
Table 5.10. Crystalline Phases Identified in KW210-MCC $(-2,000-\mu \mathrm{m} /+500-\mu \mathrm{m}$ Fraction)

\begin{tabular}{llcc} 
& & \multicolumn{2}{c}{ Peak Intensities (Rietveld Analysis) } \\
\cline { 2 - 4 } Phase Present & Chemical Formula & TI008-MH & TI008-MJ \\
\hline Quartz & $\mathrm{SiO}_{2}$ & Major & Major \\
Unassigned peaks & & $27.3^{\circ}$ & $33^{\circ}, 35^{\circ}-36^{\circ(\text { a) }}$ \\
\hline (a) A broad peak was present between $35^{\circ}$ to $36^{\circ}$ 2-theta. \\
\hline
\end{tabular}

Quartz is not expected to dissolve in the acid digestion and thus Si would not be substantially present in the acid digestate measured by ICP-OES. There was no evidence from the SCC or MCC diffraction patterns for the presence of $\mathrm{Zr}$ metal, Al hydrates, or U hydroxides.

Figure 5.13 shows the X-ray powder diffraction patterns for each of the MCC and SCC samples. All samples resulted in a raised, low-angle background between approximately 8 and $25^{\circ} 2$-theta. The backgrounds for replicate SCC samples (TI008-SH, -SJ, and -SJ with $\mathrm{TiO}_{2}$ ) appeared similar in shape and intensity. The low-angle background shape and relative intensity for sample TI008-MJ was substantially different from the duplicate sample, TI008-MH. The differences may be caused by one or both of the following phenomena: 1) less TI008-MJ sample mass was loaded on the slide relative to the sample mass loading for TI008-MH; 2) more amorphous component was present in sample TI008-MJ relative to that of sample TI008-MH. The most intense peaks of TI008-MH and TI008-MJ were equalized using the system software. Raising the sample peak height also proportionately raises all other diffraction pattern features, including the background. 


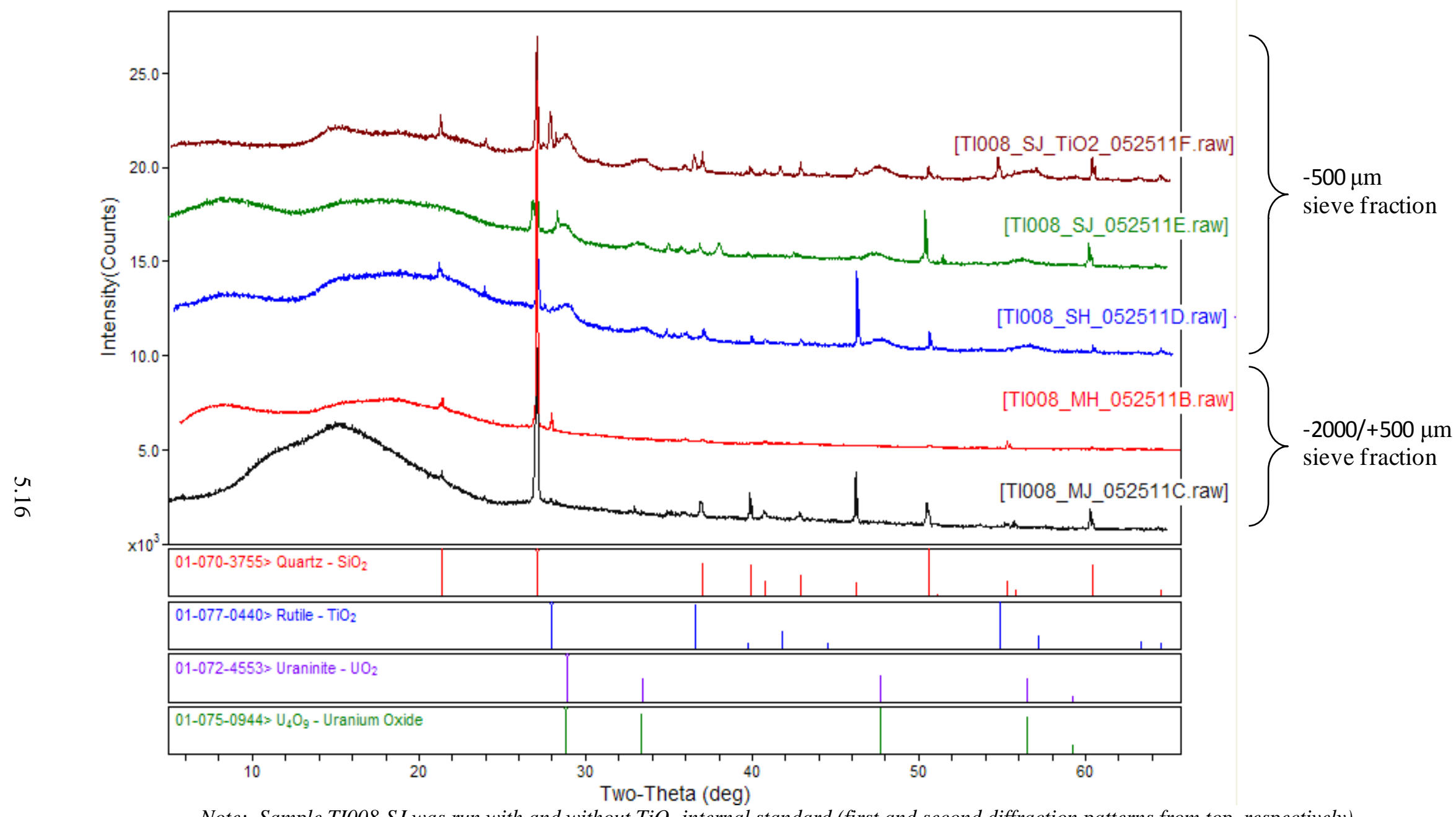

Note: Sample TI008-SJ was run with and without $\mathrm{TiO}_{2}$ internal standard (first and second diffraction patterns from top, respectively).

Figure 5.13. XRD Powder Diffraction Patterns for the KW210-MCC $(-2,000-\mu \mathrm{m} /+500-\mu \mathrm{m})$ and KW210-SCC (-500- $\mu \mathrm{m})$ Samples 


\subsection{Particle Size Distribution}

Two subsamples collected from KW210-SCC ( $-500-\mu \mathrm{m}$ sieve fraction) were evaluated for PSD, TI008-SP (primary) and TI008-SQ (duplicate). Appendix I of this report contains the detailed particle size analysis report. The parameters used for sample analysis are given in Table 5.11.

Table 5.11. Default Optical Properties Used for PSD Analysis of KW210-SCC Samples

\begin{tabular}{ll}
\hline Property & Values Employed \\
\hline Refractive Index & 2.42 \\
Absorption Index & 1 \\
Particle Shape & Irregular \\
Model & General Purpose (Broad Distribution) \\
\hline
\end{tabular}

These properties were assessed through post-measurement reanalysis of KE KW240 (TI010-SP) data collected during the initial parameter evaluation study (Fiskum et al. 2011b). Application of these parameters to the KW sludge (KW210) was considered to be appropriate.

PSD characterizations consisted of an average of three individual PSD measurements for two subaliquots (collected at the workstation) per sample. Data obtained for all sub-aliquots were averaged to obtain one PSD for each measuring condition per sample, i.e. at 1 min recirculation (unsonicated), 100\% sonication (20 watts), and post sonication.

Table 5.12 provides the matrix of conditions for both of the PSD samples analyzed. The initial measurement conditions at 1 and 3 min recirculation times are based on results obtained from a parameter evaluation study (Fiskum et al. 2011b, TI 52578-TI017). An additional data measurement set was collected (a post-sonicated measurement after 5 min of total recirculation) to further evaluate the effect of sonication on the measured PSD.

Table 5.12. Test Condition Matrix for PSD Determinations

\begin{tabular}{ccc}
\hline Recirculation Rate, RPM & Recirculation Time, min & Sonication Power, Time \\
\hline 2,000 & 1 & Pre-sonication \\
2,000 & 3 & $120 \mathrm{sec} / 100 \%$ \\
2,000 & 5 & Post-sonication \\
\hline
\end{tabular}

The cumulative \% undersize data for the 1-min recirculation time and the post-sonicated results are given in Table 5.13; these data have been plotted along with the volume contribution \% PSD and are shown in Figure 5.14 and Figure 5.15. The 100\% sonication data (see Appendix I) have also been included on Figure 5.14 and Figure 5.15. Post-sonicated data were collected as a precautionary measure, given the sample dilutions required to obtain a suitable sample obscuration. Dilute samples sometimes form bubbles and or agglomerate as a result of sonication. Neither was observed for either sample analyzed. The data obtained at 1-min recirculation time are more representative of the suspended particles present during settling of actual sludge material while the post-sonicated data better represents the true PSD of the fully suspended sample particles. Based on the similarity and consistency between the sample and duplicate, the PSDs from the samples were averaged. 
PNNL-20650, Rev. 2

Table 5.13. Cumulative Percent Undersize at 1-min Recirculation Time and Post-Sonication

\begin{tabular}{|c|c|c|c|}
\hline \multirow{2}{*}{\multicolumn{2}{|c|}{ Size, $\mu \mathrm{m}$}} & \multicolumn{2}{|c|}{$\begin{array}{c}\text { KW210 -500- } \mu \text { Size Fraction Average }{ }^{(a)} \\
\text { Cumulative Volume \% Less Than }\end{array}$} \\
\hline & & $\begin{array}{c}\text { 1-min Recirculation, } \\
\text { Unsonicated }\end{array}$ & Post-Sonication \\
\hline & 0.05 & 0 & 0 \\
\hline & 0.5 & 0.14 & 0.48 \\
\hline & 1 & 0.68 & 1.90 \\
\hline & 2.5 & 3.28 & 5.83 \\
\hline & 5 & 7.41 & 10.1 \\
\hline & 10 & 11.7 & 14.8 \\
\hline & 20 & 15.2 & 19.8 \\
\hline & 40 & 18.3 & 25.3 \\
\hline & 60 & 20.8 & 29.2 \\
\hline & 80 & 22.8 & 31.9 \\
\hline & 100 & 24.4 & 33.5 \\
\hline & 125 & 26.0 & 34.8 \\
\hline & 150 & 27.7 & 35.9 \\
\hline & 175 & 29.9 & 37.5 \\
\hline & 200 & 32.8 & 39.7 \\
\hline & 300 & 49.1 & 54.0 \\
\hline & 400 & 67.1 & 70.6 \\
\hline & 500 & 81.4 & 84.0 \\
\hline & 600 & 90.9 & 92.9 \\
\hline & 700 & 96.5 & 97.7 \\
\hline & 800 & 99.2 & 99.7 \\
\hline & 1000 & 100 & 100 \\
\hline (a) & $\begin{array}{l}\text { Based on the } \\
\text { of sample TI }\end{array}$ & $\begin{array}{l}\text { (12 PSD measurement } \\
\text { imary) and two aliquot }\end{array}$ & $\begin{array}{l}\text { ted from two aliquots } \\
\text { 08-SQ (duplicate). }\end{array}$ \\
\hline
\end{tabular}


PNNL-20650, Rev. 2

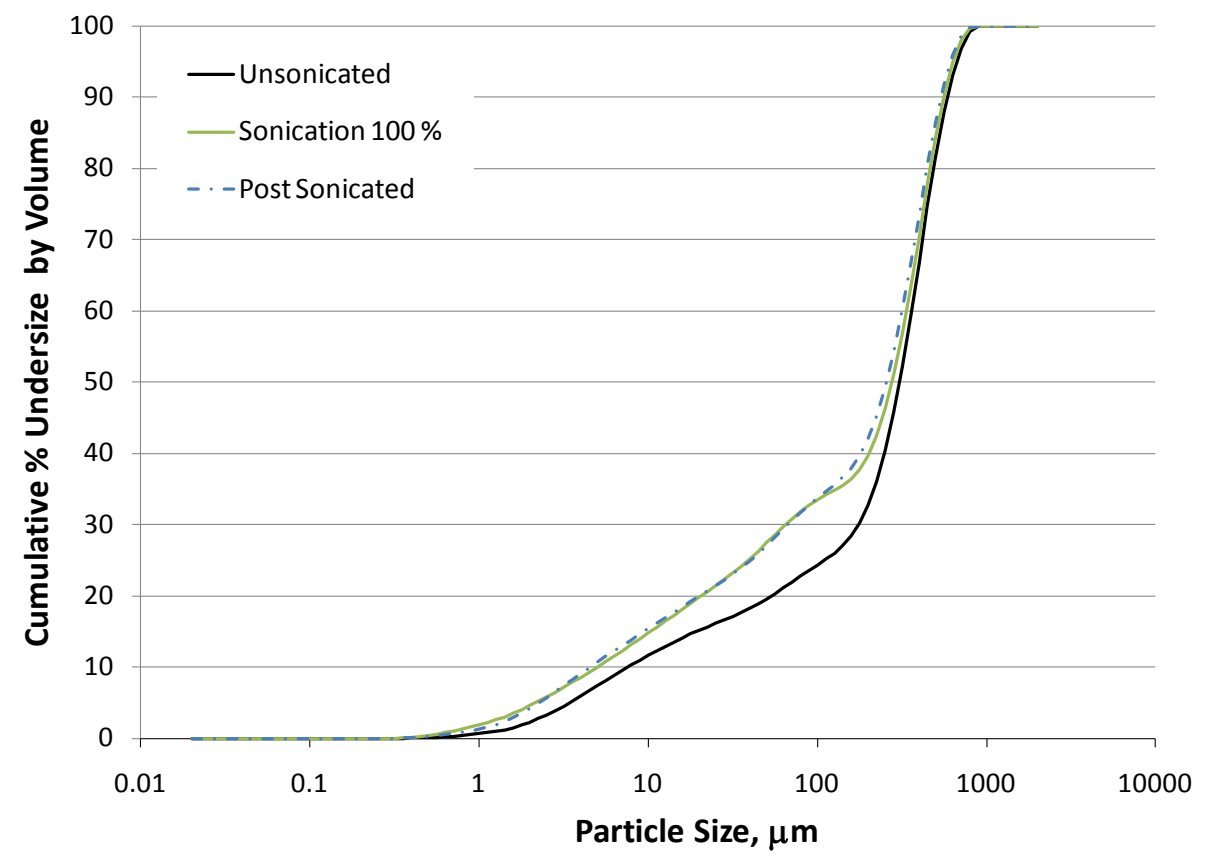

Note: The PSD is based on the average of 12 measurements generated from four aliquots, two aliquots from sample TI008-SP (primary) and two aliquots from TI008-SQ (duplicate)

Figure 5.14. Average Cumulative Volume \% Undersize Plot of KW210-SCC (TI008-SP and TI008SQ, $-500-\mu \mathrm{m}$ Size Fraction)

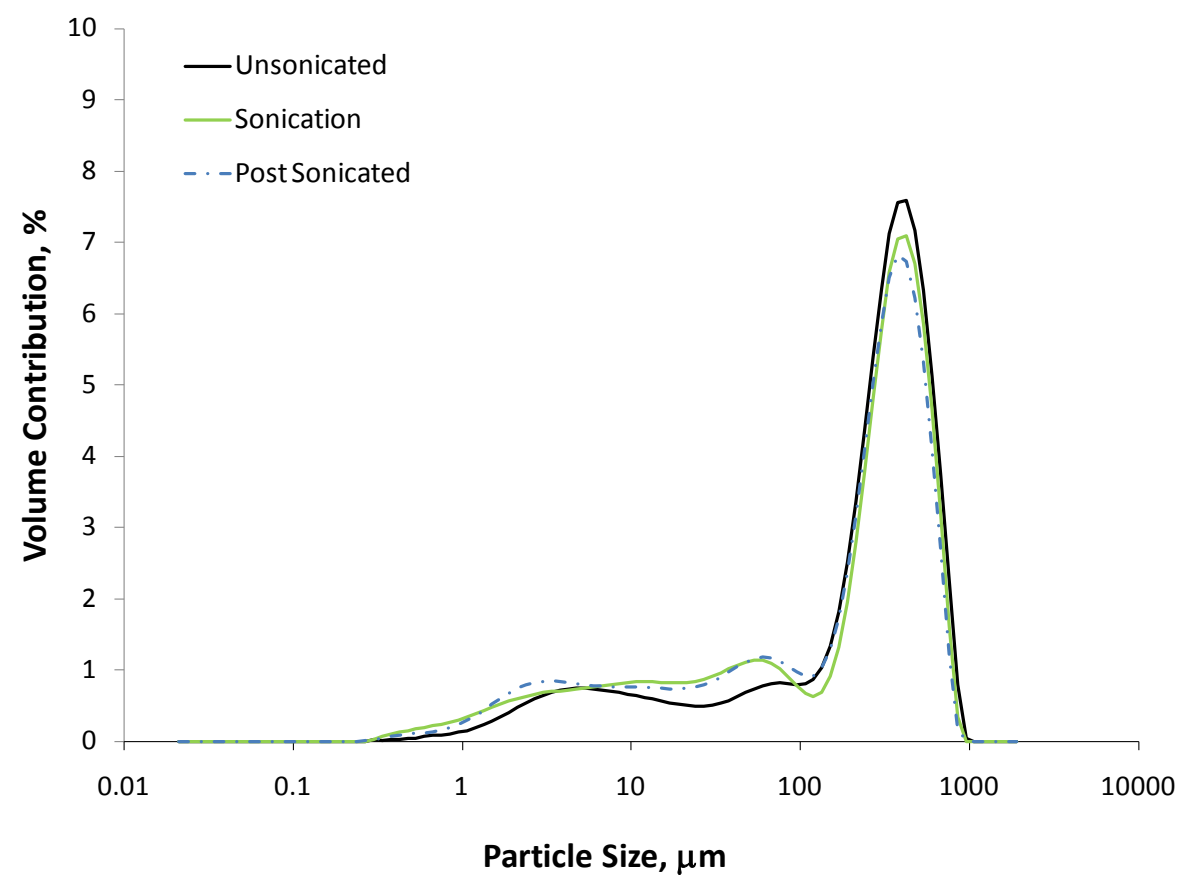

Note: The PSD is based on the average of 12 measurements generated from four aliquots, two aliquots from sample TI008-SP (primary) and two aliquots from TI008-SQ (duplicate)

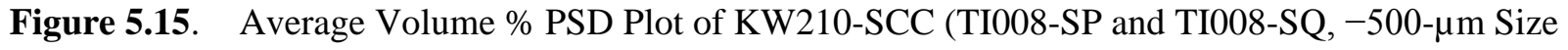
Fraction) 
A major peak in the PSD was observed between 100 and $1000 \mu \mathrm{m}$, apex at $\sim 400 \mu \mathrm{m}$. Resin beads were observed in the $-500-\mu \mathrm{m}$ sieve fraction sample (see Figure 5.17 of the UDS). Product specification for the mixed bed resin Purolite NRW37 indicates that it has a wet PSD between 425 and $1200 \mu \mathrm{m}$ and $<2 \%$ is $<425 \mu \mathrm{m}$. The $\sim 400 \mu \mathrm{m}$ peak observed in Figure 5.15 may in part be represented by the small portion of resin beads that passed through the $500-\mu \mathrm{m}$ mesh sieve.

For all sample aliquots, grinding and knocking sounds could be heard inside the dispersion unit, indicative of large particles caught-up in, and knocking on, internal parts of the dispersion unit. This would suggest that there were particles present in both samples provided for analysis with at least one linear dimension $>500 \mu \mathrm{m}$. Sieving through a $500-\mu \mathrm{m}$ mesh sieve does not always eliminate particles with one dimension $>500 \mu \mathrm{m}$. If large particles are held up in the dispersion components, they may not flow into the observation cell and be measured by the instrument. Additionally, hold-up of large particles may block the flow of smaller particles, preventing their observation. Hence the PSDs provided in Table 5.13, Figure 5.14, and Figure 5.15 may not provide an accurate representation of the sample $(<150 \mu \mathrm{m})$ with the respect to the larger size fraction. The grinding and knocking sounds were not heard once the instrument was flushed, cleaned, and filled with K Basin water for the background measurement, which suggests that the large particles did not accumulate in the system and affect subsequent measurements.

Table 5.14 summarizes selected percentiles for comparing the KW210-SCC samples with the settler sludge simulant and the KW container sludge simulant (Burns et al. 2011). The actual sludge has larger particles present when compared with the K Basin simulants. This is observed in the larger particle size for the $d(50)$ and $d(90)$ for the KW210-SCC samples analyzed. Table 5.14 provides a brief overview of the particle sizes present, in particular the tails of the PSD (upper and lower limits).

Table 5.14. Summary Table of Selected Percentile Values for KW210-SCC (-500- $\mu$ m Size Fraction) and K Basin Simulants

\begin{tabular}{cccccc}
\hline & \multicolumn{2}{c}{ KW210-SCC, $\mu \mathrm{m}$} & \multicolumn{3}{c}{ K Basin Simulants, $\mu \mathrm{m}^{(\mathrm{a})}$} \\
\cline { 2 - 6 } & $\begin{array}{c}\text { 1-min } \\
\text { Recirculation, }\end{array}$ & $\begin{array}{c}100 \% \\
\text { Unsonicated }\end{array}$ & $\begin{array}{c}\text { Settler } \\
\text { Simulant } \\
\text { ST-A }\end{array}$ & $\begin{array}{c}\text { KW Container } \\
\text { Simulant } \\
\text { KW-B } \\
(<500 \mu \mathrm{m})\end{array}$ & $\begin{array}{c}\text { KE Container } \\
\text { Simulant } \\
\text { KE-PNNL-500 } \\
(<500 \mu \mathrm{m})\end{array}$ \\
\hline $\mathrm{d}(0.01)$ & 1.24 & 0.68 & - & - & - \\
$\mathrm{d}(0.05)$ & 3.42 & 2.15 & - & - & - \\
$\mathrm{d}(0.10)$ & 7.52 & 4.96 & - & 3 & 0.7 \\
$\mathrm{~d}(0.20)$ & 53.4 & 20.5 & 5 & - & - \\
$\mathrm{d}(0.50)$ & 305 & 276 & 14 & 25 & 8 \\
$\mathrm{~d}(0.90)$ & 588 & 562 & 334 & 345 & 71 \\
$\mathrm{~d}(0.95)$ & 666 & 636 & - & - & - \\
$\mathrm{d}(0.99)$ & 787 & 750 & - & - & - \\
\hline
\end{tabular}

(a) Burns et al. 2011. Simulant samples PSD results are provided in a sonicated state. 


\subsection{Uranium Speciation}

The MCC and SCC fractions were subsampled for U speciation according to 52578-TI008. The sample collected on the 2,000- $\mu$ m mesh sieve (i.e., the LCC fraction) was not analyzed for U speciation because the sample mass was too small to split for a variety of analyses. Subsamples were processed according to ASR 8824 Special Instructions using selective acid digestion (procedure PNL-ALO-107) that provided separate aliquots for analysis: $U$ metal and $U(I V) / U(V I)$ ratio. All samples were processed (digestion, $\mathrm{U}$ metal analysis by ICP-OES, and U(IV)/U(VI) ratio by spectrometry) in one analytical batch.

Samples provided for analysis, along with cross-reference information (from Section 2.0) are identified in Table 5.15 along with the U speciation results: metal (on wt\% dry mass and wt\% settled sludge mass bases) and U(IV) and U(VI) oxidation state distribution. For reference, the wt\% U total is shown on a dry mass basis as determined from ICP-OES analysis (refer to Table 5.20). Uranium metal was measurable in all samples. The uncertainty for $U$ metal concentrations $>0.03 \mathrm{wt} \%$ is estimated to be $\pm 25 \%(2-\sigma)$ or less.

Table 5.15. Uranium Speciation in KW210-CC Sieve Fractions MCC and SCC: Metal Concentration and Oxide Fractionation (ASR 8824)

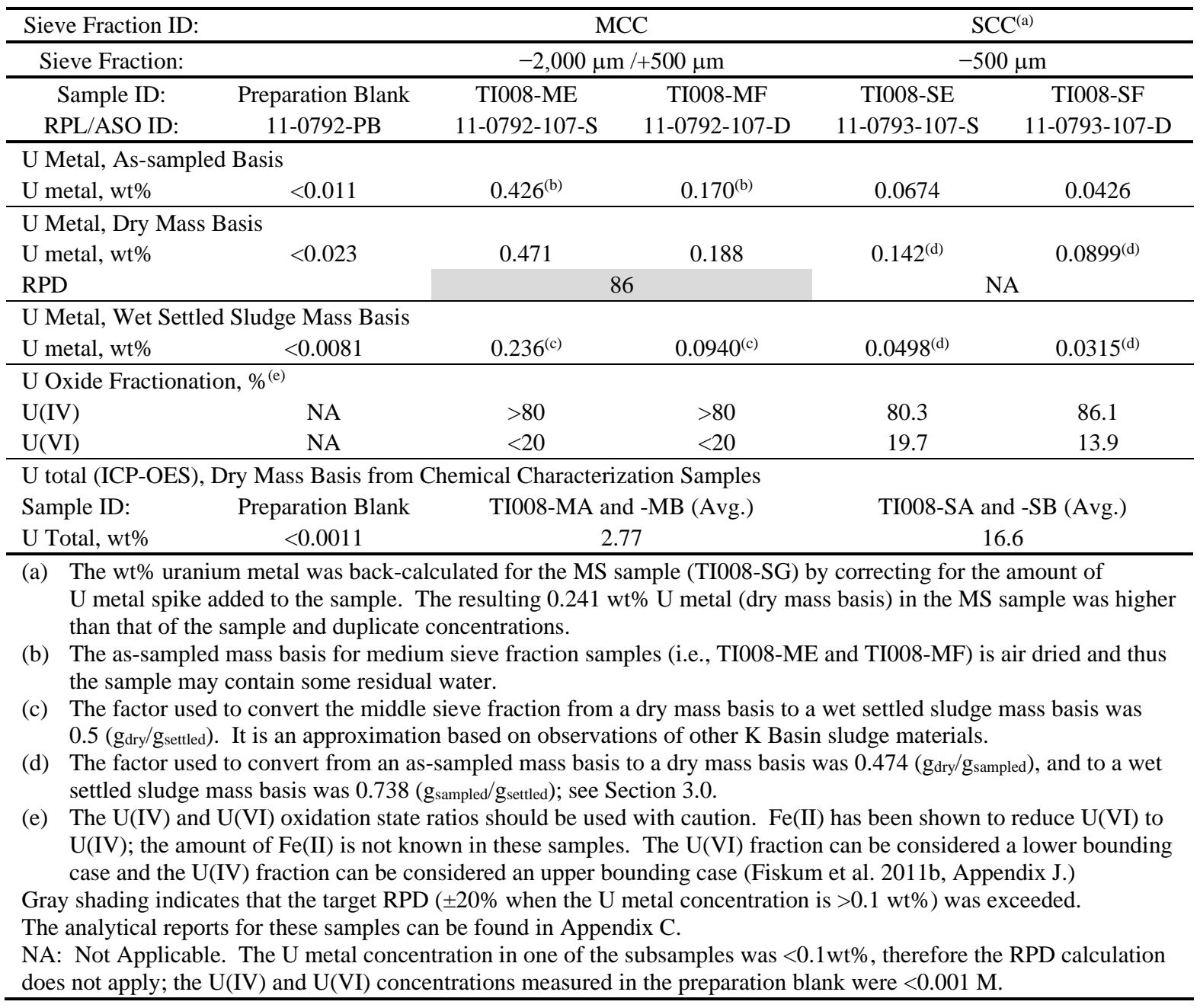


The KW210-CC sieve fraction samples were primarily U(IV). No U(VI) phases were identified by XRD in either the SCC or MCC fractions of the container composite; U(IV) phases were identified in the SCC fraction. Iron (II)-bearing phases (such as magnetite and iron oxide $\left[\mathrm{Fe}_{3} \mathrm{O}_{4}\right]$ ) were not specifically identified by XRD in these fractions; however, Figure 4.5 shows that during the sample aliquoting process, an appreciable quantity of particles were attracted to the Teflon coated magnetic stir bars. It is possible that the $\mathrm{U}$ oxidation state ratios could be confounded by reduction of U(VI) with the matrixrelated Fe(II) (Fiskum et al. 2011b, Appendix J). Thus, the reported oxidation state fractions should be used with caution. The difference between the sample and duplicate oxidation state ratios is $5.8 \%$.

The QAPjP/SAP required $\pm 20 \%$ RPD when the U metal content was $>0.1 \mathrm{wt} \%$ wet settled mass basis. Subsamples from both the MCC and SCC fractions were processed in duplicate. The MCC sample and duplicate RPD (86\%) exceeded the acceptance criterion (U metal concentration 0.471 and 0.188 wt\% dry mass basis). The variability was higher than the MCC sample/sample duplicate variability determined from ICP-OES (see Section 5.8). One of the duplicate SCC sample U metal concentrations was $<0.1 \mathrm{wt} \%$, therefore the duplicate precision calculation was not applicable.

Table 5.16 summarizes the $\mathrm{U}$ speciation QC sample results. The QAPjP/SAP required a measure of accuracy ( 75 to $125 \%$ recovery) for a LCS (U metal and $\mathrm{U}_{3} \mathrm{O}_{8}$ added to Hanford sand matrix). The $\mathrm{U}$ metal LCS recovery was $103 \%$. The U oxidation state fractionation of the LCS sample was well within the acceptance criterion. Opportunistically, a replicate SCC (TI008-SG) sample was processed as an MS sample. It recovered $123 \%$ of the added U metal. Assuming that the amount of U metal added in the MS was recovered at $100 \%$, the U metal concentration in the TI008-SG sample was back-calculated to be $0.241 \mathrm{wt} \%$ on a dry mass basis. Thus, the average U metal concentration in the three analyzed KW210SCC samples was $0.158 \mathrm{wt} \% \pm 0.077 \%$ (48\% RSD), at one $\sigma$, dry mass basis. U metal distribution heterogeneity is shown in these three separate analyses. The $U$ measured in the solids $7^{\text {th }}$ rinse solution (before nitric acid dissolution of U metal) ranged from 0.04 to $1.5 \mu \mathrm{g} / \mathrm{mL}$, well below the $100-\mu \mathrm{g} / \mathrm{mL}$ acceptance threshold, indicating the measured $U$ metal was not confounded by carryover of non-metallic $\mathrm{U}$. Detailed results and a discussion of the $\mathrm{U}$ metal and $\mathrm{U}$ oxidation state analyses can be found in Appendix C.

Table 5.16. Uranium Speciation QC Sample Results Summary

\begin{tabular}{|c|c|}
\hline & $\begin{array}{c}\text { Process Batch QC } \\
\text { Sample Results }\end{array}$ \\
\hline \multicolumn{2}{|l|}{ U Metal } \\
\hline LCS Recovery & $103 \%$ \\
\hline MS Recovery (Opportunistic) on SCC Sample (TI008-SG) & $123 \%$ \\
\hline \multicolumn{2}{|l|}{ U Oxidation State LCS Recovery } \\
\hline 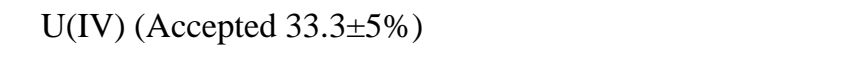 & Measured 34.2\% \\
\hline 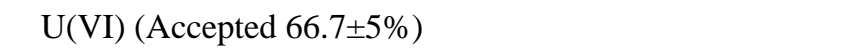 & Measured 65.8\% \\
\hline
\end{tabular}

\subsection{Chemical and Radiochemical Analysis}

Chemical and radiochemical analyses were conducted according to ASR 8824. Subsamples from the middle and small particle size sieve fractions were processed by acid digestion (PNL-ALO-129 procedure) in preparation for analysis: GEA, AEA screen, and ICP-OES. Because a small amount of 
sample was collected on the 2,000- $\mu$ m mesh sieve, the sample was measured directly by GEA and for particle density. Samples provided for chemical analysis, along with cross-reference information (from Section 2.0) are identified in Table 5.17. The ASO analytical reports for these samples can be found in Appendix C.

Table 5.17. ASR 8824 Batch Processing by PNL-ALO-129 Acid Digestion

\begin{tabular}{lclll}
\hline Sieve Fraction & Fraction Code & Sample ID & RPL/ASO ID & Analysis \\
\hline$+2,000 \mu \mathrm{m}$ & LCC & TI008-LA ${ }^{(\mathrm{a})}$ & $11-0791-\mathrm{S}^{(\mathrm{a})}$ & Direct GEA only \\
\hline$-2,000 \mu \mathrm{m} /+500 \mu \mathrm{m}$ & MCC & TI008-MA & $11-0792-129-\mathrm{S}$ & Radiochemistry, \\
& & TI008-MB & $11-0792-129-\mathrm{D}$ & ICP-OES metals \\
\hline \multirow{2}{*}{$-500 \mu \mathrm{m}$} & \multirow{2}{*}{ SCC } & TI008-SA & $11-0793-129-\mathrm{S}$ & Radiochemistry, \\
& & TI008-SB & $11-0793-129-\mathrm{D}$ & ICP-OES metals \\
& & TI008-SC & $11-0793-129-\mathrm{MS}$ & \\
\hline
\end{tabular}

(a) There was insufficient mass to conduct the acid digestion and other analyses. Sample was only analyzed directly by GEA and for particle density. No acid digestion was performed. This sample is identified as TI008-L for particle density analysis.

The holding time applicable to ICP-OES AOIs was calculated for the preparation batch samples. The sample date was defined as the date associated with the first of the core sample material that was removed from SCS-CON-210. The end of the holding time was defined as the date of the ICP-OES analysis for the sieve fractions of the container composite. In all cases, the 6-month holding time requirement was met (Table 5.18).

Table 5.18. KW210-CC Sieve Fraction Sample Holding Times Supporting WIPP Hazardous Waste Permit Metals Analysis

\begin{tabular}{c|c|c|c|c}
\hline $\begin{array}{c}\text { Core Sample } \\
\text { ID }\end{array}$ & First Sample Date & $\begin{array}{c}\text { Acid Digestion } \\
\text { Date }\end{array}$ & $\begin{array}{c}\text { ICP-OES } \\
\text { Analysis Date }\end{array}$ & $\begin{array}{c}\text { Effective Holding } \\
\text { Time, Months }\end{array}$ \\
\hline KW210-A1 & $12 / 29 / 2010$ & & & 3.93 \\
KW210-A4 & $11 / 17 / 2010$ & April 20, 2011 & April 26, 2011 & 5.33 \\
KW210-B1 & $12 / 1 / 2010$ & & & 4.87 \\
KW210-B3 & $12 / 15 / 2010$ & & & 4.40 \\
\hline
\end{tabular}

(a) The effective holding time was calculated from the first core sample collection date to the last sample analysis date (e.g., November 17, 2010 to April 26, 2011).

The pre-digestion dry masses, as-settled sludge masses (SCC fractions), and UDS dry masses following the acid digestion procedure are shown in Table 5.19. The UDS masses were also calculated relative to a wet settled solids mass basis for the SCC solids only. The applied conversion factors, $\mathrm{g}_{\mathrm{dry}} / \mathrm{g}_{\text {settled }}$, are also shown, as applicable. The UDS of the SCC and MCC fractions exceeded the $5 \mathrm{wt} \%$ threshold of the settled solids mass, thus triggering the need for GEA and XRD analyses. Additional analyses (dissolution using fusion and ICP-OES and AEA screen) of the UDS were requested by the BTR. 
Table 5.19. Chemical Analysis Characterization Samples for Acid Dissolution, Net Residue Masses, and UDS Weight Fractions (ASR 8824)

\begin{tabular}{|c|c|c|c|c|c|c|c|c|c|}
\hline \multirow[b]{2}{*}{$\begin{array}{c}\text { Sieve } \\
\text { Fraction }\end{array}$} & \multirow[b]{2}{*}{ Sample ID } & \multirow[b]{2}{*}{ RPL/ASO ID } & \multirow[b]{2}{*}{$\begin{array}{c}\text { Pre-digest } \\
\text { dry mass, } \\
\text { gdry }\end{array}$} & \multirow[b]{2}{*}{$\begin{array}{c}\text { Conversion } \\
\text { factor, } \\
\text { gdry } / \mathrm{g}_{\text {settled }} \\
\end{array}$} & \multirow{2}{*}{$\begin{array}{c}\text { Pre-digest } \\
\text { settled sample } \\
\text { mass, }{ }^{\text {(a) }} \\
\text { gsettled }^{\text {gample }}\end{array}$} & \multirow[b]{2}{*}{$\begin{array}{l}\text { UDS post- } \\
\text { digest dry } \\
\text { mass, gresidual }\end{array}$} & \multicolumn{3}{|c|}{ Weight Fraction UDS } \\
\hline & & & & & & & $\begin{array}{c}\text { Dry mass basis, } \\
\text { gresidual / gdry }\end{array}$ & $\begin{array}{l}\text { RPD } \\
\text { or } \\
\text { RSD }\end{array}$ & $\begin{array}{c}\text { Settled mass } \\
\text { basis, }{ }^{(a)} \\
\text { gresidual / } \text { g }_{\text {settled }}\end{array}$ \\
\hline \multirow[t]{2}{*}{ MCC } & TI008-MA & 11-0792-129-S & 1.3641 & -- & -- & 1.1750 & 0.8614 & 0.70 & -- \\
\hline & TI008-MB & 11-0792-129-D & 1.7754 & -- & -- & 1.5186 & 0.8554 & & -- \\
\hline \multirow[t]{3}{*}{ SCC } & TI008-SA & 11-0793-129-S & 0.8873 & 0.350 & 2.537 & 0.4087 & 0.4606 & 3.0 & 0.161 \\
\hline & TI008-SB & 11-0793-129-D & 1.0879 & 0.350 & 3.111 & 0.5009 & 0.4604 & & 0.161 \\
\hline & TI008-SC & 11-0793-129-MS & 0.8999 & 0.350 & 2.573 & 0.3934 & 0.4372 & & 0.153 \\
\hline
\end{tabular}

(a) Conversion factors, pre-digestion wet settled masses, and the weight fraction of UDS on a settled mass basis for the MCC samples were not determined as part of the sieving process/procedure.

Note: The LCC sample (TI008-LA) was not acid-digested. 


\subsubsection{ICP-OES Analysis}

Table 5.20 and Table 5.21 summarize the ICP-OES AOI results and opportunistic analyte results, respectively. The reporting mass basis is relative to the dry solids mass submitted to the acid digestion step. Also reported are the process batch QC sample results. All samples were analyzed in one process batch. The RPDs were calculated from the MCC sample and duplicate as well as the SCC sample and duplicate. A SCC sample (TI008-SC) was used as the process batch MS sample.

The ICP-OES measurements have been shown to have a general overall uncertainty of $\pm 15 \%$. Bracketed values indicate that concentrations were measured at less than the EQL but greater than the MDL; uncertainty for these values will likely be higher than $15 \%$. The less-than concentrations were determined by multiplying the instrument detection limit by the sample-specific process factor; process factor components include the sample mass, final volume of digestate, and any dilutions. All samples were diluted $10 \times$ at the ICP-OES analytical workstation. Results are based on this additional $10 \times$ dilution with the exception of $\mathrm{As}, \mathrm{Sb}$, Se, and $\mathrm{Tl}$ where results are based on the undiluted digestate.

The RPDs for all analytes from the SCC sample and duplicate met the acceptance criterion of $\pm 20 \%$. The MCC sample and duplicate analytes $\mathrm{Ba}, \mathrm{Cr}, \mathrm{Fe}, \mathrm{Ni}, \mathrm{Pb}$, and $\mathrm{Zr}$ exceeded the $\pm 20 \% \mathrm{RPD}$ criterion. All AOIs in the LCS met the accuracy acceptance criterion of $\pm 20 \%$ (80 to $120 \%$ recovery). The MS sample met the $\pm 20 \%$ accuracy acceptance criterion (80 to 120\% analyte recovery) for all AOIs except $\mathrm{Ca}, \mathrm{Na}$, and Tl. The MS sample recoveries for $\mathrm{Al}, \mathrm{Fe}$, and $\mathrm{U}$ are listed as not recovered (NR) because the spiked quantity was less than $25 \%$ of the sample analyte concentration. Sources of error for these analytes are evaluated from the post-spike recoveries and the serial dilution $(5 \times$ dilution of sample, where $10 \%$ difference is acceptable per the analysis procedure). Detection-limit requirements defined in the QAPjP/SAP were met for all AOIs, and/or the analyte was present in sufficient concentration that the detection limit was not challenged.

The mass balance was calculated to evaluate the overall consistency of the analytical data and to understand which analytes gravitate to the MCC versus SCC fractions. Table 5.22 shows the calculated AOI masses in the input container composite sample relative to the average analyte masses in the MCC and SCC fractions. The estimated input analyte mass $\left(\mathrm{A}_{\text {input }}\right)$ is based on the measured analyte concentrations (C) from each core sample multiplied by its dry mass fractionation (F) in the container composite and divided by the mass of the container composite (65 g, dry basis) according to Equation 5.3. See Table 4.10 for core sample concentrations on dry mass basis and Table 5.2 for the dry mass fractionation.

$$
\mathrm{A}_{\text {input }}=\frac{\mathrm{C}_{\mathrm{A} 1} \times \mathrm{F}_{\mathrm{A} 1}+\mathrm{C}_{\mathrm{A} 4} \times \mathrm{F}_{\mathrm{A} 4}+\mathrm{C}_{\mathrm{B} 1} \times \mathrm{F}_{\mathrm{B} 1}+\mathrm{C}_{\mathrm{B} 3} \times \mathrm{F}_{\mathrm{B} 3}}{65 \mathrm{~g}}
$$

The analyte masses of the MCC and SCC fractions were calculated by multiplying the average analyte concentration (Table 5.20) by the recovered mass in the sieve fraction (dry mass basis) (see Table 5.5). The ratio (sum/input) represents the summed analyte masses from the SCC and MCC fractions divided by the input mass. Ratios between 0.75 and 1.25 were considered to show reasonable and consistent mass balances. Most ratios were within these bounds (with the exception of Ag, Ba, Be, $\mathrm{Pb}, \mathrm{Sb}, \mathrm{V}$, and $\mathrm{Zr}$ ) indicating the mass balance was reasonably consistent. 
Table 5.20. KW210-CC Sieve Fractions ICP-OES AOI Results, Dry Mass Basis (ASR 8824)

\begin{tabular}{|c|c|c|c|c|c|c|c|c|c|c|c|}
\hline Sieve Fraction > & NA & MCC & MCC duplicate & SCC & SCC duplicate & \multicolumn{6}{|c|}{ Process Batch QC Results } \\
\hline TI Sample ID > & & TI008-MA & TI008-MB & TI008-SA & TI008-SB & & & & TI008-SC & & \\
\hline $\begin{array}{c}\text { RPL/ASO } \\
\text { ID > }\end{array}$ & $\mathrm{PB}$ & $\begin{array}{c}11-0792- \\
129-S\end{array}$ & $\begin{array}{c}11-0792- \\
129-D\end{array}$ & $\begin{array}{c}11-0793- \\
129-S\end{array}$ & $\begin{array}{c}11-0793- \\
129-\mathrm{D}\end{array}$ & $\begin{array}{c}\text { MCC \& } \\
\text { MCC dup }\end{array}$ & $\begin{array}{c}\text { SCC \& } \\
\text { SCC dup }\end{array}$ & $\operatorname{LCS}^{(\mathrm{b})}$ & $\begin{array}{l}11-0793- \\
129-\mathrm{MS}^{(\mathrm{b})}\end{array}$ & $\begin{array}{l}\text { 11-0793-S } \\
\text { Post Spike }\end{array}$ & $\begin{array}{c}\text { 11-0792-S } \\
\text { Serial } \\
\text { Dilution }\end{array}$ \\
\hline (Analyte) & $(\mu \mathrm{g} / \mathrm{g})^{(\mathrm{a})}$ & $(\mu \mathrm{g} / \mathrm{g})^{(\mathrm{a}, \mathrm{b})}$ & $(\mu \mathrm{g} / \mathrm{g})^{(\mathrm{a}, \mathrm{b})}$ & $(\mu \mathrm{g} / \mathrm{g})^{(\mathrm{a})}$ & $(\mu \mathrm{g} / \mathrm{g})^{(\mathrm{a})}$ & $\mathrm{RPD}^{(\mathrm{b})}$ & RPD & \% Recovery & $\%$ Recovery & \% Recovery & \% Difference \\
\hline Ag & $<1.5$ & [7.8] & $<1.1$ & 46.7 & 45.2 & -- & 3.3 & 101 & 102 & 103 & -- \\
\hline $\mathrm{Al}$ & $<3.6$ & 17,200 & 15,300 & 36,200 & 34,000 & 11.4 & 6.4 & 103 & NR & NR & 0.5 \\
\hline As & $<7.5$ & [35] & [31] & 182 & 167 & -- & 8.1 & 89 & 90 & 100 & -- \\
\hline $\mathrm{Ba}$ & {$[0.097]$} & 141 & 316 & 81.4 & 82.0 & 76.7 & 0.7 & 102 & 101 & 101 & 1.0 \\
\hline $\mathrm{Be}$ & $<0.052$ & 6.44 & 6.62 & 15.9 & 15.2 & 2.8 & 4.7 & 103 & 102 & 99 & 4.7 \\
\hline $\mathrm{Ca}$ & $<1.7$ & 9,220 & 9,800 & 2,080 & 1,770 & 6.0 & 16.3 & 102 & 162 & 101 & 0.2 \\
\hline $\mathrm{Cd}$ & $<1.2$ & 36.6 & 38.1 & 51.5 & 45.5 & 3.9 & 12.4 & 106 & 107 & 92 & --- \\
\hline $\mathrm{Cr}$ & $<1.6$ & 137 & 209 & 714 & 671 & 41.7 & 6.3 & 98 & 104 & 104 & 2.7 \\
\hline $\mathrm{Fe}$ & [3.8] & 37,100 & 49,500 & 135,000 & 130,000 & 28.7 & 3.7 & 104 & NR & NR & 1.2 \\
\hline $\mathrm{Mg}$ & $<0.48$ & 675 & 808 & 1,090 & 981 & 17.9 & 10.5 & 103 & 119 & 98 & 2.0 \\
\hline $\mathrm{Na}$ & 71.5 & 1,450 & 1,570 & 449 & 384 & 8.0 & 15.6 & 103 & 123 & 98 & 4.6 \\
\hline $\mathrm{Ni}$ & $<2.0$ & 99.2 & 149 & 214 & 194 & 40.2 & 9.8 & 106 & 107 & 106 & 2.6 \\
\hline $\mathrm{Pb}$ & $<16$ & 175 & 260 & 925 & 936 & 39.1 & 1.2 & 108 & 107 & 114 & -- \\
\hline $\mathrm{Sb}$ & $<1.7$ & [5.8] & [4.1] & [15] & [17] & -- & -- & 89 & 87 & 96 & -- \\
\hline Se & $<13$ & $<12$ & $<9.6$ & $<19$ & $<16$ & -- & -- & 90 & 91 & 98 & -- \\
\hline $\mathrm{Tl}$ & $<6.7$ & $<6.3$ & $<4.8$ & [15] & [12] & -- & -- & 86 & 78 & 89 & -- \\
\hline $\mathrm{U}$ & $<9.4$ & 30,300 & 25,000 & 172,000 & 160,000 & 18.9 & 7.3 & 102 & NR & 102 & 0.7 \\
\hline V & $<0.86$ & [14] & 16.6 & 58.2 & 56.0 & - & 3.9 & 103 & 103 & 97 & -- \\
\hline Zn & [6.7] & 250 & 240 & 505 & 474 & 4.1 & 6.4 & 100 & 103 & 103 & 4.5 \\
\hline $\mathrm{Zr}$ & $<0.66$ & 573 & 94.9 & 432 & 363 & 143.2 & 17.3 & 107 & NR & 102 & 1.0 \\
\hline
\end{tabular}

(a) Results shown in brackets indicate that the analyte concentrations were $>$ MDL but $<$ EQL, and measurement uncertainties were $>15 \%$. Less-than values represent the instrument detection limit multiplied by the dilution process factors.

(b) Gray shading indicates that the target QC criterion was exceeded. The targeted RPD was $\pm 20 \%$ and the LCS and MS targeted recoveries were $80 \%$ to $120 \%$.

NR: Not Recovered. This indicates the spike concentration was less than $25 \%$ of the sample concentration.

Dashed lines (--) indicate the measurement calculation was not applicable. 
Table 5.21. KW210-CC Sieve Fractions ICP-OES Opportunistic Analyte Results, Dry Mass Basis (ASR 8824)

\begin{tabular}{|c|c|c|c|c|c|c|c|c|c|c|c|}
\hline Sieve Fraction > & NA & MCC & MCC duplicate & SCC & SCC duplicate & & & Process Ba & ch QC Results & & \\
\hline TI Sample ID > & & TI008-MA & TI008-MB & TI008-SA & TI008-SB & & & & TI008-SC & 11-0793 & 11-0792 \\
\hline $\begin{array}{c}\text { RPL/ASO } \\
\mathrm{ID}>\end{array}$ & $\mathrm{PB}$ & $\begin{array}{c}11-0792- \\
129-S\end{array}$ & $\begin{array}{c}11-0792- \\
129-\mathrm{D}\end{array}$ & $\begin{array}{c}\text { 11-0793- } \\
129-S \\
\end{array}$ & $\begin{array}{c}11-0793- \\
129-\mathrm{D}\end{array}$ & $\begin{array}{c}\text { MCC \& } \\
\text { MCC dup } \\
\end{array}$ & $\begin{array}{c}\text { SCC \& } \\
\text { SCC dup } \\
\end{array}$ & LCS & $\begin{array}{c}11-0793- \\
129-M S\end{array}$ & Post Spike & $\begin{array}{c}\text { Serial } \\
\text { Dilution } \\
\end{array}$ \\
\hline (Analyte) & $(\mu \mathrm{g} / \mathrm{g})^{(\mathrm{a})}$ & $(\mu \mathrm{g} / \mathrm{g})^{(\mathrm{a}, \mathrm{b})}$ & $(\mu \mathrm{g} / \mathrm{g})^{(\mathrm{a}, \mathrm{b})}$ & $(\mu \mathrm{g} / \mathrm{g})^{(\mathrm{a})}$ & $(\mu \mathrm{g} / \mathrm{g})^{(\mathrm{a})}$ & $\mathrm{RPD}^{(\mathrm{b})}$ & $\mathrm{RPD}$ & \% Recovery & \% Recovery & \% Recovery & \% Difference \\
\hline $\mathrm{B}$ & {$[22]$} & {$[24]$} & [17] & [36] & {$[36]$} & -- & -- & 104 & 105 & 101 & -- \\
\hline $\mathrm{Bi}$ & [59] & [63] & [43] & [120] & [98] & -- & -- & -- & -- & 92 & -- \\
\hline $\mathrm{Ce}$ & $<6.8$ & [25] & [17] & 155 & 136 & -- & 12.8 & 101 & 98 & 105 & -- \\
\hline Co & $<2.0$ & [4.2] & [3.3] & {$[5.4]$} & [3.9] & -- & -- & -- & -- & 96 & -- \\
\hline $\mathrm{Cu}$ & $<1.3$ & 423 & 403 & 1,920 & 1,820 & 4.7 & 5.5 & 110 & NR & NR & 0.5 \\
\hline Dy & $<0.94$ & $<0.88$ & $<0.68$ & $<1.4$ & $<1.1$ & -- & -- & -- & -- & 101 & -- \\
\hline $\mathrm{Eu}$ & $<0.21$ & {$[0.25]$} & [0.58] & 4.09 & 4.41 & -- & 7.6 & -- & -- & 102 & -- \\
\hline $\mathrm{K}$ & [120] & 461 & 479 & 541 & 470 & 3.7 & 14.0 & 105 & 109 & 99 & -- \\
\hline $\mathrm{La}$ & $<0.94$ & 13.0 & 13.5 & 43.1 & 40.0 & 4.1 & 7.6 & 99 & 101 & 101 & -- \\
\hline $\mathrm{Li}$ & 5.39 & 5.83 & 5.25 & 14.1 & 11.8 & 10.5 & 17.6 & 113 & 108 & 105 & -- \\
\hline $\mathrm{Mn}$ & $<0.17$ & 250 & 305 & 279 & 253 & 19.8 & 10.0 & 107 & 113 & 108 & 1.8 \\
\hline Mo & $<5.5$ & [20] & [17] & [68] & [64] & -- & -- & 105 & 109 & 105 & -- \\
\hline $\mathrm{Nd}$ & $<2.1$ & 24.6 & [13] & 146 & 135 & -- & 7.3 & 97 & 101 & 103 & -- \\
\hline $\mathrm{P}$ & $<40$ & [120] & [150] & 819 & 734 & -- & 10.9 & -- & 118 & 96 & -- \\
\hline Pd & $<3.8$ & [4.6] & [3.1] & [6.9] & [6.8] & -- & -- & -- & -- & 99 & -- \\
\hline $\mathrm{Rh}$ & [7.8] & $<6.5$ & $<5.0$ & $<9.9$ & $<8.1$ & -- & -- & -- & -- & 98 & -- \\
\hline $\mathrm{Ru}$ & $<3.4$ & $<3.2$ & $<2.5$ & [37] & [31] & -- & -- & -- & -- & 101 & -- \\
\hline $\mathrm{S}$ & $<125$ & 9,130 & 9,230 & 2,260 & 1,940 & 1.1 & 15.5 & -- & 99 & 102 & 0.6 \\
\hline $\mathrm{Si}$ & [13] & 583 & 506 & 1,220 & 1,280 & 14.1 & 4.6 & 108 & $0^{(\mathrm{b})}$ & 98 & 3.4 \\
\hline Sn & $<11$ & [22] & [29] & [61] & [68] & -- & -- & -- & -- & 85 & -- \\
\hline $\mathrm{Sr}$ & [0.025] & 51.3 & 54.9 & 15.9 & 14.1 & 6.7 & 12.1 & 103 & 128 & 102 & 0.3 \\
\hline $\mathrm{Ta}$ & $<15$ & $<14$ & $<11$ & $<21$ & $<17$ & -- & -- & -- & -- & 98 & -- \\
\hline $\mathrm{Te}$ & $<13$ & $<12$ & [13] & [52] & [26] & -- & -- & -- & -- & 101 & -- \\
\hline Th & $<3.9$ & [34] & [26] & 205 & 180 & -- & 13.0 & 100 & 101 & 102 & -- \\
\hline $\mathrm{Ti}$ & $<0.41$ & 221 & 394 & 453 & 400 & 56.1 & 12.5 & 106 & 107 & 112 & 2.1 \\
\hline W & $<12$ & $<11$ & $<8.4$ & $<17$ & [26] & -- & -- & -- & -- & 98 & -- \\
\hline $\mathrm{Y}$ & $<0.20$ & [2.4] & 2.97 & 11.3 & 10.7 & -- & 5.7 & -- & -- & 98 & -- \\
\hline
\end{tabular}

(a) Results shown in brackets indicate that the analyte concentrations were $>$ MDL but $<$ EQL, and measurement uncertainties were $>15 \%$. Less-than values represent the instrument detection limit multiplied by the dilution process factors.

(b) Gray shading indicates that the RPD exceeded $\pm 20 \%$ and uncharacteristic failure of the Si MS recovery.

NR: Not Recovered. This indicates the spike concentration was less than $25 \%$ of the sample concentration.

Dashed lines (--) indicate the measurement calculation was not applicable. 
Table 5.22. Acid Soluble Analyte Distribution Between MCC $(-2,000-\mu \mathrm{m} /+500-\mu \mathrm{m})$ and SCC $(-500-\mu \mathrm{m})$ Sieve Fractions Relative to the Calculated Input Composition

\begin{tabular}{|c|c|c|c|c|}
\hline (Analyte) & $\begin{array}{c}\text { Calculated } \\
\text { KW210-CC-N } \\
(\mu \mathrm{g})\end{array}$ & $\begin{array}{c}\text { KW210-MCC } \\
(\mu \mathrm{g})\end{array}$ & $\begin{array}{c}\text { KW210-SCC } \\
(\mu g)\end{array}$ & $\begin{array}{c}\text { Ratio } \\
\text { Sum/Input }\end{array}$ \\
\hline Ag & [230] & [240] & 986 & [5.3] \\
\hline $\mathrm{Al}$ & $1,420,000$ & 897,000 & 753,000 & 1.16 \\
\hline As & [4500] & [1800] & 3,740 & [1.2] \\
\hline $\mathrm{Ba}$ & 8,410 & 12,600 & 1,750 & 1.71 \\
\hline $\mathrm{Be}$ & 526 & 360 & 334 & 1.32 \\
\hline $\mathrm{Ca}$ & 477,000 & 525,000 & 41,400 & 1.19 \\
\hline $\mathrm{Cd}$ & 2,700 & 2,060 & 1,040 & 1.15 \\
\hline $\mathrm{Cr}$ & 22,000 & 9,550 & 14,900 & 1.11 \\
\hline $\mathrm{Fe}$ & $4,480,000$ & $2,390,000$ & $2,840,000$ & 1.17 \\
\hline $\mathrm{Mg}$ & 53,100 & 40,900 & 22,300 & 1.19 \\
\hline $\mathrm{Na}$ & 84,100 & 83,300 & 8,930 & 1.10 \\
\hline $\mathrm{Ni}$ & 13,200 & 6,850 & 4,380 & 0.85 \\
\hline $\mathrm{Pb}$ & 19,500 & 12,000 & 20,000 & 1.64 \\
\hline $\mathrm{Sb}$ & [450] & [270] & [340] & [1.4] \\
\hline Se & $<970$ & $<610$ & $<370$ & -- \\
\hline $\mathrm{Tl}$ & $<550$ & $<310$ & [290] & -- \\
\hline $\mathrm{U}$ & $4,810,000$ & $1,530,000$ & $3,560,000$ & 1.06 \\
\hline V & [820] & [840] & 1220 & [2.5] \\
\hline $\mathrm{Zn}$ & 21,800 & 13,500 & 10,500 & 1.10 \\
\hline $\mathrm{Zr}$ & 16,200 & 18,400 & 8,530 & 1.66 \\
\hline \multicolumn{5}{|c|}{ Shaded ratios indicate mass balance divergence from the range of 0.75 to 1.25 . } \\
\hline \multicolumn{5}{|c|}{$\begin{array}{l}\text { Bracketed mass values indicate the ICP-OES result was }<\text { EQL. Bracketed ratios } \\
\text { indicate the ratio is calculated with a value that was }<\text { EQL. }\end{array}$} \\
\hline
\end{tabular}

Examples of analyte distribution from the data provided in Table 5.22 are as follows. Aluminum, beryllium, iron, and zinc are approximately evenly distributed between the MCC and SCC sieve fractions. Barium, calcium, and sodium are primarily in the MCC fraction. Cadmium, magnesium, nickel and zirconium split between fractions but were more heavily associated with the MCC fraction. Chromium, lead and uranium split between fractions but are more heavily associated with the SCC fraction. Silver appears to be heavily associated with the SCC fraction and measured 4 times higher than the calculated input. Note that these mass distributions only represent the acid-soluble materials. Some of these elements are likely also present in the UDS (see Section 5.8.3).

\subsubsection{Radiochemistry Analysis}

The radiochemistry analysis of the container composite acid digestates was limited to GEA and AEA screen. ${ }^{(a)}$ The GEA and AEA screen results from each sieve fraction are shown in Table 5.23. All results are reported on a dry mass basis. The PB results are also provided for direct comparison to the sample results.

(a) The AEA screen measures ${ }^{239+240} \mathrm{Pu}$ and the combined ${ }^{238} \mathrm{Pu}$ plus ${ }^{241} \mathrm{Am}$. Curium isotopes are also measured and reported opportunistically. 
PNNL-20650, Rev. 2

Table 5.23. KW210-CC Sieve Fraction Radionuclide Analysis, Dry Mass Basis (ASR 8824)

\begin{tabular}{|c|c|c|c|c|c|c|c|c|c|c|}
\hline $\begin{array}{c}\text { Sieve } \\
\text { Fraction }>\end{array}$ & NA & LCC & MCC & $\begin{array}{c}\text { MCC } \\
\text { Duplicate }\end{array}$ & SCC & $\begin{array}{c}\text { SCC } \\
\text { Duplicate }\end{array}$ & \multicolumn{4}{|c|}{ QC Sample Results } \\
\hline $\begin{array}{c}\text { TI Sample } \\
\text { ID > }\end{array}$ & & TI008-LA & TI008-MA & TI008-MB & TI008-SA & TI008-SB & $\begin{array}{c}\text { MCC \& } \\
\text { MCC dup }\end{array}$ & $\begin{array}{c}\text { SCC \& } \\
\text { SCC dup }\end{array}$ & $\begin{array}{c}\text { BS } \\
\text { recovery }\end{array}$ & $\begin{array}{c}\text { MS } \\
\text { recovery }\end{array}$ \\
\hline $\begin{array}{c}\text { RPL/ASO } \\
\text { ID }>\end{array}$ & $\mathrm{PB}$ & $11-0791$ & $\begin{array}{c}\text { 11-0792- } \\
129-S\end{array}$ & $\begin{array}{c}\text { 11-0792- } \\
129-\mathrm{D}\end{array}$ & $\begin{array}{c}\text { 11-0793- } \\
129-S\end{array}$ & $\begin{array}{c}\text { 11-0793- } \\
129-D\end{array}$ & RPD & RPD & $\%$ & $\%$ \\
\hline AEA Screen & \multicolumn{4}{|c|}{ $\mu \mathrm{Ci} / \mathrm{g}$, Reference Date May 25, 2011} & & & & & & \\
\hline${ }^{239+240} \mathrm{Pu}$ & 4.43E-5 & NA & $2.17 \mathrm{E}+0$ & $1.62 \mathrm{E}+0$ & $4.56 \mathrm{E}+1$ & $4.72 \mathrm{E}+1$ & $29 \%$ & $3 \%$ & $100 \%$ & $96 \%$ \\
\hline${ }^{238} \mathrm{Pu}+{ }^{241} \mathrm{Am}$ & $6.47 \mathrm{E}-5$ & NA & $3.61 \mathrm{E}+0$ & $2.56 \mathrm{E}+0$ & $5.03 \mathrm{E}+1$ & $5.05 E+1$ & $34 \%$ & $1 \%$ & -- & -- \\
\hline${ }^{243+244} \mathrm{Cm}^{(\mathrm{a})}$ & $1.36 \mathrm{E}-5$ & NA & $5.03 E-3$ & $2.96 \mathrm{E}-3$ & $<4$.E-2 & $5.98 \mathrm{E}-2$ & $52 \%$ & -- & -- & -- \\
\hline GEA & \multicolumn{4}{|c|}{ $\mu \mathrm{Ci} / \mathrm{g}$, Reference Date January 11, 2011} & & & & & & \\
\hline${ }^{60} \mathrm{Co}$ & $<1$. E-5 & $1.48 \mathrm{E}-1$ & $9.10 \mathrm{E}-2$ & 6.69E-2 & 2.07E-1 & $1.94 \mathrm{E}-1$ & $31 \%$ & $6 \%$ & -- & -- \\
\hline${ }^{134} \mathrm{Cs}$ & $<1 . \mathrm{E}-5$ & $<6 . \mathrm{E}-4$ & $<9 . \mathrm{E}-3$ & $<1 . \mathrm{E}-2$ & $<8 . E-3$ & $<8 . E-3$ & -- & -- & -- & -- \\
\hline${ }^{137} \mathrm{Cs}$ & $3.98 \mathrm{E}-4$ & $6.25 E+1$ & $2.96 \mathrm{E}+2$ & $3.44 \mathrm{E}+2$ & $2.08 \mathrm{E}+2$ & $2.03 E+2$ & $15 \%$ & $2 \%$ & -- & -- \\
\hline${ }^{152} \mathrm{Eu}$ & $<4 . \mathrm{E}-5$ & $<6$.E-4 & $<7 . \mathrm{E}-3$ & $<7 . \mathrm{E}-3$ & $<1$.E-2 & $<1$.E-2 & -- & -- & -- & -- \\
\hline${ }^{154} \mathrm{Eu}$ & $<3 . \mathrm{E}-5$ & $5.64 \mathrm{E}-2$ & $1.52 \mathrm{E}-1$ & 1.17E-1 & $1.52 \mathrm{E}+0$ & $1.46 \mathrm{E}+0$ & $26 \%$ & $4 \%$ & -- & -- \\
\hline${ }^{155} \mathrm{Eu}$ & $<5 . \mathrm{E}-5$ & $<9 . \mathrm{E}-3$ & $<1$.E-1 & $<1$.E-1 & $2.55 \mathrm{E}-1$ & $2.36 \mathrm{E}-1$ & -- & $8 \%$ & -- & -- \\
\hline${ }^{241} \mathrm{Am}$ & $<2 . \mathrm{E}-4$ & $1.04 \mathrm{E}+0$ & $2.45 \mathrm{E}+0$ & $2.26 \mathrm{E}+0$ & $3.94 \mathrm{E}+1$ & $3.65 E+1$ & $8 \%$ & $8 \%$ & -- & -- \\
\hline
\end{tabular}

(a) ${ }^{243+244} \mathrm{Cm}$ is an opportunistic measure, and QC sample requirements do not apply.

The analyte concentration $1-\sigma$ uncertainties varied from $2 \%$ to $32 \%$; see Appendix $\mathrm{C}$ for the full analytical report.

NA: Not Analyzed.

Dashed lines “--” indicates parameter does not apply.

Gray shading indicates that the precision criterion ( $\pm 20 \%$ RPD for GEA and $\pm 25 \%$ for AEA) was exceeded.

QC was evaluated from the PB concentration relative to the sample concentration, precision, and in the case of the AEA screen, post-MS and post-BS recoveries of ${ }^{239} \mathrm{Pu}$. The PB results are shown in Table 5.23. In all cases, the analyte concentrations in the PB were a small fraction $(<0.5 \%)$ of the sample analyte concentrations. The AOI RPDs calculated from the SCC sieve fraction duplicate samples met the $\pm 20 \%$ acceptance criteria for GEA and $\pm 25 \%$ acceptance criteria for the AEA screen. The RPDs for the MCC sieve fraction ranged from 8 to 52\% demonstrating higher variability commensurate with the greater variability in particle size and type (see Figure 5.7). The AEA screen BS and MS ${ }^{239} \mathrm{Pu}$ recoveries (100 and 96\%, respectively) met the 75 to $125 \%$ recovery acceptance criteria.

Figure 5.16 compares the total activity measured from each sieve fraction for various analytes. Comparisons are based on the dissolved analytes from the MCC and SCC sieve fractions and the gammaemitted only analytes from the LCC sieve fraction. Total activity was calculated by multiplying the average reported concentration $(\mu \mathrm{Ci} / \mathrm{g})$ by the total dry mass of the sieve fraction. The gamma emitters were measured from all sieve fractions ${ }^{60} \mathrm{Co},{ }^{154} \mathrm{Eu},{ }^{137} \mathrm{Cs}$, and ${ }^{241} \mathrm{Am}$. The ${ }^{239+240} \mathrm{Pu}$ activity was not measured in the KW210-LCC sample. The ${ }^{154} \mathrm{Eu},{ }^{239+240} \mathrm{Pu}$, and ${ }^{241} \mathrm{Am}$ activities were concentrated in the SCC sieve fraction. The ${ }^{137} \mathrm{Cs}$ activity was concentrated in the MCC sieve fraction. The ${ }^{60} \mathrm{Co}$ content appeared to be equally split between the MCC and SCC sieve fractions. 

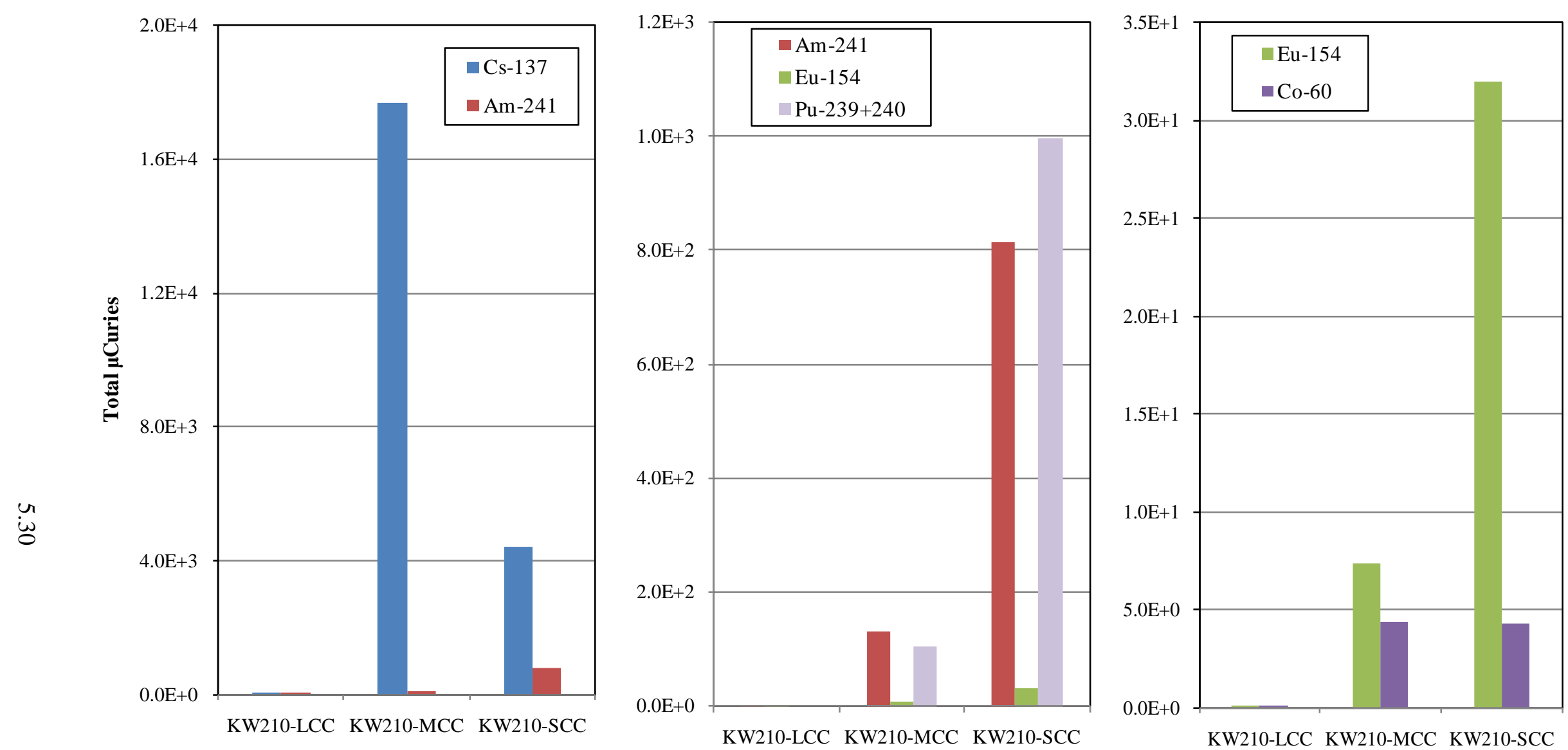

- ${ }^{239+240} \mathrm{Pu}$ was not measured in the KW210-LCC fraction per direction of the BTR; there was insufficient LCC sample mass to accommodate multiple analyses.

- Any analyte that binds to the ion exchange resin beads during the acid digestion process would not be accounted for in this comparison.

Figure 5.16. Analyte Content in KW210-CC-N Sieve Fractions 
The MCC sieve fraction was substantially comprised of resin beads and the SCC fraction was comprised of a small fraction of resin beads (see Section 5.8.3). Any analyte that exchanges onto the resin beads during the acid digestion process would not be accounted for in this comparison. Analyte exchange onto ion exchange materials in nitric acid digestions was previously reported (Schmidt et al. 1999) and is further discussed in Section 5.8.3.

\subsubsection{Undissolved Solids}

The solids that did not dissolve during the PNL-ALO-129 acid digestion were measured by GEA to assess the efficacy of the acid digestion and associated extraction of radionuclides from the solids phase. The GEA results are presented, along with the fractionation remaining with the UDS, in Table 5.24. Maximums of $0.71 \%$ of the ${ }^{137} \mathrm{Cs}$ and $0.59 \%$ of the ${ }^{241} \mathrm{Am}$ were found in the UDS.

Table 5.24. Radionuclide Results in KW210-CC-N Sieve Fraction UDS, Dry Mass Basis (ASR 8824)

\begin{tabular}{|c|c|c|c|c|}
\hline & \multicolumn{2}{|c|}{ KW210-MCC } & \multicolumn{2}{|c|}{ KW210-SCC } \\
\hline Sieve Fraction: & \multicolumn{2}{|c|}{$-2,000 \mu \mathrm{m} /+500 \mu \mathrm{m}$} & \multicolumn{2}{|c|}{$-500 \mu \mathrm{m}$} \\
\hline Sample ID: & TI008-MA & TI008-MB & TI008-SA & TI008-SB \\
\hline RPL/ASO ID: & 11-0792-129-S & 11-0792-129-D & 11-0793-129-S & 11-0793-129-D \\
\hline UDS mass, g: & 1.1750 & 1.5186 & 0.4087 & 0.5009 \\
\hline Analyte & $(\mu \mathrm{Ci} / \mathrm{g})$ & $(\mu \mathrm{Ci} / \mathrm{g})$ & $(\mu \mathrm{Ci} / \mathrm{g})$ & $(\mu \mathrm{Ci} / \mathrm{g})$ \\
\hline \multicolumn{5}{|c|}{ As-Measured $\mu \mathrm{Ci} / \mathrm{g}$ in the UDS, Reference Date: January 11, 2011} \\
\hline${ }^{60} \mathrm{Co}$ & $4.08 \mathrm{E}-4$ & 2.89E-4 & $1.12 \mathrm{E}-3$ & 7.98E-4 \\
\hline${ }^{137} \mathrm{Cs}$ & $1.19 \mathrm{E}+0$ & $2.89 \mathrm{E}+0$ & 5.83E-1 & 6.13E-1 \\
\hline${ }^{154} \mathrm{Eu}$ & $9.22 \mathrm{E}-4$ & $1.77 \mathrm{E}-3$ & $1.30 \mathrm{E}-3$ & $1.22 \mathrm{E}-3$ \\
\hline${ }^{241} \mathrm{Am}$ & $1.68 \mathrm{E}-2$ & 1.46E-2 & 3.65E-2 & 3.30E-2 \\
\hline \multicolumn{5}{|c|}{ Relative Amount of Analyte in the UDS } \\
\hline${ }^{60} \mathrm{Co}$ & $0.38 \%$ & $0.37 \%$ & $0.25 \%$ & $0.19 \%$ \\
\hline${ }^{137} \mathrm{Cs}$ & $0.35 \%$ & $0.71 \%$ & $0.13 \%$ & $0.14 \%$ \\
\hline${ }^{154} \mathrm{Eu}$ & $0.52 \%$ & $1.3 \%$ & $0.039 \%$ & $0.038 \%$ \\
\hline${ }^{241} \mathrm{Am}$ & $0.59 \%$ & $0.55 \%$ & $0.043 \%$ & $0.042 \%$ \\
\hline
\end{tabular}

All analyte concentrations are presented on a dry mass basis.

Precision criteria do not apply to the GEA analysis of the UDS.

The analyte concentration 1- $\sigma$ uncertainties varied from 3 to $7 \%$; see Appendix $\mathrm{C}$ for the full analytical report.

The BTR directed PNNL to perform additional analysis of the UDS, including fusion dissolution followed by ICP-OES and AEA screen analysis. To prepare for this analysis, the UDS were subsampled using the Y-splitter in a cascade to create small subsamples appropriate for fusion. 
One of the splits from each subsample was fractionated by panning off the rolling spherical resin beads into a vial separate from the non-rolling/non-resin material. The goal of the fractionation process was to provide a sample for XRD analysis that contained minimal resin beads and thus minimize the amorphous component background in the powder diffraction pattern. Masses from each fraction were collected. The fractionation was not perfect; some cross-contamination between resin and non-resin components was observed (see Figure 5.17 for examples). However general trends in the approximate mass distribution were recorded. The approximate OIER mass fractions in the UDS samples are provided in Table 5.25. The calculated OIER mass fraction in the sample (before acid digestion) was calculated according to Equation 4.2 and is provided in Table 5.25. This calculation approach assumes no resin mass was lost during the acid digestion processing.

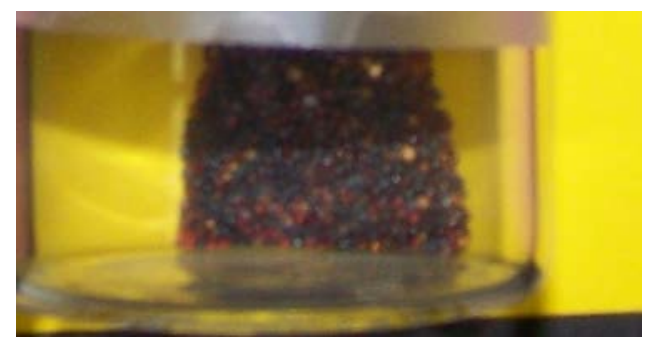

TI040-792S-2r

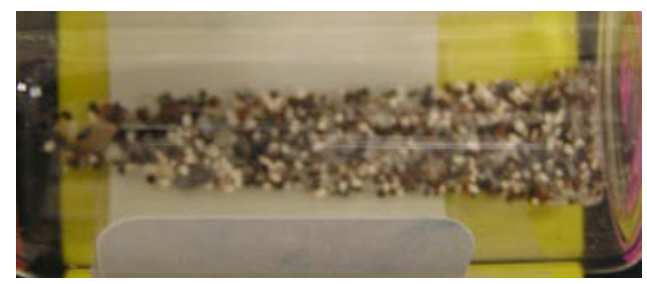

TI040-792S-2x

$-2,000-\mu \mathrm{m} /+500-\mu \mathrm{m}$ sieve Fraction

Parent sample TI008-MA

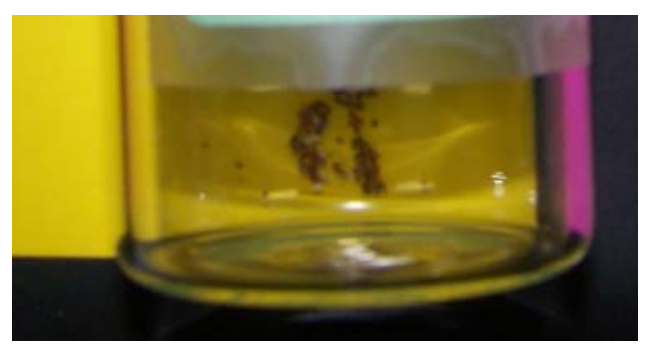

TI040-793S-2r

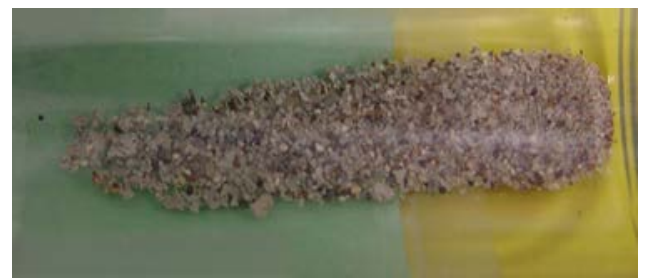

TI040-793S-2x

$-500-\mu \mathrm{m}$ sieve Fraction

Parent sample TI008-SA

Figure 5.17. KW210-CC-N UDS Phase Split Fractions (top OIER; bottom non-OIER fraction)

Table 5.25. Dry Mass Fractionation of OIER in the KW210-CC-N Sieve Fractions

\begin{tabular}{cccc}
\hline Sieve Fraction & $\begin{array}{c}\text { Parent } \\
\text { Sample ID }\end{array}$ & $\begin{array}{c}\text { OIER in UDS, } \\
\text { wt\% }\end{array}$ & $\begin{array}{c}\text { Calculated OIER in } \\
\text { Dry Parent Sample, } \\
\text { wt\% }\end{array}$ \\
\hline$-500 \mu \mathrm{m}$ & TI008-SA & 2.3 & 1.1 \\
& TI008-SB & 3.7 & 1.7 \\
& & $48 \%$ RPD & $48 \%$ RPD \\
\hline$-2,000 \mu \mathrm{m} /+500 \mu \mathrm{m}$ & TI008-MA & 51 & 44 \\
& TI008-MB & 49 & 42 \\
& & $3.4 \%$ RPD & $4.1 \%$ RPD \\
\hline
\end{tabular}

One subsample from each non-resin material sieve fraction was analyzed by XRD. The XRD patterns are compiled in Figure 5.18. Each XRD sample consisted primarily of silica quartz $\left(\mathrm{SiO}_{2}\right)$ and anorthite, $\mathrm{Ca}\left(\mathrm{Al}_{2} \mathrm{Si}_{2} \mathrm{O}_{8}\right)$. Table 5.26 lists the crystalline phases identified in the UDS, along with the sample progenitor IDs, ICDD card number, and relative intensities. All reflections were identified. 
PNNL-20650, Rev. 2

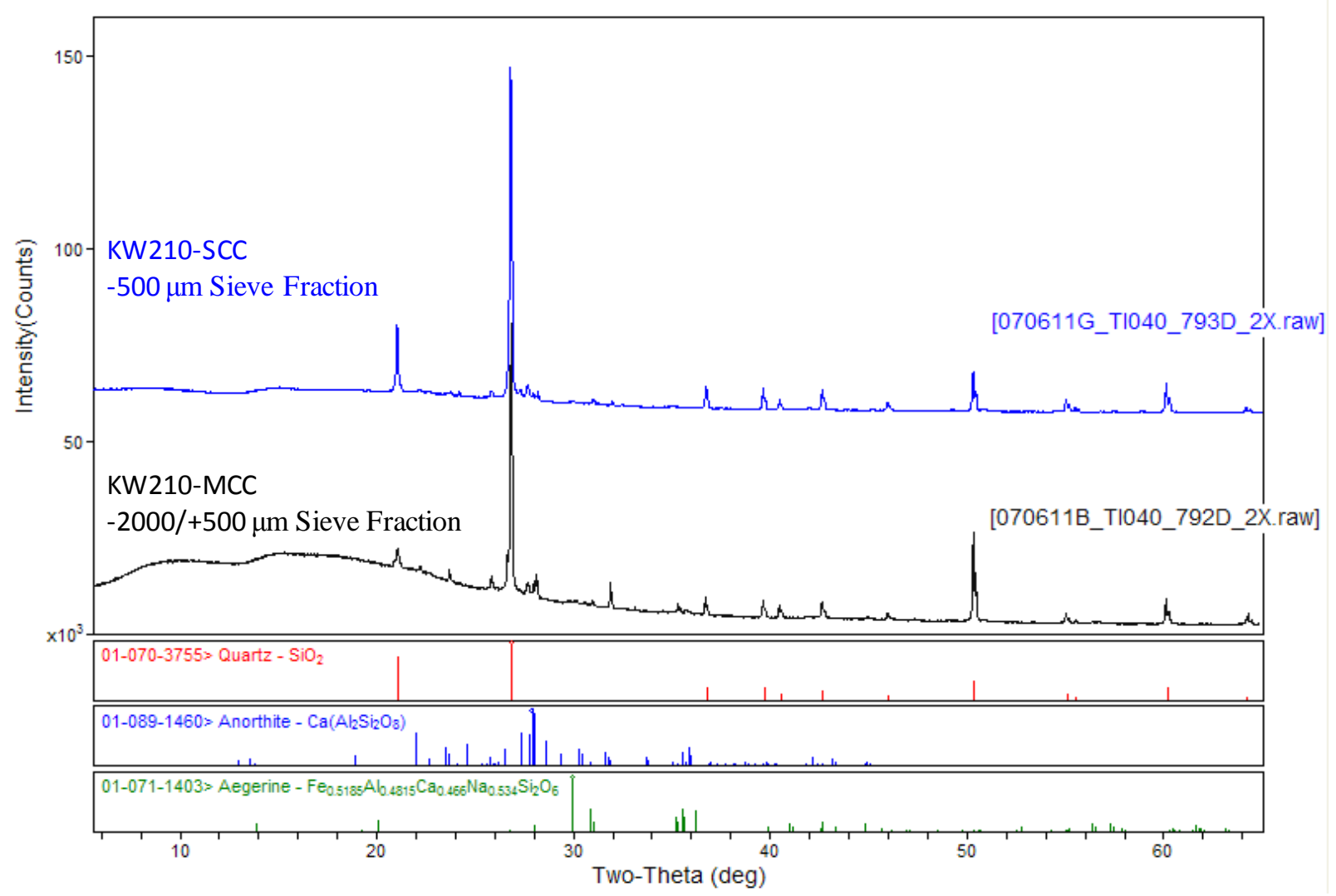

Figure 5.18. XRD Patterns of KW210-CC-N Container Composite Sieve Fraction Sample UDS

Table 5.26. Summary of Crystalline Phases Found via X-Ray Powder Diffraction on UDS Following Acid Digestion and Bulk OIER Removal from KW210-CC-N Sieve Fraction Samples

\begin{tabular}{|c|c|c|c|c|c|}
\hline $\begin{array}{l}\text { XRD } \\
\text { Sample } \\
\text { ID }\end{array}$ & $\begin{array}{c}\text { (RPL/ASO } \\
\text { Parent ID) } \\
\text { Sieve Fraction }\end{array}$ & $\begin{array}{l}\text { ICDD Card } \\
\text { Number }\end{array}$ & $\begin{array}{l}\text { Relative } \\
\text { Intensity }\end{array}$ & Formula & $\begin{array}{l}\text { Common } \\
\text { Phase } \\
\text { Name }\end{array}$ \\
\hline \multirow{3}{*}{$\begin{array}{l}\text { TI040- } \\
\text { 792D-2x }\end{array}$} & \multirow{3}{*}{$\begin{array}{c}(11-0792-129-\mathrm{D}) \\
-2,000 \mu \mathrm{m} / \\
+500 \mu \mathrm{m}\end{array}$} & 01-070-3755 & Major & $\mathrm{SiO}_{2}$ & Quartz \\
\hline & & 01-089-1460 & Major & $\mathrm{Ca}\left(\mathrm{Al}_{2} \mathrm{Si}_{2} \mathrm{O}_{8}\right)$ & Anorthite \\
\hline & & 01-071-1403 & Minor & $\mathrm{Ca}_{0.466} \mathrm{Fe}_{0.5185} \mathrm{Al}_{0.815} \mathrm{Na}_{0.534} \mathrm{O}_{6} \mathrm{Si}_{2}$ & Aegerine \\
\hline \multirow{3}{*}{$\begin{array}{l}\text { TI040- } \\
\text { 793D-2x }\end{array}$} & \multirow{3}{*}{$\begin{array}{c}(11-0793-129-\mathrm{D}) \\
-500 \mu \mathrm{m}\end{array}$} & 01-070-3755 & Major & $\mathrm{SiO}_{2}$ & Quartz \\
\hline & & 01-089-1460 & Major & $\mathrm{Ca}\left(\mathrm{Al}_{2} \mathrm{Si}_{2} \mathrm{O}_{8}\right)$ & Anorthite \\
\hline & & 01-071-1403 & Minor & $\mathrm{Ca}_{0.466} \mathrm{Fe}_{0.5185} \mathrm{Al}_{0.815} \mathrm{Na}_{0.534} \mathrm{O}_{6} \mathrm{Si}_{2}$ & Aegerine \\
\hline
\end{tabular}

(a) Crystalline components above $10 \mathrm{wt} \%$ are classified as major phases, between 1-10 wt\% as minor phases, and under $1 \mathrm{wt} \%$ as possible phases. The XRD report is provided in Appendix F. 
The UDS subsample splits (containing OIER) from the container composite MCC and SCC sieve fraction samples were further split into small representative aliquots applicable for $\mathrm{KOH} / \mathrm{KNO}_{3}$ fusion ( 0.1 to $0.2 \mathrm{~g}$ ). After fusion the digestates were split. One split was analyzed by ICP-OES for AOIs Al, $\mathrm{Ca}, \mathrm{Fe}, \mathrm{S}, \mathrm{Si}, \mathrm{U}$, and $\mathrm{Zr}$; the other split was analyzed for gross alpha and AEA screen for a ${ }^{239+240} \mathrm{Pu}$ measurement. The fusion and analyses were conducted according to ASR 8878 and Special Instructions (Appendix D). ${ }^{\text {(a) }}$ This analysis was supplemental scope beyond that defined in the QAPjP/SAP. The QC sample results were briefly discussed in Section 4.8; more detail can be found in Appendix D.

Table 5.27 provides the AOI results and their relative mass and activity fractionations to the UDS (results of ICP-OES opportunistic analytes and total alpha can be found in Appendix D). Analyte fractionation to the UDS was calculated according to Equation 4.1 where $\mathrm{C}_{\text {UDS }}$ was determined from the analysis of the fused sample. The pre-digest dry mass $\left(\mathrm{g}_{\mathrm{S}}\right)$ and UDS mass ( $\mathrm{g}_{\text {UDS }}$ ) values are reported in Table 5.19 and the concentrations of analytes in the acid digestate $\left(\mathrm{C}_{\mathrm{S}}\right)$ are reported in Table 5.20. The companion figure, Figure 5.19, presents the $\mathrm{Al}, \mathrm{Fe}, \mathrm{S}, \mathrm{U}$ and Pu retention in the UDS as a function of the OIER content in the dry parent sample (before subjection to PNL-ALO-129 acid digestion, see Table 5.25). In Figure 5.19, the MCC and SCC sample results are overlaid on the core sample results (Figure 4.17). All of the relationships regarding analyte retention in UDS described in Section 4.8 were confirmed by the additional container composite sample data, with the exception that the MCC fraction appeared to result in an enhanced $U$ retention (data points well above the trend line).

The direct relationship between the OIER content in the parent sample material to Fe, $\mathrm{U}$, and $\mathrm{Pu}$ retention in the UDS suggests that a fraction of the analyte anionic or cationic species exchanged onto the OIER during the PNL-ALO-129 acid digestion and solids rinse steps. Similarly, the exchange of analytes onto OIER was reported previously with increases in Fe and ${ }^{239+240} \mathrm{Pu}$ concentrations by factors of 17 and 290, respectively, when a K Basin OIER (NRW-37) was exposed to a $6 \mathrm{M} \mathrm{HNO}_{3}$ dissolver solution at $95^{\circ} \mathrm{C}$ for $7 \mathrm{hr}$ (Schmidt et al. 1999). In this same 1999 study, however, the U concentration increased by

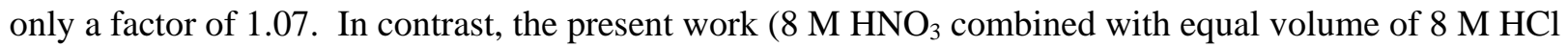
dissolver solution) resulted in a U retention in UDS that generally tracked well with the ${ }^{239+240} \mathrm{Pu}$ retention - with the exception of the OIER-rich MCC sample where U retention exceeded Pu retention by a factor of 2.6 (see Figure 5.19). Also Fe retention was greater than Pu retention. Acid type and strength are known to affect the affinity of specific cationic/anionic analyte exchange onto OIER (Coleman 1965). Therefore, the fraction of Fe, U, and ${ }^{239+240} \mathrm{Pu}$ presented in Table 5.27 likely does not represent the analyte fractions in the sludge OIER currently contained in SCS-CON-210 because these analytes were not dissolved in-situ and the contacting medium is essentially $\mathrm{pH} 7$ water (i.e., not acidic). Additional analyses of undigested OIER would be required to confirm this assessment.

(a) The UDS associated with the core samples were also processed as part of ASR 8878. Core sample 11-0420129-D and container composite sample 11-0793-129-D were split and run as batch duplicates. All process QC samples were shared between the core and container composite samples. 
PNNL-20650, Rev. 2

Table 5.27. Analytes in UDS Fusion, Dry UDS Mass Basis (ASR 8878)

\begin{tabular}{|c|c|c|c|c|c|c|}
\hline & & \multicolumn{2}{|c|}{ KW210-MCC } & \multicolumn{3}{|c|}{ KW210-SCC } \\
\hline \multicolumn{2}{|c|}{ Sieve Fraction: } & \multicolumn{2}{|c|}{$-2,000 \mu \mathrm{m} /+500 \mu \mathrm{m}$} & \multicolumn{3}{|c|}{$-500 \mu \mathrm{m}$} \\
\hline \multicolumn{2}{|c|}{ Parent Sample ID: } & TI008-MA & TI008-MB & TI008-SA & TI008-SB & TI008-SB (Dup.) \\
\hline \multicolumn{2}{|c|}{ Parent RPL/ASO ID: } & $\begin{array}{l}\text { 11-0792- } \\
129-S\end{array}$ & $\begin{array}{l}\text { 11-0792- } \\
129-\mathrm{D}\end{array}$ & $\begin{array}{l}\text { 11-0793- } \\
129-S\end{array}$ & $\begin{array}{l}\text { 11-0793- } \\
129-\mathrm{D}\end{array}$ & $\begin{array}{l}\text { 11-0793- } \\
129-\mathrm{D}\end{array}$ \\
\hline \multicolumn{3}{|c|}{ Daughter Sample ID: TI040-0792S-1c } & TI040-0792D-1c & TI040-0793S-1a & TI040-0793D-1b & TI040-0793D-2b \\
\hline $\begin{array}{c}\text { Daughter RPL/ASO } \\
\text { ID: } \\
\end{array}$ & $\begin{array}{l}\text { BLK- } \\
1279 \\
\end{array}$ & $\begin{array}{c}11-1289- \\
115-S \\
\end{array}$ & $\begin{array}{c}11-1290- \\
115-S\end{array}$ & $\begin{array}{c}11-1287- \\
115-S \\
\end{array}$ & $\begin{array}{c}11-1288- \\
115-S \\
\end{array}$ & $\begin{array}{c}11-1288- \\
115-\mathrm{D}\end{array}$ \\
\hline Analyte & \multicolumn{6}{|c|}{$(\mu \mathrm{g} / \mathrm{g})$ or $(\mu \mathrm{Ci} / \mathrm{g})$} \\
\hline $\mathrm{Al}$ & $<1,400$ & {$[11,000]$} & 12,900 & 25,000 & 26,200 & 26,900 \\
\hline $\mathrm{Ca}$ & $<1,500$ & $<1,400$ & {$[2,200]$} & {$[3,000]$} & {$[3,100]$} & {$[3,200]$} \\
\hline $\mathrm{Fe}$ & $<570$ & 20,900 & 23,000 & 8,360 & 9,780 & 8,750 \\
\hline S & $<480$ & 21,000 & 20,300 & 3,880 & {$[3,600]$} & {$[3,000]$} \\
\hline $\mathrm{Si}$ & $<1,600$ & 189,000 & 189,000 & 309,000 & 375,000 & 405,000 \\
\hline $\mathrm{U}$ & $<31$ & 8,320 & 9,910 & 1,610 & 1,270 & 1,040 \\
\hline $\mathrm{Zr}$ & $<38$ & [37] & $<27$ & [190] & [240] & [190] \\
\hline${ }^{239+240} \mathrm{Pu}$ & 6.83E-5 & 2.13E-1 & $1.82 \mathrm{E}-1$ & 4.10E-1 & 3.45E-1 & 2.39E-1 \\
\hline \multicolumn{7}{|c|}{ Relative Amount of Analyte in the UDS } \\
\hline $\mathrm{Al}$ & -- & [36\% $]$ & $42 \%$ & $24 \%$ & $26 \%$ & $26 \%$ \\
\hline $\mathrm{Ca}$ & -- & NA & [16\%] & [40\%] & [45\%] & [44\%] \\
\hline Fe & -- & $33 \%$ & $28 \%$ & $2.8 \%$ & $3.3 \%$ & $2.9 \%$ \\
\hline $\mathrm{S}$ & -- & $66 \%$ & $65 \%$ & $44 \%$ & {$[46 \%]$} & [40\%] \\
\hline $\mathrm{Si}$ & -- & NA & NA & NA & NA & NA \\
\hline $\mathrm{U}$ & -- & $19 \%$ & $25 \%$ & $0.43 \%$ & $0.36 \%$ & $0.28 \%$ \\
\hline $\mathrm{Zr}$ & -- & [5.3\%] & NA & [17\%] & [23\%] & [19\%] \\
\hline${ }^{239+240} \mathrm{Pu}$ & -- & $7.8 \%$ & $8.7 \%$ & $0.41 \%$ & $0.34 \%$ & $0.23 \%$ \\
\hline
\end{tabular}

Analyte results shown in brackets indicate that the analyte concentrations were greater than the MDL but less than the EQL. Less-than values represent the instrument detection limit multiplied by the process factors. See Appendix D for measurement uncertainties.

Percent values in brackets indicate one of the input parameters was $<$ EQL but $>$ MDL.

NA: Not Applicable; an input factor was $<\mathrm{MDL}$ or, in the case of Si, the analyte was not expected to dissolve in the PNLALO-129 acid digestion. 
PNNL-20650, Rev. 2

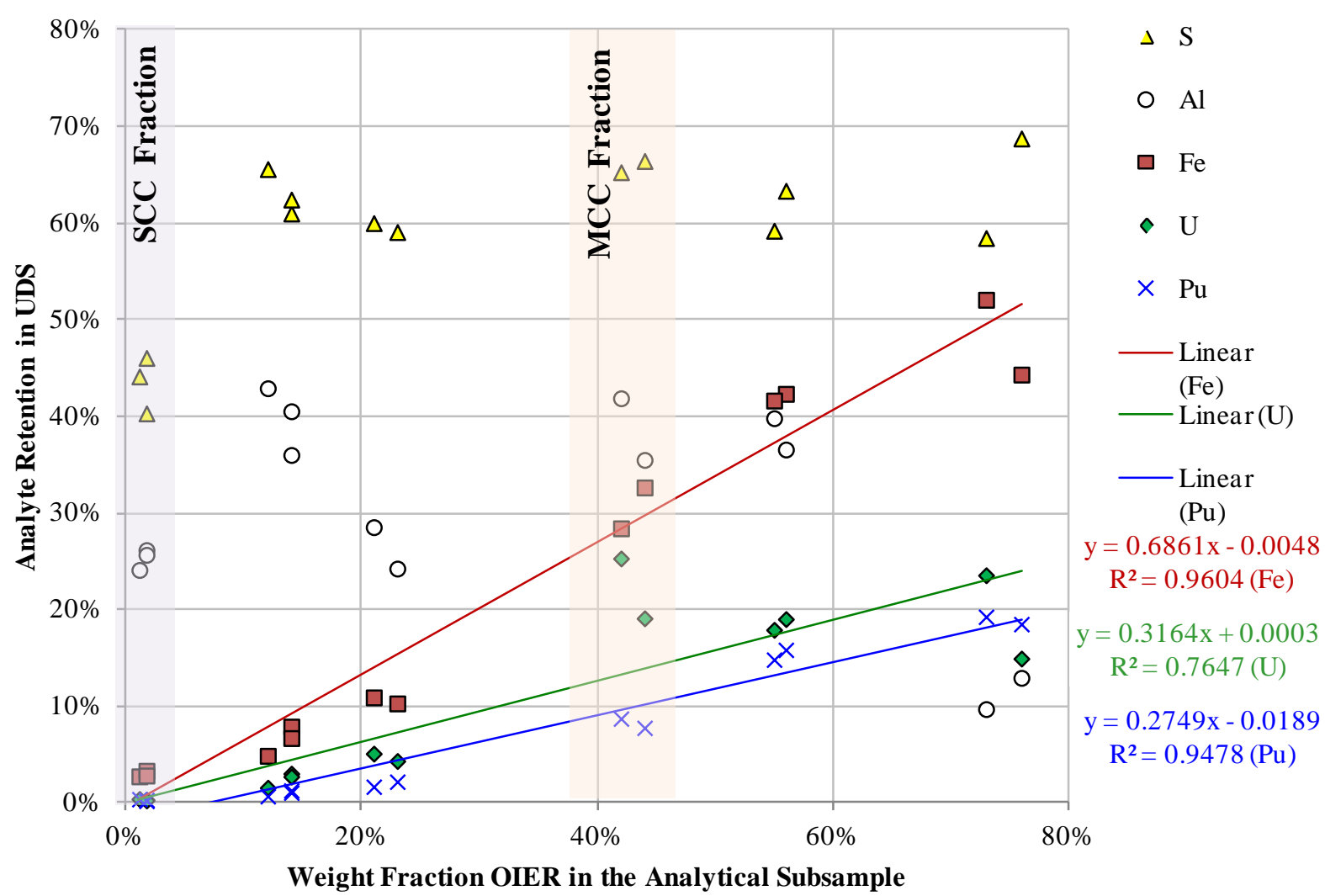

- $\quad P u, U$, and Fe increased in the UDS as OIER content increased in the parent sample.

- $\quad A l$ and $S$ in the UDS showed no relationship to the OIER content.

- Linear trend lines are provided for illustrative purposes and do not attempt to represent a model fit.

- The MCC and SCC sample results are overlaid on the core sample results.

Figure 5.19. Relationship of $\mathrm{Al}, \mathrm{Fe}, \mathrm{S}, \mathrm{U}$, and Pu to OIER Content in the Parent Analytical Sample

The total relative activity and mass distributions of $\mathrm{Pu}, \mathrm{U}$, and Fe in the MCC and SCC sieve fraction UDS versus the acid digestates were compared. This comparison required the analyte concentration be multiplied by the mass of the component (i.e., the mass and activity balances were performed relative to the calculated sum of the SCC and MCC components). The reporting mass basis of the analyte concentration and the total component mass are summarized in Table 5.28.

Table 5.28. Sieve Fraction Samples Mass Basis used to Create Figure 5.20

\begin{tabular}{|c|c|c|c|c|c|}
\hline & \multicolumn{2}{|c|}{ SCC } & \multicolumn{2}{|c|}{ MCC } & \multirow{2}{*}{$\frac{\text { LCC }}{\text { Whole Sample }}$} \\
\hline & Whole Sample & UDS & Whole Sample & UDS & \\
\hline $\begin{array}{c}\text { Dry Mass of Sieve } \\
\text { Fraction }\end{array}$ & $21.5 \mathrm{~g}$ & $9.88 \mathrm{~g}$ & $55.2 \mathrm{~g}$ & $47.4 \mathrm{~g}$ & $0.73 \mathrm{~g}$ \\
\hline $\begin{array}{l}\text { Sample Fraction } \\
\text { Analyzed }\end{array}$ & Acid Digestate & UDS & Acid Digestate & UDS & Not Analyzed \\
\hline Reporting Mass & Per Gram Dry & Per Gram & Per Gram Dry & Per Gram & Not Analyzed \\
\hline Basis of Analytes & Initial Sample & Dry UDS & Initial Sample & Dry UDS & \\
\hline
\end{tabular}


Figure 5.20 compares the ${ }^{239+240} \mathrm{Pu}, \mathrm{U}, \mathrm{Fe}$ and ${ }^{137} \mathrm{Cs}$ total analyte content in each sieve fraction (combined red and blue bars) relative to the analyte concentration that dissolved in the PNL-ALO-129 acid digestion (blue bars) and the normalized analyte concentration (see Equation 4.4) associated with the UDS (red bars). (Figure 5.20 is complimentary to Figure 5.16 and Table 5.22, with the enhancement that the UDS components and $U$ and Fe distributions are incorporated.) The total Pu content was primarily in the SCC fraction with a very small total fraction associated with the UDS. Significant U and Fe content are associated with the MCC fraction. As previously noted, the ${ }^{137} \mathrm{Cs}$ is largely associated with the MCC fraction.

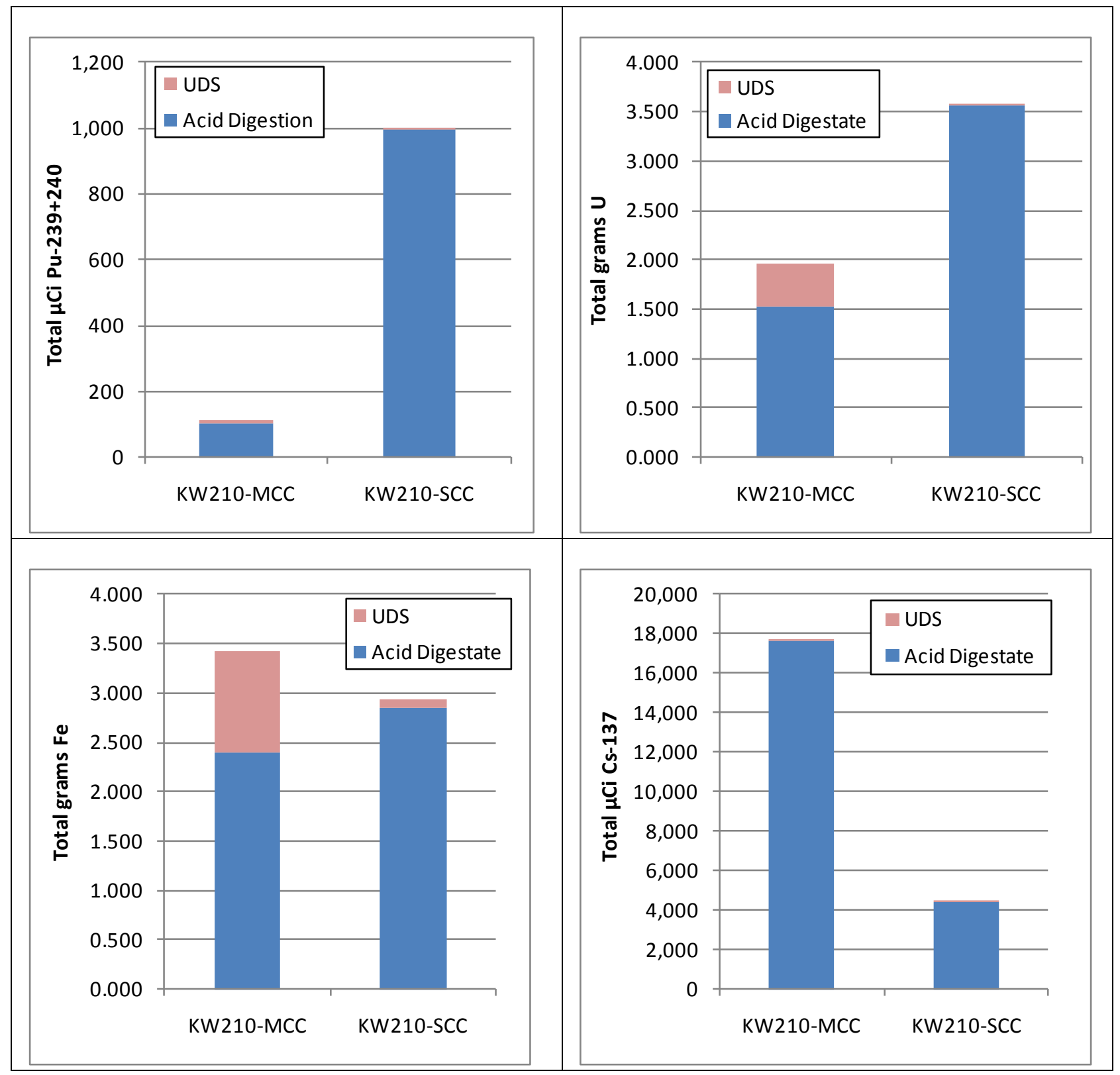

Figure 5.20. Total ${ }^{239+240} \mathrm{Pu}, \mathrm{U}, \mathrm{Fe}$ and ${ }^{137} \mathrm{Cs}$ Content and Partitions Between Acid Digestate and UDS Phases for the MCC and SCC Sieve Fractions 
An additional GEA analysis of the MCC sieve fraction samples was directed by the BTR to provide data on gamma activity in the resin bead fraction before acid dissolution. (This analysis was supplemental scope beyond that defined in the QAPjP/SAP.) Samples TI008-MK and TI008-ML (air dried, but not subjected to acid dissolution) were combined and the spherical particles (resin bead fraction) were separated from the non-spherical particles (i.e., irregular/granular-shaped particulate fraction). Particle separation was performed by placing the sample in the middle of a folded piece of paper, the paper was elevated, and spherical particles preferentially separated from less spherical particles via effects of gravity and friction. The samples were processed in this fashion over several iterations before effective separation was achieved. Some of the resin beads remained in the non-resin fraction as shown in Figure 5.21.

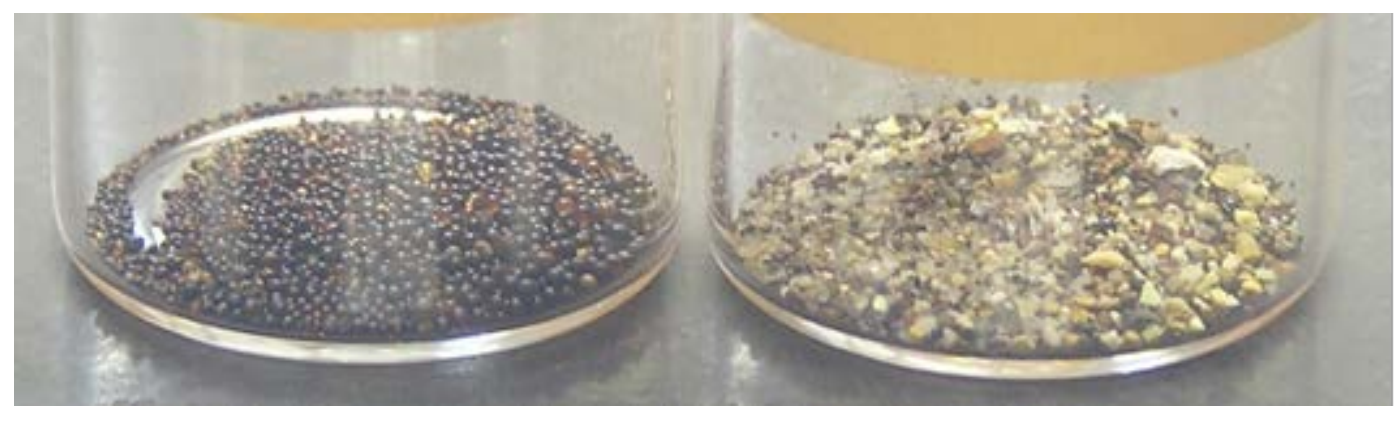

KW210-MK\&ML Resin Beads

KW210 MK \& ML Residual Solids

Figure 5.21. Undigested KW210-MCC Sample Split for GEA

Table 5.29 provides the GEA analysis results on the resin bead and irregular/granular (residual) solids fractions under ASR 8919. See Appendix D for the ASR, analysis details, and measurement uncertainties.

The distribution of the total ${ }^{137} \mathrm{Cs}$ content between the resin and the non-resin components in the MCC fraction was assessed. The total resin component in the MCC fraction was derived from the mass fraction of the resin in the MCC sample (43\% average, see Table 5.25) and the total measured MCC mass (55.2 g see Table 5.7). The non-resin mass component was calculated by difference. Each mass fraction was multiplied by the ${ }^{137} \mathrm{Cs}$ concentration assocatied with the fraction. The input data and ${ }^{137} \mathrm{Cs}$ distribtuion is summarized in Table 5.30 . Only $10 \%$ of the ${ }^{137} \mathrm{Cs}$ is associated with the OIER. A component other than OIER apparently is associated with the bulk of the ${ }^{137} \mathrm{Cs}$ activity in the MCC fraction. Mordenite is an obvious consideration; however, crystalline phases of mordenite were not observed by XRD in the MCC sample (see Tables 5.10 and 5.26). However, anorthite is prominent in the XRD pattern of the digestion heel and is known to have a high affinity for ${ }^{137} \mathrm{Cs}$ with distribution coefficients, $\mathrm{K}_{\mathrm{d}} \mathrm{S}$, of about $440 \mathrm{~mL} / \mathrm{g}$ for sorption from a $7.9 \mathrm{pH}, 0.03 \mathrm{~N} \mathrm{NaCl}$ solution (Mucciardi et al. 1979). The sorption is slightly reversible with $K_{d}$ of the desorption reaction about $44 \mathrm{~mL} / \mathrm{g}$ using $5.13 \mathrm{~N} \mathrm{NaCl}$. More generally, studies of the sorption of ${ }^{137} \mathrm{Cs}$ onto Hanford soils have been summarized and shown to be high with $\mathrm{K}_{d} \mathrm{~S}$, ranging around $1,000 \mathrm{~mL} / \mathrm{g}$, observed in tests with dilute solutions (Table 5 of Cantrell et al. 2003). However, the attribution of the high ${ }^{137}$ Cs retention in the solid phase to anorthite is speculative and would require further experimentation to confirm. 
Table 5.29. Radionuclide Results in KW210-MCC Sieve Fraction Separated Solids (Resin Beads and Irregular/Granular Particulate), Dry Mass Basis (ASR 8919)

\begin{tabular}{|c|c|c|c|}
\hline \multicolumn{4}{|c|}{ KW210-MCC } \\
\hline & Sieve Fraction: & \multicolumn{2}{|c|}{$-2,000 \mu \mathrm{m} /+500 \mu \mathrm{m}$} \\
\hline & Sample ID: & \multicolumn{2}{|c|}{ TI008-MK and TI008-ML Combined } \\
\hline & RPL/ASO ID: & 11-1555 & $11-1556$ \\
\hline & Fraction ID: & $\begin{array}{l}\text { KW210 MK \& ML } \\
\text { Resin Beads }\end{array}$ & $\begin{array}{l}\text { KW210 MK \& ML } \\
\text { Residual Solids }\end{array}$ \\
\hline & Fraction mass, $\mathrm{g}^{(\mathrm{a})}$ : & 0.3049 & 0.3591 \\
\hline & Analyte & $(\mu \mathrm{Ci} / \mathrm{g})$ & $(\mu \mathrm{Ci} / \mathrm{g})$ \\
\hline \multicolumn{4}{|c|}{ GEA, pCi/g, Reference Date January 11, 2011} \\
\hline & ${ }^{60} \mathrm{Co}$ & $2.22 \mathrm{E}-2$ & $7.60 \mathrm{E}-2$ \\
\hline & ${ }^{134} \mathrm{Cs}$ & $<4$.E-3 & $<3 . \mathrm{E}-2$ \\
\hline & ${ }^{137} \mathrm{Cs}$ & $6.97 \mathrm{E}+1$ & $4.56 \mathrm{E}+2$ \\
\hline & ${ }^{152} \mathrm{Eu}$ & $<7 . \mathrm{E}-3$ & $<4 . \mathrm{E}-2$ \\
\hline & ${ }^{154} \mathrm{Eu}$ & 9.37E-2 & $<6 . \mathrm{E}-2$ \\
\hline & ${ }^{155} \mathrm{Eu}$ & $<8 . E-2$ & $<7 . \mathrm{E}-1$ \\
\hline & ${ }^{241} \mathrm{Am}$ & 7.76E-1 & $<2 . \mathrm{E}+0$ \\
\hline (a) & \multicolumn{3}{|c|}{$\begin{array}{l}\text { The combined mass of TI008-MK and TI008-ML equals } 1.0305 \text { g. Total mass recovery equals } \\
64 \% \text {. More details of the separation work can be found in LRB BNW-60605 pages } 85-92 \text { and } \\
\text { are provided in DVD-52578-PNNL-20650 Rev. 2, Section III, September } 2013 \text {. } \\
\text { The residual solids are composed of irregular/granular particles of non-resin material. }\end{array}$} \\
\hline \multicolumn{4}{|c|}{ All analyte concentrations are presented on a dry mass basis. } \\
\hline \multicolumn{4}{|c|}{ Precision criteria do not apply to the GEA analysis of the fraction-separated MCC sample. } \\
\hline \multicolumn{4}{|c|}{$\begin{array}{l}\text { The analyte concentration } 1-\sigma \text { uncertainties varied from } 3 \text { to } 13 \% \text {; see Appendix D for the full } \\
\text { analytical report. }\end{array}$} \\
\hline
\end{tabular}

Table 5.30. ${ }^{137}$ Cs Distribution in Components of the MCC Fraction

\begin{tabular}{lcc}
\hline & \multicolumn{2}{c}{$55.2 \mathrm{~g}$ MCC Fraction } \\
\cline { 2 - 3 } Mass/Activity Balance Basis: & Resin component & Non-Resin component \\
\cline { 2 - 3 }${ }^{137} \mathrm{Cs}$ concentration & $69.7 \mu \mathrm{Ci} / \mathrm{g}$ & $456 \mu \mathrm{Ci} / \mathrm{g}$ \\
Mass of component & $23.7 \mathrm{~g}$ & $31.5 \mathrm{~g}$ \\
Total $^{137}$ Cs activity & $1,650 \mu \mathrm{Ci}$ & $14,300 \mu \mathrm{Ci}$ \\
${ }^{137} \mathrm{Cs}$ distribution in MCC & $10 \%$ & $90 \%$ \\
\hline
\end{tabular}




\subsection{Records}

Multiple record materials have been collected in the execution of the K Basin sludge processing and analysis. Records that are not ASO-specific are included in this report data package (in the appendices or DVD-52578-PNNL-20650 Rev. 2, Sections I-IV, September 2013). The contents of the DVD are stored in the PNNL records electronic database Total Records Information Management (TRIM) records system. The long-term storage of ASO-specific records are maintained in the National Archives and Records Administration (NARA) Federal Records Center (Seattle, Washington) (hardcopy), which in turn is based on DOE and other Federal records management requirements. This section describes where records are located and how they can be retrieved if not specifically included in this data package.

\subsection{ASO Records}

Sample results and supporting data are maintained in the ASO records. The records are inventoried by ASR number, PNNL project number, and RPL sample ID. The ASR and sample ID information, including the cross reference to client ID are maintained on the ASO sample tracking database (Microsoft Access database). In accordance with PNNL records management requirements, ASO records are prepared for transmittal to long-term records storage on a periodic basis, and upon project close-out. Records are boxed and the transmittal paperwork includes an inventory of the items in each box. This inventory includes a description of the record type, associated ASR number and the data type included (i.e., ICP-OES results for ASR XXXX). The ASO database is updated to reference the transmittal box ID to the associated ASR and data type sent in that transmittal. (Note: All records submitted for storage created by PNNL under the Battelle/DOE Operating Contract [DE-ACO5-76RL01830] are transferred to the NARA Federal Records Center in Seattle, Washington.) The ASO records include (but are not limited to) the following:

- bench sheets (digestion, dilutions, separation, run log records)

- analysis records (computer-generated printouts for counting instruments, ICP-OES)

- calibration records (certificates, initial and continuing calibrations, control charts)

- calculation of data to produce final summary data reports

- QE surveillance reports.

Table 6.1 summarizes the relevant record identifiers that will aid in record retrieval and points of contact/position titles that can facilitate records recovery.

\subsection{Isotopic Distribution Mass Spectrometry Records}

Radiochemical purification of the $\mathrm{U}$ and Pu fractions were conducted by the ASO; records specific to this process are handled as discussed in Section 6.1. All other records supporting the U and Pu TIMS analyses are provided in the data package, Appendix E. These include final analysis reports, sample run logs, standard certificates, computer-generated result printouts, and data calculations. 
Table 6.1. Records Summary and Contacts for Retrieval

\begin{tabular}{lccl}
\hline & $\begin{array}{c}\text { PNNL Project } \\
\text { Luboratory Name }\end{array}$ & $\begin{array}{c}\text { ASR } \\
\text { Number }\end{array}$ & \multicolumn{1}{c}{ RPL Numbers } \\
\hline Pacific Northwest & 52578 & 8798 & $11-0419-11-0422$ \\
National Laboratory & & 8824 & $11-0791-11-0967$ \\
& 8878 & $11-1279-11-1290$ \\
& 8919 & $11-1555-11-1556$ \\
\hline \multicolumn{3}{c}{ Contacts for Records Retrieval } \\
\hline Position & Name & Contact Information \\
\hline Project Manager for Project 52578 & Clark Carlson & 509-375-5334 \\
& \multicolumn{2}{c}{ clark.carlson@pnnl.gov } \\
ASO Lead & Karl Pool & 509-375-5246 \\
& & karl.pool@pnnl.gov \\
ASO Administrator & Truc Trang-Le & 509-375-5457 \\
& & truc.trangle@pnnl.gov \\
PNNL Records Management; & Terri Mooney & 509-375-3798 \\
Records Storage and Retrieval & & terri.mooney@pnnl.gov \\
\hline
\end{tabular}

\subsection{XRD Records}

Most XRD records are associated with the powder diffraction computer files. These are processed on the computer running Jade software; final output data are images of raw and processed spectra. Other records include the analysis request form, the preparation record and the scan record. Original record printouts are maintained in the project file. Reproductions of these records are provided in Appendix $\mathrm{F}$ as part of the data package.

\subsection{Particle Density Records}

All records supporting the particle density measurements are provide in the data package, Appendix G. These include the bench sheets (calibration verification, sample mass and density calculations) and standard calibration certificates.

\subsection{Rheology Records}

All records supporting rheology measurements are provide in the data package, Appendix $\mathrm{H}$. These include the bench sheets for performance verifications and standard calibration certificates.

\subsection{Particle Size Distribution Records}

All records supporting particle size distribution measurements are provided in the data package, Appendix I. These include the bench sheets for performance verifications and standard calibration certificates. 
PNNL-20650, Rev. 2

\subsection{References}

Baker RB, JL Westcott, TL Welsh, JA Pottmeyer, and AJ Schmidt. 2009. Quality Assurance Project Plan/Sampling and Analysis Plan for Sludge in the KW Engineered Containers.

KBC-33786, Rev. 2, CH2M Hill Plateau Remediation, Company, Richland, Washington.

Browne E and RB Firestone. 1986. Table of Radioactive Isotopes. ed. VS Shirley, John Wiley and Sons, New York.

Burns CA, M Luna, and AJ Schmidt. 2011. Characterization of Settler Tank, KW Container and KE Container Sludge Simulants. PNNL-18408, Rev. 1, Pacific Northwest National Laboratory, Richland, Washington.

Cantrell KJ, RJ Serne, and GV Last. 2003. Hanford Contaminant Distribution Coefficient Database and Users Guide. PNNL-13895, Rev. 1, Pacific Northwest National Laboratory, Richland, Washington.

Coleman GH. The Radiochemistry of Plutonium. National Academy of Sciences. 1965. DOE U.S. Department of Energy. 2002. Hanford Analytical Services Quality Assurance Requirements Document (HASQARD). DOE/RL-96-68 Rev. 3, Vols 1 and 4, Office of River Protection, Richland, Washington.

DOE - U.S. Department of Energy. 2009. Hanford Sitewide Transportation Document. DOE/RL-2001-36 Rev. 1-C., Richland Operations Office, Richland, Washington.

Fiskum SK, OP Bredt, CA Burns, CD Carlson, DS Coffey, RC Daniel, PJ MacFarlan, KN Pool, AJ Schmidt, GJ Sevigny, RW Shimskey, and LA Snow. 2010. Stage 1 and Stage 2 Settling Studies of K-Basin Containerized Sludge. PNNL-19213, Pacific Northwest National Laboratory, Richland, Washington.

Fiskum SK, DL Baldwin, JM Billing, CD Carlson, DS Coffey, CH Delegard, MK Edwards, LR Greenwood, D Neiner, BM Oliver, KN Pool, AJ Schmidt, RW Shimskey, SI Sinkov, and T Trang-Le. 2011a. Follow-On Characterization of Engineered Container Sludge: SCS-CON220-B4 Characterization; SCS-CON-220, -240, -250, -260 Carbon Analyses; SCS-CON-220-B1 U Metal Analysis. PNNL-20304, Pacific Northwest National Laboratory, Richland, Washington.

Fiskum SK, JM Billing, SJ Bos, CA Burns, CD Carlson, DS Coffey, JV Crum, RC Daniel, CH Delegard, MK Edwards, OT Farmer, LR Greenwood, SA Jones, D Neiner, BM Oliver, KN Pool, AJ Schmidt, RW Shimskey, SI Sinkov, SZ Soderquist, CJ Thompson, ML Thomas, T Trang-Le, and MW Urie. 2011b. Characterization Data Package for Containerized Sludge Samples Collected from Engineered Containers SCS-CON-240, 250, 260, and 220.

PNNL-19035, Rev. 1, Pacific Northwest National Laboratory, Richland, Washington.

Johnson B and SP Burke. 1995. K Basin Corrosion Program Report. WHC-EP-0877, Westinghouse Hanford Company, Richland, Washington. 
Johnson ME. 2013. Spent Nuclear Fuel Project Technical Databook, Volume 2, Sludge. HNFSD-SNF-TI-015, Volume 2, Revision 24, CH2M Hill Plateau Remediation Company, Richland Washington.

Lowell JS. 2004. Characterization of Porous Solids and Powders: Surface Area, Pore Size and Density. Volume 16 in the Particle Technology Series, Springer (Netherlands), ISBN 13:9781402023026, September 2004, 349 pp. hardcover, $2^{\text {nd }}$ printing 2006.

Makenas BJ, RB Baker, JA Pottmeyer, and TJ Welsh. 2013. Data Validation and Assessment by the Sludge Treatment Project for Characterization Data from Engineered Container SCS-CON210, PRC-STP-00560, Revision 0, 0A, and 0B, CH2M Hill Plateau Remediation Company, Richland Washington.

Makenas BJ, TL Welsh, RB Baker, DR Hansen, and GR Golcar. 1996. Analysis of Sludge from Hanford K East Basin Floor and Weasel Pit. WHC-SP-1182, Rev. 0, Westinghouse Hanford Company, Richland, Washington.

Makenas BJ, TL Welsh, RB Baker, EW Hoppe, AJ Schmidt, J Abrefah, JM Tingey, PR Bredt, and GR Golcar. 1997. Analysis of Sludge from Hanford K East Basin Canisters. HNF-SP-1201, Rev. 0, Duke Engineering \& Services Hanford, Inc., Richland, Washington.

Makenas BJ, TL Welsh, RB Baker, GR Golcar, PR Bredt, AJ Schmidt, and JM Tingey. 1998. Analysis of Sludge from Hanford K West Basin Canisters. HNF-1728, Rev. 0, Fluor Daniel Hanford, Richland, Washington.

Makenas BJ, TL Welsh, PR Bredt, GR Golcar, AJ Schmidt, KL Silvers, JM Tingey, AH Zacher, and RB Baker. 1999. Analysis of Internal Sludge and Cladding Coatings from N-Reactor Fuel Stored in Hanford K Basins. HNF-3589, Rev. 0, Fluor Daniel Hanford, Inc., Richland, Washington.

Mucciardi AN, IJ Booker, EC Orr, and D Cleveland. 1979. "Statistical Investigation of the Mechanics Controlling Radionuclide Sorption, Part II.” In Proceedings of the Task 4 Waste Isolation Safety Assessment Program, Second Contractor Information Meeting, Vol. 2, pg 333-425, PNL-SA-7352, Pacific Northwest Laboratory, Richland, Washington.

Pearce KL and SC Klimper. 2001. 105-K Basin Material Design Basis Feed Description for Spent Nuclear Fuel Project Facilities, Volume 2, Sludge. HNF-SD-SNF-TI-009, Rev. 3, Fluor Hanford, Richland, Washington.

Plys MG and AJ Schmidt. 2006. Supporting Basis for SNF Project Technical Databook. SNF-7765 Rev. 3, Fluor Hanford, Inc. Richland, Washington.

Schmidt AJ. 2010. Spent Nuclear Fuel Project Technical Databook Volume 2, Sludge. HNF-SD-TI-015, Sludge, Rev. 14A, CH2MHill Plateau Remediation Company, Richland; Washington.

Schmidt AJ, CH Delegard, KL Silvers, PR Bredt, CD Carlson, EW Hoppe, JC Hayes, DE Rinehart, SR Gano, and BM Thornton. 1999. Validation Testing of the Nitric Acid 
Dissolution Step Within the K Basin Sludge Pretreatment Process. PNNL-12120, Pacific Northwest National Laboratory, Richland, Washington.

Shimskey RW, JM Billing, SJ Bos, CA Burns, CD Carlson, DS Coffey, RC Daniel, CH Delegard, MK Edwards, SK Fiskum, LR Greenwood, SA Jones, M Luna, D Neiner, BM Oliver, AJ

Schmidt, SI Sinkov, LA Snow, CZ Soderquist, CJ Thompson, T Trang-Le, and MW Urie. 2013. Characterization Data Package for Containerized Sludge Samples Collected from Engineered

Container SCS-CON-230. PNNL-20470, Rev. 1, Pacific Northwest National Laboratory, Richland, Washington.

Thompson JF and LE Ross. 2011. Sludge Sample Collection Data Package for SCS-CON-230 and SCS-CON-210. KBC-48966, Rev. 0, CH2M Hill Plateau Remediation Company, Richland Washington.

Weast RC and MJ Astle, eds. 1979. CRC Handbook of Chemistry and Physics. $60^{\text {th }}$ Ed. CRC Press Inc., Boca Raton, Florida.

Westcott JL, BJ Makenas, TL Welsh, JA Pottmeyer, and AJ Schmidt. 2009. Data Quality Objectives for Sampling and Analysis of K Basin Sludge. HNF-36985, Rev. 3, CH2M Hill Plateau Remediation Company, Richland, Washington. 


\section{Appendix A}

\section{Completed Chain of Custody Forms}





\section{Appendix A}

\section{Completed Chain of Custody Forms}

This appendix contains images of the completed chain-of-custody (COC) forms submitted with each polybottle pair containing K Basin sludge. Table A.1 provides a cross-reference of the sample identification (ID) to the COC number, the sample date, and the receipt date at the Radiochemical Processing Laboratory in the order of sample date.

All eleven of the COC documents received with KW210 core sample bottles incorrectly listed Quality Assurance Project Plan/Sampling and Analysis Plan (QAPjP/SAP) KBC-40467. The correct QAPjP/SAP for sludge in the Engineered Container SCS-CON-210 is KBC-33786 Rev. 2 (Baker et al. 2009).

Table A.1. Listing of Individual COC Forms in Appendix A

\begin{tabular}{|c|c|c|c|}
\hline Client Sample ID & COC No. & Sample Date & Receipt Date \\
\hline KW210-A1-A & 6695 & $12 / 29 / 10$ & $1 / 4 / 11$ \\
\hline KW210-A1-B & 6695 & $12 / 29 / 10$ & $1 / 4 / 11$ \\
\hline KW210-A1-C & 6697 & $12 / 30 / 10$ & $1 / 4 / 11$ \\
\hline KW210-A1-D & 6697 & $12 / 30 / 10$ & $1 / 4 / 11$ \\
\hline KW210-A4-A & 6662 & $11 / 17 / 10$ & $11 / 18 / 10$ \\
\hline KW210-A4-B & 6662 & $11 / 17 / 10$ & $11 / 18 / 10$ \\
\hline KW210-A4-C & 6664 & $11 / 18 / 10$ & $12 / 2 / 10$ \\
\hline KW210-A4-D & 6664 & $11 / 18 / 10$ & $12 / 2 / 10$ \\
\hline KW210-A4-E & 6668 & $11 / 19 / 10$ & $12 / 6 / 10$ \\
\hline KW210-A4-F & 6668 & $11 / 19 / 10$ & $12 / 6 / 10$ \\
\hline KW210-B1-A & 6674 & $12 / 1 / 10$ & $12 / 8 / 10$ \\
\hline KW210-B1-B & 6674 & $12 / 1 / 10$ & $12 / 8 / 10$ \\
\hline KW210-B1-C & 6678 & $12 / 7 / 10$ & $12 / 14 / 10$ \\
\hline KW210-B1-D & 6678 & $12 / 7 / 10$ & $12 / 14 / 10$ \\
\hline KW210-B1-F ${ }^{(a)}$ & 6682 & $12 / 9 / 10$ & $12 / 16 / 10$ \\
\hline KW210-B1-G & 6682 & $12 / 9 / 10$ & $12 / 16 / 10$ \\
\hline KW210-B3-A & 6686 & $12 / 15 / 10$ & $12 / 27 / 10$ \\
\hline KW210-B3-B & 6686 & $12 / 15 / 10$ & $12 / 27 / 10$ \\
\hline KW210-B3-C & 6689 & $12 / 17 / 10$ & $12 / 27 / 10$ \\
\hline KW210-B3-D & 6689 & $12 / 17 / 10$ & $12 / 27 / 10$ \\
\hline KW210-B3-E & 6692 & $12 / 27 / 10$ & $12 / 29 / 10$ \\
\hline KW210-B3-F & 6692 & $12 / 27 / 10$ & $12 / 29 / 10$ \\
\hline
\end{tabular}

(a) Polybottle ID KW210-B1-E was omitted in the sequence; no such sample was collected or provided. 

PNNL-20650, Rev. 2

\section{KBC PROJECT CHAIN OF CUSTODY}

coc No. 6695

Date $12 / 29 / 10$ Field Logbook No. $n / A$

Point of Contact

Phone No.

$\operatorname{MSIN} \times 4-0 /$

Delivered to: $\square$ OS Engineer $\square 1706$ KE Counting Facility $\square$ 222-S Lab $\square$ WSCF $X O$ Other $325 \angle A B$

Sampled By $\frac{R R R \text { pmurez }}{\text { Print }}$

多

See Sample Analysis Request for individual containers and analysis.

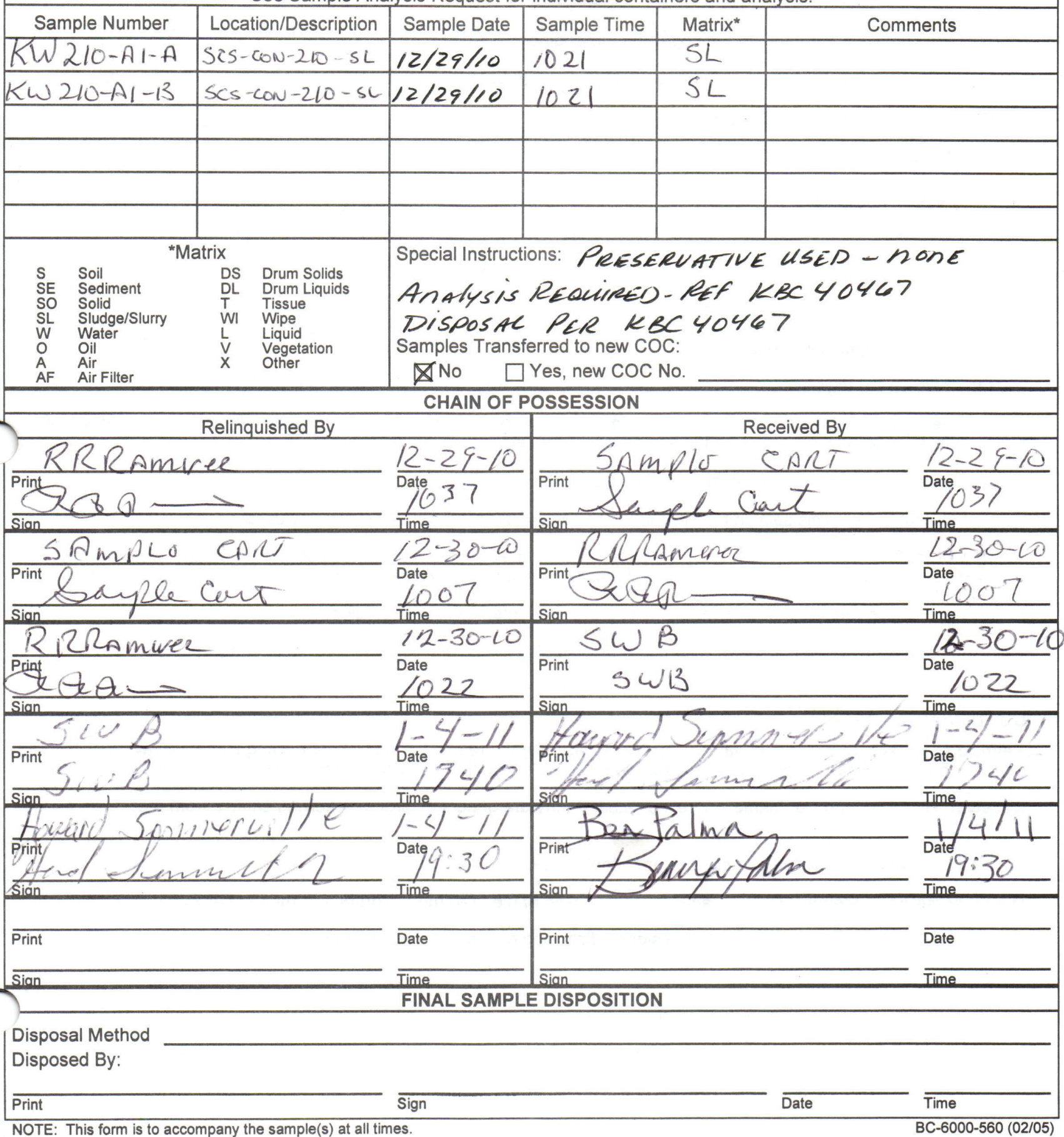




\section{KBC PROJECT CHAIN OF CUSTODY}

COC No. 669

Date $12 / 30 / 10$ Field Logbook No. N/A

point of Contact LANCE ROSS Phone No. 373-4494 MSIN $\times 4$ - of

Delivered to: $\square$ OS Engineer $\square 1706$ KE Counting Facility $\square$ 222-S Lab $\square$ WSCF 女Other 325 LAB Sampled By $\frac{R R \text { RAmirez }}{\text { Print }}$ sign 2

See Sample Analysis Request for individual containers and analysis.

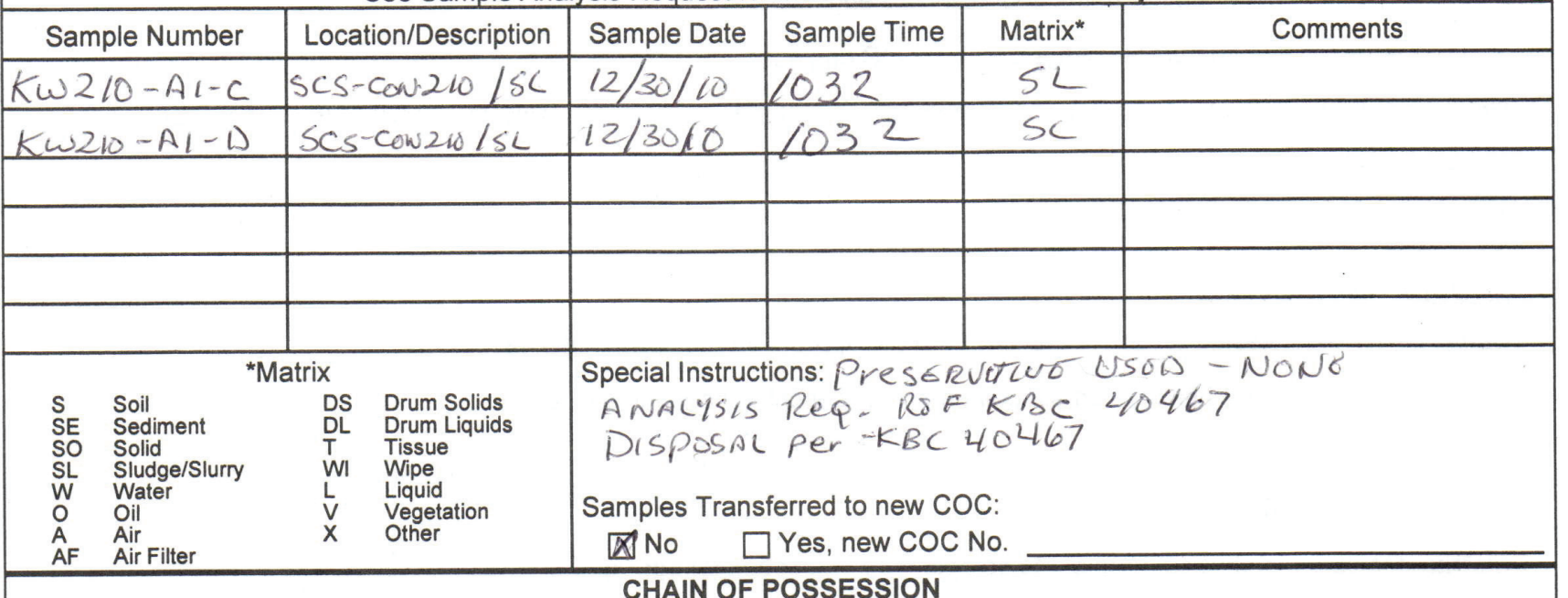

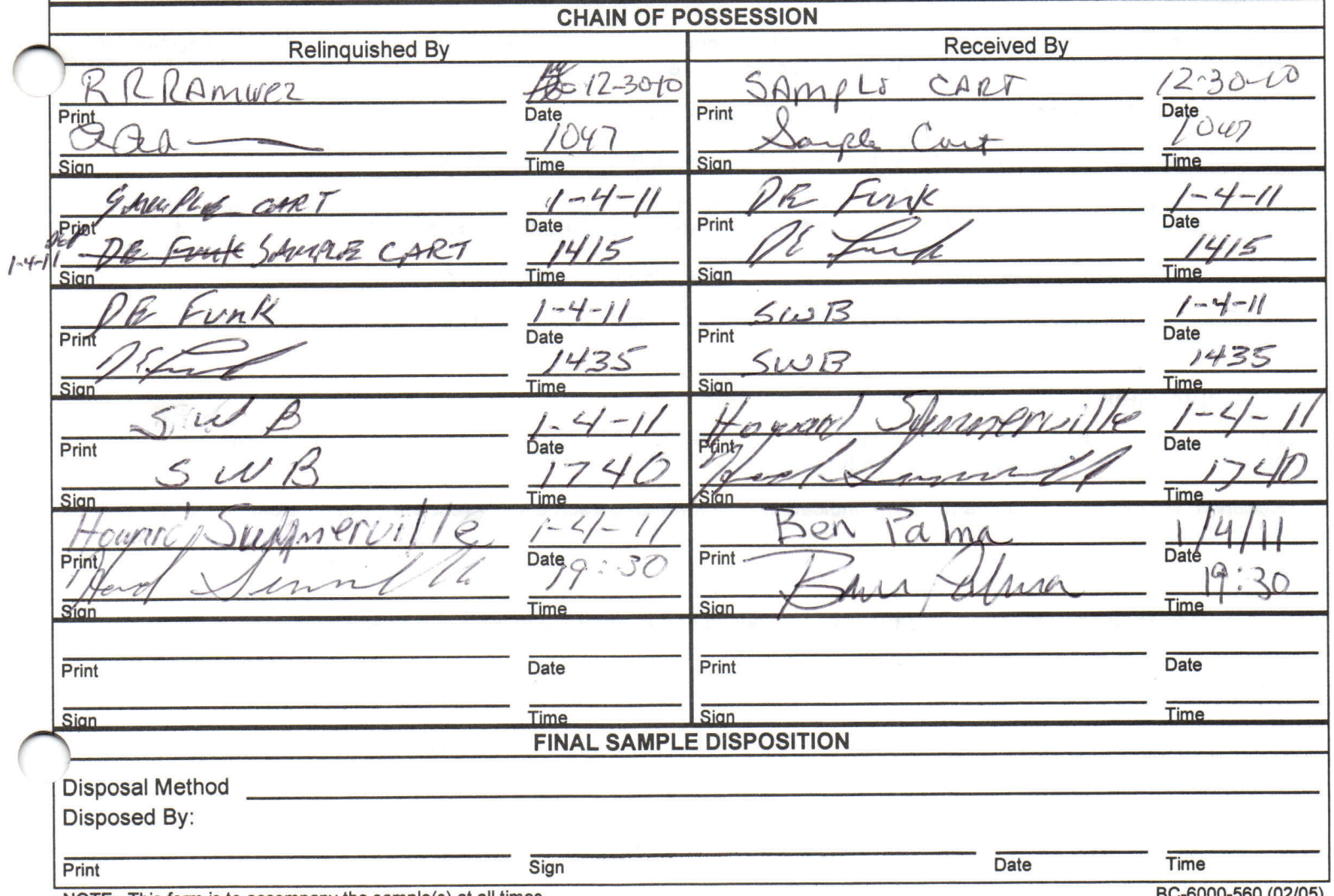

NOTE: This form is to accompany the sample(s) at all times. 


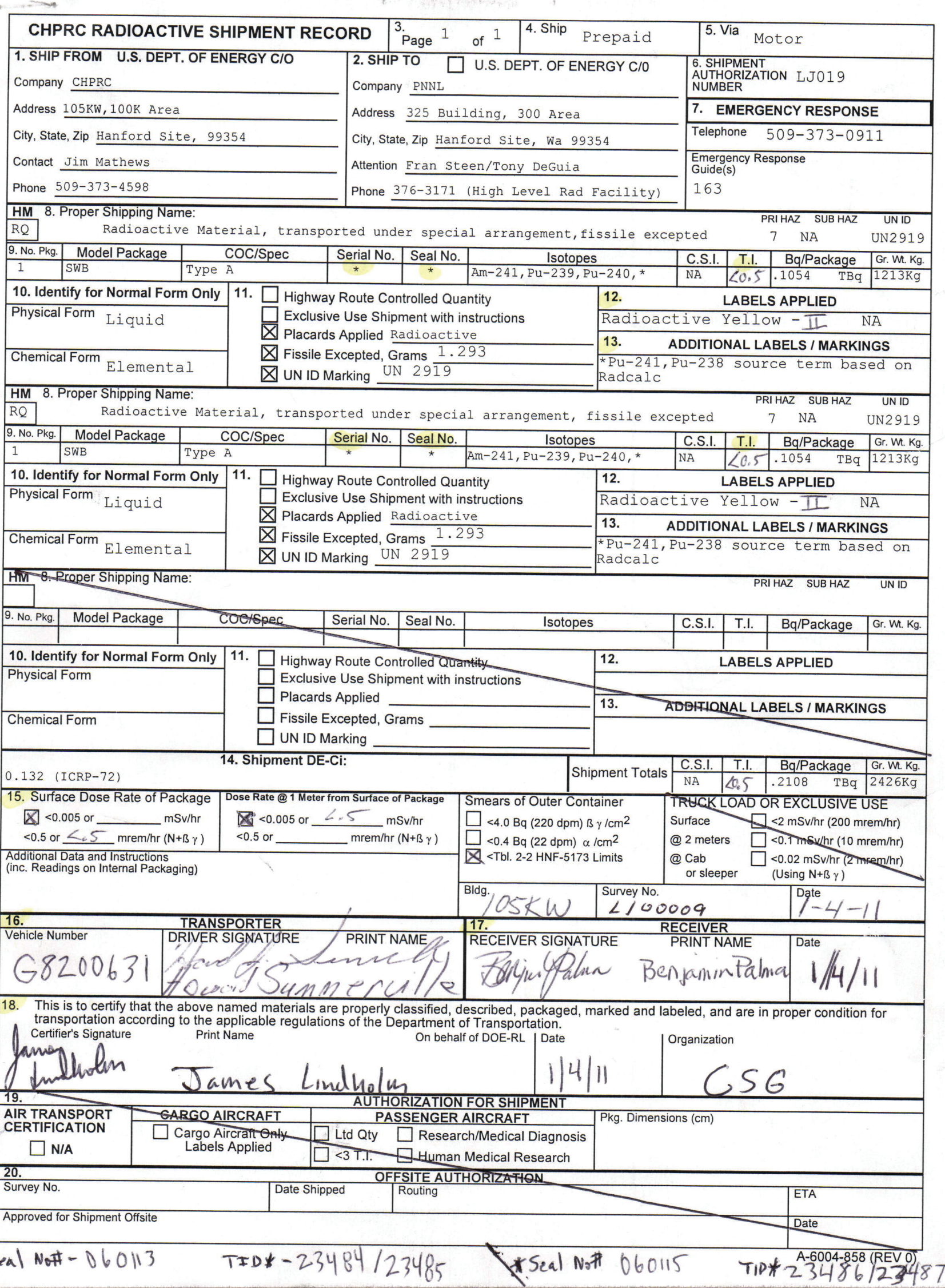




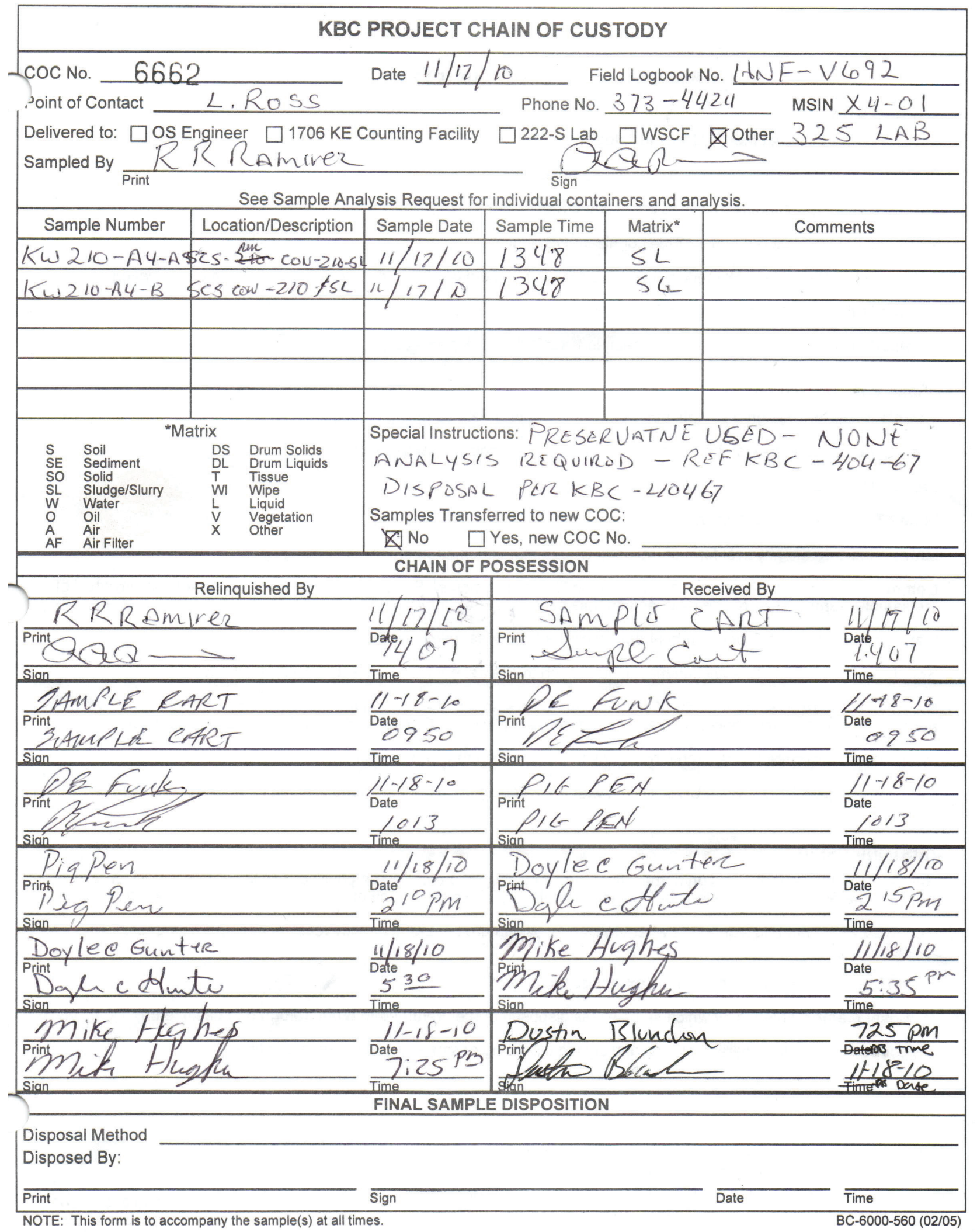




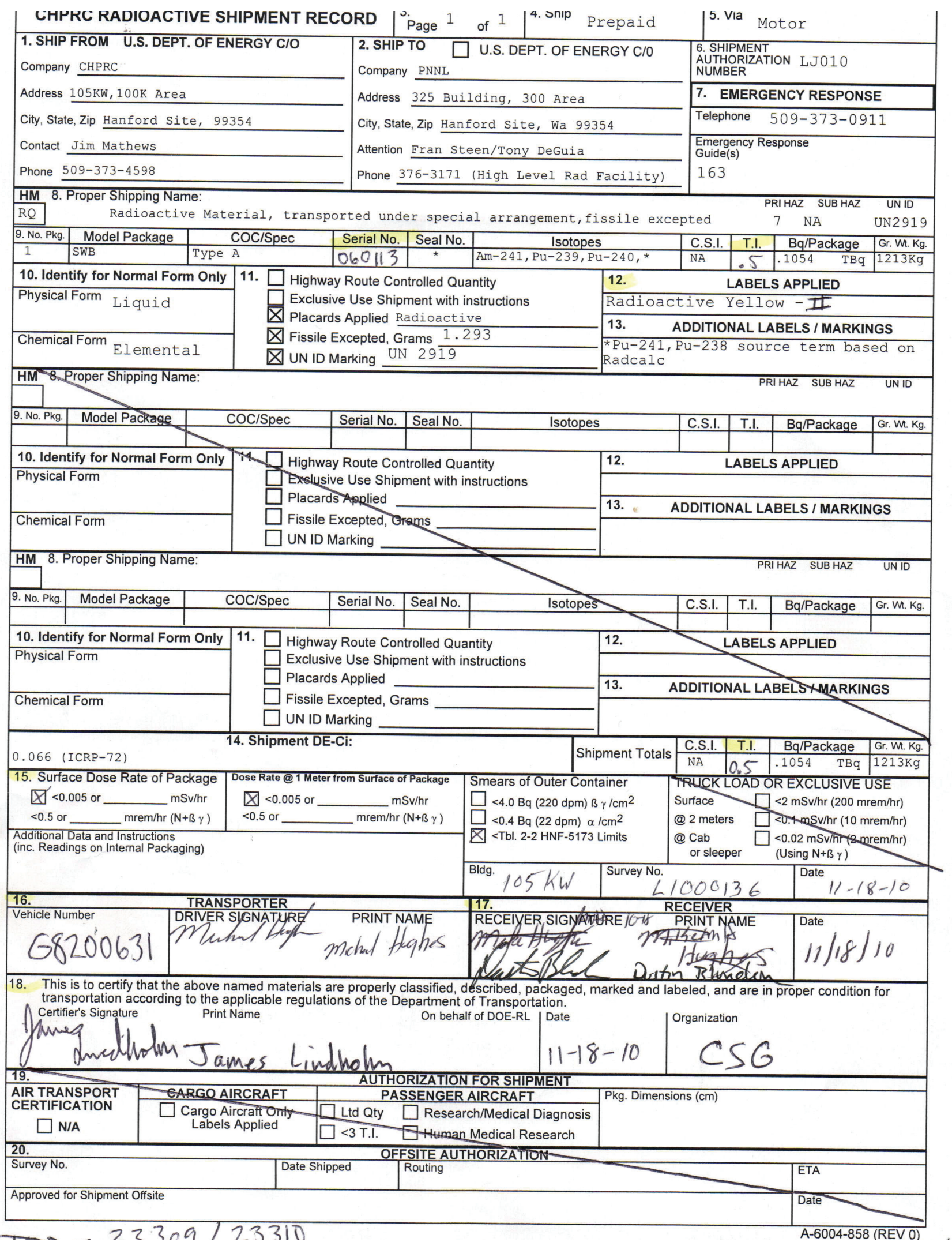




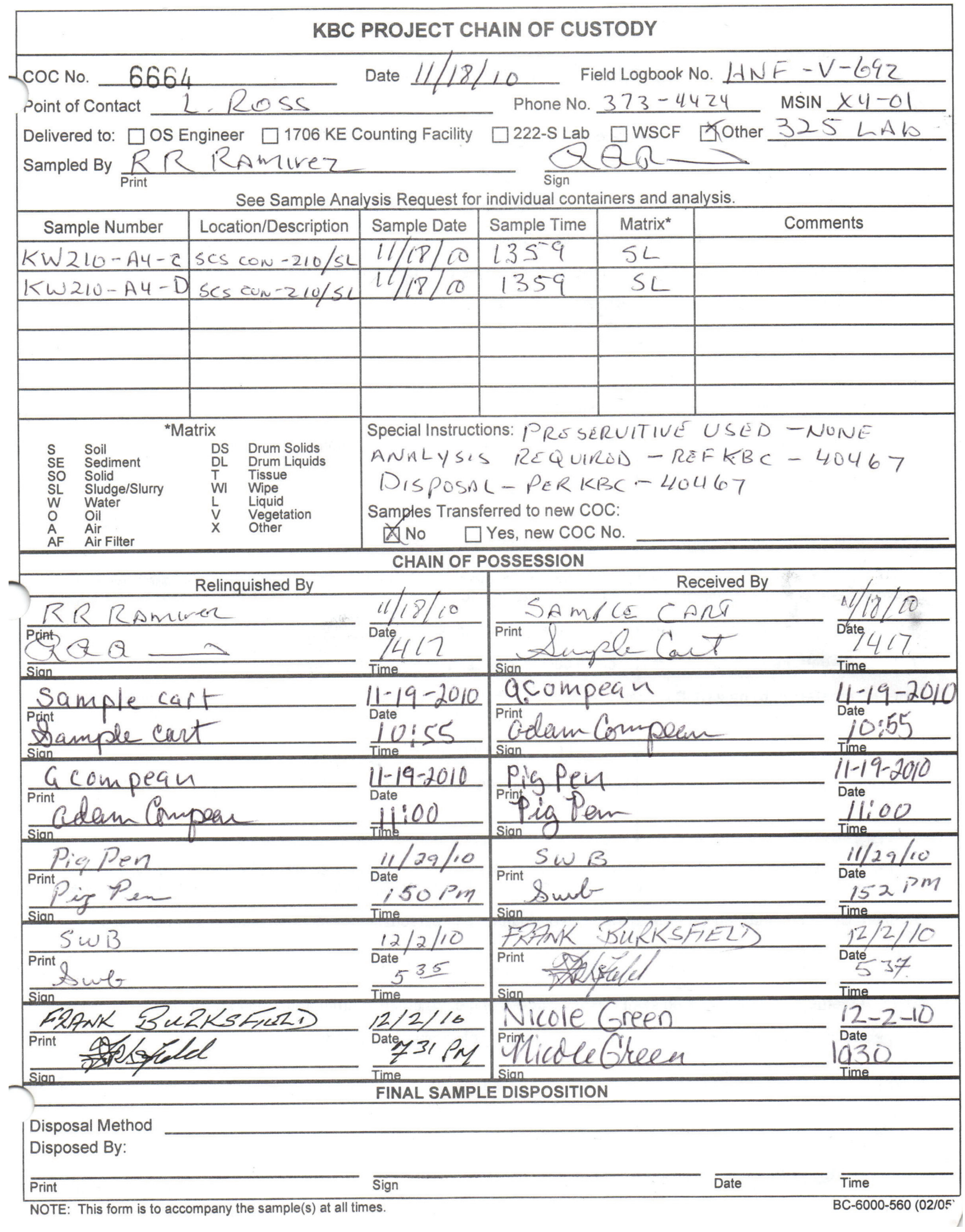




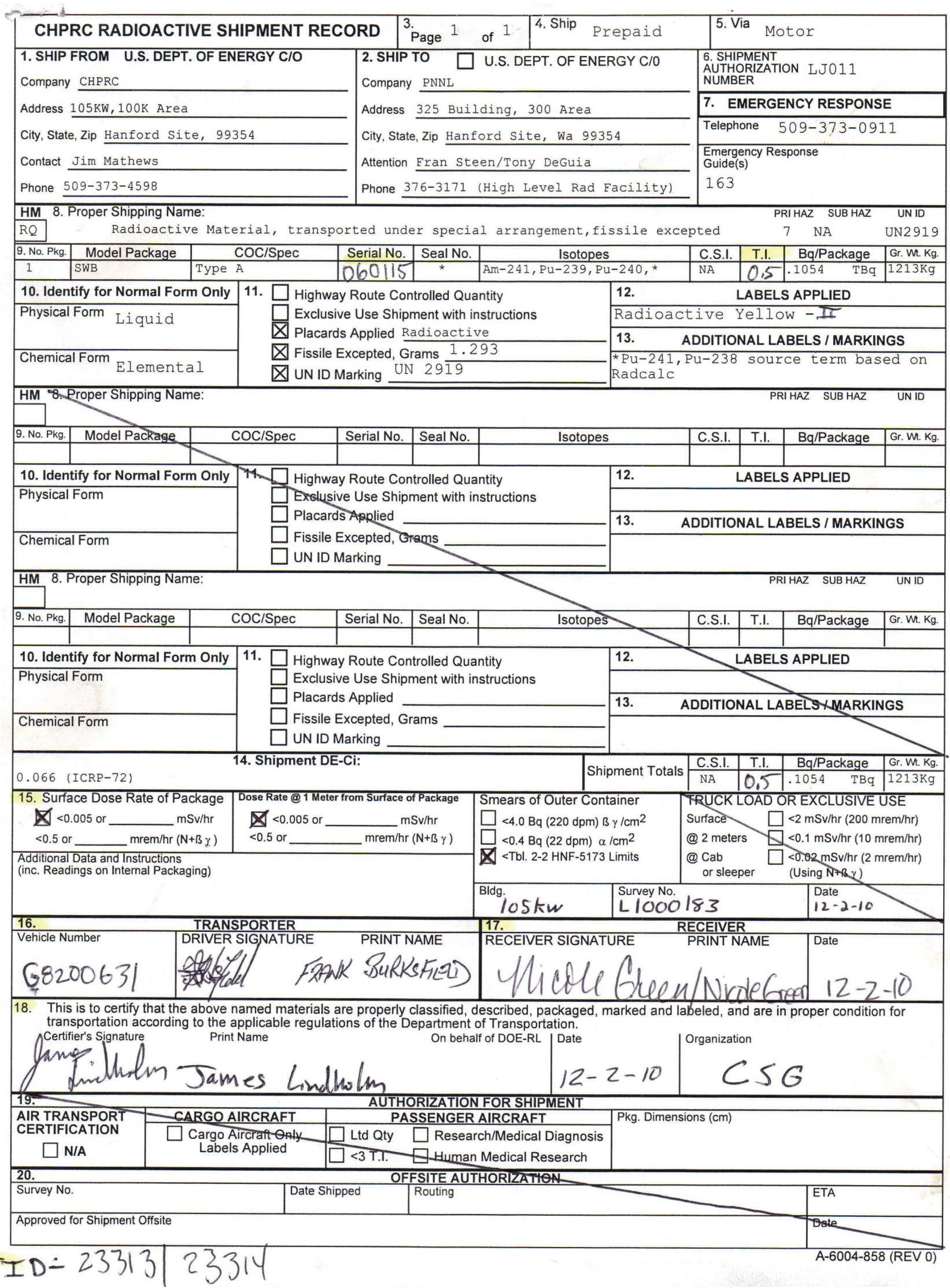




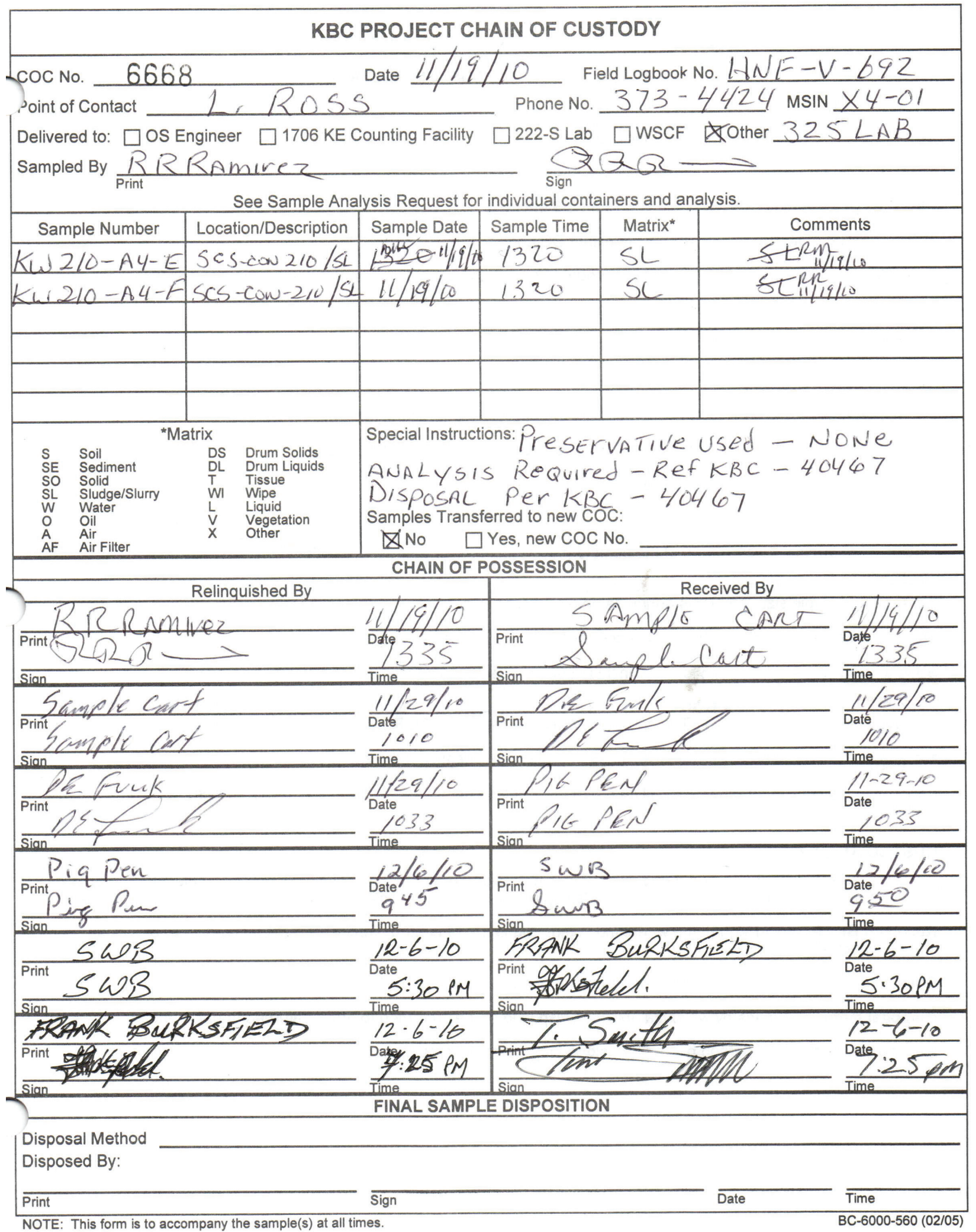




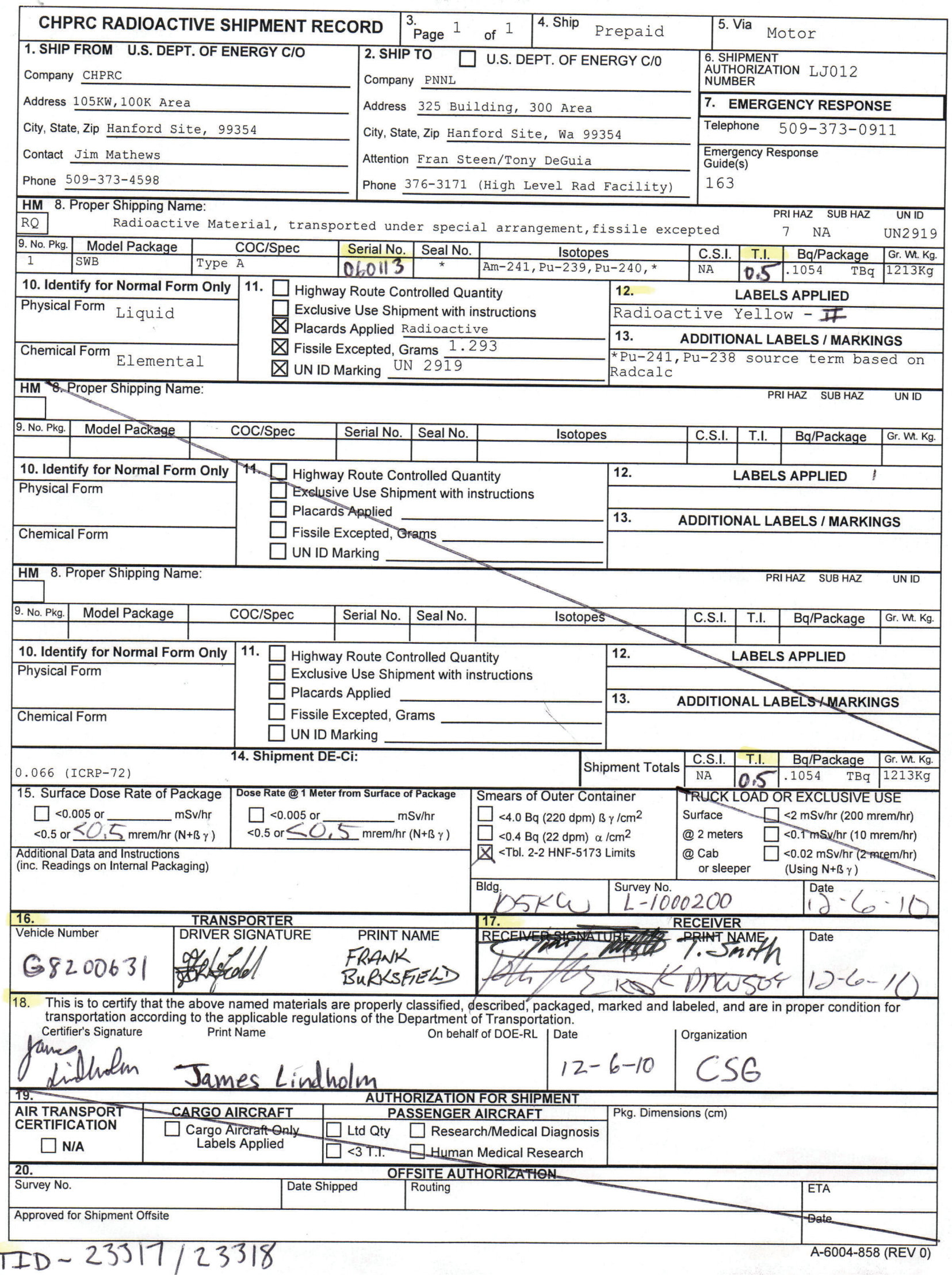




\section{KBC PROJECT CHAIN OF CUSTODY}

$\operatorname{coc}$ No. 6674 Date $12 / 1 / 10 \quad$ Field Logbook No. N/A Point of Contact Lance Ross Phone No. 373-4494 MSIN $\frac{\times 4-01}{1}$ Delivered to: $\square$ OS Engineer $\square 1706$ KE Counting Facility $\square$ 222-S Lab $\square$ WSCF $\mathbb{\text { OOther }} 3$ ZS Lab Sampled By $\frac{D F R \text { Print }}{2}$

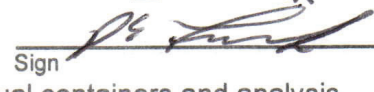

See Sample Analysis Request for individual containers and analysis.

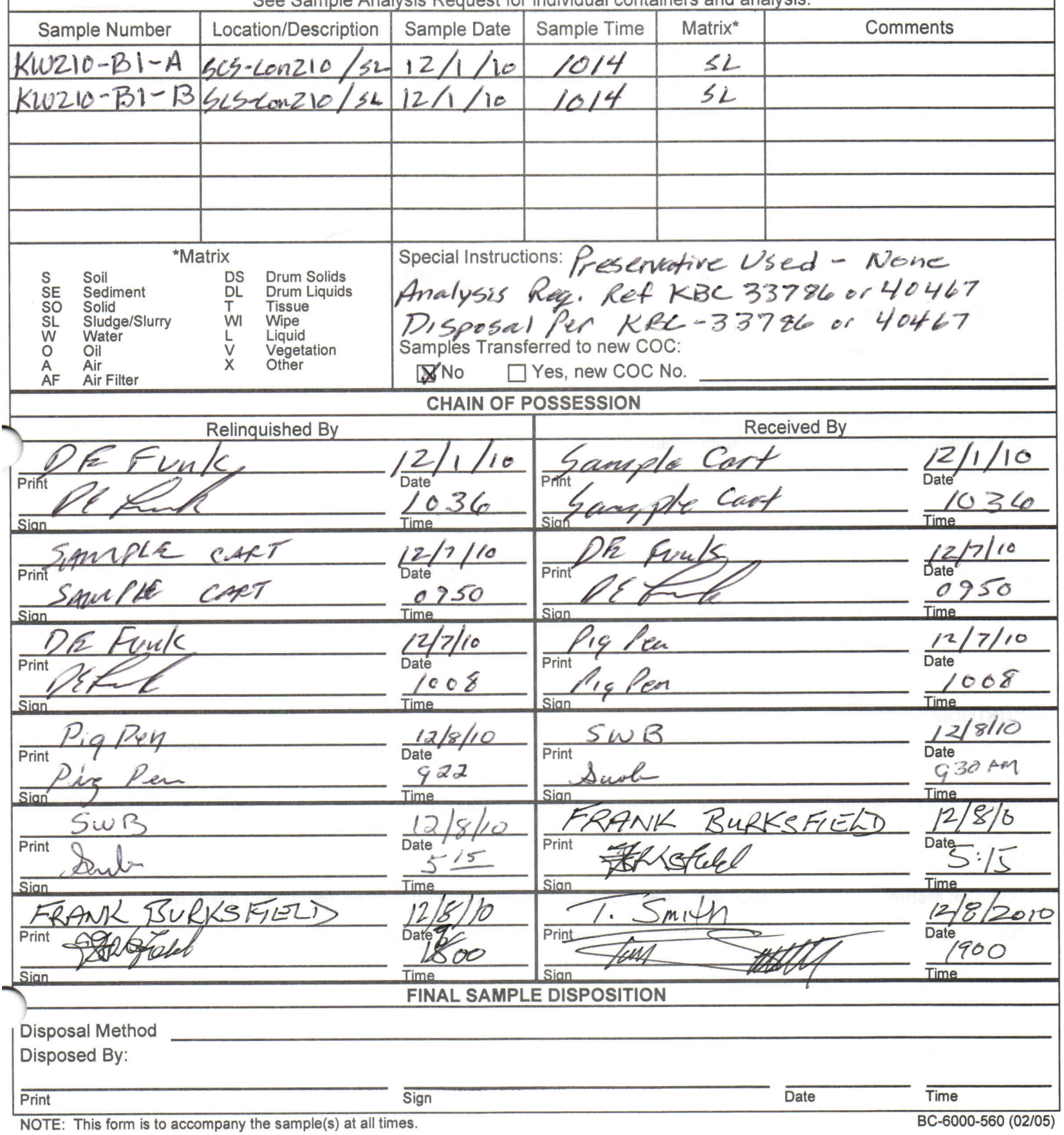




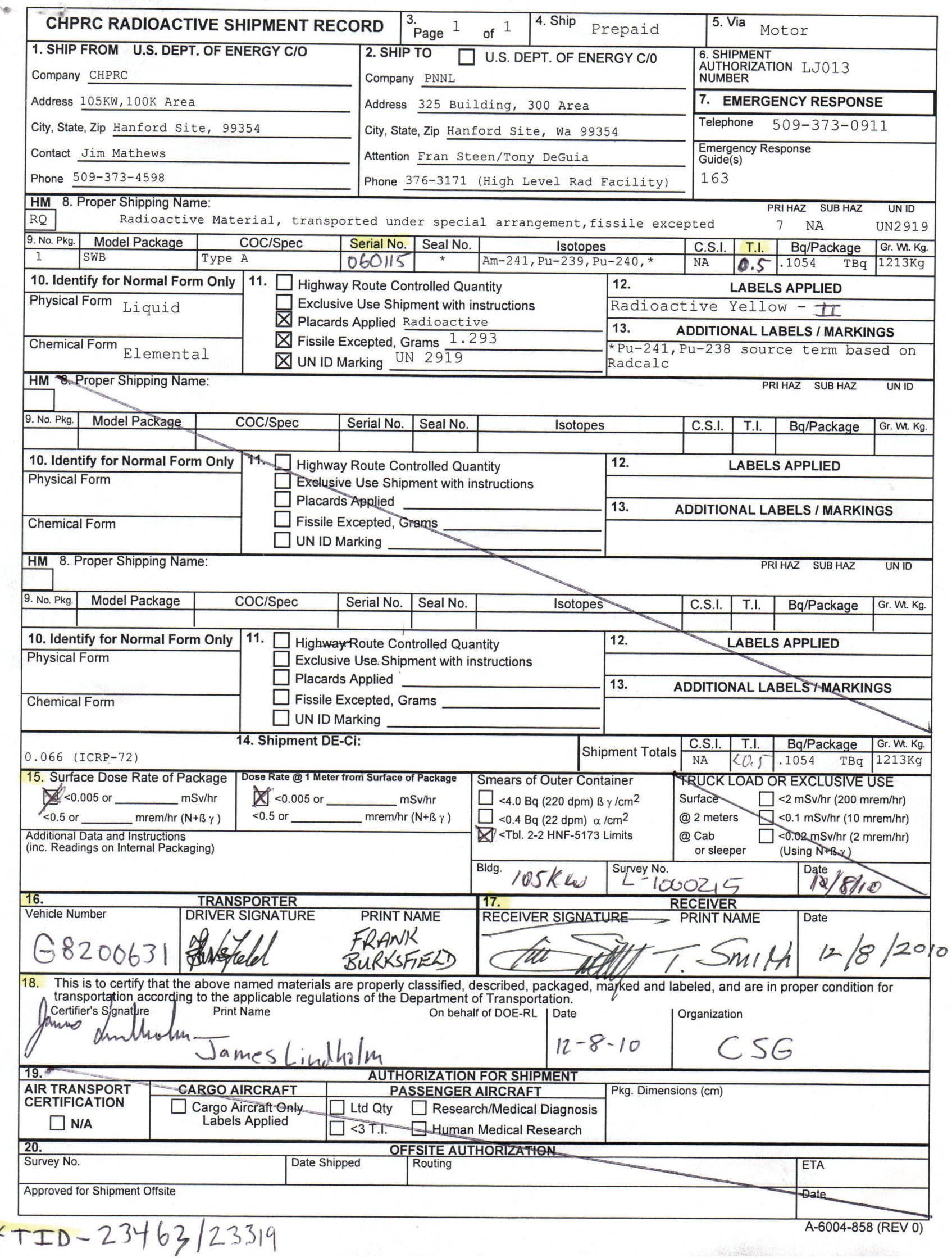




\section{KBC PROJECT CHAIN OF CUSTODY}

$\operatorname{coc}$ No. 6678

Point of Contact Lance Ross

Date $\mathrm{Z/Z/10}$ Field Logbook No. N/A

Phone No. $373-4494$ MSIN $X 4-01$

Delivered to: $\square$ OS Engineer $\square 1706 \mathrm{KE}$ Counting Facility $\square$ 222-S Lab $\square$ WSCF $\otimes$ Other $325 \mathrm{LAB}$

Sampled By $\frac{\text { Drint }}{\text { De funk }}$

sign

See Sample Analysis Request for individual containers and analysis.

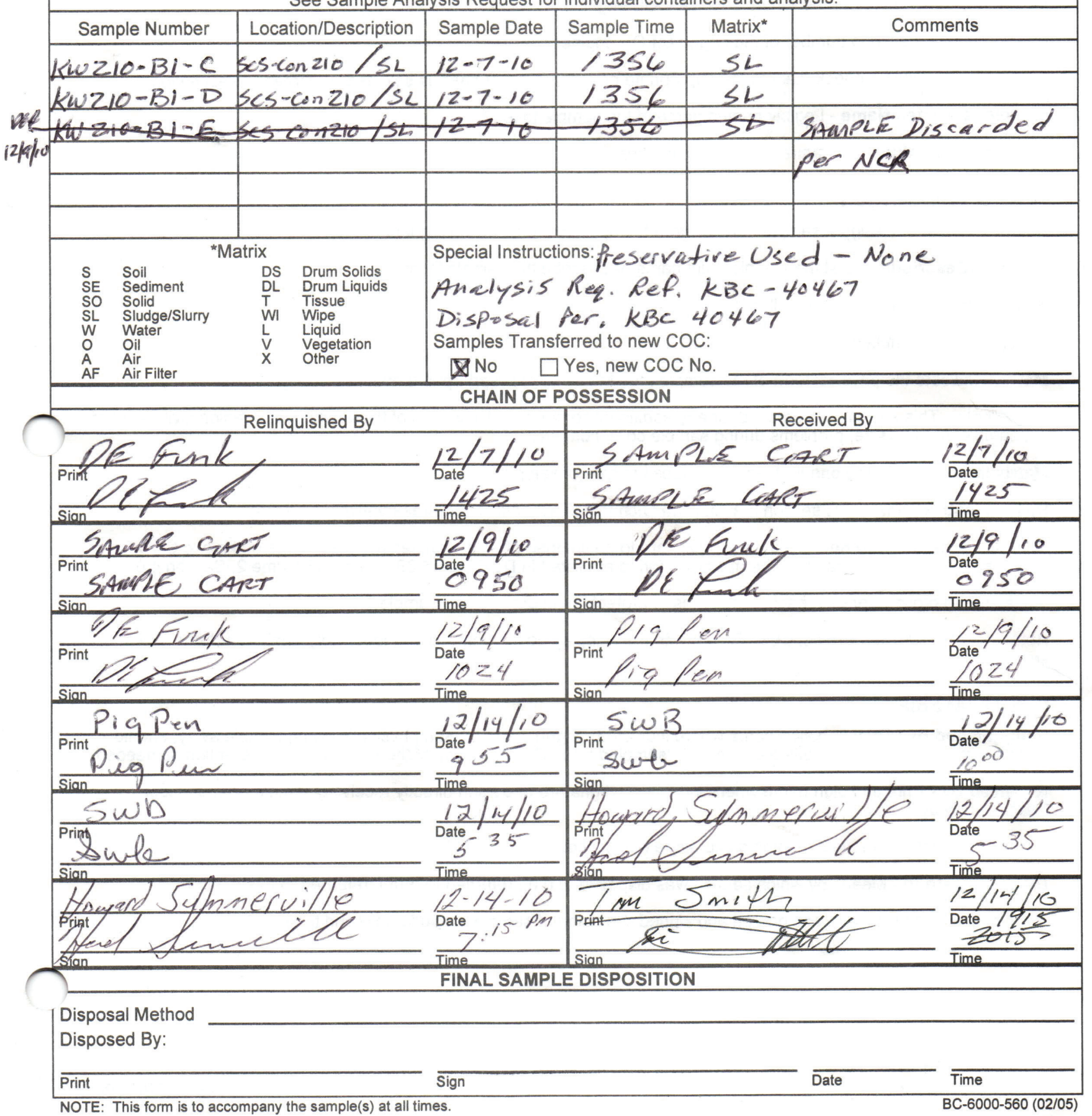




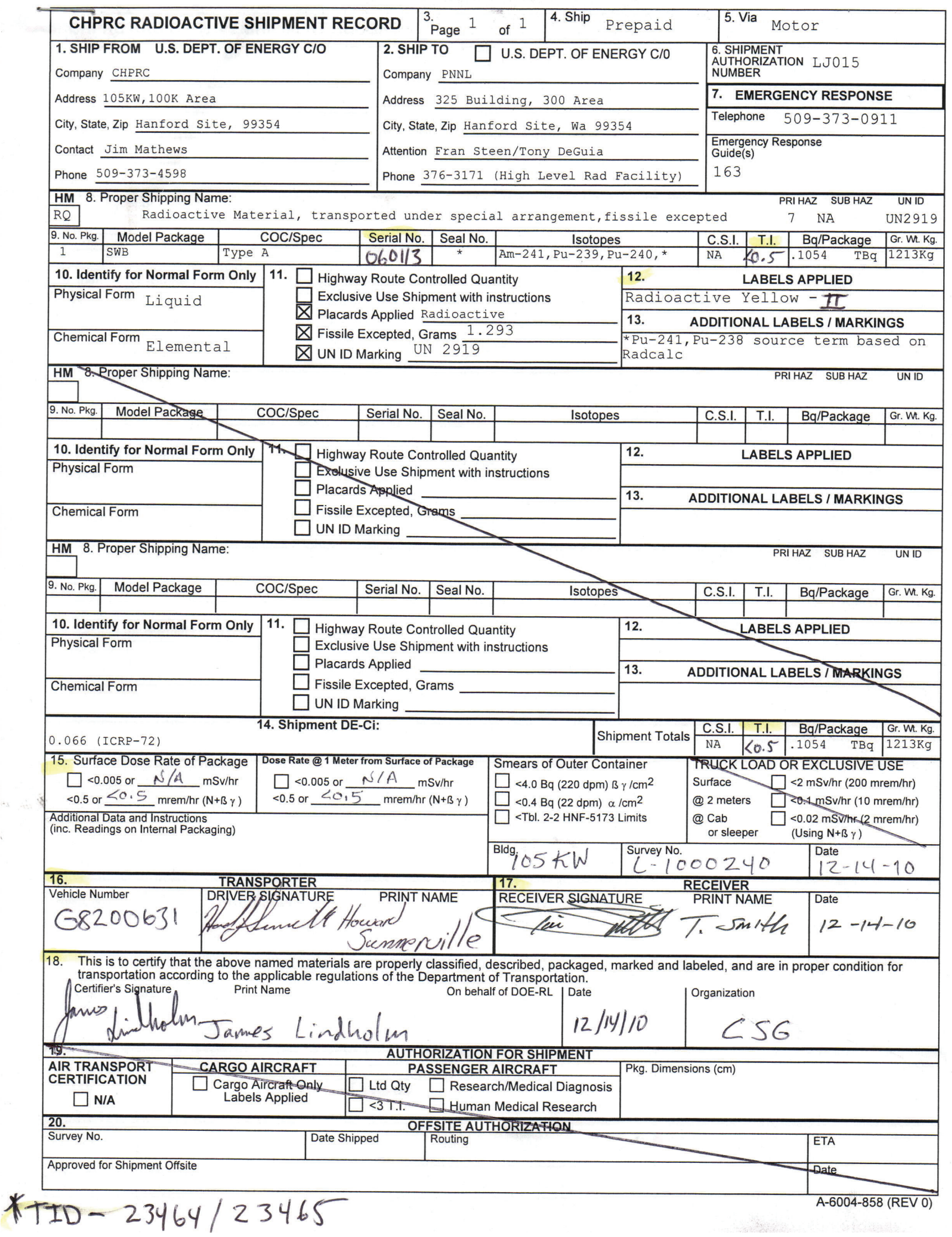




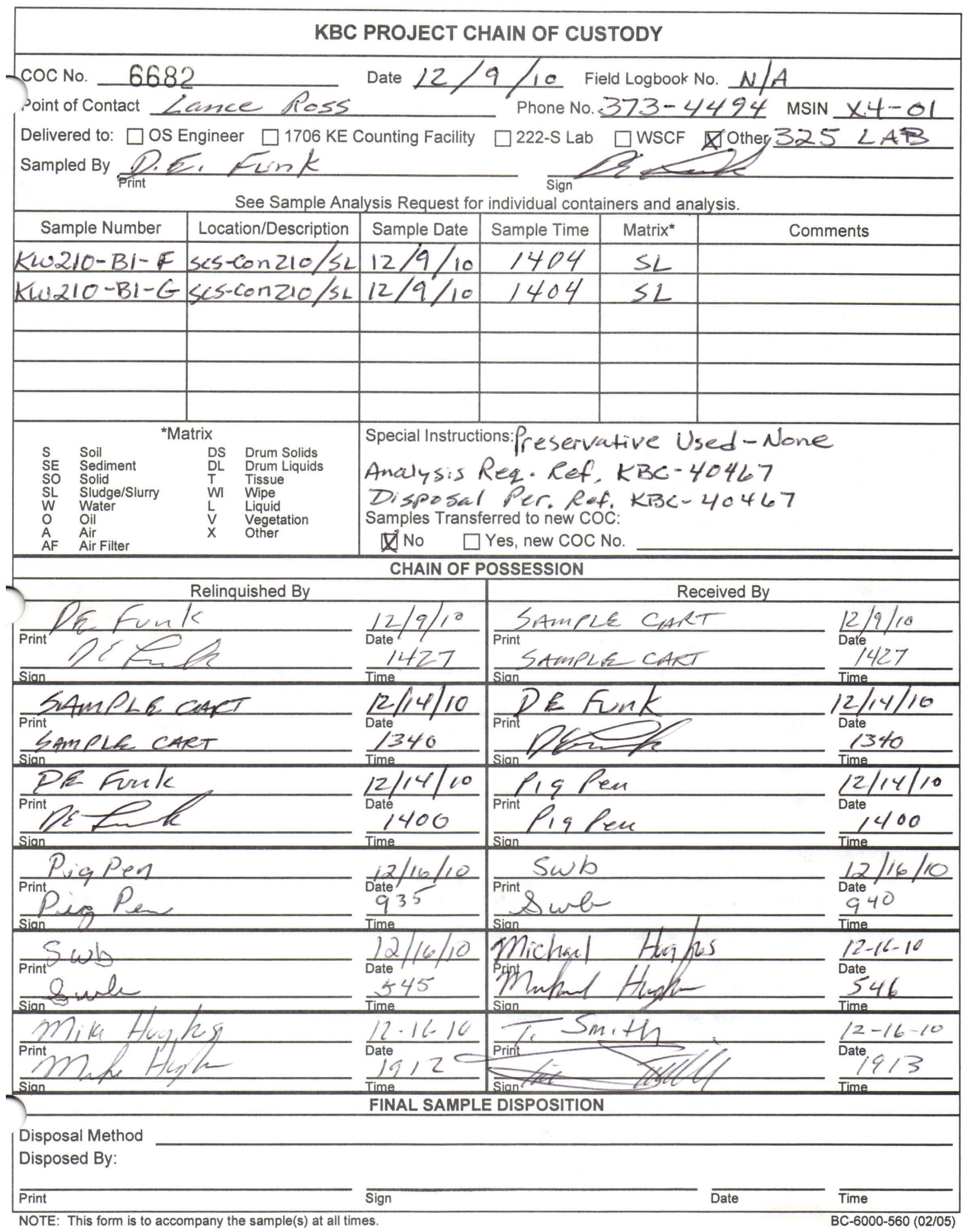




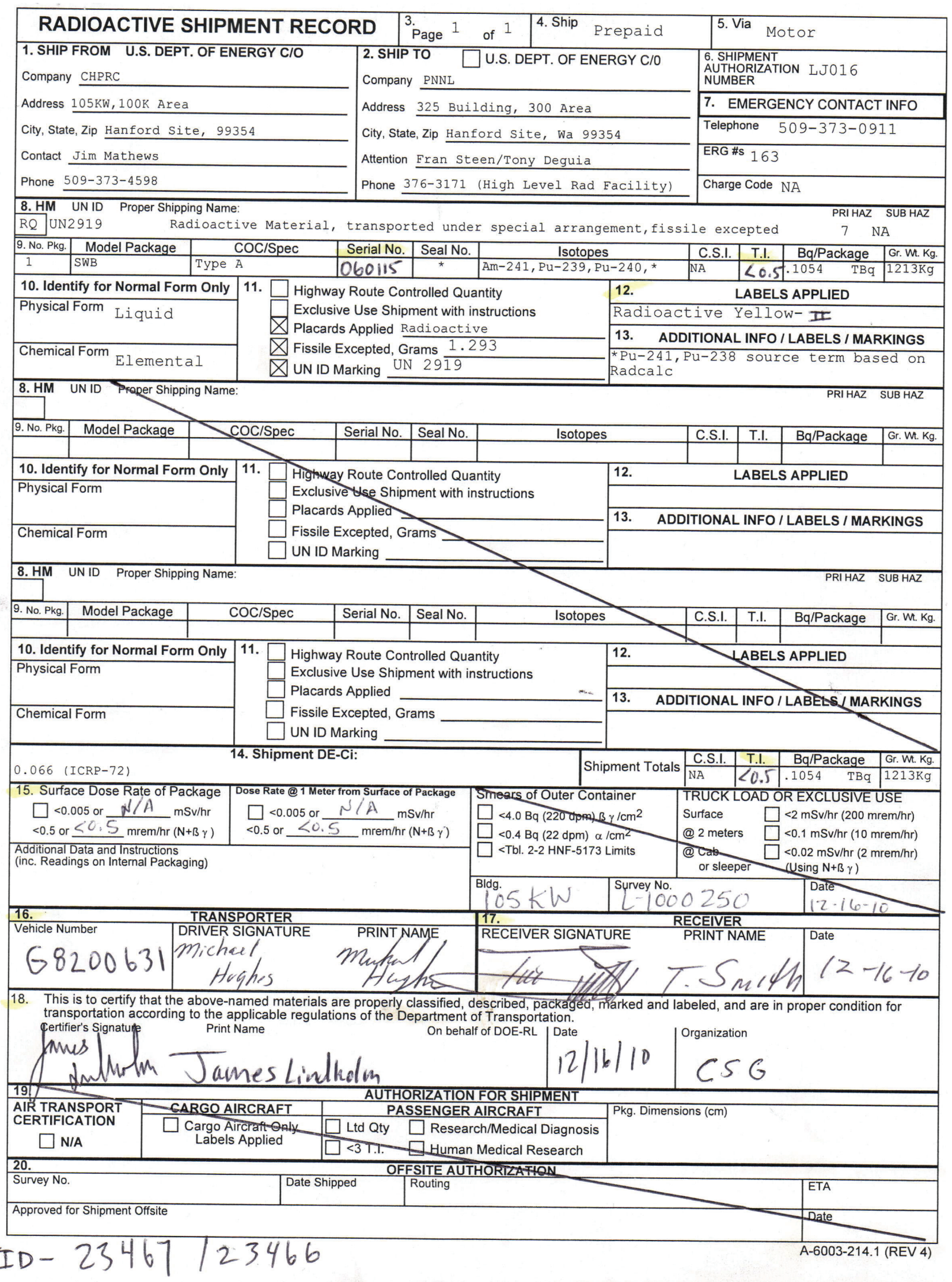




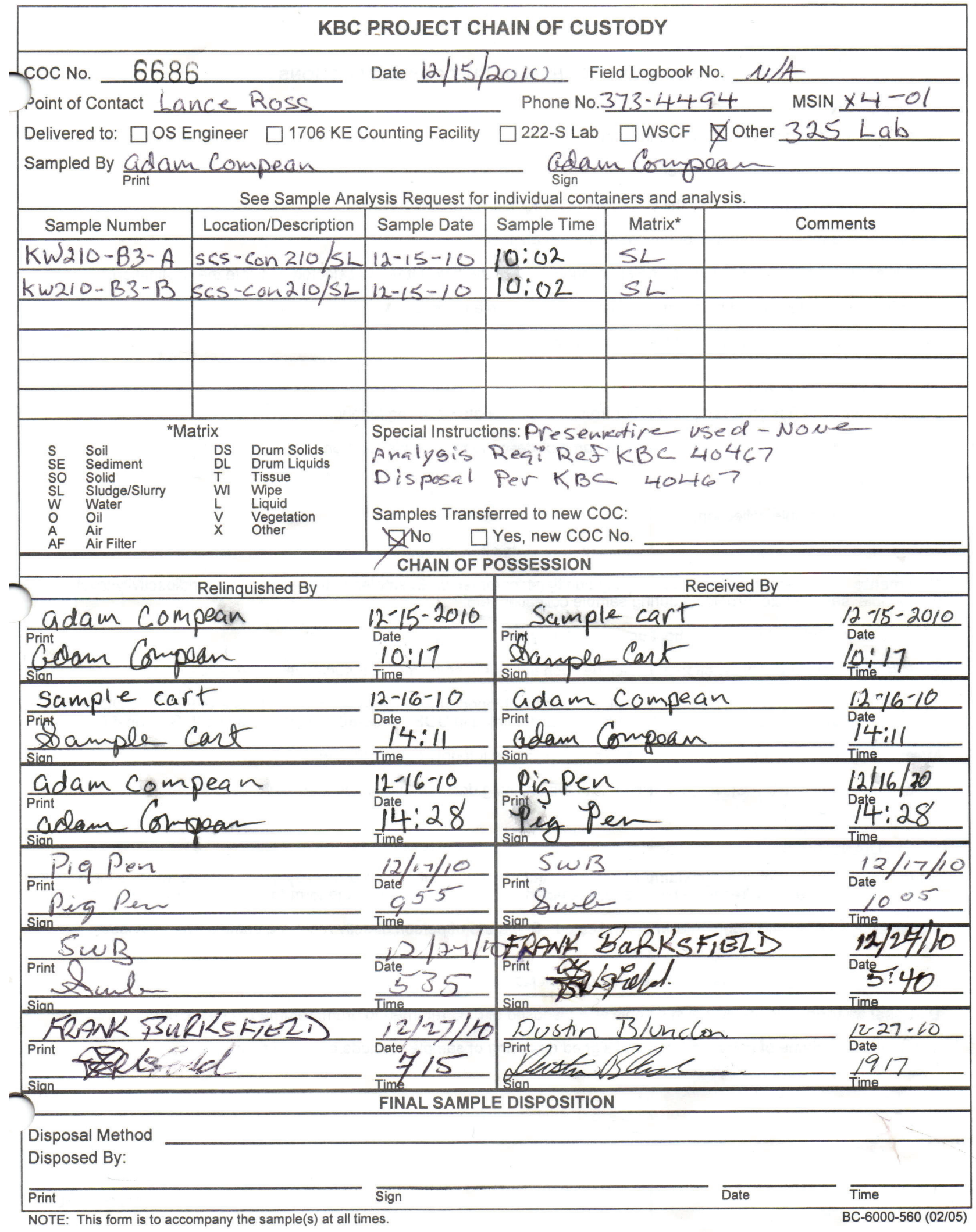




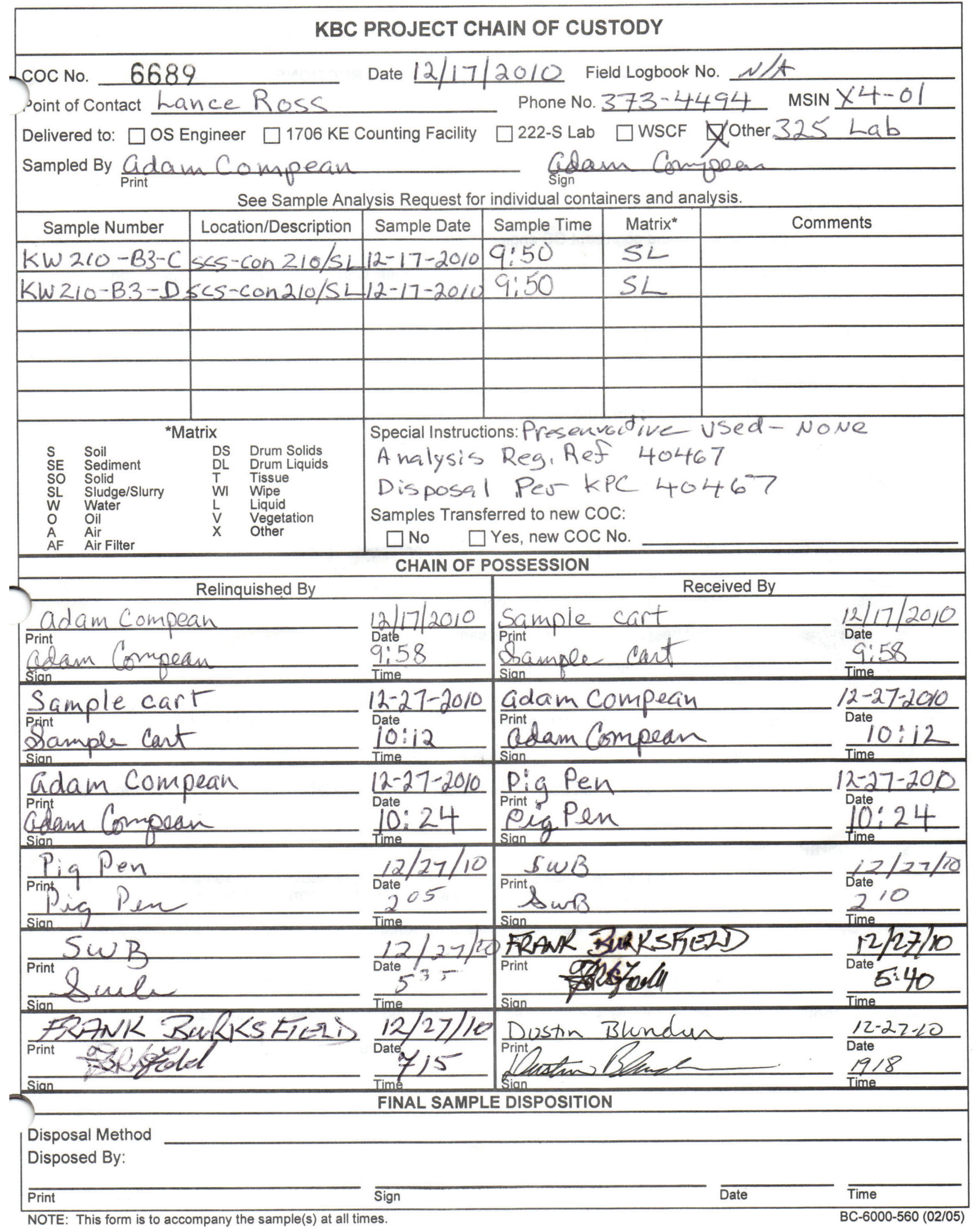




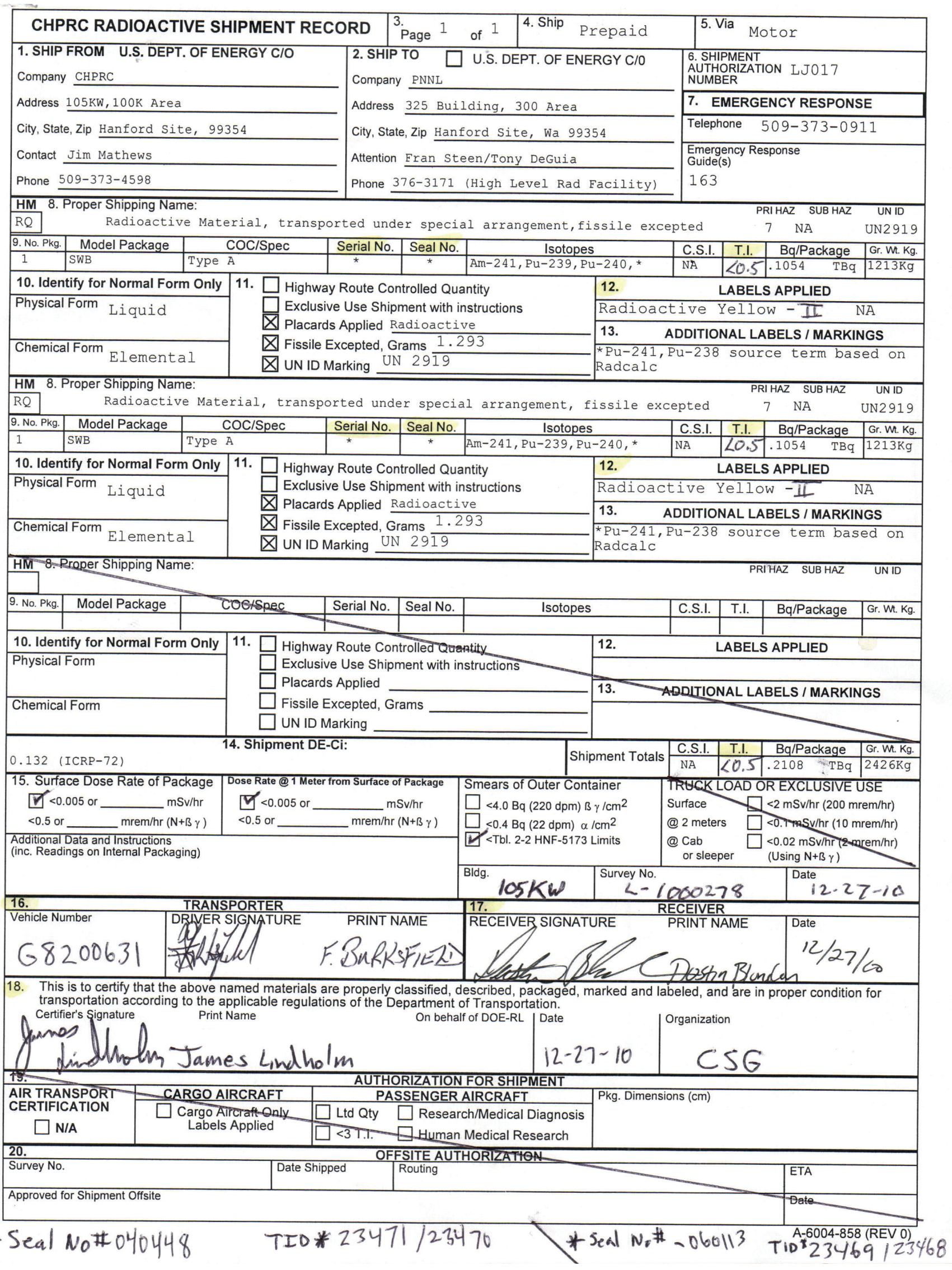




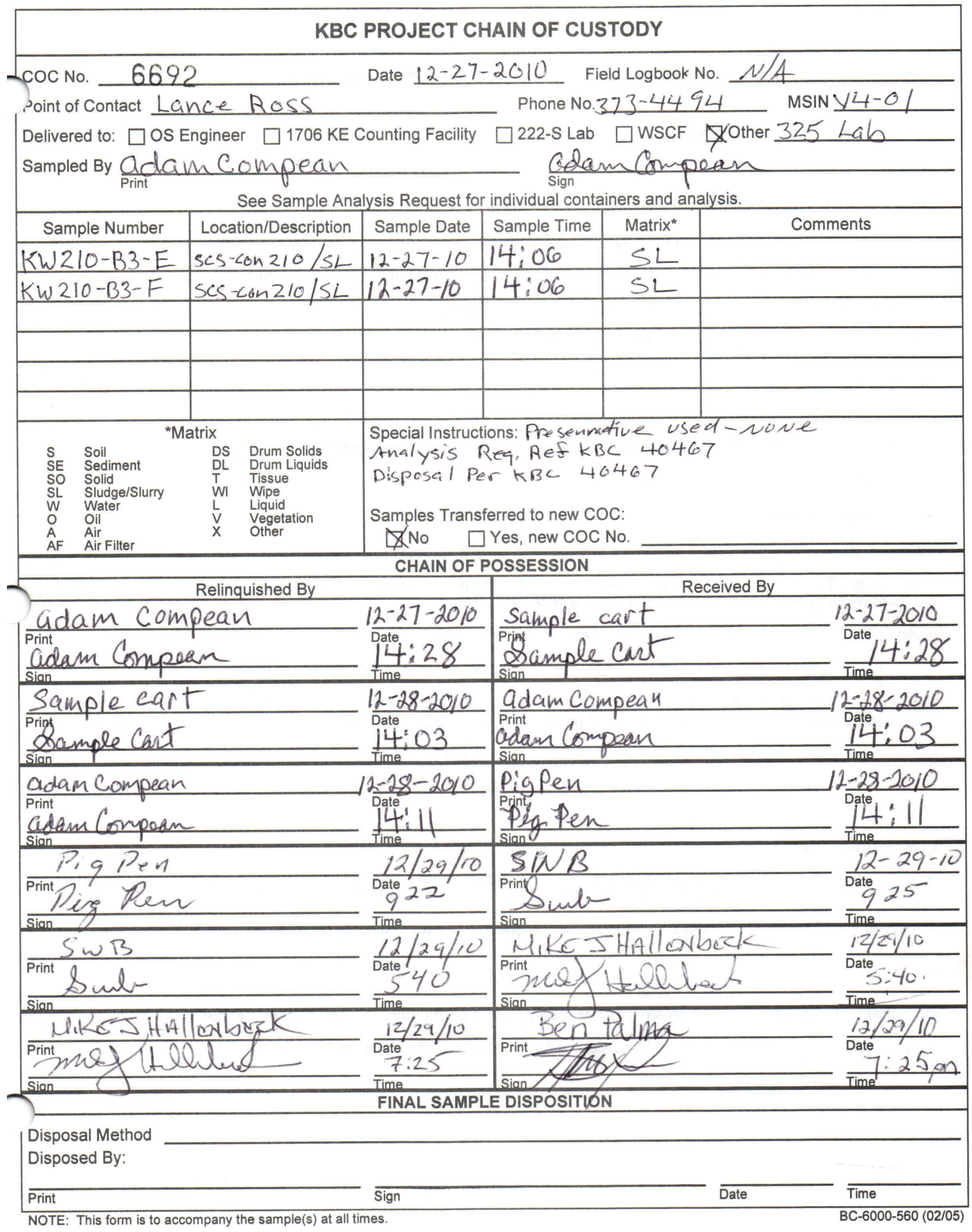




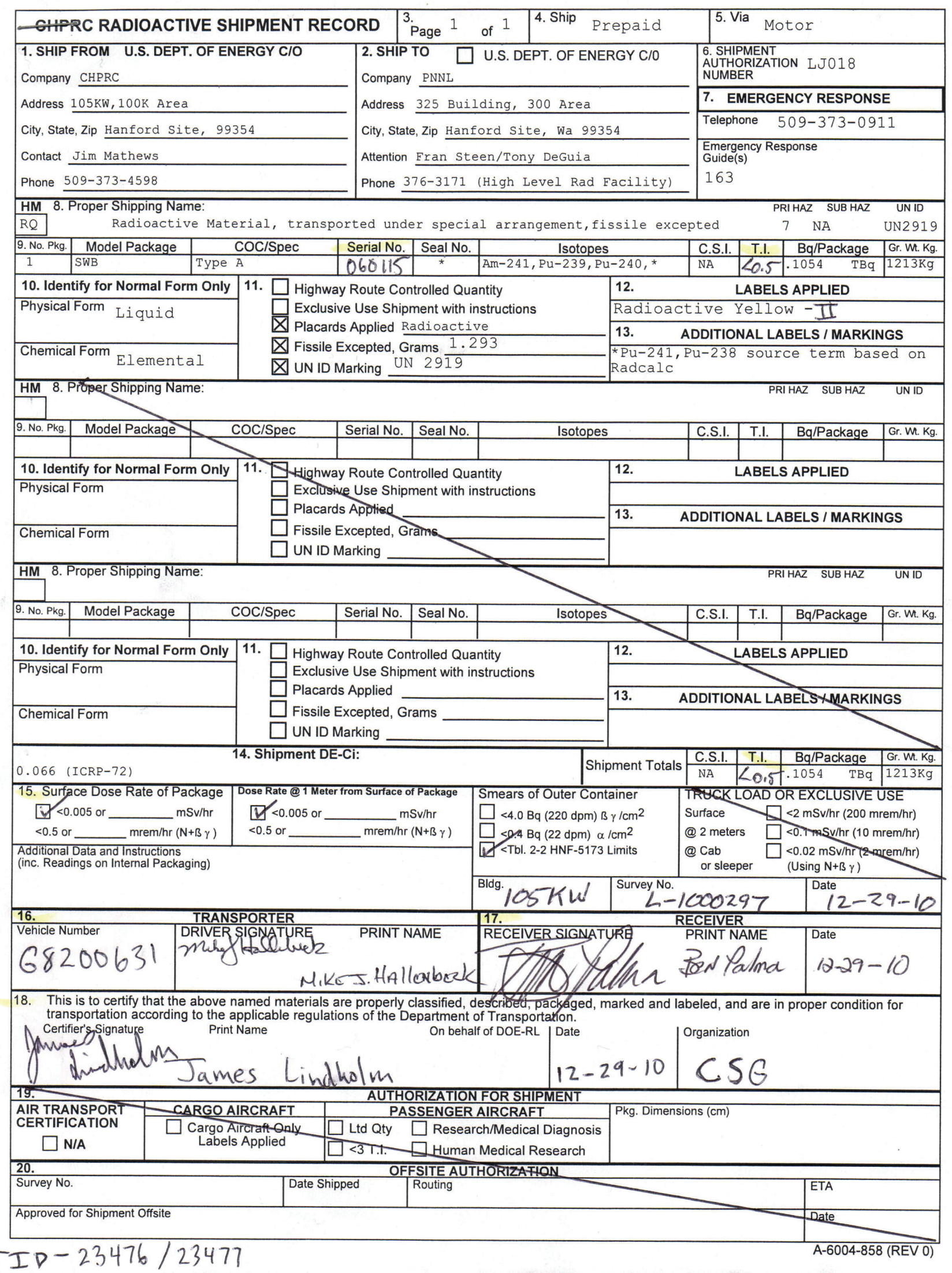


PNNL-20650, Rev. 2

\section{Appendix B}

ASR 8798 and Results 



\section{Appendix B}

\section{ASR 8798 and Results}

Appendix B contains the signed Analytical Service Request (ASR) 8798 and special instructions generated for Analytical Support Operations (ASO) laboratory analysis of the KW210 core composite samples. The special instructions delineated sample-specific information, required detection limits, quality control (QC) sample requirements, and reporting requirements. All analytical reports generated by ASO in response to the ASR are provided in Appendix B. Analytical reports provide sample results; associated uncertainties; and a discussion of sample processing, QC results, compliance with QC sample requirements, and data limitations. Occurrence Reports are also provided. These were prepared when issues were identified, and resolution was required while work was in process.

Two correspondence letters to the Buyer's Technical Representative (BTR, WW Rutherford) from the PNNL K Basin Sludge Project Manager (CD Carlson) are also provided. Letter 52578-2011-L06 details the ASO evaluation of the generally low matrix spike sample recoveries for $\mathrm{Se}, \mathrm{Sb}$, and $\mathrm{Tl}$ in the $\mathrm{K}$ Basin sludge matrix. Letter 59205-2011-L04 discusses the fact that not all of the QC sample requirements delineated by the Quality Assurance Project Plan/Sampling and Analysis Plan (QAPjP/SAP) were successfully met (some analyte spike recoveries exceeded acceptance criteria and in some cases the $\mathrm{Pb}$ detection limit was not met). A crosswalk of the QC failures with the Waste Isolation Pilot Plant Hazardous Waste Permit (Table C3-9) is included. Although this letter specifically discusses the Settler Sludge samples collected from Engineered Container SCS-CON-230, the BTR provided direction that the discussion and type of action to be taken in the event of QC sample failure was generally applicable to the sludge from SCS-CON-210 (as well as other engineered containers).

The following table of contents shows that pagination specific to Appendix B was applied to the ASR, the analytical reports and attachment, Occurrence Reports, and reference correspondence letters. This pagination is sequential and consistent (e.g., page B.\#).

\section{Appendix B Table of Contents}

ASR 8798 Request and Special Instructions.

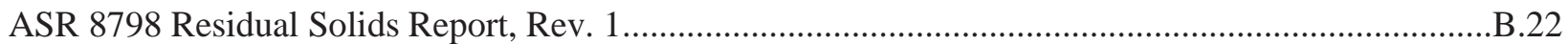

ASR 8798 Inductively Coupled Plasma-Optical Emission Spectrometry Analytical Report....................25

ASR 8798 Total Inorganic and Organic Carbon Analytical Report ......................................................33

ASR 8798 Gamma Energy Analysis Narrative Report..........................................................................

ASR 8798 Plutonium-238, -239+240 Narrative Report .........................................................................58

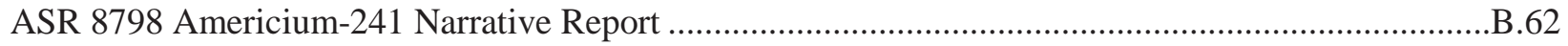

ASR 8798 Neptunium-237 Narrative Report.......................................................................................66

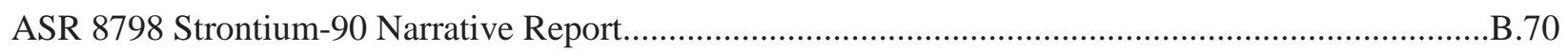

ASR 8798 Attachment 1: Radiochemistry Results Summary Tables ......................................................74

ASR 8798 Uranium Kinetic Phosphorescence Analysis Narrative Report...............................................77 
PNNL-20650, Rev. 2

ASR 8798 Uranium Metal Concentration Report, Rev. 1 B. 83

ASR 8798 Uranium Oxidative State Report B. 89

Correspondence Letter 52578-2011-L06 B.93

Correspondence Letter 59205-2011-L04 B. 103

Occurrence Report 52578-04-18-11 B. 107

Occurrence Report 52578-04-20-11 B. 108

Occurrence Report 52578-05-26-11. B.109

Occurrence Report 52578-05-27-11. B. 110

Occurrence Report 52578-06-30-11. B. 112

Occurrence Report 52578-07-19-11. B. 114 
Requestor --- Complete all fields on this COVER PAGE, unless specified as optional or ASR is a revision

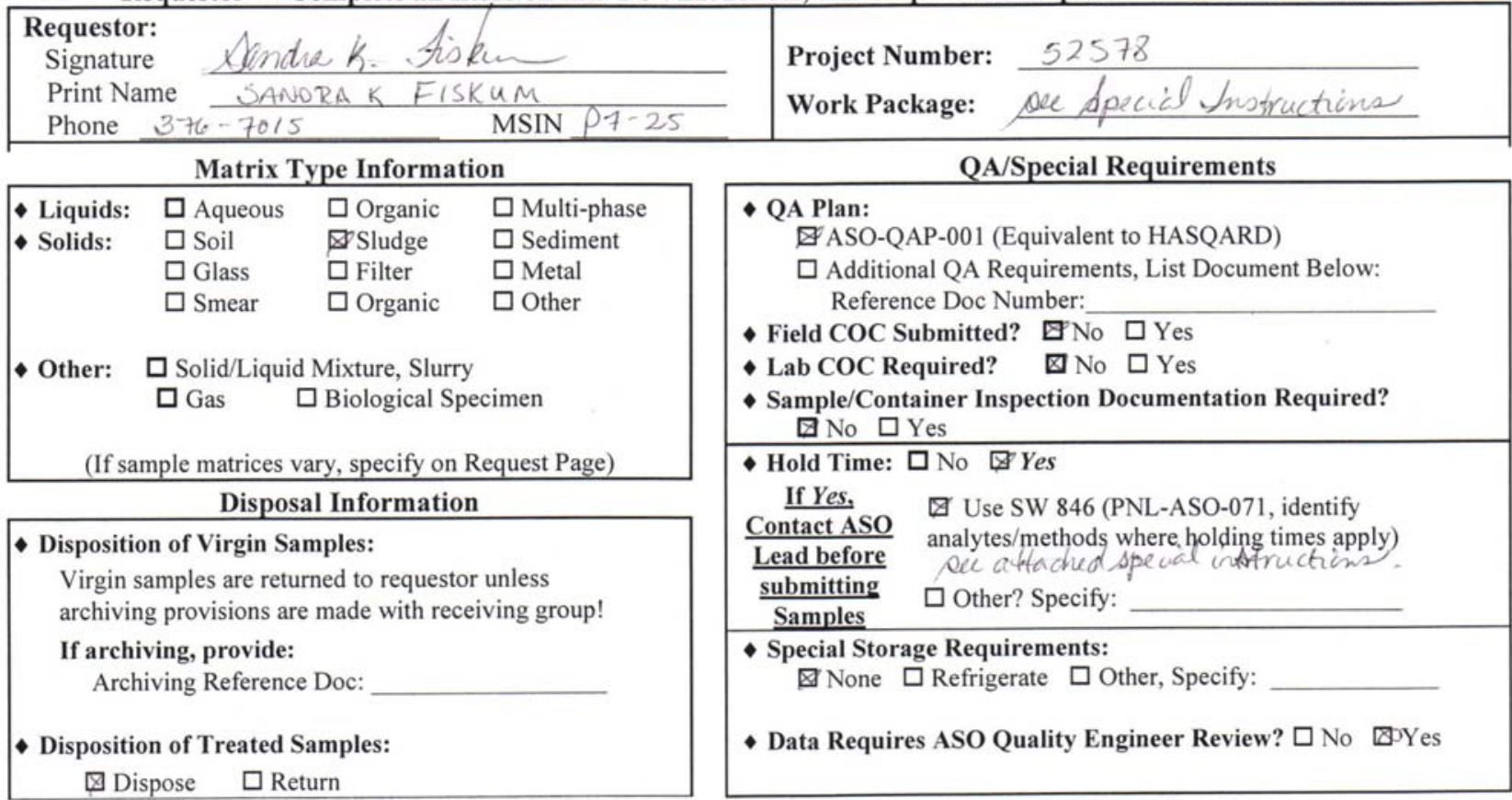

Data Reporting Information

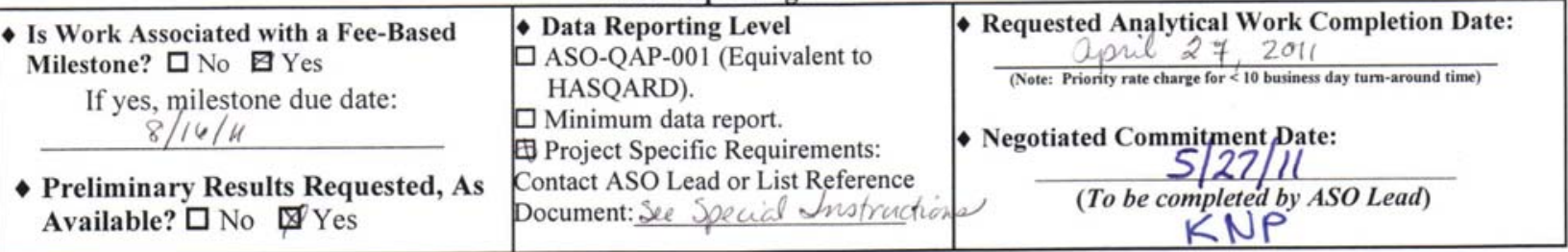

Waste Designation Information

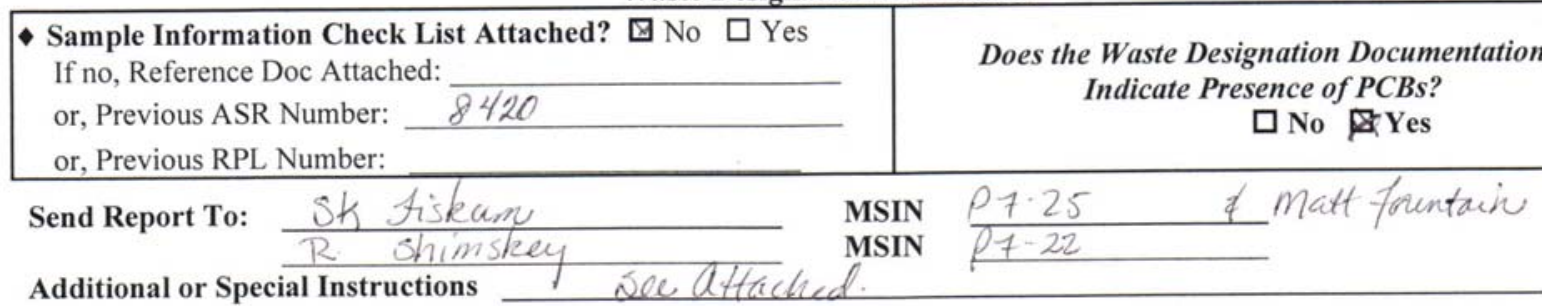

Receiving and Login Information (to be completed by ASO staff)

\begin{tabular}{|c|c|c|c|}
\hline Date Delivered: & $2 / 24 / 11$ & Received By: & \\
\hline Delivered By (optional) & SFO techs & & 00 \\
\hline Time Delivered (optional) & & & $01 / 0$ Rev.: 00 \\
\hline $\begin{array}{l}\text { Group ID (optional) } \\
\text { CMC Waste Sample? }\end{array}$ & No & RPL Numbers: & $11-0419-711-0422$ \\
\hline CMC Waste Sample? & & & . \\
\hline
\end{tabular}




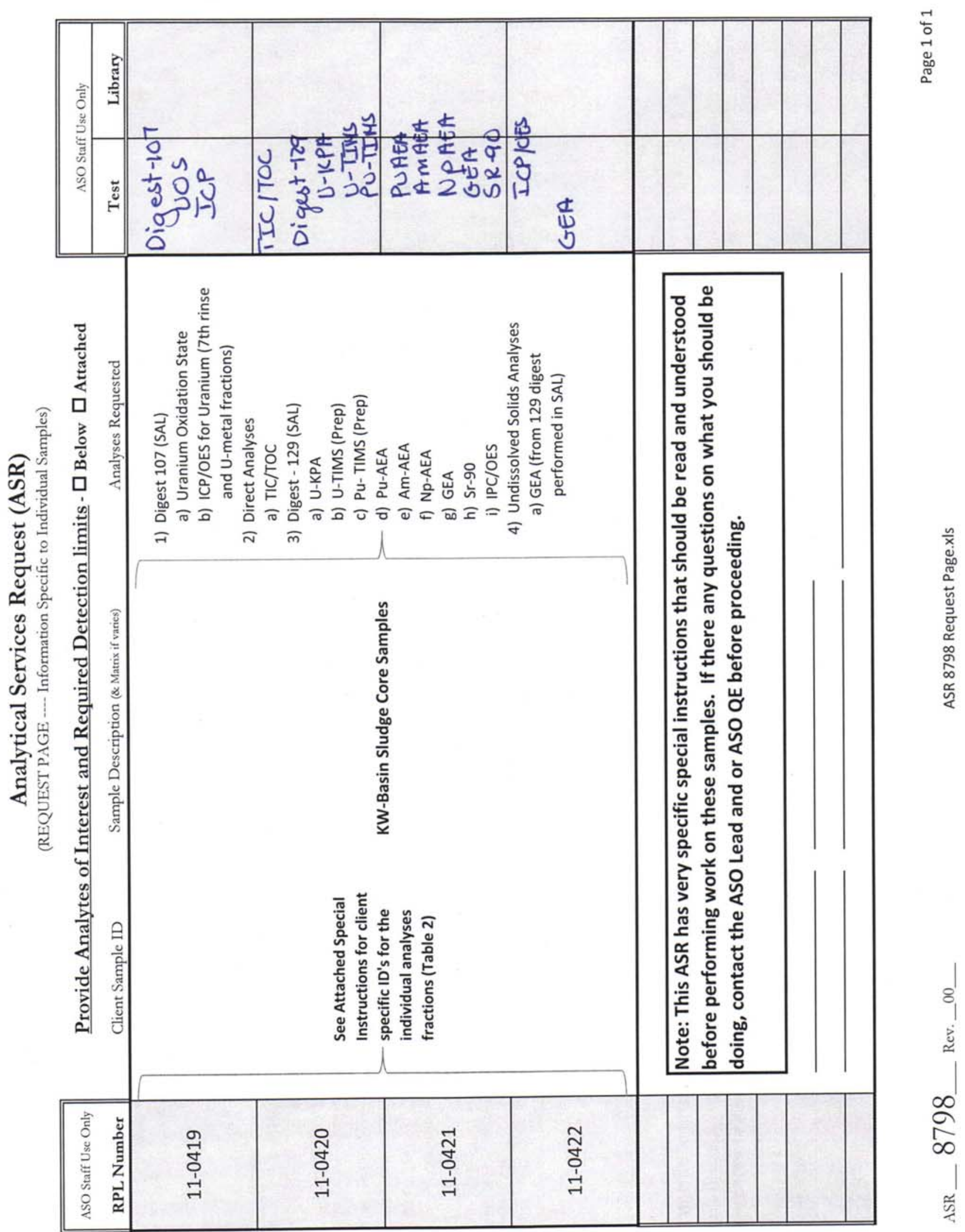


PNNL-20650, Rev. 2

\section{Special Instructions for K-Basin Container SCS-CON-210 \\ Core Sample Analysis: \\ KW210-A1, -A4, -B1, and -B3}

Cognizant Scientist:

sk. tiska

Date: $2 / 24 / 11$

\section{SK Fiskum}

Project Manager:

Date: $2 / 24 / 2311$

Project Quality Engineer: $\frac{D S \text { Collep }}{\text { DS Coffey }}$

Date: $2-24-11$

ASO Lead:

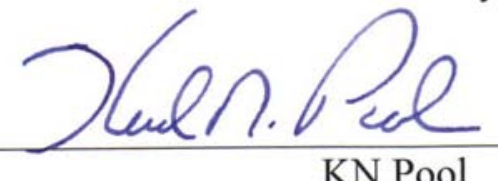

Date: $2 / 24 / 1$

STP Concurrence:

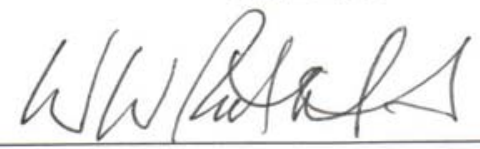

Date: $2 / 24 / 4$

WW Rutherford 
PNNL-20650, Rev. 2

ASR 8798 Special Instructions

Page 2 of 17

\section{Special Instructions for K-Basin Container SCS-CON-210 Core Sample Analysis: \\ KW210-A1, -A4, -B12, and -B3}

\section{Cognizant Scientists:}

SK Fiskum, 376-7015

RW Shimskey, 376-3183

CD Carlson, 376-2912

Four core composite materials containing liquid and sludge from $\mathrm{K}$ West $(\mathrm{KW})$ Basin Engineered Container SCS-CON-210 are presented for analysis. The sample processing and analysis schematic for a single core composite is shown in Figure 1. The processing, prior to the Analytical Support Organization (ASO)-required analyses (non-highlighted blocks), is covered in separate project-specific test instructions, 52578-TI001 and -TI002. The ASO-specific processing is shown in the highlighted blocks. The solid slurry samples have already been aliquoted.

Although most work is being performed under the ASO QA Plan, some activities are outside of the scope of the plan such as sample preparation in the High- Level Radiochemistry Facility (HLRF), X-ray diffraction (XRD), and thermal ionization mass spectrometry (TIMS). ${ }^{(a)}$

The material has been received in the Laboratory and logged into the Radioactive Material Tracking (RMT) system as RMT \#37737; the Sample Information Checklist (SICL) is tied to this RMT number. The sample labels are color coded in association with the specific container and core composite and Radiochemical Processing Laboratory (RPL) ID numbers (where possible; the Oak Ridge centrifuge tubes are not color-coded). The sample-specific IDs, the RPL assigned sample IDs, and the sampling date (at the K Basin) are cross-referenced in Table 1. Note that a 6-month hold time applies to ICP-OES metals analysis.

Table 1. Core Sample ID, RPL ID, Sampling Date, and Holding Time Cross-Reference

\begin{tabular}{|l|c|c|c|}
\hline Core Sample ID & RPL ID & $\begin{array}{c}\text { Sample Date } \\
\left(\mathbf{T}_{\mathbf{0}}\right)\end{array}$ & $\begin{array}{c}\text { End of 6-Month } \\
\text { Hold Time }\end{array}$ \\
\hline KW210-A1 & $11-0419$ & $12 / 29 / 10$ & $6 / 29 / 11$ \\
\hline KW210-A4 & $11-0420$ & $11 / 17 / 10$ & $5 / 17 / 11$ \\
\hline KW210-B1 & $11-0421$ & $12 / 01 / 10$ & $6 / 01 / 11$ \\
\hline KW210- B3 & $11-0422$ & $12 / 15 / 10$ & $6 / 15 / 11$ \\
\hline
\end{tabular}

(a) Inductively coupled plasma mass spectrometry (ICP-MS) may be used as contingency in the event TIMS is not available. 
PNNL-20650, Rev. 2

ASR 8798 Special Instructions

Page 3 of 17

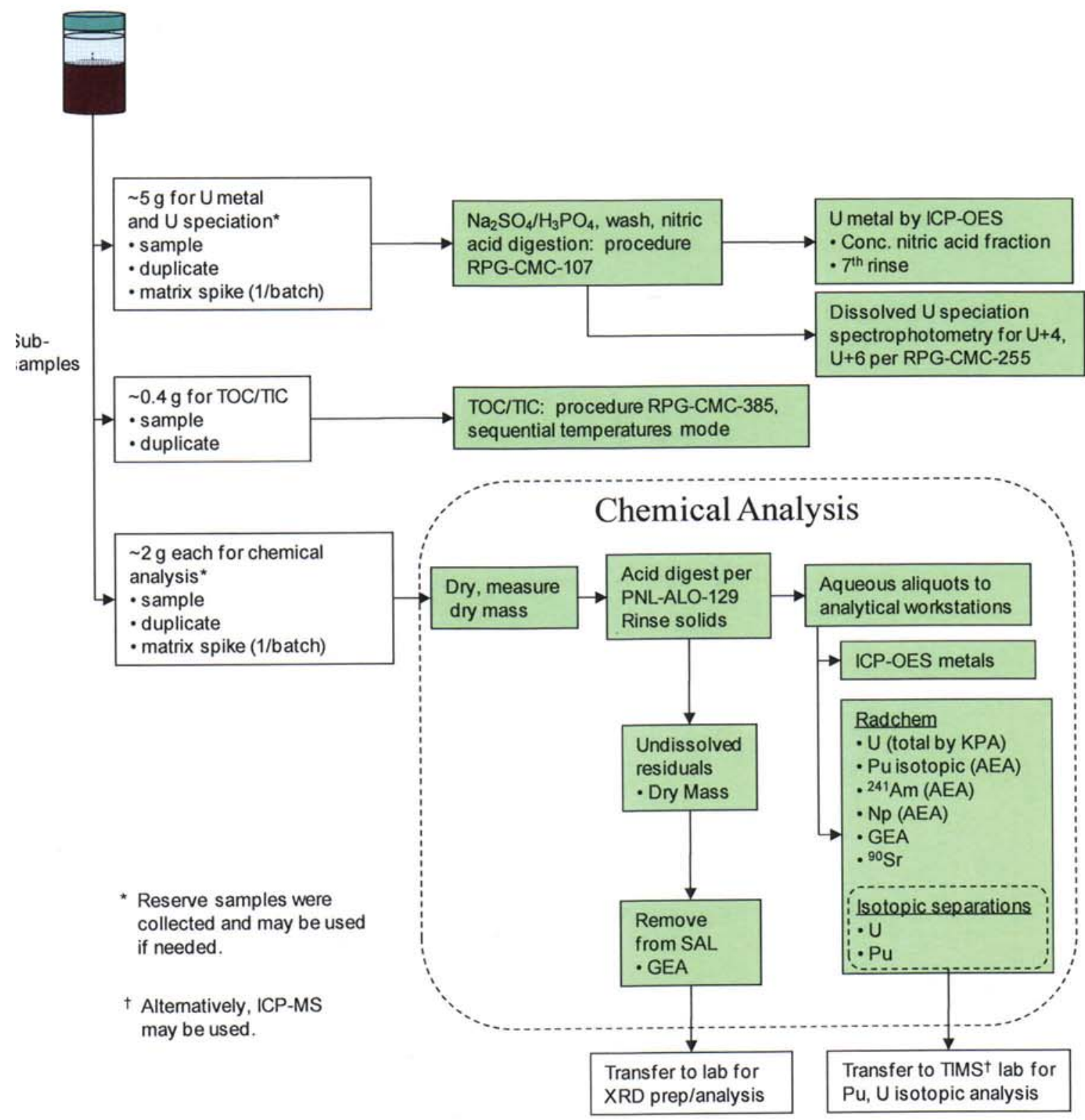

Figure 1. Analysis Schematic for Core Composites

The Chemical Measurement Center needs to charge analyses to the following work packages:

H41220 RPG-CMC-107 Rev. 1 Acid Digest

H41220 U Metal Analysis ICP-OES

H41221 U Oxide speciation

H41215 TOC/TIC

H41215 PNL-ALO-129 Rev. 0 Acid Digest

H41215 ICP-OES

H41216 $\left\{\begin{array}{l}\text { GEA } \\ \text { Pu-AEA, Am-AEA } \\ \text { Sr-90 } \\ \text { U-KPA } \\ \text { Preparations for TIMS (U and Pu) }\end{array}\right.$

All dewatered sample solids masses have been calculated based on measures recorded from HLRF processing activities as recorded in Test Instructions 52578-TI001 and -TI002; these solids masses are reproduced in Table 2 . The shaded reserve samples were collected as backups and are not expected to be analyzed. 
PNNL-20650, Rev. 2

Table 2. Cross-Reference of Sample ID, RPL ID, Dewatered Mass, and Analysis

\begin{tabular}{|c|c|c|c|c|}
\hline Sample ID & RPL ID & $\begin{array}{c}\text { Container } \\
\text { Tare, g }\end{array}$ & $\begin{array}{c}\text { Dewatered } \\
\text { Solids Mass, } \\
\text { gdewatered }^{\text {gelos }}\end{array}$ & Analysis \\
\hline KW-210-A1 & \multicolumn{4}{|l|}{$11-0419$} \\
\hline TI001-A1-B & 11-0419-129-S & 34.0686 & 2.05 & Chemical analysis \\
\hline TI001-A1-C & 11-0419-129-D & 34.3212 & 2.28 & Chemical analysis duplicate \\
\hline TI001-A1-D & & & & Chemical analysis reserve \\
\hline TI001-A1-E & & & & Chemical analysis reserve \\
\hline TI001-A1-F & & & & Chemical analysis reserve \\
\hline TI001-A1-G & & & & Chemical analysis reserve \\
\hline TI001-A1-H & 11-0419-C-S & 24.5816 & 1.3503 & TOC/TIC \\
\hline TI001-A1-J & 11-0419-C-D & 24.5729 & 1.0210 & TOC/TIC duplicate \\
\hline TI001-A1-P & $11-0419-107-\mathrm{S}$ & $37.8452 *$ & 3.89 & U metal \\
\hline TI001-A1-Q & 11-0419-107-D & $37.5330^{*}$ & 4.55 & U metal duplicate \\
\hline TI001-A1-R & & & & U metal reserve \\
\hline T1001-A1-S & & & & U metal reserve \\
\hline TI001-A1-T & & & & $\mathrm{U}$ metal reserve \\
\hline TI001-A1-U & & & & U metal reserve \\
\hline KW-210-A4 & \multicolumn{4}{|l|}{$11-0420$} \\
\hline TI002-A4-B & $11-0420-129-\mathrm{S}$ & 34.5564 & 2.30 & Chemical analysis \\
\hline TI002-A4-C & 11-0420-129-D & 35.0352 & 2.61 & Chemical analysis duplicate \\
\hline TI002-A4-D & & & & Chemical analysis reserve \\
\hline TI002-A4-E & & & & Chemical analysis reserve \\
\hline TI002-A4-F & & & & Chemical analysis reserve \\
\hline TI002-A4-G & & & & Chemical analysis reserve \\
\hline TI002-A4-H & 11-0420-C-S & 24.6518 & 1.4289 & TOC/TIC \\
\hline TI002-A4-J & 11-0420-C-D & 24.5934 & 2.0583 & TOC/TIC duplicate \\
\hline TI002-A4-P & $11-0420-107-S$ & $38.2432^{*}$ & 4.80 & $\mathrm{U}$ metal \\
\hline T1002-A4-Q & 11-0420-107-D & $37.6535^{*}$ & 3.99 & U metal \\
\hline TI002-A4-R & & & & U metal reserve \\
\hline TI002-A4-S & & & & U metal reserve \\
\hline T1002-A4-T & & & & U metal reserve \\
\hline TI002-A4-U & & & & U metal reserve \\
\hline KW-210-B1 & \multicolumn{4}{|l|}{$11-0421$} \\
\hline TI002-B1-B & $11-0421-129-\mathrm{S}$ & 33.1842 & 1.84 & Chemical analysis \\
\hline TI002-B1-C & 11-0421-129-D & 34.1788 & 1.98 & Chemical analysis duplicate \\
\hline TI002-B1-D & 11-0421-129-MS & 33.4949 & 2.15 & Chemical analysis MS \\
\hline TI002-B1-E & & & & Chemical analysis reserve \\
\hline TI002-B1-F & & & & Chemical analysis reserve \\
\hline TI002-B1-G & & & & Chemical analysis reserve \\
\hline T1002-B1-H & 11-0421-C-S & 24.5452 & 1.7868 & TOC/TIC \\
\hline
\end{tabular}




\begin{tabular}{|c|c|c|c|c|}
\hline Sample ID & RPL ID & $\begin{array}{c}\text { Container } \\
\text { Tare, g }\end{array}$ & $\begin{array}{c}\text { Dewatered } \\
\text { Solids Mass, } \\
\text { gdewatered }\end{array}$ & Analysis \\
\hline TI002-B1-J & 11-0421-C-D & 24.5051 & 2.0942 & TOC/TIC duplicate \\
\hline TI002-B1-P & 11-0421-107-S & $37.9626^{*}$ & 4.18 & U metal \\
\hline TI002-B1-Q & 11-0421-107-D & $38.1402^{*}$ & 4.62 & U metal \\
\hline TI002-B1-R & 11-0421-107-MS & $38.7078^{*}$ & 3.97 & $\mathrm{U}$ metal MS \\
\hline TI002-B1-S & & & & U metal reserve \\
\hline TI002-B1-T & & & & U metal reserve \\
\hline TI002-B1-U & & & & U metal reserve \\
\hline KW-210-B3 & $11-0422$ & & & \\
\hline TI001-B3-B & 11-0422-129-S & 33.9427 & 2.26 & Chemical analysis \\
\hline TI001-B3-C & 11-0422-129-D & 33.6711 & 2.24 & Chemical analysis duplicate \\
\hline TI001-B3-D & & & & Chemical analysis reserve \\
\hline TI001-B3-E & & & & Chemical analysis reserve \\
\hline TI001-B3-F & & & & Chemical analysis reserve \\
\hline TI001-B3-G & & & & Chemical analysis reserve \\
\hline TI001-B3-H & 11-0422-C-S & 24.6681 & 1.8483 & TOC/TIC \\
\hline TI001-B3-J & 11-0422-C-D & 24.7512 & 1.5905 & TOC/TIC duplicate \\
\hline TI001-B3-P & 11-0422-107-S & $37.4950^{*}$ & 4.33 & U metal \\
\hline TI001-B3-Q & 11-0422-107-D & $38.1267^{*}$ & 3.93 & U metal duplicate \\
\hline TI001-B3-R & & & & U metal reserve \\
\hline TI001-B3-S & & & & U metal reserve \\
\hline T1001-B3-T & & & & U metal reserve \\
\hline TI001-A1-U & & & & U metal reserve \\
\hline \multicolumn{5}{|l|}{ NA } \\
\hline $\begin{array}{l}\text { Purolite } \\
\text { Grafoil }^{\otimes}+\end{array}$ & $11-0423-\mathrm{C}-\mathrm{S}$ & -- & -- & TOC/TIC \\
\hline $\begin{array}{l}\text { Purolite }^{\otimes}+ \\
\text { Grafoil }^{\infty}\end{array}$ & 11-0423-C-D & -- & -- & TOC/TIC duplicate \\
\hline
\end{tabular}

Analytes of interest and required detection limits are provided in Table 4; quality control (QC) sample acceptance criteria are provided in Table 5. One sample per analytical batch needs to be used to prepare a matrix spike (MS) sample. Subsampling of the stock material is a laborious process; a series of reserve samples from each core composite have been collected during the initial aliquoting process. The reserve samples should be used in case of catastrophic failure (i.e., spilling) of the primary or duplicate sample. The reserve samples were also collected in case the duplicate result did not agree with the primary result and a re-analysis might be required. The reserve samples may also be forwarded for analysis in place of the primary or duplicate samples based on the prerogative of the Cognizant Scientist (pending observations of actual mass collected, or other issues).

The total organic carbon (TOC) and total inorganic carbon (TIC) samples have been delivered directly to the TOC/TIC workstation following clean removal from the HLRF. 
PNNL-20650, Rev. 2

\section{U-Metal Analysis and Uranium Oxide Speciation}

\section{U Speciation Sample Preparation}

The samples (located in the Shielded Analytical Laboratory [SAL]) are provided to ASO in tared Oak Ridge tubes (suitable for sample digestion). The ASO is responsible for preparing the preparation blank (PB), the laboratory control sample (LCS), and the MS samples.

- The PB will consist of Hanford sand or equivalent and follow all processes that the samples are subjected to.

- The LCS will consist of Hanford sand, $\mathrm{U}_{3} \mathrm{O}_{8}$, (a mixture of U[IV] and U[VI]), and U metal beads.

- The MS will consist of a sample with U metal bead(s) added to it by ASO.

The samples will be processed per ASO Procedure, RPG-CMC-107, Rev. 1, Sample Preparation for Determination of Uranium Metal Concentrations in Sludge. At least three contacts with $\mathrm{Na}_{2} \mathrm{SO}_{4} / \mathrm{H}_{3} \mathrm{PO}_{4}$ shall be conducted to dissolve non-metal $\mathrm{U}$ species and at least 7 rinses conducted on the residual solids.

The dissolved (metal) $U$ and seventh rinse solution $U$ concentrations shall be determined by ICPOES, (RPG-CMC-211, Rev. 3, Determination of Elemental Composition by Inductively Coupled Argon Plasma Optical Emission Spectrometry [ICP-OES]). The seventh rinse solution U concentrations should be $<100 \mu \mathrm{g} \mathrm{U} / \mathrm{mL}$; the ICP-OES MDL for these samples shall be $\leq 1 \mu \mathrm{g} \mathrm{U} / \mathrm{mL}$. The ASO staff is directed to promply notify SK Fiskum /CD Carlson/DS Coffey if the $7^{\text {th }}$ rinse is $>100 \mu \mathrm{g} \mathrm{U} / \mathrm{mL}$, thus enabling PNNL to fulfill its commitment to the client for prompt notication of a potential issue with the analysis. The duplicate precision criterion (Table 5) only applies to the dissolved $U$ metal sample portion; it does not apply to the $7^{\text {th }}$ rinse solution.

Submit the $\mathrm{Na}_{2} \mathrm{SO}_{4} / \mathrm{H}_{3} \mathrm{PO}_{4}$ sample solutions containing the U(IV) and U(VI) for analysis by spectrophotometry (per RPG-CMC-255, Rev. 0, Sample Preparation and Analysis for Determining Uranium Oxide Oxidation States in K Basin Sludges).

\section{U Speciation Reporting Units}

Report the $U$ metal results as $\underline{\mu} \mathbf{U}$ per g of dewatered sludge, where the mass of dewatered sludge in the analysis aliquot is taken from Table 2 . Report the seventh rinse sample $\mathbf{U}$ concentration as $\mu \mathrm{g} U$ per $\mathrm{mL}$ of solution. Report the $\mathrm{U}(\mathrm{IV})$ and $\mathrm{U}(\mathrm{VI})$ oxidation states as percentages.

\section{TOC/TIC Analysis Samples}

\section{TOC/TIC Preparation}

The TOC/TIC slurry samples need to be analyzed at the work station (fume hood). These samples pose a contact dose hazard because containment is not in the hot cell. Furthermore samples will be dried as part of the analysis processing, and will also pose a contamination hazard after processing.

Damp or wet samples will need to be dried prior to subsampling and aliquot weighing. Drying can be conducted by simple air drying or heating to $105^{\circ} \mathrm{C}$. In either case, constant dry mass will need to be ascertained prior to subsampling. The sample will need to be subsampled at the 
workstation to provide a sample, duplicate and triplicate (for each sample) for analysis. One of the samples per analytical batch will need to be subsampled again to support an analytical spike (AS). Subsampling at the workstation will need to incorporate care to provide representative subsamples to optimize chances to meet precision measurement requirements; however, it is understood that subsampling will be conducted on a best-effort basis.

Analyze the samples per RPG-CMC-385, Rev. 2, Carbon Measured in Solids, Sludge, and Liquid Matrices furnace oxidation method with sequential temperature analysis mode. The TOC will be determined from $\mathrm{C}$ combustion at $600^{\circ} \mathrm{C}$; the same sample material will then be subjected to $1000^{\circ} \mathrm{C}$ to measure all other (inorganic) $\mathrm{C}$. The inorganic $\mathrm{C}$ measurement is expected to include Grafoil $^{\otimes}$ and carbonates.

The ASO is responsible for preparing each sample in duplicate, as well as the LCS and blank spike (BS) and AS QC samples. The AS and LCS/BS samples are to include the carbon standards glucose and $\mathrm{CaCO}_{3}$.

A sample of dry Purolite ${ }^{\circledR}$ NW37 resin beads combined with a sample of Grafoil ${ }^{\circledR}$ also needs to be prepared by ASO to be run with the sample batch in duplicate, first analysis at the beginning of the analytical batch and the duplicate analysis at the end of the analytical batch. This material will be processed as a sample (11-0423) to evaluate method performance on the more-difficult-tooxidize resin beads and Grafoil ${ }^{\otimes}$. Report TOC and TIC recoveries of these two constituents. If the recovery of either of these constituents is $>125 \%$ or $<75 \%$, contact SK Fiskum/R Shimskey (Cognizant Scientists).

\section{TOC/TIC Reporting Units}

Report the TOC and TIC concentrations as $\mu \mathrm{g} \mathrm{C} / \mathrm{g}_{\mathrm{dry}}$, where $\mathrm{g}_{\mathrm{dry}}$ is determined at the workstation. Report the dry sample mass subjected to analysis.

\section{Chemical Analysis Samples}

\section{Acid Digestion}

Preparation

Note: Balances used to support the nuclear materials ( $\mathrm{Pu}, \mathrm{Am}, \mathrm{Np}$, and $\mathrm{U}$ ) analysis require stringent daily balance checks on each day the balance is used. Review section on "Quality Control" before measuring and recording sample mass data. To this end, all masses collected in SAL must have the linearity check conducted and recorded.

The analytical samples are provided to ASO in tared Oak Ridge tubes (suitable for sample digestion). Analytical samples need to be dried to constant mass at $105^{\circ} \mathrm{C}$. Determine and report the net dry sample mass.

Acid digest the dried solids according to PNL-ALO-129, Rev. 0, $\mathrm{HNO}_{3}$ - $\mathrm{HCl}$ Acid Extraction of Solids Using a Dry-Block Heater. Report observations associated with the dissolution processing, including the undissolved solids mass. Provide a copy of the ASO preparation bench sheet to SK Fiskum and RW Shimskey.

Residual solids remaining after acid digestions are expected and of great interest and shall not be lost (refer to Figure 1). Remove the acid digestate as per procedure; this may entail centrifuging and decanting. Rinse as appropriate, collecting rinse solution with the acid digestion solution. 


\section{Videotape the inside Oak Ridge tubes to show characteristics of the wet solids/residues; provide video to SK Fiskum or RW Shimskey.}

After residual solids are rinsed and isolated, dry the solids (in the digestion tube) to constant mass at $105^{\circ} \mathrm{C}$. Calculate the net residual solids mass along with the starting material dry solids mass and report to SK Fiskum/RW Shimskey. Additional processing of residual solids will depend on the mass relative to the starting (wet/dewatered) material. Additional processing may include:

- Removal from the SAL and gamma count

- Then transfer solids outside of ASO to Doinita Neiner for XRD preparation and analysis.

\section{Acid Digestion Reporting Units}

Report the dry sample mass before digestion as $\mathrm{g}_{\text {dry }}$, and report the dried residuals solids mass after digestion and rinsing as $\mathrm{g}_{\text {residual }}$.

\section{ICP-OES}

\section{ICP-OES Analysis}

Acid digestate aliquots shall be analyzed at the ICP-OES workstation according to RPG-CMC211, Rev. 3, Determination of Elemental Composition by Inductively Coupled Argon Plasma Optical Emission Spectrometry (ICP-OES).

\section{ICP-OES Reporting Units}

Report solids sample analyte concentrations as $\mu \mathrm{g} / \mathrm{g}_{\text {dry }}$ where $\mathrm{g}_{\text {dry }}$ is the initial dry sample mass determined prior to the start of the PNL-ALO-129 acid digestion.

\section{$\underline{\text { Radiochemistry }}$}

\section{Preparations and Analysis}

Acid digestate aliquots shall be directly aliquoted for GEA analysis. Acid digestate aliquots shall be aliquoted for separations, mounting, and/or analysis. Operations will be conducted according to the specific radiochemistry procedures delineated in Table 3 . No procedure or procedure revision substitutions are to be made without prior written authorization from SK Fiskum or CD Carlson to requests submitted by the ASO Lead.

Table 3. Authorized Radiochemistry Procedures for K Basin Sludge Analysis

\begin{tabular}{|c|c|c|c|}
\hline Analyte & Separation & Mounting & Counting/Analysis \\
\hline GEA & NA & NA & $\begin{array}{c}\text { RPG-CMC-450, Rev. } 1 \text { Gamma } \\
\text { Energy Analysis (GEA) and Low- } \\
\text { Energy Photon Spectrometry (LEPS) }\end{array}$ \\
\hline $\begin{array}{l}{ }^{238} \mathrm{Pu} \text { and } \\
{ }^{239+240} \mathrm{Pu} / \mathrm{AEA}\end{array}$ & $\begin{array}{l}\text { RPG-CMC-4017, Rev. 0, } \\
\text { Analysis of Environmental } \\
\text { Water Samples for Actinides } \\
\text { and Strontium-90 }\end{array}$ & $\begin{array}{l}\text { RPG-CMC-496, Rev. 0, } \\
\text { Coprecipitation } \\
\text { Mounting of Actinides } \\
\text { for Alpha Spectroscopy }\end{array}$ & $\begin{array}{l}\text { RPG-CMC-422, Rev. } 2 \text { Solutions } \\
\text { Analysis: Alpha Spectrometry }\end{array}$ \\
\hline${ }^{241} \mathrm{Am} / \mathrm{AEA}$ & $\begin{array}{l}\text { RPG-CMC-4017, Rev. 0, } \\
\text { Analysis of Environmental } \\
\text { Water Samples for Actinides } \\
\text { and Strontium-90 }\end{array}$ & $\begin{array}{l}\text { RPG-CMC-496, Rev. 0, } \\
\text { Coprecipitation } \\
\text { Mounting of Actinides } \\
\text { for Alpha Spectroscopy }\end{array}$ & $\begin{array}{l}\text { RPG-CMC-422, Rev. } 2 \text { Solutions } \\
\text { Analysis: Alpha Spectrometry }\end{array}$ \\
\hline${ }^{237} \mathrm{~Np} / \mathrm{AEA}$ & $\begin{array}{l}\text { RPG-CMC-4017, Rev. 0, } \\
\text { Analysis of Environmental } \\
\text { Water Samples for Actinides } \\
\text { and Strontium-90 }\end{array}$ & $\begin{array}{l}\text { RPG-CMC-496, Rev. 0, } \\
\text { Coprecipitation } \\
\text { Mounting of Actinides } \\
\text { for Alpha Spectroscopy }\end{array}$ & $\begin{array}{l}\text { RPG-CMC-422, Rev. } 2 \text { Solutions } \\
\text { Analysis: Alpha Spectrometry }\end{array}$ \\
\hline
\end{tabular}


PNNL-20650, Rev. 2

ASR 8798 Special Instructions

Page 9 of 17

\begin{tabular}{|c|c|c|c|}
\hline Analyte & Separation & Mounting & Counting/Analysis \\
\hline${ }^{90} \mathrm{Sr}$ & $\begin{array}{l}\text { RPG-CMC-476, Rev. } 0 \text {, } \\
\text { Strontium Separation using } \\
\text { Eichrom Strontium Resin }\end{array}$ & NA & $\begin{array}{l}\text { RPG-CMC-474, Rev. 1, Measurement } \\
\text { of Alpha and Beta Activity by Liquid } \\
\text { Scintillation Spectrometry }\end{array}$ \\
\hline $\begin{array}{l}\text { Pu isotopic } \\
\text { preparation }\end{array}$ & $\begin{array}{l}\text { RPG-CMC-455, Rev. } 0 \text {, } \\
\text { Separation of Uranium and } \\
\text { Plutonium for Isotopic Analysis } \\
\text { by Mass Spectroscopy }\end{array}$ & NA & $\begin{array}{l}\text { NA/Deliver to Pu Isotopic Analysis } \\
\text { Workstation }\end{array}$ \\
\hline $\begin{array}{l}\mathrm{U} \text { isotopic } \\
\text { preparation }\end{array}$ & $\begin{array}{l}\text { RPG-CMC-4017, Rev. 0, } \\
\text { Analysis of Environmental } \\
\text { Water Samples for Actinides } \\
\text { and Strontium-90 }\end{array}$ & NA & $\begin{array}{l}\text { NA/Deliver to U Isotopic Analysis } \\
\text { Workstation }\end{array}$ \\
\hline
\end{tabular}

The separations conducted to support $\mathrm{Pu}$ and $\mathrm{U}$ isotopic analysis need to provide purified fractions with sufficient analyte concentrations to create a measureable signal by TIMS and/or ICP-MS. Therefore, the preparation shall not be considered complete until satisfactory isotopic results are obtained. It is understood that the PB may not have sufficient analyte concentration to meet this objective. There is no LCS or MS required to be processed through separations processes to support isotopic analysis by TIMS and/or ICP-MS.

\section{Radiochemistry Reporting Units}

Report solids sample analyte concentrations as $\mu \mathrm{Ci} / \mathrm{g}_{\text {dry }}$ where $\mathrm{g}_{\text {dry }}$ is the initial dry sample mass determined prior to the start of the PNL-ALO-129 acid digestion.

Report the residual solids GEA analytes as $\mu \mathrm{Ci} / \mathrm{g}_{\text {residual }}$, where $\mathrm{g}_{\text {residual }}$ is the mass of residual solids that did not dissolve during acid digestion. The precision criteria defined in Table 5 do not apply to the undissolved solids.

\section{$\underline{\text { U KPA }}$}

\section{Preparation and Analysis}

Acid digestate aliquots shall be diluted and analyzed according to RPG-CMC-4014, Rev. 1, Uranium by Kinetic Phosphorescence Analysis. The U KPA analysis requires stringent QC sample processing (refer to Table 5) to demonstrate accuracy:

- One sample per analytical batch is to be spiked with U. This will be a sample digestate spiked with $U$ at the workstation, i.e., a post-digestion MS. The calculated $U$ recovery is required to be within $75 \%$ to $125 \%$.

- A minimum of two different calibration verification checks need to be run with each analytical batch and an assessment of their accuracies reported ( $\pm 7.5 \%$ of true value).

- An independent U BS sample needs to be run with each analytical batch. This will be an independent U BS sample processed through the acid digestion (PNL-ALO-129 acid digestion) (see Table 5). The BS recovery is required to be $80 \%$ to $120 \%$.

\section{U KPA Reporting Units}

Report solids sample analyte concentrations as $\mu \mathrm{g} / \mathrm{g}_{\text {dry }}$ (U KPA) where $\mathrm{g}_{\mathrm{dry}}$ is the initial dry sample mass determined prior to the start of the 129 acid digestion.

\section{Archive of SAL-Prepared Chemical Analysis Samples}

The acid digest preparations will result in a $\sim 50$ - to $100-\mathrm{mL}$ volume. This solution will be apportioned to the laboratory as needed to conduct work-station-specific analyses. At the time the acid digestates are apportioned to the analysis work locations, $\sim 15-\mathrm{mL}$ aliquot from each preparation should be collected and removed from SAL and stored as an archive sample. The 
PNNL-20650, Rev. 2

ASR 8798 Special Instructions

Page 10 of 17

vials need to be labeled with the following information: date, RPL-ID, sample ID, matrix, and hazard. The remaining portion of the acid digestion preparations may be disposed of.

\section{Quality Control}

All work is required to be conducted to the requirements of the Hanford Analytical Services Quality Assurance Requirements Document (HASQARD, Rev. 3). Performing work to the requirements of the ASO QA Plan (ASO-QAP-001, Rev. 7) will satisfy the HASQARD requirements. Where there is a discrepancy between the data quality objectives (DQOs) in the ASO QA Plan and these Special Instructions, the Special Instructions takes precedence as there are specific $\mathrm{K}$ Basin project requirements.

NOTE: Balances used to support the nuclear materials $(\mathrm{Pu}, \mathrm{Am}, \mathrm{Np}$, and $\mathrm{U})$ analysis require more stringent daily balance checks on each day the balance is used. In this case, the ASO SALrelated operations must use at least 3 masses (e.g., A, B, and A+B) to assess balance accuracy and linearity. The balance control checks must be traceable in the records supporting these analyses. It is suggested that the daily control check log sheets be provided as part of the analytical report; however the ASO may choose alternate means to establish traceability. Tolerances for control checks for the balance in SAL cell 2 are provided in Appendix A. If masses were inadvertently collected on a balance without the daily check, then the ASO Lead must immediately upon discovery inform the QE, Cognizant Scientist and the Project Manager.

The balance checks at the fumehood workstations may be checked per the normal process where one calibration verification check mass is measured daily when then balance is used (PNL-ASO052 , Rev. 1). This is allowed because the fumehood operations use volume measures or do not directly support nuclear materials accountability (e.g., TOC/TIC).

Solids matrix preparative analysis QC is to include a preparation blank (PB) sample, sample duplicate (provided), LCS (or BS) and a sample MS (a separate sample is provided for spiking by the ASO) as indicated in Table 5. The BS for radioisotopes will be generated after acid digestion on a sample split, i.e., post-digestion spikes. The MS for radioisotopes and U-KPA will be generated after acid digestion on a sample split, i.e., post-digestion spikes.

The ASO is responsible for preparing the $\mathrm{PB}, \mathrm{LCS} / \mathrm{BS}$, and spiking MS QC samples. The preparative MS and LCS/BS are to include all the analytes of interest: $\mathrm{Ag}, \mathrm{Al}, \mathrm{As}, \mathrm{Ba}, \mathrm{Be}, \mathrm{Ca}$, $\mathrm{Cd}, \mathrm{Cr}, \mathrm{Fe}, \mathrm{Na}, \mathrm{Ni}, \mathrm{Mg}, \mathrm{Pb}, \mathrm{Sb}, \mathrm{Se}, \mathrm{Tl}, \mathrm{U}, \mathrm{V}, \mathrm{Zn}$, and $\mathrm{Zr}$. A second preparative BS is to include U only.

The duplicate, LCS/BS, and MS sample QC acceptance criteria are provided in Table 5. The following section discusses actions to take in the event of QC failures.

Note: The ASO staff must inform/report QC failures promptly to the ASO Lead who will in turn inform the Cognizant Scientist with copy to CD Carlson and the K Basin QE (Deborah Coffey), thus enabling PNNL to fulfill its commitment to the client for prompt notification of such failures. The impact of any QC failure on data quality or project schedule (e.g., re-runs) will be assessed in conjunction with the client.

1. The preparation blank (PB) analyte concentration shall be less than the estimated quantitation limit (EQL) or the minimum detectable activity (MDA) of the associated sample. When the 
$\mathrm{PB}$ concentration is equal to or exceeds the $\mathrm{EQL}$ or $\mathrm{MDA}$, then the $\mathrm{PB}$ concentration shall not exceed $5 \%$ of the measured concentration present in the sample.

2. If and when it has been determined that the data are not useable, then the affected samples in the processing batch must be re-prepared and re-analyzed for the failed analytes, availability of samples permitting.

3. In the case of multi-elemental methods (ICP-OES), isolated QC failure(s) may be communicated to SK Fiskum, RW Shimskey, Clark Carlson and the K Basin QE by the ASO Lead for an assessment of the impact on data interpretation. If the data are acceptable as-is, the project staff will communicate in writing (e.g., e-mail message) that the data may be reported; the e-mail acceptance and resulting limitations on the data from the QC sample failure(s) shall be included in the final analytical report.

4. If and when the MS sample fails to meet the acceptance criteria, the results shall be investigated for potential sources of error by comparing the results of the MS to the BS. When the sources of error cannot be identified, the failure of the MS will be attributed to a sample matrix effect and any resulting limitations on the data shall be included in the report.

\section{Reporting}

The analytical data report shall be prepared in accordance with procedure PNL-ASO-058, Rev. 0.2, ASO Data Reporting, Section 5.3, Comprehensive Data Report. Please be sure to include action taken with respect to any identified unexpected results and discrepancies.

The Comprehensive Data Report contains three main parts; a data report cover page, a narrative, and the data summary. These elements are identified below; red text indicates project-driven scope. Black text is taken directly from the ASO procedure: PNL-ASO-058, Rev. 0.2.

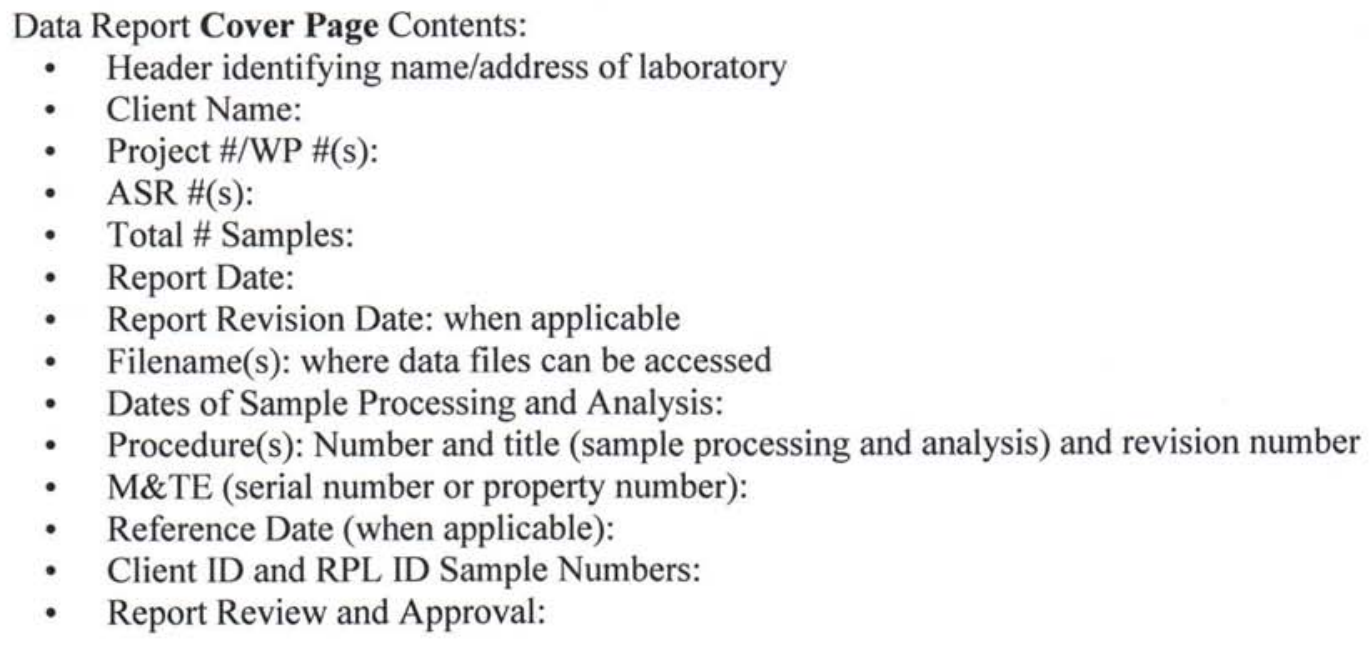

Preparer Date

(Printed name and signature of responsible individual)

Technical Reviewer Date

(Printed name and signature of technical reviewer)

A case narrative describing the analysis, limitations on the analysis results, and QA/QC issues Narrative Contents:

- Sample Description and Analyses 
PNNL-20650, Rev. 2

- Sample dissolution/preparation dates

- Sample Preparation

- Analysis dates

- Deviations from the written procedure, if any

- Method specific Sample Preparation, Separation, Mounting and Counting Methods as applicable

- Reporting Basis/Units

- QC Criteria/Results

- Limitations of the Data Interferences/Resolution Uncertainty

- Comments

\section{Data Summary Contents:}

- Client ID

- RPL ID with full ASO-related suffixes (e.g., 129-S)

- Results and result uncertainties

The following elements may be included in the final report or be traceable to the test results (usually by annotation on bench sheets) and be maintained as lifetime records:

- identification of standards used

- identification of M\&TE used

- records of daily check weight tracking

- signature and date of person who performed the test and recorded the data

- analytical bench sheets

- hand calculation review documentation.

Technical reviewers are required to be staff other than the analyst performing the work and knowledgeable of the area being reviewed. The technical review shall be completed and all reviewer issues resolved before reporting final results. Technical reviewers must receive a complete data package including narratives for review. A technical review consists of the following elements:

- evaluation of method and QC performance

- evaluation of compliance with technical (including procedural) and QC requirements, as defined in the ASR

- verification of transcription accuracy

- check for correctness of calculations

- assuring the correct reporting units (and reference dates) are used

- evaluation of overall consistency and reasonableness of data

- evidence of implementation of appropriate corrective action, when necessary.

Analytical results shall be reported both in hard copy and electronically (including excel data files to support accurate data transcription into project records). Appropriately marked "Preliminary" data reports and electronic files shall be provided by the ASO Lead as soon as practical after completion of analysis. The final ASR data report shall be provided no later than the commitment date on the ASR.

The isotopic preparations will require limited reporting since these procedures do not provide analytical results. Needed components (cover page and narrative) are described as follows: Data Report Cover Page Contents:

- Header identifying name/address of laboratory

- Client Name:

- Project \#/WP \#(s):

- $\quad$ ASR \#(s): 
PNNL-20650, Rev. 2

- Total \# Samples:

- Report Date:

- Report Revision Date: when applicable

- Filename(s): where data files can be accessed

- Dates of Sample Processing:

- Procedure(s): Number and title (sample processing and analysis) and revision number

- M\&TE (serial number or property number):

- Reference (i.e., Separation) Date (when applicable):

- Client ID and RPL ID Sample Numbers:

- Report Review and Approval:

Preparer

Date

(Printed name and signature of responsible individual)

Technical Reviewer

Date

(Printed name and signature of technical reviewer)

A case narrative describing the process and limitations on the analysis results

Narrative Contents:

- Sample Description and Analyses

- Sample dissolution/preparation dates

- Sample Preparation

- Method specific Sample Preparation, Separation

- Deviations from the written procedure, if any

- Comments 
PNNL-20650, Rev. 2

Table 4. Method Detection Limits for Solids

\begin{tabular}{|c|c|c|}
\hline Analyte & Solids & Analysis Method \\
\hline & $\mu \mathrm{Ci} / \mathrm{g}_{(d r v)}$ & \\
\hline${ }^{134} \mathrm{Cs}$ & $2.0 \mathrm{E}-01$ & \multirow{7}{*}{$\begin{array}{l}\text { GEA } \\
\text { (Reference date }=\text { date and time of the } \\
\text { first sample analysis measured by GEA.) }\end{array}$} \\
\hline${ }^{137} \mathrm{Cs}$ & $1.5 \mathrm{E}-02$ & \\
\hline${ }^{60} \mathrm{Co}$ & $1.0 \mathrm{E}-02$ & \\
\hline${ }^{152} \mathrm{Eu}$ & $1.5 \mathrm{E}+00$ & \\
\hline${ }^{154} \mathrm{Eu}$ & $1.5 \mathrm{E}+00$ & \\
\hline${ }^{155} \mathrm{Eu}$ & $2.0 \mathrm{E}+00$ & \\
\hline${ }^{241} \mathrm{Am}$ & $1.5 \mathrm{E}+01$ & \\
\hline${ }^{241} \mathrm{Am}$ & $1.0 \mathrm{E}-03$ & \multirow{4}{*}{ Separation and AEA } \\
\hline${ }^{238} \mathrm{Pu}$ & $1.0 \mathrm{E}-03$ & \\
\hline${ }^{239+240} \mathrm{Pu}$ & $1.0 \mathrm{E}-03$ & \\
\hline${ }^{237} \mathrm{~Np}$ & $1.0 \mathrm{E}-03$ & \\
\hline \multirow[t]{2}{*}{${ }^{90} \mathrm{Sr}$} & $1.0 \mathrm{E}+00$ & Separation and proportional counting \\
\hline & $\mu \mathrm{g} / \mathrm{g}_{(\mathrm{drv})}$ & \\
\hline $\mathrm{Ag}$ & $2.0 \mathrm{E}+01$ & \multirow{20}{*}{ ICP-OES } \\
\hline $\mathrm{Al}$ & $1.0 \mathrm{E}+02$ & \\
\hline As & $2.0 \mathrm{E}+01$ & \\
\hline $\mathrm{Ba}$ & $4.0 \mathrm{E}+02$ & \\
\hline $\mathrm{Be}$ & $2.0 \mathrm{E}+01$ & \\
\hline $\mathrm{Ca}$ & $2.0 \mathrm{E}+02$ & \\
\hline $\mathrm{Cd}$ & $4.0 \mathrm{E}+00$ & \\
\hline $\mathrm{Cr}$ & $2.0 \mathrm{E}+01$ & \\
\hline $\mathrm{Fe}$ & $1.0 \mathrm{E}+02$ & \\
\hline $\mathrm{Mg}$ & $2.0 \mathrm{E}+02$ & \\
\hline $\mathrm{Na}$ & $2.0 \mathrm{E}+02$ & \\
\hline $\mathrm{Ni}$ & $2.0 \mathrm{E}+01$ & \\
\hline $\mathrm{Pb}$ & $2.0 \mathrm{E}+01$ & \\
\hline $\mathrm{Sb}$ & $2.0 \mathrm{E}+01$ & \\
\hline $\mathrm{Se}$ & $2.0 \mathrm{E}+01$ & \\
\hline $\mathrm{Tl}$ & $2.0 \mathrm{E}+01$ & \\
\hline V & $2.0 \mathrm{E}+01$ & \\
\hline $\mathrm{Zn}$ & $2.0 \mathrm{E}+01$ & \\
\hline $\mathrm{Zr}$ & $2.0 \mathrm{E}+01$ & \\
\hline $\mathrm{U}$ & $1.0 \mathrm{E}+03$ & \\
\hline $\mathrm{U}$ & $1.0 \mathrm{E}+01$ & Kinetic phosphorescence analysis \\
\hline Total organic carbon & $2.0 \mathrm{E}+02$ (as C) & \multirow{2}{*}{$\begin{array}{l}\text { Furnace oxidation, sequential } \\
\text { temperatures mode }\end{array}$} \\
\hline \multirow[t]{2}{*}{ Total inorganic carbon } & $2.0 \mathrm{E}+02$ (as C) & \\
\hline & wt $\%$ of settled sludge & \\
\hline U metal & 0.01 & $\begin{array}{l}\text { Selective dissolution and } U \text { analysis by } \\
\text { KPA or ICP-OES }\end{array}$ \\
\hline U oxide speciation & N/A & $\begin{array}{l}\text { Selective dissolution and } \\
\text { spectrophotometry }\end{array}$ \\
\hline $\begin{array}{l}\text { Note: The detection lim } \\
\text { metal analysis, whereas } \\
\text { basis. These DL values }\end{array}$ & $\begin{array}{l}\mathrm{s}(\mathrm{DL}) \text { shown in this tab } \\
\text { ne DL in the QAPjP/SA } \\
\text { are therefore, tighter that }\end{array}$ & $\begin{array}{l}\text { are based on dry mass, except for the } \mathrm{U} \\
\text { are based on settled solids (wet) mass } \\
\text { those in the QAPjP/SAP. }\end{array}$ \\
\hline
\end{tabular}


PNNL-20650, Rev. 2

ASR 8798 Special Instructions

Page 15 of 17

Table 5. Analytical Quality Control Acceptance Criteria

\begin{tabular}{|c|c|c|c|c|}
\hline \multirow[t]{2}{*}{ Analyte } & \multirow[t]{2}{*}{ Analytical Technique } & $\begin{array}{l}\text { LCS or BS \% } \\
\text { Recovery }^{(a)}\end{array}$ & $\begin{array}{c}\text { MS or AS } \\
\% \text { Recovery }^{(b)}\end{array}$ & \multirow{2}{*}{$\begin{array}{c}\text { Duplicate } \\
\text { RPD }^{(\mathbf{c})}\end{array}$} \\
\hline & & \multicolumn{2}{|c|}{ Pre-digestion spike } & \\
\hline As identified in Table 4 & ICP-OES & $80-120 \%$ & $80-120 \%$ & $\leq 20 \%$ \\
\hline TOC and TIC & $\begin{array}{l}\text { Furnace oxidation, } \\
\text { sequential temperatures }\end{array}$ & $75-125 \%$ & $75-125 \%$ & $\leq 20 \%{ }^{(\mathrm{f})}$ \\
\hline U total & KPA & $80-120 \%$ & N/A & $\leq 20 \%$ \\
\hline U metal & $\begin{array}{l}\text { Selective dissolution and } \\
\text { ICP-OES or KPA }\end{array}$ & $75-125 \%$ & N/A & $\leq 20 \%$ \\
\hline U oxide speciation & $\begin{array}{l}\text { Selective dissolution and } \\
\text { spectrophotometry }\end{array}$ & (e) & N/A & N/A \\
\hline \multirow{2}{*}{ Analyte } & \multirow{2}{*}{ Analytical Technique } & $\begin{array}{l}\text { LCS or BS \% } \\
\text { Recovery }^{(a)}\end{array}$ & $\begin{array}{c}\text { Matrix Spike \% } \\
\text { Recovery }^{\left({ }^{(b)}\right.}\end{array}$ & \multirow{2}{*}{$\begin{array}{l}\text { Duplicate } \\
\text { RPD }^{\left({ }^{c}\right)}\end{array}$} \\
\hline & & \multicolumn{2}{|c|}{ Post-digestion spike } & \\
\hline $\begin{array}{l}\text { Pu isotopes } \\
\left.\text { (spike material is }{ }^{239} \mathrm{Pu}\right)\end{array}$ & AEA & $80-120 \%$ & $75-125 \%$ & $\leq 20 \%$ \\
\hline${ }^{241} \mathrm{Am}$ & AEA & $80-120 \%$ & $75-125 \%$ & $\leq 20 \%$ \\
\hline${ }^{237} \mathrm{~Np}$ & AEA & $80-120 \%$ & $75-125 \%$ & $\leq 20 \%$ \\
\hline${ }^{90} \mathrm{Sr}$ & Proportional counting & $75-125 \%$ & $75-125 \%$ & $\leq 20 \%$ \\
\hline U total & KPA & $\begin{array}{c}\text { (See pre-digestion } \\
\text { BS) Two } \\
\text { Calibration Checks: } \\
92.5-107.5 \%\end{array}$ & $75-125 \%$ & $\begin{array}{c}\leq 20 \% \\
\text { (as indicated } \\
\text { above) }\end{array}$ \\
\hline As identified in Table 4 & GEA & $\begin{array}{l}\text { Counter control } \\
\text { source is used }{ }^{(\mathrm{d})}\end{array}$ & N/A & $\leq 20 \%$ \\
\hline
\end{tabular}

N/A - not applicable

Footnotes:

(a) LCS = Laboratory Control Standard; BS = Blank Spike. A laboratory control sample (LCS) or blank spike (BS) samples are used to monitor the effectiveness of the sample preparation process and are good indicators of method accuracy. Ideally, the LCS is a material similar to the sample being processed, containing the analytes of interest (e.g., standard reference material). An LCS, if available, shall be prepared with each batch of samples processed at the same time. When an appropriate LCS is not available, a BS shall be used in lieu of the LCS. A BS is distilled or deionized water or another suitable matrix spiked with the analytes of interest. It may not be possible to prepare a single BS that contains all analytes of interest (e.g., chemical incompatibility). In such cases, an agreement with the client shall be made to identify the analytes of interest used to prepare the BS, and more than one BS may be used. The BS and AS results are expressed as percent recovery; i.e., the amount measured, divided by the known concentration, multiplied by 100 .

(b) MS = matrix spike; AS = Analytical Spike. For some methods, the sample accuracy is expressed as the percent recovery of a matrix spike (MS) or analytical spike (AS) sample. Post-digestion spikes and analytical spikes are also included under these acceptance criteria. The spiked sample result is expressed as percent recovery; i.e., the amount measured less the amount in the sample, divided by the spike added, times 100 . One MS (or post spike or analytical spike [AS]) is performed per analytical batch. Samples are batched with similar matrices. For ICP analytes, the accuracy can also be determined based on use of serial dilutions. In cases where the ICP-OES MS concentration is low relative to the sample analyte concentration (resulting in meaningless recovery calculations), matrix effects will be evaluated from a post-digestion spike.

(c) $\mathrm{RPD}=$ Relative Percent Difference between the samples. Sample precision is estimated by analyzing replicates taken separately through preparation and analysis.

(d) Per RPG-CMC-450, Rev 1, a counter control source is checked daily and must be within \pm 3 sigma or $\pm 3 \%$, whichever is greater, of the control value.

(e) The $\mathrm{LCS}_{3} \mathrm{O}_{8}$ accepted ratios are $33.3 \% \mathrm{U}(\mathrm{IV})$ and $66.6 \% \mathrm{U}(\mathrm{VI})$. The acceptable $\mathrm{U}(\mathrm{VI})$ range is 62 to $72 \%$ or $\pm 5 \%$ window. Thus the acceptable U(IV) range is 28 to $38 \%$ or $\pm 5 \%$ window.

(f) Calculate the RSD for the triplicate TIC/TOC measurements. 
PNNL-20650, Rev. 2

\section{References}

52578-TI001, Rev. 0, K West Basin Sample Receipt and Preparation of Consolidated Core Composites: SCS-CON-210-A1 and SCS-CON-210-B3

52578-TI002, Rev. 0, K West Basin Sample Receipt and Preparation of Consolidated Core Composites: SCS-CON-210-A4 and SCS-CON-210-B1

ASO-QAP-001, Rev. 7, Analytical Support Operations Quality Assurance Plan

PNL-ASO-052, Rev. 1, Balance Performance Checks

PNL-ASO-058, Rev. 0.2, ASO Data Reporting

RPG-CMC-107, Rev. 1, Sample Preparation for Determination of Uranium Metal Concentrations in Sludge

PNL-ALO-129, Rev. 0, $\mathrm{HNO}_{3}$-HCl Acid Extraction of Solids Using a Dry-Block Heater

RPG-CMC-211, Rev. 3, Determination of Elemental Composition by Inductively Coupled Argon Plasma Optical Emission Spectrometry (ICP-OES)

RPG-CMC-255, Rev. 0, Sample Preparation and Analysis for Determining Uranium Oxide Oxidation States in K Basin Sludges

RPG-CMC-385, Rev. 2, Carbon Measured in Solids, Sludge, and Liquid Matrices

RPG-CMC-422, Rev. 2, Solutions Analysis: Alpha Spectrometry

RPG-CMC-450, Rev. 1, Gamma Energy Analysis (GEA) and Low-Energy Photon Spectrometry (LEPS)

RPG-CMC-474, Rev. 1, Measurement of Alpha and Beta Activity by Liquid Scintillation Spectrometry

RPG-CMC-476, Rev. 0, Strontium Separation using Eichrom Strontium Resin

RPG-CMC-496, Rev. 0, Coprecipitation Mounting of Actinides for Alpha Spectroscopy

RPG-CMC-4014, Rev. 1 Uranium by Kinetic Phosphorescence Analysis

RPG-CMC-4017, Rev. 0, Analysis of Environmental Water Samples for Actinides and Strontium90

Hanford Analyical Services Quality Assurance Requirments Document (HASQARD), 2007, DOE/RL-96-68, Revsion 3, Department of Energy Richland Office, Richland Washington. 
PNNL-20650, Rev. 2

ASR YYYYY Special Instructions

Page 17 of 17

\section{Appendix A}

SAL Balance Control Checks and Tolerance Limits

The balance control checks will document accuracy and linearity by measure of three masses for the analytical balance Sartorius MSG234 (M\&TE 40023610) and (if needed) Mettler PR5003DR balance (SN: 1120100979).

Tolerances for the three different mass measures are provided. These tolerances have been reviewed and approved by Safeguards (client Terri Welsh, Mission Suppprt Alliance).

Radiochemical Processing Laboratory Battelle, Pacific Nor thwest National Laboratory

\begin{tabular}{|c|}
\hline Battelle, Pacific Northwest National Laboratory \\
\hline Balance Information \\
\hline Identificaiton (e.g. WD34567) \\
\hline 1120100979 \\
\hline Location (e.g. Building/Room) \\
RPL, SAL Cell 2 \\
\hline Calibration Due Date \\
Aug-2011
\end{tabular}

\section{K-Basin Project Type of Balance: Mettler PR5003DR}

\begin{tabular}{|r|c|c|c|c|c|c|}
\hline Check Weight (CW) Information & $\# 1$ & $\begin{array}{c}\mathrm{cw} \\
\text { Units }\end{array}$ & $\# 2$ & $\begin{array}{c}\mathrm{cw} \\
\text { Units }\end{array}$ & $\# 3$ & $\begin{array}{c}\mathrm{cw} \\
\text { Units }\end{array}$ \\
\hline CW Identification & Megatron & & & & & \\
\hline CW Assigned Mass: & $\mathbf{5 0 . 0 0 3}$ & $\mathrm{g}$ & 999.996 & $\mathrm{~g}$ & 2999.97 & $\mathrm{~g}$ \\
\hline & & & & & & \\
\hline Range Low Mass (+Units) \&: & $\mathbf{4 9 . 9 8 4}$ & $\mathbf{g}$ & $\mathbf{9 9 9 . 7 2 0}$ & $\mathrm{g}$ & $\mathbf{2 9 9 9 . 1 0}$ & $\mathrm{g}$ \\
Range High Mass (+Units): & $\mathbf{5 0 . 0 2 1}$ & $\mathbf{g}$ & $\mathbf{1 0 0 0 . 2 7 2}$ & $\mathbf{g}$ & $\mathbf{3 0 0 0 . 8 4}$ & $\mathrm{g}$ \\
\hline (updated on 9/30/10. skf)
\end{tabular}

Radiochemical Processing Laboratory Battelle, Pacific Northwest National Laboratory

\begin{tabular}{|c|}
\hline Balance Information \\
\hline $\begin{array}{c}\text { Identificaiton (e.g. WD34567) } \\
\text { 40023610/WD68444 }\end{array}$ \\
\hline Location (e.g. Building/Room) \\
RPL/201 Cell 2 \\
\hline Calibration Due Date \\
Feb-2011 \\
\hline
\end{tabular}

K-Basin Project Type of Balance: Sartorius MSG234

\begin{tabular}{|c|c|c|c|c|c|c|}
\hline Check Weight (CW) Information & $\# 1$ & $\begin{array}{l}\mathrm{cw} \\
\text { Units }\end{array}$ & $\# 2$ & $\begin{array}{l}\mathrm{cw} \\
\text { Units }\end{array}$ & $\# 3$ & \begin{tabular}{|l}
$\mathrm{cW}$ \\
Units
\end{tabular} \\
\hline $\mathrm{CW}$ Identification & "K-10" & & "Alien" & & $\begin{array}{c}\text { "Megatron" + } \\
\text { "Alien" }\end{array}$ & \\
\hline CW Assigned Mass: & 10.0000 & $\mathrm{~g}$ & 100.0002 & $g$ & 150.0004 & $\mathrm{~g}$ \\
\hline Range Low Mass (+Units) \&: & 10.0000 & g & 99.9998 & g & 149.9995 & g \\
\hline Range High Mass (+Units): & 10.0010 & g & 100.0015 & g & 3000.8390 & g \\
\hline
\end{tabular}


PNNL-20650, Rev. 2

Battelle PNNL/RPL/ASO Mass Measurement Analysis Report

P.O. Box 999, 902 Battelle Blvd., Richland, Washington 99352

\section{Mass Measurements}

\begin{tabular}{|l|l|}
\hline Project / WP\#: & $52578 / \mathrm{H} 41215$ \\
\hline ASR\#: & 8798 \\
\hline Client: & S. Fiskum \\
\hline Total \# of Samples: & 9 \\
\hline
\end{tabular}

\begin{tabular}{|c|c|c|}
\hline Engineered Container \& Core ID & Sample IDs & RPL Number \\
\hline KW-210-A1 & TI001-A1-B, -A1-C & $11-0419-129-\mathrm{S},-129-\mathrm{D}$ \\
\hline $\mathrm{KW}-210-\mathrm{A} 4$ & TI002-A4-B, -A4-C & $11-0420-129-\mathrm{S},-129-\mathrm{D}$ \\
\hline $\mathrm{KW}-210-\mathrm{B} 1$ & TI002-B1-B, -B1-C, -B1-D & $11-0421-129-\mathrm{S},-129-\mathrm{D},-129-\mathrm{MS}$ \\
\hline $\mathrm{KW}-210-\mathrm{B} 3$ & TI001-B3-B, -B3-C & $11-0422-129-\mathrm{S},-129-\mathrm{D}$ \\
\hline
\end{tabular}

\section{$\underline{\text { Revision } 1}$}

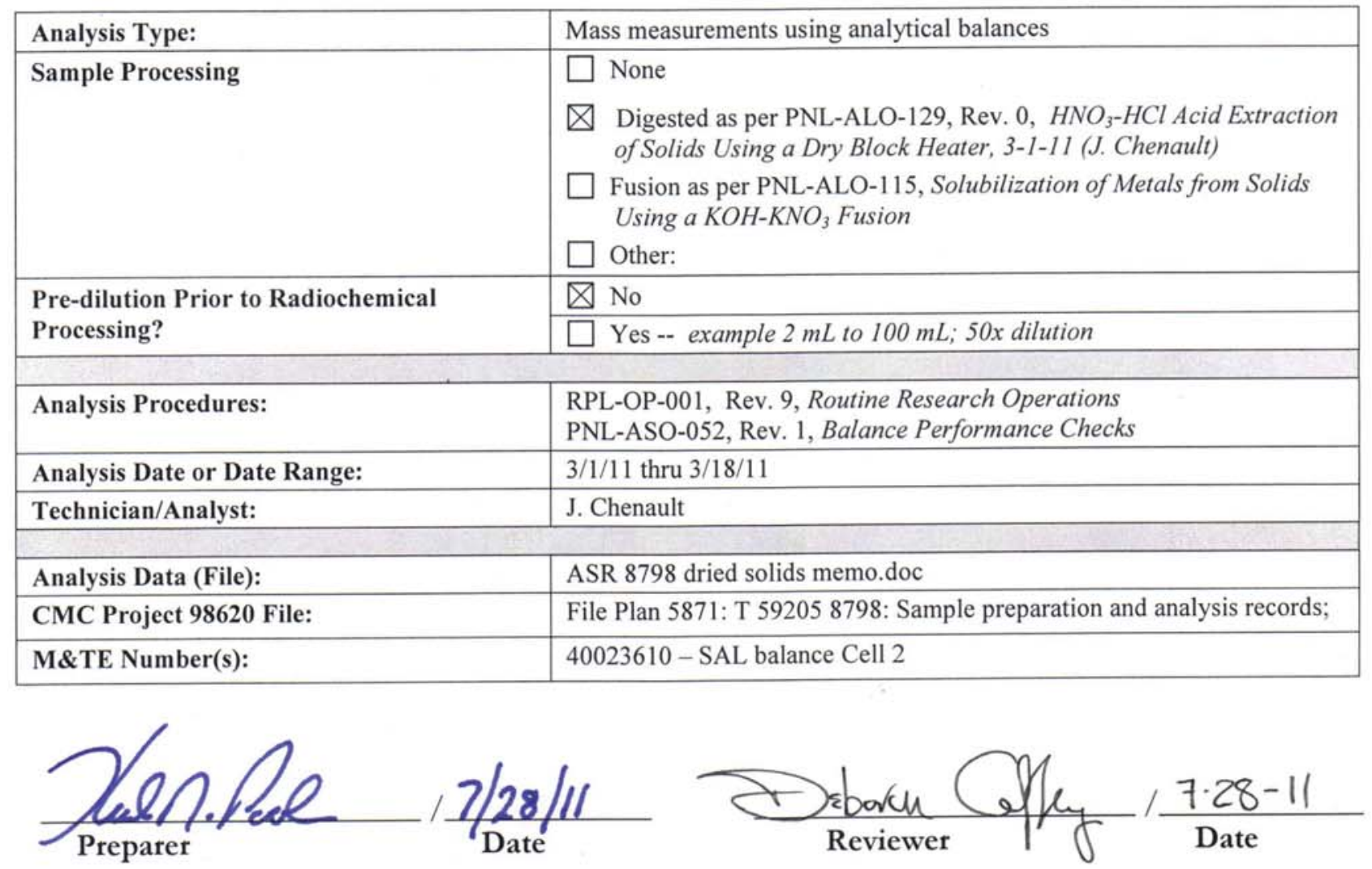




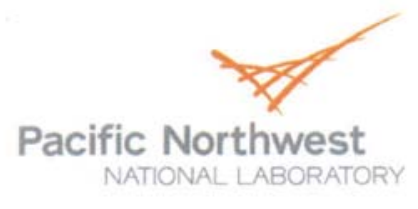

$\begin{array}{llll}\text { Date: } & \text { July 27, 2011 } & \text { Project No.: } & \mathbf{5 2 5 7 8} \\ \text { To: } & \text { Sandy Fiskum } & & \\ \text { From: } & \text { Karl Pool } & & \\ \text { Subject: } & \begin{array}{l}\text { Initial dried solids and residual } \\ \text { dried solids masses for ASR }\end{array} & \text { Internal Distribution: } & \text { File/LB } \\ & \mathbf{8 7 9 8} & & \end{array}$

Revision 1 - Report revised to correct the volumes of reagent acids used in the acid digestion of dried sludge.

Prior to ASO sample digestion, all slurry samples for ASR 8798 were dried to constant weight at $105^{\circ} \mathrm{C}$ in pre-tared Teflon Oak Ridge digestion tubes in the Shielded Analytical Laboratory (SAL) using a drying oven. The dry masses of the solids prior to digestion are presented in the Table 1. The dried solids were then acid digested using ASO procedure PNL-ALO-129, " $\mathrm{HNO}{ }_{3}-\mathrm{HCl}$ Acid Extraction of Solids Using a Dry-Block Heater". The acid volume used was proportioned to the mass of sample. Samples that had mass of $1 \mathrm{~g}$ or less used $8 \mathrm{~mL}$ of each acid. Samples that had mass of 1 to $1.5 \mathrm{~g}$ used $12 \mathrm{~mL}$ of each acid. At the completion of the acid digestion, the samples were cooled and the residual (un-dissolved solids) were allowed to settle. The digestate with settled residual was then centrifuged for approximately 10 minutes to consolidate the solids. The supernatant solution was carefully removed using transfer pipettes. The residual solids were each rinsed 3 consecutive times using $\sim 12$ to $16 \mathrm{~mL}$ of the same reagent acids $\left(1: 1\right.$ combination of $\left.8 \mathrm{M} \mathrm{HNO}_{3}+8 \mathrm{M} \mathrm{HCl}\right)$ used in the initial acid digestion. The centrifuged solids were dislodged from the bottom of the centrifuge tube using a vortex mixer after each addition of the rinse acids. The acid rinse/solids were centrifuged for approximately 10 minutes after each acid rinse addition. The remaining rinsed solids were returned to the drying oven for drying. The residual solids were dried in the drying oven for several days at $105^{\circ} \mathrm{C}$. The residual solids in the Oak Ridge tubes were dried to constant weight.

The initial acid digestate and acid rinse solutions were collected and brought to a final volume of $100 \mathrm{~mL}$ in plastic volumetric flasks. The solutions in the volumetric flask were thoroughly mixed prior to subsampling for chemical and radiochemical analyses. 


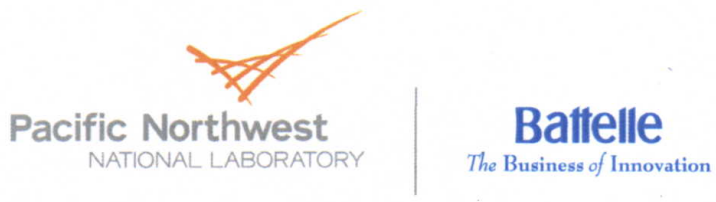

Table 1 - Dried solids masses

\begin{tabular}{|c|c|c|c|}
\hline RPL ID & Client ID & $\begin{array}{l}\text { Initial dried solids mass } \\
\text { prior to digestion (grams) }\end{array}$ & $\begin{array}{c}\text { Final dried solids mass remaining } \\
\text { after digestion (grams) }\end{array}$ \\
\hline $11-0419-129-\mathrm{S}$ & TI001-A1-B & 1.1233 & 0.7262 \\
\hline 11-0419-129-D & TI001-A1-C & 1.2773 & 0.8140 \\
\hline $11-0420-129-S$ & TI002-A4-B & 1.4258 & 1.0004 \\
\hline $11-0420-129-D$ & T1002-A4-C & 1.5994 & 1.1212 \\
\hline 11-0421-129-S & TI002-B1-B & 0.7459 & 0.6192 \\
\hline 11-0421-129-D & T1002-B1-C & 0.8192 & 0.7206 \\
\hline $11-0422-129-\mathrm{S}$ & T1001-B3-B & 1.0626 & 0.9163 \\
\hline 11-0422-129-D & Tl001-B3-C & 1.0515 & 0.9157 \\
\hline 11-0421-129-MS & TI002-B1-D & 0.8818 & 0.7621 \\
\hline
\end{tabular}

E54-1900-001 (8/98) 
Battelle PNNL/RPL/Inorganic Analysis ... ICP-OES Analysis Report PO Box 999, Richland, Washington 99352

Project / WP\#: $\quad 52578$ / H41215

ASR\#: $\quad 8798$

Client: $\quad$ S. Fiskum

Total Samples: $\quad 8$ (solids)

\begin{tabular}{|c|c|c|}
\hline & First & Last \\
\hline RPL\#: & $11-0419$ & $11-0422$ \\
\hline Client ID: & TI001-A1-B & TI001-B3-C \\
\hline
\end{tabular}

Procedure: $\quad$ RPG-CMC-211, "Determination of Elemental Composition by Inductively Coupled Argon Plasma Optical Emission Spectrometry (ICP-OES)", Rev. 3.

Analyst: J. Carter

Analysis Date (File): $\quad \underline{03-15-2011}(\mathrm{C} 0353)$

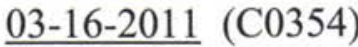

See Chemical Measurement Center 98620 file: $\quad$ ICP-325-405-3

(Calibration and Maintenance Records)

M\&TE Number: $\quad \underline{\text { N827583 }}$ (PerkinElmer 5300DV ICP-OES, S/N 077N5122002) WD66158 (Sartorius ME414S Balance, S/N 224066373)

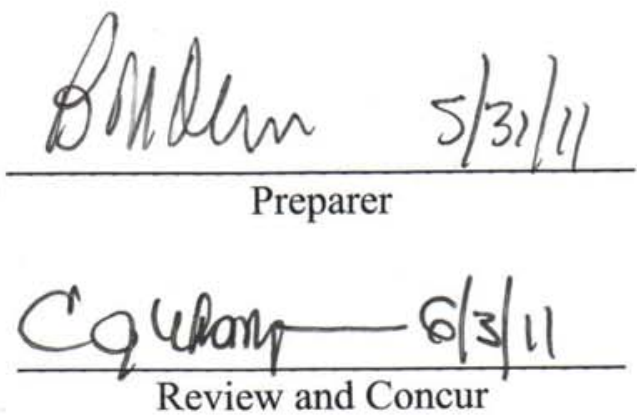




\section{Battelle PNNL/RPL/Inorganic Analysis ... ICP-OES Analysis Report}

Four samples in duplicate were provided for ICP-OES analysis under Analytical Service Request (ASR) 8798. The samples and duplicates were obtained from sub-samples prepared in the High Level Radiochemistry Facility (HLRF). Each of the samples was processed in the Shielded Analytical Laboratory (SAL) following RPL procedure PNL-ALO-129 using 1 to 2 grams of sample with dilution to a final volume of $100 \mathrm{~mL}$.

Analytes of interest (AOIs) were specified in the ASR, and are listed in the upper section of the attached ICP-OES Data Report. The quality control (QC) results for these AOIs have been evaluated and are presented below. Analytes other than AOIs are reported in the bottom section of the report, but have not been fully evaluated for QC performance. The results are reported on a mass basis $\left(\mu \mathrm{g} / \mathrm{g}_{\mathrm{dry}}\right)$ for each detected analyte based on the original sample mass. All data have been adjusted for laboratory processing factors and instrument dilutions. There were several QC issues associated with the present analyses. These issues are discussed below and also in Occurrence Report 52578-04-20-11 and PNNL Letter 52578-2011-L06.

Except for $\mathrm{As}, \mathrm{Sb}, \mathrm{Se}$, and $\mathrm{Tl}$, the results are reported for $10 \mathrm{x}$ diluted samples. As, $\mathrm{Sb}$, Se, and $\mathrm{Tl}$ are reported for undiluted samples $(1 \mathrm{x})$ in order to obtain the necessary sensitivity. Analyzing the samples at $1 \mathrm{x}$, however, does introduce potential matrix issues as both the sample density and the acid content at $1 \mathrm{x}$ are considerably higher than the normal matrix seen by the instrument ( $\sim 0.5 \mathrm{M}$ nitric acid).

Minimum Method Detection Limit (MDL) values were specified in the ASR. Except for Pb and Se in Samples 11-0421-129-S and 11-0421-129-D, MDL levels were met for all AOIs. For the $\mathrm{Pb}$, however, the observed levels exceeded the MDL requirements thereby mitigating this issue. Selenium, however, was $<$ IDL for the two samples and, therefore, the MDL was not met.

Calibration of the ICP-OES was done following the manufacturer's recommended calibration procedure and using multi-analyte custom standard solutions traceable to the National Institute of Standards and Technology (NIST). Midrange calibration verification standards (MCVA and MCVB) were used to verify acceptance of the two-point calibration curves obtained for each analyte, and also used for continuing calibration verification.

The controlling documents were ASO-QAP-001 and ASR-8798 Special Instructions. Instrument calibrations, QC checks and blanks (e.g., ICV/ICB, CCV/CCB, LLS, ICS), blank spike, matrix spike, post spike, duplicate, and serial dilution were conducted during the analysis run. The blank spike and matrix spike were prepared using a custom solution consisting of aliquots of BPNL-QC-1A and -2B, INT-QC-TCLP-A, and CG-SB1-1.

\section{Preparation Blank (PB):}

A preparation blank (reagents only) was prepared for the extraction process. The concentrations of all AOIs were within the acceptance criteria of $\leq$ EQL (estimated quantitation level) or less than $\leq 5 \%$ of the concentration in the sample. 
PNNL-20650, Rev. 2

\section{Battelle PNNL/RPL/Inorganic Analysis ... ICP-OES Analysis Report}

Blank Spike (BS)/Laboratory Control Sample (LCS):

A blank spike was prepared for the extraction process. Recovery values are listed for all analytes included in the spike that were measured at or above the EQL. The recovery values were within the acceptance criterion of $80 \%$ to $120 \%$ for all AOIs meeting the above requirement.

\section{Matrix-Spiked Sample:}

A matrix spike was prepared for the extraction process using Sample 11-0421. Recovery values are listed for all analytes included in the spike that were measured at or above the EQL. For AOIs meeting the above requirement, the recovery values for $\mathrm{Sb}(45 \%), \mathrm{Tl}$ (30\%), $\mathrm{Zn}(74 \%)$, and $\mathrm{Zr}(244 \%)$ were outside the acceptance criterion of $80 \%$ to $120 \%$. These recovery results are likely from a combination of factors, including 1) sample heterogeneity in the sludge, 2) matrix issues associated with the very high $\mathrm{Al}, \mathrm{Fe}$, and $\mathrm{U}$ in the samples, and 3) spike levels that are relatively close to the instrument EQL values. Note that all the analytes did pass the post-spike analysis acceptance range of 80 to $120 \%$ (see below).

\section{Duplicate Relative Percent Difference (RPD):}

Duplicates were prepared for each of the four samples. RPDs are listed for all analytes that were measured at or above the EQL. RPDs for Sample 11-0422 were within the acceptance criterion of $\leq 20 \%$ for AOIs meeting the above requirement. For Samples 110419 (Be-37.7\%, Cr-24.5\%, Ni-36.5\%), 11-0420 (Be-58.5\%, Cr-51.0\%, Mg-25.4\%, Ni$47.3 \%, \mathrm{Zn}-27.3 \%, 86.4 \%$ ), and 11-0421 (Be-144.1\%, Fe-24.4\%, Ni-22.0\%, Zn-47.3\%, Zr$135.0 \%$ ), however, the RPDs were outside the acceptance criterion. The high RPDs are likely from sample heterogeneity in the samples and/or from the sub-sampling in the HLRF.

\section{Post-Spike/Analytical Spike Sample (A Component):}

A post spike (A Component) was conducted on Sample 11-0421. Recovery values are listed for all analytes in the spike that were measured at or above the EQL, and that had a spike concentration $\geq 25 \%$ of that in the sample. The recovery values were within the acceptance criterion of $80 \%$ to $120 \%$ for all AOIs meeting the above requirements, including those analytes that failed the matrix-spike analysis discussed above.

\section{Post Spike/Analytical Spike Sample (B Component):}

A post spike (B Component) was conducted on Sample 11-0421. Recovery values are listed for all analytes in the spike that were measured at or above the EQL, and that had a spike concentration $\geq 25 \%$ of that in the sample. The recovery value for uranium (the only AOI in the B Component) was within the acceptance criterion of $80 \%$ to $120 \%$.

\section{Serial dilution:}

Five-fold serial dilution was conducted on Sample 11-0421. Percent differences (\%Ds) are listed for all analytes that had a concentration at or above the EQL in the diluted sample. The $\%$ Ds were within the acceptance criterion of $\leq 10 \%$ for all AOIs meeting the above requirement. 
PNNL-20650, Rev. 2

\section{Battelle PNNL/RPL/Inorganic Analysis ... ICP-OES Analysis Report}

\section{Other QC:}

Barium was slightly above the EQL $(\sim 0.002 \mu \mathrm{g} / \mathrm{mL})$ for the first of three ICSs for the $10 \mathrm{x}$ dilution run. This is believed to be from carry-over of $\mathrm{Ba}$ from the proceeding CCV. This small carry-over, however, had no statistical effect on the reported data as the level of Ba in the ICSs was negligible compared to the levels of $\mathrm{Ba}$ in the samples themselves. All other instrument-related QC tests for the AOIs passed within the appropriate acceptance criteria.

\section{Comments:}

1) The "Final Results" have been corrected for all laboratory dilutions performed on the samples during processing and analysis, unless specifically noted.

2) Instrument detection limits (IDL) and estimated quantitation limits (EQL) shown are for acidified water and/or fusion flux matrices as applicable. Detection limits for other matrices may be determined if requested. Method detection limits (MDL) can be estimated by multiplying the IDL by the "Multiplier". The estimated quantitation limit (EQL) for each concentration value can be obtained by multiplying the EQL by the "Multiplier".

3) Routine precision and bias is typically $\pm 15 \%$ or better for samples in dilute, acidified water (e.g. $2 \% \mathrm{v} / \mathrm{v}$ $\mathrm{HNO}_{3}$ or less) at analyte concentrations $>$ EQL up to the upper calibration level. This also presumes that the total dissolved solids concentration in the sample is less than $5000 \mu \mathrm{g} / \mathrm{mL}(0.5$ per cent by weight). Note that bracketed values listed in the data report are within the MDL and the EQL, and have potential uncertainties greater than $15 \%$. Concentration values $<$ MDL are listed as ". -.". Note, that calibration and QC standards are validated to a precision of $\pm 10 \%$.

4) Absolute precision, bias and detection limits may be determined on each sample if required by the client. The maximum number of significant figures for all ICP measurements is two.

5) Analytes included in the spike A component (for the AS/PS) are; Ag, Al, As, B, Ba, Be, Bi, Ca, Cd, Co, Cr, $\mathrm{Cu}, \mathrm{Fe}, \mathrm{K}, \mathrm{Li}, \mathrm{Mg}, \mathrm{Mn}, \mathrm{Mo}, \mathrm{Na}, \mathrm{Ni}, \mathrm{P}, \mathrm{Pb}, \mathrm{Sb}, \mathrm{Se}, \mathrm{Si}, \mathrm{Sn}, \mathrm{Sr}, \mathrm{Ta}, \mathrm{Ti}, \mathrm{Tl}, \mathrm{V}, \mathrm{W}, \mathrm{Y}, \mathrm{Zn}$, and $\mathrm{Zr}$. Analytes included in the spike B component are; $\mathrm{Ce}, \mathrm{Dy}, \mathrm{Eu}, \mathrm{La}, \mathrm{Nd}, \mathrm{Pd}, \mathrm{Rh}, \mathrm{Ru}, \mathrm{S}, \mathrm{Te}, \mathrm{Th}$, and $\mathrm{U}$. 
PNNL-20650, Rev. 2

Battelle PNNL/RPG/Inorganic Analysis ... ICPOES Data Report

Page 1 of 4

\begin{tabular}{|c|c|c|c|c|c|c|c|c|c|c|c|c|}
\hline \multirow{3}{*}{\multicolumn{2}{|c|}{$\mathrm{Zr}$ - }} & Run Date > & $3 / 15 / 2011$ & $3 / 16 / 2011$ & $3 / 15 / 2011$ & $3 / 16 / 2011$ & $3 / 15 / 2011$ & $3 / 16 / 2011$ & $3 / 15 / 2011$ & $3 / 16 / 2011$ & $3 / 15 / 2011$ & $3 / 16 / 2011$ \\
\hline & & Multiplier > & 878.6 & 87.9 & 890.2 & 89.0 & 782.9 & 78.3 & 701.4 & 70.1 & 625.2 & 62.5 \\
\hline & & RPLILAB > & $\begin{array}{c}11-0419-129 \\
\text { PB @ 10x }\end{array}$ & $\begin{array}{c}\text { 11-0419- } \\
129-P B\end{array}$ & $\begin{array}{c}11-0419-129 \\
\mathrm{~S} @ 10 \mathrm{x} \\
\end{array}$ & $\begin{array}{c}\begin{array}{c}11-0419- \\
129-\mathrm{S}\end{array} \\
\end{array}$ & $\begin{array}{c}11-0419-129 \\
D @ 10 x\end{array}$ & $\begin{array}{c}\begin{array}{c}11-0419- \\
129-D\end{array} \\
\end{array}$ & \begin{tabular}{|c|}
$11-0420-129$ \\
$\mathrm{~S} @ 10 \mathrm{x}$ \\
\end{tabular} & $\begin{array}{c}11-0420- \\
129-\mathrm{S}\end{array}$ & \begin{tabular}{|c|}
$11-0420-129$ \\
$D @ 10 x$
\end{tabular} & $\begin{array}{c}11-0420- \\
129-D\end{array}$ \\
\hline $\begin{array}{l}\text { Instr. Det. } \\
\text { Limit (IDL) }\end{array}$ & \begin{tabular}{|l|} 
Est. Quant. \\
Limit (EQL) \\
\end{tabular} & Client ID > & \multicolumn{2}{|c|}{ PB } & \multicolumn{2}{|c|}{ T1001-A1-B } & \multicolumn{2}{|c|}{ T1001-A1-C } & \multicolumn{2}{|c|}{ T1002-A4-B } & \multicolumn{2}{|c|}{ T1002-A4-C } \\
\hline$(\mu \mathrm{g} / \mathrm{mL})$ & $(\mu \mathrm{g} / \mathrm{mL})$ & (Analyte) & $(\mu \mathrm{g} / \mathrm{g})$ & $(\mu \mathrm{g} / \mathrm{g})$ & $(\mu \mathrm{g} / \mathrm{g})$ & $(\mu \mathrm{g} / \mathrm{g})$ & $(\mu \mathrm{g} / \mathrm{g})$ & $(\mu \mathrm{g} / \mathrm{g})$ & $(\mu \mathrm{g} / \mathrm{g})$ & $(\mu g / g)$ & $(\mu \mathrm{g} / \mathrm{g})$ & $(\mu \mathrm{g} / \mathrm{g})$ \\
\hline 0.0019 & 0.019 & $\mathbf{A g}$ & - & & [5.5] & & {$[3.8]$} & & {$[2.8]$} & & {$[2.8]$} & \\
\hline 0.0046 & 0.046 & Al & - & & 32,500 & & 32,600 & & 20,700 & & 22,400 & \\
\hline 0.0960 & 0.960 & As & & 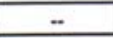 & & 89.2 & & 98.4 & & 72.3 & & 72.7 \\
\hline 0.0001 & 0.002 & $\mathrm{Ba}$ & {$[0.24]$} & & 192 & & 199 & & 135 & & 143 & \\
\hline 0.0001 & 0.001 & Be & $\because$ & & 6.99 & & 10.2 & & 12.2 & & 6.70 & \\
\hline 0.0022 & 0.044 & $\mathrm{Ca}$ & - & & 7,640 & & 6,750 & & 6,690 & & 7,740 & \\
\hline 0.0015 & 0.015 & $\mathrm{Cd}$ & $\ddot{*}$ & & 35.9 & & 33.5 & & 29.8 & & 34.6 & \\
\hline 0.0020 & 0.020 & $\mathrm{Cr}$ & {$[3.4]$} & & 354 & & 277 & & 569 & & 337 & \\
\hline 0.0045 & 0.045 & $\mathrm{Fe}$ & 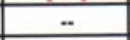 & & 86,800 & & 88,600 & & 96,400 & & 93,700 & \\
\hline 0.0006 & 0.012 & $\mathrm{Mg}$ & 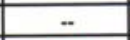 & & 1,140 & & 1,010 & & 730 & & 943 & \\
\hline 0.0036 & 0.072 & $\mathrm{Na}$ & {$[14]$} & & 708 & & 655 & & 1,420 & & 1,650 & \\
\hline 0.0026 & 0.026 & $\mathrm{Ni}$ & $\because$ & & 185 & & 128 & & 334 & & 206 & \\
\hline 0.0210 & 0.210 & $\mathrm{~Pb}$ & $\ddot{-}$ & & 345 & & 345 & & 238 & & 264 & \\
\hline 0.0220 & 0.220 & $\mathrm{Sb}$ & & 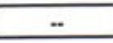 & & [8.6] & & [8.0] & & {$[9.4]$} & & {$[8.5]$} \\
\hline 0.1700 & 1.700 & Se & & 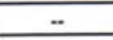 & & 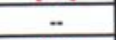 & & 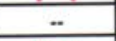 & & 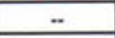 & & - \\
\hline 0.0860 & 0.860 & $\mathrm{TI}$ & & $\ldots$ & & {$[9.2]$} & & {$[8.7]$} & & {$[6.6]$} & & {$[6.8]$} \\
\hline 0.0120 & 0.120 & $u$ & 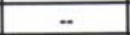 & & 93,800 & & 103,000 & & 82,800 & & 73,600 & \\
\hline 0.0011 & 0.022 & $\mathrm{v}$ & $\ddot{-}$ & & [15] & & [15] & & {$[13]$} & & 14.2 & \\
\hline 0.0056 & 0.056 & $\mathrm{Zn}$ & $\ddot{*}$ & & 469 & & 398 & & 291 & & 383 & \\
\hline 0.0008 & 0.008 & $\mathrm{Zr}$ & - & & 334 & & 369 & & 373 & & 148 & \\
\hline \multicolumn{13}{|c|}{$\begin{array}{c}0.0008 \text { I } \\
\text { Other Analytes }\end{array}$} \\
\hline 0.0057 & 0.057 & $B$ & [39] & & 52.9 & & [38] & & [26] & & [25] & \\
\hline 0.0160 & 0.160 & $\mathrm{Bi}$ & [59] & & [120] & & [110] & & {$[100]$} & & [86] & \\
\hline 0.0087 & 0.087 & Ce & $\ddot{*}$ & & 79.2 & & 74.9 & & 63.1 & & 55.9 & \\
\hline 0.0025 & 0.025 & Co & 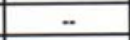 & & [4.6] & & {$[3.3]$} & & {$[6.3]$} & & {$[2.4]$} & \\
\hline 0.0016 & 0.016 & $\mathrm{Cu}$ & $\ddot{*}$ & & 1,680 & & 1,070 & & 510 & & 487 & \\
\hline 0.0012 & 0.012 & Dy & 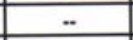 & & $\because$ & & $\because$ & & $\because$ & & - & \\
\hline 0.0003 & 0.003 & $\mathrm{Eu}$ & $\because$ & & {$[2.3]$} & & 2.34 & & 2.00 & & 2.02 & \\
\hline 0.0220 & 0.220 & $K$ & 619 & & 1,240 & & 1,230 & & 967 & & 912 & \\
\hline 0.0012 & 0.012 & La & $\ddot{-}$ & & 24.5 & & 24.7 & & 20.1 & & 20.9 & \\
\hline 0.0005 & 0.005 & $\mathrm{Li}$ & - & & [3.2] & & 3.67 & & {$[2.1]$} & & {$[2.4]$} & \\
\hline 0.0002 & 0.002 & $\mathrm{Mn}$ &.- & & 343 & & 291 & & 313 & & 297 & \\
\hline 0.0070 & 0.070 & Mo & $\because$ & & {$[43]$} & & [44] & & {$[42]$} & & [35] & \\
\hline 0.0027 & 0.027 & $\mathrm{Nd}$ & - & & 86.9 & & 92.6 & & 54.0 & & 50.6 & \\
\hline 0.0510 & 0.510 & $P$ & [47] & & {$[430]$} & & {$[370]$} & & [210] & & {$[240]$} & \\
\hline 0.0048 & 0.048 & $P d$ & $\because$ & & 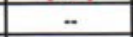 & & 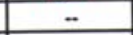 & & 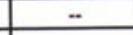 & & - & \\
\hline 0.0088 & 0.088 & $\mathbf{R h}$ & 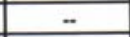 & & $-\because$ & & -- & & - & & - & \\
\hline 0.0044 & 0.044 & Ru & - & & [19] & & [21] & & [13] & & {$[18]$} & \\
\hline 0.1600 & 1.600 & $s$ & - & & 7,060 & & 6,130 & & 6,140 & & 7,120 & \\
\hline 0.0130 & 0.130 & Si & - & & 749 & & 678 & & 592 & & 610 & \\
\hline 0.0140 & 0.140 & Sn & [35] & & [88] & & [91] & & {$[67]$} & & {$[56]$} & \\
\hline 0.0000 & 0.000 & $\mathrm{Sr}$ & {$[0.041]$} & & 63.8 & & 57.8 & & 43.2 & & 49.8 & \\
\hline 0.0190 & 0.190 & $\mathrm{Ta}$ & 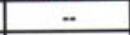 & & $\because$ & & $\because$ & & $\because$ & & - & \\
\hline 0.0160 & 0.160 & Te & - & & - & & [16] & & 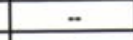 & & - & \\
\hline 0.0050 & 0.050 & Th & $\ddot{-}$ & & 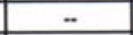 & & 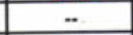 & & 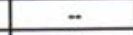 & & - & \\
\hline 0.0005 & 0.005 & $\mathrm{Ti}$ & - & & 464 & & 358 & & 382 & & 374 & \\
\hline 0.0150 & 0.150 & $w$ &.- & & - & & - & & - & & $\because$ & \\
\hline 0.0003 & 0.005 & $Y$ & - & & 7.32 & & 6.86 & & 5.43 & & 5.66 & \\
\hline
\end{tabular}

1) "-" indicates the value is $<$ MDL. The method detection limit $(M D L)=I D L$ times the "multiplier"

near the top of each column. The estimated sample quantitation limit $=E Q L$ (in Column 2)

times the "multiplier". Overall error for values 2 EQL is estimated to be within $\pm 15 \%$.

2) Values in brackets [] are $\geq M O L$ but $<E Q L$, with errors likely to exceed $15 \%$.

ASR 8798 Final from C0354-Rep S. Fiskum (ASR-8798 129's 1x).XLS 
PNNL-20650, Rev. 2

Battelle PNNL/RPG/Inorganic Analysis ... ICPOES Data Report

\begin{tabular}{|c|c|c|c|c|c|c|c|c|}
\hline Run Date > & $3 / 15 / 2011$ & $3 / 16 / 2011$ & $3 / 15 / 2011$ & $3 / 16 / 2011$ & $3 / 15 / 2011$ & $3 / 16 / 2011$ & $3 / 15 / 2011$ & $3 / 16 / 2011$ \\
\hline Multiplier > & 1340.7 & 134.1 & 1220.7 & 122.1 & 941.1 & 94.1 & 951.0 & 95.1 \\
\hline RPL/LAB > & $\begin{array}{c}11-0421-129 \\
\mathrm{~S} @ 10 \mathrm{x} \\
\end{array}$ & $\begin{array}{c}11-0421- \\
129-S \\
\end{array}$ & $\begin{array}{c}11-0421-129 \\
D @ 10 x\end{array}$ & $\begin{array}{c}11-0421- \\
129-D\end{array}$ & $\begin{array}{c}11-0422-129 \\
S @ 10 x\end{array}$ & $\begin{array}{c}11-0422- \\
129-S\end{array}$ & $\begin{array}{c}11-0422-129 \\
D @ 10 x \\
\end{array}$ & $\begin{array}{c}11-0422- \\
129-D\end{array}$ \\
\hline Client ID > & \multicolumn{2}{|c|}{ T1002-B1-B } & \multicolumn{2}{|c|}{ T1002-B1-C } & \multicolumn{2}{|c|}{ T1001-B3-B } & \multicolumn{2}{|c|}{ T1001-B3-C } \\
\hline (Analyte) & $(\mu g / g)$ & $(\mu \mathrm{g} / \mathrm{g})$ & $(\mu g / g)$ & $(\mu g / g)$ & $(\mu \mathrm{g} / \mathrm{g})$ & $(\mu \mathrm{g} / \mathrm{g})$ & $(\mu \mathrm{g} / \mathrm{g})$ & $(\mu \mathrm{g} / \mathrm{g})$ \\
\hline $\mathrm{Ag}$ & [4.2] & & [3.5] & & {$[2.0]$} & & [3.9] & \\
\hline Al & 15,600 & & 15,500 & & 12,400 & & 12,100 & \\
\hline As & & [46] & & [41] & & [54] & & {$[50]$} \\
\hline $\mathrm{Ba}$ & 75.4 & & 78.0 & & 66.8 & & 62.4 & \\
\hline $\mathrm{Be}$ & 3.03 & & 18.6 & & 2.76 & & 2.51 & \\
\hline $\mathrm{Ca}$ & 7,410 & & 6,590 & & 8,350 & & 7,510 & \\
\hline Cd & 65.5 & & 57.4 & & 48.8 & & 48.8 & \\
\hline $\mathrm{Cr}$ & 287 & & 274 & & 225 & & 218 & \\
\hline $\mathrm{Fe}$ & 35,100 & & 27,500 & & 31,800 & & 27,800 & \\
\hline $\mathrm{Mg}$ & 582 & & 648 & & 588 & & 582 & \\
\hline $\mathrm{Na}$ & 1,710 & & 1,610 & & 1,460 & & 1,340 & \\
\hline $\mathrm{Ni}$ & 223 & & 178 & & 157 & & 156 & \\
\hline $\mathrm{Pb}$ & 319 & & 322 & & 313 & & 287 & \\
\hline $\mathrm{Sb}$ & & - & & [4.6] & & [5.1] & & [3.9] \\
\hline Se & & - & & - & & - & & - \\
\hline $\mathrm{TI}$ & & $\ldots$ & & - & & - & & - \\
\hline U & 51,900 & & 45,600 & & 55,700 & & 51,000 & \\
\hline $\mathrm{V}$ & {$[9.8]$} & & [11] & & {$[9.8]$} & & {$[8.4]$} & \\
\hline $\mathrm{Zn}$ & 394 & & 243 & & 211 & & 200 & \\
\hline $\mathrm{Zr}$ & 77.6 & & 400 & & 91.8 & & 97.2 & \\
\hline
\end{tabular}

\begin{tabular}{|c|c|c|c|c|}
\hline B & [52] & [47] & [31] & [26] \\
\hline $\mathrm{Bi}$ & [140] & [150] & {$[120]$} & {$[100]$} \\
\hline $\mathrm{Ce}$ & [32] & [45] & [48] & [53] \\
\hline Co & [5.8] & [6.0] & {$[4.7]$} & {$[2.9]$} \\
\hline $\mathrm{Cu}$ & 1,190 & 1,170 & 822 & 807 \\
\hline Dy & -. & - & {$[1.7]$} & -. \\
\hline $\mathrm{Eu}$ & [1.3] & {$[1.7]$} & {$[0.86]$} & [1.1] \\
\hline $\mathbf{K}$ & 1,340 & 1,120 & 657 & 696 \\
\hline La & 19.8 & 18.9 & 21.9 & 17.9 \\
\hline $\mathrm{Li}$ & 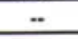 & 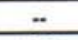 & {$[0.85]$} & {$[0.70]$} \\
\hline $\mathrm{Mn}$ & 222 & 227 & 214 & 193 \\
\hline Mo & [27] & {$[30]$} & {$[27]$} & [27] \\
\hline Nd & [35] & 41.6 & 57.6 & 45.7 \\
\hline $\mathbf{P}$ & [450] & [470] & {$[470]$} & {$[480]$} \\
\hline Pd & .. & - & - & - \\
\hline Rh & 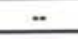 & -. & - & - \\
\hline $\mathrm{Ru}$ & [13] & [12] & [18] & [14] \\
\hline $\mathrm{s}$ & 9,720 & 8,930 & 9,470 & 8,870 \\
\hline $\mathrm{Si}$ & 697 & 656 & 492 & 482 \\
\hline $\mathrm{Sn}$ & 286 & {$[160]$} & [71] & [58] \\
\hline $\mathrm{Sr}$ & 27.3 & 26.2 & 23.7 & 21.6 \\
\hline $\mathrm{Ta}$ & - & - & - & - \\
\hline $\mathrm{Te}$ & - & - & - & - \\
\hline Th & - & - & - & - \\
\hline $\mathrm{Ti}$ & 197 & 205 & 138 & 127 \\
\hline w & - & - & - & - \\
\hline $\mathbf{Y}$ & {$[4.4]$} & {$[4.7]$} & 5.08 & [4.5] \\
\hline
\end{tabular}


PNNL-20650, Rev. 2

Battelle PNNL/RPG/Inorganic Analysis ... ICPOES Data Report

\begin{tabular}{|c|c|c|c|c|c|c|c|c|c|c|}
\hline Criteria > & $\leq 20 \%$ & $\leq 20 \%$ & $\leq 20 \%$ & $\leq 20 \%$ & $\leq 20 \%$ & $\leq 20 \%$ & $\leq 20 \%$ & $\leq 20 \%$ & $80 \%-120 \%$ & $80 \%-120 \%$ \\
\hline$Q C \mid D>$ & \multicolumn{2}{|c|}{$\begin{array}{c}11-0419 \\
\text { Dup }\end{array}$} & \multicolumn{2}{|c|}{$\begin{array}{c}11-0420 \\
\text { Dup }\end{array}$} & \multicolumn{2}{|c|}{$\begin{array}{c}11-0421 \\
\text { Dup }\end{array}$} & \multicolumn{2}{|c|}{$\begin{array}{c}11-0422 \\
\text { Dup }\end{array}$} & \multicolumn{2}{|c|}{ LCS/BS } \\
\hline Analytes & RPD (\%) & RPD (\%) & RPD (\%) & RPD (\%) & RPD (\%) & RPD (\%) & RPD (\%) & RPD (\%) & $\%$ Rec & $\%$ Rec \\
\hline $\mathrm{Ag}$ & & & & & & & & & 96 & \\
\hline Al & 0.4 & & 7.6 & & 0.8 & & 2.1 & & 101 & \\
\hline As & & 9.9 & & 0.6 & & & & & & 85 \\
\hline $\mathrm{Ba}$ & 3.8 & & 5.5 & & 3.3 & & 6.9 & & 97 & \\
\hline $\mathrm{Be}$ & 37.7 & & 58.5 & & 144.1 & & 9.3 & & 98 & \\
\hline $\mathrm{Ca}$ & 12.4 & & 14.6 & & 11.6 & & 10.6 & & 99 & \\
\hline Cd & 7.0 & & 15.0 & & 13.2 & & 0.1 & & 100 & \\
\hline $\mathrm{Cr}$ & 24.5 & & 51.0 & & 4.6 & & 3.0 & & 95 & \\
\hline $\mathrm{Fe}$ & 2.1 & & 2.9 & & 24.4 & & 13.7 & & 105 & \\
\hline $\mathrm{Mg}$ & 12.1 & & 25.4 & & 10.8 & & 1.1 & & 102 & \\
\hline $\mathrm{Na}$ & 7.8 & & 14.9 & & 6.4 & & 8.5 & & 103 & \\
\hline $\mathrm{Ni}$ & 36.5 & & 47.3 & & 22.0 & & 0.8 & & 101 & \\
\hline $\mathrm{Pb}$ & 0.0 & & 10.2 & & 0.8 & & 8.7 & & 100 & \\
\hline Sb & & & & & & & & & & 89 \\
\hline Se & & & & & & & & & & 89 \\
\hline $\mathrm{TI}$ & & & & & & & & & & 84 \\
\hline $\mathbf{U}$ & 9.6 & & 11.7 & & 13.0 & & 8.9 & & 100 & \\
\hline v & & & & & & & & & 94 & \\
\hline Zn & 16.3 & & 27.3 & & 47.3 & & 5.3 & & 93 & \\
\hline $\mathrm{Zr}$ & 9.8 & & 86.4 & & 135.0 & & 5.7 & & 104 & \\
\hline
\end{tabular}

\section{Other Analytes}

\begin{tabular}{|c|c|c|c|c|c|c|}
\hline B & & & & & 96 & \\
\hline \multicolumn{7}{|l|}{$\mathrm{Bi}$} \\
\hline Ce & 5.7 & 12.1 & & & 97 & \\
\hline \multicolumn{7}{|l|}{ Co } \\
\hline $\mathrm{Cu}$ & 44.5 & 4.7 & 1.3 & 1.8 & 105 & \\
\hline \multicolumn{7}{|l|}{ Dy } \\
\hline Eu & & 1.0 & & & & \\
\hline $\mathbf{K}$ & 0.7 & 5.8 & 18.0 & 5.6 & 101 & \\
\hline La & 0.5 & 3.6 & 4.6 & 20.0 & 103 & \\
\hline $\mathrm{Li}$ & & & & & 100 & \\
\hline $\mathrm{Mn}$ & 16.3 & 5.4 & 2.4 & 10.6 & 104 & \\
\hline Mo & & & & & 101 & \\
\hline Nd & 6.3 & 6.5 & & 23.1 & 99 & \\
\hline $\mathbf{P}$ & & & & & 97 & \\
\hline \multicolumn{7}{|l|}{$\mathrm{Pd}$} \\
\hline \multicolumn{7}{|l|}{ Rh } \\
\hline \multicolumn{7}{|l|}{$R u$} \\
\hline s & 14.1 & 14.8 & 8.5 & 6.5 & & \\
\hline Si & 9.9 & 2.9 & 6.0 & 2.0 & 103 & \\
\hline \multicolumn{7}{|l|}{ Sn } \\
\hline $\mathrm{Sr}$ & 9.8 & 14.1 & 4.2 & 9.1 & 101 & \\
\hline \multicolumn{7}{|l|}{$\mathrm{Ta}$} \\
\hline \multicolumn{7}{|l|}{$\mathrm{Te}$} \\
\hline Th & & & & & 101 & \\
\hline $\mathrm{Ti}$ & 25.6 & 2.2 & 3.7 & 8.8 & 104 & \\
\hline \multicolumn{7}{|l|}{ w } \\
\hline $\mathbf{Y}$ & 6.6 & 4.3 & & & & \\
\hline
\end{tabular}

Shaded results are outside the acceptance criteria.

$n r=$ spike concentration less than $25 \%$ of sample concentration. Matrix effects can be assessed from the serial dilution. 
PNNL-20650, Rev. 2

Battelle PNNL/RPG/Inorganic Analysis ... ICPOES Data Report

Page 4 of 4

\begin{tabular}{|c|c|c|c|c|c|c|c|c|}
\hline Criteria > & $80 \%-120 \%$ & $80 \%-120 \%$ & $80 \%-120 \%$ & $80 \%-120 \%$ & $80 \%-120 \%$ & $80 \%-120 \%$ & $\leq 10 \%$ & $\leq 10 \%$ \\
\hline$Q C \mid D>$ & \multicolumn{2}{|c|}{$\begin{array}{c}11-0421 \\
\text { MS }\end{array}$} & \multicolumn{2}{|c|}{$\begin{array}{c}11-0421+ \\
\text { AS-A }\end{array}$} & \multicolumn{2}{|c|}{$\begin{array}{c}11.0421+ \\
\text { AS-B }\end{array}$} & \multicolumn{2}{|c|}{$\begin{array}{c}11-0421 \\
5 \text {-fold } \\
\text { Serial Dil }\end{array}$} \\
\hline Analytes & \%Rec & \%Rec & $\%$ Rec & $\%$ Rec & \%Rec & \%Rec & $\%$ Diff & \%Diff \\
\hline $\mathrm{Ag}$ & 100 & & 99 & & & & & \\
\hline Al & $\mathrm{nr}$ & & 102 & & & & 1.6 & \\
\hline As & & 90 & & 97 & & & & \\
\hline $\mathrm{Ba}$ & 104 & & 101 & & & & 3.1 & \\
\hline $\mathrm{Be}$ & 105 & & 103 & & & & & \\
\hline $\mathrm{Ca}$ & $\mathrm{nr}$ & & 101 & & & & 1.8 & \\
\hline $\mathrm{Cd}$ & 90 & & 104 & & & & & \\
\hline $\mathrm{Cr}$ & 102 & & 101 & & & & 0.2 & \\
\hline $\mathrm{Fe}$ & $\mathrm{nr}$ & & 99 & & & & 2.1 & \\
\hline $\mathrm{Mg}$ & 118 & & 101 & & & & 8.3 & \\
\hline $\mathrm{Na}$ & 119 & & 100 & & & & 0.3 & \\
\hline $\mathrm{Ni}$ & 105 & & 105 & & & & 2.0 & \\
\hline $\mathrm{Pb}$ & 105 & & 100 & & & & & \\
\hline $\mathrm{Sb}$ & & 45 & & 95 & & & & \\
\hline $\mathrm{Se}$ & & 96 & & 99 & & & & \\
\hline $\mathrm{TI}$ & & 30 & & 92 & & & & \\
\hline$U$ & $\mathrm{nr}$ & & & & 98 & & 1.7 & \\
\hline $\mathrm{v}$ & 99 & & 99 & & & & & \\
\hline $\mathrm{Zn}$ & 74 & & 99 & & & & 6.8 & \\
\hline $\mathrm{Zr}$ & 244 & & 104 & & & & 3.5 & \\
\hline \multicolumn{9}{|c|}{ Other Analytes } \\
\hline B & 95 & & 104 & & & & & \\
\hline $\mathrm{Bi}$ & & & 95 & & & & & \\
\hline $\mathrm{Ce}$ & 99 & & & & 102 & & & \\
\hline Co & & & 100 & & & & & \\
\hline $\mathrm{Cu}$ & $\mathrm{nr}$ & & 104 & & & & 0.5 & \\
\hline Dy & & & & & 102 & & & \\
\hline $\mathrm{Eu}$ & & & & & 99 & & & \\
\hline$K$ & 101 & & 103 & & & & 16.5 & \\
\hline La & 103 & & & & 103 & & & \\
\hline $\mathrm{Li}$ & 109 & & 102 & & & & & \\
\hline $\mathrm{Mn}$ & 107 & & 103 & & & & 1.1 & \\
\hline Mo & 88 & & 100 & & & & & \\
\hline $\mathrm{Nd}$ & 105 & & & & 103 & & & \\
\hline$P$ & 116 & & 103 & & & & & \\
\hline $\mathrm{Pd}$ & & & & & 99 & & & \\
\hline $\mathrm{Rh}$ & & & & & 104 & & & \\
\hline $\mathrm{Ru}$ & & & & & 102 & & & \\
\hline$s$ & $\mathrm{nr}$ & & & & 102 & & & \\
\hline $\mathrm{Si}$ & 31 & & 115 & & & & 42.6 & \\
\hline Sn & & & 103 & & & & & \\
\hline $\mathrm{Sr}$ & 114 & & 104 & & & & 2.5 & \\
\hline $\mathrm{Ta}$ & & & 103 & & & & & \\
\hline Te & & & & & 101 & & & \\
\hline Th & 99 & & & & 102 & & & \\
\hline $\mathrm{Ti}$ & 92 & & 105 & & & & 13.7 & \\
\hline$w$ & & & 109 & & & & & \\
\hline$Y$ & & & 100 & & & & & \\
\hline
\end{tabular}

Shaded results are outside the acceptance criteria.

$n r=$ spike concentration less than $25 \%$ of sample concentration. Matrix effects can be assessed from the serial dilution. 
TIC/TOC Report - Furnace Oxidation Method

Project Number: Charge Code:

ASR Number:

Client:

Total Samples:
52578

H41215

8798

SK Fiskum

5

\begin{tabular}{|l|l|}
\hline RPL Sample ID & Client sample ID \\
\hline 11-0419 -C-S,-D & KW-210-A1 / TI001-A1-H,J \\
11-0420 -C-S,-D & KW-210-A4 / TI002-A4-H,J \\
11-0421 -C-S,-D & KW-210-B1 / TI002-B1-H,J \\
11-0422 -C-S,-D & KW-210-B3 / TI001-B3-H,J \\
11-0423 -C-S,-D & Purolite $^{\circledR}+$ Grafoil $^{\otimes}$ \\
\hline
\end{tabular}

\begin{tabular}{|l|l|}
\hline Analysis Procedure & $\begin{array}{l}\text { RPG-CMC-385, Rev. 2, Carbon Measured in Solids, } \\
\text { Sludges, and Liquid Matrices }\end{array}$ \\
\hline Prep Procedure & None \\
\hline Analyst & D Kallsen and C Rutherford \\
\hline Analysis Date & 6/3/2011 to 6/28/2011 \\
\hline Cal/Verify Standards & $\begin{array}{l}\text { TOC: } \text { Sigma 99.5\% Glucose CMS\#239432 } \\
\text { TIC: Mallinckrodt CMS\#239430(ICV/CCV) }\end{array}$ \\
\hline \multirow{2}{*}{ LCS Standards } & $\begin{array}{l}\text { TOC: Sigma 99.5\% Glucose CMS\#332698 (LCS) } \\
\text { TIC: JT Baker CMS\#239496 (LCS) }\end{array}$ \\
\hline Excel Data Files & TC-11-3 Fiskum/Shimsky 8798 directory (11 files) \\
\hline \multirow{2}{*}{ M\&TE Numbers } & Carbon System (WD13071-701) \\
\cline { 2 - 2 } & Balance (360-06-01-023/37050057) \\
\hline All Analysis Records & System File TC-11-3 \\
\hline
\end{tabular}

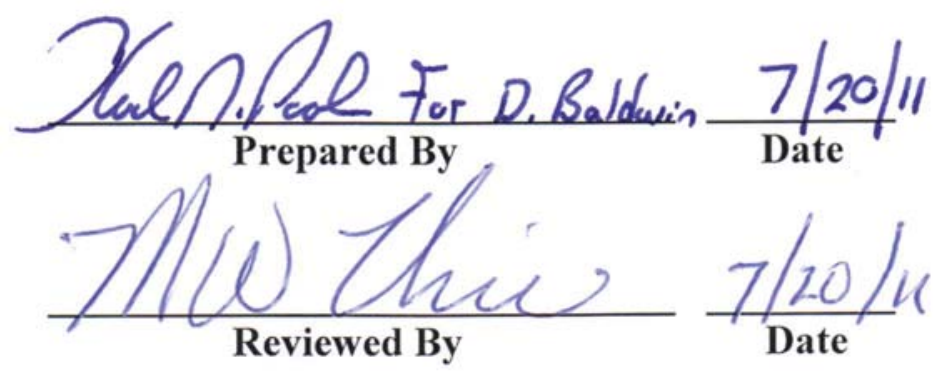




\section{TIC/TOC Report - Furnace Oxidation Method}

\section{Introduction}

The total organic carbon/total inorganic carbon (TOC/TIC) analyses of the samples submitted under Analytical Service Request (ASR) 8798 were performed by the furnace oxidation method using the "Sequential Temperatures" option described in ASO procedure, RPG-CMC-385, Rev.2, Carbon Measured in Solids, Sludges, and Liquid Matrices. Controlling documents for this work were ASO-QAP-001 and the ASR 8798 Special Instructions.

The purpose of the sequential temperatures mode is to minimize impact of possible nonhomogeneity in sample material. Therefore, instead of TOC and TIC/total carbon (TC) being run on separate samples, they are run on a single sample at sequential temperatures. The furnace is first run at the initial temperature of $600^{\circ} \mathrm{C}$ and all TOC measured, then the temperature is ramped to the final temperature of $1000^{\circ} \mathrm{C}$ and TIC is measured. TC is determined by the summation of TIC and TOC concentration values.

\section{Samples}

The core sample ID, the sample ID, RPL ID, and analysis date are shown in Table 1 . The two Purolite ${ }^{\circledR}+$ Grafoil $^{\circledR}$ mixed samples were analyzed at the beginning and end of the batches. The Special Instructions request all core samples be analyzed in at least duplicate. Because of the potential nonhomogeneous nature of these samples, all core samples were prepared and run in triplicate.

Table 1. Listing of Core Sample ID, Sample ID, RPL ID, and Analysis Date

\begin{tabular}{|l|l|c|}
\hline Sample ID & \multicolumn{1}{|c|}{ RPL ID } & $\begin{array}{c}\text { Analysis } \\
\text { date }\end{array}$ \\
\hline KW-210-A1 & $6 / 7 / 2011$ \\
\hline TI001-A1-H & $11-0419-C-S$ & $6 / 8 / 2011$ \\
\hline TI001-A1-J & $11-0419-C-D$ & $6 / 9 / 2011$ \\
\hline KW-210-A4 \\
\hline TI002-A4-H & $11-0420-C-S$ & $6 / 14 / 2011$ \\
\hline TI002-A4-J & $11-0420-C-D$ & $6 / 15 / 2011$ \\
\hline KW-210-B1 \\
\hline TI002-B1-H & $11-0421-C-S$ & $6 / 16 / 2011$ \\
\hline TI002-B1-J & $11-0421-C-D$ & $6 / 21 / 2011$ \\
\hline KW-210-B3 & $6 / 23 / 2011$ \\
\hline TI001-B3-H & $11-0422-C-S$ & $6 / 3 / 2011$ \\
\hline TI001-B3-J & $11-0422-C-D$ & $6 / 28 / 2011$ \\
\hline
\end{tabular}




\section{TIC/TOC Report - Furnace Oxidation Method}

\section{TOC/TIC Preparation}

The samples were dried prior to sub-sampling and weighing. Drying was conducted by heating to $105^{\circ} \mathrm{C}$ for several hours or overnight, until constant dry mass was obtained and documented. The samples were sub-sampled at the workstation to provide (for each sample) a sample and duplicate, then each sample or duplicate was measured in triplicate. Two of the samples per analytical batch were selected for sub-sampling again to support two analytical spikes (AS). These were 11-0419-C-S (TI001-A1-H) and 11-0422-C-D (TI001-B3-J). Sub-sampling at the workstation required care to provide representative subsamples to optimize chances to meet precision measurement requirements; however, it is understood that sub-sampling was conducted on a best-effort basis, as per the ASR special instructions.

The samples were analyzed per RPG-CMC-385, Rev. 2, Carbon Measured in Solids, Sludge, and Liquid Matrices, sequential temperatures mode. The TOC was determined from combustion at $600^{\circ} \mathrm{C}$; the same sample material was then subjected to $1000^{\circ} \mathrm{C}$ to measure all other (inorganic) C.

The ASO analyst prepared each sample in triplicate, as well as preparing the laboratory control sample (LCS) and blank spike (BS) and analytical spike (AS) QC samples. The AS and LCS/BS samples included the carbon standards: glucose and $\mathrm{CaCO}_{3}$.

A sample of dry Purolite ${ }^{\circledR}$ NRW37 resin beads combined with Grafoil ${ }^{\circledR}$ also was prepared by the ASO analyst to be run with the sample batch in duplicate at the beginning of the batches and the end of the batches - this mixed material was processed as sample 11-0423.

The TOC and TIC concentration is reported here as $\mu \mathrm{g} \mathrm{C} / \mathrm{g}_{\mathrm{dry}}$, where $\mathrm{g}_{\mathrm{dry}}$ was determined as measured at the workstation after the above-described drying process. The solids drying information is reported in Table 13.

\section{Discussion of Results}

Table 2 provides a summary of all TOC/TIC results, with a summary of attained MDL, precision, and QC sample results reported. All sample results are corrected for average percent recovery of system calibration verification check standards and are corrected for contribution from the blank, as per procedure.

The following approach was used in deriving the average result and precision for results presented in Table 2.

$\mathrm{U}$ flagged $(<\mathrm{MDL})$ data is not to be included in calculations of averaged data. $\mathrm{J}$ flagged (between MDL and $\mathrm{EQL}$; with $\mathrm{EQL}=5 * \mathrm{MDL}$ ) data will be used to calculate averaged sample results (unless there is a reason not to have confidence in the J-flagged value).

RPDs can be calculated and reported when there are two J flagged values or one $\mathrm{J}$ flagged value and one non-flagged value. 


\section{TIC/TOC Report - Furnace Oxidation Method}

Tables 3-12 provide the complete TOC/TIC individual results and average results, with sample weights, MDL, precision, QC sample results, and QC data flags reported.

Three method QC sample failures were observed in this work. 1) One procedural requirement is that the $1^{\text {st }}$ and $2^{\text {nd }}$ ICBs agree within 5ug. This QC failure was seen on one analysis (batch 6) shown on Table 8. 2) A procedural requirement is that the $1^{\text {st }}$ and $2^{\text {nd }}$ ICVs agree within $5 \%$. This QC failure was seen on 5 of the 10 batches, occurring for both TOC and TIC runs. The likely cause of this QC failure was early in the day operation. This QC failure caused no negative effect on results since all performance check standard recoveries met acceptance criteria. 3) On Table 4 (batch 2), the AS for TOC failed at $132 \%$ recovery. The DQO requirement is for $75 \%-125 \%$ recovery. The ICB/CCBs and ICV/CCVs for this batch all met requirements. The later AS for TOC (Table 11, batch 9) came in also slightly high at $115 \%$ recovery, but met the DQO requirement. There is currently no obvious reason for this AS failure. These method QC sample failures are documented in Occurrence Report 52578-07-1911.

Several instances of failure to meet MDL requirements were seen. The ASR Special Instructions requirement for MDL was $200 \mathrm{ugC/g}$. The $\mathrm{MDL}$ value for each sample is directly correlated to the sample mass used for the measurement and the instrument detection limit (IDL) for the batch. The IDL for the batch is determined using the blank data for each batch. The variation in IDL from batch to batch and the ALARA-based need to keep the sample sizes small resulted in some of the MDLs being far greater than the $200 \mu \mathrm{gC} / \mathrm{g}$ level. The MDL limit was exceeded on 34 sample analyses spread over all 10 batches. In most of these, the sample result was well above MDL, and those samples in which the result was near the MDL all occurred on TIC analyses. There is likely little impact on data quality.

Recoveries for the special materials analyzed, Purolite ${ }^{\circledR}$ and Grafoil ${ }^{\circledR}$, ranged from 91 to $108 \%$ recovery for carbon, for both TOC and TIC, using the sequential temperatures mode of operation. The percent recovery of carbon is based on theoretical percent carbon for the pure materials. The special materials were analyzed in duplicate at both the beginning of this work, in batch 1 and at the end of this work, in batch 10. The precision of measuring these materials was estimated for Purolite ${ }^{\circledR}$ with RPDs of $4-6 \%$ for TOC and for Grafoil ${ }^{\circledR}$ with RPDs of $1-3 \%$ for TIC.

Table 13 shows the sample drying weight data and percent water weight loss for each later step in drying. Each analyzed TOC/TIC sample was then sub-sampled respectively from each of these dried lots. All samples were dried to constant mass at $\sim 105^{\circ} \mathrm{C}$ to assure that the sample mass did not contain water; extra water content would contribute to low bias for TOC/TIC results. 


\section{TIC/TOC Report - Furnace Oxidation Method}

Table 2. Summary of Sample Results with MDL, Precision, and QC

\begin{tabular}{|c|c|c|c|c|c|c|c|c|c|c|}
\hline Sample ID & RPL ID & $\begin{array}{c}\text { Sample } \\
\text { Weight } \\
\text { (g) }\end{array}$ & $\begin{array}{c}\text { TOC } \\
\text { MDL } \\
(\mu \mathrm{gC} / \mathrm{g})\end{array}$ & $\begin{array}{c}\text { Average } \\
\text { TOC } \\
(\mathrm{n}=3) \\
(\mu \mathrm{gC} / \mathrm{g})\end{array}$ & $\begin{array}{l}\text { RSD/ } \\
\text { RPD } \\
(\%)\end{array}$ & $\begin{array}{c}\text { TIC } \\
\text { MDL } \\
\text { (ugC/g) }\end{array}$ & $\begin{array}{c}\text { Average } \\
\text { TIC } \\
(\mathrm{n}=3) \\
(\mu \mathrm{gC} / \mathrm{g})\end{array}$ & $\begin{array}{l}\text { RSD/ } \\
\text { RPD } \\
(\%)\end{array}$ & $\begin{array}{c}\text { QC } \\
\text { BS/LCS } \\
\text { TOC/TIC } \\
(\% \text { Rec })\end{array}$ & $\begin{array}{c}\text { QC } \\
\text { AS } \\
\text { TOC/TIC } \\
(\% \text { Rec })\end{array}$ \\
\hline \multicolumn{11}{|l|}{ KW-210-A1 } \\
\hline TI001-Al-H & 11- 0419-C-S & $\begin{array}{l}0.0326 \\
0.0165 \\
0.0058\end{array}$ & $\begin{array}{c}40 \\
80 \\
\mathbf{2 3 0} \\
\end{array}$ & 137,000 & 27 & $\begin{array}{c}59 \\
120 \\
340\end{array}$ & 4,110 & 83 & $98 / 95$ & $132 / 97$ \\
\hline Tl001-Al-J & 11-0419-C-D & $\begin{array}{l}0.0184 \\
0.0241 \\
0.0097 \\
\end{array}$ & $\begin{array}{c}62 \\
47 \\
120 \\
\end{array}$ & 140,000 & 35 & $\begin{array}{c}110 \\
84 \\
210\end{array}$ & 1,080 & 81 & $101 / 92$ & - \\
\hline \multicolumn{11}{|l|}{ KW-210-A4 } \\
\hline T1002-A4-H & 11-0420-C-S & $\begin{array}{l}0.0189 \\
0.0340 \\
0.0577 \\
\end{array}$ & $\begin{array}{l}84 \\
47 \\
27\end{array}$ & 69,300 & 39 & $\begin{array}{l}200 \\
110 \\
64\end{array}$ & 2,020 & 85 & $103 / 92$ & - \\
\hline T1002-A4-J & 11-0420-C-D & $\begin{array}{l}0.0200 \\
0.0648 \\
0.0362 \\
\end{array}$ & $\begin{array}{l}150 \\
47 \\
84 \\
\end{array}$ & 87,700 & 38 & $\begin{array}{c}\mathbf{2 5 0} \\
77 \\
140 \\
\end{array}$ & 596 & 18 & $93 / 92$ & - \\
\hline \multicolumn{11}{|l|}{ KW-210-B1 } \\
\hline TI002-B1-H & 11-0421-C-S & $\begin{array}{l}0.0132 \\
0.0035 \\
0.0188\end{array}$ & $\begin{array}{l}1500 \\
5600 \\
1100\end{array}$ & 514,000 & 1 & $\begin{array}{c}660 \\
2500 \\
460\end{array}$ & 2,790 & $\mathrm{n} / \mathrm{a}$ & $115 / 90$ &.- \\
\hline T1002-B1-J & 11-0421-C-D & $\begin{array}{l}0.0046 \\
0.0101 \\
0.0148\end{array}$ & $\begin{array}{c}2700 \\
1200 \\
830\end{array}$ & 481,000 & 8 & $\begin{array}{l}3600 \\
1600 \\
1100 \\
\end{array}$ & 1,800 & $\mathrm{n} / \mathrm{a}$ & $106 / 80$ & - \\
\hline \multicolumn{11}{|l|}{ KW-210-B3 } \\
\hline TI001-B3-H & 11-0422-C-S & $\begin{array}{l}0.0096 \\
0.0261 \\
0.0053 \\
\end{array}$ & $\begin{array}{l}540 \\
200 \\
980\end{array}$ & 395,000 & 15 & $\begin{array}{l}470 \\
170 \\
840 \\
\end{array}$ & 833 & 8 & 99/97 & - \\
\hline TI001-B3-J & 11-0422-C-D & $\begin{array}{l}0.0106 \\
0.0219 \\
0.0141 \\
\end{array}$ & $\begin{array}{l}350 \\
170 \\
260 \\
\end{array}$ & 367,000 & 3 & $\begin{array}{l}420 \\
200 \\
310 \\
\end{array}$ & 3,650 & 38 & $110 / 88$ & $115 / 87$ \\
\hline \multicolumn{3}{|c|}{ Client Control Samples } & $\begin{array}{c}\text { TOC } \\
\text { MDL } \\
(\mu \mathrm{gC} / \mathrm{g})\end{array}$ & $\begin{array}{c}\text { Average } \\
\text { TOC } \\
(\mathrm{n}=2) \\
(\mu \mathrm{gC} / \mathrm{g})\end{array}$ & $\begin{array}{l}\text { RPD } \\
(\%)\end{array}$ & $\begin{array}{c}\text { TIC } \\
\text { MDL } \\
\text { (ugC/g) }\end{array}$ & $\begin{array}{c}\text { Average } \\
\text { TIC } \\
(\mathrm{n}=2) \\
(\mu \mathrm{gC} / \mathrm{g})\end{array}$ & $\begin{array}{l}\text { RPD } \\
(\%)\end{array}$ & & \\
\hline$\underset{\text { Grafoil }^{\infty}}{\text { Purolite }^{\infty}+}$ & 11-0423-C-S & (b) & $\begin{array}{l}590 \\
320 \\
\end{array}$ & $\begin{array}{c}624,000 \\
(\% \operatorname{Rec}= \\
91.0 \text { and } \\
94.7)\end{array}$ & 4 & $\begin{array}{l}610 \\
650\end{array}$ & $\begin{array}{l}1,060,000 \\
(\% \text { Rec }= \\
106.9 \text { and } \\
105.6)^{(d)}\end{array}$ & 1 & - & - \\
\hline $\begin{array}{c}\text { Purolite }^{x}+ \\
\text { Grafoil }^{\infty}\end{array}$ & 11-0423-C-D & (c) & $\begin{array}{l}640 \\
610\end{array}$ & $\begin{array}{c}707,000 \\
(\% \operatorname{Rec}= \\
108.0 \text { and } \\
102.3)\end{array}$ & 6 & $\begin{array}{l}180 \\
330\end{array}$ & $\begin{array}{l}967,000 \\
(\% \text { Rec }= \\
95.7 \text { and } \\
98.6)^{(d)}\end{array}$ & 3 & - & - \\
\hline
\end{tabular}

(a) $\mathrm{n} / \mathrm{a}=$ one or more results were less than MDL and if only one result was affected, then an RPD and not an RSD was calculated. If two results were affected, then the RPD cannot be calculated and $\mathrm{n} / \mathrm{a}$ was reported.

(b) Purolite ${ }^{8}$ sample weight $=0.0029 \mathrm{~g}$ and $0.0053 \mathrm{~g}$; Grafoil ${ }^{\otimes}$ sample weights $=0.0046 \mathrm{~g}$ and $0.0044 \mathrm{~g}$.

(c) Purolite ${ }^{\otimes}$ sample weight $=0.0044 \mathrm{~g}$ and $0.0047 \mathrm{~g}$; Grafoil ${ }^{\otimes}$ sample weights $=0.0080 \mathrm{~g}$ and $0.0044 \mathrm{~g}$.

(d) \%Rec based on $67.2 \%$ carbon in Purolite ${ }^{\otimes}$ and $99.5 \%$ carbon in Grafoil ${ }^{\otimes}$. MDLs shown BOLD are above the requested MDL level in the Special Instructions.

\section{Quality Control Discussion}

The performance verification standards for TOC analysis are solid pure chemicals from Sigma and VWR ( $\alpha$-D-glucose for TOC) and from Mallinckrodt and JT Baker (calcium carbonate for TIC). The identification of the standards and their Chemical Management System (CMS) numbers are included on the raw data benchsheets for traceability. 
PNNL-20650, Rev. 2

\section{TIC/TOC Report - Furnace Oxidation Method}

The QC samples for the method included calibration blanks, calibration verification check samples, sample duplicates (laboratory) and/or triplicates, laboratory control sample/blank spike (LCS/BS), and analytical spike (AS). The ASR indicates that the analyses are to be performed with QC per ASO-QAP-001 and the ASR 8798 Special Instructions.

\section{Analysis QC}

Calibration Check Standards: The calibration of the coulometer analysis system was checked by analysis of calibration verification check standards analyzed at the beginning and end of the analysis run. The average recovery from the calibration check standards is applied as a correction factor to obtain the final result for the samples. The average recovery for all batches for TOC ranged from $99.9 \%$ to $107.2 \%$ recovery, and for TIC the average recovery ranged from $94.8 \%$ to $100.7 \%$; within the method DQO of 90 to $110 \%$ for the initial calibration verification (ICV) sample and 85 to $115 \%$ for the continuing calibration verification $(\mathrm{CCV})$ samples, meeting the $\mathrm{DQO}$ criteria.

Laboratory Control Sample/Blank Spike: An LCS/BS was analyzed in each of the eight core sample daily batches, not including the two special materials batches, from 6/7/2011 to $6 / 23 / 2011$, for a total of $8 \mathrm{LCS} / \mathrm{BS}$ samples. The $\%$ recovery for TOC ranged from $93.1 \%$ to $115.4 \%$ recovery, and for TIC ranged from $80.2 \%$ to $96.7 \%$ recovery. The LCS/BS recoveries are awithin acceptance criterion of $75 \%$ to $125 \%$.

Duplicates/Triplicates: Precision of the carbon measurements is demonstrated by calculating the relative percent difference (RPD) between sample and duplicate, or relative standard deviation (RSD) between sample, duplicate, and triplicate. No duplicates were provided by the client, so laboratory-prepared duplicates and triplicates were prepared and analyzed. Each core sample was analyzed in triplicate, due to potential homogeneity concerns, while the special materials of Purolite ${ }^{\circledR}$ and Grafoil ${ }^{\circledR}$ were analyzed in duplicate. Precision in each case was determined based on results for all three replicates for the core samples or duplicates for the special materials.

For the special materials Purolite ${ }^{\circledR}$ and Grafoil ${ }^{\circledR}$, the RPD met the DQO of $<20 \%$ RPD for both TOC and TIC, for all samples. The TOC RPD values ranged from $4 \%$ to $6 \%$ and the TIC RPD ranged from $1 \%$ to $3 \%$.

The RSD values for TOC ranged from $1 \%$ to $39 \%$. The RPD/RSD for TIC were $\mathrm{n} / \mathrm{a}$ for two batches, and ranged from $8-85 \% \mathrm{RPD} / \mathrm{RSD}$ in the remaining batches. The RPD/RSD exceeding acceptance limits are documented in Occurrence Report 52578-07-19-11.

Analytical Spike: The accuracy of the carbon measurements can be estimated by the recovery from the AS. An analytical spike was prepared from solid TIC standard and solid TOC standard, and was used as analytical spikes on two samples: batch 2: 11-0419-C-S (TI001$\mathrm{A} 1-\mathrm{H})$ and batch 9: 11-0422 (TI001-B3-J). The AS recovery was $131.8 \%$ and $115.4 \%$ recovery for TOC and was $97.1 \%$ and $87.5 \%$ recovery for TIC. Of the four recovery values, three met the acceptance criteria of $75 \%$ to $125 \%$, while one value for TOC, for TI001-A1-H, failed the acceptance criteria.

\section{Deviation from Procedure:}


PNNL-20650, Rev. 2

\section{TIC/TOC Report - Furnace Oxidation Method}

None.

\section{General Comments}

1) Routine precision and accuracy are typically $\pm 15 \%$ or better for non-complex samples that are free of interferences.

2) The estimated quantitation limit (EQL) is defined as 5 times the MDL. Results $<5 x M D L$ have higher uncertainties, and RPDs (or RSDs, if applicable) are not calculated if the results are $<5 \mathrm{xMDL}$.

3) For TOC and TIC, the analysis IDL, in $\mu \mathrm{gC}$, is based on the standard deviation calculated from the number $(n)$ of system blanks analyzed with the batch of samples, with the standard deviation multiplied by the Student's t values for $n-1$ degrees of freedom to establish the daily analysis. The MDL, in $\mu \mathrm{gC} / \mathrm{g}$, is sample-size dependent and based on the IDL and sample weight.

\section{Spreadsheet Attachments:}

1) 8798 Table 3

2) 8798 Table 4

3) 8798 Table 5

4) 8798 Table 6

5) 8798 Table 7

6) 8798 Table 8

7) 8798 Table 9

8) 8798 Table 10

9) 8798 Table 11

10) 8798 Table 12

11) 8798 Solids Drying Table 13 
PNNL-20650, Rev. 2

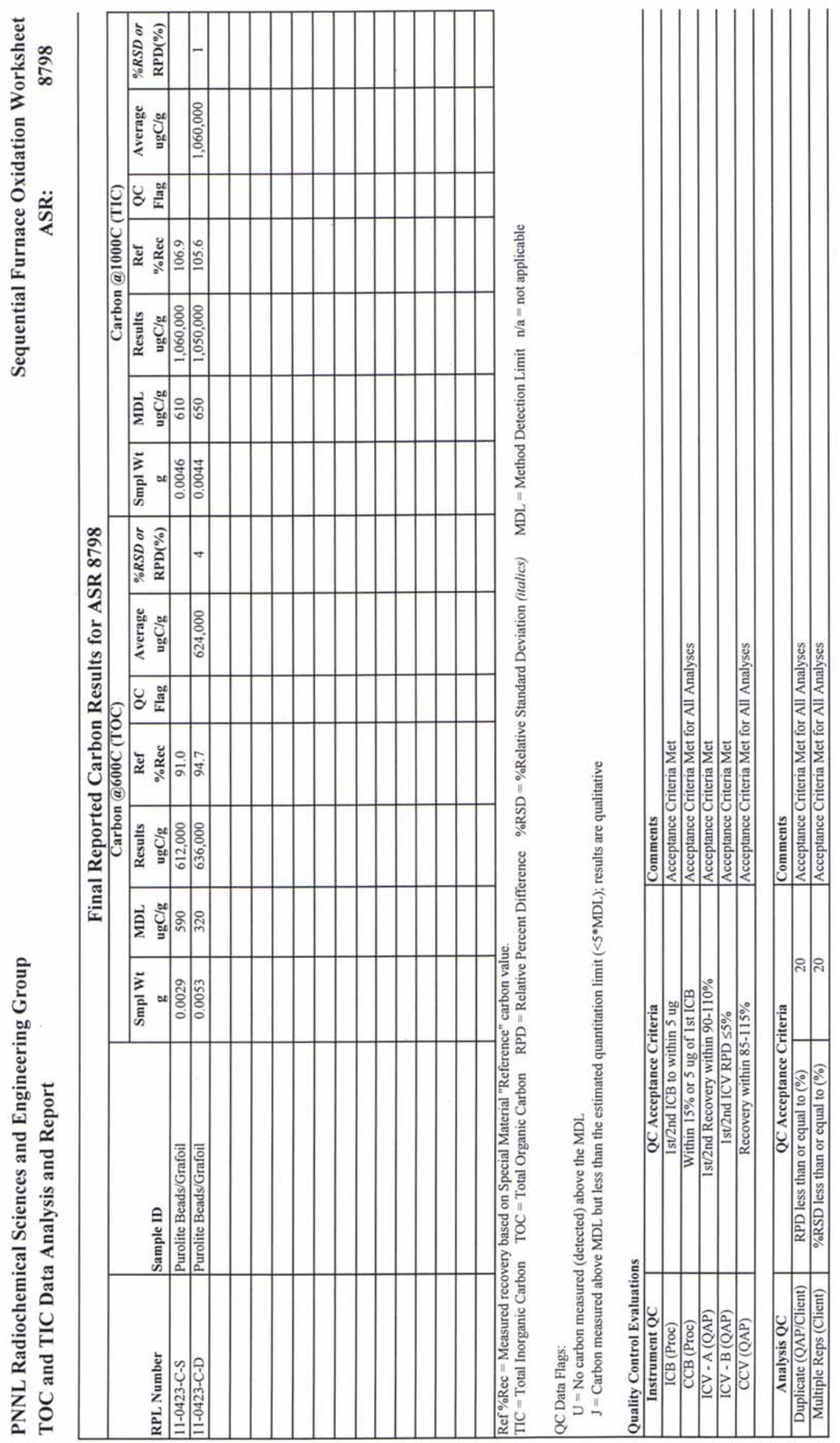

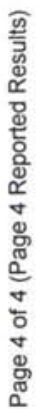

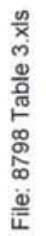

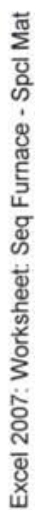


PNNL-20650, Rev. 2

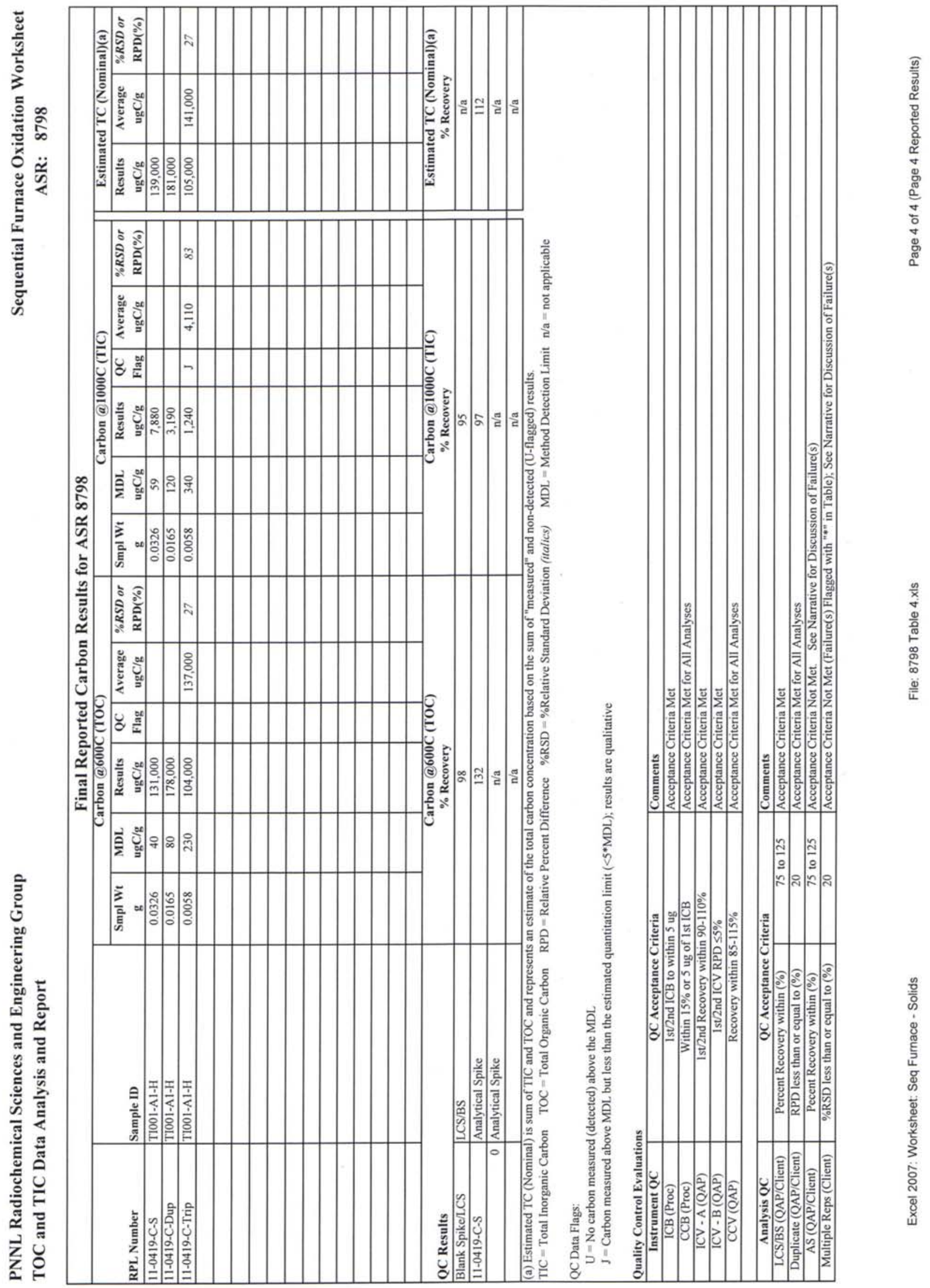


PNNL-20650, Rev. 2

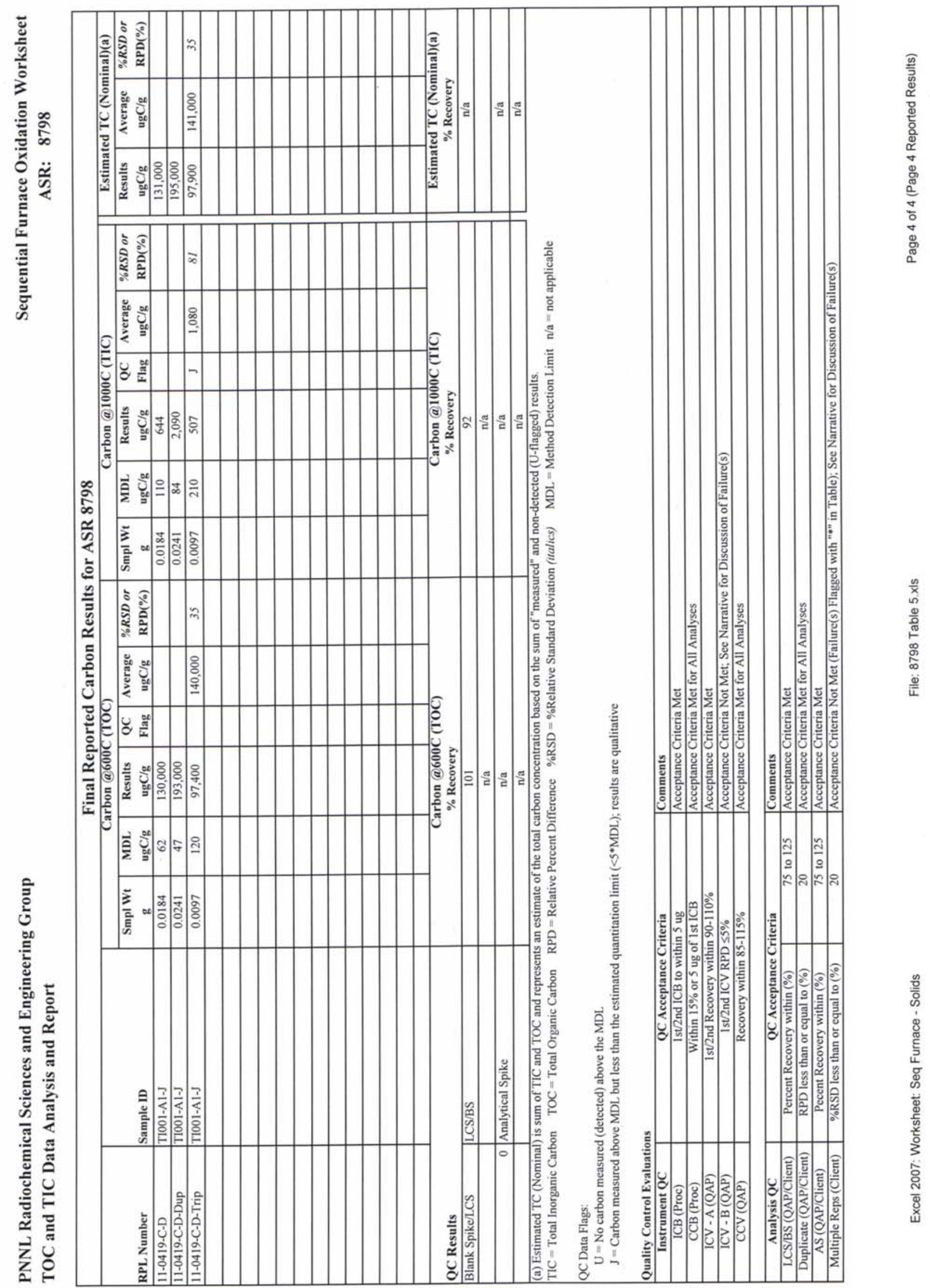


PNNL-20650, Rev. 2

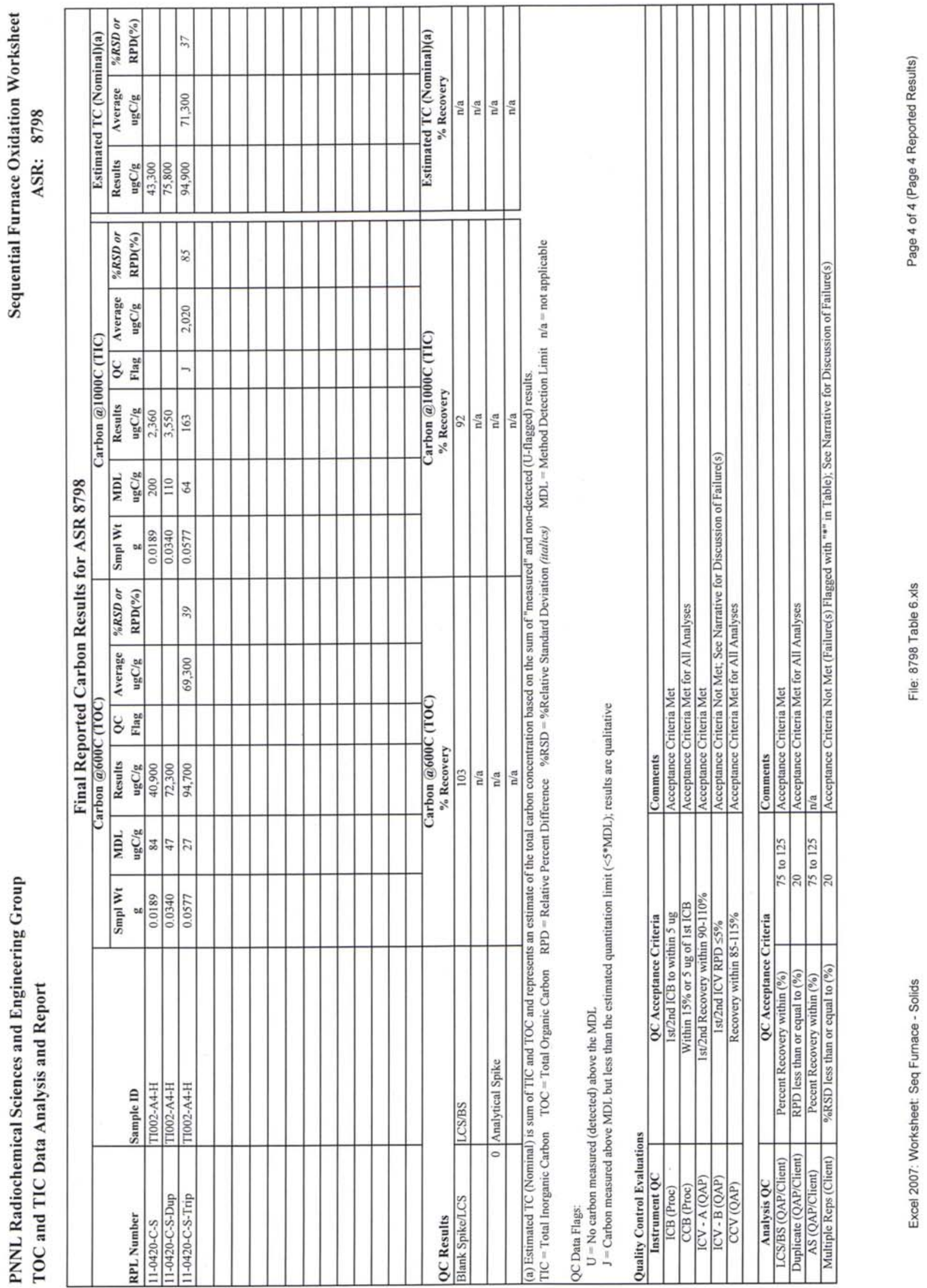


PNNL-20650, Rev. 2

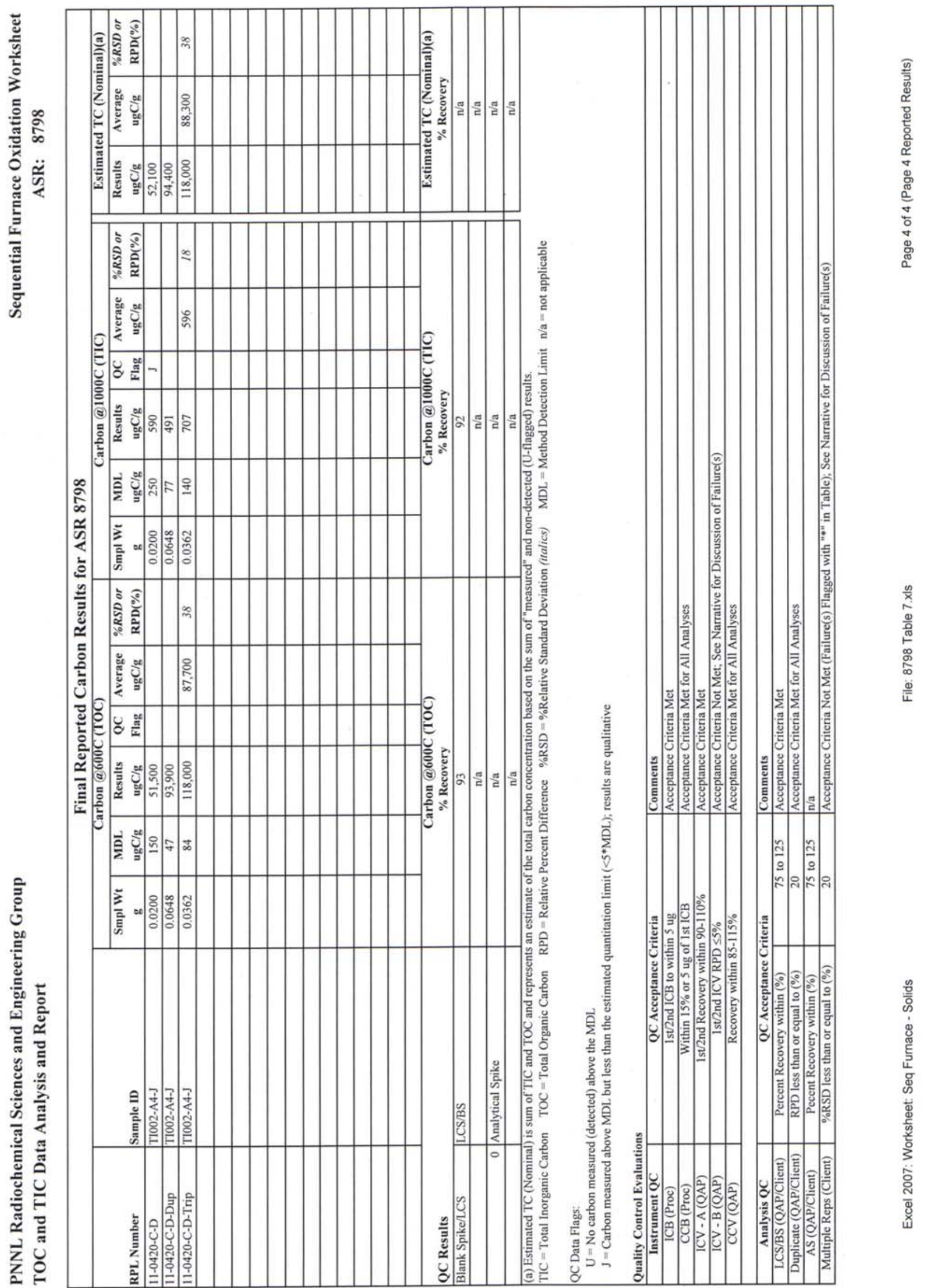


PNNL-20650, Rev. 2

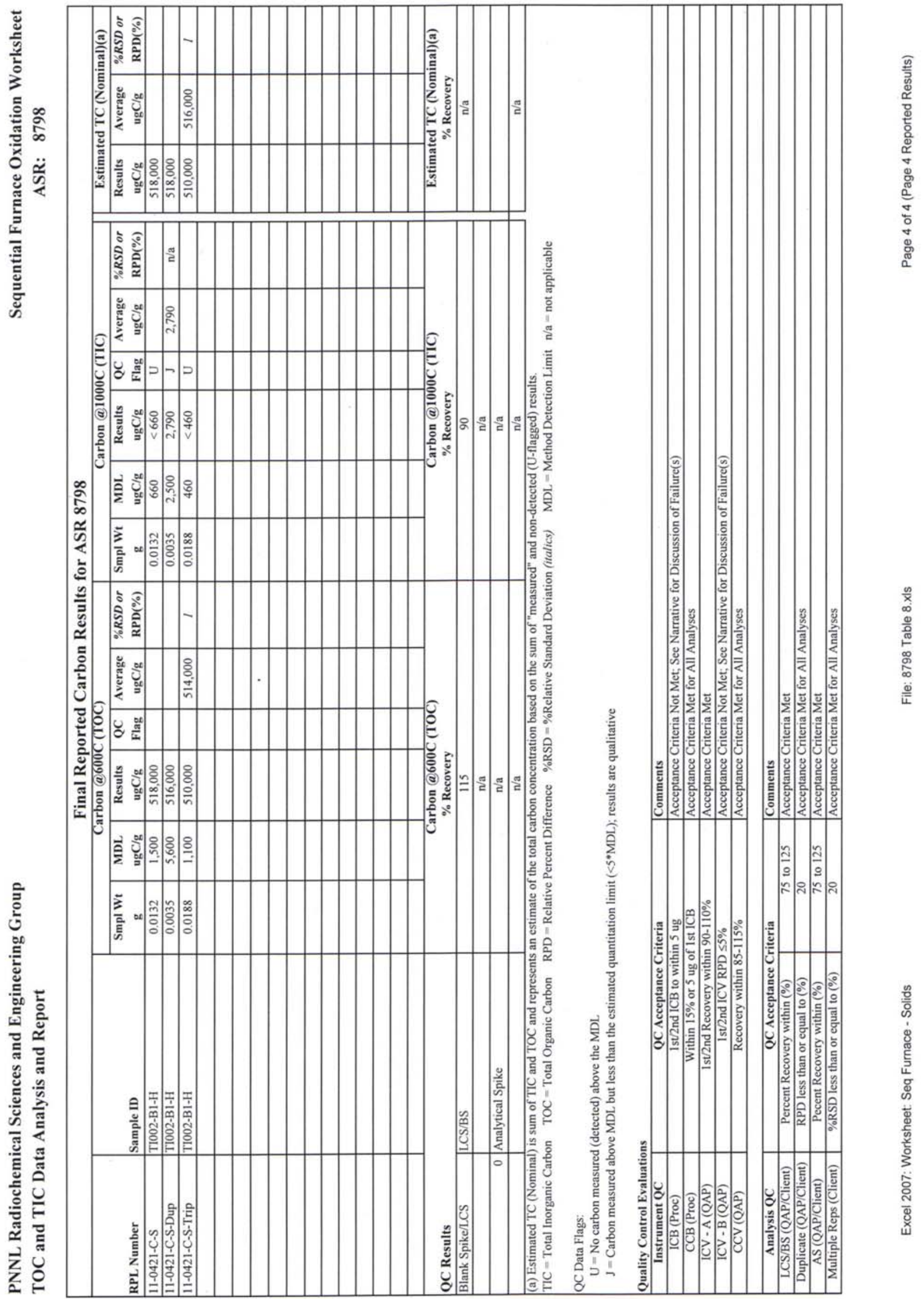


PNNL-20650, Rev. 2

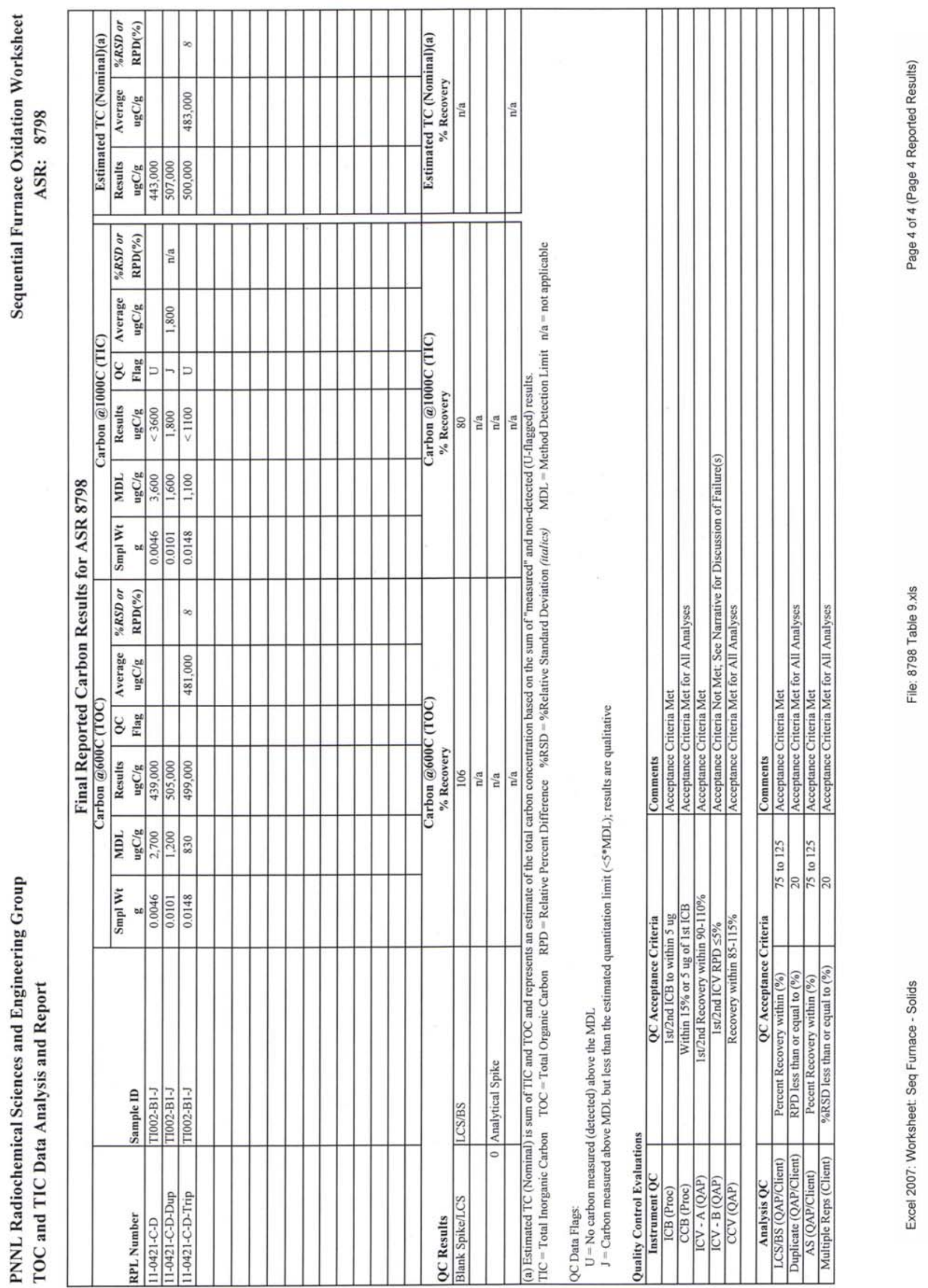




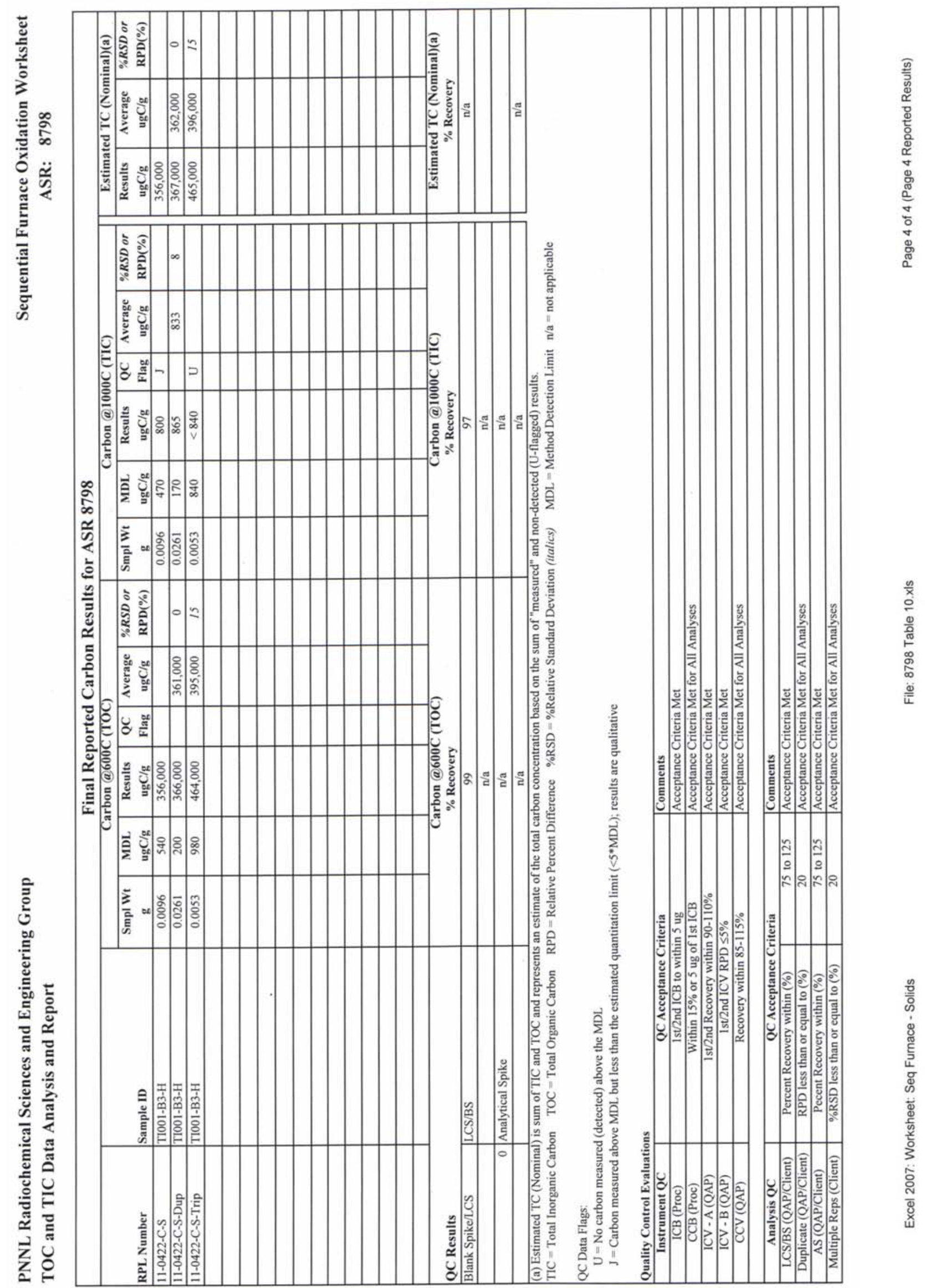


PNNL-20650, Rev. 2

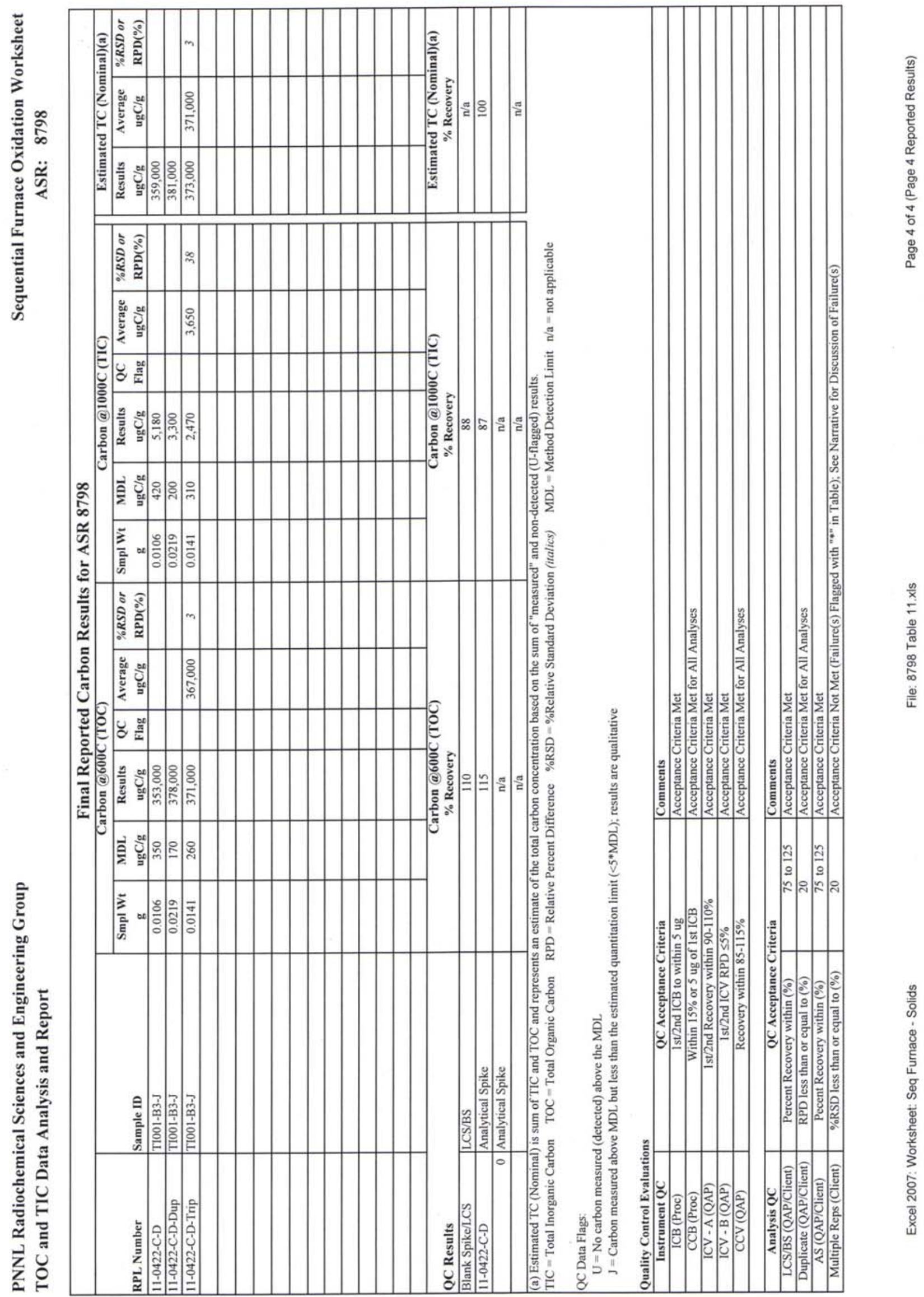


PNNL-20650, Rev. 2

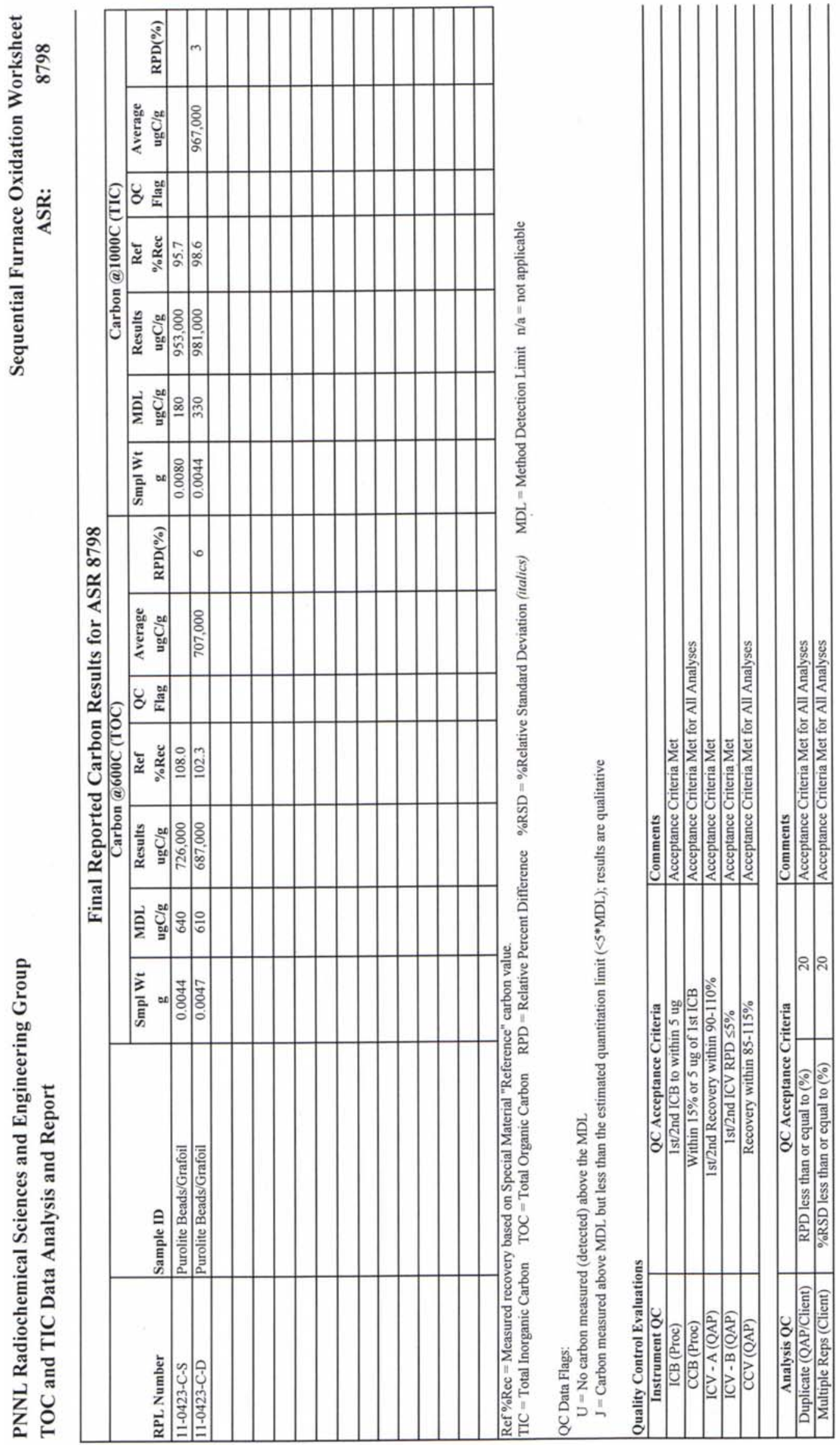

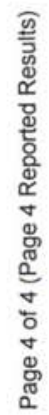

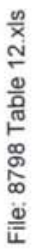

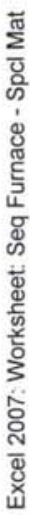


PNNL-20650, Rev. 2

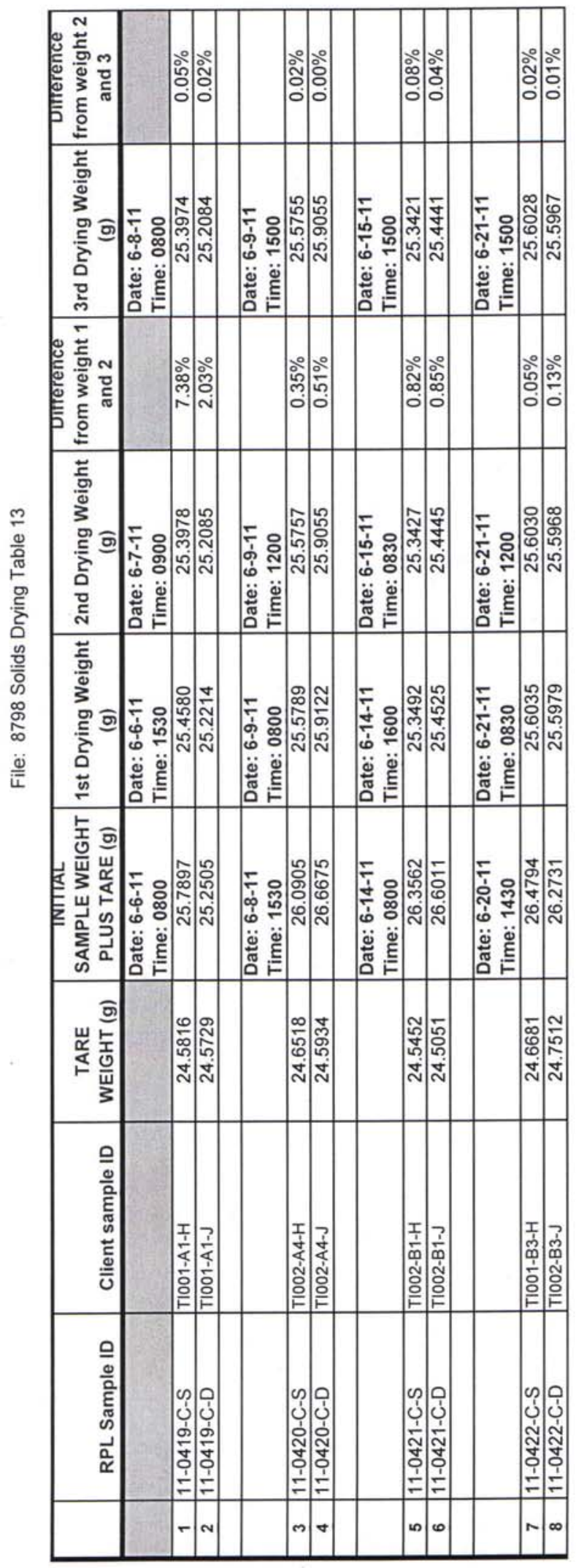


PNNL-20650, Rev. 2

Battelle PNNL/RPL/ASO Radiochemistry Analysis Report

P.O. Box 999, 902 Battelle Blvd., Richland, Washington 99352

\section{Gamma Energy Analysis (GEA)}

$\begin{array}{ll}\text { Project / WP\#: } & 52578 / \mathrm{H} 41216 \\ \text { ASR\#: } & 8798.00 \\ \text { Client: } & \text { SK Fiskum } \\ \text { Total Samples: } & 9\end{array}$

\begin{tabular}{|c|c|}
\hline RPL ID & Client Sample ID \\
\hline $11-0419-129-\mathrm{S},-\mathrm{D}$ & TI001-A1-B, -C \\
\hline $11-0420-129-\mathrm{S},-\mathrm{D}$ & TI002-A4-B, -C \\
\hline $11-0421-129-\mathrm{S},-\mathrm{D},-\mathrm{MS}$ & TI002-B1-B, -C, -D \\
\hline $11-0422-129-\mathrm{S},-\mathrm{D}$ & TI001-B3-B, -C \\
\hline
\end{tabular}

\begin{tabular}{|c|c|}
\hline Analysis Type: & GEA- for all positively measured or non-detected isotopes of interest. \\
\hline \multirow{5}{*}{$\begin{array}{l}\text { Sample Processing Prior to Radiochemical } \\
\text { Processing/Analysis }\end{array}$} & $\square$ None \\
\hline & $\begin{array}{l}\bigotimes \text { Digested as per PNL-ALO-129, Rev.0 HNO }{ }_{3} \text {-HCl Acid Extraction of } \\
\text { Solids Using a Dry Block Heater, Analyst: N. Green }(03 / 11 / 2011)\end{array}$ \\
\hline & $\begin{array}{l}\square \text { Fusion as per PNL-ALO-115, Rev. 1, Solubilization of Metals from } \\
\text { Solids Using a } \mathrm{KOH}_{-} \mathrm{KNO}_{3} \text { Fusion }\end{array}$ \\
\hline & $\square$ Other: \\
\hline & $\begin{array}{l}\text { Preparation may also involve attaining a GEA geometry that is compatible } \\
\text { with the calibration geometry. }\end{array}$ \\
\hline Analysis Procedure: & $\begin{array}{l}\text { RPG-CMC-450, Rev. 1, Gamma Energy Analysis (GEA) and Low-Energy } \\
\text { Photon Spectrometry (LEPS) }\end{array}$ \\
\hline Reference Date: & *January 11,2011@15:14:41 p.m. \\
\hline Analysis Date or Date Range: & March 14-22, 2011 \\
\hline Technician/Analyst: & T Trang-Le \\
\hline Rad Chem Electronic Data File: & 11-0419_Fiskum.xls \\
\hline ASO Project 98620 File: & $\begin{array}{l}\text { File Plan } 5871, T 4.4 \text { Technical (Radiochemistry), Gamma Calibration, } \\
\text { daily checks, and maintenance records; and T3 standard certificates and } \\
\text { preparation. Also, balance calibration and Performance check records. }\end{array}$ \\
\hline M\&TE Number(s): & $\begin{array}{l}\text { Detectors } C, D, E, G, H, K, L, N, T \text { ( } M \& T E \text { unique identifiers in attached } \\
\text { supporting documents) }\end{array}$ \\
\hline
\end{tabular}
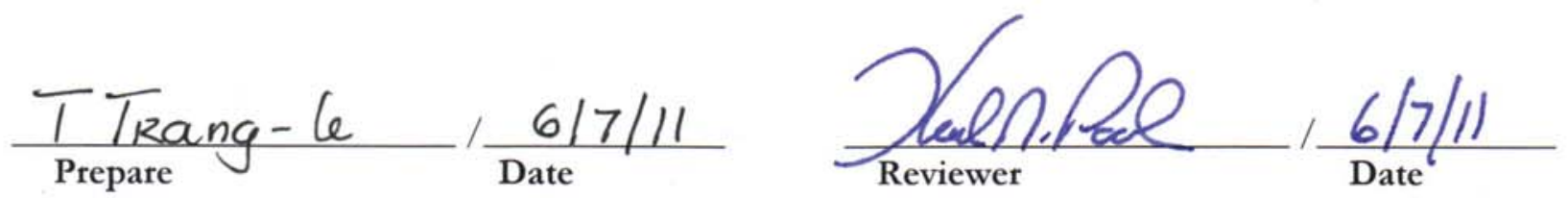

* Reference date used is the same date and time used for samples submitted under ASR 8725 as directed by S. Fiskum. 
PNNL-20650, Rev. 2

Battelle PNNL/RPL/ASO Radiochemistry Analysis Report

\section{SAMPLE RESULTS}

Activities for all gamma emitters detected in these samples are presented in an attached Excel spreadsheet for 8798.00 . All sludge data are reported in units of $\mu \mathrm{Ci} / \mathrm{g}_{\text {dry }}$ and all residual solids are reported as $\mu \mathrm{Ci} / \mathrm{g}_{\text {residual }}$ with estimates of the total propagated uncertainty reported at the 1-sigma uncertainty level. The K Basin ASR specifies that the residual solids GEA analytes are to be reported as $\mu \mathrm{Ci} / \mathrm{g}_{\text {residual, }}$ where $\mathrm{g}$ residual is the mass of residual solids that did not dissolve during acid digestion.

ASO Project File, ASR-8798 has been created for this report including all appropriate supporting records which may include the Pipette Performance Verification form, Standard Certificates, Laboratory Bench Record, Shielded Analytical Laboratory Bench Sheet, and Gamma Energy Analysis printouts. Detector calibration records, control charts and balance calibration records can be found in the ASO Records.

\section{Sample Preparation, Separation, Mounting and Counting Methods}

K-Basin dried solids samples were prepared in the shielded analytical lab (SAL) hot cells by the Project and provided to the Analytical Support Operations (ASO) under Analytical Service Request (ASR) 8798 for chemical and radio-analytical analyses.

ASO received each sample in a Teflon Oak Ridge centrifuge tube, dried the contents of each centrifuge tube to a constant weight @ $105^{\circ} \mathrm{C}$, and then leached the dried solids with hot dilute nitric and hydrochloric acids per procedure PNL-ALO-129. The leachate solution was used for radioanalytical analyses and ICP metals analysis; only GEA data are included in this report. At the completion of the acid digestion, residual unsolubilized material remained. The residual solids were dried @ $105^{\circ} \mathrm{C}$ to constant weight and the dried residuals were transferred to clean $22 \mathrm{~mL}$ glass scintillation vials for load out and GEA analyses. The residual solids do have measurable activity and those results are included in this report.

Batch quality control (QC) samples were prepared with the digestion of the initial dried solids and includes duplicates of each sample and an acid digestion blank. The quality control (QC) sample results for each of the isotopes measured above background have been evaluated and are discussed below. A summary of the GEA analysis results, including QC sample performance, is given in the attached Data Report.

The following is a list of QC sample results relative to both the $\mathrm{K}$ Basin data quality objectives and the ASO Quality Assurance (QA) Plan (ASO-QAP-001, Rev. 7); in many cases the DQOs are the same. In cases where there are differences in the DQOs, the K Basin DQO takes precedence for data reporting.

\section{QUALITY CONTROL RESULTS}

Tracer:

Tracers are not used for ASO GEA methods.

Process Blank (PB):

An acid digestion blank was prepared with the preparation of the sludge samples in the SAL, but not for the preparation of the residual solids in the SAL.

ASO-QAP-001 requires that the activities of the gamma emitting isotopes of interest measured in the PB to be within the acceptance criteria of less than $5 \%$ of the sample isotope concentration or 1 less than sample minimum detectable activity (MDA) - (See comments section). Cs-137 was 


\section{Battelle PNNL/RPL/ASO Radiochemistry Analysis Report}

detected in the blank at a level less than 500,000x the level present in the samples. For isotopes of interest that were not detected in the PB and samples, the PB MDC was less than the MDC measured in the samples. The isotopes of interest that were above the MDC in the samples are all much greater than $5 \%$ of the activity level present in the PB.

\section{$\underline{\text { Required Detection Limits }}$}

The ASR required detection limits were met for all analytes for both the digestate and residual solid sample. The GEA detection limit requirements are shown on the summary data sheets.

Blank Spike (BS)/Laboratory Control Sample (LCS)/ Matrix Spike (MS):

There are no BS, LCS or MS samples analyzed for ASO GEA analyses. Instrument performance is assessed by the analyses of daily control counts and weekly background counts, as discussed below. (The sample identified as "MS" was prepared for ICP/OES analyses only. This MS sample is also being reported for GEA as additional information for the project.)

\section{Duplicate Relative Percent Difference (RPD):}

Duplicate sample results are required to agree within $<20 \%$ relative percent difference, taking into consideration the statistical uncertainties in the data. Duplicate sample results for sludge samples ranged from 0.4 to $48 \%$ RPD. The duplicate RPD Data Quality Objective of $\pm 20 \%$ does not apply to the residual solids results. The two sludge samples results where the $\pm 20 \%$ RPD was exceeded are detailed below and are documented in Occurrence Report 52578-04-18-11.

For Sludge samples:

\begin{tabular}{|c|c|c|c|}
\hline RPL Number & Client Sample ID & Isotopes & RPD Differences \\
\hline $11-0419-129-S,-D$ & TI001-A1-B, -C & Co-60 & $48 \%$ \\
\hline $11-0421-129-S,-D$ & TI002-B1-B, -C & Eu-155 & $32 \%$ \\
\hline
\end{tabular}

\section{Instrument Calibration and Quality Control}

Gamma detectors are calibrated using multi-isotope standards that are NIST-traceable and prepared in the identical counting geometry to samples for all detectors. Control sources containing Am-241, Cs- 137 and Co- 60 are then analyzed daily before the use of each detector. There is a K Basin DQO that is referenced to ASO procedure RPG-CMC-450, that requires that a counter control source is checked daily and must be within \pm 3 sigma or $\pm 3 \%$ of the control value, whichever is greater. Gamma counting was not performed unless the control counts were within the required limits. Background counts are performed on all gamma detectors at least weekly for either an overnight or weekend count. The most recent background is subtracted from all sample counts.

\section{Assumption and Limitations of the Data}

The digestion was performed exactly as stated in procedure PNL-ALO-129. Following the digestion process, each sample had a visible quantity of residual solids. The residual solids have measureable activity. The GEA results for the residual solids are included in this report. 


\section{Battelle PNNL/RPL/ASO Radiochemistry Analysis Report}

\section{Interferences/Resolution Issues}

None.

\section{Uncertainty}

For gamma counting, the uncertainty in the counting data, calibration, photon abundance and the nuclear half-life are included in the calculation of the total uncertainty along with a systematic uncertainty for sample prep. The Canberra Genie2000 software includes both random and systematic uncertainties in the calculation of the total uncertainties which are listed on the report. We conservatively estimate that $2 \%$ is the lowest uncertainty possible for our GEA measurements taking into account systematic uncertainties in gamma calibration standards.

Solids residues were placed in glass LSC vials. Unlike liquid samples, their geometries could not be carefully controlled to match the calibration standards. The solids samples were counted at about $\sim 10 \mathrm{~cm}$ from the detector face to minimize the differences between the sample geometry and the calibration geometry. Calibrations are performed using water samples whereas the samples consisted of mostly silica (however, the exact sample mineralogical composition and associated density were not known). The small sample size $(<1 \mathrm{~g})$ minimized the gamma absorption effects; therefore, density corrections were not applied.

\section{Comments}

1. The results have been corrected for all dilution factors resulting from sample processing.

2. Minimum Detectable Activity (MDA) and Minimum Detectable Concentration (MDC). Sample results are compared to the process blank results to evaluate if the blank contains $5 \%$ or more of the analyte of interest. When samples undergo digestion and/or dilution (process factors), the MDA must be adjusted for the process factors to accurately compare activity levels in the blank to activity levels in the samples. Process blank results have been adjusted for digestion and/or process factors for evaluation of the $5 \%$ criteria.

3. Occurrence Report 52578-04-18-11 documents DQO related issue(s) with this report: RPD's outside the $<20 \%$ requirement.

For Sludge Samples:

\begin{tabular}{|c|c|c|c|}
\hline RPL Number & Client Sample ID & Isotopes & RPD Differences \\
\hline $11-0419-129-S,-D$ & TI001-A1-B, -C & Co-60 & $48 \%$ \\
\hline $11-0421-129-S,-D$ & TI002-B1-B, -C & Eu-155 & $32 \%$ \\
\hline
\end{tabular}

Attachment: Data Report Sample Results for ASR 8798. 
PNNL-20650, Rev. 2
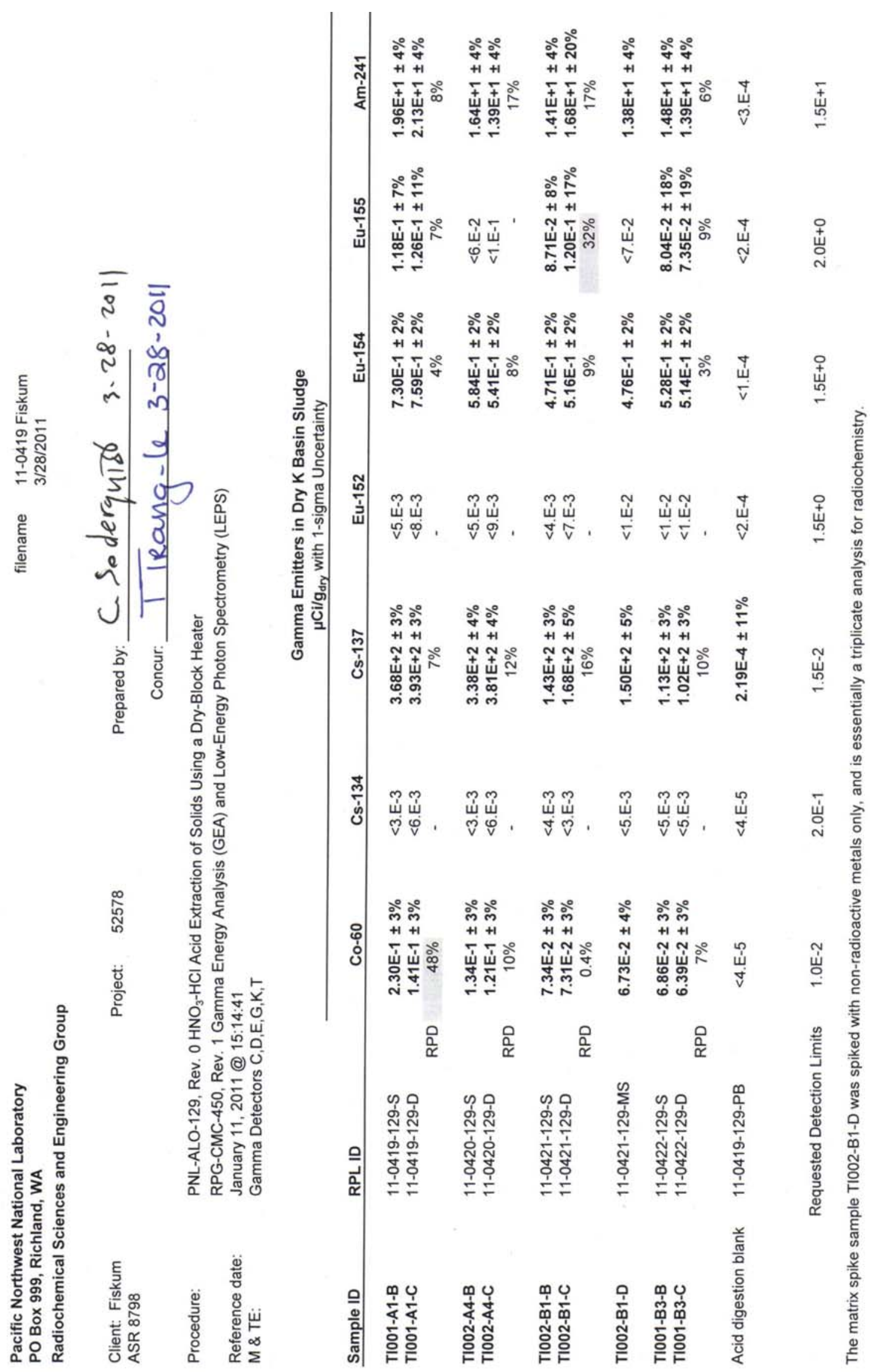

Page 1 of 1 
PNNL-20650, Rev. 2
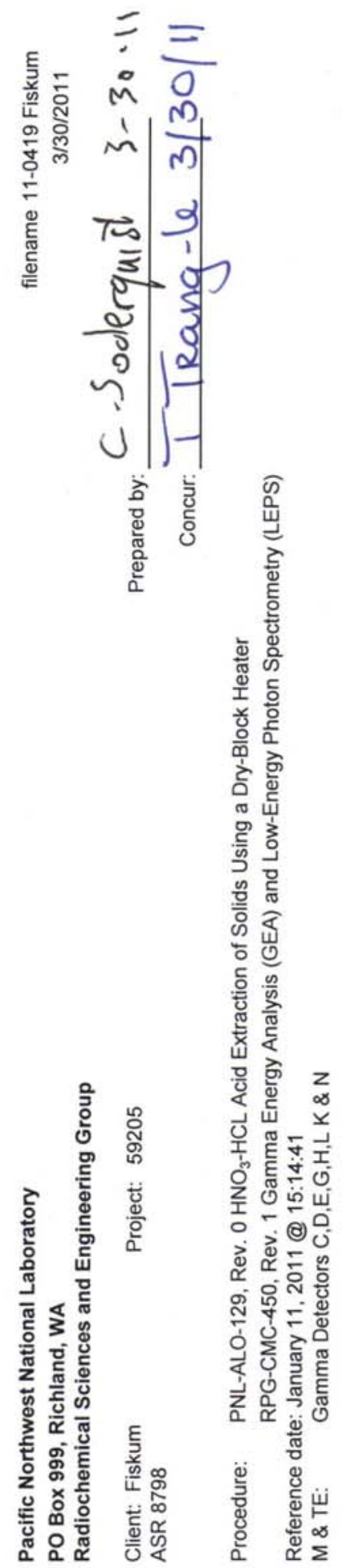

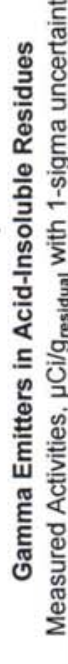

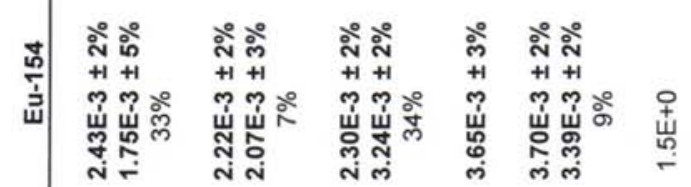

尔

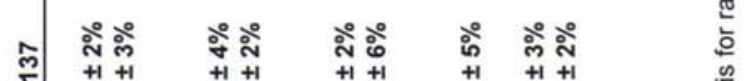

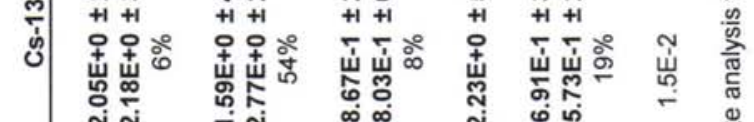
菅|

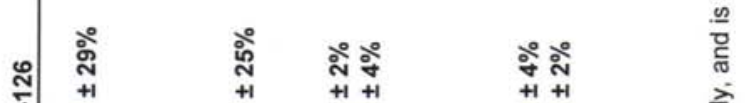
i

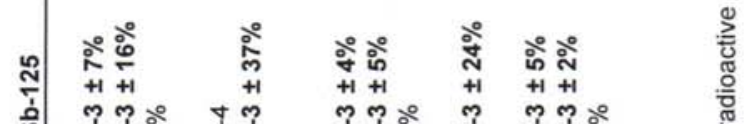

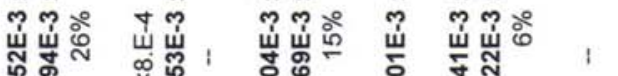

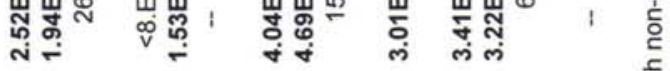

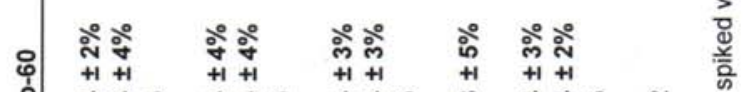

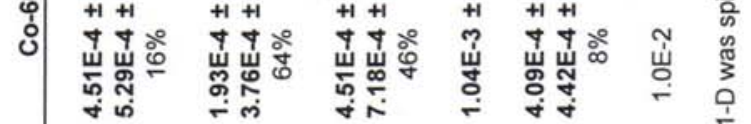

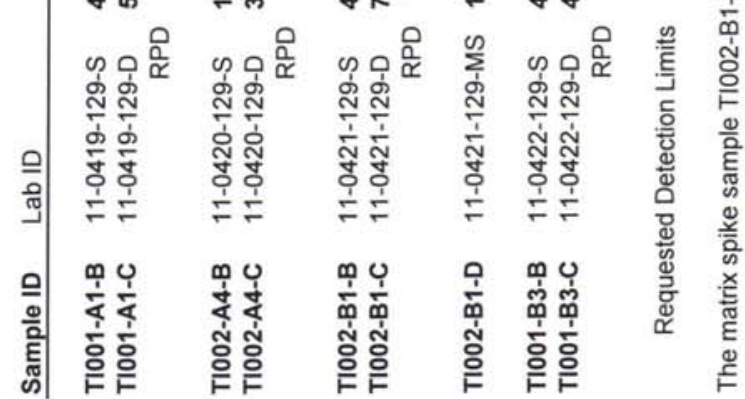


PNNL-20650, Rev. 2

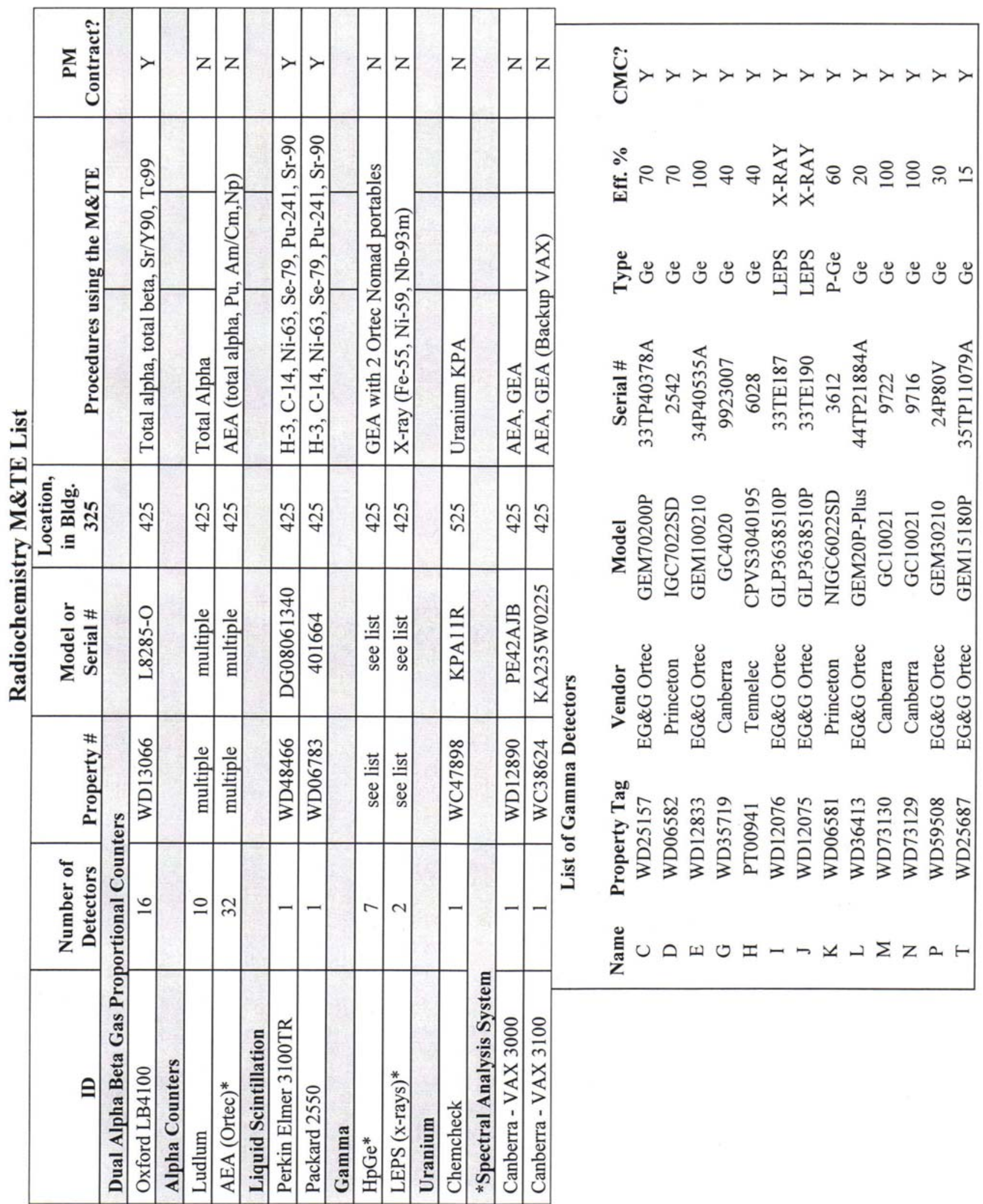


PNNL-20650, Rev. 2

Battelle PNNL/RPL/ASO Radiochemistry Analysis Report

P.O. Box 999, 902 Battelle Blvd., Richland, Washington 99352

\section{Plutonium 238, 239+240 Analysis}

\begin{tabular}{|l|l|}
\hline Project / WP\#: & $52578 / \mathrm{H} 41216$ \\
\hline ASR\#: & 8798 \\
\hline Client: & S. Fiskum \\
\hline Total \# of Samples: & 4 \\
\hline
\end{tabular}

\begin{tabular}{|c|c|c|}
\hline Engineered Container \& Core ID & Sample IDs & RPL Number \\
\hline KW-210-A1 & TI001-A1-B, -A1-C & $11-0419-129-\mathrm{S},-129-\mathrm{D}$ \\
\hline KW-210-A4 & TI002-A4-B, -A4-C & $11-0420-129-\mathrm{S},-129-\mathrm{D}$ \\
\hline KW-210-B1 & TI002-B1-B, -B1-C & $11-0421-129-\mathrm{S},-129-\mathrm{D}$ \\
\hline KW-210-B3 & TI001-B3-B, -B3-C & $11-0422-129-\mathrm{S},-129-\mathrm{D}$ \\
\hline
\end{tabular}

\begin{tabular}{|c|c|}
\hline Analysis Type: & AEA - Pu-238, Pu-239+240 \\
\hline $\begin{array}{l}\text { Sample Processing Prior to Radiochemical } \\
\text { Processing/Analysis }\end{array}$ & $\begin{array}{l}\square \text { None } \\
\square \text { Digested as per PNL-ALO-129, Rev. 0, } \mathrm{HNO}_{3} \text { - HCl Acid Extraction of } \\
\text { Solids Using a Dry Block Heater, 3-7-11 (N. Green) } \\
\square \text { Fusion as per PNL-ALO-115, Solubilization of Metals from Solids Using a } \\
\mathrm{KOH}-\mathrm{KNO}_{3} \text { Fusion } \\
\square \text { Other: }\end{array}$ \\
\hline \multirow[t]{2}{*}{ Pre-dilution Prior to Radiochemical Processing? } & $\triangle$ No \\
\hline & $\square$ Yes -- example $2 \mathrm{~mL}$ to $100 \mathrm{~mL} ; 50 x$ dilution \\
\hline Total Alpha and Beta Preparation Procedure: & $\begin{array}{l}\text { RPG-CMC-4001, Rev. 1, Source Preparation For Gross Alpha and Gross Beta } \\
\text { analyses. }\end{array}$ \\
\hline Technician/Analyst: & K. Carson, 3-28-11 \\
\hline Spike Standard ID's & R-569-a-3 (Sr-90), R-485-b-5 (Pu-239) \\
\hline Analysis Procedure & RPG-CMC-408, Rev. 2, Total Alpha and Beta Analysis \\
\hline Technician/Analyst: & T. Trang-Le, 3-29-11 \\
\hline Plutonium Separation Procedure: & $\begin{array}{l}\text { RPG-CMC-4017, Rev. 0, Analysis of Environmental Water Samples for } \\
\text { Actinides and Strontium- } 90\end{array}$ \\
\hline Technician/Analyst: & K. Carson, 4-4-11 \\
\hline Spike and Tracer Standard ID's: & R-485-b-5 (Pu-239), R-533-a-7 (Pu-242 tracer) \\
\hline Co-Precipitation Procedure: & $\begin{array}{l}\text { RPG-CMC-496, Rev. 0, Co-precipitation Mounting of Actinides for Alpha } \\
\text { Spectroscopy }\end{array}$ \\
\hline Technician/Analyst: & K. Carson, 4-8-11 \\
\hline Analysis Procedure: & RPG-CMC-422, Rev. 2, Solutions Analysis: Alpha Spectrometry \\
\hline Reference Date: & Same as counting dates \\
\hline Analysis Date or Date Range: & $4 / 8 / 11$ and $4 / 12 / 11$ \\
\hline Technician/Analyst: & T. Trang-Le \\
\hline Analysis Data (File): & 11-0419 Fiskum.xls, Alpha Beta \\
\hline CMC Project 98620 File: & $\begin{array}{l}\text { File Plan } 5871 \text { : T } 52578 \text { 8798: Sample preparation and analysis records; T- } 4.4 \\
\text { Alpha Detector calibration, calibration verification checks, and maintenance } \\
\text { records; and T3 Standard certificates and preparation. Also balance calibration } \\
\text { and instrument performance checks. }\end{array}$ \\
\hline M\&TE Number(s): & Ortec AEA counters - 32 counters - See attached M\&TE list \\
\hline
\end{tabular}


PNNL-20650, Rev. 2

\section{Battelle PNNL/RPL/ASO Radiochemistry Analysis Report}

\section{Sample Results}

See attached data report, Pu-238 and Pu-239+240 Sample Results for ASR 8798. All data are reported in units of $\mathrm{uCi} / \mathrm{g}_{- \text {dry }} \mathrm{K}-\mathrm{Basin}$ sludge with a $1-\sigma$ uncertainty (see comments).

\section{Sample Preparation, Separation, Mounting and Counting Methods}

Four K-Basin sludge samples were prepared in the shielded analytical laboratory (SAL) hot cells by the Project and provided to the Analytical Support Operations (ASO) under Analytical Service Request (ASR) 8798 for chemical and radio chemical analyses. ASO received each sample in a Teflon Oak Ridge centrifuge tube, dried the contents of each centrifuge tube to a constant weight, and then leached the dried solids with hot dilute nitric and hydrochloric acids per procedure PNL-ALO-129. The leachate solution was used for radiochemical analyses and ICP metals analysis; only Pu-AEA is reported in this report. Visible residual solids remained following the PNL-ALO-129 digestion.

Following the digestion process, the Pu was separated from the sludge leachate by anion exchange using procedure RPG-CMC-4017. The separated Pu fraction was then mounted for alpha spectrometry by coprecipitation using procedure RPG-CMC-496, and then counted using alpha spectrometry using procedure RPC-CMC-422. The samples were counted on April $8^{\text {th }}$ and $12^{\text {th }}, 2011$; no decay corrections were made.

Although not formally requested by the ASR, gross alpha and gross beta analyses were performed on each sample to obtain information to estimate aliquot sizes for other analyses and for checking the internal consistency of the Pu alpha isotopic data. The gross alpha and gross beta results are included in the data tables as supplemental information only. Gross alpha and gross beta activity were measured by evaporating small aliquots of leachate onto counting planchets per procedure RPG-CMC-4001 and counting per procedure RPG-CMC-408. These procedures are not required to be pre-approved by KBasin project staff as they are used to generate opportunistic data only. The sum of the major alpha emitters, in this case Pu-238, Pu-239+240 and Am-241, agrees very well with the gross alpha results.

\section{QUALITY CONTROL RESULTS}

Quality control (QC) samples prepared in the SAL hot cells include a hot cell process blank (PB), sample duplicates for each sample, and a matrix spike (MS - for ICP/OES only). Additional QC samples were prepared prior to separations; these include a laboratory separation blank, a reagent blank spike (BS, spiked with Pu-239), and a matrix spike (MS) made by adding Pu-239 standard to a diluted sample.

Performance acceptance criteria for these samples are established by both the K Basin data quality objectives (as provided as special instruction in the ASR) and the ASO Quality Assurance (QA) Plan (ASO-QAP-001, Rev. 7). Except where noted, the Pu-AEA K-Basin DQO and ASO QAP criteria are the same. In cases where the QC criteria are different, K-Basin DQO's will take precedence. The QC sample results for Pu-AEA have been evaluated and are discussed below. A summary of the Pu-AEA analysis results, including QC sample performance, is given in the attached data report. 


\section{Battelle PNNL/RPL/ASO Radiochemistry Analysis Report}

Tracer:

The $\mathrm{Pu}-242$ tracer is added to every sample after appropriate dilution and prior to plutonium separations. The use Pu- 242 tracer corrects for radiochemical yield and mathematically removes the detector counting efficiency from the results calculations. Tracer recover is required to be high enough to provide acceptable counting statistics. The Pu- 242 tracer counting statistics were acceptable for all samples. The tracer recoveries ranged from $94 \%$ to $108 \%$.

\section{$\underline{\text { Hot Cell Process Blank (PB) and Laboratory separations blank (LB): }}$}

The $\mathrm{Pu}-238$ and $\mathrm{Pu}-239+240$ activities measured in the $\mathrm{PB}$ are required to be within the acceptance criteria of less than $5 \%$ of the sample isotope concentration or less than sample minimum detectable activity (MDA) - see comments. The PB Pu-238 and Pu-239+240 activities are significantly less than $5 \%$ of any sample activity. There are no acceptance criteria for LB. The detection limits achieved for the $\mathrm{Pu}-238$ and $\mathrm{Pu}-239+240$ were below the required detection limit of $1.0 \mathrm{E}-03 \mu \mathrm{Ci} / \mathrm{g}_{-}$ry

Blank Spike (BS) - reagent spike:

The BS (Pu-239) recovery of $100 \%$ meets the acceptance criteria of $80 \%$ to $120 \%$ recovery.

\section{Matrix Spike (MS):}

The MS recovery of $101 \%$ meets the acceptance criterion of $75 \%$ to $125 \%$ recovery. Note: the MS sample was prepared after digestion, by adding a known Pu-239 standard quantity to an aliquot of the leachate. Sample number 11-0422-129-S (TI001-B3-B) was selected as the matrix spike sample.

\section{Duplicate -- Relative Percent Difference (RPD):}

Duplicate results are required to agree within $<20 \%$ RPD. Duplicate results range from $2 \%$ to $13 \%$ RPD; thus meeting the $<20 \%$ requirement. See Assumptions and Limitations of the Data.

\section{Instrument Quality Control}

Counter control source counting is not required when samples are spiked with tracers. When tracers are used, the absolute counting efficiencies are not used.

Detector backgrounds are determined every 4 weeks or after the last analytical run, whichever is longer. Detector background counts are subtracted from all subsequent sample counts. A process blank is analyzed with each analytical batch to evaluate for contamination in the sample preparation process.

\section{Assumption and Limitations of the Data}

Undissolved residue remained after the completion of the acid digestion/leach of all sludge samples. 
PNNL-20650, Rev. 2

\section{Battelle PNNL/RPL/ASO Radiochemistry Analysis Report}

\section{Comments}

1. The results have been corrected for all dilution factors resulting from sample processing.

2. Post-Digestion Spike (PS) - A spike made after the initial sample preparation (e.g., fusion, digestion, or leach) is considered a PS. When extremely radioactive samples are analyzed, most of the radio analytical spikes are made after the sample preparation and aliquot dilution (to avoid excessive consumption of spike and avoid creating unnecessary waste). The MS prepared with this sample batch is considered a PS, since the Pu-239 spike was not added prior to the digestion process.

3. Each alpha spectrum was examined for potential interferences from other alpha emitting isotopes. Pu-242 and Am-243 tracers are added at the start of the chemical separation process. The presence of Am-243 in the Pu-AEA would indicate interference from Am-241 on the Pu238 peak. The alpha spectra were evaluated to verify the removal of Am from the Pu. There was no evidence of interfering isotopes, including Am.

4. The 1-sigma uncertainty represents the total propagated error associated with processing and counting operations and includes weighing errors, volume uncertainties, and counting error.

5. The sample results are compared to the process blank to evaluate if the blank contains $5 \%$ or more of the measured isotope; the process blank result has been adjusted for all processing factors for evaluation of the $5 \%$ criterion.

6. The Laboratory Blank (LB) is prepared using laboratory reagents and provides data only on the cleanliness of the radiochemistry preparation process. LB results are not normalized to processing or dilution factors associated with the samples.

Attachment: Data Report -- Sample Results for ASR 8798 
PNNL-20650, Rev. 2

Battelle PNNL/RPL/ASO Radiochemistry Analysis Report

P.O. Box 999, 902 Battelle Blvd., Richland, Washington 99352

\section{Americium 241 Analysis}

\begin{tabular}{|l|l|}
\hline Project / WP\#: & $52578 / \mathrm{H} 41216$ \\
\hline ASR\#: & 8798 \\
\hline Client: & S. Fiskum \\
\hline Total \# of Samples: & 4 \\
\hline
\end{tabular}

\begin{tabular}{|c|c|c|}
\hline Engineered Container \& Core ID & Sample IDs & RPL Number \\
\hline KW-210-A1 & TI001-A1-B, -A1-C & $11-0419-129-\mathrm{S},-129-\mathrm{D}$ \\
\hline $\mathrm{KW}-210-\mathrm{A} 4$ & TI002-A4-B, -A4-C & $11-0420-129-\mathrm{S},-129-\mathrm{D}$ \\
\hline $\mathrm{KW}-210-\mathrm{B} 1$ & TI002-B1-B, -B1-C & $11-0421-129-\mathrm{S},-129-\mathrm{D}$ \\
\hline $\mathrm{KW}-210-\mathrm{B} 3$ & TI001-B3-B, -B3-C & $11-0422-129-\mathrm{S},-129-\mathrm{D}$ \\
\hline
\end{tabular}

\begin{tabular}{|c|c|}
\hline Analysis Type: & AEA - Am-241 \\
\hline $\begin{array}{l}\text { Sample Processing Prior to Radiochemical } \\
\text { Processing/Analysis }\end{array}$ & $\begin{array}{l}\square \text { None } \\
\square \text { Digested as per PNL-ALO-129, Rev. 0, } \mathrm{HNO}_{3} \text { - HCl Acid Extraction of } \\
\text { Solids Using a Dry Block Heater, 3-7-11 (N. Green) } \\
\square \text { Fusion as per PNL-ALO-115, Solubilization of Metals from Solids Using a } \\
{\mathrm{KOH}-\mathrm{KNO}_{3} \text { Fusion }}^{\square} \text { Other: }\end{array}$ \\
\hline \multirow[t]{2}{*}{ Pre-dilution Prior to Radiochemical Processing? } & $\triangle$ No \\
\hline & $\square$ Yes -- example $2 \mathrm{~mL}$ to $100 \mathrm{~mL} ; 50 x$ dilution \\
\hline Total Alpha and Beta Preparation Procedure: & $\begin{array}{l}\text { RPG-CMC-4001, Rev. 1, Source Preparation For Gross Alpha and Gross Beta } \\
\text { analyses. }\end{array}$ \\
\hline Technician/Analyst: & K. Carson, 3-28-11 \\
\hline Spike Standard ID's & R-569-a-3 (Sr-90), R-485-b-5 (Pu-239) \\
\hline Analysis Procedure & RPG-CMC-408, Rev. 2, Total Alpha and Beta Analysis \\
\hline Technician/Analyst: & T. Trang-Le, 3-29-11 \\
\hline Americium Separation Procedure: & $\begin{array}{l}\text { RPG-CMC-4017, Rev. 0, Analysis of Environmental Water Samples for } \\
\text { Actinides and Strontium- } 90\end{array}$ \\
\hline Technician/Analyst: & K. Carson, 4-4-11 \\
\hline Spike and Tracer Standard ID's: & R-542-a-3 (Am-241), R-571-a-1 (Am-243 tracer) \\
\hline Co-Precipitation Procedure: & $\begin{array}{l}\text { RPG-CMC-496, Rev. 0, Co-precipitation Mounting of Actinides for Alpha } \\
\text { Spectroscopy }\end{array}$ \\
\hline Technician/Analyst: & K. Carson, 4-8-11 \\
\hline Analysis Procedure: & RPG-CMC-422, Rev. 2, Solutions Analysis: Alpha Spectrometry \\
\hline Reference Date: & Same as counting dates \\
\hline Analysis Date or Date Range: & $4 / 11 / 11$ and $4 / 12 / 11$ \\
\hline Technician/Analyst: & T. Trang-Le \\
\hline Analysis Data (File): & 11-0419 Fiskum.xls, Alpha Beta \\
\hline CMC Project 98620 File: & $\begin{array}{l}\text { File Plan } 5871 \text { : T } 52578 \text { 8798: Sample preparation and analysis records; T-4.4 } \\
\text { Alpha Detector calibration, calibration verification checks, and maintenance } \\
\text { records; and T3 Standard certificates and preparation. Also balance calibration } \\
\text { and instrument performance checks. }\end{array}$ \\
\hline M\&TE Number(s): & Ortec AEA counters - 32 counters - See attached M\&TE list \\
\hline
\end{tabular}


PNNL-20650, Rev. 2

\section{Battelle PNNL/RPL/ASO Radiochemistry Analysis Report}

\section{Sample Results}

See attached data report, Am-241 Sample Results for ASR 8798. All data are reported in units of uCi/gdry K-Basin sludge with a $1-\sigma$ uncertainty (see comments).

\section{Sample Preparation, Separation, Mounting and Counting Methods}

Four K-Basin sludge samples were prepared in the shielded analytical laboratory (SAL) hot cells by the Project and provided to the Analytical Support Operations (ASO) under Analytical Service Request (ASR) 8798 for chemical and radiochemical analyses. ASO received each sample to a Teflon Oak Ridge centrifuge tube, dried the contents of each centrifuge tube to a constant weight, and then leached the dried solids with hot dilute nitric and hydrochloric acids per procedure PNL-ALO-129. The leachate solution was used for radiochemical analyses and ICP metals analysis; only Am isotopic results are reported in this report. Visible residual solids remained following the PNL-ALO-129 digestion.

Following the digestion process, the Pu and $\mathrm{U}$ were first removed from the leachate by anion exchange chromatography using procedure RPG-CMC-4017. The Am was separated from the load and wash solution using Eichrom TRU resin per procedure RPG-CMC-4017. The separated Am fraction was then mounted for alpha spectrometry by co-precipitation using procedure RPG-CMC-496, then counted by alpha spectroscopy using procedure RPC-CMC-422. The samples were counted on April $11^{\text {th }}$ and $12^{\text {th }}$, 2011; no decay corrections were made.

Although not formally requested by the ASR, gross alpha and gross beta analyses were performed on each sample to obtain information to estimate aliquot sizes for other analyses and for checking the internal consistency of the Am alpha isotopic data. The gross alpha and gross beta results are included in the data tables as supplemental information only. Gross alpha and gross beta activity were measured by evaporating small aliquots of leachate onto counting planchets per procedure RPG-CMC-4001 and counting per procedure RPG-CMC-408. These procedures are not required to be pre-approved by KBasin project staff as they are used to generate opportunistic data only. The sum of the major alpha emitters, in this case Pu-238, Pu-239+240 and Am-241, agrees very well with the gross alpha results.

\section{QUALITY CONTROL RESULTS}

Quality control (QC) samples prepared in the SAL hot cells include a hot cell process blank (PB), sample duplicates for each sample, and a matrix spike (MS - for ICP/OES only). Additional QC samples were prepared prior to separations; these include a laboratory separation blank, a reagent blank spike (BS), and a matrix spike (MS) made by adding Am-241 standard to a diluted sample.

Performance acceptance criteria for these samples are established by both the K Basin data quality objectives (as provided as special instruction in the ASR) and the ASO Quality Assurance (QA) Plan (ASO-QAP-001, Rev. 7). Except where noted, the Am-AEA K-Basin DQO and ASO QAP criteria are the same. In cases where the QC criteria are different, K-Basin DQO's take precedence. The QC sample results for Am-AEA have been evaluated and are discussed below. A summary of the Am-AEA analysis results, including QC sample performance, is given in the attached data report. 


\section{Battelle PNNL/RPL/ASO Radiochemistry Analysis Report}

\section{Tracer:}

The Am-243 tracer is added to every sample after appropriate dilution and prior to americium separations. The Am-243 tracer corrects for radiochemical yield and mathematically removes the detector counting efficiency from the results calculations. Tracer recover is required to be high enough to provide acceptable counting statistics. The Am- 243 tracer counting statistics were acceptable for all samples. The tracer recoveries ranged from $92 \%$ to $114 \%$.

\section{Hot Cell Process Blank (PB) and Laboratory separations blank (LB):}

The Am-241 activity measured in the PB are required to be within the acceptance criteria of less than $5 \%$ of the sample isotope concentration or less than sample minimum detectable activity (MDA) - see comments. The PB Am-241 activity is significantly less than $5 \%$ of any sample activity. There are no acceptance criteria for LB (see comment section below). The detection limit achieved for the $\mathrm{Am}-241$ is below the required detection limit of $1.0 \mathrm{E}-03 \mu \mathrm{Ci} / \mathrm{g}$-dry

\section{Blank Spike (BS) - reagent spike:}

The BS recovery of $102 \%$ meets the acceptance criteria of $80 \%$ to $120 \%$ recovery.

\section{Matrix Spike (MS):}

The MS recovery of $97 \%$ meets the acceptance criterion of $75 \%$ to $125 \%$ recovery. Note: the MS sample was prepared after digestion, by adding a known Am-241 standard quantity to an aliquot of the leachate. Sample number 11-0422-129-S (TI001-B3-B) was selected as the matrix spike sample.

\section{Duplicate -- Relative Percent Difference (RPD):}

Duplicate results are required to agree within $<20 \%$ RPD. Duplicate results range from $2 \%$ to $18 \%$ RPD; thus meeting the $<20 \%$ requirement. See Assumptions and Limitations of the Data.

\section{Instrument Quality Control}

Counter control source counting is not required when samples are spiked with tracers. When tracers are used, the absolute counting efficiencies are not used.

Detector backgrounds are determined every 4 weeks or after the last analytical run, whichever is longer. Detector background counts are subtracted from all subsequent sample counts. A process blank is analyzed with each analytical batch to evaluate for contamination in the sample preparation process.

\section{Assumption and Limitations of the Data}

Undissolved residue remained after the completion of the acid digestion/leach of all sludge samples.

\section{Comments}

1. The results have been corrected for all dilution factors resulting from sample processing. 


\section{Battelle PNNL/RPL/ASO Radiochemistry Analysis Report}

2. Post-Digestion Spike (PS) - A spike made after the initial sample preparation (e.g., fusion, digestion, or leach) is considered a PS. When extremely radioactive samples are analyzed, most of the radioanalytical spikes are made after the sample preparation and dilution (to avoid excessive consumption of spike and avoid creating unnecessary waste). The MS prepared with this batch of samples is considered a PS, since the Am-241 spike was not added prior to the digestion process.

3. The 1-sigma uncertainty represents the total propagated error associated with processing and counting operations and include; weighing errors, volume uncertainties, and counting error.

4. The sample results are compared to the process blank to evaluate if the blank contains $5 \%$ or more of the measured isotope; the process blank result has been adjusted for all processing factors for evaluation of the $5 \%$ criterion.

5. The Laboratory Blank (LB) is prepared using laboratory reagents and provides data on the cleanliness of the radiochemistry preparation/separation processes. LB results are not normalized to processing or dilution factors associated with the samples.

Attachment: Data Report -- Sample Results for ASR 8798 
PNNL-20650, Rev. 2

Battelle PNNL/RPL/ASO Radiochemistry Analysis Report

P.O. Box 999, 902 Battelle Blvd., Richland, Washington 99352

\section{Neptunium 237 Analyses}

\begin{tabular}{|l|l|}
\hline Project / WP\#: & $52578 / \mathrm{H} 41216$ \\
\hline ASR\#: & 8798 \\
\hline Client: & S. Fiskum \\
\hline Total \# of Samples: & 4 \\
\hline
\end{tabular}

\begin{tabular}{|c|c|c|}
\hline Engineered Container \& Core ID & Sample IDs & RPL Number \\
\hline KW-210-A1 & TI001-A1-B, -A1-C & $11-0419-129-\mathrm{S},-129-\mathrm{D}$ \\
\hline KW-210-A4 & TI002-A4-B, -A4-C & $11-0420-129-\mathrm{S},-129-\mathrm{D}$ \\
\hline KW-210-B1 & TI002-B1-B, -B1-C & $11-0421-129-\mathrm{S},-129-\mathrm{D}$ \\
\hline KW-210-B3 & TI001-B3-B, -B3-C & $11-0422-129-\mathrm{S},-129-\mathrm{D}$ \\
\hline
\end{tabular}

\begin{tabular}{|c|c|}
\hline Analysis Type: & $\mathrm{AEA}-\mathrm{Np}-237$ \\
\hline $\begin{array}{l}\text { Sample Processing Prior to Radiochemical } \\
\text { Processing/Analysis }\end{array}$ & $\begin{array}{l}\square \text { None } \\
\square \text { Digested as per PNL-ALO-129,Rev. 0, } \mathrm{HNO}_{3} \text { - HCl Acid Extraction of Solids } \\
\text { Using a Dry Block Heater, 3-7-11 (N. Green) } \\
\square \text { Fusion as per PNL-ALO-115, Solubilization of Metals from Solids Using a } \\
\mathrm{KOH}-\mathrm{KNO}_{3} \text { Fusion } \\
\square \text { Other: }\end{array}$ \\
\hline \multirow[t]{2}{*}{ Pre-dilution Prior to Radiochemical Processing? } & $\triangle$ No \\
\hline & $\square$ Yes -- example $2 \mathrm{~mL}$ to $100 \mathrm{~mL} ; 50 x$ dilution \\
\hline Total Alpha and Beta Preparation Procedure: & $\begin{array}{l}\text { RPG-CMC-4001, Rev. 1, Source Preparation For Gross Alpha and Gross Beta } \\
\text { analyses. }\end{array}$ \\
\hline Technician/Analyst: & $\mathrm{K}$, Carson, 3-28-11 \\
\hline Spike Standard ID's & R-569-a-3 (Sr-90), R-485-b-5 (Pu-239) \\
\hline Analysis Procedure & RPG-CMC-408, Rev. 2, Total Alpha and Beta Analysis \\
\hline Technician/Analyst: & T. Trang-Le, 3-29-11 \\
\hline Neptunium Separation Procedure: & $\begin{array}{l}\text { RPG-CMC-4017, Rev. 0, Analysis of Environmental Water Samples for } \\
\text { Actinides and Strontium- } 90\end{array}$ \\
\hline Technician/Analyst: & K. Carson, 4-8-11 \\
\hline Spike Standard ID's: & $\mathrm{R}-439-\mathrm{c}-13(\mathrm{~Np}-237)$ \\
\hline Co-Precipitation Procedure: & $\begin{array}{l}\text { RPG-CMC-496, Rev. 0, Co-precipitation Mounting of Actinides for Alpha } \\
\text { Spectroscopy }\end{array}$ \\
\hline Technician/Analyst: & K. Carson, 4-14-11 \\
\hline Analysis Procedure: & RPG-CMC-422, Rev. 2, Solutions Analysis: Alpha Spectrometry \\
\hline Reference Date: & Same as counting dates \\
\hline Analysis Date or Date Range: & $4 / 14 / 11$ to $4 / 19 / 11$ \\
\hline Technician/Analyst: & T. Trang-Le \\
\hline Analysis Data (File): & 11-0419 Fiskum.xls, Alpha Beta \\
\hline CMC Project 98620 File: & $\begin{array}{l}\text { File Plan 5871: T } 52578 \text { 8798: Sample preparation and analysis records; T- } 4.4 \\
\text { Alpha Detector calibration, calibration verification checks, and maintenance } \\
\text { records; and T3 Standard certificates and preparation. Also balance calibration } \\
\text { and instrument performance checks. }\end{array}$ \\
\hline M\&TE Number(s): & Ortec AEA counters - 32 counters - See attached M\&TE list \\
\hline
\end{tabular}




\section{Battelle PNNL/RPL/ASO Radiochemistry Analysis Report}

\section{Sample Results}

See attached data report, Sample Results for ASR 8798. All data are reported in units of uCi/g-dry KBasin sludge with a $1-\sigma$ uncertainty unless noted otherwise (see comments).

\section{Sample Preparation, Separation, Mounting and Counting Methods}

Four K-Basin sludge samples were prepared in the shielded analytical laboratory (SAL) hot cells by the Project and provided to the Analytical Support Operations (ASO) under Analytical Service Request (ASR) 8798 for chemical and radio chemical analyses. ASO received each sample in a Teflon Oak Ridge centrifuge tube, dried the contents of each centrifuge tube to a constant weight, and then leached the dried solids with hot dilute nitric and hydrochloric acids per procedure PNL-ALO-129. The leachate solution was used for radio chemical analyses and ICP metals analysis; only Np-AEA is reported in this report. Visible residual solids remained following the PNL-ALO-129.

Following the digestion process, the Np was separated from the sludge leachate using anion exchange chromatography using procedure RPG-CMC-4017. The ion exchange separation process was repeated to in order to eliminate the interference from U-234. The separated $\mathrm{Np}$ fraction was mounted for alpha spectrometry by co-precipitation using procedure RPG-CMC-496, and then counted by alpha spectrometry using procedure RPC-CMC-422. U-238 was observed at very low levels in all of the samples alpha spectra. The highest level of U-238 observed in the alpha spectra corresponds to $\sim 9 \%$ of the Np-237 activity for the following three samples: TI001-A1-C (11-0419-129-D), TI002-B1-B (110421-129-S) and TI001-B3-B (11-0422-129-S).

Although not formally requested by the ASR, gross alpha and gross beta analyses were performed on each sample to obtain information to estimate aliquot sizes for other analyses and for checking the internal consistency of the $\mathrm{Np}$ alpha isotopic data. The gross alpha and gross beta results are included in the data tables as supplemental information only. Gross alpha and gross beta activity were measured by evaporating small aliquots of leachate onto counting planchets per procedure RPG-CMC-4001 and counting per procedure RPG-CMC-408. These procedures are not required to be pre-approved by $\mathrm{K}$ Basin project staff as they are used to generate informational data only. The sum of the major alpha emitters, in this case $\mathrm{Pu}-238, \mathrm{Pu}-239+240$ and $\mathrm{Am}-241$, agree very well with the gross alpha results.

\section{QUALITY CONTROL RESULTS}

Quality control (QC) samples prepared in the SAL hot cells include a hot cell process blank (PB), sample duplicates for each sample, and a matrix spike (MS - ICP/OES only). Additional QC samples were prepared prior to separations; these include a laboratory separation blank, a reagent blank spike (BS), and a matrix spike (MS) made by adding $\mathrm{Np}-237$ standard to a diluted sample. 


\section{Battelle PNNL/RPL/ASO Radiochemistry Analysis Report}

Performance acceptance criteria for these samples are established by both the K Basin data quality objectives (as provided as special instruction in the ASR) and the ASO Quality Assurance (QA) Plan (ASO-QAP-001, Rev. 7). Except where noted, the Np-AEA K-Basin DQO and ASO QAP criteria are the same. In cases where the QC criteria are different, K-Basin DQO's will take precedence. The QC sample results for Np-AEA have been evaluated and are discussed below. A summary of the Np-AEA analysis results, including QC sample performance, is given in the attached data report.

\section{Tracer:}

Tracer is not used for analyses of $\mathrm{Np}$.

\section{Hot Cell Process Blank (PB) and Laboratory separations blank (LB):}

The Np-237 activity measured in the PB is required to be within the acceptance criteria of less than $5 \%$ of the sample isotope concentration or less than sample minimum detectable activity (MDA) (see comments) The PB Np-237 activity is significantly less than $5 \%$ of any sample activity. There are no acceptance criteria for LB. The detection limit achieved for the Np-237 is below the required detection limit of $1.0 \mathrm{E}-03 \mu \mathrm{Ci} / \mathrm{g}_{-}$dry

\section{Blank Spike (BS) - reagent spike:}

The BS recovery of $102 \%$ meets the acceptance criteria of $80 \%$ to $120 \%$ recovery.

\section{Matrix Spike (MS):}

The MS recovery of $103 \%$ meets the acceptance criterion of $75 \%$ to $125 \%$ recovery. Note: the MS sample was prepared "after" digestion, by adding a known Np-237 standard quantity to an aliquot of the leachate. Sample number 11-0422-129-S (TI001-B3-B) was selected as the matrix spike sample.

\section{Duplicate -- Relative Percent Difference (RPD):}

Duplicate results are required to agree within $<20 \%$ RPD. Duplicate results range from $2 \%$ to $82 \%$ RPD. The RPD for sample pair TI001-A1-B (11-0419-129-S) and TI001-A1-C (11-0419$129-\mathrm{D})$ is $22 \%$ and for sample pair TI002-B1-B (11-0421-129-S) and TI002-B1-C (11-0421-129D) is $82 \%$. The Np-237 activity detected in the samples all have $1-\sigma$ counting error of $10 \%$ or greater. $1-\sigma$ counting error at these levels indicates the activity measured in the samples is nearing the minimum detectable activity for the samples. The duplicate RPD's exceeding the $20 \%$ acceptance limit are documented in occurrence report 52578-05-27-11.

\section{Instrument Quality Control}

Alpha counters undergo calibration annually to determine the counter's efficiency over the normal calibration range of 3 to $6 \mathrm{MeV}$. The vendor software determines a constant detector efficiency for this energy range. Np samples are counted and results calculated using the established detector efficiency.

Detector backgrounds are determined every 4 weeks or after the last analytical run, whichever is longer. Detector background counts are subtracted from all subsequent sample counts. A process blank is analyzed with each analytical batch to evaluate for contamination in the sample preparation process. 
PNNL-20650, Rev. 2

\section{Battelle PNNL/RPL/ASO Radiochemistry Analysis Report}

\section{Assumption and Limitations of the Data}

Undissolved residue remained after the completion of the acid digestion/leach of all sludge samples.

\section{Comments}

1. The results have been corrected for all dilution factors resulting from sample processing.

2. Post-Digestion Spike (PS) - A spike made after the initial sample preparation (e.g., fusion, digestion, or leach) is considered a PS. When extremely radioactive samples are analyzed, most of the radioanalytical spikes are made after the sample preparation (to avoid excessive consumption of spike and avoid creating unnecessary waste) and are post-digestion spikes. The MS prepared with this batch of sample is considered a PS, since the Np-237 spike was not added prior to the digestion process.

3. The 1-sigma uncertainty represents the total propagated error associated with processing and counting operations and include; weighing errors, volume uncertainties, and counting error.

4. The sample results are compared to the process blank to evaluate if the blank contains $5 \%$ or more of the measured isotope; the process blank result has been adjusted for all processing factors for evaluation of the $5 \%$ criterion.

5. The Laboratory Blank (LB) is prepared using laboratory reagents and provides data on the cleanliness of the radiochemistry preparation/separation processes. LB results are not normalized to processing or dilution factors associated with the samples.

6. The sample results are compared to the process blank to evaluate if the blank contains $5 \%$ or more of the measured isotope; the process blank result has been adjusted for all processing factors for evaluation of the $5 \%$ criterion.

Attachment: Data Report -- Sample Results for ASR 8798. 
Battelle PNNL/RPL/ASO Radiochemistry Analysis Report

P.O. Box 999, 902 Battelle Blvd., Richland, Washington 99352

Sr-90 by Liquid Scintillation Spectrometry
\begin{tabular}{|l|l|}
\hline Project / WP\#: & $52578 / \mathrm{H} 41216$ \\
\hline ASR\#: & 8798 \\
\hline Client: & S. Fiskum \\
\hline Total \# of Samples: & 4 \\
\hline
\end{tabular}

\begin{tabular}{|c|c|c|}
\hline Engineered Container \& Core ID & Sample IDs & RPL Number \\
\hline KW-210-A1 & TI001-A1-B, -A1-C & $11-0419-129-\mathrm{S},-129-\mathrm{D}$ \\
\hline $\mathrm{KW}-210-\mathrm{A} 4$ & TI002-A4-B, -A4-C & $11-0420-129-\mathrm{S},-129-\mathrm{D}$ \\
\hline $\mathrm{KW}-210-\mathrm{B} 1$ & TI002-B1-B, -B1-C & $11-0421-129-\mathrm{S},-129-\mathrm{D}$ \\
\hline $\mathrm{KW}-210-\mathrm{B} 3$ & TI001-B3-B, -B3-C & $11-0422-129-\mathrm{S},-129-\mathrm{D}$ \\
\hline
\end{tabular}

\begin{tabular}{|c|c|}
\hline Analysis Type: & Sr-90 \\
\hline $\begin{array}{l}\text { Sample Processing Prior to Radiochemical } \\
\text { Processing/Analysis }\end{array}$ & $\begin{array}{l}\square \text { None } \\
\square \text { Digested as per PNL-ALO-129, Rev. 0, HNO } \mathrm{H}_{3} \text { HCl Acid Extraction of Solids } \\
\text { Using a Dry Block Heater, 3-7-11 (N. Green) } \\
\square \text { Fusion as per PNL-ALO-115, Solubilization of Metals from Solids Using a } \\
\text { KOH-KNO} \mathrm{H}_{3} \text { Fusion } \\
\square \text { Other: }\end{array}$ \\
\hline \multirow{2}{*}{$\begin{array}{l}\text { Pre-dilution Prior to Radiochemical } \\
\text { Processing? }\end{array}$} & $\triangle$ No \\
\hline & $\square$ Yes \\
\hline $\begin{array}{l}\text { Total Alpha and Beta Preparation } \\
\text { Procedure: }\end{array}$ & $\begin{array}{l}\text { RPG-CMC-4001, Rev. 1, Source Preparation For Gross Alpha and Gross Beta } \\
\text { Analyses. }\end{array}$ \\
\hline Technician/Analyst: & K. Carson, 3-28-11 \\
\hline Spike Standard ID's & R-569-a-3 (Sr-90), R-485-b-5 (Pu-239) \\
\hline Analysis Procedure & RPG-CMC-408, Rev. 2, Total Alpha and Beta Analysis \\
\hline Technician/Analyst: & T. Trang-Le, 3-29-11 \\
\hline Separation Procedure: & RPG-CMC-476, Rev. 0, Strontium Separation Using Eichrom Strontium Resin \\
\hline Spike Standard ID: & R-569-a-3 (Sr-90) \\
\hline Separation Date: & 4/1/11 10:45 a.m. \\
\hline Technician/Analyst: & K. Carson \\
\hline Analysis Procedure: & $\begin{array}{l}\text { RPG-CMC-474, Rev. 1, Measurement of Alpha and Beta Activity by Liquid } \\
\text { Scintillation Spectrometry }\end{array}$ \\
\hline Reference Date: & N/A \\
\hline Analysis Date or Date Range: & 4/7/11 (first count), 4/13/11 \& 4/14/11 (second count) \\
\hline Technician/Analyst: & T. Trang-Le \\
\hline Rad Chem Electronic Data File: & 11-0419 Fiskum.xls; Worksheet Alpha Beta \\
\hline ASO Project 98620 File: & $\begin{array}{l}\text { File Plan } 5871 \text { : T } 52578 \text { 8798: Sample preparation and analysis records; T-4.4 LSC } \\
3100 \text { calibration, daily checks, and maintenance records; and T3 standard certificates } \\
\text { and preparation. Also, balance calibration and performance check records. }\end{array}$ \\
\hline M\&TE Number(s): & $\begin{array}{l}\text { Perkin Elmer Tri-Carb 3100, Serial \# DG08061340, RPL 425, Tri-Carb 2700TR } \\
\text { software version } 1.04 \text { dated 9/99. }\end{array}$ \\
\hline
\end{tabular}




\section{Battelle PNNL/RPL/ASO Radiochemistry Analysis Report}

\section{SAMPLE RESULTS}

See attached data report, Sample Results for ASR 8798. All sample data are reported in $\mu \mathrm{Ci} / \mathrm{g}_{\mathrm{dry}}$ K-Basin sludge with a $1-\sigma$ uncertainty (see Comments).

\section{Sample preparation, separation, mounting, and counting}

Four K-Basin sludge samples were prepared in the shielded analytical laboratory (SAL) hot cells by the Project and provided to the Analytical Support Operations (ASO) under Analytical Service Request (ASR) 8798 for chemical and radioanalytical analyses. ASO received each sample in a Teflon Oak Ridge centrifuge tube, dried the contents of each centrifuge tube to a constant weight, and then leached the dried solids with hot dilute nitric and hydrochloric acids per procedure PNL-ALO-129. This digestion process was conducted in the SAL hot cells. The leachate solution was used for radio-analytical analyses and ICP metals analysis; only $\mathrm{Sr}-90$ is discussed in this report. Visible residual solids remained following the PNL-ALO-129 digestion.

Following the digestion process, the $\mathrm{Sr}-90$ was separated from the leachate using Eichrom strontium resin per procedure RPG-CMC-476, then counted by liquid scintillation counting (LSC) per procedure RPG-CMC-474.

Although not formally requested by the ASR, gross alpha and gross beta analyses were performed on each sample to obtain information to estimate aliquot sizes for other analyses and for checking the internal consistency of the Sr-90 data. The gross alpha and gross beta results are included in the data tables as supplemental information only. Gross alpha and gross beta activity were measured by evaporating small aliquots of leachate onto counting planchets per procedure RPG-CMC-4001 and counting per procedure RPG-CMC-408. These procedures are not required to be pre-approved by KBasin project staff as they are used to generate informational data only. The sum of the major beta emitters, in this case $\mathrm{Sr} / \mathrm{Y}-90$ and $\mathrm{Cs}-137$, agree very well with the gross beta results.

\section{QUALITY CONTROL RESULTS}

Radioanalytical quality control (QC) samples prepared in the SAL hot cells include a hot cell process blank (PB), and sample duplicates for each sample; no Sr-90 MS was prepared at time of digestion. Additional laboratory QC samples were prepared prior to separations; these include a laboratory separation blank, a reagent blank spike (BS), and a matrix spike (i.e., addition of Sr-90 standard to an aliquot of one of the samples).

Performance acceptance criteria for these samples are established by both the K Basin data quality objectives (as provided as special instruction in the ASR) and the ASO Quality Assurance (QA) Plan (ASO-QAP-001, Rev. 7). Except where noted, the Sr-90 K-Basin DQO and ASO QAP criteria are the same. The quality control (QC) sample results for Sr-90 have been evaluated and are discussed below. A summary of the Sr-90 analysis results, including QC sample performance, is given in the attached data report. The calculated Sr-90 MDC (minimum detectable concentration) for all samples was less than the $\mathrm{K}-\mathrm{Basin}$ method detection limit requirement of $1.0 \mathrm{E} 00 \mu \mathrm{Ci} / \mathrm{g}_{\text {dry }}$ 


\section{Battelle PNNL/RPL/ASO Radiochemistry Analysis Report}

\section{Instrument Calibration Control}

\section{Hot Cell Process Blank (PB) and Laboratory separations blank (LB):}

The Sr-90 activity measured in the PB is required to be within the acceptance criteria of less than sample minimum detectable activity (MDA) or less than $5 \%$ of the sample isotope concentration. The Sr-90 PB is $<5 \%$ of all sample results; thus meeting the acceptance criteria. There are no acceptance criteria for LB (see Comments).

Blank Spike (BS) - reagent spike:

The BS recovery of $99 \%$ meets the procedure acceptance criteria of $80 \%$ to $120 \%$ recovery.

\section{Matrix Spike (MS):}

The MS recovery of $98 \%$ meets the acceptance criterion of $75 \%$ to $125 \%$ recovery. Note: the MS sample was prepared "after" digestion (see comments), by adding a known Sr-90 standard quantity to an aliquot of TI001-B3-B (11-0422-129-S).

\section{Duplicate -- Relative Percent Difference (RPD):}

Duplicate results are required to agree within $<20 \%$ RPD. The ASO QAP further specifies that the two results need to be $>5$ times the MDA or have individual uncertainties $<20 \%$. Duplicate results range from $0.5 \%$ to $11 \% \mathrm{RPD}$; thus meeting the $<20 \%$ requirement.

\section{Instrument Quality Control}

The liquid scintillation counter is calibrated for tritium and C-14 using quenched standard sets that are purchased from the vendor. Daily control counts are then performed using a tritium, C-14, and a background count sample. The instrument software assesses the performance of the control counts and provides control charts to ensure the continuing calibration of the instrument. If the daily performance check fails, then the instrument is not used. Preventative maintenance (PM) and repairs are performed by the vendor under our PM contract. The counting efficiency for Sr-90 is assumed to be $100 \%$; therefore no specific $\mathrm{Sr}-90$ calibration is performed. The LSC system calibration and performance is verified by assessing the recovery of a reagent spike and a matrix spike that are included in every batch of samples. A preparation blank (i.e., digestion blank) and a laboratory separations blank are also included with every batch of samples; the instrument background is subtracted from all results and the preparation and separation blanks are used to assess sample contamination during sample processing steps.

\section{Assumption and Limitations of the Data}

The 1- $\sigma$ uncertainty reported for each Sr-90 result has been set at $2 \%$. Although the calculated uncertainty values are less than $2 \%$ for all samples, the radiochemistry convention is to not report calculated uncertainties less than $2 \%$, but to provide a more realistic estimate of the uncertainty in view of systematic uncertainties that are not fully accounted for in the uncertainty calculations. 
PNNL-20650, Rev. 2

\section{Battelle PNNL/RPL/ASO Radiochemistry Analysis Report}

\section{Comments}

1. The results have been corrected for all dilution factors resulting from sample processing.

2. Post-Digestion Spike (PS) - A spike made after the initial sample preparation (e.g., fusion, digestion, or leach) is considered a PS. When extremely radioactive samples are analyzed, most of the radio-analytical spikes are made after the sample preparation (to avoid excessive consumption of spike and avoid creating unnecessary waste) and are post-digestion spikes. The MS prepared with this batch of sample is considered a PS, since the Sr-90 spike was not added prior to the digestion process.

3. Radiochemistry Electronic Systems File "11-0419 Fiskum" has been created for this report. Supporting records such as Pipette Performance Verification forms, Laboratory Bench Record, Shielded Analytical Laboratory (SAL) Bench Sheet, Standards Certifications and preparation records, and balance calibration and performance check records are maintained per RS\&E Group ASO File Plan 5871.

4. Sample results are compared to the process blank results to evaluate if the blank contains $5 \%$ or more of the measured isotope; the process blank results have been adjusted for all processing factors for the evaluation of the $5 \%$ criterion.

5. The stated $1-\sigma$ uncertainty represents the total propagated error associated with processing and counting operations and includes weighing errors, volume uncertainties, and counting error.

Attachment: Data Report -- Sample Results for ASR 8798 


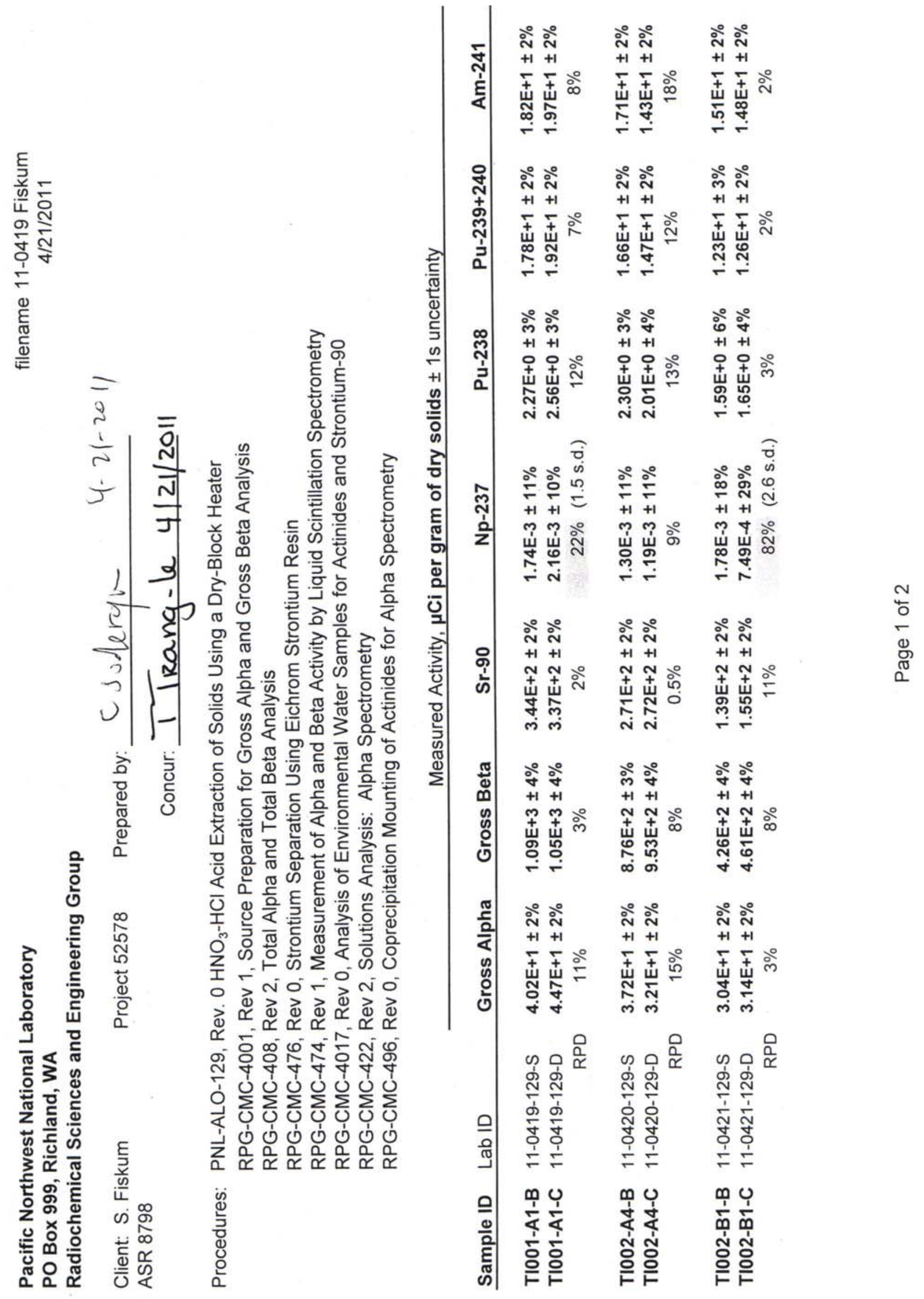


PNNL-20650, Rev. 2

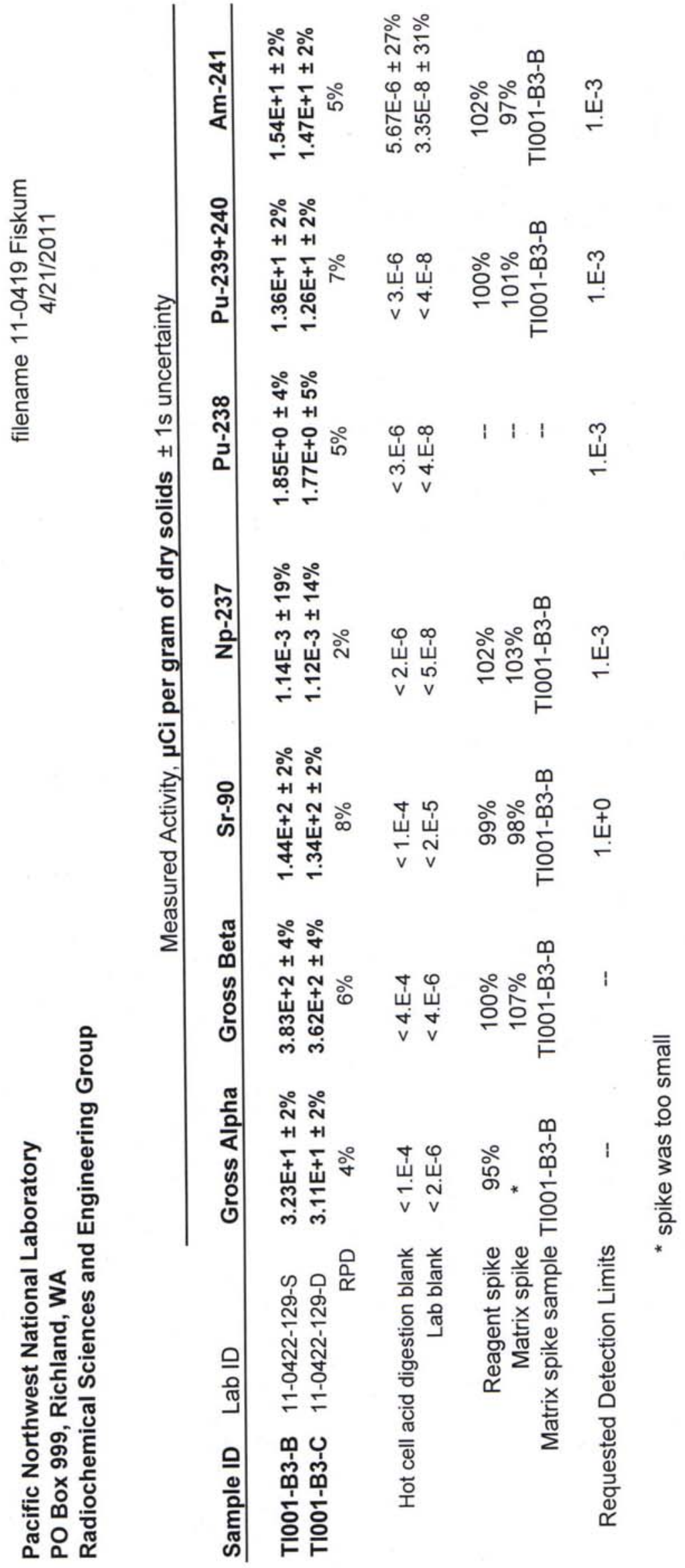

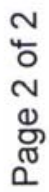


PNNL-20650, Rev. 2

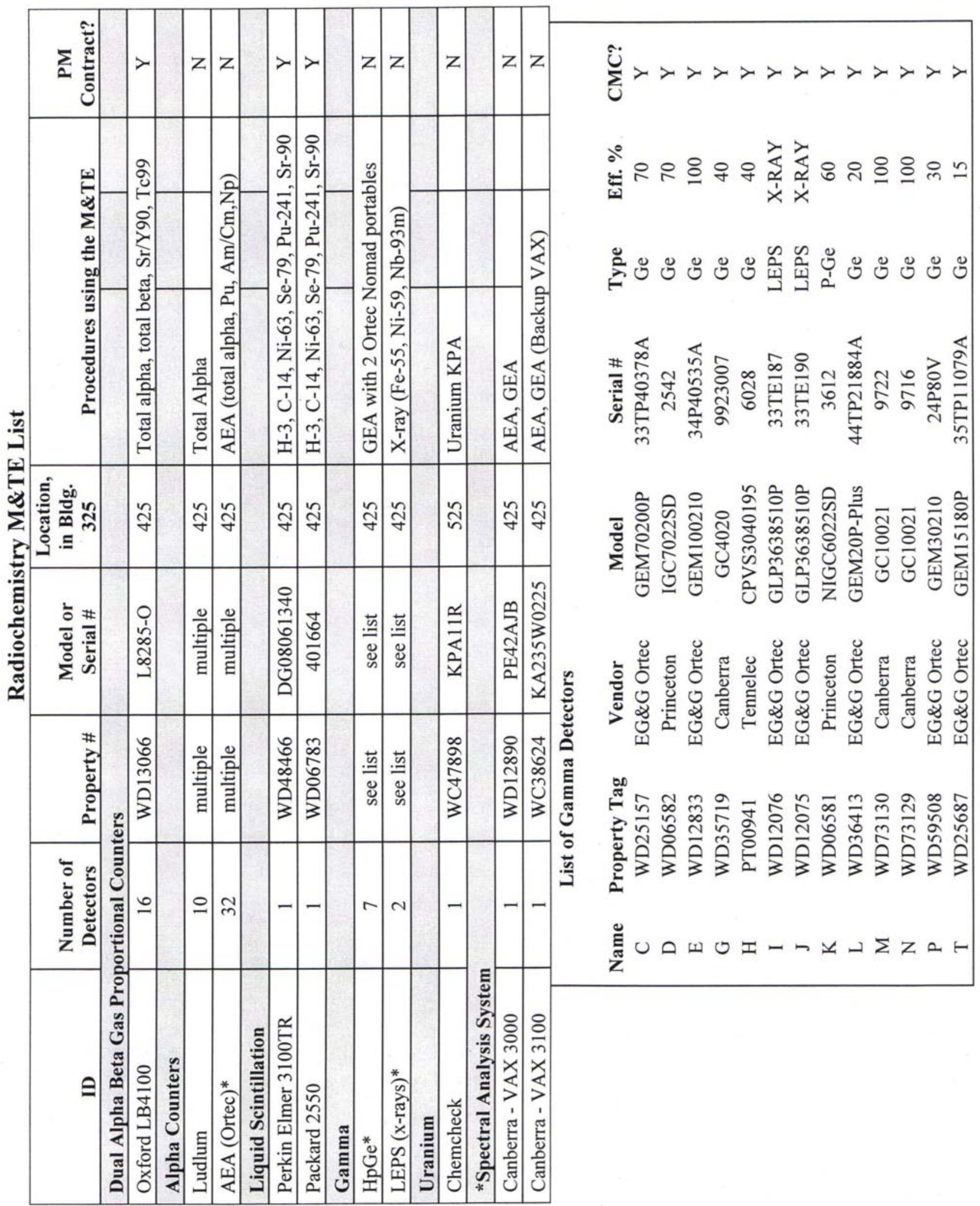


PNNL-20650, Rev. 2

Battelle PNNL/RPL/ASO Radiochemistry Analysis Report

P.O. Box 999, 902 Battelle Blvd., Richland, Washington 99352

\section{Uranium by Kinetic Phosphorescence Analysis (KPA)}

\begin{tabular}{|l|l|}
\hline Project / WP\#: & $52578 / \mathrm{H} 41216$ \\
\hline ASR\#: & 8798 \\
\hline Client: & S. Fiskum \\
\hline Total Samples: & 4 \\
\hline
\end{tabular}

\begin{tabular}{|c|c|c|}
\hline Engineered Container \& Core ID & Sample IDs & RPL Number \\
\hline KW-210-A1 & TI001-A1-B, -A1-C & $11-0419-129-\mathrm{S},-129-\mathrm{D}$ \\
\hline KW-210-A4 & TI002-A4-B, -A4-C & $11-0420-129-\mathrm{S},-129-\mathrm{D}$ \\
\hline $\mathrm{KW}-210-\mathrm{B} 1$ & TI002-B1-B, -B1-C & $11-0421-129-\mathrm{S},-129-\mathrm{D}$ \\
\hline $\mathrm{KW}-210-\mathrm{B} 3$ & TI001-B3-B, -B3-C & $11-0422-129-\mathrm{S},-129-\mathrm{D}$ \\
\hline
\end{tabular}

\begin{tabular}{|c|c|}
\hline Analysis Type: & $\mathrm{U} / \mathrm{KPA}$ \\
\hline $\begin{array}{l}\text { Sample Processing Prior to Radiochemical } \\
\text { Processing/Analysis }\end{array}$ & $\begin{array}{l}\square \text { None } \\
\square \text { Digested as per PNL-ALO-129, Rev. 0, } \mathrm{HNO}_{3} \text { - } \mathrm{HCl} \text { Acid Extraction } \\
\text { of Solids Using a Dry Block Heater, 3-7-11 (N. Green) } \\
\square \text { Other: }\end{array}$ \\
\hline Matrix Spike: & $\begin{array}{l}\text { R608c - Matrix Spike, Expiration 1/1/12 } \\
\text { R608e - Lab Reagent Spike, Expiration - 1/1/12 } \\
\text { RS-301 - Blank Spike, Expiration 1/3/12 }\end{array}$ \\
\hline Analysis Procedure: & RPG-CMC-4014, Rev. 1, Uranium by Kinetic Phosphorescence Analysis \\
\hline Analysis Date: & $5 / 26 / 11$ \\
\hline Technician/Analyst: & K. Carson \\
\hline Rad Chem Electronic Data File: & 11-0419 Fiskum.xls; UKPA \\
\hline ASO Project 98620 File: & $\begin{array}{l}\text { File Plan 5871: T } 52578 \text { 8798: Sample preparation and analysis records; T3 } \\
\text { standard certificates and preparation. Also, balance calibration and } \\
\text { performance check records. }\end{array}$ \\
\hline M\&TE Number(s): & $\begin{array}{l}\text { Chemcheck Instruments, Inc., Model: KPA 11, WC47898, RPL 525; } \\
\text { KPAWIN Software, Version 1.2.8 Release 19 July } 2000 .\end{array}$ \\
\hline
\end{tabular}
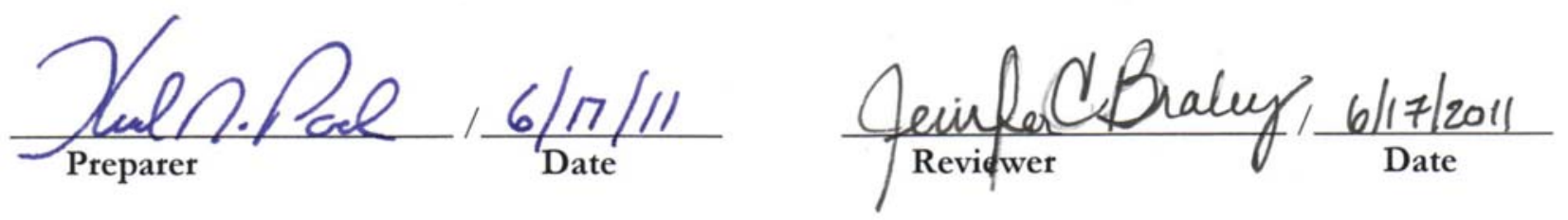


\section{Battelle PNNL/RPL/ASO Radiochemistry Analysis Report}

\section{Sample Results}

See attached data report, Sample Results for ASR 8798. All data are reported in units of $\mu \mathrm{g} \mathrm{U} / \mathrm{g}_{\text {dry }}$ $\mathrm{K}$-Basin sludge with uncertainty reported as \pm 1 -sigma error. Calculated uncertainty includes measurement error of the instrument and dilution error.

Radiochemistry Systems file, 11-0419 Fiskum.xls, has been created for this report. Supporting records include Pipette Performance Verification forms, standard certificates, Laboratory Bench Records, and Shielded Analytical Laboratory Bench Sheet. Balance calibration records can be found in the ASO Records.

\section{Sample Preparation and Analysis}

Four K-Basin sludge samples were prepared in the shielded analytical lab (SAL) hot cells by the Project and provided to the Analytical Support Operations (ASO) under Analytical Service Request (ASR) 8798 for chemical and radio-analytical analyses. ASO received each sample in a Teflon Oak Ridge centrifuge tube, dried the contents of each centrifuge tube to a constant weight, and then leached the dried solids with hot dilute nitric and hydrochloric acids per procedure PNL-ALO-129. The leachate solution was used for radio-analytical analyses and ICP metals analysis; only $\mathrm{U}$ is reported in this report. Visible residual solids remained following the PNL-ALO-129 digestion.

Total uranium was measured directly in the acid digestions by kinetic phosphorescence analysis, without chemical separation. Sample digestates required an additional dilution of $10,000 \mathrm{x}$ to decrease sample concentrations to be within the measurable calibration range.

At the project's request, the results of all of the KPA uranium standards run concurrent with this ASR have been tabulated and attached to this report.

Quality control (QC) samples prepared include a hot cell blank, a laboratory blank, a hot cell reagent spike (BS, prepared with the samples in the hot cell), reagent (blank) spike (BS, at the instrument), a post digestion matrix spike (MS), and sample duplicates of all four samples analyzed. Minimum QC sample frequency was $2 /$ batch.

The quality control (QC) sample results for the Uranium/KPA analysis have been evaluated and are discussed below.

The following is a list of QC sample results relative to both the $\mathrm{K}$ Basin data quality objectives and the ASO Quality Assurance (QA) Plan (ASO-QAP-001, Rev. 7); in many cases the DQOs are the same. In cases where there are differences in the DQOs, the K Basin DQO takes precedence for data reporting. 
PNNL-20650, Rev. 2

\section{Battelle PNNL/RPL/ASO Radiochemistry Analysis Report}

\section{QUALITY CONTROL RESULTS}

\section{Instrument Calibration Control}

The U/KPA analysis has a DQO for the two calibration verification check standards analyzed at the beginning of the batch analysis. The DQO is $92.5 \%$ to $107.5 \%$ recovery of the expected value. The run was bracketed by analysis of 2 sets of the 2 calibration verification check standards. The $\%$ recovery results from the high and low pairs respectively were as follows: $103 \%$ and $102 \%$, and $102 \%$ and $102 \%$.

\section{Tracer:}

There is no tracer used in ASO U KPA analyses.

\section{Hot Cell and Reagent Blank (PB):}

For UKPA, the $\mathrm{PB}$ is required to be $<\mathrm{EQL}$ (estimated quantitation limit, here $0.10 \mu \mathrm{g} / \mathrm{mL}$ ); sample results associated with the analytical run can generally be reported when the sample concentration exceeds 20 times the PB concentration. The hot cell blank was $1.99 \mathrm{E}-2 \pm 7 \%(\mu \mathrm{g} / \mathrm{g}$ dry sludge) and the lab blank was $<2 \mathrm{E}-5(\mu \mathrm{g} / \mathrm{mL})$. Samples were reported at a range from $4.88 \mathrm{E}+4$ to $1.08 \mathrm{E}+5 \mu \mathrm{g} / \mathrm{g}$ dry sludge exceeding the blank concentrations more than 20 times, thereby meeting the MDL requirements.

Blank Spike (BS):

The hot cell reagent spike recovery was $103 \%$ and the reagent spike prepared at the instrument was $107 \%$, within the acceptance criteria of $80 \%$ to $120 \%$.

\section{Matrix Spike (MS):}

The matrix spike (TI001-B3-C) recovery was $103 \%$, within the acceptance criteria of $75-125 \%$.

Note: The MS sample was prepared "after" digestion, by adding a known U standard quantity to an aliquot of the leachate.

\section{Duplicate Relative Percent Difference (RPD):}

Duplicate results are required to agree within $<20 \%$ relative percent difference. Duplicate results ranged from $6 \%$ to $13 \%$ RPD and were within the acceptance limits.

\section{Assumption and Limitations of the Data}

None

\section{Comments}

1. The results have been corrected for all dilution factors resulting from sample processing.

2. Post-Digestion Spike (PS) - A spike made after the initial sample preparation (e.g., fusion, digestion, or leach) is considered a PS. When extremely radioactive samples are analyzed, most of the radioanalytical spikes are made after the sample preparation (to avoid excessive consumption of spike and avoid creating unnecessary waste) and are post-digestion spikes. 
PNNL-20650, Rev. 2

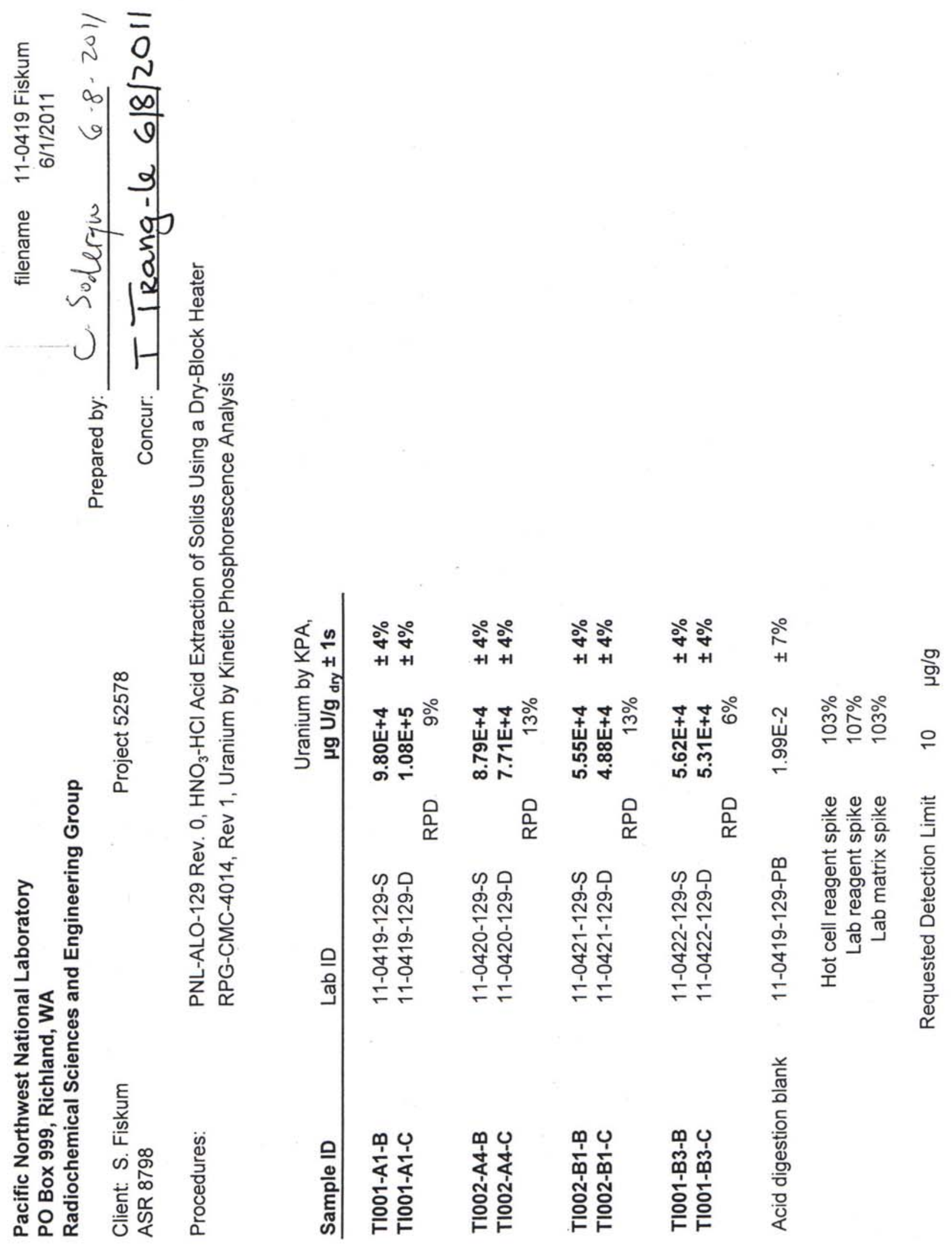

Page 1 of 1 
PNNL-20650, Rev. 2

$\frac{N}{1}$

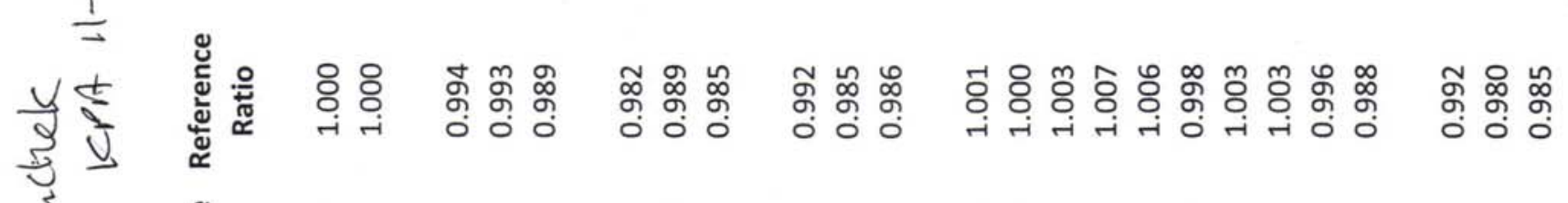

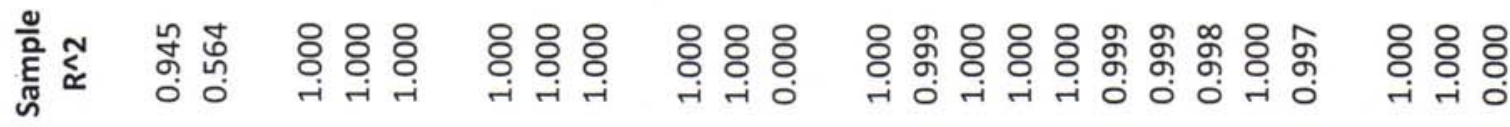

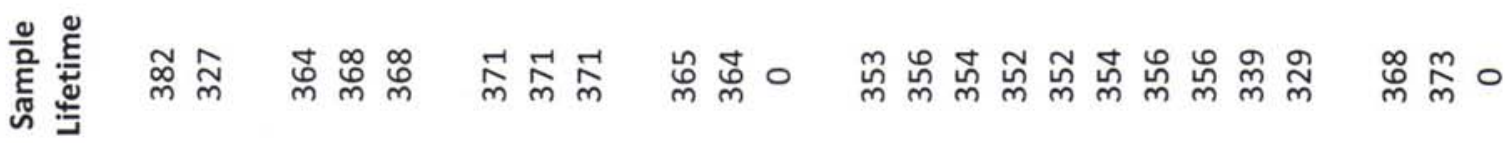

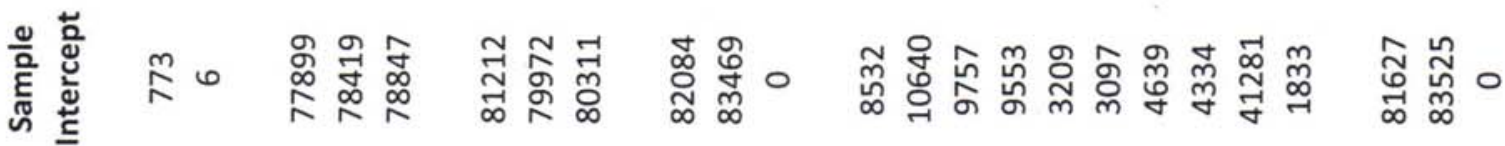
毫喜

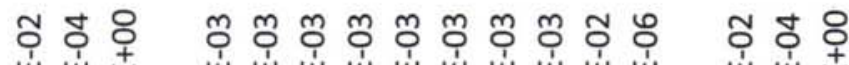

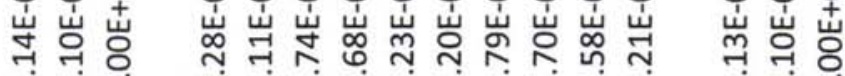
में

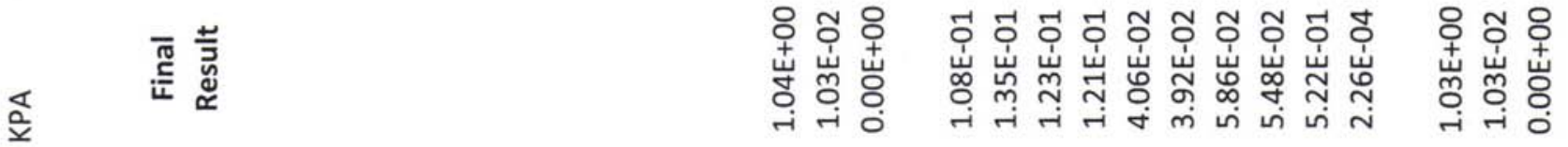

고ำ

$\stackrel{2}{2}$

党

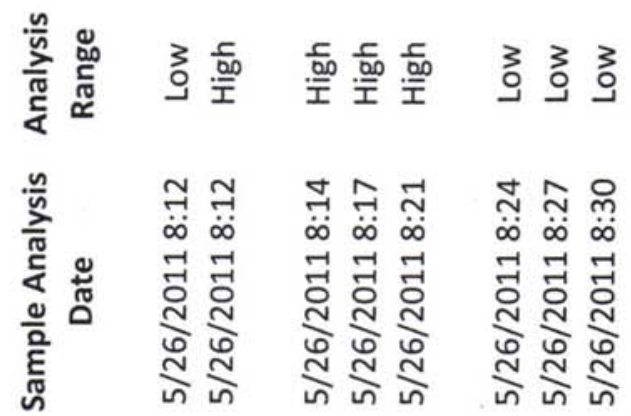

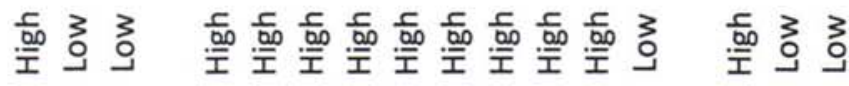

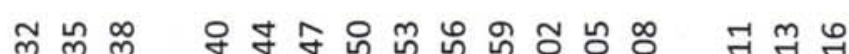

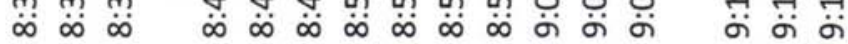

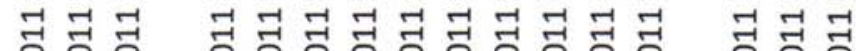

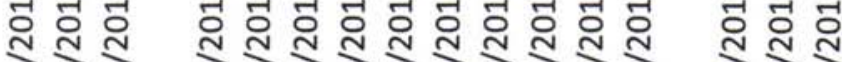

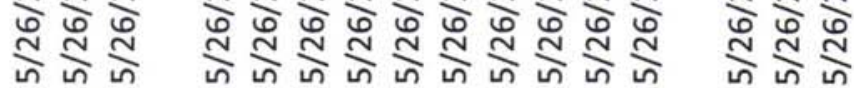

مำ

ํㅗ 은

ค ก゚

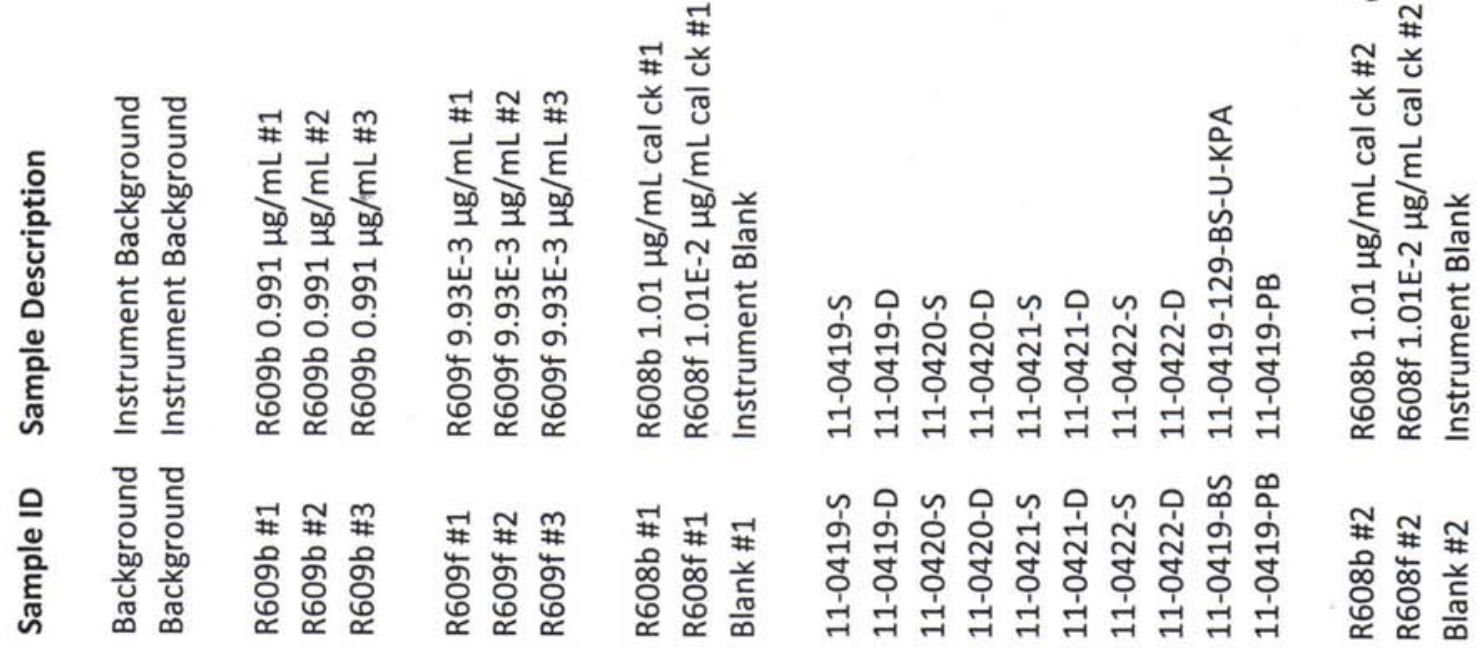


PNNL-20650, Rev. 2

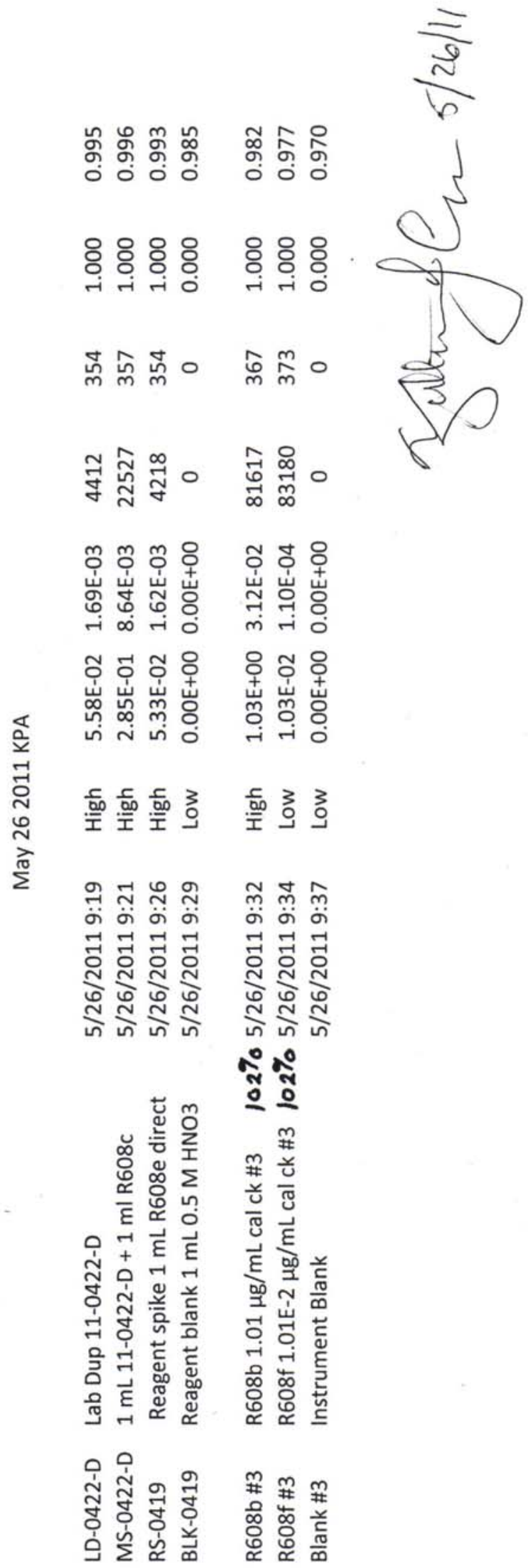

Page 2 of 2 


\section{Battelle - Pacific Northwest National Laboratory Analytical Support Operations -}

\section{Uranium Metal Concentration in K Basin Sludge Report - Revision 1} PO Box 999, Richland, Washington 99352

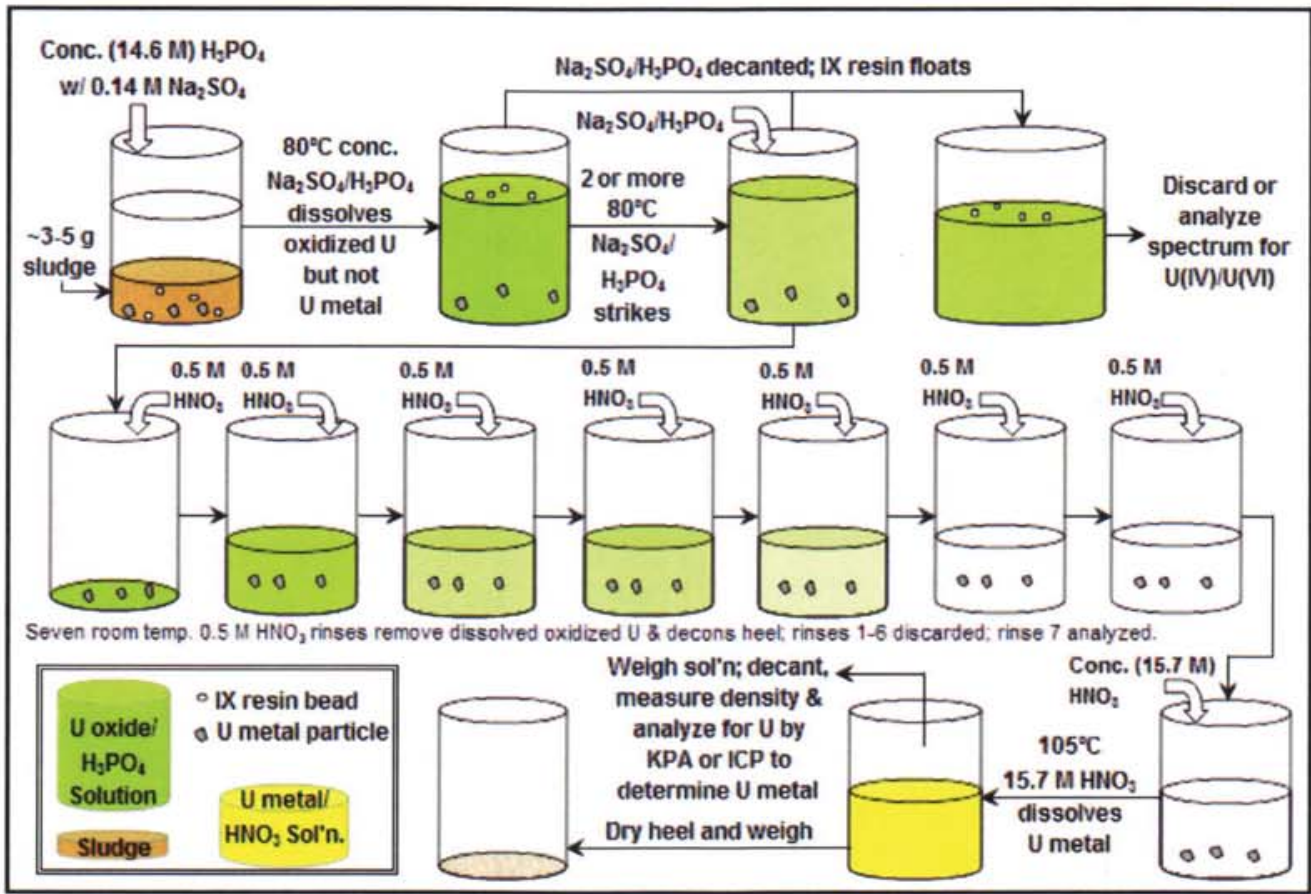

\begin{tabular}{|l|l|l|l|}
\hline Client: & Sandy Fiskum & ASR \#: & 8798 \\
\hline Project \#: & 52578 & \# Samples: & $9+1$ standard+1 process blank \\
\hline Charge Code: & H41220 & \\
\hline RPL Numbers: & $\begin{array}{l}\text { 11-0419-107-LCS, -B2, -S, -D; 11-0420-107-S, -D; 11-0421-107-S, -D, -MS; } \\
\text { 11-0422-107-S, -D }\end{array}$ \\
\hline
\end{tabular}

Procedure, Analysis, System, and Records Information

\begin{tabular}{|l|l|}
\hline Analysis Procedure: & $\begin{array}{l}\text { RPG-CMC-211, Rev. 3. Determination of Elemental Composition by Inductively } \\
\text { Coupled Argon Plasma Optical Emission Spectrometry (ICP-OES) }\end{array}$ \\
\hline Sample Prep: & $\begin{array}{l}\text { RPG-CMC-107, Rev. 1, Sample Preparation for Determination of Uranium Metal } \\
\text { Concentrations in Sludge } \\
\text { PNL-ALO-501, Rev. 0, Laboratory Procedure for Measurement of Physical and } \\
\text { Rheological Properties of Solutions, Slurries, and Sludges }\end{array}$ \\
\hline Analyst: & Cal Delegard/Nicole Green (digestion) and Jenn Carter (ICP-OES) \\
\hline Analysis Dates: & ICP-325-405-3 in Chemical Measurement Center 98620 file; 4 March 2011 \\
\hline $\begin{array}{l}\text { Calibration Date/ } \\
\text { Filename: }\end{array}$ & LCS: 25 February 2011 \\
\hline $\begin{array}{l}\text { Cal/Ver Stds Prep } \\
\text { Date: }\end{array}$ & $\begin{array}{l}\text { ICP: C0350, 4 March 2011 } \\
\text { Sample Analysis Excel } \\
\text { Data Files: }\end{array}$ \\
\hline M \&TE Numbers & $\begin{array}{l}\text { Balances 1114123894, Lab 516 and 25608154, Lab 312 (U metal beads; LCS and MS) } \\
\text { Balance N04143 (Container Tare) } \\
\text { Balance 40023610, SAL (solution weights); Balance 360-06-01-026 (solution density) } \\
\text { Balance Mettler AT400 M19445 (ICP-OES) } \\
\text { ICP-OES instrument N827583 (Lab 405) }\end{array}$ \\
\hline All Analysis Records & Chemical Measurement Center 98620: RIDS U-Metal System File \\
\hline
\end{tabular}


PNNL-20650, Rev. 2

\section{Battelle - Pacific Northwest National Laboratory Analytical Support Operations -}

Uranium Metal Concentration in K Basin Sludge Report - Revision 1

PO Box 999, Richland, Washington 99352

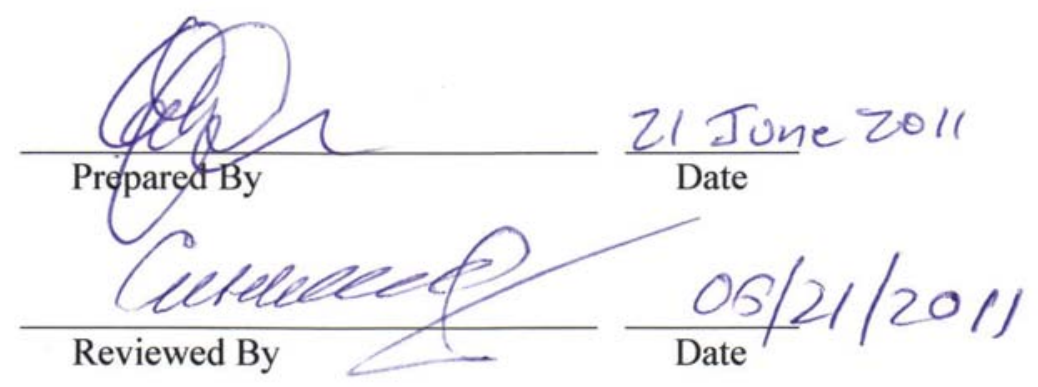

Page 2 of 6 


\section{Battelle - Pacific Northwest National Laboratory Analytical Support Operations - \\ Uranium Metal Concentration in K Basin Sludge Report-Revision 1 PO Box 999, Richland, Washington 99352}

\section{Revision 1}

The report was revised to provide amended values for $\left[\mathrm{U}_{\text {metal }}\right]$ in Table A.1 for the Process Blank 2 sample.

\section{Sample Results}

See Attachment 1: Uranium Metal Sample Results ASR 8798.

\section{Sample Analysis/Results Discussion}

The results of uranium metal concentration analysis of nine samples of K-Basin settler tube sludge submitted under Analytical Service Request (ASR) 8798 are reported. Nine samples were delivered for analysis as wet slurries with an overburden of supernatant water. The samples were analyzed in a single batch.

The samples for uranium metal analysis were digested according to the PNNL Technical Procedure, RPG-CMC-107, Rev. 1, Sample Preparation for Determination of Uranium Metal Concentrations in Sludge. The contacts with $0.14 \mathrm{M} \mathrm{Na}_{2} \mathrm{SO}_{4}$ in concentrated $\mathrm{H}_{3} \mathrm{PO}_{4}$ are designed to dissolve and remove the accompanying uranium oxides. ASR 8798 Special Instructions specified a minimum of three contacts with $0.14 \mathrm{M} \mathrm{Na}_{2} \mathrm{SO}_{4}$ in concentrated $\mathrm{H}_{3} \mathrm{PO}_{4}$. The second and third contact solutions were colorless to the eye, indicating good dissolution of the contained uranium oxides.

The residues from the $\mathrm{Na}_{2} \mathrm{SO}_{4} / \mathrm{H}_{3} \mathrm{PO}_{4}$ contacts were rinsed sequentially seven times in $0.5 \mathrm{M} \mathrm{HNO}_{3}$ and the rinsed heels, containing the undissolved uranium metal, were digested in concentrated $\mathrm{HNO}_{3}$ to dissolve the uranium metal, all according to the digestion procedure, RPG-CMC-107, Rev. 1. For each concentrated $\mathrm{HNO}_{3}$ digestate sample, mass and density were measured and uranium concentration in the digestate was measured by inductively coupled plasma optical emission spectrometry (ICP-OES) according to RPG-CMC-211, Rev. 3, Determination of Elemental Composition by Inductively Coupled Argon Plasma Optical Emission Spectrometry (ICP-OES). The sample digestion procedure followed under RPG-CMC-107 is outlined in the Figure at the top of page 1.

\section{Data Limitations}

None.

\section{Quality Control Discussion}

The method performance is evaluated against the acceptance criteria provided in ASR 8798. Values at the Process Quantitation Limit $(0.03 \mathrm{wt} \% \mathrm{U}$ in sludge $)$ or greater are considered to be quantified with an uncertainty of $\pm 25 \%$ (at $2 \sigma$, relative) or better.

Blanks: Reagent blanks are analyzed at least once in every batch of 20 samples. Blanks of the $0.5 \mathrm{M}$ $\mathrm{HNO}_{3}$ rinse solution and the concentrated $\mathrm{HNO}_{3}$ metal digestion solution were prepared with the sample batch; this result is in the supporting ICP-OES report. A blank containing only Hanford sand was included in the batch and was taken through all of the procedure steps. The blanks were near or below the

Page 3 of 6 
PNNL-20650, Rev. 2

\section{Battelle - Pacific Northwest National Laboratory Analytical Support Operations - \\ Uranium Metal Concentration in K Basin Sludge Report-Revision 1 PO Box 999, Richland, Washington 99352}

analytical detection limits established for the ICP-OES $(0.012 \mu \mathrm{g} / \mathrm{mL})$. The blank level for the $0.5 \mathrm{M}$ $\mathrm{HNO}_{3}$ reagent solution (Table A.1) was generally only a small contributor to the observed $7^{\text {th }}$ rinse solution concentrations and, even when near the observed rinse solution concentrations, did not interfere with the key observations of rinse solution concentration which become important at values greater than about $100 \mu \mathrm{g} / \mathrm{mL}$. Similarly, the concentrated $\mathrm{HNO}_{3}$ blank solution concentration contributed negligibly to the 17.8 to $5,920 \mu \mathrm{g} / \mathrm{mL}$ digestate concentrations. Because the objective of the $7^{\text {th }}$ rinse is to achieve low uranium concentrations (indicating low carryover from dissolved uranium oxides), the requirement that the prep blank concentrations be $<5 \%$ of the measured concentration values in the samples does not apply to the $7^{\text {th }}$ rinse concentrations.

Seventh Rinses: The uranium concentrations in the $7^{\text {th }} 0.5 \mathrm{M} \mathrm{HNO}_{3}$ rinses indicate the thoroughness of oxidized (i.e., non-metallic) uranium removal from the sludge. In developing this analytical technique (Delegard et al. 2008), uranium concentrations in the $7^{\text {th }}$ rinse less than $\sim 100 \mu \mathrm{g} \mathrm{U} / \mathrm{mL}$ gave good indication that the uranium oxides were dissolved and their solutions removed from the heel.

As shown in Table A.1, all of the $7^{\text {th }}$ rinse solution concentrations were less than $100 \mu \mathrm{g} / \mathrm{mL}$ in uranium. The rinse concentrations ranged from 0.08 to $0.81 \mu \mathrm{g} \mathrm{U} / \mathrm{mL}$ and indicate complete removal of uranium oxide from the sample. The uranium concentrations in the $7^{\text {th }}$ rinse were examined along with other batch quality control indicators, including duplicates, LCS results, and process blanks, in making data quality judgments.

Laboratory Control Samples (LCS): A single LCS was analyzed with the K Basin sludge samples. The LCS contained $\sim 0.5 \mathrm{~g}$ of $\mathrm{U}_{3} \mathrm{O}_{8}$ (weighed to the nearest $0.001 \mathrm{~g}$ ), $\sim 0.6 \mathrm{~g}$ of Hanford sand (from Arid Lands Ecology, ALE, Reserve; weighed to the nearest $0.001 \mathrm{~g}$ ), and $\sim 0.011 \mathrm{~g}$ of uranium metal (weighed to the nearest $0.00001 \mathrm{~g}$ ) present as a single near-spherical bead.

The LCS uranium metal recovery was $102 \%$ and was within the acceptance limits of $75-125 \%$ specified in the procedure RPG-CMC-107 and in the ASR 8798 Special Instructions.

Duplicate (Precision): A duplicate sample is required to be analyzed in every batch of 20 samples or fewer. This requirement was met.

Prior to this Project work, the MDL, $0.011 \mathrm{wt} \% \mathrm{U}_{\text {metal }}$ on a settled sludge basis, was determined by evaluating uranium metal analysis recovery data (defined as PDL in Attachment J of Fiskum et al. 2009). The ASR specifies an MDL of $0.01 \mathrm{wt} \%$, settled sludge basis. With the exception of one sample, all wt $\%$ results were above the ASR-specified MDL. Refer to footnote (a) of Table A.1.

The QAPjP/SAP (KBC-33786, Rev. 2) specifies that a $\pm 20 \%$ precision criterion for duplicates is to be applied when $\left[\mathrm{U}_{\text {metal }}\right]>0.1 \mathrm{wt} \%$. The $\left[\mathrm{U}_{\text {metal }}\right]>0.1 \mathrm{wt} \%$ threshold was exceeded for one $\mathrm{KW}-210$ duplicate sample set. The RPD for duplicate KW-210-A4 samples was 54\%. The Occurrence Report 52578-05-26-11 was generated to document the duplicate RPD values that did not meet the acceptance limits.

Matrix Spike: Based on the QAPjP/SAP (KBC-33786, Rev. 2), uranium metal measurement accuracy is to be assessed by evaluation of the LCS recoveries. However, a matrix spike (MS) sample was included to provide additional indicators of method performance. To prepare the MS sample, a uranium metal bead spike was added to one of three sludge subsamples taken from the same (B1) core. The amount of $U$ metal added as MS was about 20-times greater than the average amount of uranium metal found present in the other two B1 core subsamples. The MS recovery, adjusted for the uranium metal quantity expected based on the average concentration found in the companion subsamples, was $99 \%$.

Page 4 of 6 


\section{Battelle - Pacific Northwest National Laboratory Analytical Support Operations - \\ Uranium Metal Concentration in K Basin Sludge Report-Revision 1 \\ PO Box 999, Richland, Washington 99352}

\section{Deviations from Procedure}

There were no deviations from the procedure.

\section{General Comments}

In June 2009, based on discussions between PNNL and CHPRC, it was determined that matrix spikes were to be included within each analytical batch to be processed for uranium metal and uranium oxide oxidation state distribution. While the matrix spike samples were intended to provide an additional assessment of the procedure performance, performance or failure criteria for matrix spike recoveries were not established.

\section{References}

ASO-QAP-001, Rev. 7. 2009. Analytical Support Operations (ASO) Quality Assurance Plan, Pacific Northwest National Laboratory, Richland, WA.

Delegard CH, SI Sinkov, AJ Schmidt, and JW Chenault. 2008. Uranium Metal Analysis via Selective Dissolution. PNNL-17800, Pacific Northwest National Laboratory, Richland, WA.

Fiskum, SK, JV Crum, D Neiner, CJ Thompson, JM Billing, RC Daniel, BM Oliver, T Trang-Le, SJ Bos, CH Delegard, KN Pool, MW Urie, CA Burns, MK Edwards, AJ Schmidt, CD Carlson, LR Greenwood, RW Shimskey, DS Coffey, SA Jones, and CZ Soderquist, 2009, Characterization of K-Basin Containerized Sludge Samples Collected from Engineered Containers SCS-CON-240, 250, 260, and 220, PNNL-19035, Pacific Northwest National Laboratory, Richland, WA.

KBC-33786, Rev. 2, Quality Assurance Project Plan / Sampling and Analysis Plan for Sludge in the KW Engineered Containers, CH2M Hill Plateau Remediation Company, Richland, WA. December 2009. 
PNNL-20650, Rev. 2

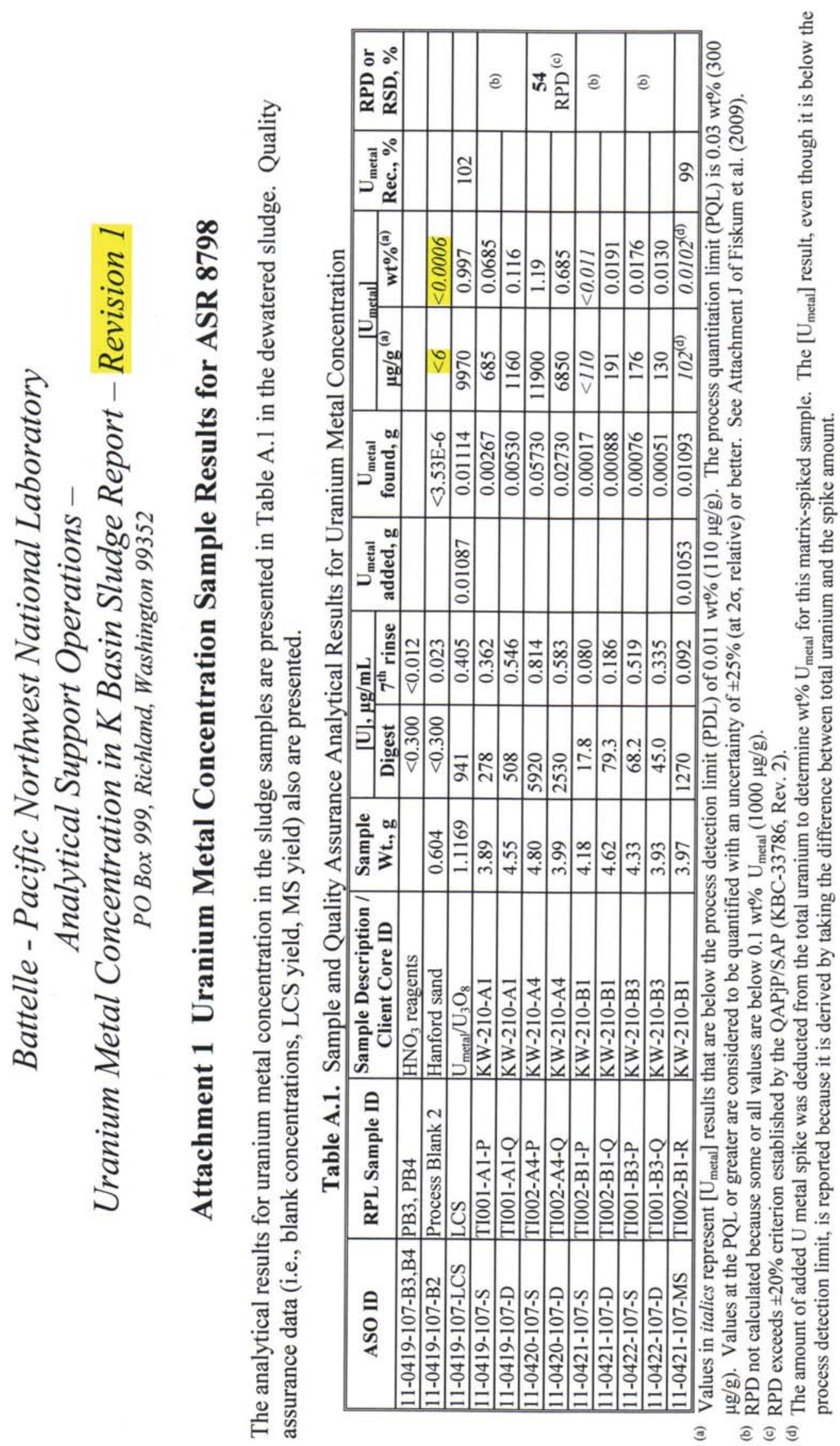

Page 6 of 6 
PNNL-20650, Rev. 2

\section{Battelle - Pacific Northwest National Laboratory Analytical Support Operations - \\ Uranium Oxidation States in K Basin Sludge Report \\ PO Box 999, Richland, Washington 99352}

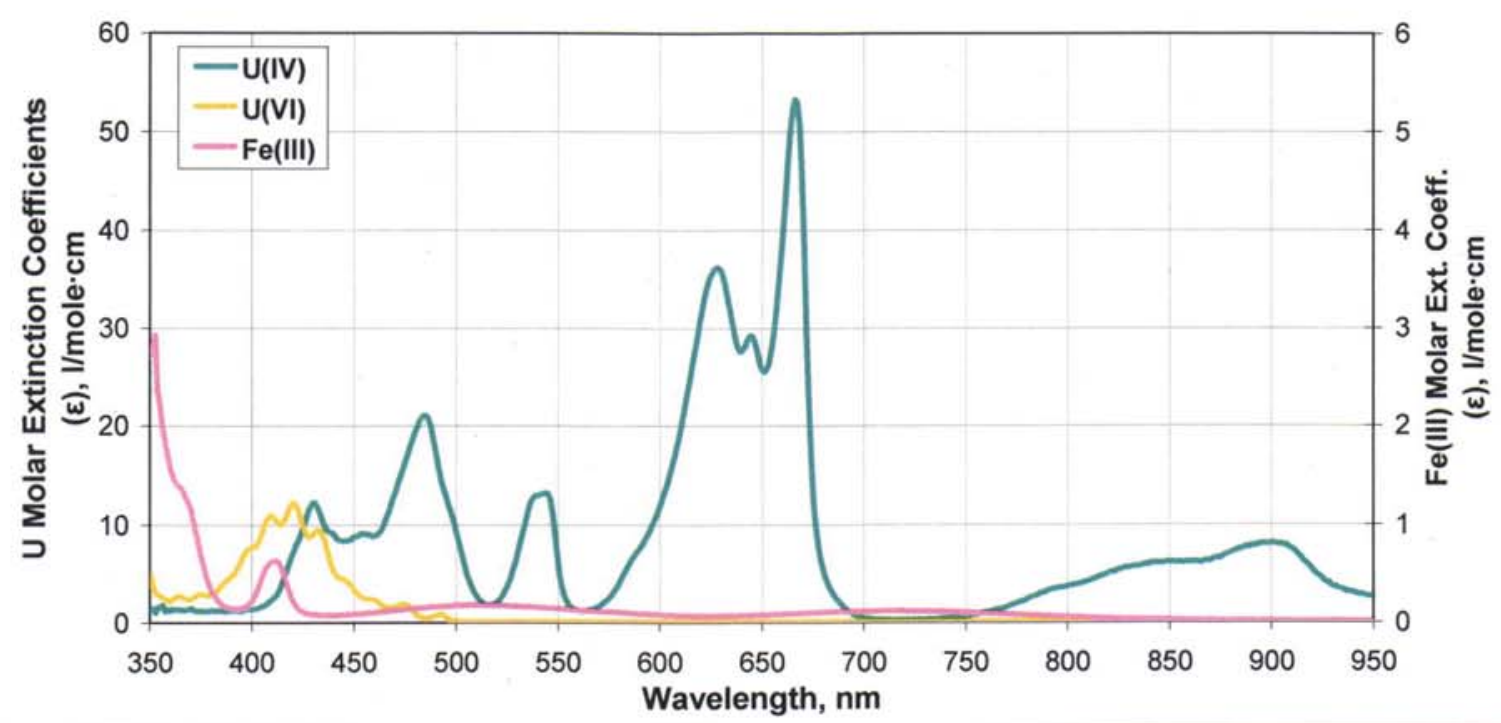

\begin{tabular}{|l|l|l|l|}
\hline Client: & Sandy Fiskum & ASR \#: & 8798 \\
\hline Project \#: & 52578 & \# Samples: & 9 samples, 1 standard, 1 blank \\
\hline Charge Code: & H41221 & \\
\hline RPL Numbers: & $\begin{array}{l}\text { 11-0419-107-LCS, -B2, -S, -D; 11-0420-107-S, -D; 11-0421-107-S, -D, -MS; } \\
\text { 11-0422-107-S, -D }\end{array}$ \\
\hline
\end{tabular}

Procedure, Analysis, System, and Records Information

\begin{tabular}{|l|l|}
\hline Analysis Procedure: & $\begin{array}{l}\text { RPG-CMC-255, Rev. 0, Sample Preparation and Analysis for Determining } \\
\text { Uranium Oxide Oxidation States in K Basin Sludges }\end{array}$ \\
\hline Sample Prep: & $\begin{array}{l}\text { RPG-CMC-107, Rev. 1, Sample Preparation for Determination of Uranium Metal } \\
\text { Concentrations in Sludge }\end{array}$ \\
\hline Analyst: & Sergey Sinkov \\
\hline Analysis Dates: & 8 March 2011 \\
\hline Calibration Date/ & $12 / 2008$ \\
Filename: & RPG-CMC-255 - U Oxidation State Calculation - Final.xls \\
\hline Cal/Ver Stds Prep Date: & 25 February 2011 \\
\hline Sample Analysis Excel & 030811 Spectra of H3PO4 digestates from hot cell - ASR 8798.xls \\
Data Files: & RPG-CMC-255 - U Oxidation State Calculation - ASR8798.xls \\
\hline M \&TE Numbers & Spectral Instruments SI 400 (property number WD13494) in Lab 516 \\
\hline All Analysis Records & Chemical Measurement Center 98620: Fileplan U Oxide System File \\
\hline
\end{tabular}

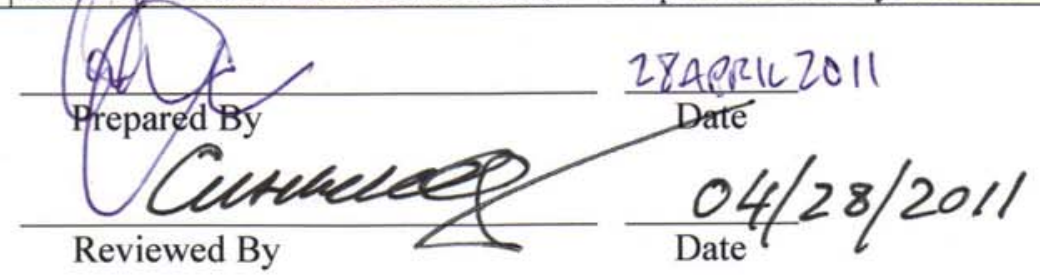

Page 1 of 4 


\section{Battelle - Pacific Northwest National Laboratory Analytical Support Operations - Uranium Oxidation States in K Basin Sludge Report PO Box 999, Richland, Washington 99352}

\section{Sample Results}

See Attachment: Uranium Oxide Oxidation States Sample Results for ASRs 8798.

\section{Sample Analysis/Results Discussion}

The results for the uranium oxidation state distribution of the uranium oxides in nine samples of K-Basin settler tube sludge submitted under Analytical Service Request (ASR) 8798 are reported. All samples were from container KW210.

The samples were prepared according to the PNNL Technical Procedure, RPG-CMC-107, Rev. 1, Sample Preparation for Determination of Uranium Metal Concentrations in Sludge, to dissolve and remove the accompanying uranium oxides. The digestates from the uranium oxide dissolutions were analyzed by spectrophotometry to determine the oxidation state distributions [to the U(IV) and U(VI) oxidation states] of the constituent uranium oxides according to the PNNL Technical Procedure, RPG-CMC-255, Rev. 0, Sample Preparation and Analysis for Determining Uranium Oxide Oxidation States in K Basin Sludges.

\section{Data Limitations}

The U(IV) and U(VI) oxidation state ratios should be used with caution. Ferrous iron, Fe(II), which is present in magnetite that has been observed in $\mathrm{K}$ Basin sludge, has been shown to reduce U(VI) to U(IV) in $\mathrm{Na}_{2} \mathrm{SO}_{4} / \mathrm{H}_{3} \mathrm{PO}_{4}$ solutions; the amount of $\mathrm{Fe}(\mathrm{II})$ is not known in these samples. The $\mathrm{U}(\mathrm{VI})$ fraction can be considered a lower bounding case and the U(IV) fraction can be considered upper bounding case.

\section{Quality Control Discussion}

The uranium oxidation state method performance is evaluated against the acceptance criteria established within procedure RPG-CMC-255, Rev. 0, Sample Preparation and Analysis for Determining Uranium Oxide Oxidation States in K Basin Sludge, any client specified special instructions provided in the ASR.

Blanks: The uranium oxide and many of the accompanying sludge phases were dissolved at $\sim 80^{\circ} \mathrm{C}$ by three sequential contacts with $0.14 \mathrm{M} \mathrm{Na}_{2} \mathrm{SO}_{4}$ in concentrated $\mathrm{H}_{3} \mathrm{PO}_{4}$. In accordance with the procedure, RPG-CMC-107, Rev. 1, Sample Preparation for Determination of Uranium Metal Concentrations in Sludge, a process blank of $0.14 \mathrm{M} \mathrm{Na}_{2} \mathrm{SO}_{4}$ in concentrated $\mathrm{H}_{3} \mathrm{PO}_{4}$ was prepared per the requirement that one process blank be prepared and analyzed at least once in every batch of 20 samples. The process blank contained $\sim 0.6 \mathrm{~g}$ of Hanford sand and showed $<0.001 \mathrm{M}$ each of $\mathrm{U}(\mathrm{IV})$ and $\mathrm{U}(\mathrm{VI})$, below the minimum concentrations detectible by the uranium oxidation state method, thus indicating the desired lack of interference by U(IV) and U(VI) contamination.

Laboratory Control Samples (LCS): A single Laboratory Control Sample (LCS) must be analyzed at least once in every batch of 20 samples. One LCS QC sample was analyzed in parallel with the K Basin sludge samples within the batch of 9 samples. The LCS QC sample contained $\sim 0.5 \mathrm{~g}$ of $\mathrm{U}_{3} \mathrm{O}_{8}$ and $\sim 0.6 \mathrm{~g}$ of Hanford sand (each weighed to the nearest $0.001 \mathrm{~g}$ ) and $\sim 0.01 \mathrm{~g}$ of uranium metal (weighed to the nearest $0.00001 \mathrm{~g}$ ) present as a single near-spherical bead. The U(IV) and U(VI) uranium oxidation state distributions in the dissolved $\mathrm{U}_{3} \mathrm{O}_{8}$ were measured to determine analytical performances of the uranium oxide oxidation state distribution analyses. Because $\mathrm{U}_{3} \mathrm{O}_{8}$ is effectively one part $\mathrm{U}(\mathrm{IV})$ and two parts 
PNNL-20650, Rev. 2

\section{Battelle - Pacific Northwest National Laboratory Analytical Support Operations - Uranium Oxidation States in K Basin Sludge Report PO Box 999, Richland, Washington 99352}

$\mathrm{U}(\mathrm{VI})$ on a mole basis, the $\mathrm{U}_{3} \mathrm{O}_{8}$ dissolved in the $\mathrm{Na}_{2} \mathrm{SO}_{4} / \mathrm{H}_{3} \mathrm{PO}_{4}$ solution ideally yields $33.3 \% \mathrm{U}(\mathrm{IV})$ and $66.7 \% \mathrm{U}(\mathrm{VI})$. Per the procedure, the relative amount of $\mathrm{U}(\mathrm{IV})$ in the $\mathrm{U}_{3} \mathrm{O}_{8} \mathrm{LCS}$ should vary by no more than $5 \%$, absolute, from the ideal $33.3 \%$ (i.e., from 28.3 to $38.3 \%$ ). The QAPjP/SAP specifies that the LCS values differ by no more than $25 \%$, relative, from the true values and thus affords greater tolerance margins (KBC-33786, Rev. 2). The LCS for this batch contained $33.7 \% \mathrm{U}(\mathrm{IV})$ and $66.3 \% \mathrm{U}(\mathrm{VI})$ and thus met the target performances for both the procedure and the less stringent QAPjP/SAP.

Duplicate (Precision): A duplicate sample is required to be analyzed in every batch (of 20 samples or fewer) to allow determination of method precision. This requirement was met. Each of the four samples was measured at least in duplicate. One sample pair yielded results that could be used to estimate sample duplicate precision for U(IV), 1.7 RPD, and U(VI), 14 RPD. There was no precision Data Quality Objective specified by the Project.

Per the ASO QA Plan (Pool and Coffey 2009), the relative percent difference (RPD) is calculated in cases where both U(IV) and U(VI) concentrations are measured at or above the $0.001 \mathrm{M}$ minimum detectible concentration for two samples. The $\geq 0.001 \mathrm{M} \mathrm{U}(\mathrm{IV})$ and U(VI) concentration criteria were met for one of the four samples (from core KW210-A1). The other three samples had $<0.001 \mathrm{M} \mathrm{U}(\mathrm{VI})$. The reported RPD for the U(IV) mole\%, shown in Table A.1, was $1.7 \%$ and the RPD for U(VI) was $14 \%$. No requirement on uranium oxidation state RPD was provided in the ASR special instructions.

The QAPjP/SAP states that results should agree $\pm 5 \%$ (absolute) if concentrations greater than $0.001 \mathrm{M}$ in each oxidation state are present (KBC-33786, Rev. 2). The individual KW210-A1 uranium oxidation state values are within $\pm 5 \%$, absolute, of their average or 84.2 to $94.2 \%$ for U(IV) and 5.8 to $15.8 \%$ for $\mathrm{U}(\mathrm{VI})$. Therefore, this objective was met.

A single matrix spike (MS) sample was run for KW210-B1. This MS contained an added bead of uranium metal. The uranium metal spike should not alter the uranium oxide oxidation state distribution. Like the associated sample and duplicate uranium oxidation state analyses for KW210-B1, the U(VI) concentration for the MS was $<0.001 \mathrm{M} \mathrm{U}(\mathrm{VI})$. No Data Quality Objectives are associated with the oxidation state result for the MS sample.

\section{Deviations from Procedure}

There were no deviations from the procedure RPG-CMC-255, Rev. 0, Sample Preparation and Analysis for Determining Uranium Oxide Oxidation States in K Basin Sludges.

\section{References}

KBC-33786, Rev. 2, Quality Assurance Project Plan / Sampling and Analysis Plan for Sludge in the KW Engineered Containers, CH2M Hill Plateau Remediation Company, Richland, WA. December 2009.

Pool, KN, and DS Coffey. 2009. Analytical Support Operations (ASO) Quality Assurance Plan, ASOQAP-001, Rev. 7, Pacific Northwest National Laboratory, Richland, WA. 
PNNL-20650, Rev. 2

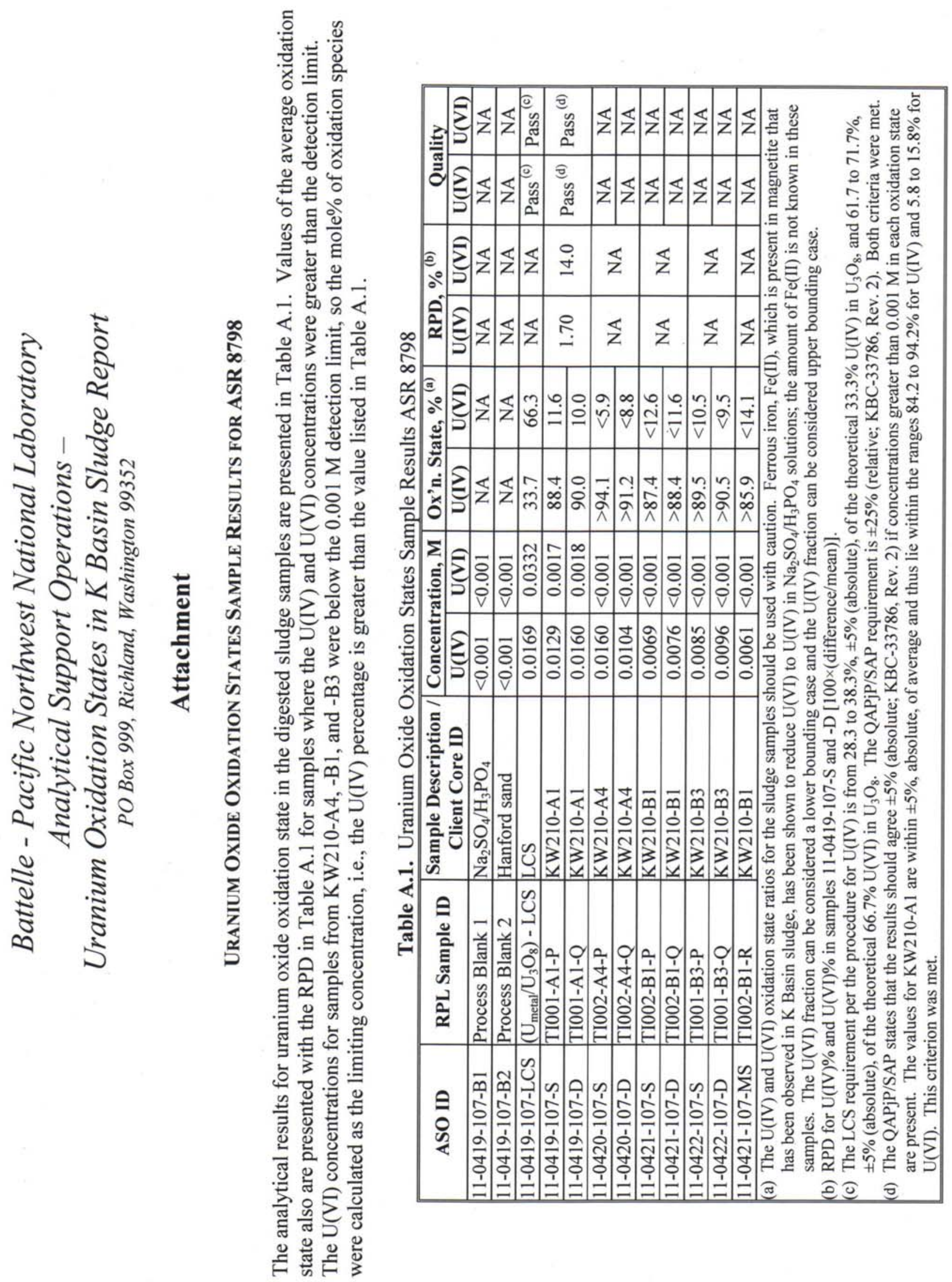

Page 4 of 4 
WW Rutherford

K Basin Sludge Treatment Project, Manager Sampling and Characterization

CH2M Hill Plateau Remediation Company

P.O. Box 1600

Richland, WA 99352

Dear Mr. Rutherford:

\section{EVALUATION OF LOW Sb, Se, and TI RECOVERIES IN K-BASIN MATRIX QC SAMPLES}

During the inductively coupled plasma optical emission spectrometry (ICP-OES) analyses of KBasin samples, the recovery quality control samples of antimony, selenium and thallium have not met the requirements of the QjPAP/SAP. The Analytical Services Organization (ASO) staff evaluated the issues involved and have prepared the attached document provide a summary of the issues leading to this failure per your request.

This letter will be referenced in future ASO ICP-OES reports when similar low matrix spiked analyte recoveries are found as the means to help explain the underpinning issues.

Should you have any questions please feel free to call me (376-2912) or Sandy Fiskum (3767015).

\section{Sincerely,}

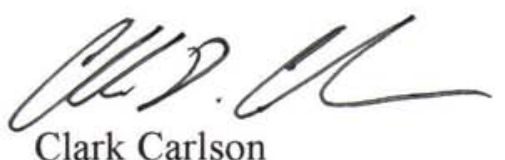

PNNL Project Manager

Enclosures 


\section{WW Rutherford}

February 15, 2011

Page 2

cc: WW Rutherford, MS A3-06

RB Baker, MS A3-06

STP Project File, (N. Fouad, H6-08)

bcc: CD Carlson, P7-25

SK Fiskum, P7-25

RM Shimskey, P7-27

AJ Schmidt, P8-60

KN Pool, P7-25

BM Oliver, P7-22

52578 Project File (PDF) 


\section{Evaluation of Low Sb, Se, and TI Recoveries in K-Basin Matrix QC Samples}

Inductively coupled argon plasma optical emission spectroscopy (ICP/OES) analyses of K-basin samples have demonstrated less than ideal performance in the quantification of $\mathrm{Se}, \mathrm{Sb}$ and $\mathrm{Tl}$ at the detection limits requested by the K-basin project. Specifically, the matrix spike (MS) (and Se post spike [PS]) quality control samples run with the sample batches showed recoveries ranging from $95 \%$ to as low as $48 \%$ (summarized in Table 1). The acceptance criteria for the MS and PS are 80 to $120 \%$ recoveries. The batch laboratory control sample (LCS) recoveries (matrix-free condition) met acceptance criteria of 80 to $120 \%$. Sample heterogeneity was considered to have factored into poor MS recoveries; however these elements in the MS samples recovered consistently $<100 \%$ indicating a consistent negative bias as opposed to a random variation where some recoveries $>100 \%$ would be expected. The generally consistent low MS and PS recoveries for these elements prompted a more detailed look at the method capability, instrument performance, and spectral interferences for these analytes in the K Basin sludge matrix.

Table 1. QC Summary of Selected ASRs (\% Recovery)

\begin{tabular}{cccccccccc}
\hline & \multicolumn{3}{c}{ MS } & \multicolumn{3}{c}{ LCS } & \multicolumn{3}{c}{ PS } \\
& $\mathrm{Sb}$ & $\mathrm{Se}$ & $\mathrm{Tl}$ & $\mathrm{Sb}$ & $\mathrm{Se}$ & $\mathrm{Tl}$ & $\mathrm{Sb}$ & $\mathrm{Se}$ & $\mathrm{Tl}$ \\
\hline ASR 8420 & -- & -- & -- & -- & -- & -- & 86 & 77 & 83 \\
ASR 8429 & 79 & Not & 76 & 104 & 108 & 95 & 88 & Not & 80 \\
ASR 8453/8487 & 83 & 81 & 70 & 86 & 84 & 80 & 94 & 95 & 85 \\
ASR 8608 & 59 & 48 & 61 & 99 & 107 & 98 & 91 & 79 & 87 \\
\hline Average & 74 & 65 & 69 & 96 & 100 & 91 & 90 & 84 & 84 \\
\hline
\end{tabular}

Method Capability - The ICP/OES technique is limited in detection capability for $\mathrm{Se}$, $\mathrm{Sb}$ and $\mathrm{TI}$ due to their poor emission intensities. The current instrument detection limits (IDL) for these elements by the Analytical Support Operations (ASO) ICP/OES are $0.17 \mathrm{ppm}, 0.022 \mathrm{ppm}$ and $0.086 \mathrm{ppm}$ respectively. The IDL values are determined annually using solutions that are matrix matched to the matrices most commonly analyzed by the ASO. The method detection limit for these elements is directly affected by the processing factor associated with the sample preparation. To determine the method detection limit (MDL) for each sample, the IDL is multiplied by the processing factor associated with the sample preparation. To obtain the best possible detection capability for the K-basin samples, the digest solutions coming from the hotcell were run without additional dilution (run direct as-received) on the ICP/OES.

A more detailed evaluation was conducted on ASR 8608 samples, which demonstrated the highest variance from the QAPjP/SAP acceptance criteria. Table 2 summarizes the actual detection limits achieved for each ASR 8608 sample compared to the required detection limit requested by the project. ${ }^{(a)}$ The sample specific MDL's were $1.1 x$ to $10 x$ lower than the client-requested detection limits.

(a) Larger sample size and smaller digestate volumes might be realized; however the concentration of matrix analytes (e.g., $\mathrm{U}$ and $\mathrm{Fe}$ ) can cause additional interferences. 
Selenium is the most challenging of the elements with MDL levels 1.1x (TI21-MC) to 1.7x (TI021-A) of the client-required MDL, leaving little room for additional dilution. With $\mathrm{K}$ Basin samples containing upwards of $34 \mathrm{wt} \% \mathrm{Fe}$ and $\mathrm{U}$, application of a nominal process factor of 100 results in a ICP/OES analysis solution containing 3,300ppm Fe and 3,500ppm U.

Table 2. Achieved Detection Limits for Each K-Basin Sample (ASR 8608)

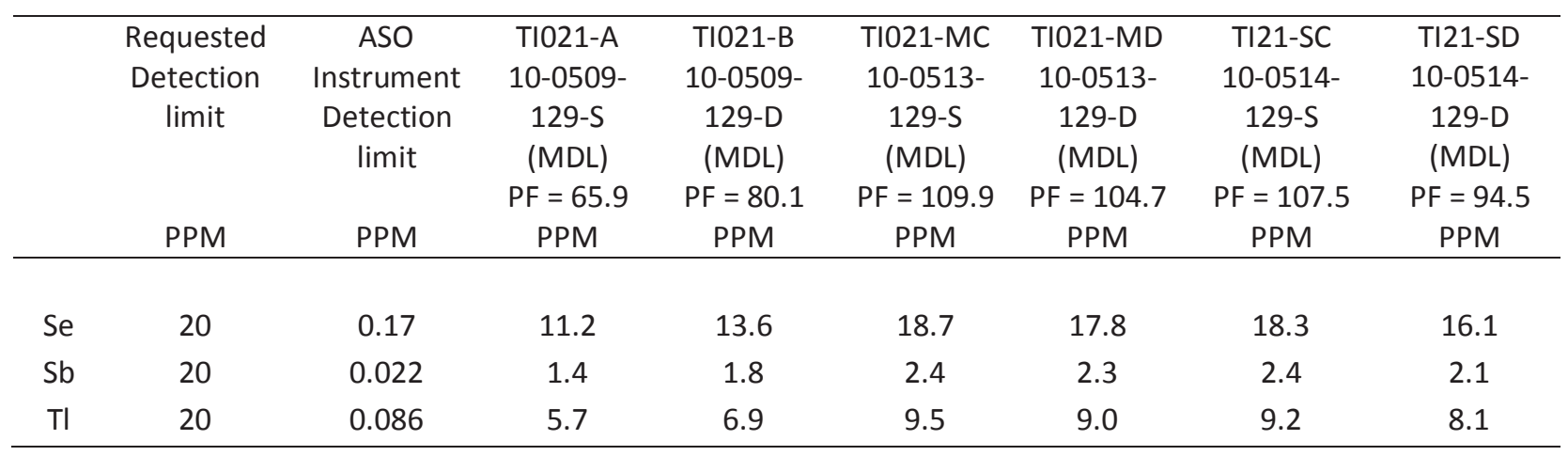

Instrument Performance - During the analyses of the K-basin samples, numerous instrument quality control samples were run to verify the instrument performed within specification during the entire analyses process. Instrument QC samples include initial and continuing calibration verification standards, initial and continuing calibration blanks, low level standard and interference check standard (ICS). All instrument QC passed the method acceptance criteria for Se, Sb, and TI for all the ASR's listed in Table 1. Ongoing instrument performance has also been confirmed through performance evaluation (PE) sample programs. The ASO has passed the most current PE sample for $\mathrm{Se}, \mathrm{Sb}$ and $\mathrm{TI}$ and historically has passed on these 4 elements.

Spectral Interferences - The ICP/OES system software captures the spectral data collected for each sample during the analyses run. The spectral data can be retrieved and evaluated by the ICP/OES scientist. The K-basin samples, where the undiluted digestates were analyzed for $\mathrm{Se}, \mathrm{Sb}$ and $\mathrm{Tl}$, were processed knowing the sample solution matrix did not match the matrix used for calibration and calibration verification standards. In this case, the K-basin samples contained a mix of $\mathrm{HNO}_{3}$ and $\mathrm{HCl}$ diluted to a nominal concentration of $1 \mathrm{M}$ to $2 \mathrm{M}$. The ICP/OES calibration and calibration verification solutions use $0.8 \mathrm{M} \mathrm{HNO}_{3}$ sample matrix. Additionally the high concentrations of Fe and $\mathrm{U}$ in the undiluted digestate samples typically caused these analytes to saturate the detector resulting in the instrument not using the inter-element correction (IEC) factors for Fe and $U^{(b)}$. To mitigate the effect of not applying IECs, the ICP/OES scientist carefully evaluated the spectral lines to be used for $\mathrm{Se}$, $\mathrm{Sb}$ and $\mathrm{TI}$ in the presence of high concentrations of Fe and $\mathrm{U}$. The spectral lines selected for $\mathrm{Se}, \mathrm{Sb}$ and $\mathrm{TI}$ provided the highest sensitivity with the lowest spectral interference potential.

(b) The instrument software does not perform the inter-element correction because the concentration of the saturating element(s) is unknown, thus the appropriate factor to apply to the other elements present in the spectra cannot be determined. The instrument flags the element as "Saturated" so the user is aware the IEC are not being applied for that element. 
Critical to the proper quantification of all elements by the ICP/OES technique is determination of the back ground subtraction points used for each spectral peak. Background subtraction points are carefully selected by the ICP/OES scientist to optimize the analysis method. These points are selected using the solution matrix that is most commonly run on the instrument, $0.8 \mathrm{M} \mathrm{HNO}_{3}$. As noted earlier, the sample matrix analyzed for the $\mathrm{K}$-basin samples was a mix of $\mathrm{HNO}_{3}$ and $\mathrm{HCl}$. The spectra showed a substantial increase in background levels in the spectral regions of the 4 target analytes. The background levels not only increased but the shape of the background baseline also changed, showing more sloping and irregular contour. The Se was especially impacted due to a large peak shoulder coming from the Fe emission peak pulling the background levels up. This change in background baseline impacted how the background subtraction corrects the spectral peaks for the region of interest used for sample quantification.

As an example of these matrix effects, the Se spectra for samples 10-0509-129-S (blue) and matrix spike sample 10-0509-129-MS (white) are shown in Fig. 1. The highlighted green data points represent the detector array pixels sampled for Se for 10-0509-129-S. The green "cross" indicates the location of the single spectral point in the method used for background subtraction. The large peak at the right side is a secondary peak from the Fe component in the sample. As is evident, the signal intensities for the five highlighted points are all lower than the background subtraction line represented by the horizontal white dashed line, which results in a negative concentration value for Se of -1.8ppm after background subtraction. No other points near the Se peak emission signal are less than the observed Se peak area intensity or less than the selected background point. Note that defining the background within the integration peak area (which would result in a calculated 0 ppm Se as opposed to a negative Se concentration) is not a defensible practice. The corresponding five pixels for 10-0509-129-MS (not highlighted) result in a background-corrected concentration of $1.9 \mathrm{ppm}$. For this particular sample matrix, there is no background location within the integration window which would provide for a positive corrected concentration value for the sample. 


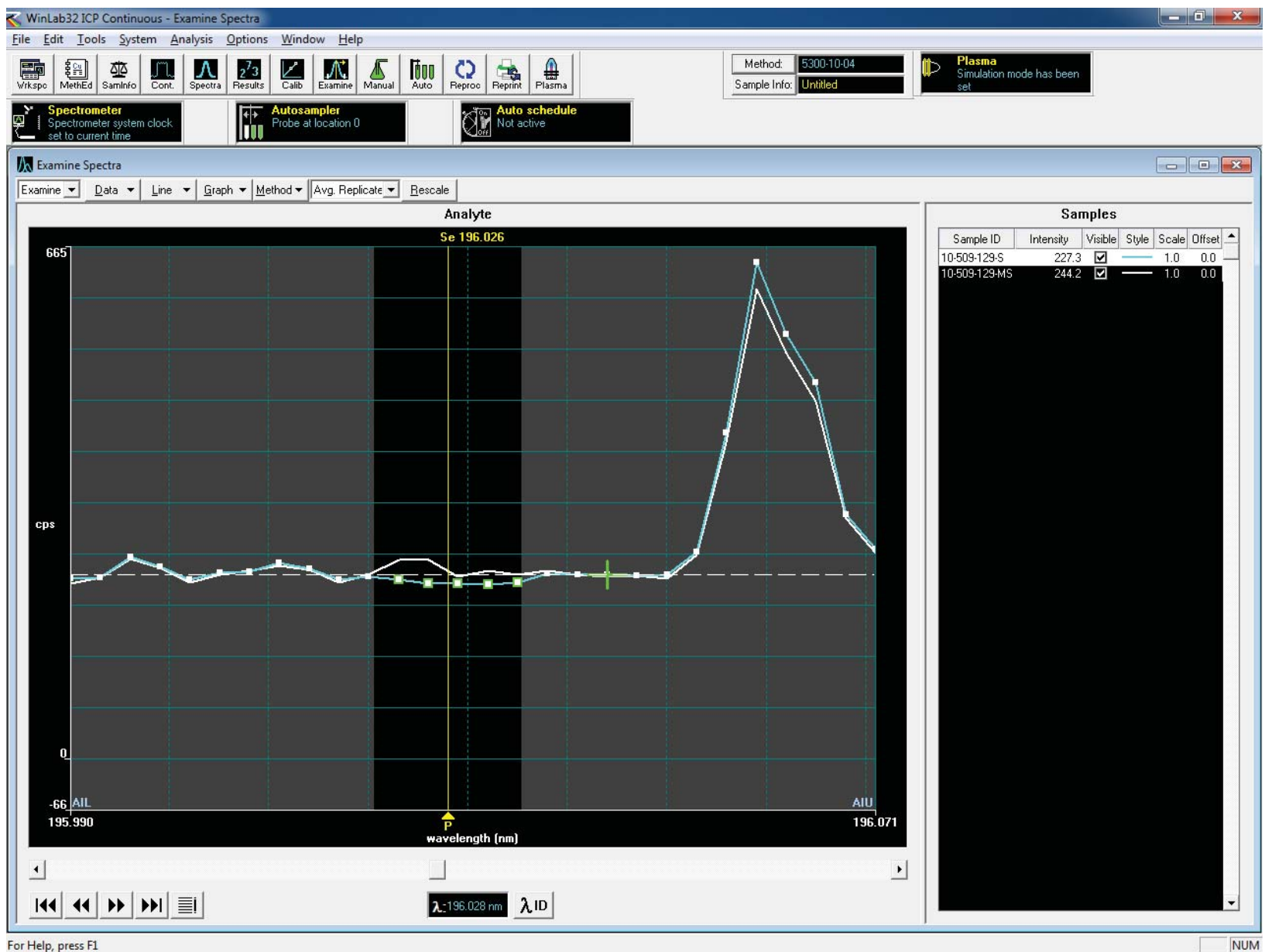

Figure 1. ICP-OES Spectra for Sample 10-0509-129-S (Blue and Green Highlight) and Matrix Spike Sample 10-0509-129-MS (White)

The matrix-specific QC samples were equally affected by the background baseline shape and the resulting over-correction from the background subtraction. The ASO does not use negative concentration results when determining sample and matrix QC concentrations. Results that are below the optimized background subtraction points indicate the concentration present is so close to the background that slight fluctuations in the integrations of the signal will cause some results to be slightly negative while others may be slightly positive. All negative concentration results are treated as $0.0 p p m$ concentration for the specific element. Samples from ASR 8608 were investigated more thoroughly. The Se concentrations detected at the instrument (not dilution-corrected) are summarized in Table 3.

Table 3. Se Results for ASR 8608, Sample 10-0509

\begin{tabular}{|l|l|l|l|}
\hline & $\begin{array}{l}\text { Measured } \\
\mathrm{ppm}\end{array}$ & $\begin{array}{l}\text { Reported Values and } \\
\text { Recoveries }\end{array}$ & $\begin{array}{l}\text { Alternative Recovery } \\
\text { Calculation }\end{array}$ \\
\hline $10-0509-129-\mathrm{S}$ & -1.8 & $<\mathrm{MDL}$ & $<\mathrm{MDL}$ \\
\hline $10-0509-129-\mathrm{D}$ & -1.1 & $<\mathrm{MDL}$ & $<\mathrm{MDL}$ \\
\hline $10-0509-129-\mathrm{MS}$ & 1.9 & $+(1.9-0) / 4.0=48 \%$ & $(1.9--1.8) / 4.0=93 \%$ \\
\hline $\mathrm{PS}^{(\mathrm{a})}$ & 3.97 & $(3.97-0) / 5.0=79 \%$ & $(3.97--0.9) / 5.0=97 \%$ \\
\hline (a) The action of post-spiking resulted in $2 \times$ sample dilution.
\end{tabular}


The negative Se concentration (measured at the instrument, not dilution corrected) calculated for the sample (10-0509-129-S) was -1.8ppm and for the duplicate (10-0509-129-D) was -1.1ppm. The actual amount of Se spike present in the matrix spike sample was 4ppm. The measured concentration of Se in the MS sample (at the instrument and not dilution corrected) was $1.9 \mathrm{ppm}$. The actual sample result of $-1.8 \mathrm{ppm}$ gets zeroed out instead of added back into the MS result. If the sample result of $-1.8 \mathrm{ppm}$ is not zeroed out and added back to the MS results, the MS Se concentration would be $3.7 \mathrm{ppm}$ (93\% recovery). The situation is similar for the PS sample. In this case, sample 10-0509-129-D was diluted 2x with the PS solution. The Se concentration in the PS solution was $10 \mathrm{ppm}$; after $2 \times$ dilution it was 5ppm at the instrument. The measured Se concentration of the PS was $3.97 \mathrm{ppm}$. The measured sample Se concentration is $-1.8 \mathrm{ppm}$, thus $-0.90 \mathrm{ppm}$ in the PS solution due to the $2 \times$ dilution. Since the sample result is zeroed out, the PS result is calculated using the $3.96 \mathrm{ppm}$ result and does not add back in the $-0.90 \mathrm{ppm}$ from the sample. Had the $-0.90 \mathrm{ppm}$ been added back to the PS sample, the result would have been $4.87 p p m$ or $97 \%$ recovery.

Similar spectral plots of Sb and TI for sample 10-0509-129-S (blue trace with green highlight) and 100509-129-MS (white trace) are shown in Figs. 2 and 3, respectively. In Fig. 2, the Sb spectra are positive for both the sample and MS, and thus the location of the background point did not contribute to the MS under-recovery. In Fig. 3, the TI value is slightly negative for the sample, but this was not a significant contributor to the MS under-recovery. Thus, relocation of the background point to provide a positive TI signal for the sample would not have increased the MS TI recovery to any significant extent. Thus there appear to be additional effects associated with the sample matrix that are influencing the calculated under-recovery of the Sb and TI matrix spikes that are not well understood. 


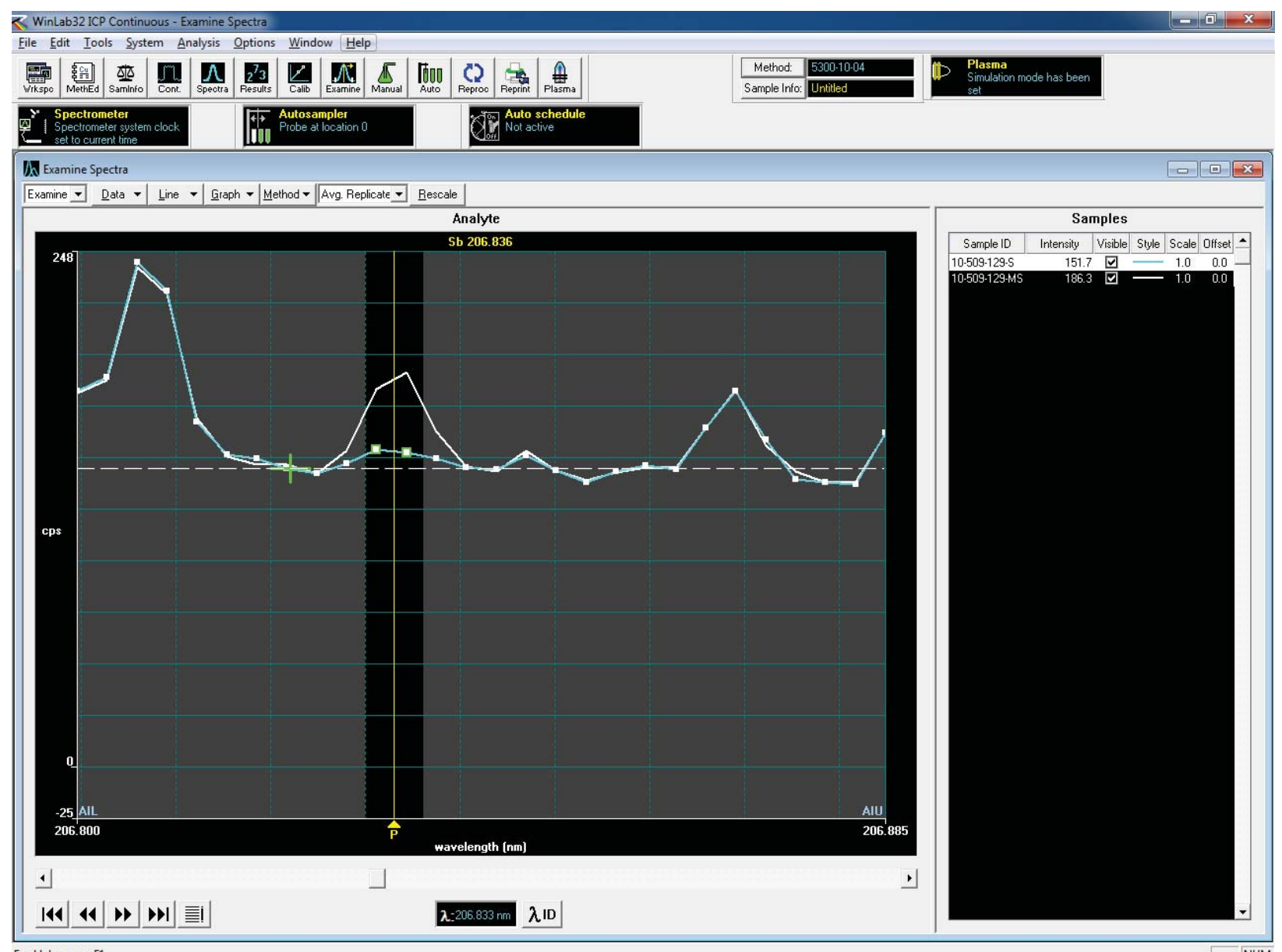

For Help, press F1

Figure 2. Sb Spectra for 10-0509-129-S (Blue and Green Highlight) and Matrix Spike Sample 10-0509-129-MS (White) 


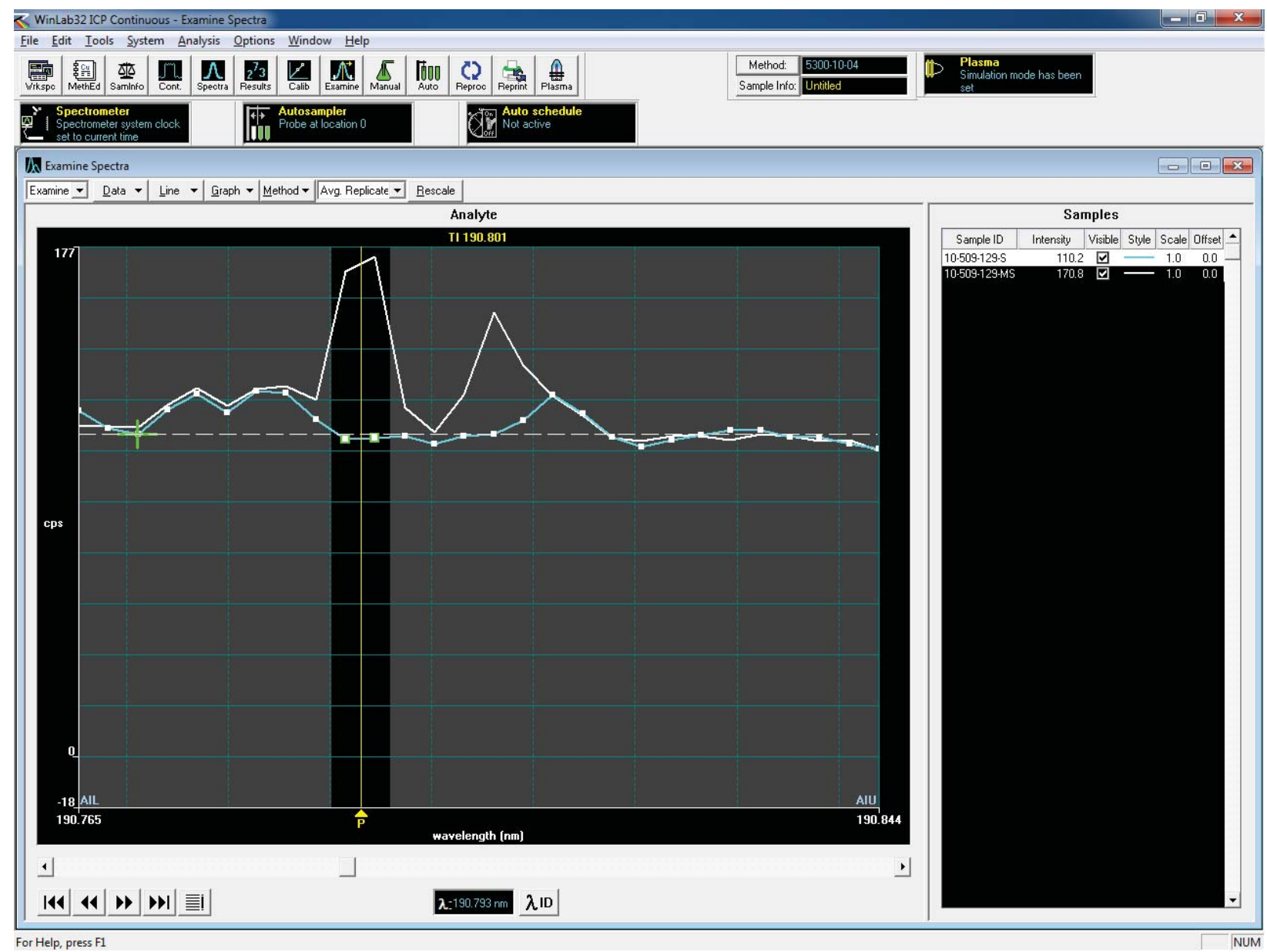

Figure 3. TI Spectra for 10-0509-129-S (Blue and Green Highlight) and Matrix Spike Sample 10-0509-129-MS (White)

The same affects in spectral background were observed in previously analyzed K-basin samples from earlier core sample analyses. The background corrections applied to the $\mathrm{Se}$, Sb and $\mathrm{Tl}$ for the current Kbasin work are consistent with back ground corrections applied to the earlier K-basin work.

Spiking Levels - The matrix related issues are confounded by the spiking levels (i.e., low concentrations) that are optimally designed for Toxic Characteristic Leaching Procedure (TCLP) related work. Some laboratories overcome shifting background issues by using matrix QC standards at higher concentrations, thus the analyte emission signal is substantially above background and shifting background changes have minor impacts on matrix QC sample recoveries. The ASO has not taken this approach, but instead has built our matrix QC standards to be near the regulatory threshold levels for the TCLP samples. ${ }^{(c)}$

(c) Spiking at levels near the regulatory threshold provides more accurate representation on how the analytes of interest are behaving in the sample matrix near the regulatory limit. Over-spiking (spikes for TCLP analytes significantly above the regulatory threshold) can provide a false representation of quantification of the target analyte near the regulatory threshold. 
Alternate analytical techniques that provide lower detection capability for $\mathrm{Se}$, $\mathrm{Sb}$ and $\mathrm{Tl}$ (graphite furnace atomic absorption) are commonly used in the regulatory community to quantify these elements. This capability is not currently available through the PNNL ASO.

Conclusions - The limitations of method capability for these elements and the low levels present in the matrix QC standards are expected to produce similar low recoveries for the MS and PS samples associated with the $\mathrm{K}$ Basin samples high in Fe and $\mathrm{U}$ concentrations. The low Se recovery in the MS samples would suggest a persistent low bias in the measured sample concentration at or near the detection limit. Future analyses of K-Basin samples for $\mathrm{Se}$, Sb and TI will likely result in matrix QC sample spike recoveries falling near or below the $80 \%$ acceptance threshold. Future reports for these analytes may include a statement such as "Se, Sb, Tl analyte concentrations at or near the MDL are likely biased low" depending on the batch-specific MS and PS sample recovery evaluations. Reference to this letter transmittal will be included in the future reports as means for explaining low bias issues. 
PNNL-20650, Rev. 2

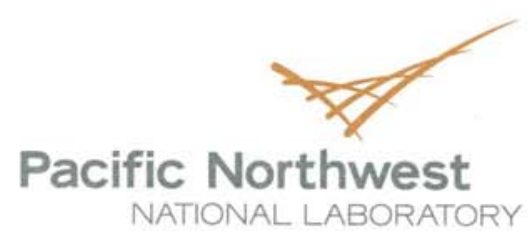

$\begin{array}{ll}\text { Tel: } & (509) 376-2319 \\ \text { Fax: } & (509) 373-6001 \\ \text { MSIN: P7-25 } \\ \text { clark.carison@ipnl.gov }\end{array}$

March 14, 2011

59205-2011-L04

WW Rutherford

K Basin Sludge Treatment Project, Manager Sampling and Characterization

$\mathrm{CH} 2 \mathrm{M}$ Hill Plateau Remediation Company

P.O. Box 1600

Richland, WA 99352

Dear Mr. Rutherford:

\section{ISSUES WITH MEETING LEAD METHOD DETECTION LIMIT AND QUALITY CONTROL SAMPLE ACCEPTANCE CRITERIA}

During the analytical campaigns PNNL has been performing for the Sludge Treatment Project of CHPRC, there have been several cases where we have not met various Quality Control (QC) requirements, including detection limits and spike recoveries. The QC samples which have not been missed are those related to the functioning of the instruments and the appropriateness of the methods used (i.e. preparation blanks, laboratory control samples, initial and continuing verification samples). The samples that have been missed have generally been the result of sample inhomogeneity (whether associated with subsampling in the hot cells or at the basins) and/or complications from the sample matrix. A point that needs to be made at this juncture is that just because a QC sample result did not meet its acceptance criteria, it does not mean there was a failure of the analysis. Two main reasons that we have observed failures in the QC sample results are associated with the 1) sample matrix complexity and the potential for inhomogeneity when the sample is subdivided into small subsamples-samples sizes are limited to reduce dose consequences to analysts and to accommodate the digestion or processing procedure sample size limitations; and 2) sample matrix inter-element effects. This letter will concentrate on the failures of meeting the required inductively coupled plasma-optical emission spectroscopy (ICP-OES) QC acceptance limits for the samples analyzed under ASR 8725, but also apply to past QC issues and may be indicated in future results. The table at the end of this letter shows the crosswalk between the results and responses by PNNL and the WIPP Table C3-9. As a part of the corrective actions for not meeting the QC criteria, this letter will be included in the final data packages for both Containers SCS-CON 230 and SCSCON 210.

An issue that needs to be addressed when discussing QC sample analysis failure is the intended application of the digestion and analysis procedures. SW-846 and other USEPA procedures are generally intended for common matrices, such as soils and surface and ground waters. The samples from the K- 
WW Rutherford

March 14, 2011

Page 2

Basins are more complex containing target analytes in a U-rich environment $;^{(1)}$ these matrices are not considered when the USEPA developed method QC sample acceptance criteria.

During the ICP-OES analysis performed on ASR 8725 samples, there were four kinds of QC failures observed:

1) the lead $(\mathrm{Pb})$ method detection limit (MDL) was higher than the QAPjP/SAP-required MDL $(20 \mu \mathrm{g} / \mathrm{g})$;

2) the precision of $\pm 20 \%$ relative percent difference (RPD, based on duplicate sample analysis) was not met for beryllium $(\mathrm{Be})$ in core $\mathrm{A} 3$ and for calcium $(\mathrm{Ca})$, chromium $(\mathrm{Cr})$, nickel $(\mathrm{Ni})$, and zirconium $(\mathrm{Zr})$ in core $\mathrm{B} 4$; and

3) the accuracy ( 80 to $120 \%$, matrix spike sample analyte recovery on core sample A3) was not met for arsenic (As), selenium (Se), chromium (Cr) and thallium ( $\mathrm{Tl})$; and

4) the accuracy $(<10 \%$, serial dilution on core sample A2) was not met for arsenic (As).

The failure to meet the MDL for lead $(\mathrm{Pb})$ is directly related to the process factor (overall dilution the sample is subjected before measuring ICP-OES analytes). The dilutions required to bring the samples to the bench for handling (addressing ALARA concerns) in addition to the dilutions required to reduce the effect of interferents (uranium in this case) make the effective sample-specific MDL slightly higher than the limit required by the QAPjP/SAP of $20 \mu \mathrm{g} / \mathrm{g}$, but still below the value of $100 \mu \mathrm{g} / \mathrm{g}$ required by WIPP in Table B3-8. ${ }^{(2)}$ Efficacy of measuring the Pb concentration on less-diluted samples was evaluated. The $\mathrm{U}$ interference on the $\mathrm{Pb}$ emission line was significant and required an inter-element correction; however the $U$ concentration in the less-diluted sample exceeded the linear range on the ICP-OES, precluding the correct application of an inter-element correction. In order to rectify this issue, two possible corrections could be made. The first would be to perform a different analysis technique, such as Atomic Absorption Spectroscopy, which we are not set up to perform and would require sending the samples off for analysis at another laboratory. The second potential correction would be to separate out the interferents, which would require significant expense and lead time for method qualification.

The precision failures of three analytes $(\mathrm{Cr}, \mathrm{Ni}$, and $\mathrm{Zr})$ in the $\mathrm{B} 4$ core samples are indicative of the variable presence of stainless steel and/or zircaloy cladding. A slight inhomogeneity issue with stainless steel and/or zircaloy particles and their partition to the analytical subsamples would explain the differences between duplicates. The failure of the precision for Ca cannot be simply explained. Reanalysis could be performed, but the likelihood of inhomogeneity in any additional analyses would be present and may affect other analytes as well.

The accuracy failures for selenium and thallium in the MS sample are associated with the spectral background issues discussed in the memo sent to you earlier. ${ }^{(3)}$ The matrix spike and serial dilution for arsenic did not meet the required limits, but both of these together indicate a matrix interference. This could be adjusted by using a higher dilution, but this will again cause a missing of the required detection

( $\left.{ }^{1}\right)$ The $\mathrm{U}$ emission spectrum is replete with lines; high-U concentration solutions require extensive dilution such that inter-element corrections can be applied.

$\left(^{2}\right)$ WIPP document for $100 \mathrm{ug} / \mathrm{g} \mathrm{Pb}$ dl.

$\left({ }^{3}\right)$ 52578-2011-L06, to WW Rutherford from CD Carlson, 2/15/11. 
PNNL-20650, Rev. 2

WW Rutherford

March 14, 2011

Page 3

limit. The blank spike sample analyte recoveries met all acceptance criteria, further indicating the MS sample analyte recovery failures were confounded by the sample matrix.

The crosswalk of the QC failures with the WIPP Hazardous Waste Permit Table C3-9 is as follows:

\begin{tabular}{|c|c|c|c|}
\hline QC Sample & $\begin{array}{l}\text { Results obtained by } \\
\text { PNNL on WIPP Metals } \\
\text { under ASR- } 8725\end{array}$ & $\begin{array}{l}\text { Recommend Action based } \\
\text { on WIPP Criteria (Table } \\
3 \text { C-9) if criteria failed }\end{array}$ & $\begin{array}{l}\text { Actual Action taken by } \\
\text { PNNL } \\
\text { (if a failure occurred) }\end{array}$ \\
\hline $\begin{array}{l}\text { Method Performance } \\
\text { Samples }\end{array}$ & $\begin{array}{l}\text { Semi-Annual } \\
\text { Performance samples } \\
\text { meet criteria }\end{array}$ & NA & None \\
\hline Laboratory blanks & $\begin{array}{l}\mathrm{Ca} \text { and } \mathrm{U} \text { greater than } \\
\text { detection limit }\end{array}$ & $\begin{array}{l}\text { If analyte values are } \leq 10 \mathrm{x} \\
\text { blank value and } \geq 5 \mathrm{X} \\
\text { PRQL- samples must be } \\
\text { re-digested and reanalyzed }\end{array}$ & $\begin{array}{l}\text { All sample analyses } \\
\text { for Ca and } U \text { are } \geq 10 \mathrm{x} \\
\text { blank value and } \geq 5 \mathrm{X} \\
\text { PRQL - no action } \\
\text { needed }\end{array}$ \\
\hline Matrix spikes & $\begin{array}{l}\text { As, Se and } \mathrm{Tl} \text { all have } \\
\text { low recovery. } \mathrm{Cr} \text { has } \\
\text { high recovery }\end{array}$ & $\begin{array}{l}\text { No action required for } \\
\text { matrix related exceedances } \\
\text { - only listed as a non- } \\
\text { conformance }\end{array}$ & $\begin{array}{l}\text { Se and } \mathrm{Tl} \text { issue } \\
\text { evaluated in PNNL } \\
\text { letter 52578-2011-L06. } \\
\text { As low, so result is } \\
\text { reported as suspect. Cr } \\
\text { high, so value is upper } \\
\text { bounding case - no } \\
\text { action taken but result } \\
\text { indicated in the final } \\
\text { verified data package }\end{array}$ \\
\hline $\begin{array}{l}\text { Matrix Spike } \\
\text { duplicate }\end{array}$ & NA & $\begin{array}{l}\text { No action required for } \\
\text { matrix related exceedances } \\
\text { - only listed as a non- } \\
\text { conformance }\end{array}$ & NA \\
\hline Initial Calibration & All calibration completed & Correct issue and repeat & NA \\
\hline $\begin{array}{l}\text { Continuing } \\
\text { Calibration }\end{array}$ & $\begin{array}{l}\mathrm{CCV}^{\prime} \text { complete and } \\
\text { within specifications }\end{array}$ & $\begin{array}{l}\text { Correct issue, recalibrate } \\
\text { and repeat }\end{array}$ & NA \\
\hline Serial Dilution & Dilution failure for As & $\begin{array}{l}\text { Flag data if result is }>50 \mathrm{x} \\
\text { IDL }\end{array}$ & $\begin{array}{l}\text { Some of the As sample } \\
\text { results are }>50 \mathrm{x} \text { IDL - } \\
\text { failure will be } \\
\text { indicated in the final } \\
\text { verified data package. }\end{array}$ \\
\hline $\begin{array}{l}\text { Interference } \\
\text { Correction } \\
\text { verification }\end{array}$ & $\begin{array}{l}\mathrm{ICV}^{2} \text { complete and within } \\
\text { specification }\end{array}$ & $\begin{array}{l}\text { Recalibrate and repeat } \\
\text { analysis - if this does not } \\
\text { correct, identify as a } \\
\text { nonconformance }\end{array}$ & NA \\
\hline
\end{tabular}


PNNL-20650, Rev. 2

WW Rutherford

March 14, 2011

Page 4

\begin{tabular}{|l|l|l|l|}
\hline $\begin{array}{l}\text { Laboratory Control } \\
\text { Samples }\end{array}$ & $\begin{array}{l}\text { LCS all within } \\
\text { specifications }\end{array}$ & $\begin{array}{l}\text { Re-digest and reanalyze all } \\
\text { samples }\end{array}$ & NA \\
\hline Blind Audit Samples & NA & NA & NA \\
\hline
\end{tabular}

Initial Calibration Verification sample

${ }^{2}$ Continuing Calibration Verification sample

Sincerely,

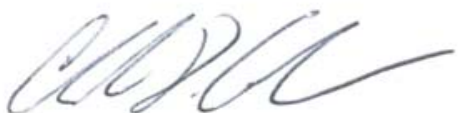

Clark Carlson

PNNL Project Manager

Enclosures

cc: WW Rutherford, MS A3-06

RB Baker, MS A3-06

JA Pottmeyer, MS A3-06

STP Project File, (FE Wickstrand, R1-29)

bec: CD Carlson, P7-25

SK Fiskum, P7-25

RM Shimskey, P7-27

AJ Schmidt, P8-60

KN Pool, P7-25

DS Coffey, P7-25

52578 Project File (PDF) 
PNNL-20650, Rev. 2

\section{$\mathbb{K}=$ BBasin Occurrence $\mathbb{R e p o r t}$}

\begin{tabular}{|c|c|c|c|}
\hline \multicolumn{3}{|c|}{ Title: ASR 8798 GEA, Duplicate resulths exceed 20\% requirement } & Date: $4018-11$ \\
\hline \multicolumn{4}{|l|}{ Unique Idemtemififier: 52578-04-18-11 } \\
\hline \multicolumn{4}{|c|}{ Primariry Person Idententifying Issue: True Trang } \\
\hline \multirow{4}{*}{$\begin{array}{l}\text { Distributiom: Email and posting on } \mathbb{K}- \\
\text { Basin Share-Point }\end{array}$} & Clark Carlson & \multirow{2}{*}{\multicolumn{2}{|c|}{ Andy Schmidt }} \\
\hline & Deborah Coffey & & \\
\hline & \begin{tabular}{|l|l} 
Sandy Fiskum \\
\end{tabular} & \multicolumn{2}{|c|}{ Rick Shimskey } \\
\hline & Karl Pool & \multicolumn{2}{|c|}{ Truc Trang Le, Project file for ASR 8798} \\
\hline
\end{tabular}

Occurrence Descriptiom: ASR\#: 8798

The duplicate relative percent difference (RPD) for Co-60 on samples TI-001-A1-B and TI001-A1-C was 48\%. The duplicate RPD for Eu-155 on samples TI-002-B1-B and TI002-B1-C was $32 \%$. Both of these RPDs exceed the acceptance limit of $\pm 20 \%$.

Impact of Decurrence:

There is no impact due to the RPDs exceeding the acceptance limits for these two isotopes.

The GEA results for the other 4 measured isotopes for the duplicate pair of TI-001-A1-A and TIO01-A1-B ranged from 4 to $7 \%$. This is a good indication that the majority of the target isotopes are agreeing well between sample and duplicate.

The GEA results for the other 4 measured isotopes for the duplicate pair of TI-001-B1-B and TIO01-B 1-C ranged from 0.4 to $16 \%$. This is a good indication that the majority of the target isotopes are agreeing well between sample and duplicate.

The overall activity $\left(\mu \mathrm{Ci} / \mathrm{g}_{\text {dry }}\right.$ ) present in each sample is dominated by Cs-137 and $\mathrm{Am}-241$ (over $100 \mathrm{x}$ more activity than present from the Co-60 or Eu-155).

Sigmificame Comdition Adverse to Qunality? Yes _ No Y Y

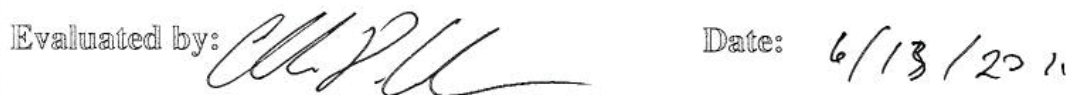

Furither Dispositiom: [X] No $\square$ Yes If yes, $\square$ Nomconformamce? $\square$ Deficiency?

Daite Occunrence Cllosed: $6-13-2011$

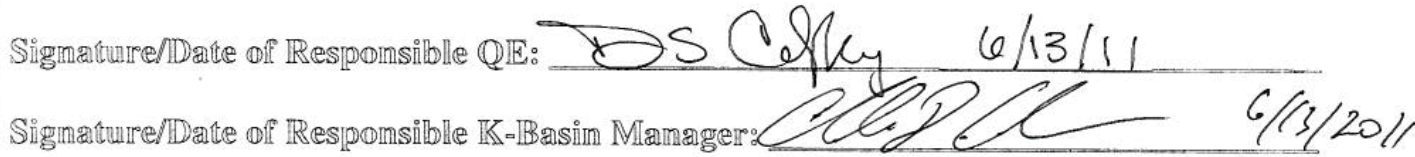

Comments: 
PNNL-20650, Rev. 2

$\mathbb{K} \cdot \mathbb{B}$ asim Occunrence Report

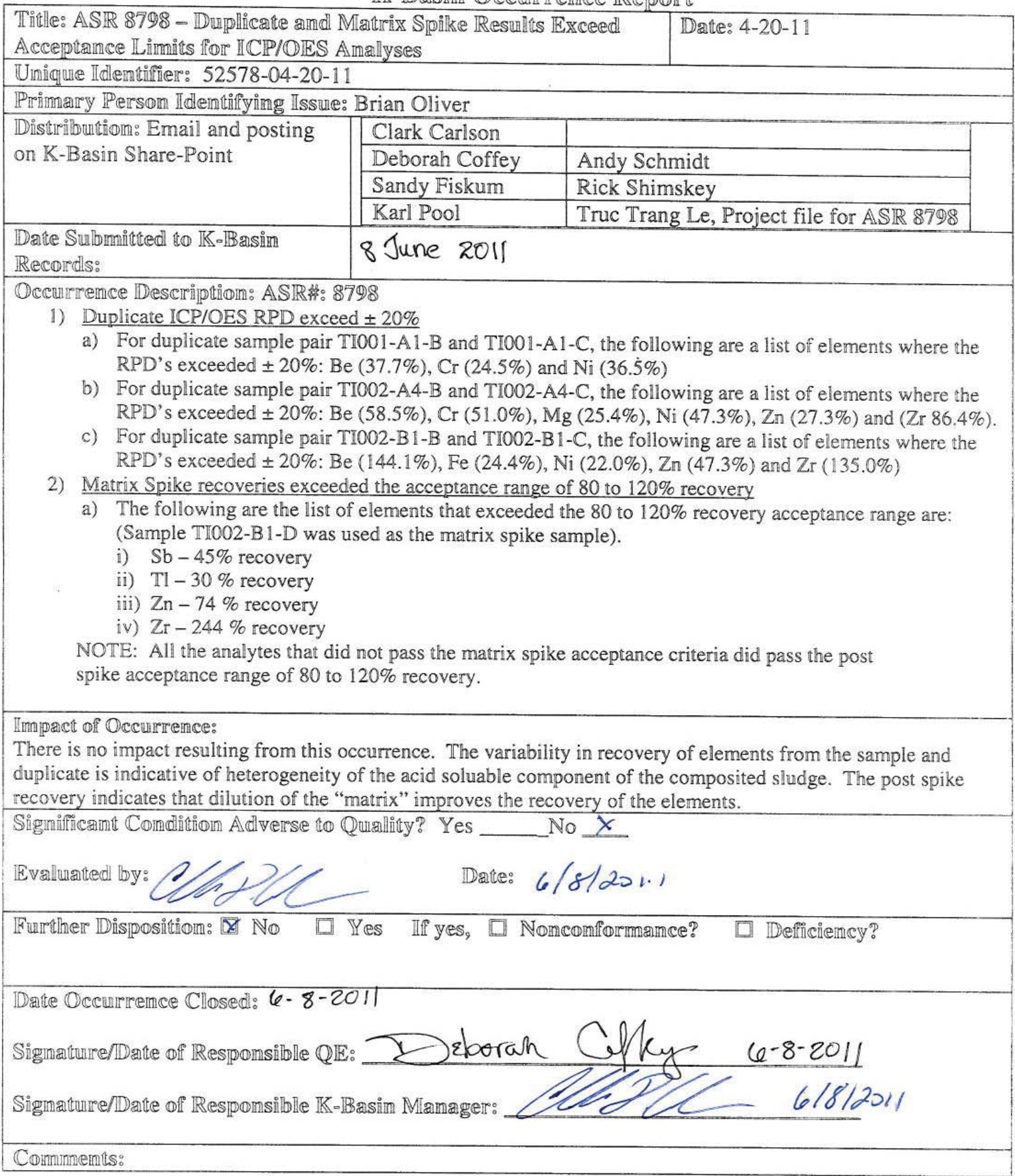


PNNL-20650, Rev. 2

$\mathbb{K}=\mathbb{B a s i n}$ Occurremee Report

\begin{tabular}{|c|c|c|}
\hline Title: ASR 8798 Duplicate U.Metal & esunft Exceeds 20\% & Date: $5-26-11$ \\
\hline $\begin{array}{l}\text { Unique Idlemtifier: 52578-05-26-11 } \\
\text { Primmary Person Identifying Issue: }\end{array}$ & Delegard & \\
\hline Distribibution: Email and posting on & Clark Carlson & \\
\hline K-Basin Share-Point & Deborah Coffey & Andy Schmidt \\
\hline & Sandy Fiskum & Rick Shimskey \\
\hline & Karl Pool & Truc Trang Le, Project file for ASR 8798 \\
\hline $\begin{array}{l}\text { Date Submituted to } \mathbb{K} \text {-BBasim } \\
\text { Records: }\end{array}$ & 8 June 2011 & \\
\hline Occurrence Description: ASR 87 & & \\
\hline The duplicate $\mathbb{R P D}$ for sample pair & A4-P $(11-0420-$ & $\begin{array}{l}\text { and TIO02-A4-Q (11-0420-407-D) is } 5 \text { - } \\
\text { esult for sample TIO02-A4-P is } 1.19 \mathrm{w}\end{array}$ \\
\hline
\end{tabular}

Impact of Occurrence:

There is no impact due to this occurrence. Variation in the U-metal content of $\mathrm{K}$-Basin sludge samples is not unexpected.

Signifficamt Comdintion Adverse to Qualinty? Yes__ No Y Y

Eivaluated by: Date: $6 / 8 / 201$,

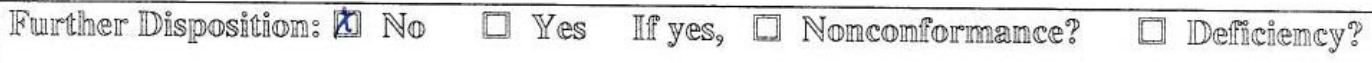

Date Occurrence $\mathbb{C}$ losed: 8 June 2011

Sigmature/Date of $\mathbb{R e s p o n s i b l e ~} \mathbb{Q}$ LE: Deborah offey $6 / 8 / 2011$

Comments: 
PNNL-20650, Rev. 2

$\mathrm{K}$-BBasim Occurrence Report

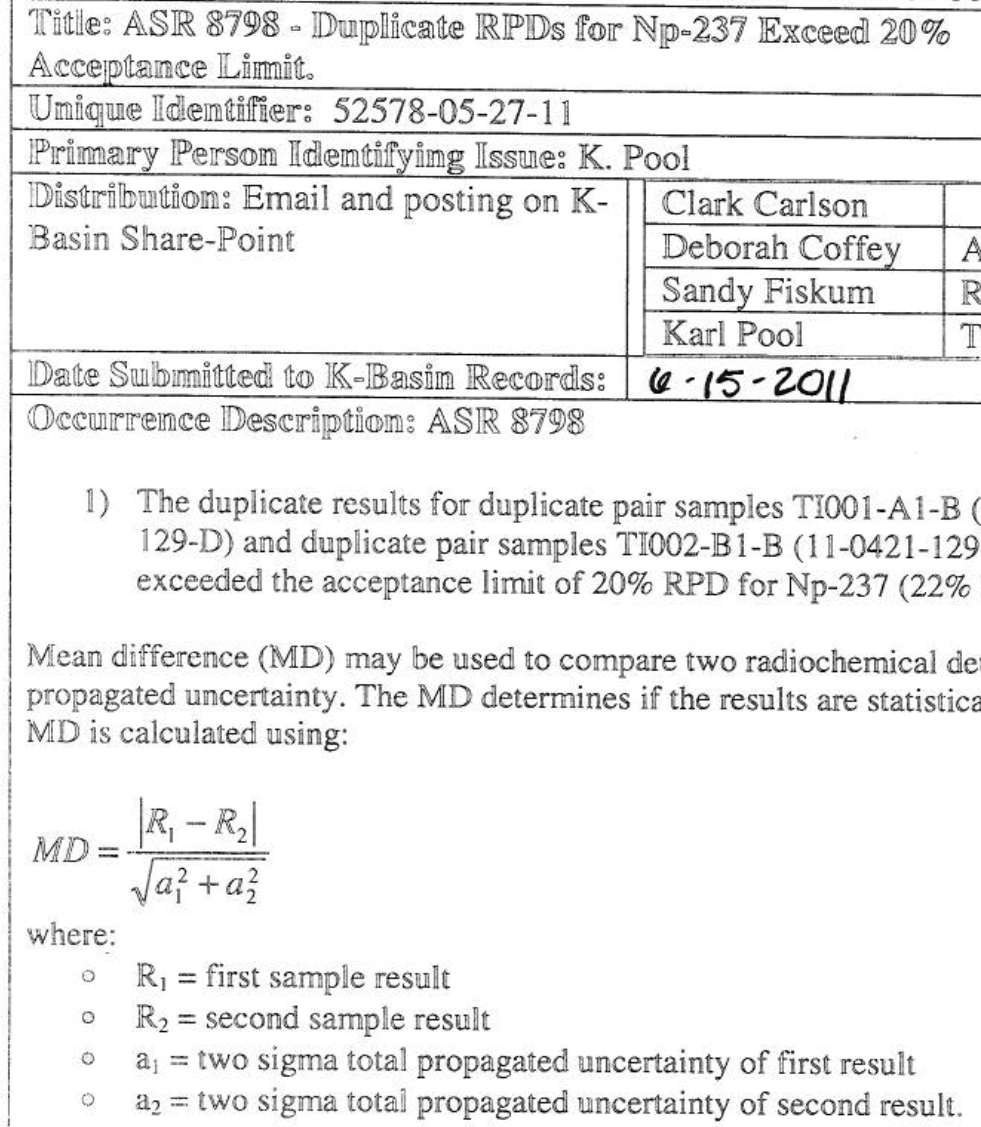

When the MD result is greater than or equal to 1.96 , there is $95 \%$ confidence that the two results are not equal.

The mean difference for the duplicate pairs that did not meet the acceptance criteria are as follows:

Np-237 (TIO01-Al pair) $M D=\frac{|(1.74 E-3)-(2.16 E-3)|}{\sqrt{(3.83 E-4)^{2}+(4.32 E-4)^{2}}}=7.27 \mathrm{E}-01$

$\mathrm{Np}-237\left(\mathrm{TI} 1002-\mathrm{B} 1\right.$ pair) $M D=\frac{|(1.78 E-3)-(7.49 E-4)|}{\sqrt{(6.23 E-4)^{2}+(4.33 E-4)^{2}}}=1.36 \mathrm{E}+00$

In both cases, the MD calculation shows the results are less than 1.96 , indicating the sample and duplicate results are statistically equal.

Itmpact of Occurremce:

There is no impact from this occurrence.

The sample and duplicate results have $1-\sigma$ counting uncertainties that range from 10 to $29 \%$. This indicates the sample and duplicate results are close to the detection limit. The mean difference calculation shows the sample and duplicate results are statistically equal. 
PNNL-20650, Rev. 2

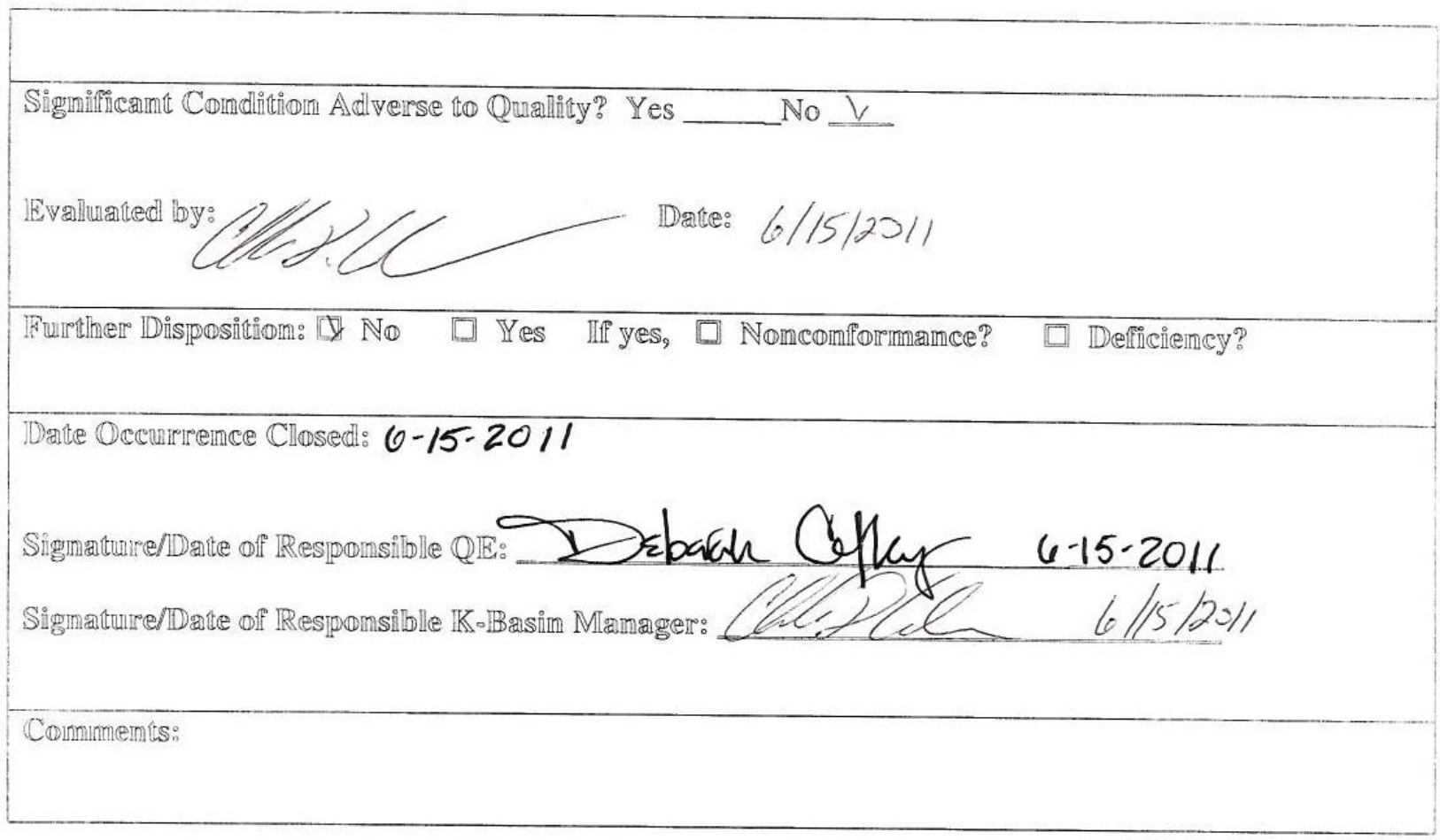


PNNL-20650, Rev. 2

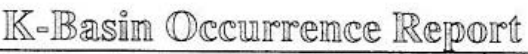

\begin{tabular}{|c|c|c|c|}
\hline \multicolumn{3}{|c|}{ Tithle: Drying of Swmples for TOC/TIC Amallyses, ASR 879\& } & Date: $6-30-11$ \\
\hline \multicolumn{4}{|l|}{ Umique Identinfirier: 52578-06-30-11 } \\
\hline \multicolumn{4}{|c|}{ Proimairy Person Idemtifying Issue: Clark Carlson } \\
\hline \multirow{4}{*}{$\begin{array}{l}\text { Distroiburtion: Email and posting } \\
\text { on } \mathbb{K}-B \text { asin Share-Point }\end{array}$} & Clark Carlson & \multicolumn{2}{|c|}{ Karl Pool } \\
\hline & Deborah Coffey & \multicolumn{2}{|c|}{ Andy Schmidt } \\
\hline & Sandy Fiskum & \multicolumn{2}{|c|}{ Rick Shimskey } \\
\hline & Matt Fountain & \multicolumn{2}{|c|}{ Truc Trang Le, Project file for ASIR $\$ 798$} \\
\hline $\begin{array}{l}\text { Date Subminitted to } \mathbb{K} \cdot \mathbb{B} \text { isin } \\
\text { Recordls: }\end{array}$ & \multicolumn{3}{|l|}{20 July 2011} \\
\hline
\end{tabular}

Occurrence IDescriptiom:

QAPJP/SAP KBC-33786, Rev. 2 specified sample analysis for total organic carbon (TOC) only (refer to Table 3-4a and Figure 3-4) and indicated that the samples were to be analyzed on a wet (settled basis). However, in accordance with the ASO ASR 8798 Special Instructions reviewed and approved by the Buyer's Technical Representative (BTR), the samples were dried at $105^{\circ} \mathrm{C}$ before implementing ASO procedure, RPG-CMC-385, Carbon Measured in Solids, Sludge, and Liquid Matrices for TOC and TIC analyses.

IImpact off Oecunrremce:

Drying of the samples to constant weight would remove any organic species (e.g. organic solvents) that have an appreciable vapor pressure at or near $105^{\circ} \mathrm{C}$. However, due to the small sample size and the environment in which the samples were weighed, the loss of water during weighing operation would be a potential issue, leading to large uncertainty on the mass basis of the analysis results. Please see the attached discussion for a complete analysis of this issue.

Signifficant Comdlitiom Adverse to $\mathbb{Q}$ unallity? Yes__ No

Evilluwited by:

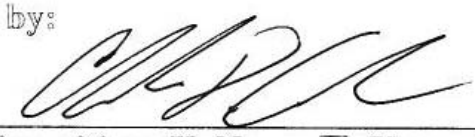

Date: $7 / 20 / 2011$
Furither $\mathbb{D}$ ispositiom: $\mathbb{X} \mathbb{N} \oplus$
10] Yes IIf yes,
Nonconformance?
[1 Defficiemey?

Date Occurrence Closed: $7-20-11$

Sigmature/Dite of Responsible QR:

Sigmaturire/Date of $\mathbb{R}$ esponsi̊ble $\mathbb{K} \cdot \mathbb{B}$ asim Mamager:

Comments: 
PNNL-20650, Rev. 2

Total Carbon/Total Organic Carbon Analysis

In the QAPjP/SAP, KBC-33786, Revision 2 for Container $\mathbb{K W}-210$, Table 3-4a on page 3.21, the sample preparation step for determining total organic carbon (TOC) is to collect settled sludge sub-samples for testing. Samples were actually collected from what is termed an "as-sampled" basis, where water has been removed from the sludge such that the sludge has enough strength to suspend dense particles such as $\mathbb{U}$ metal during the course of the subsampling activity. The as-settled sludge density is smaller than the as-sampled sludge density, A small subsample of strengthened sludge is transferred to a vial for clean transfer from the hot cell; the air headspace is substantial. During analysis, the TOC samples are dried prior to placing them in the furnace to perform the total combustion of the samples. It is assumed that the reason for requesting the analysis on the wet sample mass is to minimize the loss of the more volatile organic species and to assess the carbon concentration on an as-settled sludge mass basis. The reason the laboratory dries the samples prior to analysis will be discussed in the following paragraph.

During the initial analysis of the containerized sludges from Containers SCS-CON-220, 240, 250 and 260, the TOC was performed using the hot persulfate method, which is a wet chemical method and lends itself well to analyzing wet samples, although the samples were dried to minimize the issue of water evaporation during weighing and confounding the reporting mass basis. Because of the presence of chemical oxidation resistant OIER beads, the hot persulfate method was shown to be inadequate to get a true reading of organic carbon content and the high temperature furnace method was used on subsequent samples. The high temperature method is generally used to determine total carbon and total organic carbon. This procedure was later modified to include a two-step heating regimen to differentiate the contribution of $\mathrm{Grafoil}^{\circledR}$ from the organic carbon in the individual samples. The high temperature carbon combustion apparatus is in an open hood so there are potential issues with contamination and dose to staff. Generally, samples are heat-dried $\left(105^{\circ} \mathrm{C}\right)$ to constant weight prior to analysis since wet samples in the high temperature furnace may spatter during initial heating, which could send some of the material out of the combustion area of the furnace, resulting in incomplete combustion of the sample and underestimation of carbon in the sample.

Many of the samples analyzed for this project have high carbon content due to the presence of resin beads and Grafoil ${ }^{\circledR}$, so small sample sizes are required to prevent a swamping of the detector. Small samples sizes are also desirable from an ALARA perspective because the samples are relatively high dose. When small sample sizes are used, the analysis of wet samples is problematic. During the transfer of a wet sample with a relatively high surface area to mass ratio from the sample vial to the analysis boat followed by weighing, a measureable amount of water is lost to evaporation. This water loss will affect the true sample mass and any correlation to the as-settled solid mass will not be possible, giving results that cannot be tied back to a known water content.

One final consideration is that any of the more volatile organic species will have losses due to the handling of the samples during all steps from collection in the basin to processing in the HLRF. Sample temperatures were not controlled, head spaces during processing were not minimized, and holding times were not applied, therefore an accurate determination of more volatile species would not be possible for these samples.

For the reasons discussed above, all of the samples for carbon analysis were dried prior to sample analysis. 
PNNL-20650, Rev. 2

\section{K-Basin Occurrence Report}

\begin{tabular}{|c|c|c|c|}
\hline \multicolumn{3}{|c|}{$\begin{array}{l}\text { Title: TOC/TIC method QC, Duplicate/Replicate and Analytical } \\
\text { Spike not meeting method and project specific acceptance } \\
\text { criteria. }\end{array}$} & Date: $7-19-11$ \\
\hline \multicolumn{4}{|c|}{ Unique Identifier: 52578-07-19-11 } \\
\hline \multicolumn{4}{|c|}{ Primary Person Identifying Issue: D. Baldwin } \\
\hline \multirow{4}{*}{$\begin{array}{l}\text { Distribution: Email and posting } \\
\text { on K-Basin Share-Point }\end{array}$} & Clark Carlson & \\
\hline & $\begin{array}{l}\text { Deborah } \\
\text { Coffey }\end{array}$ & \multicolumn{2}{|l|}{ Andy Schmidt } \\
\hline & $\begin{array}{l}\text { Sandy } \\
\text { Fiskum }\end{array}$ & \multicolumn{2}{|l|}{ Rick Shimskey } \\
\hline & Karl Pool & \multicolumn{2}{|c|}{ Truc Trang Le, Project file for ASR 8798} \\
\hline $\begin{array}{l}\text { Date Submitted to K-Basin } \\
\text { Records: }\end{array}$ & \multicolumn{3}{|l|}{$7-21-11$} \\
\hline
\end{tabular}

Occurrence Description: ASR 8798:

The following list of items detail the issues identified with the TIC/TOC analyses:

1) Method QC related issues:

a) $1^{\text {st }}$ and $2^{\text {nd }}$ Initial Calibration Blanks (ICB) are to agree within $5 \mu \mathrm{g}$. The first and second ICB's for run \#6 (TI002-B1-H) differed by $5.6 \mu \mathrm{g}$.

b) $1^{\text {st }}$ and $2^{\text {nd }}$ Initial Calibration Verification (ICV) standards are to agree within 5\%. In 4 of the 10 TOC runs, the $1^{\text {st }}$ and $2^{\text {nd }}$ ICV's exceeded the $5 \%$ agreement criteria.

i) Run \#2 (TI001-A1-H) - The ICV's are $104 \%$ and $110 \%$.

ii) Run \#4 (TI002-A4-H) - The ICV's are $100 \%$ and $107 \%$.

iii) Run \#5 (TI002-A4-J) - The ICV's are $100 \%$ and $108 \%$.

iv) Run \#6 (TI002-B1-H) - The ICV's are $91 \%$ and $105 \%$.

In 4 of the 10 TIC runs, the $1^{\text {st }}$ and $2^{\text {nd }}$ ICV's exceeded the $5 \%$ agreement criteria.

i) Run \#3 (TI001-A1-J) - The ICV's are $95 \%$ and $103 \%$.

ii) Run \#5 (TI002-A4-J) - The ICV's are $92 \%$ and $100 \%$.

iii) Run \#6 (TI002-B1-H) - The ICV's are 95\% and $110 \%$.

iv) Run \#7 (TI002-B1-J) - The ICV's are $90 \%$ and $97 \%$.

NOTE: All ICV results are within the method acceptance limits of $90 \%$ to $110 \%$.

2) Project specific acceptance criteria issues:

a) The method detection limit (MDL) is required to be $200 \mu \mathrm{gC} / \mathrm{g}$.

A total of 56 sample runs (TOC and TIC combined) were performed for the samples in the ASR. The $200 \mu \mathrm{g} / \mathrm{g}$ MDL requirement was exceeded in 34 of those runs. The MDL for each sample run is mathematically derived from the sample size used and the instrument detection limit for the batch (run\#). Sample sizes were intentionally kept small to limit technician exposure to radiation (ALARA). The small sample sizes along with the variation in the IDL from batch to batch caused the MDL values to exceed the $200 \mu \mathrm{g} / \mathrm{g}$. The method is capable of running larger sample sizes and thus able to achieve MDL values much lower than $200 \mu \mathrm{g} / \mathrm{g}$. In this case, due to the intentional use of small sample sizes, the MDL of $200 \mu \mathrm{g} / \mathrm{g}$ could not be 
met for all sample runs.

3) Analyses QC issues:

a) The Analytical Spike (AS) recovery is required to be within $75 \%$ to $125 \%$. The AS recovered at $132 \%$ (TOC) for the AS in run \#2. The other AS run with this batch (run \#9) recovered at $115 \%$ (TOC). The TIC recoveries are $97 \%$ for run $\# 2$ and $88 \%$ for run $\# 9$. There is currently no obvious reason for the high AS recovery for TOC in run \#2.

4) Duplicate and Triplicate precision is required to be $\leq 20 \%$.

Each core sample was analyzed in triplicate, due to potential homogeneity concerns, while the special materials of Purolite ${ }^{\circledR}$ and Grafoil ${ }^{\circledR}$ were analyzed in duplicate. Precision in each case was determined based on results for all three replicates for the core samples or duplicates for the special materials.

The following approach was used in deriving the precision for results.

Relative Percent Differences (RPDs) can be calculated and reported when there are two J flagged values or one J flagged value and one non-flagged value.

NOTE: The customary reporting basis for RPD's uses only results that are greater than the Estimated Quantitation Limit (EQL) (not J-flagged). For this report, it was agreed to use results that were greater than the instrument detection limit and less than the EQL in the RPD/Relative Standard Deviation (RSD) calculations.

The following summarized the RPD/RSD issues:

a) Run \#2 (TI001-A1-H) - TOC (27\%), TIC (83\%)

b) Run \#3 (TI001-A1-J) - TOC (35\%), TIC (81\%)

c) Run \#4 (TI002-A4-H) - TOC (39\%), TIC (85\%)

d) Run \#5 (TI002-A4-J) - TOC (38\%)

e) Run \#9 (TI001-B3-J) - TIC (38\%)

\section{Impact of Occurrence:}

1) Method QC related issues:

a) For run \#6, the two initial TOC ICB results differed by $5.6 \mu \mathrm{gC}$ and are required to be no greater than $5 \mu \mathrm{gC}$. The run IDL for TOC is determined by the average of the blanks run for the day. The end of day blank was between the two initial blanks. The average blank is subtracted from the sample result to correct for the background. In this case the run $\# 6$ average blank for the day is $10 \mu \mathrm{gC}$. The TOC triplicate sample results ranged from $1850 \mu \mathrm{gC}$ to 9690 $\mu \mathrm{gC}$. The small change that resulted in the two ICB differing by $5.6 \mu \mathrm{gC}$ has negligible impact on the final TOC results.

b) The $1^{\text {st }}$ and $2^{\text {nd }}$ ICV's are to agree within $5 \%$ - The cases where the initial two ICV's did not achieve agreement within $5 \%$ (TOC and/or TIC); the ICV results were all within the method acceptance range of $90 \%$ to $110 \%$. No impact to reported results.

2) Project specific acceptance criteria :

a) MDL is required to be $200 \mu \mathrm{gC} / \mathrm{g}$ of lower - The IDL for the batch is determined using the 
blank data for each batch. The variation in IDL from batch to batch and the ALARA-based need to keep the sample sizes small resulted in some of the MDLs being far greater than the 200 $\mu \mathrm{gC} / \mathrm{g}$ level. Most of the sample result was well above MDL, and those samples in which the result was near the MDL all occurred on TIC analyses. There is likely little impact.

3) Analysis QC Issues:

a) The Analytical Spike (AS) recovery is required to be within $75 \%$ to $125 \%$. The AS recovered at $132 \%$ (TOC) for the AS in run \#2. The other AS run with this batch (run \#9) recovered at $115 \%$ (TOC). The TIC recoveries are $97 \%$ for run $\# 2$ and $88 \%$ for run $\# 9$. There is currently no obvious reason for the high AS recovery for TOC in run \#2. The ICV's and LCS/BS all recovered within the acceptance limits for this run.

4) Duplicate and Triplicate precision is required to be $\leq 20 \%$.

a) The variability observed with the duplicate and triplicate data is indicative of heterogeneity of the parent sample coupled with the small sample sizes used for the analyses. The data for the triplicate measurements provides some indication of the range of $\mathrm{C}$ from each subsample of the parent.

\section{Significant Condition Adverse to Quality? Yes

No $X$

This determination will be made by Clark Carlson. This section may not apply to all systems evaluated. When used, document who made the determination of significant condition.

Review other ORs to evaluate if this is a recurring problem. Provide a short summary of what was done to determine the impact of the occurrence and the extent of the problem.

Evaluated by: Date: $7 / 201201$

Further Disposition: $₫$ No $\square$ Yes If yes, $\square$ Nonconformance? $\square$ Deficiency?

This will be partially based on project managers' discussions with the client.

Date Occurrence Closed: $7-20-11$

Signature/Date of Responsible QE:

\section{Comments:}

Attachments may be added as needed. Identification of specific affected samples is useful. 
PNNL-20650, Rev. 2

\section{Appendix C}

\section{ASR 8824 and Results}





\section{Appendix C}

\section{ASR 8824 and Results}

Appendix C contains the signed Analytical Service Request (ASR) 8824 and special instructions generated for Analytical Support Operations (ASO) laboratory analysis of the KW210 container composite samples. The special instructions delineated sample-specific information, required detection limits, quality control (QC) sample requirements, and reporting requirements. All analytical reports generated by the ASO in response to the ASR are provided in Appendix C. Analytical reports provide sample results; associated uncertainties; and a discussion of sample processing, QC results, compliance with QC sample requirements, and data limitations. Occurrence Reports are also provided. These were prepared when issues were identified, and resolution was required while work was in process.

The following table of contents shows that pagination specific to Appendix C was applied to the ASR, the analytical reports, and Occurrence Reports. This pagination is sequential and consistent (e.g., page C.\#).

\section{Appendix C Table of Contents}

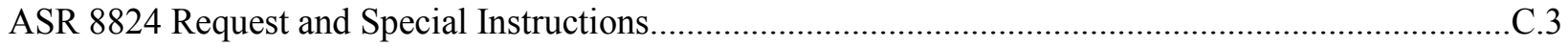

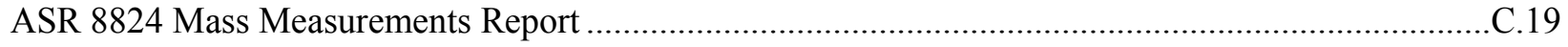

ASR 8824 Inductively Coupled Plasma-Optical Emission Spectrometry Analysis Report .....................22

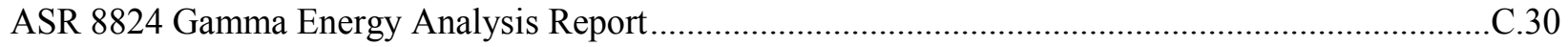

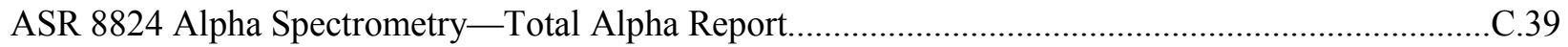

ASR 8824 Uranium Metal Concentration in K Basin Sludge Report, Rev. 2 ......................................45

ASR 8824 Uranium Oxidative State in K Basin Sludge Report.............................................................51

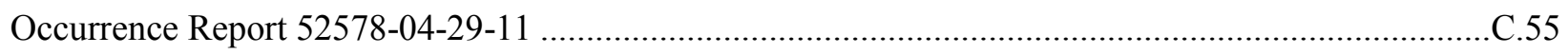

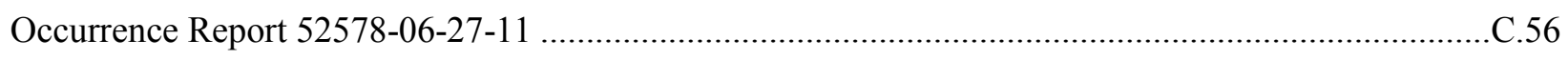

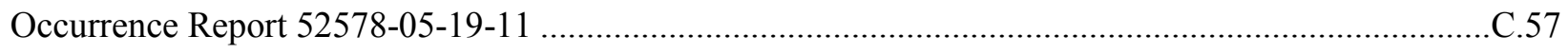



Requestor --- Complete all fields on this COVER PAGE, unless specified as optional or ASR is a revision

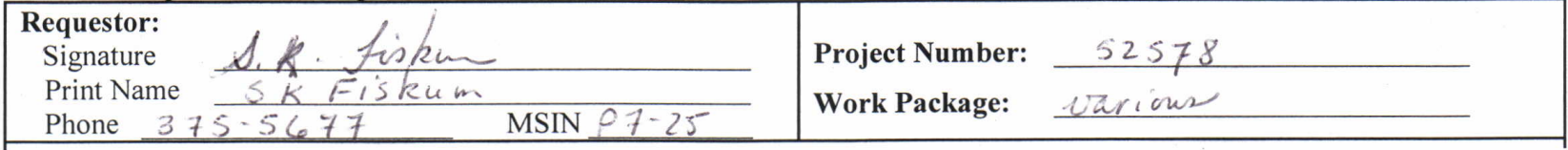

Matrix Type Information

\begin{tabular}{cccc|}
\hline - Liquids: & $\square$ Aqueous & $\square$ Organic & $\square$ Multi-phase \\
& $\square$ Soil & $\square$ Sludge & $\square$ Sediment \\
& $\square$ Glass & $\square$ Filter & $\square$ Metal \\
& $\square$ Smear & $\square$ Organic & $\square$ Other \\
- Other: & $\square$ Solid/Liquid Mixture, Slurry \\
& $\square$ Gas $\quad \square$ Biological Specimen \\
(If sample matrices vary, specify on Request Page)
\end{tabular}

Disposal Information

- Disposition of Virgin Samples:

Virgin samples are returned to requestor unless archiving provisions are made with receiving group!

If archiving, provide:

Archiving Reference Doc:

- Disposition of Treated Samples: 囚Dispose $\square$ Return

\section{Data Reporting Information}

- Is Work Associated with a Fee-Based

Milestone? $\square$ No $\square^{\prime}$ Yes

If yes, milestone due date:

July 1, 2011

- Preliminary Results Requested, As Available? $\square$ No $ه$ Yes

\begin{tabular}{|c|c|}
\hline $\begin{array}{l}\text { Data Reporting Level } \\
\square \text { ASO-QAP-001 (Equivalent to } \\
\text { HASQARD). } \\
\square \text { Minimum data report. } \\
\text { هroject Specific Requirements: } \\
\text { Contact ASO Lead or List Reference } \\
\text { Document:Specicl Imprecti }\end{array}$ & $\begin{array}{l}\text { - Requested Analytical Work Completion Date: } \\
\frac{\text { may } 20}{\text { (Note: Priority rate charge for }<10 \text { business day turn-around time) }} \\
\text { - Negotiated Commitmept Date: } \\
\frac{5 / 20 / 11}{\text { (To be completed by ASO Lead) }} \\
\text { LNP }\end{array}$ \\
\hline
\end{tabular}

Waste Designation Information

\section{QA/Special Requirements}

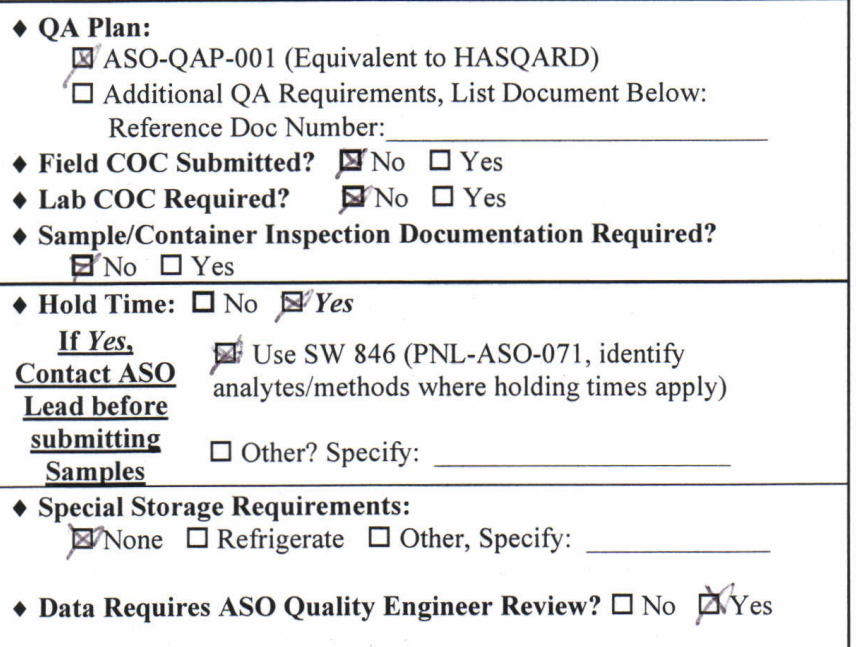

QA Plan:

Reference Doc Number:

- Field COC Submitted? 叉No $\square$ Yes

Lab COC Required? $\square$ No $\square$ Yes

Sample/Container Inspection Documentation Required?

Hold Time: $\square$ No $\mathbb{8}$ Yes

Lead before

submitting

4. Use SW 846 (PNL-ASO-071, identify tes/methods where holding times apply)

$\square$ Other, Specify:

- Data Requires ASO Quality Engineer Review? $\square$ No Ø̋Yes

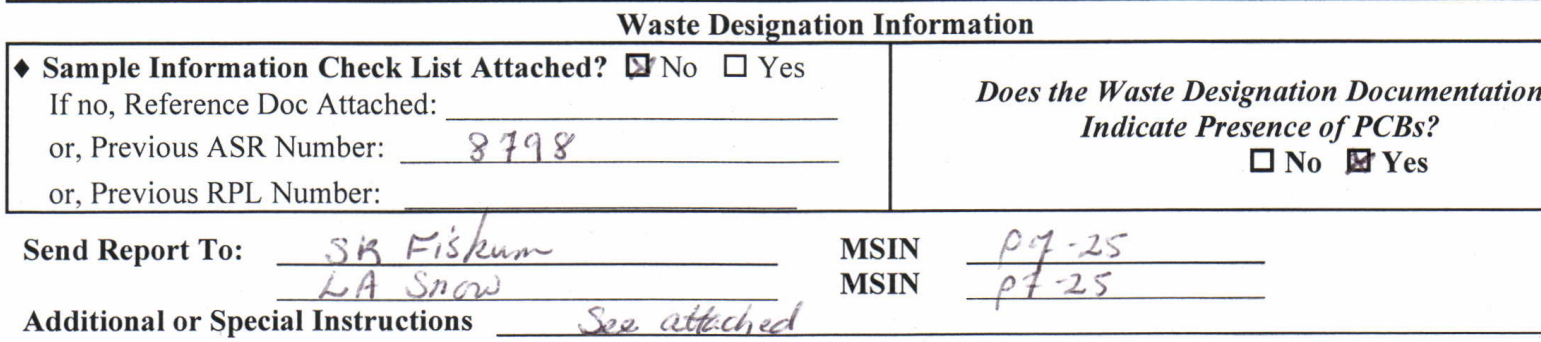

Receiving and Login Information (to be completed by ASO staff)

\begin{tabular}{|lc}
\hline Date Delivered: & \multicolumn{1}{c}{$4 / 6 / 11$} \\
\cline { 2 - 2 } Delivered By (optional) \\
Time Delivered (optional) \\
Group ID (optional) \\
\cline { 2 - 2 } \\
CMC Waste Sample?
\end{tabular}

ASO Work Accepted By: $\mathrm{K} / 6$ oel Signature/Date: Received By:
ASR Number: $\quad 882 \mathrm{R}$ Rev. 00
RP Numbers: $\quad 11-0791,-0792,0793,0967$

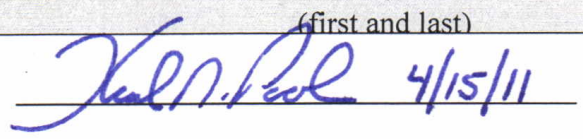


PNNL-20650, Rev. 2

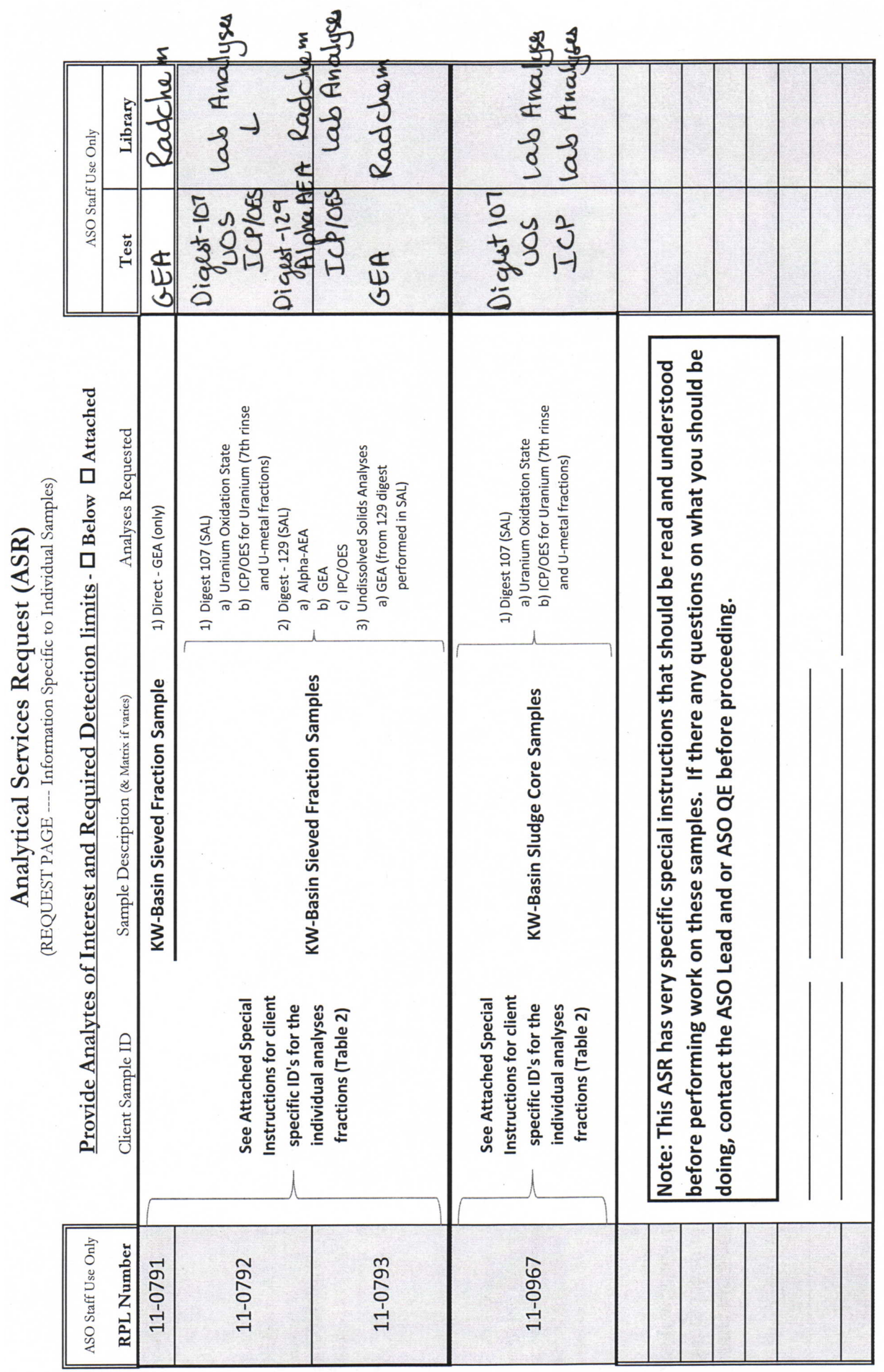

-1
4
0
0
0
0
0
0

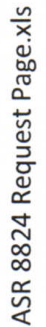

家 
PNNL-20650, Rev. 2

\section{Special Instructions for K-Basin Container SCS-CON-210 \\ Container Composite Sample Analysis (Project 52578)}

Cognizant Scientist:

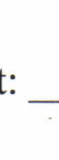

Uk. Hiskm

Date: $4 / 5 / 11$

Project Manager:

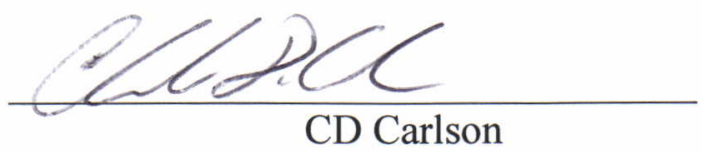

Date: $4 / 11 / 1$

Project Quality Engineer: $\frac{D S C \text { M }}{\text { DS Coffey }}$

Date: $4 \cdot 5-2011$

ASO Lead:

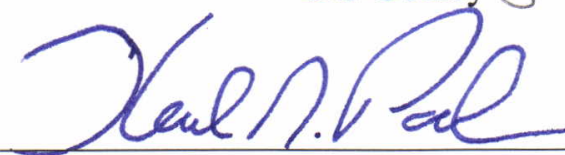

KN Pool

Date: $4 / 5 / 11$

STP Concurrence:

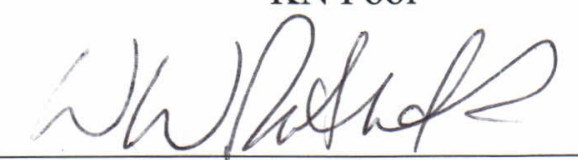

WW Rutherford

Date: $4 / 5 / 4$ 
PNNL-20650, Rev. 2

ASR 8824 Special Instructions

Page 2 of 14

\title{
Special Instructions for K-Basin Container SCS-CON-210 Container Composite Sample Analysis
}

\author{
Cognizant Scientists: \\ SK Fiskum, 376-7015 \\ RW Shimskey, 376-3183 \\ LA Snow, 376-4628 \\ CD Carlson, 376-2912
}

Material from the K West (KW) Basin Engineered Container SCS-CON-210 was combined into a container composite and then sieve-fractionated. Materials from $+2000 \mu \mathrm{m}$, between 500 and $2000 \mu \mathrm{m}$, and $-500 \mu \mathrm{m}$ are presented for analysis. One of the core samples from this container, KW210-A4, will also be analyzed in duplicate. (This sample was analyzed per ASR 8798 and preliminary results indicated high RPDs were obtained; the re-analysis will better clarify the U metal content.)

The material has been received in the Radiochemistry Processing Laboratory (RPL) and logged into the Radioactive Material Tracking (RMT) system as RMT \#37737; the Sample Information Checklist (SICL) is tied to this RMT number. The sample-specific IDs, the RPL/Analytical Support Organization (ASO) assigned sample IDs, and the first core sampling date (at the K Basin) are cross-referenced in Table 1 . Note that the 6-month hold time noted below applies to ICP-OES metals analysis on the PNL-ALO-129, Rev. 0 acid digest.

Table 1. Core Sample ID, RPL ID, Sampling Date, and Holding Time Cross-Reference

\begin{tabular}{|c|c|c|c|}
\hline Core Sample ID & RPL/ASO ID & $\begin{array}{c}\text { Sample Date } \\
\left(\mathbf{T}_{\mathbf{0}}\right)\end{array}$ & $\begin{array}{c}\text { End of 6-Month } \\
\text { Hold Time }\end{array}$ \\
\cline { 1 - 2 } KW210-LCC & $11-0791$ & & \\
\cline { 1 - 2 } KW210-MCC & $11-0792$ & $11 / 17 / 10$ & $5 / 17 / 11$ \\
\cline { 1 - 2 } KW210-SCC & $11-0793$ & & NA \\
\hline KW210-A4 & $11-0967$ & NA & \\
\hline
\end{tabular}

The sample processing and analysis schematic is shown in Figure 1. The processing, prior to the ASO-required analyses (non-highlighted blocks), is covered in a separate project-specific test instruction, 52578-TI008. The ASO-specific processing is shown in the highlighted blocks. The samples have already been aliquoted, so no further subdivision by ASO is required.

Although most work is being performed under the ASO Quality Assurance (QA) Plan, some activities shown in Figure lare outside of the scope of the plan such as X-ray diffraction (XRD) particle density, and particle size distribution (PSD). 
PNNL-20650, Rev. 2
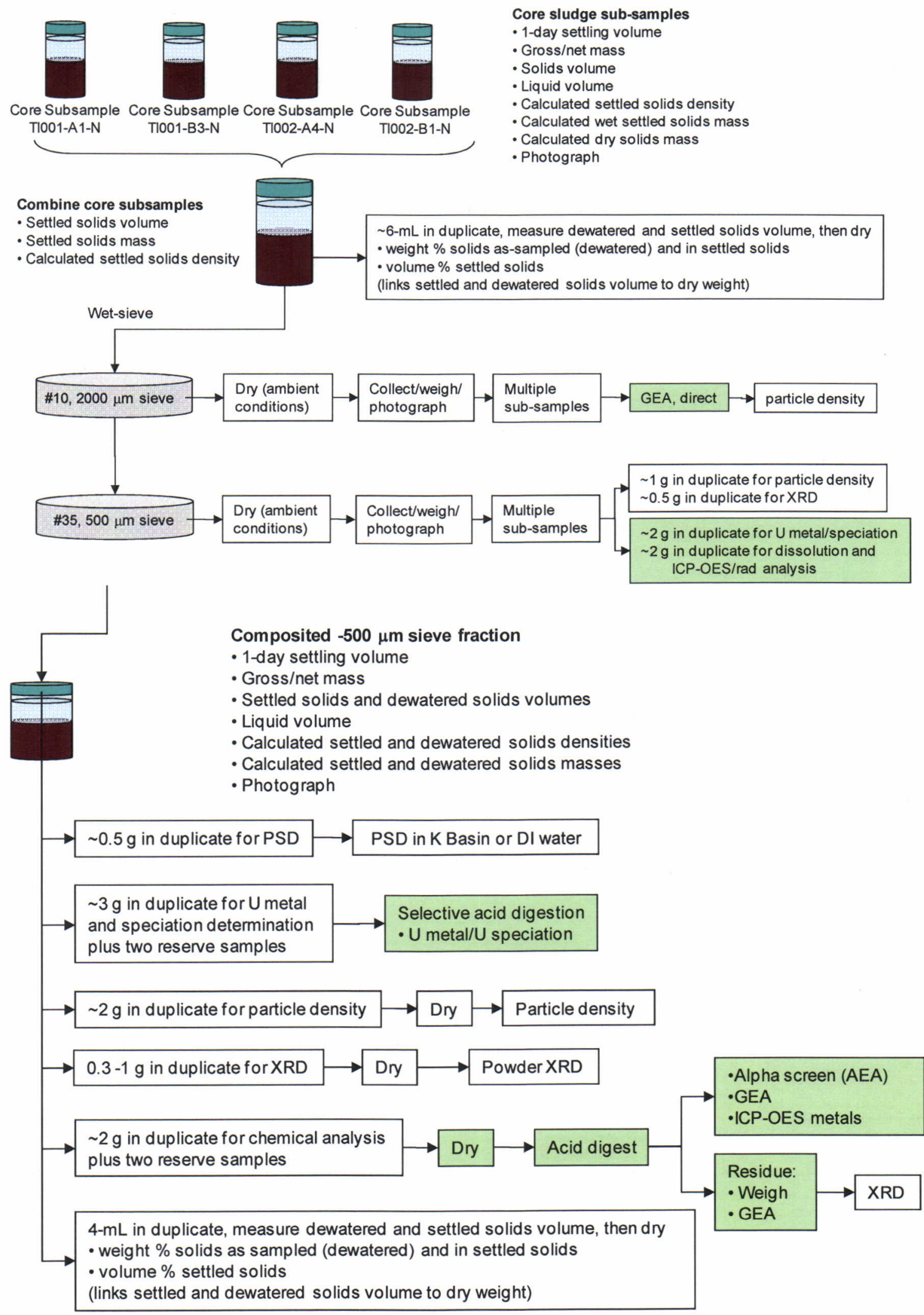

Figure 1. Analysis Schematic for KW210 Container Composite

C.7 
PNNL-20650, Rev. 2

The Chemical Measurement Center needs to charge analyses to the following work packages:

\begin{tabular}{|l|l|}
\hline H41220 & RPG-CMC-107, Rev. 1, Acid Digest \\
\hline H41220 & U Metal Analysis ICP-OES \\
\hline H41221 & U Oxide speciation \\
\hline \multirow{2}{*}{ H41216 } & GEA \\
\cline { 2 - 2 } & Alpha-AEA \\
\hline \multirow{2}{*}{ H41215 } & PNL-ALO-129, Rev. 0, Acid Digest \\
\cline { 2 - 2 } & ICP-OES \\
\hline
\end{tabular}

All sample solids masses have been calculated based on measures recorded from the Shielded Analytical Laboratory (SAL) processing activities as recorded in Test Instruction, 52578-TI008; these solids masses are reproduced in Table 2. The shaded (reserve) samples were collected as back-up samples and are not expected to be analyzed.

Table 2. Cross-Reference of Sample ID, RPL ID, Dewatered Mass, and Analysis

\begin{tabular}{|c|c|c|c|l||}
\hline Sample ID & RPL ID & $\begin{array}{c}\text { Dry or } \\
\text { Oak Ridge } \\
\text { Tube Tare, } \\
\text { g }\end{array}$ & $\begin{array}{c}\text { Dewatered } \\
\text { Solids Mass, } \\
\text { gdewatered }\end{array}$ & Analysis \\
\hline KW210-LCC & $11-0791$ & & dry & \\
\hline TI008-LA & $11-0791-S$ & NA & 0.7263 & Analytical (direct GEA only) \\
\hline KW210-MCC & $11-0792$ & & dry & \\
\hline TI008-MA & $11-0792-129-S$ & NA & 1.5122 & Analytical \\
\hline TI008-MB & $11-0792-129-D$ & NA & 1.9603 & Analytical duplicate \\
\hline TI008-MC & $11-0792-129-M S$ & NA & 1.3960 & Analytical matrix spike/reserve \\
\hline TI008-MD & $11-0792-$ Archive & NA & 1.1887 & Analytical reserve \\
\hline TI008-ME & $11-0792-107-S$ & NA & 2.5202 & U metal/ speciation \\
\hline TI008-MF & $11-0792-107-D$ & NA & 2.6176 & U metal/ speciation dup \\
\hline TI008-MG & $11-0792-107-M S$ & NA & 2.4524 & U metal matrix spike/reserve \\
\hline KW210-SCC & $11-0793$ & & dewatered & \\
\hline TI008-SA & $11-0793-129-S$ & 32.4119 & 1.9371 & Analytical \\
\hline TI008-SB & $11-0793-129-D$ & 32.1468 & 2.2705 & Analytical duplicate \\
\hline TI008-SC & $11-0793-129-M S$ & 33.0204 & 1.9678 & Analytical matrix spike \\
\hline TI008-SD & $11-0793-A r c h i v e$ & 33.4496 & 2.8118 & Analytical reserve \\
\hline TI008-SE & $11-0793-107-S$ & 38.9594 & 2.1878 & U metal/ speciation \\
\hline TI008-SF & $11-0793-107-D$ & 38.8666 & 3.5278 & U metal/speciation dup. \\
\hline TI008-SG & $11-0793-107-M S$ & 38.8473 & 4.1801 & U metal matrix spike \\
\hline KW210-A4 & & & & \\
\hline TI002-A4-R & $11-0967-107-S$ & 34.3532 & 4.37 & U metal only \\
\hline TI002-A4-S & $11-0967-107-D$ & 33.1604 & 4.36 & U metal only, duplicate \\
\hline
\end{tabular}

Analytes of interest and required detection limits are provided in Table 4; quality control (QC) sample acceptance criteria are provided in Table 5. One sample per analytical batch needs to be used to prepare a matrix spike (MS) sample. Subsampling of the stock material is a laborious 
process; a series of reserve samples from each core composite have been collected during the initial aliquoting process. The reserve samples should be used in case of catastrophic failure (i.e., spilling) of the primary or duplicate sample. The reserve samples were also collected in case the duplicate result did not agree with the primary result and a re-analysis might be required. The reserve samples may also be forwarded for analysis in place of the primary or duplicate samples based on the prerogative of the Cognizant Scientist (pending observations of actual mass collected, or other issues).

\section{Uranium Metal Analysis and Uranium Oxide Speciation}

\section{U Metal/U Speciation Sample Preparation}

The wet sample slurries (located in the SAL) are provided to ASO in tared Oak Ridge tubes (suitable for sample digestion). The dry samples are provided in 5-mL plastic vials (pour solids into Oak Ridge centrifuge tubes). The ASO is responsible for preparing the preparation blank (PB), the laboratory control sample (LCS), and the MS samples.

- The PB will consist of Hanford sand or equivalent and follow all processes that the samples are subjected to.

- The LCS will consist of Hanford sand, $\mathrm{U}_{3} \mathrm{O}_{8}$, (a mixture of U[IV] and U[VI]), and U metal beads.

- The MS will consist of a sample with U metal bead(s) added to it by ASO.

The samples will be processed per ASO Procedure, RPG-CMC-107, Rev. 1, Sample Preparation for Determination of Uranium Metal Concentrations in Sludge. At least three contacts with $\mathrm{Na}_{2} \mathrm{SO}_{4} / \mathrm{H}_{3} \mathrm{PO}_{4}$ shall be conducted to dissolve non-metal $\mathrm{U}$ species and at least 7 rinses conducted on the residual solids.

The dissolved (metal) $U$ and seventh rinse solution $U$ concentrations shall be determined by ICPOES, (RPG-CMC-211, Rev. 3, Determination of Elemental Composition by Inductively Coupled Argon Plasma Optical Emission Spectrometry [ICP-OES]). The seventh rinse solution U concentrations should be $<100 \mu \mathrm{g} \mathrm{U} / \mathrm{mL}$; the ICP-OES MDL for these samples shall be $\leq 1 \mu \mathrm{g} \mathrm{U} / \mathrm{mL}$. The ASO staff is directed to promply notify SK Fiskum /CD Carlson/DS Coffey if the $7^{\text {th }}$ rinse is $>100 \mu \mathrm{g} \mathrm{U} / \mathrm{mL}$, thus enabling PNNL to fulfill its commitment to the client for prompt notication of a potential issue with the analysis. The duplicate precision criterion (Table 5) only applies to the dissolved $U$ metal sample portion; it does not apply to the $7^{\text {th }}$ rinse solution.

Submit the $\mathrm{Na}_{2} \mathrm{SO}_{4} / \mathrm{H}_{3} \mathrm{PO}_{4}$ sample solutions containing the U(IV) and U(VI) for analysis by spectrophotometry (per RPG-CMC-255, Rev. 0, Sample Preparation and Analysis for Determining Uranium Oxide Oxidation States in K Basin Sludges). Note that the re-analysis of $\mathrm{KW} 210-\mathrm{A} 4$ samples do not require the $\mathrm{U}(\mathrm{IV}) / \mathrm{U}(\mathrm{VI})$ ratio analysis.

\section{U Metal and Speciation Reporting Units}

Report the $U$ metal results as $\mu \mathrm{g} \mathbf{U}$ per $\mathbf{g}$ of dewatered sludge, where the mass of dewatered sludge in the analysis aliquot is taken from Table 2 . Report the seventh rinse sample $\mathbf{U}$ concentration as $\mu \mathrm{g} U$ per $\mathrm{mL}$ of solution. Report the $\mathrm{U}(\mathrm{IV})$ and $\mathrm{U}(\mathrm{VI})$ oxidation states as percentages. 
PNNL-20650, Rev. 2

\section{Chemical Analysis Samples}

\section{Acid Digestion \\ Preparation}

Note: Balances used to support the nuclear materials $(\mathrm{Pu}, \mathrm{Am}, \mathrm{Np}$, and $\mathrm{U})$ analysis require stringent daily balance checks on each day the balance is used . Review section on "Quality Control" before measuring and recording sample mass data. To this end, all masses collected in SAL must have the linearity check conducted and recorded.

The analytical slurry samples are provided to ASO in tared Oak Ridge tubes (suitable for sample digestion); the analytical dry solids samples are provided in 5-mL plastic vials. The wet slurry analytical samples need to be dried to constant mass at $105^{\circ} \mathrm{C}$. Determine and report the net dry sample mass.

Acid digest the dried solids according to PNL-ALO-129, Rev. 0, $\mathrm{HNO}_{3}$ - $\mathrm{HCl}$ Acid Extraction of Solids Using a Dry-Block Heater. Report observations associated with the dissolution processing, including the undissolved solids mass. Provide a copy of the ASO preparation bench sheet to SK Fiskum and LA Snow.

Residual solids remaining after acid digestions are expected and of great interest and shall not be lost (refer to Figure 1). Remove the acid digestate as per procedure; this may entail centrifuging and decanting. Rinse as appropriate, collecting rinse solution with the acid digestion solution.

After residual solids are rinsed and isolated, dry the solids (in the digestion tube) to constant mass at $105^{\circ} \mathrm{C}$. Calculate the net residual solids mass along with the starting material dry solids mass and report to SK Fiskum/LA Snow. Additional processing of residual solids will depend on the mass relative to the starting (wet/dewatered) material. Additional processing may include:

- Remove from the SAL for pictures

- Direct gamma count

- Transfer solids outside of ASO to Doinita Neiner for XRD preparation and analysis.

\section{Acid Digestion Reporting Units}

Report the dry sample mass before digestion as $\mathrm{g}_{\mathrm{dry}}$, and report the dried residuals solids mass after digestion and rinsing as $\mathrm{g}_{\text {residual }}$.

\section{ICP-OES}

\section{ICP-OES Analysis}

Acid digestate aliquots (PNL-ALO-129) shall be analyzed for the Table 4 analytes of interest (Ag, Al, As, Ba, Be, Ca, Cd, Cr, Fe, Na, Ni, Mg, Pb, Sb, Se, Tl, U, V, Zn, and Zr) at the ICP-OES workstation according to RPG-CMC-211, Rev. 3, Determination of Elemental Composition by Inductively Coupled Argon Plasma Optical Emission Spectrometry (ICP-OES).

\section{ICP-OES Reporting Units}

Report solids sample analyte concentrations as $\mu \mathrm{g} / \mathrm{g}_{\mathrm{dry}}$ where $\mathrm{g}_{\mathrm{dry}}$ is the initial dry sample mass determined prior to the start of the PNL-ALO-129 acid digestion.

\section{Radiochemistry}

Preparations and Analysis 
Acid digestate aliquots (PNL-ALO-129) shall be directly aliquoted for GEA analysis. Acid digestate aliquots shall be aliquoted for separations, mounting, and/or analysis. Operations will be conducted according to the specific radiochemistry procedures delineated in Table 3 . No procedure or procedure revision substitutions are to be made without prior written authorization; requests shall be made by the ASO Lead and submitted to SK Fiskum or CD Carlson to forward to the client for approval.

Report analytes defined in Table 4 plus any other observed analytes.

Table 3. Authorized Radiochemistry Procedures for K Basin Sludge Analysis

\begin{tabular}{|l|c|c|c|}
\hline Analyte & Separation & Mounting & Counting/Analysis \\
\hline GEA & NA & NA & $\begin{array}{c}\text { RPG-CMC-450, Rev. 1 Gamma } \\
\text { Energy Analysis (GEA) and Low- } \\
\text { Energy Photon Spectrometry (LEPS) }\end{array}$ \\
\hline $\begin{array}{l}\text { Alpha screen } \\
\text { /AEA }\end{array}$ & NA & $\begin{array}{c}\text { RPG-CMC-496, Rev. 0, } \\
\text { Coprecipitation Mounting of } \\
\text { Actinides for Alpha Spectroscopy }\end{array}$ & $\begin{array}{c}\text { RPG-CMC-422, Rev. 2 Solutions } \\
\text { Analysis: Alpha Spectrometry }\end{array}$ \\
\hline
\end{tabular}

\section{Radiochemistry Reporting Units}

Report solids sample analyte concentrations as $\mu \mathrm{Ci} / \mathrm{g}_{\text {dry }}$ where $\mathrm{g}_{\text {dry }}$ is the initial dry sample mass determined prior to the start of the PNL-ALO-129 acid digestion.

Report the residual solids GEA analytes as $\mu \mathrm{Ci} / \mathrm{g}_{\text {residual }}$, where $\mathrm{g}_{\text {residual }}$ is the mass of residual solids that did not dissolve during acid digestion. The precision criteria defined in Table 5 do not apply to the undissolved solids.

\section{Archive of SAL-Prepared Chemical Analysis Samples}

The PNL-ALO-129 acid digest preparations will result in a $\sim 50$ - to $100-\mathrm{mL}$ volume. This solution will be apportioned to the laboratory as needed to conduct work-station-specific analyses. At the time the acid digestates are apportioned to the analysis work locations, $\sim 15-\mathrm{mL}$ aliquot from each preparation should be collected and removed from SAL and stored as an archive sample. The vials need to be labeled with the following information: date, RPL-ID, sample ID, matrix, and hazard. The remaining portion of the acid digestion preparations may be disposed of.

\section{Quality Control}

All work is required to be conducted to the requirements of the Hanford Analytical Services Quality Assurance Requirements Document (HASQARD, Rev. 3). Performing work to the requirements of the ASO QA Plan (ASO-QAP-001, Rev. 7) will satisfy the HASQARD requirements. Where there is a discrepancy between the data quality objectives (DQOs) in the ASO QA Plan and these Special Instructions, the Special Instructions takes precedence as there are specific $\mathrm{K}$ Basin project requirements. Where there is a discrepancy between the data quality objectives (DQOs) in the Special Instructions and the Quality Assurance Project Plan/Sampling and Analysis Plan (QAPjP/ SAP)for Sludge in the KW Engineered Containers; KBC-33786, Rev. 2, December 2009, the QAPjP/SAP takes precedence. 
NOTE: Balances used to support the nuclear materials $(\mathrm{Pu}, \mathrm{Am}, \mathrm{Np}$, and $\mathrm{U})$ analysis require more stringent daily balance checks on each day the balance is used. To maintain consistency with project-established hot cell practices, the ASO SAL-related operations must use at least 3 masses (e.g., A, B, and A+B) to provide an indication of balance accuracy and linearity. The balance control checks must be traceable in the records supporting these analyses. It is suggested that the daily control check log sheets be provided as part of the analytical report; however the ASO may choose alternate means to establish traceability. Acceptance limits for control checks for the balance in SAL cell 2 are provided in Appendix A. If masses are inadvertently collected on a balance without the daily calibration verification check, then the ASO Lead must immediately upon discovery inform the QE, Cognizant Scientist and the Project Manager.

The balance calibration verification checks at the fumehood workstations may be made per the normal process where one calibration verification check weight (mass) is measured daily when then balance is used (as per the ASO procedure, PNL-ASO-052, Rev. 1, Balance Performance Checks). This is allowed because the fumehood operations use volume measures or do not directly support nuclear materials accountability.

Solids matrix preparative analysis QC is to include a PB sample, sample duplicate (provided), LCS (or BS) and a sample MS (a separate sample is provided for spiking by the ASO) as indicated in Table 2 and Table 5. The BS for the AEA screen will be generated after acid digestion on a sample split, i.e., post-digestion spike (PS). The MS for alpha AEA screen will be generated after acid digestion on a sample split, i.e., PS.

The ASO is responsible for preparing the $\mathrm{PB}, \mathrm{LCS} / \mathrm{BS}$, and spiking MS QC samples. The preparative MS and LCS/BS are to include all the analytes of interest: $\mathrm{Ag}, \mathrm{Al}, \mathrm{As}, \mathrm{Ba}, \mathrm{Be}, \mathrm{Ca}$, $\mathrm{Cd}, \mathrm{Cr}, \mathrm{Fe}, \mathrm{Na}, \mathrm{Ni}, \mathrm{Mg}, \mathrm{Pb}, \mathrm{Sb}, \mathrm{Se}, \mathrm{Tl}, \mathrm{U}, \mathrm{V}, \mathrm{Zn}$, and $\mathrm{Zr}$.

The duplicate, LCS/BS, and MS sample QC acceptance criteria are provided in Table 5. The following section discusses actions to take in the event of QC failures.

Note: The ASO staff must inform/report QC failures promptly to the ASO Lead who will in turn inform the Cognizant Scientist with copy to CD Carlson and the K Basin QE (Deborah Coffey), thus enabling PNNL to fulfill its commitment to the client for prompt notification of such failures. The impact of any QC failure on data quality or project schedule (e.g., re-runs) will be assessed in conjunction with the client.

1. The preparation blank (PB) analyte concentration shall be less than the estimated quantitation limit (EQL) or the minimum detectable activity (MDA) of the associated sample. When the $\mathrm{PB}$ concentration is equal to or exceeds the EQL or MDA, then the $\mathrm{PB}$ concentration shall not exceed $5 \%$ of the measured concentration present in the sample.

2. If and when it has been determined that the data are not useable, then the affected samples in the processing batch must be re-prepared and re-analyzed for the failed analytes, availability of samples permitting.

3. In the case of multi-elemental methods (ICP-OES), isolated QC failure(s) may be communicated to SK Fiskum, RW Shimskey, Clark Carlson and the K Basin QE, DS Coffey, by the ASO Lead for an assessment of the impact on data interpretation. If the data are acceptable as-is, the project staff will communicate in writing (e.g., e-mail message) that the 
PNNL-20650, Rev. 2

ASR 8824 Special Instructions

Page 9 of 14

data may be reported; the e-mail acceptance and resulting limitations on the data from the QC sample failure(s) shall be included in the final analytical report.

4. If and when the MS sample fails to meet the acceptance criteria, the results shall be investigated for potential sources of error by comparing the results of the MS to the BS. When the sources of error cannot be identified, the failure of the MS will be attributed to a sample matrix effect and any resulting limitations on the data shall be included in the report.

\section{Reporting}

The analytical data report shall be prepared in accordance with procedure PNL-ASO-058, Rev. 0.2, ASO Data Reporting, Section 5.3, Comprehensive Data Report. Please be sure to include action taken with respect to any identified unexpected results and discrepancies.

The Comprehensive Data Report contains three main parts; a data report cover page, a narrative, and the data summary. These elements are identified below; red text indicates project-driven scope. Black text is taken directly from the ASO procedure: PNL-ASO-058, Rev. 0.2.

Data Report Cover Page Contents:

- Header identifying name/address of laboratory

- Client Name:

- $\quad$ Project \#/WP \#(s):

- $\quad$ ASR \#(s):

- Total \# Samples:

- Report Date:

- Report Revision Date: when applicable

- Filename(s): where data files can be accessed

- Dates of Sample Processing and Analysis:

- Procedure(s): Number and title (sample processing and analysis) and revision number

- M\&TE (serial number or property number):

- Reference Date (when applicable):

- Client ID and RPL ID Sample Numbers:

- Report Review and Approval:

Preparer Date

(Printed name and signature of responsible individual)

Technical Reviewer Date

(Printed name and signature of technical reviewer)

A case narrative describing the analysis, limitations on the analysis results, and QA/QC issues Narrative Contents:

- Sample Description and Analyses

- Sample dissolution/preparation dates

- Sample Preparation

- Analysis dates

- Deviations from the written procedure, if any

- Method specific Sample Preparation, Separation, Mounting and Counting Methods as applicable

- Reporting Basis/Units

- QC Criteria/Results 
PNNL-20650, Rev. 2

ASR 8824 Special Instructions

Page 10 of 14

- Limitations of the Data Interferences/Resolution Uncertainty

- Comments

Data Summary Contents:

- Client ID

- RPL ID with full ASO-related suffixes (e.g., 129-S)

- Results and result uncertainties

The following elements may be included in the final report or be traceable to the test results (usually by annotation on bench sheets) and be maintained as lifetime records:

- identification of standards used

- identification of M\&TE used

- records of daily check weight tracking

- signature and date of person who performed the test and recorded the data

- analytical bench sheets

- hand calculation review documentation.

Technical reviewers are required to be staff other than the analyst performing the work and knowledgeable of the area being reviewed. The technical review shall be completed and all reviewer issues resolved before reporting final results. Technical reviewers must receive a complete data package including narratives for review. A technical review consists of the following elements:

- evaluation of method and QC performance

- evaluation of compliance with technical (including procedural) and QC requirements, as defined in the ASR

- verification of transcription accuracy

- check for correctness of calculations

- assuring the correct reporting units (and reference dates) are used

- evaluation of overall consistency and reasonableness of data

- evidence of implementation of appropriate corrective action, when necessary.

Analytical results shall be reported both in hard copy and electronically (including excel data files to support accurate data transcription into project records). Appropriately marked "Preliminary" data reports and electronic files shall be provided by the ASO Lead as soon as practical after completion of analysis. The final ASR data report shall be provided no later than the commitment date on the ASR. 
PNNL-20650, Rev. 2

ASR 8824 Special Instructions

Page 11 of 14

Table 4. Method Detection Limits for Solids

\begin{tabular}{|c|c|c|}
\hline Analyte & Solids & Analysis Method \\
\hline & $\mu \mathrm{Ci} / \mathrm{g}_{(\mathrm{dry})}$ & \\
\hline${ }^{134} \mathrm{Cs}$ & $2.0 \mathrm{E}-01$ & \multirow{7}{*}{$\begin{array}{l}\text { GEA } \\
\text { (Reference date = date and time of the } \\
\text { first KW210 core sample analysis } \\
\text { measured by GEA for ASR } 8798 \text {.) }\end{array}$} \\
\hline${ }^{137} \mathrm{Cs}$ & $1.5 \mathrm{E}-02$ & \\
\hline${ }^{60} \mathrm{Co}$ & $1.0 \mathrm{E}-02$ & \\
\hline${ }^{152} \mathrm{Eu}$ & $1.5 \mathrm{E}+00$ & \\
\hline${ }^{154} \mathrm{Eu}$ & $1.5 \mathrm{E}+00$ & \\
\hline${ }^{155} \mathrm{Eu}$ & $2.0 \mathrm{E}+00$ & \\
\hline${ }^{241} \mathrm{Am}$ & $1.5 \mathrm{E}+01$ & \\
\hline${ }^{241} \mathrm{Am} /{ }^{238} \mathrm{Pu}$ & $1.0 \mathrm{E}-03$ & \multirow{2}{*}{ Alpha screen/ AEA } \\
\hline \multirow[t]{2}{*}{${ }^{239+240} \mathrm{Pu}$} & $1.0 \mathrm{E}-03$ & \\
\hline & $\mu \mathrm{g} / \mathrm{g}_{(\mathrm{dry})}$ & \\
\hline $\mathrm{Ag}$ & $2.0 \mathrm{E}+01$ & \multirow{20}{*}{ ICP-OES } \\
\hline $\mathrm{Al}$ & $1.0 \mathrm{E}+02$ & \\
\hline As & $2.0 \mathrm{E}+01$ & \\
\hline $\mathrm{Ba}$ & $4.0 \mathrm{E}+02$ & \\
\hline $\mathrm{Be}$ & $2.0 \mathrm{E}+01$ & \\
\hline $\mathrm{Ca}$ & $2.0 \mathrm{E}+02$ & \\
\hline $\mathrm{Cd}$ & $4.0 \mathrm{E}+00$ & \\
\hline $\mathrm{Cr}$ & $2.0 \mathrm{E}+01$ & \\
\hline $\mathrm{Fe}$ & $1.0 \mathrm{E}+02$ & \\
\hline $\mathrm{Mg}$ & $2.0 \mathrm{E}+02$ & \\
\hline $\mathrm{Na}$ & $2.0 \mathrm{E}+02$ & \\
\hline $\mathrm{Ni}$ & $2.0 \mathrm{E}+01$ & \\
\hline $\mathrm{Pb}$ & $2.0 \mathrm{E}+02$ & \\
\hline $\mathrm{Sb}$ & $2.0 \mathrm{E}+01$ & \\
\hline $\mathrm{Se}$ & $2.0 \mathrm{E}+02$ & \\
\hline $\mathrm{Tl}$ & $2.0 \mathrm{E}+01$ & \\
\hline V & $2.0 \mathrm{E}+01$ & \\
\hline $\mathrm{Zn}$ & $2.0 \mathrm{E}+01$ & \\
\hline $\mathrm{Zr}$ & $2.0 \mathrm{E}+01$ & \\
\hline \multirow[t]{2}{*}{$\mathrm{U}$} & $1.0 \mathrm{E}+03$ & \\
\hline & $\begin{array}{l}\text { wt\% of as-sampled } \\
\text { sludge }\end{array}$ & \\
\hline U metal & 0.01 & $\begin{array}{l}\text { Selective dissolution and } U \text { analysis by } \\
\text { KPA or ICP-OES }\end{array}$ \\
\hline U oxide speciation & N/A & $\begin{array}{l}\text { Selective dissolution and } \\
\text { spectrophotometry }\end{array}$ \\
\hline
\end{tabular}


PNNL-20650, Rev. 2

ASR 8824 Special Instructions

Page 12 of 14

Table 5. Analytical Quality Control Acceptance Criteria

\begin{tabular}{|c|c|c|c|c|}
\hline \multirow[t]{2}{*}{ Analyte } & \multirow[t]{2}{*}{ Analytical Technique } & $\begin{array}{l}\text { LCS or BS \% } \\
\text { Recovery }^{\left({ }^{2}\right)}\end{array}$ & $\begin{array}{c}\text { MS or AS } \\
\text { \% Recovery }^{(b)}\end{array}$ & \multirow{2}{*}{$\begin{array}{c}\text { Duplicate } \\
\text { RPD }^{\text {(c) }}\end{array}$} \\
\hline & & \multicolumn{2}{|c|}{ Pre-digestion spike } & \\
\hline As identified in Table 4 & ICP-OES & $80-120 \%$ & $80-120 \%$ & $\leq 20 \%$ \\
\hline U metal & $\begin{array}{l}\text { Selective dissolution and } \\
\text { ICP-OES }\end{array}$ & $75-125 \%$ & N/A & $\begin{array}{c}\leq 20 \% \text { where } \\
{[\mathrm{U}]>0.1 \mathrm{wt} \%}\end{array}$ \\
\hline U oxide speciation & $\begin{array}{l}\text { Selective dissolution and } \\
\text { spectrophotometry }\end{array}$ & (e) & N/A & N/A \\
\hline \multirow[t]{2}{*}{ Analyte } & \multirow[t]{2}{*}{ Analytical Technique } & $\begin{array}{l}\text { LCS or BS \% } \\
\text { Recovery }^{(\mathbf{a})}\end{array}$ & $\begin{array}{c}\text { Matrix Spike \% } \\
\text { Recovery }^{(\mathrm{b})}\end{array}$ & \multirow{2}{*}{$\begin{array}{l}\text { Duplicate } \\
\text { RPD }^{\left(c^{2}\right)}\end{array}$} \\
\hline & & \multicolumn{2}{|c|}{ Post-digestion spike } & \\
\hline $\begin{array}{c}\text { Alpha screen } \\
\text { (spike material is }{ }^{239} \mathrm{Pu} \text { ) }\end{array}$ & AEA & $80-120 \%$ & $75-125 \%$ & $\leq 25 \%$ \\
\hline As identified in Table 4 & GEA & $\begin{array}{l}\text { Counter control } \\
\text { source is used }^{(d)}\end{array}$ & N/A & $\leq 20 \%$ \\
\hline
\end{tabular}

N/A - not applicable

Footnotes:

(a) LCS = Laboratory Control Standard; BS = Blank Spike. A laboratory control sample (LCS) or blank spike (BS) samples are used to monitor the effectiveness of the sample preparation process and are good indicators of method accuracy. Ideally, the LCS is a material similar to the sample being processed, containing the analytes of interest (e.g., standard reference material). An LCS, if available, shall be prepared with each batch of samples processed at the same time. When an appropriate LCS is not available, a BS shall be used in lieu of the LCS. A BS is distilled or deionized water or another suitable matrix spiked with the analytes of interest. It may not be possible to prepare a single BS that contains all analytes of interest (e.g., chemical incompatibility). In such cases, an agreement with the client shall be made to identify the analytes of interest used to prepare the BS, and more than one BS may be used. The BS and AS results are expressed as percent recovery; i.e., the amount measured, divided by the known concentration, multiplied by 100 .

(b) MS = matrix spike; AS = Analytical Spike. For some methods, the sample accuracy is expressed as the percent recovery of a matrix spike (MS) or analytical spike (AS) sample. Post-digestion spikes and analytical spikes are also included under these acceptance criteria. The spiked sample result is expressed as percent recovery; i.e., the amount measured less the amount in the sample, divided by the spike added, times 100 . One MS (or post spike or analytical spike [AS]) is performed per analytical batch. Samples are batched with similar matrices. For ICP analytes, the accuracy can also be determined based on use of serial dilutions. In cases where the ICP-OES MS concentration is low relative to the sample analyte concentration (resulting in meaningless recovery calculations), matrix effects will be evaluated from a post-digestion spike.

(c) RPD = Relative Percent Difference between the samples. Sample precision is estimated by analyzing replicates taken separately through preparation and analysis. The ASO calculates RPD from the absolute value of the difference between a sample and duplicate.

(d) Per RPG-CMC-450, Rev 1, a counter control source is checked daily and must be within \pm 3 sigma or $\pm 3 \%$, whichever is greater, of the control value.

(e) The $\mathrm{LCS} \mathrm{U}_{3} \mathrm{O}_{8}$ accepted ratios are $33.3 \% \mathrm{U}(\mathrm{IV})$ and $66.6 \% \mathrm{U}(\mathrm{VI})$. The acceptable $\mathrm{U}(\mathrm{VI})$ range is 62 to $72 \%$ or $\pm 5 \%$ window. Thus the acceptable U(IV) range is 28 to $38 \%$ or $\pm 5 \%$ window.

\section{References}

52578-TI008, Rev. 0, Preparation of K West Basin Consolidated Container Composite for SCS$\mathrm{CON}-210$

ASO-QAP-001, Rev. 7, Analytical Support Operations Quality Assurance Plan

PNL-ASO-052, Rev. 1, Balance Performance Checks 
PNNL-20650, Rev. 2

ASR 8824 Special Instructions

Page 13 of 14

PNL-ASO-058, Rev. 0.2, ASO Data Reporting

Hanford Analyical Services Quality Assurance Requirments Document (HASQARD), 2007, DOE/RL-96-68, Revsion 3, Department of Energy Richland Office, Richland Washington

RPG-CMC-107, Rev. 1, Sample Preparation for Determination of Uranium Metal Concentrations in Sludge

PNL-ALO-129, Rev. 0, $\mathrm{HNO}_{3}$-HCl Acid Extraction of Solids Using a Dry-Block Heater

RPG-CMC-211, Rev. 3, Determination of Elemental Composition by Inductively Coupled Argon Plasma Optical Emission Spectrometry (ICP-OES)

RPG-CMC-255, Rev. 0, Sample Preparation and Analysis for Determining Uranium Oxide Oxidation States in K Basin Sludges

RPG-CMC-422, Rev. 2, Solutions Analysis: Alpha Spectrometry

RPG-CMC-450, Rev. 1, Gamma Energy Analysis (GEA) and Low-Energy Photon Spectrometry (LEPS)

RPG-CMC-496, Rev. 0, Coprecipitation Mounting of Actinides for Alpha Spectroscopy

Quality Assurance Project Plan (QAPjP)/Sampling and Analysis Plan (SAP)for Sludge in the KW Engineered Containers; KBC-33786, Rev. 2, December 2009 
PNNL-20650, Rev. 2

ASR YYYYY Special Instructions

Page 14 of 14

\section{Appendix A}

SAL Balance Control Checks and Acceptance Limits

The balance control checks will document accuracy and linearity by measure of three masses for the analytical balance Sartorius MSG234 (M\&TE 40023610).

Acceptance limits for the three different mass measures are provided. These acceptance limits have been reviewed and approved by Safeguards (client Terri Welsh, Mission Suppprt Alliance) in 2010. Re-evaluation of the check weights following the 2/2011 calibration on the MSG234 were conducted. The previously approved acceptance limit were slightly more restrictive than the recalculated acceptance limits from 10 measures of each weight following the February 2011 re-calibration. The more restrictive acceptance limit will be applied for conservativism.

Radiochemical Processing Laboratory Battelle, Pacific Northwest National Laboratory

K-Basin Project Type of Balance: Sartorius MSG234

\begin{tabular}{|c|}
\hline Balance Information \\
\hline \hline$\frac{\text { Identificaiton (e.g. WD34567) }}{40023610 / W D 68444}$ \\
\hline$\frac{\text { Location (e.g. Building/Room) }}{R P L / 201 \text { Cell 2 }}$ \\
\hline Calibration Due Date \\
Feb-2012 \\
\hline
\end{tabular}

\begin{tabular}{|c|c|c|c|c|c|c|}
\hline Check Weight (CW) Information & $\# 1$ & \begin{tabular}{|cc}
$\mathrm{cw}$ \\
Units
\end{tabular} & $\# \mathbf{2}$ & \begin{tabular}{|c|}
$c w$ \\
Units
\end{tabular} & $\# 3$ & $\begin{array}{c}c \mathrm{cw} \\
\text { Units }\end{array}$ \\
\hline $\mathrm{CW}$ Identification & "K-10" & & "Alien" & & $\begin{array}{c}\text { "Megatron" + } \\
\text { "Alien" }\end{array}$ & \\
\hline CW Assigned Mass: & 10.0000 & $g$ & 100.0002 & $\mathrm{~g}$ & 150.0004 & g \\
\hline Range Low Mass (+Units) \&: & 10.0000 & g & 99.9998 & g & 149.9995 & g \\
\hline Range High Mass (+Units): & 10.0010 & g & 100.0015 & g & 3000.8390 & g \\
\hline
\end{tabular}


Battelle PNNL/RPL/ASO Mass Measurement Analysis Report

P.O. Box 999, 902 Battelle Blvd., Richland, Washington 99352

\section{Mass Measurements}

\begin{tabular}{|l|l|}
\hline Project / WP\#: & $52578 / \mathrm{H} 41215$ \\
\hline ASR\#: & 8824 \\
\hline Client: & S. Fiskum \\
\hline Total \# of Samples: & 5 \\
\hline
\end{tabular}

\begin{tabular}{|c|c|c|}
\hline Engineered Container \& Core ID & Sample IDs & RPL Number \\
\hline KW-210-MCC & TI008-MA, -MB & $11-0792-129-\mathrm{S},-129-\mathrm{D}$ \\
\hline KW-210-SCC & TI008-SA, -SB, -SC & $11-0793-129-\mathrm{S},-129-\mathrm{D},-129-\mathrm{MS}$ \\
\hline
\end{tabular}

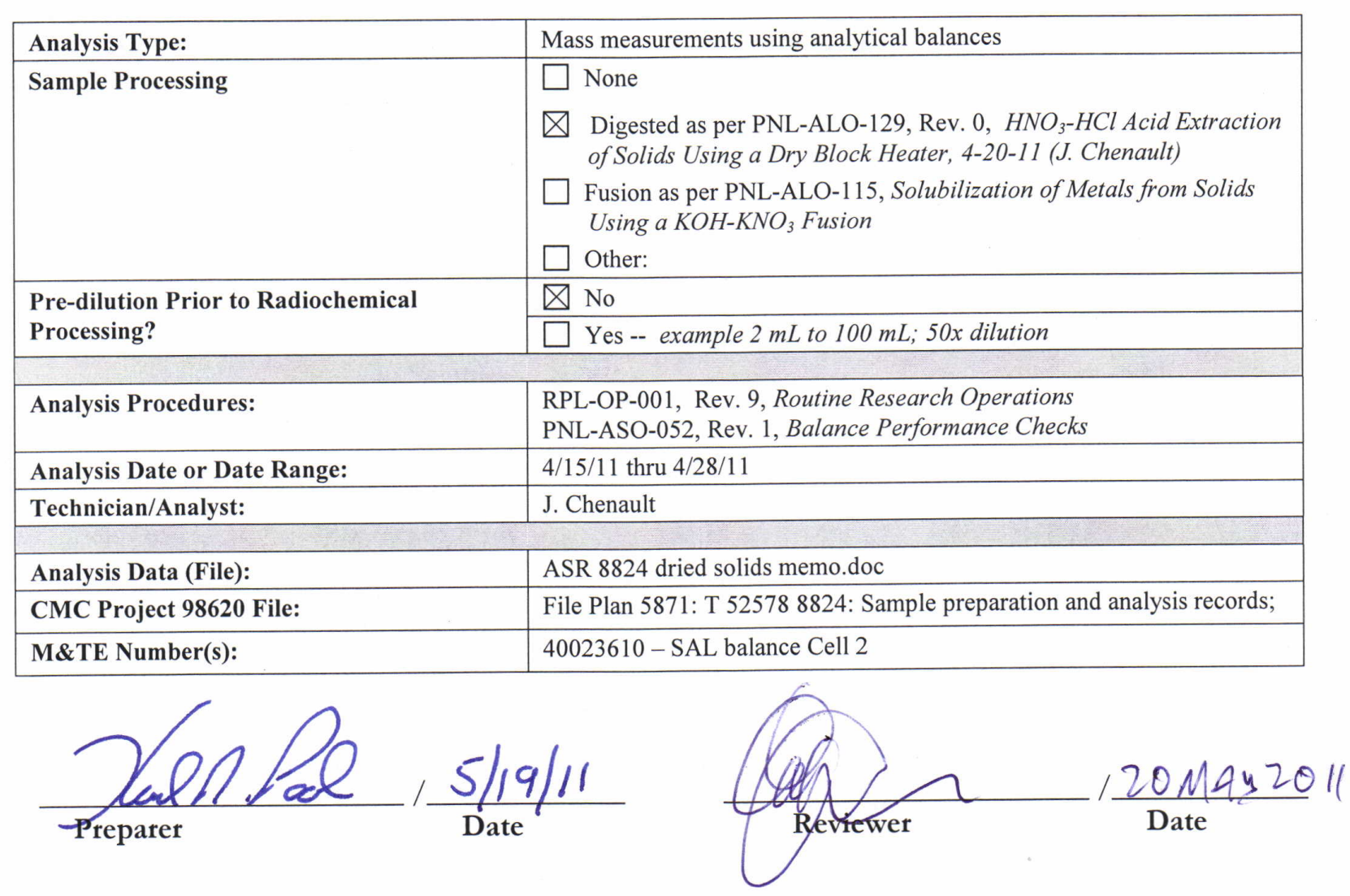




$\begin{array}{llll}\text { Date: } & \text { May 18, 2011 } & \text { Project No.: } & \mathbf{5 2 5 7 8} \\ \text { To: } & \text { Sandy Fiskum } & & \\ \text { From: } & \text { Karl Pool } & & \\ & \text { Initial dried solids and residual } & \text { Internal Distribution: } & \text { File/LB } \\ \text { Subject: } & \begin{array}{l}\text { dried solids masses for ASR } \\ \mathbf{8 8 2 4}\end{array} & \\ & \mathbf{8} & & \end{array}$

Prior to ASO sample digestion, the previously air dried and slurry samples for ASR 8824 were dried to constant weight at $105^{\circ} \mathrm{C}$ in pre-tared Teflon Oak Ridge digestion tubes in the Shielded Analytical Laboratory (SAL) using a drying oven. The dry masses of the solids prior to digestion are presented in the Table 1. The dried solids were then acid digested using ASO procedure PNL-ALO-129, " $\mathrm{HNO}$ - $\mathrm{HCl}$ Acid Extraction of Solids Using a Dry-Block Heater". The acid volume used was proportioned to the mass of sample. Samples that had mass of $1 \mathrm{~g}$ or less used $8 \mathrm{~mL}$ of each acid, samples that had mass of 1 to $1.5 \mathrm{~g}$ used $12 \mathrm{~mL}$ of each acid and samples which had mass of 1.5 to $2.0 \mathrm{~g}$ used $16 \mathrm{~mL}$ of each acid. At the completion of the acid digestion, the samples were cooled and the residual (un-dissolved solids) were allowed to settle. The digestate with settled residual was then centrifuged for approximately 10 minutes to consolidate the solids. The supernatant solution was carefully removed using transfer pipettes. The residual solids were each rinsed 3 consecutive times using $\sim 12$ to $16 \mathrm{~mL}$ of the same reagent acids $(1: 1$ combination of $\left.8 \mathrm{M} \mathrm{HNO}_{3}+8 \mathrm{M} \mathrm{HCl}\right)$ used in the initial acid digestion. The centrifuged solids were dislodged from the bottom of the centrifuge tube using a vortex mixer after each addition of the rinse acids. The acid rinse/solids were centrifuged for approximately 10 minutes after each acid rinse addition. The remaining rinsed solids were returned to the drying oven for drying. The residual solids in the Oak Ridge tubes were dried in the drying oven for several days at $105^{\circ} \mathrm{C}$ to constant weight. The dry masses of the solids after digestion are presented in the Table 1.

The initial acid digestate and acid rinse solutions were collected and brought to a final volume of $100 \mathrm{~mL}$ in plastic volumetric flasks. The solutions in the volumetric flask were thoroughly mixed prior to subsampling for chemical and radiochemical analyses. 
PNNL-20650, Rev. 2

MEMORANDUM

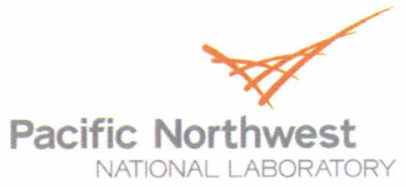

\section{Battelle}

The Business of Innovation

Table 1 - Dried solids masses

\begin{tabular}{|l|l|c|c|}
\hline \multicolumn{1}{|c|}{ RPL ID } & \multicolumn{1}{|c|}{ Client ID } & $\begin{array}{c}\text { Initial dried solids mass } \\
\text { prior to digestion (grams) }\end{array}$ & $\begin{array}{c}\text { Final dried solids mass remaining } \\
\text { after digestion (grams) }\end{array}$ \\
\hline $11-0792-129-S$ & TI008-MA & 1.3641 & 1.1750 \\
\hline $11-0792-129-D$ & T1008-MB & 1.7754 & $\mathbf{1 . 5 1 8 6}$ \\
\hline $11-0793-129-S$ & T1008-SA & $\mathbf{0 . 8 8 7 3}$ & $\mathbf{0 . 4 0 8 7}$ \\
\hline $11-0793-129-D$ & Tl008-SB & $\mathbf{1 . 0 8 7 9}$ & $\mathbf{0 . 5 0 0 9}$ \\
\hline $11-0793-129-M S$ & Tl008-SC & $\mathbf{0 . 8 9 9 9}$ & $\mathbf{0 . 3 9 3 4}$ \\
\hline
\end{tabular}

E54-1900-001 (8/98) 
Battelle PNNL/RPL/Inorganic Analysis ... ICP-OES Analysis Report PO Box 999, Richland, Washington 99352

Project / WP\#: $\quad 52578$ / H41220

ASR\#:

8824

Client:

S. Fiskum

Total Samples: $\quad 4$ (solids)

\begin{tabular}{|c|c|c|}
\hline & First & Last \\
\hline RPL\#: & 11-0792-S & 11-0793-D \\
\hline Client ID: & TI008-MA & TI008-SB \\
\hline
\end{tabular}

Procedure: $\quad$ RPG-CMC-211, "Determination of Elemental Composition by Inductively Coupled Argon Plasma Optical Emission Spectrometry (ICP-OES)", Rev. 3.

Analyst: J. Carter

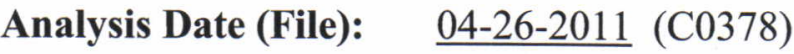

See Chemical Measurement Center 98620 file: $\quad \underline{I C P-325-405-3}$

(Calibration and Maintenance Records)

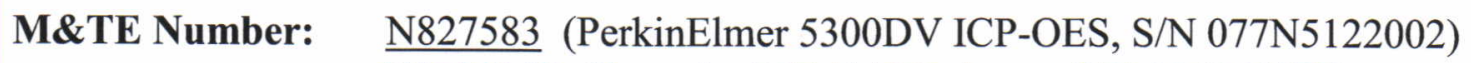

$\underline{\text { WD66158 }}$ (Sartorius ME414S Balance, S/N 224066373)

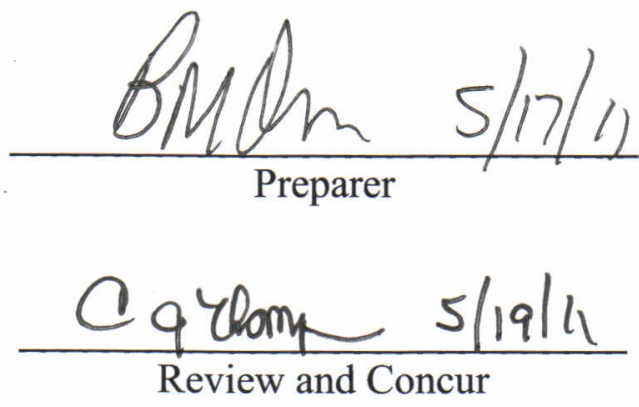




\section{Battelle PNNL/RPL/Inorganic Analysis ... ICP-OES Analysis Report}

Two samples in duplicate were provided for ICP-OES analysis under Analytical Service Request (ASR) 8824. The samples and duplicates were obtained from sub-samples prepared in the High Level Radiochemistry Facility (HLRF). Each of the samples was processed in the Shielded Analytical Laboratory (SAL) following RPL procedure PNL-ALO-129 using 1 to 2 grams of sample with dilution to a final volume of $100 \mathrm{~mL}$.

Analytes of interest (AOIs) were specified in the ASR, and are listed in the upper section of the attached ICP-OES Data Report. The quality control (QC) results for these AOIs have been evaluated and are presented below. Analytes other than AOIs are reported in the bottom section of the report, but have not been fully evaluated for QC performance. The results are reported on a mass basis $\left(\mu \mathrm{g} / \mathrm{g}_{\text {dry }}\right)$ for each detected analyte based on the original sample mass. All data have been adjusted for laboratory processing factors and instrument dilutions. There were several QC issues associated with the present analyses. These issues are discussed below and also in Occurrence Report (OR) 52578-04-29-11.

Except for As, Sb, Se, and Tl, the results are reported for 10x diluted samples. As, Sb, Se, and Tl are reported for undiluted samples (1x) in order to obtain the necessary sensitivity. Analyzing the samples at $1 \mathrm{x}$, however, does introduce potential matrix issues as both the sample density and the acid content at $1 \mathrm{x}$ are considerably higher than the normal matrix seen by the instrument ( 0.5 M nitric acid).

Minimum Method Detection Limit (MDL) values were specified in the ASR. Except for Pb in Sample 11-0793-S, MDL levels were met for all AOIs. However, the observed level of Pb exceeded the MDL requirements thereby making this issue moot.

Calibration of the ICP-OES was done following the manufacturer's recommended calibration procedure and using multi-analyte custom standard solutions traceable to the National Institute of Standards and Technology (NIST). Midrange calibration verification standards (MCVA and MCVB) were used to verify acceptance of the two-point calibration curves obtained for each analyte, and also used for continuing calibration verification.

The controlling documents were ASO-QAP-001 and ASR-8824 Special Instructions. Instrument calibrations, QC checks and blanks (e.g., ICV/ICB, CCV/CCB, LLS, ICS), blank spike, matrix spike, post spike, duplicates, and serial dilution were conducted during the analysis run. The blank spike and matrix spike were prepared using a custom solution consisting of aliquots of BPNL-QC-1A and -2B, INT-QC-TCLP-A, and CG-SB1-1.

\section{Preparation Blank (PB):}

A preparation blank (reagents only) was prepared for the extraction process. Except for $\mathrm{Na}$ $(71.5 \mu \mathrm{g} / \mathrm{g})$, the concentrations of all AOIs were within the acceptance criteria of $\leq \mathrm{EQL}$ (estimated quantitation level) or less than $\leq 5 \%$ of the concentration in the sample. Sodium exceeded the 5\% requirement for Samples 11-0793-S and 11-0793-D, and is addressed in OR 52578-04-29-11. 
PNNL-20650, Rev. 2

\section{Battelle PNNL/RPL/Inorganic Analysis ... ICP-OES Analysis Report}

\section{Blank Spike (BS)/Laboratory Control Sample (LCS):}

A blank spike was prepared for the extraction process. Recovery values are listed for all analytes included in the spike that were measured at or above the EQL. Recovery values for the AOIs meeting this requirement ranged from 86 to $108 \%$, and were within the acceptance criterion of $80 \%$ to $120 \%$.

\section{Matrix-Spiked Sample:}

A matrix spike was prepared for the extraction process using Sample 11-0793. Recovery values are listed for all analytes included in the spike that were measured at or above the EQL. The recovery values for the AOIs meeting these requirements ranged from 78 to $162 \%$, and except for $\mathrm{Ca}, \mathrm{Na}$, and $\mathrm{Tl}$, were within the acceptance criterion of $80 \%$ to $120 \%$. However, $\mathrm{Ca}, \mathrm{Na}$, and $\mathrm{Tl}$ were also analyzed in the post-spike (discussed below) and were all recovered within the acceptance criterion.

\section{Duplicate Relative Percent Difference (RPD):}

Duplicates were prepared for each of the two samples. RPDs are listed for all analytes that were measured at or above the EQL. RPDs for the AOIs meeting this requirement ranged from 0.7 to $143.2 \%$, and except for $\mathrm{Ba}, \mathrm{Cr}, \mathrm{Fe}, \mathrm{Ni}, \mathrm{Pb}$, and $\mathrm{Zr}$ for Sample 11-0792, were within the acceptance criterion of $\leq 20 \%$. The high RPDs for 11-0792 are likely from sample heterogeneity and/or from the sub-sampling in the HLRF, and are addressed in OR 52578-04-29-11.

\section{Post-Spike/Analytical Spike Sample (A Component):}

A post spike (A Component) was conducted on Sample 11-0793-S. Recovery values are listed for all analytes in the spike that were measured at or above the EQL, and that had a spike concentration $\geq 25 \%$ of that in the sample. Recovery values for the AOIs meeting these requirements ranged from 92 to $114 \%$, and were within the acceptance criterion of $80 \%$ to $120 \%$.

\section{Post Spike/Analytical Spike Sample (B Component):}

A post spike (B Component) was conducted on Sample 11-0793-S. Recovery values are listed for all analytes in the spike that were measured at or above the EQL, and that had a spike concentration $\geq 25 \%$ of that in the sample. The recovery value for $U$ was $102 \%$, and was within the acceptance criterion of $80 \%$ to 120 .

\section{Serial dilution:}

Five-fold serial dilution was conducted on Sample 11-0792-S. Percent differences (\%Ds) are listed for all analytes that had a concentration at or above the EQL in the diluted sample. The $\% \mathrm{Ds}$ for the AOIs meeting this requirement ranged from 0.2 to $4.7 \%$, and were within the acceptance criterion of $\leq 10 \%$.

\section{Other QC:}

$\mathrm{Ba}, \mathrm{Be}$, and $\mathrm{Zn}$ were slightly above the EQL for some of the Interference Check Standards (ICSs) analyzed during the run. In the case of $\mathrm{Ba}$ and $\mathrm{Zn}$, the issue is believed to be from small impurities in the ICS solution. In the case of $\mathrm{Be}$, the issue is from a small 


\section{Battelle PNNL/RPL/Inorganic Analysis ... ICP-OES Analysis Report}

background shift in the instrument for the ICS runs. In all three cases, these results had no statistical effect on the reported data. All other instrument-related QC tests for the AOIs passed within the appropriate acceptance criteria.

\section{Comments:}

1) The "Final Results" have been corrected for all laboratory dilutions performed on the samples during processing and analysis, unless specifically noted.

2) Instrument detection limits (IDL) and estimated quantitation limits (EQL) shown are for acidified water and/or fusion flux matrices as applicable. Detection limits for other matrices may be determined if requested. Method detection limits (MDL) can be estimated by multiplying the IDL by the "Multiplier". The estimated quantitation limit (EQL) for each concentration value can be obtained by multiplying the EQL by the "Multiplier".

3) Routine precision and bias is typically $\pm 15 \%$ or better for samples in dilute, acidified water (e.g. $2 \% \mathrm{v} / \mathrm{v}$ $\mathrm{HNO}_{3}$ or less) at analyte concentrations $>$ EQL up to the upper calibration level. This also presumes that the total dissolved solids concentration in the sample is less than $5000 \mu \mathrm{g} / \mathrm{mL}(0.5$ per cent by weight). Note that bracketed values listed in the data report are within the MDL and the EQL, and have potential uncertainties greater than $15 \%$. Concentration values $<$ MDL are listed as " . .". Note, that calibration and QC standards are validated to a precision of $\pm 10 \%$.

4) Absolute precision, bias and detection limits may be determined on each sample if required by the client. The maximum number of significant figures for all ICP measurements is two.

5) Analytes included in the spike A component (for the AS/PS) are; $\mathrm{Ag}, \mathrm{Al}, \mathrm{As}, \mathrm{B}, \mathrm{Ba}, \mathrm{Be}, \mathrm{Bi}, \mathrm{Ca}, \mathrm{Cd}, \mathrm{Co}, \mathrm{Cr}$, $\mathrm{Cu}, \mathrm{Fe}, \mathrm{K}, \mathrm{Li}, \mathrm{Mg}, \mathrm{Mn}, \mathrm{Mo}, \mathrm{Na}, \mathrm{Ni}, \mathrm{P}, \mathrm{Pb}, \mathrm{Sb}, \mathrm{Se}, \mathrm{Si}, \mathrm{Sn}, \mathrm{Sr}, \mathrm{Ta}, \mathrm{Ti}, \mathrm{Tl}, \mathrm{V}, \mathrm{W}, \mathrm{Y}, \mathrm{Zn}$, and Zr. Analytes included in the spike B component are; $\mathrm{Ce}, \mathrm{Dy}, \mathrm{Eu}, \mathrm{La}, \mathrm{Nd}, \mathrm{Pd}, \mathrm{Rh}, \mathrm{Ru}, \mathrm{S}, \mathrm{Te}, \mathrm{Th}$, and $\mathrm{U}$. 
PNNL-20650, Rev. 2

Battelle PNNL/RPL/Inorganic Analysis ... ICPOES Data Report

\begin{tabular}{|c|c|c|c|c|c|c|c|c|c|}
\hline & Run Date > & $4 / 26 / 2011$ & $4 / 26 / 2011$ & $4 / 26 / 2011$ & $4 / 26 / 2011$ & 4/26/2011 & 4/26/2011 & $4 / 26 / 2011$ \\
\hline & & Multiplier > & 1.0 & 78.2 & 782.1 & 73.3 & 733.1 & 56.3 & 563.3 \\
\hline & & RPL/LAB > & 405 diluent & $\begin{array}{c}11-0792- \\
129-P B\end{array}$ & $\begin{array}{c}11-0792- \\
129-P B @ \\
10 x\end{array}$ & $\begin{array}{c}\text { 11-0792- } \\
129-S\end{array}$ & $\begin{array}{c}11-0792- \\
129-S @ \\
10 x\end{array}$ & $\begin{array}{c}\text { 11-0792- } \\
129-D\end{array}$ & $\begin{array}{c}11-0792- \\
129-D @ \\
10 x\end{array}$ \\
\hline $\begin{array}{l}\text { Instr. Det. } \\
\text { Limit (IDL) }\end{array}$ & $\begin{array}{l}\text { Est. Quant. } \\
\text { Limit (EQL) }\end{array}$ & Client ID > & Lab diluent & \multicolumn{2}{|c|}{ PB } & \multicolumn{2}{|c|}{ T1008-MA } & \multicolumn{2}{|c|}{ T1008-MB } \\
\hline$(\mu \mathrm{g} / \mathrm{mL})$ & $(\mu \mathrm{g} / \mathrm{mL})$ & Analytes & $(\mu \mathrm{g} / \mathrm{mL})$ & $(\mu \mathrm{g} / \mathrm{g})$ & $(\mu \mathrm{g} / \mathrm{g})$ & $(\mu \mathrm{g} / \mathrm{g})$ & $(\mu \mathrm{g} / \mathrm{g})$ & $(\mu \mathrm{g} / \mathrm{g})$ & $(\mu \mathrm{g} / \mathrm{g})$ \\
\hline 0.0019 & 0.019 & $\mathrm{Ag}$ & -- & & -- & & {$[7.8]$} & & -- \\
\hline 0.0046 & 0.046 & $\mathrm{Al}$ & -- & & -- & & 17,200 & & 15,300 \\
\hline 0.0960 & 0.960 & As & -- & - & & [35] & & [31] & \\
\hline 0.0001 & 0.002 & $\mathrm{Ba}$ & {$[0.0001]$} & & {$[0.097]$} & & 141 & & 316 \\
\hline 0.0001 & 0.001 & $\mathrm{Be}$ & -- & & -- & & 6.44 & & 6.62 \\
\hline 0.0022 & 0.044 & $\mathrm{Ca}$ & -- & & -- & & 9,220 & & 9,800 \\
\hline 0.0015 & 0.015 & Cd & - & & -- & & 36.6 & & 38.1 \\
\hline 0.0020 & 0.020 & $\mathrm{Cr}$ & -- & & -- & & 137 & & 209 \\
\hline 0.0045 & 0.045 & $\mathrm{Fe}$ & -- & & [3.8] & & 37,100 & & 49,500 \\
\hline 0.0006 & 0.012 & $\mathrm{Mg}$ & {$[0.0008]$} & & -- & & 675 & & 808 \\
\hline 0.0036 & 0.072 & $\mathrm{Na}$ & {$[0.015]$} & & 71.5 & & 1,450 & & 1,570 \\
\hline 0.0026 & 0.026 & $\mathrm{Ni}$ & -- & & -- & & 99.2 & & 149 \\
\hline 0.0210 & 0.210 & $\mathrm{~Pb}$ & -- & & -- & & 175 & & 260 \\
\hline 0.0220 & 0.220 & $\mathbf{S b}$ & -- & -- & & [5.8] & & [4.1] & \\
\hline 0.1700 & 1.700 & Se & -- & -- & & -- & & -- & \\
\hline 0.0860 & 0.860 & $\mathrm{TI}$ & -- & -- & & -- & & -- & \\
\hline 0.0120 & 0.120 & $U$ & -- & & - & & 30,300 & & 25,000 \\
\hline 0.0011 & 0.022 & $\mathrm{v}$ & - & & - & & [14] & & 16.6 \\
\hline 0.0056 & 0.056 & $\mathrm{Zn}$ & -- & & [6.7] & & 250 & & 240 \\
\hline 0.0008 & 0.008 & $\mathrm{Zr}$ & -- & & - & & 573 & & 94.9 \\
\hline \multicolumn{2}{|c|}{ Other Analytes } & Other Analyte & & & & & & & \\
\hline 0.0057 & 0.057 & B & {$[0.027]$} & & [22] & & [24] & & [17] \\
\hline 0.0160 & 0.160 & $\mathrm{Bi}$ & -- & & [59] & & [63] & & [43] \\
\hline 0.0087 & 0.087 & $\mathrm{Ce}$ & -- & & -- & & [25] & & [17] \\
\hline 0.0025 & 0.025 & Co & - & & - & & [4.2] & & [3.3] \\
\hline 0.0016 & 0.016 & $\mathrm{Cu}$ & -- & & - & & 423 & & 403 \\
\hline 0.0012 & 0.012 & Dy & -- & & - & & -- & & -- \\
\hline 0.0003 & 0.003 & $\mathrm{Eu}$ & -- & & -- & & {$[0.25]$} & & {$[0.58]$} \\
\hline 0.0220 & 0.220 & $\mathrm{~K}$ & -- & & [120] & & 461 & & 479 \\
\hline 0.0012 & 0.012 & La & -- & & -- & & 13.0 & & 13.5 \\
\hline 0.0005 & 0.005 & $\mathrm{Li}$ & -- & & 5.39 & & 5.83 & & 5.25 \\
\hline 0.0002 & 0.002 & $\mathrm{Mn}$ & - & & -- & & 250 & & 305 \\
\hline 0.0070 & 0.070 & Mo & - & & -- & & [20] & & [17] \\
\hline 0.0027 & 0.027 & $\mathrm{Nd}$ & {$[0.0028]$} & & -- & & 24.6 & & [13] \\
\hline 0.0510 & 0.510 & $\mathbf{P}$ & - & & - & & [120] & & [150] \\
\hline 0.0048 & 0.048 & $P d$ & - & & -- & & [4.6] & & [3.1] \\
\hline 0.0088 & 0.088 & $\mathrm{Rh}$ & -- & & [7.8] & & -- & & - \\
\hline 0.0044 & 0.044 & Ru & -- & & -- & & -- & & -- \\
\hline 0.1600 & 1.600 & $s$ & -- & & -- & & 9,130 & & 9,230 \\
\hline 0.0130 & 0.130 & $\mathrm{Si}$ & -- & & [13] & & 583 & & 506 \\
\hline 0.0140 & 0.140 & Sn & -- & & -- & & [22] & & [29] \\
\hline 0.0000 & 0.000 & $\mathrm{Sr}$ & {$[0.0000]$} & & {$[0.025]$} & & 51.3 & & 54.9 \\
\hline 0.0190 & 0.190 & $\mathrm{Ta}$ & -- & & -- & & -- & & - \\
\hline 0.0160 & 0.160 & Te & -- & & -- & & -- & & [13] \\
\hline 0.0050 & 0.050 & Th & -- & & -- & & [34] & & [26] \\
\hline 0.0005 & 0.005 & $\mathrm{Ti}$ & -- & & -- & & 221 & & 394 \\
\hline 0.0150 & 0.150 & w & -- & & -- & & -- & & -- \\
\hline 0.0003 & 0.005 & $Y$ & -- & & - & & {$[2.4]$} & & 2.97 \\
\hline
\end{tabular}

1) "--" indicates the value is Shaded results are outside the acceptance criteria.

near the top of each column. The estimated sample quantitation limit $=E Q L$ (in Column 2)

times the "multiplier". Overall error for values $\geq E Q L$ is estimated to be within $\pm 15 \%$.

2) Values in brackets [] are $\geq M D L$ but $<E Q L$, with errors likely to exceed $15 \%$.

Page 1 of 4 
PNNL-20650, Rev. 2

Battelle PNNL/RPL/Inorganic Analysis ... ICPOES Data Report

\begin{tabular}{|c|c|c|c|c|}
\hline Run Date > & $4 / 26 / 2011$ & $4 / 26 / 2011$ & $4 / 26 / 2011$ & 4/26/2011 \\
\hline Multiplier > & 112.7 & 1127.0 & 91.9 & 919.2 \\
\hline RPL/LAB > & $\begin{array}{c}11-0793-129 \\
S\end{array}$ & $\begin{array}{c}11-0793-129 \\
S @ 10 x\end{array}$ & $\begin{array}{c}11-0793-129 \\
D\end{array}$ & $\begin{array}{c}11-0793-129 \\
\text { D @ 10x }\end{array}$ \\
\hline Client ID > & \multicolumn{2}{|c|}{ T1008-SA } & \multicolumn{2}{|c|}{ T1008-SB } \\
\hline (Analyte) & $(\mu \mathrm{g} / \mathrm{g})$ & $(\mu \mathrm{g} / \mathrm{g})$ & $(\mu \mathrm{g} / \mathrm{g})$ & $(\mu \mathrm{g} / \mathrm{g})$ \\
\hline $\mathrm{Ag}$ & & 46.7 & & 45.2 \\
\hline Al & & 36,200 & & 34,000 \\
\hline As & 182 & & 167 & \\
\hline $\mathrm{Ba}$ & & 81.4 & & 82.0 \\
\hline $\mathrm{Be}$ & & 15.9 & & 15.2 \\
\hline $\mathrm{Ca}$ & & 2,080 & & 1,770 \\
\hline Cd & & 51.5 & & 45.5 \\
\hline $\mathrm{Cr}$ & & 714 & & 671 \\
\hline $\mathrm{Fe}$ & & 135,000 & & 130,000 \\
\hline $\mathrm{Mg}$ & & 1,090 & & 981 \\
\hline $\mathrm{Na}$ & & 449 & & 384 \\
\hline $\mathrm{Ni}$ & & 214 & & 194 \\
\hline $\mathrm{Pb}$ & & 925 & & 936 \\
\hline $\mathbf{S b}$ & [15] & & [17] & \\
\hline Se & -- & & -- & \\
\hline TI & [15] & & [12] & \\
\hline$u$ & & 172,000 & & 160,000 \\
\hline v & & 58.2 & & 56.0 \\
\hline $\mathrm{Zn}$ & & 505 & & 474 \\
\hline $\mathrm{Zr}$ & & 432 & & 363 \\
\hline
\end{tabular}

\begin{tabular}{|c|c|c|c|c|}
\hline $\mathrm{B}$ & & {$[36]$} & & {$[36]$} \\
\hline $\mathrm{Bi}$ & & {$[120]$} & & {$[98]$} \\
\hline $\mathrm{Ce}$ & & 155 & & 136 \\
\hline $\mathrm{Co}$ & & {$[5.4]$} & & {$[3.9]$} \\
\hline $\mathrm{Cu}$ & & 1,920 & & 1,820 \\
\hline $\mathrm{Dy}$ & & -- & & -- \\
\hline $\mathrm{Eu}$ & & 4.09 & & 4.41 \\
\hline $\mathrm{K}$ & & 541 & & 470 \\
\hline $\mathrm{La}$ & & 43.1 & & 40.0 \\
\hline $\mathrm{Li}$ & & 14.1 & & 11.8 \\
\hline $\mathrm{Mn}$ & & 279 & & 253 \\
\hline $\mathrm{Mo}$ & & {$[68]$} & & {$[64]$} \\
\hline $\mathrm{Nd}$ & & 146 & & 135 \\
\hline $\mathrm{P}$ & & 819 & & 734 \\
\hline $\mathrm{Pd}$ & & {$[6.9]$} & & {$[6.8]$} \\
\hline $\mathrm{Rh}$ & & -- & & -- \\
\hline $\mathrm{Ru}$ & & {$[37]$} & & {$[31]$} \\
\hline $\mathrm{S}$ & & 2,260 & & 1,940 \\
\hline $\mathrm{Si}$ & & 1,220 & & 1,280 \\
\hline $\mathrm{Sn}$ & & {$[61]$} & & {$[68]$} \\
\hline $\mathrm{Sr}$ & & 15.9 & & 14.1 \\
\hline $\mathrm{Ta}$ & & -- & & -- \\
\hline $\mathrm{Te}$ & & {$[52]$} & & {$[26]$} \\
\hline $\mathrm{Th}$ & & 205 & & 180 \\
\hline $\mathrm{Ti}$ & & 453 & & 400 \\
\hline $\mathrm{W}$ & & -- & & {$[26]$} \\
\hline $\mathrm{Y}$ & & 11.3 & & 10.7 \\
\hline
\end{tabular}


PNNL-20650, Rev. 2

\section{Battelle PNNL/RPL/Inorganic Analysis ... ICPOES Data Report}

\begin{tabular}{|c|c|c|c|c|c|c|c|c|}
\hline Criteria > & $\leq 20 \%$ & $\leq 20 \%$ & $\leq 20 \%$ & $\leq 20 \%$ & $80 \%-120 \%$ & $80 \%-120 \%$ & $80 \%-120 \%$ & $80 \%-120 \%$ \\
\hline$Q C$ ID > & \multicolumn{2}{|c|}{ 11-0792 Dup } & \multicolumn{2}{|c|}{ 11-0793 Dup } & \multicolumn{2}{|c|}{ LCS/BS } & \multicolumn{2}{|c|}{ 11-0793 MS } \\
\hline Analytes & RPD (\%) & RPD (\%) & RPD (\%) & RPD (\%) & $\% \operatorname{Rec}$ & $\% \operatorname{Rec}$ & $\% \operatorname{Rec}$ & $\% \operatorname{Rec}$ \\
\hline $\mathrm{Ag}$ & & & & 3.3 & & 101 & & 102 \\
\hline Al & & 11.4 & & 6.4 & & 103 & & $\mathrm{nr}$ \\
\hline As & & & 8.1 & & 89 & & 90 & \\
\hline $\mathrm{Ba}$ & & 76.7 & & 0.7 & & 102 & & 101 \\
\hline $\mathrm{Be}$ & & 2.8 & & 4.7 & & 103 & & 102 \\
\hline $\mathrm{Ca}$ & & 6.0 & & 16.3 & & 102 & & 162 \\
\hline $\mathrm{Cd}$ & & 3.9 & & 12.4 & & 106 & & 107 \\
\hline $\mathrm{Cr}$ & & 41.7 & & 6.3 & & 98 & & 104 \\
\hline $\mathrm{Fe}$ & & 28.7 & & 3.7 & & 104 & & $\mathrm{nr}$ \\
\hline Mg & & 17.9 & & 10.5 & & 103 & & 119 \\
\hline $\mathrm{Na}$ & & 8.0 & & 15.6 & & 103 & & 123 \\
\hline $\mathrm{Ni}$ & & 40.2 & & 9.8 & & 106 & & 107 \\
\hline $\mathrm{Pb}$ & & 39.1 & & 1.2 & & 108 & & 107 \\
\hline $\mathrm{Sb}$ & & & & & 89 & & 87 & \\
\hline Se & & & & & 90 & & 91 & \\
\hline TI & & & & & 86 & & 78 & \\
\hline$U$ & & 18.9 & & 7.3 & & 102 & & $\mathrm{nr}$ \\
\hline $\mathrm{v}$ & & & & 3.9 & & 103 & & 103 \\
\hline $\mathrm{Zn}$ & & 4.1 & & 6.4 & & 100 & & 103 \\
\hline $\mathrm{Zr}$ & & 143.2 & & 17.3 & & 107 & & $\mathrm{nr}$ \\
\hline
\end{tabular}

\begin{tabular}{|c|c|c|c|c|}
\hline B & & & 104 & 105 \\
\hline \multicolumn{5}{|l|}{$\mathrm{Bi}$} \\
\hline $\mathrm{Ce}$ & & 12.8 & 101 & 98 \\
\hline \multicolumn{5}{|l|}{ Co } \\
\hline $\mathrm{Cu}$ & 4.7 & 5.5 & 110 & $\mathrm{nr}$ \\
\hline \multicolumn{5}{|l|}{ Dy } \\
\hline Eu & & 7.6 & & \\
\hline $\mathbf{K}$ & 3.7 & 14.0 & 105 & 109 \\
\hline La & 4.1 & 7.6 & 99 & 101 \\
\hline $\mathrm{Li}$ & 10.5 & 17.6 & 113 & 108 \\
\hline $\mathrm{Mn}$ & 19.8 & 10.0 & 107 & 113 \\
\hline Mo & & & 105 & 109 \\
\hline Nd & & 7.3 & 97 & 101 \\
\hline $\mathbf{P}$ & & 10.9 & & 118 \\
\hline \multicolumn{5}{|l|}{$\mathbf{P d}$} \\
\hline \multicolumn{5}{|l|}{ Rh } \\
\hline \multicolumn{5}{|l|}{ Ru } \\
\hline $\mathrm{s}$ & 1.1 & 15.5 & & 99 \\
\hline Si & 14.1 & 4.6 & 108 & 0 \\
\hline \multicolumn{5}{|l|}{ Sn } \\
\hline $\mathrm{Sr}$ & 6.7 & 12.1 & 103 & 128 \\
\hline \multicolumn{5}{|l|}{$\mathrm{Ta}$} \\
\hline \multicolumn{5}{|l|}{ Te } \\
\hline Th & & 13.0 & 100 & 101 \\
\hline $\mathrm{Ti}$ & 56.1 & 12.5 & 106 & 107 \\
\hline \multicolumn{5}{|l|}{ w } \\
\hline $\mathbf{Y}$ & & 5.7 & & \\
\hline
\end{tabular}


PNNL-20650, Rev. 2

Battelle PNNL/RPL/Inorganic Analysis ... ICPOES Data Report

\begin{tabular}{|c|c|c|c|c|c|}
\hline Criteria > & $80 \%-120 \%$ & $80 \%-120 \%$ & $80 \%-120 \%$ & $\leq 10 \%$ & $\leq 10 \%$ \\
\hline QC ID > & \multicolumn{2}{|c|}{$11-0793+$ AS-A } & $\begin{array}{c}11-0793+\text { AS } \\
B\end{array}$ & \multicolumn{2}{|c|}{ 11-0792 5-Fold Serial Dil. } \\
\hline Analytes & $\% \operatorname{Rec}$ & $\% \operatorname{Rec}$ & $\%$ Rec & $\%$ Diff & \%Diff \\
\hline $\mathbf{A g}$ & & 103 & & & \\
\hline Al & & $\mathrm{nr}$ & & & 0.5 \\
\hline As & 100 & & & & \\
\hline $\mathrm{Ba}$ & & 101 & & & 1.0 \\
\hline $\mathrm{Be}$ & & 99 & & & 4.7 \\
\hline $\mathrm{Ca}$ & & 101 & & & 0.2 \\
\hline $\mathrm{Cd}$ & & 92 & & & \\
\hline $\mathrm{Cr}$ & & 104 & & & 2.7 \\
\hline $\mathrm{Fe}$ & & $\mathrm{nr}$ & & & 1.2 \\
\hline $\mathbf{M g}$ & & 98 & & & 2.0 \\
\hline $\mathrm{Na}$ & & 98 & & & 4.6 \\
\hline $\mathrm{Ni}$ & & 106 & & & 2.6 \\
\hline $\mathrm{Pb}$ & & 114 & & & \\
\hline $\mathrm{Sb}$ & 96 & & & & \\
\hline Se & 98 & & & & \\
\hline $\mathrm{TI}$ & 89 & & & & \\
\hline $\mathbf{U}$ & & & 102 & & 0.7 \\
\hline v & & 97 & & & \\
\hline $\mathrm{Zn}$ & & 103 & & & 4.5 \\
\hline $\mathrm{Zr}$ & & 102 & & & 1.0 \\
\hline \multicolumn{6}{|c|}{ ther Analytes } \\
\hline B & & 101 & & & \\
\hline $\mathrm{Bi}$ & & 92 & & & \\
\hline $\mathrm{Ce}$ & & & 105 & & \\
\hline Co & & 96 & & & \\
\hline $\mathrm{Cu}$ & & $\mathrm{nr}$ & & & 0.5 \\
\hline Dy & & & 101 & & \\
\hline $\mathrm{Eu}$ & & & 102 & & \\
\hline K & & 99 & & & \\
\hline $\mathrm{La}$ & & & 101 & & \\
\hline $\mathrm{Li}$ & & 105 & & & \\
\hline $\mathrm{Mn}$ & & 108 & & & 1.8 \\
\hline Mo & & 105 & & & \\
\hline Nd & & & 103 & & \\
\hline $\mathbf{P}$ & & 96 & & & \\
\hline $\mathbf{P d}$ & & & 99 & & \\
\hline Rh & & & 98 & & \\
\hline $\mathbf{R u}$ & & & 101 & & \\
\hline $\mathrm{s}$ & & & 102 & & 0.6 \\
\hline Si & & 98 & & & 3.4 \\
\hline Sn & & 85 & & & \\
\hline $\mathrm{Sr}$ & & 102 & & & 0.3 \\
\hline $\mathrm{Ta}$ & & 98 & & & \\
\hline $\mathrm{Te}$ & & & 101 & & \\
\hline Th & & & 102 & & \\
\hline $\mathrm{Ti}$ & & 112 & & & 2.1 \\
\hline$w$ & & 98 & & & \\
\hline $\mathbf{Y}$ & & 98 & & & \\
\hline
\end{tabular}

Shaded results are outside the acceptance criteria.

$n r=$ spike concentration less than $25 \%$ of sample concentration. Matrix effects can be assessed from the serial dilu. 
Battelle PNNL/RPL/ASO Radiochemistry Analysis Report

P.O. Box 999, 902 Battelle Blvd., Richland, Washington 99352

\section{Gamma Energy Analysis (GEA)}

$\begin{array}{ll}\text { Project / WP\#: } & 52578 / \mathrm{H} 41216 \\ \text { ASR\#: } & 8824.00 \\ \text { Client: } & \text { SK Fiskum } \\ \text { Total Samples: } & \text { 3 Samples }\end{array}$

\begin{tabular}{|c|c|c|}
\hline $\begin{array}{c}\text { Engineered Container and } \\
\text { Core ID }\end{array}$ & RPL ID & Client Sample ID \\
\hline KW210-LCC & $11-0791-129-\mathrm{S}$ & TI008-LA \\
\hline KW210-MCC & $11-0792-129-\mathrm{S},-\mathrm{D}$ & TI008-MA, -MB \\
\hline KW210-SCC & $11-0793-129-\mathrm{S},-\mathrm{D},-\mathrm{MS}$ & TI008-SA, -SB, -SC \\
\hline
\end{tabular}

\begin{tabular}{|c|c|}
\hline Analysis Type: & GEA- for all positively measured or non-detected isotopes \\
\hline $\begin{array}{l}\text { Sample Processing Prior to Radiochemical } \\
\text { Processing/Analysis }\end{array}$ & $\begin{array}{l}\square \text { None (TI008-LA) Only } \\
\square \text { Digested as per RPG-CMC-128, Rev. } \mathrm{OHNO}_{3}-\mathrm{HCl} \text { Acid Extraction of } \\
\text { Liquids for Metals Analysis Using a Dry Block Heater } \\
\bigotimes \text { Digested as per PNL-ALO-129, Rev. } 0 \mathrm{HNO}_{3} \text { - } \mathrm{HCl} \text { Acid Extraction of } \\
\text { Solids Using a Dry Block Heater } \\
\square \text { Fusion as per PNL-ALO-115, Solubilization of Metals from Solids } \\
\text { Using a } \mathrm{KOH}-\mathrm{KNO}_{3} \text { Fusion } \\
\square \text { Other: } \\
\text { Preparation may also involve attaining a GEA geometry that is compatible } \\
\text { with the calibration geometry. }\end{array}$ \\
\hline Analysis Procedure: & $\begin{array}{l}\text { RPG-CMC-450, Rev. } 1 \text { Gamma Energy Analysis (GEA) and Low-Energy } \\
\text { Photon Spectrometry (LEPS) }\end{array}$ \\
\hline Reference Date: & *January 11,2011@15:14:41 \\
\hline Analysis Date or Date Range: & May 3-24, 2011 \\
\hline Technician/Analyst: & T Trang-Le \\
\hline Rad Chem Electronic Data File: & 11-0792 Fiskum.xls \\
\hline ASO Project 98620 File: & $\begin{array}{l}\text { File Plan 5871, T4.4 Technical (Radiochemistry), Gamma Calibration, } \\
\text { daily checks, and maintenance records; and T3 standard certificates and } \\
\text { preparation. Also, balance calibration and Performance check records. }\end{array}$ \\
\hline M\&TE Number(s): & $\begin{array}{l}\text { Detector } C, D, E, G, K, T \text { ( } M \& T E \text { unique identifiers in attached supporting } \\
\text { documents) }\end{array}$ \\
\hline
\end{tabular}

*Reference date used is the same date and time used for samples submitted under ASR 8725 as directed by S. Fiskum.
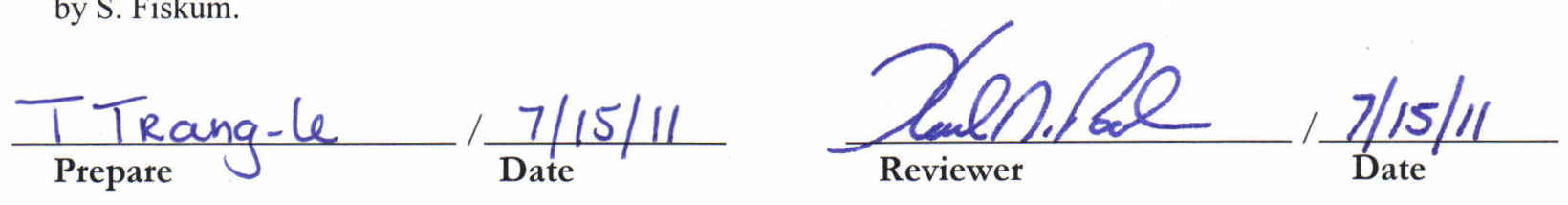
PNNL-20650, Rev. 2

Battelle PNNL/RPL/ASO Radiochemistry Analysis Report

\section{SAMPLE RESULTS}

Activities for all gamma emitters detected in these samples are presented in an attached Excel spreadsheets for ASR 8824.00. All sludge data are reported in units of $\mu \mathrm{Ci} / \mathrm{g}$ dry and all residual solids are reported as $\mu \mathrm{Ci} / \mathrm{g}_{\text {residual, }}$, both with estimates of the total propagated uncertainty reported at the 1sigma level.

The K Basin ASR specifies that the residual solids GEA analytes are to be reported as $\mu \mathrm{Ci} / \mathrm{g}$ residual, where $\mathrm{g}$ residual is the mass of residual solids that did not dissolve during acid digestion.

ASO Project File, ASR 8824 has been created for this report including all appropriate supporting records which may include the Pipette Performance Verification form, Standard Certificates, Laboratory Bench Record, Shielded Analytical Laboratory Bench Sheet, and Gamma Energy Analysis printouts. Detector calibration records, control charts and balance calibration records can be found in the ASO Records.

\section{Sample Preparation, Separation, Mounting and Counting Methods}

K-Basin sludge samples were prepared in the shielded analytical lab (SAL) hot cells by the Project and provided to the Analytical Support Operations (ASO) under Analytical Service Request (ASR) 8824 for chemical and radioanalytical analyses.

Sample TI008-LA was received from client pre-dried and analyzed by GEA "as received."

ASO received each sample requiring acid digestion in Teflon Oak Ridge centrifuge tubes, dried the contents of each centrifuge tube to a constant weight, and then leached the dried solids with hot dilute nitric and hydrochloric acids per procedure PNL-ALO-129. The leachate solution was used for radioanalytical analyses and ICP metals analysis; only GEA data are included in this report. At the completion of the acid digestion, residual unsolubilized material remained. The residual solids were dried to constant weight and the dried residuals were transferred to clean $22 \mathrm{~mL}$ glass scintillation vials for load out and GEA analyses. The residual solids do have measurable activity and those results are included in this report.

Quality control (QC) samples prepared include: 1) the duplicate pairs; TI008-MA, -MB and TI008-SA, $-\mathrm{SB}, 2)$ the reagent blank.

The quality control (QC) sample results for each of the isotopes measured above background have been evaluated and are discussed below. A summary of the GEA analysis results, including QC sample performance, is given in the attached Data Report.

The following is a list of QC sample results relative to both the K Basin data quality objectives and the ASO Quality Assurance (QA) Plan (ASO-QAP-001, Rev. 7); in many cases the DQOs are the same. In cases where there are differences in the DQOs, the K Basin DQO takes precedence for data reporting.

\section{QUALITY CONTROL RESULTS}

\section{Tracer:}

Tracers are not used for ASO GEA methods. 


\section{Process Blank (PB):}

A reagent blank was prepared with the preparation of the sludge samples in the SAL, but not for the preparation of the residual solids in the SAL.

The ASO-QAP-001 requires the activities of the gamma emitting isotopes of interest measured in the PB to be within the acceptance criteria of less than $5 \%$ of the sample isotope concentration or less than sample minimum detectable activity (MDA) - (See comments). For isotopes of interest that were not detected in the PB and samples, the PB minimum detectable concentration (MDC) was less than the MDC measured in the samples. The isotopes of interest that were detected in the samples above the MDC are all greater than $5 \%$ of the activity level present in the PB.

\section{$\underline{\text { Required Detection Limits }}$}

The ASR required detection limits were met for all analytes for both the digestate and residual solid sample. The GEA detection limit requirements are shown on the summary data sheets.

\section{Blank Spike (BS)/Laboratory Control Sample (LCS)/ Matrix Spike (MS):}

There are no BS or LCS samples analyzed for ASO GEA analyses. Instrument performance is assessed by the analyses of daily control counts and weekly background counts, as discussed below. (The sample identified as "MS" was prepared for ICP/OES analyses only. This MS sample is also being reported for GEA as additional information for the project.)

\section{Duplicate Relative Percent Difference (RPD):}

Duplicate results for the sludge are required to agree within $\leq 20 \%$ relative percent difference, taking into consideration the statistical uncertainties in the data. The residual solids results are not subject to the duplicate acceptance criteria. Duplicate results for the sludge samples ranged from $4 \%$ to $31 \%$

For Sludge samples:

\begin{tabular}{|c|c|c|c|}
\hline RPL Number & Client Sample ID & Isotopes & RPD Differences \\
\hline \multirow{2}{*}{$11-0792-129-S,-D$} & \multirow{2}{*}{ TI008-MA, -MB } & Co-60 & $31 \%$ \\
& & Eu-154 & $26 \%$ \\
\hline
\end{tabular}

Occurrence report (52578-06-27-11) documents DQO related issue(s) with this report: RPD's outside the $\leq 20 \%$ requirement.

\section{Replicate Relative Standard Deviation (RSD):}

Replicate results are required to agree within $<20 \%$ relative percent difference or within 2-sigma standard deviation taking into account the uncertainties for each measurement. 
PNNL-20650, Rev. 2

\section{Battelle PNNL/RPL/ASO Radiochemistry Analysis Report}

\section{Instrument Calibration and Quality Control}

Gamma detectors are calibrated using multi-isotope standards that are NIST-traceable and prepared in the identical counting geometry used for the samples for all detectors. Counter control sources containing Am-241, Cs- 137 and Co-60 are then analyzed daily before the use of each detector. There is an ASO procedure RPG-CMC-450, that requires that a counter control source is checked daily and must be within \pm 3 sigma or $\pm 3 \%$ of the control value, whichever is greater. Gamma counting was not performed unless the control counts were within the required limits. Background counts are performed on all gamma detectors at least weekly for either an overnight or weekend count. The most recent background is subtracted from all sample counts.

\section{Assumption and Limitations of the Data}

The digestion was performed exactly as stated in procedure PNL-ALO-129. Following the digestion process, each sample had a visible quantity of residual solids. The residual solids have measureable activity. The GEA results for the residual solids are included in this report.

\section{Interferences/Resolution}

None.

\section{Uncertainty}

For gamma counting, the uncertainty in the efficiency, counting data, photon abundance and the nuclear half-life are included in the calculation of the total uncertainty along with a systematic uncertainty for sample preparation. The Canberra Genie software includes both random and systematic uncertainties in the calculation of the total uncertainties which are listed on the report. A conservative estimate is that $2 \%$ is the lowest uncertainty possible for our GEA measurements taking into account systematic uncertainties in gamma calibration standards.

The samples were counted at about $10 \mathrm{~cm}$ from the detector to minimize dead time due to the high sample activities. Counting at such long distances also minimizes the differences between the sample geometry and the calibration geometry. Calibrations are performed using water samples whereas the samples consisted of mostly silica (however, the exact sample mineralogical composition and associated density were not known). The small sample size $(<1 \mathrm{~g})$ minimized the gamma absorption effects; therefore, density corrections were not applied.

\section{Comments}

1. The results have been corrected for all dilution factors resulting from sample processing.

2. Minimum Detectable Activity (MDA) and Minimum Detectable Concentration (MDC). Sample results are compared to the process blank results to evaluate if the blank contains $5 \%$ or more of the analyte of interest. When samples undergo digestion and/or dilution (process factors), the MDA must be adjusted for the process factors to accurately compare activity levels in the blank to activity levels in the samples. Process blank results have been adjusted for digestion and/or process factors for evaluation of the $5 \%$ criteria.

3. Occurrence report (52578-06-27-11) documents DQO related issue(s) with this report: RPD's outside the $\leq 20 \%$ requirement. 
PNNL-20650, Rev. 2

Battelle PNNL/RPL/ASO Radiochemistry Analysis Report

For Sludge samples:

\begin{tabular}{|c|c|c|c|}
\hline RPL Number & Client Sample ID & Isotopes & RPD Differences \\
\hline 11-0792-129-S, -D & TI008-MA, -MB & Co-60 & $31 \%$ \\
& & Eu-154 & $26 \%$ \\
\hline
\end{tabular}

Attachment: Data Report Sample Results for ASR 8824. 
PNNL-20650, Rev. 2

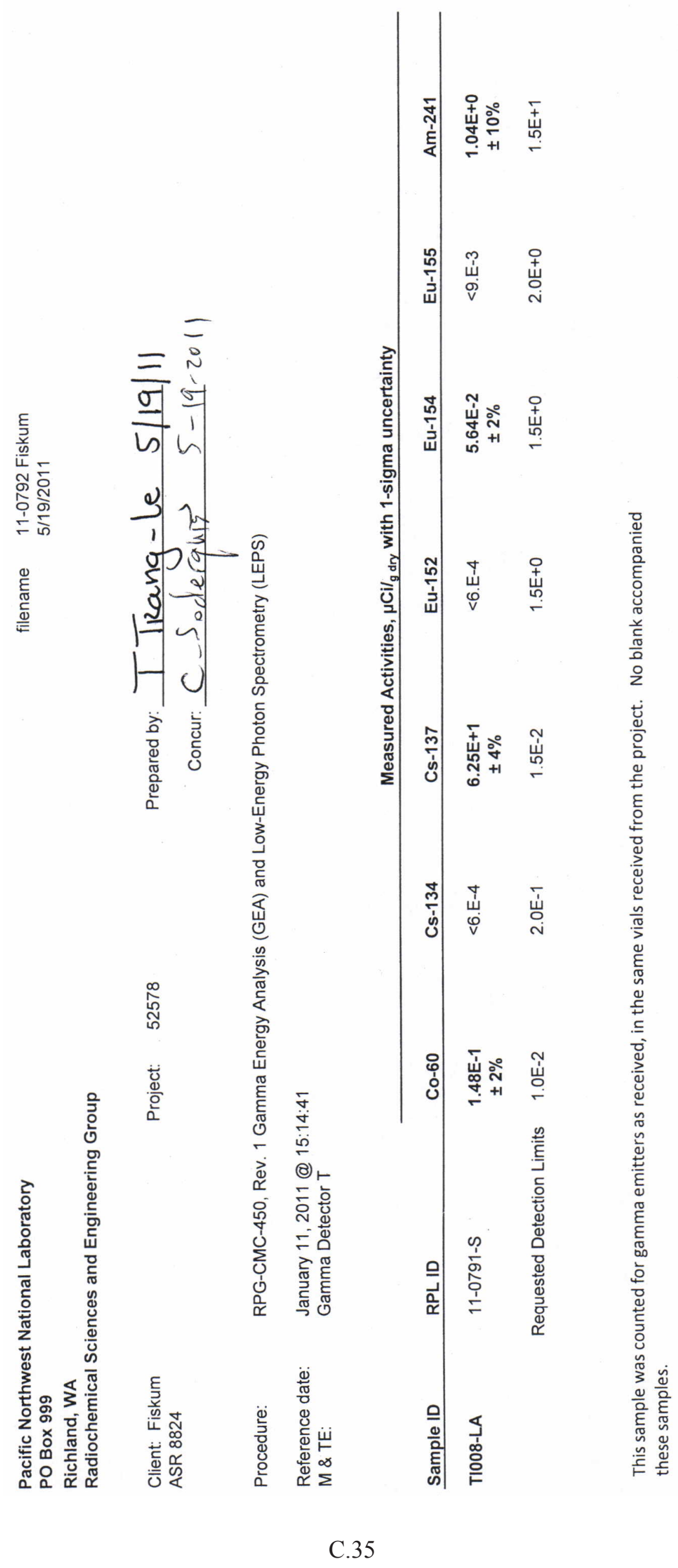

Page 1 of 1 
PNNL-20650, Rev. 2
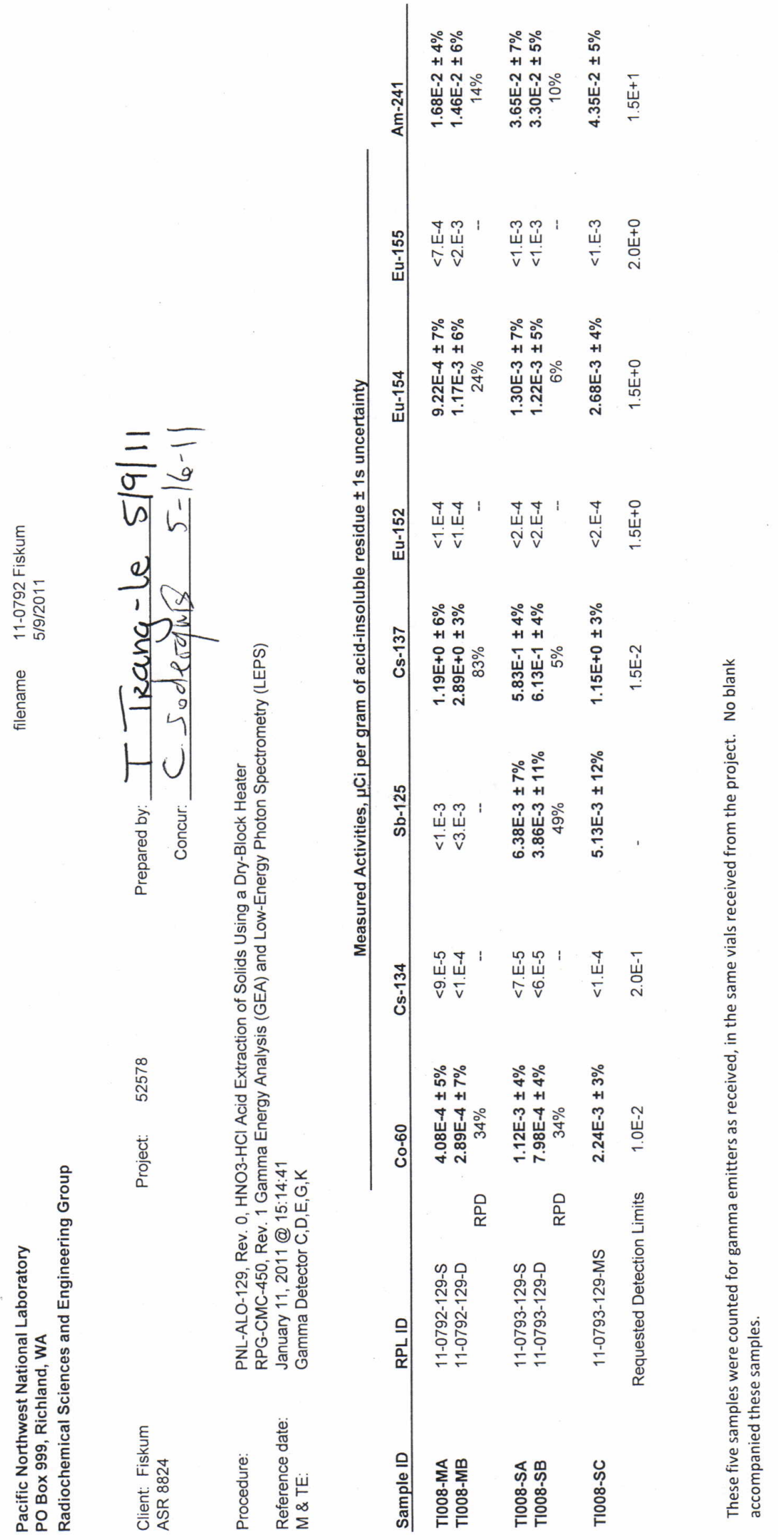

C. 36 
PNNL-20650, Rev. 2

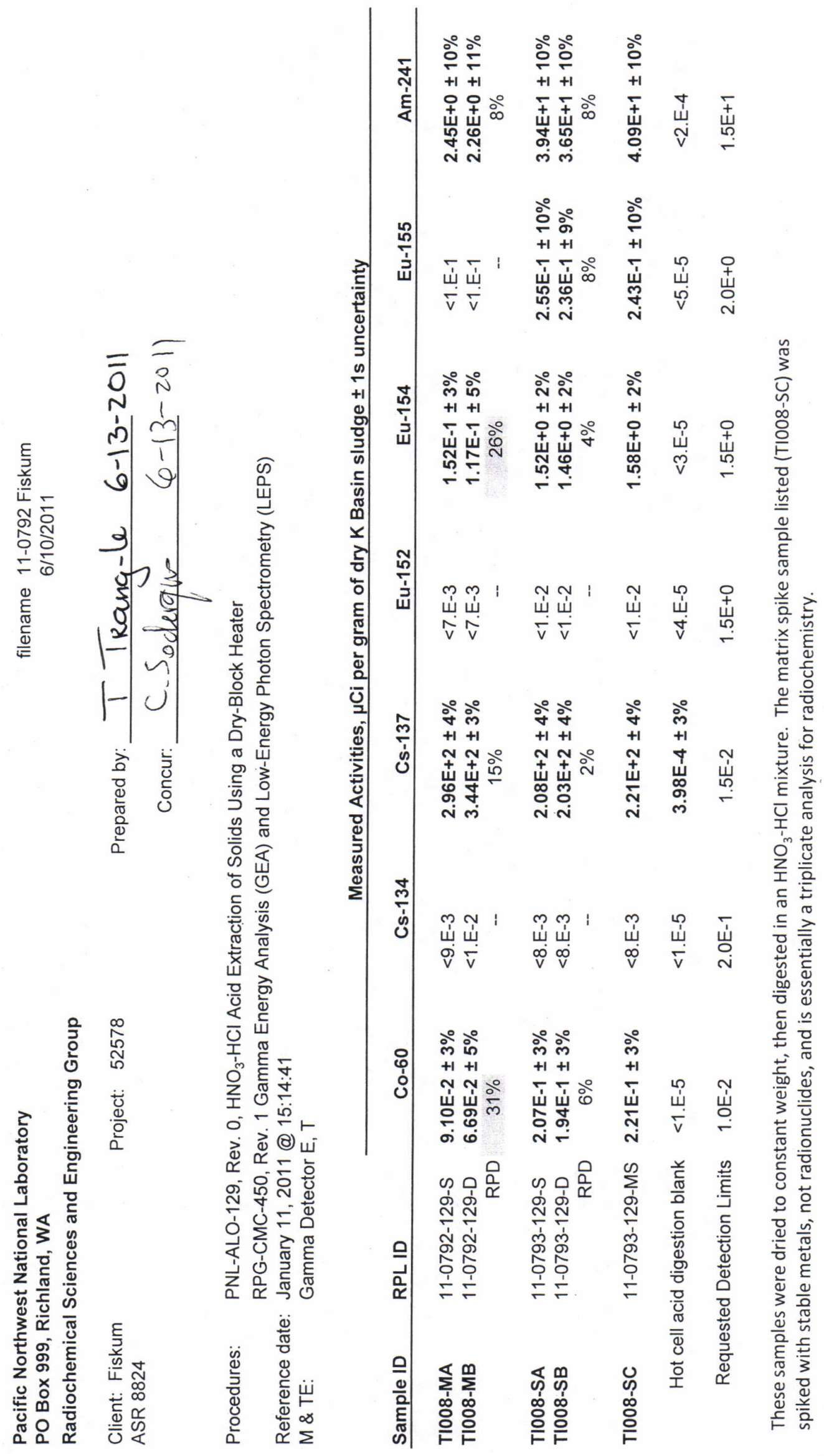

Page 1 of 1 
PNNL-20650, Rev. 2

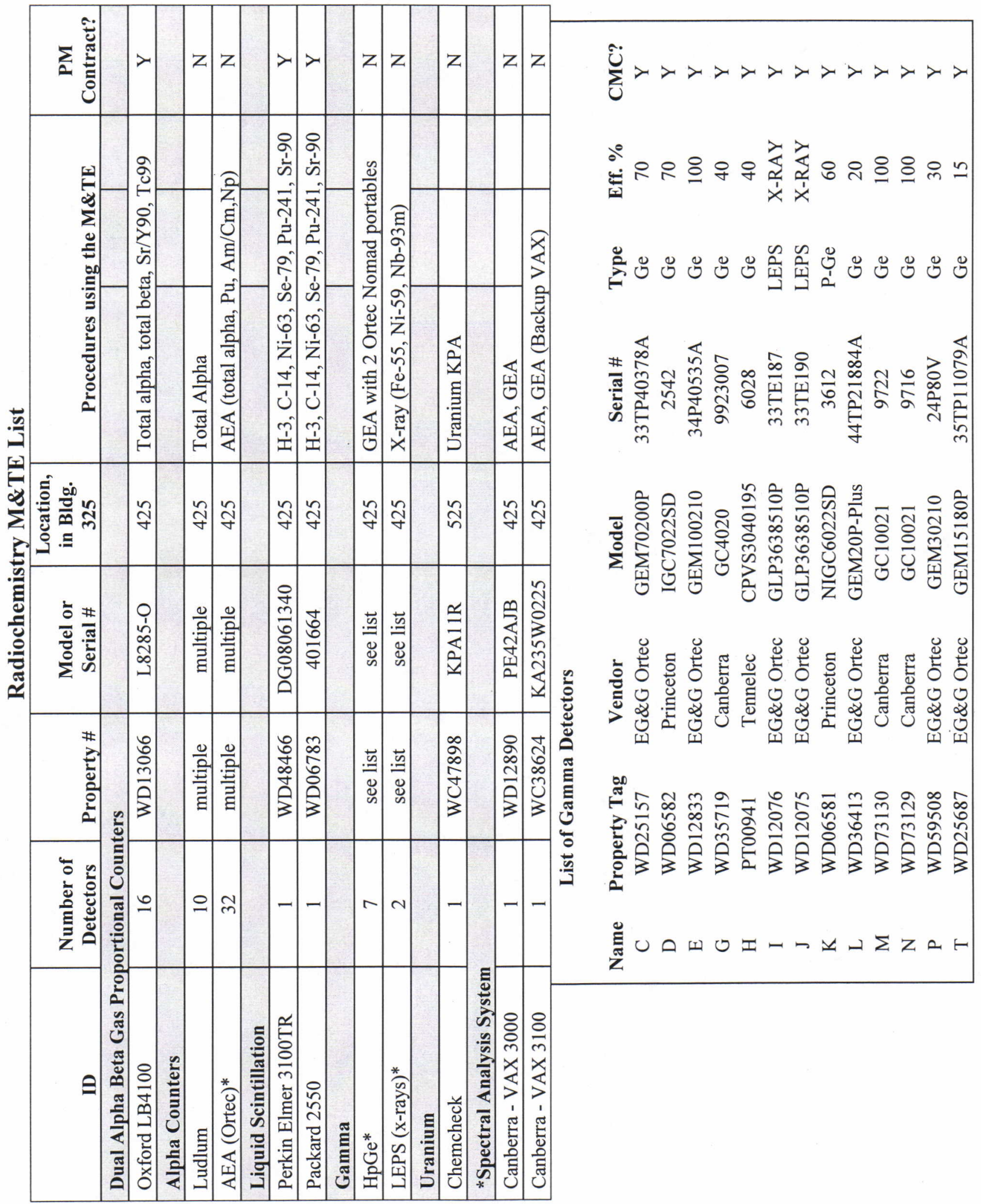


Battelle PNNL/RPL/ASO Radiochemistry Analysis Report

P.O. Box 999, 902 Battelle Blvd., Richland, Washington 99352

\section{Alpha Spectrometry - Total Alpha}

\begin{tabular}{|l|l|}
\hline Project / WP\#: & $52578 / \mathrm{H} 41216$ \\
\hline ASR\#: & 8824 \\
\hline Client: & S. Fiskum \\
\hline Total \# of Samples: & 4 \\
\hline
\end{tabular}

\begin{tabular}{|c|c|c|}
\hline Engineered Container \& Core ID & Sample IDs & RPL Number \\
\hline \multirow{2}{*}{ KW-210-MCC } & TI008-MA & $11-0792-129-\mathrm{S}$ \\
\cline { 2 - 3 } & TI008-MB & $11-0792-129-\mathrm{D}$ \\
\hline \multirow{2}{*}{ KW-210-SCC } & TI008-SA & $11-0793-129-\mathrm{S}$ \\
\cline { 2 - 3 } & TI008-SB & $11-0793-129-\mathrm{D}$ \\
\hline
\end{tabular}

\begin{tabular}{|c|c|}
\hline Analysis Type: & AEA - Total Alpha \\
\hline $\begin{array}{l}\text { Sample Processing Prior to Radiochemical } \\
\text { Processing/Analysis }\end{array}$ & $\begin{array}{l}\square \text { None } \\
\square \text { Digested as per PNL-ALO-129, Rev. 0, } \mathrm{HNO}_{3} \text {-HCl Acid Extraction of } \\
\text { Solids Using a Dry Block Heater, 4-20-11 (N. Green) } \\
\square \text { Fusion as per PNL-ALO-115 Rev. 1.1, Solubilization of Metals from Solids } \\
\text { Using a } \mathrm{KOH}-\mathrm{KNO}_{3} \text { Fusion } \\
\square \text { Other: }\end{array}$ \\
\hline \multirow[t]{2}{*}{ Pre-dilution Prior to Radiochemical Processing? } & 区 No \\
\hline & $\square$ Yes -- example $2 \mathrm{~mL}$ to $100 \mathrm{~mL} ; 50 x$ dilution \\
\hline Total Alpha Preparation Procedure: & $\begin{array}{l}\text { RPG-CMC-4001, Rev. 1, Source Preparation For Gross Alpha and Gross Beta } \\
\text { analyses. }\end{array}$ \\
\hline Technician/Analyst: & L. Darnell, 5-25-11 \\
\hline Spike Standard ID's & $\mathrm{R}-485-\mathrm{a}-10(\mathrm{Pu}-239)$ \\
\hline Analysis Procedure & RPG-CMC-408, Rev. 2, Total Alpha and Beta Analysis \\
\hline Technician/Analyst: & L. Darnell, 5-25-11 \\
\hline Co-Precipitation Procedure: & $\begin{array}{l}\text { RPG-CMC-496, Rev. 0, Co-precipitation Mounting of Actinides for Alpha } \\
\text { Spectroscopy }\end{array}$ \\
\hline Technician/Analyst: & L. Darnell, 5-31-11 \\
\hline Spike and Tracer Standard ID's: & $\mathrm{R}-485-\mathrm{a}-10$ (Pu-239) \\
\hline Analysis Procedure: & RPG-CMC-422, Rev. 2, Solutions Analysis: Alpha Spectrometry \\
\hline Reference Date: & Same as analyses dates \\
\hline Analysis Date or Date Range: & $6 / 1 / 11$ \\
\hline Technician/Analyst: & T. Trang-Le \\
\hline Analysis Data (File): & 11-0792 Fiskum.xls, Alpha Beta \\
\hline CMC Project 98620 File: & $\begin{array}{l}\text { File Plan 5871: T } 52578 \text { 8824: Sample preparation and analysis records; T-4.4 } \\
\text { Alpha Detector calibration, calibration verification checks, and maintenance } \\
\text { records; and T3 Standard certificates and preparation. Also balance calibration } \\
\text { and instrument performance checks. }\end{array}$ \\
\hline M\&TE Number(s): & Ortec AEA counters - 32 counters - See attached M\&TE list \\
\hline
\end{tabular}


PNNL-20650, Rev. 2

Battelle PNNL/RPL/ASO Radiochemistry Analysis Report

\section{Sample Results}

See attached data report, Sample Results for ASR 8824. All data are reported in units of $\mu \mathrm{Ci} / \mathrm{g} \mathrm{K}$-Basin dry sludge with a $1-\sigma$ uncertainty (see comments).

\section{Sample Preparation, Separation, Mounting and Counting Methods}

Four K-Basin sludge samples were prepared in the shielded analytical laboratory (SAL) hot cells by the Project and provided to the Analytical Support Operations (ASO) under Analytical Service Request (ASR) 8824 for chemical and radiochemical analyses. ASO received each sample in a Teflon Oak Ridge centrifuge tube, dried the contents of each centrifuge tube to a constant weight, and then leached the dried solids with hot dilute nitric and hydrochloric acids per procedure PNL-ALO-129. The leachate solution was used for radiochemical analyses and ICP metals analysis; only Alpha-AEA is reported in this report. Visible residual solids remained following the PNL-ALO-129 digestion.

Following the digestion process, an aliquot of the digestate was mounted for alpha spectrometry by coprecipitation using procedure RPG-CMC-496, then counted by alpha spectroscopy using procedure RPC-CMC-422. The samples were counted on June $1^{\text {st }}, 2011$; no decay corrections were made.

Although not formally requested by the ASR, gross alpha analyses was performed on each sample to obtain information to estimate aliquot size for the alpha-AEA and for checking the internal consistency of the alpha isotopic data. The gross alpha results are included in the data tables as supplemental information only. Gross alpha activity was measured by evaporating small aliquots of leachate onto counting planchets per procedure RPG-CMC-4001 and counting per procedure RPG-CMC-408. These procedures are not required to be pre-approved by K-Basin project staff as they are used to generate opportunistic data only. The sum of the major alpha emitters, in this case Pu-239+240 and Pu-238 + Am241 , agrees very well with the gross alpha results.

\section{QUALITY CONTROL RESULTS}

Quality control (QC) samples prepared in the SAL hot cells include a hot cell process blank (PB), sample duplicate, and a matrix spike (MS). Additional QC samples were prepared prior to alpha counting including a laboratory blank, a reagent blank spike (BS, Pu-239), and addition of $\mathrm{Pu}-239$ standard to an aliquot of the sample digestate selected as the matrix spike (MS).

Performance acceptance criteria for these samples are established by both the K Basin data quality objectives (as provided as special instruction in the ASR) and the ASO Quality Assurance (QA) Plan (ASO-QAP-001, Rev. 7). Except where noted, the Alpha-AEA K-Basin DQO and ASO QAP criteria are the same. In cases where the QC criteria are different, K-Basin DQO's take precedence. The QC sample results for Am-AEA have been evaluated and are discussed below. A summary of the Alpha-AEA analysis results, including QC sample performance, is given in the attached data report. 


\section{Battelle PNNL/RPL/ASO Radiochemistry Analysis Report}

Tracer:

Tracer is not used for this analysis.

Hot Cell Process Blank (PB) and Laboratory separations blank (LB):

The alpha isotopic $(\mathrm{Pu}-239+240, \mathrm{Pu}-238+\mathrm{Am}-241$ and $\mathrm{Cm}-243+244)$ activities measured in the PB are required to be within the acceptance criteria of less than $5 \%$ of the sample isotope concentration or less than sample minimum detectable activity (MDA) - (See comments). The PB alpha isotopic activities are significantly less than $5 \%$ of any sample activity. There are no acceptance criteria for LB. The detection limit achieved in the PNL-ALO-129 acid digestion blank for Pu-239+240 is $4.43 \mathrm{E}-5 \mu \mathrm{Ci} / \mathrm{g}_{\text {dry sludge, }}$ for $\mathrm{Am}-241+\mathrm{Pu}-238$ is $6.47 \mathrm{E}-5 \mu \mathrm{Ci} / \mathrm{g}_{\text {dry sludge }}$ and for Cm$243+244$ is $1.36 \mathrm{E}-5 \mu \mathrm{Ci} / \mathrm{g}_{\text {dry sludge. }}$. The achieved detection limits are all below the requested detection limit of $1.0 \mathrm{E}-3 \mu \mathrm{Ci} / \mathrm{g}_{\text {dry sludge. }}$

Blank Spike (BS) - reagent spike:

The BS recovery of $100 \%$ (Pu-239) meets the acceptance criteria of $80 \%$ to $120 \%$ recovery.

\section{Matrix Spike (MS):}

The MS recovery of $96 \%$ (Pu-239) meets the acceptance criterion of $75 \%$ to $125 \%$ recovery. Note: the MS sample was prepared after digestion, by adding a known quantity of $\mathrm{Pu}-239$ standard to a diluted aliquot of the digestate. Sample number 11-0792-129-D (TI008-MB) was selected as the matrix spike sample.

\section{Duplicate - Relative Percent Difference (RPD):}

Duplicate results are required to agree within $<20 \%$ RPD. Duplicate results (samples TI008-MA and TI008-MB duplicate) for Pu-238+Am-241 is 34\%, and for Pu-239+240 is $29 \%$ and for Cm$243+244$ is $52 \%$ thus exceeding the acceptance criteria for duplicate precision. Occurrence Report 52578-06-27-11 has been generated to document the duplicate results exceeding the acceptance limits.

\section{Instrument Quality Control}

Alpha counters receive initial calibration with NIST traceable sources to determine the counter efficiency. When internal tracers are not used, the counter efficiency is used in calculation of final results.

Detector backgrounds are determined every 4 weeks or after the last analytical run, whichever is longer. Detector background counts are subtracted from all subsequent sample counts. A process blank is analyzed with each analytical batch to evaluate for contamination in the sample preparation process.

\section{Assumption and Limitations of the Data}

None 
PNNL-20650, Rev. 2

\section{Battelle PNNL/RPL/ASO Radiochemistry Analysis Report}

1. The results have been corrected for all dilution factors resulting from sample processing.

2. Post-Digestion Spike (PS) - A spike made after the initial sample preparation (e.g., fusion, digestion, or leach) is considered a PS. When extremely radioactive samples are analyzed, most of the radioanalytical spikes are made after the sample preparation and dilution (to avoid excessive consumption of spike and avoid creating unnecessary waste). The MS prepared with this batch of samples is considered a PS, since the Pu-239 spike was not added prior to the digestion process.

3. The 1-sigma uncertainty represents the total propagated error associated with processing and counting operations and include; weighing errors, volume uncertainties, and counting error.

4. The sample results are compared to the process blank to evaluate if the blank contains $5 \%$ or more of the measured isotope; the process blank result has been adjusted for all processing factors for evaluation of the $5 \%$ criterion.

5. The Laboratory Blank (LB) is prepared using laboratory reagents and provides data on the cleanliness of the radiochemistry preparation/separation processes. LB results are not normalized to processing or dilution factors associated with the samples.

Attachment: Data Report -- Sample Results for ASR 8824 
PNNL-20650, Rev. 2

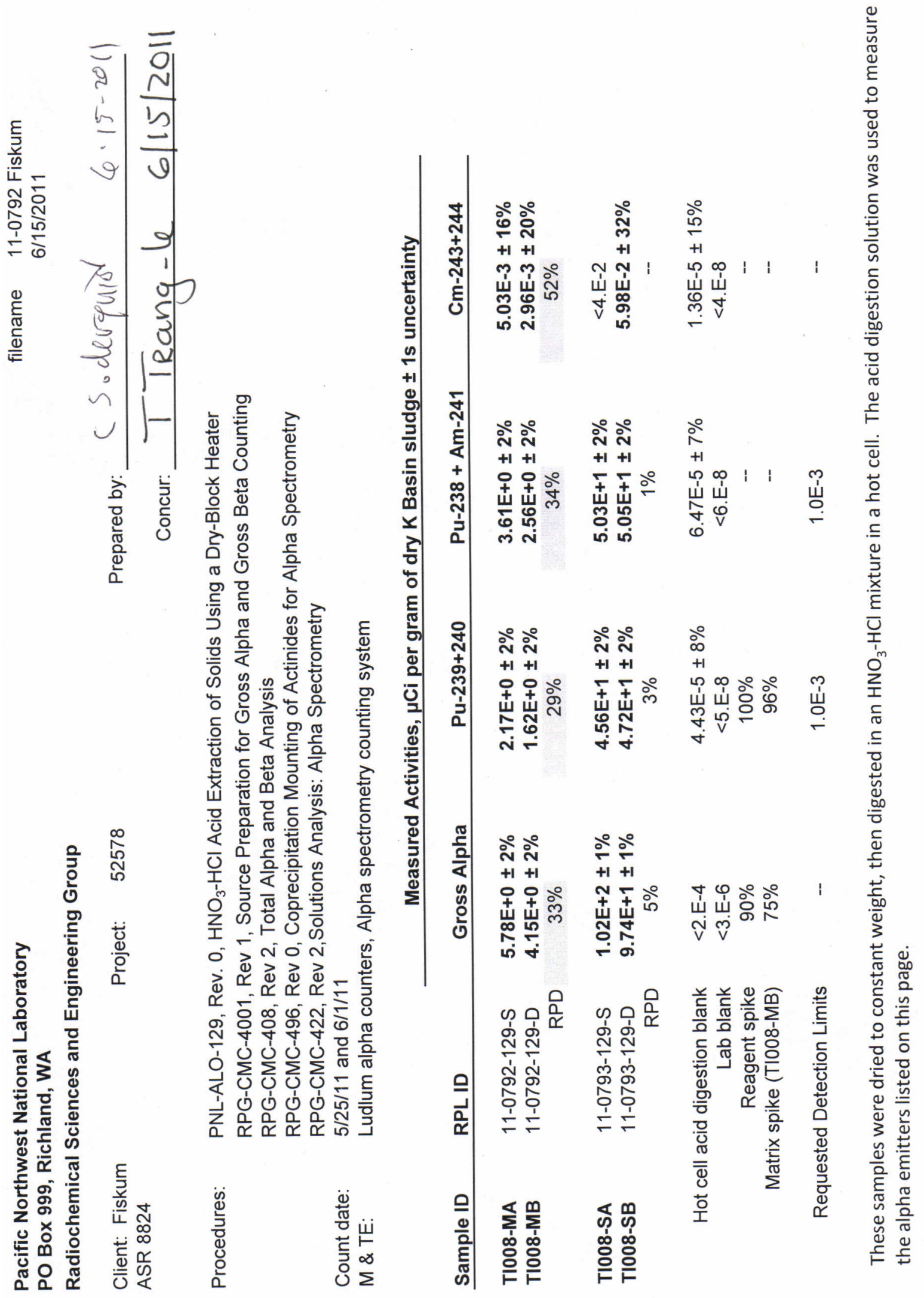


PNNL-20650, Rev. 2

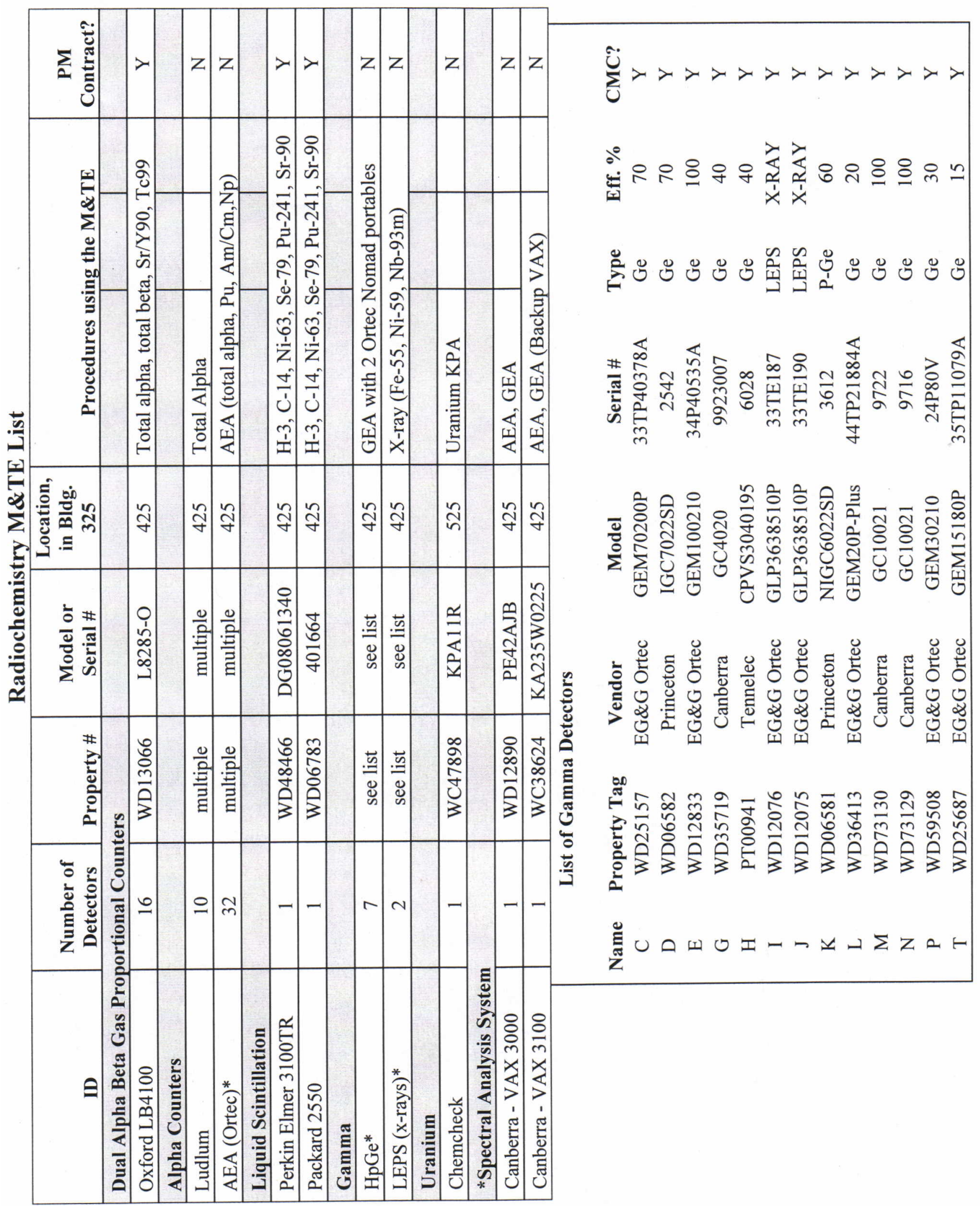




\section{Battelle - Pacific Northwest National Laboratory Analytical Support Operations - \\ Uranium Metal Concentration in K Basin Sludge Report-Revision 2 PO Box 999, Richland, Washington 99352}

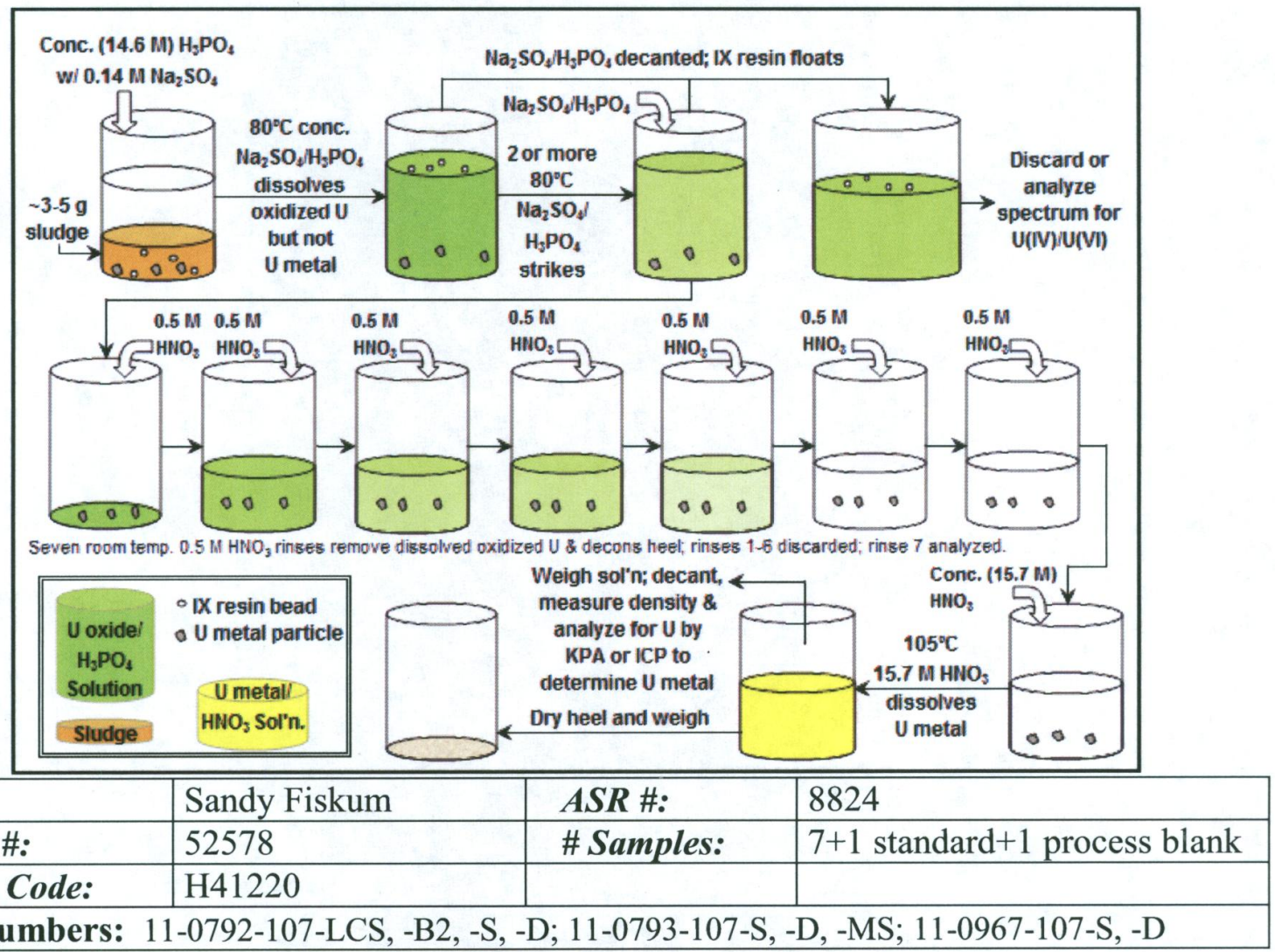

\begin{tabular}{|l|l|l|l|}
\hline Client: & Sandy Fiskum & ASR \#: & 8824 \\
\hline Project \#: & 52578 & \# Samples: & $7+1$ standard+1 process blank \\
\hline Charge Code: & H41220 & \\
\hline RPL Numbers: & 11-0792-107-LCS, -B2, -S, -D; 11-0793-107-S, -D, -MS; 11-0967-107-S, -D \\
\hline
\end{tabular}

Procedure, Analysis, System, and Records Information

\begin{tabular}{|c|c|}
\hline Sample Prep: & $\begin{array}{l}\text { RPG-CMC-107, Rev. 1, Sample Preparation for Determination of Uranium Metal } \\
\text { Concentrations in Sludge } \\
\text { PNL-ALO-501, Rev. 0, Laboratory Procedure for Measurement of Physical and } \\
\text { Rheological Properties of Solutions, Slurries, and Sludges }\end{array}$ \\
\hline Analysis Procedure: & $\begin{array}{l}\text { RPG-CMC-211, Rev. 3. Determination of Elemental Composition by Inductively } \\
\text { Coupled Argon Plasma Optical Emission Spectrometry (ICP-OES) }\end{array}$ \\
\hline Analyst: & Cal Delegard/Nicole Green (digestion) and Jenn Carter (ICP-OES) \\
\hline Analysis Dates: & Digestion - 13 April 2011; ICP - 19 April 2011 \\
\hline $\begin{array}{l}\text { Calibration Date/ } \\
\text { Filename: }\end{array}$ & ICP-325-405-3 in Chemical Measurement Center 98620 file; 4 March 2011 \\
\hline Cal/Ver Stds Prep Date: & LCS: 8 April 2011 \\
\hline $\begin{array}{l}\text { Sample Analysis Excel } \\
\text { Data Files: }\end{array}$ & ICP: C0374, 19 April 2011 \\
\hline M \&TE Numbers & $\begin{array}{l}\text { Balances } 1114123894, \text { Lab } 516 \text { and 25608154, Lab } 312 \text { (U metal beads--LCS \& MS) } \\
\text { Balance N04143 (Container Tare) } \\
\text { Balance 40023610, SAL (solution weights); Balance 360-06-01-037 (sol'n. density) } \\
\text { Balance Mettler AT400 M19445 (ICP-OES) } \\
\text { ICP-OES instrument N827583 (Lab 405) }\end{array}$ \\
\hline All Analysis Records & Chemical Measurement Center 98620: $\quad$ RIDS U-Metal System File \\
\hline
\end{tabular}


PNNL-20650, Rev. 2

\section{Battelle - Pacific Northwest National Laboratory Analytical Support Operations -}

Uranium Metal Concentration in K Basin Sludge Report - Revision 2

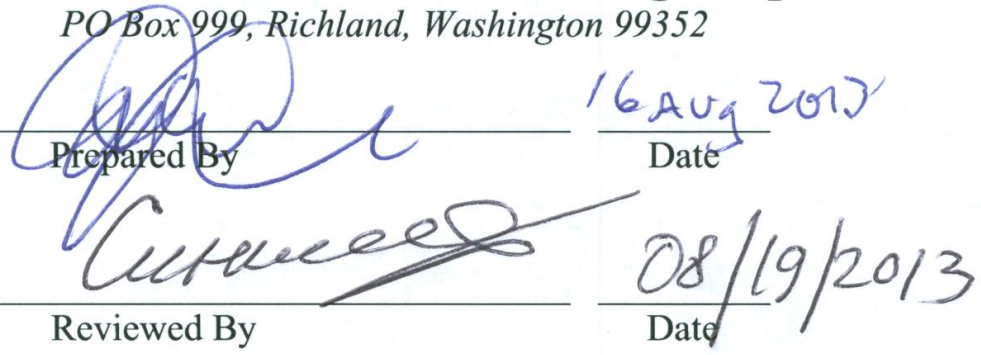


PNNL-20650, Rev. 2

\section{Battelle - Pacific Northwest National Laboratory Analytical Support Operations - \\ Uranium Metal Concentration in K Basin Sludge Report-Revision 2 \\ PO Box 999, Richland, Washington 99352}

\section{Revisions 1 and 2}

Revision 1 to the report was made to remove "less than" symbols $(<)$ from the values for $U_{\text {metal }}$ found, $g$, and $\left[\mathrm{U}_{\text {metal }}\right]$, expressed in $\mu \mathrm{g} / \mathrm{g}$ and $\mathrm{wt} \%$, in Table A.1 for the Process Blank 2 sample.

Revision 2 to the report was made to correct the number of samples delivered as wet slurries from two to five and the number delivered as dried solids from five to two in the first paragraph of the section "Sample Analysis/Results Discussion". The drying method also was further clarified to be "air-dried". A new second paragraph in the "Sample Analysis/Results Discussion" section was added to explain the mass differences for the KW-210-MCC samples between those reported in ASR 8824 and those presented in Table A.1 of Attachment 1. Finally, the explanatory paragraph in Attachment 1 was corrected to describe the KW-210-SCC samples as being dewatered (not dried) and the drying method further clarified to be "air-dried".

\section{Sample Results}

See Attachment 1: Uranium Metal Sample Results ASR 8824.

\section{Sample Analysis/Results Discussion}

The results of uranium metal concentration analysis of seven samples of K-Basin sludge submitted under Analytical Service Request (ASR) 8824 are reported. Five samples were delivered for analysis as wet slurries with an overburden of supernatant water and two as air-dried solids. The samples were analyzed in a single batch.

The bulk KW-210-MCC material was air-dried in the hot cell before mixing, aliquoting, and weighing into 5-mL plastic vials. The MCC splits used for the uranium metal digestions then were transferred to Oak Ridge centrifuge tubes, as required under RPG-CMC-107, Rev. 1, and the amounts transferred determined by weighing. The transferred amounts were nearly quantitative ( $\sim 99 \mathrm{wt} \%)$ with the materials not transferred likely retained within the plastic $5-\mathrm{mL}$ vials by electrostatics. Because of this retention, the aliquot masses in ASR 8824 are slightly higher than those given in Table A.1 of Attachment 1.

The samples for uranium metal analysis were digested according to the PNNL Technical Procedure, RPG-CMC-107, Rev. 1, Sample Preparation for Determination of Uranium Metal Concentrations in Sludge. The contacts with $0.14 \mathrm{M} \mathrm{Na}_{2} \mathrm{SO}_{4}$ in concentrated $\mathrm{H}_{3} \mathrm{PO}_{4}$ are designed to dissolve and remove the accompanying uranium oxides. ASR 8824 Special Instructions specified a minimum of three contacts with $0.14 \mathrm{M} \mathrm{Na}_{2} \mathrm{SO}_{4}$ in concentrated $\mathrm{H}_{3} \mathrm{PO}_{4}$. The second and third contact solutions were colorless to the eye, indicating good dissolution of the contained uranium oxides.

The residues from the $\mathrm{Na}_{2} \mathrm{SO}_{4} / \mathrm{H}_{3} \mathrm{PO}_{4}$ contacts were rinsed sequentially seven times in $0.5 \mathrm{M} \mathrm{HNO}_{3}$ and the rinsed heels, containing the undissolved uranium metal, were digested in concentrated $\mathrm{HNO}_{3}$ to dissolve the uranium metal, all according to the digestion procedure, RPG-CMC-107. For each concentrated $\mathrm{HNO}_{3}$ digestate sample, mass and density were measured and uranium concentration in the digestate was measured by inductively coupled plasma optical emission spectrometry (ICP-OES) according to RPG-CMC-211, Rev. 3, Determination of Elemental Composition by Inductively Coupled 


\section{Battelle - Pacific Northwest National Laboratory Analytical Support Operations - \\ Uranium Metal Concentration in K Basin Sludge Report-Revision 2 PO Box 999, Richland, Washington 99352}

Argon Plasma Optical Emission Spectrometry (ICP-OES). The sample digestion procedure followed under RPG-CMC-107 is outlined in the Figure at the top of page 1.

\section{Data Limitations}

None.

\section{Quality Control Discussion}

The method performance is evaluated against the acceptance criteria provided in ASR 8824 . Values at the Process Quantitation Limit ( $0.03 \mathrm{wt} \% \mathrm{U}$ in sludge) or greater are considered to be quantified with an uncertainty of $\pm 25 \%$ (at $2 \sigma$, relative) or better.

Blanks: Reagent blanks are analyzed at least once in every batch of 20 samples. Blanks of the $0.5 \mathrm{M}$ $\mathrm{HNO}_{3}$ rinse solution and the concentrated $\mathrm{HNO}_{3}$ metal digestion solution were prepared with the sample batch; this result is in the supporting ICP-OES report. A blank containing only Hanford sand was included in the batch and was taken through all of the procedure steps. The blanks were near or below the analytical detection limits established for the ICP-OES $(0.012 \mu \mathrm{g} / \mathrm{mL})$. The blank level for the $0.5 \mathrm{M}$ $\mathrm{HNO}_{3}$ reagent solution (Table A.1) was generally only a small contributor to the observed $7^{\text {th }}$ rinse solution concentrations and, even when near the observed rinse solution concentrations, did not interfere with the key observations of rinse solution concentration which become important at values greater than about $100 \mu \mathrm{g} / \mathrm{mL}$. Similarly, the concentrated $\mathrm{HNO}_{3}$ blank solution concentration contributed negligibly to the 177 to $5,340 \mu \mathrm{g} / \mathrm{mL}$ digestate concentrations.

Seventh Rinses: The uranium concentrations in the $7^{\text {th }} 0.5 \mathrm{M} \mathrm{HNO}_{3}$ rinses indicate the thoroughness of oxidized (i.e., non-metallic) uranium removal from the sludge. In developing this analytical technique (Delegard et al. 2008), uranium concentrations in the $7^{\text {th }}$ rinse less than $\sim 100 \mu \mathrm{g} \mathrm{U} / \mathrm{mL}$ gave good indication that the uranium oxides were dissolved and their solutions removed from the heel.

As shown in Table A.1, all of the $7^{\text {th }}$ rinse solution concentrations were less than $100 \mu \mathrm{g} / \mathrm{mL}$ in uranium. The rinse concentrations ranged from 0.04 to $1.5 \mu \mathrm{g} \mathrm{U} / \mathrm{mL}$ and indicate complete removal of uranium oxide from the sample. The uranium concentrations in the $7^{\text {th }}$ rinse were examined along with other batch quality control indicators, including duplicates, LCS results, and process blanks, in making data quality judgments.

Laboratory Control Samples (LCS): A single LCS was analyzed with the K Basin sludge samples. The LCS contained $\sim 0.5 \mathrm{~g}$ of $\mathrm{U}_{3} \mathrm{O}_{8}$ (weighed to the nearest $0.001 \mathrm{~g}$ ), $\sim 0.6 \mathrm{~g}$ of Hanford sand (from Arid Lands Ecology, ALE, Reserve; weighed to the nearest $0.001 \mathrm{~g}$ ), and $\sim 0.012 \mathrm{~g}$ of uranium metal (weighed to the nearest $0.00001 \mathrm{~g}$ ) present as a single near-spherical bead.

The LCS uranium metal recovery was $103 \%$ and was within the acceptance limits of $75-125 \%$ specified in the procedure RPG-CMC-107 and in the ASR 8824 Special Instructions.

Duplicate (Precision): A duplicate sample is required to be analyzed in every batch of 20 samples or fewer. This requirement was met.

Prior to this Project work, the MDL, $0.011 \mathrm{wt} \% \mathrm{U}_{\text {metal }}$ on a settled sludge basis, was determined by evaluating uranium metal analysis recovery data (defined as PDL in Attachment J of Fiskum et al. 2011). The ASR specifies an MDL of $0.01 \mathrm{wt} \%$, settled sludge basis. All sample wt $\%$ results were above the ASR-specified MDL. Refer to footnote (a) of Table A.1. 
PNNL-20650, Rev. 2

\section{Battelle - Pacific Northwest National Laboratory Analytical Support Operations - \\ Uranium Metal Concentration in K Basin Sludge Report-Revision 2 PO Box 999, Richland, Washington 99352}

The QAPjP/SAP (KBC-33786, Rev. 2) specifies that a $\pm 20 \%$ precision criterion for duplicates is to be applied when $\left[\mathrm{U}_{\text {metal }}\right]>0.1 \mathrm{wt} \%$. The $\left[\mathrm{U}_{\text {metal }}\right]>0.1 \mathrm{wt} \%$ threshold was exceeded for the $\mathrm{KW}-210-\mathrm{MCC}$ and KW-210-A4 duplicate sample sets. The RPD for duplicate KW-210-MCC samples was $86 \%$ and the RPD for KW-210-A4 was 4\%. Occurrence Report 52578-05-19-2011 was generated to document that the duplicate RPD values for KW-210-MCC did not meet the acceptance limits.

Matrix Spike: Based on the QAPjP/SAP (KBC-33786, Rev. 2), uranium metal measurement accuracy is to be assessed by evaluation of the LCS recoveries. However, a matrix spike (MS) sample was included to provide additional indicators of method performance. To prepare the MS sample, a uranium metal bead spike was added to one of three sludge subsamples taken from the same set of KW-210-SCC samples. The amount of $U$ metal added as MS was about 7-times greater than the average amount of uranium metal found present in the other two -SCC subsamples. The MS recovery, adjusted for the uranium metal quantity expected based on the average concentration found in the companion subsamples, was $123 \%$.

\section{Deviations from Procedure}

There were no deviations from the procedure.

\section{General Comments}

In June 2009, based on discussions between PNNL and CHPRC, it was determined that matrix spikes were to be included within each analytical batch to be processed for uranium metal and uranium oxide oxidation state distribution. While the matrix spike samples were intended to provide an additional assessment of the procedure performance, performance or failure criteria for matrix spike recoveries were not established.

\section{References}

ASO-QAP-001, Rev. 7. 2009. Analytical Support Operations (ASO) Quality Assurance Plan, Pacific Northwest National Laboratory, Richland, WA.

Delegard CH, SI Sinkov, AJ Schmidt, and JW Chenault. 2008. Uranium Metal Analysis via Selective Dissolution. PNNL-17800, Pacific Northwest National Laboratory, Richland, WA.

Fiskum SK, JM Billing, SJ Bos, CA Burns, CD Carlson, DS Coffey, JV Crum, RC Daniel, CH Delegard, MK Edwards, OT Farmer, LR Greenwood, SA Jones, D Neiner, BM Oliver, KN Pool, AJ Schmidt, RW Shimskey, SI Sinkov, SZ Soderquist, CJ Thompson, ML Thomas, T Trang-Le, and MW Urie. 2011. Characterization Data Package for Containerized Sludge Samples Collected from Engineered Containers SCS-CON-240, 250, 260, and 220. PNNL-19035, Rev. 1, Pacific Northwest National Laboratory, Richland, WA.

KBC-33786, Rev. 2, Quality Assurance Project Plan / Sampling and Analysis Plan for Sludge in the KW Engineered Containers, CH2M Hill Plateau Remediation Company, Richland, WA. December 2009. 
PNNL-20650, Rev. 2

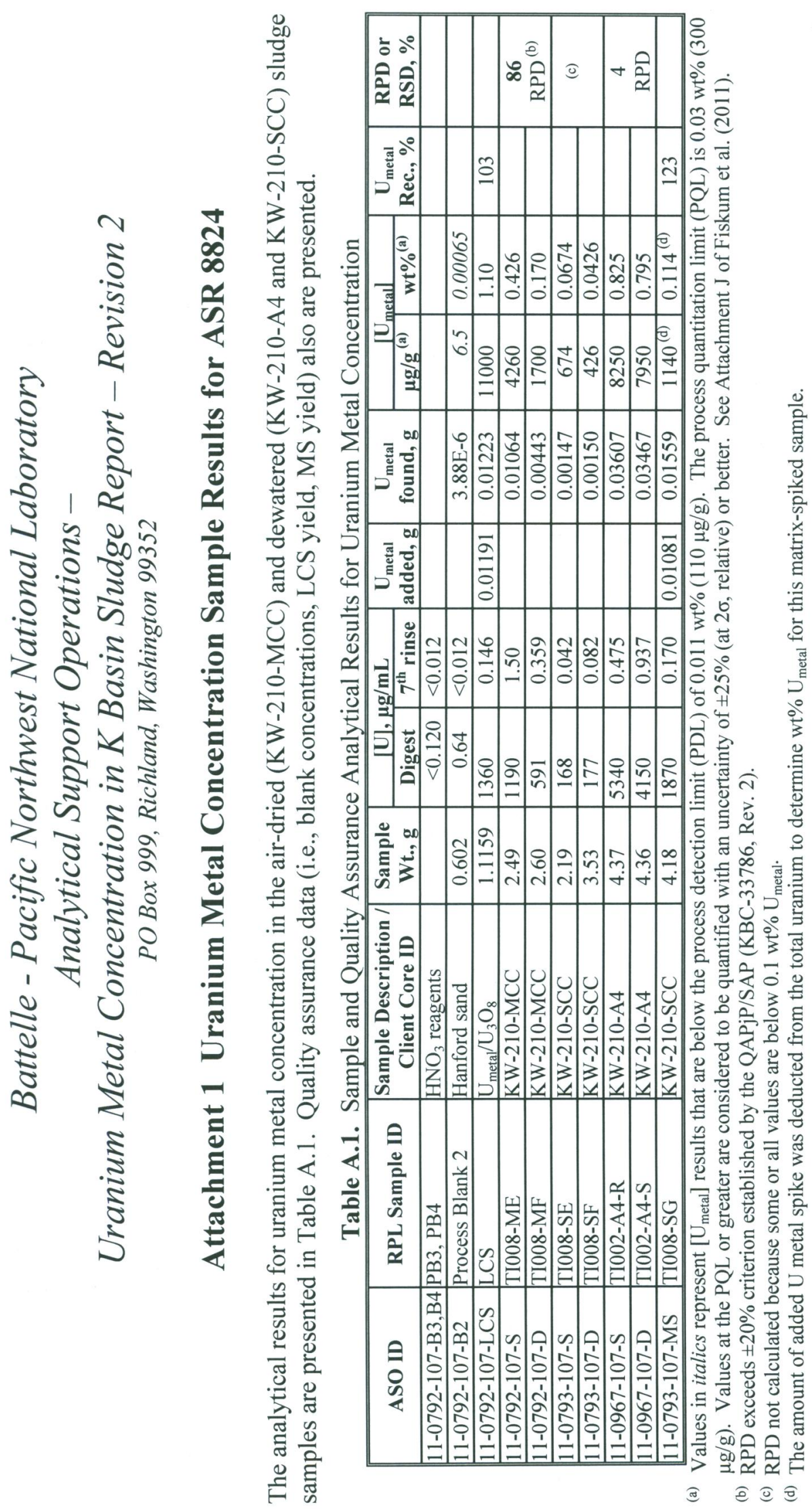




\section{Battelle - Pacific Northwest National Laboratory Analytical Support Operations - \\ Uranium Oxidation States in K Basin Sludge Report \\ PO Box 999, Richland, Washington 99352}

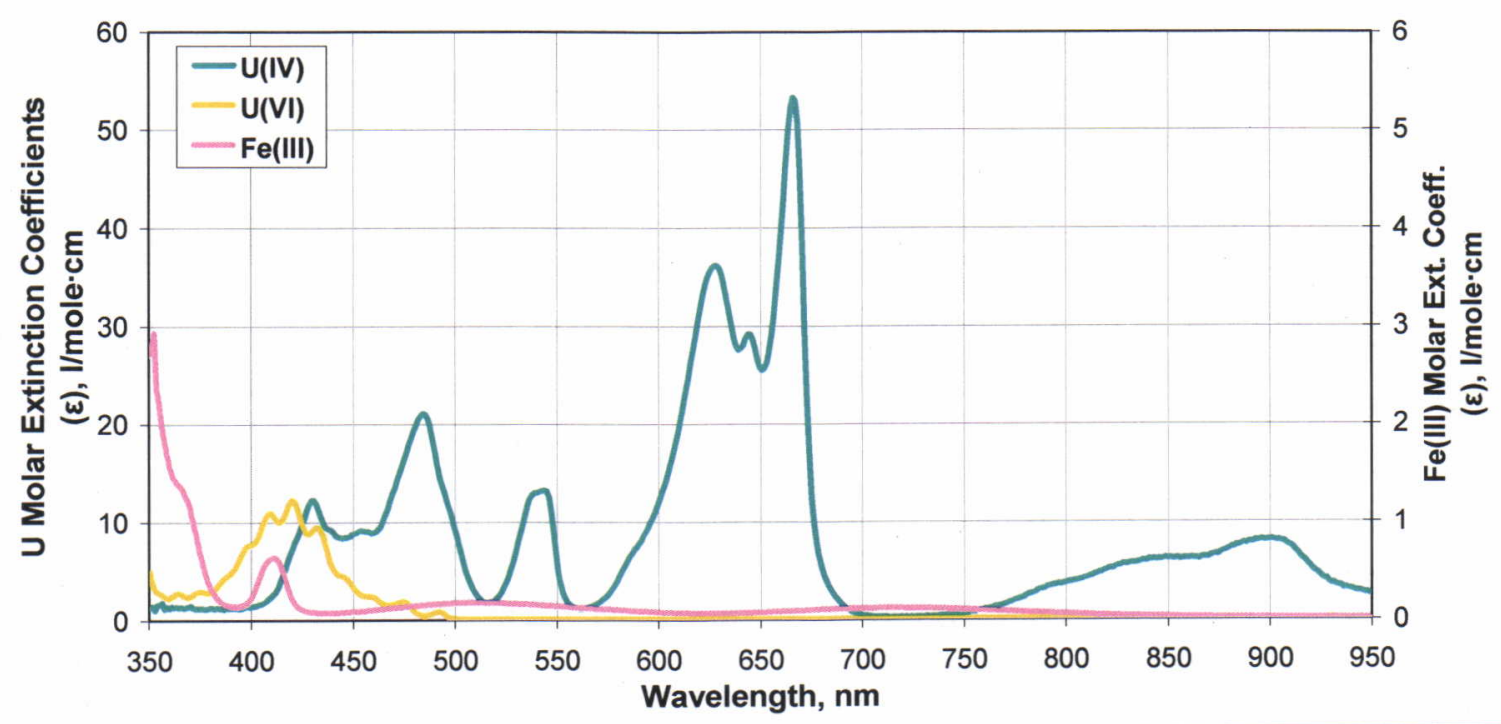

\begin{tabular}{|c|c|c|c|}
\hline Client: & Sandy Fiskum & ASR \#: & 8824 \\
\hline Project \#: & 52578 & \# Samples: & 7 samples, 1 standard, 1 blank \\
\hline Charge Code: & $\mathrm{H} 41221$ & & \\
\hline
\end{tabular}

Procedure, Analysis, System, and Records Information

\begin{tabular}{|c|c|}
\hline Analysis Procedure: & $\begin{array}{l}\text { RPG-CMC-255, Rev. 0, Sample Preparation and Analysis for Determining } \\
\text { Uranium Oxide Oxidation States in K Basin Sludges }\end{array}$ \\
\hline Sample Prep: & $\begin{array}{l}\text { RPG-CMC-107, Rev. 1, Sample Preparation for Determination of Uranium } \\
\text { Concentrations in Sludge }\end{array}$ \\
\hline Analyst: & Sergey Sinkov \\
\hline Analysis Dates: & 15-19 April 2011 \\
\hline $\begin{array}{l}\text { Calibration Date/ } \\
\text { Filename: }\end{array}$ & $\begin{array}{l}\text { 12/2008 } \\
\text { RPG-CMC-255 - U Oxidation State Calculation - Final.xls }\end{array}$ \\
\hline Cal/Ver Stds Prep Date: & 8 April 2011 \\
\hline $\begin{array}{l}\text { Sample Analysis Excel } \\
\text { Data Files: }\end{array}$ & $\begin{array}{l}\text { RPG-CMC-255 - U Oxidation State Calculation - ASR 8824.xls } \\
041811 \text { Spectra of H3PO4 digestates from hot cell - ASR 8824.xls }\end{array}$ \\
\hline M \&TE Numbers & Spectral Instruments SI 400 (property number WD13494) in Lab 516 \\
\hline All Analysis Records & Chemical Measurement Center 98620: Fileplan U Oxide System File \\
\hline
\end{tabular}




\section{Battelle - Pacific Northwest National Laboratory Analytical Support Operations - \\ Uranium Oxidation States in K Basin Sludge Report \\ PO Box 999, Richland, Washington 99352}

\section{Sample Results}

See Attachment: Uranium Oxide Oxidation States Sample Results for ASR 8824.

\section{Sample Analysis/Results Discussion}

The results for the uranium oxidation state distribution of the uranium oxides in seven samples of KBasin settler tube sludge submitted under Analytical Service Request (ASR) 8824 are reported. All samples were from container KW210.

The samples were prepared according to the PNNL Technical Procedure, RPG-CMC-107, Rev. 1, Sample Preparation for Determination of Uranium Metal Concentrations in Sludge, to dissolve and remove the accompanying uranium oxides. There were no problems with the digestions. The digestates from the uranium oxide dissolutions were analyzed by spectrophotometry to determine the oxidation state distributions [to the U(IV) and U(VI) oxidation states] of the constituent uranium oxides according to the ASO procedure RPG-CMC-255, Rev. 0, Sample Preparation and Analysis for Determining Uranium Oxide Oxidation States in K Basin Sludges.

Two additional samples from KW210-A4 also were run with the present batch to provide supplemental information to results obtained under ASR 8798 for KW210-A4.

\section{Data Limitations}

The U(IV) and U(VI) oxidation state ratios should be used with caution. Ferrous iron, Fe(II), which is present in magnetite that has been observed in $\mathrm{K}$ Basin sludge, has been shown to reduce U(VI) to U(IV) in $\mathrm{Na}_{2} \mathrm{SO}_{4} / \mathrm{H}_{3} \mathrm{PO}_{4}$ solutions; the amount of $\mathrm{Fe}(\mathrm{II})$ is not known in these samples. The U(VI) fraction can be considered a lower bounding case and the U(IV) fraction can be considered upper bounding case.

\section{Quality Control Discussion}

The uranium oxidation state method performance is evaluated against the acceptance criteria established within procedure RPG-CMC-255, Rev. 0, Sample Preparation and Analysis for Determining Uranium Oxide Oxidation States in K Basin Sludge, and any client specified special instructions provided in the ASR.

Blanks: The uranium oxide and many of the accompanying sludge phases were dissolved at $\sim 80^{\circ} \mathrm{C}$ by three sequential contacts with $0.14 \mathrm{M} \mathrm{Na}_{2} \mathrm{SO}_{4}$ in concentrated $\mathrm{H}_{3} \mathrm{PO}_{4}$. In accordance with the procedure, RPG-CMC-107, Rev. 1, Sample Preparation for Determination of Uranium Metal Concentrations in Sludge, a process blank of $0.14 \mathrm{M} \mathrm{Na}_{2} \mathrm{SO}_{4}$ in concentrated $\mathrm{H}_{3} \mathrm{PO}_{4}$ was prepared per the requirement that one process blank be prepared and analyzed at least once in every batch of 20 samples. The process blank contained $\sim 0.6 \mathrm{~g}$ of Hanford sand and showed $<0.001 \mathrm{M}$ each of U(IV) and U(VI), below the minimum concentrations detectible by the uranium oxidation state method. The process blank showed negligible uranium concentration indicating negligible contamination.

Laboratory Control Samples (LCS): A single Laboratory Control Sample (LCS) was analyzed, meeting the requirement that at least one LCS to be analyzed in parallel with every batch of 20 samples. The 


\section{Battelle - Pacific Northwest National Laboratory Analytical Support Operations - \\ Uranium Oxidation States in K Basin Sludge Report \\ PO Box 999, Richland, Washington 99352}

present batch had $9 \mathrm{~K}$ Basin sludge samples. The LCS sample contained $\sim 0.5 \mathrm{~g}$ of $\mathrm{U}_{3} \mathrm{O}_{8}$ and $\sim 0.6 \mathrm{~g}$ of Hanford sand (each weighed to the nearest $0.001 \mathrm{~g}$ ) and $\sim 0.01 \mathrm{~g}$ of uranium metal (weighed to the nearest $0.00001 \mathrm{~g}$ ) present as a single near-spherical bead. The U(IV) and U(VI) uranium oxidation state distributions in the dissolved $\mathrm{U}_{3} \mathrm{O}_{8}$ were measured to determine analytical performances of the uranium oxide oxidation state distribution analyses. Because $\mathrm{U}_{3} \mathrm{O}_{8}$ is effectively one part $\mathrm{U}(\mathrm{IV})$ and two parts $\mathrm{U}(\mathrm{VI})$ on a mole basis, the $\mathrm{U}_{3} \mathrm{O}_{8}$ dissolved in the $\mathrm{Na}_{2} \mathrm{SO}_{4} / \mathrm{H}_{3} \mathrm{PO}_{4}$ solution ideally yields $33.3 \% \mathrm{U}(\mathrm{IV})$ and $66.7 \% \mathrm{U}(\mathrm{VI})$. Per the procedure, the relative amounts of U(IV) and U(VI) in the $\mathrm{U}_{3} \mathrm{O}_{8}$ LCS should vary by no more than $5 \%$, absolute, from the ideal $33.3 \%$ (i.e., from 28 to $38 \%$ ) and $66.7 \%$ (i.e., from 62 to $72 \%$ ), respectively. The QAPjP/SAP (KBC-33786, Rev. 2) specifies that the LCS values differ by no more than $25 \%$, relative, from the true values and thus affords greater tolerance margins. The LCS sample for this batch contained $34.2 \% \mathrm{U}(\mathrm{IV})$ and $65.8 \% \mathrm{U}(\mathrm{VI})$ and thus met the target performances for both the procedure and the less stringent QAPjP/SAP.

Duplicate (Precision): A duplicate sample is required to be analyzed in every batch (of 20 samples or fewer) to allow determination of method precision. This requirement was met. Each of the three samples was measured at least in duplicate.

Per the ASO QA Plan (ASO-QAP-001, Rev. 7), the relative percent difference (RPD) is calculated in cases where both U(IV) and U(VI) concentrations are measured at or above the $0.001 \mathrm{M}$ minimum detectible concentration for two samples. The $\geq 0.001 \mathrm{M} \mathrm{U}(\mathrm{IV})$ and $\mathrm{U}(\mathrm{VI})$ concentration criteria were met for one of the three samples (from the small size fraction of the KW210 core composite, KW210-SCC). The other two samples had $<0.001 \mathrm{M}$ U(VI). The reported RPD for KW210-SCC for the U(IV) mole\%, shown in Table A.1, was $7.0 \%$ and the RPD for U(VI) was 35\%. No RPD requirement for uranium oxidation state is provided in the ASR special instructions.

The QAPjP/SAP states that the results should agree $\pm 5 \%$ (absolute) if concentrations greater than 0.001 $\mathrm{M}$ in each oxidation state are present (KBC-33786, Rev. 2). The individual KW210-SCC uranium oxidation state values are within $\pm 5 \%$, absolute, of their average or 78.2 to $88.2 \%$ for U(IV) and 11.8 to $21.8 \%$ for U(VI). Therefore, this objective was met.

A single matrix spike (MS) sample was run for KW210-SCC. This MS contained an added bead of uranium metal. The uranium metal spike should not alter the uranium oxide oxidation state distribution. The U(IV) and U(VI) percentage values for the MS were between, and thus were consistent with, the values found for the associated sample and duplicate uranium oxidation state analyses for KW210-SCC. No Data Quality Objectives are associated with the oxidation state result for the MS sample.

\section{Deviations from Procedure}

There were no deviations from the procedure RPG-CMC-255, Rev. 0, Sample Preparation and Analysis for Determining Uranium Oxide Oxidation States in K Basin Sludges.

\section{References}

ASO-QAP-001, Rev. 7. 2009. Analytical Support Operations (ASO) Quality Assurance Plan, Pacific Northwest National Laboratory, Richland, WA.

KBC-33786, Rev. 2, Quality Assurance Project Plan / Sampling and Analysis Plan for Sludge in the KW Engineered Containers, CH2M Hill Plateau Remediation Company, Richland, WA. December 2009. 
PNNL-20650, Rev. 2

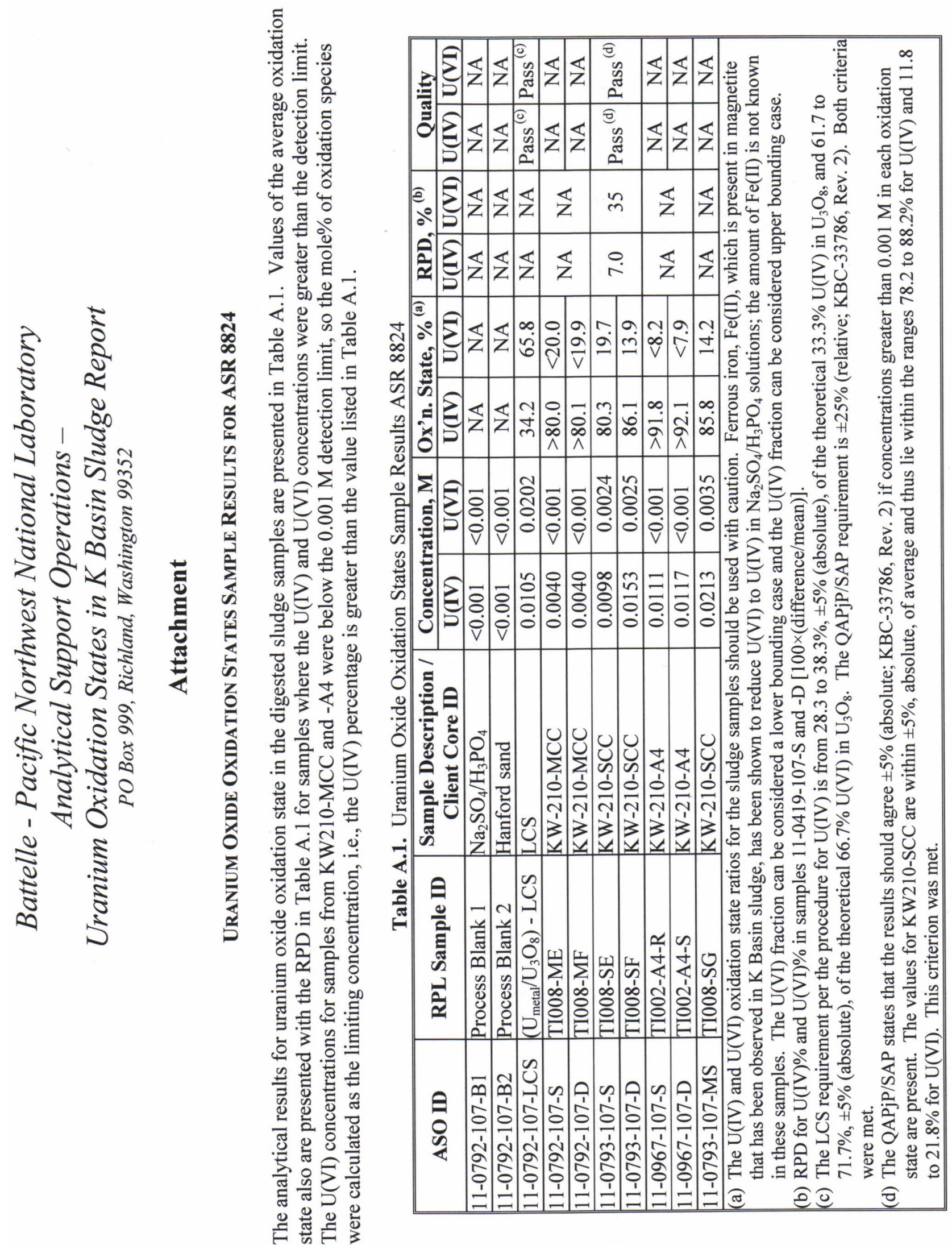

Page 4 of 4 
PNNL-20650, Rev. 2

$\mathbb{K} \cdot \mathbb{B}$ asim Occurirence $\mathbb{R e p}$ Orot

\begin{tabular}{|c|c|}
\hline 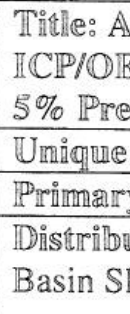 & $\begin{array}{l}\text { SR } 8824 \text { Dup } \\
\text { ES frrom -129) } \\
\text { esent in the S2 } \\
\text { Identifier: } 52 \\
\text { y Persom IIdem } \\
\text { utiom: Email at } \\
\text { hare-Point }\end{array}$ \\
\hline Date Sn & Ibmitted to $\mathbb{K}$ \\
\hline Occurre & ence Descr \\
\hline 1) $\mathbb{I}$ & $\begin{array}{l}\text { ICP/OES duplic } \\
\text { RPD requiremer } \\
\text { a) } \mathrm{Ba}-76.7 \% \\
\text { b) } \mathrm{Cr}-41.7 \% \\
\text { c) } \mathrm{Fe}-28.7 \% \\
\text { d) } \mathrm{Ni}-40.2 \% \\
\text { e) } \mathrm{Pb}-39.1 \% \\
\text { f) } \mathrm{Zr}-143.2 \%\end{array}$ \\
\hline
\end{tabular}

2) The $\mathrm{Na}$ concentration in the process blank (71.5ppm) exceeds $5 \%$ of the $\mathrm{Na}$ level present in samples TI008-SA (Na blank is $15.9 \%$ of the sample result) and TIO08-SB (Na blank is $18.6 \%$ of the sample result).

Impact of Occurrence:

The variability between sample and duplicate is an indication of primary sample heterogeneity.

The elevated $\mathrm{Na}$ in the process blank could results in a positive bias for the Na results for this batch rum.

Sigmifificant Condition Adverse to Qualicy? Yes__ No $X$

Evalluated by: Date: $6 / 812021$

Further Disposition: $\bar{\emptyset}$ No $\square$ Yes Iff yes, $\square$ Nomconformance? $\square$ Deficiency?

Date Occurrence Closed: $6 / 8 / 2011$

Sigmature/Date of $\mathbb{R}$ esponsible $\mathbb{Q} E: \sum$ sbarah Coffey $6 / 8 / 2011$

Sigmature/Date of $\mathbb{R}$ esponsible $\mathbb{K}$-Basim Mamager: 1 /8/201/

Comments: 
PNNL-20650, Rev. 2

$8 d s c 6-30-11$

$\underline{52578-06-27-11}$

Page 1 of $\mathbb{1}$

$\mathbb{K}$-Basin Occunrremce $\mathbb{R}$ epont

Tithle: ASR 8824 GEA and Alpha-AEA duplicate results exeed $\pm 20 \%$ requirement.

Umique Idemtififer: 52578-06-27-11

Primary Persom Identifying Issue: Trune Tramg-Le

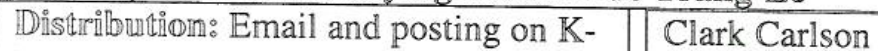

Basin Share-Point

Date Submirtted to $\mathbb{K}-\mathbb{B}$ asin $\mathbb{R e c o r d s : ~}$

Deborah Coffey

Sandy Fiskum

Karl Pool

Decurrence Description: $\mathbb{A S R}:$ \$24

$6-30-2011$

Andy Schmidt

Rick Shimskey

Truc Trang Le, Project file for ASR \$824

GEA Amalyses:

The duplicate relative percent difference (RPD) for Co-60 and Eu-154 on samples TI008-MA and T1008-

MBB is $31 \%$ and $26 \%$ respectively. Both of these RPDs exceed the acceptance limit of $\pm 20 \%$.

Allpha AEA Amalyses:

The duplicate relative percent difference (RPD) for Pu-238+Am-241 is $34 \%$, for Pu- $239+240$ is $29 \%$ and for $\mathrm{Cm}-243+244$ is $52 \%$ for samples TI008-MA and TI008-MB. These RPDs exceed the accetance limit of $\pm 20 \%$.

Ilmpact of Occunremce:

There is no impact due to the RPDs exceeding the acceptance limit for these two isotopes.

The variation observed is reflective of the heterogeneous nature of the mid fraction samples.

Sigmifficamt Condition Adverse to Qunaliety? Yes __ No $x$

Eivalluated by:

Furither Dispositiom: $\mathbb{W}$ No $\square$ Yes IIf yes, $\square$ Nomeomformamce? $\square$ Defficiency?

Datte Occurremce Closed: $\varphi-30-2011$

Sigmature $/ \mathrm{Date}$ of $\mathbb{R e s p o m s i b l e ~} \mathbb{Q E}:$ Crebcrah Cofky $6-30-2011$

Signature/IDate of $\mathbb{R e s p o n s i b l e ~} \mathbb{K} \cdot \mathbb{B}$ asim $M$ Manager: $6 / 30 / 20 / 1$

Comments: 


\section{K-Basin Occurrence Report}

\begin{tabular}{|l|l|l|}
\hline Title: U-metal RPD exceeds 20\% & Date: 5-19-11 \\
\hline Unique Identifier: 52578-05-19-11 & \\
\hline Primary Person Identifying Issue: C. Delegard & \\
\hline $\begin{array}{l}\text { Distribution: Email and posting on K- } \\
\text { Basin Share-Point }\end{array}$ & Clark Carlson & \\
\cline { 2 - 3 } & Deborah Coffey & Andy Schmidt \\
\cline { 2 - 3 } & Sandy Fiskum & Rick Shimskey \\
\cline { 2 - 3 } & Karl Pool & $\begin{array}{l}\text { Truc Trang Le, Project file for ASR } \\
\mathbf{8 8 2 4}\end{array}$ \\
\hline Date Submitted to K-Basin Records: & $-7-28 \cdot 201 j$ \\
\hline Occurrence Description: ASR 8824:
\end{tabular}

The duplicate RPD for results for samples TI008-ME (11-0792-107-S) and TI008-MF (11-0792-107-D) was $86 \%$, exceeding the acceptance criteria of $20 \%$. Both the sample and duplicate sample have U-metal concentrations greater than 0.1 weight $\%$, TI008-ME (0.426 wt. \%) and TI008-MF (0.170 wt. \%).

\section{Impact of Occurrence:}

The sample masses used for the U-metal analyses were $2.49 \mathrm{~g}$ for the sample and $2.60 \mathrm{~g}$ for the duplicate. Both the sample and duplicate sample have U-metal concentrations greater than 0.1 weight $\%$, TI008-ME $(0.426$ wt. $\%)$ and TI008-MF (0.170 wt. \%). Both results are above the process quantitation limit of 0.03 weight $\% \mathrm{U}$. The variability in the results is likely due to sample inhomogeniety.

Significant Condition Adverse to Quality? Yes ___ No $\not \underline{ }$

Evaluated by:

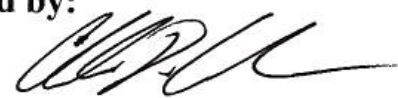

Date:<smiles>[124IH]</smiles>

\section{Comments:}



PNNL-20650, Rev. 2

Appendix D

ASR 8878 and ASR 8919 Results 



\section{Appendix D}

\section{ASR 8878 and ASR 8919 Results}

Appendix D contains the signed Analytical Services Request (ASR) 8878 and Special Instructions for the undissolved solids (UDS) of selected subsamples from the SCS-CON-210The UDS were obtained following the acid digestion conducted according to PNL-ALO-129, Rev. 0, $\mathrm{HNO}_{3}$-HCl Acid Extraction of Solids Using a Dry-Block Heater. Weighed UDS samples were fused and the fusion flux dissolved according to procedure PNL-ALO-115, Rev. 1, Solubilization of Metals from Solids Using a $\mathrm{KOH}_{-} \mathrm{KNO}_{3}$ Fusion. The resulting solutions were analyzed for gross alpha, alpha isotopes by alpha energy analysis (AEA screen), and inductively coupled plasma-optical emission spectrometry (ICP-OES).

Appendix D also includes the signed ASR 8919 Request for the GEA analysis of the spherical (resin bead fraction) and non-spherical (residual solids fraction) particle fractions separated from the merge of $-2,000-\mu \mathrm{m} /+500-\mu \mathrm{m}$ (MCC) sieve fraction samples (TI008-MK and TI008-ML).

All analytical reports generated by the Analytical Support Operations (ASO) laboratory in response to these ASRs are provided in Appendix D. Analytical reports provide sample results, associated uncertainties, and a discussion of sample processing, quality control results, compliance with QC sample requirements, and data limitations. An Occurrence Report was also provided. These was prepared when issues were identified, and resolution was required while work was in process.

The following table of contents shows that pagination specific to Appendix D was applied to the ASRs, the analytical reports, and Occurrence Report. This pagination is sequential and consistent (e.g., page D.\#).

\section{Appendix D Table of Contents}

ASR 8878 Request and Special Instructions D.3

ASR 8878 Inductively Coupled Plasma-Optical Emission Spectrometry Analytical Report. D. 14

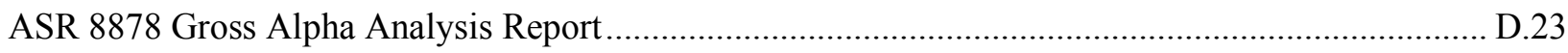

ASR 8878 Alpha Spectrometry-Total Alpha Report Analysis Report............................................ D.26

Radiochemistry Data Report—Sample Results for ASR 8878 …..................................................... D.30

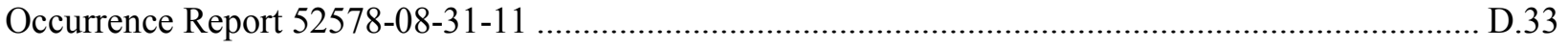

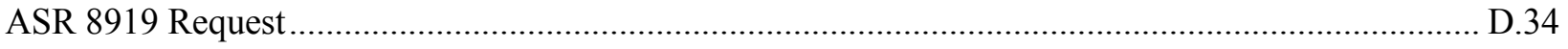

ASR 8919 Gamma Energy Analysis Narrative Report....................................................................... D.36 

Requestor --- Complete all fields on this COVER PAGE, unless specified as optional or ASR is a revision

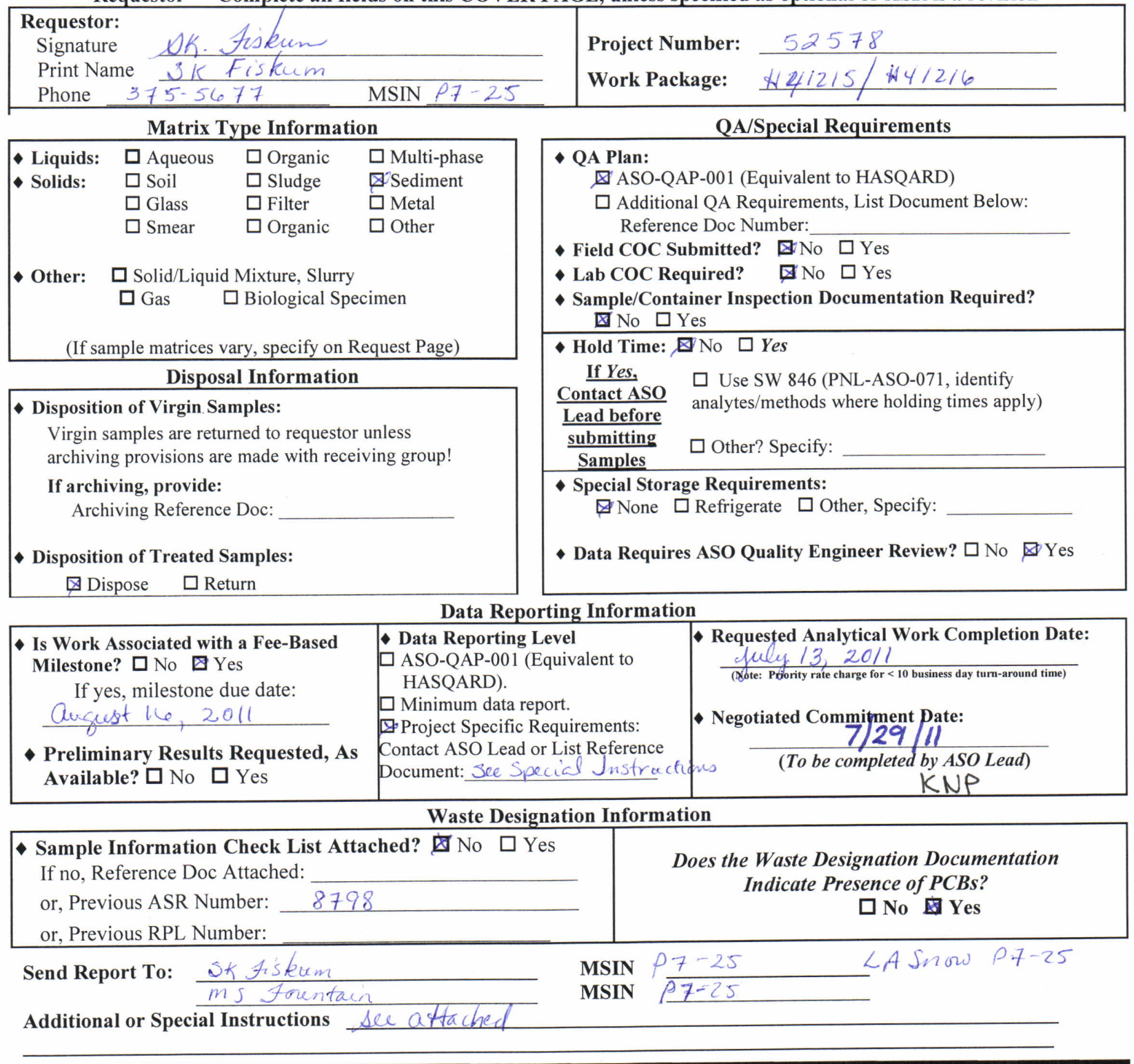

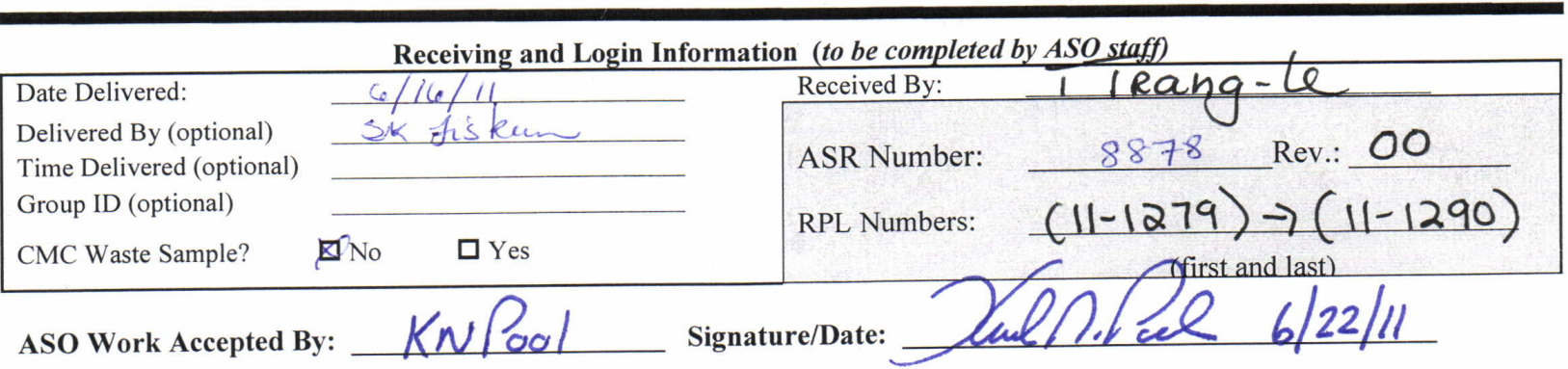


PNNL-20650, Rev. 2

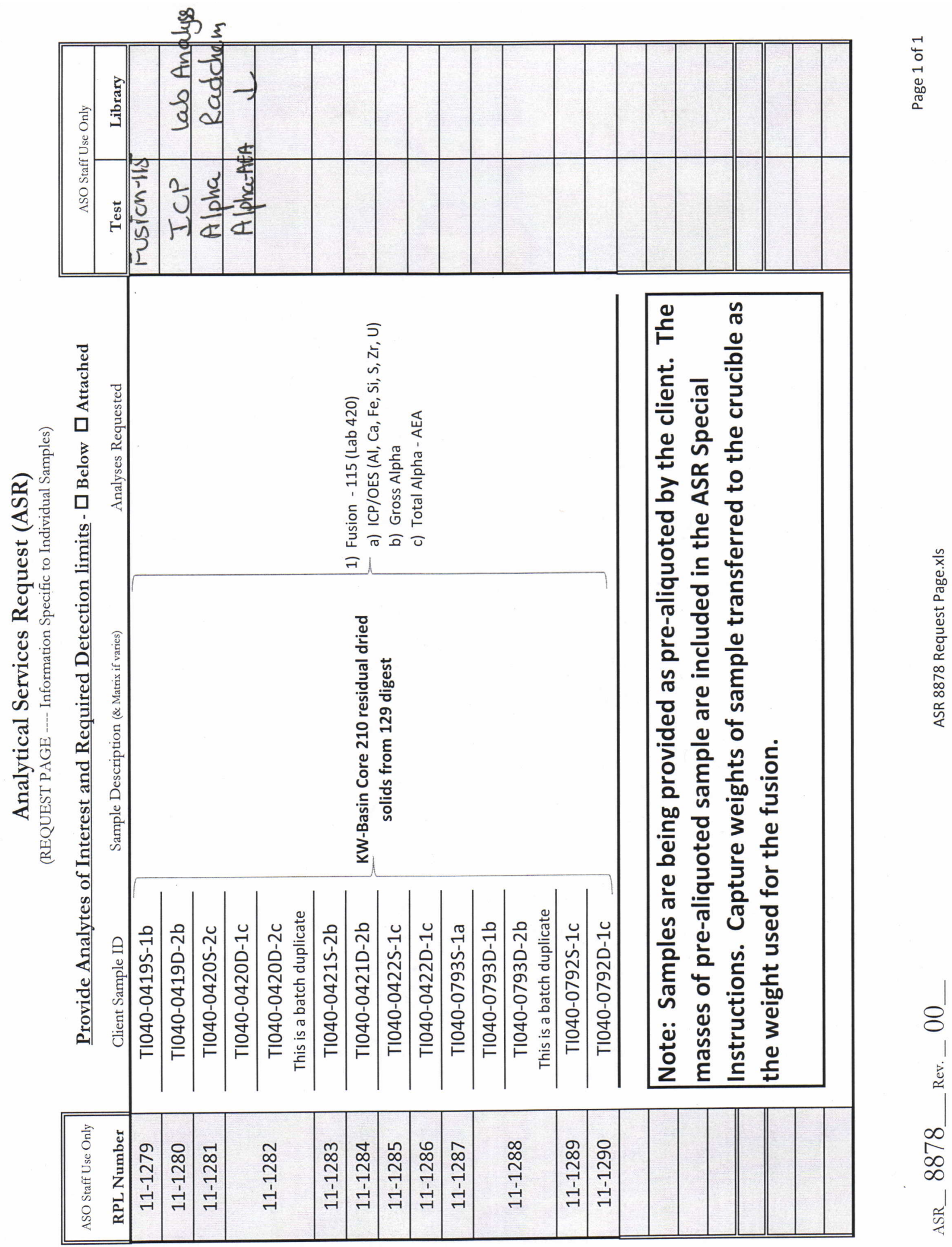


PNNL-20650, Rev. 2

\section{Special Instructions for K-Basin SCS-CON-210 Core Sample Undissolved Sludge: \\ Fusion Preparation and Analysis \\ Project 52578}

Cognizant Scientist: Uande R. Josken Date: $6 / 16 / 11$

Project Manager:

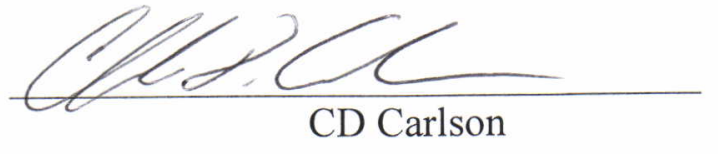

Date: $6 / 16 / 201 /$

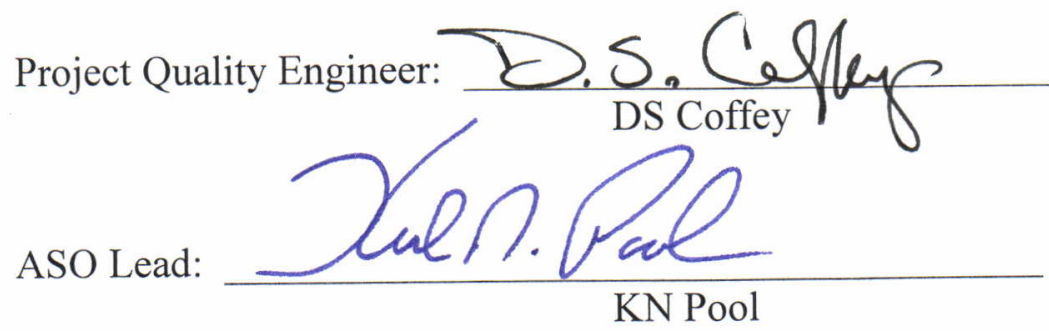

Date: $6-16 \cdot 2011$

Date: $6 / 16 / 11$

STP Concurrence:

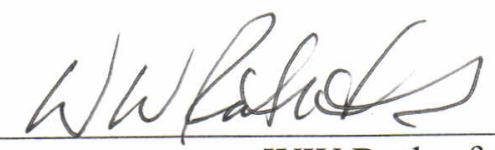

WW Rutherford
Date: $6 / 16 / 1$ 
PNNL-20650, Rev. 2

ASR 8878 Special Instructions

Page 2 of 9

\section{Special Instructions for K-Basin SCS-CON-210 Core Sample Undissolved Sludge: Fusion Preparation and Analysis Project 52578}

Cognizant Scientists:

SK Fiskum, 375-5677

MS Fountain, 375-5311

CD Carlson, 375-5334

Core and composite materials containing liquid and sludge from $\mathrm{K}$ West (KW) Basin Engineered Container 210 (i.e., SCS-CON-210) were processed for analysis using the ASO procedure PNLALO-129 acid digestion according to ASR 8798 (core samples) and ASR 8824 (container composite samples). Significant dry mass fraction of material did not dissolve. The composition of these undissolved solids (UDS) is of interest. The UDS are presented for fusion-aided dissolution followed by ICP-OES analysis, gross alpha, and alpha energy analysis (AEA) screen. The samples are provided in masses suitable for direct fusion processing (splitting conducted per Test Instruction 52578-TI040); no additional subsampling should be attempted due to the heterogenous nature of the material. The sample batch duplicates (2) have already been aliquoted. All samples probably contain significant sand and organic ion exchange resin beads.

A cross-reference of sample IDs and sample mass is provided in Table 1.

Table 1. Associated Core Sample ID, Sample ID, ASO/RPL ID, and Sample Mass CrossReference

\begin{tabular}{|c|c|c|c|c|}
\hline $\begin{array}{c}\text { Associated Core } \\
\text { Sample ID }\end{array}$ & Sample ID & ASO/RPL ID & & Sample Mass, \\
\hline \multirow{2}{*}{ KW210-A1 } & TI040-0419S-1b & $11-1279$ & & 0.1589 \\
\hline & TI040-0419D-2b & $11-1280$ & & 0.1559 \\
\hline \multirow{2}{*}{ KW210-A4 } & TI040-0420S-2c & $11-1281$ & & 0.1331 \\
\cline { 2 - 5 } & TI040-0420D-1c & $11-1282$ & & 0.1116 \\
\cline { 2 - 5 } & TI040-0420D-2c & $11-1282 \mathrm{D}$ & Batch duplicate & 0.1140 \\
\hline \multirow{2}{*}{ KW210-B1 } & TI040-0421S-2b & $11-1283$ & & 0.1554 \\
\cline { 2 - 5 } & TI040-0421D-1b & $11-1284$ & & 0.1698 \\
\hline \multirow{2}{*}{ KW210-B3 } & TI040-0422S-1c & $11-1285$ & & 0.1238 \\
\cline { 2 - 5 } & TI040-0422D-1c & $11-1286$ & & 0.1360 \\
\hline \multirow{2}{*}{ KW210-CC } & TI040-0793S-1a & $11-1287$ & & 0.1795 \\
\cline { 2 - 5 } \multirow{2}{*}{$-500 \mu \mathrm{m}$} & TI040-0793D-1b & $11-1288$ & & 0.1202 \\
\hline & TI040-0793D-2b & $11-1288 \mathrm{D}$ & Batch duplicate & 0.1124 \\
\hline \multirow{2}{*}{ KW210-CC } & TI040-0792S-1c & $11-1289$ & & 0.1485 \\
\hline & TI040-0792D-1c & $11-1290$ & & 0.1967 \\
\hline
\end{tabular}

D.6 
The following work packages apply

- Sample processing by PNL-ALO-115 fusion and ICP-OES analysis: H41215

- Radiochemistry analysis: H41216

Analytes of interest and targeted detection limits are provided in Table 3; the QAPjP/SAP KBC33786, Rev. 2 did not delineate these follow-on analyses for UDS and as such, MDLs were not specified. The quality control (QC) sample acceptance criteria are provided in Table 4; these criteria are consistent with Hanford Analytical Services Quality Assurance Requirements (HASQARD) requirements. Although a 6-month holding time was implemented for the original sample ICP-OES analysis, a holding time does not apply to these undissolved solids.

\section{Chemical Analysis Samples}

\section{Fusion}

\section{Preparation}

Fusion of the solids is to be conducted according to the ASO procedure, PNL-ALO-115, Rev. 1, Solubilization of Metals from Solids Using a $\mathrm{KOH}-\mathrm{KNO}_{3}$ Fusion. Report observations associated with the dissolution processing.

\section{$\underline{\text { ICP-OES }}$}

\section{ICP-OES Analysis}

Fusion-prepared aliquots shall be analyzed at the ICP-OES workstation according to RPG-CMC211, Rev. 3, Determination of Elemental Composition by Inductively Coupled Argon Plasma Optical Emission Spectrometry (ICP-OES).

\section{ICP-OES Reporting Units}

Report solids sample analyte concentrations as $\mu \mathrm{g} / \mathrm{g}$.

\section{$\underline{\text { Radiochemistry }}$}

Preparations and Analysis

Fusion-prepared aliquots shall be prepared according to procedures identified in Table 2. No procedure or procedure revision substitutions are to be made without prior written authorization from SK Fiskum or CD Carlson to requests submitted by the ASO Lead.

Table 2. Authorized Radiochemistry Procedures for K Basin Sludge Analysis

\begin{tabular}{|c|c|c|}
\hline Analyte & Mounting & Counting/Analysis \\
\hline Gross Alpha & $\begin{array}{c}\text { RPG-CMC-4001, Rev. 1, Source } \\
\text { Preparation for Gross Alpha and Gross } \\
\text { Beta Analysis }\end{array}$ & $\begin{array}{c}\text { RPG-CMC-408, Rev. 2, Total Alpha and } \\
\text { Beta Analysis }\end{array}$ \\
\hline AEA Screen & $\begin{array}{c}\text { RPG-CMC-496, Rev. 0, Coprecipitation } \\
\text { Mounting of Actinides for Alpha } \\
\text { Spectroscopy }\end{array}$ & $\begin{array}{l}\text { RPG-CMC-422, Rev. } 2 \text { Solutions Analysis } \\
\text { Alpha Spectrometry }\end{array}$ \\
\hline
\end{tabular}

\section{Radiochemistry Reporting Units}

Report solids sample analyte concentrations as $\mu \mathrm{Ci} / \mathrm{g}$. 
PNNL-20650, Rev. 2

\section{Archive of Prepared Chemical Analysis Samples}

The fusion preparations will result in a $\sim 50$ - to $100-\mathrm{mL}$ volume. This solution will be apportioned to the laboratory as needed to conduct work-station-specific analyses. At the time the fusion preparations are apportioned to the analysis work locations, $\sim 15-\mathrm{mL}$ aliquot from each preparation should be collected and and reserved as an archive sample. The vials need to be labeled with the following information: date, RPL-ID, sample ID, matrix, and hazard. The remaining portion of the fusion preparations may be disposed of.

\section{Quality Control}

All work is required to be conducted to the requirements of the HASQARD, Rev. 3 document. Performing work to the requirements of the ASO QA Plan (ASO-QAP-001, Rev. 7) will satisfy the HASQARD requirements. Where there is a discrepancy between the data quality objectives (DQOs) in the ASO QA Plan and these Special Instructions, the Special Instructions takes precedence as there are specific $\mathrm{K}$ Basin project requirements.

NOTE: The balance checks at the fumehood workstations shall be checked per the normal process where one calibration verification check mass is measured daily when then balance is used (PNL-ASO-052, Rev. 1).

Matrix preparative analysis QC is to include a preparation blank (PB), sample/sample duplicate (2 sample duplicates provided), and an LCS (Montana Soil, SRM 2710) as indicated in Table 4. The BS and MS for the AEA will be generated after fusion on a sample split, i.e., post-fusion spikes.

The ASO is responsible for preparing the PB and LCS. The LCS, and post-fusion BS/MS sample QC acceptance criteria are provided in Table 4.

Note: The ASO staff must inform/report QC failures promptly to the Cognizant Scientists with copy to CD Carlson and the K Basin QE (Deborah Coffey), thus enabling PNNL to fulfill its commitment to the client for prompt notification of such failures. The impact of any QC failure on data quality or project schedule (e.g., re-runs) will be assessed in conjunction with the client.

1. The preparation blank $(\mathrm{PB})$ analyte concentration shall be less than the estimated quantitation limit (EQL) or the minimum detectable activity (MDA) of the associated sample. When the $\mathrm{PB}$ concentration is equal to or exceeds the $\mathrm{EQL}$ or MDA, then the $\mathrm{PB}$ concentration shall not exceed $5 \%$ of the measured concentration present in the sample.

2. If and when it has been determined that the data are not useable, then the affected samples in the processing batch must be re-prepared and re-analyzed for the failed analytes, availability of samples permitting.

3. In the case of multi-elemental methods (ICP-OES), isolated QC failure(s) may be communicated to SK Fiskum, MS Fountain, CD Carlson and the K Basin QE by the ASO Lead for an assessment of the impact on data interpretation.

QAPjP/SAP KBC-33786, Rev. 2 does not apply precision criteria to analysis of the UDS, therefore the QAPjP/SAP DQOs for sample duplicate pairs do not apply. Precision will be calculated in terms of relative percent difference (RPD) for two sample split duplicate pairs (111282/11-1282D and 11-1288/11-1288D). 
PNNL-20650, Rev. 2

\section{Reporting}

The analytical data report shall be prepared in accordance with procedure PNL-ASO-058, Rev. 0.2, ASO Data Reporting, Section 5.3, Comprehensive Data Report. Please be sure to include action taken with respect to any identified unexpected results and discrepancies.

The Comprehensive Data Report contains three main parts; a data report cover page, a narrative, and the data summary. These elements are identified below; red text indicates project-driven scope. Black text is taken directly from the ASO procedure: PNL-ASO-058, Rev. 0.2.

Data Report Cover Page Contents:

- Header identifying name/address of laboratory

- Client Name:

- Project \#/WP \#(s):

- $\operatorname{ASR} \#(\mathrm{~s})$ :

- Total \# Samples:

- Report Date:

- Report Revision Date: when applicable

- Filename(s): where data files can be accessed

- Dates of Sample Processing and Analysis:

- Procedure(s): Number and title (sample processing and analysis) and revision number

- M\&TE (serial number or property number):

- Reference Date (when applicable):

- Client ID and RPL ID Sample Numbers:

- Report Review and Approval:

Preparer Date

(Printed name and signature of responsible individual)

Technical Reviewer Date

(Printed name and signature of technical reviewer)

A case narrative describing the analysis, limitations on the analysis results, and QA/QC issues

Narrative Contents:

- Sample Description and Analyses

- Sample dissolution/preparation dates

- Sample Preparation

- Analysis dates

- Deviations from the written procedure, if any

- Method specific Sample Preparation, Separation, Mounting and Counting Methods as applicable

- Reporting Basis/Units

- QC Criteria/Results

- Limitations of the Data Interferences/Resolution Uncertainty

- Comments

\section{Data Summary Contents:}

- Client ID

- RPL ID with full ASO-related suffixes (e.g., 115-S)

- Results and result uncertainties 
PNNL-20650, Rev. 2

The following elements may be included in the final report or be traceable to the test results (usually by annotation on bench sheets) and be maintained as lifetime records:

- identification of standards used

- identification of M\&TE used

- records of daily check weight tracking

- signature and date of person who performed the test and recorded the data

- analytical bench sheets

- hand calculation review documentation.

Technical reviewers are required to be staff other than the analyst performing the work and knowledgeable of the area being reviewed. The technical review shall be completed and all reviewer issues resolved before reporting final results. Technical reviewers must receive a complete data package including narratives for review. A technical review consists of the following elements:

- evaluation of method and QC performance

- evaluation of compliance with technical (including procedural) and QC requirements, as defined in the ASR

- verification of transcription accuracy

- check for correctness of calculations

- assuring the correct reporting units (and reference dates) are used

- evaluation of overall consistency and reasonableness of data

- evidence of implementation of appropriate corrective action, when necessary.

Analytical results shall be reported both in hard copy and electronically (including excel data files to support accurate data transcription into project records). Appropriately marked "Preliminary" data reports and electronic files shall be provided by the ASO Lead as soon as practical after completion of analysis. The final ASR data report shall be provided no later than the commitment date on the ASR. 
PNNL-20650, Rev. 2

Table 3. Target Method Detection Limits for Solids

\begin{tabular}{|c|c|c|}
\hline \multirow[t]{2}{*}{ Analyte } & Solids & Analysis Method \\
\hline & $\mu \mathrm{Ci} / \mathrm{g}$ & \\
\hline Total Alpha & $1.0 \mathrm{E}-03$ & Gross Alpha \\
\hline${ }^{241} \mathrm{Am} /{ }^{238} \mathrm{Pu}$ & $1.0 \mathrm{E}-03$ & \\
\hline${ }^{239+240} \mathrm{Pu}$ & $1.0 \mathrm{E}-03$ & AEA Screen \\
\hline \multicolumn{3}{|c|}{ Identify and quantify any other spectral peak } \\
\hline \multicolumn{3}{|c|}{\begin{tabular}{l|l}
$\mu \mathrm{g} / \mathrm{g}$ \\
\end{tabular}} \\
\hline $\mathrm{Al}$ & $3.0 \mathrm{E}+03$ & \\
\hline $\mathrm{Ca}$ & $3.0 \mathrm{E}+03$ & ' \\
\hline $\mathrm{Fe}$ & $1.0 \mathrm{E}+03$ & \\
\hline S & $1.0 \mathrm{E}+03$ & ICP-OES $^{(\mathrm{a})}$ \\
\hline $\mathrm{Si}$ & $3.0 \mathrm{E}+03$ & \\
\hline $\mathrm{Zr}$ & $2.0 \mathrm{E}+02$ & \\
\hline $\mathrm{U}$ & $1.0 \mathrm{E}+03$ & \\
\hline \multicolumn{3}{|c|}{$\begin{array}{l}\text { (a) Selected analytes were measured in the parent sample at }>5,000 \mu \mathrm{g} / \mathrm{g} \text { (dry mass basis). } \\
\text { (b) The targeted MDL is based on the fusion flux matrix adjusted instrument detection limit } \\
\text { (IDL) multiplied by a nominal process factor of 5,000. The MDL is intended to be a guide to } \\
\text { the analytical staff when selecting aliquot sizes and conducting dilutions. It is understood that } \\
\text { matrix effects may confound the achieved MDL. }\end{array}$} \\
\hline
\end{tabular}


PNNL-20650, Rev. 2

ASR 8878 Special Instructions

Page 8 of 9

Table 4. Analytical Quality Control Sample Acceptance Criteria

\begin{tabular}{|c|c|c|c|}
\hline \multirow{2}{*}{ Analyte } & \multirow{2}{*}{ Analytical Technique } & \multicolumn{2}{|c|}{ LCS \% Recovery ${ }^{(\mathrm{a})}$} \\
\hline & & \multicolumn{2}{|c|}{ Pre-digestion spike } \\
\hline $\begin{array}{c}\mathrm{Al}, \mathrm{Fe}, \mathrm{Si}, \mathrm{Ca} \text { in } \\
\text { Montana I Soil } \\
\text { SRM } 2710 \\
\end{array}$ & ICP-OES & \multicolumn{2}{|c|}{$80-120 \%$} \\
\hline \multirow{2}{*}{ Analyte } & \multirow{2}{*}{ Analytical Technique } & BS \% Recovery ${ }^{(a)}$ & MS \% Recovery ${ }^{(b)}$ \\
\hline & & \multicolumn{2}{|c|}{ Post-digestion spike } \\
\hline $\begin{array}{c}\text { Gross Alpha } \\
\text { (spike material is }{ }^{239} \mathrm{Pu} \text { ) }\end{array}$ & Alpha scintillation & $80-120 \%$ & $75-125 \%$ \\
\hline $\begin{array}{c}\text { AEA Screen } \\
\text { (spike material is }{ }^{239} \mathrm{Pu} \text { ) }\end{array}$ & AEA & $80-120 \%$ & $75-125 \%$ \\
\hline
\end{tabular}

N/A - not applicable

Footnotes:

(a) LCS = Laboratory Control Standard; BS = Blank Spike. A laboratory control sample (LCS) or blank spike (BS) samples are used to monitor the effectiveness of the sample preparation process and are good indicators of method accuracy. Ideally, the LCS is a material similar to the sample being processed, containing the analytes of interest (e.g., standard reference material). An LCS, if available, shall be prepared with each batch of samples processed at the same time. When an appropriate LCS is not available, a BS shall be used in lieu of the LCS. A BS is distilled or deionized water or another suitable matrix spiked with the analytes of interest. It may not be possible to prepare a single BS that contains all analytes of interest (e.g., chemical incompatibility). In such cases, an agreement with the client shall be made to identify the analytes of interest used to prepare the BS, and more than one BS may be used. The BS and AS results are expressed as percent recovery; i.e., the amount measured, divided by the known concentration, multiplied by 100 .

(b) MS = matrix spike. For some methods, the sample accuracy is expressed as the percent recovery of a matrix spike (MS) or analytical spike (AS) sample. Post-digestion spikes and analytical spikes are also included under these acceptance criteria. The spiked sample result is expressed as percent recovery; i.e., the amount measured less the amount in the sample, divided by the spike added, times 100. One MS (or post spike or analytical spike [AS]) is performed per analytical batch. Samples are batched with similar matrices. For ICP analytes, the accuracy can also be determined based on use of serial dilutions. In cases where the ICP-OES MS concentration is low relative to the sample analyte concentration (resulting in meaningless recovery calculations), matrix effects will be evaluated from a post-digestion spike. 
PNNL-20650, Rev. 2

ASR 8878 Special Instructions

Page 9 of 9

\section{References}

52578-TI040, Rev. 0, Processing of the Undissolved Solids Samples from the K West Basin Core Samples Collected from SCS-CON-210 for Additional Analysis

ASO-QAP-001, Rev. 7, Analytical Support Operations Quality Assurance Plan

PNL-ASO-052, Rev. 1, Balance Performance Checks

PNL-ASO-058, Rev. 0.2, ASO Data Reporting

PNL-ALO-115, Rev. 1, Solubilization of Metals from Solids Using a $\mathrm{KOH}-\mathrm{KNO}_{3}$ Fusion

PNL-ALO-129, Rev. 0, $\mathrm{HNO}_{3}$-HCl Acid Extraction of Solids Using a Dry-Block Heater

RPG-CMC-211, Rev. 3, Determination of Elemental Composition by Inductively Coupled Argon Plasma Optical Emission Spectrometry (ICP-OES)

RPG-CMC-408, Rev. 2, Total Alpha and Beta Analysis

RPG-CMC-422, Rev. 2, Solutions Analysis: Alpha Spectrometry

RPG-CMC-496, Rev. 0, Coprecipitation Mounting of Actinides for Alpha Spectroscopy

RPG-CMC-4001, Rev. 1, Source Preparation for Gross Alpha and Gross Beta Analysis

Hanford Analytical Services Quality Assurance Requirements Documents (HASQARD), 2007, DOE/RL-96-68, Revision 3, Department of Energy Richland Office, Richland, Washington

KBC-33786, Rev. 2, Baker RB, JL Westcott, TL Welsh, JA Pottmeter, and AJ Schmidt. 2009 Quality Assurance Project Plan/Sampling and Analysis Plan for Sludge in the KW

Engineered Containers. CH2M Hill Plateau Remediation, Company, Richland, Washington. 
Battelle PNNL/RPL/Inorganic Analysis ... ICP-OES Analysis Report PO Box 999, Richland, Washington 99352

Project / WP\#: $\quad 52578$ / H41215

ASR\#: $\quad 8878$

Client: $\quad$ S. Fiskum

Total Samples: 14 (solids)

\begin{tabular}{|c|c|c|}
\hline & First & Last \\
\hline RPL\#: & $11-1279$ & $11-1290$ \\
\hline Client ID: & TI040-0419S-1b & TI040-0792D-1c \\
\hline
\end{tabular}

Procedure: $\quad$ RPG-CMC-211, Rev. 3, "Determination of Elemental Composition by Inductively Coupled Argon Plasma Optical Emission Spectrometry (ICP-OES)",.

Analyst: J. Carter

Analysis Date (File): $\quad \underline{07-29-2011}$ (C0399)

08-01-2011 (C0400)

See Chemical Measurement Center 98620 file: $\quad \underline{\text { ICP-325-405-3 }}$

(Calibration and Maintenance Records)

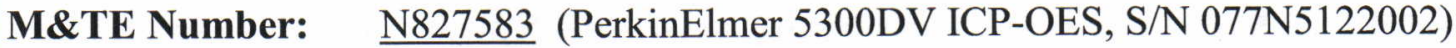

$\underline{\text { WD66158 }}$ (Sartorius ME414S Balance, S/N 224066373)
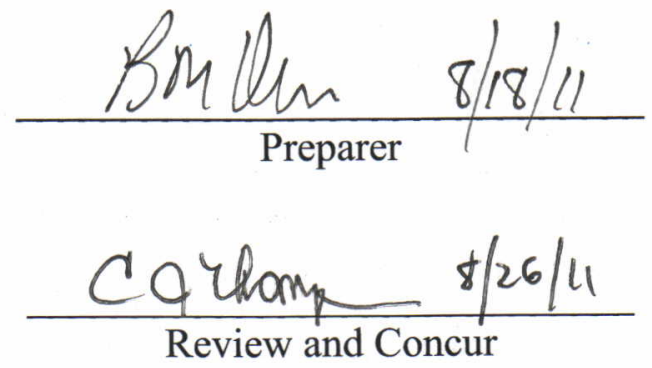


\section{Battelle PNNL/RPL/Inorganic Analysis ... ICP-OES Analysis Report}

Fourteen samples were provided for ICP-OES analysis under Analytical Service Request (ASR) 8878. The samples were residual dried solids from an earlier digestion preparation. Each of the samples was processed in the Sample Receiving and Preparation Laboratory (SRPL) following RPL procedure PNL-ALO-115 using $\sim 0.1$ gram of sample with dilution to a final volume of 100 $\mathrm{mL}$.

Analytes of interest (AOIs) were specified in the ASR, and are listed in the upper section of the attached ICP-OES Data Report. The quality control (QC) results for these AOIs have been evaluated and are presented below. Analytes other than AOIs are reported in the bottom section of the report, but have not been fully evaluated for QC performance. The results for $\mathrm{Sr}$ are not reported because of issues with the instrument calibration for that analyte.

The results are reported on a mass basis $(\mu \mathrm{g} / \mathrm{g})$ and have been adjusted for laboratory processing factors and instrument dilutions. Minimum Method Detection Limit (MDL) values were specified in the ASR. MDL levels were met for all AOIs.

Calibration of the ICP-OES was done following the manufacturer's recommended calibration procedure and using multi-analyte custom standard solutions traceable to the National Institute of Standards and Technology (NIST). Midrange calibration verification standards (MCVA and MCVB) were used to verify acceptance of the two-point calibration curves obtained for each analyte, and also used for continuing calibration verification.

The controlling documents were ASO-QAP-001 and ASR-8878 Special Instructions. Instrument calibrations, QC checks and blanks (e.g., ICV/ICB, CCV/CCB, LLS, ICS), blank spike, post spike, duplicates, and serial dilution were conducted during the analysis run. The blank spike was prepared using NIST SRM-2710 (Montana Soil).

\section{Preparation Blank (PB):}

A preparation blank (reagents only) was prepared for the extraction process. The concentrations of all AOIs were within the acceptance criteria of $\leq \mathrm{EQL}$ (estimated quantitation level) or less than $\leq 5 \%$ of the concentration in the sample.

\section{Blank Spike (BS)/Laboratory Control Sample (LCS):}

An LCS was prepared for the extraction process. Recovery values are listed for all analytes included in the spike that were measured at or above the EQL. Recovery values for AOIs meeting this requirement ranged from 95 to $107 \%$, and were within the acceptance criterion of $80 \%$ to $120 \%$.

\section{Matrix-Spiked Sample:}

No matrix spike was prepared for the extraction process. 


\section{Battelle PNNL/RPL/Inorganic Analysis ... ICP-OES Analysis Report}

\section{Duplicate Relative Percent Difference (RPD):}

Duplicates were prepared for two of the samples (11-1282 and 11-1288). RPDs are listed for all analytes that were measured at or above the EQL. RPDs for AOIs meeting this requirement ranged from 2.6 to $20.6 \%$. Per the ASR, no acceptance criterion are specified for the duplicate RPDs.

\section{Post-Spike/Analytical Spike Sample (A Component):}

Post-spike (A Component) analyses were performed on Samples 11-1279 and 11-1285. Recovery values are listed for all analytes in the spike that were measured at or above the $E Q L$, and that had a spike concentration $\geq 25 \%$ of that in the sample. Recovery values for AOIs meeting these requirements ranged from 99 to $119 \%$, and were within the acceptance criterion of $80 \%$ to $120 \%$.

\section{Post Spike/Analytical Spike Sample (B Component):}

Post-spike (B Component) analyses were performed on Samples 11-1279 and 11-1285. Recovery values are listed for all analytes in the spike that were measured at or above the EQL, and that had a spike concentration $\geq 25 \%$ of that in the sample. Recovery values for AOIs meeting these requirements ranged from 96 to $107 \%$, and were within the acceptance criterion of $80 \%$ to $120 \%$.

\section{Serial dilution:}

Five-fold serial dilution was performed on Samples 11-1279 and 11-1285. Percent differences $(\% \mathrm{Ds})$ are listed for all analytes that had a concentration at or above the EQL in the diluted sample. The $\%$ Ds for AOIs meeting this requirement ranged from 0.5 to $6.1 \%$, and were within the acceptance criterion of $\leq 10 \%$.

\section{Other QC:}

Silicon was outside the acceptance range for two of the CCV's, and was the result of carryover from a previous HF rinse applied to the system nebulizer and quartz spray chamber as part of in-process preventative maintenance. After a suitable rinse-out period, the Si fell within the acceptance criterion. All other instrument-related QC tests for the AOIs passed within the appropriate acceptance criteria.

\section{Comments:}

1) The "Final Results" have been corrected for all laboratory dilutions performed on the samples during processing and analysis, unless specifically noted.

2) Instrument detection limits (IDL) and estimated quantitation limits (EQL) shown are for acidified water and/or fusion flux matrices as applicable. Detection limits for other matrices may be determined if requested. Method detection limits (MDL) can be estimated by multiplying the IDL by the "Multiplier". The estimated quantitation limit (EQL) for each concentration value can be obtained by multiplying the EQL by the "Multiplier".

3) Routine precision and bias is typically $\pm 15 \%$ or better for samples in dilute, acidified water (e.g. $2 \% \mathrm{v} / \mathrm{v}$ $\mathrm{HNO}_{3}$ or less) at analyte concentrations $>E Q L$ up to the upper calibration level. This also presumes that the total dissolved solids concentration in the sample is less than $5000 \mu \mathrm{g} / \mathrm{mL}(0.5$ per cent by weight). Note that bracketed values listed in the data report are within the MDL and the EQL, and have potential uncertainties greater than $15 \%$. Concentration values $<$ MDL are listed as ".$- "$. Note, that calibration and QC standards are validated to a precision of $\pm 10 \%$. 


\section{Battelle PNNL/RPL/Inorganic Analysis ... ICP-OES Analysis Report}

4) Absolute precision, bias and detection limits may be determined on each sample if required by the client. The maximum number of significant figures for all ICP measurements is two.

5) Analytes included in the spike $\mathrm{A}$ component (for the $\mathrm{AS} / \mathrm{PS}$ ) are; $\mathrm{Ag}, \mathrm{Al}, \mathrm{As}, \mathrm{B}, \mathrm{Ba}, \mathrm{Be}, \mathrm{Bi}, \mathrm{Ca}, \mathrm{Cd}, \mathrm{Co}, \mathrm{Cr}$, $\mathrm{Cu}, \mathrm{Fe}, \mathrm{K}, \mathrm{Li}, \mathrm{Mg}, \mathrm{Mn}, \mathrm{Mo}, \mathrm{Na}, \mathrm{Ni}, \mathrm{P}, \mathrm{Pb}, \mathrm{Sb}, \mathrm{Se}, \mathrm{Si}, \mathrm{Sn}, \mathrm{Sr}, \mathrm{Ta}, \mathrm{Ti}, \mathrm{Tl}, \mathrm{V}, \mathrm{W}, \mathrm{Y}, \mathrm{Zn}$, and Zr. Analytes included in the spike B component are; Ce, Dy, Eu, La, Nd, Pd, Rh, Ru, S, Te, Th, and U. 
PNNL-20650, Rev. 2

Battelle PNNL/RPG/Inorganic Analysis ... ICPOES Data Report

\begin{tabular}{|c|c|c|c|c|c|c|}
\hline & Run Date > & $7 / 29 / 2011$ & $7 / 29 / 2011$ & $7 / 29 / 2011$ & $7 / 29 / 2011$ \\
\hline & & Multiplier > & 3518.6 & 3148.6 & 3205.1 & 3782.1 \\
\hline & & RPL/LAB > & \begin{tabular}{|c} 
BLK-1279 @ \\
$5 x$ \\
\end{tabular} & \begin{tabular}{|c|}
$11-1279 @$ \\
$5 x$
\end{tabular} & \begin{tabular}{|c|}
$11-1280 @$ \\
$5 x$
\end{tabular} & $\begin{array}{c}11-1281 @ \text { @x } \\
\end{array}$ \\
\hline \multirow{2}{*}{$\begin{array}{l}\text { Instr. Det. } \\
\text { Limit (IDL) }\end{array}$} & Est. Quant. & & & T1040-0419S & TI040-0419D & $\underline{T 1040-0420 \mathrm{~S}}$ \\
\hline & Limit (EQL) & Client ID > & Prep Blank & $\underline{1 \mathrm{~b}}$ & $\underline{2 b}$ & $\underline{2 c}$ \\
\hline$(\mu \mathrm{g} / \mathrm{mL})$ & $(\mu \mathrm{g} / \mathrm{mL})$ & (Analyte) & $(\mu \mathrm{g} / \mathrm{g})$ & $(\mu \mathrm{g} / \mathrm{g})$ & $(\mu \mathrm{g} / \mathrm{g})$ & $(\mu \mathrm{g} / \mathrm{g})$ \\
\hline 0.4064 & 4.064 & Al & $\cdots$ & 20,100 & 16,400 & 22,200 \\
\hline 0.4345 & 4.345 & $\mathrm{Ca}$ & $\cdots$ & {$[3,400]$} & {$[2,100]$} & {$[2,700]$} \\
\hline 0.1614 & 1.614 & $\mathrm{Fe}$ & -- & 16,500 & 16,000 & 7,080 \\
\hline 0.1356 & 1.356 & $\mathbf{s}$ & - & 16,400 & 13,900 & 16,700 \\
\hline 0.4424 & 4.424 & Si & $\cdots$ & 276,000 & 268,000 & 350,000 \\
\hline 0.0088 & 0.176 & $\mathbf{U}$ & -- & 7,840 & 7,370 & 1,920 \\
\hline 0.0109 & 0.109 & $\mathrm{Zr}$ & -. & 694 & 579 & 623 \\
\hline
\end{tabular}

Other Analytes

\begin{tabular}{|c|c|c|c|c|c|c|}
\hline 0.0014 & 0.014 & $\mathrm{Ag}$ & {$[10.0]$} & -- & -- & - \\
\hline 0.0730 & 0.730 & $\mathrm{As}$ & -- & -- & -- & - \\
\hline 0.0046 & 0.046 & $\mathrm{~B}$ & {$[27]$} & {$[52]$} & {$[110]$} & {$[42]$} \\
\hline 0.0078 & 0.078 & $\mathrm{Ba}$ & -- & 656 & 511 & 840 \\
\hline
\end{tabular}

\begin{tabular}{|c|c|c|c|c|c|c|}
\hline 0.0078 & 0.078 & Ba & $=$ & 656 & 511 & 840 \\
\hline 0.0001 & 0.001 & $\mathrm{Be}$ & $=$ & {$[0.32]$} & {$[0.22]$} & -- \\
\hline 0.0120 & 0.120 & $\mathrm{Bi}$ & -- & -- & - & -- \\
\hline
\end{tabular}

\begin{tabular}{|l|l|l|l|l|l|c|}
\hline 0.0120 & 0.120 & $\mathrm{Bi}$ & - & -- & -- & - \\
\hline 0.0022 & 0.022 & $\mathrm{Cd}$ & -- & -- & -- & {$[63]$} \\
\hline 0.0066 & 0.066 & $\mathrm{Ce}$ & -- & -- & -- & - \\
\hline
\end{tabular}

\begin{tabular}{|c|c|c|c|c|c|c|}
\hline 0.0066 & 0.066 & $\mathrm{Ce}$ & -- & -- & -- & -- \\
\hline 0.0027 & 0.027 & Co & [32] & [14] & -- & -- \\
\hline 0.0016 & 0.016 & $\mathrm{Cr}$ & {$[6.0]$} & [40] & [35] & [19] \\
\hline
\end{tabular}

\begin{tabular}{l|l|c|c|c|c|c|}
\hline 0.0016 & 0.016 & $\mathrm{Cr}$ & {$[6.0]$} & {$[40]$} & {$[35]$} & {$[19]$} \\
\hline 0.0543 & 0.543 & $\mathrm{Cu}$ & -- & -- & - & - \\
\hline 0.0014 & 0.014 & $\mathrm{Dy}$ & - & - & -- & \\
\hline
\end{tabular}

\begin{tabular}{|c|c|c|c|c|c|c|}
\hline & & & & & & \\
\hline 0.0014 & 0.014 & Dy & -- & - & - & - \\
\hline 0.0004 & 0.004 & $\mathrm{Eu}$ &.- & [2.1] & [2.1] & 17.9 \\
\hline 0.0000 & 0.000 & K & na & na & na & na \\
\hline
\end{tabular}

\begin{tabular}{|c|c|c|c|c|c|c|}
\hline 0.0000 & 0.000 & $\boldsymbol{K}$ & na & na & na & na \\
\hline 0.0017 & 0.017 & La & -. & 174 & 232 & 369 \\
\hline 0.0003 & 0.003 & $\mathbf{L i}$ & -- & 9.45 & -- & - \\
\hline
\end{tabular}

\begin{tabular}{l|l}
\hline 0.0003 & 0.003 \\
\hline 0.0150 & 0.150
\end{tabular}

\begin{tabular}{|c|c|c|c|c|c|c|}
\hline 103 & 0003 & Ii & & 9.45 & & \\
\hline 0.0150 & 0.150 & $\mathrm{Ma}$ & -. & 1540 & 1100 & 1.090 \\
\hline 0 & 01 & 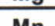 & - & . & 1 & \\
\hline
\end{tabular}

\begin{tabular}{|c|c|c|c|c|c|c|}
\hline 0.0150 & 0.150 & $\mathrm{Mg}$ & - & 1,540 & 1,100 & 1,090 \\
\hline 0.0166 & 0.166 & $\mathrm{Mn}$ & -- & {$[92]$} & {$[62]$} & -- \\
\hline
\end{tabular}

\begin{tabular}{|c|c|c|c|c|c|c|}
\hline 0.0166 & 0.166 & Mn & -- & [92] & [62] & - \\
\hline 0.0063 & 0.063 & Mo & -- & -- & -- & -. \\
\hline 2.0840 & 20.840 & $\mathrm{Na}$ & -- & -- & -- & -- \\
\hline
\end{tabular}

\begin{tabular}{|c|c|c|c|c|c|c|}
\hline & & & & & & \\
\hline 2.0840 & 20.840 & $\mathrm{Na}$ & -- & - & - & - \\
\hline 0.0041 & 0.041 & Nd & -. & -- & -- & -- \\
\hline
\end{tabular}

\begin{tabular}{c|c|c|c|}
0.0041 & 0.041 & $\mathrm{Nd}$ & -- \\
\hline 0.0000 & 0.000 & $\mathrm{Ni}$ & na \\
\hline
\end{tabular}

\begin{tabular}{|c|c|c|c|c|c|c|}
\hline & & & & & & \\
\hline 0.0000 & 0.000 & $\mathrm{Ni}$ & na & na & na & na \\
\hline 0.0690 & 0.690 & $\mathbf{P}$ & - & -- & - & -- \\
\hline
\end{tabular}

\begin{tabular}{|c|c|c|c|c|c|c}
0.0690 & 0.690 & $\mathrm{P}$ & -- & -- & -- & -- \\
\hline 0.0603 & 0.603 & $\mathrm{~Pb}$ & -- & -- & -- & -- \\
\hline 0.0052 & 0.052 & $\mathrm{Pd}$ & -- & -- & -- & -- \\
\hline 0.0098 & 0.098 & $\mathrm{Rh}$ & -- & -- & -- & -- \\
\hline
\end{tabular}

\begin{tabular}{|c|c|c|c|c|c|c|}
\hline 0.0052 & 0.052 & Pd & -- & - & -- & - \\
\hline 0.0098 & 0.098 & $\mathbf{R h}$ & - & - & - & - \\
\hline 0.0066 & 0.066 & $\mathbf{R u}$ & - & - & - & - \\
\hline 0.0340 & 0.340 & $\mathrm{Sb}$ & - & - & - & - \\
\hline 0.2400 & 2.400 & $\mathrm{Se}$ & - & - & - & - \\
\hline 0.0240 & 0.240 & Sn & - & - & - & - \\
\hline 0.0019 & 0.019 & $\mathrm{Sr}$ & - & 89.8 & 78.5 & 111 \\
\hline 0.0200 & 0.200 & $\mathrm{Ta}$ & - & - & $-\cdot$ & $\cdots$ \\
\hline 0.0160 & 0.160 & $\mathrm{Te}$ & - & - & {$[60]$} & [65] \\
\hline 0.0028 & 0.028 & Th & -- & - & -- & $-\cdot$ \\
\hline 0.0035 & 0.035 & $\mathrm{Ti}$ & - & 2,370 & 1,940 & 716 \\
\hline 0.0460 & 0.460 & TI & -- & - & -. & - \\
\hline 0.0007 & 0.021 & v & [3.5] & {$[8.6]$} & [6.7] & [15] \\
\hline 0.0190 & 0.190 & $\mathbf{w}$ & -- & -- & -- & -- \\
\hline 0.0002 & 0.002 & $\mathbf{Y}$ & - & [4.5] & [2.2] & [2.7] \\
\hline 0.1315 & 1.315 & $\mathrm{Zn}$ & -- & -. & -- & 9,160 \\
\hline
\end{tabular}

1) "--" indicates the value is $<M D L$. The method detection limit $(M D L)=I D L$ times the "multiplier" near the top of each column. The estimated sample quantitation limit $=E Q L$ (in Column 2) times the "multiplier". Overall error for values $\geq E Q L$ is estimated to be within $\pm 15 \%$. 2) Values in brackets [] are $\geq M D L$ but $<E Q L$, with errors likely to exceed $15 \%$.

na = not applicable; $\mathrm{KOH}$ flux and Ni crucible or $\mathrm{Na}_{2} \mathrm{O}_{2}$ flux and $\mathrm{Zr}$ crucible for fusion preparations, or Si for HF assisted digests. 
PNNL-20650, Rev. 2

Battelle PNNL/RPG/Inorganic Analysis ... ICPOES Data Report

Page 2 of 2

\begin{tabular}{|c|c|c|c|c|c|c|}
\hline Criteria > & $\leq 20 \%$ & $80 \%-120 \%$ & $80 \%-120 \%$ & $80 \%-120 \%$ & $80 \%-120 \%$ & $\leq 10 \%$ \\
\hline QC ID > & Dup (none) & LCS/BS & MS (none) & $\begin{array}{c}11-1279+ \\
\text { AS-A }\end{array}$ & $\begin{array}{c}11-1279+ \\
\text { AS-B }\end{array}$ & $\begin{array}{c}11-1279 \\
5-\text { fold } \\
\text { Serial Dil }\end{array}$ \\
\hline Analytes & RPD (\%) & \%Rec & $\% \operatorname{Rec}$ & $\% \operatorname{Rec}$ & \%Rec & $\%$ Diff \\
\hline Al & & 95 & & 104 & & \\
\hline $\mathrm{Ca}$ & & [99] & & 100 & & \\
\hline $\mathrm{Fe}$ & & 97 & & 99 & & \\
\hline $\mathbf{s}$ & & & & & 98 & \\
\hline Si & & 107 & & 101 & & 0.5 \\
\hline U & & & & & 96 & 6.1 \\
\hline $\mathrm{Zr}$ & & & & 102 & & \\
\hline \multicolumn{7}{|c|}{ Other Analytes } \\
\hline $\mathrm{Ag}$ & & & & 91 & & \\
\hline As & & & & 100 & & \\
\hline B & & & & 99 & & \\
\hline $\mathrm{Ba}$ & & 93 & & 92 & & \\
\hline $\mathrm{Be}$ & & & & 101 & & \\
\hline $\mathrm{Bi}$ & & & & 92 & & \\
\hline Cd & & & & 106 & & \\
\hline $\mathrm{Ce}$ & & & & & 96 & \\
\hline Co & & & & 97 & & \\
\hline $\mathrm{Cr}$ & & & & 102 & & \\
\hline $\mathrm{Cu}$ & & 99 & & 106 & & \\
\hline Dy & & & & & 100 & \\
\hline $\mathrm{Eu}$ & & & & & 97 & \\
\hline$K$ & & na & na & na & na & na \\
\hline La & & & & & 100 & \\
\hline Li & & & & 102 & & \\
\hline $\mathrm{Mg}$ & & 96 & & 101 & & \\
\hline $\mathrm{Mn}$ & & 100 & & 100 & & \\
\hline Mo & & & & 97 & & \\
\hline $\mathrm{Na}$ & & & & 97 & & \\
\hline $\mathrm{Nd}$ & & & & & 94 & \\
\hline $\mathrm{Ni}$ & & na & na & na & na & na \\
\hline $\mathbf{P}$ & & & & 100 & & \\
\hline $\mathrm{Pb}$ & & 97 & & 100 & & \\
\hline $\mathbf{P d}$ & & & & & 89 & \\
\hline $\mathbf{R h}$ & & & & & 99 & \\
\hline $\mathbf{R u}$ & & & & & 93 & \\
\hline Sb & & & & 96 & & \\
\hline $\mathrm{Se}$ & & & & 101 & & \\
\hline Sn & & & & 96 & & \\
\hline $\mathrm{Sr}$ & & 97 & & 103 & & \\
\hline $\mathrm{Ta}$ & & & & 99 & & \\
\hline $\mathrm{Te}$ & & & & & 98 & \\
\hline Th & & & & & 95 & \\
\hline $\mathrm{Ti}$ & & 93 & & 100 & & 2.5 \\
\hline $\mathrm{TI}$ & & & & 91 & & \\
\hline V & & & & 97 & & \\
\hline W & & & & 101 & & \\
\hline$Y$ & & 84 & & 99 & & \\
\hline $\mathrm{Zn}$ & & 100 & & 100 & & \\
\hline
\end{tabular}

Shaded results arè outside the acceptance criteria.

$n r=$ spike concentration less than $25 \%$ of sample concentration. Matrix effects can be assessed from the serial dilution.

$n a=$ not applicable; $\mathrm{KOH}$ flux and Ni crucible or $\mathrm{Na}_{2} \mathrm{O}_{2}$ flux and $\mathrm{Zr}$ crucible for fusion preparations, or Si for HF assisted digests. 
PNNL-20650, Rev. 2

Battelle PNNL/RPG/Inorganic Analysis ... ICPOES Data Report

Page 1 of 3

\begin{tabular}{|c|c|c|c|c|c|c|c|c|c|}
\hline & & Run Date > & $8 / 1 / 2011$ & $8 / 1 / 2011$ & $8 / 1 / 2011$ & $8 / 1 / 2011$ & $8 / 1 / 2011$ & $8 / 1 / 2011$ & $8 / 1 / 2011$ \\
\hline & & Multiplier > & 4533.1 & 4456.3 & 3217.5 & 2944.6 & 4061.7 & 3684.6 & 2796.4 \\
\hline & & RPL/LAB > & \begin{tabular}{|c|}
$11-1282 @$ \\
$5 x$ \\
\end{tabular} & $\begin{array}{c}\text { DUP-1282 } \\
@ 5 \times \\
\end{array}$ & $\begin{array}{c}11-1283 @ \\
5 x\end{array}$ & \begin{tabular}{|c|}
$11-1284 @$ \\
$5 x$ \\
\end{tabular} & \begin{tabular}{|c|}
$11-1285 @$ \\
$5 x$
\end{tabular} & $\begin{array}{c}11-1286 @ \\
5 x \\
\end{array}$ & \begin{tabular}{|c|}
$11-1287 @$ \\
$5 x$ \\
\end{tabular} \\
\hline Instr. Det. & Est. Quant. & & T1040-0420D & TI $040-0420 \mathrm{D}$ & T1040-0421S & T1040-0421D & T1040-0422s & T1040-0422D & TI1040-0793S \\
\hline Limit (IDL) & Limit (EQL) & Client ID > & $\underline{1 \mathrm{C}}$ & $\underline{\underline{2 c}}$ & $\underline{\underline{2 b}}$ & $\underline{1 \mathrm{~b}}$ & $\underline{1 \mathrm{c}}$ & $\underline{1 c}$ & $\underline{1 \mathrm{a}}$ \\
\hline$(\mu \mathrm{g} / \mathrm{mL})$ & $(\mu \mathrm{g} / \mathrm{mL})$ & (Analyte) & $(\mu \mathrm{g} / \mathrm{g})$ & $(\mu \mathrm{g} / \mathrm{g})$ & $(\mu \mathrm{g} / \mathrm{g})$ & $(\mu \mathrm{g} / \mathrm{g})$ & $(\mu \mathrm{g} / \mathrm{g})$ & $(\mu \mathrm{g} / \mathrm{g})$ & $(\mu \mathrm{g} / \mathrm{g})$ \\
\hline 0.4064 & 4.064 & Al & {$[18,000]$} & 21,800 & {$[2,800]$} & {$[1,900]$} & {$[8,300]$} & {$[9,200]$} & 25,000 \\
\hline 0.4345 & 4.345 & $\mathrm{Ca}$ & {$[3,300]$} & {$[2,900]$} & - & - & - & - & {$[3,000]$} \\
\hline 0.1614 & 1.614 & $\mathrm{Fe}$ & 11,500 & 9,650 & 33,700 & 34,000 & 27,100 & 22,800 & 8,360 \\
\hline 0.1356 & 1.356 & $s$ & 15,900 & 16,900 & 25,800 & 14,300 & 19,000 & 14,800 & 3,880 \\
\hline 0.4424 & 4.424 & Si & 316,000 & 355,000 & 38,200 & 22,100 & 127,000 & 161,000 & 309,000 \\
\hline 0.0088 & 0.176 & $\mathbf{U}$ & 3,310 & 2,970 & 11,000 & 16,000 & 15,200 & 12,800 & 1,610 \\
\hline 0.0109 & 0.109 & $\mathrm{Zr}$ & 847 & -- & -- & - & - & - & [190] \\
\hline \multicolumn{10}{|c|}{ Other Analytes } \\
\hline 0.0014 & 0.014 & $\mathrm{Ag}$ & - & - & $=$ & - & [6.2] & - & - \\
\hline 0.0730 & 0.730 & As & - & - & - & - & - & - & - \\
\hline 0.0046 & 0.046 & B & [25] & - & [32] & [19] & [51] & - & [44] \\
\hline 0.0078 & 0.078 & $\mathrm{Ba}$ & 737 & 785 & [68] & [43] & 363 & 467 & 947 \\
\hline 0.0001 & 0.001 & $\mathrm{Be}$ & - & {$[0.50]$} & - & {$[0.19]$} & - & - & {$[0.39]$} \\
\hline 0.0120 & 0.120 & $\mathrm{Bi}$ & - & - & - & - & - & - & -- \\
\hline 0.0022 & 0.022 & Cd & - & - & -- & - & - & - & - \\
\hline 0.0066 & 0.066 & $\mathrm{Ce}$ & - & - & - & - & - & - & - \\
\hline 0.0027 & 0.027 & Co & - & [15] & [37] & [43] & [34] & [21] & [24] \\
\hline 0.0016 & 0.016 & $\mathrm{Cr}$ & 72.9 & [31] & [38] & 47.5 & [39] & [41] & 139 \\
\hline 0.0543 & 0.543 & $\mathrm{Cu}$ & - & - & - & - & - & - & -- \\
\hline 0.0014 & 0.014 & Dy & [9.6] & - & $=$ & - & $=$ & - & [4.9] \\
\hline 0.0004 & 0.004 & Eu & {$[3.2]$} & [1.8] & [1.8] & [1.7] & - & [1.6] & - \\
\hline 0.0000 & 0.000 & $\mathrm{~K}$ & na & na & na & na & na & na & na \\
\hline 0.0017 & 0.017 & $\mathrm{La}$ & 670 & - & - & -- & -- & - & -- \\
\hline 0.0003 & 0.003 & $\mathbf{L i}$ & [3.5] & [1.2] & - & - & - & - & {$[1.4]$} \\
\hline 0.0150 & 0.150 & Mg & 1,410 & 947 & [350] & [140] & {$[120]$} & [140] & 2,210 \\
\hline 0.0166 & 0.166 & Mn & [110] & [95] & - & [110] & - & -- & [130] \\
\hline 0.0063 & 0.063 & Mo & - & $=$ & - & - & - & $\cdots$ & -- \\
\hline 2.0840 & 20.840 & $\mathrm{Na}$ & -- & - & - & - & - & - & - \\
\hline 0.0041 & 0.041 & Nd & - & - & - & - & - & - & - \\
\hline 0.0000 & 0.000 & $\mathrm{Ni}$ & na & na & na & na & na & na & na \\
\hline 0.0690 & 0.690 & $\mathbf{P}$ & - & - & - & - & - & - & - \\
\hline 0.0603 & 0.603 & $\mathrm{~Pb}$ & -- & - & - & - & -- & -- & - \\
\hline 0.0052 & 0.052 & Pd & - & - & - & - & - & - & - \\
\hline 0.0098 & 0.098 & $\mathbf{R h}$ & - & [48] & - &.- & - & - & - \\
\hline 0.0066 & 0.066 & $\mathbf{R u}$ & [35] & - & - & - & - & -- & - \\
\hline 0.0340 & 0.340 & $\mathrm{Sb}$ & -- & - & - & - & - & -- & - \\
\hline 0.2400 & 2.400 & Se & - & - & - & - & - & - & - \\
\hline 0.0240 & 0.240 & Sn & - & - & - & - & - & - & - \\
\hline 0.0019 & 0.019 & $\mathrm{Sr}$ & (a) & (a) & (a) & (a) & (a) & (a) & (a) \\
\hline 0.0200 & 0.200 & $\mathrm{Ta}$ & - & -- & - & - & - & - & - \\
\hline 0.0160 & 0.160 & $\mathrm{Te}$ & - & - & - & - & - & - & - \\
\hline 0.0028 & 0.028 & Th & - & - & - & - & - & - & - \\
\hline 0.0035 & 0.035 & $\mathrm{Ti}$ & 1,470 & 1,010 & 345 & 385 & 351 & 455 & 3,540 \\
\hline 0.0460 & 0.460 & $\mathrm{TI}$ & - & - & - & - & - & - & - \\
\hline 0.0007 & 0.021 & V & [21] & [18] & [11] & [2.1] & [7.0] & - & [11] \\
\hline 0.0190 & 0.190 & w & - & - & - & - & - & - & - \\
\hline 0.0002 & 0.002 & $Y$ & {$[6.6]$} & {$[4.4]$} & [1.3] & - & {$[0.81]$} & - & 13.0 \\
\hline 0.1315 & 1.315 & $\mathrm{Zn}$ & - & - & -- & - & - & - & -- \\
\hline
\end{tabular}

1) "--" indicates the value is < MDL. The method detection limit (MDL) =IDL times the "multiplier"

near the top of each column. The estimated sample quantitation limit $=E Q L$ (in Column 2)

times the "multiplier". Overall error for values $\geq E Q L$ is estimated to be within $\pm 15 \%$.

2) Values in brackets [] are $\geq M D L$ but $<E Q L$, with errors likely to exceed $15 \%$.

na = not applicable; $\mathrm{KOH}$ flux and Ni crucible or $\mathrm{Na}_{2} \mathrm{O}_{2}$ flux and $\mathrm{Zr}$ crucible for fusion preparations, or Si for HF assisted digests.

(a) $=\mathrm{Sr}$ results are not reported (calibration issues). 
PNNL-20650, Rev. 2

Battelle PNNL/RPG/Inorganic Analysis ... ICPOES Data Report

Page 2 of 3

\begin{tabular}{|c|c|c|c|c|}
\hline Run Date > & $8 / 1 / 2011$ & $8 / 1 / 2011$ & $8 / 1 / 2011$ & $8 / 1 / 2011$ \\
\hline Multiplier > & 4223.0 & 4537.2 & 3328.9 & 2510.0 \\
\hline RPL/LAB > & $\begin{array}{c}11-1288 @ \\
5 x\end{array}$ & $\begin{array}{c}\text { DUP-1288 } \\
\text { @ 5x }\end{array}$ & \begin{tabular}{|c|}
$11-1289$ \\
$5 x$
\end{tabular} & $\begin{array}{c}11-1290 @ \\
5 \mathrm{x}\end{array}$ \\
\hline & T1040-0793D & T1040-0793D & T1040-0792S & $T 1040-0792 D$ \\
\hline Client ID > & $\underline{1 b}$ & $\underline{2 b}$ & $1 \mathrm{c}$ & 1c \\
\hline (Analyte) & $(\mu \mathrm{g} / \mathrm{g})$ & $(\mu \mathrm{g} / \mathrm{g})$ & $(\mu \mathrm{g} / \mathrm{g})$ & $(\mu \mathrm{g} / \mathrm{g})$ \\
\hline Al & 26,200 & 26,900 & {$[11,000]$} & 12,900 \\
\hline $\mathrm{Ca}$ & {$[3,100]$} & {$[3,200]$} & -- & {$[2,200]$} \\
\hline $\mathrm{Fe}$ & 9,780 & 8,750 & 20,900 & 23,000 \\
\hline s & {$[3,600]$} & {$[3,000]$} & 21,000 & 20,300 \\
\hline Si & 375,000 & 405,000 & 189,000 & 189,000 \\
\hline$u$ & 1,270 & 1,040 & 8,320 & 9,910 \\
\hline $\mathrm{Zr}$ & [240] & [190] & [37] & -- \\
\hline
\end{tabular}

\begin{tabular}{|c|c|c|c|c|}
\hline $\mathbf{A g}$ & - & - & {$[4.8]$} & {$[4.8]$} \\
\hline As &.- & 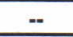 & - & $=$ \\
\hline B & [31] & - & - & 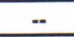 \\
\hline $\mathrm{Ba}$ & 966 & 1,050 & 423 & 499 \\
\hline $\mathrm{Be}$ & {$[0.57]$} & {$[0.36]$} & {$[0.23]$} & {$[0.30]$} \\
\hline $\mathrm{Bi}$ & - & $=$ & - & - \\
\hline Cd & -. & $=$ & - & -. \\
\hline $\mathrm{Ce}$ & -- & - & - & - \\
\hline Co & [26] & [36] & [15] & [16] \\
\hline $\mathrm{Cr}$ & [67] & 84.0 & 1,290 & [34] \\
\hline $\mathrm{Cu}$ & $\ldots$ & - & - & -. \\
\hline Dy & -. & - & - & [4.9] \\
\hline $\mathrm{Eu}$ & - & - & - & {$[1.2]$} \\
\hline$K$ & na & na & na & na \\
\hline La &.- & $=$ & - & - \\
\hline $\mathbf{L i}$ & [1.2] & $=$ & {$[2.1]$} & - \\
\hline $\mathrm{Mg}$ & 2,470 & 2,110 & 551 & 728 \\
\hline$M n$ & [140] & [140] & 727 & [52] \\
\hline Mo & - & - & - & - \\
\hline $\mathrm{Na}$ & $-\cdot$ & - & - & - \\
\hline Nd & -- & -- & - & - \\
\hline $\mathrm{Ni}$ & na & na & na & na \\
\hline $\mathbf{P}$ & -- & -. & - & $=$ \\
\hline $\mathrm{Pb}$ & -. & - & - & - \\
\hline $\mathbf{P d}$ & - & - & - & - \\
\hline $\mathbf{R h}$ & - & - & - & - \\
\hline $\mathbf{R u}$ & $=$ & $=$ & - & $=$ \\
\hline Sb & - & -. & - & -- \\
\hline $\mathrm{Se}$ & $=$ & - & - & - \\
\hline Sn & - & - & - & - \\
\hline $\mathrm{Sr}$ & (a) & (a) & (a) & (a) \\
\hline $\mathrm{Ta}$ & - & $=$ & - & $=$ \\
\hline $\mathrm{Te}$ & -- & - & - & - \\
\hline Th & - & -. & - & - \\
\hline $\mathrm{Ti}$ & 3,390 & 3,060 & 1,190 & 1,190 \\
\hline $\mathrm{TI}$ & -- & - & - & - \\
\hline v & [15] & [16] & [19] & [14] \\
\hline $\mathbf{W}$ & -- & - & - & $=$ \\
\hline$Y$ & {$[5.3]$} & {$[6.4]$} & {$[3.5]$} & {$[1.4]$} \\
\hline $\mathrm{Zn}$ & -. & -. & -. & -- \\
\hline
\end{tabular}


PNNL-20650, Rev. 2

Battelle PNNL/RPG/Inorganic Analysis ... ICPOES Data Report

Page 3 of 3

QC Performance 8/1/2011

\begin{tabular}{|c|c|c|c|c|c|c|c|}
\hline Criteria > & $\leq 20 \%$ & $\leq 20 \%$ & $80 \%-120 \%$ & $80 \%-120 \%$ & $80 \%-120 \%$ & $80 \%-120 \%$ & $\leq 10 \%$ \\
\hline QC ID > & $\begin{array}{c}11-1282 \\
\text { Dup }\end{array}$ & $\begin{array}{c}11-1288 \\
\text { Dup }\end{array}$ & $\begin{array}{c}\text { LCS/BS } \\
\text { (none) }\end{array}$ & MS (none) & $\begin{array}{c}11-1285+ \\
\text { AS-A }\end{array}$ & $\begin{array}{c}11-1285+ \\
\text { AS-B }\end{array}$ & $\begin{array}{c}11-1285 \\
5-f o l d \\
\text { Serial Dil }\end{array}$ \\
\hline Analytes & RPD (\%) & RPD (\%) & \%Rec & \%Rec & \%Rec & \%Rec & $\%$ Diff \\
\hline Al & & 2.6 & & & 106 & & \\
\hline $\mathrm{Ca}$ & & & & & 107 & & \\
\hline $\mathrm{Fe}$ & 17.8 & 11.1 & & & 113 & & \\
\hline$s$ & 6.0 & & & & & 107 & \\
\hline Si & 11.8 & 7.7 & & & 119 & & 1.7 \\
\hline $\mathrm{U}$ & 10.9 & 20.6 & & & & 99 & 1.2 \\
\hline $\mathrm{Zr}$ & & & & & 111 & & \\
\hline
\end{tabular}

Other Analytes

\begin{tabular}{|c|c|c|c|c|c|c|c|}
\hline $\mathbf{A g}$ & & & & & 103 & & \\
\hline As & & & & & 115 & & \\
\hline B & & & & & 107 & & \\
\hline $\mathrm{Ba}$ & 6.4 & 8.1 & & & 109 & & \\
\hline $\mathrm{Be}$ & & & & & 108 & & \\
\hline $\mathbf{B i}$ & & & & & 104 & & \\
\hline Cd & & & & & 113 & & \\
\hline $\mathrm{Ce}$ & & & & & & 100 & \\
\hline Co & & & & & 111 & & \\
\hline $\mathrm{Cr}$ & & & & & 110 & & \\
\hline $\mathrm{Cu}$ & & & & & 116 & & \\
\hline Dy & & & & & & 103 & \\
\hline $\mathrm{Eu}$ & & & & & & 103 & \\
\hline $\mathbf{K}$ & na & na & na & na & na & na & na \\
\hline $\mathrm{La}$ & & & & & & 103 & \\
\hline Li & & & & & 104 & & \\
\hline $\mathbf{M g}$ & 39.4 & 15.5 & & & 108 & & \\
\hline Mn & & & & & 109 & & \\
\hline Mo & & & & & 109 & & \\
\hline $\mathrm{Na}$ & & & & & 105 & & \\
\hline Nd & & & & & & 99 & \\
\hline $\mathrm{Ni}$ & na & na & na & na & na & na & na \\
\hline $\mathbf{P}$ & & & & & 111 & & \\
\hline $\mathrm{Pb}$ & & & & & 113 & & \\
\hline $\mathrm{Pd}$ & & & & & & 96 & \\
\hline Rh & & & & & & 97 & \\
\hline $\mathbf{R u}$ & & & & & & 102 & \\
\hline $\mathrm{Sb}$ & & & & & 110 & & \\
\hline $\mathrm{Se}$ & & & & & 113 & & \\
\hline $\mathrm{Sn}$ & & & & & 106 & & \\
\hline $\mathrm{Sr}$ & (a) & (a) & (a) & (a) & (a) & (a) & (a) \\
\hline $\mathrm{Ta}$ & & & & & 113 & & \\
\hline $\mathrm{Te}$ & & & & & & 105 & \\
\hline Th & & & & & & 105 & \\
\hline $\mathrm{Ti}$ & 37.8 & 10.3 & & & 111 & & \\
\hline $\mathrm{TI}$ & & & & & 102 & & \\
\hline$v$ & & & & & 104 & & \\
\hline $\mathbf{W}$ & & & & & 111 & & \\
\hline$Y$ & & & & & 107 & & \\
\hline $\mathrm{Zn}$ & & & & & 116 & & \\
\hline
\end{tabular}

Shaded results are outside the acceptance criteria.

$n r=$ spike concentration less than $25 \%$ of sample concentration. Matrix effects can be assessed from the serial dilution.

na = not applicable; $\mathrm{KOH}$ flux and Ni crucible or $\mathrm{Na}_{2} \mathrm{O}_{2}$ flux and $\mathrm{Zr}$ crucible for fusion preparations, or Si for HF assisted digests.

(a) $=\mathrm{Sr}$ results are not reported (calibration issues). 
PNNL-20650, Rev. 2

Battelle PNNL/RPL/ASO Radiochemistry Analysis Report

P.O. Box 999, 902 Battelle Blvd., Richland, Washington 99352

\section{Gross Alpha}

\begin{tabular}{|l|l|}
\hline Project / WP\#: & $52578 / \mathrm{H} 41216$ \\
\hline ASR\#: & 8878 \\
\hline Client: & S. Fiskum \\
\hline Total \# of Samples: & 14 \\
\hline
\end{tabular}

\begin{tabular}{|c|c|c|c|c|}
\hline Sample IDs & RPL Number & & Sample IDs & RPL Number \\
\hline TI040-0419S-1b & $11-1279-115-S$ & & TI040-0422S-1c & $11-1285-115-S$ \\
\hline TI040-0419D-2b & $11-1280-115-S$ & & TI040-0422D-1c & $11-1286-115-S$ \\
\hline TI040-0420S-2c & $11-1281-115-S$ & & TI040-0793S-1a & $11-1287-115-S$ \\
\hline TI040-0420D-1c & $11-1282-115-S$ & & TI040-0793D-1b & $11-1288-115-S$ \\
\hline TI040-0420D-2c & $11-1282-115-D$ & & TI040-0793D-2b & $11-1288-115-D$ \\
\hline TI040-0421S-2b & $11-1283-115-S$ & & TI040-0792S-1c & $11-1289-115-S$ \\
\hline TI040-0421D-1b & $11-1284-115-S$ & & TI040-0792D-1c & $11-1290-115-S$ \\
\hline
\end{tabular}

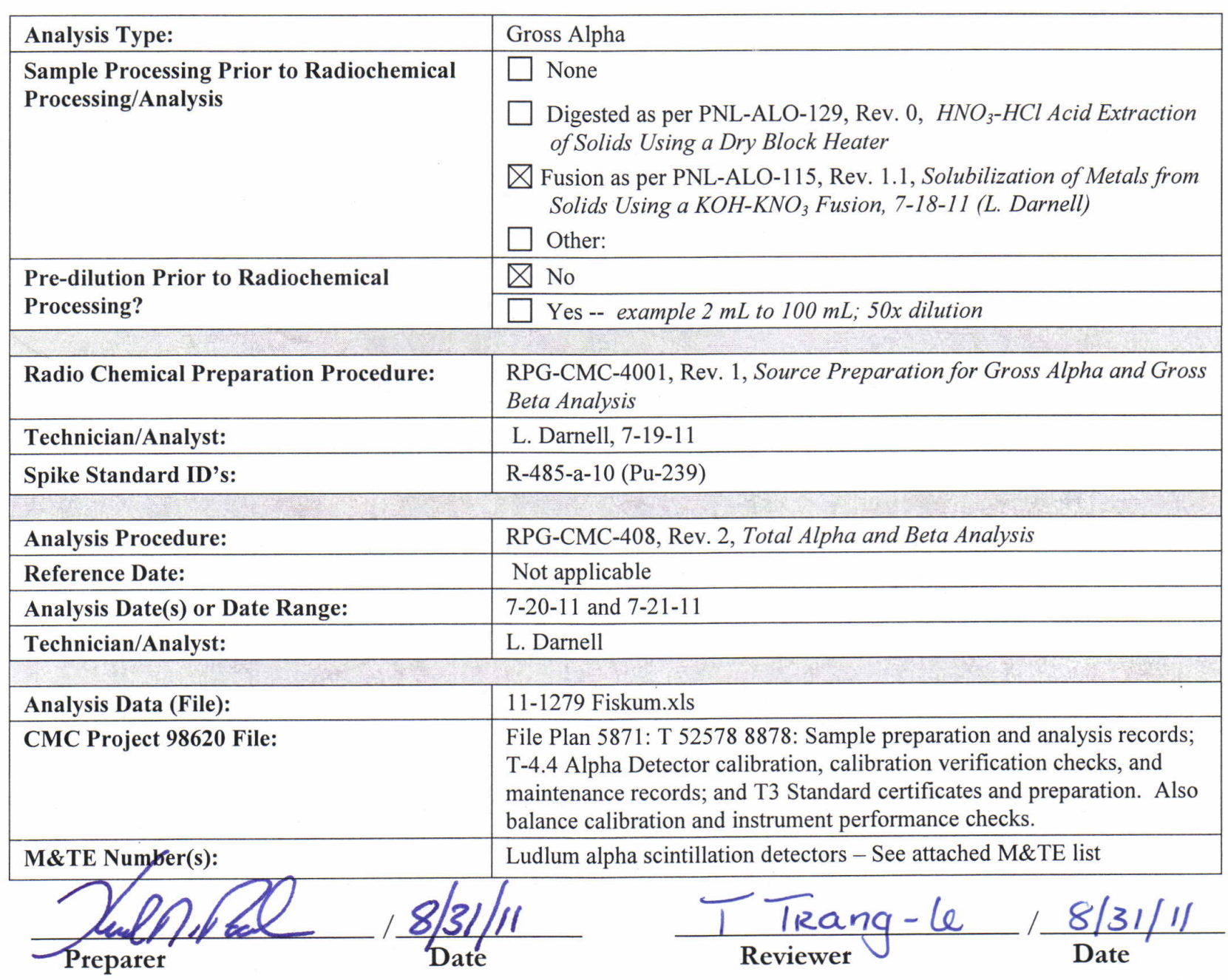


PNNL-20650, Rev. 2

Battelle PNNL/RPL/ASO Radiochemistry Analysis Report

\section{Sample Results}

See attached data report, Sample Results for ASR 8878. All data are reported in units of $\mu \mathrm{Ci} / \mathrm{g}$ dry sample with a $1-\sigma$ uncertainty (see comments).

\section{Sample Preparation, Separation, Mounting and Counting Methods}

Fourteen samples submitted under Analytical Service Request (ASR) 8878 were analyzed for Gross Alpha. All the samples were prepared in laboratory 420 following RPL procedure PNL-ALO-115. Each sample was provided to the laboratory such that the entire mass of the sample could be used for the fusion dissolution. The entire contents of each vial was emptied into tared Ni crubles and then weighed. The weight obtained by the laboratory is the weight used for all dilution calculations. The resulting solution was used for radiochemical analyses; only Gross Alpha is reported under this report.

Following the fusion dissolution process, the digestates were prepared for counting without chemical separation. The digestates were diluted to bring the alpha activity down to levels needed for alpha counting and reduce the solids loading that occurs due to the fusion flux matrix. The diluted digestate was mounted for gross alpha counting using procedure RPG-CMC-4001, then counted using alpha counters per procedure RPG-CMC-408.

\section{QUALITY CONTROL RESULTS}

Quality control (QC) samples prepared in laboratory 420 include a process blank (PB) and sample duplicates (identified and provided by the client). Additional QC samples were prepared prior to alpha counting including a laboratory blank, a reagent blank spike (BS, Pu-239), and addition of Pu-239 standard to a diluted aliquot of the sample selected as the matrix spike (MS).

Performance acceptance criteria for these samples are established by both the K Basin data quality objectives (as provided as special instruction in the ASR) and the ASO Quality Assurance (QA) Plan (ASO-QAP-001, Rev. 7). Except where noted, the Gross Alpha K-Basin DQO and ASO QAP criteria are the same. In cases where the QC criteria are different, K-Basin DQO's will take precedence. The QC sample results for Gross Alpha have been evaluated and are discussed below. A summary of the Gross Alpha analysis results, including QC sample performance, is given in the attached data report.

\section{Tracer:}

Tracer is not used for this analysis.

\section{Laboratory Preparation Blank (PB) and Laboratory blank (LB):}

The gross alpha activity measured in the PB is required to be within the acceptance criteria of less than $5 \%$ of the sample isotope concentration or less than sample minimum detectable activity (MDA) - see comments. The PB gross alpha activity is less than the MDA. There are no acceptance criteria for LB. The requested detection limit of $1.0 \mathrm{E}-03 \mu \mathrm{Ci} / \mathrm{g}$ is lower than what can be achieved for this sample set. The sample matrix of dissolved fusion flux results in solid formation on the counting disc upon evaporation that causes absorption of alpha. Each sample counting disc was counted for 120 minutes. The achievable detection limit for the samples was $2.0 \mathrm{E}-02 \mu \mathrm{Ci} / \mathrm{g}$. Occurrence report 52578-08-31-11 has been generated to document the achieved MDL not meeting the requested MDL. 


\section{Battelle PNNL/RPL/ASO Radiochemistry Analysis Report}

Blank Spike (BS) - reagent spike:

The BS (Pu-239) recovery of $96 \%$ meets the acceptance criteria of $80 \%$ to $120 \%$ recovery.

\section{Matrix Spike (MS):}

The MS (Pu-239) recovery of $90 \%$ meets the acceptance criterion of $75 \%$ to $125 \%$ recovery.

Note: the MS sample was prepared after digestion, by adding a known Pu-239 standard quantity to an aliquot of the leachate. Sample number 11-1290 (TI040-0792D-1c) was selected as the matrix spike sample.

\section{Duplicate -- Relative Percent Difference (RPD):}

Duplicate precision was not a required DQO for this samples set.

\section{Instrument Quality Control}

Alpha Ludlum detectors undergo initial calibration to determine the detector efficiency. The established efficiency for each detector is used in the final calculation of the sample gross alpha activity. Continuing calibration verification checks are performed on the detectors once per day as the system is used.

Detector backgrounds are obtained once per day or as the system is used or per batch.

\section{Assumption and Limitations of the Data}

None

\section{Comments}

1. The results have been corrected for all dilution factors resulting from sample processing.

2. Post-Digestion Spike (PS) - A spike made after the initial sample preparation (e.g., fusion, digestion, or leach) is considered a PS. When extremely radioactive samples are analyzed, most of the radioanalytical spikes are made after the sample preparation (to avoid excessive consumption of spike and avoid creating unnecessary waste) and are post-digestion spikes. The MS prepared with this batch of sample is considered a PS, since the Pu-239 spike was not added prior to the digestion process.

3. The 1-sigma uncertainty represents the total propagated error associated with processing and counting operations and includes weighing errors, volume uncertainties, and counting error.

4. The Laboratory Blank (LB) is prepared using laboratory reagents and provides data only on the cleanliness of the radiochemistry preparation process. LB results are not normalized to processing or dilution factors associated with the samples.

5. The sample results are compared to the process blank to evaluate if the blank contains $5 \%$ or more of the measured isotope; the process blank result has been adjusted for all processing factors for evaluation of the $5 \%$ criterion.

Attachment: Data Report -- Sample Results for ASR 8878 
PNNL-20650, Rev. 2

Battelle PNNL/RPL/ASO Radiochemistry Analysis Report

P.O. Box 999, 902 Battelle Blvd., Richland, Washington 99352

\section{Alpha Spectrometry - Total Alpha}

\begin{tabular}{|l|l|}
\hline Project / WP\#: & $52578 / \mathrm{H} 41216$ \\
\hline ASR\#: & 8878 \\
\hline Client: & S. Fiskum \\
\hline Total \# of Samples: & 14 \\
\hline
\end{tabular}

\begin{tabular}{|c|c|c|c|c|}
\hline Sample IDs & RPL Number & & Sample IDs & RPL Number \\
\hline TI040-0419S-1b & $11-1279-115-\mathrm{S}$ & & TI040-0422S-1c & $11-1285-115-\mathrm{S}$ \\
\hline TI040-0419D-2b & $11-1280-115-\mathrm{S}$ & & TI040-0422D-1c & $11-1286-115-\mathrm{S}$ \\
\hline TI040-0420S-2c & $11-1281-115-\mathrm{S}$ & & TI040-0793S-1a & $11-1287-115-\mathrm{S}$ \\
\hline TI040-0420D-1c & $11-1282-115-\mathrm{S}$ & & TI040-0793D-1b & $11-1288-115-\mathrm{S}$ \\
\hline TI040-0420D-2c & $11-1282-115-\mathrm{D}$ & & TI040-0793D-2b & $11-1288-115-\mathrm{D}$ \\
\hline TI040-0421S-2b & $11-1283-115-\mathrm{S}$ & & TI040-0792S-1c & $11-1289-115-\mathrm{S}$ \\
\hline TI040-0421D-1b & $11-1284-115-\mathrm{S}$ & & TI040-0792D-1c & $11-1290-115-\mathrm{S}$ \\
\hline
\end{tabular}

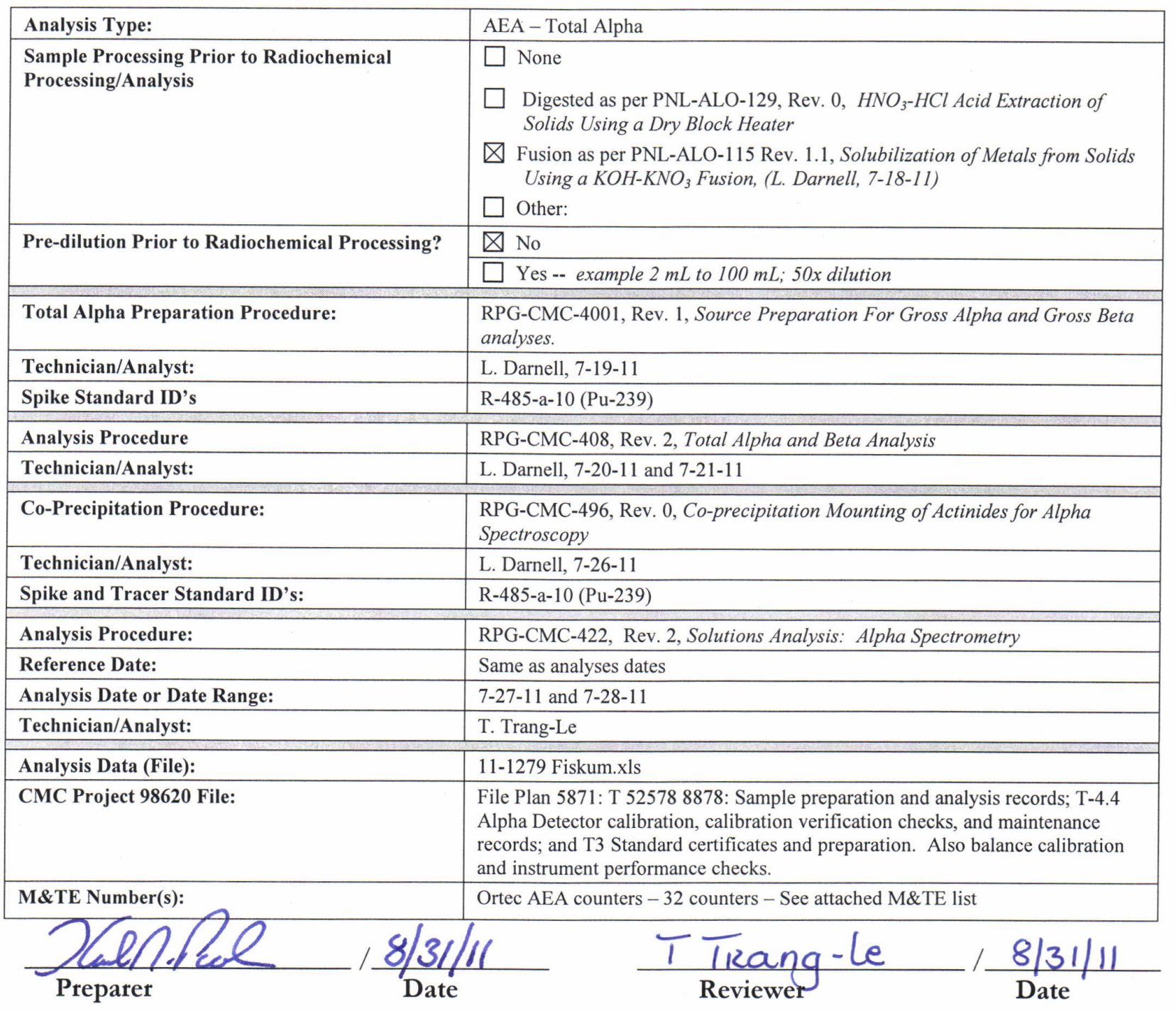




\section{Battelle PNNL/RPL/ASO Radiochemistry Analysis Report}

\section{Sample Results}

See attached data report, Sample Results for ASR 8878. All data are reported in units of $\mu \mathrm{Ci} / \mathrm{g}$ dry sample with a $1-\sigma$ uncertainty (see comments).

\section{Sample Preparation, Separation, Mounting and Counting Methods}

Fourteen samples submitted under Analytical Service Request (ASR) 8878 were analyzed for AlphaAEA. All the samples were prepared in laboratory 420 following RPL procedure PNL-ALO-115. Each sample was provided to the laboratory such that the entire mass of the sample could be used for the fusion dissolution. The entire contents of each vial was emptied into tared $\mathrm{Ni}$ crubles and then weighed. The weight obtained by the laboratory is the weight used for all dilution calculations.

Following the fusion digestion process, an aliquot of the digestate was mounted for alpha spectrometry by co-precipitation using procedure RPG-CMC-496, then counted by alpha spectroscopy using procedure RPC-CMC-422. The samples were counted on from July $27^{\text {th }}$ to July $28^{\text {th }}, 2011$; no decay corrections were made.

\section{QUALITY CONTROL RESULTS}

Quality control (QC) samples prepared in laboratory 420 include a process blank (PB) and sample duplicates (identified and provided by the client). Additional QC samples were prepared prior to alpha counting including a laboratory blank, a reagent blank spike (BS, Pu-239), and addition of Pu-239 standard to an aliquot of the sample digestate selected as the matrix spike (MS).

Performance acceptance criteria for these samples are established by both the K Basin data quality objectives (as provided as special instruction in the ASR) and the ASO Quality Assurance (QA) Plan (ASO-QAP-001, Rev. 7). Except where noted, the Alpha-AEA K-Basin DQO and ASO QAP criteria are the same. In cases where the QC criteria are different, K-Basin DQO's take precedence. The QC sample results for Am-AEA have been evaluated and are discussed below. A summary of the Alpha-AEA analysis results, including QC sample performance, is given in the attached data report. 


\section{Battelle PNNL/RPL/ASO Radiochemistry Analysis Report}

Tracer:

Tracer is not used for this analysis.

Process Blank (PB) and Laboratory separations blank (LB):

The alpha isotopic ( $\mathrm{Pu}-239+240, \mathrm{Pu}-238+\mathrm{Am}-241$ and $\mathrm{Cm}-243+244)$ activities measured in the $\mathrm{PB}$ are required to be within the acceptance criteria of less than $5 \%$ of the sample isotope concentration or less than sample minimum detectable activity (MDA) - (See comments). The PB alpha isotopic activities are significantly less than $5 \%$ of any sample activity. There are no acceptance criteria for LB. The detection limit achieved in the fusion preparation blank for Pu$239+240$ is $6.83 \mathrm{E}-5 \mu \mathrm{Ci} / \mathrm{g}$, for Pu- $238+\mathrm{Am}-241$ is $<5.0 \mathrm{E}-5 \mu \mathrm{Ci} / \mathrm{g}$ and for $\mathrm{Cm}-243+244$ is $<1.0 \mathrm{E}-$ $5 \mu \mathrm{Ci} / \mathrm{g}$. The achieved detection limits are all below the requested detection limit of $1.0 \mathrm{E}-3$ $\mu \mathrm{Ci} / \mathrm{g}_{\text {residual }}$

\section{Blank Spike (BS) - reagent spike:}

The BS recovery of $92 \%(\mathrm{Pu}-239)$ meets the acceptance criteria of $80 \%$ to $120 \%$ recovery.

\section{Matrix Spike (MS):}

The MS recovery of $100 \%$ (Pu-239) meets the acceptance criterion of $75 \%$ to $125 \%$ recovery. Note: the MS sample was prepared after digestion, by adding a known quantity of Pu-239 standard to a diluted aliquot of the digestate. Sample number 11-1290-115-S (TI040-0792D-1c) was selected as the matrix spike sample.

Duplicate - Relative Percent Difference (RPD):

Duplicate precision was not a required DQO for this samples set.

\section{Instrument Quality Control}

Alpha counters receive initial calibration with NIST traceable sources to determine the counter efficiency. When internal tracers are not used, the counter efficiency is used in calculation of final results.

Detector backgrounds are determined every 4 weeks or after the last analytical run, whichever is longer. Detector background counts are subtracted from all subsequent sample counts. A process blank is analyzed with each analytical batch to evaluate for contamination in the sample preparation process.

\section{Assumption and Limitations of the Data}

\section{None}

\section{Comments}

1. The results have been corrected for all dilution factors resulting from sample processing. 


\section{Battelle PNNL/RPL/ASO Radiochemistry Analysis Report}

2. Post-Digestion Spike (PS) - A spike made after the initial sample preparation (e.g., fusion, digestion, or leach) is considered a PS. When extremely radioactive samples are analyzed, most of the radioanalytical spikes are made after the sample preparation and dilution (to avoid excessive consumption of spike and avoid creating unnecessary waste). The MS prepared with this batch of samples is considered a PS, since the Pu-239 spike was not added prior to the digestion process.

3. The 1-sigma uncertainty represents the total propagated error associated with processing and counting operations and include; weighing errors, volume uncertainties, and counting error.

4. The sample results are compared to the process blank to evaluate if the blank contains $5 \%$ or more of the measured isotope; the process blank result has been adjusted for all processing factors for evaluation of the $5 \%$ criterion.

5. The Laboratory Blank (LB) is prepared using laboratory reagents and provides data on the cleanliness of the radiochemistry preparation/separation processes. LB results are not normalized to processing or dilution factors associated with the samples.

Attachment: Data Report -- Sample Results for ASR 8878 


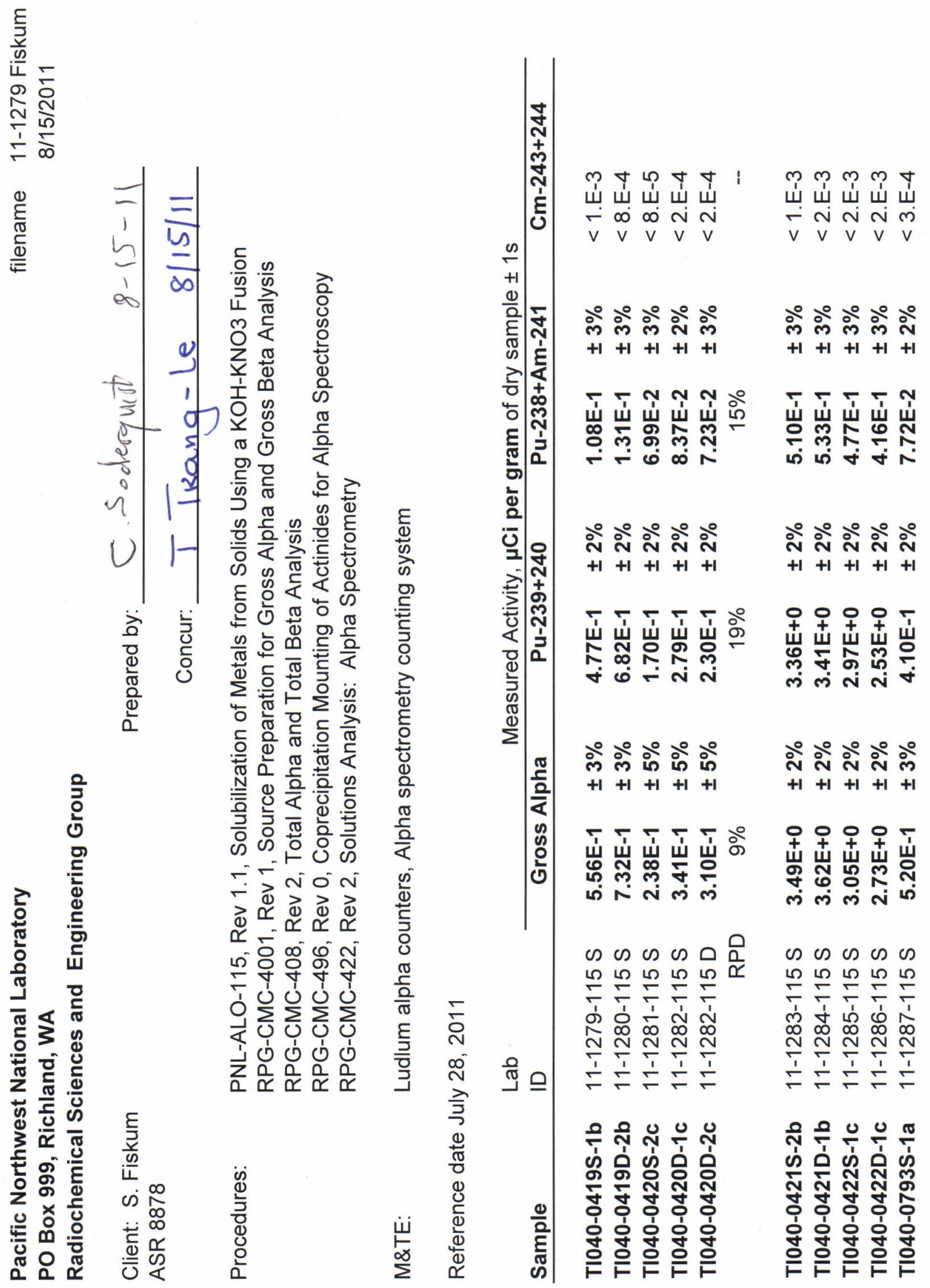


PNNL-20650, Rev. 2

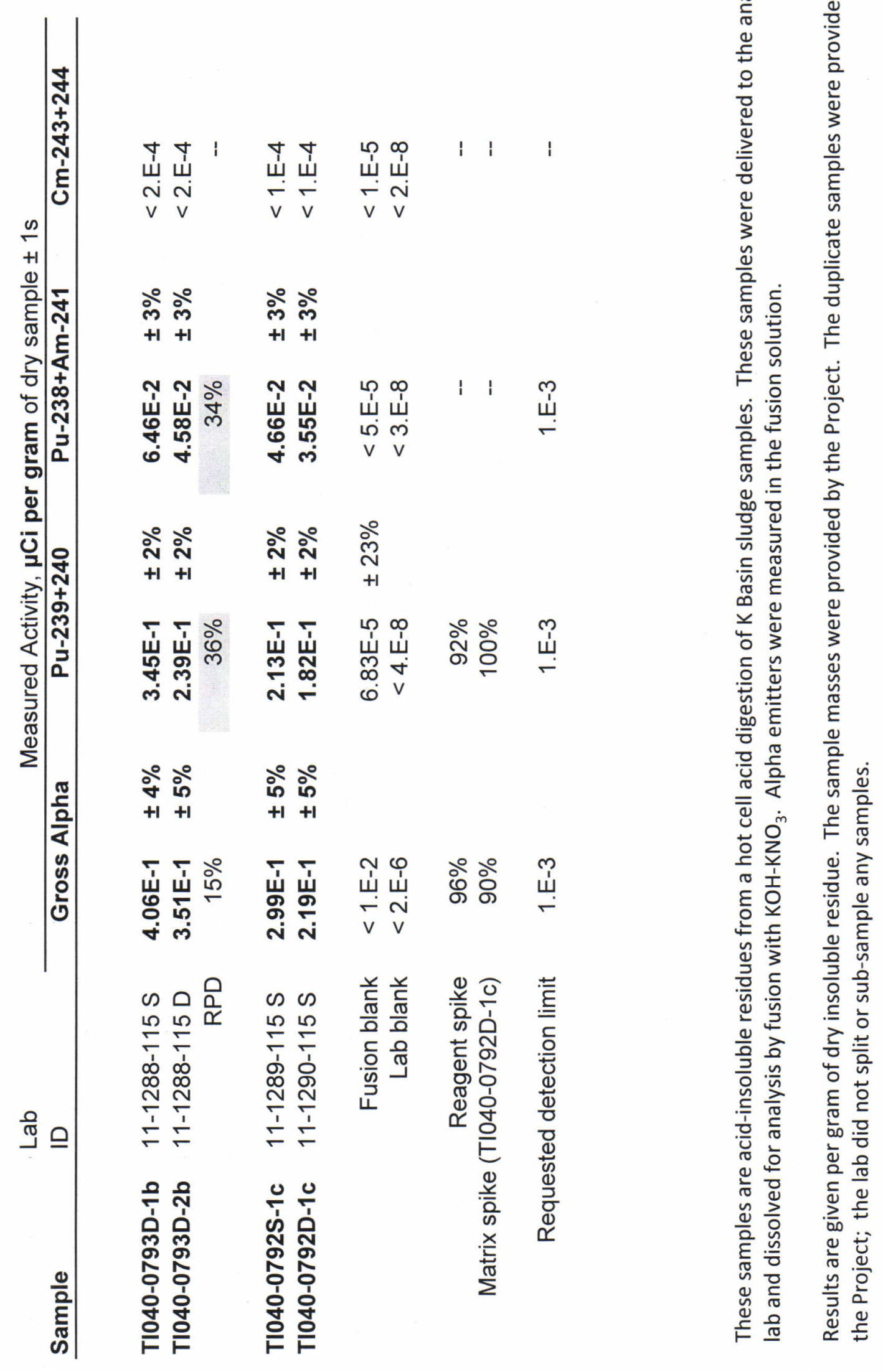


PNNL-20650, Rev. 2

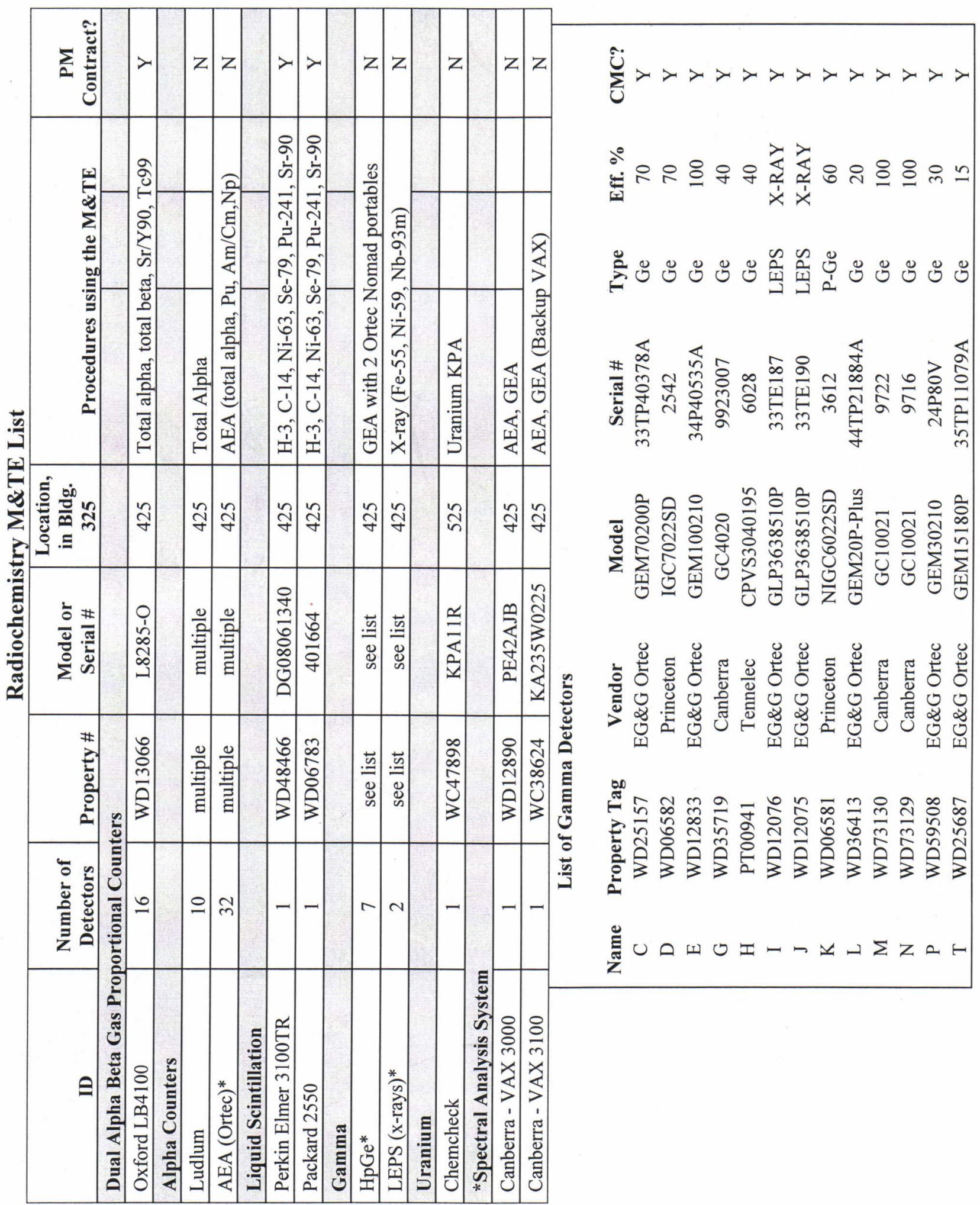




\section{K-Basin Occurrence Report}

\begin{tabular}{|c|c|c|c|}
\hline \multicolumn{3}{|c|}{$\begin{array}{l}\text { Title: ASR } 8878 \text { - Gross Alpha - Achieved detection limit for } \\
\text { samples was greater than the requested detection limit of } 1.0 \mathrm{E} \text { - } \\
03 \mu \mathrm{Ci} / \mathrm{g} \text {. }\end{array}$} & Date: 08-31-11 \\
\hline \multicolumn{4}{|c|}{ Unique Identifier: $52578-08-31-11$} \\
\hline \multicolumn{4}{|c|}{ Primary Person Identifying Issue: Karl Pool } \\
\hline \multirow{4}{*}{$\begin{array}{l}\text { Distribution: Email and posting } \\
\text { on K-Basin Share-Point }\end{array}$} & Clark Carlson & Karl Pool & \\
\hline & Deborah Coffey & Andy Schmid & \\
\hline & Sandy Fiskum & Rick Shimske & \\
\hline & Matt Fountain & Truc Trang Le & Project file for ASR 8878 \\
\hline $\begin{array}{l}\text { Date Submitted to K-Basin } \\
\text { Records: }\end{array}$ & \multicolumn{3}{|l|}{$9 \cdot 1-2011$} \\
\hline \multicolumn{4}{|c|}{ Occurrence Description: ASR 8878: } \\
\hline \multirow[t]{2}{*}{ 1) Requested detection limit } & $1.0 \mathrm{E}-03 \mu \mathrm{Ci} / \mathrm{g}$ wa & s not achieved & the gross alpha samples - \\
\hline & $\begin{array}{l}\text { detection limit of } \\
\text { lids samples are d } \\
\text { solids, primarily f } \\
\text { e sample size used } \\
\text { counting disk. T } \\
\text { A small sample }\end{array}$ & $\begin{array}{l}1.0 \mathrm{E}-03 \mu \mathrm{Ci} / \mathrm{g} \mathrm{f} \\
\text { issolved using } \\
\text { rom the fusion } \\
\text { takes into acco } \\
\text { he solids loadin } \\
\text { size is thus nee }\end{array}$ & $\begin{array}{l}\text { r gross alpha that is not achievable } \\
\mathrm{OH} / \mathrm{KNO}_{3} \text { fusion. The resulting } \\
\text { ux used. When gross alpha samples } \\
\text { nt the solids loading that will occur } \\
\text { significantly attenuates the alpha } \\
\text { ed to minimize solids loading. Each } \\
\text { igh as } 2.0 \mathrm{E}-02 \mu \mathrm{Ci} / \mathrm{g} \text {. }\end{array}$ \\
\hline
\end{tabular}

Impact of Occurrence:

There is no impact associated with this occurrence. Each sample in the set had measureable alpha activity. The lowest alpha activity measured in the sample set was $2.19 \mathrm{E}-01 \mu \mathrm{Ci} / \mathrm{g}$.

Significant Condition Adverse to Quality? Yes __ No $\underline{\text { _ }}$

Evaluated by: Date: $9 / 1 / 2011$

Further Disposition: $\square$ No $\square$ Yes If yes, $\square$ Nonconformance? $\square$ Deficiency?

Date Occurrence Closed: $9 \cdot i \cdot 201 /$

Signature/Date of Responsible QE: $\frac{1-2 \text { - }}{\text { Signature/Date of Responsible K-Basin Manager: }}$

Comments: 
Requestor --- Complete all fields on this COVER PAGE, unless specified as optional or ASR is a revision

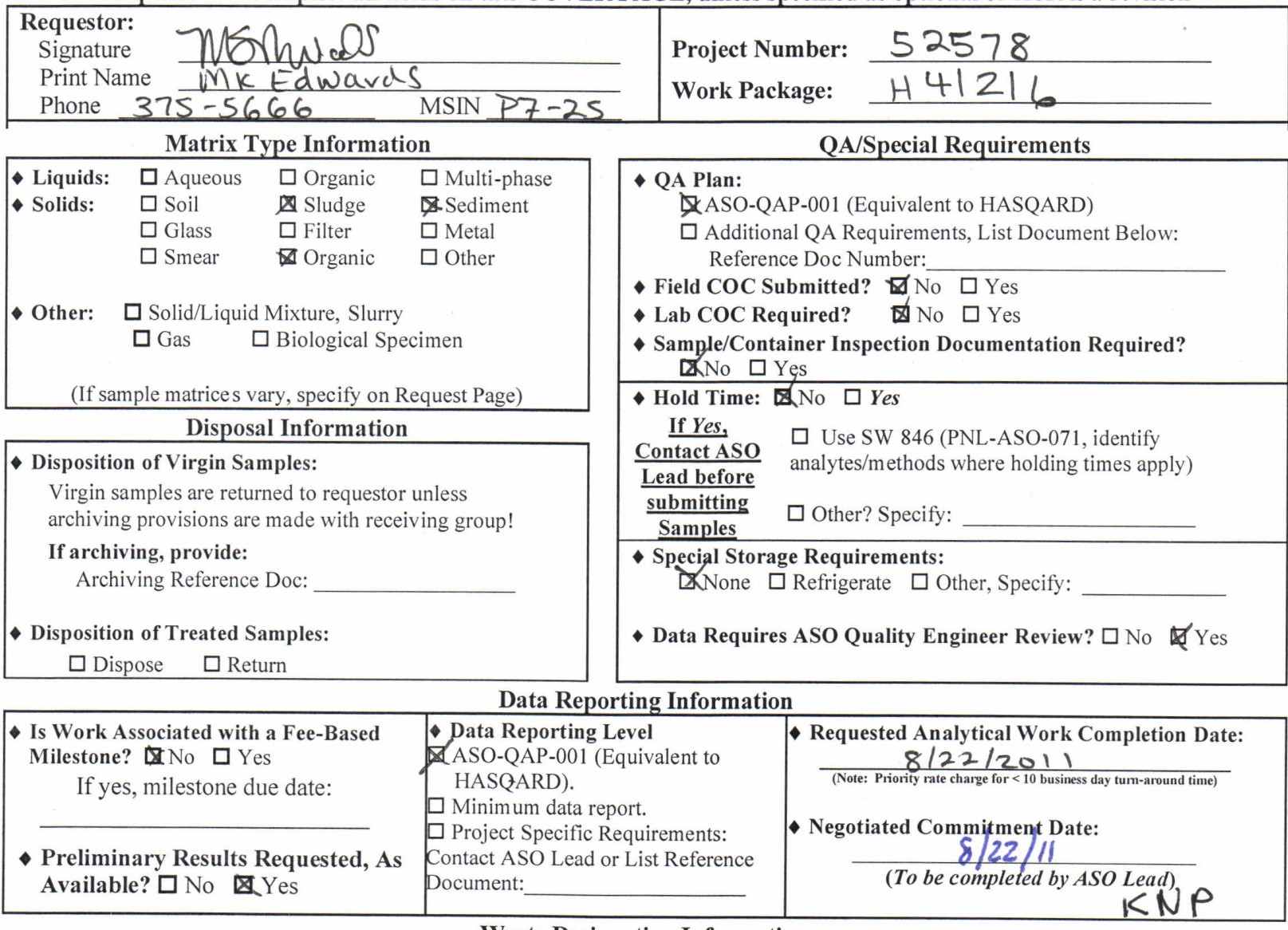

Waste Designation Information

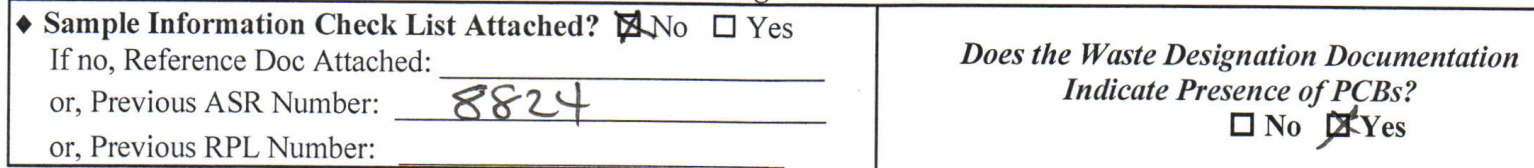

$\begin{array}{llll}\text { Send Report To: } \frac{M K E d w a r d s}{S K \text { Fisk }} & \text { MSIN } & \text { MSIN } & \frac{\$ P 7-25}{P 7-25} \\ \text { Additional or Special Instructions } & & \end{array}$

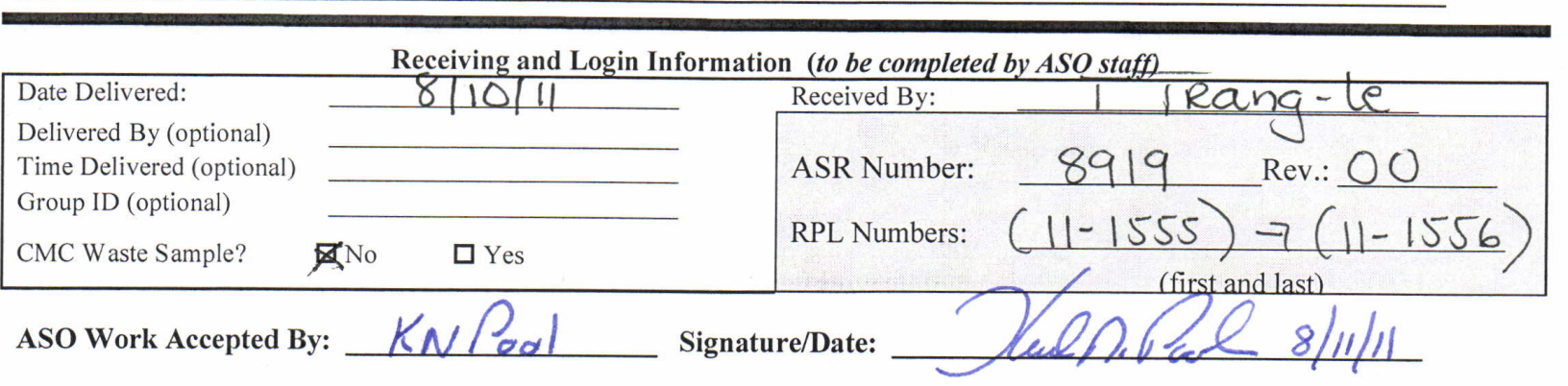


PNNL-20650, Rev. 2
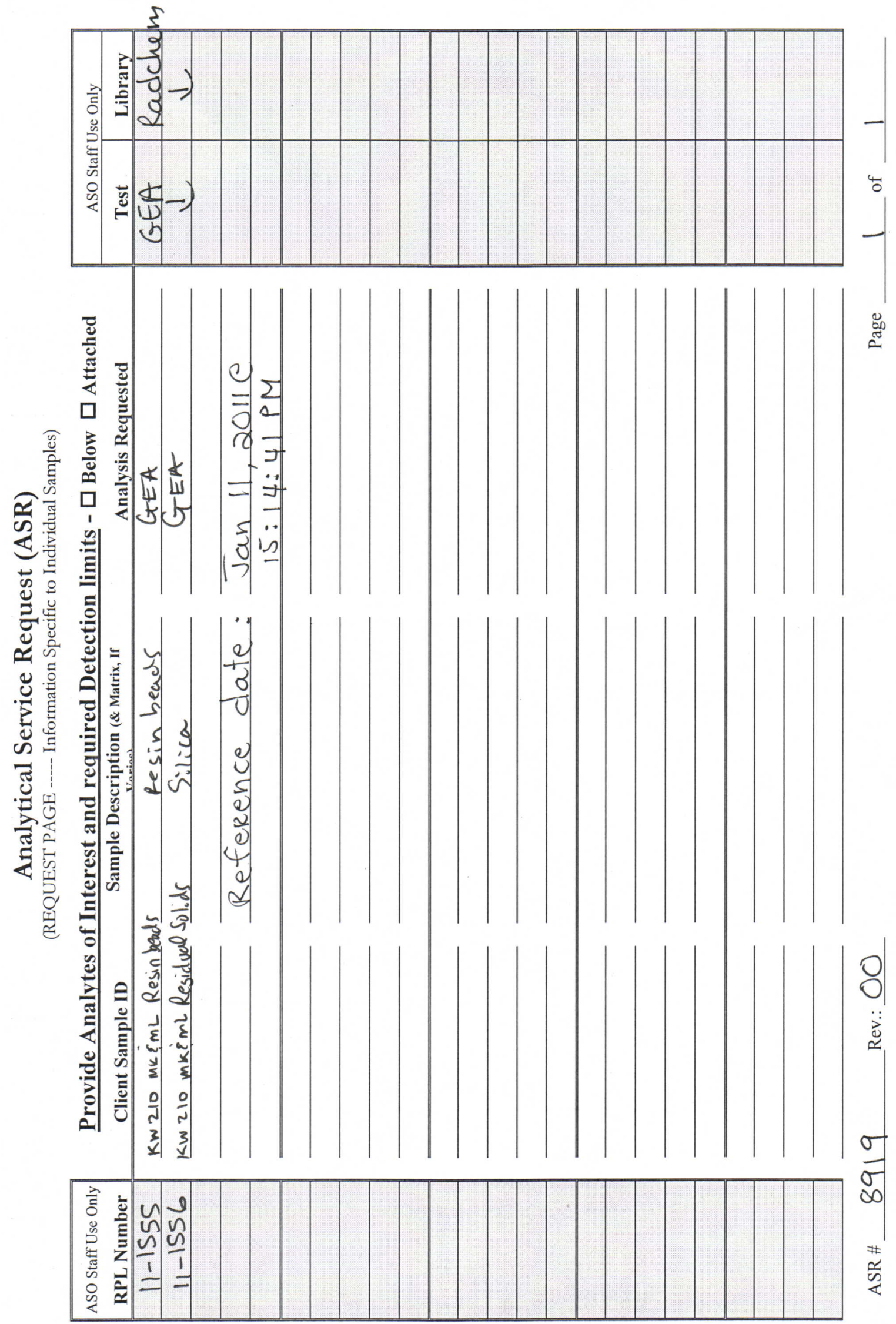
Battelle PNNL/RPL/ASO Radiochemistry Analysis Report

P.O. Box 999, 902 Battelle Blvd., Richland, Washington 99352

\section{Gamma Energy Analysis (GEA)}

$\begin{array}{ll}\text { Project / WP\#: } & 52578 / \mathrm{H} 41216 \\ \text { ASR\#: } & 8919.00 \\ \text { Client: } & \text { MK Edwards } \\ \text { Total Samples: } & \text { 2 Samples }\end{array}$

\begin{tabular}{|c|c|}
\hline RPL ID & Client Sample ID \\
\hline $11-1555$ & KW210 MK \& ML Resin Beads \\
\hline $11-1556$ & KW210 MK \& ML Residual Solids \\
\hline
\end{tabular}

\begin{tabular}{|c|c|}
\hline Analysis Type: & GEA- for all positively measured or non-detected isotopes \\
\hline $\begin{array}{l}\text { Sample Processing Prior to Radiochemical } \\
\text { Processing/Analysis }\end{array}$ & $\begin{array}{l}\square \text { None } \\
\square \text { Digested as per RPG-CMC-128, Rev. } \mathrm{OHNO}_{3} \text { - } \mathrm{HCl} \text { Acid Extraction of } \\
\text { Liquids for Metals Analysis Using a Dry Block Heater } \\
\square \text { Digested as per PNL-ALO-129, Rev. } 0 \mathrm{HNO}_{3}-\mathrm{HCl} \text { Acid Extraction of } \\
\text { Solids Using a Dry Block Heater } \\
\square \text { Fusion as per PNL-ALO-115, Solubilization of Metals from Solids } \\
\text { Using a } \mathrm{KOH}-\mathrm{KNO}_{3} \text { Fusion } \\
\square \text { Other: } \\
\text { Preparation may also involve attaining a GEA geometry that is compatible } \\
\text { with the calibration geometry. }\end{array}$ \\
\hline Analysis Procedure: & $\begin{array}{l}\text { RPG-CMC-450, Rev. } 1 \text { Gamma Energy Analysis (GEA) and Low-Energy } \\
\text { Photon Spectrometry (LEPS) }\end{array}$ \\
\hline Reference Date: & *January 11,2011@15:14:41 \\
\hline Analysis Date or Date Range: & August 10, 2011 \\
\hline Technician/Analyst: & MG Cantaloub \\
\hline Rad Chem Electronic Data File: & 11-1555 Edwards.xls \\
\hline ASO Project 98620 File: & $\begin{array}{l}\text { File Plan 5871, T4.4 Technical (Radiochemistry), Gamma Calibration, } \\
\text { daily checks, and maintenance records; and T3 standard certificates and } \\
\text { preparation. Also, balance calibration and Performance check records. }\end{array}$ \\
\hline M\&TE Number(s): & $\begin{array}{l}\text { Detector } T \text { ( } M \& T E \text { unique identifiers in attached supporting } \\
\text { documents) }\end{array}$ \\
\hline
\end{tabular}

*Reference date used is the same date and time used for samples submitted under ASR 8725 as directed by S. Fiskum.
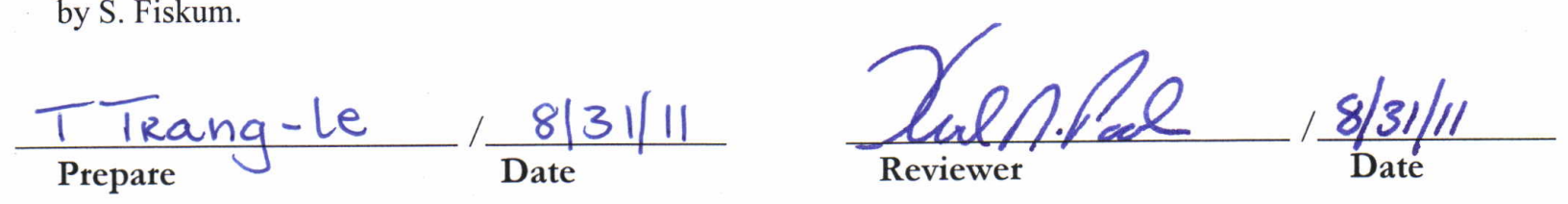
Battelle PNNL/RPL/ASO Radiochemistry Analysis Report

\section{SAMPLE RESULTS}

Activities for all gamma emitters detected in these samples are presented in an attached Excel spreadsheets for ASR 8919.00. All residual solids are reported as $\mu \mathrm{Ci} / \mathrm{g}$, both with estimates of the total propagated uncertainty reported at the 1-sigma level.

The $\mathrm{K}$ Basin ASR specifies that the residual solids GEA analytes are to be reported as $\mu \mathrm{Ci} / \mathrm{g}$ where $\mathrm{g}$ residual is the mass of residual solids that did not dissolve during acid digestion.

ASO Project File, ASR 8919 has been created for this report including all appropriate supporting records which may include the Pipette Performance Verification form, Standard Certificates, Laboratory Bench Record, Shielded Analytical Laboratory Bench Sheet, and Gamma Energy Analysis printouts. Detector calibration records, control charts and balance calibration records can be found in the ASO Records.

\section{Sample Preparation, Separation, Mounting and Counting Methods}

Solid samples were received from client pre-dried and analyzed by GEA "as received."

The quality control (QC) sample results for each of the isotopes measured above background have been evaluated and are discussed below. A summary of the GEA analysis results, including QC sample performance, is given in the attached Data Report.

The following is a list of QC sample results relative to both the $\mathrm{K}$ Basin data quality objectives and the ASO Quality Assurance (QA) Plan (ASO-QAP-001, Rev. 7); in many cases the DQOs are the same. In cases where there are differences in the DQOs, the K Basin DQO takes precedence for data reporting.

\section{QUALITY CONTROL RESULTS}

Tracer:

Tracers are not used for ASO GEA methods.

\section{Process Blank (PB):}

There is no process blank in this batch. No sample preparation was performed, the samples were analyzed "as received".

\section{$\underline{\text { Required Detection Limits }}$}

The ASR doesn't have detection limit requirements for residual solid or resin samples.

\section{Blank Spike (BS)/Laboratory Control Sample (LCS)/ Matrix Spike (MS):}

There were no BS, MS or LCS samples analyzed for ASO GEA analyses. Instrument performance is assessed by the analyses of daily control counts and weekly background counts, as discussed below.

\section{Duplicate Relative Percent Difference (RPD):}

There is no duplicate sample pairs for this ASR. 
PNNL-20650, Rev. 2

Battelle PNNL/RPL/ASO Radiochemistry Analysis Report

Replicate Relative Standard Deviation (RSD):

There were no replicate samples for this ASR.

\section{Instrument Calibration and Quality Control}

Gamma detectors are calibrated using multi-isotope standards that are NIST-traceable and prepared in the identical counting geometry used for the samples for all detectors. Counter control sources containing Am-241, Cs-137 and Co-60 are then analyzed daily before the use of each detector. There is an ASO procedure RPG-CMC-450, that requires that a counter control source is checked daily and must be within \pm 3 sigma or $\pm 3 \%$ of the control value, whichever is greater. Gamma counting was not performed unless the control counts were within the required limits. Background counts are performed on all gamma detectors at least weekly for either an overnight or weekend count. The most recent background is subtracted from all sample counts.

\section{Assumption and Limitations of the Data}

None.

\section{Interferences/Resolution}

None.

\section{Uncertainty}

For gamma counting, the uncertainty in the efficiency, counting data, photon abundance and the nuclear half-life are included in the calculation of the total uncertainty along with a systematic uncertainty for sample preparation. The Canberra Genie software includes both random and systematic uncertainties in the calculation of the total uncertainties which are listed on the report. A conservative estimate is that $2 \%$ is the lowest uncertainty possible for our GEA measurements taking into account systematic uncertainties in gamma calibration standards.

The samples were counted at about 20.5 inches from the detector to minimize dead time due to the high sample activities. Counting at such long distances also minimizes the differences between the sample geometry and the calibration geometry. Calibrations are performed using water samples whereas the samples consisted of mostly silica (however, the exact sample mineralogical composition and associated density were not known). The small sample size $(<1 \mathrm{~g})$ minimized the gamma absorption effects; therefore, density corrections were not applied.

\section{Comments}

1. The results have been corrected for all dilution factors resulting from sample processing.

2. Minimum Detectable Activity (MDA) and Minimum Detectable Concentration (MDC). Sample results are compared to the process blank results to evaluate if the blank contains $5 \%$ or more of the analyte of interest. When samples undergo digestion and/or dilution (process factors), the MDA must be adjusted for the process factors to accurately compare activity levels in the blank to activity levels in the samples. Process blank results have been adjusted for digestion and/or process factors for evaluation of the $5 \%$ criteria.

Attachment: Data Report Sample Results for ASR 8919. 
PNNL-20650, Rev. 2

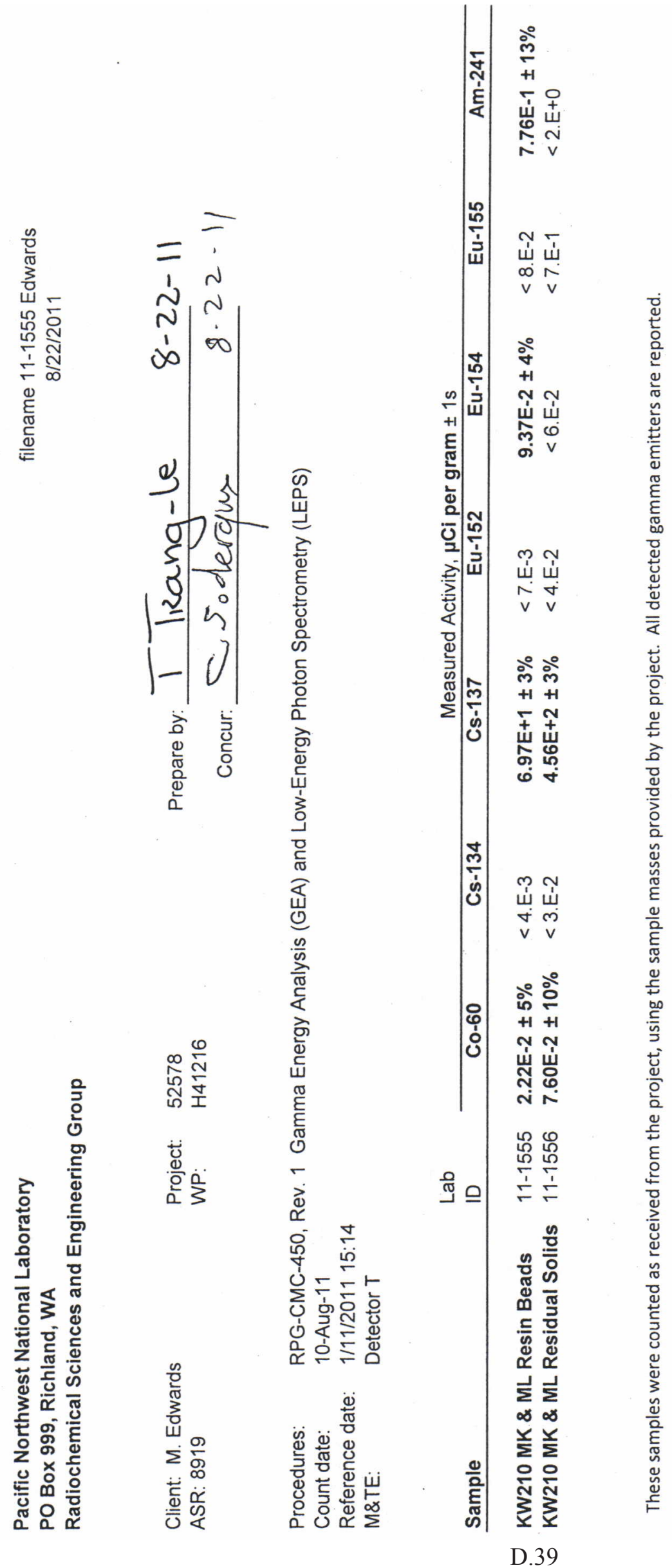


PNNL-20650, Rev. 2

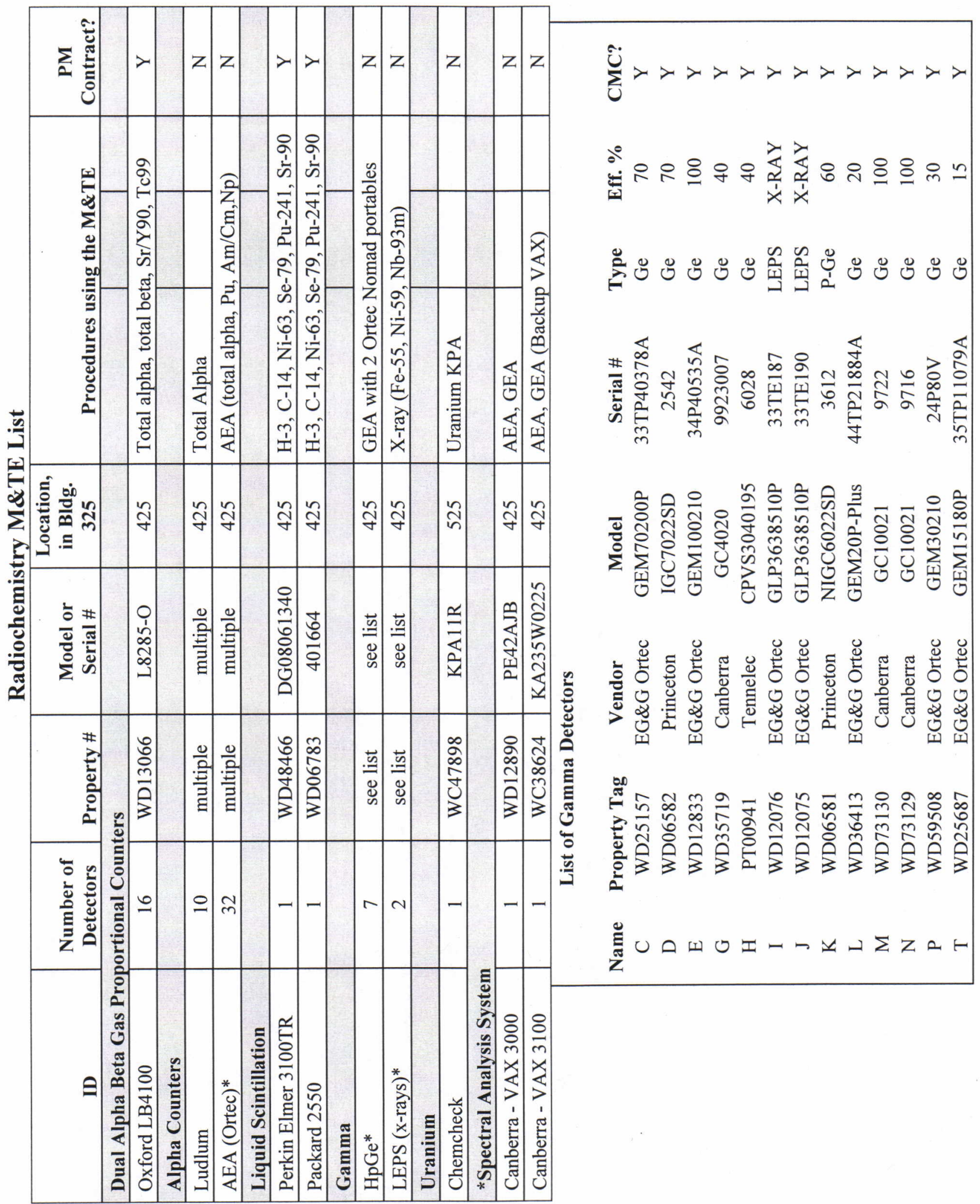

Printed 5/04/2011 
PNNL-20650, Rev. 2

\section{Appendix E}

Isotopic Analysis Request and Results 

PNNL-20650, Rev. 2

\section{Appendix E}

\section{Isotopic Analysis Request and Results}

Appendix E contains the signed analysis request for the $\mathrm{Pu}$ and $\mathrm{U}$ isotopic analyses of Engineered Container SCS-CON-210 core samples. The analysis request delineates sample-specific information, required precision, quality control (QC) sample requirements, and reporting requirements. The core samples were initially processed by the Analytical Services Operations (ASO) laboratory according to Analytical Services Request (ASR) 8798 (see Appendix B). This included acid digestion according to PNL-ALO-129, Rev. 0, $\mathrm{HNO}_{3}-\mathrm{HCl}$ Acid Extraction of Solids Using a Dry-Block Heater. The resulting solutions were processed by the ASO to isolate the $\mathrm{U}$ component and the Pu component as two separate fractions per sample. The ASO staff then provided the purified fractions to the thermal ionization mass spectrometry (TIMS) workstation for analysis. The ASO documented the TIMS preparative work in the “Thermal Ionization Mass Spectrometry Sample Preparation” summary report (provided in Appendix E).

The TIMS analytical report with the sample isotopic distributions, associated uncertainties, and a discussion of sample processing, QC results, data limitations, and Excel data reduction spreadsheet (KW210 TIMS data June 23, 2011.xls) are provided in Appendix E. Occurrence Reports are prepared when issues are identified, and resolution is required while work is in process; one Occurrence Report was issued and is also provided. A portion of the data reduction spreadsheet, ASR 8798 data reduction Rev. 1.xlsx, which is relevant to the isotopic calculations, is also provided. The isotopic calculations data reduction spreadsheet renormalized the TIMS-reported isotopic distribution with ${ }^{238} \mathrm{Pu}$ concentration from $\mathrm{Pu}$ alpha energy analysis, converted $\mathrm{U}$ and $\mathrm{Pu}$ isotopic results from atom percent to mass percent, and then converted isotope fraction to activity and mass concentrations (dry and wet settled sludge mass basis). The full calculation spreadsheet is provided with the supplemental information as part of the complete data package.

The following table of contents shows that pagination specific to Appendix E was applied to the TIMS analysis request, the ASO preparation and TIMS analysis reports, the supporting TIMS data, Occurrence Report 52578-05-09-11, and the surveillance report (generated by the Pacific Northwest National Laboratory Quality Engineer). This pagination is sequential and consistent (e.g., page E.\#). 


\section{Appendix E Table of Contents}

Thermal Ionization Mass Spectrometry Analysis Request for Plutonium and Uranium Isotopes

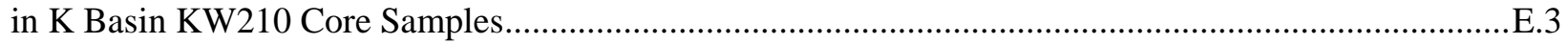

ASR 8798 Thermal Ionization Mass Spectrometry Sample Preparation Report ...................................... E.9

Plutonium and Uranium Isotopic Analysis for KW210 Core Samples..................................................11

Report Document Review Record ................................................................................................. 20

TIMS Data Reduction File (KW210 TIMS data June 23, 2011.xls) ..............................................21

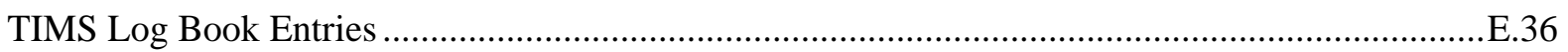

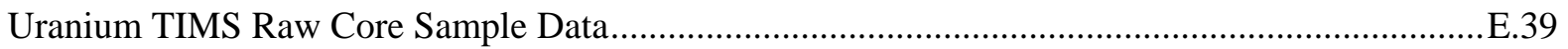

Certificate Standard Reference Material U-030 Uranium Isotopic Standard ....................................48

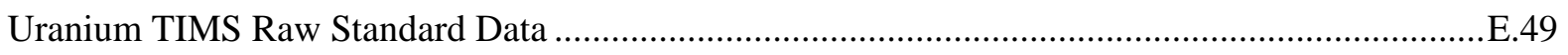

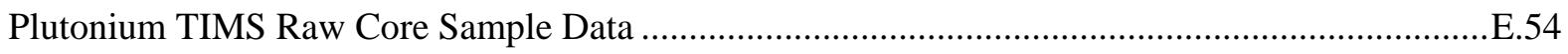

National Bureau of Standards Certificate of Analysis Standard Reference Material 946

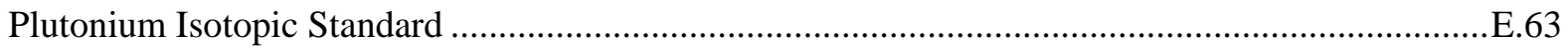

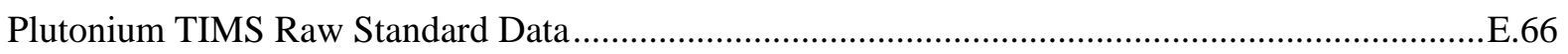

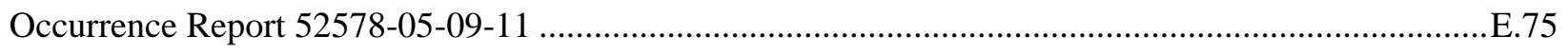

Surveillance Report SR-52578-2011-27 Rev. 1 .............................................................................8.

Data Reduction Spreadsheet ASR 8798 Data Reduction Rev. 1.xlsx (TIMS portion only)......................96 
Thermal Ionization Mass Spectrometry

Analysis Request for Plutonium and Uranium Isotopes in K Basin KW210 Core Samples

Cognizant Scientist:

Analyst:

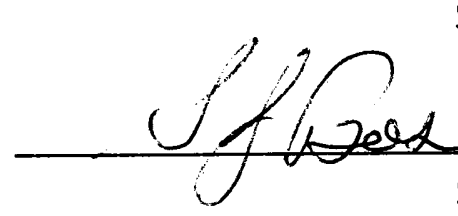

SJ Bos

Project Quality Engineer: $\frac{25 \text { Coffey }}{\text { DS Coffey }}$

Project Manager:

STP Concurrence:
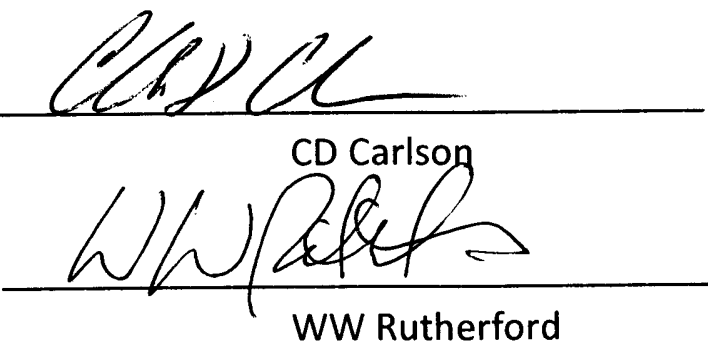

Date: $4 / 13 / 11$

Date: $4 / 14 / 11$

Date: $\underline{4-14-11}$

Date: $4 / 1 / / 25 / 1$

Date: $4 / 14 / 11$ 


\section{Thermal Ionization Mass Spectrometry}

\section{Analysis Request for Plutonium and Uranium Isotopes in}

\section{K Basin KW210 Core Samples}

\begin{tabular}{|c|c|c|c|}
\hline Requester & SK Fiskum & Work Package & H41217 \\
\hline \multirow{2}{*}{ Signature } & & Project Number & 52578 \\
\hline & & Subtask & TIMS \\
\hline $\begin{array}{l}\text { Date } \\
\text { Submitted: }\end{array}$ & & Date Required: & $4 / 11 / 11$ \\
\hline $\begin{array}{l}\text { Sample } \\
\text { Description: }\end{array}$ & \multicolumn{3}{|c|}{$\begin{array}{l}\text { Four core samples were collected from the Large Engineered Container, SCS-CON- } \\
\text { 210, containing sludge located at the K West Basin. Subsamples have been } \\
\text { collected from each of the core samples and dissolved according to PNL-ALO-129, } \\
\text { Rev. 0, } \mathrm{HNO}_{3}-\mathrm{HCl} \text { Acid Extraction of Solids Using a Dry-Block Heater. } \\
\text { Aliquots of the sample digestates were prepared for Pu TIMS analysis by the ASO } \\
\text { using the separation procedure RPG-CMC-455, Rev. 0, Separation of Uranium and } \\
\text { Plutonium for Isotopic Analysis by Mass Spectroscopy. } \\
\text { Aliquots of the sample digestates were prepared for U TIMS analysis by the ASO } \\
\text { using the separation procedure RPG-CMC-4017, Rev. 0, Analysis of Environmental } \\
\text { Water Samples for Actinides and Strontium-90. } \\
\text { The sample IDs and corresponding core sample IDs are provided. }\end{array}$} \\
\hline $\begin{array}{l}\text { Project } \\
\text { Sample ID }\end{array}$ & $\begin{array}{l}\text { RPL/ASO Sample ID- } \\
\text { Uranium Isotopic }\end{array}$ & $\begin{array}{l}\text { RPL/ASO Sample ID- } \\
\text { Plutonium Isotopic }\end{array}$ & K Basin Core Sample ID \\
\hline TI001-A1-B & 11- 0419-129-S-U & 11-0419-129-S-Pu & KW210-A1 \\
\hline TI001-A1-C & 11-0419 -129-D-U & 11-0419-129-D-Pu & KW210-A1 duplicate \\
\hline TI002-A4-B & 11-0420-129-S-U & 11-0420-129-S-Pu & KW210-A4 \\
\hline TI002-A4-C & 11-0420-129-D-U & 11-0420-129-D-Pu & KW210-A4 duplicate \\
\hline TI002-B1-B & 11-0421-129-S-U & 11-0421-129-S-Pu & KW210-B1 \\
\hline TI002-B1-C & 11-0421-129-D-U & 11-0421-129-D-Pu & KW210-B1 duplicate \\
\hline TI001-B3-B & 11-0422-129-S-U & 11-0422-129-S-Pu & KW210-B3 \\
\hline TI001-B3-C & 11-0422-129-D-U & 11-0422-129-D-Pu & KW210-B3 duplicate \\
\hline NA & 11-0419-129-PB-U & 11-0419-129-PB-Pu & ASO preparation blank \\
\hline
\end{tabular}

\section{Preparations and Analysis}

The Analytical Support Operations (ASO) was directed to provide purified sample fractions with sufficient analyte concentrations to create a measureable signal by Thermal Ionization Mass Spectrometry 
(TIMS). Therefore, the ASO preparation shall not be considered complete until satisfactory isotopic results are obtained. It is understood that the preparation blank (PB) may not have sufficient analyte concentration to meet this objective. The ASO does not provide a laboratory control sample (LCS) or a matrix spike (MS) sample.

TIMS-specific sample preparation, processing, and analyses shall be conducted according to PNNL98523-264, Mass Spectrometer Isotopic Analysis, Rev. 2. Procedure substitutions or procedure revision substitutions are not to be made without prior written authorization from SK Fiskum or CD Carlson and may result in the need to issue a K Basin Occurrence Report. Should insufficient analyte concentration be found to conduct an analysis, contact SK Fiskum and KA Pool, who will set up an additional sample purification process to be performed by the ASO.

\section{Quality Control}

The TIMS analysis processing will need to adhere to the QC defined in the technical procedure PNNL98523-264, Mass Spectrometer Isotopic Analysis, Rev. 2 and project-specific quality control as follows:

\begin{tabular}{|c|c|}
\hline \multirow{2}{*}{ Duplicates } & $\begin{array}{l}\text { The }{ }^{239} \mathrm{Pu},{ }^{240} \mathrm{Pu},{ }^{241} \mathrm{Pu} \text {, and }{ }^{242} \mathrm{Pu} \text { atom percents for a given core } \\
\text { sample and duplicate shall agree within } \pm 20 \% \text { where the isotopic } \\
\text { abundance is greater than } 1 \% \text {. }\end{array}$ \\
\hline & $\begin{array}{l}\text { The }{ }^{233} \mathrm{U},{ }^{234} \mathrm{U},{ }^{235} \mathrm{U},{ }^{236} \mathrm{U} \text {, and }{ }^{238} \mathrm{U} \text { atom percents shall agree within } \\
\pm 20 \% \text { for a given core and duplicate sample where the isotopic } \\
\text { abundance is greater than } 1 \% \text {. }\end{array}$ \\
\hline \multirow{2}{*}{$\begin{array}{l}\text { Reference } \\
\text { Standards }\end{array}$} & $\begin{array}{l}\text { At least one traceable reference standard must be analyzed at the } \\
\text { beginning of the analytical batch. The results for }{ }^{238} \mathrm{Pu},{ }^{239} \mathrm{Pu} \text {, } \\
{ }^{240} \mathrm{Pu},{ }^{241} \mathrm{Pu},{ }^{242} \mathrm{Pu} \text { atom percents shall be within } 6 \% \text { of the certified } \\
\text { value where the isotope atom percent of the certified value is }>0.1 \text {. }\end{array}$ \\
\hline & $\begin{array}{l}\text { Traceable reference standards must be analyzed at the beginning of } \\
\text { the analytical batch. The results for }{ }^{234} \mathrm{U},{ }^{235} \mathrm{U},{ }^{236} \mathrm{U} \text {, and }{ }^{238} \mathrm{U} \text { atom } \\
\text { percents shall be within } 2 \% \text { of the certified value where the isotope } \\
\text { atom percent of the certified value is }>0.1 \text {. }\end{array}$ \\
\hline
\end{tabular}

Note that the Reference Standard acceptance criteria identified in the QAPjP/SAP KBC33786, Rev. 2 were developed specifically for ${ }^{239} \mathrm{Pu}$ and ${ }^{240} \mathrm{Pu}( \pm 6 \%)$ and for ${ }^{235} \mathrm{U}$ and ${ }^{238} \mathrm{U}( \pm 2 \%)$. These criteria are conservatively applied to other (minor) isotopes in the Standard Reference Material (SRM) is where the minor isotope concentration exceeds 0.1 atom $\%$.

Note: The TIMS analysis staff must inform/report QC failures promptly to the Cognizant Scientists with copy to CD Carlson and the K Basin QE (Deborah Coffey), thus enabling PNNL to fulfill its commitment to the client for prompt notification of such failures. The impact of any QC failure on data quality or project schedule (e.g., re-runs) will be assessed in conjunction with the client. 


\section{TIMS Reporting}

Report ${ }^{238} \mathrm{Pu},{ }^{239} \mathrm{Pu},{ }^{240} \mathrm{Pu},{ }^{241} \mathrm{Pu}$, and ${ }^{242} \mathrm{Pu}$ atom percents for each sample in a tabular format.

Report ${ }^{233} U,{ }^{234} U,{ }^{235} U,{ }^{236} U$, and ${ }^{238} U$ atom percents for each sample in a tabular format.

Provide a report with the following elements: a data report Cover Page, a Narrative, the Data

Summary, the signed Analysis Request Form and the Supporting Data.

Data Report Cover Page Contents (see 52578-ADM-001 K Basin Requests for Analysis and Guidelines for Data Reporting and Technical, QE, and Spreadsheet Reviews and Attachment 1 for an example):

- Header identifying name/address of laboratory

- Client Name:

- $\quad$ Project \#/WP \#(s):

- Total \# Samples:

- Report Date:

- Report Revision Date: when applicable

- Filename(s): where data files can be accessed

- Dates of Sample Analysis:

- Procedure(s): Number and title (sample processing and analysis) and revision number

- M\&TE (serial number or property number):

- Reference Date (e.g., sample run date):

- Client ID and RPL/ASO ID Sample Numbers:

- Report Review and Approval:

Preparer Date

(Printed name and signature of responsible individual)

Technical Review Date (Printed name and signature of technical reviewer)

Quality Engineer Review Date (Printed name and signature of quality engineering reviewer)

Project Manager Review Date (Printed name and signature of Project Management reviewer)

\section{Narrative Contents:}

- Sample Description and Analyses

- Sample dissolution/preparation dates

- Sample Preparation

- Analysis dates

- Deviations from the written procedure, if any

- Method specific Sample Preparation, Separation, Mounting and Counting Methods as applicable

- Reporting Basis/Units

- QC Criteria/Results

- Limitations of the Data Interferences/Resolution Uncertainty

- Comments 
Data Summary Contents: (This is a tabular summary with the following components)

- Client ID

- RPL ID with full ASO-related suffixes (e.g., 129-S)

- TIMS ID if used

- Sample results and result uncertainties

- Standard results and result uncertainties

\section{Supporting Data}

- Standards certificates

- Signature and date of person who performed the test and recorded the data

- Analytical bench sheets or copies of LRB pages (including evidence of technical review)

- Hand/spreadsheet calculation review documentation

- Computer printouts of the raw data

Technical reviewers are required to be staff other than the analyst performing the work and knowledgeable of the area being reviewed. The technical review shall be completed and all reviewer issues resolved before reporting final results. Technical reviewers must receive a complete data package including narratives for review. A technical review consists of the following elements:

- Evaluation of method and QC performance

- Evaluation of compliance with technical (including procedural) and QC requirements, as defined in the ASR

- Verification of transcription accuracy

- Check for correctness of calculations

- Assuring the correct reporting units (and reference dates) are used

- Evaluation of overall consistency and reasonableness of data

- Evidence of implementation of appropriate corrective action, when necessary.

Analytical results shall be reported both in hard copy and electronically (including excel data files to support accurate data transcription into project records). Appropriately marked "Preliminary" data reports and electronic files shall be provided as soon as practical after completion of analysis. 
Attachment 1. Example Data Reporting Cover Sheet

\title{
Plutonium and Uranium Isotopic Analysis for KW210 Core Samples
}

\author{
Battelle-Pacific Northwest National Laboratory \\ Radiochemical Sciences and Engineering Group \\ PO Box 999, Richland, Washington 99352 \\ Project: \#52578
}

Client: SK Fiskum

Sample ID's:

\begin{tabular}{|l|l|l|l|}
\hline $\begin{array}{l}\text { Project } \\
\text { Sample ID }\end{array}$ & $\begin{array}{l}\text { RPL/ASO Sample ID- } \\
\text { Uranium Isotopic }\end{array}$ & $\begin{array}{l}\text { RPL/ASO Sample ID- } \\
\text { Plutonium Isotopic }\end{array}$ & K Basin Core Sample ID \\
\hline TI001-A1-B & $11-0419-129-S-U$ & $11-0419-129-S-P u$ & KW210-A1 \\
\hline TI001-A1-C & $11-0419-129-D-U$ & $11-0419-129-D-P u$ & KW210-A1 duplicate \\
\hline TI002-A4-B & $11-0420-129-S-U$ & $11-0420-129-S-P u$ & KW210-A4 \\
\hline TI002-A4-C & $11-0420-129-D-U$ & $11-0420-129-D-P u$ & KW210-A4 duplicate \\
\hline TI002-B1-B & $11-0421-129-S-U$ & $11-0421-129-S-P u$ & KW210-B1 \\
\hline TI002-B1-C & $11-0421-129-D-U$ & $11-0421-129-D-P u$ & KW210-B1 duplicate \\
\hline TI001-B3-B & $11-0422-129-S-U$ & $11-0422-129-S-P u$ & KW210-B3 \\
\hline TI001-B3-C & $11-0422-129-D-U$ & $11-0422-129-D-P u$ & KW210-B3 duplicate \\
\hline NA & $11-0419-129-P B-U$ & $11-0419-129-P B-P u$ & ASO preparation blank \\
\hline
\end{tabular}

Total Samples: 18

Procedure: PNNL-98523-264, Mass Spectrometer Isotopic Analysis, Rev. 2

Analyst:

Analysis (Reference) Dates:

Instrument: Manufacturer, Model, Unique Identifier (property or serial number), Instrument Software (name, version, date)

Electronic Report File name: File name and file location

Prepared By: \{type name here $\}$ / Date

Technical Review By:\{type name here\} / Date

QE Review By: \{type name here $\}$ / Date

Project Management Release / Date

Page 6 of 6

E. 8 
Battelle PNNL/RPL/ASO Radiochemistry Analysis Report

P.O. Box 999, 902 Battelle Blvd., Richland, Washington 99352

\section{Thermal Ionization Mass Spectrometry Sample Preparation}

\begin{tabular}{|l|l|}
\hline Project /WP\#: & $52578 / \mathrm{H} 41216$ \\
\hline ASR\#: & 8798 \\
\hline Client: & S. Fiskum \\
\hline Total \# of Samples: & 4 \\
\hline
\end{tabular}

Revision 1

\begin{tabular}{|c|c|c|}
\hline Engineered Container \& Core ID & Sample IDs & RPL Number \\
\hline KW-210-A1 & TI001-A1-B, -A1-C & $11-0419-129-\mathrm{S},-129-\mathrm{D}$ \\
\hline KW-210-A4 & TI002-A4-B, -A4-C & $11-0420-129-\mathrm{S},-129-\mathrm{D}$ \\
\hline KW-210-B1 & TI002-B1-B, -B1-C & $11-0421-129-\mathrm{S},-129-\mathrm{D}$ \\
\hline KW-210-B3 & TI001-B3-B, -B3-C & $11-0422-129-\mathrm{S},-129-\mathrm{D}$ \\
\hline
\end{tabular}

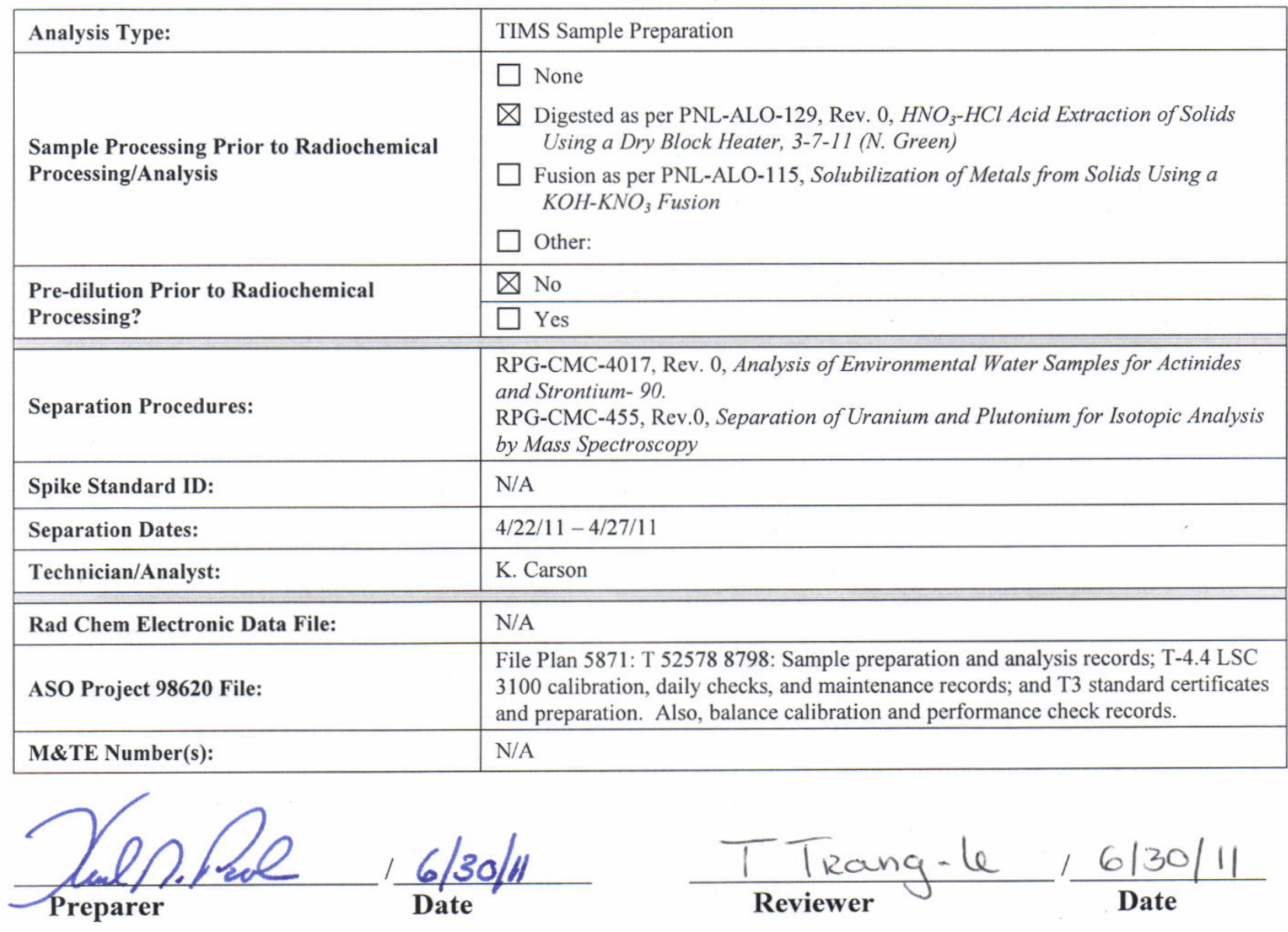




\section{Battelle PNNL/RPL/ASO Radiochemistry Analysis Report}

\section{$\underline{\text { Revision } 1}$}

This revision is being issued to correct the procedure references for the $\mathrm{Pu}$ and $\mathrm{U}$ Thermal Ionization Mass Spectrometry (TIMS) preparations.

\section{Sample preparation and separation}

Four K-Basin sludge samples were prepared in the Shielded Analytical Laboratory (SAL) hot cells by the Project and provided to the Analytical Support Operations (ASO) under Analytical Service Request (ASR) 8798 for chemical and radioanalytical analyses. ASO received each sample in a Teflon Oak Ridge centrifuge tube, dried the contents of each centrifuge tube to a constant weight, and then leached the dried solids with hot dilute nitric and hydrochloric acids per procedure PNL-ALO-129, Rev. 0, $\mathrm{HNO}_{3}-\mathrm{HCl}$ Acid Extraction of Solids Using a Dry Block Heater. This digestion process was conducted in the SAL hot cells. The leachate solution was used for radioanalytical analysis, ICP metals analysis and isotopic distribution of Pu and U by (TIMS); only TIMS sample preparation is discussed in this report.

Analytical Support Operations (ASO) performed the initial chemical separation for sample aliquouts that are transferred to the TIMS for isotopic ratio determinations from the PNL-ALO-129 digests. The text below provides a description of the chemical separation performed on samples for TIMS analyses.

The ASO utilizes procedure RPG-CMC-4017, Rev. 0, Analysis of Environmental Water Samples for Actinides and Sr-90 to obtain the uranium chemical fraction used for TIMS analyses. The uranium is chemically separated using ion exchange chromatography; the uranium chemical fraction is collected and then concentrated in Teflon conical vials. The uranium fraction samples are then sent to the TIMS laboratory for analysis.

The ASO utilizes procedure RPG-CMC-455, Rev. 0, Separation of Uranium and Plutonium for Isotopic Analysis by Mass Spectrometry to obtain the plutonium chemical fraction used for TIMS analysis. The plutonium is chemically separated using ion exchange chromatography; the plutonium chemical fraction is collected and then concentrated in Teflon conical vials. The plutonium fraction samples are then sent to the TIMS laboratory for analysis.

\section{Preparation Blank}

A preparation blank (PB) was included in the SAL digestion of the K-Basin sludge samples and the PB sample was also processed for Pu and U TIMS. The aliquot size used for the preparation blank was 1.0 $\mathrm{mL}$ of undiluted digestate while the aliquot sized used for the samples was $0.1 \mathrm{~mL}$ of the undiluted digestate. 
PNNL-20650, Rev. 2

\title{
Plutonium and Uranium Isotopic Analysis for KW210 Core Samples
}

\author{
Battelle-Pacific Northwest National Laboratory \\ Radiochemical Sciences and Engineering Group \\ PO Box 999, Richland, Washington 99352 \\ Project: \#52578
}

July 15, 2011

Sample ID's:

\begin{tabular}{|c|l|l|l|}
\hline Project Sample ID & ASO Sample ID & ASO Sample ID & Sample Description \\
\hline TI001-A1-B & $11-0419-129-S-U$ & $11-0419-129-S-P u$ & KW210-A1 \\
\hline TI001-A1-C & $11-0419-129-D-U$ & $11-0419-129-D-P u$ & KW210-A1 duplicate \\
\hline TI002-A4-B & $11-0420-129-S-U$ & $11-0420-129-S-P u$ & KW210-A4 \\
\hline TI002-A4-C & $11-0420-129-D-U$ & $11-0420-129-D-P u$ & KW210-A4 duplicate \\
\hline TI002-B1-B & $11-0421-129-S-U$ & $11-0421-129-S-P u$ & KW210-B1 \\
\hline TI002-B1-C & $11-0421-129-D-U$ & $11-0421-129-D-P u$ & KW210-B1 duplicate \\
\hline TI001-B3-B & $11-0422-129-S-U$ & $11-0422-129-S-P u$ & KW210-B3 \\
\hline TI001-B3-C & $11-0422-129-D-U$ & $11-0422-129-D-P u$ & KW210-B3 duplicate \\
\hline NA & $11-0419-129-P B-U$ & $11-0419-129-P B-P u$ & ASO preparation blank \\
\hline
\end{tabular}

Procedure: PNNL-98523-264 Rev. 2, Mass Spectrometer Isotopic Analysis

Analyst: Stan Bos

Analysis Dates: U-TIMS 5/6/11 and 5/7/2011, Pu-TIMS 5/18 and 5/25/2011

Instrument: CEC, model 21-703 (M\&TE\# 467806) Property \# WB76849, Instrument Software Isoto_26.prg Rev. A, (3/2000)

Electronic Report File name: https://pnlweb.pnl.gov/projects/KBasin_2lPlutonium and Uranium Isotopic Analysis for KW210 Core Samples

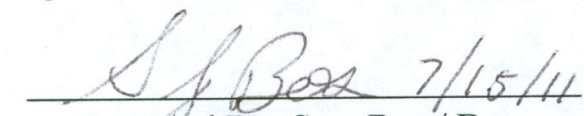

Prepared By: Stan Bos / Date

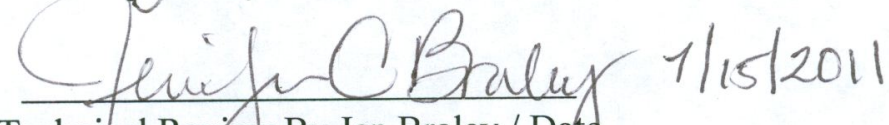

Technical Review By Jen Braley / Date

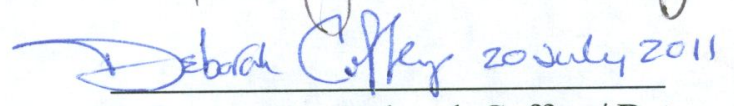

QE Review By: Deborah Coffey / Date

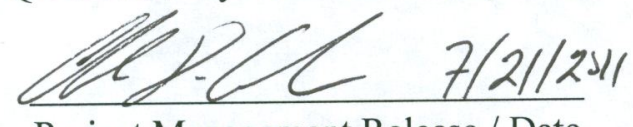

Project Management Release / Date

E.11 


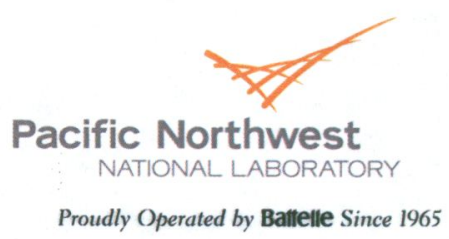

Project No:

Internal Distribution: File/LB

To: Sandy Fiskum

Stan Bos

Subject:

$$
\mathrm{Pu} \text { and } \mathrm{U} \text { Isotopic analysis }
$$

Samples of K-Basin sludge material were analyzed for uranium and plutonium isotopic abundance by thermal ionization mass spectrometry (TIMS). Sample sets consisted of four samples each prepared in duplicate along with one preparation blank. Samples were delivered on May 3, 2011 in two sets; one containing the uranium fraction and the second containing the plutonium fraction.

The uranium preparations arrived at the mass spectrometer lab in $20 \mathrm{cc}$ glass vials. One milliliter of nitric acid was added to each vial and $5 \mu \mathrm{L}$ of sample was pipetted and dried onto rhenium filaments for analysis. Uranium isotopic analysis began on May 3, 2011 running NIST uranium isotopic standard U-030 and continued on May 6 and 9, 2010. Seven samples were analyzed on May 6, 2011, but due to a temperature swing in the laboratory analysis had to be stopped where the mass spectrometer went out of calibration. Analyses were restarted on May 9, 2011 rerunning samples 11-0422-129SU, 11-420-129-D-U and running 11-0422-129-D-U. The uranium isotopic abundance results and are reported in Table 1.

Plutonium preparations were received either dry or with up to $30 \mu \mathrm{L}$ of solution in conical shaped Teflon vials. For the samples that were dry or nearly dry, $25 \mu \mathrm{L}$ of $4 \mathrm{M}$ nitric acid was added to the vial and $10 \mu \mathrm{L}$ of solution were pipetted and dried on a rhenium filament for analysis. Plutonium isotopic analysis was performed on May 17 and 18, 2011 running NIST isotopic standard Pu-946 before and after sample analysis. Three samples 11-0420-129-S-P, 11-0420-129-D-P, and 11-0421-129-D-P, had inconsistent results and were reanalyzed on May 25, 2011. Results of the analyses are reported in Table 3. Sample signal was adequate for the analyses but was not comparable to the signal strength of the standards. While the instrument was producing $\sim 10$ volt signals on Pu-946 standards, the samples were providing $\sim 1$ volt signals. It was noted that there was a buildup of residue on the filament which might have caused the signal to be depressed.

A preparation blank was included with each set of samples. In both sample sets the preparation blank arrived dry and were diluted the same as the associated samples. Five microliters of the preparation blank for uranium and $10 \mu \mathrm{L}$ for plutonium was loaded onto the rhenium filament and installed in the mass spectrometer. For both the $U$ and Pu preparation blanks, there was not adequate signal at the major analyte mass to 
PNNL-20650, Rev. 2

Sandy Fiskum

June 15, 2011

Page 2

warrant an attempt at isotopic analysis. Preparation blanks are reported as not detected (ND) for all isotopes.

Separations:

In preparation for TIMS analyses, the plutonium and uranium were chemically separated by Analytical Support Operations (ASO) staff using ion exchange procedures RPG-CMC-4017 (Rev 0) for uranium and RPG-CMC-455 (Rev 0) for the plutonium. Separations procedures were run concurrently on April 22, 2011. These procedures segregate the uranium and plutonium into separate and purified fractions. This process is necessary to eliminate mass interferences common to both elements (e.g., mass 238 as $\mathrm{Pu}$ and $\mathrm{U}$ ) as well as to reduce other materials that otherwise would create mass loading on the filament. The PNL-ALO-129 acid digestion preparation blank (generated during processing in the hot cells) was also prepared for TIMS in parallel with the samples

Analysis:

The mass spectrometer is designed to analyze one sample at a time. Samples arrive at the mass spectrometry laboratory in either $20 \mathrm{cc}$ glass vials or $5 \mathrm{cc}$ conical Teflon vials. Depending on the condition of the sample they may be diluted in nitric acid or may be run without dilution. A portion of the sample solution is pipetted onto a rhenium filament then taken to dryness by electrically heating the filament. Samples are then loaded into the mass spectrometer for analysis.

TIMS measurements are made with the instrument cycling through each isotope analyte, taking measurements at each stop and averaging the results at a given mass. This routine is repeated 9 times calculating the isotope ratios after each cycle. An average isotope ratio is computed at the end of the analytical routine. From the isotopic ratios, atom percent and mass percent are calculated. Estimates of uncertainty are calculated from the standard deviation of the isotopic ratios for the minor peaks. For the major peak the uncertainty is calculated from the sum of the uncertainties in minor peaks.

Isotopic abundance analysis was performed using the CEC 21-703 thermal ionization mass spectrometer (M\&TE 467806) following procedure PNNL-98523-264 (Rev 2). A record of the analyses was recorded in laboratory record book 56999 pages 77 and 78 . Samples were assigned RPL numbers 11-0419 through 11-0422. Analytical requests forms were prepared for the $\mathrm{U}$ and $\mathrm{Pu}$ TIMS analyses and are attached to this report. Work package $\mathrm{H} 41217$ has been charged for this analysis. 
PNNL-20650, Rev. 2

Sandy Fiskum

June 15, 2011

Page 3

Calibration:

Instrument performance checks for uranium isotopic analysis are made using an isotopic reference standard supplied by NIST. Uranium isotopic standard U-030 was analyzed on May 3, 2011 to verify the instrument output and testing the mass discrimination correction. No adjustment was made to the mass discrimination correction. Standards were analyzed on May 6, and 9, 2011 with a standard being run before the samples on May 6 and before and after samples on May 9, 2011. Results of 5 runs of $\mathrm{U}-030$ are reported and averaged in the uranium standards Table 2 . The performance criterion for the TIMS instrument is agreement of $\pm 2 \%$ for the major ( $>0.1$ atom percent) isotopes to the certified standard. Pooled averages for all U-030 standard runs met the $\pm 2 \%$ criteria for the major isotopes, $\mathrm{U}^{235}$ and $\mathrm{U}^{238}$, but did not meet the criteria for minor isotope $\mathrm{U}^{236}$ where the percent difference was $-2.66 \%$. Occurrence Report 52578-05-09-11 has been issued to address the impact of reference standard failure.

Instrument performance checks for plutonium isotopic analysis are made using NIST Pu-946 isotopic reference standard. Prior to starting sample analysis on May 17, 2011 three runs of Pu-946 were made to verify the instrument output and the mass discrimination setting. No adjustment was made to the mass discrimination correction. Standards were also analyzed before and after samples on May 18 and 25, 2011. Results of nine Pu-946 analyses are reported in the Pu isotopic standards Table 4. The performance criterion for the TIMS analysis is agreement of $\pm 6 \%$ for the major $(>0.1$ atom percent) isotopes. This criterion was met for all isotopes.

Relative percent difference of duplicate samples:

Duplicate preparations were analyzed and relative percent differences of the results were calculated. A performance agreement of $\pm 20 \%$ was requested. This criterion was met for the major isotopes of uranium but was not attained for isotope U-234 on samples 11-0422-129-S-U and -D-U. The \pm 20 RPD was not attained on the U-233 isotope likely due to the low abundance of the isotope in the sample. Performance agreement for Pu-241 was not met on one set of duplicate pairs (TI001-A1-B and TI001-A1-C at 27\% RPD). Per the analysis request, duplicate precision criteria did not apply to $\mathrm{Pu}^{238}$ because of the recognition that the $\mathrm{U}^{238}$ isobar may interfere (despite efforts to create a clean Pu-U separation by the ASO. The QAPjP/SAP KBC-33786, Rev. 2 Tables 3-7 indicates that the duplicate precision only applies to major isotopes where a "major isotope" was defined as isotope abundance greater than $1 \%$ of the total sample. The $\mathrm{U}^{233}, \mathrm{U}^{234}, \mathrm{Pu}^{238}, \mathrm{Pu}^{241}$ and $\mathrm{Pu}^{242}$ were each present at $<0.7$ atom\%, therefore the precision criterion does not apply to these isotopes. Occurrence Report 52578-05-09-11 has been issued to address the impact of duplicate sample RPD failures. 
PNNL-20650, Rev. 2

Sandy Fiskum

June 15, 2011

Page 4

Sample Identification:

\begin{tabular}{|c|l|l|l|}
\hline Project Sample ID & ASO Sample ID & ASO Sample ID & Sample Description \\
\hline TI001-A1-B & $11-0419-129-S-U$ & $11-0419-129-S-P u$ & KW210-A1 \\
\hline TI001-A1-C & $11-0419-129-D-U$ & $11-0419-129-D-P u$ & KW210-A1 duplicate \\
\hline TI002-A4-B & $11-0420-129-S-U$ & $11-0420-129-S-P u$ & KW210-A4 \\
\hline TI002-A4-C & $11-0420-129-D-U$ & $11-0420-129-D-P u$ & KW210-A4 duplicate \\
\hline TI002-B1-B & $11-0421-129-S-U$ & $11-0421-129-S-P u$ & KW210-B1 \\
\hline TI002-B1-C & $11-0421-129-D-U$ & $11-0421-129-D-P u$ & KW210-B1 duplicate \\
\hline TI001-B3-B & $11-0422-129-S-U$ & $11-0422-129-S-P u$ & KW210-B3 \\
\hline TI001-B3-C & $11-0422-129-D-U$ & $11-0422-129-D-P u$ & KW210-B3 duplicate \\
\hline NA & $11-0419-129-P B-U$ & $11-0419-129-P B-P u$ & ASO preparation \\
\hline
\end{tabular}


PNNL-20650, Rev. 2
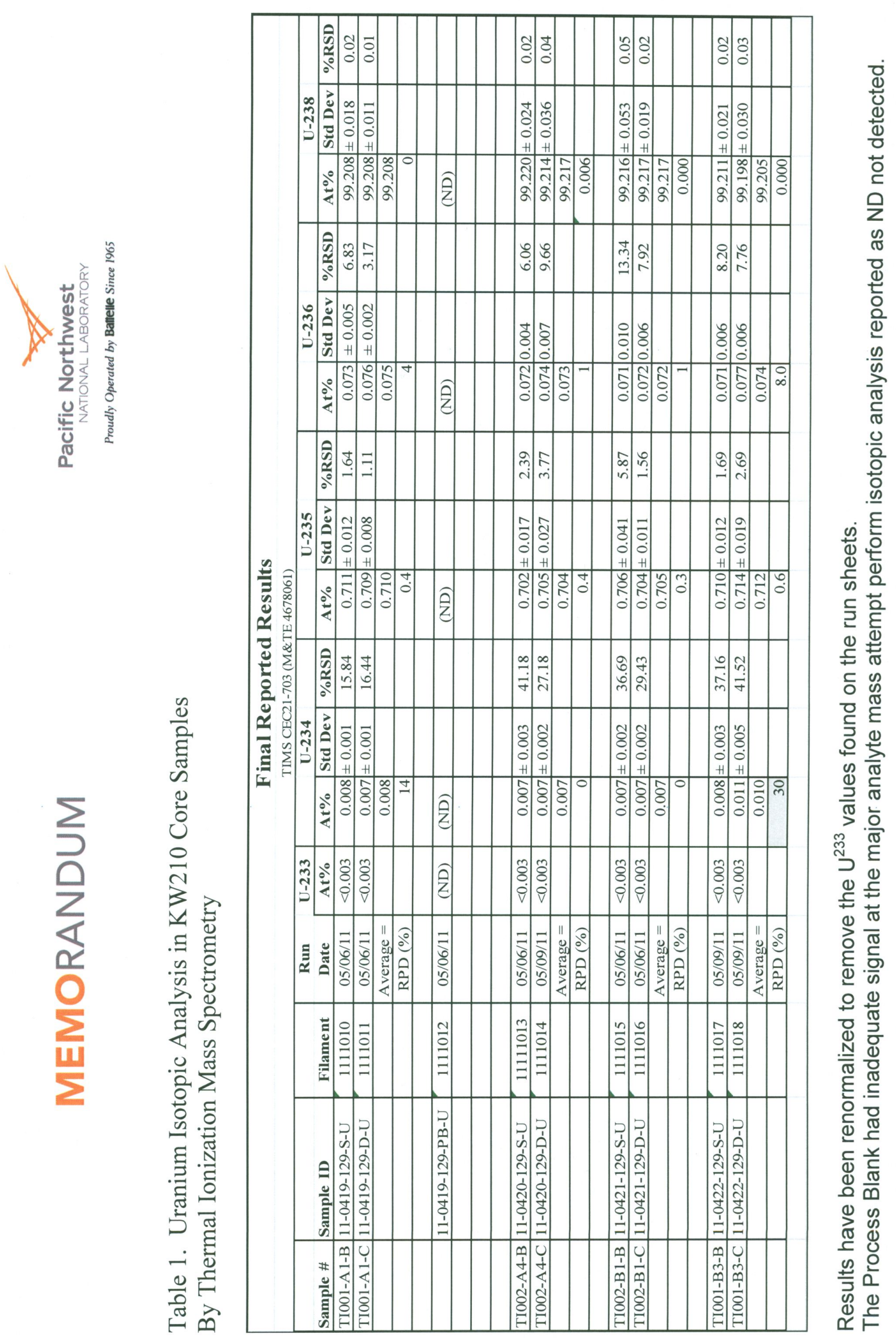

E.16 
PNNL-20650, Rev. 2

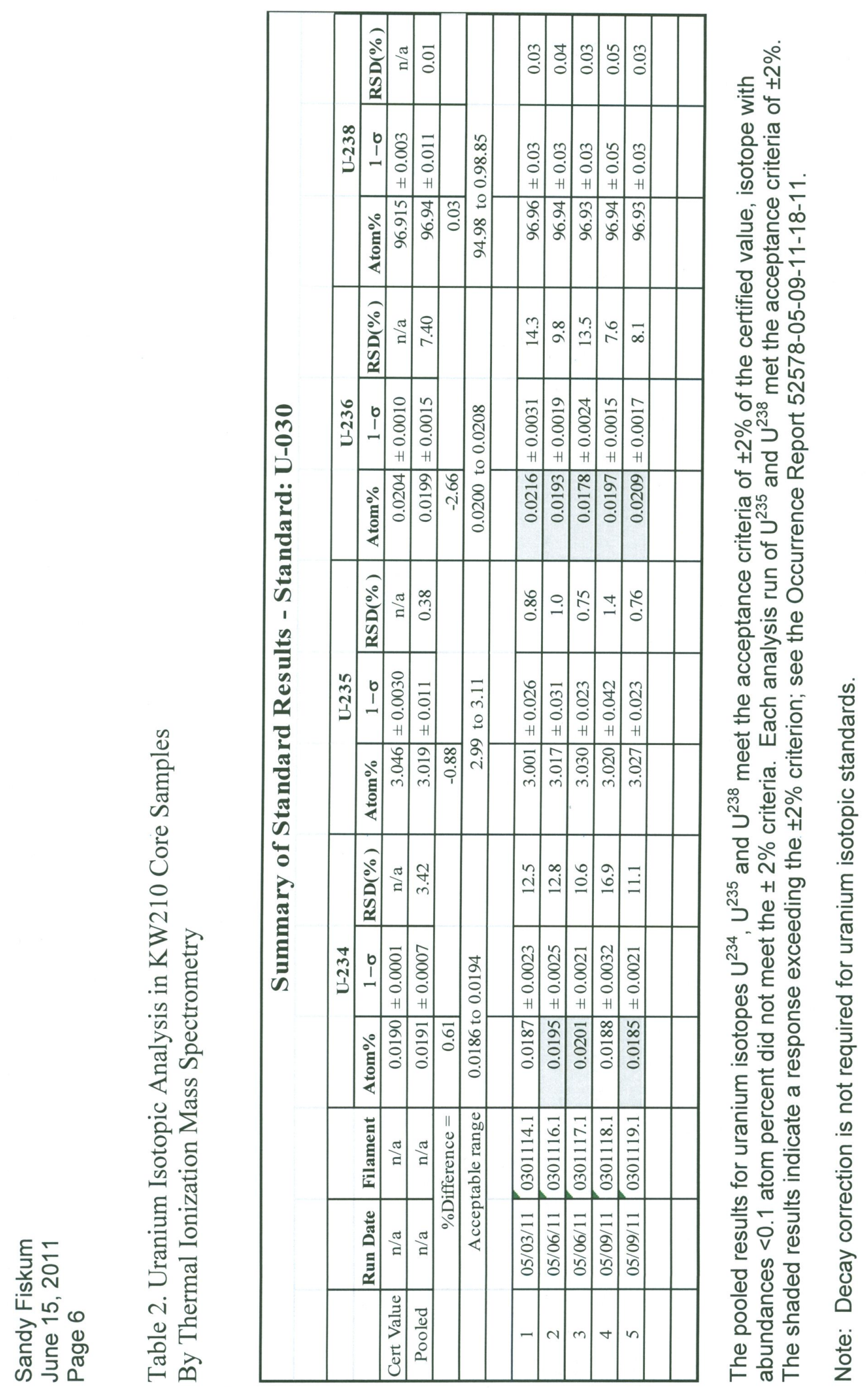


PNNL-20650, Rev. 2

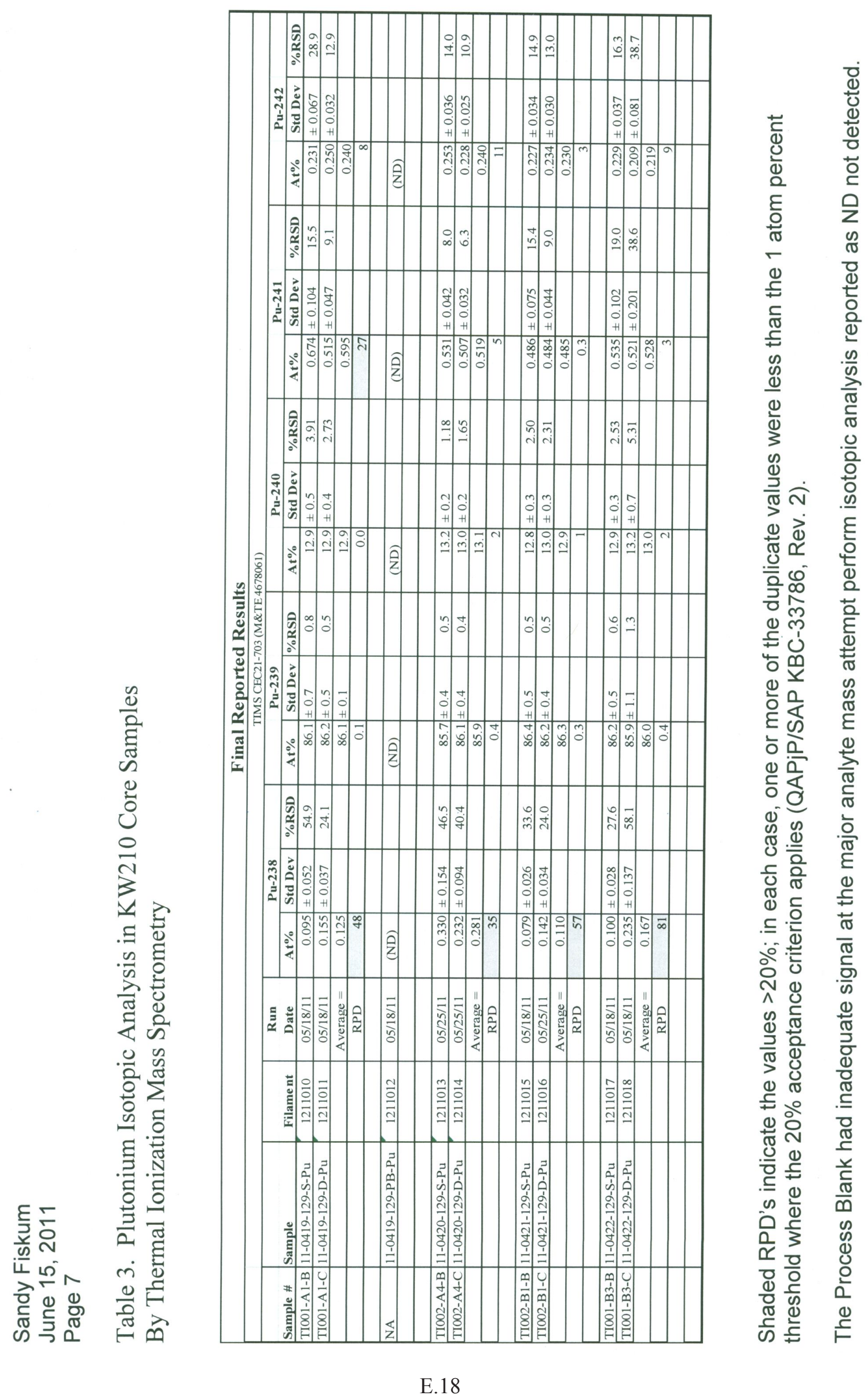


PNNL-20650, Rev. 2

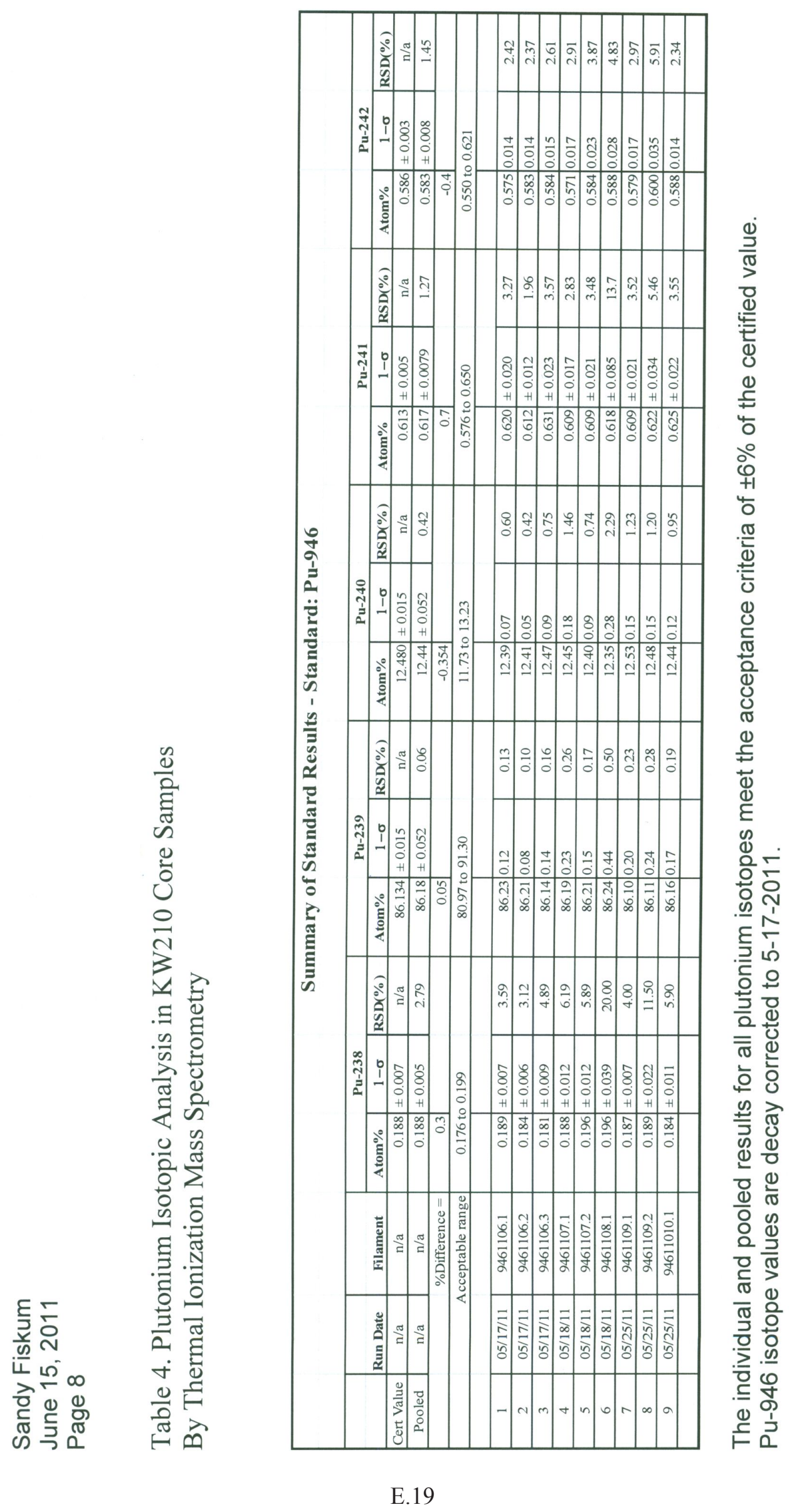




\section{RPL K Basin Project 52578}

\section{Document Review Record}

\section{Title: $\quad$ Plutonium and Uranium Isotopic Analysis for KW210 Core Samples}

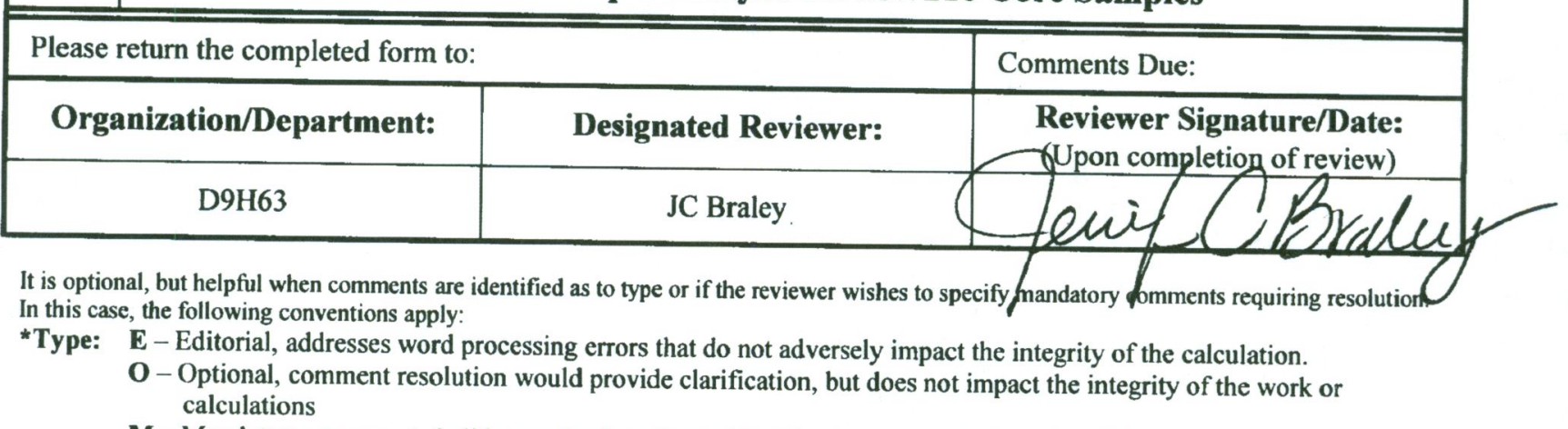

$\mathbf{M}$ - Mandatory, comment shall be resolved; reviewer identifies impact on the integrity of the work or calculations

\begin{tabular}{|c|c|c|c|c|}
\hline $\begin{array}{l}\text { Comment } \\
\text { Number }\end{array}$ & $\begin{array}{l}\text { Section/ } \\
\text { Page }\end{array}$ & Type & Comment & Comment Resolution \\
\hline 1 & $1 / 1$ & $\mathrm{E}$ & $\begin{array}{l}\text { A 'was' was present when it } \\
\text { should be a 'were' }\end{array}$ & cirrsident \\
\hline 2 & $1 / 1$ & $E$ & table 3 should be Table 3 & coureditel \\
\hline 3 & $1 / 2$ & $\mathrm{E}$ & $\begin{array}{l}\text { Period after one Rev and not the } \\
\text { other, should be stricken for } \\
\text { consistency }\end{array}$ & ond \\
\hline 4 & $1 / 3$ & $\mathrm{E}$ & Table $3-7$ should be Tables $3-7$ & Cones \\
\hline 5 & & & & $A$ \\
\hline 6 & & & & \\
\hline 7 & & & & \\
\hline 8 & & & & \\
\hline END & & & & \\
\hline
\end{tabular}

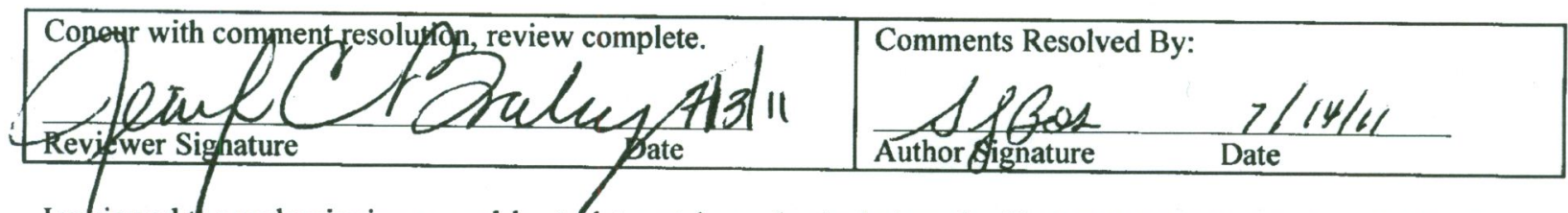

I refiewed the underpinning spreadsheet (data entries and calculations for file KW210 TIMS data June 2011.xls) and data incorporation to the report. Discussions in the report are accurate and the conclusions are sound. I also acknowledge that while Sandy Fiskum suggested the verbiage for this disclosure, I, Jenifer C. Braley, concur with this statement. 
PNNL-20650, Rev. 2

Title: TIMS Isotopic Data for KW Basin Core KW230 (?)

\begin{tabular}{|c|c|}
\hline Title: & TIMS Isotopic Data for KW Basin Core KW230 (?) \\
\hline File Name & \\
\hline Revision Number: & 1 \\
\hline Date Prepared: & $6 / 15 / 2011$ \\
\hline Prepared by: & Stan Bos \\
\hline $\begin{array}{r}\text { General Statement of } \\
\text { Purpose: }\end{array}$ & $\begin{array}{l}\text { Spreadsheet calculates renormalizes data when run cycles or isotopes must be } \\
\text { removed for the data reduction, uncertainties and presents the data in a format that } \\
\text { can be pasted into reports. }\end{array}$ \\
\hline \multicolumn{2}{|c|}{ General Description of Approach: } \\
\hline Urainium std & Uranium isotopic standards \\
\hline U TIMS & Isotope ratio analysis of the urainium fractions \\
\hline Final Results 1 & Report table for plutioium fractions \\
\hline ----------- & \\
\hline Plutonium std & Plutonium 946 isotope ratio standards \\
\hline Pu TIMS & Isotope ratio analysis of the plutonium fractions \\
\hline Final Results 2 & Report table for plutioium fractions \\
\hline
\end{tabular}


PNNL-20650, Rev. 2

$\frac{3}{3}$

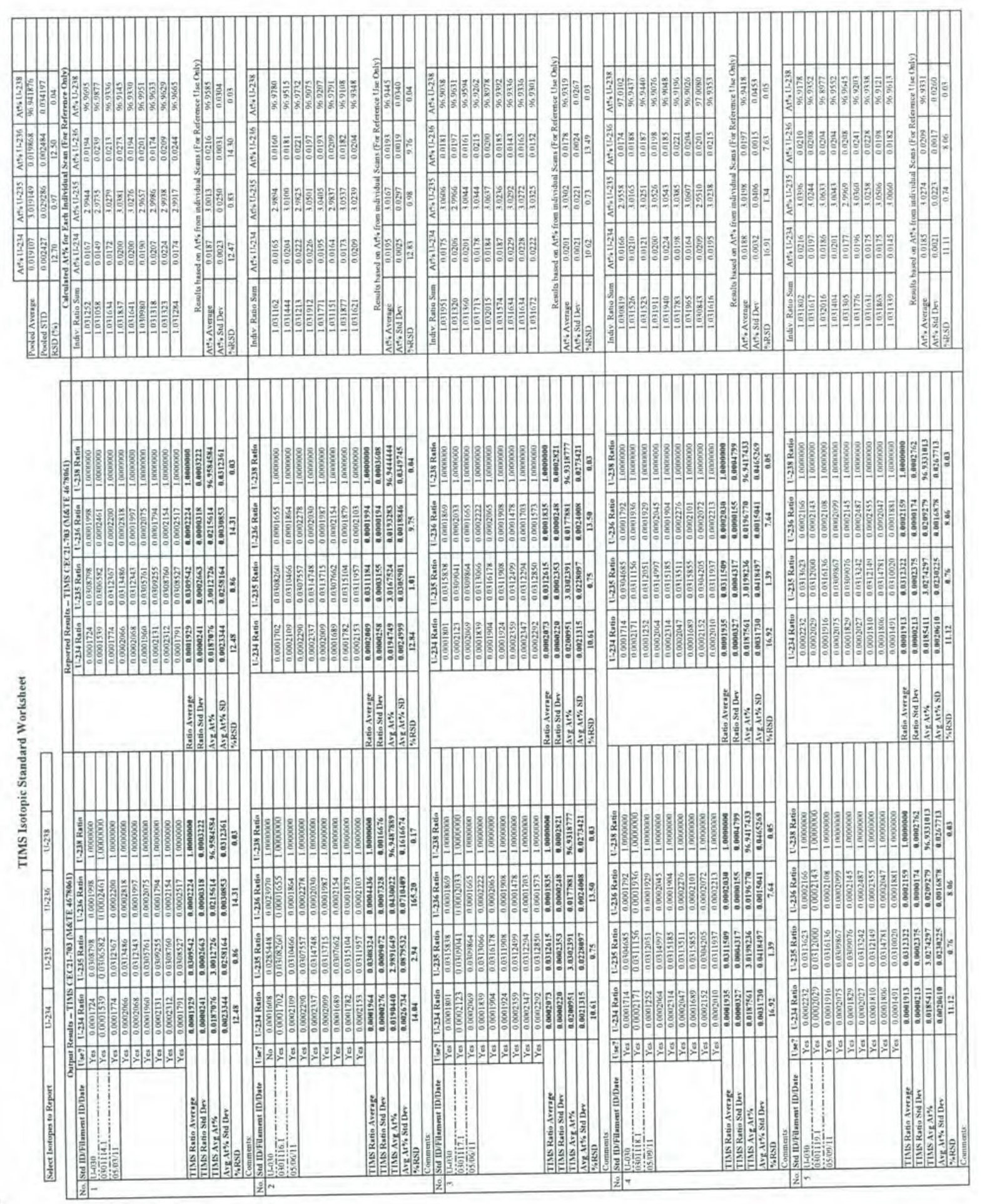

है 
PNNL-20650, Rev. 2

בे

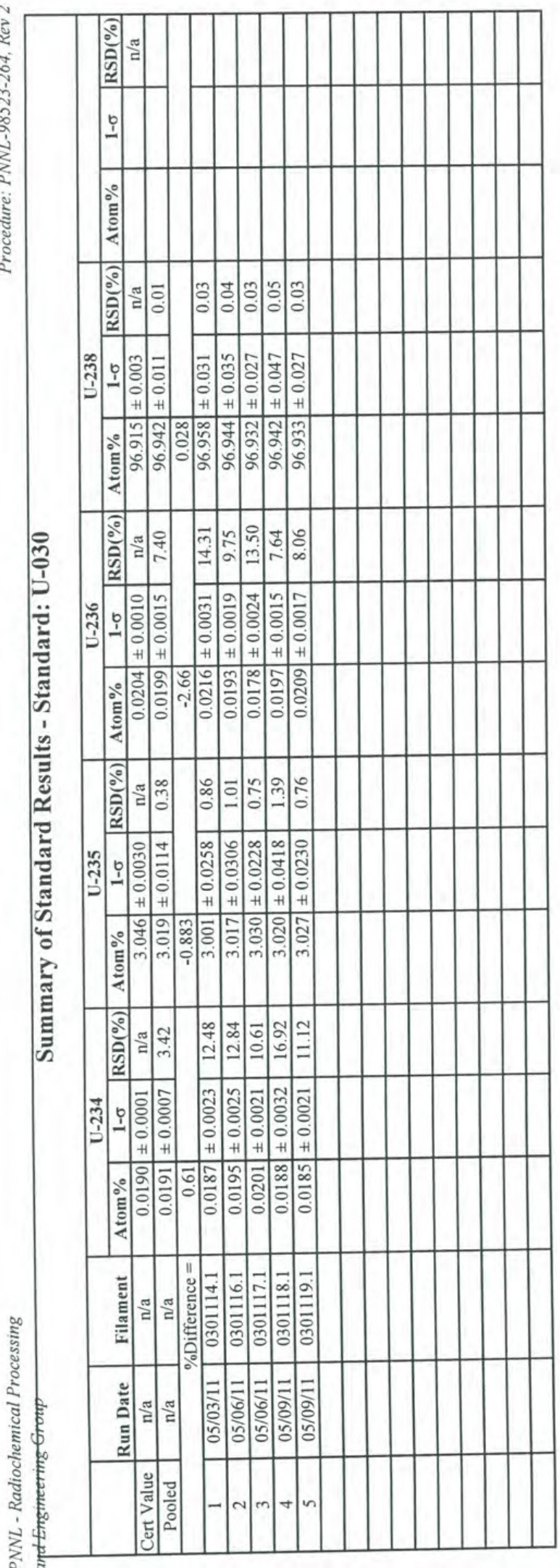


PNNL-20650, Rev. 2

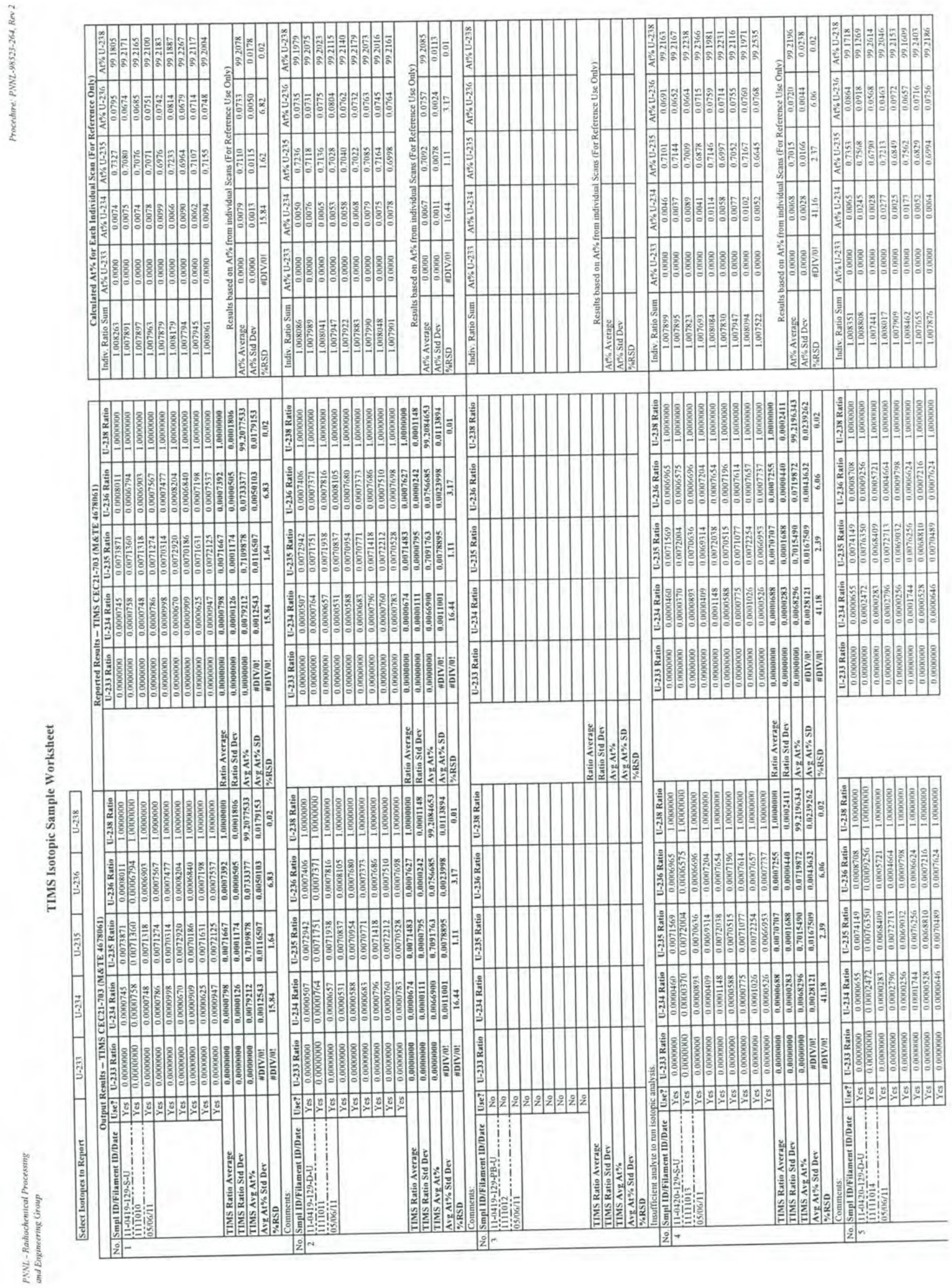


PNNL-20650, Rev. 2

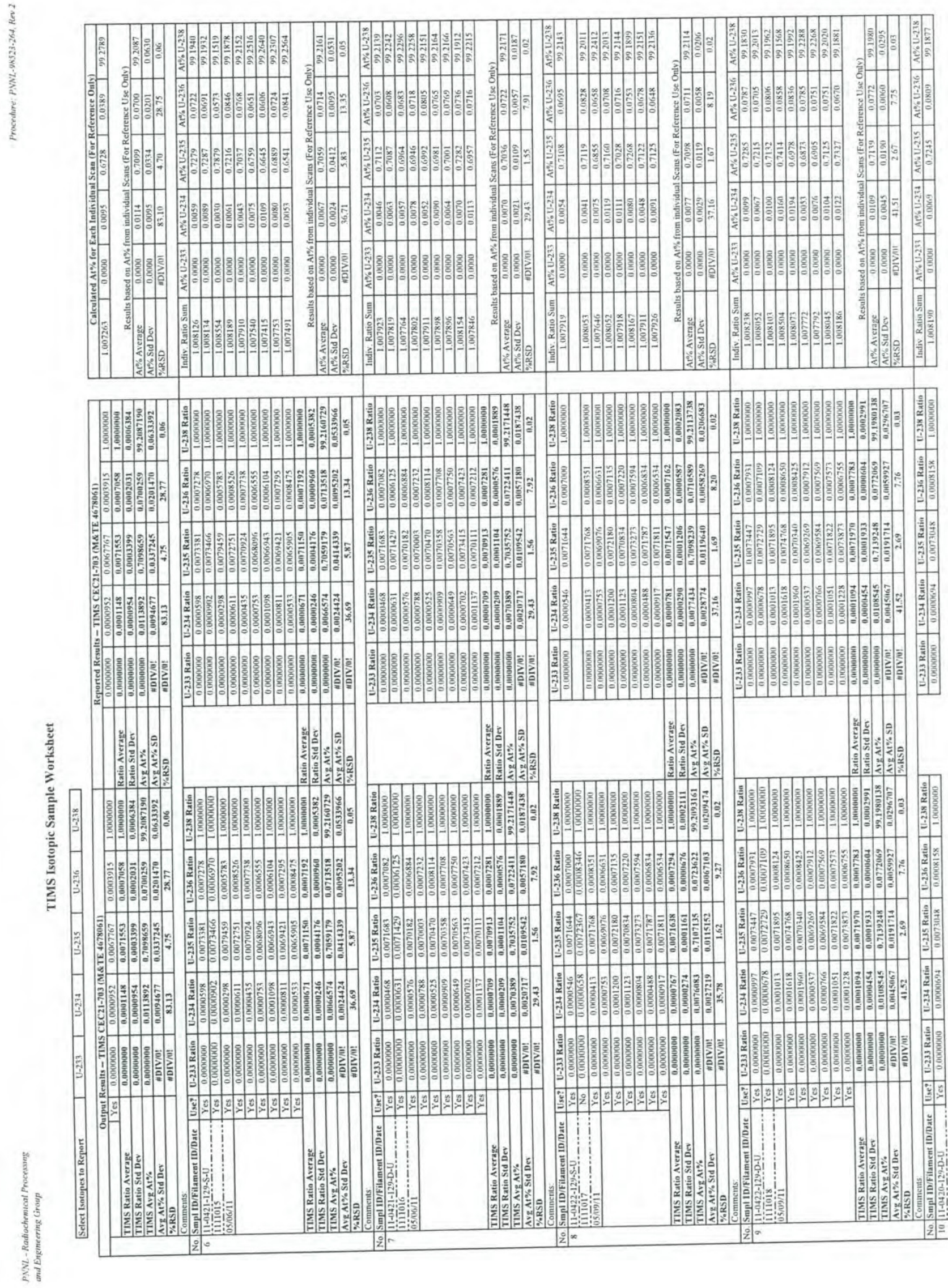


PNNL-20650, Rev. 2

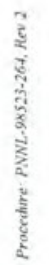
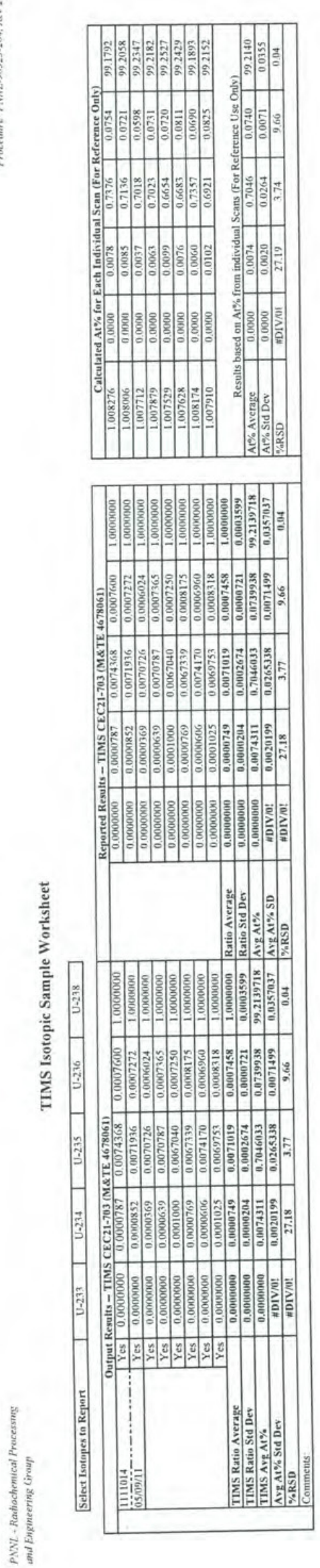

E.26

Puge 3of 3 
PNNL-20650, Rev. 2

है

(1)

: |

:

죠요:

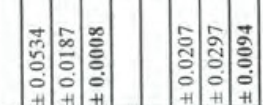

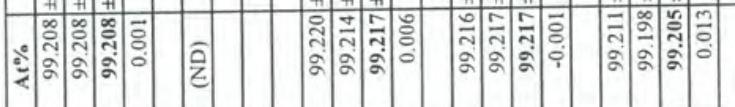

(2)

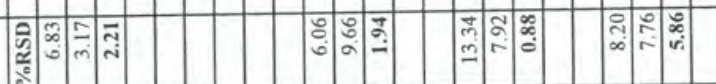

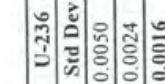

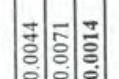

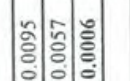

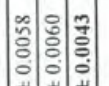

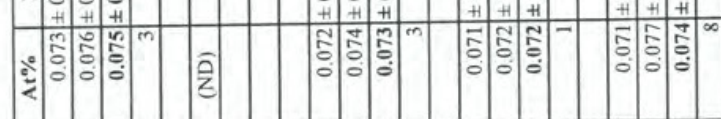

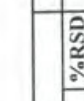

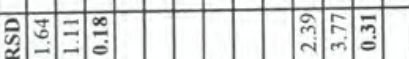

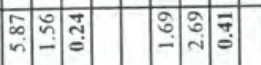

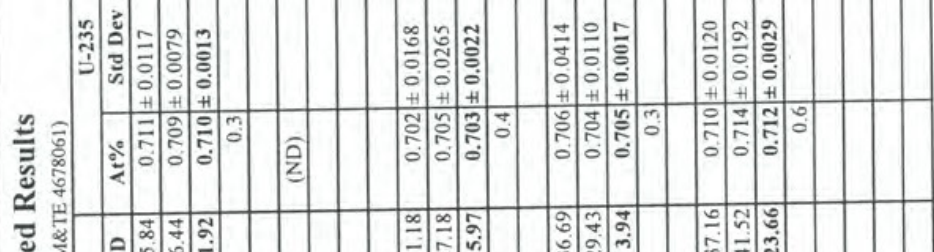

(1)

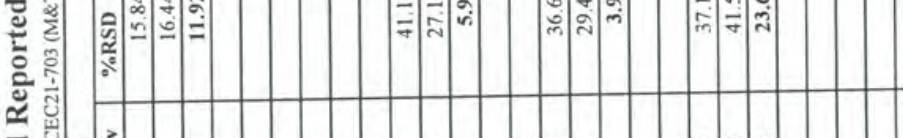

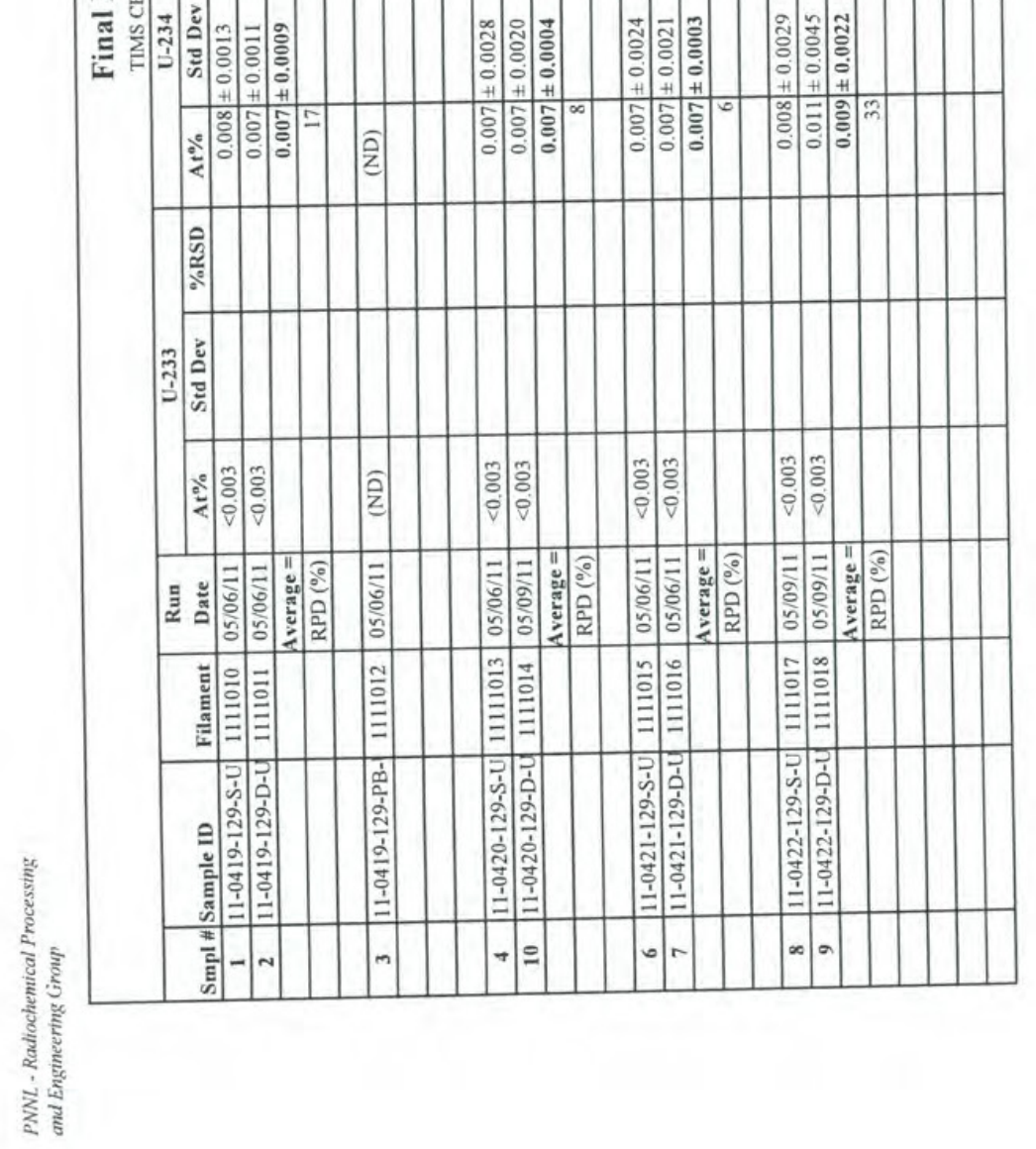

E. 27 
PNNL-20650, Rev. 2

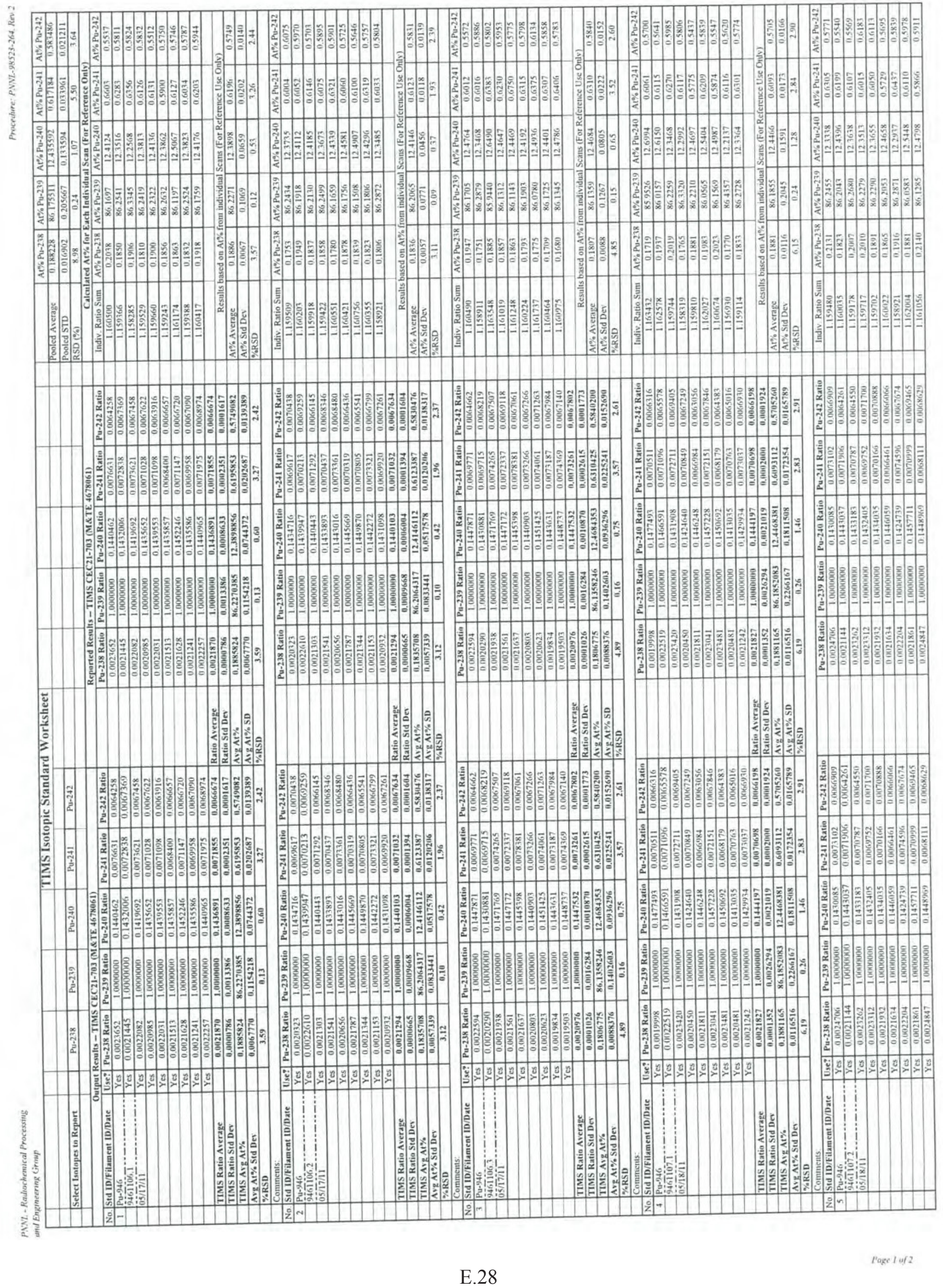


PNNL-20650, Rev. 2

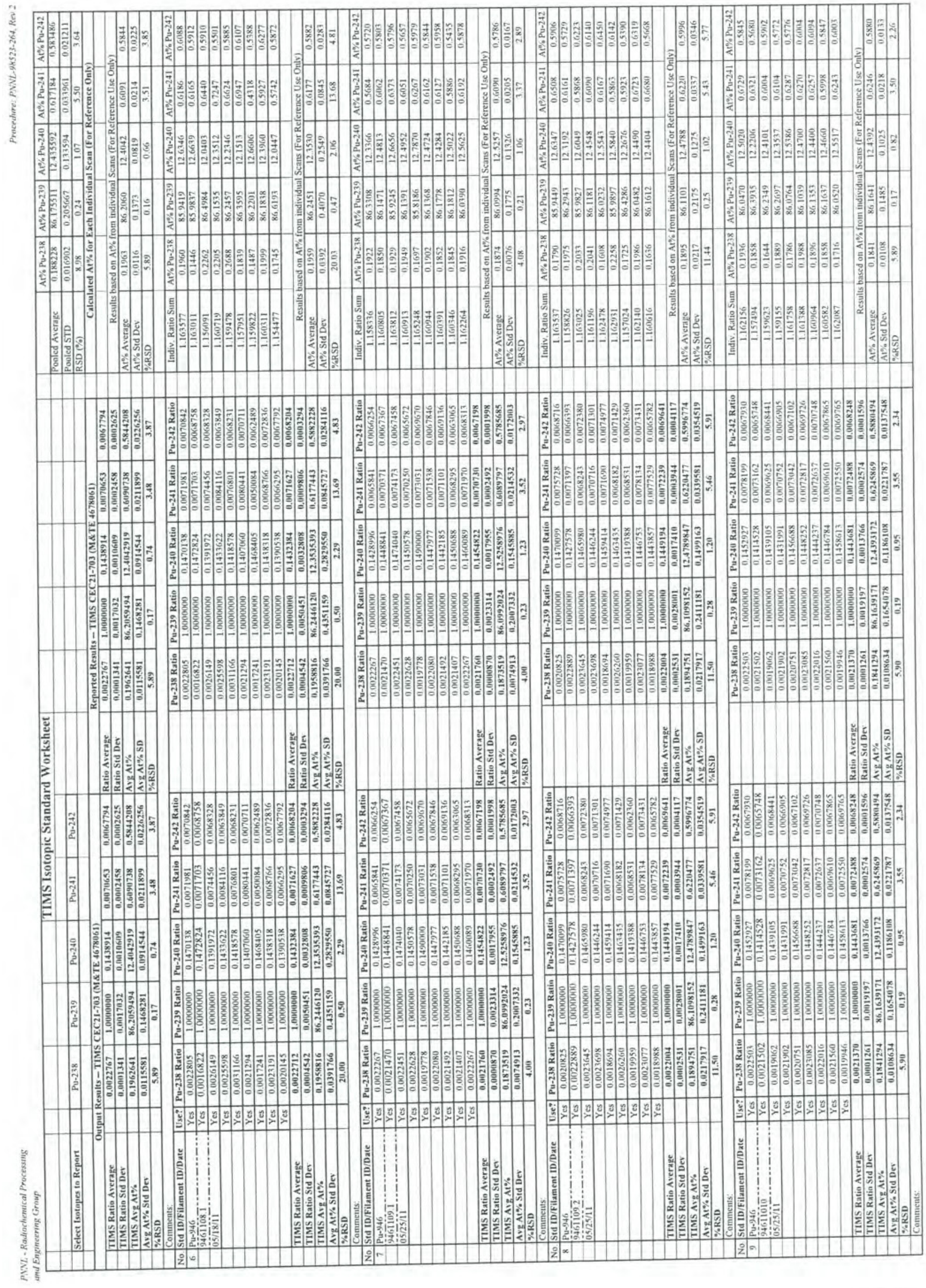


PNNL-20650, Rev. 2

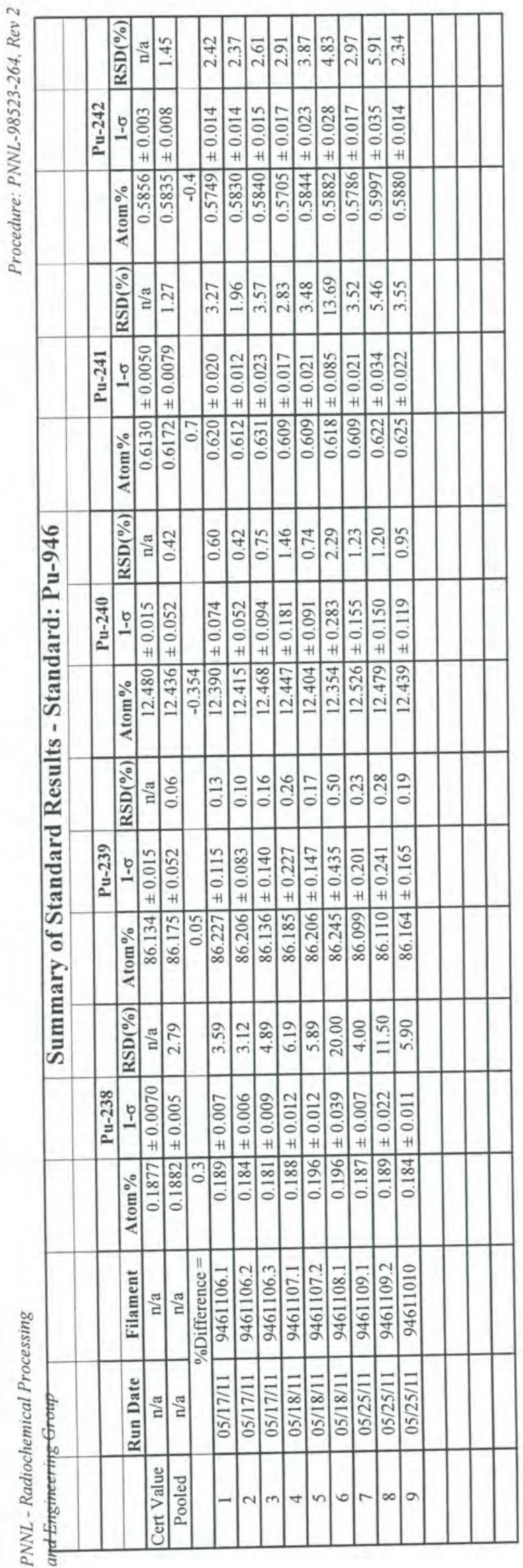

E.30 
PNNL-20650, Rev. 2

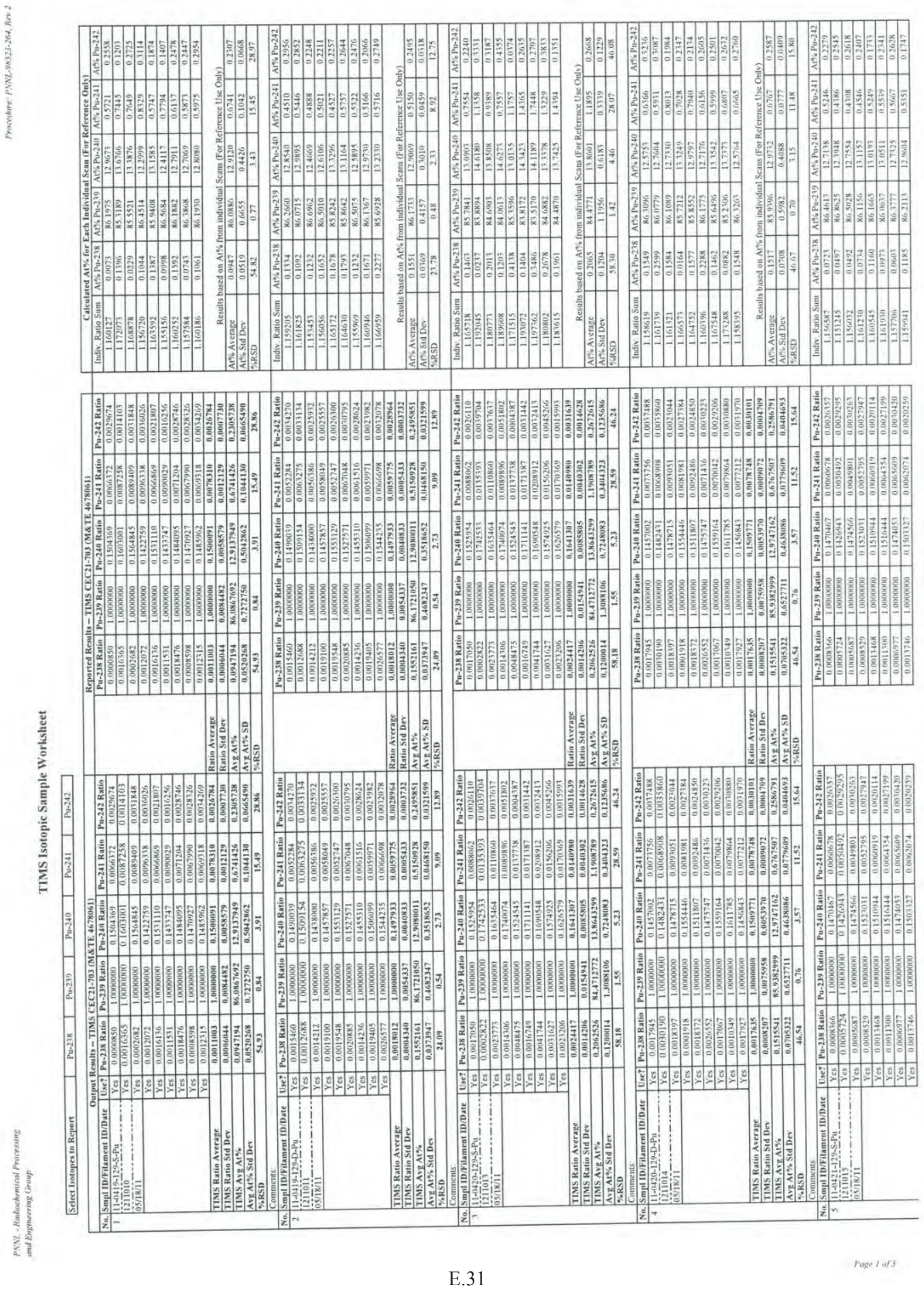


PNNL-20650, Rev. 2

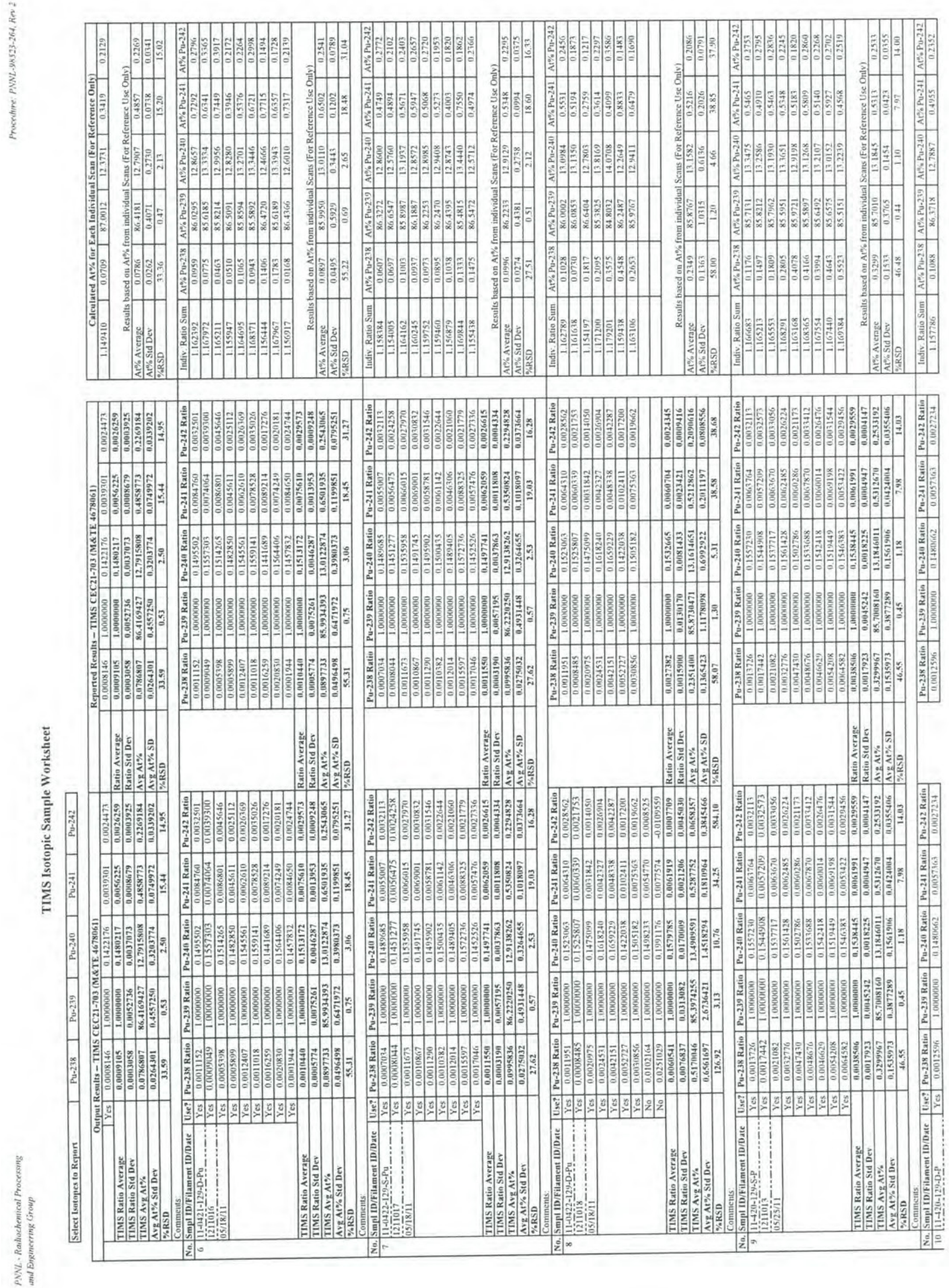


PNNL-20650, Rev. 2

है
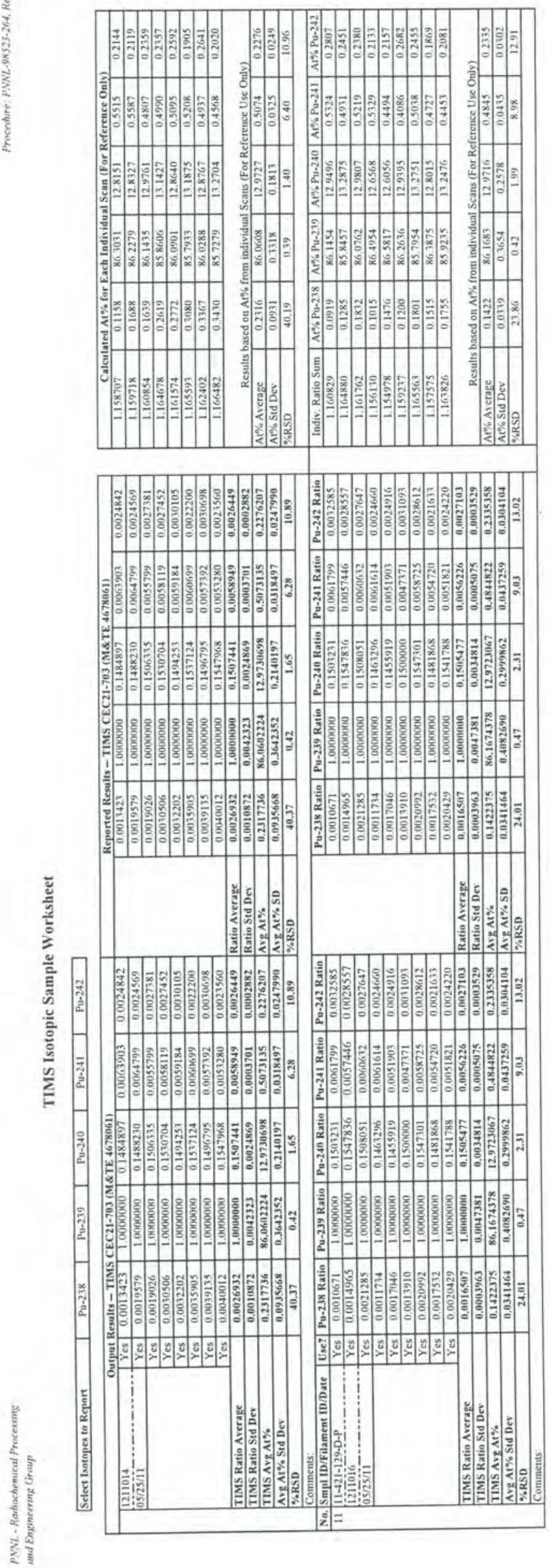

E.33 
PNNL-20650, Rev. 2

हैं

$\frac{\sqrt{2}}{8}$

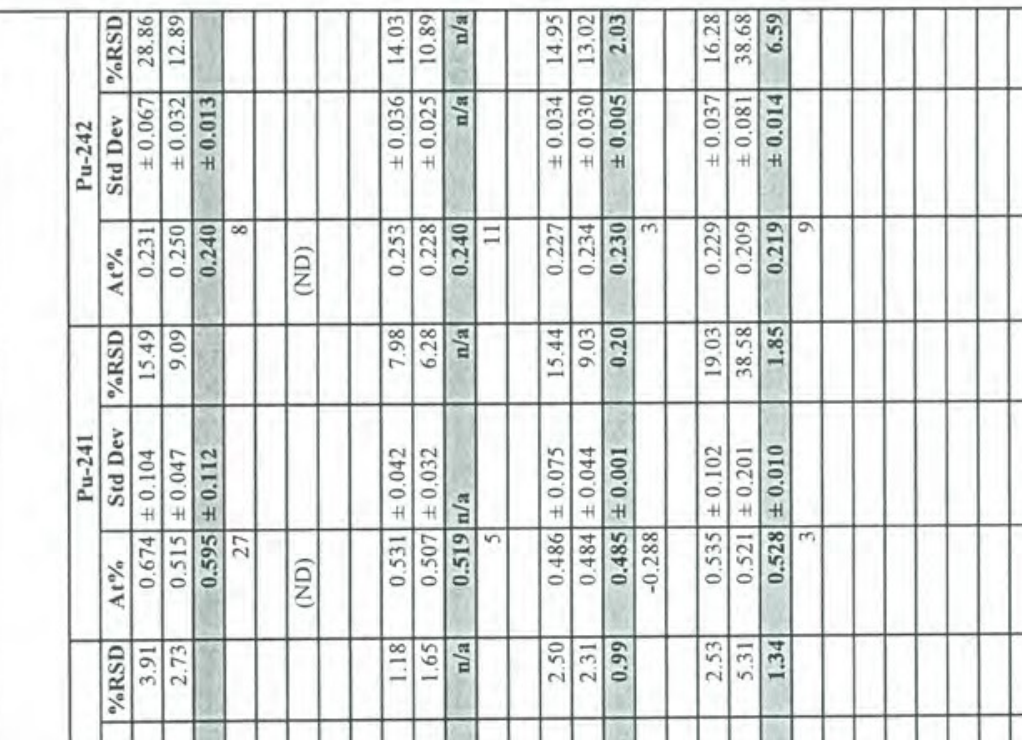

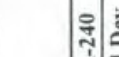

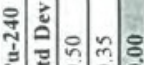

능 ิㅗㅇ

กิ

ำㅇํㅇ응

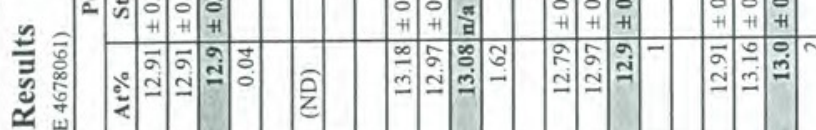

는

ํㅡㄹ

ज्ञे:

जि.

:

ตุ: ㅎํㅇ증

जิ స్లి స్తి

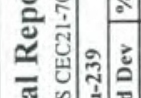

吾该

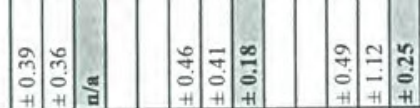

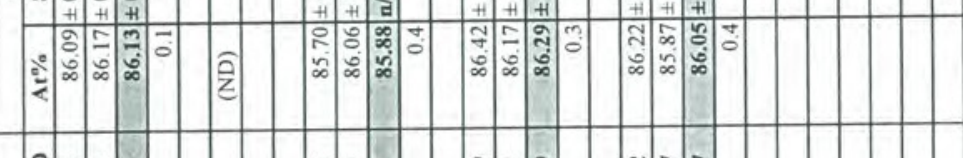

की ₹

ลับे

ஸे

ฟิ

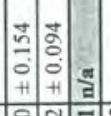

范

कि $\begin{array}{ll}0 \\ 0 \\ +1\end{array}$

वर्व

¿

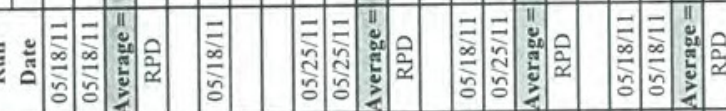

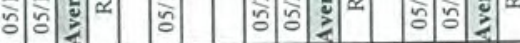

힌을

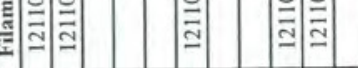

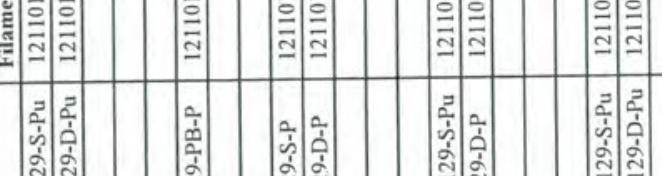

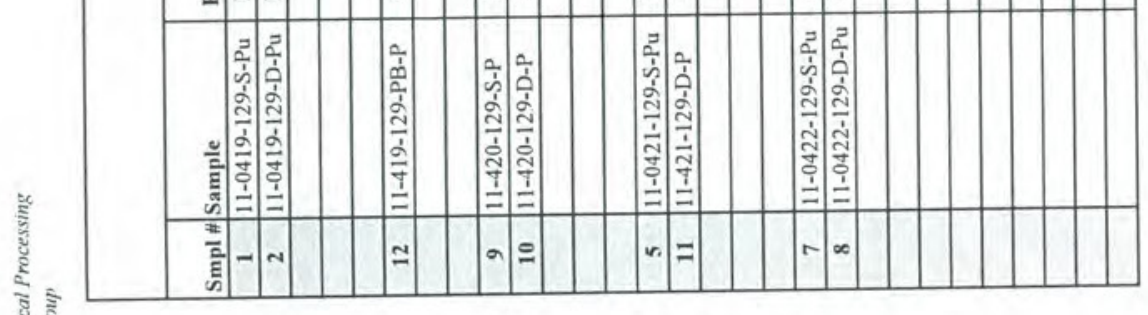


Spreadsheet Review Form

\begin{tabular}{|l|c|l|c|}
\hline Spreadsheet Author Name: & SJ Bos & Review Date: & June 17, 2011 \\
\hline Date Prepared: & 6/15/2011 & Spreadsheet Subject: & Thermal ionization mass spectrometery data \\
\hline Reviewer Name: & JC Braley & File Name: & KW210 TIMS data June 2011 \\
\hline Reviewer Title: & Scientist & Revision No. & Rev 1 \\
\hline
\end{tabular}

Scope of Spreadsheet Review: (Check one or more of the following)

\begin{tabular}{|l|l|l|l|}
\hline$\square$ & $\begin{array}{l}\text { General Validation Review: (General review } \\
\text { and spot checks) }\end{array}$ & $\square$ & $\begin{array}{l}\text { Independent calculation check (With hand calculations or independent } \\
\text { spreadsheet software) }\end{array}$ \\
\hline$\square$ & $\begin{array}{l}\text { Review of updated spreadsheet/calc (Revised } \\
\text { portion only) }\end{array}$ & $\square$ & Other: \\
\hline$\square$ & $\begin{array}{l}100 \% \text { Verification Review (Verification of } \\
\text { all cells/calculations) }\end{array}$ & & \\
\hline
\end{tabular}

\section{REVIEW CHECK LIST}

\begin{tabular}{|c|c|c|c|c|c|c|c|}
\hline \multicolumn{8}{|c|}{ Spreadsheet/Calculation Identification } \\
\hline Spreadsheet Information & Yes & No & NA & \multirow{3}{*}{$\begin{array}{l}\text { Are input parameters correct (verified with } \\
\text { source?) }\end{array}$} & Yes & No & NA \\
\hline Title: & 7 & & & & & & \\
\hline Revision Number: & $<$ & & & & & & \\
\hline Date Prepared: & 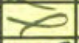 & & & Are parameter units consistent? & $x$ & & \\
\hline Prepared by: & 72 & & & Are input values properly referenced? & $>2$ & & \\
\hline General Statement of Purpose: & 7 & & & Are input uncertainties correct? & 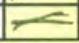 & & \\
\hline General Description of Approach: & -1 & & & \multirow{2}{*}{\multicolumn{2}{|c|}{ Comments: }} & & \\
\hline \multicolumn{4}{|l|}{ Comments: } & & & & \\
\hline Assumptions & Yes & No & NA & Equations/Approach & Yes & No & NA \\
\hline Are assumptions clearly stated? & 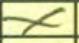 & & & \multirow{2}{*}{\multicolumn{2}{|c|}{\begin{tabular}{|l} 
Are equation algorithms adequately \\
Are equations properly referenced? \\
\end{tabular}}} & & \\
\hline Are assumptions supported and & $x$ & & & & & & \\
\hline Are assumptions reasonable? & $<$ & & & Are limitations of approach/equations & $>$ & & \\
\hline \multicolumn{4}{|l|}{ Comments: } & Are equations appropriate? & 8 & & \\
\hline Results/Conclusions & Yes & No & NA & Are units consistent? & $\infty$ & & \\
\hline Are formulas consistent in cells? & $<$ & & & \multirow{3}{*}{\multicolumn{2}{|c|}{ Comments: }} & & \\
\hline Are calculations correct? & $x$ & & & & & & \\
\hline $\begin{array}{l}\text { Are conclusions consistent with } \\
\text { results? }\end{array}$ & $x$ & & & & & & \\
\hline $\begin{array}{l}\text { Are conclusions consistent with } \\
\text { applicable limits? }\end{array}$ & $x$ & & & \multirow{2}{*}{\multicolumn{2}{|c|}{ Reviewer Sign/Date: }} & & \\
\hline Comments: & & & & & & & \\
\hline
\end{tabular}


PNNL-20650, Rev. 2

76

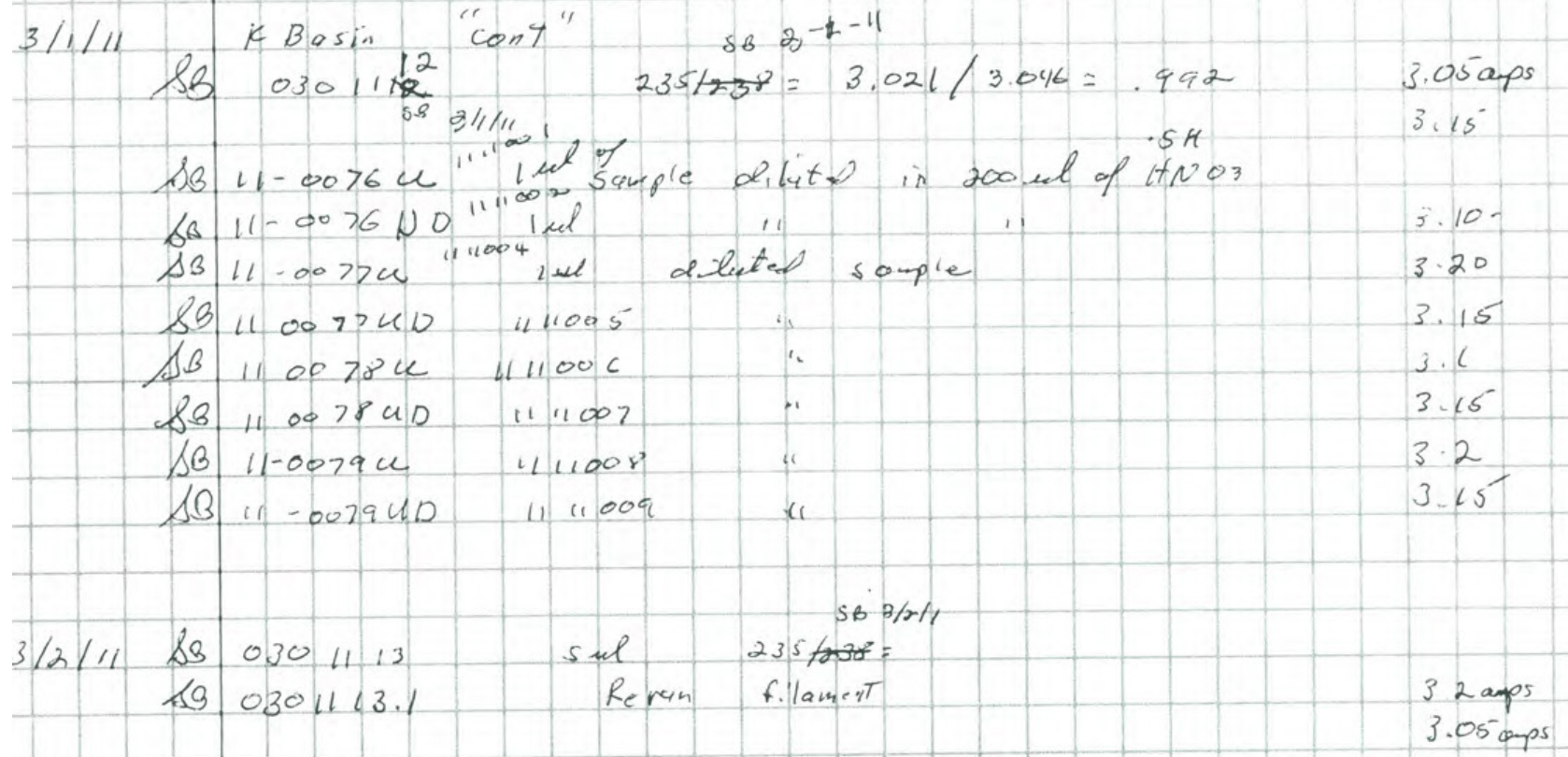
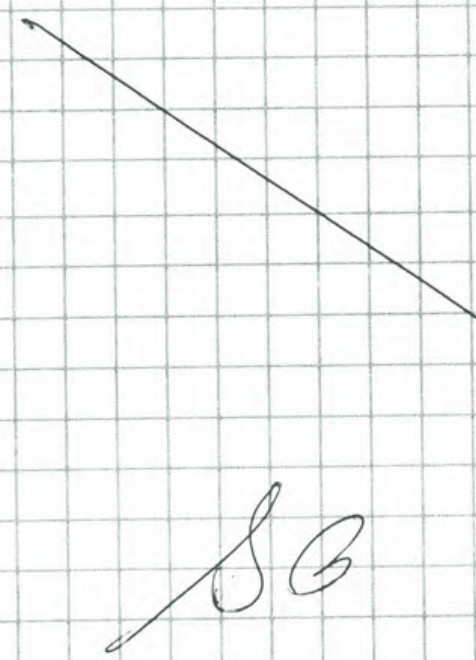

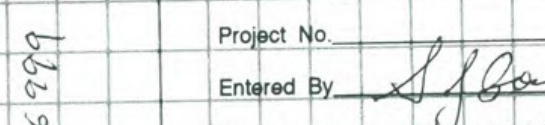

Date of Work

Disclosed To and understogd By 1.

Date $5-4-11$

Dato $4 \cdot 4 \cdot 11$ 
$\therefore 160301616.1 \quad 5 \mathrm{~d} \quad 2351238$ o3t

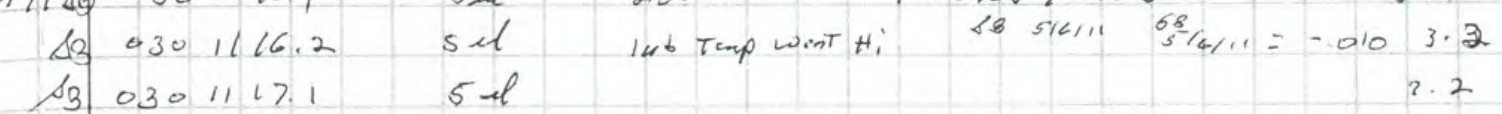

\begin{tabular}{l|llll}
$B_{B}$ & 03011 & 11 & 1 & $5 \mathrm{el}$ \\
$A_{B}$ & 03011 & 17.2 & $5 \mathrm{el}$
\end{tabular}

3. 2

Samples dilutal $\operatorname{lml}$ ir $\mathrm{HHOH}_{3}$

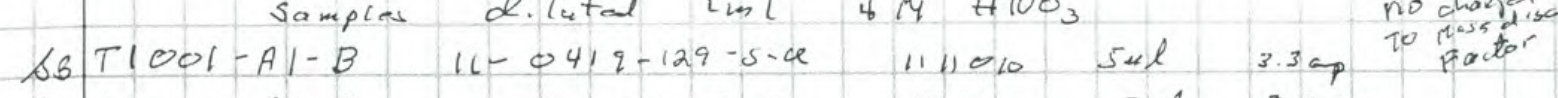

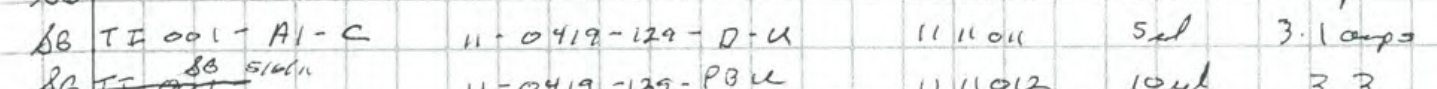

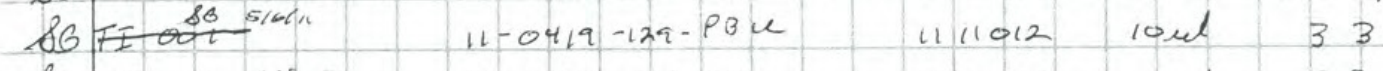

SB II Doi-A4-B $11-0420-129 \mathrm{su} \quad 411013 \mathrm{sm} 3.3$

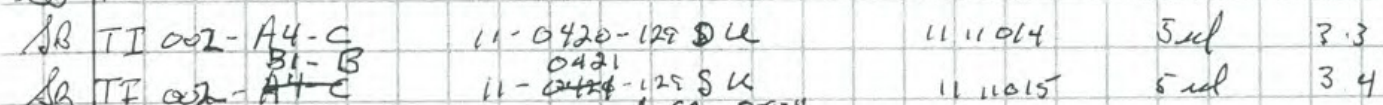

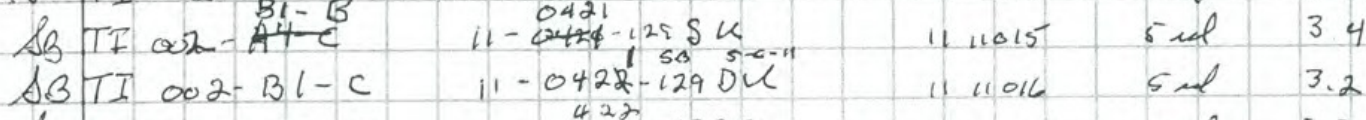

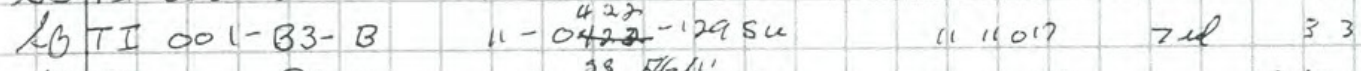

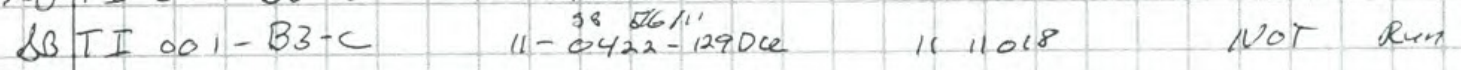

9/11SB II ool-B3-B Repect No 234 ferun sample

AB $0301118.1 \quad 5 \mathrm{ml} \quad 2351236 \quad 3.019813 .046=$

SB TI. oor - B 3-B 5al $11-0422-129-54 \quad 11-11017$

S6. II-001-B3-C i $16-0422-1290 \mathrm{C} \quad 11-11018$

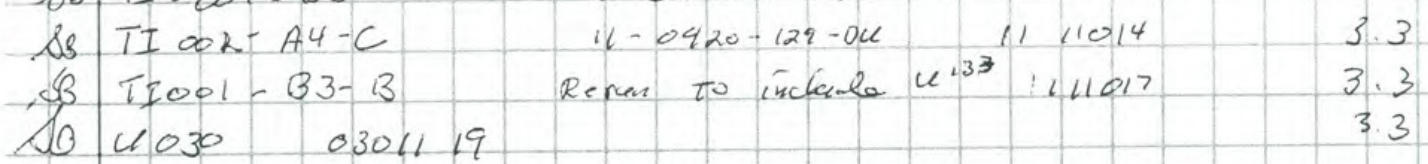

$104030 \quad 0301119$

so Pu $946 \quad 94011006.124 / 239 \quad, 14369 /, 14489=$

SB " $\quad 946 i 1006.2$

So pu $946 \quad 94611006.3$
$=$
$1437 / .1449$

\author{
3.a args \\ 3. 3 \\ 3. 3 \\ 3. 3
}

$\%$ s3 Pu $9469461 / 007.1+0072585 / 1 \%$

Ss TLEOAL $\quad 41.0419-129-5-Q$ Phe

S3TIOON-ALC i1-0419-129-B-Pa

SB $\quad 11-0419-129-P B-P_{k}$

942

.992
2.25 omps 4
1211012 no signd Date of Work
$835 / \% 1 / 1$ 2.25 2.5 2,25 2.2 .5 
PNNL-20650, Rev. 2

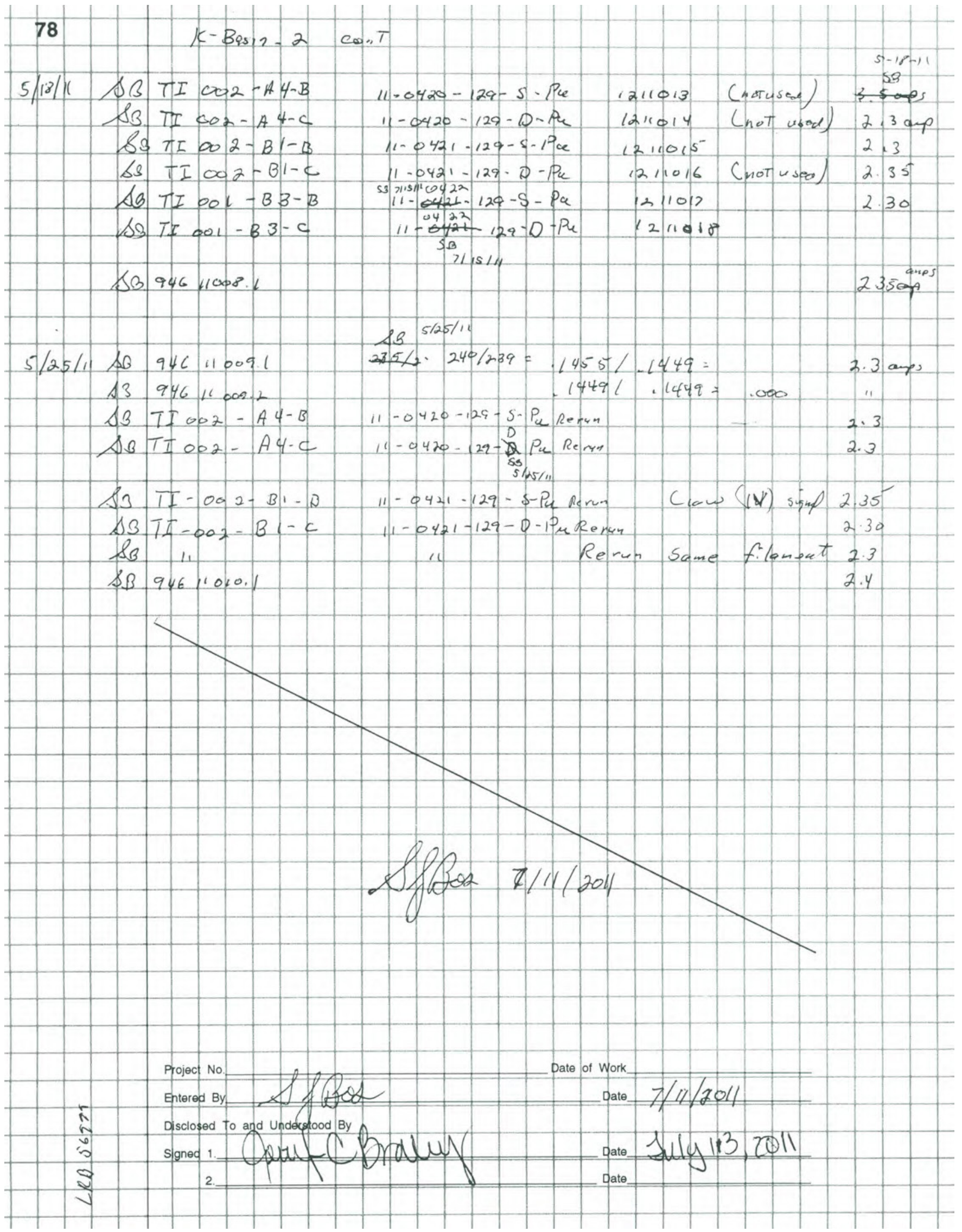


PNNL-20650, Rev. 2

MASS SPECTROMETER LABORATORIES

\author{
ANALYTICAL CHEMISTRY \\ 325 BUILDING \\ CEC21-703A (M\&TE 467806) \\ PROCEDURE NO.: PNL-MA-599, PNNL-98523-264
}

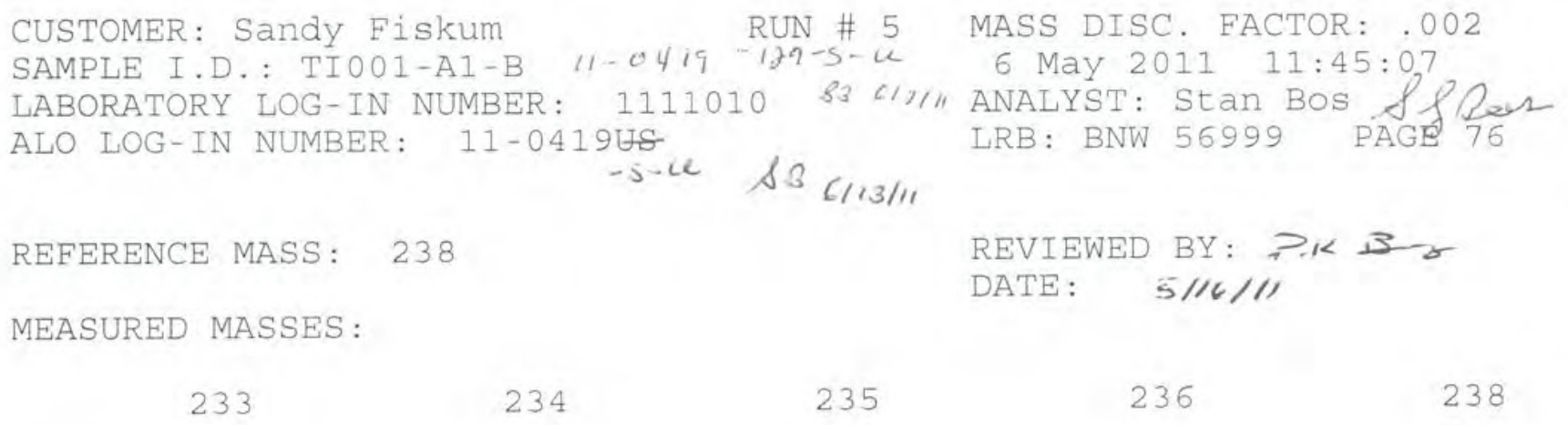

RATIOS:

$\begin{array}{lllll}1.26 E-5 & 7.45 \mathrm{E}-5 & .0073871 & .0008011 & 1 \\ 2.04 \mathrm{E}-5 & 7.58 \mathrm{E}-5 & .007136 & .0006794 & 1 \\ 1.06 \mathrm{E}-5 & 7.48 \mathrm{E}-5 & .0071318 & .0006903 & 1 \\ 3.2 \mathrm{E}-6 & 7.86 \mathrm{E}-5 & .0071274 & .0007567 & 1 \\ 3.3 \mathrm{E}-6 & 9.98 \mathrm{E}-5 & .0070314 & .0007477 & 1 \\ 1.43 \mathrm{E}-5 & 6.7 \mathrm{E}-5 & .007292 & .0008204 & 1 \\ 1 . \mathrm{E}-7 & 9.09 \mathrm{E}-5 & .0070186 & .000684 & 1 \\ 2.29 \mathrm{E}-5 & 6.25 \mathrm{E}-5 & .0071631 & .0007198 & 1 \\ 4.39 \mathrm{E}-5 & 9.47 \mathrm{E}-5 & .0072125 & .0007537 & 1\end{array}$

NO SCANS REJECTED AT THE 95\% LEVEL

MEAN RATIO FOR EACH ISOTOPE IS:
$1.46 \mathrm{E}-5$
$7.99 E-5$
.0071667
.0007392
1

STANDARD DEVIATION OF RATIO FOR EACH ISOTOPE IS:
1.35E-5
1. $26 E-5$
.0001174
$5.05 E-5$
0

$\begin{array}{llcrc}\text { MASS } & \text { ATOM } & \text { MASS } \% & \text { Mean Ratio } & \text { STD } \\ 233 & .0014465 & .0014162 & .0000146 & .0000135 \\ 234 & .0079220 & .0077897 & .0000799 & .0000126 \\ 235 & .7109772 & .7020837 & .0071667 & .0001174 \\ 236 & .0733360 & .0727268 & .0007392 & .0000505 \\ 238 & 99.2063184 & 99.2159837 & 1.0000000 & 0.0000000\end{array}$


PNNL-20650, Rev. 2

\author{
MASS SPECTROMETER LABORATORIES \\ ANALYTICAL CHEMISTRY \\ 325 BUILDING \\ CEC21-703A (M\&TE 467806) \\ PROCEDURE NO.: PNL-MA-599, PNNL-98523-264
}

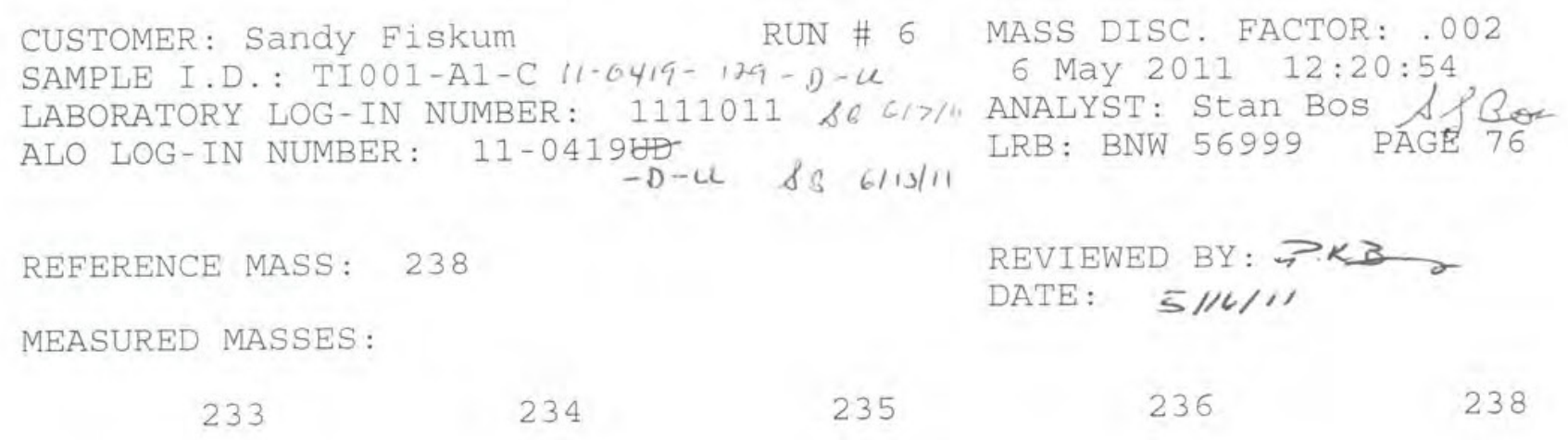

RATIOS:

$\begin{array}{lllll}-3.3 \mathrm{E}-6 & 5.07 \mathrm{E}-5 & .0072942 & .0007406 & 1 \\ 3.5 \mathrm{E}-6 & 7.64 \mathrm{E}-5 & .0071751 & .0007371 & 1 \\ 9.7 \mathrm{E}-6 & 6.57 \mathrm{E}-5 & .0071938 & .0007816 & 1 \\ 2.7 \mathrm{E}-6 & 5.31 \mathrm{E}-5 & .0070837 & .0008105 & 1 \\ 1.1 \mathrm{E}-6 & 5.88 \mathrm{E}-5 & .0070954 & .000768 & 1 \\ 1.17 \mathrm{E}-5 & 6.83 \mathrm{E}-5 & .0070771 & .0007373 & 1 \\ 1.48 \mathrm{E}-5 & 7.96 \mathrm{E}-5 & .0071418 & .0007686 & 1 \\ 8.5 \mathrm{E}-6 & 7.6 \mathrm{E}-5 & .0072212 & .000751 & 1 \\ 1.26 \mathrm{E}-5 & 7.83 \mathrm{E}-5 & .0070528 & .0007698 & 1\end{array}$

NO SCANS REJECTED AT THE 95\% LEVEL

MEAN RATIO FOR EACH ISOTOPE IS:
6. $8 E-6$
6. $74 \mathrm{E}-5$
.0071484
.0007627
1

STANDARD DEVIATION OF RATIO FOR EACH ISOTOPE IS:
$6.1 E-6$
1. 11E-5
7. $95 \mathrm{E}-5$
2. $42 E-5$
0

$\begin{array}{ccccc}\text { MASS } & \text { ATOM } & \text { MASS } \% & \text { Mean Ratio } & \text { STD } \\ 233 & .0006754 & .0006613 & .0000068 & .0000061 \\ 234 & .0066902 & .0065784 & .0000674 & .0000111 \\ 235 & .7091722 & .7003010 & .0071484 & .0000795 \\ 236 & .0756665 & .0750379 & .0007627 & .0000242 \\ 238 & 99.2077957 & 99.2174214 & 1.0000000 & 0.0000000\end{array}$


PNNL-20650, Rev. 2

\author{
MASS SPECTROMETER LABORATORIES \\ ANALYTICAL CHEMISTRY \\ 325 BUILDING \\ CEC21-703A (M\&TE 467806) \\ PROCEDURE NO.: PNL-MA-599, PNNL-98523-264
}

CUSTOMER: Sandy Fiskum RUN \# 7 MASS DISC. FACTOR: .002

SAMPLE I.D.: Process Blank 11-0419-129-pB-k 6 May 2011 12:45:46,

LABORATORY LOG-IN NUMBER: 1111012 SG GIII ANALYST: Stan BOS OfGo

ALO LOG-IN NUMBER: 11-0419PB LRB: BNW 56999 PAGE 76

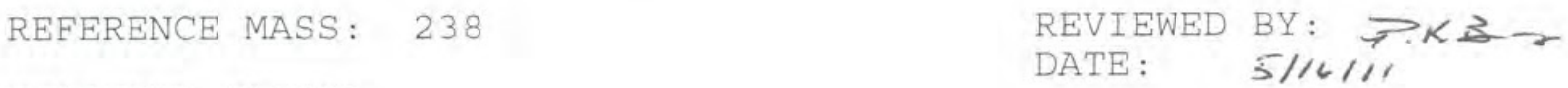

MEASURED MASSES :
233
234
235
236
238

RATIOS :

$\begin{array}{lllll}2.6 \mathrm{E}-6 & -.0006611 & .0085665 & .0002158 & 1 \\ 1.48 \mathrm{E}-5 & 1.74 \mathrm{E}-5 & .0101087 & .0003697 & 1 \\ 3.06 \mathrm{E}-5 & 3.01 \mathrm{E}-5 & .0079469 & .0003888 & 1\end{array}$

MEAN RATIO FOR EACH ISOTOPE IS:

$\begin{array}{lllll}1.6 \mathrm{E}-5 & -.0002045 & .008874 & .0003248\end{array}$

STANDARD DEVIATION OF RATIO FOR EACH ISOTOPE IS:
1. $4 E-5$
.0003955
.0011132
$9.49 \mathrm{E}-5$
0

MASS

$\begin{array}{rrrc}\text { ATOM } & \text { MASS } \% & \text { Mean Ratio } & \text { STD } \\ .0015884 & .0015552 & .0000160 & .0000140 \\ -.0202700 & -.0199315 & -.0002045 & .0003955 \\ .8794804 & .8684904 & .0088740 & .0011132 \\ .0321881 & .0319211 & .0003248 & .0000949 \\ 9.1070132 & 99.1179648 & 1.0000000 & 0.0000000\end{array}$

$$
\begin{aligned}
& 1 \text { volt } 238 \text { signel to ran isetwopic cerselysis. } \\
& \text { nor enouis sample to kath s/Ber } 5 / 6 / 1
\end{aligned}
$$


PNNL-20650, Rev. 2

MASS SPECTROMETER LABORATORIES

\author{
ANALYTICAL CHEMISTRY \\ 325 BUILDING \\ CEC21-703A (M\&TE 467806) \\ PROCEDURE NO.: PNL-MA-599, PNNL-98523-264
}

CUSTOMER: Sandy Fiskum

RUN \# 8

MASS DISC. FACTOR: .002

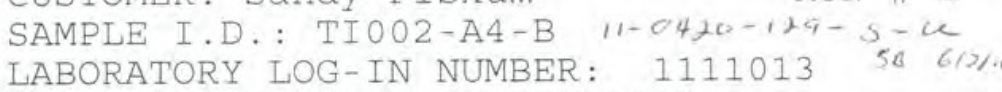

ALO LOG-IN NUMBER: 11-0420SU

6 May 2011 13:21:55

ALO LOG-IN NUMBER: 11-0A2OSU

ANALYST: Stan BOs \& $\mathrm{f}$ Bor

LRB: BNW 56999 PAGE 76

REFERENCE MASS: 238

REVIEWED BY: ?.K及>

MEASURED MASSES :

DATE: $5 / / 6 / 1 /$
233
234
235
236
238

RATIOS:
6. $7 E-6$
4. $6 \mathrm{E}-5$
.0071569
.0006965
.0072004
$-1.37 E-5$
8. $93 E-5$
.0070636
$-2.9 E-6$
4. $09 \mathrm{E}-5$
.0069314
.0006575
.0006696
.0072038
5. $6 \mathrm{E}-6$
.0001148
.0007204
.0007654
.0070515
$5.6 \mathrm{E}-6$
$5.88 \mathrm{E}-5$
.0071077
$-5 \cdot 1 \mathrm{E}-6$
$7.75 \mathrm{E}-5$
.0072254
5. $8 \mathrm{E}-6$
.0001026
$5.26 E-5$
.0066953
.0007196
.0007614
.0007657
.0007737

1
1
1
1
1
1
1
1
1

NO SCANS REJECTED AT THE 95\% LEVEL

MEAN RATIO FOR EACH ISOTOPE IS:
$2 \cdot 8 \mathrm{E}-6$
$6.88 \mathrm{E}-5$
.0070707
.0007255
1

STANDARD DEVIATION OF RATIO FOR EACH ISOTOPE IS:
9. $3 E-6$
$2.83 E-5$
.0001688
4. $4 E-5$
0

$\begin{array}{lllrc}\text { MASS } & \text { ATOM } & \text { MASS } \% & \text { Mean Ratio } & \text { STD } \\ 233 & .0002799 & .0002741 & .0000028 & .0000093 \\ 234 & .0068289 & .0067147 & .0000688 & .0000283 \\ 235 & .7015473 & .6927705 & .0070707 & .0001688 \\ 236 & .0719858 & .0713877 & .0007255 & .0000440 \\ 238 & 99.2193582 & 99.2288530 & 1.0000000 & 0.0000000\end{array}$


PNNL-20650, Rev. 2

\author{
MASS SPECTROMETER LABORATORIES \\ ANALYTICAL CHEMISTRY \\ 325 BUILDING \\ CEC21-703A (M\&TE 467806) \\ PROCEDURE NO.: PNL-MA-599, PNNL-98523-264
}

CUSTOMER: Sandy Fiskum RUN \# 5 MASS DISC. FACTOR: .002 SAMPLE I.D.: TIO02-A4-C $11-0420-129-D-u$

LABORATORY LOG-IN NUMBER: 1111014 ALO LOG-IN NUMBER: 11-0420DU 9 May 2011 15:04:19 ANALYST: Stan BOS S J Q OA
LRB: BNW 56999 PAG 77 REFERENCE MASS: 238 REVIEWED BY: $\Rightarrow K \mathrm{~B}_{2}$ MEASURED MASSES: DATE: $\quad 5 / / 6 / 1 /$
233
234
235
236
238

RATIOS:
1. $73 E-5$
3. $52 \mathrm{E}-5$
1. $1 \mathrm{E}-6$
1. $76 \mathrm{E}-5$
$-2.7 E-6$
1. $71 \mathrm{E}-5$
1. $5 E-5$
1. $06 \mathrm{E}-5$
$-5 \cdot \mathrm{E}-7$
6. $94 \mathrm{E}-5$
7. $87 \mathrm{E}-5$
8. $52 E-5$
3. $69 \mathrm{E}-5$
6. $39 E-5$
.0001
7. $69 \mathrm{E}-5$
$6.06 \mathrm{E}-5$
.0001025
.0073048
.0074368
.0071936
.0070726
.0070787
.006704
.0067339
.007417
.0069753
.0008158
.00076
.0007272
.0006024
.0007365
.000725
.0008175
.000696
.0008318

NO SCANS REJECTED AT THE 95\% LEVEL

MEAN RATIO FOR EACH ISOTOPE IS:
1. $23 E-5$
$7.49 \mathrm{E}-5$
.0071019
.0007458
1

STANDARD DEVIATION OF RATIO FOR EACH ISOTOPE IS:
1. $19 \mathrm{E}-5$
$2.04 E-5$
.0002675
7. $21 E-5$
0

MASS

\section{ATOM \%}

$$
.0012199
$$

.0074303

.7045946

.0739927

99.2127626

233
234
235
236
238
$2.04 \mathrm{E}-5$

MASS \%
.0011944
.0073061
.6957803
.0733780
99.2223412

99.2223412
Mean Ratio

$$
\begin{array}{r}
.0000123 \\
.0000749 \\
.0071019 \\
.0007458 \\
1.0000000
\end{array}
$$

STD

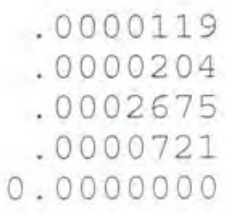


PNNL-20650, Rev. 2

\section{MASS SPECTROMETER LABORATORIES}

\section{ANALYTICAL CHEMISTRY \\ 325 BUILDING}

\section{CEC21-703A (M\&TE 467806)}

PROCEDURE NO.: PNL-MA-599, PNNL-98523-264

CUSTOMER: Sandy Fiskum SAMPLE I.D.: TIO02-B1-B LABORATORY LOG-IN NUMBER ALO LOG-IN NUMBER: 11-0421SU

REFERENCE MASS: 238

MEASURED MASSES :
RUN \# 10
$11 .+421-129-5-4$

1111015 U $55^{25}$
612111 chth

MASS DISC. FACTOR: .002

6 May 2011 14:39:12 ANALYST: Stan BOS
LRB: BNW 56999 PAGE 78 Lot REVIEWED BY: アイ\&\&ー DATE: $\quad 5 / / 6 / / 1$
233
234
235
236
238

RATIOS:

$5.89 E-5$
$9.02 E-5$
$2.98 E-5$
$6.11 E-5$
$4.35 E-5$
$7.53 E-5$
.0001098
$8.11 E-5$
$5.33 E-5$

.0073381

.0073466

.0079459

.0072751

.0070924

.0068096

.0066943

.0069421

.0065905

$\begin{array}{ll}.0007278 & 1 \\ .000697 & 1 \\ .0005783 & 1 \\ .0008526 & 1 \\ .0007738 & 1 \\ .0006555 & 1 \\ .0006104 & 1 \\ .0007295 & 1 \\ .0008475 & 1\end{array}$

NO SCANS REJECTED AT THE 95\% LEVEL

MEAN RATIO FOR EACH ISOTOPE IS:
1. $71 \mathrm{E}-5$
6. $7 E-5$
.007115
.0007192
1

STANDARD DEVIATION OF RATIO FOR EACH ISOTOPE IS:
3. $1 E-5$
2. $47 E-5$
.0004176
9. $6 E-5$
0

MASS

233

234

235

236

238

ATOM \%
.0016931
.0066464
.7059076
.0713519
99.2144010

MASS \%
.0016577
.0065353
.6970769
.0707591
99.2239710

Mean Ratio

$$
\begin{array}{r}
.0000171 \\
.0000670 \\
.0071150 \\
.0007192 \\
1.0000000
\end{array}
$$

STD
.0000310
.0000247
.0004176
.0000960
.0000000 
PNNL-20650, Rev. 2

MASS SPECTROMETER LABORATORIES

\author{
ANALYTICAL CHEMISTRY \\ 325 BUILDING \\ CEC21-703A (M\&TE 467806) \\ PROCEDURE NO.: PNL-MA-599, PNNL-98523-264
}

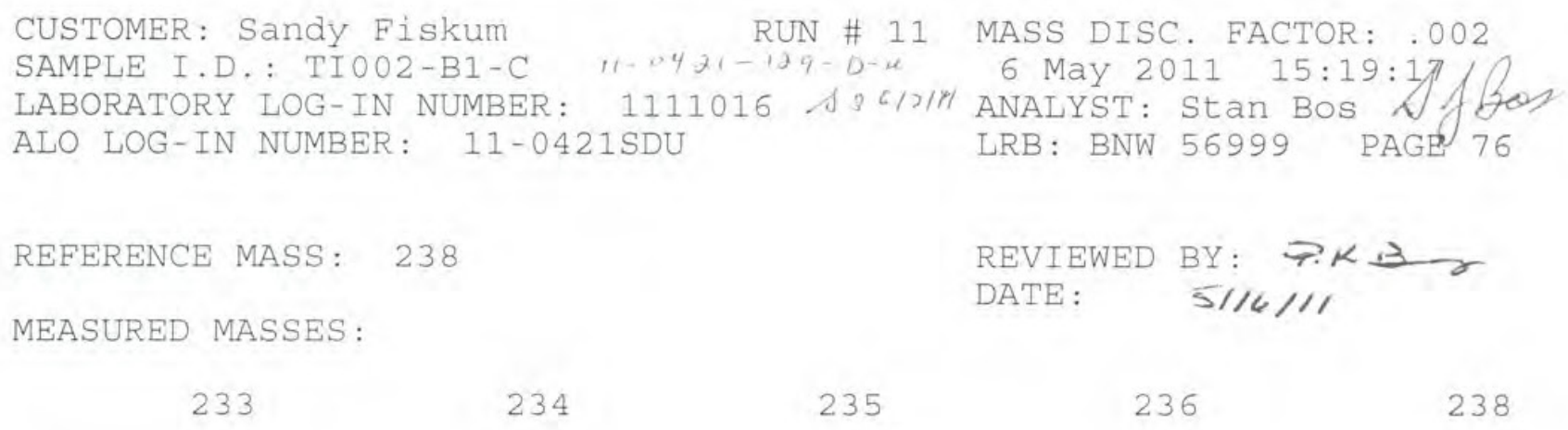

RATIOS:

$\begin{array}{lllll}8 . E-7 & 4.68 E-5 & .0071683 & .0007082 & 1 \\ 1.55 E-5 & 6.31 E-5 & .0071429 & .0006125 & 1 \\ 1.56 E-5 & 5.76 E-5 & .0070182 & .0006884 & 1 \\ 1.54 E-5 & 7.88 E-5 & .0070003 & .0007232 & 1 \\ 3.54 E-5 & 5.25 E-5 & .007047 & .0008114 & 1 \\ 3.97 E-5 & 9.09 E-5 & .0070358 & .0007708 & 1 \\ 4.6 E-5 & 6.49 E-5 & .0070563 & .000775 & 1 \\ 4.84 E-5 & 7.02 E-5 & .0073415 & .0007423 & 1 \\ 3.81 E-5 & .0001137 & .0070111 & .0007212 & 1\end{array}$

NO SCANS REJECTED AT THE 95\% LEVEL

MEAN RATIO FOR EACH ISOTOPE IS:
$2.83 E-5$
7. $09 \mathrm{E}-5$
.0070913
.0007281
1

STANDARD DEVIATION OF RATIO FOR EACH ISOTOPE IS:
1. $67 \mathrm{E}-5$
$2.09 E-5$
.0001104
$5.76 \mathrm{E}-5$
0

MASS

$$
\text { ATOM \% }
$$

MASS \%

Mean Ratio

STD

233
234

.0028093

.0027506

.0069198

.6947547

.7035560

.0716397

236

.0722399

99.2239352

.0000283
.0000709
.0070913
.0007281
1.0000000

.0000167

.0000209

.0001104

.0000576

99.2143574

1.0000000

0.0000000 
PNNL-20650, Rev. 2

\author{
MASS SPECTROMETER LABORATORIES

\section{ANALYTICAL CHEMISTRY \\ 325 BUILDING} \\ CEC21-703A (M\&TE 467806) \\ PROCEDURE NO.: PNL-MA-599, PNNL-98523-264
}

CUSTOMER: Sandy Fiskum

SAMPLE I.D.: TIO01-B3-B

LABORATORY LOG-IN NUMBER :

ALO LOG-IN NUMBER: 11-0422SU
RUN \# 6

$1-42 \alpha-129-5-4$
1111017 \& $617 / 4$

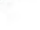

REFERENCE MASS: 238

MEASURED MASSES:
233
234
235
236
238

REVIEWED BY: $\overrightarrow{2 K}$ DATE: 5/14/1/
MASS DISC. FACTOR: .002

9 May 2011 15:39:26

ANALYST: Stan Bos A f Bor

LRB : BNW 56999 PAGE 77

RATIOS:

$\begin{array}{lllll}2.29 E-5 & 5.46 E-5 & .0071644 & .0007 & 1 \\ 8.1 \mathrm{E}-6 & 6.58 \mathrm{E}-5 & .0072367 & .0008346 & 1 \\ -6.3 \mathrm{E}-6 & 4.13 \mathrm{E}-5 & .0071768 & .0008351 & 1 \\ 9.5 \mathrm{E}-6 & 7.53 \mathrm{E}-5 & .0069076 & .0006631 & 1 \\ 8.9 \mathrm{E}-6 & .00012 & .007218 & .0007135 & 1 \\ 6.2 \mathrm{E}-6 & .0001123 & .0070834 & .000722 & 1 \\ 3.96 \mathrm{E}-5 & 8.04 \mathrm{E}-5 & .0073273 & .0007594 & 1 \\ 1.67 \mathrm{E}-5 & 4.88 \mathrm{E}-5 & .0071787 & .0006834 & 1 \\ 1.79 \mathrm{E}-5 & 9.17 \mathrm{E}-5 & .0071811 & .0006534 & 1\end{array}$

NO SCANS REJECTED AT THE 95\% LEVEL

MEAN RATIO FOR EACH ISOTOPE IS:
1. $37 \mathrm{E}-5$
$7.67 E-5$
.0071638
.0007294
1

STANDARD DEVIATION OF RATIO FOR EACH ISOTOPE IS:
1. $28 \mathrm{E}-5$
$2.74 \mathrm{E}-5$
.0001161
$6.76 E-5$
0

MASS

233
234

235

236

238

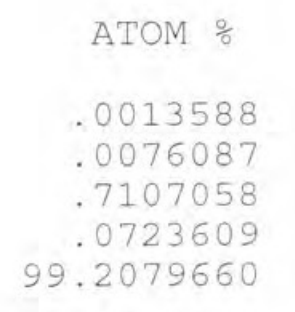

.0013588

.0076087

.7107058

99.2079660

\begin{abstract}
MASS \%
.0013303

.0074815

.7018155

.0717598

99.2176129
\end{abstract}

Mean Ratio

$$
\begin{array}{r}
.0000137 \\
.0000767 \\
.0071638 \\
.0007294 \\
1.0000000
\end{array}
$$

STD

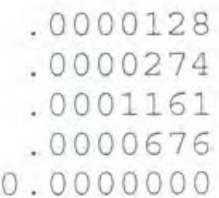

.0000128

001161

76

0.0000000 
PNNL-20650, Rev. 2

\author{
MASS SPECTROMETER LABORATORIES \\ ANALYTICAL CHEMISTRY \\ 325 BUILDING \\ CEC21-703A (M\&TE 467806) \\ PROCEDURE NO.: PNL-MA-599, PNNL-98523-264
}

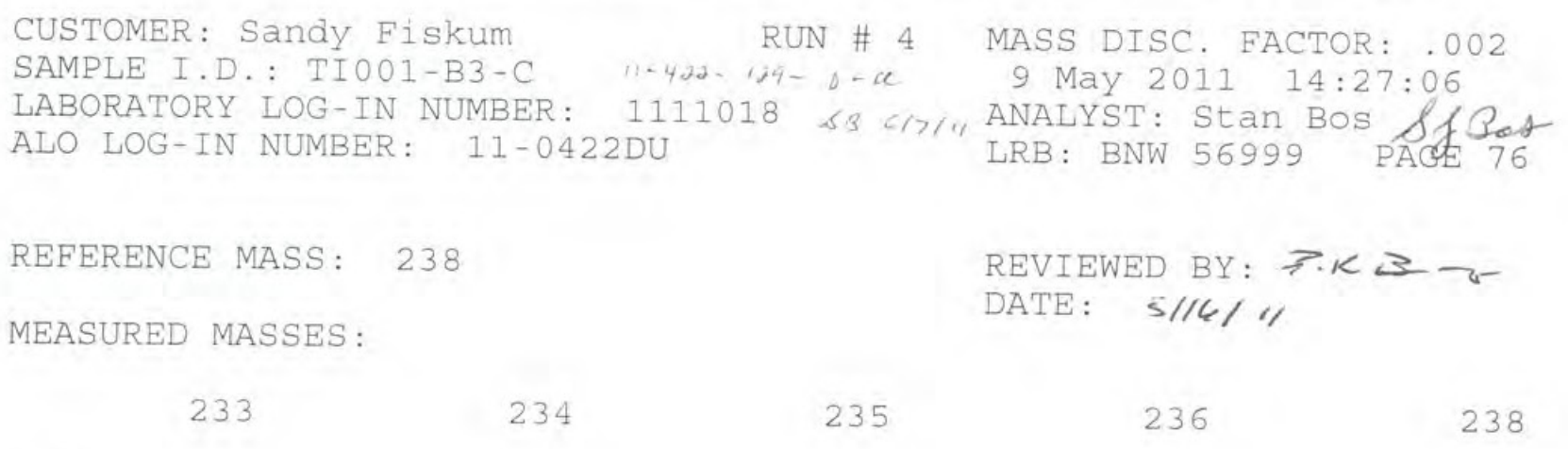

RATIOS:

$\begin{array}{lllll}1.94 \mathrm{E}-5 & 9.97 \mathrm{E}-5 & .0073447 & .0007931 & 1 \\ 7 . \mathrm{E}-6 & 6.78 \mathrm{E}-5 & .0072729 & .0007109 & 1 \\ -1.06 \mathrm{E}-5 & .0001013 & .0071895 & .0008124 & 1 \\ -4.9 \mathrm{E}-6 & .0001618 & .0074768 & .000865 & 1 \\ 6.1 \mathrm{E}-6 & .000196 & .007034 & .0008425 & 1 \\ 2 . \mathrm{E}-6 & 5.37 \mathrm{E}-5 & .0069269 & .0007912 & 1 \\ -5.7 \mathrm{E}-6 & 7.66 \mathrm{E}-5 & .0069584 & .0007569 & 1 \\ -1.28 \mathrm{E}-5 & .0001051 & .0071822 & .0007573 & 1 \\ 2.96 \mathrm{E}-5 & .0001228 & .0073873 & .0006755 & 1\end{array}$

NO SCANS REJECTED AT THE 95\% LEVEL

MEAN RATIO FOR EACH ISOTOPE IS:
3. $3 E-6$
.0001094
.007197
.0007783
1

STANDARD DEVIATION OF RATIO FOR EACH ISOTOPE IS:
1. $4 E-5$
4. $54 \mathrm{E}-5$
.0001933
$6.04 \mathrm{E}-5$
0

$\begin{array}{ccccc}\text { MASS } & \text { ATOM } & \text { MASS } \% & \text { Mean Ratio } & \text { STD } \\ 233 & .0003318 & .0003248 & .0000033 & .0000140 \\ 234 & .0108518 & .0106704 & .0001094 & .0000454 \\ 235 & .7139215 & .7049918 & .0071970 & .0001933 \\ 236 & .0772069 & .0765656 & .0007783 & .0000604 \\ 238 & 99.1976881 & 99.2074473 & 1.0000000 & 0.0000000\end{array}$


PNNL-20650, Rev. 2

U. S. Department of Commerce
Maurice. H. Stans
Secretary

A. V. Astibo Director

\section{(Tertifitate}

\section{Standard Reference Material U-030 Uranium Isotopic Standard}

\begin{tabular}{lcccr} 
& ${ }^{234} \mathrm{U}$ & ${ }^{235} \mathrm{U}$ & ${ }^{236} \mathrm{U}$ & \multicolumn{1}{c}{${ }^{238} \mathrm{U}$} \\
Atom pereent & 0.0190 & 3.046 & 0.0204 & 96.915 \\
& $\pm .000 \mathrm{I}$ & \pm .003 & \pm .0001 & \pm .003 \\
Weight pereent & 0.0187 & 3.009 & 0.0202 & 96.953
\end{tabular}

The material consists of highly purified oxide, $\mathrm{L}_{3} \mathrm{O}_{8}$. The atomic weight of the material is calculated to be 237.958 using the nuclidic masses $234.0409 ; 235.0439$ : 236.0457 and 238.0508 .

The values for ${ }^{234} \mathrm{U}$ and ${ }^{236} \mathrm{U}$ were calculated from measurements at the National Bureau of Standards. The samples were spiked with high-purity ${ }^{23}{ }^{3}$ t to approximate the ${ }^{23}{ }^{4} \mathrm{U}$ concentration, the ratios ${ }^{233} \mathrm{U}$ to ${ }^{234} \mathrm{U}$ and ${ }^{233} \mathrm{~L}$ to ${ }^{236} \mathrm{U}$ were measured on a triple-filament equipped surface ionization mass spectrometer with ion-multiplier amplifier circuits.

The values for ${ }^{235} \mathrm{U}$ and ${ }^{238} \mathrm{U}$ were calculated from measurements of the ${ }^{235} \mathrm{U}$ to ${ }^{238} \mathrm{U}$ ratio made at the National Bureau of Standards on a triple-filament. surface ionization mass spectrometer equipped with de amplifier circuits. The observed ratios were corrected for mass discrimination effeets by intercomparison with synthetic mixtures prepared at the 3 percent ${ }^{235} \mathrm{U}$ level from high-purity ${ }^{235} \mathrm{U}$ and ${ }^{238} \mathrm{U}$.

The limits indicated for the isotopic concentrations are at least as large as the 95-percent confidence limits for a single determination, and include terms for inhomogeneities in the material as well as analytical error. The ${ }^{235} \mathrm{U}$ to ${ }^{238} \mathrm{U}$ ratio for this standart. 0.03143 , is known to at least 0.1 percent.

Mass spectrometry measurements at NBS were made by E. L. Garner on solutions prepared by L. A. Nachlan.

The overall direction and coordination of the technical measurements leading to certification were performed under the chairmanship of W. R. Shields.

The technical and support aspects in the preparation, certification, and issuance of this Standard Reference Material were coordinated through the Office of Standard Reference Materials by J. L. Hague.

Washington. D. C. 20234

W. Wayne Meinke, Chief

April 21, 1969

Office of Standard Reference Materials

(This certificate supersedes certificate of October 1, 1958) 
PNNL-20650, Rev. 2

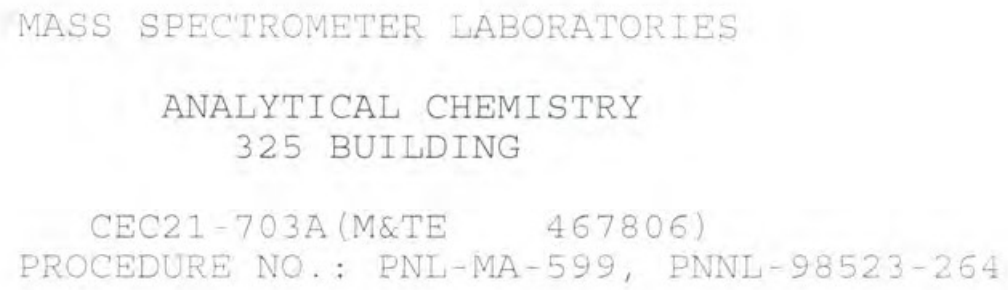

CUSTOMER: Sandy Eiskum

SAMPLE I.D.: U-030

LABORATORY LOG-IN NUMBER: 0301114.1

ALO LOG-IN NUMBER:

REFERENCE MASS: 238

MEASURED MASSES :
234
235
236
238

RATIOS:

$\begin{array}{llll}.0001724 & .0308798 & .0001998 & 1 \\ .0001539 & .0306582 & .0002461 & 1 \\ .0001774 & .0312367 & .00022 & 1 \\ .0002066 & .0313486 & .0002818 & 1 \\ .0002068 & .0312343 & .0001997 & 1 \\ .000196 & .0305761 & .0002075 & 1 \\ .0002131 & .0309255 & .0001794 & 1 \\ .0002312 & .030876 & .0002154 & 1 \\ .0001791 & .0308527 & .0002517 & 1\end{array}$

NO SCANS REJECTED AT THE 95\% LEVEL

MEAN RATIO FOR EACH ISOTOPE IS:
.0001929
.0309542
.0002224
1

STANDARD DEVIATION OF RATIO FOR EACH ISOTOPE IS:
$2.41 E-5$
.0002663
$3 \cdot 18 \mathrm{E}-5$

\begin{tabular}{rrrrr} 
MASS & \multicolumn{1}{c}{ ATOM } & \multicolumn{1}{c}{ MASS } & Mean Ratio & STD \\
234 & .0187075 & .0184001 & .0001929 & .0000241 \\
235 & 3.0012722 & 2.9645773 & .0309542 & .0002663 \\
236 & .0215610 & .0213881 & .0002224 & .0000318 \\
238 & 96.9584592 & 96.9956345 & 1.0000000 & 0.0000000
\end{tabular}


PNNL-20650, Rev. 2

MASS SPECTROMETER LABORATORIES

\author{
ANALYTICAL CHEMISTRY \\ 325 BUILDING \\ CEC21-703A (M\&TE 467806) \\ PROCEDURE NO.: PNL-MA-599, PNNL-98523-264
}

CUSTOMER: Sandy Fiskum

SAMPLE I.D.: U-030

LABORATORY LOG-IN NUMBER: 0301116.1

ALO LOG-IN NUMBER:

REFERENCE MASS: 238

MEASURED MASSES:

$$
234
$$

235
RUN \# 1

MASS DISC. FACTOR: .002

6 May 2011 09:53:08

ANALYST: Stan BOS If gen LRB: BNW 56999 PAGE 76

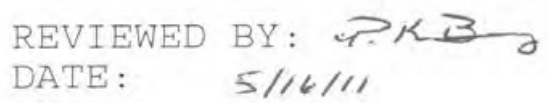

RATIOS:

$\begin{array}{llll}.0001608 & .0285448 & .0002397 & 1 \\ .0001702 & .030826 & .0001655 & 1 \\ .0002109 & .0310466 & .0001864 & 1 \\ .000229 & .0307557 & .0002278 & 1 \\ .0002337 & .0314748 & .000203 & 1 \\ .0002009 & .0313715 & .0001987 & 1 \\ .0001689 & .0307662 & .0002154 & 1 \\ .0001782 & .0315104 & .0001879 & 1 \\ .0002153 & .0311957 & .0002102 & 1\end{array}$

CYCLE \# 1 REJECTED AT THE 95\% LEVEL DUE TO THE 235/238 RATIO

MEAN RATIO FOR EACH ISOTOPE IS:

$$
\begin{array}{llll}
.0002009 & .0311184 & .0001994
\end{array}
$$

STANDARD DEVIATION OF RATIO FOR EACH ISOTOPE IS:
$2.58 \mathrm{E}-5$
.0003155
1. $94 E-5$
0 
PNNL-20650, Rev. 2

MASS SPECTROMETER LABORATORIES

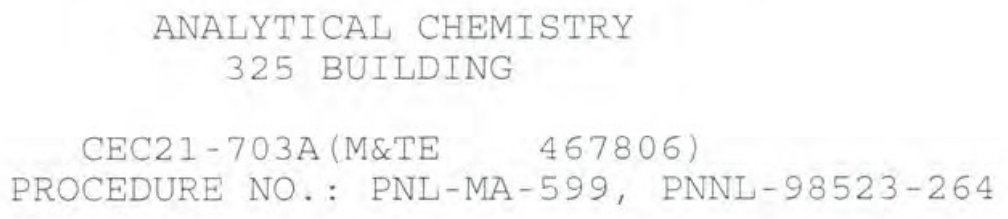

CUSTOMER: Sandy Fiskum RUN \# 3 MASS DISC. FACTOR: .002 SAMPLE I.D.: U-030 LABORATORY LOG-IN NUMBER: 0301117.1 ALO LOG-IN NUMBER : 6 May $2011 \quad 10: 48: 28$ ANALYST: Stan BOS S/JBer LRB: BNW 56999 PAGE 76

REFERENCE MASS: 238

REVIEWED BY: ア.K及ح MEASURED MASSES :
234
235
236
238

RATIOS:

$\begin{array}{llll}.0001801 & .0315838 & .0001869 & 1 \\ .0002123 & .0309041 & .0002033 & 1 \\ .0002069 & .0309864 & .0001665 & 1 \\ .0001839 & .0313066 & .0002222 & 1 \\ .0001904 & .0316178 & .0002065 & 1 \\ .0001924 & .0311908 & .0001908 & 1 \\ .0002359 & .0312499 & .0001478 & 1 \\ .0002347 & .0312294 & .0001703 & 1 \\ .0002292 & .031285 & .0001573 & 1\end{array}$

NO SCANS REJECTED AT THE 95\% LEVEL

MEAN RATIO FOR EACH ISOTOPE IS:

$\begin{array}{llll}.0002073 & .0312615 & .0001835 & 1\end{array}$

STANDARD DEVIATION OF RATIO FOR EACH ISOTOPE IS:
2. $2 E-5$
.0002353
$2.48 \mathrm{E}-5$
0

\begin{tabular}{rrrrr} 
MASS & \multicolumn{1}{c}{ ATOM } & MASS & Mean Ratio & STD \\
234 & .0200955 & .0197655 & .0002073 & .0000220 \\
235 & 3.0302398 & 2.9932013 & .0312615 & .0002353 \\
236 & .0177867 & .0176440 & .0001835 & .0000248 \\
238 & 96.9318780 & 96.9693892 & 1.0000000 & 0.0000000
\end{tabular}


PNNL-20650, Rev. 2

\author{
MASS SPECTROMETER LABORATORIES \\ ANALYTICAL CHEMISTRY \\ 325 BUILDING \\ CEC21-703A (M\&TE 467806) \\ PROCEDURE NO.: PNL-MA-599, PNNL-98523-264
}

\begin{tabular}{|c|c|c|c|}
\hline \multicolumn{2}{|c|}{$\begin{array}{l}\text { CUSTOMER: Sandy Fiskum } \\
\text { SAMPLE I.D.: U-0 } 30 \\
\text { LABORATORY LOG-IN NUMBER: } \\
\text { ALO LOG-IN NUMBER: }\end{array}$} & 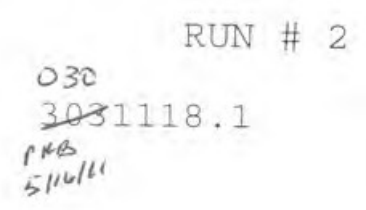 & $\begin{array}{l}\text { MASS DISC. FACTOR: .002 } \\
9 \text { MaY } 2011 \quad 12: 53: 55 \\
\text { ANALYST: Stan BOS J fBot } \\
\text { LRB: BNW } 56999 \text { PAGE } 76\end{array}$ \\
\hline $\begin{array}{l}\text { REFERENCE MASS: } \\
\text { MEASURED MASSES: }\end{array}$ & 238 & & $\begin{array}{l}\text { REVIEWED BY: PKß } \\
\text { DATE: } 5 / / 4 / 1\end{array}$ \\
\hline 234 & 235 & 236 & 238 \\
\hline \multicolumn{4}{|l|}{ RATIOS: } \\
\hline $\begin{array}{l}.0001714 \\
.0002171 \\
.0001252 \\
.0002064 \\
.0002314 \\
.0002047 \\
.0001689 \\
.0002152 \\
.000201\end{array}$ & $\begin{array}{l}.0304685 \\
.0311156 \\
.0312051 \\
.0314997 \\
.0315185 \\
.0313511 \\
.0315855 \\
.0304205 \\
.0311937\end{array}$ & $\begin{array}{l}.0001792 \\
.0001936 \\
.0001929 \\
.0002045 \\
.0001904 \\
.0002276 \\
.0002101 \\
.0002072 \\
.0002213\end{array}$ & $\begin{array}{l}1 \\
1 \\
1 \\
1 \\
1 \\
1 \\
1 \\
1 \\
1\end{array}$ \\
\hline
\end{tabular}

NO SCANS REJECTED AT THE 95\% LEVEL

MEAN RATIO FOR EACH ISOTOPE IS:

$\begin{array}{llll}.0001935 & .0311509 & .000203 & 1\end{array}$

STANDARD DEVIATION OF RATIO FOR EACH ISOTOPE IS:
3. $27 \mathrm{E}-5$
.0004317
1. $55 E-5$
0

MASS

234

235

236

238

\begin{abstract}
ATOM 응
.0187572

3.0198226

.0196759

96.9417443
\end{abstract}

\begin{abstract}
MASS \%
.0184491

2.9829074

.0195180

96.9791255
\end{abstract}

Mean Ratio

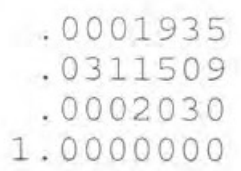

.0001935

.0002030

1.0000000

\title{
PNL-20650, Rev. 2
}

(1)


PNNL-20650, Rev. 2

\author{
MASS SPECTROMETER LABORATORIES \\ ANALYTICAL CHEMISTRY \\ 325 BUILDING \\ CEC21-703A (M\&TE 467806) \\ PROCEDURE NO.: PNL-MA-599, PNNL-98523-264
}

CUSTOMER: Sandy Fiskum

SAMPLE I.D. : U-030

LABORATORY LOG-IN NUMBER: 0301119

ALO LOG-IN NUMBER:

REFERENCE MASS: 238

MEASURED MASSES :
RUN \# 7

MASS DISC. FACTOR: .002 9 May 2011 16:09:54 ANALYST: Stan BOs J 1 pos LRB: BNW 56999 PAGE 77
234
235
236
238

RATIOS:

$\begin{array}{llll}.0002232 & .0313623 & .0002166 & 1 \\ .0002029 & .0312 & .0002143 & 1 \\ .0001916 & .0316136 & .0002108 & 1 \\ .0002075 & .0309867 & .0002099 & 1 \\ .0001829 & .0309076 & .0002145 & 1 \\ .0002027 & .0313242 & .0002487 & 1 \\ .000181 & .0312149 & .0002355 & 1 \\ .0001806 & .0314781 & .0002047 & 1 \\ .0001491 & .031002 & .0001881 & 1\end{array}$

NO SCANS REJECTED AT THE 95\% LEVEL

MEAN RATIO FOR EACH ISOTOPE IS:
.0001913
.0312321
.0002159
1

STANDARD DEVIATION OF RATIO FOR EACH ISOTOPE IS:
$2 \cdot 13 E-5$
.0002375
1. $74 \mathrm{E}-5$
0

MASS

234

235

236

238

\begin{abstract}
ATOM \%
.0185408

3.0274292

.0209280

96.9331020
\end{abstract}

\begin{abstract}
MASS \%
.0182362

2. 9904241

.0207602

96.9705795
\end{abstract}

Mean Ratio

$$
\begin{array}{r}
.0001913 \\
.0312321 \\
.0002159 \\
1.0000000
\end{array}
$$

STD

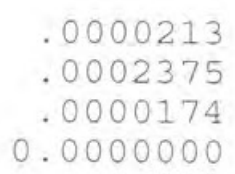


PNNL-20650, Rev. 2

\title{
MASS SPECTROMETER LABORATORIES
}

\author{
ANALYTICAL CHEMISTRY \\ 325 BUILDING \\ CEC21-703A (M\&TE 467806) \\ PROCEDURE NO.: PNL-MA-599, DNLL- $93523-264$
}

CUSTOMER: Sandy Fiskum $53^{55^{13}}$ RUN \# 3 MASS DISC. FACTOR: 0

SAMPLE I.D.: 11-0419-129-S-UPU 18 MaY 2011 10:34:02

LABORATORY LOG-IN NUMBER: 1211010

ALO LOG-IN NUMBER: 11-0419DS

SP

18 MaY $2011 \quad 10: 34: 02$
ANALYST: Stan Bos 1300 LRB: BNW 56999 PAGE 77

REFERENCE MASS: 239

REVIEWED BY: Pर r

MEASURED MASSES:
238
239
240
241
242

RATIOS:
$8 \cdot 5 E-5$
.1504369
.0066372
.0087258
.0029674
.0016365
.1603001
.0087258
.0014103
.0002682
.1564845
.0096338
.0031848
.0012072
.1422759
.0066869
.0036026
.0016136
.153111
.1433747
.0090029
.0021807
.0011531
.1484095
.0071204
.0016256
.0018476
.1470927
.006799
.0028746
.0008598
.1485962
.0028326
.0012315
1
.0069318
.0034269

NO SCANS REJECTED AT THE 95\% LEVEL

MEAN RATIO FOR EACH ISOTOPE IS:
.0011003
1
.1500091
.007831
.0026784

STANDARD DEVIATION OF RATIO FOR EACH ISOTOPE IS:
.0006044
0
.0058579
.0012129
.000773

Mean Ratio

STD

$238 \quad .0947206$

.0942657

.0011003

.0006044

86.0867677

86.0332792

12.9597719

1.0000000

0.0000000

12.9137967

.1500091

.0058579

.6741419

.6793609

.0078310

.0012129

.2305731

.2333223

.0026784

.0007730 
PNNL-20650, Rev. 2

\section{MASS SPECTROMETER LABORATORIES}

$$
\begin{aligned}
& \text { ANALYTICAL CHEMISTRY } \\
& \text { 325 BUILDING } \\
& \text { CEC21-703A (M\&TE 467806) PNIL-40.76 } 26-264 \\
& \text { PROCEDURE NO.: PNL-MA-599, P//4/" }
\end{aligned}
$$

CUSTOMER: Sandy Fiskum RUN \# 5

SAMPLE I.D. : 11-0419-129-D-Pu

LABORATORY LOG-IN NUMBER: 1211011

ALO LOG-IN NUMBER: 11-0419,DX

$$
\text { DP } 2,43
$$

REFERENCE MASS: 239

MEASURED MASSES:

$$
238
$$

239

240
MASS DISC. FACTOR: 0

18 May 2011 11:17:33

ANALYST: Stan BOS af f Beor LRB : BNW 56999 PAGE 77

REVIEWED BY: PK DATE: $\quad 5 / 15 / 11$

RATIOS:

$\begin{array}{ll}.001546 & 1 \\ .0012688 & 1 \\ .0014212 & 1 \\ .00191 & 1 \\ .0019548 & 1 \\ .0020885 & 1 \\ .0014236 & 1 \\ .0019405 & 1 \\ .0026577 & 1\end{array}$

$$
\begin{aligned}
& .1490039 \\
& .1509154 \\
& .1438 \\
& .1457857 \\
& .1553129 \\
& .1527571 \\
& .145531 \\
& .1506099 \\
& .1544235
\end{aligned}
$$

.0052284

.0063275

.0056386

.0058049

.0052747

.0067048

.0061516

.0059971

.0066698

NO SCANS REJECTED AT THE 95\% LEVEL

MEAN RATIO FOR EACH ISOTOPE IS:

$$
\begin{array}{llllll}
.0018012 & 1 & .002897933 & .0059775 & .0028964
\end{array}
$$

STANDARD DEVIATION OF RATIO FOR EACH ISOTOPE IS:

$$
\begin{array}{lllll}
.0004339 & 0 & .0040833 & .0005433 & .0003732
\end{array}
$$

MASS

238

239

240

241

242

\section{ATOM $\frac{5}{\circ}$}

.1552157

86.1721060

12.9080005

.5150926

.2495853
MASS $\frac{5}{0}$

.1544723

86.1197433

12.9541324

.5190874

.2525646
Mean Ratio

$$
\begin{array}{r}
.0018012 \\
1.0000000 \\
.1497933 \\
.0059775 \\
.0028964
\end{array}
$$

.003427

.0033134

.0025932

.0025557

.00263

.0030795

.0028624

.0023982

.0032078 
PNNL-20650, Rev. 2

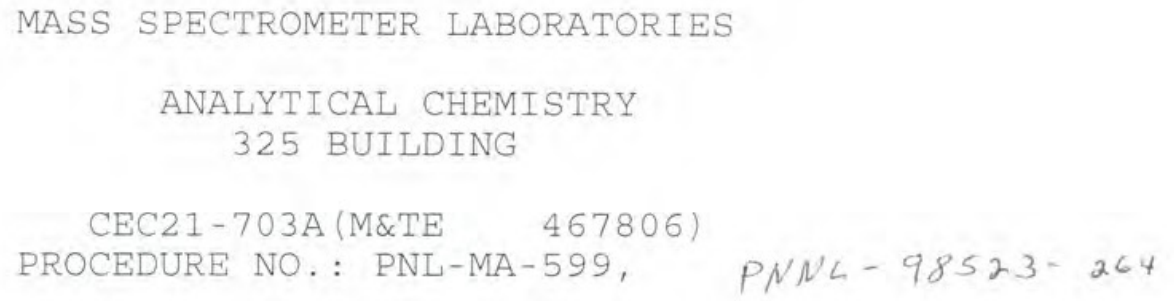

241242

RATIOS:

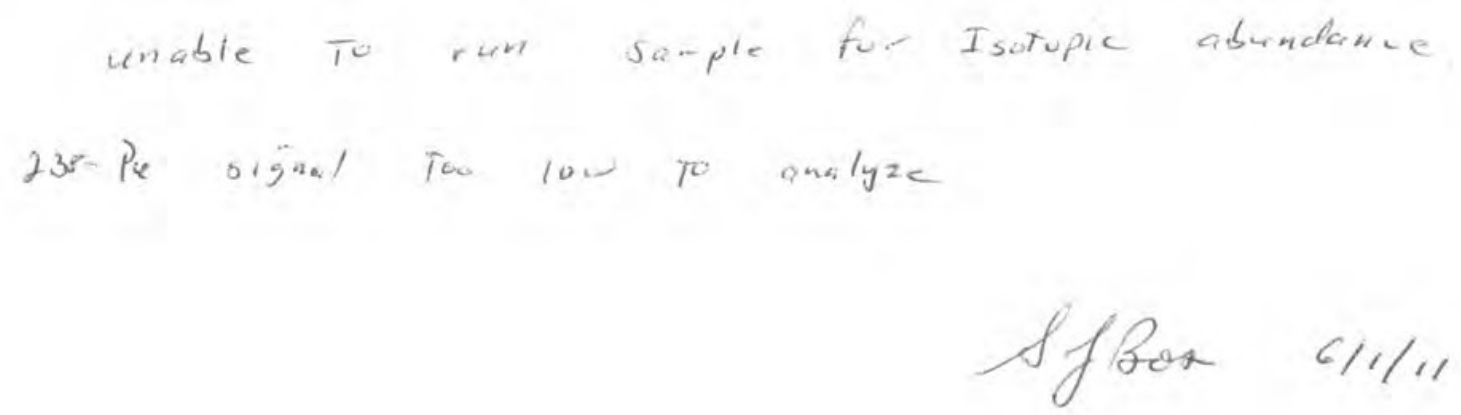


PNNL-20650, Rev. 2

MASS SPECTROMETER LABORATORIES

CUSTOMER: Sandy Fiskum

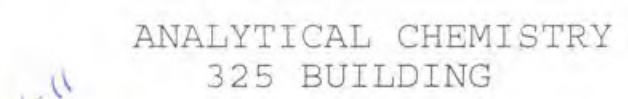

REFERENCE MASS: 239

REVIEWED BY: $\Rightarrow<\& 3$

MEASURED MASSES: DATE: $\quad 6 / 1 / 1 /$
238
239
240
241
242

RATIOS :

$\begin{array}{lllll}.0013726 & 1 & .155723 & .0063764 & .0032113 \\ .0017442 & 1 & .1544908 & .0057209 & .0032573 \\ .0021082 & 1 & .1537717 & .006367 & .0033056 \\ .0032776 & 1 & .1561428 & .0062485 & .0026224 \\ .004743 & 1 & .1502786 & .0060286 & .0021173 \\ .0048676 & 1 & .1533688 & .006787 & .0033412 \\ .0046629 & 1 & .1542418 & .0060014 & .0026476 \\ .0054208 & 1 & .1519449 & .0069198 & .0031544 \\ .0064582 & 1 & .1546383 & .0053422 & .0029456\end{array}$

NO SCANS REJECTED AT THE 95\% LEVEL

MEAN RATIO FOR EACH ISOTOPE IS:
.0038506
1
.1538445
.0061991
.0029559

STANDARD DEVIATION OF RATIO FOR EACH ISOTOPE IS:
.0017923
0
.0018225
.0004948
.0004147

MASS

238

239

240

241

242

ATOM
.3299960
85.7008174
13.1846007
.5312662
.2533196

MASS \%

.3284136

85.6482205

13.2316408

.5353832

.2563419
Mean Ratio

$$
\begin{array}{r}
.0038506 \\
1.0000000 \\
.1538445 \\
.0061991 \\
.0029559
\end{array}
$$

STD

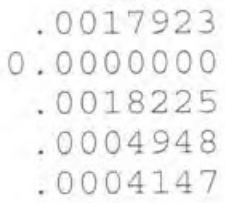


PNNL-20650, Rev. 2

\section{MASS SPECTROMETER LABORATORIES}

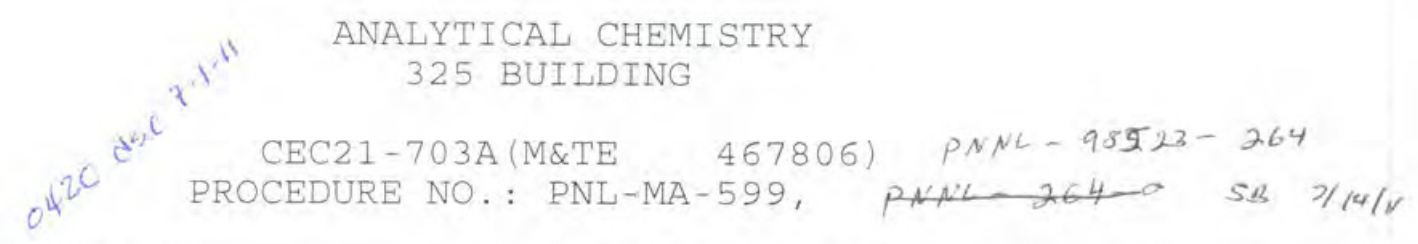

CUSTOMER: Sandy Fiskum

SAMPLE I.D.: 11-420-129-D-Pa

LABORATORY LOG-IN NUMBER: 1211014

ALO LOG-IN NUMBER: 11-0420DP

REFERENCE MASS: 239

MEASURED MASSES:
MASS DISC. FACTOR: 0 25 May 2011 14:03:40 ANALYST: Stan Bos of Bos REVIEWED BY: $P$ P B DATE: $6 / 1 / 1$,
239
240
238
241
242

RATIOS :

$\begin{array}{lllll}.0012596 & 1 & .1480662 & .0057363 & .0027234 \\ .0013423 & 1 & .1484897 & .0063903 & .0024842 \\ .0019579 & 1 & .148823 & .0064799 & .0024569 \\ .0019026 & 1 & .1506335 & .0055799 & .0027381 \\ .0030506 & 1 & .1530704 & .0058119 & .0027452 \\ .0032202 & 1 & .1494253 & .0059184 & .0030105 \\ .0035905 & 1 & .1537124 & .0060699 & .00222 \\ .0039135 & 1 & .1496795 & .0057392 & .0030698 \\ .0040012 & 1 & .1547968 & .005328 & .002356\end{array}$

NO SCANS REJECTED AT THE 95\% LEVEL

MEAN RATIO FOR EACH ISOTOPE IS:
.0026932
1
.1507441
.0058949
.0026449

STANDARD DEVIATION OF RATIO FOR EACH ISOTOPE IS:
.0010872
0
.0024869
.0003701
.0002882

MASS

238

239

240

241

242
ATOM \%

.2317737

86.0602213

12.9730700

.5073140

.2276210
MASS \%

.2306646

86.0082608

13.0194852

.5112505

.2303390
Mean Ratio

.0026932

1.0000000

.1507441

.0058949

.0026449
STD

.0010872

0.0000000

.0024869

.0003701

.0002882 
PNNL-20650, Rev. 2

\section{MASS SPECTROMETER LABORATORIES}

ANALYTICAL CHEMISTRY

325 BUILDING

CEC21-703A (M\&TE 467806)
PROCEDURE NO.: PNL-MA-599, PNNL- $98523-264$

CUSTOMER: Sandy Fiskum

SAMPLE I.D.: 11-0421-129-S-PU

LABORATORY LOG-IN NUMBER: 1211015

ALO LOG-IN NUMBER: 11-0421SP

REFERENCE MASS: 239

MEASURED MASSES :
RUN \# 9

MASS DISC. FACTOR: 0

18 MaY 2011 13:43:09

ANALYST: Stan BOS flos LRB: BNW 56999 PAGE 77

238

239

240

241

242

RATIOS:

.0008366
.0005724
.0005687
.0008529
.0013468
.00113
.0006977
.0013746
.0008146

$$
\begin{array}{r}
.1470467 \\
.1426943 \\
.1474566 \\
.1523031 \\
.1510944 \\
.1516444 \\
.1474053 \\
.1503327 \\
.1422176
\end{array}
$$

REVIEWED BY: DATE : $5 / / 8 / 11$

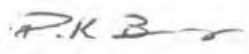

.0008146

1

.0026357

.0029295

.0030263

.0027947

.0020114

.0027199

.003042

.0020259

.0024473

NO SCANS REJECTED AT THE 95\% LEVEL

MEAN RATIO FOR EACH ISOTOPE IS:
.0009105
1
.1480217
.0056225
.0026258

STANDARD DEVIATION OF RATIO FOR EACH ISOTOPE IS:
.0003058
0
.0037073
.0008679
.0003925

MASS

238

239

240

241

242

ATOM
.0786802
86.4169435
12.7915816
.4858774
.2269174

MASS \%

.0783039

86.3650326

12.8373868

.4896490

.2296277
Mean Ratio

$$
\begin{array}{r}
.0009105 \\
1.0000000 \\
.1480217 \\
.0056225 \\
.0026258
\end{array}
$$

STD

.0003058

0.0000000

.0037073

.0008679

.0003925 
PNNL-20650, Rev. 2

\section{MASS SPECTROMETER LABORATORIES}

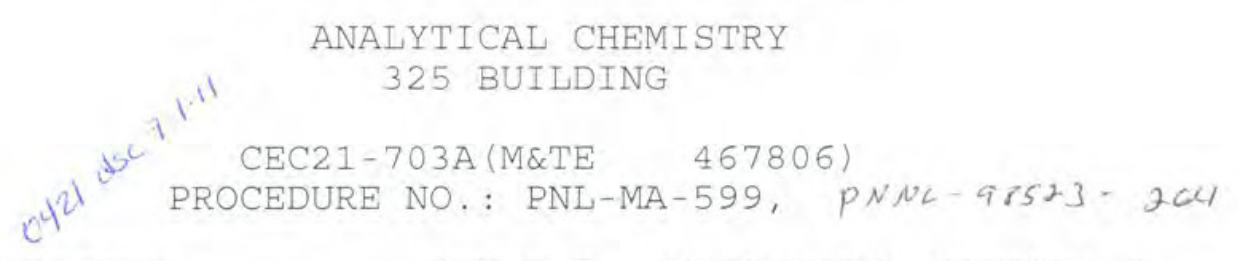

CUSTOMER: Sandy Fiskum

SAMPLE I.D.: 11-42I-129-D-P

LABORATORY LOG-IN NUMBER: 1211016

ALO LOG-IN NUMBER: 11-0421DP

REFERENCE MASS: 239

MEASURED MASSES:

\section{RUN \# 7}

MASS DISC. FACTOR: 0

25 May 2011 15:26:09, ANALYST: Stan BOS LRB: BNW 56999
PAGE 78

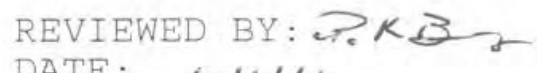

DATE: $6 / 1 / 1$
238
239
240
241
242

RATIOS:

$\begin{array}{lllll}.0010671 & 1 & .1503231 & .0061799 & .0032585 \\ .0014965 & 1 & .1547836 & .0057446 & .0028557 \\ .0021285 & 1 & .1508051 & .0060632 & .0027647 \\ .0011734 & 1 & .1463296 & .0061614 & .002466 \\ .0017046 & 1 & .1455919 & .0051903 & .0024916 \\ .001391 & 1 & .15 & .0047371 & .0031093 \\ .0020992 & 1 & .1547301 & .0058725 & .0028612 \\ .0017532 & 1 & .1481868 & .005472 & .0021633 \\ .0020429 & 1 & .1541788 & .0051821 & .002422\end{array}$

NO SCANS REJECTED AT THE 95\% LEVEL

MEAN RATIO FOR EACH ISOTOPE IS:
.0016507
1
.1505477
.0056225
.0027103

STANDARD DEVIATION OF RATIO FOR EACH ISOTOPE IS:

.0003963

MASS

238

239

240

241

242
0

$$
\text { ATOM \% }
$$

.1422372

86.1674394

12.9723060

.4844804

.2335370
.0034814

.0005074

.0003529 
PNNL-20650, Rev. 2

\section{MASS SPECTROMETER LABORATORIES}

\section{ANALYTICAL CHEMISTRY \\ 325 BUILDING \\ CEC21-703A (M\&TE 467806)}

PROCEDURE NO.: PNL-MA-599, DA

CUSTOMER: Sandy Fiskum

SAMPLE I.D.: 11-0422-129-S-PU

LABORATORY LOG-IN NUMBER: 1211017

ALO LOG-IN NUMBER: 11-0422SP

REFERENCE MASS: 239

MEASURED MASSES :
RUN \# 11 MASS DISC. FACTOR: 0 18 May 2011 14:53:07 ANALYST: Stan BOS Af 304 LRB : BNW 56999 PAGE 77

REVIEWED BY: $ج K$ B 1 DATE : $\quad(/ /, 1$
238
239
240
241
242

RATIOS:

$\begin{array}{lllll}.0007034 & 1 & .1489685 & .0055007 & .0032113 \\ .0008044 & 1 & .1451277 & .0056475 & .0024258 \\ .0011673 & 1 & .1535958 & .0066015 & .002797 \\ .0010867 & 1 & .1491745 & .0069001 & .0030832 \\ .001129 & 1 & .1495902 & .0058781 & .0031546 \\ .0010382 & 1 & .1500435 & .0061142 & .0022644 \\ .0012014 & 1 & .1489405 & .0046306 & .002106 \\ .0015597 & 1 & .1572736 & .0088325 & .0021779 \\ .0017046 & 1 & .1452526 & .0057476 & .0027336\end{array}$

NO SCANS REJECTED AT THE 95\% LEVEL

MEAN RATIO FOR EACH ISOTOPE IS:
.001155
1
.1497741
.0062058
.0026615

STANDARD DEVIATION OF RATIO FOR EACH ISOTOPE IS:

$$
\begin{array}{lllll}
.000319 & 0 & .0037864 & .0011808 & .0004334
\end{array}
$$

MASS
238
239
240
241
242

$$
\begin{aligned}
& \text { ATOM } \div \quad \text { MASS } \% \text { Mean Ratio STD }
\end{aligned}
$$

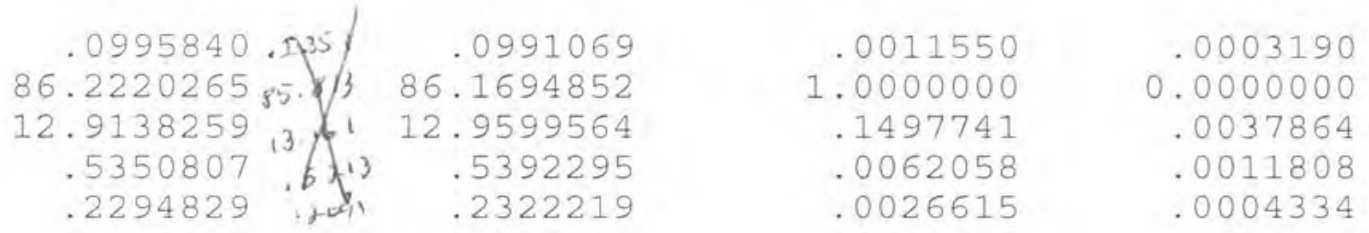

$$
\begin{aligned}
& \text { Is } 6 / 13 / 11 \\
& \text { entew on wrong ibret } \\
& \text { SB E/BII }
\end{aligned}
$$


PNNL-20650, Rev. 2

\author{
MASS SPECTROMETER LABORATORIES \\ ANALYTICAL CHEMISTRY \\ 325 BUILDING \\ CEC21-703A (M\&TE 467806) \\ PROCEDURE NO.: PNL-MA-599, PNFL- $98523-264$
}

CUSTOMER: Sandy Fiskum RUN \# 12 MASS DISC. FACTOR: 0

SAMPLE I.D.: 11-0422-129-D-Pu 18 May 2011 15:20:5,7

LABORATORY LOG-IN NUMBER: 1211018

ALO LOG-IN NUMBER: 11-0422DP

ANALYST: Stan BOS 1 Rer

LRB: BNW 56999 PAG 77

REFERENCE MASS: 239

REVIEWED BY: ? 2 \& $r$

MEASURED MASSES:

DATE: $\quad 6 / / / \%$
238
239
240
241
242

RATIOS :

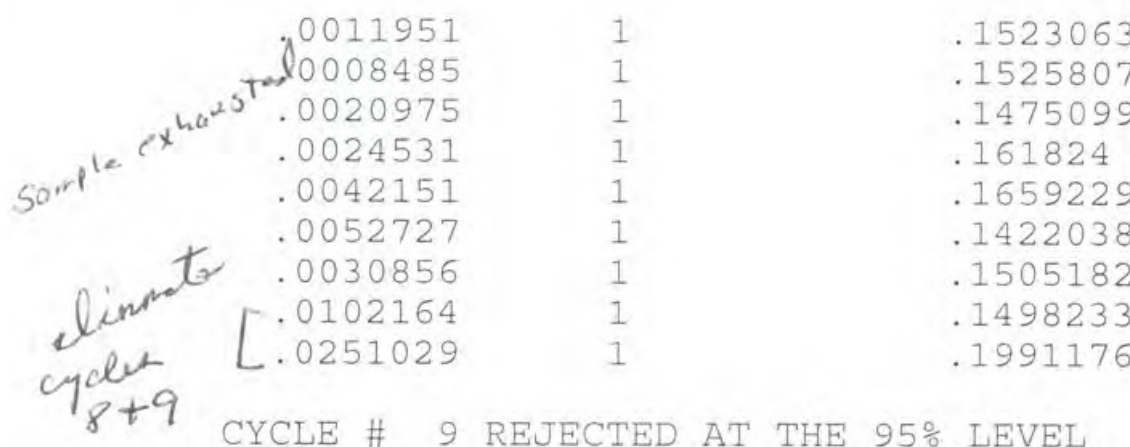

DUE TO THE 242/ 239 RATIO

MEAN RATIO FOR EACH ISOTOPE IS:

$\begin{array}{ll}.006431 & .0028562 \\ .0060339 & .0021753 \\ .0031842 & .001405 \\ .0042327 & .0026904 \\ .0048338 & .0042287 \\ .0102411 & .00172 \\ .0075363 & .0019662 \\ .005477 & .0008525 \\ .0077574 & -.0109559\end{array}$

$.006431 \quad .0028562$

$.0060339 \quad .0021753$

$.0042327 \quad .0026904$

$.0048338 \quad .0042287$

$.0102411 \quad .00172$

$-.0109559$

$$
\begin{array}{llllll}
.003673 & 1 & .00528361 & .005963 & .0022368
\end{array}
$$

STANDARD DEVIATION OF RATIO FOR EACH ISOTOPE IS:

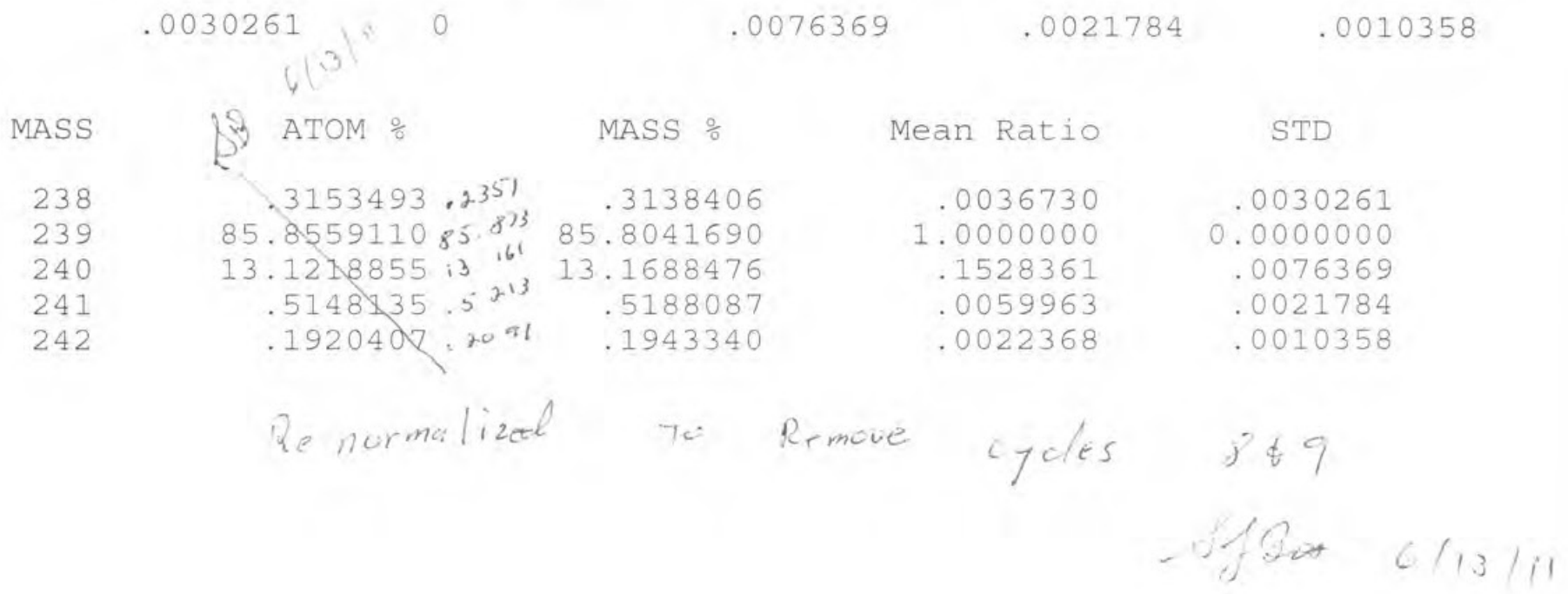


PNNL-20650, Rev. 2

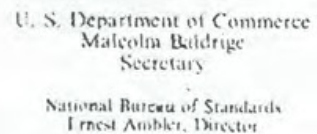

RECEIVED

MAY 171983

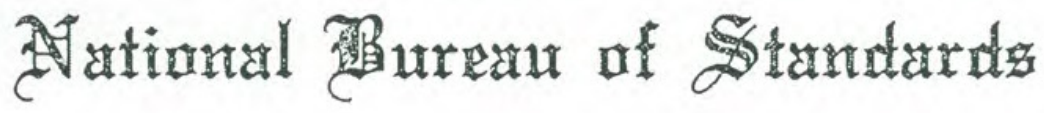

W.Y. MATSUMOTO

\section{UTertitititate of Ânulugis}

\section{Standard Reference Material 946}

\section{Plutonium Isotopic Standard}

This Standard Reference Material (SRM) is certified as an isotopic standard for use in isotopic measurements of plutonium. SRM 946 consists of approximately $250 \mathrm{mg}$ of plutonium in the form of plutonium sulfate tetrahydrate packaged in a glass microbottle.

\begin{tabular}{|c|c|c|c|c|c|}
\hline & ${ }^{238} \mathrm{Pu}$ & ${ }^{239} \mathrm{Pu}$ & ${ }^{240} \mathrm{Pu}$ & ${ }^{241} \mathrm{Pu}$ & ${ }^{242} \mathrm{Pu}$ \\
\hline Atom Percent* & $\begin{array}{r}0.232 \\
\pm 0.007\end{array}$ & $\begin{array}{r}84.464 \\
\pm 0.015\end{array}$ & $\begin{array}{r}12.253 \\
\pm 0.015\end{array}$ & $\begin{array}{r}2.477 \\
\pm 0.005\end{array}$ & $\begin{array}{r}0.574 \\
\pm 0.003\end{array}$ \\
\hline
\end{tabular}

* As of January 1, 1982, refer to Table I for quarterly decay-adjusted values.

The plut onium isotopic distribution was determined by thermal ionization mass spectrometry at the National Bureau of Standards (NBS) on samples from which the americium and uranium were removed. Because high-purity plutonium isotopes have not been used to prepare known synthetic mixtures, the accuracy is dependent on uranium and plutonium exhibiting similar behavior. The observed mass spectrometer data were corrected for mass discrimination effects using data from the analysis of uranium isotopic SRM's that had been analyzed under similar conditions.

SRM 946 contains uranium and americium isotopes, including growing-in daughters of plutonium that are isobaric with the plutonium isotopes. In addition, there may be radiation damage to the glass bottle and the teflon cap liner resulting in small glass slivers. Therefore, in its use, a chemical separation that provides a purified plutonium fraction is essential to the attainment of high accuracy.

Measurements leading 10 the certitication of this SRM were made in the Inorganic Analytical Research Division by E.L. Garner and L.A. Machlan.

The technical and support aspects involved in the revision of this Certificate were coordinated through the Office of Standard Reference Materials by T.E. Gills.

August 19, 1982

Washington, D.C. 20234

(Revision of Certificate

dated 12-3-71)
George A. Uriano, Chief

Office of Standard Reference Materials

(over) 
PNNL-20650, Rev. 2

The decay-adjusted values for the plutonium isotopic composition, in atompercent, are tabulated below in Table 1 . The half-life values, in years, used for the decay-adjustment are: ${ }^{238} \mathrm{Pu}, 87.74 ;{ }^{239} \mathrm{Pu}, 24,119 ;{ }^{240} \mathrm{Pu}, 6,560 ;{ }^{24 !} \mathrm{Pu}, 14.34$; and ${ }^{242} \mathrm{Pu}, 387,000$.

Table 1

Decay-Adjusted Plutonium Isotopic Composition

Date

January I, 1982

April 1, 1982

July 1, 1982

October 1, 1982

January I, 1983

April I, 1983

July 1, 1983

October I, 1983

January 1, 1984

April I, 1984

July I, 1984

October 1, 1984

January I, 1985

April 1, 1985

July 1,1985

October 1, 1985

January I, 1986

April 1, 1986

July 1, 1986

October 1, 1986

95\% Confidence Limit:

\begin{tabular}{l}
${ }^{238} \mathrm{Pu}$ \\
\hline 0.232 \\
.231 \\
.231 \\
.230 \\
.230 \\
.230 \\
.229 .225 \\
.229 \\
.229 \\
.228 \\
.228
\end{tabular}

.227

.227

.227

.226

.226

.225

.225 .00265

.225 .00265

.224

$\pm 0.007$

\section{Atom Percent}

\begin{tabular}{|c|c|c|c|c|c|c|}
\hline${ }^{239} \mathrm{Pu}$ & & ${ }^{240} \mathrm{Pu}$ & & ${ }^{241} \mathrm{Pu}$ & & ${ }^{242} \mathrm{Pu}$ \\
\hline 84.464 & & 12.253 & & 2.477 & & 0.574 \\
\hline 84.490 & & 12.257 & & 2.448 & & .574 \\
\hline 84.515 & & 12.260 & & 2.419 & & .575 \\
\hline 84.540 & & 12.2 .64 & & 2.391 & & .575 \\
\hline 84.565 & & 12.267 & & 2.363 & & .575 \\
\hline 84.590 & & 12.270 & & 2.335 & & .575 \\
\hline 84.614 & .888 & 12.274 & .311 & 2.308 & .000 & $.575,577$ \\
\hline 84.638 & & 12.277 & & 2.281 & & .575 \\
\hline 84.662 & & 12.280 & & 2.253 & & .576 \\
\hline 84.685 & & 12.283 & & 2.227 & & .576 \\
\hline 84.709 & & 12.286 & & 2.201 & & .576 \\
\hline 84.732 & & 12.290 & & 2.175 & & .576 \\
\hline 84.755 & & 12.293 & & 2.149 & & .576 \\
\hline 84.777 & & 12.296 & & 2.125 & & .576 \\
\hline 84.799 & & 12.299 & & 2.100 & & .577 \\
\hline 84.821 & & 12.302 & & 2.075 & & .577 \\
\hline 84.843 & & 12.305 & & 2.050 & & .577 \\
\hline 84.864 & .14502 & 12.307 & & 2.026 & 0238 & .577 .00 \\
\hline 84.885 & & 12.310 & & 2.003 & & .577 \\
\hline 84.906 & & 12.313 & & 1.979 & & .577 \\
\hline \pm 0.015 & & \pm 0.015 & & \pm 0.005 & & \pm 0.003 \\
\hline 85.08 & & 12136 & & & & \\
\hline
\end{tabular}


PNNL-20650, Rev. 2

\begin{tabular}{|c|c|c|c|c|}
\hline Pu SRM 94 & & & Renorm & Decay Correcte \\
\hline & $\begin{array}{c}10 / 1 / 1997 \\
\text { at } \%\end{array}$ & $\begin{array}{c}5 / 17 / 2011 \\
\text { at } \%\end{array}$ & $\begin{array}{c}5 / 17 / 2011 \\
\text { at } \%\end{array}$ & $\begin{array}{c}\text { at } \% \\
\text { Pu Only }\end{array}$ \\
\hline 238 & 0.2078 & 0.186588 & 0.186625 & 0.187685 \\
\hline 239 & 85.63 & 85.630000 & 85.647226 & 86.133715 \\
\hline 240 & 12.407 & 12.407000 & 12.409496 & 12.479984 \\
\hline 241 & 1.1741 & 0.609406 & 0.609529 & 0.612991 \\
\hline Am-241 & 0 & 0.564694 & 0.564808 & \\
\hline 242 & 0.5822 & 0.582200 & 0.582317 & 0.585625 \\
\hline & 100.0011 & 99.979888 & $\begin{array}{r}100.000000 \\
99.435192\end{array}$ & $\begin{array}{l}100.000000 \\
\text { Pu only }\end{array}$ \\
\hline
\end{tabular}


PNNL-20650, Rev. 2

MASS SPECTROMETER LABORATORIES

\section{ANALYTICAL CHEMISTRY \\ 325 BUILDING}

CEC21-703A (M\&TE 467806)

PROCEDURE NO.: PNL-MA-599, PNN $96523-264$

CUSTOMER: Sandy Fiskum

SAMPLE I.D.: PU-946

LABORATORY LOG-IN NUMBER: 9461106 .

ALO LOG-IN NUMBER:

REFERENCE MASS: 239

MEASURED MASSES:
RUN \# 1

MASS DISC. FACTOR: 0 17 May $2011 \quad 13: 21: 28$ ANALYST: Stan BOS f Gery
LRB: BNW 56999 PAGE 77

REVIEWED BY: $\supset$, DATE: $5 /, 7 / 1$,
238
239
240
241
242

RATIOS:

$\begin{array}{lllll}.0023652 & 1 & .1440462 & .0076631 & .0064258 \\ .0021445 & 1 & .1432006 & .0072838 & .0067369 \\ .0022082 & 1 & .1419692 & .0073621 & .0067458 \\ .0020985 & 1 & .1435652 & .0071028 & .0067622 \\ .0022031 & 1 & .1439553 & .0071098 & .0063916 \\ .0021513 & 1 & .1435857 & .00684 & .0066657 \\ .0021628 & 1 & .1452246 & .0071147 & .006672 \\ .0021241 & 1 & .1435586 & .0069958 & .006709 \\ .0022257 & 1 & .1440954 & .0071975 & .0068974\end{array}$

NO SCANS REJECTED AT THE 95\% LEVEL

MEAN RATIO FOR EACH ISOTOPE IS:
.002187
1
.143689
.0071855
.0066674

STANDARD DEVIATION OF RATIO FOR EACH ISOTOPE IS:
$7.86 E-5$
0
.0008632
.0002351
.0001617

MASS \%

Mean Ratio

STD

238

.1876741

86.1723697

12.4338272

.6243730

.5817559
.0021870
1.0000000
.1436890
.0071855
.0066674

.0000786

0.0000000

.0008632

.0002351

.0001617 
PNNL-20650, Rev. 2

MASS SPECTROMETER LABORATORIES

\section{ANALYTICAL CHEMISTRY \\ 325 BUILDING}

CEC21-703A (M\&TE 467806)
PROCEDURE NO.: PNL-MA-599, PNHL-98523-264

CUSTOMER: Sandy Fiskum

SAMPLE I.D.: PU-946

LABORATORY LOG-IN NUMBER: 9461106.2

ALO LOG-IN NUMBER:

REFERENCE MASS: 239

MEASURED MASSES:

238

239

240

241

242

REVIEWED BY: جK\& DATE: $\quad s / 17 / 1$,

MASS DISC. FACTOR: 0 17 May 2011 13:37:27 ANALYST: Stan BOS
LRB: BNW 56999 PAGE 770 \%

RATIOS:

$\begin{array}{lllll}.0020323 & 1 & .1434716 & .0069617 & .0070438 \\ .002261 & 1 & .1439947 & .0070213 & .0069259 \\ .0021303 & 1 & .1440443 & .0071292 & .0066145 \\ .0021541 & 1 & .1433893 & .0070437 & .0068346 \\ .0020656 & 1 & .1443016 & .0073361 & .006848 \\ .0021787 & 1 & .1445669 & .0070319 & .0066436 \\ .0021344 & 1 & .144987 & .0070805 & .0065541 \\ .0021153 & 1 & .1442272 & .0073321 & .0066799 \\ .0020932 & 1 & .1431098 & .006992 & .0067261\end{array}$

NO SCANS REJECTED AT THE 95\% LEVEL

MEAN RATIO FOR EACH ISOTOPE IS:
.0021294
1
.1440103
.0071032
.0067634

STANDARD DEVIATION OF RATIO FOR EACH ISOTOPE IS:
$6.65 E-5$
0
.0006004
.0001395
.0001604

MASS

238

239

240

241

242

\section{ATOM :}

.1835709

86.2064313

12.4146099

.6123394

.5830485
MASS \%

.1826866

86.1516228

12.4586279

.6170710

.5899918
Mean Ratio

.0021294

1.0000000

.1440103

.0071032

.0067634
STD

.0000665
0.0000000
.0006004
.0001395
.0001604 
PNNL-20650, Rev. 2

MASS SPECTROMETER LABORATORIES

\author{
ANALYTICAL CHEMISTRY \\ 325 BUILDING \\ CEC21-703A (M\&TE 467806) \\ PROCEDURE NO.: PNL-MA-599, PANL-18533- 264
}

CUSTOMER: Sandy Fiskum

SAMPLE I.D.: PU-946

LABORATORY LOG-IN NUMBER: 9461106.3

ALO LOG-IN NUMBER:

REFERENCE MASS: 239

MEASURED MASSES:
RUN \# 3

MASS DISC. FACTOR: 0

17 May 2011 13:54:47,

ANALYST: Stan BOS 30 LRB : BNW 56999

REVIEWED BY: $\rightarrow$ ? $3-2$ DATE: $5 /, 7 / 1$
238
239
240
241

RATIOS:

$\begin{array}{lllll}.0022594 & 1 & .1447871 & .0069771 & .0064662 \\ .002029 & 1 & .1430881 & .0069715 & .0068219 \\ .0021938 & 1 & .1471769 & .0074265 & .0067507 \\ .0021561 & 1 & .1447172 & .0072337 & .0069118 \\ .0021637 & 1 & .1445398 & .0078381 & .0067061 \\ .0020803 & 1 & .1440903 & .0073266 & .0067266 \\ .0020623 & 1 & .1451425 & .0074061 & .0071263 \\ .0019834 & 1 & .1443631 & .0073187 & .0067984 \\ .0019503 & 1 & .1448737 & .0074369 & .006714\end{array}$

NO SCANS REJECTED AT THE 95\% LEVEL

MEAN RATIO FOR EACH ISOTOPE IS:
.0020976
1
.1447532
.0073261
.0067802

STANDARD DEVIATION OF RATIO FOR EACH ISOTOPE IS:
.0001026
0
.001087
.0002615
.0001773

\begin{tabular}{rr} 
MASS & \multicolumn{1}{r}{ ATOM \% } \\
238 & .1806782 \\
239 & 86.1358230 \\
240 & 12.4684360 \\
241 & .6310425 \\
242 & .5840203
\end{tabular}

MASS \%
.1798071
86.0807101
12.5125940
.6359160
.5909728

Mean Ratio

STD

86.1358230
12.4684360
.6310425
.5840203
.0020976
1.0000000
.1447532
.0073261
.0067802
.0001026
0.0000000
.0010870
.0002615
.0001773

\title{
E.68
}


PNNL-20650, Rev. 2

MASS SPECTROMETER LABORATORIES

\author{
ANALYTICAL CHEMISTRY \\ 325 BUILDING \\ CEC21-703A (M\&TE 467806) \\ PROCEDURE NO.: PNL-MA-599, DNNL $98525-264$
}

CUSTOMER: Sandy Fiskum

SAMPLE I.D.: PU-946

LABORATORY LOG-IN NUMBER: 9461107.1

ALO LOG-IN NUMBER:

REFERENCE MASS: 239

MEASURED MASSES:

$$
238
$$

239

240
MASS DISC. FACTOR: 0 18 May 2011 09:26:44 ANALYST: Stan BOS 1 PRB: BNW 56999 PAGE 77

REVIEWED BY: PK R 2 DATE: $\quad 5 / 18 / 11$

RATIOS:

$\begin{array}{ll}.0019998 & 1 \\ .0022519 & 1 \\ .002342 & 1 \\ .002045 & 1 \\ .0021811 & 1 \\ .0023041 & 1 \\ .0023481 & 1 \\ .0020481 & 1 \\ .0021242 & 1\end{array}$

.1477493

.1466591

.1431908

.142464

.1446248

.1457228

.1450692

.1413035

.1429934

241

242

NO SCANS REJECTED AT THE 95\% LEVEL

MEAN RATIO FOR EACH ISOTOPE IS:

$$
\begin{array}{lllll}
.0021827 & 1 & .1444196 & .0070698 & .0066198
\end{array}
$$

STANDARD DEVIATION OF RATIO FOR EACH ISOTOPE IS:
.0001352
0
.0021019
.0002
.0001924

MASS

238

239

240

241

242

\section{ATOM $\div$}

.1881173

86.1852074

12.4468369

.6093116

.5705268
MASS \%

.1872112

86.1304698

12. 4909775

.6140202

.5773213
Mean Ratio

$$
\begin{array}{r}
.0021827 \\
1.0000000 \\
.1444196 \\
.0070698 \\
.0066198
\end{array}
$$

.0066316

.0065578

.0069405

.0067249

.0063056

.0067846

.0064383

.0065016

.006693 
PNNL-20650, Rev. 2

MASS SPECTROMETER LABORATORIES

\section{ANALYTICAL CHEMISTRY \\ 325 BUILDING \\ CEC21-703A (M\&TE 467806)}

PROCEDURE NO.: PNL-MA-599, PNy - $98523-264$

CUSTOMER: Sandy Fiskum

SAMPLE I.D.: PU-946

LABORATORY LOG-IN NUMBER: $\quad 9461107.2$

ALO LOG-IN NUMBER:

REFERENCE MASS: 239

MEASURED MASSES :
MASS DISC. FACTOR: 0 18 May 2011 09:43:03 ANALYST: Stan BOS LRB: BNW 56999

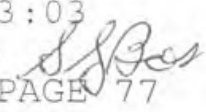

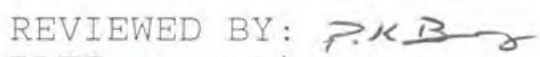
DATE: $\quad 5 / 18 / 1$,

$$
238
$$

240

241

242

RATIOS:

.0024706
.0021144
.0023262
.0023312
.0021932
.0021634
.0022204
.0021861
.0024847

$$
\begin{array}{r}
.1430085 \\
.1443037 \\
.1433183 \\
.1432405 \\
.1434035 \\
.1446059 \\
.1424739 \\
.1457711 \\
.1448969
\end{array}
$$

$$
\begin{aligned}
& .0073102 \\
& .0071906 \\
& .0070787 \\
& .0069752 \\
& .0070166 \\
& .0066461 \\
& .0074596 \\
& .0070999 \\
& .0068111
\end{aligned}
$$

.0066909 .0064261 .006455 .00717 .0070888 .0066066 .0067674 .0069465 .0068629

NO SCANS REJECTED AT THE 95\% LEVEL

MEAN RATIO FOR EACH ISOTOPE IS:

$$
\begin{array}{lllll}
.0022767 & 1 & .1438914 & .0070653 & .0067793
\end{array}
$$

STANDARD DEVIATION OF RATIO FOR EACH ISOTOPE IS:

$$
\begin{array}{lllll}
.0001341 & 0 & .0010609 & .0002458 & .0002625
\end{array}
$$

MASS

238

239

240

241

242

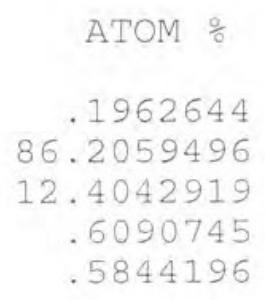

MASS $\frac{\circ}{2}$

.1953191

86.1512330

12.4482865

.6137815

.5913798
Mean Ratio

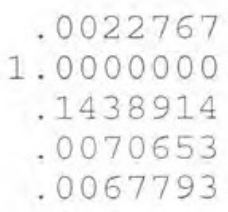

STD

$$
\begin{array}{r}
.0001341 \\
0.0000000 \\
.0010609 \\
.0002458 \\
.0002625
\end{array}
$$


PNNL-20650, Rev. 2

\section{MASS SPECTROMETER LABORATORIES \\ ANALYTICAL CHEMISTRY \\ 325 BUILDING \\ CEC21-703A (M\&TE 467806)}

PROCEDURE NO.: PNL-MA-599, PPNL-98523. 264

CUSTOMER: Sandy Fiskum

SAMPLE I.D.: PU-946

LABORATORY LOG-IN NUMBER:

ALO LOG-IN NUMBER:

REFERENCE MASS: 239

MEASURED MASSES :

238

239

240

RUN \# $\# 13$

$946110 \not 8^{2} .1$

MASS DISC. FACTOR: 0

18 May 2011 15:54:4j

ANALYST: Stan BOS J Bad

REVIEWED BY: Pיא DATE: $5 / 25 / 11$

RATIOS :

$\begin{array}{ll}.0022805 & 1 \\ .0016822 & 1 \\ .0026149 & 1 \\ .0025598 & 1 \\ .0031166 & 1 \\ .0021294 & 1 \\ .0017241 & 1 \\ .0023191 & 1 \\ .0020145 & 1\end{array}$

.1470138

.0071981

.0070842

.1472824

.0071703

.0068758

.1391972

.0074456

.0068328

.1433622

.0084116

.0063849

.1418578

.0076801

.0068231

.140706

.0080441

.0070711

.1468405

.0050084

.0062489

.1438318

.0068766

.0072836

.1390538

.0066295

.0067792

NO SCANS REJECTED AT THE 95\% LEVEL

MEAN RATIO FOR EACH ISOTOPE IS:

$$
\begin{array}{lllll}
.0022712 & 1 & .1432384 & .0071627 & .0068204
\end{array}
$$

STANDARD DEVIATION OF RATIO FOR EACH ISOTOPE IS:
.0004542
0
.0032809
.0009806
.0003294

MASS
238
239
240
241
242

ATOM \%
.1958812
86.2446118
12.3535396
.6177442
.5882232

MASS $\frac{1}{\circ}$

.1949380

86.1899486

12.3973654

.6225188

.5952293
Mean Ratio

$$
\begin{array}{r}
.0022712 \\
1.0000000 \\
.1432384 \\
.0071627 \\
.0068204
\end{array}
$$

STD

$$
\begin{array}{r}
.0004542 \\
0.0000000 \\
.0032809 \\
.0009806 \\
.0003294
\end{array}
$$


PNNL-20650, Rev. 2

MASS SPECTROMETER LABORATORIES

\author{
ANALYTICAL CHEMISTRY \\ 325 BUILDING \\ CEC21-703A (M\&TE 467806) \\ PROCEDURE NO.: PNL-MA-599, PNLC- $98520-264$
}

CUSTOMER: Sandy Fiskum

SAMPLE I.D.: PU-946

LABORATORY LOG-IN NUMBER: $946110 \not 09.1$

ALO LOG-IN NUMBER:

REFERENCE MASS: 239

MEASURED MASSES:

$$
238
$$

240
MASS DISC. FACTOR: 0 25 May 2011 11:23:54

ANALYST: Stan BOS Of Bes LRB: BNW 56999 PAGE 78 REVIEWED BY: $>x+2$
DATE: $5 / 25 / 1$

\title{
RATIOS:
}

$\begin{array}{lllll}.0022267 & 1 & .1428996 & .0065841 & .0066254 \\ .002147 & 1 & .1448841 & .0070371 & .0067367 \\ .0022451 & 1 & .147404 & .0074173 & .0067458 \\ .0022628 & 1 & .1450578 & .007025 & .0065672 \\ .0019778 & 1 & .149 & .0073031 & .006967 \\ .002208 & 1 & .1447977 & .0071538 & .0067846 \\ .0021492 & 1 & .1450688 & .0071101 & .0069136 \\ .0021407 & 1 & .1460089 & .0068295 & .0063065 \\ .0022267 & 1 & .007197 & .0068313\end{array}$

NO SCANS REJECTED AT THE 95\% LEVEL

MEAN RATIO FOR EACH ISOTOPE IS:
.002176
1
.1454821
.007073
.0067198

STANDARD DEVIATION OE RATIO FOR EACH ISOTOPE IS:
$8 \cdot 7 \mathrm{E}-5$
0
.0017955
.0002492
.0001998

MASS

238

239

240

241

242

ATOM \%
.1873524
86.0992015
12.5258964
.6089794
.5785703

MASS $\frac{\circ}{0}$

.1864492

86.0441469

12.5702630

.6136828

.5854581
Mean Ratio

$$
\begin{array}{r}
.0021760 \\
1.0000000 \\
.1454821 \\
.0070730 \\
.0067198
\end{array}
$$

STD

.0000870

0.0000000

.0017955

.0002492

.0001998 
PNNL-20650, Rev. 2

MASS SPECTROMETER LABORATORIES

\author{
ANALYTICAL CHEMISTRY \\ 325 BUILDING \\ CEC21-703A (M\&TE 467806) \\ PROCEDURE NO.: PNL-MA-599, PN1K- $98523-264$
}

CUSTOMER: Sandy Fiskum

SAMPLE I.D. : PU-946

LABORATORY LOG-IN NUMBER

ALO LOG-IN NUMBER:

REFERENCE MASS: 239

MEASURED MASSES :

238

239

240

RUN \# 2

Aas $5 / 25 / 4$

$946110 \not 09 \cdot 2$

MASS DISC. FACTOR: 0

25 May 2011 11:40:26,

ANALYST: Stan BOS of Pes

LRB: BNW 56999 PAGE 78

242

RATIOS:

$\begin{array}{ll}.0020825 & 1 \\ .0022889 & 1 \\ .0023645 & 1 \\ .0023698 & 1 \\ .0018694 & 1 \\ .002626 & 1 \\ .0019959 & 1 \\ .0023077 & 1 \\ .0018988 & 1\end{array}$

.1470099

.0075728

.0068716

.1427578

.0071397

.0068243

.0066393

.146598

.1446244

.0070716

.007238

.1459414

.007169

.0068182

.1463435

.0068531

419388

.0078134

.0077529

NO SCANS REJECTED AT THE 95\% LEVEL

MEAN RATIO FOR EACH ISOTOPE IS:

$$
\begin{array}{lllll}
.0022004 & 1 & .1449194 & .0072239 & .0069641
\end{array}
$$

STANDARD DEVIATION OF RATIO FOR EACH ISOTOPE IS:

.0002531

0

.001741

.0003944

.0004117

MASS

238

239

240

241

242

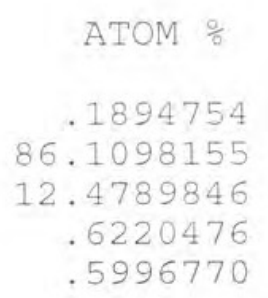

.5996770

\begin{abstract}
MASS \%
\end{abstract}
.1885616

86.0546086

12.5231639

.6268508

.6068150
Mean Ratio

$$
\begin{array}{r}
.0022004 \\
1.0000000 \\
.1449194 \\
.0072239 \\
.0069641
\end{array}
$$

STD

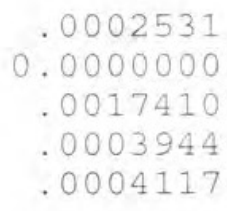


PNNL-20650, Rev. 2

MASS SPECTROMETER LABORATORIES

ANALYTICAL CHEMISTRY
325 BUILDING
CEC21-703A (M\&TE 467806)
PROCEDURE NO.: PNL-MA-599, PNOL.98523-264

CUSTOMER: Sandy Fiskum

SAMPLE I.D.: PU-946

RUN \# 8 MASS DISC. FACTOR: 0

LABORATORY LOG-IN NUMBER: 94611010.

ALO LOG-IN NUMBER:

25 May 2011 15:55:53

ANALYST: Stan BOS If Bey

LRB: BNW 56999 PAGEO 8

REFERENCE MASS: 239

REVIEWED BY: P.KB 2

MEASURED MASSES :

DATE: $5 / 25 / 11$
238
239
240
241
242

RATIOS:

$\begin{array}{ll}.0022503 & 1 \\ .0021502 & 1 \\ .0019062 & 1 \\ .0021902 & 1 \\ .0020751 & 1 \\ .0023085 & 1 \\ .0022016 & 1 \\ .002156 & 1 \\ .0019946 & 1\end{array}$

.1452927

.0078199

.0073162

.0069625

.1439105

.0070752

.1431991

.0073042

.1456688

.0072817

.1448252

.0072637

.1446784

.006961

.1458613

.007255

.0067963

.0065748

.0068441

.0066905

.0067102

.0069726

.0070748

.0067865

.0069765

NO SCANS REJECTED AT THE 95\% LEVEL

MEAN RATIO FOR EACH ISOTOPE IS:
.002137
1
.144368
.0072488
.0068251

STANDARD DEVIATION OF RATIO FOR EACH ISOTOPE IS:

.0001261

0

.0013766

.0002574

.0001596

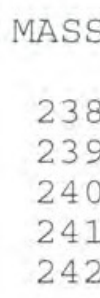

MASS

238

239

240

241

242

\section{ATOM \%}

.1841289

86.1638925

12.4393117

.6245859

.5880810
MASS \%

.1832415

86.1088815

12.4833840

.6294105

.5950826
Mean Ratio

.0021370

1.0000000

.1443680

.0072488

.0068251
STD

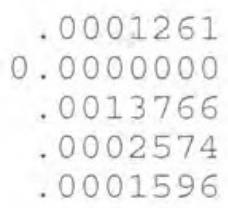


PNNL-20650, Rev. 2

\section{K-Basin Occurrence Report}

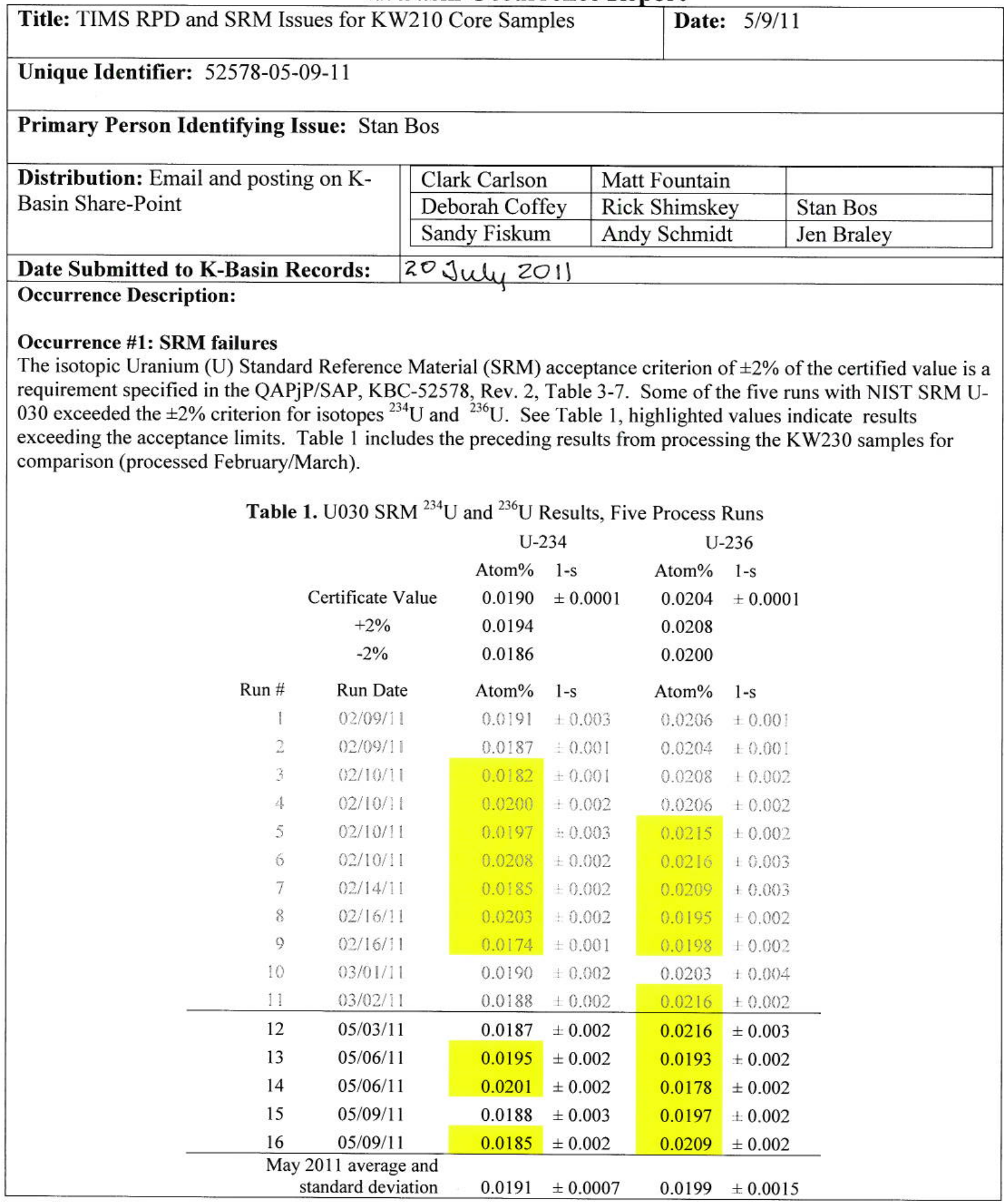




\begin{tabular}{|c|c|c|c|c|}
\hline $\begin{array}{l}\text { Grand average and } \\
\text { standard deviation }\end{array}$ & 0.0191 & \pm 0.0009 & 0.0204 & \pm 0.0010 \\
\hline Occurrence \#2: RPD Failures & & & & \\
\hline
\end{tabular}

\section{Impact:}

\section{Impact of Occurrence \#1:}

No impact. The specified $\pm 2 \%$ acceptance criterion was intended to apply to ${ }^{235} \mathrm{U}$ and ${ }^{238} \mathrm{U}$ (see attached correspondence). The QAPjP/SAP KBC-33786, Rev. 2, Table 3-7 did not specifiy which isotopes were were subject to this criterion. Therefore the sample analyisis request specified the $\pm 2 \%$ criterion applied to isotopes at concentrations $>0.1$ atom percent. This analysis request had been reviewed by the $\mathrm{CH} 2 \mathrm{M}$ Hill Plataeu Remeidation Co. (CHPRC) Safeguards representative and signed by the Buyer's Technical Representative (BTR); the BTR signature indicated concurrence that the DQOs were properly identified and flowed down to the analytical laboratory/staff.

The ${ }^{234} U$ and ${ }^{236} U$ isotopic ratios were significantly less than 0.1 atom \%. The $1-\sigma$ uncertainties of the ${ }^{234} U$ and ${ }^{236} U$ values were large (much greater than $2 \%$, relative) and generally bracketed the $\pm 2 \%$ criterion range as shown in Figure 1. The averaged results for isotopes ${ }^{234} \mathrm{U}$ and ${ }^{236} \mathrm{U}$ for the 11 standard runs (processed in February/March 2011 ) and the 5 additional standard runs (processed in May 2011 to support KW210 samples) met the acceptance limit.
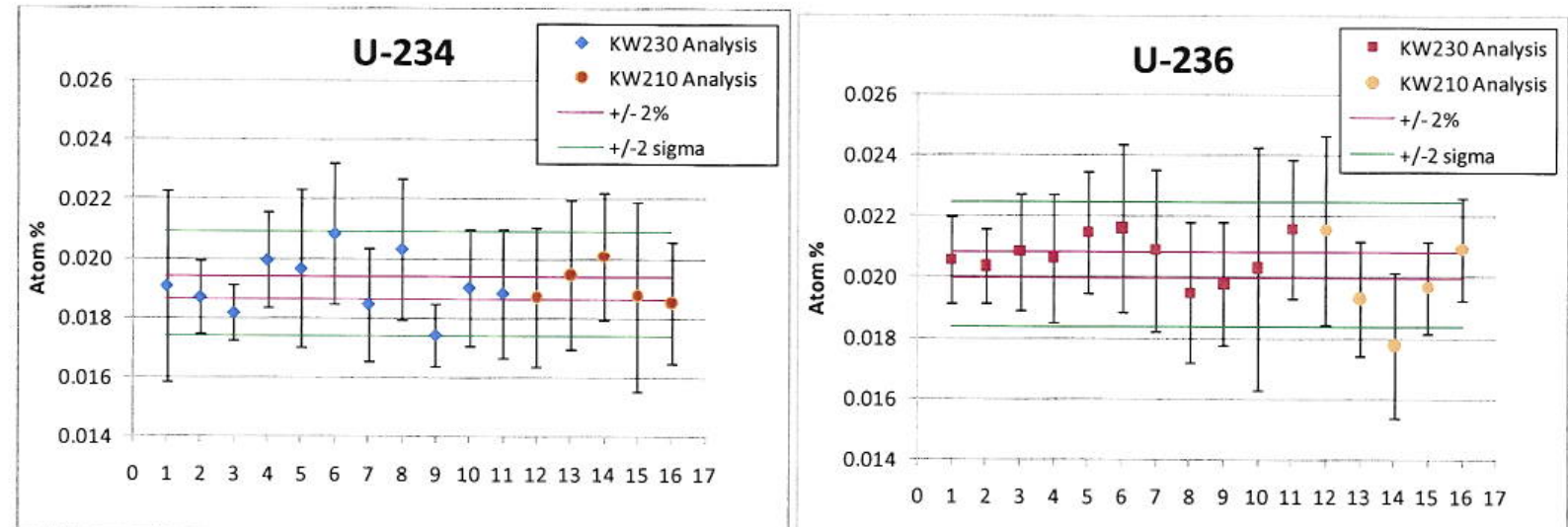

Figure 1 SRM U-030 ${ }^{234} \mathrm{U}$ and ${ }^{236} \mathrm{U}$ Atom \% and Associated $1-\sigma$ Measurement Uncertainty (Also shown are the $\pm 2 \%$ criterion and the \pm 2 times the standard deviation.)

\section{Impact of Occurrence \#2}

Plutonium isotopic precision requirements were provided to the analyst as follows: The ${ }^{239} \mathrm{Pu},{ }^{240} \mathrm{Pu},{ }^{241} \mathrm{Pu}$, and ${ }^{242} \mathrm{Pu}$ atom percents for a given core sample and duplicate shall agree within $\pm 20 \%$ where the isotopic abundance is greater than $1 \%$.

The analysis request specifically excluded precision criteria for the ${ }^{238} \mathrm{Pu}$ isotope because potential isobaric interference from ${ }^{238} \mathrm{U}$ was recognized as a likely possibility. The ${ }^{238} \mathrm{Pu}$ concentration will be determined strictly from the alpha energy analysis results. 
There is no project impact for the ${ }^{241} \mathrm{Pu}$ high RPD result because the sample pair results were $<1$ atom $\%$ (specifically 0.674 and 0.515 atom \%). The QAPjP/SAP specifies "For TIMS analyses, the $\pm 20 \%$ precision applies to the isotopic abundance of major isotopes in the Main and Client Duplicate samples for each core (major isotope $=$ isotope abundance greater than $1 \%$ of the total sample)." That said, it is noted that the value of 0.674 atom $\%{ }^{241} \mathrm{Pu}$ determined from sample TI001-A1-B was greater than two standard deviations of all ${ }^{241} \mathrm{Pu}$ sample results $(0.532$ atom $\% \pm 0.0605$, where 0.0605 is the standard deviation determined from Excel function [STDEV]).

Significant Condition Adverse to Quality? Yes ___ No _ $\quad 4$

Evaluated by:

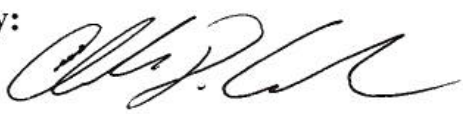

Date: $7 / 20 / 2011$

Further Disposition: $\varangle$ No $\square$ Yes If yes, $\square$ Nonconformance? $\square$ Deficiency?

Date Occurrence Closed: $7 \cdot 20-11$

Signature/Date of Responsible QE: DoS Ceflay

Signature/Date of Responsible K-Basin Manager:

\section{Comments:}

E-mail discussion with the client describing the genesis of the standard acceptance criteria for $\mathrm{U}$ and $\mathrm{Pu}$ in the QAPjP/SAP KBC-33786 Rev. 2 is attached to this occurrence report. 
PNNL-20650, Rev. 2

Fiskum, Sandra K

$\begin{array}{ll}\text { From: } & \text { Schmidt, Andrew J } \\ \text { Sent: } & \text { Monday, April 11, 2011 11:59 AM } \\ \text { To: } & \text { Welsh, Terri L; Baker, Ronald B (Ron) } \\ \text { Cc: } & \text { Carlson, Clark D; Fiskum, Sandra K } \\ \text { Subject: } & \text { FW: Pu and U control charts for TIMS } \\ \text { Attachments: } & \text { TIMS Control chart Restored 1.xls; ARF-KW230_TIMS_Pu[1].pdf; ARF-KW230\%20TIMS\% } \\ & \text { 20U[1].pdf }\end{array}$

Terri:

Thank you! I corrected the date in the note below from 4/12/11 vs. 4/11/11).

Andy

From: Welsh, Terri L

Sent: Monday, April 11, 2011 11:48 AM

To: Schmidt, Andrew J; Baker, Ronald B (Ron)

Subject: RE: Pu and U control charts for TIMS

Andy,

The words below reflect our conversation this morning.

Terri Welsh

From: Schmidt, Andrew ] [mailto:andy.schmidt@pnl.gov]

Sent: Monday, April 11, 2011 10:14 AM

To: Welsh, Terri L; Baker, Ronald B (Ron)

Subject: FW: Pu and U control charts for TIMS

Importance: High

Terri and Ron:

The purpose of this note is to confirm the technical basis for the LCS recoveries used in Pu and U TIMS, and the applicability of the QAPJP LCS accuracy (recoveries) to minor isotopes (very low abundance).

Based on our brief discussion this morning (4-11-11; AJ Schmidt and TL Welsh), the recoveries in the LCS/standard reference material for U-234 and U-236 are non-issues with respect to the QAPjP and the Safeguard's needs.

Technical basis of LCS recoveries in the QAPjP:

For $\mathrm{U}$, the $2 \%$ recovery was based on the TIMS historical performance for $\mathrm{U}-238$ and $\mathrm{U}-235$. (Control Chart) For $\mathrm{Pu}$, the $6 \%$ recovery was based on TIMS historical performance for Pu-239 and Pu-240. (Control Chart) Control charts we not prepared for other $\mathrm{U}$ and $\mathrm{Pu}$ isotopes.

The QAPjP is silent on the LCS/SRM recovery, in terms of applicability to low abundance isotopes. The only guidance provided is that given with respect to the $20 \%$ duplicate criterion (only applicable to isotopes greater than $1 \%$ abundance). 
PNNL-20650, Rev. 2

52578-05-09-11 (Attachment 1)

Page 5 of 13

Consequently, a conservative and achievable approach was recommended by PNNL for LCS/SRM accuracy within the TIMS ARFs. In the TIMS ARFs, a threshold was added that the $U$ and Pu LCS accuracies only applied to isotopes present at $>0.1 \%$ abundance. The adaptation of the $>0.1 \%$ threshold was previously discussed in a conversation between Fiskum and Welsh. The outcome of the discussion was reflected in the ARFs, which were approved by the BTR.

Recoveries for U-234 and U-236.

LCS recoveries for U-234 and U-236 (less than $0.1 \%$ abundance) exceeded $2 \%$. The isotopic Uranium (U) Standard Reference Material (SRM) acceptance criterion of $\pm 2 \%$ of the certified value is a requirement specified in the QAPjP/SAP, KBC-40467, Rev. 1, Table 3-7, but only applicable to $U-235$ and $U-238$. The ${ }^{234} \mathrm{U}$ and ${ }^{236} \mathrm{U}$ isotopic ratios were significantly less than 0.1 atom $\%$; the sample analysis request specified that $\pm 2 \%$ of the certified value criterion applies to values $>0.1$ atom \%. The request had been reviewed and signed by the Buyer's Technical Representative (BTR). The 1-s uncertainties of the ${ }^{234} \mathrm{U}$ and ${ }^{236} \mathrm{U}$ values were large (much greater than $2 \%$, relative) and generally bracketed the $\pm 2 \%$ criterion range as shown in Figure 1.

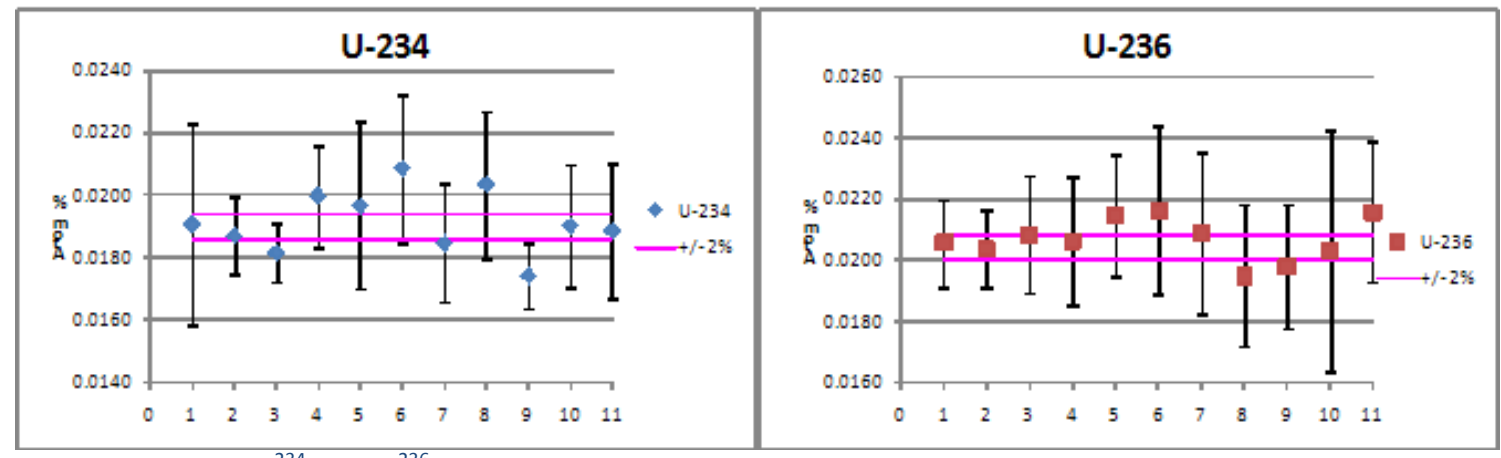

Figure 1 SRM $\cup 030{ }^{234} \mathrm{U}$ and ${ }^{236} \mathrm{U}$ Atom \% and Associated 1-s Measurement Uncertainty

- Andy

From ARF-KW-230 TIMS Pu:

Quality Control

The TIMS analysis processing will need to adhere to the QC defined in the technical procedure PNNL98523-264, Mass Spectrometer Isotopic Analysis, Rev. 2 and project-specific quality control as follows:

Duplicates The ${ }^{239} \mathrm{Pu},{ }^{240} \mathrm{Pu},{ }^{241} \mathrm{Pu}$, and ${ }^{242} \mathrm{Pu}$ atom percents for a given core sample and duplicate shall agree within $\pm 20 \%$.

Reference At least one traceable reference standard must be analyzed at the

Standard beginning of the analytical batch. The results for ${ }^{238} \mathrm{Pu},{ }^{239} \mathrm{Pu}$

${ }^{240} \mathrm{Pu},{ }^{241} \mathrm{Pu},{ }^{242} \mathrm{Pu}$ atom percents shall be within $6 \%$ of the certified

value where the isotope atom percent of the certified value is $>0,1$.

From ARF-KW-230 TIMS U: 
PNNL-20650, Rev. 2

\section{Quality Control}

The TIMS analysis processing will need to adhere to the QC defined in the technical procedure PNNL98523-264, Mass Spectrometer Isotopic Analysis, Rev. 2 and project-specific quality control sample requirements as follows:

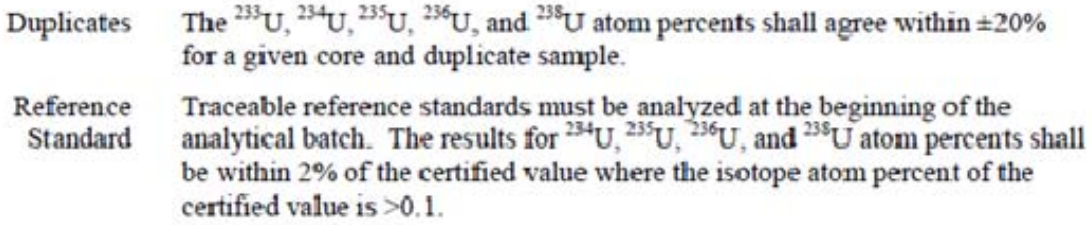

From: Schmidt, Andrew J

Sent: Wednesday, February 02, 2011 3:14 PM

To: Welsh, Terri L

Subject: FW: Pu and U control charts for TIMS

Terri:

Stan was able to dig up the original file.

- Andy

From: Bos, Stanley J

Sent: Wednesday, February 02, 2011 2:49 PM

To: Schmidt, Andrew J

Subject: RE: Pu and U control charts for TIMS

Andy

Looks like I lucky, and found the old control chart.

Stan

From: Welsh, Terri L

Sent: Tuesday, February 01, 2011 2:58 PM

To: Schmidt, Andrew J

Subject: RE: Pu control charts

Andy,

We both should have a copy in our files. If we do other work, then the applicable SAP could reference the correct isotope for the $2 \%$ and $6 \%$ limits.

Terri 
PNNL-20650, Rev. 2

From: Schmidt, Andrew ] [mailto:andy.schmidt@pnl.gov]

Sent: Monday, January 31, 2011 5:49 PM

To: Welsh, Terri L

Cc: Bos, Stanley J

Subject: FW: Pu control charts

Terri:

I have not been able to find the PDFs of Stan's Control charts in my files. Do you need these?

Stan: Do you have these available? I must have deleted the original message.

Andy

From: Welsh, Terri L

Sent: Wednesday, December 19, 2007 4:40 PM

To: Schmidt, Andrew J

Subject: RE: Pu control charts

Andy,

For the Uranium data there is definitely one data point that is a significant player in the standard deviation (also in the mean). I believe that it is an outlier and should not be included for the control limits. I think we should stay with $2 \%$ for the U TIMS.

The Pu TIMS data definitely shows some scatter and we should use the $6 \%$.

Terri

From: Schmidt, Andrew ] [mailto:andy.schmidt@pnl.gov]

Sent: Wednesday, December 19, 2007 4:34 PM

To: Carlson, Clark D; Welsh, Terri L

Cc: Baker, Ronald B (Ron)

Subject: FW: Pu control charts

Clark and Terri:

Stan performed a more detailed review of the TIMS data.

Based on his recommendation, I propose we change the LSC Recovery Values in Table 3 as follows:

U TIMS, = +/- 4\% (Stnd Dev. = 1.4) (note Terri: This could be dropped to $2 \%$ as we only have one outlier.... your call).

Pu TIMS, +/- 6\% (Stnd. Dev = 2\%)

- Andy

From: Bos, Stanley ]

Sent: Wednesday, December 19, 2007 3:42 PM

To: Schmidt, Andrew J

Subject: Pu control charts

Andy 
PNNL-20650, Rev. 2

Attached is another version of the Pu control chart and the spreadsheet used to develop the chart. After our discussions concerning the variance of the Pu TIMS data, I went through the lab book and culled out a number of the values I used in the earlier control chart. The current version is data from Jan 2004 to present, I've eliminated some values where duplicate or replacement runs were made. I think this current list accurately reflects the Pu standards data that was used during analytical runs.

After reviewing the data, my recommendation is to use a 3 sigma uncertainty band. I believe that better reflects the scatter in the Pu standards.

Stan Bos

$<$ TIMS Control chart Pu .pdf >> <<TIMS Control chart.xls >> 
PNNL-20650, Rev. 2

CEC 21-703

TIMS Mass Spectrometer

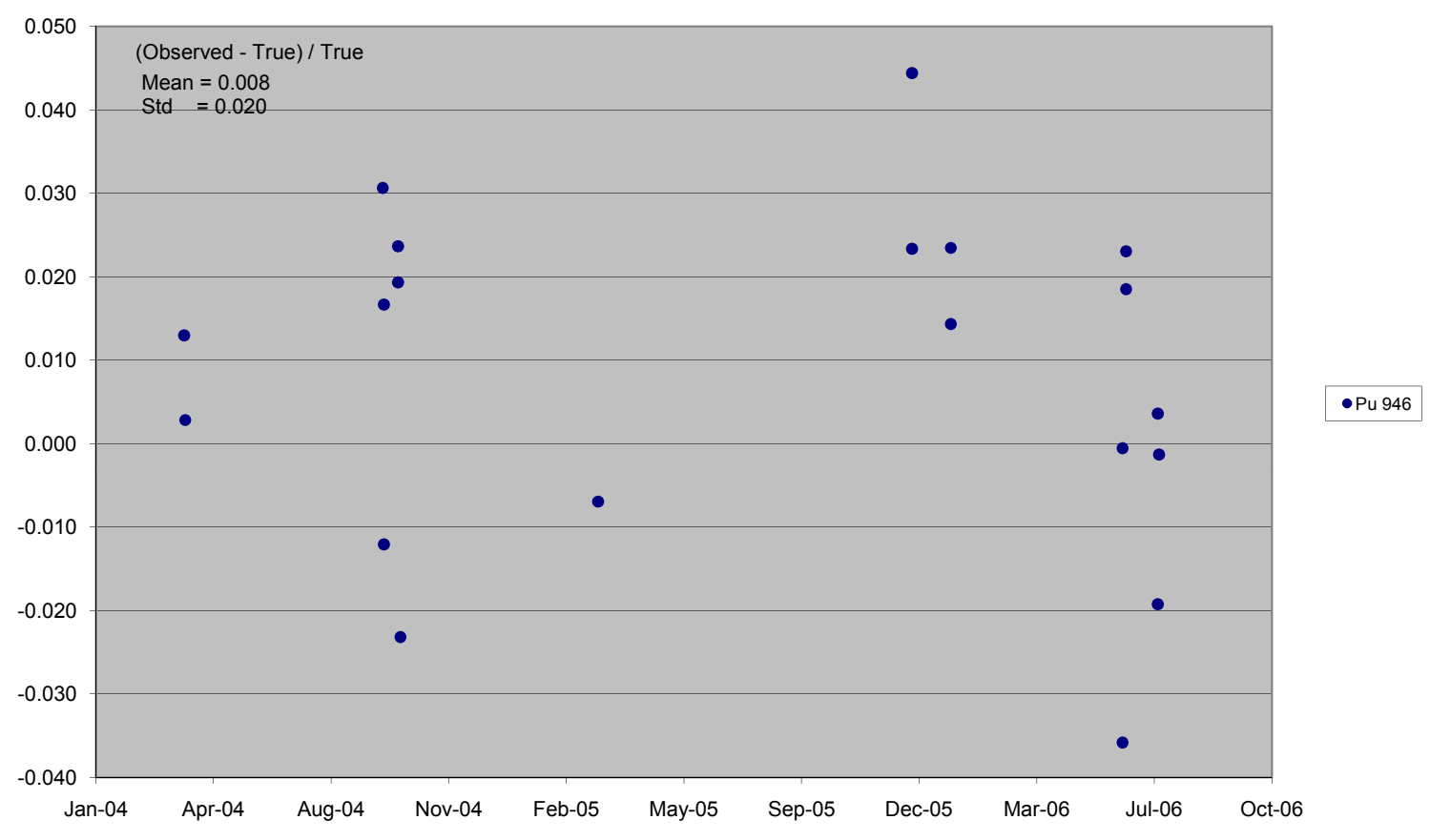


PNNL-20650, Rev. 2

52578-05-09-11 (Attachment 1)

CEC 21-703

TIMS Mass Spectrometer

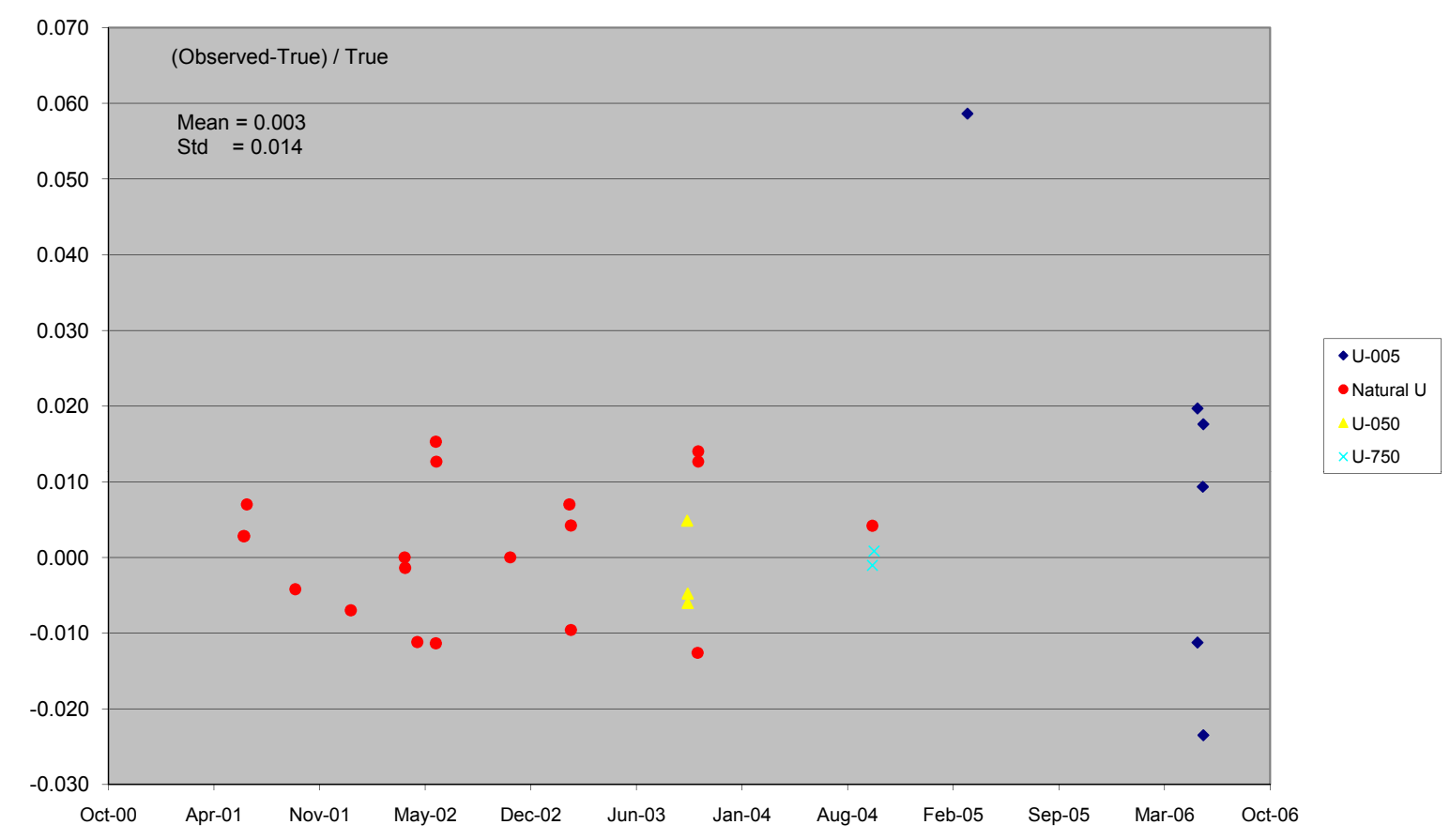


PNNL-20650, Rev. 2

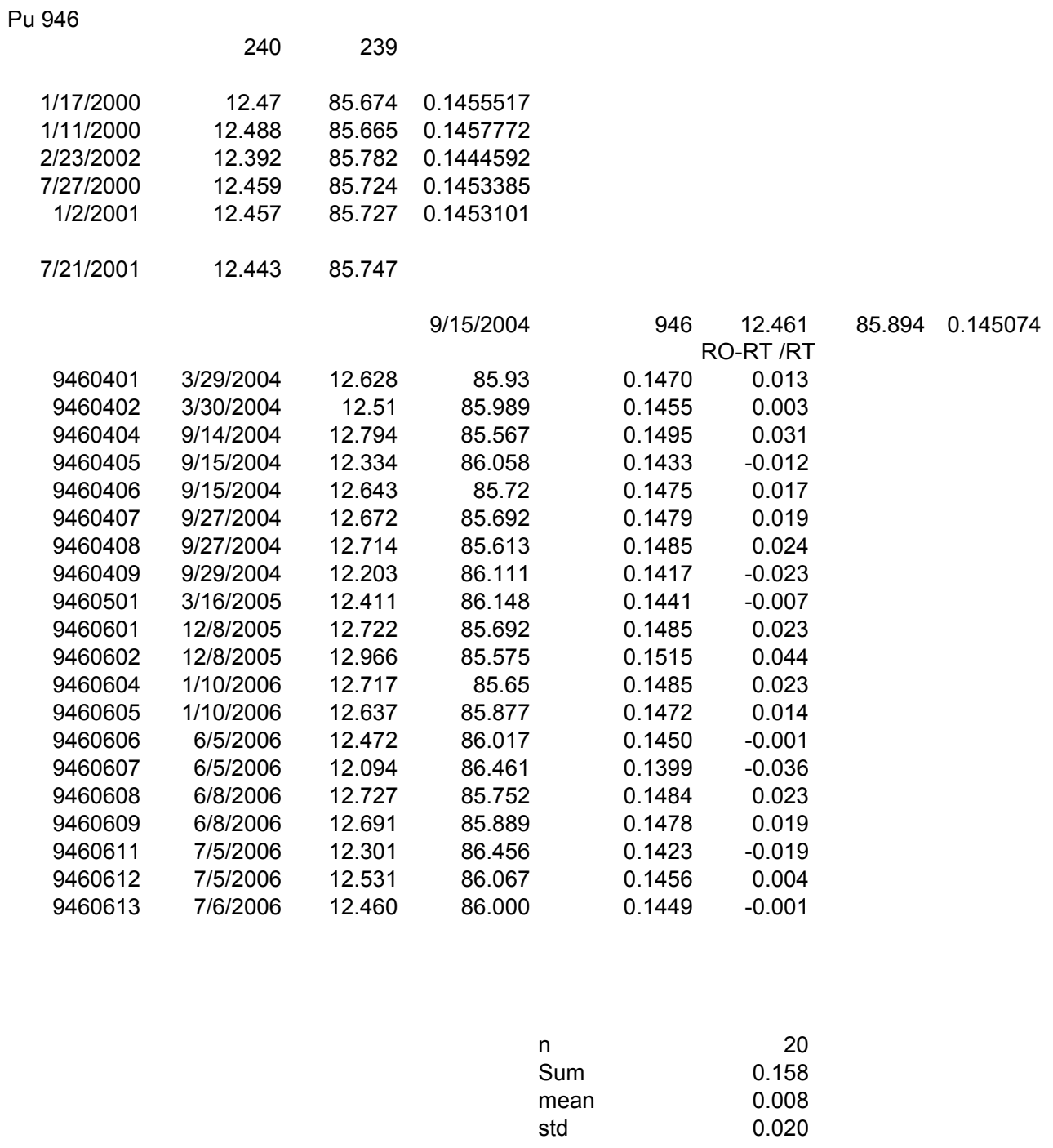


PNNL-20650, Rev. 2

\subsection{8}

0.484

0.499

0.494

0.498

0.478

nat

0.717

0.722

0.722

0.725

0.715

nat

0.711

0.729

0.73

nat

0.725

0.72

0.712

0.731

0.729

0.72

0.719

0.712

0.713

0.723

0.723

5.033

4.987

4.981

75.367

75.395

\begin{abstract}
$\begin{array}{ll}99.464 & 0.005208\end{array}$
$\begin{array}{lll}99.503 & 0.004864\end{array}$

$\begin{array}{lll}99.476 & 0.005016\end{array}$

$99.489 \quad 0.004965$

$99.48 \quad 0.005006$

$\begin{array}{lll}99.503 & 0.004804\end{array}$

$\begin{array}{lll}99.278 & 0.007222\end{array}$

$\begin{array}{ll}99.272 & 0.007273\end{array}$

$99.272 \quad 0.007273$

$99.269 \quad 0.007303$

$\begin{array}{lll}99.278 & 0.007202\end{array}$

$\begin{array}{lll}99.285 & 0.007161\end{array}$

$\begin{array}{lll}99.26 & 0.007344\end{array}$

$\begin{array}{lll}99.263 & 0.007354\end{array}$

$\begin{array}{lll}99.268 & 0.007303\end{array}$

$99.274 \quad 0.007253$

$\begin{array}{ll}99.298 & 0.00717\end{array}$

$99.274 \quad 0.007363$

$\begin{array}{lll}99.26 & 0.007344\end{array}$

$99.274 \quad 0.007253$

$99.274 \quad 0.007243$

$99.281 \quad 0.007172$

$99.261 \quad 0.007183$

$\begin{array}{lll}99.27 & 0.007283\end{array}$

$99.273 \quad 0.007283$

$\begin{array}{ll}94.89 & 0.05304\end{array}$

$94.933 \quad 0.052532$

$94.938 \quad 0.052466$

$23.829 \quad 3.162827$

$23.793 \quad 3.168789$
\end{abstract}

0.059

$-0.011$

0.020

0.009

0.018

$-0.023$

$-0.004$

0.003

0.003

0.007

$-0.007$

$-0.013$

0.013

0.014

0.007

0.000

$-0.011$

0.015

0.013

0.000

$-0.001$

$-0.011$

$-0.010$

0.004

0.004

0.005

$-0.005$

$-0.006$

$-0.001$

0.001

31

0.090

$\begin{array}{ll}\text { mean } & 0.003 \\ \text { Std } & 0.015\end{array}$
$9.504 \quad 0.004919$

$0.72 \quad 99.275 \quad 0.007253$

$\begin{array}{lll}5.01 & 94.915 & 0.052784\end{array}$

$\begin{array}{lll}75.357 & 23.801 \quad 3.166127\end{array}$ 
PNNL-20650, Rev. 2

\begin{tabular}{|c|c|c|c|c|c|c|c|}
\hline & & & $15 / 2004$ & 946 & 12.461 & 85.894 & 0.145074 \\
\hline & & & & & -RT /RT & & \\
\hline 9460401 & $3 / 29 / 2004$ & 12.628 & 85.93 & 0.146957 & 0.013 & & \\
\hline 9460402 & $3 / 30 / 2004$ & 12.51 & 85.989 & 0.145484 & 0.003 & & \\
\hline 9460404 & $9 / 14 / 2004$ & 12.794 & 85.567 & 0.14952 & 0.031 & & \\
\hline 9460405 & $9 / 15 / 2004$ & 12.334 & 86.058 & 0.143322 & -0.012 & & \\
\hline 9460406 & $9 / 15 / 2004$ & 12.643 & 85.72 & 0.147492 & 0.017 & & \\
\hline 9460407 & 9/27/2004 & 12.672 & 85.692 & 0.147878 & 0.019 & & \\
\hline 9460408 & 9/27/2004 & 12.714 & 85.613 & 0.148505 & 0.024 & & \\
\hline 9460409 & $9 / 29 / 2004$ & 12.203 & 86.111 & 0.141712 & -0.023 & & \\
\hline 9460501 & $3 / 16 / 2005$ & 12.411 & 86.148 & 0.144066 & -0.007 & & \\
\hline 9460601 & $12 / 8 / 2005$ & 12.722 & 85.692 & 0.148462 & 0.023 & & \\
\hline 9460601 & $12 / 8 / 2005$ & 12.722 & 85.692 & 0.148462 & 0.023 & & \\
\hline 9460602 & $12 / 8 / 2005$ & 12.966 & 85.575 & 0.151516 & 0.044 & & \\
\hline 9460604 & $1 / 10 / 2006$ & 12.717 & 85.65 & 0.148476 & 0.023 & & \\
\hline 9460605 & $1 / 10 / 2006$ & 12.637 & 85.877 & 0.147152 & 0.014 & & \\
\hline 9460606 & $6 / 5 / 2006$ & 12.472 & 86.017 & 0.144995 & -0.001 & & \\
\hline 9460607 & $6 / 5 / 2006$ & 12.094 & 86.461 & 0.139878 & -0.036 & & \\
\hline 9460608 & $6 / 8 / 2006$ & 12.727 & 85.752 & 0.148416 & 0.023 & & \\
\hline 9460609 & $6 / 8 / 2006$ & 12.691 & 85.889 & 0.14776 & 0.019 & & \\
\hline 9460611 & $7 / 5 / 2006$ & 12.301 & 86.456 & 0.14228 & -0.019 & & \\
\hline 9460612 & $7 / 5 / 2006$ & 12.531 & 86.067 & 0.145596 & 0.004 & & \\
\hline 9460613 & $7 / 6 / 2006$ & 12.460 & 86.000 & 0.144884 & -0.001 & & \\
\hline
\end{tabular}

$\begin{array}{lr}\mathrm{n} & 21 \\ \text { Sum } & 0.181 \\ \text { mean } & 0.009 \\ \text { std } & 0.020\end{array}$




\section{PLANNING}

Topic/Issue to be evaluated: QE Review of Draft Report, Isotopic Analysis in KW230 [Sic] Core Samples (June 2011)

This report has been revised to include the addition of a data reduction spreadsheet that was included in Appendix $\mathbf{E}$ to the draft -210 final report and was not included in the TIMS data package submitted for $\mathrm{QE}$ review.

This surveillance was undertaken to evaluate the draft Report, that was wrongly titled "Isotopic Analysis in KW230 Core Samples (June 2011)" that should rightly be titled "Isotopic Analysis in KW210 Core Samples from the Settler Sludge" for the samples listed in Table 1 below.

Table 1. Sample IDs for Isotopic Analysis in KW210 Core Samples from the Settler Sludge

\begin{tabular}{|c|c|c|c|}
\hline $\begin{array}{c}\text { Project } \\
\text { Sample ID }\end{array}$ & $\begin{array}{c}\text { RPL/ASO Sample } \\
\text { ID-Uranium }\end{array}$ & $\begin{array}{c}\text { RPL/ASO Sample ID- } \\
\text { Plutonium Isotopic }\end{array}$ & K Basin Core Sample ID \\
\hline TI001-A1-B & $11-0419-129-\mathrm{S}-\mathrm{U}$ & $11-0419-129-\mathrm{S}-\mathrm{Pu}$ & KW210-A1 \\
\hline TI001-A1-C & $11-0419-129-\mathrm{D}-\mathrm{U}$ & $11-0419-129-\mathrm{D}-\mathrm{Pu}$ & KW210-A1 duplicate \\
\hline TI002-A4-B & $11-0420-129-\mathrm{S}-\mathrm{U}$ & $11-0420-129-\mathrm{S}-\mathrm{Pu}$ & KW210-A4 \\
\hline TI002-A4-C & $11-0420-129-\mathrm{D}-\mathrm{U}$ & $11-0420-129-\mathrm{D}-\mathrm{Pu}$ & KW210-A4 duplicate \\
\hline TI002-B1-B & $11-0421-129-\mathrm{S}-\mathrm{U}$ & $11-0421-129-\mathrm{S}-\mathrm{Pu}$ & KW210-B1 \\
\hline TI002-B1-C & $11-0421-129-\mathrm{D}-\mathrm{U}$ & $11-0421-129-\mathrm{D}-\mathrm{Pu}$ & KW210-B1 duplicate \\
\hline TI001-B3-B & $11-0422-129-\mathrm{S}-\mathrm{U}$ & $11-0422-129-\mathrm{S}-\mathrm{Pu}$ & KW210-B3 \\
\hline TI001-B3-C & $11-0422-129-\mathrm{D}-\mathrm{U}$ & $11-0422-129-\mathrm{D}-\mathrm{Pu}$ & KW210-B3 duplicate \\
\hline NA & $11-0419-129-\mathrm{PB}-\mathrm{U}$ & $11-0419-129-\mathrm{PB}-\mathrm{Pu}$ & ASO preparation blank \\
\hline
\end{tabular}

\section{PERFORMANCE}

\begin{tabular}{|l|l|}
\hline Date Initiated: 28 June 2011 & Location: RPL \\
\hline Date Completed: 1 July 2011 & $\begin{array}{l}\text { DRAFT Report Issued: 1 July 2011 } \\
\text { FINAL Report Issued: 21 July 2011 } \\
\text { Revised Report Issued: 3 August 2011 }\end{array}$ \\
\hline $\begin{array}{l}\text { Contacts: Stan Bos, Clark Carlson, Sandy } \\
\text { Fiskum, and Jen Braley }\end{array}$ & Org Code: PE 137, D9H63 \\
\hline Surveillance Team: Deborah Coffey & \\
\hline
\end{tabular}




\section{PRELIMINARY INFORMATION}

\section{Source of Requirement(s):}

KBC-33786, Rev. 2, Quality Assurance Project Plan/Sampling and Analysis Plan (QAPjP/SAP for Sludge in the $K W$ Engineered Containers, $\mathrm{CH} 2 \mathrm{M}$ Hill Plateau Remediation Company, Richland, Washington.

HNF-36985, Rev. 3, Data Quality Objectives for Sampling and Analysis of K Basin Sludge (DQO), , $\mathrm{CH} 2 \mathrm{M}$ Hill Plateau Remediation Company, Richland, Washington.

\section{Other Applicable Documents:}

- $\quad$ RPL-OP-001, Rev. 9, Routine Research Operations

- PNNL-98523-264, Rev. 2, Mass Spectrometer Isotopic Analysis

- 52578-ADM-001, Rev. 0, K Basin Requests for Analysis and Guidelines for Data Reporting and Technical, QE, and Spreadsheet Reviews.

- Analysis Request for Plutonium and Uranium Isotopes in K Basin Core Samples from the Settler Sludge Origin, KW210 (S Fiskum to S. Bos, signed off 4/14/11)

\section{Purpose/Scope:}

The 52578 Project QE is required via the QA Program to perform a review of K Basin reports generated within the Radiochemical Processing Laboratory (RPL) to determine traceability and accuracy of data contributing to the completed report.

The draft report was reviewed to evaluate that the data are what they purport to be, that the reported results reflect what was actually done and the results meet applicable requirements. When deficiencies in the data are identified, then those deficiencies need to be documented as a K Basin Occurrence Report for the data user's review and, when applicable, resolved by corrective action.

The requirements for this work are defined in Table 3-6 and Table 3-7, KBC-33786, Rev. 2, Quality Assurance Project Plan/Sampling and Analysis Plan (QAPjP/SAP for Sludge in the KW Engineered Containers and in Analysis Request for Plutonium and Uranium Isotopes in K Basin Core Samples from the Settler Sludge Origin, KW210.

\section{Requirements:}

Requirements from KBC-33786, Rev. 2, the applicable QAPjP/SAP s are the following:

- Reporting requirements - atom \% of $\mathrm{U}$ and $\mathrm{Pu}$ for ${ }^{233} \mathrm{U},{ }^{234} \mathrm{U},{ }^{235} \mathrm{U},{ }^{236} \mathrm{U}$, and ${ }^{238} \mathrm{U}$; and ${ }^{238} \mathrm{Pu},{ }^{239} \mathrm{Pu},{ }^{240} \mathrm{Pu},{ }^{241} \mathrm{Pu}$, and ${ }^{242} \mathrm{Pu}$.

- There is no specified reporting limit

- The DQO for preparative duplicate precision is $\pm 20 \%$.

- There is no accuracy DQO for a post-prep MS 
- There are DQOs for a LCS sample to be analyzed at a frequency of $1 /$ batch $(2 \%$ for U-TIMS and $6 \%$ for Pu-TIMS)

Requirements from the analysis request were the following:

\begin{tabular}{|c|l|}
\hline \multirow{5}{*}{ Duplicates } & $\begin{array}{l}\text { The }{ }^{239} \mathrm{Pu},{ }^{240} \mathrm{Pu},{ }^{241} \mathrm{Pu}, \text { and }{ }^{242} \mathrm{Pu} \text { atom percents for a given core sample and } \\
\text { duplicate shall agree within } \pm 20 \% \text { where the isotopic abundance is greater } \\
\text { than } 1 \% .\end{array}$ \\
\cline { 2 - 3 } & $\begin{array}{l}\text { The }{ }^{233} \mathrm{U},{ }^{234} \mathrm{U},{ }^{235} \mathrm{U},{ }^{236} \mathrm{U}, \text { and }{ }^{238} \mathrm{U} \text { atom percents shall agree within } \pm 20 \% \\
\text { for a given core and duplicate sample where the isotopic abundance is } \\
\text { greater than } 1 \% .\end{array}$ \\
\hline \multirow{5}{*}{$\begin{array}{l}\text { Reference } \\
\text { Standards }\end{array}$} & $\begin{array}{l}\text { At least one traceable reference standard must be analyzed at the beginning } \\
\text { of the analytical batch. The results for }{ }^{238} \mathrm{Pu},{ }^{239} \mathrm{Pu},{ }^{240} \mathrm{Pu},{ }^{241} \mathrm{Pu},{ }^{242} \mathrm{Pu} \text { atom } \\
\text { percents shall be within } 6 \% \text { of the certified value where the isotope atom } \\
\text { percent of the certified value is }>0.1 .\end{array}$ \\
\cline { 2 - 2 } & $\begin{array}{l}\text { Traceable reference standards must be analyzed at the beginning of the } \\
\text { analytical batch. The results for }{ }^{234} \mathrm{U},{ }^{235} \mathrm{U},{ }^{236} \mathrm{U}, \text { and } \\
{ }^{238} \mathrm{U} \text { atom percents shall } \\
\text { be within } 2 \% \text { of the certified value where the isotope atom percent of the } \\
\text { certified value is }>0.1 .\end{array}$ \\
\hline
\end{tabular}

Note that the Reference Standard acceptance criteria identified in the QAPjP/SAP KBC33786, Rev. 2 were developed specifically for ${ }^{239} \mathrm{Pu}$ and ${ }^{240} \mathrm{Pu}( \pm 6 \%)$ and for ${ }^{235} \mathrm{U}$ and ${ }^{238} \mathrm{U}( \pm 2 \%)$. These criteria are conservatively applied to other (minor) isotopes in the Standard Reference Material (SRM) is where the minor isotope concentration exceeds 0.1 atom $\%$.

\section{TIMS Reporting:}

Report ${ }^{238} \mathrm{Pu},{ }^{239} \mathrm{Pu},{ }^{240} \mathrm{Pu},{ }^{241} \mathrm{Pu}$, and ${ }^{242} \mathrm{Pu}$ atom percents for each sample in a tabular format.

Report ${ }^{233} \mathrm{U},{ }^{234} \mathrm{U},{ }^{235} \mathrm{U},{ }^{236} \mathrm{U}$, and ${ }^{238} \mathrm{U}$ atom percents for each sample in a tabular format.

Provide a report with the following elements: a data report Cover Page, a Narrative, the Data Summary, the signed Analysis Request Form and the Supporting Data.

When issues were found, the cognizant staff were contacted to resolve issues prior to completion of the review.

\section{APPROACH:}

The report and supporting data package contained the following information:

1. Analysis Request for Plutonium and Uranium Isotopes in K Basin Core Samples from the Settler Sludge Origin, KW210 (S Fiskum to S. Bos, signed off 4/14/11)

2. Email submittal of draft report and Excel spreadsheets on $6 / 23 / 11$ 
3. DRR form for Technical Reviewer, Jen Braley - This form was incomplete and lacked a title for the document reviewed and the organization of the Technical Reviewer. Although the DRR states that there were editorial comments, there was no indication what those comments were and the form did not show that the comments were resolved.

4. Cover page -Present in report attached to DRR, but missing in electronic version submitted. Cover page did not have the right title; says "Isotopic Analysis in KW230 Core Samples (June 2011 )" that should rightly be titled "Isotopic Analysis in KW210 Core Samples from the Settler Sludge". Cover page did not have signatures.

5. Memo Report, Pu and U isotopic analysis (pp. 4, plus four (4) tables )

6. Mass Spec data print-outs for $8 \mathrm{U}$ samples plus PB analyzed on 5/6 and $9 / 11$; references LRB 56999 p. 76-77. Technical Review by P Berry is noted on each sample data report.

7. NBS SRM U-030 Certificate, and SRM analysis data for 5/3/11 (1);5/6/11 (2) and 5/9/11 (2); references LRB 56999 pp. 76 \& 77.

8. Mass Spec data print-outs for 8 Pu samples plus PB analyzed on 5/18 \& 25/11; references LRB 56999 p. $77 \& 78$. Technical Review by P Berry is noted on each sample data report.

9. Summary of Standard results - for Pu 946; NBS Certificate of Analysis for SRM 946, Plutonium Isotopic Standard; and SRM analysis data for 5/17, 18 \& 25/11 references LRB 56999 pp. 77 \&78. See decay-corrected SRM 946 values with technical review by LR Greenwood.

10. Copies of 56999 LRB pages 77 and 78. Missing a copy of LRB page 76. Pages were not signed as to completion of a Technical Review.

11. Copy of ASR 8798 Thermal Ionization Mass Spectrometry Sample Preparation, Rev. 1 -dated 6/3011- added by QE Reviewer

12. TIMS Isotopic Data for KW Basin Cores KW230 (?), prepared 6/15/2011 by Stan Bos; Excel file KW210TIMS data June 2011.xls with worksheets:

- Uranium Std.

- U TIMS

- Final Results 1

- Plutonium Std

- Pu TIMS

- Final Results 2

Included a Spreadsheet Review Form completed by J Braley that was signed, but not dated.

13. Missing a copy of draft OR 52578-05-09-11.

14. Excel Spreadsheet ASR 8798 Data Reduction Rev. 1.xlsx, pp.4; prepared by SK Fiskum/M Fountain 7/18/2011; Technical review provided by Cal Delegard 23 July 2011; titled K Basin Radionuclide Calculations; plus a spreadsheet titled ASR 8798 Data reduction, Rev.1.xlsx TIMS Rad wet mass basis; with no indication in the data package of technical review status

- Worksheet - Spreadsheet Review Form - 


\section{- TIMS Rad Calc}

Jen Braley was identified as the technical reviewer of the data package with the exception of item 14 above, and this was documented with a DRR.

The completed surveillance report will be posted to the SharePoint site and submitted as a project record. Any documentation associated with this surveillance will be available in the Lead Auditor's office, Building 325, 5-G.

\section{RESULTS}

Samples were prepared by ASO and submitted to S. Bos for TIMS analysis. ASO TIMS sample preparation activities are documented in an ASO report:

- ASR 8798 Thermal Ionization Mass Spectrometry Sample Preparation, Rev. 1 -dated 6/3011

The draft report initiated by the $\mathrm{K}$ Basin analysis request dated 4/14/11 was provided as the basis for the data quality review. Eight (8) samples and an ASO preparation blank (PB) were provided for analysis on $5 / 3 / 11$ as noted above in Table 1 .

\section{Procedure:}

- PNNL-98523-264, Rev. 2, Mass Spectrometer Isotopic Analysis

\section{M\&TE used:}

\begin{tabular}{|c|c|c|c|c|}
\hline Location & Description & Manufacturer & Model & Unique ID \\
\hline RPL/320 & Mass spec & CEC & $21-703$ & 467806 \\
& & & & WB76849 \\
& & & & \\
& NBN:21793 \\
\hline & U-030 & NBS & & \\
\hline & NBS SRM & NBS & & \\
& 946 & & & \\
\hline
\end{tabular}

Findings are defined as a statement of fact relating to noncompliance with previously agreed-upon procedures, plans, codes, standards, specifications, or other forms of contractual or legal obligation. It should be understood that any lack of a finding in a specific area is not considered an indication that deficiencies do not exist.

Observations are defined as a weakness that if not corrected, could yield a departure from a requirement although the weakness is not necessarily a departure from requirements. 
PNNL-20650, Rev. 2

PROJECT 52578 - SURVEILLANCE REPORT

Number: SR-52578-2011-027, Rev. 1

Page 6 of 8

\section{Summary of Identified Concerns:}

Note: Concerns identified in the review are expected to be addressed in a comment resolution meeting to be scheduled by the report author when the comments have been addressed.

A comment resolution meeting was convened on 15 July 2011; , Stan Bos and Deborah Coffey attended to disposition review comments and discuss the draft Occurrence Report, OR 52578-05-09-11. The report was re-submitted on 15 July 2011. There were no outstanding identified findings or observations.

Issues of concern:

1. Apparent data transcription errors in a few places on $U$ results; refer to comment copy. Discussed and tracked correct data to spreadsheet/normalized data. No further action required. Closed.

2. Add footnotes to both $U$ and Pu reporting tables to explain why PB data are ND; define ND in the table. The PB sample did not have a measureable single so that the data could not be collected. The $\mathrm{U}$ and Pu results are appropriately tagged with ND in the results section but ND is not defined in the table nor footnoted regarding the lack of signal. This was done; no further action required. Closed.

3. Typo in Pu-238 acceptance criteria for Pu-946 std. Says 0.176 to 00.199 ; should be 0.199 . This was corrected no further action required. Closed.

4. DRR form for Technical Reviewer, Jen Braley - This form is incomplete and lacks a title for the document reviewed and the organization of the Technical Reviewer. Although the DRR states that there were editorial comments, there is no indication what those comments were and the form does not show that the comments were resolved. There also appeared to be information added that is not initialed and dated that was added by someone other than J. Braley. Re-done DRR provided and initialed/dated. Closed.

5. Cover page does not have the right title; says "Isotopic Analysis in KW230 Core Samples (June 2011)" that should rightly be titled "Isotopic Analysis in KW210 Core Samples from the Settler Sludge". This was corrected no further action required. Cover page does not have signatures.

6. 56999 LRB pages 77 and 78 . Will need copy of LRB page 76 . Pages are not signed as to completion of a Technical Review. Obtained a copy of page 76 and re-reviewed pages 77 and 78 against the data to reconcile log \# issues. See issue \#8. Closed.

7. Lack of Page 76 of LRB affects traceability of results for the following samples: Obtained a copy of page 76 and re-reviewed pages 77 and 78 against the data to reconcile log \# issues. See issue \#8. Closed. 


\begin{tabular}{|l|l|l|l|}
\hline $\begin{array}{l}\text { Project } \\
\text { Sample ID }\end{array}$ & $\begin{array}{l}\text { RPL/ASO Sample } \\
\text { ID-Uranium }\end{array}$ & $\begin{array}{l}\text { RPL/ASO Sample ID- } \\
\text { Plutonium Isotopic }\end{array}$ & K Basin Core Sample ID \\
\hline TI001-A1-B & $11-0419-129-\mathrm{S}-\mathrm{U}$ & $11-0419-129-\mathrm{S}-\mathrm{Pu}$ & KW210-A1 \\
\hline TI001-A1-C & $11-0419-129-\mathrm{D}-\mathrm{U}$ & $11-0419-129-\mathrm{D}-\mathrm{Pu}$ & KW210-A1 duplicate \\
\hline TI002-A4-B & $11-0420-129-\mathrm{S}-\mathrm{U}$ & $11-0420-129-\mathrm{S}-\mathrm{Pu}$ & KW210-A4 \\
\hline TI002-B1-C & $11-0421-129-\mathrm{D}-\mathrm{U}$ & $11-0421-129-\mathrm{D}-\mathrm{Pu}$ & KW210-B1 duplicate \\
\hline TI001-B3-B & $11-0422-129-\mathrm{S}-\mathrm{U}$ & $11-0422-129-\mathrm{S}-\mathrm{Pu}$ & KW210-B3 \\
\hline TI001-B3-C & $11-0422-129-\mathrm{D}-\mathrm{U}$ & $11-0422-129-\mathrm{D}-\mathrm{Pu}$ & KW210-B3 duplicate \\
\hline NA & $11-0419-129-\mathrm{PB}-\mathrm{U}$ & $11-0419-129-\mathrm{PB}-\mathrm{Pu}$ & ASO preparation blank \\
\hline
\end{tabular}

8. Two samples documented on LRB page 78 do not match the data print-out results for sample log entries 1211017 and 1211018; LRB says these were samples 11-0421-129-S-Pu and 11-0421129D-Pu, but data print-out calls these samples 11-0422-129-S-Pu and 11-0422-129D-Pu. This discrepancy needs to be unraveled. This problem was resolved and log entries on page 78 required corrections by line-out, initial/date. Page 78 replaced in record. Closed.

9. Data print-outs for May $18^{\text {th }}$ list the incorrect procedure - Should be PNNL-98523-264, Rev. 2, Mass Spectrometer Isotopic Analysis, but "PNL-MA-599" is listed. Correct procedure added by hand correction, initial and date. Closed.

10. Why are there no corresponding data print-outs for standards samples? Discussed. Logbook works OK. Closed.

11. Spreadsheet Review Form completed by J Braley and signed, but not dated. Corrected. Closed.

12. The practice of calculating RPD values using more significant figures than are reported leads to apparent errors in data reporting. For example, for two samples the sample and the duplicate are the same reported values which would of course be a relative percent difference of $0 \%$, but these are reported as $8 \%$ and $6 \%$ RPD which is ridiculous. Corrected. Closed.

13. The standard analyzed for $U$ reporting failed rather profoundly for two isotopes $\left({ }^{234} U-3\right.$ of 5 and ${ }^{236} \mathrm{U} 5$ of 5); How does this impact the accuracy of the data collected? An Occurrence Report has been issued to address the impact of reference standard failures (OR 52578-05-0911). Closed.

14. Although a draft $\mathrm{K}$ Basin Occurrence Report is mentioned in the narrative, it was not included in the data package provided for review, and due to PNNL network issues could not be reviewed as part of this surveillance. A completed and signed copy is needed to complete the surveillance. Obtained. Closed.

15. Also RPD failures needed to be added to the OR and referenced in the text. Email sent $7 / 18 / 11$ on this issue. Added to OR 52578-05-09-11. Corrected. Closed.

16. Data reduction and reporting is being performed separately from data package review, was not reviewed by the TIMS analyst, was not submitted for $Q E$ review, and the basis for data reduction, namely normalizing $\mathrm{Pu}$ isotopic results to the ${ }^{238} \mathrm{Pu}$ AEA results was not technically established in the TIMS review process. Data reduction and reporting also 
Number: SR-52578-2011-027, Rev. 1

involved conversion of wt $\%$ results to units of $\mu \mathrm{g} / \mathrm{g}$ and $\mu \mathrm{Ci} / \mathrm{g}$ on a dry mass and settled mass basis. Any errors are the responsibility of the authors and technical reviewer. Basis for normalizing Pu isotopic results to the ${ }^{238} \mathrm{Pu}$ AEA was discussed with Karl Pool w.r.t. selection of ${ }_{238} \mathrm{Pu}$ over other isotopes analyzed. No further action.

\section{CORRECTIVE ACTION}

[X] None Required. [ ] Complete [ ] Follow-up Corrective Action -

\section{Surveillance Performed By:}

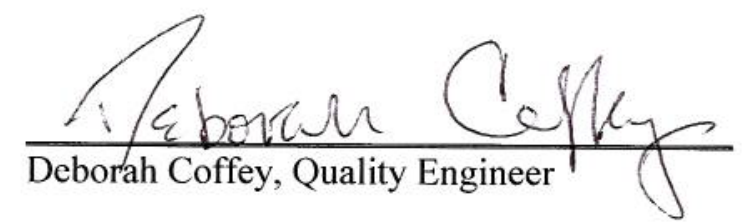

\section{DISTRIBUTION:}

Clark Carlson - P7-25

Sandy Fiskum - P7-25

Matt Fountain, P7-27

Rick Shimskey - P7-27

Andy Schmidt - P8-60

Stan Bos - P7-22

Jen Braley - P7-25

Cal Delegard, P7-25

Deborah Coffey - P7-25

Lisa Middleton - K9-75
Date:

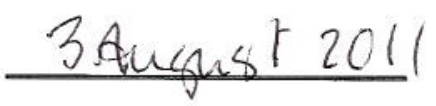




\section{Spreadsheet Review Form}

\begin{tabular}{|l|c|l|c|}
\hline Spreadsheet Author Name: & SK Fiskum/M Fountain & Review Date: & 23 July 2011 \\
\hline Date Prepared: & $7 / 18 / 2011$ & Spreadsheet Subject: & KW210 Core Characterization \\
\hline Reviewer Name: & Cal Delegard & File Name: & ASR 8798 data reduction.xlsx \\
\hline Reviewer Title: & Senior Research Scientist & Revision No. & 1 \\
\hline
\end{tabular}

\section{Scope of Spreadsheet Review: (Check one or more of the following)}

\begin{tabular}{|c|c|c|c|}
\hline ㄷ & $\begin{array}{l}\text { General Validation Review: (General review and } \\
\text { spot checks) }\end{array}$ & $\square$ & $\begin{array}{l}\text { Independent calculation check (With hand calculations } \\
\text { or independent spreadsheet software) }\end{array}$ \\
\hline L & $\begin{array}{l}\text { Review of updated spreadsheet/calc (Revised } \\
\text { portion only) }\end{array}$ & 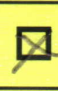 & $\begin{array}{l}\text { Other: Vertfieation of data inpurt to tabs "TIMS } \\
\text { RodCalc." and "TIMSRod wet moss basis" and }\end{array}$ \\
\hline [ & $\begin{array}{l}100 \% \text { Verification Review (Verification of all } \\
\text { cells/calculations) }\end{array}$ & & $\begin{array}{l}\text { calculations for "TIMs Rod wet mass basis" } \\
\text { Note that cale.cells locked for "Tms Rad Calc." }\end{array}$ \\
\hline
\end{tabular}

\section{REVIEW CHECK LIST}

\begin{tabular}{|c|c|c|c|c|c|c|c|}
\hline \multicolumn{8}{|l|}{ Spreadsheet/Calculation Identification } \\
\hline Spreadsheet Information & Yes & No & NA & Input Values & Yes & \multirow[t]{3}{*}{ No } & NA \\
\hline Title: & & & & \multirow{2}{*}{$\begin{array}{l}\text { Are input parameters correct (verified } \\
\text { with source?) }\end{array}$} & \multirow[b]{2}{*}{$\checkmark$} & & \\
\hline Revision Number: & & & & & & & \\
\hline Date Prepared: & & & & Are parameter units consistent? & $\checkmark$ & & \\
\hline Prepared by: & & & & Are input values properly referenced? & $\checkmark$ & & \\
\hline General Statement of Purpose: & & & & Are input uncertainties correct? & & & $\checkmark$ \\
\hline General Description of Approach: & & & & \multirow{2}{*}{\multicolumn{4}{|c|}{$\begin{array}{l}\text { Comments: } \\
\text { Input values copied from ASO and TIMS electronic files, } \\
\text { which are properly referenced. Cover sheet references } \\
\text { source data for physical properties. }\end{array}$}} \\
\hline \multicolumn{4}{|c|}{$\begin{array}{l}\text { Comments: } \\
\text { "Overview" worksheet contains all this information. }\end{array}$} & & & & \\
\hline Assumptions & Yes & No & NA & Equations/Approach & Yes & No & NA \\
\hline Are assumptions clearly stated? & & & & $\begin{array}{l}\text { Are equation algorithms adequately } \\
\text { defined? }\end{array}$ & $\checkmark$ & & \\
\hline Are assumptions supported and justified? & & & & Are equations properly referenced? & $\checkmark$ & & \\
\hline Are assumptions reasonable? & & & & $\begin{array}{l}\text { Are limitations of approach/equations } \\
\text { identified? }\end{array}$ & $\checkmark$ & & \\
\hline $\begin{array}{l}\text { Comments: } \\
\text { "Overview" worksheet contains all this info }\end{array}$ & & & & Are equations appropriate? & $\checkmark$ & & \\
\hline Results/Conclusions & Yes & No & NA & Are units consistent? & $\checkmark$ & & \\
\hline Are formulas consistent in cells? & $r$ & & & \multirow{3}{*}{\multicolumn{3}{|c|}{ Comments: }} & \\
\hline Are calculations correct? & $\checkmark$ & & & & & & \\
\hline Are conclusions consistent with results? & $\checkmark$ & & & & & & \\
\hline $\begin{array}{l}\text { Are conclusions consistent with applicable } \\
\text { limits? }\end{array}$ & $\checkmark$ & & & \multirow{2}{*}{\multicolumn{3}{|c|}{ Reviewer Sign/Date: }} & \\
\hline \multicolumn{4}{|c|}{$\begin{array}{l}\text { Comments: Seecomments to "Other" in } \\
\text { "Scope of Spreadshect Review", above, }\end{array}$} & & & & \\
\hline
\end{tabular}




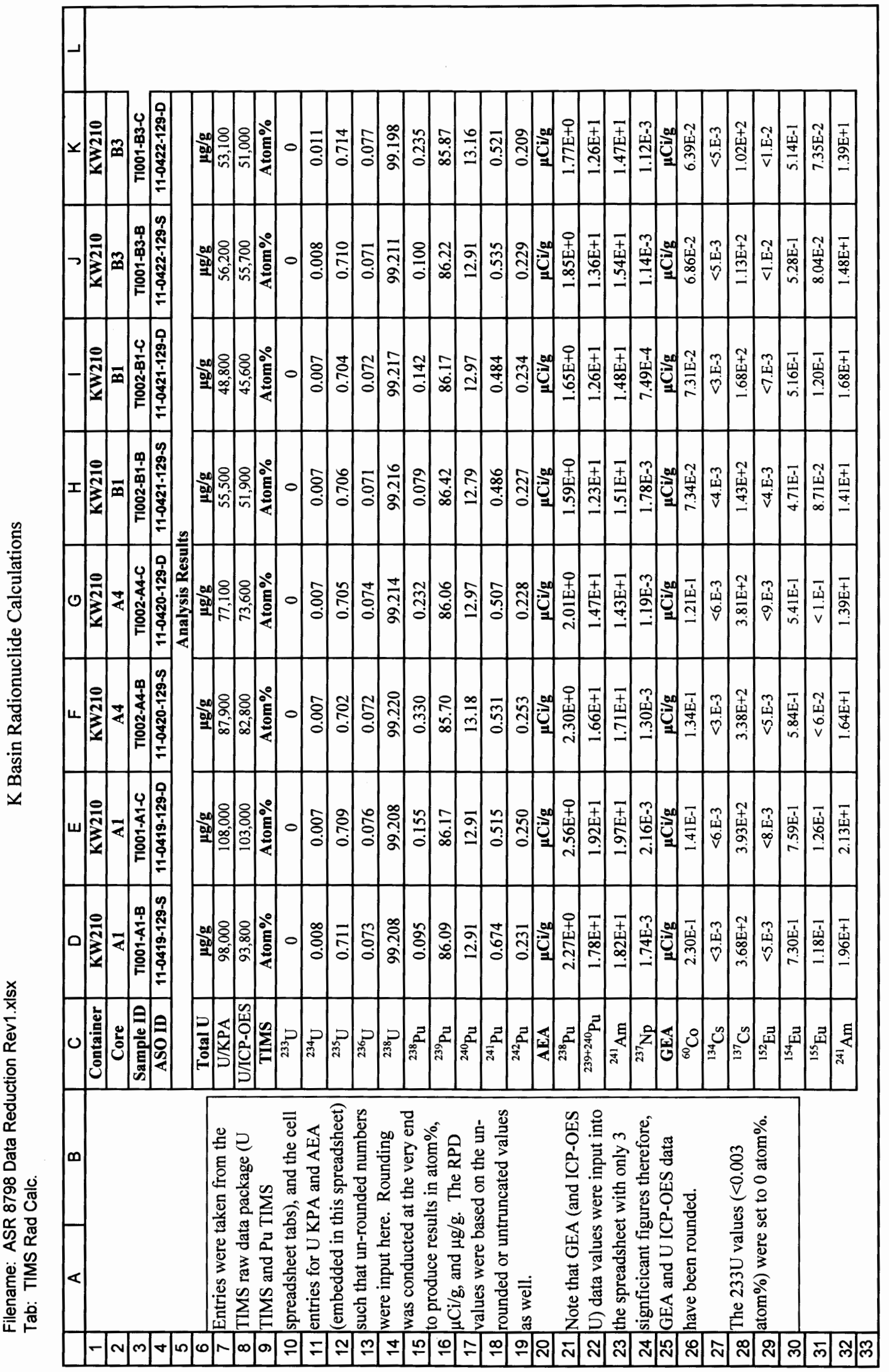




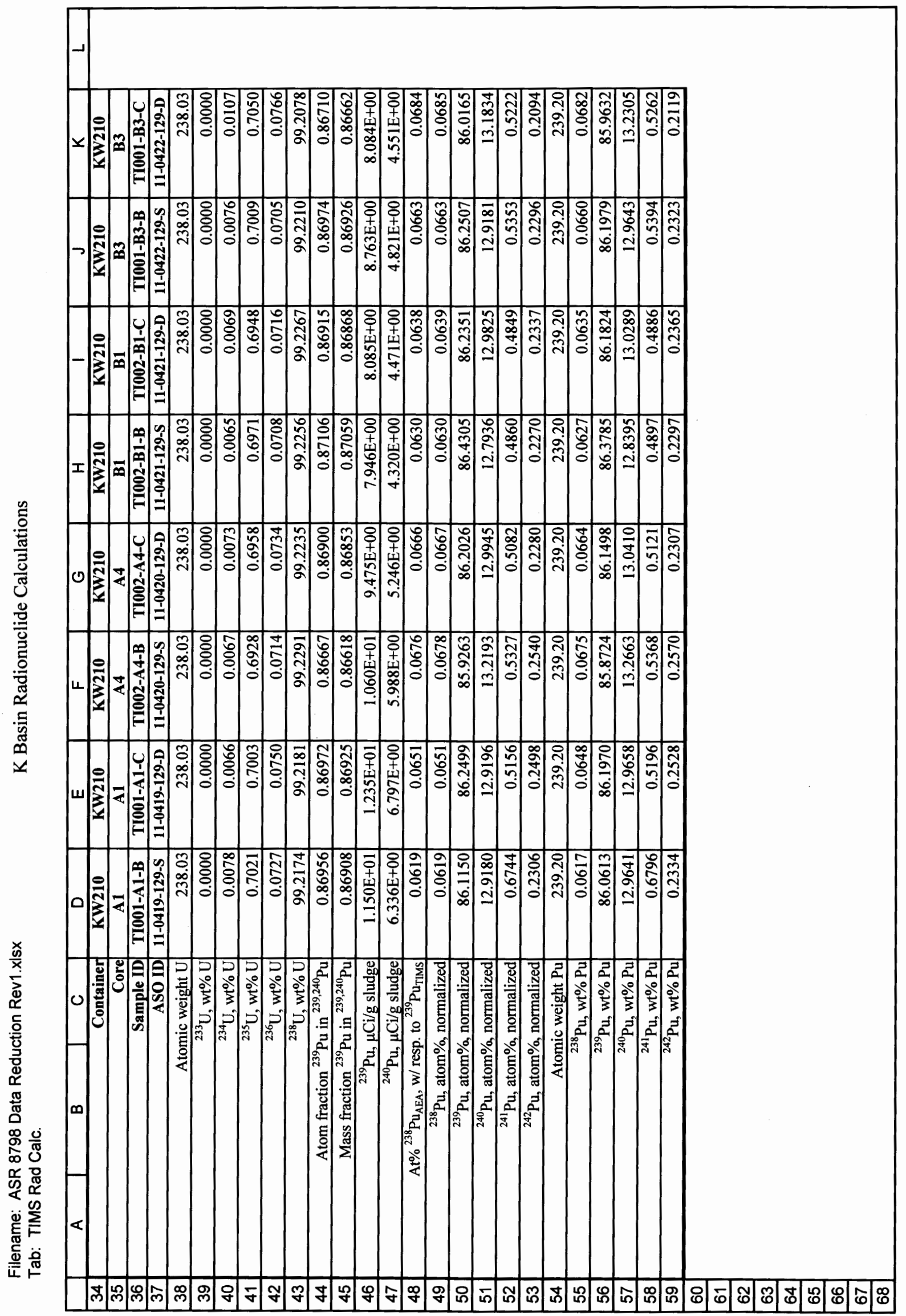




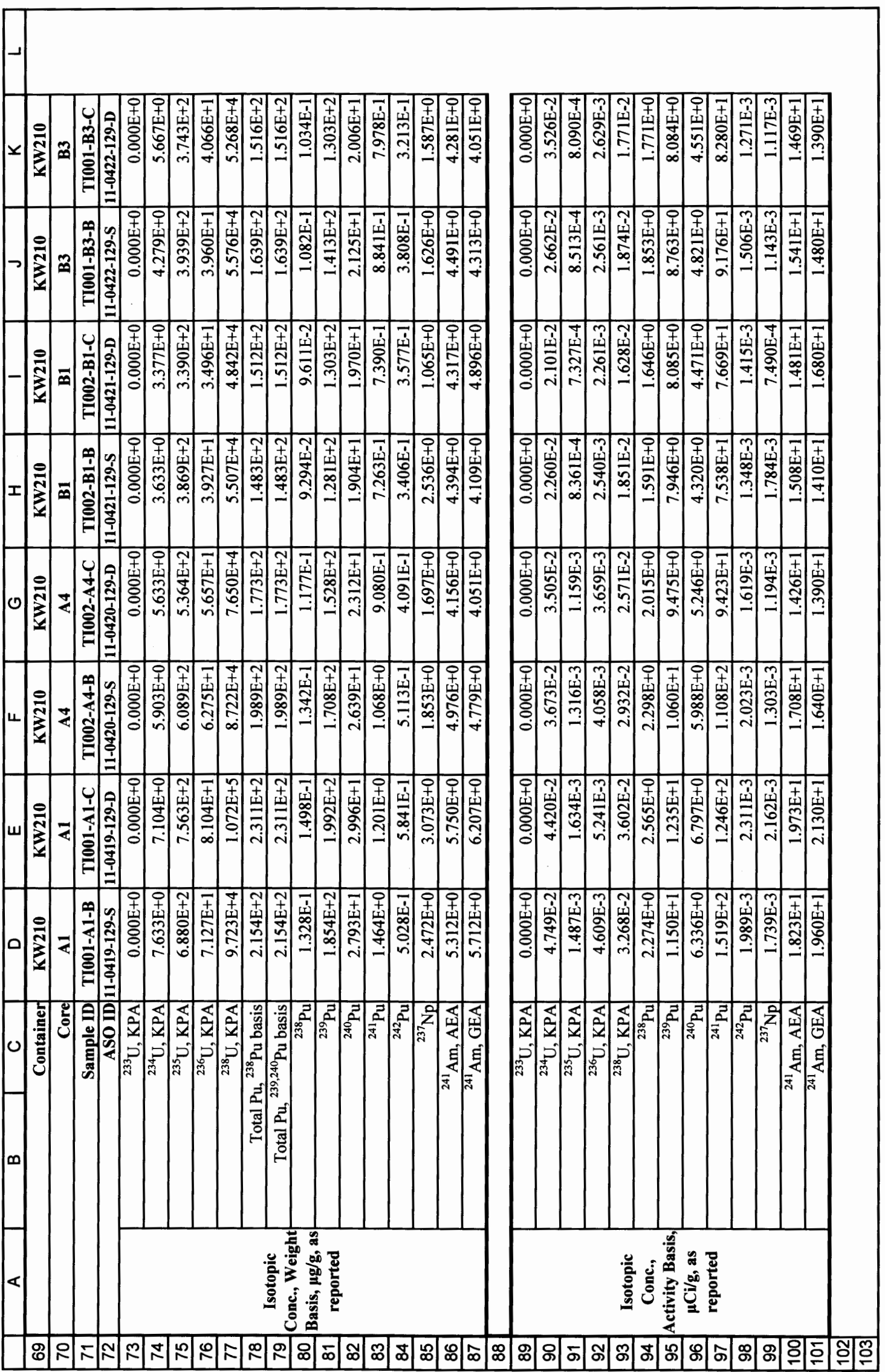




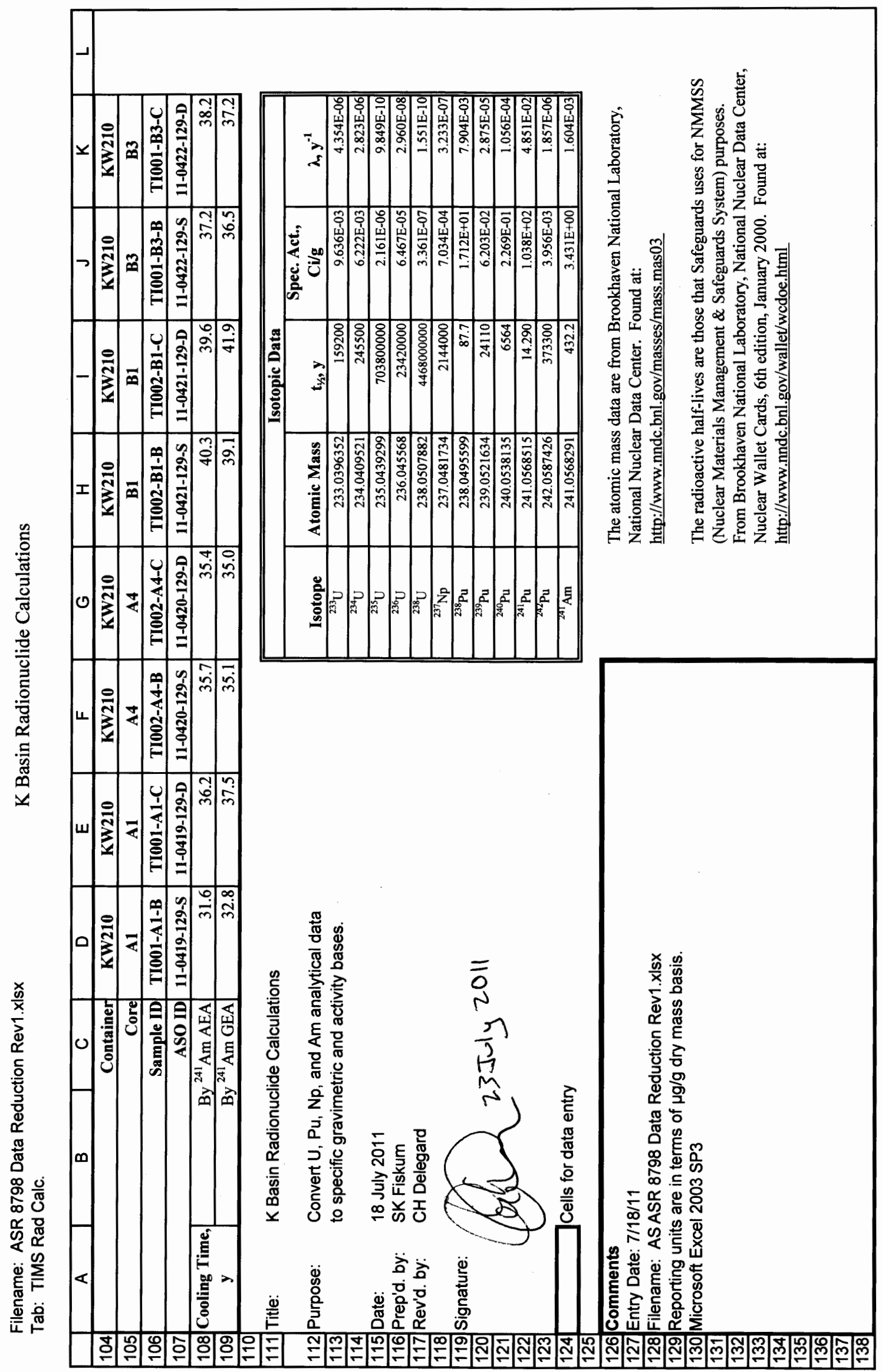


PNNL-20650, Rev. 2

Filename: ASR 8798 Data Reduction Rev1.xlsx

Date: TIMS Rad wet mass basis

K Basin Radionuclide Calculations

Wet settled solids mass basis

\begin{tabular}{|c|c|c|c|c|c|c|c|c|c|}
\hline $\begin{array}{l}\text { Conversion factor, } \\
\mathrm{R}_{\mathrm{dr}} / \mathrm{g}_{\text {gescetiled }}\end{array}$ & & 0.447 & 0.447 & 0.567 & 0.567 & 0.328 & 0.328 & 0.363 & 0.363 \\
\hline & 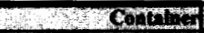 & Kiva & Trutos & RUTOU & minas & KWIn & Dubrow & WinT & WII \\
\hline & Cost & SIX: & 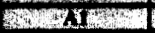 & $\mathrm{x}_{\mathrm{X}}$ & $2 \times 3$ & BJW & & & Ex: \\
\hline & Bponin & Tro,derra: & Wicingeren & 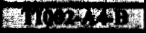 & duistara & 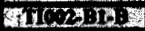 & 2.125100 & Minas: & 10:34 \\
\hline & XSD & FonISIXS & IIFIIS624 & 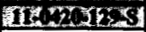 & 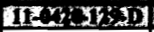 & Tromersas & 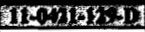 & 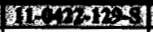 & 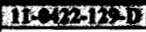 \\
\hline \multirow{15}{*}{$\begin{array}{c}\text { Isotopic Conc., } \\
\text { Weight Basis, } \\
\mu \mathrm{g} / \mathrm{g}, \text { wet settled } \\
\text { solids }\end{array}$} & ${ }^{233} \mathrm{U}, \mathrm{KPA}$ & $0.000 \mathrm{E}+0$ & $0.000 \mathrm{E}+0$ & $0.000 \mathrm{E}+0$ & $0.000 \mathrm{E}+0$ & $0.000 \mathrm{E}+0$ & $0.000 \mathrm{E}+0$ & $0.000 \mathrm{E}+0$ & $0.000 \mathrm{E}+0$ \\
\hline & ${ }^{234} \mathrm{U}, \mathrm{KPA}$ & $3.412 \mathrm{E}+0$ & $3.176 \mathrm{E}+0$ & $3.345 \mathrm{E}+0$ & $3.192 \mathrm{E}+0$ & $1.192 \mathrm{E}+0$ & $1.108 \mathrm{E}+0$ & $1.551 \mathrm{E}+0$ & $2.054 \mathrm{E}+0$ \\
\hline & ${ }^{235} \mathrm{U}, \mathrm{KPA}$ & $3.075 \mathrm{E}+2$ & $3.381 \mathrm{E}+2$ & $3.451 \mathrm{E}+2$ & $3.040 \mathrm{E}+2$ & $1.269 \mathrm{E}+2$ & $1.112 \mathrm{E}+2$ & $1.428 \mathrm{E}+2$ & $1.357 \mathrm{E}+2$ \\
\hline & ${ }^{236} \mathrm{U}, \mathrm{KPA}$ & $3.186 \mathrm{E}+1$ & $3.623 \mathrm{E}+1$ & $3.556 \mathrm{E}+1$ & $3.206 \mathrm{E}+1$ & $1.288 \mathrm{E}+1$ & $1.147 \mathrm{E}+1$ & $1.436 \mathrm{E}+1$ & $1.474 \mathrm{E}+1$ \\
\hline & ${ }^{238} \mathrm{U}, \mathrm{KPA}$ & $4.346 \mathrm{E}+4$ & $4.790 \mathrm{E}+4$ & $4.943 \mathrm{E}+4$ & $4.335 \mathrm{E}+4$ & $1.807 \mathrm{E}+4$ & $1.589 \mathrm{E}+4$ & $2.021 \mathrm{E}+4$ & $1.910 \mathrm{E}+4$ \\
\hline & Total $\mathrm{Pu}_{2}{ }^{238} \mathrm{Pu}$ basis & $9.629 \mathrm{E}+1$ & $1.033 \mathrm{E}+2$ & $1.127 \mathrm{E}+2$ & $1.005 \mathrm{E}+2$ & $4.866 \mathrm{E}+1$ & $4.962 \mathrm{E}+1$ & $5.942 \mathrm{E}+1$ & $5.496 \mathrm{E}+1$ \\
\hline & Total $\mathrm{Pu},{ }^{239,240} \mathrm{Pu}$ basis & $9.629 \mathrm{E}+1$ & $1.033 \mathrm{E}+2$ & $1.127 \mathrm{E}+2$ & $1.005 \mathrm{E}+2$ & $4.866 \mathrm{E}+1$ & $4.962 \mathrm{E}+1$ & $5.942 \mathrm{E}+1$ & $5.496 \mathrm{E}+1$ \\
\hline & ${ }^{238} \mathrm{Pu}$ & $5.936 \mathrm{E}-2$ & $6.694 \mathrm{E}-2$ & $7.606 \mathrm{E}-2$ & $6.667 \mathrm{E}-2$ & $3.049 \mathrm{E}-2$ & $3.153 \mathrm{E}-2$ & $3.923 \mathrm{E}-2$ & $3.749 \mathrm{E}-2$ \\
\hline & ${ }^{239} \mathrm{Pu}$ & $8.287 \mathrm{E}+1$ & $8.903 \mathrm{E}+1$ & $9.681 \mathrm{E}+1$ & $8.656 \mathrm{E}+1$ & $4.203 \mathrm{E}+1$ & $4.276 \mathrm{E}+1$ & $5.122 \mathrm{E}+1$ & $4.725 \mathrm{E}+1$ \\
\hline & ${ }^{240} \mathrm{Pu}$ & $1.248 \mathrm{E}+1$ & $1.339 \mathrm{E}+1$ & $1.496 \mathrm{E}+1$ & $1.310 \mathrm{E}+1$ & $6.247 \mathrm{E}+0$ & $6.465 \mathrm{E}+0$ & $7.703 \mathrm{E}+0$ & $7.272 \mathrm{E}+0$ \\
\hline & ${ }^{241} \mathrm{Pu}$ & $6.544 \mathrm{E}-1$ & $5.366 \mathrm{E}-1$ & $6.052 \mathrm{E}-1$ & $5.146 \mathrm{E}-1$ & $2.383 \mathrm{E}-1$ & $2.425 \mathrm{E}-1$ & $3.205 \mathrm{E}-1$ & $2.892 \mathrm{E}-1$ \\
\hline & ${ }^{242} \mathrm{Pu}$ & $2.247 \mathrm{E}-1$ & $2.611 \mathrm{E}-1$ & $2.898 \mathrm{E}-1$ & $2.318 \mathrm{E}-1$ & $1.118 \mathrm{E}-1$ & $1.174 \mathrm{E}-1$ & $1.380 \mathrm{E}-1$ & $1.165 \mathrm{E}-1$ \\
\hline & ${ }^{237} \mathrm{~Np}$ & $1.105 \mathrm{E}+0$ & $1.374 \mathrm{E}+0$ & $1.050 \mathrm{E}+0$ & $9.616 \mathrm{E}-1$ & $8.321 \mathrm{E}-1$ & $3.494 \mathrm{E}-1$ & $5.893 \mathrm{E}-1$ & $5.754 \mathrm{E}-1$ \\
\hline & ${ }^{241} \mathrm{Am}, \mathrm{AEA}$ & $2.374 \mathrm{E}+0$ & $2.570 \mathrm{E}+0$ & $2.820 \mathrm{E}+0$ & $2.355 \mathrm{E}+0$ & $1.442 \mathrm{E}+0$ & $1.416 \mathrm{E}+0$ & $1.628 \mathrm{E}+0$ & $1.552 \mathrm{E}+0$ \\
\hline & ${ }^{241} \mathrm{Am}, \mathrm{GEA}$ & $2.553 \mathrm{E}+0$ & $2.775 \mathrm{E}+\mathrm{C}$ & $2.708 \mathrm{E}+0$ & $2.296 \mathrm{E}+0$ & $1.348 \mathrm{E}+0$ & $1.606 \mathrm{E}+0$ & $1.564 \mathrm{E}+0$ & $1.468 \mathrm{E}+0$ \\
\hline \multirow{13}{*}{$\begin{array}{l}\text { Isotopic Conc., } \\
\text { Activity Basis, } \\
\mu \mathrm{Ci} / \mathrm{g} \text {, wet settled } \\
\text { solids }\end{array}$} & ${ }^{233} \mathrm{U}, \mathrm{KPA}$ & $0.000 \mathrm{E}+0$ & $0.000 \mathrm{E}+0$ & $0.000 \mathrm{E}+0$ & $0.000 \mathrm{E}+0$ & $0.000 \mathrm{E}+0$ & $0.000 \mathrm{E}+0$ & $0.000 \mathrm{E}+0$ & $0.000 \mathrm{E}+0$ \\
\hline & ${ }^{234} \mathrm{U}, \mathrm{KPA}$ & $2.123 \mathrm{E}-2$ & $1.976 \mathrm{E}-2$ & $2.081 \mathrm{E}-2$ & $1.986 \mathrm{E}-2$ & $7.416 \mathrm{E}-3$ & $6.894 \mathrm{E}-3$ & $9.651 \mathrm{E}-3$ & $1.278 \mathrm{E}-2$ \\
\hline & ${ }^{235} \mathrm{U}, \mathrm{KPA}$ & $6.646 \mathrm{E}-4$ & $7.306 \mathrm{E}-4$ & $7.457 \mathrm{E}-4$ & $6.570 \mathrm{E}-4$ & $2.743 \mathrm{E}-4$ & $2.404 \mathrm{E}-4$ & $3.086 \mathrm{E}-4$ & $2.933 \mathrm{E}-4$ \\
\hline & ${ }^{236} \mathrm{U}, \mathrm{KPA}$ & $2.060 \mathrm{E}-3$ & $2.343 \mathrm{E}-3$ & $2.300 \mathrm{E}-3$ & $2.073 \mathrm{E}-3$ & $8.332 \mathrm{E}-4$ & $7.417 \mathrm{E}-4$ & $9.284 \mathrm{E}-4$ & $9.531 \mathrm{E}-4$ \\
\hline & ${ }^{238} \mathrm{U}, \mathrm{KPA}$ & $1.461 \mathrm{E}-2$ & $1.610 \mathrm{E}-2$ & $1.661 \mathrm{E}-2$ & $1.457 \mathrm{E}-2$ & $6.073 \mathrm{E}-3$ & $5.340 \mathrm{E}-3$ & $6.794 \mathrm{E}-3$ & $6.419 \mathrm{E}-3$ \\
\hline & ${ }^{238} \mathrm{Pu}$ & $1.017 \mathrm{E}+0$ & $1.146 \mathrm{E}+0$ & $1.302 \mathrm{E}+0$ & $1.142 \mathrm{E}+0$ & $5.221 \mathrm{E}-1$ & $5.400 \mathrm{E}-1$ & $6.718 \mathrm{E}-1$ & $6.420 \mathrm{E}-1$ \\
\hline & ${ }^{239} \mathrm{Pu}$ & $5.140 \mathrm{E}+0$ & $5.522 \mathrm{E}+0$ & $6.005 \mathrm{E}+0$ & $5.369 \mathrm{E}+0$ & $2.607 \mathrm{E}+0$ & $2.652 \mathrm{E}+0$ & $3.177 \mathrm{E}+0$ & $2.931 \mathrm{E}+0$ \\
\hline & ${ }^{240} \mathrm{Pu}$ & $2.832 \mathrm{E}+0$ & $3.038 \mathrm{E}+0$ & $3.393 \mathrm{E}+0$ & $2.973 \mathrm{E}+0$ & $1.417 \mathrm{E}+0$ & $1.467 \mathrm{E}+0$ & $1.748 \mathrm{E}+0$ & $1.650 \mathrm{E}+0$ \\
\hline & ${ }^{241} \mathrm{Pu}$ & $6.791 \mathrm{E}+1$ & $5.569 \mathrm{E}+1$ & $6.280 \mathrm{E}+1$ & $5.340 \mathrm{E}+1$ & $2.473 \mathrm{E}+1$ & $2.516 \mathrm{E}+1$ & $3.326 \mathrm{E}+1$ & $3.002 \mathrm{E}+1$ \\
\hline & ${ }^{242} \mathrm{Pu}$ & $8.892 \mathrm{E}-4$ & $1.033 \mathrm{E}-3$ & $1.146 \mathrm{E}-3$ & $9.172 \mathrm{E}-4$ & $4.421 \mathrm{E}-4$ & $4.643 \mathrm{E}-4$ & $5.461 \mathrm{E}-4$ & $4.608 \mathrm{E}-4$ \\
\hline & ${ }^{237} \mathrm{~Np}$ & $7.774 \mathrm{E}-4$ & $9.663 \mathrm{E}-4$ & $7.386 \mathrm{E}-4$ & $6.764 \mathrm{E}-4$ & $5.853 \mathrm{E}-4$ & $2.457 \mathrm{E}-4$ & $4.145 \mathrm{E}-4$ & $4.048 \mathrm{E}-4$ \\
\hline & ${ }^{241} \mathrm{Am}, \mathrm{AEA}$ & $8.147 E+0$ & $8.819 \mathrm{E}+0$ & $9.676 \mathrm{E}+0$ & $8.082 \mathrm{E}+0$ & $4.946 \mathrm{E}+0$ & $4.860 \mathrm{E}+0$ & $5.586 \mathrm{E}+0$ & $5.325 \mathrm{E}+0$ \\
\hline & ${ }^{241} \mathrm{Am}, \mathrm{GEA}$ & $8.761 E+0$ & $9.521 \mathrm{E}+0$ & $9.294 \mathrm{E}+0$ & $7.877 \mathrm{E}+0$ & $4.626 \mathrm{E}+0$ & $5.512 E+0$ & $5.365 \mathrm{E}+0$ & $5.039 \mathrm{E}+0$ \\
\hline
\end{tabular}

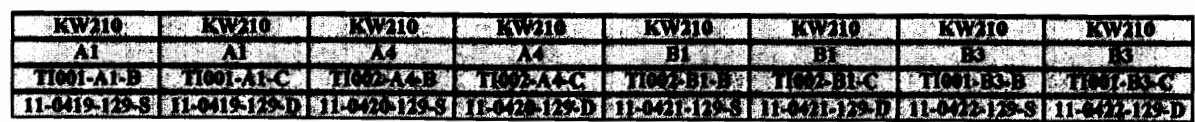

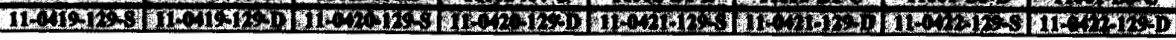

\begin{tabular}{cc}
$\mathrm{ug} / \mathrm{g}$ & RPD \\
\hline${ }^{233} \mathrm{U}, \mathrm{KPA}$ & $\mathrm{NA}$ \\
${ }^{234} \mathrm{U}, \mathrm{KPA}$ & $7.2 \%$ \\
${ }^{235} \mathrm{U}, \mathrm{KPA}$ & $9.5 \%$ \\
${ }^{236} \mathrm{U}, \mathrm{KPA}$ & $13 \%$ \\
${ }^{238} \mathrm{U}, \mathrm{KPA}$ & $9.7 \%$
\end{tabular}

Normalized $\mu \mathrm{Ci} / \mathrm{g}$ or $\mu \mathrm{g} / \mathrm{g}$

$\begin{array}{ll}{ }^{238} \mathrm{Pu} & 12 \% \\ { }^{239} \mathrm{Pu} & 7.2 \%\end{array}$

${ }^{240} \mathrm{Pu} \quad 7.0 \%$

${ }^{241} \mathrm{Pu} \quad 20 \%$

${ }^{242} \mathrm{Pu} \quad 15 \%$

Atom\%

${ }^{233} \mathrm{U}$, TIMS

${ }^{234}$ U, TIMS NA

${ }^{235} \mathrm{U}$, TIMS $\quad 0.26 \%$

${ }^{236} \mathrm{U}$, TIMS $\quad 3.1 \%$

${ }^{238} \mathrm{U}$, TIMS $\quad 0.0007 \%$

Normalized Atom\%

${ }^{238} \mathrm{Pu}$ TIMS $\quad 5.0 \%$

${ }^{239} \mathrm{Pu}$ TIMS $\quad 0.16 \%$

${ }^{240} \mathrm{Pu}$ TIMS $\quad 0.013 \%$

${ }^{241} \mathrm{Pu}$ TIMS $\quad 27 \%$

${ }^{242} \mathrm{Pu}$ TIMS $\quad 8.0 \%$

${ }^{238} \mathrm{Pu}$ renormalization and it's associated variation.)

Gray shading indicates the RPD exceeded $\pm 20 \%$ criterion defined by the QAPjP/SAP.

$\begin{array}{cc}\text { RPD } & \text { RPD } \\ \text { NA } & \text { NA } \\ 7.3 \% & \mathbf{2 8 \%} \\ 13 \% & 5.1 \% \\ 12 \% & 2.6 \% \\ 13 \% & 5.7 \% \\ & \\ & \\ 3.4 \% & 4.5 \% \\ 1.7 \% & 8.1 \% \\ 3.4 \% & 5.8 \% \\ 1.7 \% & 10 \% \\ 4.9 \% & 17 \%\end{array}$

$\begin{array}{lcc}\text { NA } & \text { NA } & \text { NA } \\ 8.4 \% & 5.6 \% & 33 \% \\ 0.43 \% & 0.33 \% & 0.58 \% \\ 2.7 \% & 1.2 \% & 8.3 \% \\ 0057 \% & 0.0011 \% & 0.0135 \%\end{array}$

\begin{tabular}{lrc}
$1.7 \%$ & $1.4 \%$ & $3.3 \%$ \\
$0.32 \%$ & $0.23 \%$ & $0.27 \%$ \\
$1.7 \%$ & $1.5 \%$ & $2.0 \%$ \\
$4.7 \%$ & $0.2 \%$ & $2.5 \%$ \\
$11 \%$ & $2.9 \%$ & $9.2 \%$ \\
ore it will be affected relative to that reported by & \\
\multicolumn{2}{l}{} \\
TIMS because of the effect of \\
rion defined by the QAPjP/SAP.
\end{tabular}
$2.9 \% \quad 9.2 \%$ NA

$5.1 \%$

$2.6 \%$

$4.5 \%$

$5.8 \%$

$10 \%$

E.101 
PNNL-20650, Rev. 2

\section{Appendix F}

\section{X-Ray Diffraction (XRD) Analysis}





\section{Appendix F}

\section{X-Ray Diffraction (XRD) Analysis}

Appendix F contains the X-ray diffraction (XRD) reports. The XRD analyses were conducted on the size-fractionated samples from SCS-CON-210 and the undissolved solids generated from the acid digestions resulting from the completion of Analytical Service Requests (ASR) 8798 (core samples) and 8824 (size-fractionated container composite samples). Both reports reference appendices, which are selfcontained within the XRD reports.

The following table of contents shows that pagination specific to Appendix F was applied to the reports (including self-contained appendices), document review records, instrument log sheets, and surveillance reports (generated by the PNNL Quality Engineers). This pagination is sequential and consistent (e.g., page F.\#).

\section{Appendix F Table of Contents}

XRD Report Supporting Analysis of KW210 Sieve Fractions .........................................................

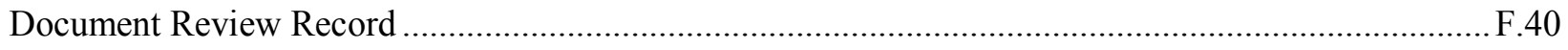

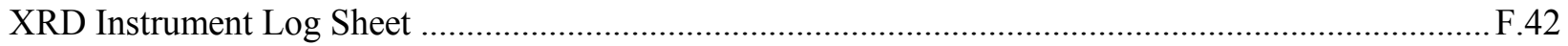

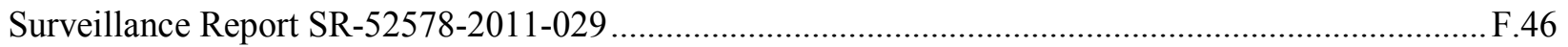

XRD Report Supporting Analysis of Undissolved Solids (from ASR 8798 and 8824) ........................F.52

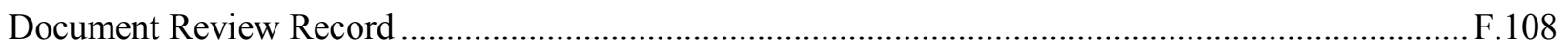

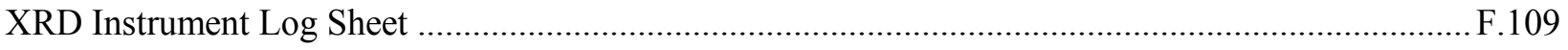

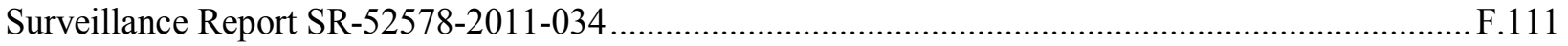





\section{X-Ray Diffraction Analysis Report}

\section{Battelle-Pacific Northwest National Laboratory Radiochemical Sciences and Engineering Group PO Box 999, Richland, Washington 99352}

Project: \#52578

Sample ID: KW 210 Container Composites

Procedure: PNNL-RPG-268 Rev. 4, "Solids Analysis by X-Ray Diffraction"

Analyst: Doinita Neiner

Analysis Dates: 05/25/2011

Instrument: RGD \# 105

Property Number: WD 59716

Serial Number: GD 3827N

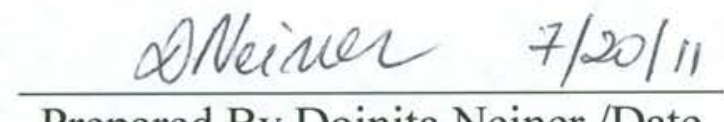

Prepared By Doinita Neiner /Date
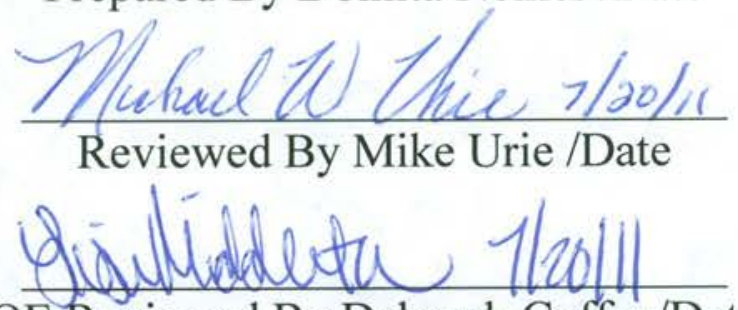

QE Reviewed By Deborah Coffey/Date $7 / 20 / 11$

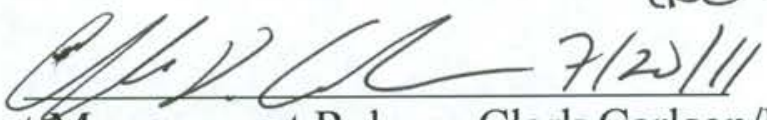

Project Management Release Clark Carlson/Date 
PNNL-20650, Rev. 2

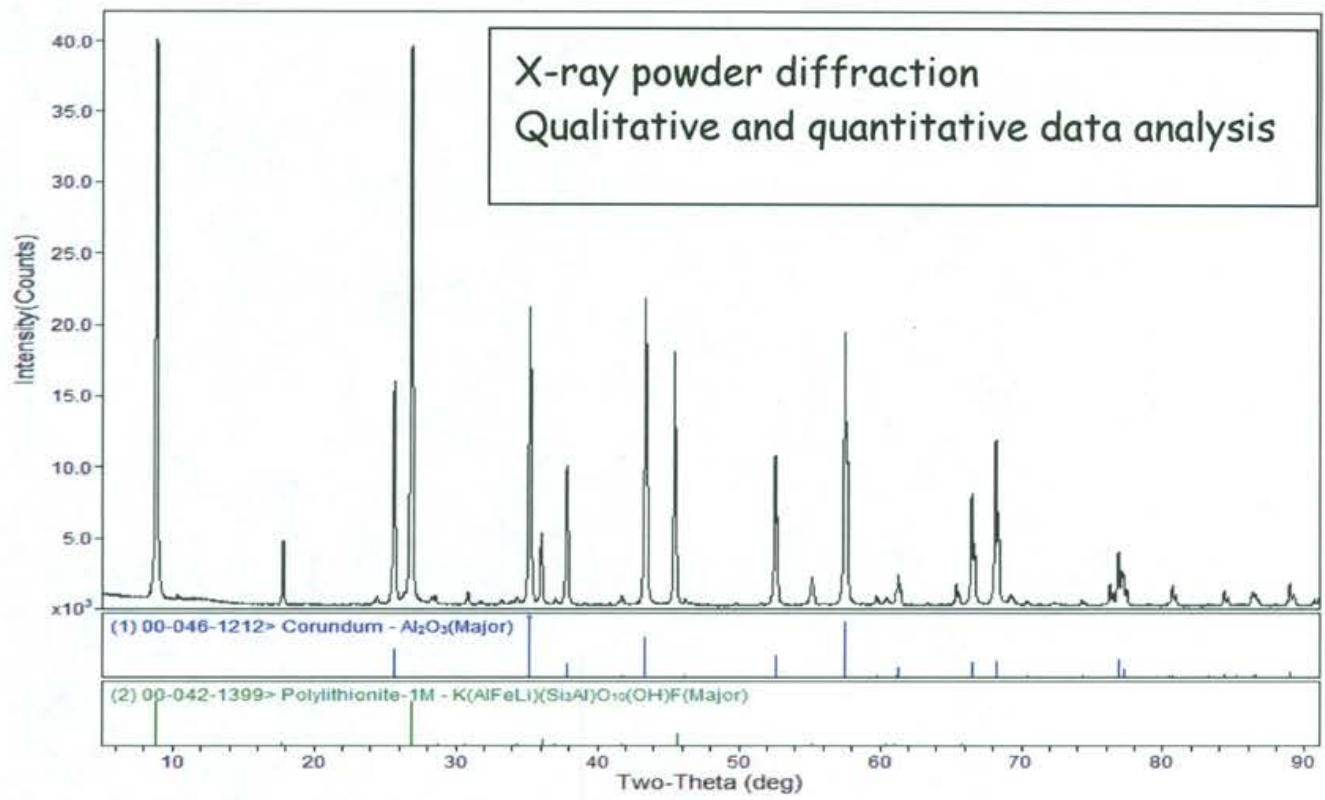

Project Number: Charge Code: Client:

Total Samples:
52578

H41223

S. Fiskum

4 solids (See results for Client IDs)

\begin{tabular}{|l|l|}
\hline Analysis Procedure & PNNL-RPG-268 Rev. 4, "Solids Analysis by X-Ray Diffraction" \\
\hline Prep Procedure & Slurry of amyl acetate and Krylon on a glass slide covered with Kapton \\
\hline Analyst & Doinita Neiner \\
\hline Analysis Date & May 25, 2011 \\
\hline Sample Prep. Date & May 19, 2011 through May 24, 2011 \\
\hline Reference Standards & Rutile - SRM 674- PDF 01-077-0440 \\
\hline $\begin{array}{l}\text { Software } \\
\text { Reference database }\end{array}$ & $\begin{array}{l}\text { Jade MDI 9 } \\
\text { ICDD PDF -2 2009 }\end{array}$ \\
\hline M\&TE Numbers & XRD System (WD 59716), RPL/317 \\
\hline
\end{tabular}




\section{XRD Analysis Request Form}

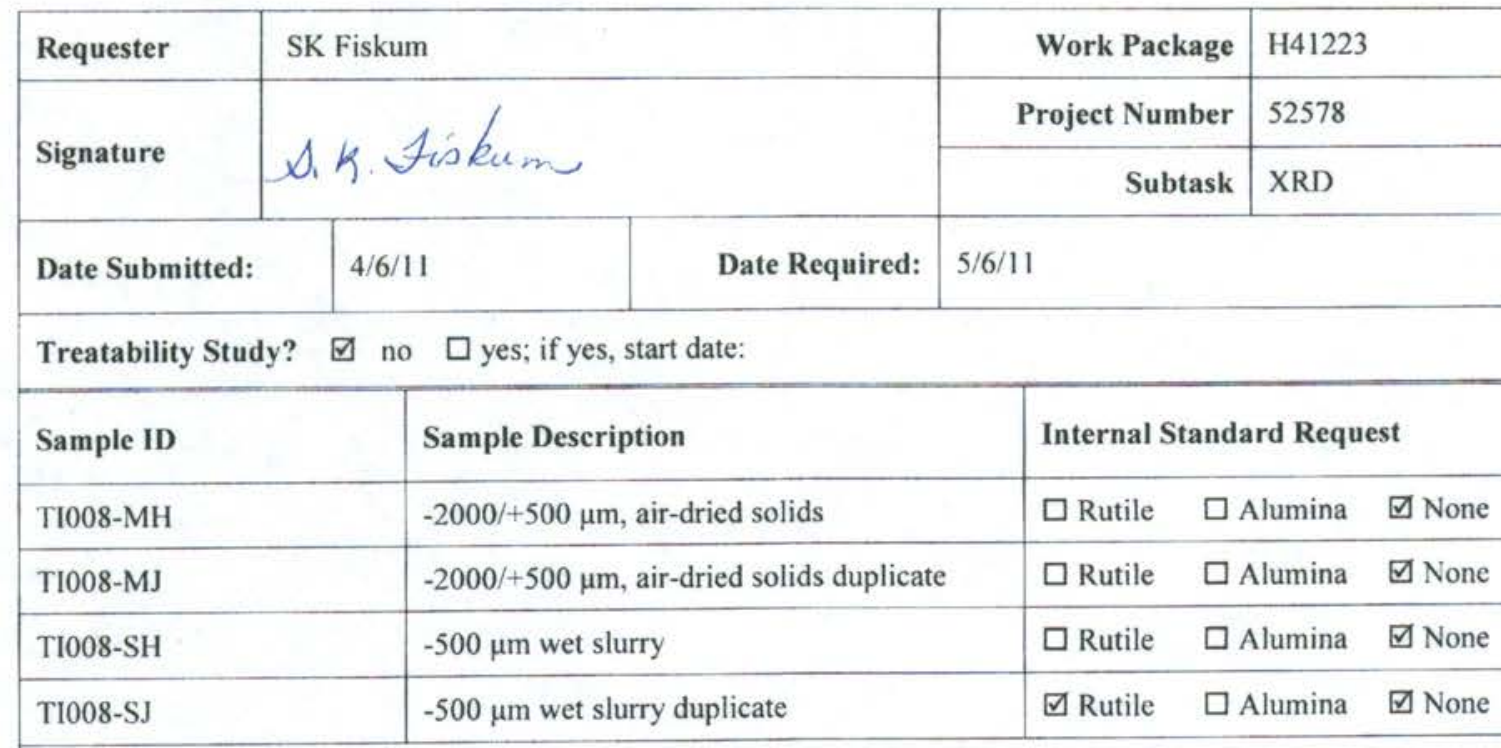

Comments: All samples are from the KW210 container composite that had been wet-sieved into three size fractions. Only samples from two fractions are provided herein. A large quantity of resin beads have been observed in the $-2000 /+500 \mu \mathrm{m}$ fraction. The $-500 \mu \mathrm{m}$ sample fractions are wet slurries that will require air drying.

Identify crystalline structures in room-temperature-dried sludge. Identify hydrate compounds plus any other identifiable compounds. This includes noting the presence (or absence) of aluminum hydrates, uranium hydrides, uranium oxides as well as zirconium metal. The analytical composition (ICP-OES analytes) will be provided upon availability. Uranium is expected to be a dominant component.

All samples are to be analyzed without internal standard; one sample (selected choice above can be altered depending on quantity of sample available) are to also be run with rutile $\left(\mathrm{TiO}_{2}\right)$. Rutile X-ray diffraction angles and relative peak magnitudes will be used to help demonstrate acceptable system performance within the sample matrix and analysis process.

Please use the new Rigaku system for powder diffraction analysis if at all possible. Please use a bench sheet you develop to support sample processing and analysis and provide this original bench sheet as part of the data package.

Notes for Pattern Analyst:

\section{XRD Mount preparation}

Standard Reference Materials ID's: $\mathrm{TiO}_{2}: \underline{\mathrm{SRM}} 674 \quad \mathrm{Al}_{2} \mathrm{O}_{3}: \underline{\mathrm{SRM}} 676$ Other:

Fixative: $\square$ Collodion 凶Krylon $\square$ Other

Mount: G Glass Slide, $\square$ Kapton covered $\square$ PIP Holder $\square$ Other

Prepared by: $\frac{0 \text { Mecine }}{\text { Signature }}$

$5 / 24 / 11$

XRD Operating Parameters

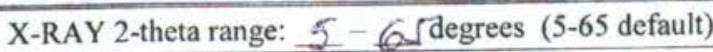

\begin{tabular}{lll} 
Step size: & degrees $(0.02$ default $)$ & \\
\hline Count time: & second(s) $(2$ default $)$ & $\square$ Multi-Scans
\end{tabular}

(Shaded areas to be filled out by the XRD analysis staff.) 
PNNL-20650, Rev. 2

\section{XRD Analysis Bench Sheet \\ For Procedure PNNL-RPG-268, Rev. 4, Solids Analysis by X-Ray Diffraction Sample Preparation}

Print and complete pages as needed in order to document sample preparation for all samples submitted under one Analysis Request.

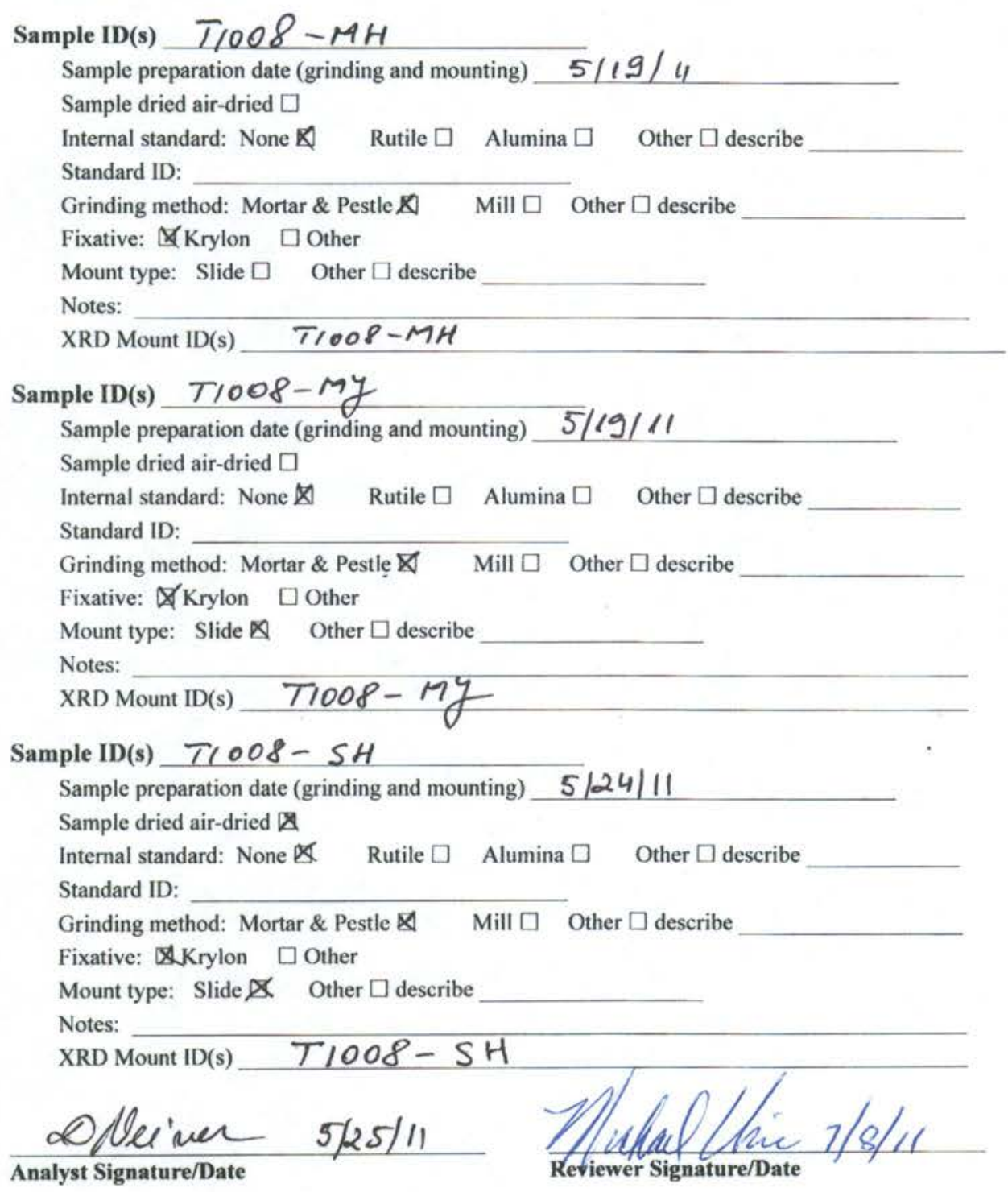


PNNL-20650, Rev. 2

Battelle - Pacific Northwest National Laboratory

Radiochemical Sciences and Engineering Group X-Ray Diffraction Data Analysis . - ene

\section{XRD Analysis Bench Sheet \\ For Procedure PNNL-RPG-268, Rev. 4, \\ Solids Analysis by X-Ray Diffraction \\ Sample Preparation}

Print and complete pages as needed in order to document sample preparation for all samples submitted under one Analysis Request.

\section{Sample ID(s) $\quad 7 / 008-5 y$}

Sample preparation date (grinding and mounting) $5 / 24 / 11$

Sample dried air-dried $\triangle$

Internal standard: None $\bigotimes \quad$ Rutile $\square \quad$ Alumina $\square \quad$ Other $\square$ describe

Standard ID:

Grinding method: Mortar \& Pestle $\mathbb{Q} \quad$ Mill $\square \quad$ Other $\square$ describe

Fixative: $₫$ Krylon $\square$ Other

Mount type: Slide $\mathbb{C}$ Other $\square$ describe

Notes:

XRD Mount ID(s) T/008-S

Sample ID(s) T/1008-S

Sample preparation date (grinding and mounting)

Sample dried air-dried $\bigotimes$

Internal standard: None $\square \quad$ Rutile $\bowtie \quad$ Alumina $\square \quad$ Other $\square$ describe

Standard ID: $\quad \mathrm{TiO}_{2}$

Grinding method: Mortar \& Pestle $\bigotimes \quad$ Mill $\square \quad$ Other $\square$ describe

Fixative: $\mathbb{X}$ Krylon $\square$ Other

Mount type: Slide $\mathbb{X}$ Other $\square$ describe

Notes:

XRD Mount ID(s) T1008-SJ-TiOz

Sample ID(s)

Sample preparation date (grinding and mounting)

Sample dried air-dried $\square$

Internal standard: None $\square \quad$ Rutile $\square \quad$ Alumina $\square \quad$ Other $\square$ describe

Standard ID:

Grinding method: Mortar \& Pestle $\square \quad$ Mill $\square \quad$ Other $\square$ describe

Fixative: $\square$ Krylon $\square$ Other

Mount type: Slide $\square$ Other $\square$ describe

Notes:

XRD Mount ID(s)

onciner 5/25/11

Analyst Signature/Date

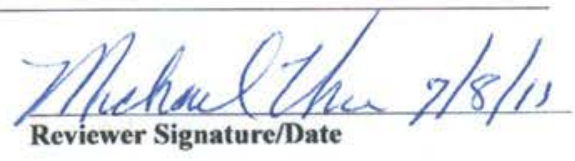


PNNL-20650, Rev. 2

Battelle - Pacific Northwest National Laboratory

Radiochemical Sciences and Engineering Group

X-Ray Diffraction Data Analysis

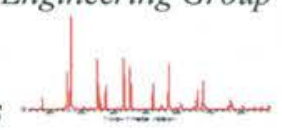

\section{XRD Analysis Bench Sheet \\ For Procedure PNNL-RPG-268, Rev. 4, \\ Solids Analysis by X-Ray Diffraction \\ Diffraction Measurements}

Print and complete pages as needed in order to document sample analysis and daily calibration verification for all samples submitted under one Analysis Request.

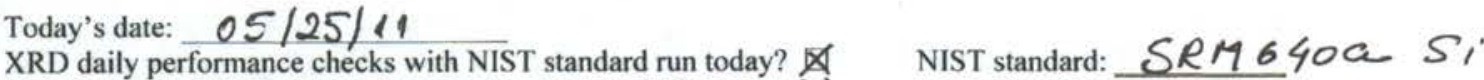
Associated daily calibration check filename $052511 a \ldots$

Logbook updated?

\begin{tabular}{|c|c|c|c|c|}
\hline XRD mount ID & \multicolumn{2}{|c|}{ XRD filename } & Notes & Analyst initials \\
\hline $\mathrm{T} / 008-\mathrm{MH}$ & \multicolumn{2}{|c|}{$T 1008-M H-0525116$} & & $\Delta N$ \\
\hline $71008-M$ & \multicolumn{2}{|c|}{$T 1008-M y-052511 C$} & & $\Delta N$ \\
\hline$T 1008-S H$ & \multicolumn{2}{|c|}{$T 1008-5 H-052511 D$} & & $\Delta N$ \\
\hline$T 1008-57$ & \multicolumn{2}{|c|}{ T1008-SJ-052511E } & & sov \\
\hline$T 1008-5 f$ & \multicolumn{3}{|c|}{$T 1008-5 y-1,02-0525117$} & $\Delta N$ \\
\hline \multirow{3}{*}{\multicolumn{2}{|c|}{ XRD Operating Parameters }} & \multicolumn{3}{|c|}{ X-RAY 2-theta range: $5-65$ degrees (5-65 default) } \\
\hline & & \multicolumn{3}{|c|}{ Step size: 0.02 degrees $(0.02$ default $)$} \\
\hline & & \multicolumn{3}{|c|}{$\begin{array}{l}\text { Other XRD scan parameters: } \\
2 \mathrm{dy} / \text { min, } D T E x, 40 \mathrm{kV}, 44 \mathrm{~mA}\end{array}$} \\
\hline
\end{tabular}

Today's date:

XRD daily performance checks with NIST standard run today? $\square \quad$ NIST standard:

Associated daily calibration check filename

Logbook updated? $\square$

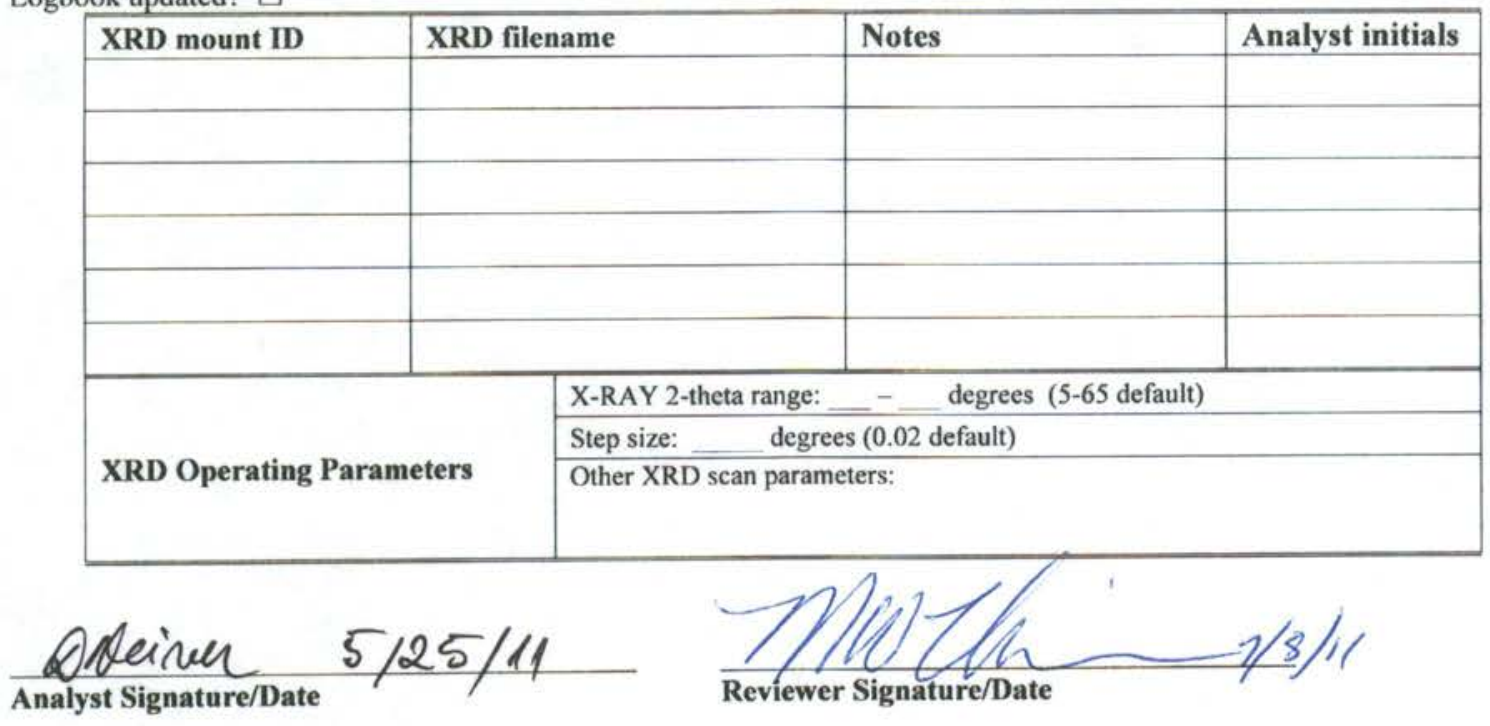


PNNL-20650, Rev. 2

X-Ray Diffraction Data Analysis

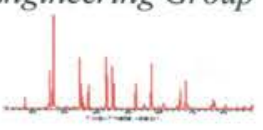

Table 1. Summary of Phases Identified in KW210 Container Composites

\begin{tabular}{|c|c|c|c|c|}
\hline $\begin{array}{l}\text { Sample ID } \\
\text { RPL ID/(Client } \\
\text { ID) }\end{array}$ & $\begin{array}{l}\text { ICDD } \\
\text { Card Number }\end{array}$ & $\begin{array}{c}\text { Relative } \\
\text { Intensity }\end{array}$ & Formula & $\begin{array}{l}\text { Common Phase } \\
\text { Name }\end{array}$ \\
\hline \multirow[t]{2}{*}{ TI008-MH } & $01-070-3755$ & Major & $\mathrm{SiO}_{2}$ & Quartz \\
\hline & -- & Unassigned & $27.3^{\circ}$ & \\
\hline \multirow[t]{2}{*}{ TI008-MJ } & $01-070-3755$ & Major & $\mathrm{SiO}_{2}$ & Quartz \\
\hline & -- & Unassigned & $33^{\circ}, 35^{\circ}-36^{\circ(b)}$ & \\
\hline \multirow[t]{4}{*}{ TI008-SH } & $01-070-3755$ & Major & $\mathrm{SiO}_{2}$ & Quartz \\
\hline & $01-072-4553$ & Major & $\mathrm{UO}_{2}$ & Uraninite \\
\hline & 01-075-0944 & Major & $\mathrm{U}_{4} \mathrm{O}_{9}$ & Uranium oxide \\
\hline & -- & Unassigned & $\begin{array}{l}23.6^{\circ}, 34.5^{\circ}, \\
35.6^{\circ}\end{array}$ & \\
\hline \multirow[t]{4}{*}{ TI008-SJ } & $01-070-3755$ & Major & $\mathrm{SiO}_{2}$ & Quartz \\
\hline & $01-072-4553$ & Major & $\mathrm{UO}_{2}$ & Uraninite \\
\hline & 01-075-0944 & Major & $\mathrm{U}_{4} \mathrm{O}_{9}$ & Uranium oxide \\
\hline & -- & Unassigned & $\begin{array}{l}24^{\circ}, 35^{\circ}, 35.8^{\circ}, \\
37^{\circ}, 38^{\circ}, 51.5^{\circ}\end{array}$ & \\
\hline
\end{tabular}

(a) Crystalline components above $10 \mathrm{wt} . \%$ are classified as Major phases, between 1-10 wt. $\%$ as Minor phases, and under $1 \mathrm{wt} . \%$ as Possible phases.

(b) A broad peak was present between $35^{\circ}$ to $36^{\circ} 2$-theta. 


\section{Sample preparation}

X-ray powder diffraction (XRD) data from the samples identified in Table 2 have been examined for identification of crystalline phases present. The samples were KW210 container composite that was wet sieved into three size fractions prior to submitting for XRD analysis. Only two sieve fractions were submitted for analysis. The samples TI008-SH and TI008-SJ were air dried prior to XRD slide preparation. The samples TI008-MH and TI008-MJ were used as received.

All the samples were processed and analyzed according to procedure, Solids Analysis by X-Ray Diffraction, PNNL-RPG-268, Rev. 4. A sample was mixed with approximately $2 \mathrm{~mL}$ of amyl acetate in a 20 -mL glass scintillation vial to produce a slurry. A small aliquot of the slurry was transferred into a mortar and ground with a pestle (all tungsten carbide components). After grinding, a small fraction of the sample was pipetted onto a Krylon-covered glass slide and allowed to dry. The dried samples were then covered with Kapton. One client sample, TI008-SJ was run in duplicate (one without internal standard and one with 3-4 mg TiO 2 , rutile as internal standard). Titania (rutile structure) internal standard was used, SRM 674; the standard certificate is provided in Appendix 1.

Table 2. Sample Identification

\begin{tabular}{|l|l|l|c|}
\hline Sample ID & Sample Description & XRD Sample ID & $\begin{array}{l}\text { Internal } \\
\text { Standard }\end{array}$ \\
\hline \multirow{2}{*}{ TI008-MH } & KW210 $(500-2000 \mu \mathrm{m})$ & TI008-MH & None \\
\hline TI008-MJ & KW210 $(500-2000 \mu \mathrm{m})$ & TI008-MJ & None \\
\hline \multirow{2}{*}{ TI008-SH } & $\mathrm{KW} 210(<500 \mu \mathrm{m})$ & TI008-SH & None \\
\hline & & TI008-SJ & None \\
\cline { 3 - 4 } & $\mathrm{KW} 210(<500 \mu \mathrm{m})$ & TI008-SJ-TiO & $\mathrm{TiO}_{2}$ \\
\hline
\end{tabular}


PNNL-20650, Rev. 2

Battelle - Pacific Northwest National Laboratory

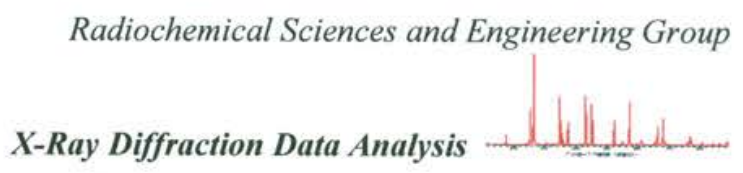

\section{X-ray powder diffraction data collection}

X-ray powder diffraction analyses were performed on a Rigaku Ultima IV X-ray Diffractometer ( $\mathrm{Cu} \mathrm{K} \alpha$ radiation, $\lambda=1.5418 \AA$ ). Data were collected in a step scan mode between 5 and $65^{\circ} 2$ theta with a step size of $0.02^{\circ}$, a speed of $2^{\circ}$ minute, and using a $2 \mathrm{D}$ detector DTEX. The X-ray tube operating conditions were $40 \mathrm{kV}$ and $44 \mathrm{~mA}$.

The daily performance check associated with the XRD sample files is presented in Appendix 2. This performance check meets the performance criteria (i.e., $111 \mathrm{Si}$ 2-theta $=28.44^{\circ} \pm 0.06^{\circ}$ ) specified in the XRD procedure PNNL-RPG-268 Rev. 4. The NIST certificate for the standard used for the daily performance check (SRM 640a) is presented in Appendix 3.

The quality control/calibration of the XRD instrument results are provided in Appendix 4 and the standard certificate, SRM 1976 is presented in Appendix 5. The requirement for the semiannual calibration of the diffractometer (as stated in the PNNL-RPG-268 Rev. 4, is that observed d-interplanar distance for any given crystal lattice Miller indices be within $\pm 0.02 \AA$ (Del-d value - difference between the calculated and the observed interplanar distance) of the d-values from the ICDD (International Centre for Diffraction Data) card for alumina (corundum structure). For this quality control semiannual calibration, all Del-d values are well within the aforementioned criterion $( \pm 0.02 \AA$ Del-d value).

\section{X-ray powder diffraction data analysis}

\subsection{Database Search Details}

Phase identification was conducted using JADE search match routines. The search was done including the following elements: $\mathrm{C}, \mathrm{Na}, \mathrm{K}, \mathrm{Mg}, \mathrm{Ca}, \mathrm{Al}, \mathrm{Sr}, \mathrm{Si}, \mathrm{O}, \mathrm{H}, \mathrm{Cd}, \mathrm{Cr}, \mathrm{Ba}, \mathrm{Cu}, \mathrm{Ni}, \mathrm{Pb}, \mathrm{Mn}$, $\mathrm{Fe}, \mathrm{Zn}, \mathrm{Zr}, \mathrm{Ti}$ and $\mathrm{U}$. All diffraction patterns have been compared against three ICDD PDF-2 databases: RDB-ICSD, Inorganics, and Minerals. All of the XRD analyses included the search for uranium oxides, oxyhydroxides, hydrates and hydroxides; aluminum oxides, oxyhydroxides and hydrates; and zirconium metal and zirconia (from cladding materials). The PDF (Powder Diffraction File) cards of the crystalline phases identified in the samples analyzed in this report are given in Appendix 6.

\subsection{Rietveld Refinement Details}

The Rietveld method effectively separates overlapping data, thereby allowing an accurate determination of the structure/composition. Rietveld refinement on powder materials is a widely used application in determining the components of chemical mixtures. The refinement takes into account the PDF cards of the identified phases, and compares those with the calculated peaks from the observed powder pattern. The refinement generates a difference plot between an observed and a calculated powder pattern. In addition, because it calculates a powder pattern from the PDF cards of the phases taken into consideration it gives a goodness of fit for that particular phase, which is a reflection of how good the fit is between the observed and the calculated powder patterns. The refined parameters are the unit cell parameters, background, and 
PNNL-20650, Rev. 2

preferred orientation. The refinement helps decide which one of the examined phases is present in the observed powder pattern, by comparing all peak positions and intensities with calculated values (from the PDF card). The quality of the refinement can be judged based on the R factors (e.g., $\mathrm{R}_{\mathrm{w}}$ ) and the difference map (i.e., the difference between an observed and a calculated powder pattern). Typically, the better the Rietveld fit the lower the $R_{w}$ factor; $R_{w}<20 \%$ suggests a good fit, $R_{w}<10 \%$ suggests an excellent fit. $R_{w}$ values $<20 \%$ have to be supported by a small difference between the calculated and observed powder patterns (i.e., minor deviations within the difference map).

\section{Summary of findings}

The samples represent $>500 /<2000$ micron particle size (medium fraction) samples TI008-MH and TI008-MJ, and $<500$ micron particle size (small fraction) samples, TI008-SH and TI008-SJ. The medium fraction is primarily composed of quartz. The small fraction contains uraninite in addition to quartz. In addition some reflections present in the powder diffraction data were unidentified. The subsamples do not contain uranium oxyhydroxides, hydrates and hydroxides; aluminum oxides, oxyhydroxides and hydrates, or zirconium metal and zirconia (from cladding materials) as crystalline phases. A summary of phases is presented in Table 1.

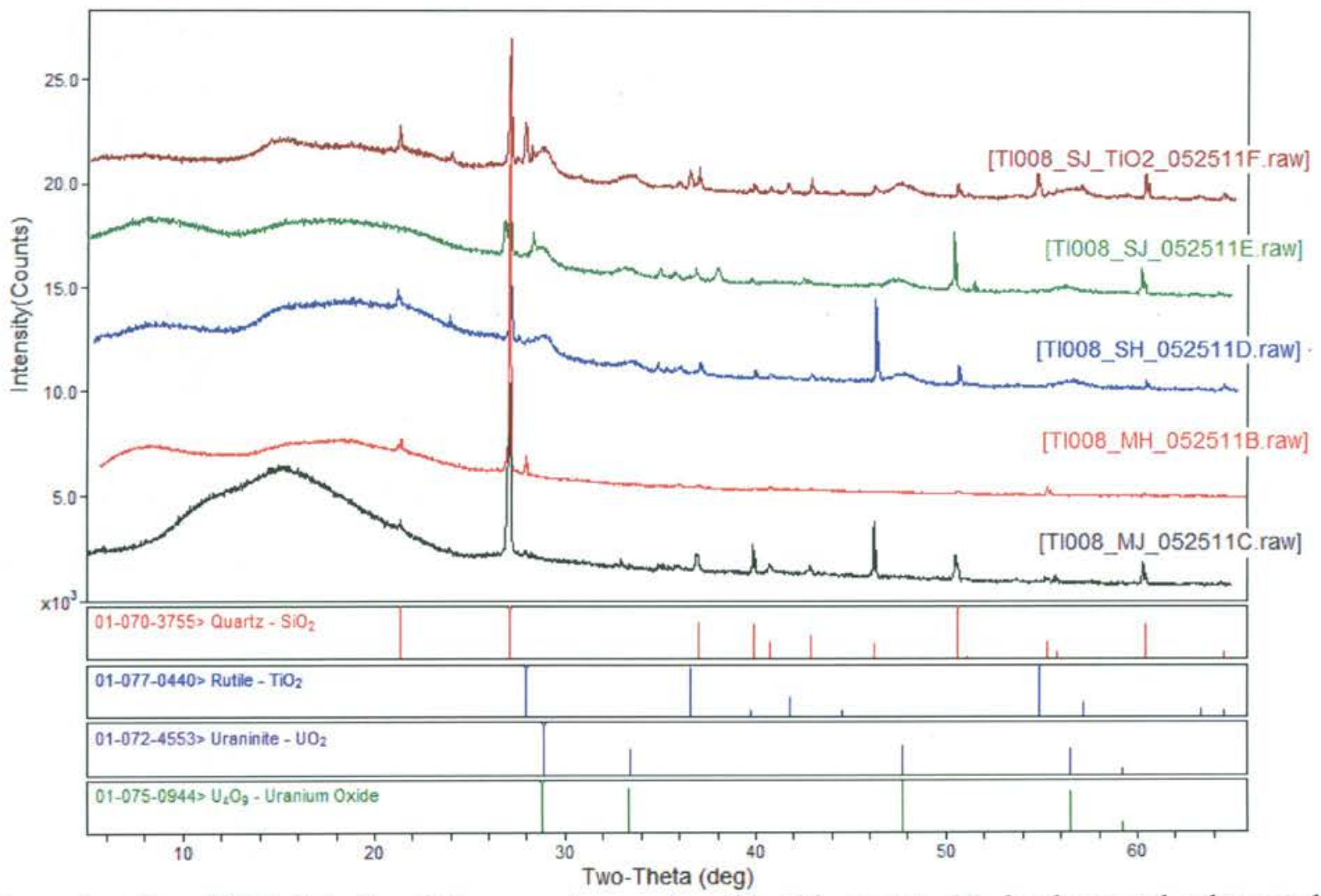

Figure 1. Raw XRD data for all the samples analyzed in this report. No background subtracted. 


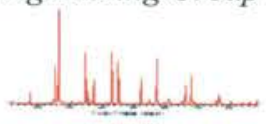

The following table summarizes the figures present in this report.

Table 3. Summary of Figures

\begin{tabular}{|l|l|c|}
\hline Client Sample ID & Sample Description & Figures \\
\hline TI008-MH & KW210 $(500-2000 \mu \mathrm{m})$ & 2 \\
\hline TI008-MJ & KW210 $(500-2000 \mu \mathrm{m})$ & 3 \\
\hline TI008-SH & KW210 $(<500 \mu \mathrm{m})$ & 4 \\
\hline & KW210 $(<500 \mu \mathrm{m})$ & 5,6 \\
\hline
\end{tabular}

\section{XRD Sample Analysis}

\subsection{XRD Samples TI008-MH}

Figure 2 presents the raw XRD data for sample TI008-MH along with the ICDD cards of the crystalline phases identified based on the Rietveld refinement. This sample is composed of silica (quartz). There is an unidentified peak at $27.3^{\circ} 2$ theta and a broad peak from $5^{\circ}$ to $12^{\circ} 2$-theta. The broad peak may be attributable to resin beads which could be present as an amorphous lowangle hump. The amorphous hump contributes to the high $\mathrm{R}_{\mathrm{w}}=13 \%$ seen in the Rietveld refinement presented in Figure 2.

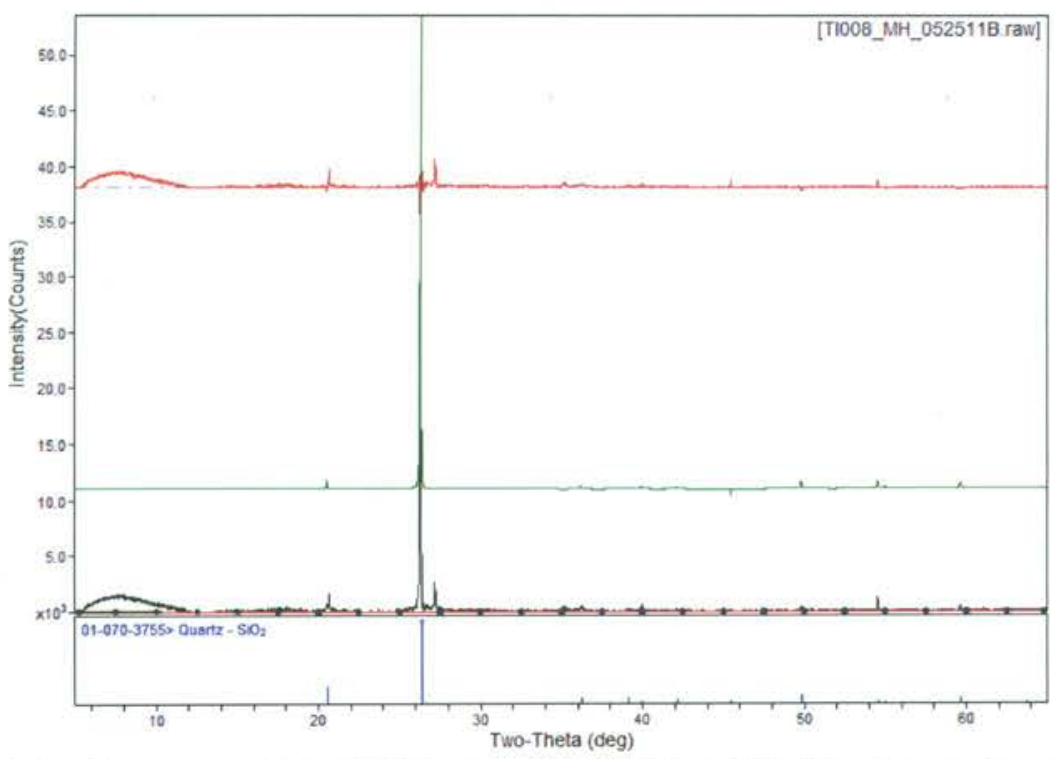

Figure 2. Rietveld refinement of the XRD data for TI008-MH. The black line is the observed powder pattern, the green line is the simulated powder pattern based on the model, and the red line is the difference between the calculated and the observed powder patterns. $R_{w}=13 \%$ (See Section 3.2). Background subtracted spectra shown. 
PNNL-20650, Rev. 2

\subsection{XRD Sample TI008-MJ}

Figure 3 presents the raw XRD data for samples TI008-MJ along with the ICDD card of the crystalline phase identified based on the Rietveld refinement $\left(\mathrm{R}_{\mathrm{w}}=5 \%\right)$, silica (quartz). In addition the peaks at $33^{\circ}$ and a small broad peak between $35^{\circ}-36^{\circ} 2$-theta remain unidentified.

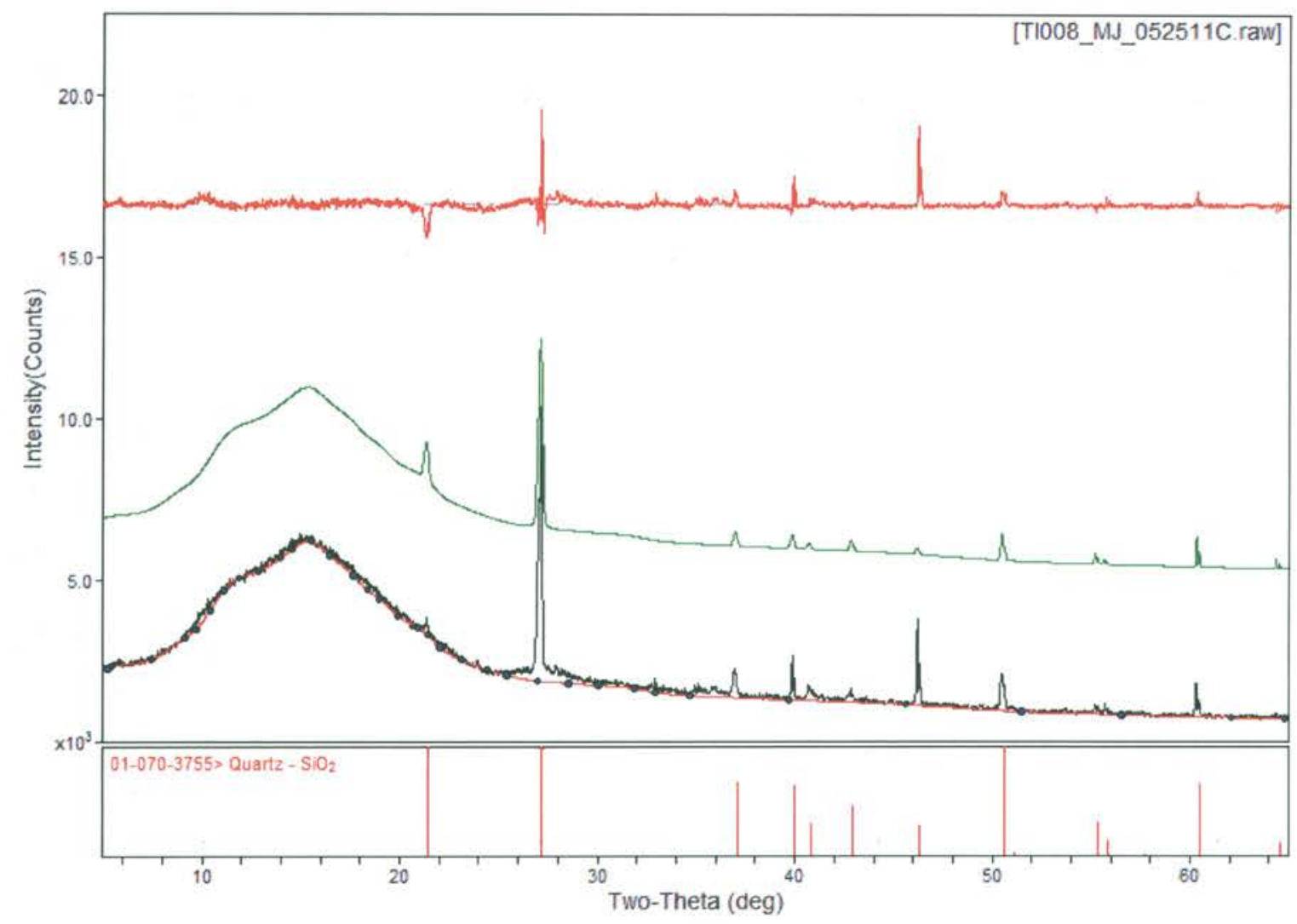

Figure 3. Rietveld refinement of the XRD data for TI008-MJ. The black line is the observed powder pattern, the green line is the simulated powder pattern based on the model, and the red line is the difference between the calculated and the observed powder patterns. $R_{w}=5 \%$ (See Section 3.2). Background subtracted spectra shown. 
PNNL-20650, Rev. 2

\subsection{XRD Sample TI008-SH}

Figure 4 presents the Rietveld refinement $\left(\mathrm{R}_{\mathrm{w}}=5 \%\right)$ of the raw XRD data for sample TI008-SH with the ICDD cards of the crystalline phases identified in this material. Based on this analysis the sample contains silica (quartz) and uranium oxides $\left(\mathrm{UO}_{2}\right.$ and $\left.\mathrm{U}_{4} \mathrm{O}_{9}\right)$ as major phases. In addition the peaks at $23.6^{\circ}, 34.5^{\circ}$ and $35.6^{\circ}$ 2-theta, remain unidentified. The relatively big difference between the calculated and the observed diffraction patterns is due to preferred orientation of the quartz crystals.

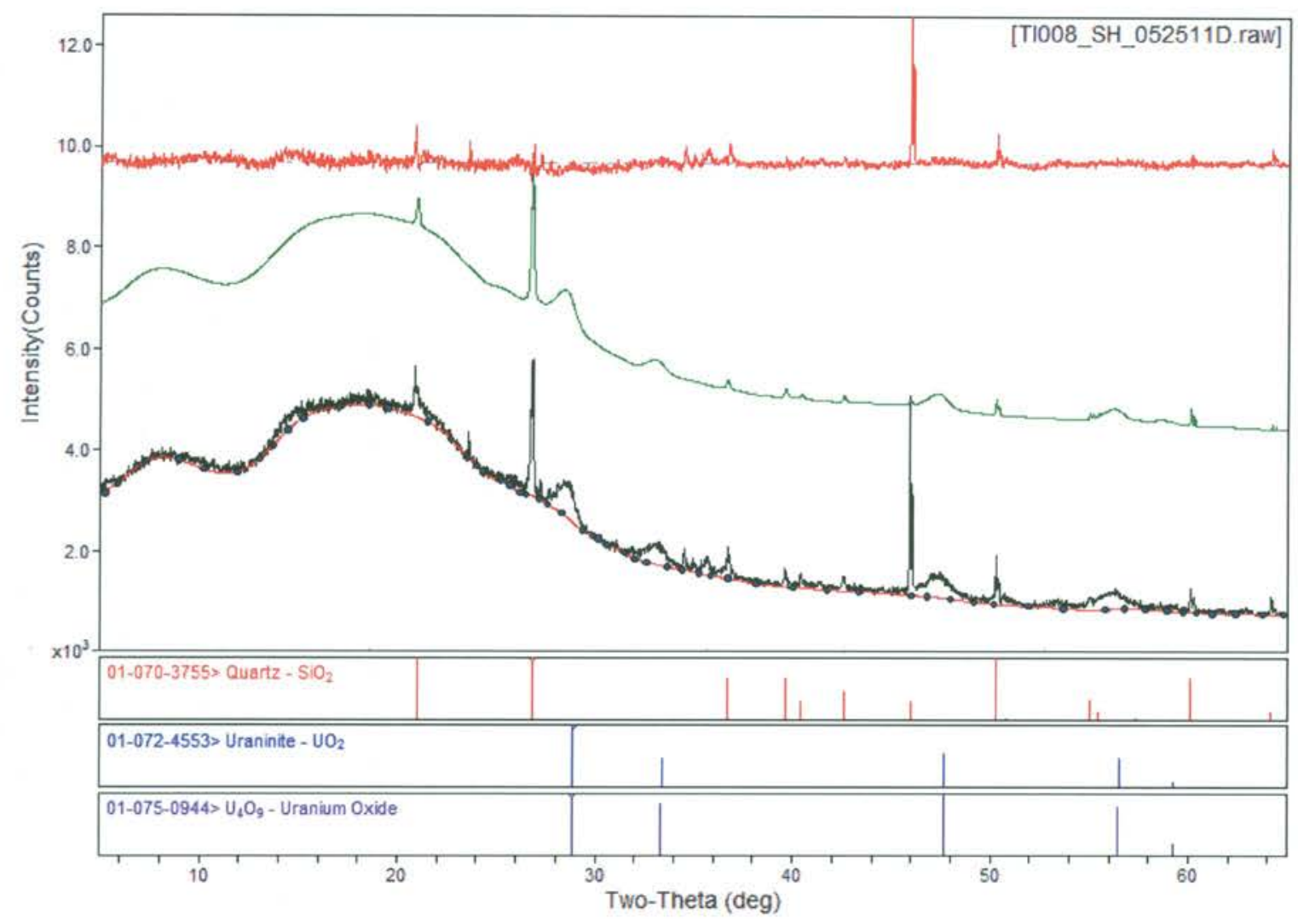

Figure 4. Rietveld refinement of the XRD data for TI008-SH. The black line is the observed powder pattern, the green line is the simulated powder pattern based on the model, and the red line is the difference between the calculated and the observed powder patterns. $R_{w}=5 \%$ (See Section 3.2). No background subtracted spectra shown. 


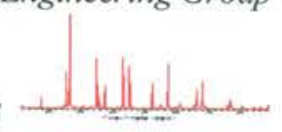

\subsection{XRD Samples TI008-SJ and TI008-SJ-TiO}

Figure 5 presents the Rietveld refinement $\left(\mathrm{R}_{\mathrm{w}}=8 \%\right)$ of the XRD data for sample TI008-SJ with the ICDD cards of the corresponding crystalline phases. Figure 6 presents the Rietveld refinement $\left(\mathrm{R}_{\mathrm{w}}=5 \%\right)$ data for TI008-SJ- $\mathrm{TiO}_{2}$ with the ICDD cards of the corresponding crystalline phases. The major phases present in these samples are silica (quartz) and uranium oxides $\left(\mathrm{UO}_{2}\right.$ and $\left.\mathrm{U}_{4} \mathrm{O}_{9}\right)$. In addition sample TI008-SJ-TiO 2 contains $\mathrm{TiO}_{2}$ (rutile) that was added as an internal standard. Rutile internal standard peaks, SRM 674, are consistent with the ones reported in the ICDD 01-077-0440, which means that the peak positions in the analyzed samples are reliable. In addition sample TI008-SJ has unidentified peaks at $24,35^{\circ}, 35.8^{\circ}, 37^{\circ}, 38^{\circ}$ and $51.5^{\circ} 2$-theta. Although not as intense, these unidentified peaks are also present in TI008-SJ$\mathrm{TiO}_{2}$.

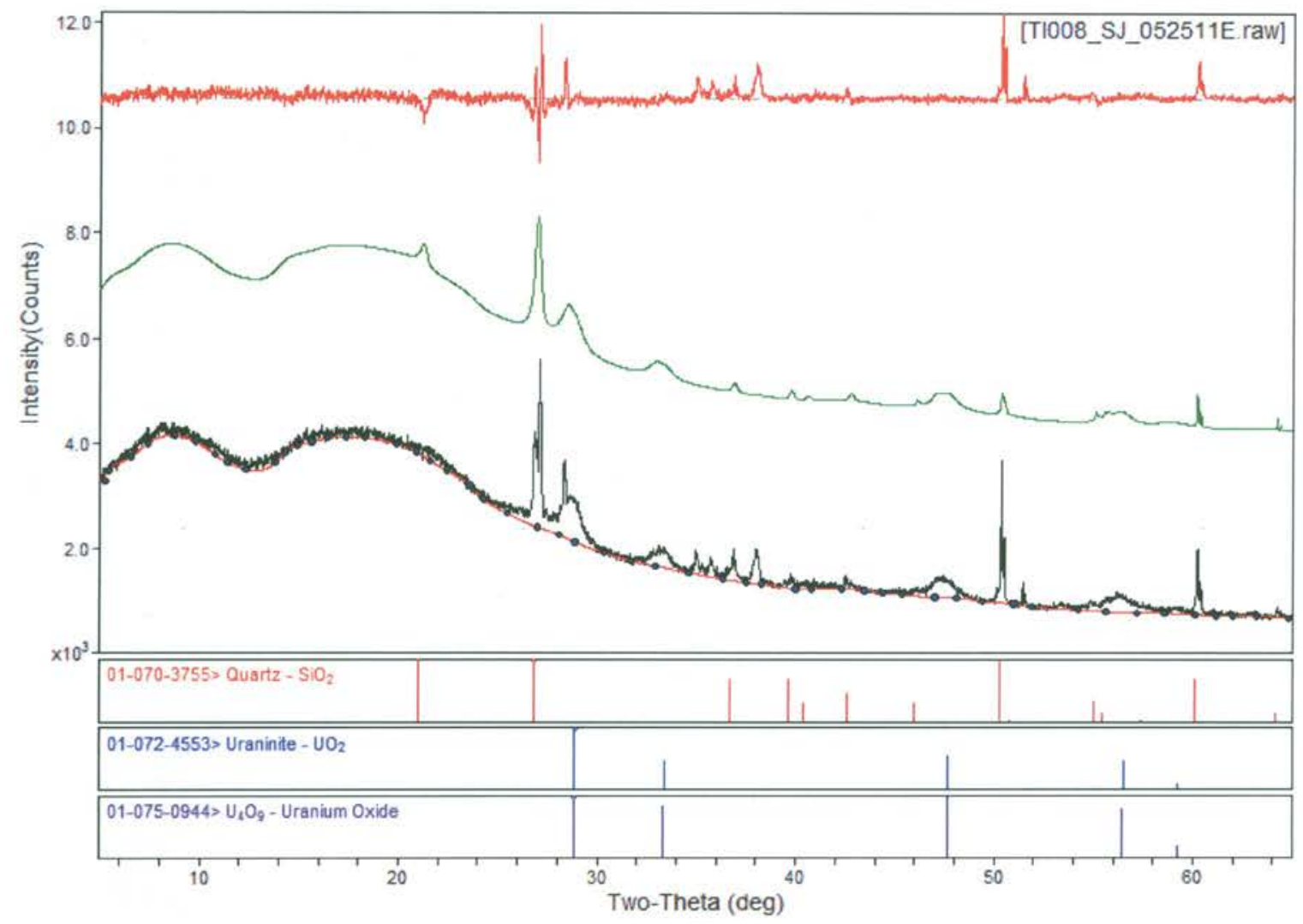

Figure 5. Rietveld refinement of the XRD data for TI008-SJ. The black line is the observed powder pattern, the green line is the simulated powder pattern based on the model, and the red line is the difference between the calculated and the observed powder patterns. $R_{w}=8 \%$ (See Section 3.2). No background subtracted spectra shown. 
PNNL-20650, Rev. 2
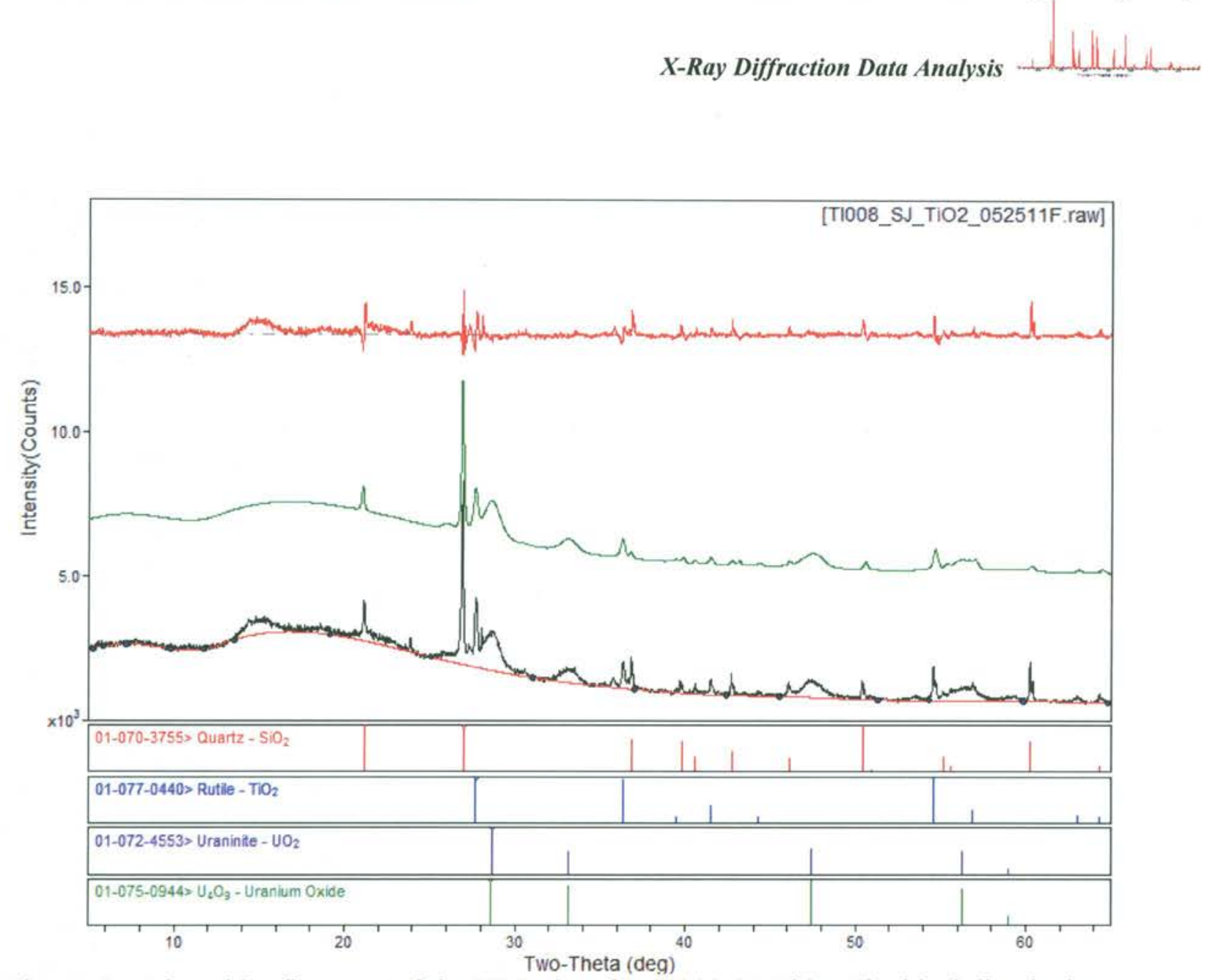

Figure 6. Rietveld refinement of the XRD data for TI008-SJ-TiO ${ }_{2}$. The black line is the observed powder pattern, the green line is the simulated powder pattern based on the model, and the red line is the difference between the calculated and the observed powder patterns. $R_{w}=5 \%$ (See Section 3.2 ). No background subtracted spectra shown. 
PNNL-20650, Rev. 2

Appendix 1

SRM 674 NIST Certificate

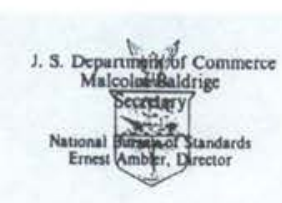

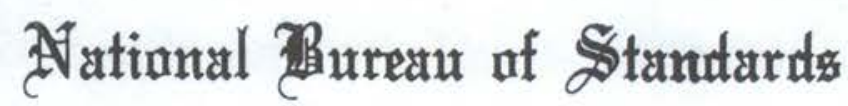

\author{
(Tertifitiate
}

\section{Standard Reference Material 674}

\author{
X-Ray Powder Diffraction \\ Intensity Set
}

\section{R. Hubbard}

This Standard Reference Material (SRM) consists of five different phases. The phases, separately bottled, are $\alpha-\mathrm{Al}_{2} \mathrm{O}_{3}$ (corundum structure), $\mathrm{ZnO}$ (wurtzite structure), $\mathrm{TiO}_{2}$ (rutile structure), $\mathrm{Cr}_{2} \mathrm{O}_{3}$ (corundum structure), and $\mathrm{CeO}_{2}$ (fluorite structure). These phases can be used as internal standards for quantitative analysis and as external standards for checking the intensity response of $\mathrm{x}$-ray diffraction instruments. The five phases cover the range of linear absorption coefficient from 100 to $1000 \mathrm{~cm}^{-1}$ for $\mathrm{CuK} \alpha$ radiation. The intensity values are for a constant diffracting volume (fixed divergent slit and infinitely thick mounting or equivalent).

The certified relative intensity values ( $\mathrm{CuK} \alpha$ radiation), for the major diffraction lines below $70^{\circ} 2(\theta)$, for each phase are given in Table $\mathrm{l}$. The certified referance intensitv ratios $(\mathrm{I} / \mathrm{Ic})$ for $\mathrm{ZnO}, \mathrm{TiO}_{2}, \mathrm{Cr}_{2} \mathrm{O}_{3}$, and $\mathrm{CeO}_{2}$, relative to $\alpha-\mathrm{Al}_{2} \mathrm{O}_{3}$ and the lattice parameters are given in Tables 2 and 3 . The uncertainties of the relative intensity and lattice parameter values are the standard deviations derived from multiple measurements. The uncertainties of the reference intensity ratio values were determined from the value of the internal inconsistency derived from all possible hinary mixtures

The average crystallite size for each of the five phases is near $2.0 \mu \mathrm{m}$. Crystallite size broadening of the diffraction peaks is small but present. This is particularly apparent for $\alpha-\mathrm{Al}_{2} \mathrm{O}_{3}$, which has a nominal crystallite size less than $1.0 \mu \mathrm{m}$. The lattice parameters, uncorrected for refraction, were determined with a relative uncertainty of about three parts in $10^{5}$ using an internal standard. This uncertainty in the lattice parameter must be increased by a factor of two or three if one wishes to determine absolute d-spacings as the uncertainties due to the internal standard $26{ }^{\circ} \mathrm{s}$ and to thermal expansion would need to be added.

The technical and support aspects concerning the preparation, certification, and issuance of this Standard Reference Material were coordinated through the Office of Standard Reference Materials by R.K. Kirby.

Washington, D.C. 20234

June I. 1983 (over)
Stanley D. Rasberry, Chief

Office of Standard Reference Materials 
PNNL-20650, Rev. 2

Table 1. Relative Intensitites of Major Lines

CuK $\alpha$ Radiation $\left(\mu=124.1 \mathrm{~cm}^{-1}\right)$

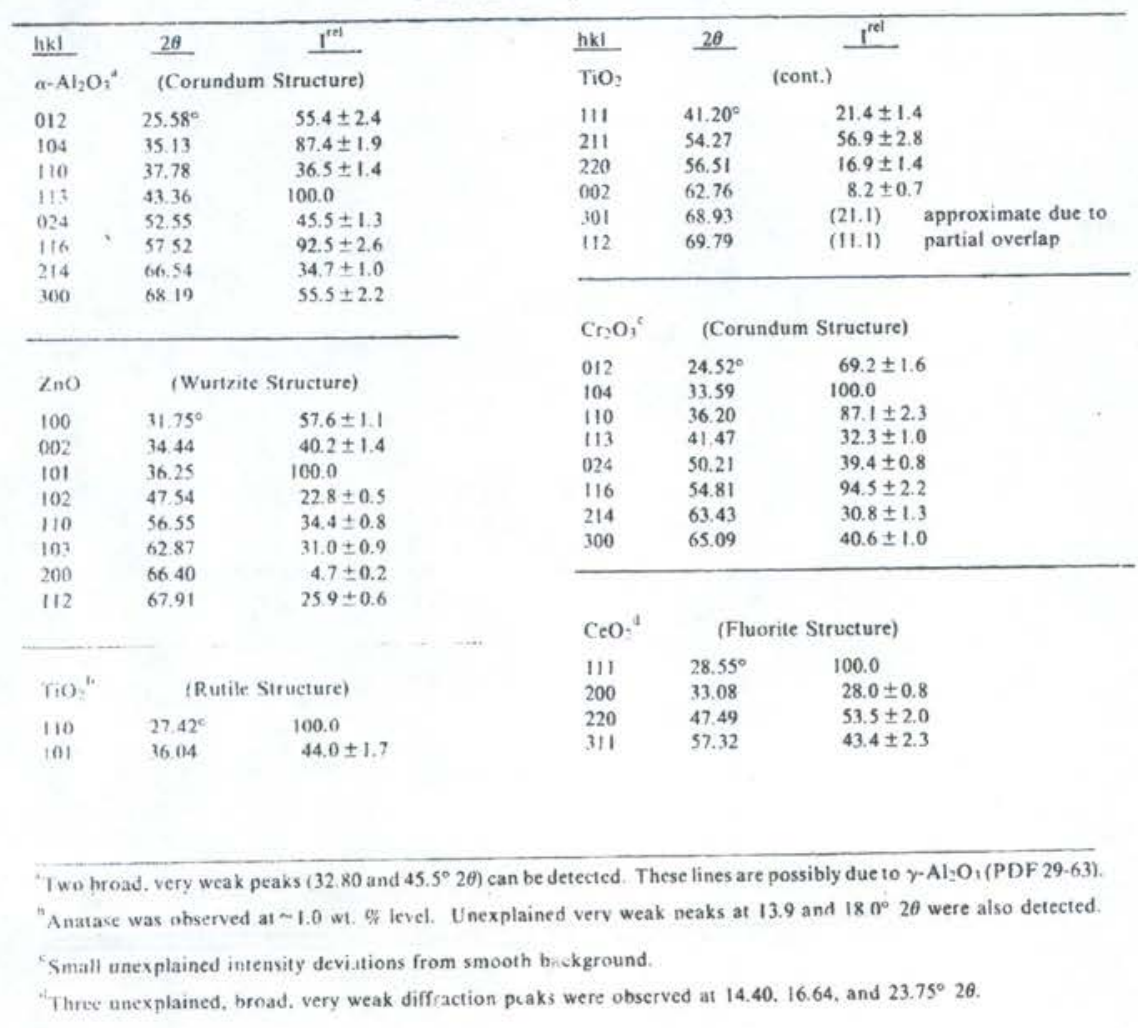




\section{Appendix 2 Daily Performance Checks}

The daily performance check shows that the Rigaku Ultima IV diffractometer is within calibration per PNNL-RPG-268 Rev. 4, "Solids Analysis by X-Ray Diffraction" so no adjustments have been made. Figure 7 presents the XRD data obtained on the silicon SRM 640a standard. Table 4 contains summarized results of the daily performance check with this standard. The columns in Table 4 have the following significance:

1) $2 \mathrm{~T}(\mathrm{r})$ gives the 2-theta of the reference powder pattern (ICDD card),

2) $2 \mathrm{~T}(\mathrm{o})$ is the observed 2 -theta,

3) Delta is the difference between the observed and the reference 2-theta,

4) $d(r)$ is the interplanar distance $d$ for the reference pattern (in $\AA$ ),

5) d(o) is the observed d,

6) Del-d is the difference between the observed and the reference d,

7) I \% is the intensity in percent (the most intense peak is always $100 \%$ and all other peaks are normalized).

Per procedure the measured 2-theta of the performance check standard (i.e., the $111 \mathrm{Si}$ reference line) must be $28.44^{\circ} \pm 0.06^{\circ}$ 2-theta; the observed 2-theta difference was 0.012 and met this requirement. Thus the performance check conducted on May 25, 2011 met this criterion.

\section{Daily calibration report (27 to 29 2-theta) - Date 05-25-2011}

Theta Calibration Report

[052511a_si.raw] si 640a - Full Chart

Reference Lines $=01-073-6978>$ Silicon $-\mathrm{Si}$

Two-Theta Range of Fit $=27.0 / 29.0(\mathrm{deg})$

Zero Offset $=-0.012(\mathrm{deg})$

ESD of Fit $=0.0^{\circ}, \mid$ Delta $2-$ Theta $\mid=0.012(\mathrm{deg})$

Table 4. Calibration Report Summary

\begin{tabular}{|l|l|l|l|l|l|l|}
\hline $\mathbf{2 T}(\mathbf{r})$ & $\mathbf{2 T}(\mathbf{0})$ & Delta & $\mathbf{d}(\mathbf{r})$ & $\mathbf{d}(\mathbf{0})$ & Del-d & $\mathbf{I} \%$ \\
\hline 28.447 & 28.459 & 0.012 & 3.1350 & 3.1337 & 0.0013 & 100.0 \\
\hline
\end{tabular}


PNNL-20650, Rev. 2
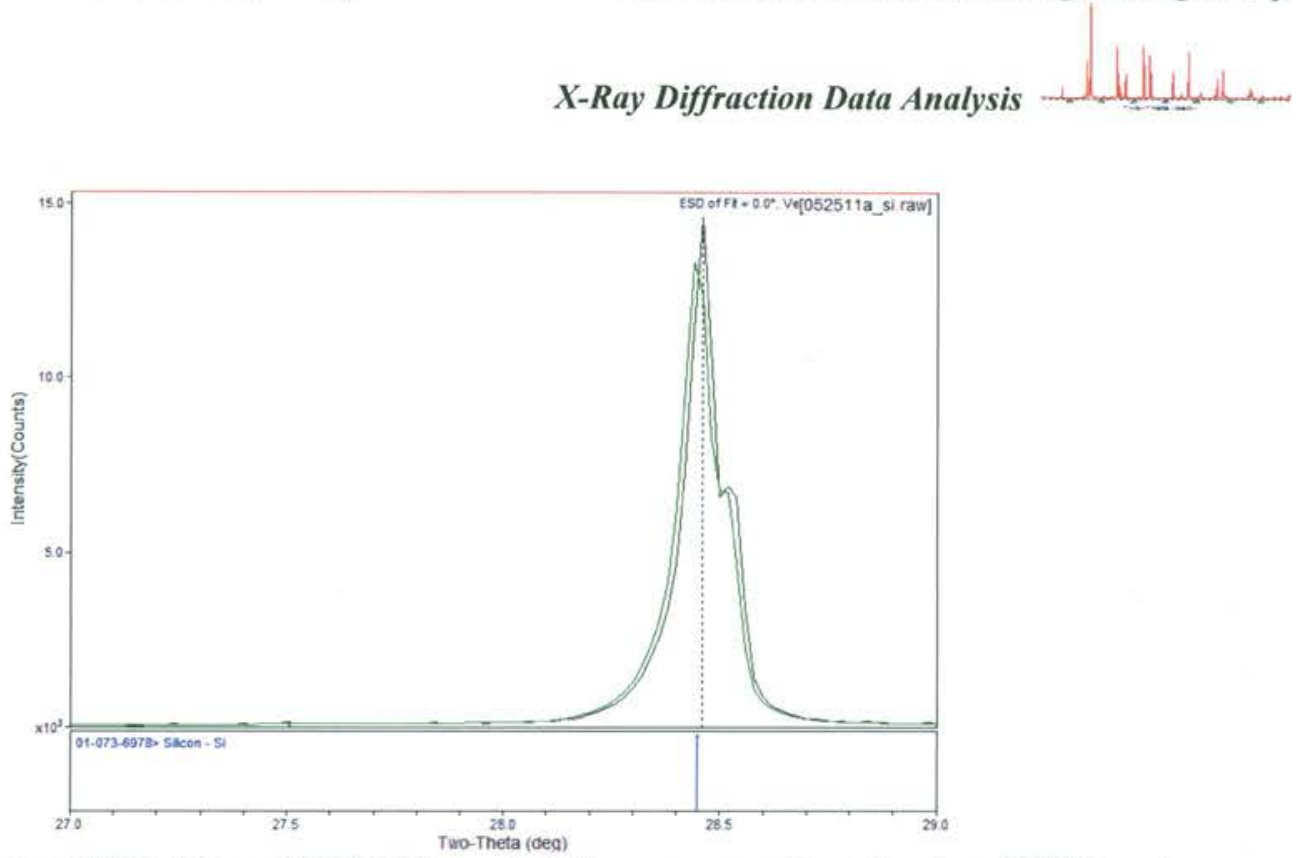

Figure 7. The raw XRD data on SRM 640a against the corresponding structure ICDD card. 
PNNL-20650, Rev. 2

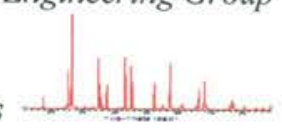

\author{
Appendix 3 \\ SRM 640a NIST Certificate
}

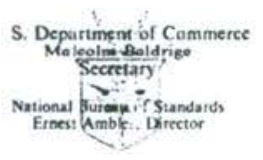

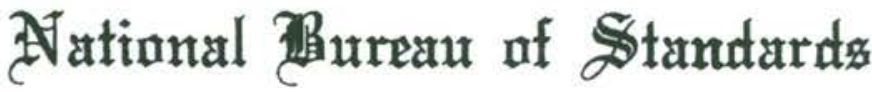 \\ Uertifitate
}

\author{
Standard Reference Material 640a \\ Silicon Powder $2 \theta / d-S p a c i n g$ Standard \\ for X-Ray Diffraction
}

\begin{abstract}
Camden R. Hubbard
This Standand Refocnec Matcrial (SRM) was prepared for usc as an external on intcinal 20/N-spaciug calibuatiun standard for powder diffractometry.

Electronic grade float-zone prepared silicon boules, $99.999+\%$ pure, were ground to pass a $75 \mu \mathrm{m}$ (200 mesh) sieve. This powder, which was identical to SRM 640, was then jet-milled to reduce the mean particle size to about $2 \mu \mathrm{m}$.

A total of twelve samples, mixed with tungsten and silver internal standards [1]. were measured using a high angle goniometer controlled by a minicomr uter. The $\mathrm{CuK} \alpha_{1}$ peak position was determined by profile fitting procedures and then corrected for sample. instrumental, and physical aberrations (except refraction) through use of the internal standard lines [2]. Silicon peak positions were then corrected for effects of thermal expansion. Each corrected set was refined by a least-square routine that minimized $\Sigma\left(\theta_{\text {obs }}-\theta_{\text {calc }}\right)^{2}$ to obtain estimates of $a_{i}$ and their estimated standard errors, $\mathrm{s}$. . The weighted average of the twelve lattice parameters, uncorrected for refraction, at $25^{\circ} \mathrm{C}$ is
\end{abstract}

$$
\text { (a) }=5.430825 \pm 0.000036 \AA
$$

where $\lambda\left(\mathrm{CuK} \alpha_{1}\right)=1.5405981 \AA$ [3]. The estimated total uncertainty given above includes contributions from three sources (listed in decreasing importance): (1) uncertainty of the lattice parameters of the tungsten and silver standards; (2) random errors of the measurements; and (3) the uncertainty in $\lambda\left(\mathrm{CuK} \alpha_{1}\right)$.

The technical and support aspects concerning the preparation, certification, and issuance of this Standard Reference Material were coordinated through the Office of Standard Reference Materials by R.K. Kirby.

Washington. D.C. 20234

February 19. 1982
George A. Uriano, Chief Office of Standard Reference Materials

(over) 
PNNL-20650, Rev. 2

The $2 \theta$ values given in Table I were calculated from the certified value of the lattice parameter. The relative intensities [4], I (rel), also given in Table I can be used as an aid in identifying the silicon lines when SRM 640a is mixed with test materials. The uncertainty of each value of 1 (rel) may be as large as \pm 3 . Suggested methods for use of this SRM are given in references [2], [5], and [6].

Tabie 1

Calculated Diffraction Angles and Relative Intensities

$$
\left(\mathrm{T}=25.0^{\circ} \mathrm{C}\right)
$$

These Values Are Not Certified

\begin{tabular}{|c|c|c|c|c|c|}
\hline hkl & 1 (rel) & 20peak & hkl & I (rel) & 20 peak \\
\hline 111 & 100 & $28.443^{\circ}$ & $511 / 333$ & 6 & $94.955^{\circ}$ \\
\hline 220 & 55 & 47.304 & 440 & 3 & 106.712 \\
\hline 311 & 30 & 56.124 & 531 & 7 & 114.096 \\
\hline 400 & 6 & 69.132 & 620 & 8 & 127.550 \\
\hline 331 & 11 & 76.378 & 533 & 3 & 136.900 \\
\hline 422 & 12 & 88.033 & 444 & * & 158.644 \\
\hline
\end{tabular}

*Not measured

[1] Swanson, H.E.; McMurdie, H.F; Morris, M.C.; and Evans, E.H. (1966), Standard X-Ray Diffraction Powder Patterns, National Bureau of Standards Monograph 25. Section 4, NBS, Washington. D.C. 20234.

[2] Hubbard, C.R. (1982), submitted for publication in J. Appl. Cryst.

[3] Deslattes. R.D. and Henins. A. (1973), Phys. Rev. Letters, 31, 972-975.

[4] Morris, M.C.; McMurdic, H.F.; Evans, E.H.; Paretzkin, B.; deGroot, J.H.; Hubbard, C.R : and Carmel, S.J. (1976) Standard X-Ray Diffraction Powder Patterns. National Bureau of Standards Monograph 25, Section 13, NBS. Washington. D.C. 20234.

[5] Snyder. R.L.; Hubbard. C.R.; and Panagiotopoulos, N.C., Advances in X-Ray Analysis, Vol. 25, (in press).

[6] Hubbard. C.R. (1980) Accuracy in Powder Diffraction, NBS Special Publication 567, p 489-502. 
PNNL-20650, Rev. 2

Battelle - Pacific Northwest National Laboratory

Radiochemical Sciences and Engineering Group

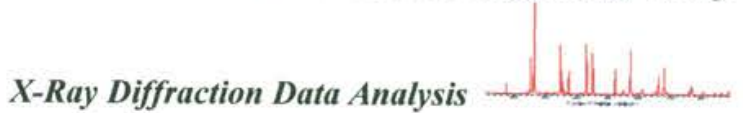

\section{Appendix 4 \\ Quality Control/Calibration}

This control calibration measurement shows that the Rigaku Ultima IV diffractometer is within calibration per PNNL-RPG-268 Rev. 4, "Solids Analysis by X-Ray Diffraction" so no

adjustments have been made. The XRD data obtained on this standard are represented in Figure 8. Table 5 contains summarized results of the quality control/calibration results obtained using NIST SRM 1976 (Corundum).

The columns in Table 5 have the following significance:

1) $2 \mathrm{~T}(\mathrm{r})$ gives the 2 -theta of the reference powder pattern (ICDD card),

2) $2 \mathrm{~T}(\mathrm{o})$ is the observed 2-theta,

3) Delta is the difference between the observed and the reference 2-theta,

4) d(r) is the interplanar distance $d$ for the reference pattern (in $\AA$ ),

5) $d(o)$ is the observed $d$,

6) Del-d is the difference between the observed and the reference d,

7) $\mathrm{I} \%$ is the intensity in percent (the most intense peak is always $100 \%$ and all other peaks are normalized).

The requirement for this quality check (semiannual calibration verification of the diffractometer as stated in the PNNL-RPG-268 Rev. 4, is that observed d-interplanar distance for any given crystal lattice Miller indices be within $\pm 0.02 \AA$ of the NIST SRM 1976 ICDD card d-values. For this quality performance check, all Del-d values are well within this criterion. The ICDD card for alumina (corundum structure) is provided in Appendix 6.

\section{Control Calibration Report (10 to 602 theta) - Date 01-06-2011}

Theta Calibration Report

[nist_1976_dtex_010611d.raw] corundum - Full Chart

Reference Lines $=01-071-6478>$ Corundum $-\mathrm{Al} 2 \mathrm{O} 3$

Two-Theta Range of Fit $=5.0 / 65.0(\mathrm{deg})$

Zero Offset $=0.032(\mathrm{deg})$

Table 5. Summary of the semiannual calibration report

\begin{tabular}{|r|r|r|r|r|r|r|}
\hline \multicolumn{1}{l|}{ 2T(r) } & \multicolumn{1}{l|}{ 2T(o) } & \multicolumn{1}{l|}{ Delta } & d(r) & \multicolumn{1}{l|}{$\mathrm{d}(\mathrm{o})$} & \multicolumn{1}{l|}{ Del-d } & \multicolumn{1}{c|}{ \% } \\
\hline 25.573 & 25.541 & 0.032 & 3.4805 & 3.4848 & 0.0043 & 32.7 \\
\hline 35.147 & 35.116 & 0.031 & 2.5512 & 2.5534 & 0.0022 & 100 \\
\hline 37.77 & 37.739 & 0.03 & 2.3799 & 2.3817 & 0.0018 & 13 \\
\hline 41.676 & 41.639 & 0.037 & 2.1654 & 2.1673 & 0.0019 & 3 \\
\hline 43.347 & 43.317 & 0.03 & 2.0857 & 2.0871 & 0.0014 & 49.6 \\
\hline 46.17 & 46.14 & 0.031 & 1.9646 & 1.9658 & 0.0012 & 0.5 \\
\hline 52.544 & 52.515 & 0.029 & 1.7403 & 1.7412 & 0.0009 & 24.2 \\
\hline 57.494 & 57.46 & 0.034 & 1.6016 & 1.6025 & 0.0009 & 80.4 \\
\hline 59.729 & 59.7 & 0.029 & 1.5469 & 1.5476 & 0.0007 & 0.8 \\
\hline 61.118 & 61.099 & 0.019 & 1.5151 & 1.5155 & 0.0004 & 1.4 \\
\hline 61.299 & 61.261 & 0.039 & 1.511 & 1.5119 & 0.0009 & 14 \\
\hline
\end{tabular}


PNNL-20650, Rev. 2

Battelle - Pacific Northwest National Laboratory
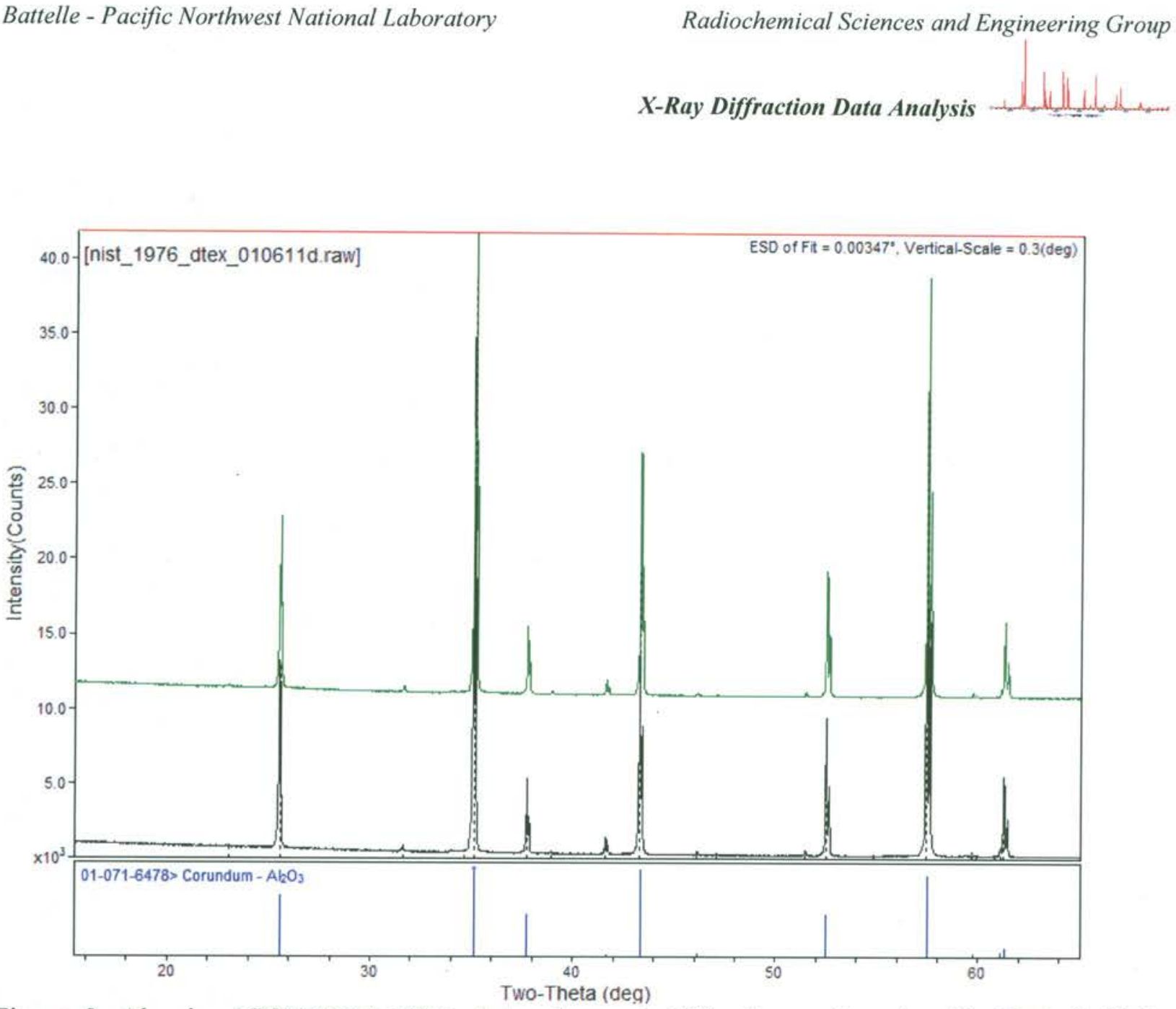

Figure 8. Alumina NIST SRM 1976 plate, observed diffraction pattern is with black (middle image) the simulated reference diffraction pattern is with green (top image) and the ICDD card is with blue (bottom image). 
PNNL-20650, Rev. 2

Battelle - Pacific Northwest National Laboratory

Radiochemical Sciences and Engineering Group

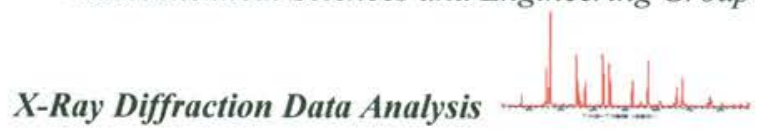

\author{
Appendix 5 \\ SRM 1976 NIST Certificate
}

i

\title{
Eirational Institute of Standards \& Uprhnology Tertifitrate of Analustis
}

Standard Reference Material 1976

\author{
Instrument Sensitivity Standard for X-ray Powder Diffraction
}

\begin{abstract}
This Standard Reference Material (SRM) consists of a sintered alumina plate, approximately $45 \mathrm{~mm}$ on a side by $1.6 \mathrm{~mm}$ in thickness, intended for use in calibration of powder X-ray equipment for diffraction intensity as a function of $2 \theta$ angle (instrument sensitivity). The form of the SRM serves to eliminate the variability of sample loading procedure from intensity measurements. This SRM material was chosen for consistency of microstructure with respect to grain size, shape, micro-strain and orientation. The platelets comprising this microstructure are 3 to $7 \mu \mathrm{m}$ in diameter by 1 to $2 \mu \mathrm{m}$ in thickness and are highly oriented with the basal plane paraliel to the surface of the plate. Some amount of amorphous phase is present though no crystalline impurities could be detected.
\end{abstract}

The proper use of this SRM requires the measurement of intensity values from test equipment in a manner analogous to either of the two measurement methods used for certification. The certified parameters consist of absolute variation in intensity, 12 relative intensity values calculated from integrated intensity and peak height measurements from 25.5 to 145 degrees $2 \Theta$, and the lattice parameters. Graphical evaluation of the ratio of data from the test equipment to the certified values will allow for an appropriate judgment as to the condition of the test equipment. This may be followed by the calculation of a correction curve, yielding standardization of instrument sensitivity and thus more accurate inter-laboratory comparisons of data involving $\mathrm{X}$-ray diffraction intensity measurements.

The overall coordination of the preparation and the technical measurements leading to certification was performed by J.P. Cline of the NIST Ceramics Division. The project was the result of a round robin study carried out by R. Jenkins and W. Schreiner of the International Centre for Diffraction Data, Swarthmore, Pennsylvania.

The alumina substrates for this SRM were donated to NIST by International Business Machines Corporation, East Fishkill, New York, through the efforts of R. Anderson.

Statistical analysis was provided by S.B. Schiller and K.R. Eberhardt of the NIST Statistical Engineering Division.

The technical and support aspects involved in the preparation. certification, and issuance of this Standard Reference Material were coordinated through the Standard Reference Materials Program by N.M. Trahey.

Gaithersburg, MD 20899

November 8,1991
William P. Reed, Chief Standard Reference Materials Program

(over) 
PNNL-20650, Rev. 2

Battelle - Pacific Northwest National Laboratory

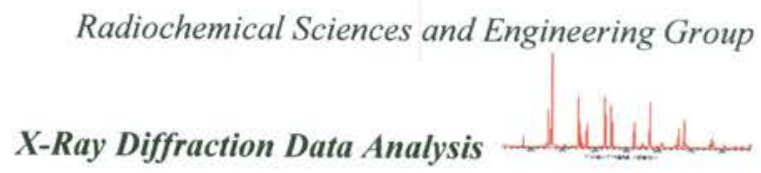

Certified intensity data were collected from 25 randomly selected samples on a Philips' diffractometer using an $\mathrm{AEG}^{\prime}$ long fine-focus $\mathrm{Cu} \mathrm{X}$-ray tube operated at 1800 watts with a constant potential gencrator. The diffractometer was equipped with incident and diffracted beam sollar slits, graphite diffracted beam monochromator in anti-parallel geometry, and scintillation detector. The incident beam slits were of the theta compensating type, locked such that the illumination arca was $12.5 \mathrm{~mm}$ wide at 25 degrees $2 \Theta$. This corresponds to a slit size of 0.93 degrees; the receiving slit was 0.066 degrees. The take-off angle was 4 degrees. Samples were spun at approximately $52 \mathrm{rpm}$ during data collection.

Data were collected and processed with the Siemens Diffrac500' version of NBS ${ }^{*}$ Quant. This algorithim scans specific peaks for an integrated intensity measurement, with extended count times for each of ten points on either side of the scan range. Scan angles are indicated in Table 1; the step width was 0.005 degrees. Scan time ranged from 30 to 90 minutes per peak, for a total of 16 hours per sample. The seventh and eleventh scan ranges actually include two peaks, as noted by the two sets of miller indices listed for them in Table 1. The use of two peaks in these scan ranges ensured that intensity data were reported evenly through the desired $2 \Theta$ range.

Peak height values were manually determined, with a background subtraction, from the same scans as were the integrated intensity data. Five background data points from each end of the scan range were averaged to determine the background value. Peak height values were determined from an average of the three most intense points for data below 55 degrees $2 \Theta$ : four data points were used for the remainder of the data. Due to the proximity of the two reflections used in the seventh and eleventh relative intensity values, peak height values for these data reflect the superposition of the $\mathrm{Cu} \mathrm{K} \alpha_{i}$ and $\mathrm{K} \alpha_{2}$ : peaks. Thus, peak height data for these two pairs of reflections will be valid only with the use of $\mathrm{Cu} \mathrm{K \alpha}$ radiation.

Lattice parameters were determined from the 25 samples by means of leasi squares refinement from 47 peak positions determined with the spline method, ranging from 22 to 152 degrees $2 \Theta$. Peak positions were corrected for optical aberrations with the use of SRM $640 \mathrm{~b}$ silicon powder, to generate a 2 nd order polynomial external standard correction curve which was applied to peak location determinations. Measurements were made on a Siemens D500' diffractometer equipped with a focusing Ge incident beam monochromator, sample spinner, and a position sensitive proportional detector. Copper Ka radiation was used. Measurements were made within a period of 48 hours during which operating conditions were maintained. The machine was stabilized under such conditions for several days prior to measurements.

The intensity measurements from the SRM material are expected to show heterogeneity due to variations in preferred orientation. The heterogeneous nature of the samples requires a certification of a population of true relative intensities rather than the average relative intensity for each peak. The preferred orientation will uniformly effect the variability of all the peaks so analysis of the data assumed the intensity measurements have the same true relative variance. This allowed for pooling of the variances for both absolute and relative intensities. The exception to this assumption is the $(300)$ peak; the crystallites in the diffracting position for this refection must be perpendicular to the preferred orientation direction. Increased variability of intensity from this reflection is thus expected. The nominal value of the relative intensity is reported for information only; it was not used in subsequent analysis.

'Certain manufacturers are mentioned for informative purposes only and do not represent an endorsement by NIST. 
PNNL-20650, Rev. 2

Battelle - Pacific Northwest National Laboratory

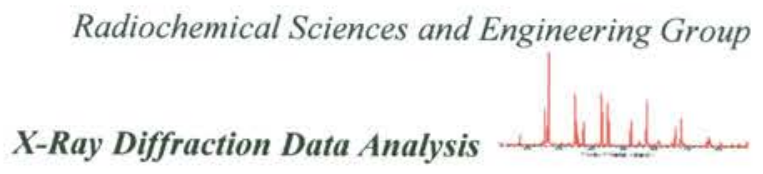

\section{ABSOLUTE VARIATION IN INTENSITY}

Analysis of the raw integrated intensity values indicated a drift in the instrument. This drift was modeled with a quadratic equation and removed from subsequent anainses. The relative standard deviation of the raw counts, pooled from the 13 peaks is:

$$
\operatorname{RSD}(\text { raw counts })=2.21 \%
$$

This value incorporates both material variability and machine imprecision. This parameter can be used to determine differences in absolute sensitivity with different SRM samples.

\section{RELATIVE INTENSITY VARIATION}

The relative intensity values displayed no drift. The pooled relative standard deviation from integrated intensity measurements of the 12 peaks is:

$$
\text { RSD (integrated area, relative intensity) }=2.06 \%
$$

The pooled relative standard deviation of the relative intensity values determined from peak height measurements is:

$$
\text { RSD (peak height, relative intensity) }=2.62 \%
$$

The total relative uncertainty is a multiple of the relative standard deviation. For each relative intensity, this value is:

$$
\begin{array}{ll}
\text { Total Uncertainty (integrated intensity) } & =6.12 \% \\
\text { Total Uncertainty (peak height) } & =7.85 \%
\end{array}
$$

This uncertainty is the half-width of a $90 \%$ confidence, $95 \%$ coverage tolerance interval. With $90 \%$ confidence, $95 \%$ of the true relative intensities for all of the peaks of this SRM should fall within the uncertainty of the certified values.

Each SRM plate is sintered to a relatively low density allowing it to be scored and broken easily into a desired size. Care should be taken to ensure that the side opposite the label is illuminated; otherwise, results are invalid. Each plate is cylindrically symmetrical, thus orientation of the plate is not of concern. However, errors due to particle counting statistics will be reduced if a sample spinner is used. Consistency in the degree of size and micro-strain induced peak broadening of the SRM allow for the use of peak heights as a substitution for integrated intensity measurements, though precision is seen to suffer as a result. The ratio of experimental data to the certified values should be plotted as a function of $2 \Theta$ angle. If this data is patternless, and if all of the ratios fall within the total uncertainty $(0.0612,0.0785)$ of 1 , then the test equipment is in control. However, if there is a clear pattern in the data, or if the ratio for at least one peak is more than $0.0612 / 0.0785$ from 1, then the system is out of control (sec Appendix). 
PNNL-20650, Rev. 2

Battelle - Pacific Northwest National Laboratory

Refiection(s) (h
$(012)$
$(104)$
$(113)$
$(024)$
$(116)$
$(300)$
$(1.0 .10)(119)$
$(0.2 .10)$
$(226)$
$(2.1 .10)$
$(324)(0.1 .14)$
$(1.3 .10)$
$(146)$
$(4.0 .10)$

\begin{tabular}{|c|c|}
\hline \multicolumn{2}{|c|}{$\begin{array}{c}\text { Scan } \\
\text { Angles (low:high) } 2 \Theta\end{array}$} \\
\hline - $\quad 24.7$ & 26.2 \\
\hline - 34.0 & 36.2 \\
\hline - 42.4 & 44.2 \\
\hline - 51.8 & 53.3 \\
\hline - 56.0 & 59.0 \\
\hline s $\quad 67.4$ & 69.0 \\
\hline 75.7 & 78.2 \\
\hline - 88.1 & 89.7 \\
\hline $9: 3$ & 96.0 \\
\hline 100.1 & 102.0 \\
\hline 115.4 & 117.4 \\
\hline 126.8 & 128.95 \\
\hline 135.2 & 137.4 \\
\hline 144.3 & 146.7 \\
\hline
\end{tabular}

\section{LATTICE PARAMETERS}

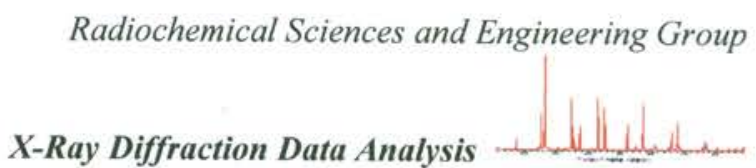

\begin{tabular}{cc}
\multicolumn{2}{c}{ Relative Intensitv } \\
\hline Integrated Area & Peak Height \\
\cline { 2 - 2 } 32.34 & 33.31 \\
100.0 & 100.0 \\
51.06 & 49.57 \\
26.69 & 25.17 \\
92.13 & 83.6 \\
19.13 & 16.89 \\
55.57 & 34.61 \\
11.76 & 8.99 \\
10.14 & 7.25 \\
16.13 & 10.94 \\
20.86 & 10.09 \\
15.58 & 7.56 \\
15.47 & 6.55 \\
11.29 & 4.06
\end{tabular}

The certified lattice parameters, from the mean of the 25 samples are:

$$
\begin{aligned}
& a=0.4758846 \mathrm{~nm}(4.758846 \AA) \\
& a=0.0000109(0.000109) \\
& c=1.299306 \mathrm{~nm}(12.99306 \dot{A}) \\
& a=0.0000238(0.000238)
\end{aligned}
$$

where $\lambda\left(\mathrm{CuKN}_{1}\right)=0.1540629 \mathrm{~nm}(1.540629 \dot{\mathrm{A}})$ 
PNNL-20650, Rev. 2

Battelle - Pacific Northwest National Laboratory

Radiochemical Sciences and Engineering Group

X-Ray Diffraction Data Analysis . -

Appendix: How to assess and correct instrument bias using this SRM

When looking for instrumental problems, the peaks should be considered jointly, not individually. First, plot control bands as $1 \pm U$ over the range of $2 \Theta$, where $U$ is the relative uncertainty $(\mathrm{U}=0.0612$ if peak areas were used, or 0.0785 if peak heights were used). Next, plot the ratio of each new measurement to the certified value on the vertical axis against the $2 \Theta$ peak location on the horizontal axis. As long as these ratios fall within the control bands and are patternless, the systern cannot be labelled "out of control."

However, if there is a pattern in the ratios, then the device is introducing a systematic error, even if all of the ratios fall within the control bands. This systematic error should be corrected by modelling the ratios as a function of peak location and using the model to correct future observations on unknowns.

Let the measured relative intensities on the 12 peaks be $y_{i}$ and the certified values be $\mathrm{c}_{\mathrm{i}}$, so the ratios to model are

$$
r_{i}=\frac{y_{i}}{c_{i}} .
$$

Fit a simple model, using ordinary least squares regression, which describes these ratios adequately as a function of peak location $\left(\mathrm{x}_{\mathrm{i}}\right)$. The fitted model has $\mathrm{m}$ parameters: $\mathrm{m}=1$ for a horizontal straight line, $m=2$ for a slanted straight line, $m=3$ for a quadratic, etc. The residual sum of squares from the fit, plus an allowance for the uncertainty in the certified values on the certificate, is:

$$
\mathrm{RSS}_{\text {fuil }}=\sum_{1}^{\mathrm{n}}\left(\mathrm{r}_{\mathrm{i}}-\hat{\mathrm{r}}_{\mathrm{i}}\right)^{2}+(\mathrm{n}-\mathrm{m}) \cdot \frac{(\mathrm{RSD})^{2}}{25}
$$

and the residual degrees of freedom for this "full" model are $n-m$. The RSD $=0.0206$ if peak areas were used, or 0.0262 if peak heights were used. The residual sum of squares for the horizontal line at 1 (which describes "ideal" data), plus an allowance for the uncerainty in the certified values on the certificate, is:

$$
\operatorname{RSS}_{1}=\sum_{1}^{n}\left(\mathrm{r}_{\mathrm{i}}-1\right)^{2}+(\mathrm{n}-1) \frac{(\mathrm{RSD})^{2}}{25}
$$

and the residual degrees of freedom are $n-1$. Then if the test statistic

$$
F=\frac{\left\{R^{2} S_{1}-R_{\text {full }}+(m-1) \frac{(R S D)^{2}}{25}\right\} /(m-1)}{\left\{R_{\text {f }} S_{\text {full }}+(n-m) \frac{(R S D)^{2}}{25}\right\} /(n-m)}
$$

is larger than the upper 0.05 percentage point from the $\mathrm{F}$ distribution with $\mathrm{m}-1$ numerator and $\mathrm{n}-\mathrm{m}$ denominator degrees of freedom (commonly provided in table form in introductory statistics texts), the correction model is needed.

To use the correction model: A new peak angle $x^{*}$ and a new relative intensity $y^{*}$ are measured on an unknown sample. If $\hat{\mathrm{r}}^{*}$ is the predicted value of the fitted model at $x^{*}$, the corrected relative intensity for the new peak angle on the unknown sample is

$$
\text { corrected relative intensity }=\frac{y^{*}}{\hat{\mathrm{r}}^{*}}
$$


PNNL-20650, Rev. 2

Battelle - Pacific Northwest National Laboratory

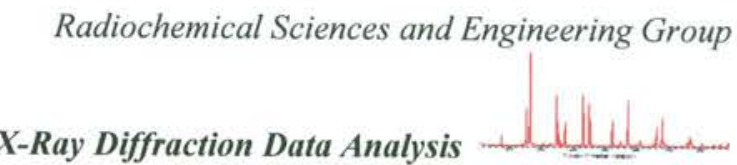

\section{Appendix 6 \\ ICDD cards for the identified crystalline phases presented in this report}

The following ICDD cards are for the crystalline phases identified in this report.

The ICDD card includes information about the crystal structure, the reference, the X-ray radiation wavelength, the calculated density and the observed density (if published), the strongest lines and the corresponding 2 theta angles. The table presented at the end of each ICDD card includes information about the 2 theta angle positions, interplanar distances at that angle, the intensity of that particular reflection and Miller indices at that particular 2 theta. The compounds identified in this report match the ICDD card peaks, which are the stick figures in the report.

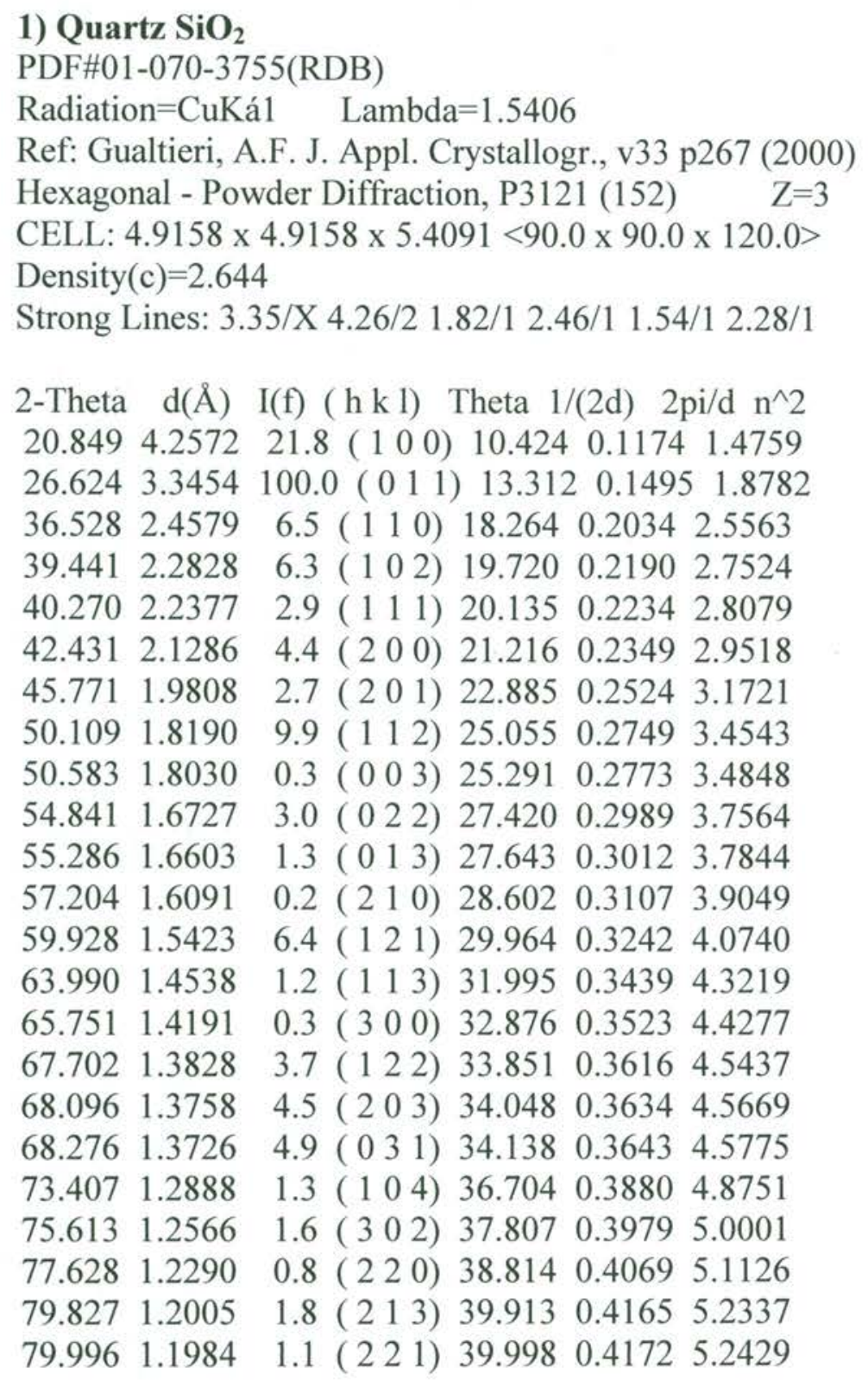

F.31 
PNNL-20650, Rev. 2

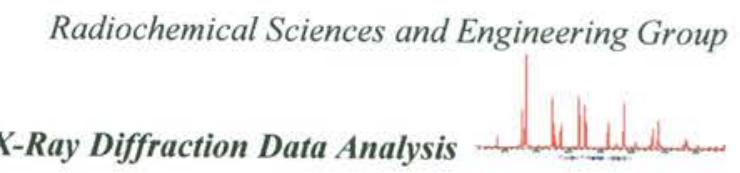

\begin{tabular}{|c|c|c|c|c|c|}
\hline 05 & 48 & $1.4\left(\begin{array}{lll}1 & 14\end{array}\right)$ & 40.553 & 0.4220 & 5.3032 \\
\hline 81.443 & 1.1807 & $1.7\left(\begin{array}{lll}3 & 10\end{array}\right)$ & 40.722 & 0.4235 & 3214 \\
\hline 83.787 & 1.1536 & $0.9\left(\begin{array}{lll}1 & 3 & 1\end{array}\right)$ & 41.894 & 0.4334 & 5.4467 \\
\hline 84.886 & 1.1414 & $0.2(204)$ & 42.443 & 0.4381 & 5.5047 \\
\hline 87.017 & 1.1189 & $0.1(222)$ & 43.509 & 0.4469 & 5.6157 \\
\hline 87.383 & 1.1151 & $0.2\left(\begin{array}{lll}3 & 0 & 3\end{array}\right)$ & 43.691 & 0.4484 & 5.6345 \\
\hline 90.771 & 1.0821 & $1.4\left(\begin{array}{lll}1 & 3 & 2\end{array}\right)$ & 45.385 & 0.4621 & 5.8064 \\
\hline 92.731 & 1.0643 & $0.2\left(\begin{array}{lll}4 & 0 & 0\end{array}\right)$ & 46.366 & 0.4698 & 5.9036 \\
\hline 94.558 & 1.0485 & $0.7\left(\begin{array}{lll}0 & 1 & 5\end{array}\right)$ & 47.279 & 0.4769 & 5.9925 \\
\hline 95.060 & 1.0443 & $0.5\left(\begin{array}{lll}4 & 0 & 1\end{array}\right)$ & 47.530 & 0.4788 & 6.0168 \\
\hline 96.159 & 1.0352 & $0.7(214)$ & 48.080 & 0.4830 & 6.0693 \\
\hline 98.672 & 1.0155 & $0.6(223)$ & 49.336 & 0.4924 & 6.1873 \\
\hline 102.147 & 0.9902 & $0.5\left(\begin{array}{lll}1 & 1 & 5\end{array}\right)$ & 51.074 & 0.5050 & 6.3457 \\
\hline 102.147 & 0.9902 & $0.5\left(\begin{array}{lll}0 & 4 & 2\end{array}\right)$ & 51.074 & 0.5050 & 6.3457 \\
\hline 102.489 & 0.9878 & $0.4\left(\begin{array}{lll}1 & 3 & 3\end{array}\right)$ & 51.244 & 0.5062 & 6.3609 \\
\hline 103.784 & 0.9790 & $0.1\left(\begin{array}{lll}0 & 3 & 4\end{array}\right)$ & 51.892 & 0.5107 & 6.4182 \\
\hline 104.128 & 0.9767 & $0.4(320)$ & 52.064 & 0.5119 & 6.4333 \\
\hline 106.016 & 0.9644 & $0.1\left(\begin{array}{ll}0 & 25)\end{array}\right.$ & 53.008 & 0.5 & 6.5150 \\
\hline 106.537 & 0.9611 & $0.6(231)$ & 53.269 & 0.5202 & 6.5373 \\
\hline 112.027 & 0.9290 & $0.1\left(\begin{array}{lll}4 & 1 & 0\end{array}\right)$ & 56.013 & 0.5 & 6.763 \\
\hline 113.974 & 0.9186 & $0.2\left(\begin{array}{lll}3 & 2 & 2\end{array}\right)$ & 56.987 & 0.5443 & 6.8399 \\
\hline 114.373 & 0.9165 & $0.5\left(\begin{array}{lll}4 & 0 & 3\end{array}\right)$ & 57.187 & 0.5 & 6.8 \\
\hline 114.557 & 0.9156 & $0.6\left(\begin{array}{lll}4 & 1 & 1\end{array}\right)$ & 57.278 & 0.5461 & 6.8624 \\
\hline 115.766 & 0.9095 & $0.2(224)$ & 57.883 & 0.5498 & 6.9085 \\
\hline 117.397 & 0.9015 & $0.1\left(\begin{array}{lll}0 & 0 & 6\end{array}\right)$ & 58.698 & 0.5546 & 6.9696 \\
\hline 118.186 & 0.8978 & $0.4(125)$ & 59.093 & 0.5569 & 6.9986 \\
\hline 120.011 & 0.8894 & $0.3\left(\begin{array}{lll}3 & 1 & 4\end{array}\right)$ & 60.005 & 0.5622 & 7.0644 \\
\hline 121.710 & 0.8820 & $0.1\left(\begin{array}{lll}1 & 0 & 6\end{array}\right)$ & 60.855 & 0.5669 & 7.1241 \\
\hline 122.498 & 0.8786 & $0.2(142)$ & 61.249 & 0.5691 & 7.1513 \\
\hline 127.106 & 0.8603 & $0.1 \quad\left(\begin{array}{lll}3 & 0 & 5\end{array}\right)$ & 63.553 & 0.5812 & 7.3032 \\
\hline 127.526 & 0.8588 & $0.1 \quad(233)$ & 63.763 & 0.5822 & 7.3165 \\
\hline 131.040 & 0.8464 & $0.1\left(\begin{array}{lll}1 & 1 & 6\end{array}\right)$ & 65.520 & 0.5908 & 7.4236 \\
\hline 132.649 & 0.8411 & $0.1(0.51)$ & 66.325 & 0.5945 & 7.4703 \\
\hline 134.155 & 0.8363 & $0.1(044)$ & 67.077 & 0.5978 & 7.5127 \\
\hline 136.225 & 0.8301 & $0.4\left(\begin{array}{ll}0 & 2 \\
6\end{array}\right)$ & 68.112 & 0.6023 & 7.5689 \\
\hline 137.738 & 0.8258 & $0.5(143)$ & 68.869 & 0.6055 & 7.6084 \\
\hline 140.166 & 0.8193 & 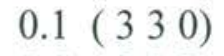 & 70.083 & 0.6103 & 7.6690 \\
\hline 143.052 & 0.8121 & $0.6\left(\begin{array}{lll}5 & 0 & 2\end{array}\right)$ & 71.526 & 0.6157 & 7.7365 \\
\hline 143.052 & 0.8121 & $0.6(225)$ & 71.526 & 0.6157 & 7.7365 \\
\hline 143.946 & 0.8101 & $0.5\left(\begin{array}{lll}3 & 3 & 1\end{array}\right)$ & 71.973 & 0.6172 & 7.7564 \\
\hline 146.447 & 0.8045 & $0.3(420)$ & 73.224 & 0.6215 & 7.8097 \\
\hline 149.90 & 0.7976 & $0.2\left(\begin{array}{lll}1 & 3 & 5\end{array}\right)$ & 74.953 & 0.6268 & 7.8772 \\
\hline
\end{tabular}


PNNL-20650, Rev. 2

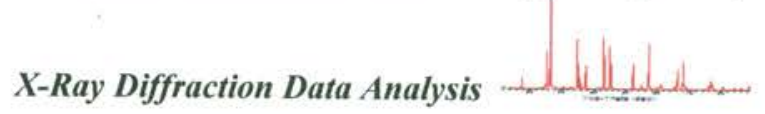

2) Rutile, syn (Titanium Oxide) Ti O2PDF\#01-077-0440(RDB)

Radiation $=$ CuKál $\quad$ Lambda $=1.5406 \quad$ Filter $=$

Calibration $=2 \mathrm{~T}=27.438-143.112 \quad \mathrm{I} / \mathrm{Ic}(\mathrm{RIR})=3.6$

Ref: Seki, H., Ishizawa, N., Mizutani, N., Kato, M. Yogyo Kyokaishi (J. Ceram. Assoc.

Jpn.), v92 p219 (1984)

Tetragonal - Single Crystal, P42/mnm (136) Z=2

CELL: $4.5934 \times 4.5934 \times 2.9575<90.0 \times 90.0 \times 90.0>\quad P . S=t P 6.00$

$\operatorname{Density}(\mathrm{c})=4.252$

Strong Lines: 3.25/X 1.69/5 2.49/4 2.19/2 1.36/2 1.62/1 1.35/1 1.48/1

Calculated Pattern Original Remarks: Stable from 800 to $1800 \mathrm{~K}$ (2nd ref.,

Tomaszewski), 300-800 K: Pbca.

Temperature of Data Collection: $300 \mathrm{~K}$.

Wyckoff Sequence: f a(P42/MNM).

Unit Cell Data Source: Single Crystal.

2-Theta $\mathrm{d}(\AA) \quad \mathrm{I}(\mathrm{f}) \quad(\mathrm{h} \mathrm{k} \mathrm{l})$ Theta $1 /(2 \mathrm{~d}) \quad 2 \mathrm{pi} / \mathrm{d} \mathrm{n} \mathrm{n}^{\wedge} 2$

27.4383 .2480100 .0 ( $\begin{array}{lllllll}1 & 1 & 0 & 13.719 & 0.1539 & 1.9345\end{array}$

$\begin{array}{lllllllll}36.091 & 2.4867 & 43.6 & \left(\begin{array}{lllll}1 & 0 & 1\end{array}\right) & 18.046 & 0.2011 & 2.5268\end{array}$

$\begin{array}{llllllll}39.193 & 2.2967 & 6.4 & \left(\begin{array}{llll}2 & 0 & 0\end{array}\right) & 19.596 & 0.2177 & 2.7357\end{array}$

$\begin{array}{lllllll}41.250 & 2.1868 & 17.0 & \left.\text { (l } \begin{array}{lllll}1 & 1 & 1\end{array}\right) & 20.625 & 0.2286 & 2.8733\end{array}$

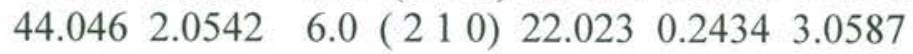

$\begin{array}{llllllll}54.331 & 1.6872 & 47.8 & \text { (2 } 2111 \text { ) } & 27.165 & 0.2964 & 3.7241\end{array}$

$\begin{array}{llllllll}56.630 & 1.6240 & 13.7 & \text { (2 } 220) & 28.315 & 0.3079 & 3.8689\end{array}$

$\begin{array}{lllllllll}62.787 & 1.4788 & 6.5 & \left(\begin{array}{llll}0 & 0 & 2\end{array}\right) & 31.393 & 0.3381 & 4.2490\end{array}$

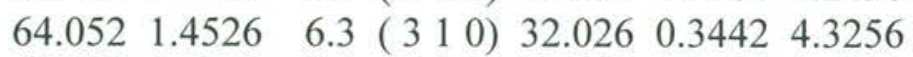

$\begin{array}{lllllllll}65.520 & 1.4235 & 0.5 & \left(\begin{array}{lll}2 & 2 & 1\end{array}\right) & 32.760 & 0.3512 & 4.4138\end{array}$

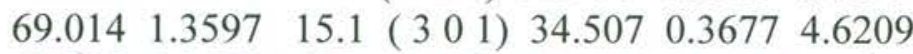

$\begin{array}{llllllll}69.829 & 1.3458 & 7.7 & \left(\begin{array}{llll}1 & 1 & 2\end{array}\right) & 34.915 & 0.3715 & 4.6686\end{array}$

$\begin{array}{lllllllll}72.429 & 1.3038 & 0.8 & \left(\begin{array}{llll}3 & 1 & 1\end{array}\right) & 36.214 & 0.3835 & 4.8191\end{array}$

$\begin{array}{llllllll}74.405 & 1.2740 & 0.2 & \left(\begin{array}{l}3 \\ 3\end{array} 20\right) & 37.203 & 0.3925 & 4.9319\end{array}$

$\begin{array}{llllllll}76.565 & 1.2433 & 1.6 & (202) & 38.283 & 0.4021 & 5.0535\end{array}$

$\begin{array}{llllllll}79.858 & 1.2001 & 0.9 & \left(\begin{array}{llll}2 & 1 & 2\end{array}\right) & 39.929 & 0.4166 & 5.2354\end{array}$

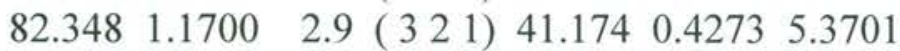

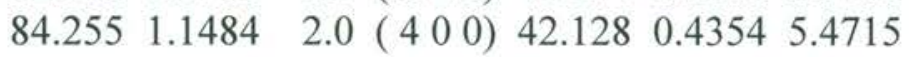

$\begin{array}{llllllll}87.487 & 1.1141 & 0.7 & \left(\begin{array}{llll}4 & 1 & 0\end{array}\right) & 43.743 & 0.4488 & 5.6399\end{array}$

$\begin{array}{llllllll}89.579 & 1.0934 & 4.8 & (222) & 44.789 & 0.4573 & 5.7465\end{array}$

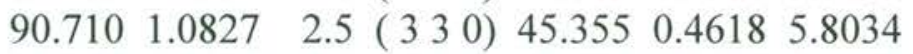

$\begin{array}{lllllll}95.268 & 1.0426 & 4.1 & \left(\begin{array}{llll}4 & 1 & 1\end{array}\right) & 47.634 & 0.4796 & 6.0267\end{array}$

$\begin{array}{llllllll}96.035 & 1.0363 & 3.3 & \left(\begin{array}{llll}3 & 1 & 2\end{array}\right) & 48.017 & 0.4825 & 6.0634\end{array}$

$\begin{array}{llllllll}97.173 & 1.0271 & 1.8 & (420) & 48.587 & 0.4868 & 6.1173\end{array}$

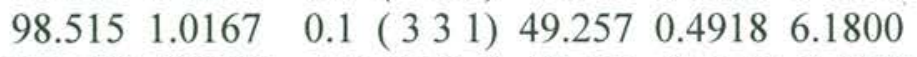

$\begin{array}{llllllll}105.103 & 0.9703 & 0.4 & (421) & 52.551 & 0.5153 & 6.4757\end{array}$

$\begin{array}{lllllllll}106.099 & 0.9639 & 1.3 & \left(\begin{array}{llll}1 & 0 & 3\end{array}\right) & 53.050 & 0.5187 & 6.5186\end{array}$

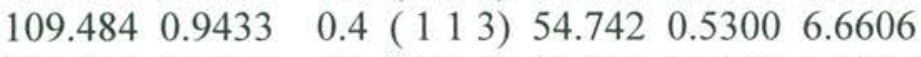

$\begin{array}{llllllll}113.960 & 0.9187 & 0.1 & \left(\begin{array}{l}4 \\ 4\end{array} \text { 0) }\right. & 56.980 & 0.5443 & 6.8394\end{array}$ 
PNNL-20650, Rev. 2

Battelle - Pacific Northwest National Laboratory

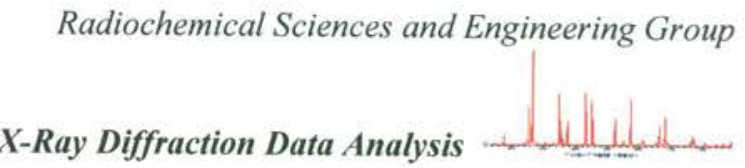

\begin{tabular}{|c|c|c|c|c|c|}
\hline 16.270 & & 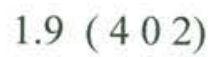 & & & \\
\hline & 0.9008 & $2.1\left(\begin{array}{lll}5 & 1 & 0\end{array}\right)$ & 58.769 & 0.555 & 0.577 \\
\hline & 0.8898 & $1.0\left(\begin{array}{lll}4 & 1 & 2\end{array}\right)$ & 59.962 & 0.5619 & 1.001 \\
\hline & 0.8888 & $4.2\left(\begin{array}{lll}2 & 1 & 3\end{array}\right)$ & 6 & & \\
\hline & 0.8773 & $3.9\left(\begin{array}{lll}4 & 3 & 1\end{array}\right)$ & 61.402 & 0.5699 & 7. \\
\hline & 736 & $2.8(3$ & & & \\
\hline & 0.8618 & $0.1\left(\begin{array}{lll}5 & 1 & 1\end{array}\right)$ & 63. & 0. & 1.2 \\
\hline & & & 64 & & \\
\hline & 0.84 & $2.5(422$ & 65. & 0. & 1.4 \\
\hline & 087 & $3.0(3$ & 68. & 0. & $I^{\prime}$ \\
\hline & & 5.1 & 70. & 0.6 & 7.66 \\
\hline & & 0.2 & & & \\
\hline & & 0.7 & 71.556 & 0.6158 & .73 \\
\hline
\end{tabular}

3) Corundum (Aluminum Oxide), $\mathrm{Al}_{2} \mathrm{O}_{3}, \mathrm{PDF} \# 01-071-6478$

Radiation $=$ CuKál Lambda $=1.5406$

Ref: Riello, P., Canton, P., Fagherazzi, G. Powder Diffraction, v12 p160 (1997)

Rhombohedral - Powder Diffraction, R-3c (167) $\quad Z=6$

CELL: $4.7598 \times 4.7598 \times 12.9924<90.0 \times 90.0 \times 120.0>\quad$ P.S $=$ hR $10.00(A ́)$

$\operatorname{Density}(\mathrm{c})=3.985$

Strong Lines: 2.55/X 2.09/X 1.60/9 3.48/7 1.37/5 1.74/5 2.38/5 1.40/3

ICSD Collection Code: 77810.

Calculated Pattern Original Remarks: $R($ Bragg $)=0.03$.

Minor Warning: $10 \%<\mathrm{R}$ factor $<15 \%$ (for powder).

Wyckoff Sequence: e c(R3-CH)

2-Theta $\mathrm{d}(\AA) \quad \mathrm{I}(\mathrm{f}) \quad(\mathrm{h} \mathrm{k} \mathrm{l})$ Theta $1 /(2 \mathrm{~d}) \quad 2 \mathrm{pi} / \mathrm{d} \mathrm{n} \mathrm{n}^{\wedge} 2$

$\begin{array}{llllllll}25.573 & 3.4805 & 67.0 & \left(\begin{array}{llll}0 & 1 & 2\end{array}\right) & 12.786 & 0.1437 & 1.8052\end{array}$

$\begin{array}{llllllll}35.147 & 2.5512 & 100.0 & \left(\begin{array}{lll}1 & 0 & 4\end{array}\right) & 17.574 & 0.1960 & 2.4628\end{array}$

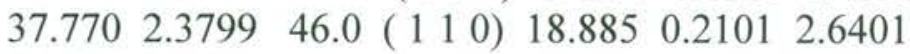

$\begin{array}{llllllll}41.676 & 2.1654 & 0.5 & \left(\begin{array}{llll}0 & 0 & 6\end{array}\right) & 20.838 & 0.2309 & 2.9016\end{array}$

$\begin{array}{llllllll}43.347 & 2.0857 & 96.5 & \left.\text { (l } \begin{array}{lllll}1 & 1 & 3\end{array}\right) & 21.674 & 0.2397 & 3.0125\end{array}$

$\begin{array}{llllllll}46.170 & 1.9646 & 1.5 & \left(\begin{array}{lll}2 & 0 & 2\end{array}\right) & 23.085 & 0.2545 & 3.1983\end{array}$

$\begin{array}{lllllllll}52.544 & 1.7403 & 46.2 & \left(\begin{array}{lll}0 & 2 & 4\end{array}\right) & 26.272 & 0.2873 & 3.6105\end{array}$

$\begin{array}{llllllll}57.494 & 1.6016 & 91.0 & \text { ( } 1116) & 28.747 & 0.3122 & 3.9230\end{array}$

$\begin{array}{llllllll}59.729 & 1.5469 & 2.2 & \left(\begin{array}{llll}2 & 1 & 1\end{array}\right) & 29.865 & 0.3232 & 4.0617\end{array}$

$\begin{array}{llllllll}61.118 & 1.5151 & 3.4 & \left(\begin{array}{llll}1 & 2 & 2\end{array}\right) & 30.559 & 0.3300 & 4.1472\end{array}$

$\begin{array}{llllllll}61.299 & 1.5110 & 8.2 & \left(\begin{array}{llll}0 & 1 & 8\end{array}\right) & 30.650 & 0.3309 & 4.1583\end{array}$

$\begin{array}{llllllll}66.507 & 1.4048 & 34.4 & (214) & 33.254 & 0.3559 & 4.4728\end{array}$

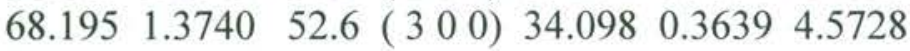

$\begin{array}{llllllll}70.405 & 1.3362 & 1.1 & \left(\begin{array}{llll}1 & 2 & 5\end{array}\right) & 35.203 & 0.3742 & 4.7022\end{array}$

$\begin{array}{llllllll}74.294 & 1.2756 & 1.3 & (208) & 37.147 & 0.3920 & 4.9256\end{array}$

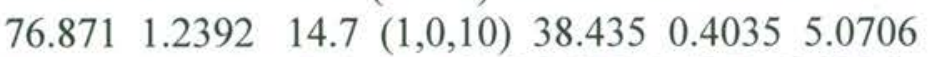

$\begin{array}{lllllll}77.230 & 1.2343 & 8.6 & \left(\begin{array}{ll}1 & 1\end{array}\right) & 38.615 & 0.4051 & 5.0906\end{array}$

$\begin{array}{llllllll}80.407 & 1.1933 & 0.7 & (217) & 40.204 & 0.4190 & 5.2653\end{array}$

F.34 
PNNL-20650, Rev. 2

\begin{tabular}{|c|c|c|c|}
\hline & & $5.7(2$ & 202 \\
\hline & 602 & 0.6 & 3105 \\
\hline & 1.1474 & $4.6(223) 4$ & 585 \\
\hline & 1.1389 & 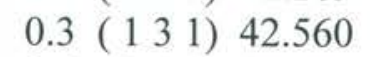 & 905 \\
\hline & 1.1260 & $.4\left(\begin{array}{lll}3 & 1 & 2\end{array}\right) 43.167$ & 44415 \\
\hline & 1.1 & $2.7(128) 43.246$ & 14475 \\
\hline & & $.3(0,2,10) 4$ & $0.4549 \quad 5.7167$ \\
\hline 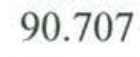 & 1.0 & $1.6(0,0,12) 4$ & 6185.8033 \\
\hline & & 4) 45.585 & 0.46365 \\
\hline & 1.0 & $15) 47.4$ & 0.47786 \\
\hline & & 6) 47.615 & 0.47956 \\
\hline 870 & 1.0 & 2) 49.1 & \\
\hline & & 0) 5( & 2969 \\
\hline & 0.9855 & 12) 51. & 0.50746 .3756 \\
\hline & & 4) 51 . & 906.3966 \\
\hline & 0.9734 & 7) 52. & $0.5137 \quad 6.4548$ \\
\hline & & 1) 54 & 016.6617 \\
\hline & & 11) 54 & 126.6755 \\
\hline & & & 86.7210 \\
\hline & 0.9 & 9) 57 . & 56 \\
\hline 200 & 0.9 & t) 58 & 76 \\
\hline & & 4) 58 & 236.9399 \\
\hline 7. & 0.8 & 0) 58 . & 0.55596 .9 \\
\hline & & 5) 60 & 267. \\
\hline 0 & 0.8 & 3) 61 . & 777.1341 \\
\hline & & 8) 62 . & 467.2209 \\
\hline & 3 & 10.5 & 0.58267 .3206 \\
\hline & & $4.2(0$ & 0.58797 .3884 \\
\hline & 0.8 & $4.1(2,0,14) 65$. & 0.59097 .4251 \\
\hline & & 7) 66.089 & 0.59347 .4568 \\
\hline 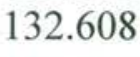 & 0.84 & $0.3(2,1,13) 66$ & 0.59447 .4692 \\
\hline & 0.8307 & 15.7 ( 1468. & 97.5638 \\
\hline & 0.8215 & $0.1 \quad(3,1,11) 69.669$ & 0.60877 .6487 \\
\hline & 0.8172 & $0.4(238) 70.489$ & 0.61187 .6885 \\
\hline & 0.81 & $2.1(1,1,15) 71.154$ & 0.61437 .7196 \\
\hline & 080 & $6.1(4,0,10) 72.565$ & 0.61937 .7821 \\
\hline & & $0.2(2,2,12) 74.129$ & 0.62447 .8459 \\
\hline & 70 & $4.1(054) 74.574$ & $0.6257 \quad 7.8630$ \\
\hline
\end{tabular}


PNNL-20650, Rev. 2

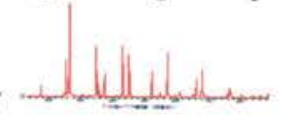

\section{4) Uranium Oxide, U4 O9, PDF\#01-075-0944(RDB)}

Radiation $=$ CuKál $\quad$ Lambda $=1.5406$

Calibration $=2 \mathrm{~T}=9.963-101.026 \quad \mathrm{I} / \mathrm{Ic}(\mathrm{RIR})=22.4$

Ref: Belbboch, B., Piekarski, C., Perio, P. Bull. Soc. Fr. Mineral. Cristallogr., v83 p206 (1960)

Cubic - Single Crystal, I-43d (220) $\quad Z=64$

CELL: $21.73 \times 21.73 \times 21.73<90.0 \times 90.0 \times 90.0>$ P.S $=$ cI832.00

$\operatorname{Density}(\mathrm{c})=11.353$

Strong Lines: 3.14/X 1.92/4 2.72/4 1.64/4 1.25/1 1.11/1 1.21/1 1.57/1

Calculated Pattern Original Remarks: Stable above 338 K (2nd ref., Tomaszewski), below trigonal.

Minor Warning: No e.s.d reported/abstracted on the cell dimension.

No R factors reported/abstracted.

Wyckoff Sequence: e14 d3 c4 b a(I4-3D).

Unit Cell Data Source: Single Crystal.

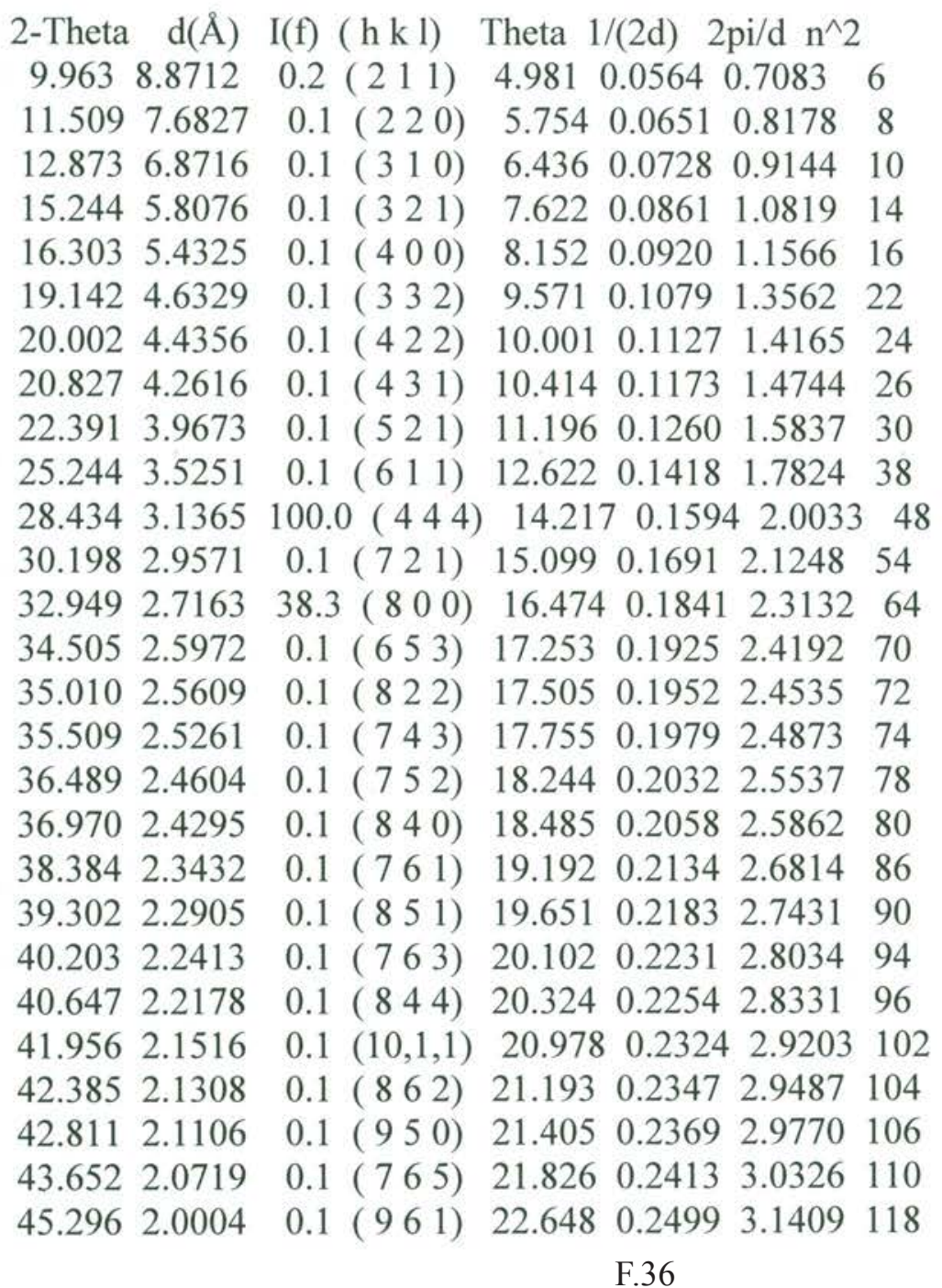


PNNL-20650, Rev. 2

$\begin{array}{llllllll}47.288 & 1.9207 & 44.0 & (880) & 23.644 & 0.2603 & 3.2713 & 128\end{array}$

$\begin{array}{lllllllll}48.453 & 1.8772 & 0.1 & (972) & 24.226 & 0.2664 & 3.3471 & 134\end{array}$

$\begin{array}{lllllllll}49.975 & 1.8235 & 0.1 & (965) & 24.987 & 0.2742 & 3.4456 & 142\end{array}$

$\begin{array}{lllllllll}50.350 & 1.8108 & 0.1 & (12,0,0) & 25.175 & 0.2761 & 3.4698 & 144\end{array}$

$\begin{array}{llllllll}51.463 & 1.7743 & 0.1 & (10,7,1) & 25.731 & 0.2818 & 3.5413 & 150\end{array}$

$\begin{array}{lllllllll}52.196 & 1.7511 & 0.1 & (12,3,1) & 26.098 & 0.2855 & 3.5882 & 154\end{array}$

$\begin{array}{lllllllll}52.921 & 1.7288 & 0.1 & (11,6,1) & 26.460 & 0.2892 & 3.6345 & 158\end{array}$

$\begin{array}{lllllllll}53.640 & 1.7073 & 0.1 & (11,5,4) & 26.820 & 0.2929 & 3.6803 & 162\end{array}$

$\begin{array}{llllllll}54.352 & 1.6866 & 0.1 & (976) & 27.176 & 0.2965 & 3.7254 & 166\end{array}$

$\begin{array}{llllllll}56.105 & 1.6380 & 35.0 & (12,4,4) & 28.052 & 0.3053 & 3.8360 & 176\end{array}$

$\begin{array}{lllllllll}58.837 & 1.5682 & 8.2 & \left(\begin{array}{l}8 \\ 88\end{array} 8\right) & 29.419 & 0.3188 & 4.0065 & 192\end{array}$

$\begin{array}{lllllllll}59.842 & 1.5443 & 0.1 & (13,5,2) & 29.921 & 0.3238 & 4.0687 & 198\end{array}$

$\begin{array}{llllllll}61.165 & 1.5140 & 0.1 & (14,3,1) & 30.583 & 0.3303 & 4.1501 & 206\end{array}$

$\begin{array}{llllllll}62.472 & 1.4854 & 0.1 & (13,6,3) & 31.236 & 0.3366 & 4.2299 & 214\end{array}$

$\begin{array}{llllllll}62.797 & 1.4785 & 0.1 & (10,10,4) & 31.398 & 0.3382 & 4.2496 & 216\end{array}$

$\begin{array}{lllllllll}63.120 & 1.4717 & 0.1 & (12,7,5) & 31.560 & 0.3397 & 4.2692 & 218\end{array}$

$\begin{array}{lllllllll}63.764 & 1.4584 & 0.1 & (14,5,1) & 31.882 & 0.3428 & 4.3082 & 222\end{array}$

$\begin{array}{llllllll}65.041 & 1.4328 & 0.1 & (13,6,5) & 32.521 & 0.3490 & 4.3852 & 230\end{array}$

$\begin{array}{lllllllll}65.675 & 1.4205 & 0.1 & (11,8,7) & 32.838 & 0.3520 & 4.4231 & 234\end{array}$

$\begin{array}{lllllllll}66.305 & 1.4086 & 0.1 & (15,3,2) & 33.153 & 0.3550 & 4.4607 & 238\end{array}$

$\begin{array}{lllllllll}67.558 & 1.3855 & 0.1 & (14,7,1) & 33.779 & 0.3609 & 4.5351 & 246\end{array}$

$\begin{array}{llllllll}69.107 & 1.3581 & 5.4 & (16,0,0) & 34.554 & 0.3682 & 4.6264 & 256\end{array}$

$\begin{array}{lllllllll}71.250 & 1.3225 & 0.1 & (14,7,5) & 35.625 & 0.3781 & 4.7512 & 270\end{array}$

$\begin{array}{lllllllll}72.462 & 1.3033 & 0.1 & (15,7,2) & 36.231 & 0.3836 & 4.8211 & 278\end{array}$

$\begin{array}{lllllllll}73.065 & 1.2940 & 0.1 & (16,5,1) & 36.533 & 0.3864 & 4.8556 & 282\end{array}$

$\begin{array}{lllllllll}73.667 & 1.2849 & 0.1 & (14,9,3) & 36.833 & 0.3891 & 4.8899 & 286\end{array}$

$\begin{array}{lllllllll}73.966 & 1.2805 & 0.1 & (16,4,4) & 36.983 & 0.3905 & 4.9070 & 288\end{array}$

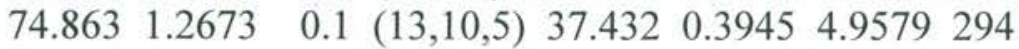

$\begin{array}{llllllll}76.350 & 1.2463 & 11.6 & (12,12,4) & 38.175 & 0.4012 & 5.0415 & 304\end{array}$

$\begin{array}{llllllll}76.646 & 1.2422 & 6.0 & (17,4,1) & 38.323 & 0.4025 & 5.0580 & 306\end{array}$

$\begin{array}{llllllll}78.710 & 1.2147 & 8.9 & (16,8,0) & 39.355 & 0.4116 & 5.1725 & 320\end{array}$

$\begin{array}{llllllll}79.003 & 1.2110 & 4.6 & (13,12,3) & 39.501 & 0.4129 & 5.1886 & 322\end{array}$

$\begin{array}{llllllll}81.924 & 1.1750 & 0.1 & (15,9,6) & 40.962 & 0.4255 & 5.3473 & 342\end{array}$

$\begin{array}{llllllll}82.215 & 1.1716 & 0.1 & (18,4,2) & 41.107 & 0.4268 & 5.3629 & 344\end{array}$

$\begin{array}{llllllll}82.505 & 1.1682 & 0.1 & (15,11,0) & 41.253 & 0.4280 & 5.3785 & 346\end{array}$

$\begin{array}{llllllll}83.085 & 1.1615 & 0.1 & (17,6,5) & 41.543 & 0.4305 & 5.4095 & 350\end{array}$

$\begin{array}{llllllll}83.376 & 1.1582 & 0.1 & (12,12,8) & 41.688 & 0.4317 & 5.4249 & 352\end{array}$

$\begin{array}{llllllll}83.665 & 1.1549 & 0.1 & (17,8,1) & 41.833 & 0.4329 & 5.4403 & 354\end{array}$

$\begin{array}{lllllllll}84.244 & 1.1485 & 0.1 & (18,5,3) & 42.122 & 0.4354 & 5.4709 & 358\end{array}$

$\begin{array}{llllllll}84.534 & 1.1453 & 0.1 & (16,10,2) & 42.267 & 0.4366 & 5.4862 & 360\end{array}$

$\begin{array}{llllllll}84.824 & 1.1421 & 0.1 & (15,11,4) & 42.412 & 0.4378 & 5.5014 & 362\end{array}$

$\begin{array}{llllllll}85.113 & 1.1390 & 0.1 & (18,6,2) & 42.556 & 0.4390 & 5.5166 & 364\end{array}$

$\begin{array}{lllllllll}85.401 & 1.1359 & 0.1 & (19,2,1) & 42.701 & 0.4402 & 5.5317 & 366\end{array}$

$\begin{array}{llllllll}86.556 & 1.1236 & 0.1 & (14,13,3) & 43.278 & 0.4450 & 5.5919 & 374\end{array}$

$\begin{array}{llllllll}87.998 & 1.1089 & 9.1 & (16,8,8) & 43.999 & 0.4509 & 5.6661 & 384\end{array}$ 
PNNL-20650, Rev. 2

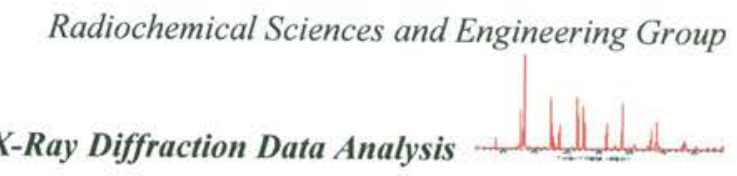
88.2861 .1060
$\begin{array}{llllll}4.7 & (19,4,3) & 44.143 & 0.4521 & 5.6808 & 386\end{array}$
$91.166 \quad 1.0784$
$0.1(15,10,9) 45.583 \quad 0.46365 .8262406$
91.4541 .0758
$\begin{array}{lllllll}0.1 & (20,2,2) & 45.727 & 0.4648 & 5.8405 & 408\end{array}$
$91.742 \quad 1.0732$
$\begin{array}{lllllll}0.1 & (19,7,0) & 45.871 & 0.4659 & 5.8548 & 410\end{array}$
92.3191 .0680
$0.1 \quad(17,11,2) \quad 46.159 \quad 0.4682 \quad 5.8833 \quad 414$
94.9151 .0455
$\begin{array}{llllll}7.7 & (20,4,4) & 47.458 & 0.4782 & 6.0098 & 432\end{array}$
$95.206 \quad 1.0431$
$\begin{array}{llllll}4.0 & (19,8,3) & 47.603 & 0.4794 & 6.0237 & 434\end{array}$
98.1051 .0198
$\begin{array}{lllllll}0.1 & (21,3,2) & 49.052 & 0.4903 & 6.1610 & 454\end{array}$
98.3961 .0176
$\begin{array}{llllll}0.1 & (14,14,8) & 49.198 & 0.4914 & 6.1745 & 456\end{array}$
$98.687 \quad 1.0154$
$\begin{array}{lllllll}0.1 & (20,7,3) & 49.343 & 0.4924 & 6.1880 & 458\end{array}$
98.9781 .0132
$\begin{array}{llllll}0.1 & (18,10,6) & 49.489 & 0.4935 & 6.2015 & 460\end{array}$
99.2701 .0110
$\begin{array}{llllll}0.1 & (17,13,2) & 49.635 & 0.4946 & 6.2150 & 462\end{array}$
$99.562 \quad 1.0088$
$\begin{array}{lllllll}0.1 & (20,8,0) & 49.781 & 0.4956 & 6.2284 & 464\end{array}$
99.8551 .0066
$\begin{array}{llllll}0.1 & (21,5,0) & 49.927 & 0.4967 & 6.2419 & 466\end{array}$
100.4401 .0023
$\begin{array}{lllllll}0.1 & (21,5,2) & 50.220 & 0.4988 & 6.2686 & 470\end{array}$
100.7331 .0002
$\begin{array}{llllll}0.1 & (18,12,2) & 50.367 & 0.4999 & 6.2819 & 472\end{array}$
$101.026 \quad 0.9981$
$\begin{array}{llllll}0.1 & (17,13,4) & 50.513 & 0.5010 & 6.2952 & 474\end{array}$

\section{5) Uraninite (Uranium Oxide) U O2, PDF\#01-072-4553(RDB)}

Radiation $=$ CuKál $\quad$ Lambda $=1.5406$

Calibration $=2 \mathrm{~T}=28.373-139.625 \quad \mathrm{I} / \mathrm{Ic}(\mathrm{RIR})=23.7$

Ref: Wasserstein, B. Nature (London), v168 p380 (1951)

Cubic - Single Crystal, Fm-3m (225) Z=4

CELL: $5.444 \times 5.444 \times 5.444<90.0 \times 90.0 \times 90.0>$ P.S $=\mathrm{cF} 12.00$ (Á)

$\operatorname{Density}(\mathrm{c})=11.116$

Strong Lines: $3.14 / X$ 1.92/4 2.72/4 1.64/4 1.25/1 1.11/1 1.22/1 0.92/1

Calculated Pattern Original Remarks: Stable above $30 \mathrm{~K}$ (2nd ref., Tomaszewski).

Fluorite type.

Minor Warning: No e.s.d reported/abstracted on the cell dimension.

No R factors reported/abstracted.

Wyckoff Sequence: c a(FM3-M).

Unit Cell Data Source: Single Crystal.

2-Theta d( $(\AA) \quad \mathrm{I}(\mathrm{f})(\mathrm{h} \mathrm{k} \mathrm{l})$ Theta $1 /(2 \mathrm{~d}) \quad 2 \mathrm{pi} / \mathrm{d} \mathrm{n} \mathrm{n}^{\wedge} 2$

$\begin{array}{llllllllll}28.373 & 3.1431 & 100.0 & \left(\begin{array}{lllll}1 & 1 & 1\end{array}\right) & 14.186 & 0.1591 & 1.9990 & 3\end{array}$

$\begin{array}{llllllllll}32.877 & 2.7220 & 35.2 & \left(\begin{array}{lllll}2 & 0 & 0\end{array}\right) & 16.439 & 0.1837 & 2.3083 & 4\end{array}$

$\begin{array}{llllllllll}47.182 & 1.9247 & 41.1 & \text { (2 } 220) & 23.591 & 0.2598 & 3.2644 & 8\end{array}$

$\begin{array}{lllllllll}55.976 & 1.6414 & 35.1 & \left(\begin{array}{lllll}3 & 1 & 1\end{array}\right) & 27.988 & 0.3046 & 3.8279 & 11\end{array}$

$\begin{array}{lllllllll}58.701 & 1.5716 & 7.7 & \text { (2 2 2 2) } & 29.350 & 0.3182 & 3.9981 & 12\end{array}$

$\begin{array}{llllllll}68.940 & 1.3610 & 5.1 & \left(\begin{array}{llll}4 & 0 & 0\end{array}\right) & 34.470 & 0.3674 & 4.6166 & 16\end{array}$

$\begin{array}{lllllllll}76.160 & 1.2489 & 11.5 & (33 & 3 & 38.080 & 0.4003 & 5.0308 & 19\end{array}$

$\begin{array}{lllllllll}78.511 & 1.2173 & 8.4 & (420) & 39.255 & 0.4107 & 5.1615 & 20\end{array}$

$\begin{array}{llllllll}87.765 & 1.1113 & 8.5 & (422) & 43.882 & 0.4499 & 5.6542 & 24\end{array}$

$\begin{array}{lllllllll}94.652 & 1.0477 & 7.6 & \left(\begin{array}{llll}5 & 1 & 1\end{array}\right) & 47.326 & 0.4772 & 5.9971 & 27\end{array}$ 
PNNL-20650, Rev. 2

Battelle - Pacific Northwest National Laboratory

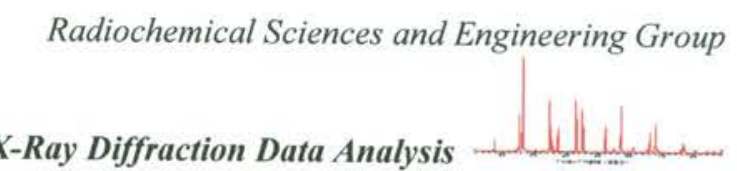

$\begin{array}{llllllll}106.339 & 0.9624 & 2.6 & (440) & 53.170 & 0.5195 & 6.5289 & 32\end{array}$

$\begin{array}{lllllllll}113.669 & 0.9202 & 7.9 & \left(\begin{array}{llll}5 & 3 & 1\end{array}\right) & 56.834 & 0.5434 & 6.8280 & 35\end{array}$

$\begin{array}{lllllllll}116.199 & 0.9073 & 4.0 & \left(\begin{array}{lll}6 & 0 & 0\end{array}\right) & 58.099 & 0.5511 & 6.9249 & 36\end{array}$

$\begin{array}{llllllllll}126.988 & 0.8608 & 4.1 & (620) & 63.494 & 0.5809 & 7.2995 & 40\end{array}$

$\begin{array}{llllllllll}136.201 & 0.8302 & 3.3 & \left(\begin{array}{lllll}5 & 3 & 3\end{array}\right) & 68.101 & 0.6023 & 7.5683 & 43\end{array}$

$\begin{array}{lllllllll}139.625 & 0.8207 & 2.8 & \left(\begin{array}{llll}6 & 2 & 2\end{array}\right) & 69.812 & 0.6092 & 7.6558 & 44\end{array}$ 
PNNL-20650, Rev. 2

\begin{tabular}{|c|c|c|c|}
\hline & PL K Basin Proje & \multicolumn{2}{|c|}{ Document Review Record } \\
\hline Title: & \multicolumn{3}{|c|}{ XRD Report: KW 210 Container Composite } \\
\hline \multicolumn{3}{|c|}{ Please return the completed form to: Doinita Neiner } & Comments Due: \\
\hline Org: & nization/Department: & Designated Reviewer: & $\begin{array}{l}\text { Reviewer Signature/Date: } \\
\text { (Upon completigh of review) }\end{array}$ \\
\hline & D9H63 & Mike Urie & $1 / 1 / N$ \\
\hline
\end{tabular}

It is optional, but helpful when comments are identified as to type or if the reviewer wishes to specify mandatory comments requiring resolution. In this case, the following conventions apply:

*Type: $\quad \mathbf{E}$ - Editorial, addresses word processing errors that do not adversely impact the integrity of the calculation.

$\mathbf{O}$-Optional, comment resolution would provide clarification, but does not impact the integrity of the work or calculations

M - Mandatory, comment shall be resolved; reviewer identifies impact on the integrity of the work or calculations

\begin{tabular}{|c|c|c|c|c|}
\hline $\begin{array}{l}\text { Comment } \\
\text { Number }\end{array}$ & $\begin{array}{l}\text { Section/ } \\
\text { Page }\end{array}$ & Type & Comment & Comment Resolution \\
\hline \multicolumn{5}{|c|}{ See attached "Track Changes" Copy of Report for all Comments/Corrections/Edits. } \\
\hline 1 & General & $\mathrm{O}$ & $\begin{array}{l}\text { Throughout report references are made to specific } 2 \text {-theta angles. It } \\
\text { would be very beneficial for all detected } 2 \text {-theta angles above a } \\
\text { threshold be included for each sample in the appendices. }\end{array}$ & $\begin{array}{l}\text { Reject } \rightarrow \text { we have } \\
\text { a PDF cand. }\end{array}$ \\
\hline 2 & Page 1 & $\mathrm{E}$ & Analysis date does not correspond with analysis date on page 2 & Dowe \\
\hline 3 & Page 3 & E & $\begin{array}{l}\text { Lots of spelling errors in "Comment" section. Also, should the } \\
\text { "XRD Operating Parameter" section be completed? }\end{array}$ & \\
\hline 4 & Page 5 & E & $\begin{array}{l}\text { Sample ID(s) TI008-SJ TiO }{ }_{2} \text { : There is inconsistency in } \\
\text { terminology, such as Sample ID(s) are called "XRD Mount ID" on } \\
\text { page } 6 \text { and sample is shown as TI008-SJ_TiO }{ }_{2} \text {. Similar } \\
\text { inconsistencies in sample naming are throughout the report. Use of } \\
\text { underscore vs hyphen is routine. How sample IDs or XRD Mount } \\
\text { IDs are presented in the report need to be consistent so that there is } \\
\text { no possibility of confusion of sample names. This DRR form will } \\
\text { not address this issue for every inconsistency ... attached report } \\
\text { highlights many of these sample naming inconsistencies. }\end{array}$ & \\
\hline 5 & Page 6 & M & $\begin{array}{l}\text { Page } 9 \text { states "... 2-theta with a step size of } 0.02 \% \text { minute. Page } 6 \\
\text { under "Other XRD scan parameters" states " } 2 \text { deg/minute...." }\end{array}$ & courected to 0.02 stepcife \\
\hline 6 & Page 7,Tab 1 & M & $\begin{array}{l}\text { Two places: } 01-070-3755 \text { shown as ICDD for } \mathrm{UO}_{2} \ldots \text { this should } \\
\text { be } 01-072-4553\end{array}$ & \\
\hline 7 & Page 7,Tab 1 & $\mathrm{E}$ & Under Formula, degrees are not displayed consistently. & Dos ne \\
\hline 8 & Page 7,Tab 1 & $\mathrm{E}$ & $\begin{array}{l}\text { Table references ICDD Card Number. Appendix } 6 \text { references PDF } \\
\text { Cards. Need to be consistent. }\end{array}$ & et to $\mid C D D$ \\
\hline 9 & Page7, Tab1 & E & $\begin{array}{l}\text { Title is confusion. Suggest "Summary of Phases identified in } \\
\text { KW210 Container Composite" }\end{array}$ & e \\
\hline 10 & \begin{tabular}{|l} 
Page $8,1^{\text {st }}$ \\
Para
\end{tabular} & E & $\begin{array}{l}\text { Change "has been" to "was", "XRD slides preparation" to either } \\
\text { "XRD slide preparation" or "XRD slide preparations" }\end{array}$ & \\
\hline 11 & $\begin{array}{l}\text { Page } 8,1^{\text {s }} \\
\text { Para }\end{array}$ & E & $\begin{array}{l}\text { Suggest adding "prior to samples being submitted for XRD } \\
\text { analysis" at end of second sentence. }\end{array}$ & \\
\hline 12 & $\begin{array}{l}\text { Page } 8,2^{\text {nd }} \\
\text { Para }\end{array}$ & E & $\begin{array}{l}\text { Second sentence ... "Typically" implies that some other method } \\
\text { other than the one described was used on one or more sample } \\
\text { preparations. Suggest deleting "Typically". }\end{array}$ & \\
\hline 13 & Page 8,Tab2 & E & "Client Sample ID" is shown as "Sample ID" on Request Form & Dowe \\
\hline
\end{tabular}


PNNL-20650, Rev. 2

\begin{tabular}{|c|c|c|c|c|}
\hline 14 & Page 8,Tab2 & $\mathrm{E}$ & $\begin{array}{l}\text { Sample Description: In all tables and all text, the sample } \\
\text { description should be consistently presented. }\end{array}$ & dove \\
\hline 15 & Page 9 & E & "2 theta" or "2-theta" ... need to be consistent throughout report & e \\
\hline 16 & Page 9 & M & $\begin{array}{l}44 \mathrm{kV} \text { and } 40 \mathrm{~mA} \text { shown as } 40 \mathrm{kV} \text { and } 44 \mathrm{~mA} \text { on benchsheet; one } \\
\text { is incorrect. }\end{array}$ & corrected \\
\hline 17 & $\begin{array}{l}\text { Page } 9 \text { and } \\
\text { after }\end{array}$ & $\mathrm{E}$ & $\begin{array}{l}\text { There is no reason to write out the entire name of the procedure } \\
\text { every time that procedure number is referenced. This simply adds } \\
\text { superfluous text! This is especially objectionable when the } \\
\text { procedure is referenced multiple times in same paragraph. }\end{array}$ & Bo 2 \\
\hline 18 & $\begin{array}{l}\text { Page } 9,2^{\text {nd }} \\
\text { Para }\end{array}$ & M & SRM 1976 appendix reference is incorrect. It should be " 5 " & Dome \\
\hline 19 & $\begin{array}{l}\text { Page } 9,2^{\text {nd }} \\
\text { Para }\end{array}$ & $\mathrm{E}$ & Aforementioned is one word & \\
\hline 20 & Page 9, S 3.1 & E & $\begin{array}{l}\text { If ICP data was available, a statement should be made that all major } \\
\text { element identified by ICP were included in the search element list }\end{array}$ & No i it verasnot. \\
\hline 21 & Page 9, S 3.1 & E & $\begin{array}{l}\text { " ... hydrates; zirconium metal, and zirconia..." Recommend } \\
\text { changing to "... hydrates; and zirconium metal and zirconia ..." to } \\
\text { be consistent with other entries in the sentence. }\end{array}$ & \\
\hline 22 & Page 9, S 3.2 & $\mathrm{E}$ & What does "account for instrument profile" mean in this sentence. & Deyes \\
\hline 23 & Page 10, S 4 & $\mathrm{E}$ & $\begin{array}{l}\text { Suggestions: Replace "made" with "comprised". Change "The } \\
\text { small fraction contains in addition to quartz, uraninite" to "The } \\
\text { small fraction samples contain uraninite in addition to quartz. }\end{array}$ & \\
\hline 24 & Page $10, \mathrm{~S} 4$ & $\mathrm{E}$ & $\begin{array}{l}\text { Probably need to insert "uranium" prior to first "oxyhydroxide". } \\
\text { Suggestion: Add semicolon before "aluminum" and add "; or" } \\
\text { before "zirconium" }\end{array}$ & \\
\hline 25 & Page 11, Tab3 & E & $\begin{array}{l}\text { Sample descriptions need to be consistent throughout report; e.g., } \\
\text { use " }<500 \text { " or "less than } 500 \text { ". }\end{array}$ & \\
\hline 26 & Page 12 & $\mathrm{E}$ & $\begin{array}{l}35^{\circ}-36^{\circ} ? \text { are there numerous peaks identified in this "range" or } \\
\text { should this be " } 35^{\circ}, 36^{\circ} \text { ? }\end{array}$ & corrected \\
\hline 27 & Page 13 & $\mathrm{E}$ & $\begin{array}{l}\text { Suggest changing "and uranium oxide" to "and uranium oxides } \\
\left(\mathrm{UO}_{2}, \mathrm{U}_{4} \mathrm{O}_{9}\right) \text { " }\end{array}$ & D \\
\hline 28 & Page 14 & $\mathrm{E}$ & $\begin{array}{l}\text { Suggest changing " } \ldots(\text { quartz), uranium oxide" to "... (quartz) and } \\
\text { uranium oxides }\left(\mathrm{UO}_{2}, \mathrm{U}_{4} \mathrm{O}_{9}\right) \text { " }\end{array}$ & Dove \\
\hline 29 & Page 19 & M & $\begin{array}{l}\text { First sentence references Scintag, last sentence references date } 02- \\
09-2011 \text {. Need to ensure Daily calibration data presented is from } \\
\text { Rigaku and need to change system and date references. Figure } 15 \\
\text { needs to be Figure } 8 \text {. }\end{array}$ & \\
\hline 30 & Page 19 & $\mathrm{E}$ & $\begin{array}{l}\text { The explanation in paragraph that describes table headings would } \\
\text { be better if each heading was in a list (e.g., a) } 2 \mathrm{~T}(\mathrm{r})=\ldots \text { b) } 2 \mathrm{~T}(\mathrm{o})= \\
\text {... etc. }\end{array}$ & $\$$ \\
\hline 31 & Page 22 & $\mathrm{M}$ & $\begin{array}{l}\text { First sentence references Scintag and there is a reference to Figure } \\
17 \text { (which should be } 8 \text { ).. Need to ensure Quality } \\
\text { Control/Calibration data presented is from Rigaku and need to } \\
\text { change system and date references. Is } 01-06-2011 \text { date correct? }\end{array}$ & \\
\hline 32 & Page 22 & M & $\begin{array}{l}\text { Last sentence reference PDF card being in Appendix } 5 \text {. Appendix } 5 \\
\text { does not contain the } 2 \mathrm{~T}(\mathrm{r}) \text { displayed in Table } 5 \text {; these values come } \\
\text { from PDF card in Appendix } 6 \text {. Appendix } 5 \text { seems to merely } \\
\text { provide acceptance } 2 \mathrm{~T} \text { ranges. }\end{array}$ & \\
\hline 33 & Page 22 & $\mathrm{E}$ & $\begin{array}{l}\text { The explanation in paragraph that describes table headings would } \\
\text { be better if each heading was in a list (e.g., a) } 2 \mathrm{~T}(\mathrm{r})=\ldots \text { b) } 2 \mathrm{~T}(\mathrm{o})= \\
\ldots \text { etc. }\end{array}$ & \\
\hline 34 & Page 23 & $\mathrm{M}$ & Figure 17 should be Figure 8. & Dowe \\
\hline
\end{tabular}

Concur with comment resolutiog, review complete.


PNNL-20650, Rev. 2

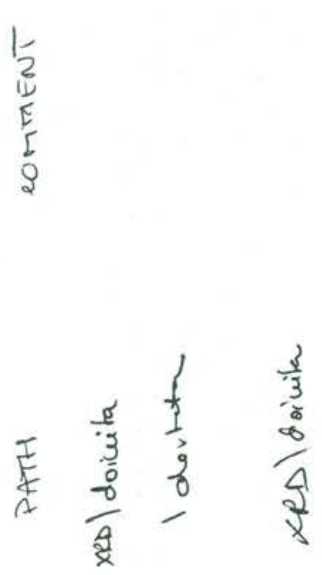

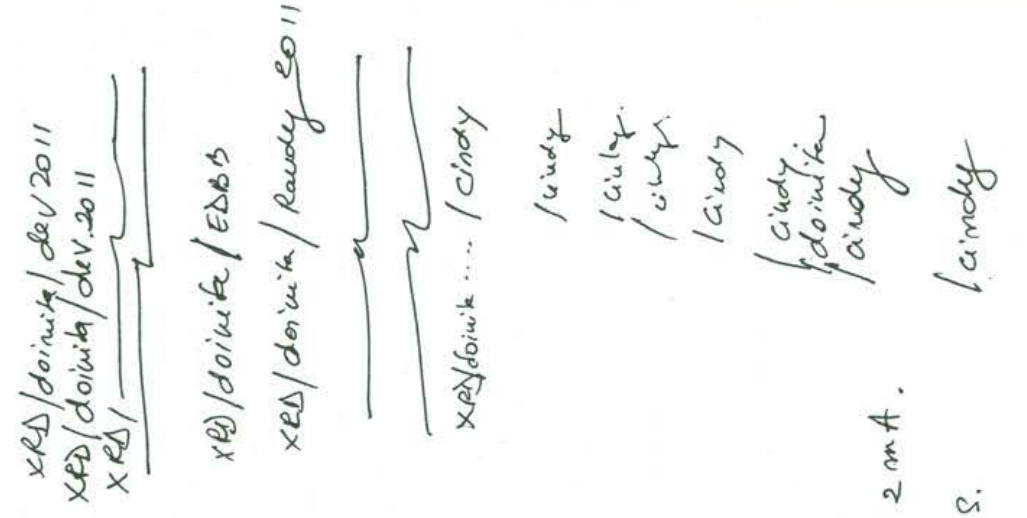

$n$
0
2
E
w

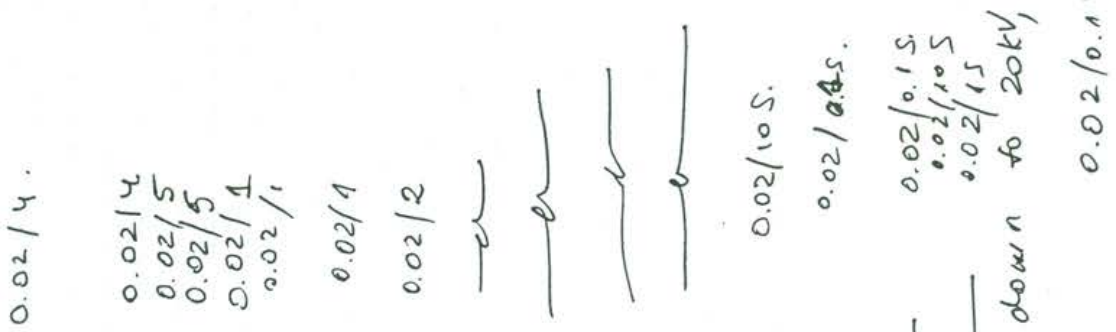

$\left\{\left[\begin{array}{l}1 \\ 1 \\ 3 \\ 3 \\ 8 \\ 8 \\ 3\end{array}\right.\right.$

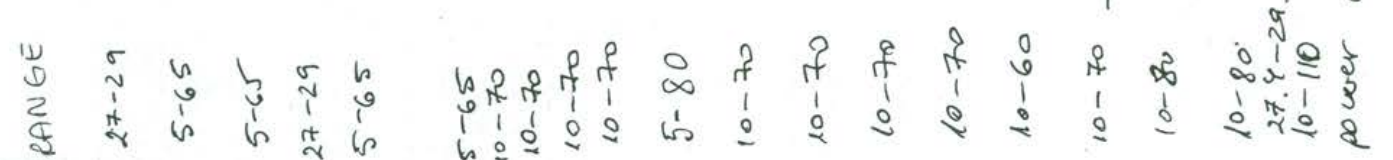

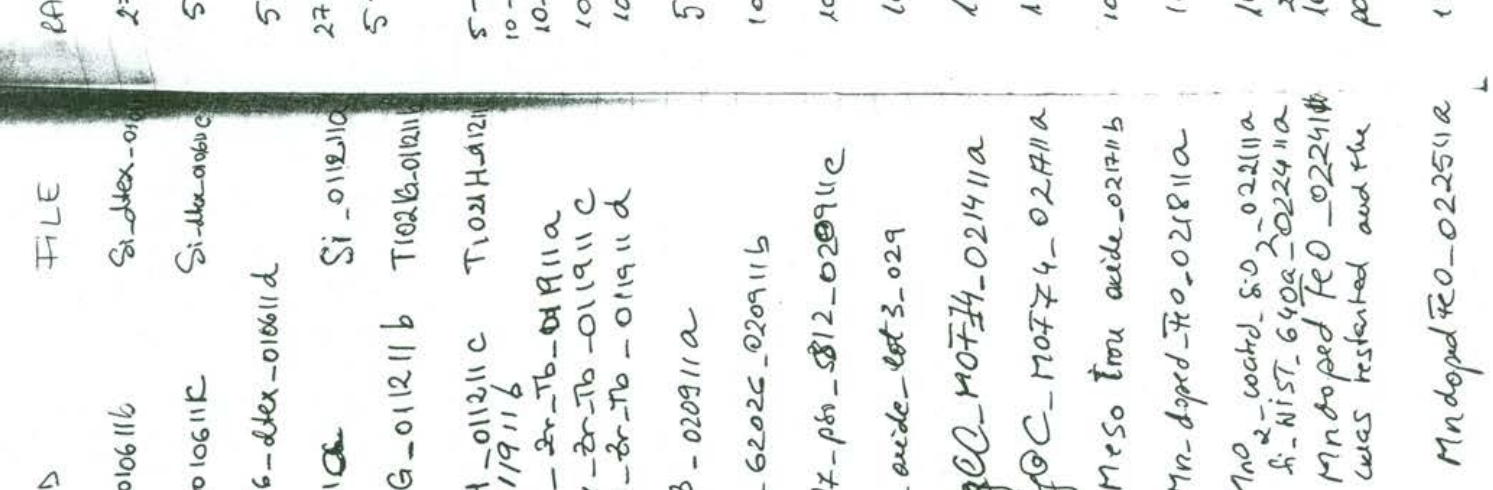

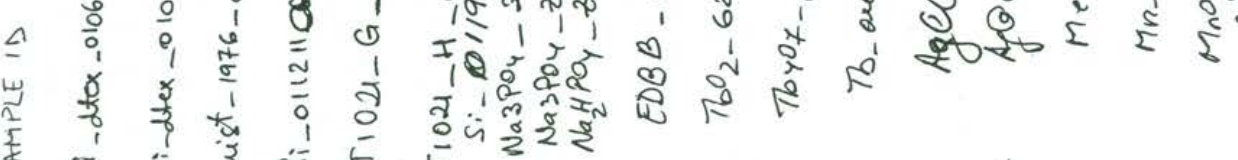

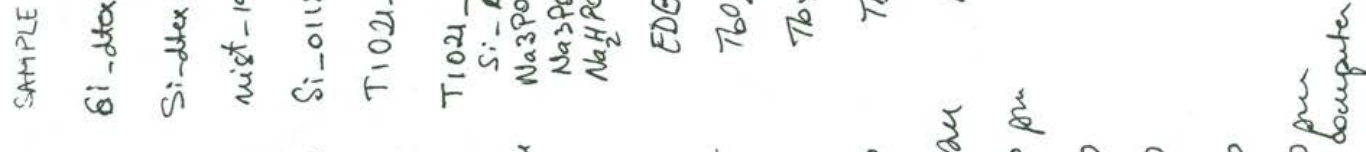

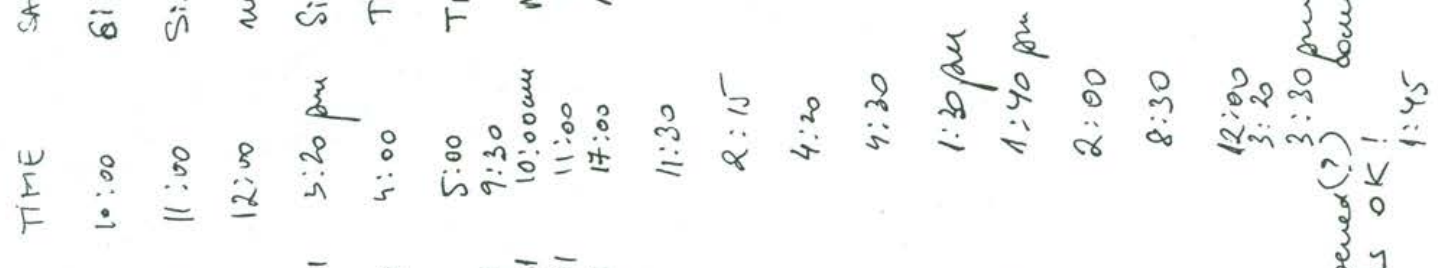

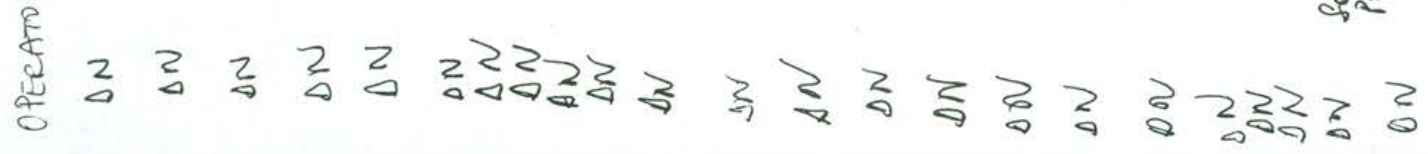


PNNL-20650, Rev. 2

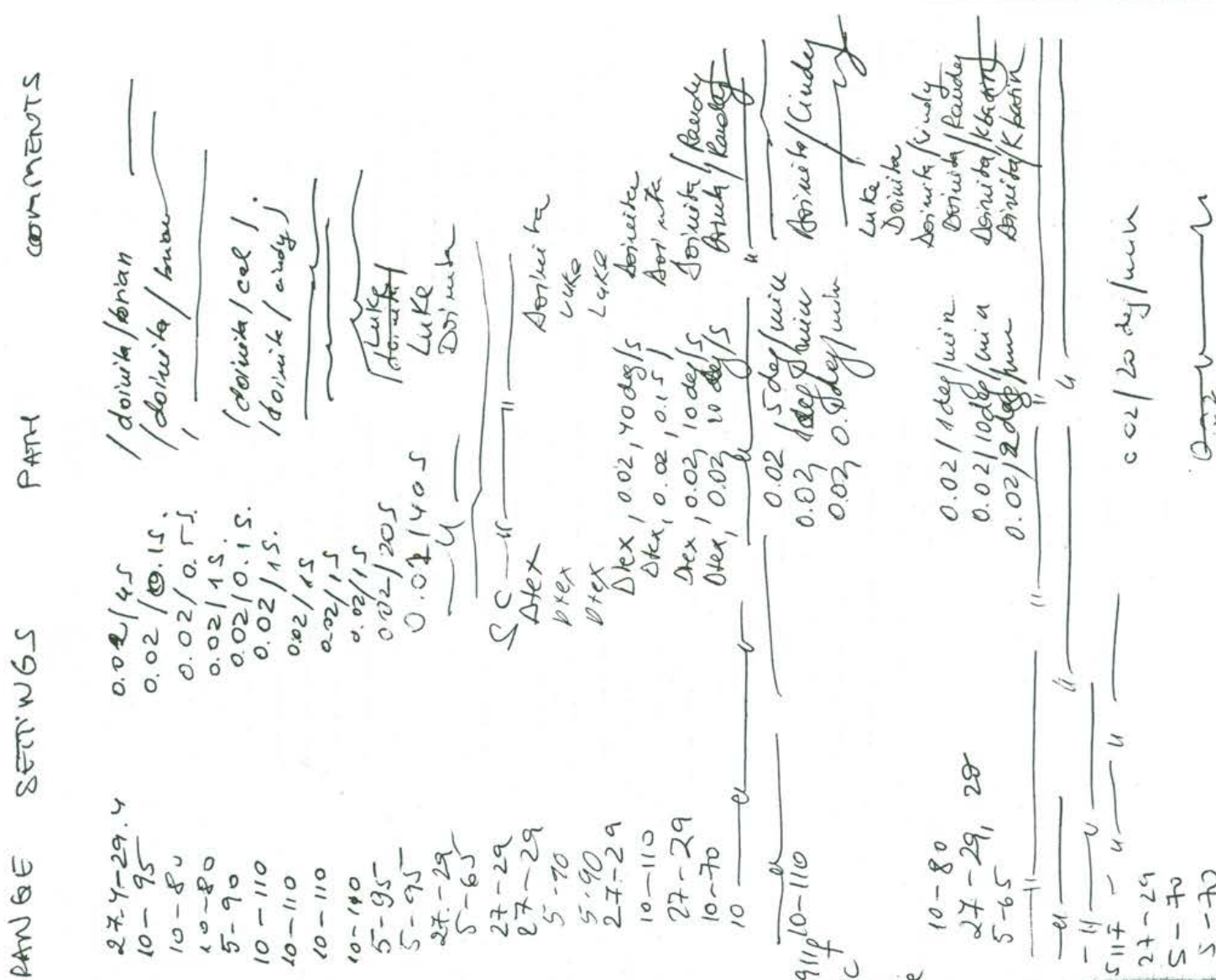

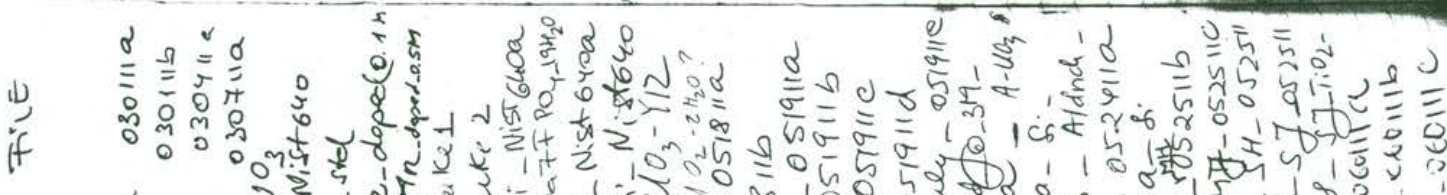

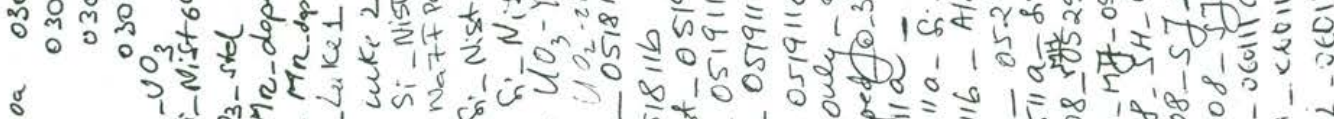

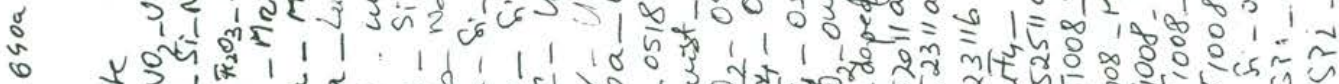
\%

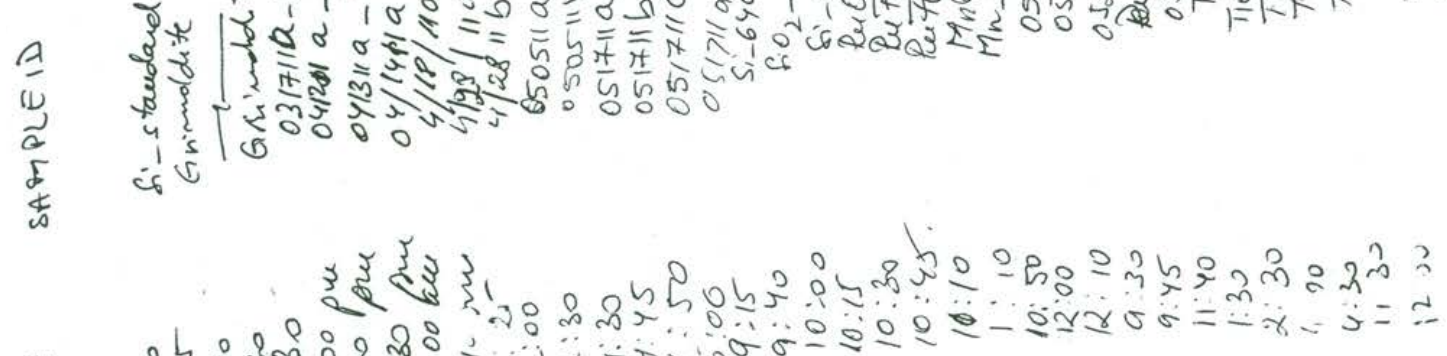

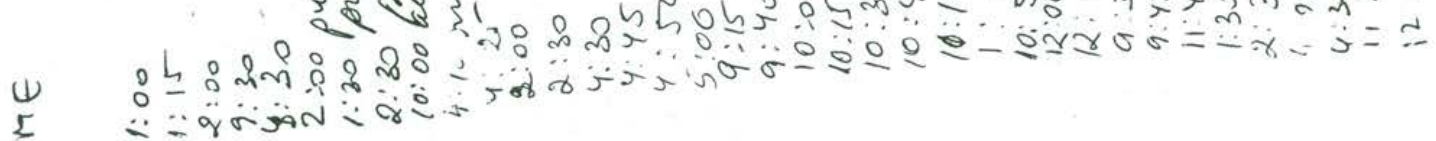
卢

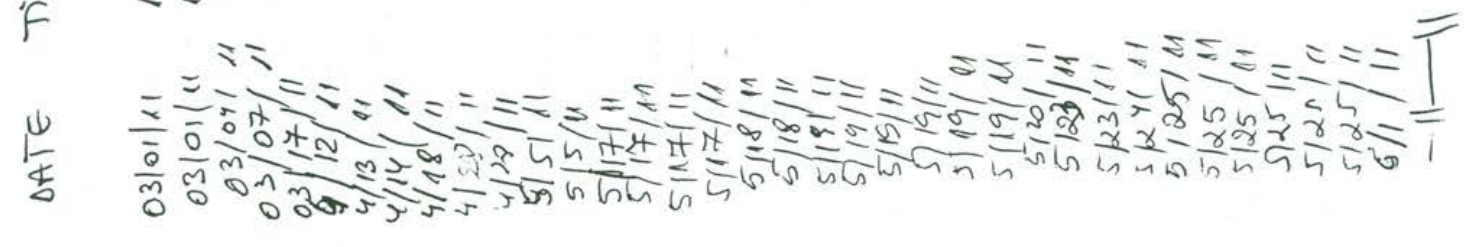

$\frac{d}{2}$ $j^{2}$
$j^{2}$
0 
PNNL-20650, Rev. 2

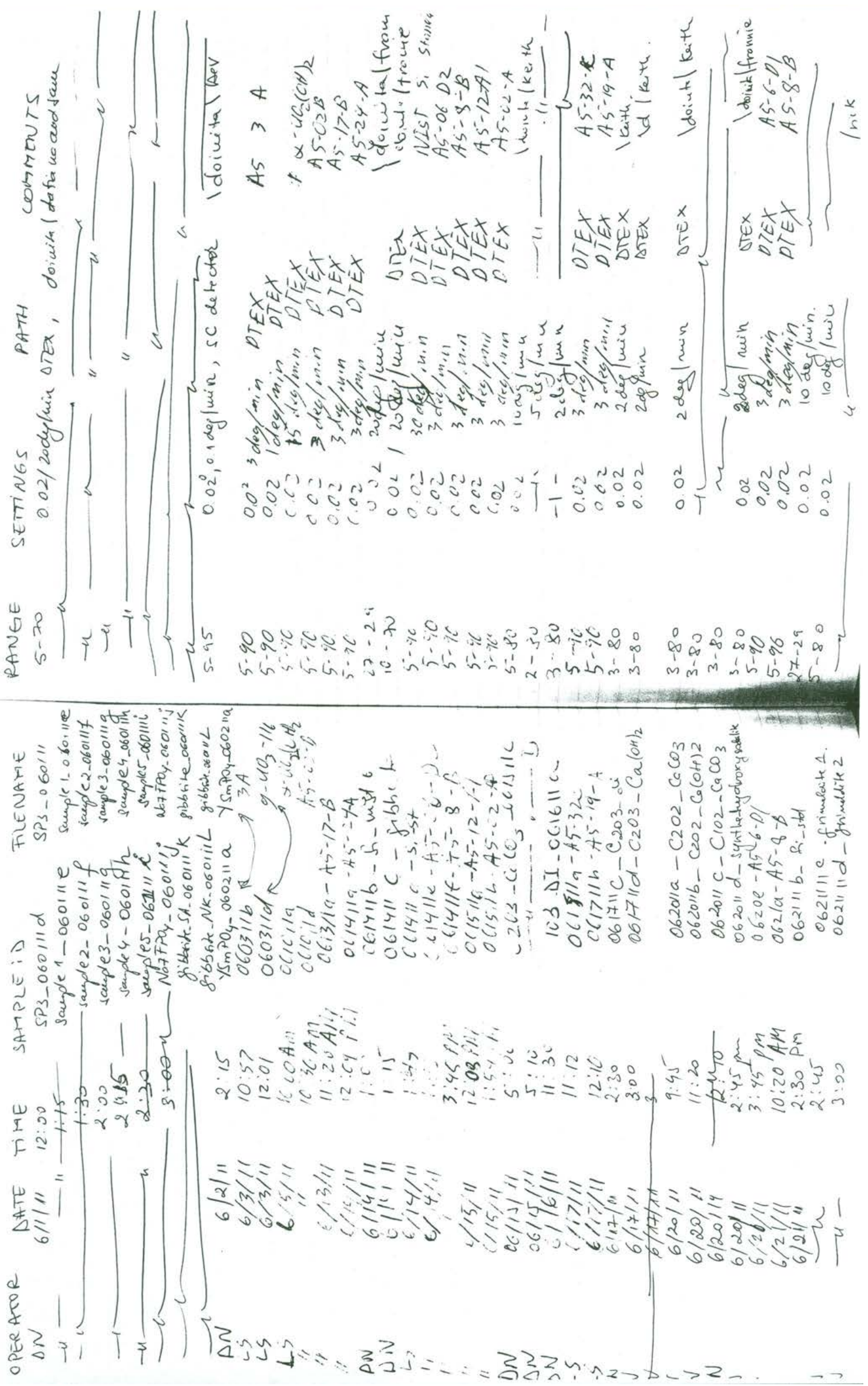


PNNL-20650, Rev. 2
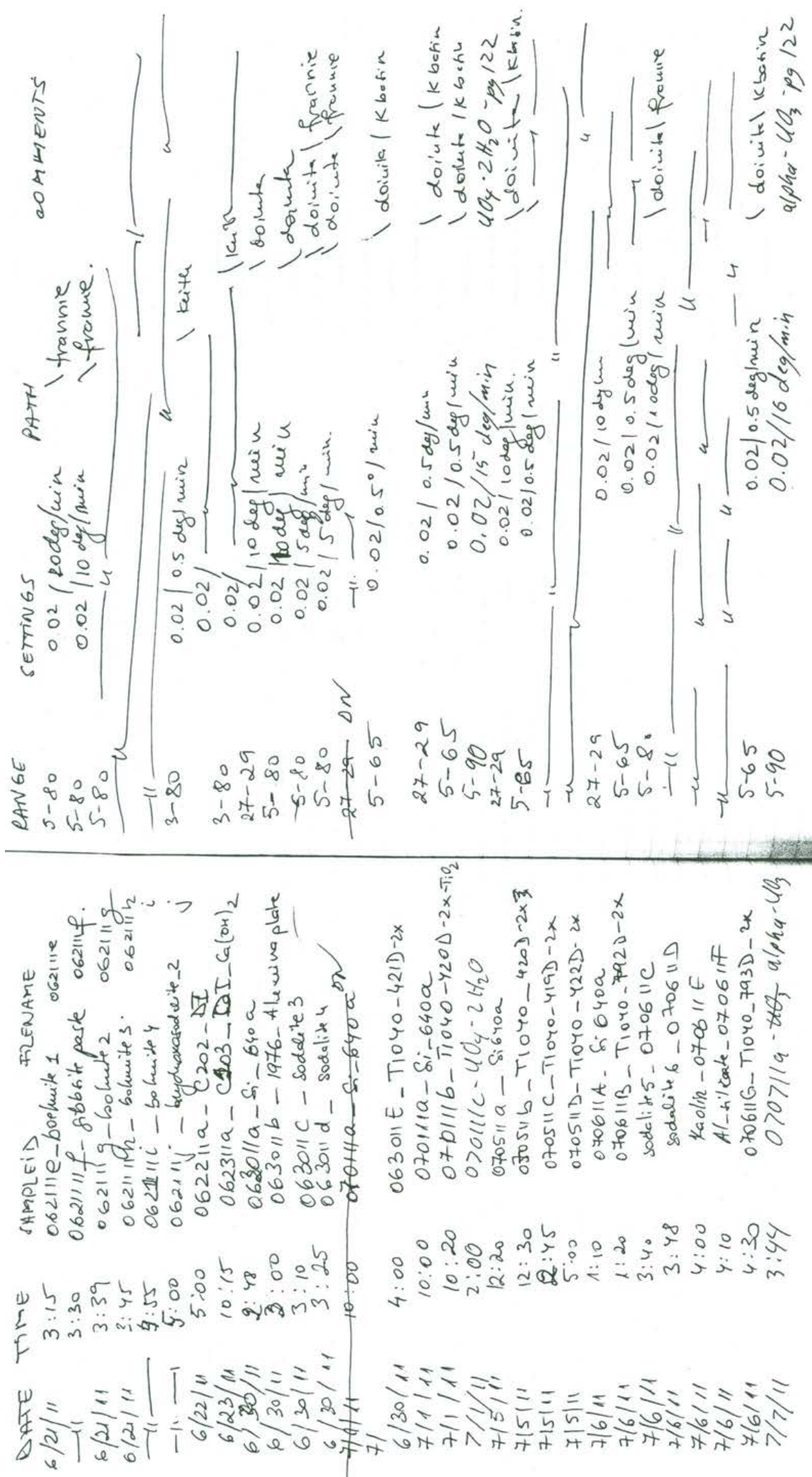

$d$
0
$d$
$d$

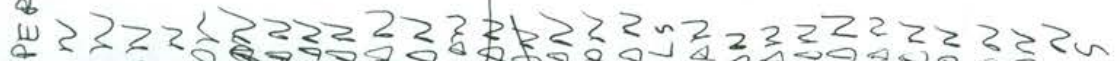




\section{PLANNING}

\section{Topic/Issue to be Evaluated: Surveillance of K Basin X-Ray Diffraction Analysis Report;} KW 210 Container Composites

This surveillance was undertaken to evaluate the draft X-Ray Diffraction (XRD) Analysis Report, KW 210 Container Composites for the samples listed in Table 1.

\section{PERFORMANCE}

\begin{tabular}{|l|l|}
\hline Date Initiated: 6 April 2011 & Location: RPL \\
\hline Date Received: 1 July 2011 & Date Reviewed: 6 \& 7 July 2011 \\
\hline Date Resubmitted: 20 July 2011 & Date Closed: 20 July 2011 \\
\hline Date Completed: 20 July 2011 & $\begin{array}{l}\text { DRAFT Report Issued: } 8 \text { July } 2011 \\
\text { FINAL Report Issued: } 20 \text { July } 2011\end{array}$ \\
\hline $\begin{array}{l}\text { Contacts:, Clark Carlson, Sandy Fiskum, } \\
\text { Doinita Neiner, Mike Urie }\end{array}$ & Org Code: PE137, D9H63 \\
\hline Surveillance Team: Lisa Middleton & \\
\hline
\end{tabular}

\section{PRELIMINARY INFORMATION}

Source of Requirement(s):

- KBC-33786, Rev. 2, December 2009; Quality Assurance Project Plan/Sampling and Analysis Plan (QAPjP/SAP): Quality Assurance Project Plan/Sampling and Analysis Plan for Containerized KW Settler Sludge

\section{Other Applicable Documents:}

- RPL-OP-001, Rev. 9, Routine Research Operations

- PNNL-RPG-268, Rev. 4, Solids Analysis by X-Ray Diffraction

- 52578-ADM-001, Rev. 0, K Basin Requests for Analysis and Guidelines for Data Reporting and Technical, $Q E$, and Spreadsheet Reviews. 
PNNL-20650, Rev. 2

Number: SR-52578-2011-029

Page 2 of 6

\section{Purpose/Scope:}

The 52578 Project QE staff are required via the QA Program to perform a review of K Basin reports generated within the Radiochemical Processing Laboratory (RPL) to determine traceability and accuracy of data contributing to the completed report.

The draft report was reviewed to evaluate that the data are what they purport to be, that is, that the reported results reflect what was actually done and the results meet applicable requirements. When deficiencies in the data are identified, then those deficiencies need to be documented as a K Basin Occurrence Report for the data user's review and, when applicable, resolved by corrective action. The XRD analysis under this Analysis Request was performed to identify crystalline structures in roomtemperature-dried sludge, to identify hydrate compounds plus any other identifiable compounds. This includes noting the presence (or absence) of aluminum hydrates, uranium hydrates, uranium oxides as well as zirconium metal.

When issues were found, the cognizant staff were contacted to resolve issues prior to completion of the review.

\section{Approach:}

The draft report initiated by a K Basin Analysis Request form dated 4/6/11 was provided as the basis for the data quality review. Four (4) samples were submitted on $4 / 6 / 11$ for analysis as noted in Table 1 :

Table 1: XRD Samples IDs \& Descriptions

\begin{tabular}{|c|c|c|c|c|c|}
\hline $\begin{array}{c}\text { Client } \\
\text { Sample ID }\end{array}$ & Sample Description & $\begin{array}{c}\text { XRD Sample } \\
\text { ID }\end{array}$ & $\begin{array}{c}\text { Internal } \\
\text { Standard }\end{array}$ & $\begin{array}{c}\text { Date } \\
\text { Analyzed }\end{array}$ & $\begin{array}{c}\text { Daily Performance } \\
\text { Check w/ 640a? }\end{array}$ \\
\hline TI008-MH & $\begin{array}{c}-2000 /+500 \mu \mathrm{m}, \text { air- } \\
\text { dried solids }\end{array}$ & TI008-MH & None & $5 / 25 / 11$ & $\mathrm{Y}$ \\
\hline TI008-MJ & $\begin{array}{c}-2000 /+500 \mu \mathrm{m}, \text { air- } \\
\text { dried solids duplicate }\end{array}$ & TI008-MJ & None & $5 / 25 / 11$ & $\mathrm{Y}$ \\
\hline TI008-SH & $-500 \mu \mathrm{m}$, wet slurry & T1008-SH & None & $5 / 25 / 11$ & $\mathrm{Y}$ \\
\hline \multirow{2}{*}{ TI008-SJ } & $\begin{array}{c}-500 \mu \mathrm{m}, \text { wet slurry } \\
\text { duplicate }\end{array}$ & TI008-SJ & None & $5 / 25 / 11$ & $\mathrm{Y}$ \\
\cline { 3 - 6 } & & TI008-SJ-TiO2 & Rutile & $5 / 25 / 11$ & $\mathrm{Y}$ \\
\hline
\end{tabular}

All samples were to be analyzed without internal standard, one sample (TI008-SJ) was to also be run with rutile, creating 5 samples total.

The report contained the following information:

1. Cover page

2. XRD Analysis Request Form/ XRD Mount Preparation Signed by Sandy Fiskum

3. XRD Analysis Bench Sheet - Sample Preparation

4. XRD Analysis Bench Sheet - Diffraction Measurements 
PNNL-20650, Rev. 2

Number: SR-52578-2011-029

Page 3 of 6

5. Report
a. Table 1 - Summary of Phases Identified in KW210 Container Composites
b. Sample preparation
c. X-Ray powder diffraction data collection
d. X-Ray powder diffraction data analysis
e. Summary of findings
f. Sample Analyses
g. Appendix 1 -SRM 674 NIST Certificate
h. Appendix 2 - Daily Performance Checks - 5/25/11
i. Appendix 3-SRM 640a NIST Certificate
j. Appendix 4-Quality Control/Calibration - 1/6/11
k. Appendix 5 - -SRM 1976 NIST Certificate
1. Appendix 6-PDF cards for the identified crystalline phases presented in report
m. DRR form signed by the Technical Reviewer, Mike Urie 4/29/11
n. Copy of XRD run log, 2 pages

M\&TE used:

\begin{tabular}{|c|c|c|c|c|}
\hline Location & Description & Manufacturer & Model & Inique ID \\
\hline RPL/409 & $\begin{array}{c}\text { X-Ray } \\
\text { Diffractometer }\end{array}$ & Rigaku & Ultima IV & $\begin{array}{c}\text { GD 3827N } \\
\text { /WD59716 }\end{array}$ \\
\hline & RGD & & & \#105 \\
\hline & SRM & NIST & $640 \mathrm{a}$ & $640 \mathrm{a}$ \\
\hline & SRM & NIST & 674 & 674 \\
\hline & SRM & NIST & 1976 & 1976 \\
\hline
\end{tabular}

Mike Urie was identified as the technical reviewer and this was documented with a DRR. During the QE review, it was found that 11 of the 34 comments from the DRR had not been addressed.

The completed surveillance report will be posted to the SharePoint site and submitted as a project record. Any documentation associated with this surveillance will be available in the Lead Auditor's office, Building 325, 5-G.

\section{RESULTS}

Findings are defined as a statement of fact relating to noncompliance with previously agreed-upon procedures, plans, codes, standards, specifications, or other forms of contractual or legal obligation. It should be understood that any lack of a finding in a specific area is not considered an indication that deficiencies do not exist.

Observations are defined as a weakness that if not corrected, could yield a departure from a requirement although the weakness is not necessarily a departure from requirements.

\section{Summary of Identified Concerns:}

Note: All concerns identified in the review were addressed in a comment resolution meeting on 20 July 2011, Deborah Coffey, Lisa Middleton and Doinita Neiner attended to disposition review comments. There were no outstanding identified findings or observations. 
PNNL-20650, Rev. 2

PROJECT 52578 - SURVEILLANCE REPORT

Number: SR-52578-2011-029

Page 4 of 6

1. A copy of the XRD logbook needs to be provided for review to verify calibration runs. Provided. Closed.

2. Analysis dates on cover page includes the sample preparation dates. Analysis was only done on $5 / 25 / 11$. This was also noted on the DRR (\#2), but not addressed. Analysis dates corrected to $5 / 25 / 11$ only. No further action required. Closed.

3. Page 2: The sample prep days are incorrect according to the bench sheets provided in pages $4 \& 5$ of the report. The only days listed for sample preparation are $5 / 19 / 11 \& 5 / 24 / 11$. There was no sample prep on $5 / 25 / 11$ so the sample prep dates should be $5 / 19 / 11-5 / 24 / 11$, not $5 / 19 / 11$ $5 / 25 / 11$. Sample prep fixed $(5 / 19 / 11-5 / 24 / 11)$. No further action required. Closed.

4. XRD Analysis Request Form: There are multiple typos in the Comments section; see comment copy of report. This was also noted on the DRR (\#3), but not addressed. Errors fixed by S Fiskum, new version of XRD Analysis Request Form included in report. No further action required. Closed.

5. XRD Analysis Request Form: The XRD Mount Preparation Section \& XRD Operating Parameters Section has not been completed. These sections should have been completed before analysis. Bottom section completed; section is redundant with use of new bench sheets. No further action required. Closed.

6. XRD Analysis Bench Sheets, pages 4-6: These are not the correct bench sheets. The format that was agreed upon has been resent in an e-mail dated 7/7/11. The section identifying which standard was used has been omitted. Correct bench sheets were provided, however, they still need to be paginated. Sheets paginated. No further action required. Closed.

7. Page 8: Table 1 is never addressed in the report, it needs an introduction. Figures can't just be put into a report without discussing them. Discussed. No further action required. Closed.

8. Page 8: Typo in $1^{\text {st }}$ paragraph. Change "has been" to "was". This was also noted on the DRR $(\# 10)$, but not addressed. Changed. No further action required. Closed.

9. Page 8: Add "prior to samples being submitted for XRD analysis" at the end of the second sentence. This was also noted on the DRR (\#11), but not addressed. Fixed adequately. No further action required. Closed.

10. Page 8: Typo: "slides" in the $4^{\text {th }}$ sentence of the $1^{\text {st }}$ paragraph should be singular, "slide". This was also noted on the DRR (\#10), but not addressed. Corrected. No further action required. Closed.

11. Sample Preparation, page 8: In the second sentence of the second paragraph, it says "Typically, the sample was mixed with approximately $2 \mathrm{~mL}$ of amyl acetate in a $20-\mathrm{mL}$ glass scintillation vial to produce a slurry." This statement implies that the above is the general protocol for sample preparation but that maybe it wasn't followed. Either the sample was mixed with amyl acetate to produce a slurry or it wasn't. This ambiguity needs to be cleared up. This was also noted on the 
PNNL-20650, Rev. 2

\section{PROJECT 52578 - SURVEILLANCE REPORT}

Number: SR-52578-2011-029

Page 5 of 6

DRR (\#12), but not addressed. Removed "typically," sample was mixed with amyl acetate. No further action required. Closed.

12. Page 9, Section $2,3^{\text {rd }}$ paragraph: Do not write out the name of the procedure after the $1^{\text {st }}$ time, only the procedure \# needs to be used. This was also noted on the DRR (\#17), but not addressed. Removed. No further action required. Closed.

13. Page 9 , Section $2,3^{\text {rd }}$ paragraph: The acronym ICDD needs to be defined. Defined as "International Center for Diffraction Data". No further action required. Closed.

14. Page 9 section $2,3^{\text {rd }}$ paragraph: Aforementioned is one word. This was also noted on the DRR (\#19), but not addressed. Corrected. No further action required. Closed.

15. Page 9, section 3.1: "...hydrates; zirconium metal, and zirconia..." should be changed to "...hydrates; and zirconium metal and zirconia..." to be consistent with the other entries in the sentence. This was also noted on the DRR (\#21), but not addressed. Changed. No further action required. Closed.

16. Page 9, section 3.1: The acronym PDF needs to be defined or changed to ICDD if they are interchangeable. Just using one term eliminates confusion. PDF defined as Powder Diffraction File. Not interchangeable. No further action required. Closed.

17. Page 9, section 3.2: What does "account for instrument profile" mean in this sentence? This was also noted on the DRR (\#22), but not addressed. Sentence removed. No further action required. Closed.

18. Page $10,1^{\text {st }}$ paragraph: Change PDF to ICDD. Discussed, leave as-is. No further action required. Closed.

19. Page 13: Add chemical formulas for uranium oxides as DRR \#27 suggests and is noted as "done". Formulas added. No further action required. Closed.

20. Page 22, Appendix 4: "Is" in the second sentence should be changed to "are". "Data" is plural. Changed to "are." No further action required. Closed. 
PNNL-20650, Rev. 2

PROJECT 52578 - SURVEILLANCE REPORT

Number: SR-52578-2011-029

Page 6 of 6

CORRECTIVE ACTION

[X] None Required. [ ] Complete [ ] Follow up Corrective Action

Surveillance Performed By:

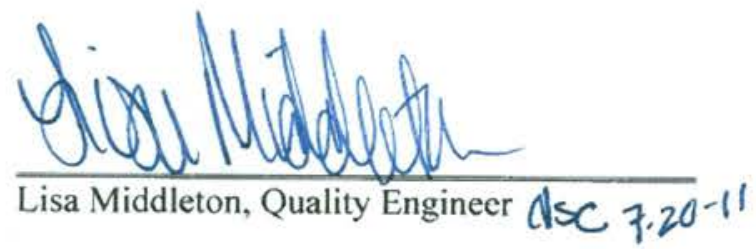

Date:

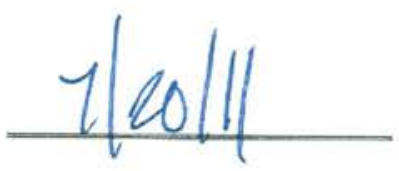

DISTRIBUTION: Clark Carlson, Sandy Fiskum, Doinita Neiner, Andy Schmidt, Rick Shimskey, Mike Urie, Deborah Coffey, Lisa Middleton 


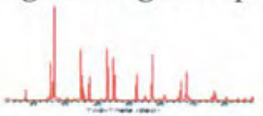

\section{X-Ray Diffraction Analysis Report}

\section{Battelle-Pacific Northwest National Laboratory Radiochemical Sciences and Engineering Group PO Box 999, Richland, Washington 99352}

Project: \#52578

Sample ID: KW 210 Undissolved Solids

Procedure: PNNL-RPG-268 Rev. 4, "Solids Analysis by X-Ray Diffraction"

Analyst: Doinita Neiner

Analysis Dates: 06/30/2011-07/06/2011

Instrument: RGD \# 105

Property Number: WD 59716

Serial Number: GD 3827N

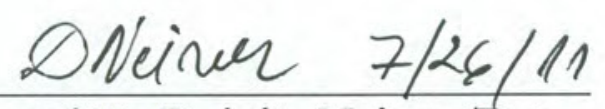

Prepared By Doinita Neiner/Date
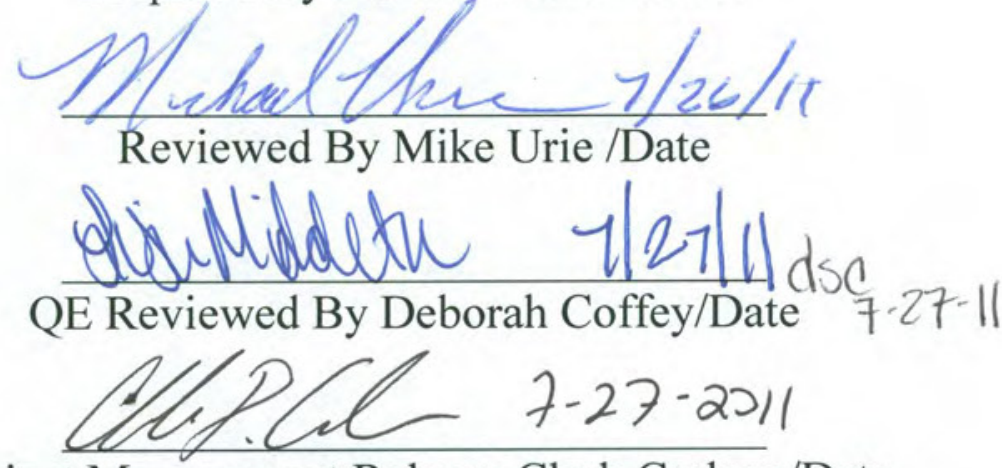

Project Management Release Clark Carlson/Date 
PNNL-20650, Rev. 2

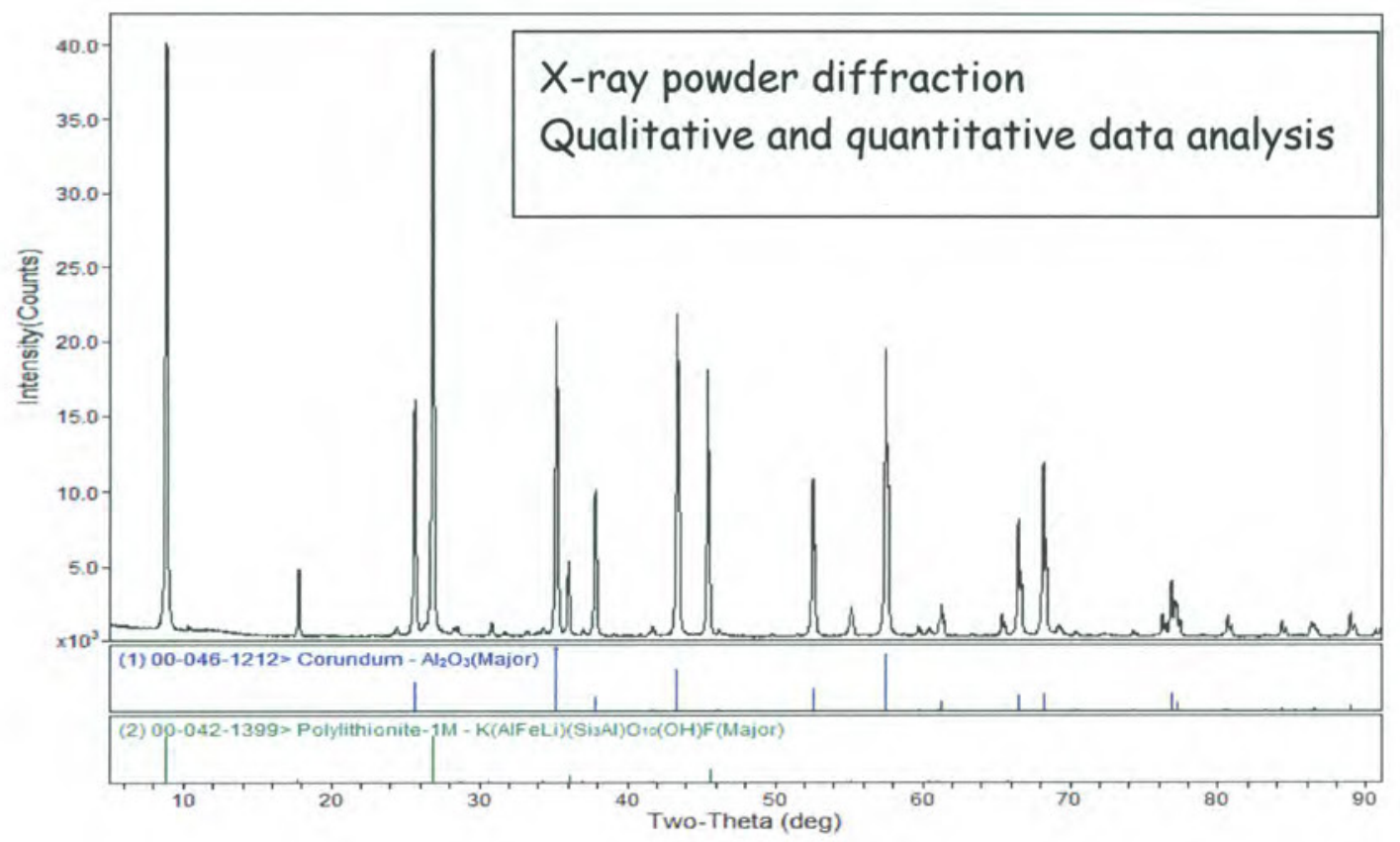

Project Number:

52578

Charge Code:

H41223

Client:

S. Fiskum

Total Samples:

6 solids (See results for Client IDs)

\begin{tabular}{|l|l|}
\hline Analysis Procedure & PNNL-RPG-268 Rev. 4, "Solids Analysis by X-Ray Diffraction" \\
\hline Prep Procedure & Slurry of amyl acetate and Krylon on a glass slide covered with Kapton \\
\hline Analyst & Doinita Neiner \\
\hline Analysis Date & June 30, 2011 through July 6, 2011 \\
\hline Sample Prep. Date & June 23, 2011 through June 30, 2011 \\
\hline Reference Standards & Rutile - SRM 674, PDF 01-077-0440 \\
\hline $\begin{array}{l}\text { Software } \\
\text { Reference database }\end{array}$ & Jade MDI 9 \\
\hline ICDD PDF-2 2009 \\
\hline
\end{tabular}


PNNL-20650, Rev. 2

XRD Analysis Request Form

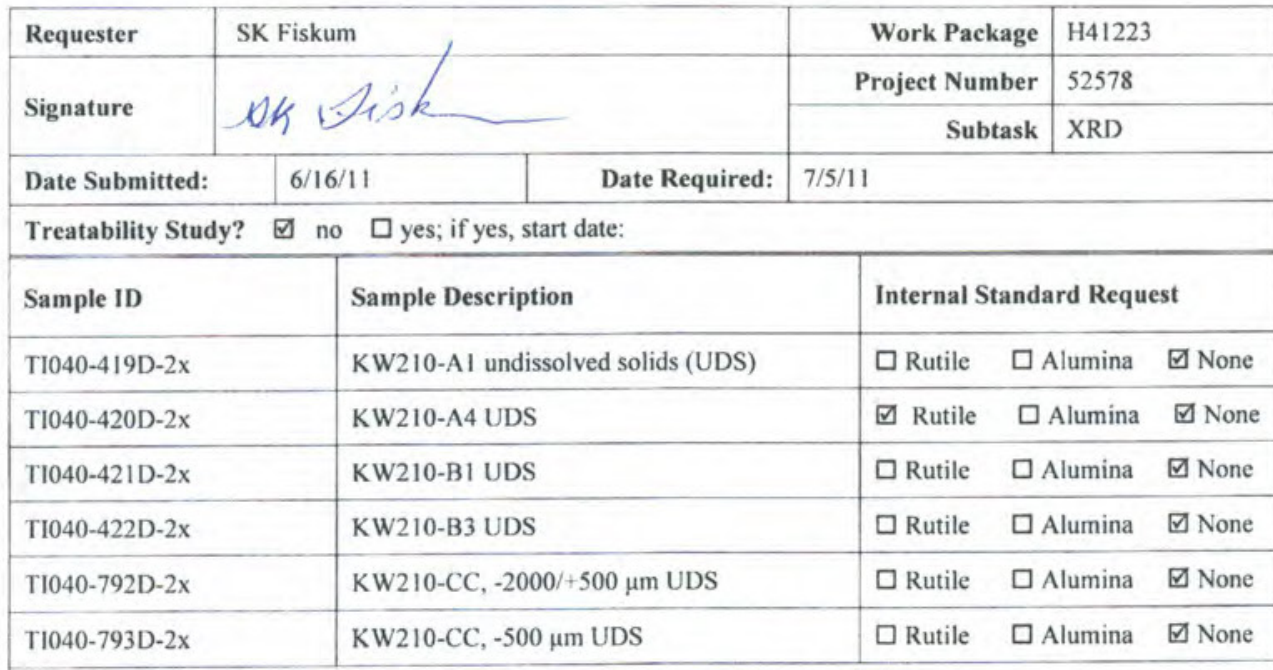

Comments: All samples are undissolved solids (UDS) remaining from the KW210 core samples and container composite samples that had been acid digested according to PNL-ALO-129. The UDS contained resin beads; these beads were manually removed (best effort basis) from the non-resin material.

Identify crystalline structures in room-temperature-dried sludge. Identify hydrate compounds plus any other identifiable compounds. This includes noting the presence (or absence) of aluminum hydrates, uranium hydrides, uranium oxides as well as zirconium metal. The analytical composition (ICP-OES analytes) will be provided upon availability. Uranium is expected to be a dominant component.

All samples are to be analyzed without internal standard; one sample (selected choice above can be altered depending on quantity of sample available) is to also be run with internal standard rutile $\left(\mathrm{TiO}_{2}\right)$. Rutile $\mathrm{X}$-ray diffraction angles and relative peak magnitudes will be used to help demonstrate acceptable system performance within the sample matrix and analysis process

Please use the new Rigaku system for powder diffraction analysis if at all possible. Please use a bench sheet you have developed to support sample processing and analysis and provide this original bench sheet as part of the data package.

Notes for Pattern Analyst:

XRD Mount preparation

Standard Reference Materials ID's: $\mathrm{TiO}_{2}: \underline{\mathrm{SRM} 674} \quad \mathrm{Al}_{2} \mathrm{O}_{3}: \underline{\mathrm{SRM} 676}$ Other:

Fixative: $\square$ Collodion Krylon $\square$ Other

Mount: Glass Slide, Kapton covered $\square$ PIP Holder $\square$ Other

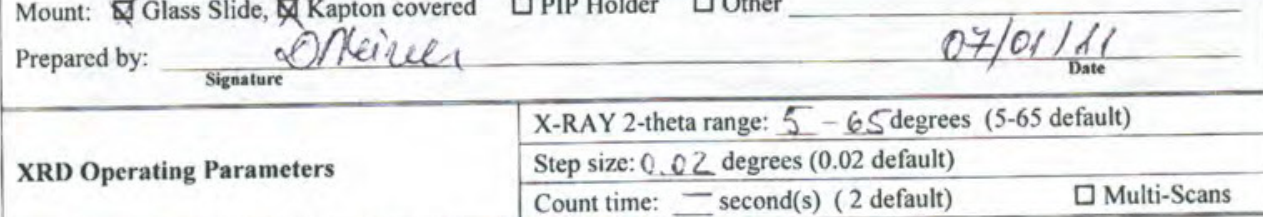

(Shaded areas to be filled out by the XRD analysis staff.) 
PNNL-20650, Rev. 2

Battelle - Pacific Northwest National Laboratory

Radiochemical Sciences and Engineering Group

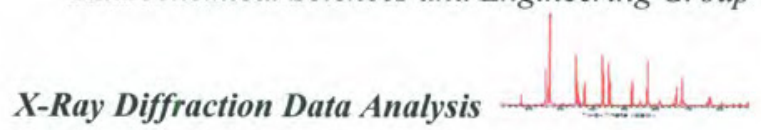

\section{XRD Analysis Bench Sheet \\ For Procedure PNNL-RPG-268, Rev. 4, \\ Solids Analysis by $X$-Ray Diffraction \\ Sample Preparation}

Print and complete pages as needed in order to document sample preparation for all samples submitted under one Analysis Request.

Sample ID(s) $T / 040-419 D-2 x$

Sample preparation date (grinding and mounting)

Sample dried air-dried $\square$

Internal standard: None $₫ \quad$ Rutile $\square \quad$ Alumina $\square \quad$ Other $\square$ describe

Standard ID:

Grinding method: Mortar \& Pestle $₫ \quad$ Mill $\square \quad$ Other $\square$ describe

Fixative: \Krylon $\square$ Other

Mount type: Slide $\not$ Other $\square$ describe

Notes:

XRD Mount ID(s) T1040-419D $-2 X$

Sample ID(s) T1040-4200-2x

Sample preparation date (grinding and mounting) $6 / 23 / 11$

Sample dried air-dried $\square$

Internal standard: None $₫ \quad$ Rutile $\square \quad$ Alumina $\square \quad$ Other $\square$ describe

Standard ID:

Grinding method: Mortar \& Pestle \ Mill $\square$ Other $\square$ describe

Fixative: $\bowtie$ Krylon $\square$ Other

Mount type: Slide $\bowtie \quad$ Other $\square$ describe

Notes:

XRD Mount ID(s) T/O40-420D-2X

Sample ID(s) $T / 040-4210-2 x$

Sample preparation date (grinding and mounting)

$6 / 23 / 11$

Sample dried air-dried $\square$

Internal standard: None $₫ \quad$ Rutile $\square \quad$ Alumina $\square \quad$ Other $\square$ describe

Standard ID:

Grinding method: Mortar \& Pestle $\bigotimes \quad$ Mill $\square \quad$ Other $\square$ describe

Fixative: \Krylon $\square$ Other

Mount type: Slide $\square \quad$ Other $\square$ describe

Notes:

XRD Mount ID(s) T/040-421D-2X

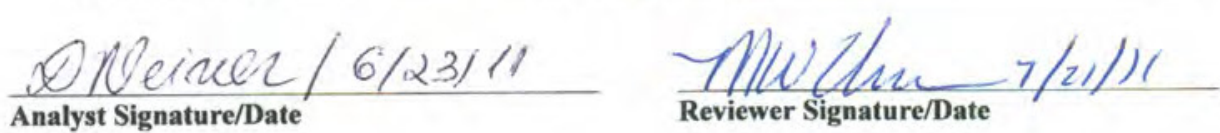


PNNL-20650, Rev. 2

Sample ID(s) T1040-420D-2X

Sample preparation date (grinding and mounting)

Sample dried air-dried $\square$

Internal standard: None $\square \quad$ Rutile $\mathbb{\bigotimes}$ Alumina $\square \quad$ Other $\square$ describe

Standard ID: $\mathrm{TiO}_{2}$

Grinding method: Mortar \& Pestle $\otimes \quad$ Mill $\square$ Other $\square$ describe

Fixative: बKrylon $\square$ Other

Mount type: Slide 风 Other $\square$ describe

Notes:

XRD Mount ID(s) $\quad 7 / 040-420 D-2 x-\mathrm{TiO}_{2}$

Sample ID(s) T, 040-422D-2x

Sample preparation date (grinding and mounting) $6 / 30 / 11$

Sample dried air-dried $\square$

Internal standard: None $\backslash \backslash \quad$ Rutile $\square \quad$ Alumina $\square \quad$ Other $\square$ describe

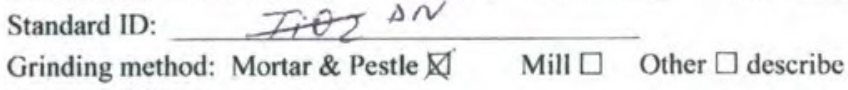

Fixative: $\triangle$ Krylon $\square$ Other

Mount type: Slide $\mathbb{W}$ Other $\square$ describe

Notes:

XRD Mount ID(s) T1040-422 $1-2 x$

Sample ID(s) T/040-792D-2x

Sample preparation date (grinding and mounting) $6 / 30 / 11$

Sample dried air-dried $\square$

Internal standard: None $凶 \quad$ Rutile $\square \quad$ Alumina $\square \quad$ Other $\square$ describe

Standard ID:

Grinding method: Mortar \& Pestle $\$ \quad$ Mill $\square \quad$ Other $\square$ describe

Fixative: $\triangle$ Krylon $\square$ Other

Mount type: Slide Other $\square$ describe

Notes:

XRD Mount ID(s) T/1040-792-2x

Sample ID(s) T/040-7931 - 2x

Sample preparation date (grinding and mounting)

Sample dried air-dried $₫ \square N$

Internal standard: None $\bowtie \quad$ Rutile $\square \quad$ Alumina $\square \quad$ Other $\square$ describe

Standard ID:

Grinding method: Mortar \& Pestle $\ \quad$ Mill $\square \quad$ Other $\square$ describe

Fixative: XKrylon $\square$ Other

Mount type: Slide $\triangle$ Other $\square$ describe

Notes:

XRD Mount ID(s) T/040-793-2x

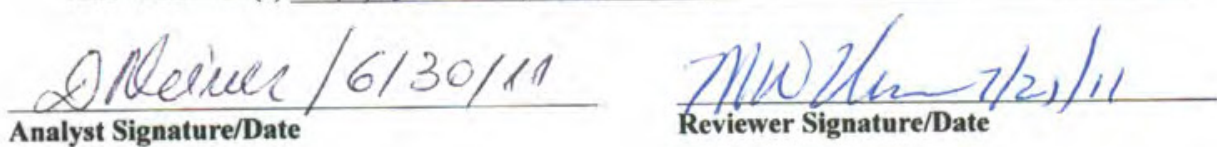


PNNL-20650, Rev. 2

\section{XRD Analysis Bench Sheet \\ For Procedure PNNL-RPG-268, Rev. 4, Solids Analysis by X-Ray Diffraction Diffraction Measurements}

Print and complete pages as needed in order to document sample analysis and daily calibration verification for all samples submitted under one Analysis Request.

Today's date: $06 / 30 / 11$

XRD daily performance checks with NIST standard run today? $\triangle \quad$ NIST standard: _Si 640a

Associated daily calibration check filename $063011 a-5,640 a$

Logbook updated? IX

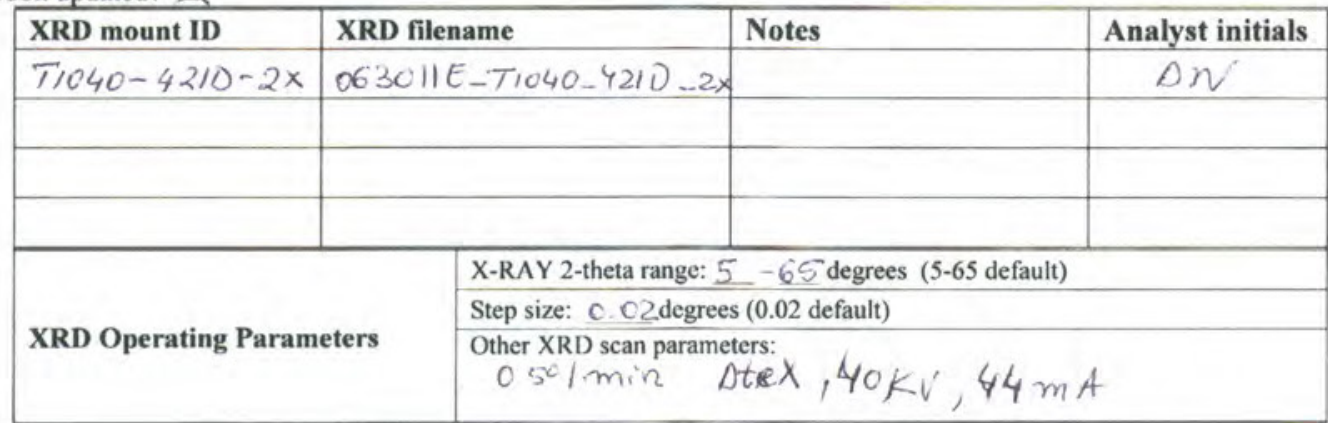

Today's date: 07/01/11

XRD daily performance checks with NIST standard run today? 叉 NIST standard: Si 640a Associated daily calibration check filename $070.111 \mathrm{a}-5,640$ a Logbook updated? $\mathbb{X}$

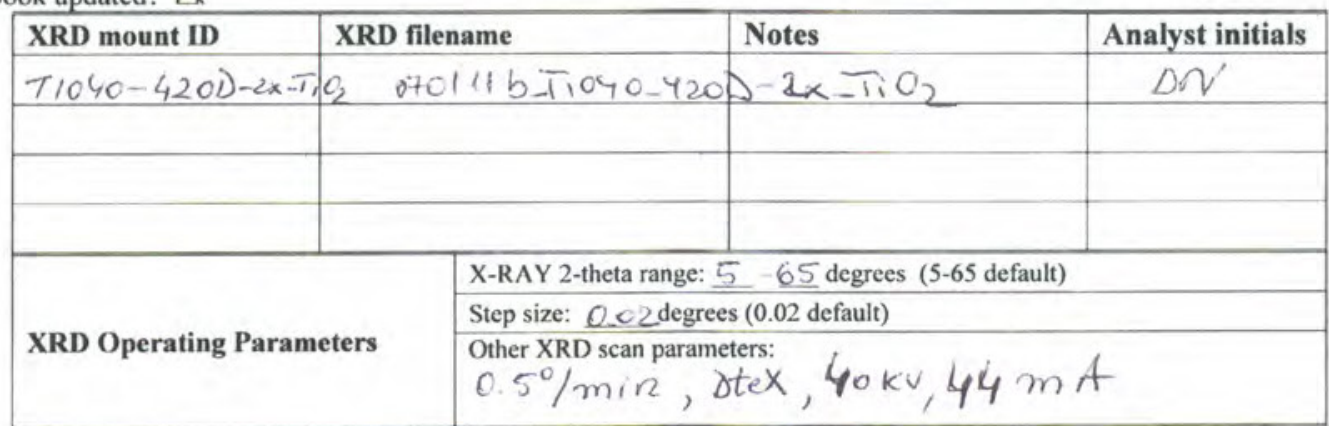
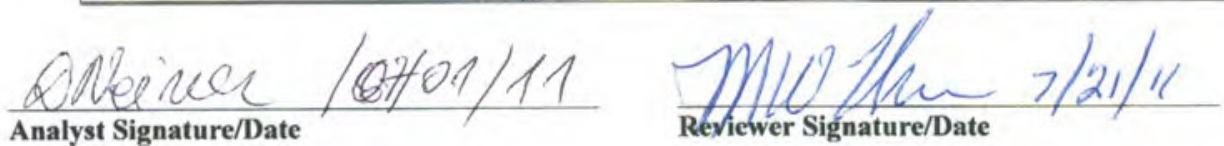
PNNL-20650, Rev. 2

Today's date: $07 / 05 / 11$

XRD daily performance checks with NIST standard run today? $\otimes \quad$ NIST standard: _Si 640a

Associated daily calibration check filename $070511 a-5 i 6$ Y0a

Logbook updated? $₫$

\begin{tabular}{|c|c|c|c|c|}
\hline XRD mount ID & \multicolumn{2}{|c|}{ XRD filename } & Notes & Analyst initials \\
\hline $71040-420-2 x$ & \multicolumn{3}{|c|}{$070511 b-11040-1201-2 x$} & $\Delta N$ \\
\hline $11040-4191]-2 x$ & \multicolumn{3}{|c|}{$07 \cos 110.11040-4(91)-2 x$} & DN \\
\hline $14040-422 D-2 x$ & \multicolumn{3}{|c|}{$070511 D-71040-422 D-2 x$} & $\Delta r$ \\
\hline \multirow{3}{*}{\multicolumn{2}{|c|}{ XRD Operating Parameters }} & \multicolumn{3}{|c|}{ X-RAY 2-theta range: $5 \quad 65$ degrees (5-65 default) } \\
\hline & & \multicolumn{3}{|c|}{ Step size: 0.02 degrees $(0.02$ default $)$} \\
\hline & & \multicolumn{3}{|c|}{$\begin{array}{l}\text { Other XRD scan parameters: } \\
0.5 \% \mathrm{~min}, D t e x, \text { yo } \mathrm{KV}, 4 \mathrm{~mA}\end{array}$} \\
\hline
\end{tabular}

Today's date: 07/06/11

XRD daily performance checks with NIST standard run today? $₫ \quad$ NIST standard: _Si 640a

Associated daily calibration check filename $070611 a-5 i 640 a$

Logbook updated? $\otimes$

\begin{tabular}{|c|c|c|c|c|}
\hline XRD mount ID & \multicolumn{2}{|c|}{ XRD filename } & Notes & Analyst initials \\
\hline$T 1040-792 D-2$ & \multicolumn{3}{|c|}{$x \quad 070611 B=-11040-792 D-2 x$} & $\Delta N$ \\
\hline$\pi 040-793 D-28$ & \multicolumn{3}{|c|}{$x 070611 G-11040=793 D-2 x$} & DN \\
\hline \multirow{3}{*}{\multicolumn{2}{|c|}{ XRD Operating Parameters }} & \multicolumn{3}{|c|}{ X-RAY 2-theta range: $5-65$ degrees (5-65 default) } \\
\hline & & \multicolumn{3}{|c|}{ Step size: 0.02 degrees $(0.02$ default $)$} \\
\hline & & \multicolumn{3}{|c|}{ 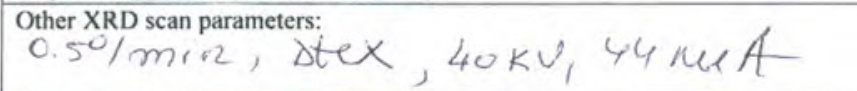 } \\
\hline
\end{tabular}

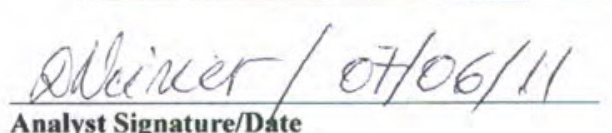

Analyst Signature/Date

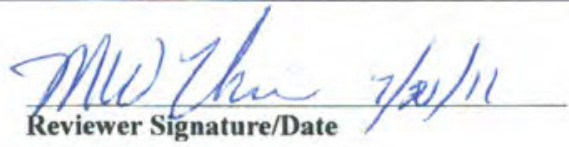


PNNL-20650, Rev. 2

Battelle - Pacific Northwest National Laboratory

Radiochemical Sciences and Engineering Group

X-Ray Diffraction Data Analysis

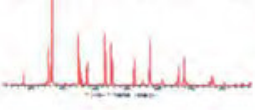

Table 1. Summary of Phases Identified in KW210 Undissolved Solids

\begin{tabular}{|c|c|c|c|c|}
\hline Sample ID & $\begin{array}{l}\text { PDF } \\
\text { Card } \\
\text { Number }\end{array}$ & $\begin{array}{l}\text { Relative } \\
\text { Intensity }^{(\text {a) }}\end{array}$ & Formula & $\begin{array}{l}\text { Common } \\
\text { Phase Name }\end{array}$ \\
\hline \multirow[t]{3}{*}{ TI040-419D-2X } & $01-070-3755$ & Major & $\mathrm{SiO}_{2}$ & Quartz \\
\hline & $01-089-1460$ & Major & $\mathrm{Ca}\left(\mathrm{Al}_{2} \mathrm{Si}_{2} \mathrm{O}_{8}\right)$ & Anorthite \\
\hline & -- & Unassigned & & \\
\hline \multirow[t]{5}{*}{ TI040-420D-2X } & $01-070-3755$ & Major & $\mathrm{SiO}_{2}$ & Quartz \\
\hline & 01-089-1460 & Major & $\mathrm{Ca}\left(\mathrm{Al}_{2} \mathrm{Si}_{2} \mathrm{O}_{8}\right)$ & Anorthite \\
\hline & 01-071-1403 & Minor & $\mathrm{Ca}_{0.466} \mathrm{Fe}_{0.5185} \mathrm{Al}_{0.815} \mathrm{Na}_{0.534} \mathrm{O}_{6} \mathrm{Si}_{2}$ & Aegerine \\
\hline & 01-070-0909 & Minor & $\mathrm{CaSO}_{4}$ & Calcium sulfate \\
\hline & -- & Unassigned & & \\
\hline \multirow[t]{3}{*}{ TI040-421D-2X } & $01-070-3755$ & Major & $\mathrm{SiO}_{2}$ & Quartz \\
\hline & 01-089-1460 & Major & $\mathrm{Ca}\left(\mathrm{Al}_{2} \mathrm{Si}_{2} \mathrm{O}_{8}\right)$ & Anorthite \\
\hline & -- & Unassigned & & \\
\hline \multirow[t]{4}{*}{ TI040-422D-2X } & 01-070-3755 & Major & $\mathrm{SiO}_{2}$ & Quartz \\
\hline & 01-089-1460 & Major & $\mathrm{Ca}\left(\mathrm{Al}_{2} \mathrm{Si}_{2} \mathrm{O}_{8}\right)$ & Anorthite \\
\hline & 01-071-1403 & Minor & $\mathrm{Ca}_{0.466} \mathrm{Fe}_{0.5185} \mathrm{Al}_{0.815} \mathrm{Na}_{0.534} \mathrm{O}_{6} \mathrm{Si}_{2}$ & Aegerine \\
\hline & -- & Unassigned & & \\
\hline \multirow{4}{*}{ TI040-792D-2X } & 01-070-3755 & Major & $\mathrm{SiO}_{2}$ & Quartz \\
\hline & $01-089-1460$ & Major & $\mathrm{Ca}\left(\mathrm{Al}_{2} \mathrm{Si}_{2} \mathrm{O}_{8}\right)$ & Anorthite \\
\hline & 01-071-1403 & Minor & $\mathrm{Ca}_{0.466} \mathrm{Fe}_{0.5185} \mathrm{Al}_{0.815} \mathrm{Na}_{0.534} \mathrm{O}_{6} \mathrm{Si}_{2}$ & Aegerine \\
\hline & -- & Unassigned & & \\
\hline \multirow{4}{*}{ TI040-793D-2X } & 01-070-3755 & Major & $\mathrm{SiO}_{2}$ & Quartz \\
\hline & 01-089-1460 & Major & $\mathrm{Ca}\left(\mathrm{Al}_{2} \mathrm{Si}_{2} \mathrm{O}_{8}\right)$ & Anorthite \\
\hline & $01-071-1403$ & Minor & $\mathrm{Ca}_{0.466} \mathrm{Fe}_{0.5185} \mathrm{Al}_{0.815} \mathrm{Na}_{0.534} \mathrm{O}_{6} \mathrm{Si}_{2}$ & Aegerine \\
\hline & -- & Unassigned & & \\
\hline
\end{tabular}

(a) Crystalline components above $10 \mathrm{wt} . \%$ are classified as Major phases, between 1-10 wt.\% as Minor phases, and under $1 \mathrm{wt} . \%$ as Possible phases. 


\section{Sample preparation}

X-ray powder diffraction (XRD) data from the samples identified in Table 2 have been examined for identification of crystalline phases present. The samples were undisolved solids and they were used as received.

All the samples were processed and analyzed according to procedure, Solids Analysis by X-Ray Diffraction, PNNL-RPG-268, Rev. 4. Each sample was mixed with approximately $2 \mathrm{~mL}$ of amyl acetate in a $20-\mathrm{mL}$ glass scintillation vial to produce a slurry. A small aliquot of the slurry was transferred into a mortar and ground with a pestle (all tungsten carbide components). After grinding, a small fraction of the sample was pipetted onto a Krylon-covered glass slide and allowed to dry. The dried samples were then covered with Kapton. One client sample, TI040$420 \mathrm{D}-2 \mathrm{X}$ was run in duplicate (one without internal standard and one with 3-4 $\mathrm{mg} \mathrm{TiO}_{2}$, rutile as internal standard). Titania (rutile structure) internal standard SRM 674 was used; the standard certificate is provided in Appendix 1.

Table 2. Sample Identification

\begin{tabular}{|l|l|c|}
\hline Sample ID & XRD Sample ID & Internal Standard \\
\hline \multirow{2}{*}{ TI040-419D-2X } & TI040-419D-2X & None \\
\hline & TI040-420D-2X & None \\
\cline { 2 - 3 } TI040-420D-2X & TI040-420D-2X-TiO 2 & TiO $_{2}$ \\
\hline \multirow{2}{*}{ TI040-421D-2X } & TI040-421D-2X & None \\
\hline \multirow{2}{*}{ TI040-422D-2X } & TI040-422D-2X & None \\
\hline TI040-792D-2X & TI040-792D-2X & None \\
\hline TI040-793D-2X & TI040-793D-2X & None \\
\hline
\end{tabular}


PNNL-20650, Rev. 2

Battelle - Pacific Northwest National Laboratory

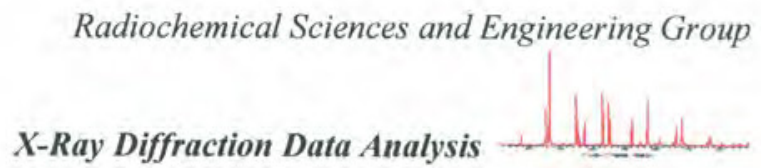

\section{X-ray powder diffraction data collection}

Each sample was analyzed using a Rigaku Ultima IV diffractometer XRD unit equipped with a high speed position sensitive detector system $\mathrm{D} / \mathrm{teX}$ and a copper X-ray tube $(\mathrm{Cu} \mathrm{K} \alpha$ radiation, $\lambda$ $=1.5418 \AA$ ). Data were collected in a step scan mode between 5 and $65^{\circ} 2$-theta with a step size of $0.02^{\circ}$, a speed of $0.5^{\circ} /$ minute. The X-ray tube operating conditions were $40 \mathrm{kV}$ and $44 \mathrm{~mA}$.

The daily performance checks associated with the XRD sample files are presented in Appendix 2. These performance checks meet the performance criteria (i.e., $111 \mathrm{Si} 2$-theta $=$ $28.44^{\circ} \pm 0.06^{\circ}$ ) specified in the XRD procedure PNNL-RPG-268 Rev. 4. The NIST certificate for the standard used for the daily performance check (SRM 640a) is presented in Appendix 3.

The quality control/calibration of the XRD instrument results are provided in Appendix 4 and the certificate for the standard used, SRM 1976, is presented in Appendix 5. The requirement for the semiannual calibration of the diffractometer (as stated in the PNNL-RPG-268 Rev. 4, is that observed d-interplanar distance for any given crystal lattice Miller indices be within $\pm 0.02 \AA$ (Del-d value - difference between the calculated and the observed interplanar distance) of the dvalues from the International Centre for Diffraction Data (ICDD) card for alumina (corundum structure). For this quality control semiannual calibration, all Del-d values are well within the aforementioned criterion $( \pm 0.02 \AA$ Del-d value $)$.

\section{X-ray powder diffraction data analysis}

\subsection{Database Search Details}

Phase identification was conducted using JADE search match routines. The search was done including the following elements: $\mathrm{C}, \mathrm{Na}, \mathrm{K}, \mathrm{Mg}, \mathrm{Ca}, \mathrm{Al}, \mathrm{Sr}, \mathrm{Si}, \mathrm{O}, \mathrm{H}, \mathrm{Cd}, \mathrm{Cr}, \mathrm{Ba}, \mathrm{Cu}, \mathrm{Ni}, \mathrm{Pb}, \mathrm{Mn}$, $\mathrm{Fe}, \mathrm{Zn}, \mathrm{Zr}$, Ti and $\mathrm{U}$. All diffraction patterns have been compared against International Centre for Diffraction Data Powder Diffraction File (ICDD PDF-2) 2009 database. All of the XRD analyses included the search for uranium oxides, oxyhydroxides, hydrates and hydroxides; aluminum oxides, oxyhydroxides and hydrates; and zirconium metal and zirconia (from cladding materials). The PDF cards of the crystalline phases identified in the samples analyzed in this report are given in Appendix 6.

\subsection{Rietveld Refinement Details}

The Rietveld method effectively separates overlapping data, thereby allowing an accurate determination of the structure/composition. Rietveld refinement on powder materials is a widely used application in determining the components of chemical mixtures. The refinement takes into account the PDF cards of the identified phases, and compares those with the calculated peaks from the observed powder pattern. The refinement generates a difference plot between an observed and a calculated powder pattern. In addition, because it calculates a powder pattern from the PDF cards of the phases taken into consideration it gives a goodness of fit for that particular phase, which is a reflection of how good the fit is between the observed and the calculated powder patterns. The refined parameters are the unit cell parameters, background, and 


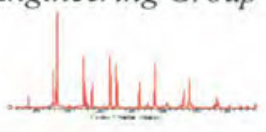

in the observed powder pattern, by comparing all peak positions and intensities with calculated values (from the PDF card). The quality of the refinement can be judged based on the R factors (e.g., $R_{w}$ ) and the difference map (i.e., the difference between an observed and a calculated powder pattern). Typically, the better the Rietveld fit the lower the $R_{w}$ factor; $R_{w}<20 \%$ suggests a good fit, $R_{w}<10 \%$ suggests an excellent fit. $R_{w}$ values $<20 \%$ have to be supported by a small difference between the calculated and observed powder patterns (i.e., minor deviations within the difference map).

\section{Summary of findings}

The analysis samples represent the undissolved solids from the KW210 core and container composite after gross resin bead removal. The XRD data on these samples are presented in Figure 1. The samples contain primarily quartz. The samples do not contain uranium oxyhydroxides, hydrates and hydroxides; aluminum oxides, oxyhydroxides and hydrates; or zirconium metal and zirconia as crystalline phases. A summary of phases is presented in Table 1. Per request the powder patterns of the core samples and the container composite samples were presented in two different figures: Figures 15 and 16 (see Appendix 7).

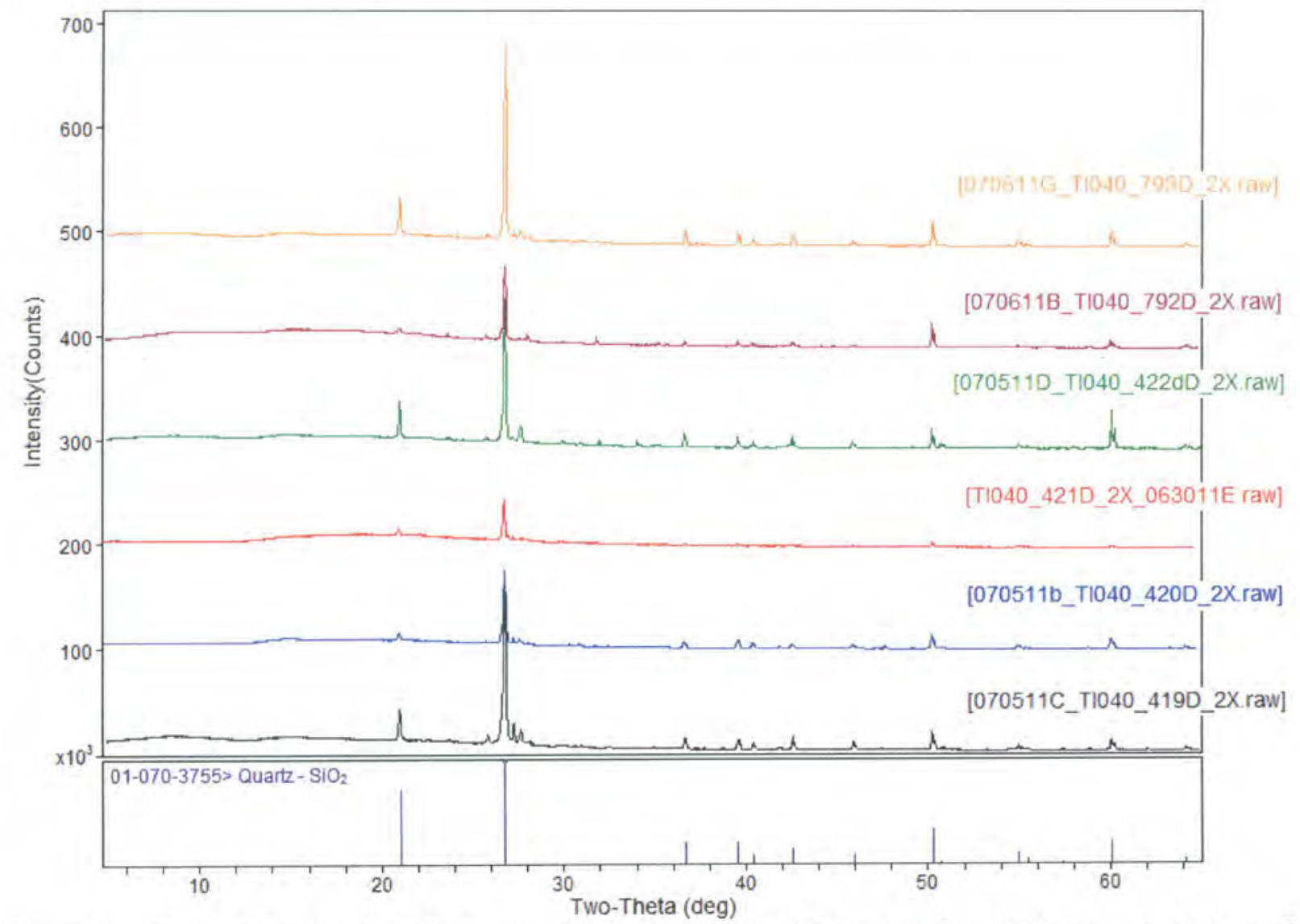

Figure 1. Raw XRD data for all the samples analyzed in this report. No background subtracted. 
PNNL-20650, Rev. 2

Battelle - Pacific Northwest National Laboratory

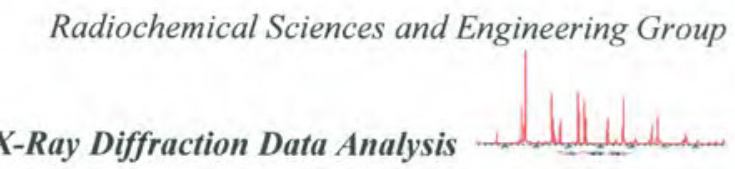

The following table summarizes the sample analysis-related figures present in this report.

Table 3. Summary of Figures

\begin{tabular}{|l|l|}
\hline Client Sample ID & Figures \\
\hline TI040-419D-2X & $2,3,15$ \\
\hline TI040-420D-2X & $4,5,15$ \\
\hline TI040-421D-2X & 6,15 \\
\hline TI040-422D-2X & 7,15 \\
\hline TI040-792D-2X & 8,16 \\
\hline TI040-793D-2X & 9,16 \\
\hline
\end{tabular}


PNNL-20650, Rev. 2

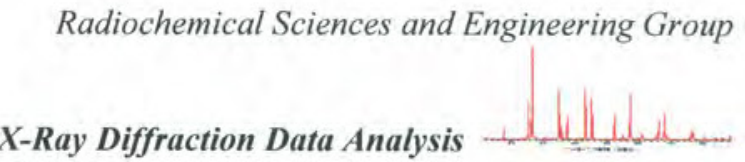

\section{XRD Sample Analysis}

\subsection{XRD Sample TI040-419D-2X}

Figure 2 presents the raw XRD data for sample TI040-419D-2X along with the PDF cards of the crystalline phases identified based on the Rietveld refinement $\left(R_{w}=13 \%\right)$. This sample is composed of silica (quartz) and anorthite as major phases. This composition has been previously observed in Hanford site sand. Figure 3 presents a plot of the Hanford site sand and sample TI040-419D-2X for comparison.

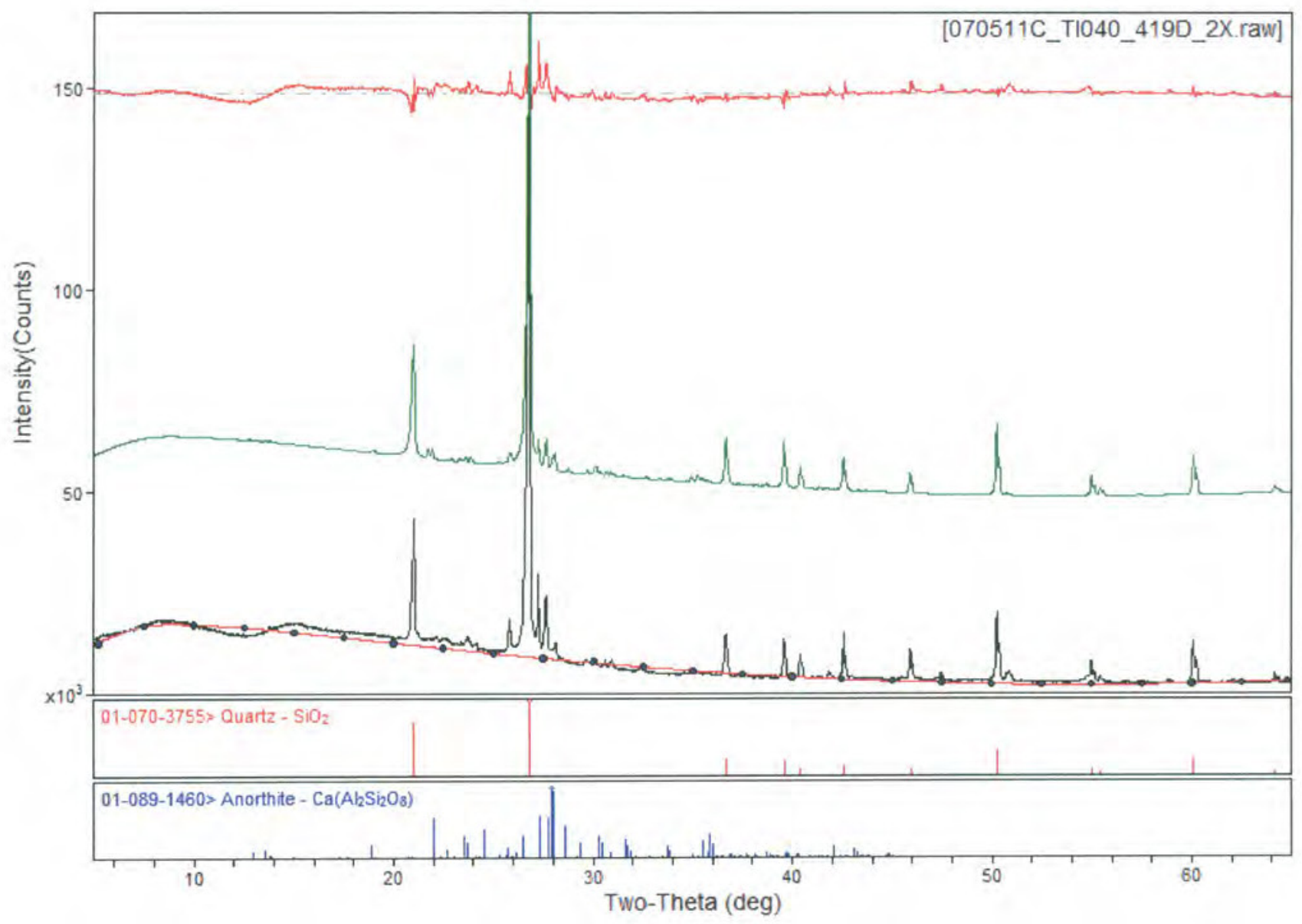

Figure 2. Rietveld refinement of the XRD data for TI040-419D-2X. The black line is the observed powder pattern (the red line underneath the black line is the background), the green line is the simulated powder pattern based on the model, and the top red line is the difference between the calculated and the observed powder patterns. $\mathrm{R}_{\mathrm{w}}=13 \%$ (See Section 3.2). 
PNNL-20650, Rev. 2
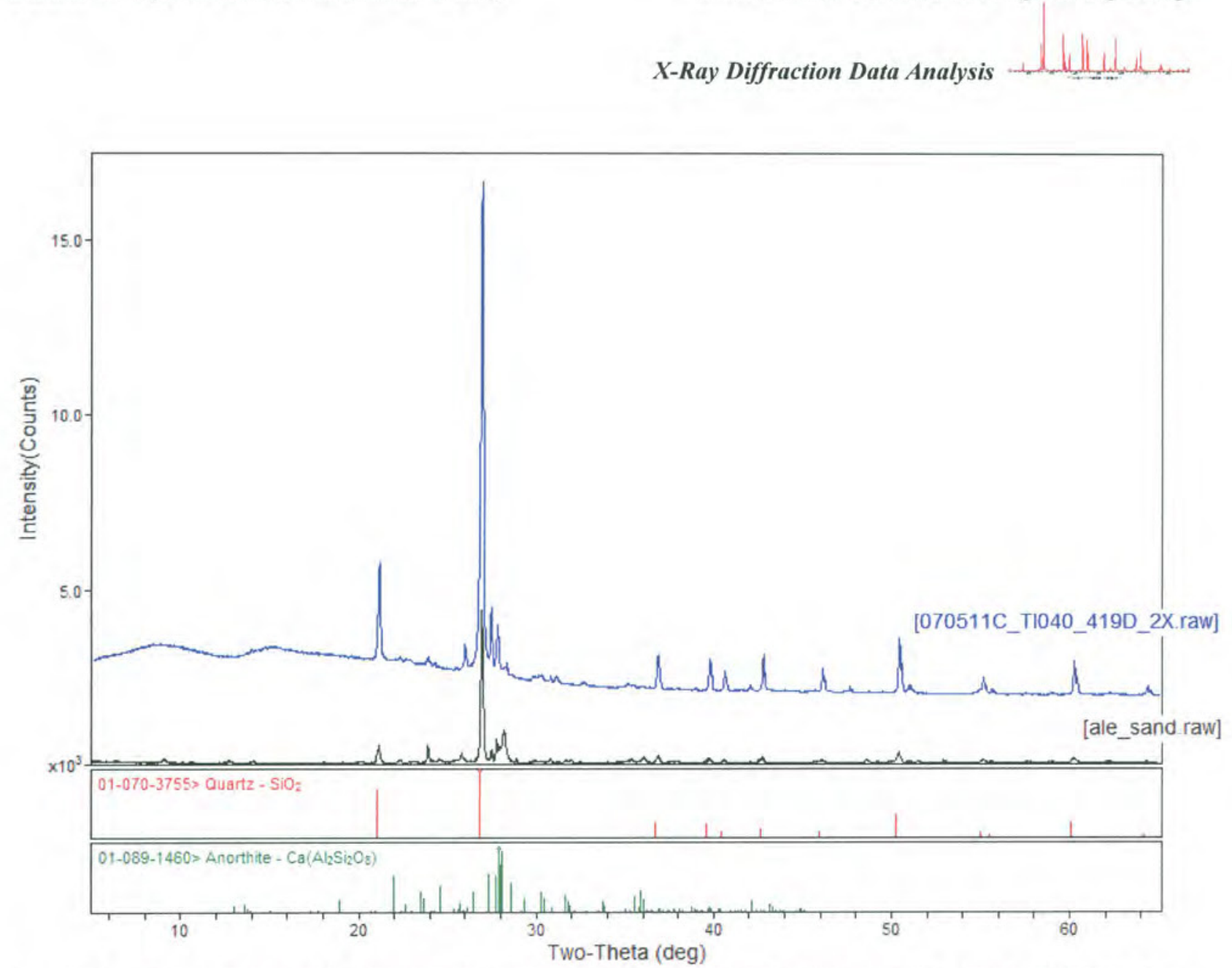

Figure 3. X-ray powder diffraction data for Hanford site sand and sample TI040-419D-2X. No background subtracted spectra are shown. 


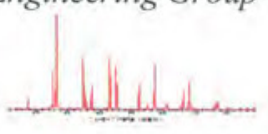

\subsection{XRD Sample TI040-420D-2X and TI040-420D-2X-TiO}

Figure 4 presents the raw XRD data for samples TI040-420D-2X along with the PDF card of the crystalline phase identified based on the Rietveld refinement $\left(\mathrm{R}_{\mathrm{w}}=10 \%\right)$. This sample is composed of silica (quartz) and anorthite as major phases. In addition the sample contains aegerine and calcium sulfate as minor crystalline phases. Figure 5 presents the background subtracted XRD data for TI040-420D-2X and TI040-420D-2X-TiO2. Rutile internal standard peaks, SRM 674, are consistent with the ones reported in the PDF card 01-077-0440, which means that the peak positions in the analyzed samples are reliable. The two duplicate samples confirm the diffraction patterns given the overall analysis method uncertainty.

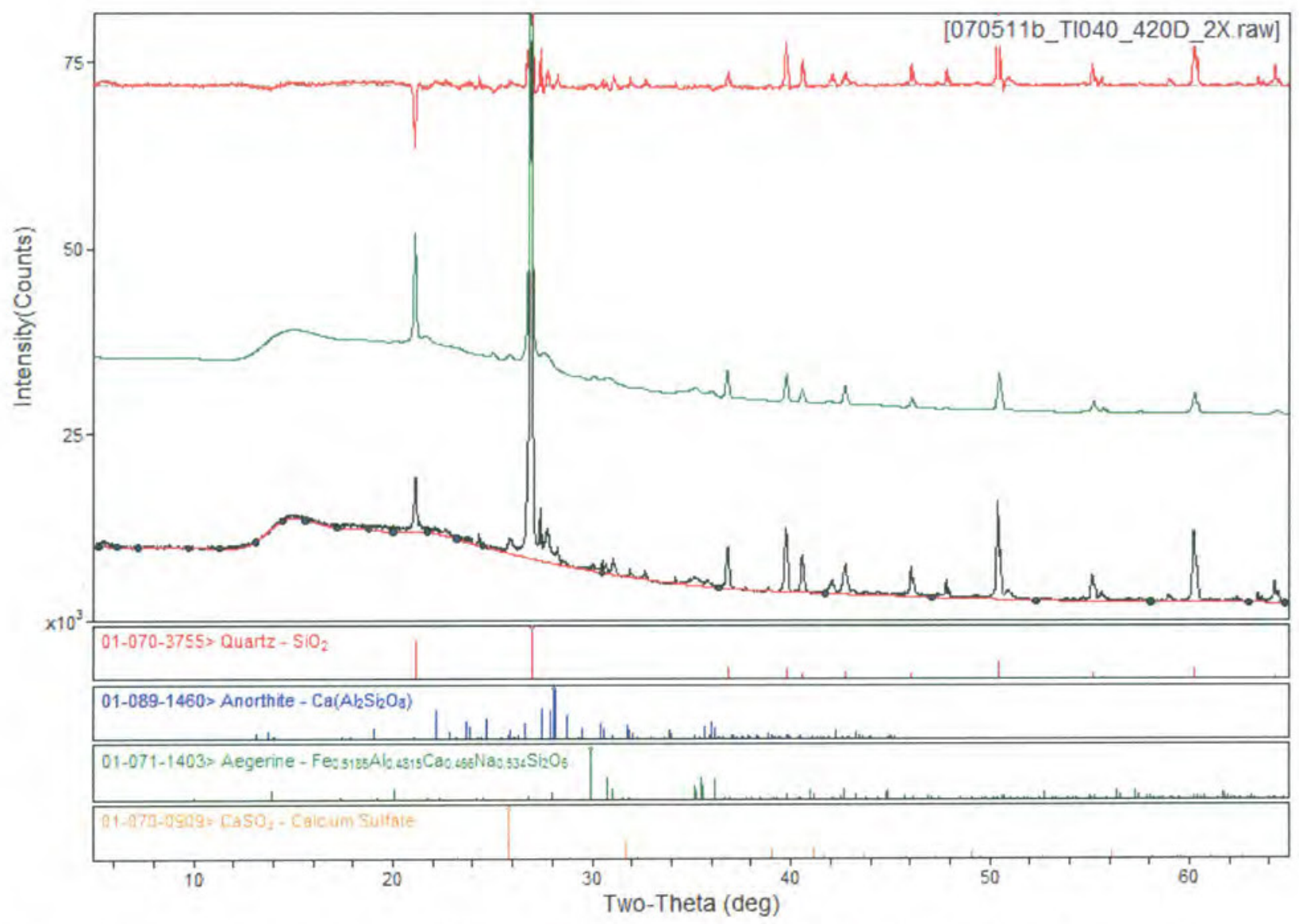

Figure 4. Rietveld refinement of the XRD data for TI040-420D-2X. The black line is the observed powder pattern (the red line underneath the black line is the background), the green line is the simulated powder pattern based on the model, and the red line is the difference between the calculated and the observed powder patterns. $R_{w}=10 \%$ (See Section 3.2). 
PNNL-20650, Rev. 2

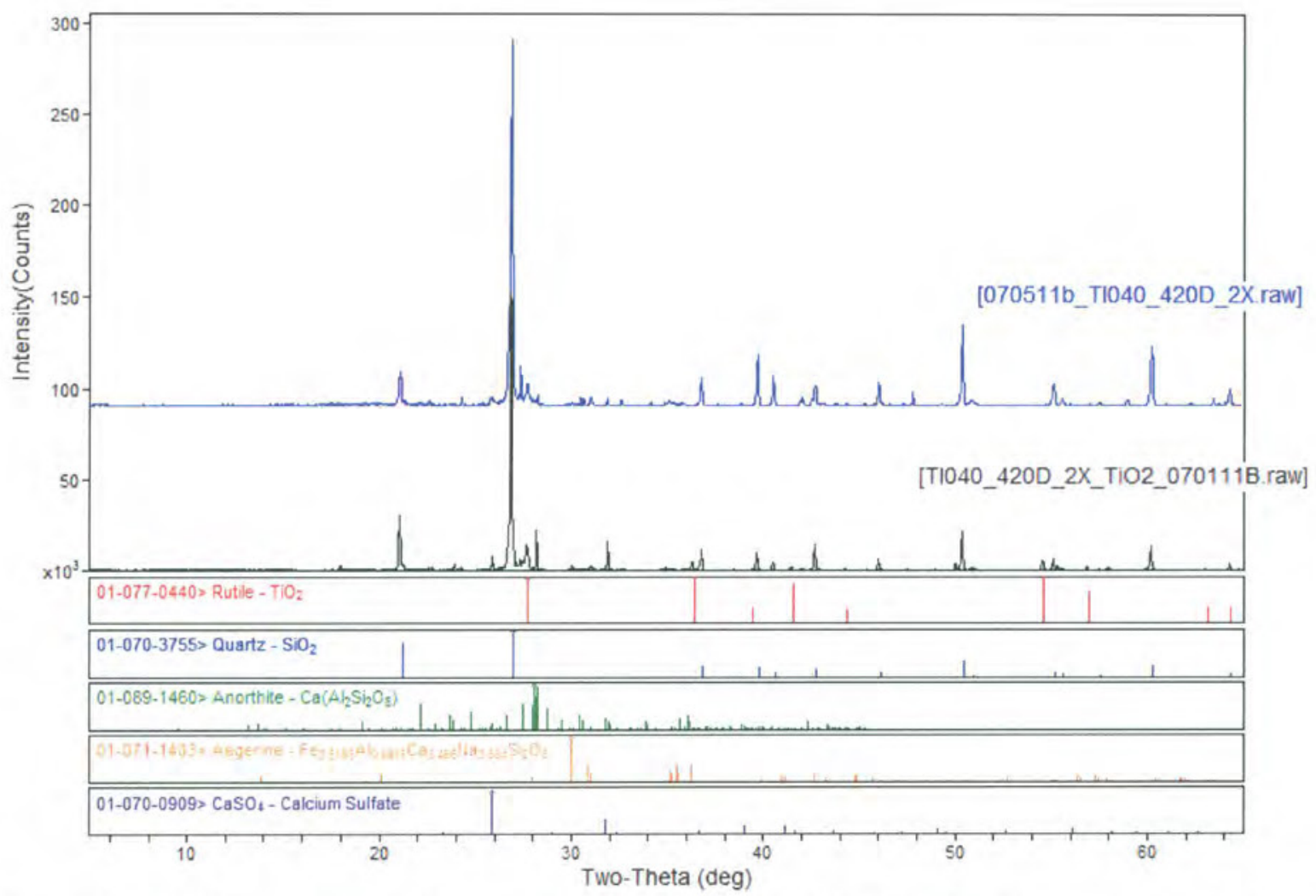

Figure 5. XRD data for TI040-420D-2X and TI040-420D-2X-TiO . Background subtracted spectra are shown. 
PNNL-20650, Rev. 2

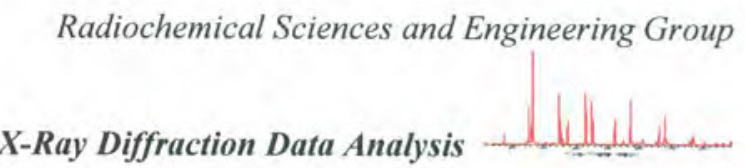

\subsection{XRD Sample TI040-421D-2X}

Figure 6 presents the raw XRD data for sample TI040-421D-2X along with the PDF cards of the crystalline phases identified based on the Rietveld refinement $\left(\mathrm{R}_{\mathrm{w}}=6 \%\right)$. This sample is composed of silica (quartz) and anorthite as major crystalline phases.

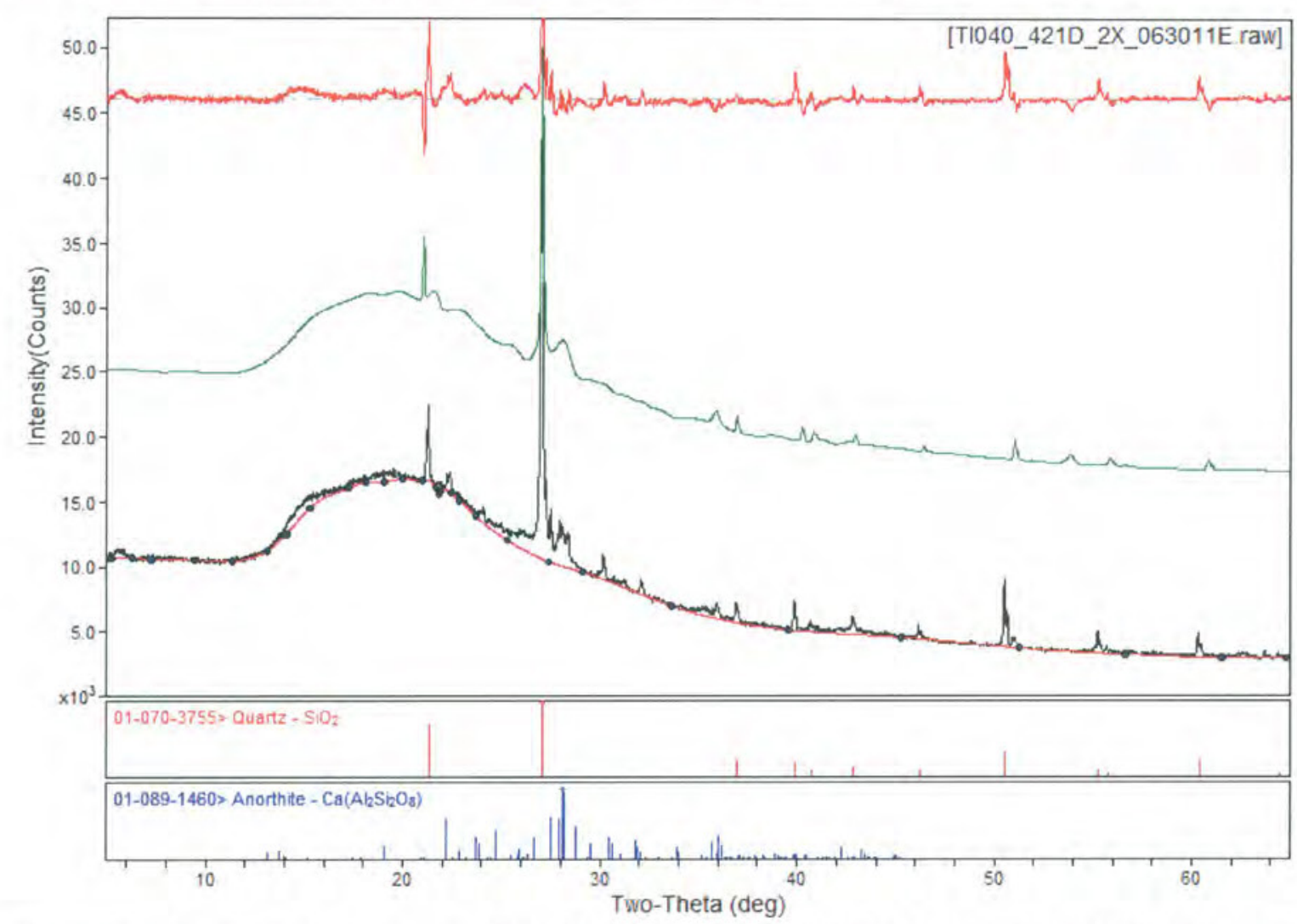

Figure 6. Rietveld refinement of the XRD data for TI040-421D-2X. The black line is the observed powder pattern, the green line is the simulated powder pattern based on the model, and the red line is the difference between the calculated and the observed powder patterns. $R_{w}=6 \%$ (See Section 3.2). No background subtracted spectra shown. 
PNNL-20650, Rev. 2

\subsection{XRD Sample TI040-422D-2X}

Figure 7 presents the Rietveld refinement $\left(\mathrm{R}_{\mathrm{w}}=11 \%\right)$ of the XRD data for sample TI040-422D$2 \mathrm{X}$ with the PDF cards of the corresponding crystalline phases. The major phases present in this sample are silica (quartz) and anorthite. In addition the sample contains aegerine as a minor crystalline phase.

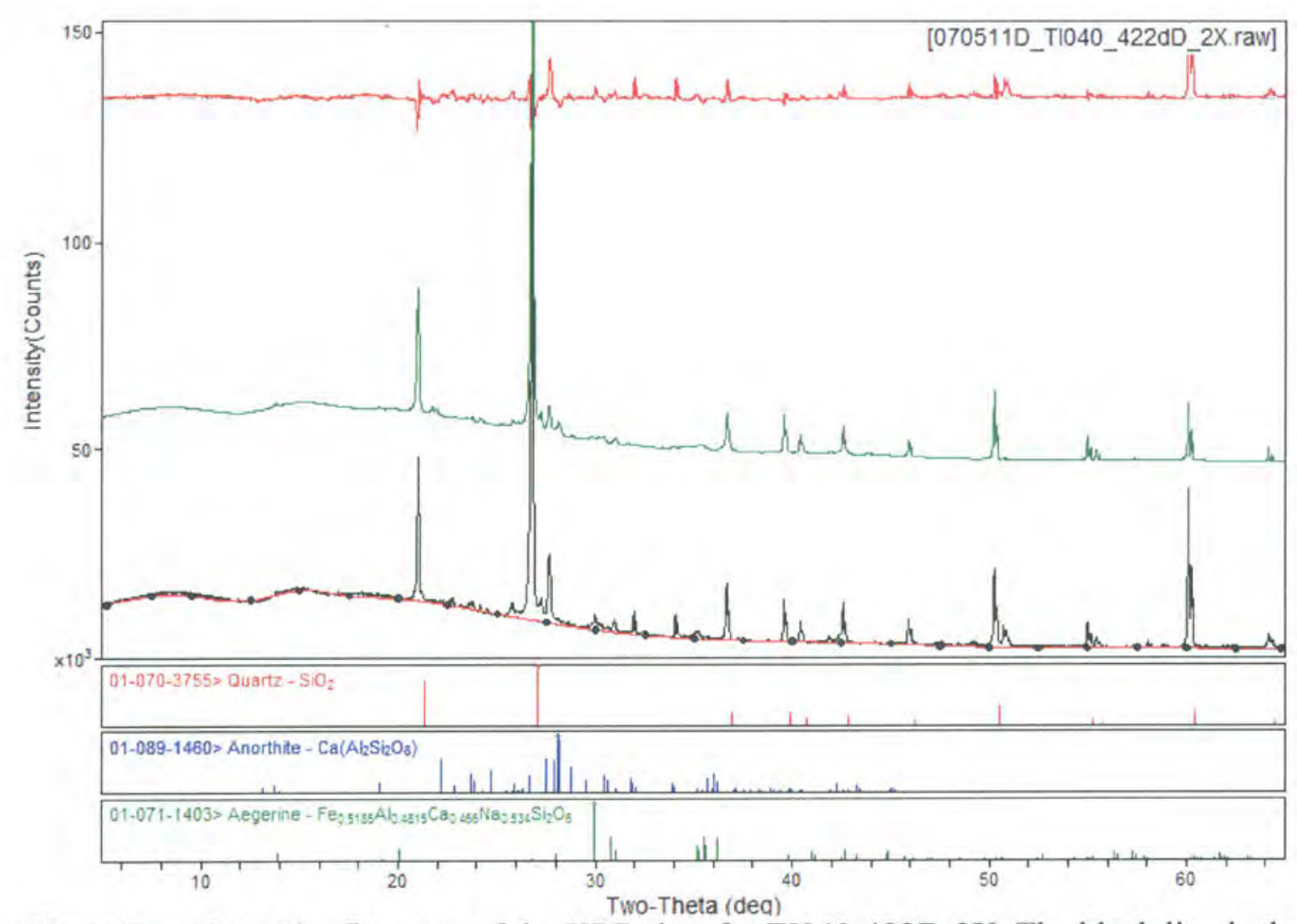

Figure 7. Rietveld refinement of the XRD data for TI040-422D-2X. The black line is the observed powder pattern, the green line is the simulated powder pattern based on the model, and the red line is the difference between the calculated and the observed powder patterns. $R_{\mathrm{w}}=11 \%$ (See Section 3.2). No background subtracted spectra shown. 
PNNL-20650, Rev. 2

\subsection{XRD Sample TI040-792D-2X}

Figure 8 presents the Rietveld refinement $\left(\mathrm{R}_{\mathrm{w}}=7 \%\right)$ of the XRD data for sample TI040-792D$2 \mathrm{X}$ with the PDF cards of the corresponding crystalline phases. The major phases present in this sample are silica (quartz) and anorthite. In addition the sample contains aegerine as a minor crystalline contribution.

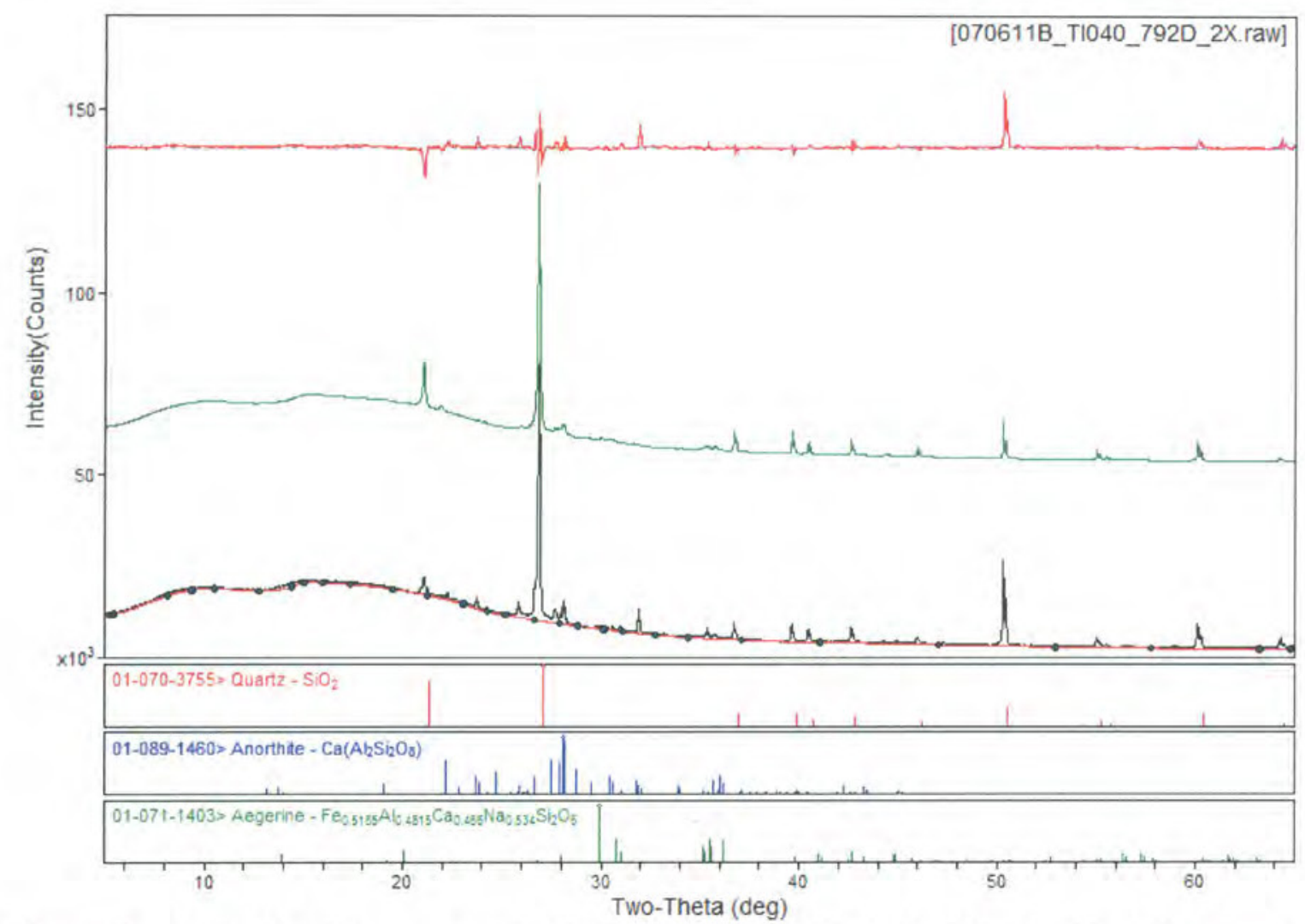

Figure 8. Rietveld refinement of the XRD data for TI040-792D-2X. The black line is the observed powder pattern, the green line is the simulated powder pattern based on the model, and the red line is the difference between the calculated and the observed powder patterns. $R_{w}=7 \%$ (See Section 3.2). No background subtracted spectra shown. 
PNNL-20650, Rev. 2

\subsection{XRD Sample TI040-793D-2X}

Figure 9 presents the Rietveld refinement $\left(\mathrm{R}_{\mathrm{w}}=7 \%\right)$ of the XRD data for sample TI040-793D$2 \mathrm{X}$ with the PDF cards of the corresponding crystalline phases. The major phase present in this sample is silica (quartz). In addition the sample contains anorthite and aegerine as minor crystalline phases.

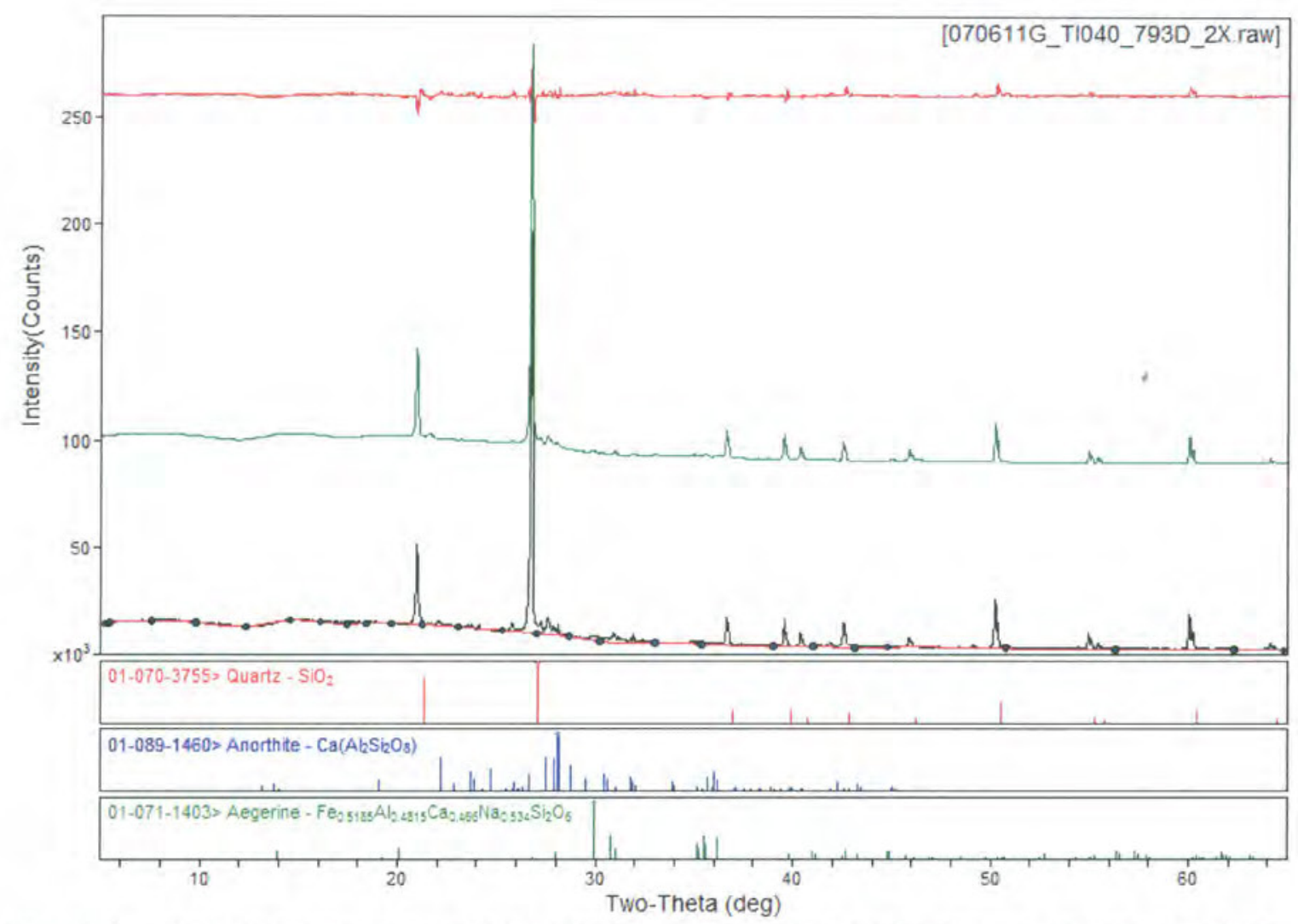

Figure 9. Rietveld refinement of the XRD data for TI040-793D-2X. The black line is the observed powder pattern, the green line is the simulated powder pattern based on the model, and the red line is the difference between the calculated and the observed powder patterns. $R_{w}=7 \%$ (See Section 3.2). No background subtracted spectra shown. 
PNNL-20650, Rev. 2

\title{
Appendix 1 \\ SRM 674 NIST Certificate
}

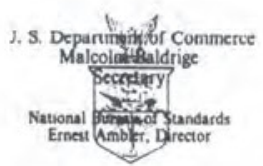

\section{A}

\author{
(Tertifitate
}

\section{Standard Reference Material 674}

\author{
X-Ray Powder Diffraction \\ Intensity Set
}

\section{R. Hubbard}

This Standard Reference Material (SR M) consists of five different phases. The phases, separately bottled, are $\alpha-\mathrm{Al}_{2} \mathrm{O}_{3}$ (corundum structure), $\mathrm{ZnO}$ (wurtzite structure), $\mathrm{TiO}_{2}$ (rutile structure), $\mathrm{Cr}_{2} \mathrm{O}_{3}$ (corundum structure), and $\mathrm{CeO}_{2}$ (fluorite structure). These phases can be used as internal standards for quantitative analysis and as external standards for checking the intensity response of $x$-ray diffraction instruments. The five phases cover the range of linear absorption coefficient from 100 to $1000 \mathrm{~cm}^{-1}$ for CuK $\alpha$ radiation. The intensity values are for a constant diffracting volume (fixed divergent slit and infinitely thick mounting or equivalent).

The certified relative intensity values ( $\mathrm{CuK} \alpha$ radiation), for the major diffraction lines below $70^{\circ} 2(\theta)$, for each phase are given in Table 1. The certified referance intensity ratios (I/Ic) for $\mathrm{ZnO}, \mathrm{TiO}_{2}, \mathrm{Cr}_{2} \mathrm{O}_{3}$, and $\mathrm{CeO}_{2}$, relative to $\alpha-\mathrm{Al}_{2} \mathrm{O}_{3}$ and the lattice parameters are given in Tables 2 and 3 . The uncertainties of the ielative intensity and lattice parameter values are the standard deviations derived from muittple measurements. The uncertainties of the reference intensity ratio values were determined from the value of the internal inennsistency derived from all possible hinary mixtures

The average crystallite size for each of the five phases is near $2.0 \mu \mathrm{m}$. Crystallite size broadening of the diffraction peaks is small but present. This is particularly apparent for $\alpha-\mathrm{Al}_{2} \mathrm{O}_{3}$, which has a nominal crystallite size less than $1.0 \mu \mathrm{m}$. The lattice parameters, uncorrected for refraction, were determined with a relative uncertainty of about three parts in $10^{5}$ using an internal standard. This uncertainty in the lattice parameter must be increased by a factor of two or three if one wishes to determine absolute $\mathrm{d}$-spacings as the uncertainties due to the internal standard $2 \theta^{\circ} \mathrm{s}$ and to thermal expansion would need to be added.

The technical and support aspects concerning the preparation, certification, and issuance of this Standard Reference Material were coordinated through the Office of Standard Reference Materials by R.K. Kirby.

Washington. D.C. 20234

June 1, 1983 (over)

Stanley D. Rasberry, Chief

Office of Standard Reference Materials 
PNNL-20650, Rev. 2

Table 1. Relative Intensitites of Major lines

CuKa Radiation ( $\left.\mu=124.1 \mathrm{~cm}^{-1}\right)$

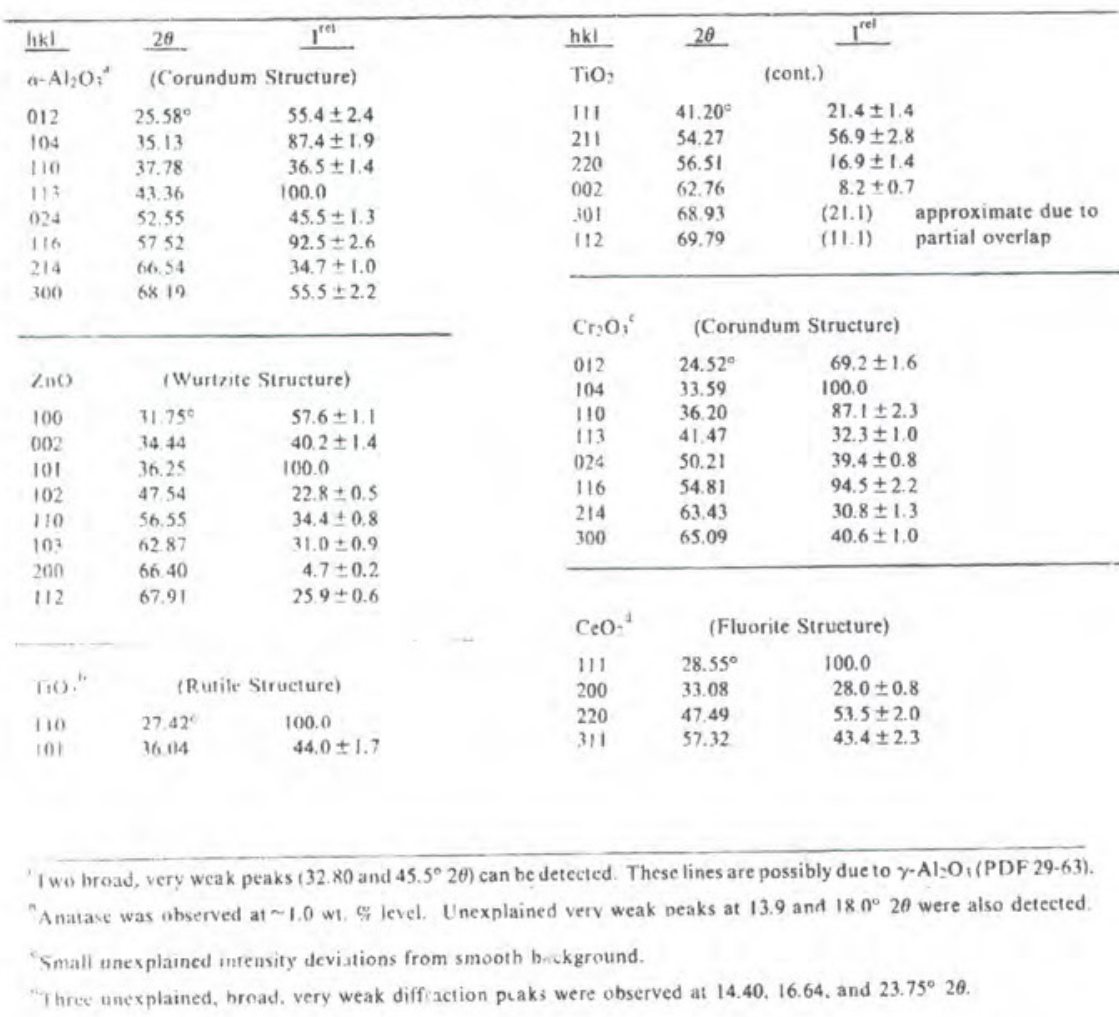




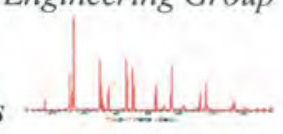

\section{Appendix 2 Daily Performance Checks}

The daily performance check shows that the Rigaku Ultima IV diffractometer is within calibration per PNNL-RPG-268 Rev. 4, "Solids Analysis by X-Ray Diffraction" so no adjustments have been made. Figure 10,11, 12 and 13 present the XRD data obtained on the silicon SRM 640a standard. In Figures 10-13, the black line represents the observed XRD powder pattern, and the green line represents a simulated Si pattern using the PDF card 01-0713770 . Tables $4,5,6,7$ contain summarized results of the daily performance check with this standard.

The columns in Tables 4-7 have the following significance:

1) $2 \mathrm{~T}(\mathrm{r})$ gives the 2-theta of the reference powder pattern (PDF card)

2) $2 \mathrm{~T}(\mathrm{o})$ is the observed 2-theta

3) Delta is the difference between the observed and the reference 2-theta

4) $d(r)$ is the interplanar distance $d$ for the reference pattern (in $\AA$ )

5) $d(o)$ is the observed $d$

6) Del-d is the difference between the observed and the reference $d$

7) $\mathrm{I} \%$ is the intensity in percent (the most intense peak is always $100 \%$ and all other peaks are normalized)

Per procedure the measured 2-theta of the performance check standard (i.e., the 111 Si reference line) must be $28.44^{\circ} \pm 0.06^{\circ}$ 2-theta; the observed 2-theta difference (Delta) was $0.014-0.015$ and met this requirement. Thus the performance checks met this criterion. 
PNNL-20650, Rev. 2

Battelle - Pacific Northwest National Laboratory

Radiochemical Sciences and Engineering Group X-Ray Diffraction Data Analysis

Daily performance check $\left(27^{\circ}\right.$ to $29^{\circ}$ 2-theta) - Date 06-30-2011

Theta Calibration Report

[063011a_si_640a.raw] - Full Chart

Reference Lines $=01-071-3770>$ Silicon $-\mathrm{Si}$

Two-Theta Range of Fit $=27.0 / 29.0(\mathrm{deg})$

Zero Offset $=-0.019(\mathrm{deg})$

Table 4. Daily performance check report summary

\begin{tabular}{|l|l|l|l|l|l|l|}
\hline $\mathbf{2 T}(\mathbf{r})$ & $\mathbf{2 T}(\mathbf{0})$ & Delta & d(r) & d(o) & Del-d & I\% \\
\hline 28.443 & 28.462 & -0.019 & 3.1355 & 3.1334 & 0.0020 & 100.0 \\
\hline
\end{tabular}

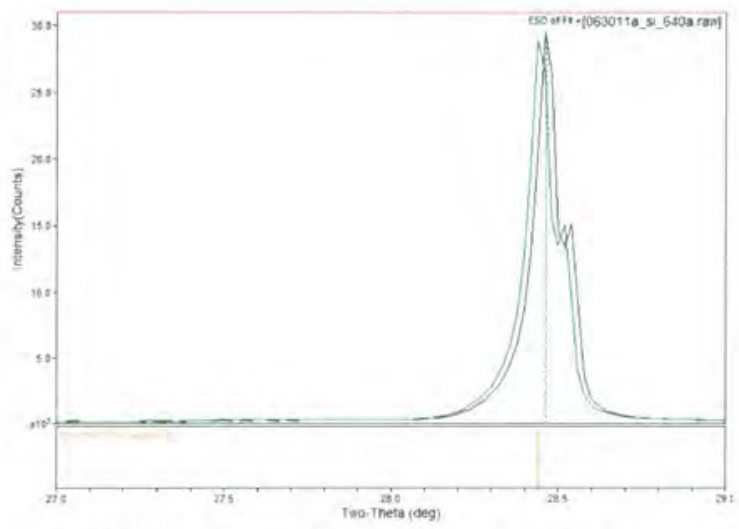

Figure 10. The raw XRD data on SRM 640a against the corresponding PDF card.

Daily performance check $\left(27^{\circ}\right.$ to $29^{\circ}$ 2-theta) - Date 07-01-2011

Theta Calibration Report

[070111A_SI_640A.raw] STD - Full Chart

Reference Lines $=$ 01-071-3770 $>$ Silicon $-\mathrm{Si}$

Two-Theta Range of Fit $=27.0 / 29.0(\mathrm{deg})$

Zero Offset $=-0.019(\mathrm{deg})$

Table 5. Daily performance check report summary

\begin{tabular}{|l|l|l|l|l|l|l|}
\hline $\mathbf{2 T}(\mathbf{r})$ & $\mathbf{2 T}(\mathbf{o})$ & Delta & d(r) & $\mathbf{d}(\mathbf{o})$ & Del-d & I\% \\
\hline 28.443 & 28.462 & -0.019 & 3.1355 & 3.1334 & 0.0020 & 100.0 \\
\hline
\end{tabular}


PNNL-20650, Rev. 2
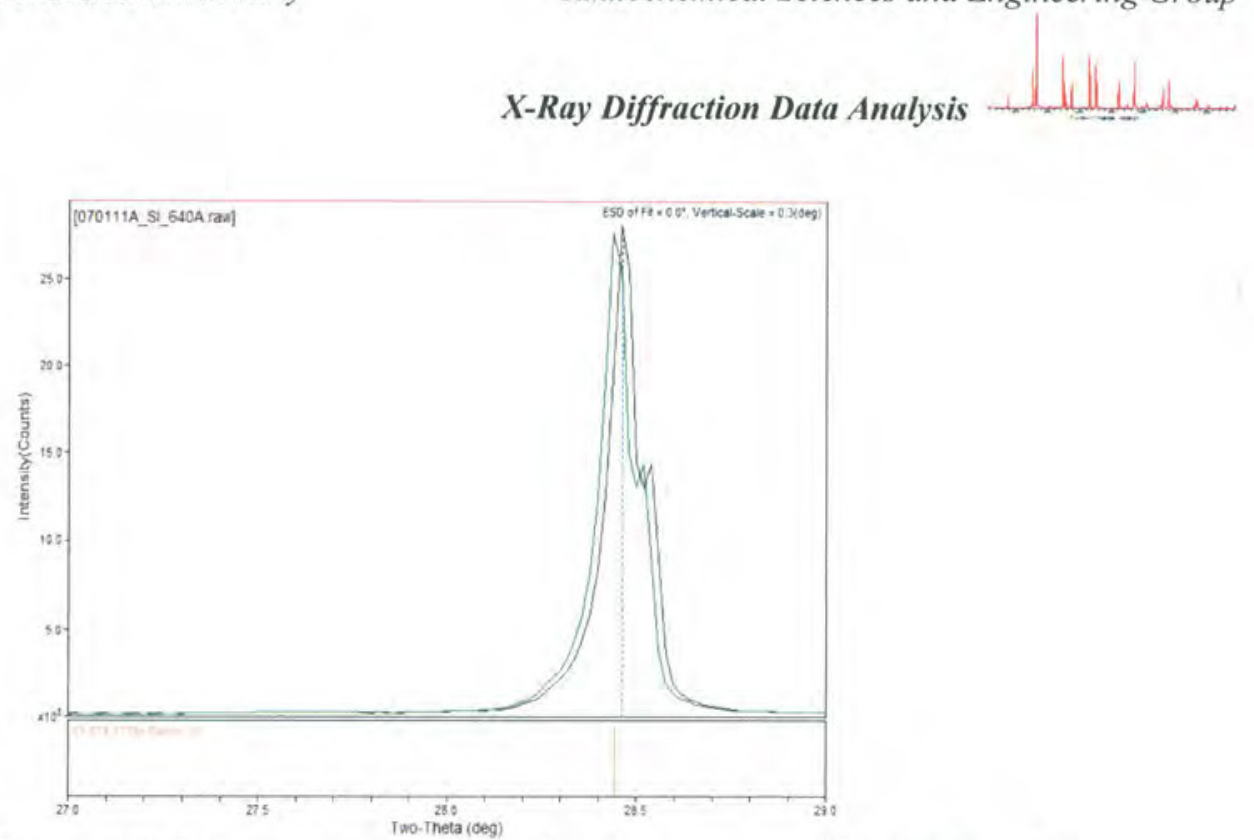

Figure 11. The raw XRD data on SRM 640a against the corresponding PDF card.

Daily performance check $\left(27^{\circ}\right.$ to $29^{\circ}$ 2-theta $)$ - Date 07-05-2011

Theta Calibration Report [070511a_si_640a.raw] standard si - Full Chart Reference Lines $=01-071-3770>$ Silicon $-\mathrm{Si}$

Two-Theta Range of Fit $=27.0 / 29.0(\mathrm{deg})$

Zero Offset $=-0.018(\mathrm{deg})$

Table 6. Daily performance check report summary

\begin{tabular}{|l|l|l|l|l|l|l|}
\hline $\mathbf{2 T}(\mathbf{r})$ & $\mathbf{2 T}(\mathbf{0})$ & Delta & d(r) & d(o) & Del-d & I\% \\
\hline 28.443 & 28.461 & -0.018 & 3.1355 & 3.1335 & 0.0020 & 100.0 \\
\hline
\end{tabular}

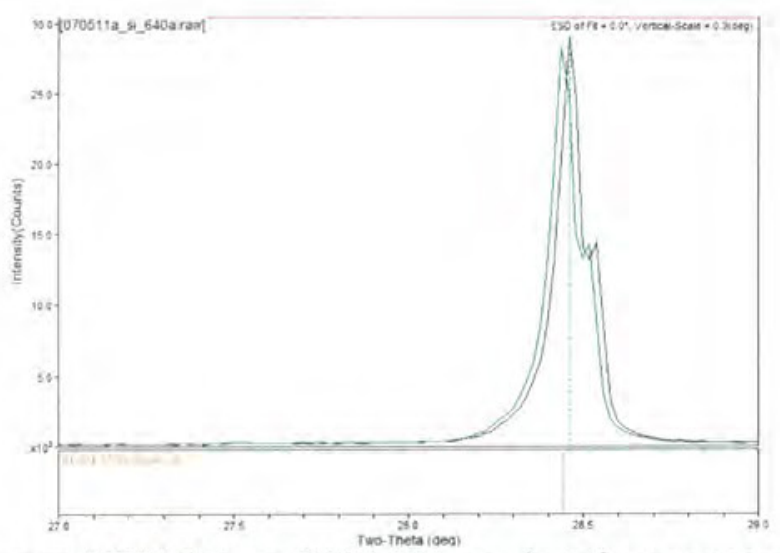

Figure 12. The raw XRD data on SRM 640a against the corresponding PDF card.

Daily performance check $\left(27^{\circ}\right.$ to $29^{\circ}$ 2-theta) - Date 07-06-2011

Theta Calibration Report

[070611A SI640A.raw] - Full Chart 
PNNL-20650, Rev. 2

Battelle - Pacific Northwest National Laboratory

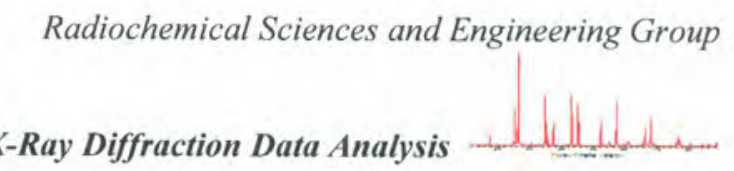

Reference Lines $=01-071-3770>$ Silicon $-\mathrm{Si}$

Two-Theta Range of Fit $=27.0 / 29.0(\mathrm{deg})$

Zero Offset $=-0.018(\mathrm{deg})$

Table 7. Daily performance check report summary

\begin{tabular}{|l|l|l|l|l|l|l|}
\hline $\mathbf{2 T}(\mathbf{r})$ & 2T(o) & Delta & d(r) & d(o) & Del-d & I\% \\
\hline 28.443 & 28.461 & -0.018 & 3.1355 & 3.1335 & 0.0019 & 100.0 \\
\hline
\end{tabular}

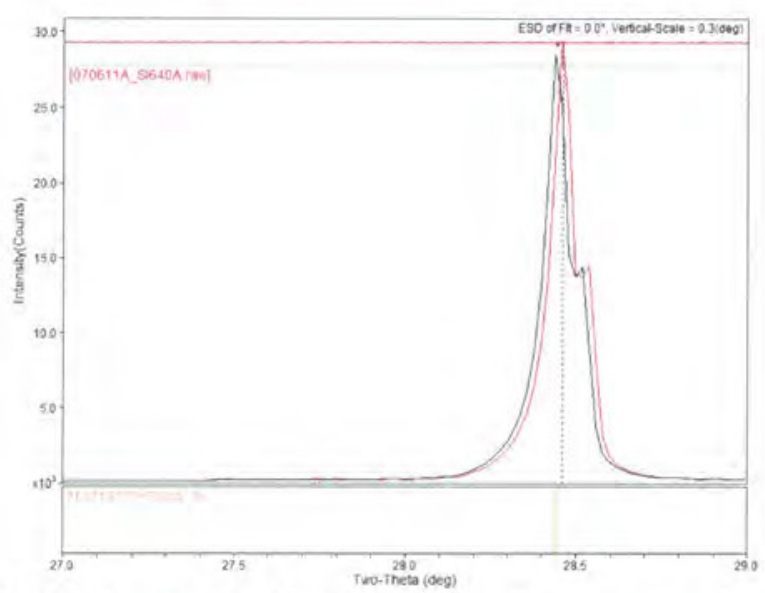

Figure 13. The raw XRD data on SRM 640a against the corresponding structure PDF card. 
PNNL-20650, Rev. 2

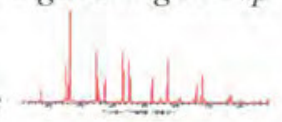

\title{
Appendix 3 \\ SRM 640a NIST Certificate
}

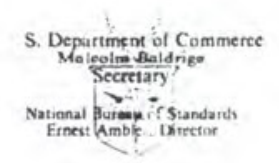

\section{ANatiunal 色ureau of SStantarts}

\author{
Uertifitate
}

\author{
Standard Reference Material 640a \\ Silicon Powder $20 / \mathrm{d}-$-Spacing Standard \\ for X-Ray Diffraction
}

\begin{abstract}
Camden R. Hubbard
This Standand Refercuec Material (GRM) was prepared for usc as an external on interual 20N-spaviug valituativu standard for powder diffractometry.

Electronic grade float-zone prepared silicon boules, $99.999+\%$ pure, were ground to pass a $75 \mu \mathrm{m}(200 \mathrm{mesh})$ sieve. This powder, which was identical to SRM 640, was then jet-milled to reduce the mean particle size to about $2 \mu \mathrm{m}$.

A total of twelve samples. mixed wi h tungsten and silver internal standards [1]. were measured using a high angle goniometer controlled by a minicomr uter. The $\mathrm{CuK} \alpha$, peak position was determined by profile fitting procedures and then corrected for sample, instrumental, and physical aberrations (except refraction) through use of the internal standard lines [2]. Silicon peak positions were then corrected for effects of thermal expansion. Each corrected set was refined by a least-square routine that minimized $\Sigma\left(\theta_{0 b s}-\theta_{c a l c}\right)^{2}$ to obtain estimates of $a_{1}$ and their estimated standard errors, 5 . The weighted average of the twelve lattice parameters, uncorrected for refraction, at $25^{\circ} \mathrm{C}$ is
\end{abstract}

$$
\text { (a) }=5.430825 \pm 0.000036 \AA
$$

where $\lambda\left(C_{U K} \alpha_{1}\right)=1.5405981 \AA[3]$. The estimated total uncertainty given above includes contributions from three sources (listed in decreasing importance): (1) uncertainty of the lattice parameters of the tungsten and silver standards; (2) random errors of the measurements; and (3) the uncertainty in $\lambda\left(\mathrm{CuK} \alpha_{1}\right)$.

The technical and support aspects concerning the preparation, certification. and issuance of this Standard Reference Material were coordinated through the Office of Standard Reference Materiais by R.K. Kirby.

Washington, D.C. 20234

February 19. 1982
George A. Uriano, Chief Office of Standard Reference Materials 
PNNL-20650, Rev. 2

Battelle - Pacific Northwest National Laboratory

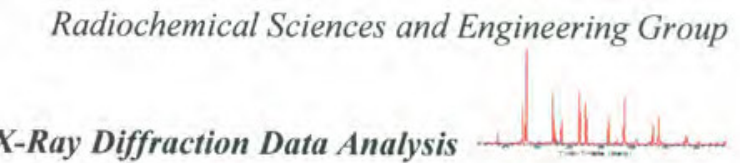

The $2 \theta$ values given in Table 1 were calculated from the certified value of the lattice parameter. The relative intensities [4]. I (rel), also given in Table I can be used as an aid in identifying the silicon lines when SRM 640a is mixed with test materials. The uncertainty of each value of 1 (rel) may be as large as \pm 3 . Suggested methods for use of this SRM are given in references [2], [5], and [6].

Tabie 1

Calculated Diffraction Angles and Relative Intensities

$$
\left(\mathrm{T}=25.0^{\circ} \mathrm{C}\right)
$$

These Values Are Not Certified

\begin{tabular}{|c|c|c|c|c|c|}
\hline hkl & I (rel) & $2 \theta$ peak & hkl & I (rel) & 20peak \\
\hline 111 & 100 & $28.443^{\circ}$ & $511 / 333$ & 6 & $94.955^{\circ}$ \\
\hline 220 & 55 & 47.304 & 440 & 3 & 106.712 \\
\hline 311 & 30 & 56.124 & 531 & 7 & 114.096 \\
\hline 400 & 6 & 69.132 & 620 & 8 & 127.550 \\
\hline 331 & 11 & 76.378 & 533 & 3 & 136.900 \\
\hline 422 & 12 & 88.033 & 444 & * & 158.644 \\
\hline
\end{tabular}

*Not measured

[1] Swanson, H.E.; McMurdie, H.F; Morris, M.C.; and Evans, E.H. (1966), Standard X-Ray Diffraction Powder Patterns, National Bureau of Standards Monograph 25, Section 4, NBS, Washington. D.C. 20234.

[2] Hubbard, C.R. (1982), submitted for publication in J. Appl. Cryst.

[3] Deslattes. R.D. and Henins, A. (1973), Phys. Rev. Letters, 31, 972-975.

[4] Morris, M.C;: McMurdic. H.F.: Evans, E.H.; Paretzkin, B.; deGroot, J.H.; Hubbard, C.R tand Carmel, S.J. (1976) Standard X-Ray Diffraction Powder Patterns. National Bureau of Standards Monograph 25, Sertion 13, NBS, Washington. D.C. 20234.

[5] Snyder. R.L., Hubbard. C.R.; and Panagiotopoulos, N.C., Advances in X-Ray Analysis, Vol. 25, (in press).

[6] Hubbard. C.R. (1980) Accuracy in Powder Diffraction. NBS Special Publication 567, p 489-502. 
PNNL-20650, Rev. 2

Radiochemical Sciences and Engineering Group

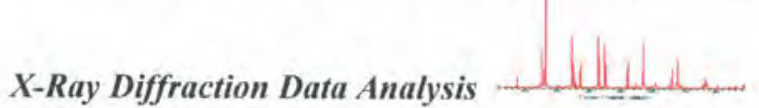

\section{Appendix 4 \\ Quality Control/Calibration}

This control calibration measurement shows that the Rigaku Ultima IV diffractometer is within calibration per PNNL-RPG-268 Rev. 4, "Solids Analysis by X-Ray Diffraction" so no adjustments have been made. The XRD data obtained on this standard are represented in Figure 14. Table 8 contains summarized results of the quality control/calibration results obtained using NIST SRM 1976 (Corundum).

The columns in Table 8 have the following significance:

1) $2 \mathrm{~T}(\mathrm{r})$ gives the 2-theta of the reference powder pattern (PDF card)

2) $2 \mathrm{~T}(\mathrm{o})$ is the observed 2 -theta

3) Delta is the difference between the observed and the reference 2-theta

4) $d(r)$ is the interplanar distance $d$ for the reference pattern (in $\AA$ )

5) $d(o)$ is the observed $d$

6) Del-d is the difference between the observed and the reference $d$

7) $I \%$ is the intensity in percent (the most intense peak is always $100 \%$ and all other peaks are normalized)

The requirement for this quality check (semiannual calibration verification of the diffractometer as stated in the PNNL-RPG-268 Rev. 4, is that observed d-interplanar distance for any given crystal lattice Miller indices be within $\pm 0.02 \AA$ of the NIST SRM 1976 PDF card d-values. For this quality performance check, all Del-d values are well within this criterion. The PDF card for alumina (corundum structure) is provided in Appendix 6.

\section{Control Calibration Report $\left(10^{\circ}\right.$ to $60^{\circ} 2$ theta) - Date 06-30-2011}

Theta Calibration Report

[063011b_1976_alumina_plate.raw] - Full Chart

Reference Lines $=01-071-6478>$ Corundum $-\mathrm{Al} 2 \mathrm{O} 3$

Two-Theta Range of Fit $=5.0 / 80.0(\mathrm{deg})$

Zero Offset $=0.026(\mathrm{deg})$

ESD of Fit $=0.00647^{\circ}, \mid$ Delta $2-$ Theta $\mid=0.026(\mathrm{deg})$ 
PNNL-20650, Rev. 2

X-Ray Diffraction Data Analysis

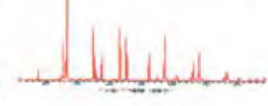

Table 8. Summary of the semiannual calibration report

\begin{tabular}{|r|r|r|r|r|r|r|}
\hline \multicolumn{1}{|c|}{ 2T(r) } & \multicolumn{1}{l|}{ 2T(o) } & \multicolumn{1}{l|}{ Delta } & \multicolumn{1}{l|}{ d(r) } & d(o) & Del-d & \multicolumn{1}{l|}{ \% } \\
\hline 25.573 & 25.543 & 0.03 & 3.4805 & 3.4845 & 0.004 & 31.1 \\
\hline 35.147 & 35.118 & 0.029 & 2.5512 & 2.5533 & 0.002 & 100 \\
\hline 37.77 & 37.743 & 0.027 & 2.3799 & 2.3815 & 0.0016 & 12.6 \\
\hline 41.676 & 41.642 & 0.034 & 2.1654 & 2.1671 & 0.0017 & 2.7 \\
\hline 43.347 & 43.32 & 0.027 & 2.0857 & 2.087 & 0.0013 & 50.2 \\
\hline 46.17 & 46.147 & 0.023 & 1.9646 & 1.9655 & 0.0009 & 0.4 \\
\hline 52.544 & 52.519 & 0.025 & 1.7403 & 1.741 & 0.0008 & 26 \\
\hline 57.494 & 57.463 & 0.031 & 1.6016 & 1.6024 & 0.0008 & 76.1 \\
\hline 59.729 & 59.71 & 0.019 & 1.5469 & 1.5474 & 0.0005 & 0.8 \\
\hline 61.118 & 61.103 & 0.015 & 1.5151 & 1.5154 & 0.0003 & 1.4 \\
\hline 61.299 & 61.264 & 0.036 & 1.511 & 1.5118 & 0.0008 & 13.2 \\
\hline
\end{tabular}

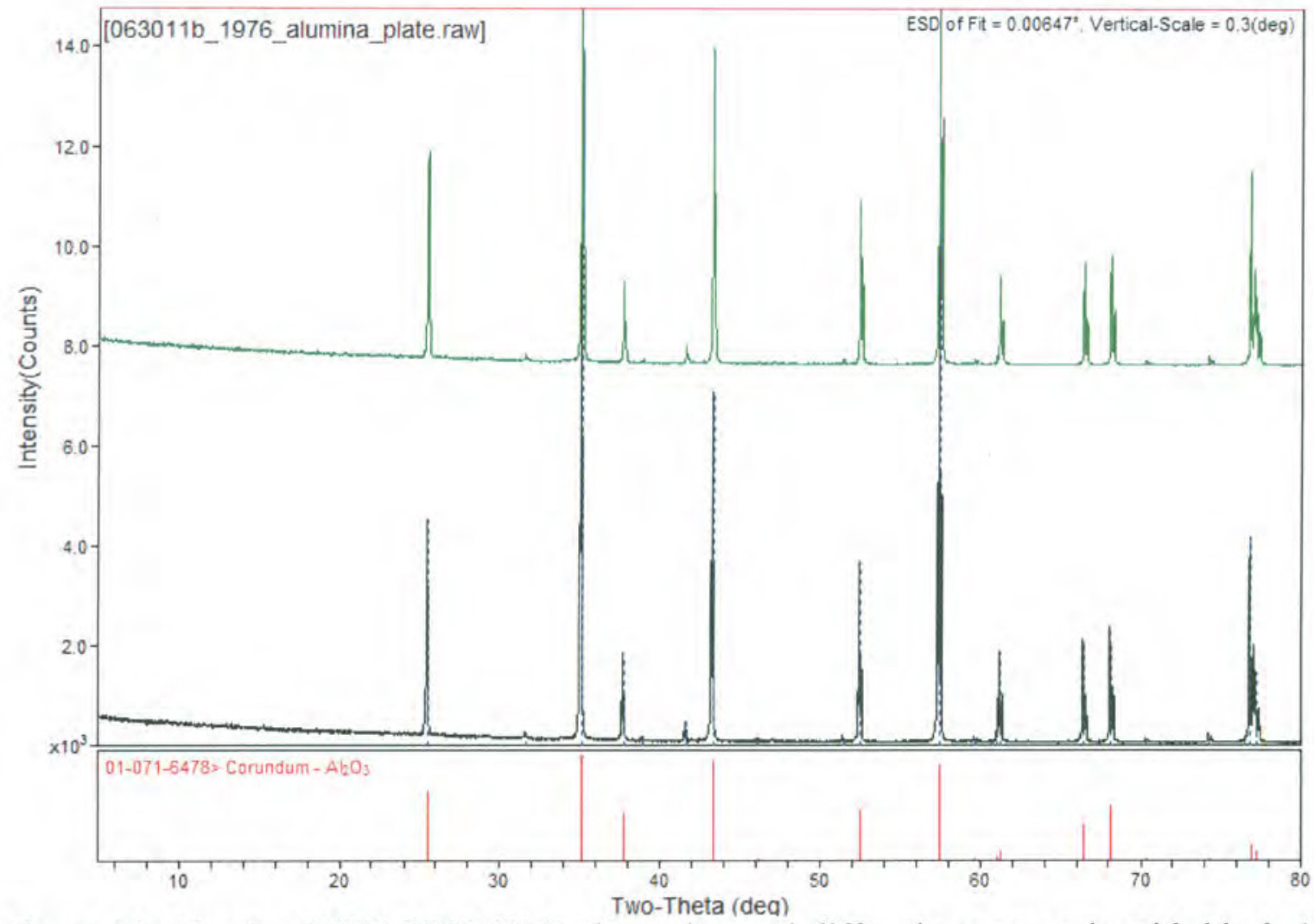

Figure 14. Alumina NIST SRM 1976 plate, observed diffraction pattern is with black (middle image) the simulated reference diffraction pattern is with green (top image) and the PDF card is with red (bottom image). 
PNNL-20650, Rev. 2

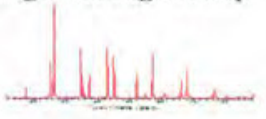

\author{
Appendix 5 \\ SRM 1976 NIST Certificate
}

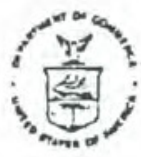

\title{
Eutimal Institute of Standards \& Urrhnology Tertifturate of Analusts
}

\author{
Standard Reference Material 1976
}

\author{
Instrument Sensitivity Standard for X-ray Powder Diffraction
}

\begin{abstract}
This Standard Reference Material (SRM) consists of a sintered alumina plate, approximately $45 \mathrm{~mm}$ on a side by $1.6 \mathrm{~mm}$ in thickness, intended for use in calibration of powder X-ray equipment for diffraction intensity as a function of $2 \theta$ angle (instrument sensitivity). The form of the SRM serves to eliminate the variability of sample loading procedure from intensity measurements. This SRM material was chosen for consistency of microstructure with respect to grain size, shape, micro-strain and orientation. The platelets comprising this microstructure are 3 to $7 \mu \mathrm{m}$ in diameter by 1 to $2 \mu \mathrm{m}$ in thickness and are highly oriented with the basal plane parallel to the surface of the plate. Some amount of amorphous phase is present though no crystalline impurities could be detected.
\end{abstract}

The proper use of this SR.M requires the measurement of intensity values from test equipment in a manner analogous to either of the two measurement methods used for certification. The certified parameters consis! of absolute variation in intensity, 12 relative intensity values calculated from integrated intensity and peak height measurements from 25.5 to 145 degrees $2 \Theta$, and the lattice parameters. Graphical evaluation of the ratio of data from the test equipment to the certified values will allow for an appropriate judgment as to the condition of the test equipment. This may be followed by the calculation of a correction curve, yielding standardization of instrument sensitivity and thus more accurate inter-laboratory comparisons of data involving $X$-ray diffraction intensity measurements.

The overall coordination of the preparation and the technical measurements leading to certification was performed by J.P. Cline of the NIST Ceramics Division. The project was the result of a round robin study carried out by R. Jenkins and W. Schreiner of the International Centre for Diffraction Data, Swarthmore, Pennsylvania.

The alumina substrates for this SRM were donated to NIST by International Business Machines Corporation. East Fishkill, New York, through the efforts of R. Anderson.

Statistical analysis was provided by S.B. Schiller and K.R. Eberhardt of the NIST Statistical Engineering Division.

The technical and support aspects involved in the preparation, certification, and issuance of this Standard Reference Material were coordinated through the Standard Reference Materials Program by N.M. Trahey.

Gaithersburg, MD 20899

November 8,1991
William P. Reed, Chief Standard Reference Materials Program 
PNNL-20650, Rev. 2

Battelle - Pacific Northwest National Laboratory

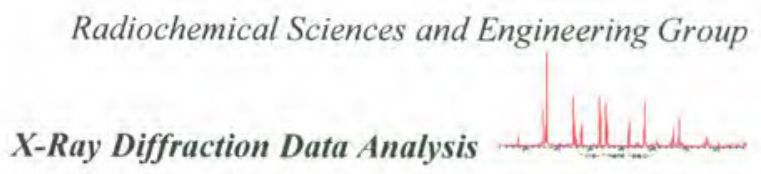

Certified intensity data were collected from 25 randomly selected samples on a Philips' diffractometer using an $\mathrm{AEG}^{\prime}$ long fine-focus $\mathrm{Cu} X$-ray tube operated at 1800 watts with a constant potential generator. The diffractometer was equipped with incident and diffracted beam sollar slits, graphite diffracted beam monochromator in anti-parallel geometry, and scintillation detector. The incident beam slits were of the theta compensating type, locked such that the illumination arca was $12.5 \mathrm{~mm}$ wide at 25 degrees $2 \Theta$. This corresponds to a slit size of 0.93 degrees; the receiving slit was 0.066 degrees. The take-off angle was 4 degrees. Samples were spun at approximately $52 \mathrm{rpm}$ during data collection.

Data were collected and processed with the Siemens Diffrac $500^{\prime}$ version of NBS ${ }^{*}$ Quant. This algorithim scans specific peaks for an integrated intensity measurement, with extended count times for each of ten points on either side of the scan range. Scan angles are indicated in Table 1; the step widah was 0.005 degrees. Scan time ranged from 30 to 90 minutes per peak, for a total of 16 hours per sample. The seventh and eleventh scan ranges actually include two peaks, as noted by the two sets of miller indices listed for them in Table 1. The use of two peaks in these scan ranges ensured that intensity data were reported evenly through the desired $2 \Theta$ range.

Peak height values were manually determined, with a hackground subtraction, from the same scans as were the integrated intensity data. Five background data points from each end of the scan range were averaged to determine the background value. Peak height values were determined from an average of the three most intense points for data below 55 degrees $2 \Theta$ : four data points were used for the remainder of the data. Due to the proximity of the two reflections used in the seventh and eleventh relative intensity values, peak height values for these data reflect the superposition of the $\mathrm{Cu} \mathrm{K} \alpha$, and $\mathrm{K} \alpha$ : peaks. Thus, peak height data for these two pairs of reflections will be valid only with the use of $\mathrm{Cu} \mathrm{K} \alpha$ radiation.

Lattice parameters were determined from the 25 samples by means of least squares refinement from 47 peak positions determined with the spline method, ranging from 22 10 152 degrees $2 \Theta$. Peak positions were corrected for optical aberrations with the use of SRM $640 \mathrm{~b}$ silicon powder, 10 generate a 2 nd order polynomial external standard correction curve which was applied to peak location determinations. Measurements were made on a Siemens D500' diffractometer equipped with a focusing Ge incident beam monochromator. sample spinner, and a position sensitive proportional detector. Copper $\mathrm{K} \alpha$ radiation was used. Measurements were made within a period of 48 hours during which operating conditions were maintained. The machine was stabilized under such conditions for several days prior to measurements.

The intensity measurements from the SRM material are expected to show heterogeneity due to variations in preferred orientation. The heterogeneous nature of the samples requires a certification of a population of true relative intensities rather than the average relative intensity for each peak. The preferred orientation will uniformly effect the variability of all the peaks so analysis of the data assumed the intensity measurements have the same true relative variance. This allowed for pooling of the variances for both absolute and relative intensities. The exception to this assumption is the (300) peak; the crystallites in the diffracting position for this reflection must be perpendicular to the preferred orientation direction. Increased variability of intensity from this reflection is thus expected. The nominal value of the relative intensity is reported for information only, it was not used in subsequeni analysis.

\footnotetext{
'Certain manufacturers are mentioned for informative purposes only and do not represent an endorsement by NIST.
} 
PNNL-20650, Rev. 2

Battelle - Pacific Northwest National Laboratory

Radiochemical Sciences and Engineering Group

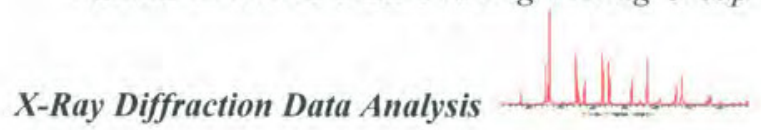

\section{ABSOLUTE VARIATION IN INTENSITY}

Analysis of the raw integrated intensity values indicated a drift in the instrument. This drift was modeled with a quadratic equation and removed from subsequent anaisses. The relative standard deviation of the raw counts, pooled from the 13 peaks is:

$$
\operatorname{RSD}(\text { raw counts })=2.21 \%
$$

This value incorporates both material variability and machine imprecision. This parameter can be used to determine differences in absolute sensitivity with different SRM samples.

\section{RELATIVE INTENSITY VARIATION}

The relative intensity values displayed no drift. The pooled relative standard deviation from integrated intensity measurements of the 12 peaks is:

$$
\text { RSD (integrated area, relative intensity) }=2.06 \%
$$

The pooled relative standard deviation of the relative intensity values determined from peak height measurements is:

$$
\text { RSD (peak height, relative intensity) }=2.62 \%
$$

The total relative uncertainty is a multiple of the relative standard deviation. For each relative intensity, this value is:

$$
\begin{array}{ll}
\text { Total Uncertainty (integrated intensity) } & =6.12 \% \\
\text { Total Uncertainty (peak height) } & =7.85 \%
\end{array}
$$

This uncertainty is the hall-width of a $90 \%$ conlidence, $95 \%$ coverage tolerance interva!. With $90 \%$ confidence, $95 \%$ of the true relative intensities for all of the peaks of this SRM should fall within the uncertainty of the certified values.

Each SRM plate is sintered to a relatively low density allowing it to be scored and broken easily into a desired size. Care should be taken to ensure that the side opposite the label is illuminated; otherwise, results are invalid. Each plate is cylindrically symmetrical, thus orientation of the plate is not of concern. However, errors due to particle counting statistics will be reduced if a sample spinner is used. Consistency in the degree of size and micro-strain induced peak broadening of the SRM allow for the use of peak heights as a substitution for integrated intensity measurements, though precision is seen to suffer as a result. The ratio of experimental data to the certified values should be plotied as a function of $2 \Theta$ angle. If this data is patiernless, and if all of the ratios fall within the total uncertainty $(0.0612 / 0.0785)$ of 1 , then the test equipment is in control. However, if there is a clear pattern in the data, or if the ratio for at least one peak is more than $0.0612 / 0.0785$ from 1, then the system is out of control (sec Appendix). 
PNNL-20650, Rev. 2

Battelle - Pacific Northwest National Laboratory

Table !

Refiection(s) (h
$(012)$
$(104)$
$(113)$
$(024)$
$(116)$
$(300)$
$(1.0 .10)(119)$
$(0.2 .10)$
$(226)$
$(2.1 .10)$
$(324)(0.1 .14)$
$(1.3 .10)$
$(146)$
$(4.0 .10)$

Scan Angles (low:high) $2 \Theta$

- 24.7

26.2

- 34.0

36.2

- 42.4

44.2

- 51.8

53.3

- 56.0

59.0

- 67.4

69.0

75.7

78.2

- 88.1

89.7

$9: 3$

96.0

100.1

102.0

115.4

117.4

$126.8 \quad 128.95$

135.2

137.4

144.3

146.7

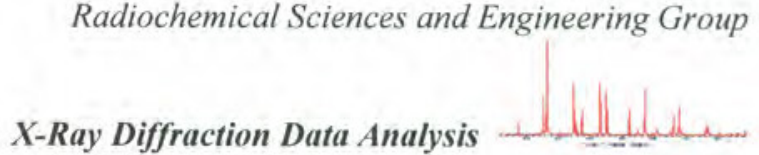

Relative Intensity

\begin{tabular}{cc}
\hline Integrated Area & Peak Height \\
\cline { 2 - 2 } 32.34 & 33.31 \\
100.0 & 100.0 \\
51.06 & 49.57 \\
26.69 & 25.17 \\
92.13 & 83.6 \\
19.13 & 16.89 \\
55.57 & 34.61 \\
11.76 & 8.99 \\
10.14 & 7.25 \\
16.13 & 10.94 \\
20.86 & 10.09 \\
15.58 & 7.56 \\
15.47 & 6.55 \\
11.29 & 4.06
\end{tabular}

\section{LATTICE PARAMETERS}

The certified lattice parameters, from the mean of the 25 samples are:

$$
\begin{aligned}
& a=0.4758846 \mathrm{~nm}(4.758846 \AA) \\
& a=0.0000109(0.000109) \\
& c=1.299306 \mathrm{~nm}(12.99306 \AA) \\
& a=0.0000238(0.000238)
\end{aligned}
$$

where $\lambda\left(\mathrm{CuK}_{1}\right)=0.1540629 \mathrm{~nm}(1.540629 \AA)$ 
PNNL-20650, Rev. 2

Appendix: How to assess and correct instrument bias using this SRM

When looking for instrumental problems, the peaks should be considered joindly, not individually. First, plot control bands as $1 \pm U$ over the range of $2 \Theta$, where $U$ is the relative uncerainty $(\mathrm{U}=0.0612$ if peak areas were used, or 0.0785 if peak heights were used). Next, plot the ratio of each new measurement to the certified value on the vertical axis against the $2 \Theta$ peak location on the horizontal axis. As long as these ratios fall within the control bands and are patternless, the system cannot be labelled "out of control."

However, if there is a pattern in the ratios, then the device is introducing a systemaric error, even if all of the ratios fall within the control bands. This systematic error should be corrected by modelling the ratios as a function of peak location and using the model to correct future observations on unknowns.

Let the measured relative intensities on the 12 peaks be $y_{i}$ and the certified values be $c_{i}$, so the ratios to model are

$$
\mathrm{r}_{\mathrm{i}}=\frac{\mathrm{y}_{\mathrm{i}}}{\mathrm{c}_{\mathrm{i}}}
$$

Fit a simple model, using ordinary least squares regression, which describes these ratios adequately as a function of peak location $\left(\mathrm{x}_{\mathrm{i}}\right)$. The fitted model has $m$ parameters: $m=1$ for a horizontal straight line, $m=2$ for a slanted straight line, $m=3$ for a quadratic, etc. The residual sum of squares from the fit, plus an allowance for the uncertainty in the certified values on the certificate, is:

$$
\operatorname{RSS}_{\text {full }}=\sum_{1}^{n}\left(r_{i}-\hat{r}_{i}\right)^{2}+(n-m) \frac{(R S D)^{2}}{25}
$$

and the residual degrees of freedom for this "full" model are $n-m$. The RSD $=0.0206$ if peak areas were used, or 0.0262 if peak heights were used. The residual sum of squares for the horizontal line at 1 (which describes "ideal" data), plus an allowance for the uncertainty in the certified values on the certificate, is:

$$
\operatorname{RSS}_{1}=\sum_{1}^{n}\left(x_{i}-1\right)^{2}+(n-1) \frac{(R S D)^{2}}{25}
$$

and the residual degrees of freedom are $n-1$. Then if the test statistic

$$
F=\frac{\left\{\text { RSS }_{1}-\mathrm{RSS}_{\text {full }}+(\mathrm{m}-1) \frac{(\mathrm{RSD})^{2}}{25}\right\} /(\mathrm{m}-1)}{\left\{\mathrm{RSS}_{\text {full }}+(\mathrm{n}-\mathrm{m}) \frac{(\mathrm{RSD})^{2}}{25}\right\} /(\mathrm{n}-\mathrm{m})}
$$

is larger than the upper 0.05 percentage point from the $\mathrm{F}$ distribution with $\mathrm{m}-\mathrm{I}$ numerator and $\mathrm{n}-\mathrm{m}$ denominator degrees of freedom (commonly provided in table form in introductory statistics texts), the correction model is needed.

To use the correction model: A new peak angle $x^{*}$ and a new relative intensity $y^{*}$ are measured on an unknown sample. If $\hat{r}^{*}$ is the predicted value of the firted model at $x^{*}$, the corrected relative intensity for the new peak angle on the unknown sample is

$$
\text { corrected relative intensity }=\frac{y^{*}}{\hat{\mathrm{r}}^{*}} \text {. }
$$


PNNL-20650, Rev. 2

Battelle - Pacific Northwest National Laboratory

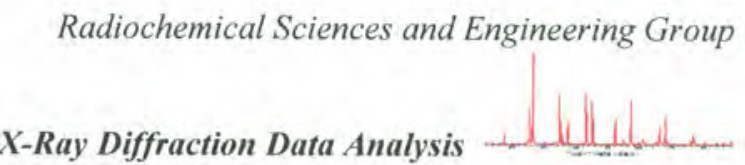

\section{Appendix 6 \\ PDF cards for the identified crystalline phases presented in this report}

The following PDF cards are for the crystalline phases identified in this report.

The PDF card includes information about the crystal structure, the reference, the X-ray radiation wavelength, the calculated density and the observed density (if published), the strongest lines and the corresponding 2 theta angles. The table presented at the end of each PDF card includes information about the 2 theta angle positions, interplanar distances at that angle, the intensity of that particular reflection and Miller indices at that particular 2 theta. The compounds identified in this report match the PDF card peaks, which are the stick figures in the report.

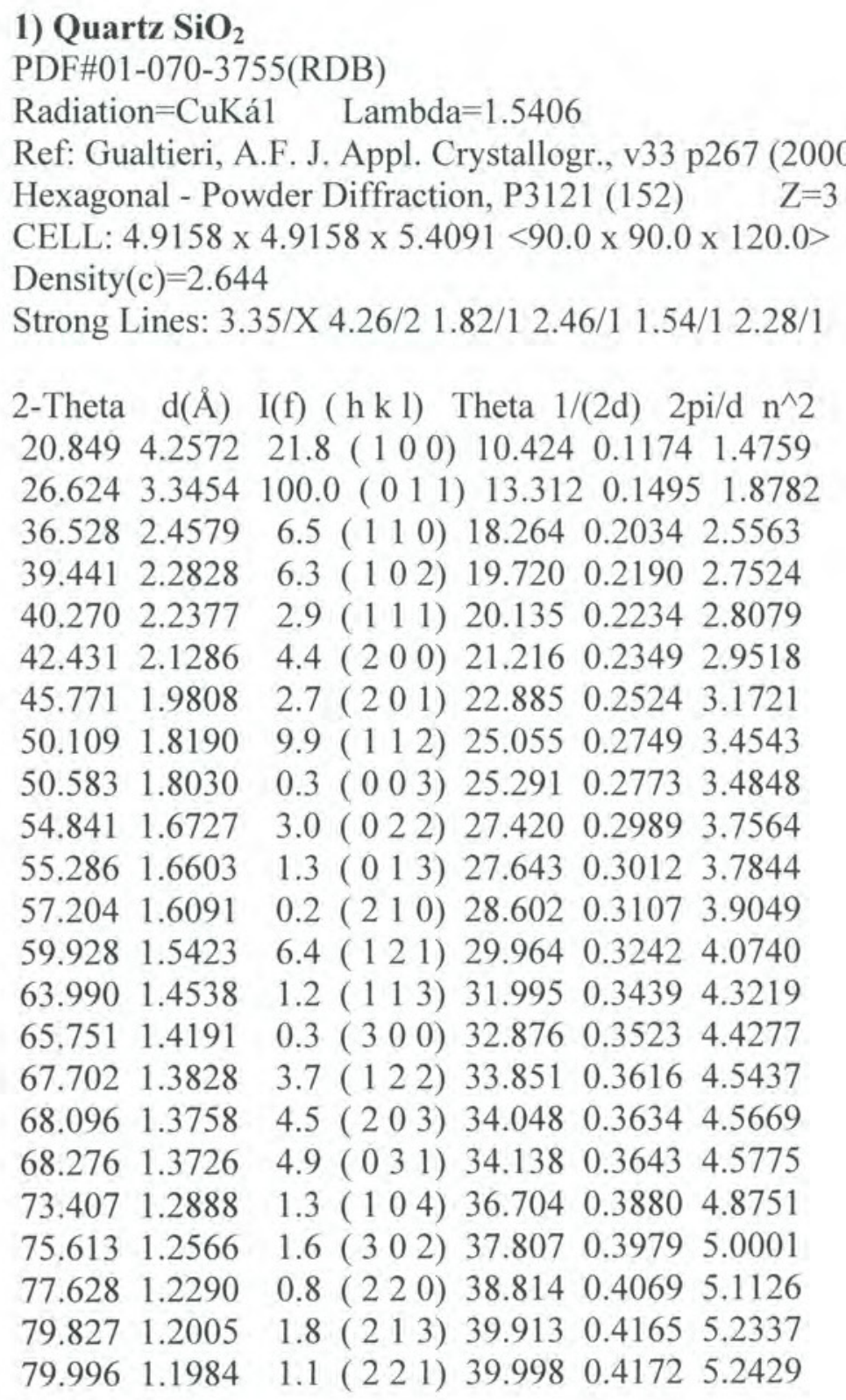


PNNL-20650, Rev. 2

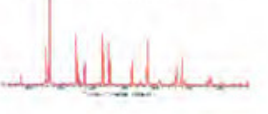

\begin{tabular}{|c|c|c|c|c|c|}
\hline & & & & & \\
\hline & & 7 & & & \\
\hline & & 9 & & & \\
\hline & & & & & \\
\hline & & & & & \\
\hline & & & & & \\
\hline & & & & & \\
\hline & & & & & \\
\hline & & & & & \\
\hline & & & & & \\
\hline & & & & & \\
\hline & & & & & \\
\hline & & & & & \\
\hline & & & & & \\
\hline & & & & & \\
\hline & & & & & \\
\hline & & & & & \\
\hline & & & & & \\
\hline & & & & & \\
\hline & & & & & \\
\hline & & & & & \\
\hline & & & & & \\
\hline & & & & & \\
\hline & & & & & \\
\hline & & & & & \\
\hline & & & & & \\
\hline & & & & & \\
\hline & & & & & \\
\hline & & 0.2 & & & \\
\hline & & 0.1 & & & \\
\hline & & 0.1 & & & \\
\hline & & & & & \\
\hline & & 0.1 & & & \\
\hline & & 0.1 & & & \\
\hline & & 0.4 & & & \\
\hline & & 0.5 & & & \\
\hline & & 0.1 & & & \\
\hline & & 0.6 & & & \\
\hline & & 0.6 & & & \\
\hline & & & & & \\
\hline & & 0.3 & & & \\
\hline & & 0.2 & 74. & 0. & 7.877 \\
\hline
\end{tabular}


PNNL-20650, Rev. 2

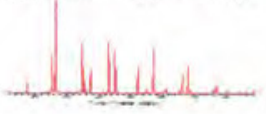

2) Rutile, syn (Titanium Oxide) Ti O2PDF\#01-077-0440(RDB)

Radiation $=$ CuKál Lambda $=1.5406 \quad$ Filter $=$

Calibration $=2 \mathrm{~T}=27.438-143.112 \quad \mathrm{I} / \mathrm{Ic}(\mathrm{RIR})=3.6$

Ref: Seki, H., Ishizawa, N., Mizutani, N., Kato, M. Yogyo Kyokaishi (J. Ceram. Assoc. Jpn.), v92 p219 (1984)

Tetragonal - Single Crystal, P42/mnm (136) Z=2

CELL: $4.5934 \times 4.5934 \times 2.9575<90.0 \times 90.0 \times 90.0>\quad P . S=t P 6.00$

$\operatorname{Density}(\mathrm{c})=4.252$

Strong Lines: 3.25/X 1.69/5 2.49/4 2.19/2 1.36/2 1.62/1 1.35/1 1.48/1

Calculated Pattern Original Remarks: Stable from 800 to 1800 K (2nd ref.,

Tomaszewski), 300-800 K: Pbca.

Temperature of Data Collection: $300 \mathrm{~K}$.

Wyckoff Sequence: f a(P42/MNM).

Unit Cell Data Source: Single Crystal.

2-Theta $\mathrm{d}(\AA) \quad \mathrm{I}(\mathrm{f}) \quad(\mathrm{h} \mathrm{k} \mathrm{l})$ Theta $1 /(2 \mathrm{~d}) \quad 2 \mathrm{pi} / \mathrm{d} \mathrm{n} \mathrm{n}^{\wedge} 2$

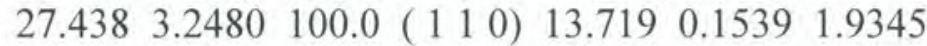

$\begin{array}{llllllll}36.091 & 2.4867 & 43.6 & \left(\begin{array}{lll}1 & 0 & 1\end{array}\right) & 18.046 & 0.2011 & 2.5268\end{array}$

$\begin{array}{llllllll}39.193 & 2.2967 & 6.4 & (200) & 19.596 & 0.2177 & 2.7357\end{array}$

$\begin{array}{lllllll}41.250 & 2.1868 & 17.0 & \left(\begin{array}{lllll}1 & 1 & 1\end{array}\right) & 20.625 & 0.2286 & 2.8733\end{array}$

$\begin{array}{lllllll}44.046 & 2.0542 & 6.0 & \text { (2 } 210) & 22.023 & 0.2434 & 3.0587\end{array}$

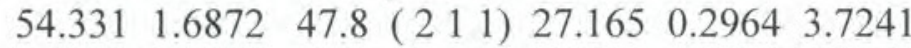

$\begin{array}{lllllllll}56.630 & 1.6240 & 13.7 & \text { (2 } 220) & 28.315 & 0.3079 & 3.8689\end{array}$

$\begin{array}{lllllllll}62.787 & 1.4788 & 6.5 & \left(\begin{array}{llll}0 & 0 & 2\end{array}\right) & 31.393 & 0.3381 & 4.2490\end{array}$

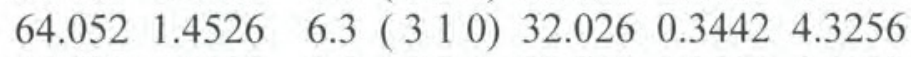

$\begin{array}{lllllllll}65.520 & 1.4235 & 0.5 & \text { (2 } 2 \text { 2 } 1) & 32.760 & 0.3512 & 4.4138\end{array}$

$69.014 \quad 1.3597 \quad 15.1 \quad\left(\begin{array}{lllll}3 & 0 & 1\end{array}\right) 34.507 \quad 0.3677 \quad 4.6209$

$\begin{array}{lllllll}69.829 & 1.3458 & 7.7 & \left(\begin{array}{llll}1 & 1 & 2\end{array}\right) & 34.915 & 0.3715 & 4.6686\end{array}$

$\begin{array}{llllllll}72.429 & 1.3038 & 0.8 & \left(\begin{array}{llll}3 & 1 & 1\end{array}\right) & 36.214 & 0.3835 & 4.8191\end{array}$

$\begin{array}{llllllll}74.405 & 1.2740 & 0.2 & \text { (3 } 220) & 37.203 & 0.3925 & 4.9319\end{array}$

$\begin{array}{llllllll}76.565 & 1.2433 & 1.6 & \text { (2 } 202 \text { 2) } & 38.283 & 0.4021 & 5.0535\end{array}$

$\begin{array}{llllllll}79.858 & 1.2001 & 0.9 & \text { (2 } 12 \text { 2) } & 39.929 & 0.4166 & 5.2354\end{array}$

$\begin{array}{llllllll}82.348 & 1.1700 & 2.9 & \left(\begin{array}{l}3 \\ 3\end{array} 21\right) & 41.174 & 0.4273 & 5.3701\end{array}$

$\begin{array}{lllllll}84.255 & 1.1484 & 2.0 & \left(\begin{array}{lll}4 & 0 & 0\end{array}\right) & 42.128 & 0.4354 & 5.4715\end{array}$

$\begin{array}{llllllll}87.487 & 1.1141 & 0.7 & (410) & 43.743 & 0.4488 & 5.6399\end{array}$

$\begin{array}{lllllll}89.579 & 1.0934 & 4.8 & \text { (2 2 2) } & 44.789 & 0.4573 & 5.7465\end{array}$

$\begin{array}{lllllllll}90.710 & 1.0827 & 2.5 & \left(\begin{array}{l}3 \\ 3\end{array} 0\right) & 45.355 & 0.4618 & 5.8034\end{array}$

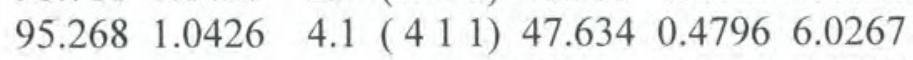

$\begin{array}{llllllll}96.035 & 1.0363 & 3.3 & \left(\begin{array}{llll}3 & 1 & 2\end{array}\right) & 48.017 & 0.4825 & 6.0634\end{array}$

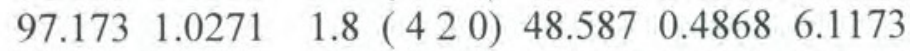

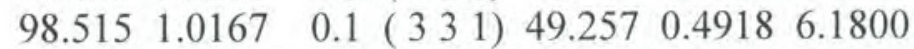

$\begin{array}{llllllll}105.103 & 0.9703 & 0.4 & (421) & 52.551 & 0.5153 & 6.4757\end{array}$

$\begin{array}{lllllllll}106.099 & 0.9639 & 1.3 & \left(\begin{array}{lllll}1 & 0 & 3\end{array}\right) & 53.050 & 0.5187 & 6.5186\end{array}$

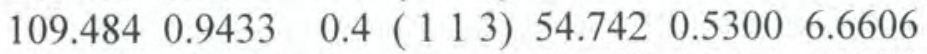

$\begin{array}{llllllll}113.960 & 0.9187 & 0.1 & (430) & 56.980 & 0.5443 & 6.8394\end{array}$ 
PNNL-20650, Rev. 2

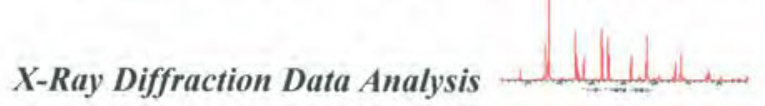
116.2700 .9070
1.9 (4 $\left.\begin{array}{lll}4 & 2\end{array}\right) 58.1350 .5513 \quad 6.9276$
$\begin{array}{lll}117.538 & 0.9008\end{array}$
$2.1\left(\begin{array}{lllll}5 & 10) & 58.769 & 0.5550 & 6.9748\end{array}\right.$
119.9230 .8898
1.0 (4 $\left.\begin{array}{ll}4 & 2\end{array}\right) 59.9620 .56197 .0613$
$\begin{array}{lll}120.151 & 0.8888\end{array}$

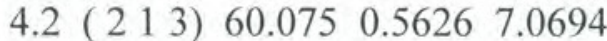
$122.804 \quad 0.8773$
3.9 (4 3 1) 61.4020 .56997 .1617
$\begin{array}{lll}123.716 & 0.8736\end{array}$
2.8 (3 332 2) $61.858 \quad 0.5724 \quad 7.1926$
$\begin{array}{lll}126.728 & 0.8618\end{array}$
$0.1\left(\begin{array}{llll}5 & 1 & 1\end{array}\right) 63.364 \quad 0.5802 \quad 7.2912$
129.1290 .8530

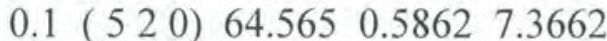
$131.880 \quad 0.8436$
2.5 (4 2 2 2) $65.940 \quad 0.5927 \quad 7.4482$
$\begin{array}{lll}136.657 & 0.8289\end{array}$
3.0 (3 $\left.\begin{array}{lllll}3 & 0 & 3\end{array}\right) 68.3280 .60327 .5803$
140.0630 .8196
$5.1\left(\begin{array}{lll}5 & 2 & 1\end{array}\right) 70.0310 .61017 .6665$
141.5820 .8157

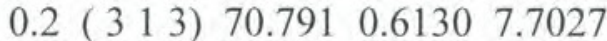
143.1120 .8120
0.7 ( 440$) 71.556 \quad 0.6158 \quad 7.7379$

\section{3) Corundum (Aluminum Oxide), $\mathrm{Al}_{2} \mathrm{O}_{3}, \mathrm{PDF}$ 01-071-6478}

\section{Radiation $=$ CuKál $\quad$ Lambda $=1.5406$}

Ref: Riello, P., Canton, P., Fagherazzi, G. Powder Diffraction, v12 p160 (1997)

Rhombohedral - Powder Diffraction, R-3c (167) $\quad Z=6$

CELL: $4.7598 \times 4.7598 \times 12.9924<90.0 \times 90.0 \times 120.0>\quad$ P.S $=h R 10.00(A ́)$

$\operatorname{Density}(\mathrm{c})=3.985$

Strong Lines: 2.55/X 2.09/X 1.60/9 3.48/7 1.37/5 1.74/5 2.38/5 1.40/3

ICSD Collection Code: 77810.

Calculated Pattern Original Remarks: R(Bragg) $=0.03$.

Minor Warning: $10 \%<\mathrm{R}$ factor $<15 \%$ (for powder).

Wyckoff Sequence: e c(R3-CH)

2-Theta $\mathrm{d}(\AA)$ I(f) (h k l) Theta $1 /(2 \mathrm{~d}) \quad 2 \mathrm{pi} / \mathrm{d} \mathrm{n} \mathrm{n}^{\wedge} 2$

$\begin{array}{lllllllll}25.573 & 3.4805 & 67.0 & \left(\begin{array}{llll}0 & 1 & 2\end{array}\right) & 12.786 & 0.1437 & 1.8052\end{array}$

$35.1472 .5512100 .0\left(\begin{array}{lllll}1 & 0 & 4\end{array}\right) \quad 17.5740 .19602 .4628$

$\begin{array}{lllllll}37.770 & 2.3799 & 46.0 & \left(\begin{array}{llll}1 & 1 & 0\end{array}\right) & 18.885 & 0.2101 & 2.6401\end{array}$

$\begin{array}{lllllll}41.676 & 2.1654 & 0.5 \cdot\left(\begin{array}{llll}0 & 0 & 6\end{array}\right) & 20.838 & 0.2309 & 2.9016\end{array}$

$\begin{array}{lllllll}43.347 & 2.0857 & 96.5 & \left(\begin{array}{llll}1 & 1 & 3\end{array}\right) & 21.674 & 0.2397 & 3.0125\end{array}$

$\begin{array}{lllllll}46.170 & 1.9646 & 1.5 & \left(\begin{array}{llll}2 & 0 & 2\end{array}\right) & 23.085 & 0.2545 & 3.1983\end{array}$

$\begin{array}{llllllll}52.544 & 1.7403 & 46.2 & \left(\begin{array}{llll}0 & 2 & 4\end{array}\right) & 26.272 & 0.2873 & 3.6105\end{array}$

$\begin{array}{lllllll}57.494 & 1.6016 & 91.0 & \left(\begin{array}{llll}1 & 1 & 6\end{array}\right) & 28.747 & 0.3122 & 3.9230\end{array}$

$\begin{array}{llllllll}59.729 & 1.5469 & 2.2 & \left(\begin{array}{llll}2 & 1 & 1\end{array}\right) & 29.865 & 0.3232 & 4.0617\end{array}$

$\begin{array}{llllllll}61.118 & 1.5151 & 3.4 & \left(\begin{array}{llll}1 & 2 & 2\end{array}\right) & 30.559 & 0.3300 & 4.1472\end{array}$

$\begin{array}{llllllll}61.299 & 1.5110 & 8.2 & \left(\begin{array}{llll}0 & 1 & 8\end{array}\right) & 30.650 & 0.3309 & 4.1583\end{array}$

$\begin{array}{lllllll}66.507 & 1.4048 & 34.4 & \text { (2 } 14 \text { ) } & 33.254 & 0.3559 & 4.4728\end{array}$

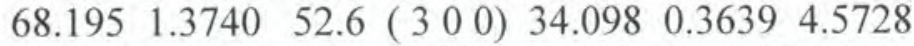

$\begin{array}{lllllllll}70.405 & 1.3362 & 1.1 & \left(\begin{array}{llll}1 & 2 & 5\end{array}\right) & 35.203 & 0.3742 & 4.7022\end{array}$

$\begin{array}{llllllll}74.294 & 1.2756 & 1.3 & (208) & 37.147 & 0.3920 & 4.9256\end{array}$

$\begin{array}{lllllll}76.871 & 1.2392 & 14.7 & (1,0,10) & 38.435 & 0.4035 & 5.0706\end{array}$

$\begin{array}{lllllll}77.230 & 1.2343 & 8.6 & \left(\begin{array}{lll}1 & 1 & 9\end{array}\right) & 38.615 & 0.4051 & 5.0906\end{array}$

$80.407 \quad 1.1933 \quad 0.7$ (2 217 ) $40.204 \quad 0.4190 \quad 5.2653$ 
PNNL-20650, Rev. 2

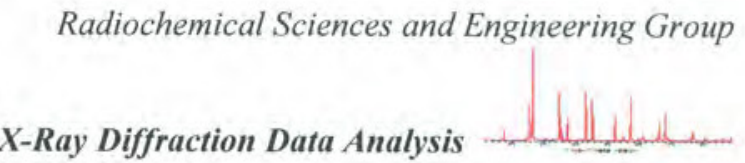

\begin{tabular}{|c|c|c|c|}
\hline 682 & 00 & $5.7(220) 40.341$ & 2025 \\
\hline 3.203 & 1.1602 & $0.6(036) 41.601$ & $0.4310 \quad 5.4157$ \\
\hline 338 & 1.1474 & $4.6(223) 42.169$ & $0.4358 \quad 5.4759$ \\
\hline 121 & 1.1389 & $0.3(131) 42.560$ & $0.4390 \quad 5.5170$ \\
\hline 86.333 & 1.1260 & $3.4\left(\begin{array}{lll}3 & 1 & 2\end{array}\right) 43.167$ & $0.4441 \quad 5.5803$ \\
\hline 86.492 & 1.1243 & $2.7(128) 43.246$ & 0.44475 .5885 \\
\hline 88.990 & 1.0991 & $6.3(0,2,10) 44.495$ & 0.45495 .7167 \\
\hline 90.707 & 1.0827 & $1.6(0,0,12) 45.354$ & 0.46185 .8033 \\
\hline 91.170 & 1.0784 & $7.4(134) 45.585$ & $0.4636 \quad 5.8263$ \\
\hline 94.800 & 1.0465 & $0.4(315) 47.400$ & $0.4778 \quad 6.0042$ \\
\hline 95.231 & 1.0429 & $14.4(226) 47.615$ & 0.47956 .0250 \\
\hline 98.370 & 1.0178 & $1.8(042) 49.185$ & 0.49136 .1733 \\
\hline 101.06 & 0.9978 & $10.3(2,1,10) 50.532$ & 20.50116 .2969 \\
\hline 102.819 & 0.9855 & $0.3(1,1,12) 51.409$ & 90.50746 .3756 \\
\hline 103.293 & 0.9823 & $1.9(404) 51.646$ & 0.50906 .3966 \\
\hline 104.620 & 0.9734 & $0.1\left(\begin{array}{lll}1 & 37 & 7\end{array}\right) 52.310$ & 0.51376 .4548 \\
\hline 109.511 & 0.9432 & $0.4(321) 54.756$ & $0.5301 \quad 6.6617$ \\
\hline 109.848 & 0.9412 & $0.3(1,2,11) 54.924$ & $4 \quad 0.5312 \quad 6.6755$ \\
\hline 110.96 & 0.9349 & $2.9(318) 55.484$ & $0.5348 \quad 6.7210$ \\
\hline 114.050 & 0.9182 & $2.0(229) 57.025$ & $0.5445 \quad 6.8428$ \\
\hline 116.069 & 0.9080 & $9.4(324) 58.034$ & 0.55076 .9200 \\
\hline 116.600 & 0.9054 & $6.5(0,1,14) 58.300$ & $0 \quad 0.5523 \quad 6.9399$ \\
\hline 117.817 & 0.8995 & $5.2\left(\begin{array}{lll}4 & 10 & 58.908\end{array}\right.$ & 0.55596 .9851 \\
\hline 120.180 & 0.8887 & $0.3(235) 60.090$ & 0.56267 .0704 \\
\hline 122.000 & 0.8807 & 3.2 ( 4 1 1 3) 61.000 & 0.56777 .1341 \\
\hline 124.567 & 0.8701 & $1.9(048) 62.284$ & 0.57467 .2209 \\
\hline 127.657 & 0.8583 & $10.5(1,3,10) 63.829$ & $90.5826 \quad 7.3206$ \\
\hline 129.859 & 0.8504 & $4.2(0,3,12) 64.930$ & $0 \quad 0.5879 \quad 7.3884$ \\
\hline 131.092 & 0.8462 & $4.1(2,0,14) 65.546$ & $60.5909 \quad 7.4251$ \\
\hline 132.178 & 0.8426 & $0.4(327) 66.089$ & 0.59347 .4568 \\
\hline 132.608 & 0.8412 & $0.3(2,1,13) 66.304$ & $4 \quad 0.5944 \quad 7.4692$ \\
\hline 136.032 & 0.8307 & $15.7(146) 68.016$ & 60.60197 .5638 \\
\hline 139.339 & 0.8215 & $0.1 \quad(3,1,11) 69.669$ & $90.6087 \quad 7.6487$ \\
\hline 140.977 & 0.8172 & $0.4(238) 70.489$ & 0.61187 .6885 \\
\hline 142.308 & 0.8139 & $2.1(1,1,15) 71.154$ & $40.6143 \quad 7.7196$ \\
\hline & 0.8074 & $6.1(4,0,10) 72.565$ & $5 \quad 0.6193 \quad 7.7821$ \\
\hline 148.258 & 0.8008 & $0.2(2,2,12) 74.129$ & 90.62447 .8459 \\
\hline 149.14 & 0.7991 & $4.1(054) 74.574$ & 0.62577 .8630 \\
\hline
\end{tabular}


PNNL-20650, Rev. 2

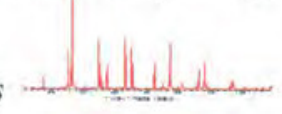

4) PDF\#01-070-0909(RDB): Calcium Sulfate, Ca S O4

Radiation $=$ CuKál $\quad$ Lambda $=1.5406$

Ref: Morikawa, H., Tomita, T., Minato, I., Iwai, S. Acta Crystallogr., Sec. B: Struct.

Crystallogr. Cryst. Chem., v31 p2164 (1975)

Orthorhombic - Single Crystal, Bmmb (63) $\mathrm{Z}=4$

CELL: $6.992 \times 6.999 \times 6.24<90.0 \times 90.0 \times 90.0>\quad P . S=0 C 24.00$

Density $(c)=2.961$

Strong Lines: 3.50/X 3.50/X 2.85/3 2.21/2 2.33/2 2.33/2 1.87/1 1.65/1

2-Theta d( $(\AA)$ I(f) $(\mathrm{h} \mathrm{k} \mathrm{l})$ Theta $1 /(2 \mathrm{~d}) \quad 2 \mathrm{pi} / \mathrm{d} \mathrm{n} \mathrm{n}^{\wedge}$

$\begin{array}{llllllll}19.047 & 4.6556 & 0.1 & \left(\begin{array}{lllll}1 & 0 & 1\end{array}\right) & 9.524 & 0.1074 & 1.3496\end{array}$

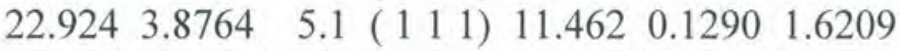

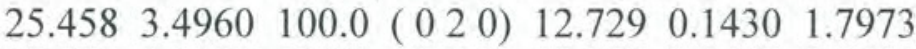

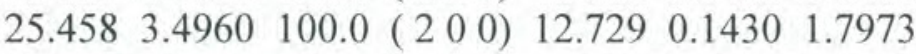

$\begin{array}{llllllllllll}28.587 & 3.1200 & 2.2 & \left(\begin{array}{llll}0 & 0 & 2\end{array}\right) & 14.294 & 0.1603 & 2.0138\end{array}$

$\begin{array}{llllllllllll}31.365 & 2.8497 & 31.8 & \left(\begin{array}{lllllll}0 & 1 & 2\end{array}\right) & 15.683 & 0.1755 & 2.2049\end{array}$

$\begin{array}{llllllllll}31.968 & 2.7974 & 3.1 & \left(\begin{array}{lllll}1 & 2 & 1\end{array}\right) & 15.984 & 0.1787 & 2.2461\end{array}$

$\begin{array}{lllllllll}36.293 & 2.4733 & 7.2 & \left(\begin{array}{lllll}2 & 2 & 0\end{array}\right) & 18.146 & 0.2022 & 2.5404\end{array}$

$\begin{array}{llllllllll}38.648 & 2.3278 & 17.9 & \left(\begin{array}{llllll}2 & 0 & 2\end{array}\right) & 19.324 & 0.2148 & 2.6992\end{array}$

$\begin{array}{lllllllllllllllll}38.648 & 2.3278 & 17.9 & \left(\begin{array}{llll}0 & 2 & 2\end{array}\right) & 19.324 & 0.2148 & 2.6992\end{array}$

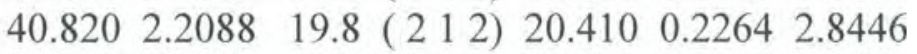

$\begin{array}{lllllllll}41.318 & 2.1833 & 6.1 & \left(\begin{array}{llll}3 & 0 & 1\end{array}\right) & 20.659 & 0.2290 & 2.8778\end{array}$

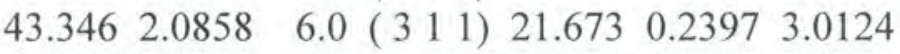

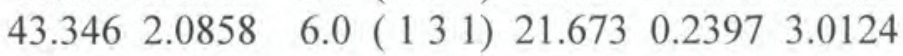

$\begin{array}{llllllllll}45.458 & 1.9937 & 4.3 & \left(\begin{array}{llllll}1 & 0 & 3\end{array}\right) & 22.729 & 0.2508 & 3.1516\end{array}$

$\begin{array}{lllllllll}46.836 & 1.9382 & 2.9 & \left(\begin{array}{llll}2 & 2 & 2\end{array}\right) & 23.418 & 0.2580 & 3.2418\end{array}$

$\begin{array}{llllllll}47.374 & 1.9174 & 0.4 & \left(\begin{array}{lllll}1 & 1 & 3\end{array}\right) & 23.687 & 0.2608 & 3.2770\end{array}$

$\begin{array}{llllllllll}48.695 & 1.8684 & 12.9 & \left(\begin{array}{lllll}0 & 3 & 2\end{array}\right) & 24.348 & 0.2676 & 3.3629\end{array}$

$\begin{array}{lllllll}49.144 & 1.8524 & 2.9 & \left(\begin{array}{llll}3 & 2 & 1\end{array}\right) & 24.572 & 0.2699 & 3.3919\end{array}$

$\begin{array}{lllllllll}52.294 & 1.7480 & 6.7 & (04 & 4 & 0 & 26.147 & 0.2860 & 3.5945\end{array}$

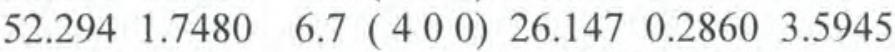

$\begin{array}{lllllllll}52.805 & 1.7323 & 0.3 & \left(\begin{array}{lllll}1 & 2 & 3\end{array}\right) & 26.402 & 0.2886 & 3.6271\end{array}$

$\begin{array}{llllllll}55.739 & 1.6478 & 12.6 & (232) & 27.869 & 0.3034 & 3.8130\end{array}$

$\begin{array}{llllllll}57.790 & 1.5941 & 2.1 & \left(\begin{array}{lll}3 & 3 & 1\end{array}\right) & 28.895 & 0.3136 & 3.9414\end{array}$

$\begin{array}{llllllll}59.022 & 1.5638 & 2.9 & (420) & 29.511 & 0.3197 & 4.0180\end{array}$

$\begin{array}{llllllll}59.022 & 1.5638 & 2.9 & (240) & 29.511 & 0.3197 & 4.0180\end{array}$

$\begin{array}{lllllllll}59.179 & 1.5600 & 1.7 & \left(\begin{array}{lllll}0 & 0 & 4\end{array}\right) & 29.589 & 0.3205 & 4.0277\end{array}$

$\begin{array}{llllllll}60.679 & 1.5250 & 2.5 & \left(\begin{array}{llll}0 & 4 & 2\end{array}\right) & 30.339 & 0.3279 & 4.1202\end{array}$

$\begin{array}{llllllll}60.679 & 1.5250 & 2.5 & \left(\begin{array}{llll}4 & 0 & 2\end{array}\right) & 30.339 & 0.3279 & 4.1202\end{array}$

$\begin{array}{lllllllll}60.781 & 1.5226 & 1.5 & \left(\begin{array}{llllll}0 & 1 & 4\end{array}\right) & 30.391 & 0.3284 & 4.1265\end{array}$

$\begin{array}{lllllllll}61.118 & 1.5151 & 0.9 & \left(\begin{array}{lllll}3 & 1 & 3\end{array}\right) & 30.559 & 0.3300 & 4.1471\end{array}$

$\begin{array}{lllllllll}61.118 & 1.5151 & 0.9 & \left(\begin{array}{llllll}1 & 3 & 3\end{array}\right) & 30.559 & 0.3300 & 4.1471\end{array}$

$\begin{array}{llllllll}62.258 & 1.4900 & 4.3 & \left(\begin{array}{llll}4 & 1 & 2\end{array}\right) & 31.129 & 0.3356 & 4.2168\end{array}$

$\begin{array}{lllllllll}65.464 & 1.4246 & 2.5 & \left(\begin{array}{llll}0 & 2 & 4\end{array}\right) & 32.732 & 0.3510 & 4.4105\end{array}$

$\begin{array}{lllllll}65.464 & 1.4246 & 2.5 & (204) & 32.732 & 0.3510 & 4.4105\end{array}$ 
PNNL-20650, Rev. 2

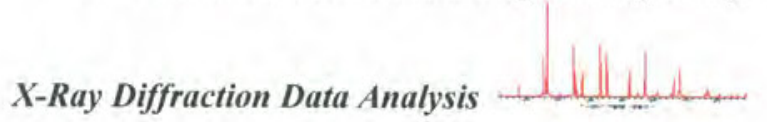

\begin{tabular}{|c|c|c|c|c|c|}
\hline & & 0.9 & & & \\
\hline & 1.3987 & $1.3(242)$ & (T) & 3575 & 4 \\
\hline & 1.3987 & $1.3(422)$ & .417 & & \\
\hline & 1.3960 & $2.1(214)$ & 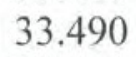 & & \\
\hline & 1.3654 & $0.5\left(\begin{array}{lll}5 & 0 & 1\end{array}\right)$ & .5 & 662 & 0 \\
\hline & 1.3654 & 0.5 & & - & 4 \\
\hline .147 & 1.3405 & $0.1\left(\begin{array}{lll}1 & 5 & 1\end{array}\right)$ & .073 & 13 & 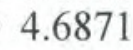 \\
\hline & 1.3393 & $0.1\left(\begin{array}{lll}5 & 1 & 1\end{array}\right)$ & & & \\
\hline & 1.3195 & $3.9(224)$ & 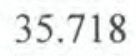 & 0 & . \\
\hline & & 2.1 & & & \\
\hline & 1.2968 & 1.4( & 0.7 & & \\
\hline 6 & 1.2765 & $4.8(052)$ & 37.1 & 0 & \\
\hline & 1.2 & 4.8 & & & \\
\hline 0 & 1.2366 & 0.8 & 38.528 & 0 . & \\
\hline & 1.2 & 0.1 & & & \\
\hline$f$ & 1.2159 & 3.0 & .312 & 0 . & \\
\hline & 1.2 & 0.2 & & & \\
\hline 900 & 1.1996 & 1.7( & 39.950 & 0 . & \\
\hline & 1.1 & 0.3 & & & \\
\hline 4 & 1.17 & 0.3 & 40. & & \\
\hline 653 & 1.16 & 1.7 & 4 & & \\
\hline & 1.1 & 2.0 & & & \\
\hline .878 & 1.1 & 1.8 & 41 & & \\
\hline 3 & 1.1 & 1.8 & & & \\
\hline .174 & 1.16 & 1.0 & 41 . & 0 . & \\
\hline 4 & 1.16 & 1.0 & & & \\
\hline 287 & 1.1592 & 0.4 & 41. & 0. & \\
\hline 10 & 1.1496 & 0.4 & 42. & & \\
\hline 84.275 & 1.14 & 0.8 & 42. & & \\
\hline & & 0.4 & 42.2 & & \\
\hline 84.503 & 1.14 & 0.4 & 42.2 & & \\
\hline 500 & 1.13 & 0.1 ( & 42.903 & & \\
\hline 8.236 & 1.10 & 1.8 & 44.118 & 0 . & \\
\hline (1) & 1.10 & 3.6 & 44.2 & 0. & \\
\hline 88.415 & 1.10 & 3.6 & 44.207 & 0.4526 & \\
\hline 88.742 & 1.10 & 1.8 & 44.371 & 0.4 & \\
\hline 88.877 & 1.10 & 0.5 & 44. & 0. & \\
\hline 00650 & 1.0926 & $0.2(0$ & 44.8 & 0. & 5. \\
\hline 89.758 & 1.09 & 0.1 & & 0 . & \\
\hline 90.243 & 1.0871 & 0.1 & 45.1 & 0.4600 & 5.7 \\
\hline 90.243 & 1.08 & $0.1\left(\begin{array}{lll}1 & 3 & 5\end{array}\right)$ & 45.1 & 0.4600 & \\
\hline 91.146 & 1.0786 & 1.4 ( 6 & 45.573 & 0.4636 & 5.8 \\
\hline 91.429 & 1.0760 & $0.8(5$ & 45.7 & 0.4647 & \\
\hline 94.434 & 1.04 & 0.1 (3 & 47.2 & 0.4764 & 5.98 \\
\hline 95.228 & 1.0429 & $0.9(262)$ & 47.6 & 0.4794 & 6.0248 \\
\hline
\end{tabular}


PNNL-20650, Rev. 2

\begin{tabular}{|c|c|c|c|c|c|}
\hline & & r & & & \\
\hline & & 1.7 & 47.698 & 4801 & \\
\hline & 400 & $1.1(353)$ & 47.78 & 1000 & \\
\hline & 400 & $1.1\left(\begin{array}{lll}0 & 0 & 6\end{array}\right)$ & 47788 & 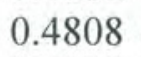 & \\
\hline & 1.0391 & 1.0 & 47.846 & 812 & \\
\hline & 312 & 0 & & 49 & \\
\hline & 289 & 0.9 & .477 & 60 & \\
\hline & & 0.9( & 477 & 60 & \\
\hline & & 0.2 ( 1 & (1) & 00 & \\
\hline & & & & & \\
\hline & & 0.5( & 50.4 & & \\
\hline & 9 & 1.4 & 50 & 0. & 6 \\
\hline & & 1.4( & & & \\
\hline & 0 & 0.7 & 50. & & 6. \\
\hline & & 1.7 & & & \\
\hline & 0 & 1.1 & 51 & & 6. \\
\hline & & 1.1 & & & \\
\hline & 1 & 0.1 & 5 & & 6. \\
\hline & & 0.1 & & & \\
\hline & & 1.4 & 5 & & \\
\hline & & 1.4 & & & \\
\hline & & 1.2 & & & \\
\hline & 0.9 & 0.8 & 5 & & \\
\hline & & 0.7 & & & \\
\hline & 0.9 & 0.5 & 5 & & 6. \\
\hline & & 0.2( & & & \\
\hline & 0.9 & 0.2( & 5 & & 6. \\
\hline & & 0.1 ( & & & \\
\hline & 0.9 & 0.1 & & & 6. \\
\hline & & 0.1 & & & \\
\hline & 0.9 & 0.1 & & & \\
\hline & 0.9 & 0.1 & 55 & & 6. \\
\hline & 0.9 & 0.5 & 56. & & 6. \\
\hline & 0.9 & 0.5( & 56.2 & & 6.7 \\
\hline ) & 0.9 & 0.3 & 56 & & \\
\hline & $0.9 ?$ & 0.2( & 56. & & 6.8 \\
\hline 111 & 0.9 & 0.2( & 56 & & \\
\hline & 0.91 & 0.8 & 56.9 & & 6.8 \\
\hline & 0.9 & 0.4 & & & \\
\hline & 0.90 & 0.1 & 57.986 & 0.5 & 6.9164 \\
\hline & & $0.1(371)$ & 57.9 & & 6.9164 \\
\hline 117.18 & 0.90 & $0.7(264)$ & 58.592 & 0.5 & 6.9617 \\
\hline & 0.90 & $0.7(624)$ & 58.592 & 0.5540 & 6.961 \\
\hline 177 & 0.89 & $0.5(525)$ & 58.8 & 0.5 & 6.982 \\
\hline & 000 & $0.5\left(\begin{array}{lll}7 & 0 & 3\end{array}\right)$ & 58.8 & 0.5557 & 6.982 \\
\hline
\end{tabular}


PNNL-20650, Rev. 2

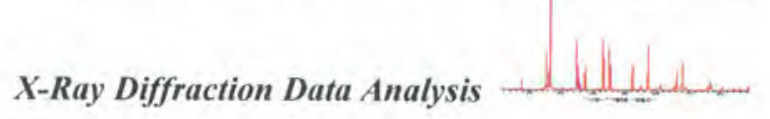

\begin{tabular}{|c|c|c|c|c|c|}
\hline & & 0.4 & & & \\
\hline 0 & 0.8940 & $1.0(046)$ & 59.499 & 93 & \\
\hline 9 & 0.8940 & 1.0 & & & \\
\hline & 866 & .5 & & & \\
\hline & 8866 & .5( & & & , \\
\hline & & & & & \\
\hline & 8773 & 7) & & & \\
\hline & & & & & \\
\hline & 40 & & & & \\
\hline & & & & & \\
\hline & & & & & \\
\hline & & & & & \\
\hline & & & & & \\
\hline & & & & & \\
\hline & & & & & \\
\hline & & & & & \\
\hline & & & & & \\
\hline & & & & & \\
\hline & & & & & \\
\hline & & 0.2 & & & \\
\hline & & & & & \\
\hline & & 0.4 & & & 57 \\
\hline & & & & & \\
\hline & & & & & \\
\hline & & & & & \\
\hline & & & & & \\
\hline & & & & & \\
\hline & & - & & & \\
\hline & & 0.3 & & & \\
\hline & & 0.1 & & & \\
\hline & & 1.1 & & & \\
\hline & & 0.9 & & & \\
\hline & 0.8 & 0.9 & & & 60 \\
\hline & & 0.7 & & & \\
\hline & 0.8 & 0.5 & 69 & & 7.6440 \\
\hline & & 0 . & & & 6774 \\
\hline & 0.8 & 0.5 & 70. & & 7.6774 \\
\hline & & 0 . & & & 7.7381 \\
\hline & 0.8 & 0.5 & 71. & & 7.7381 \\
\hline & & 0.4 & & & 7.7571 \\
\hline & 0.80 & 0.1 & 72. & & 7.7930 \\
\hline & & 0.1 ( 7 & 72.8 & & 7.7930 \\
\hline & & 0.2 & & & \\
\hline & 0.8004 & $0.2(7$ & 74.243 & 0.6247 & 7.850 \\
\hline
\end{tabular}


PNNL-20650, Rev. 2

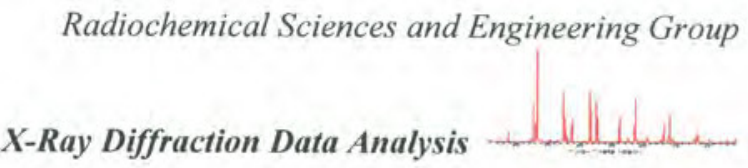

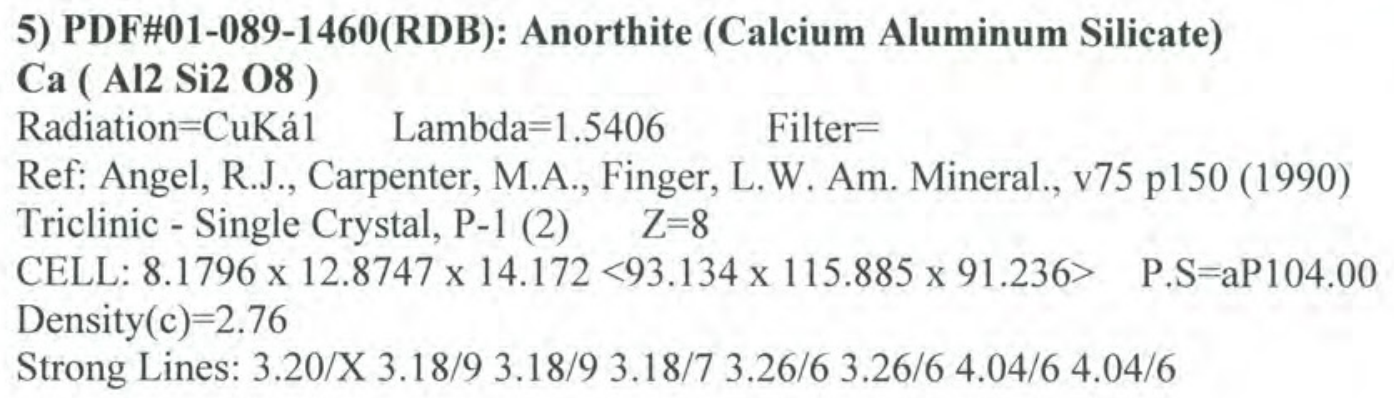

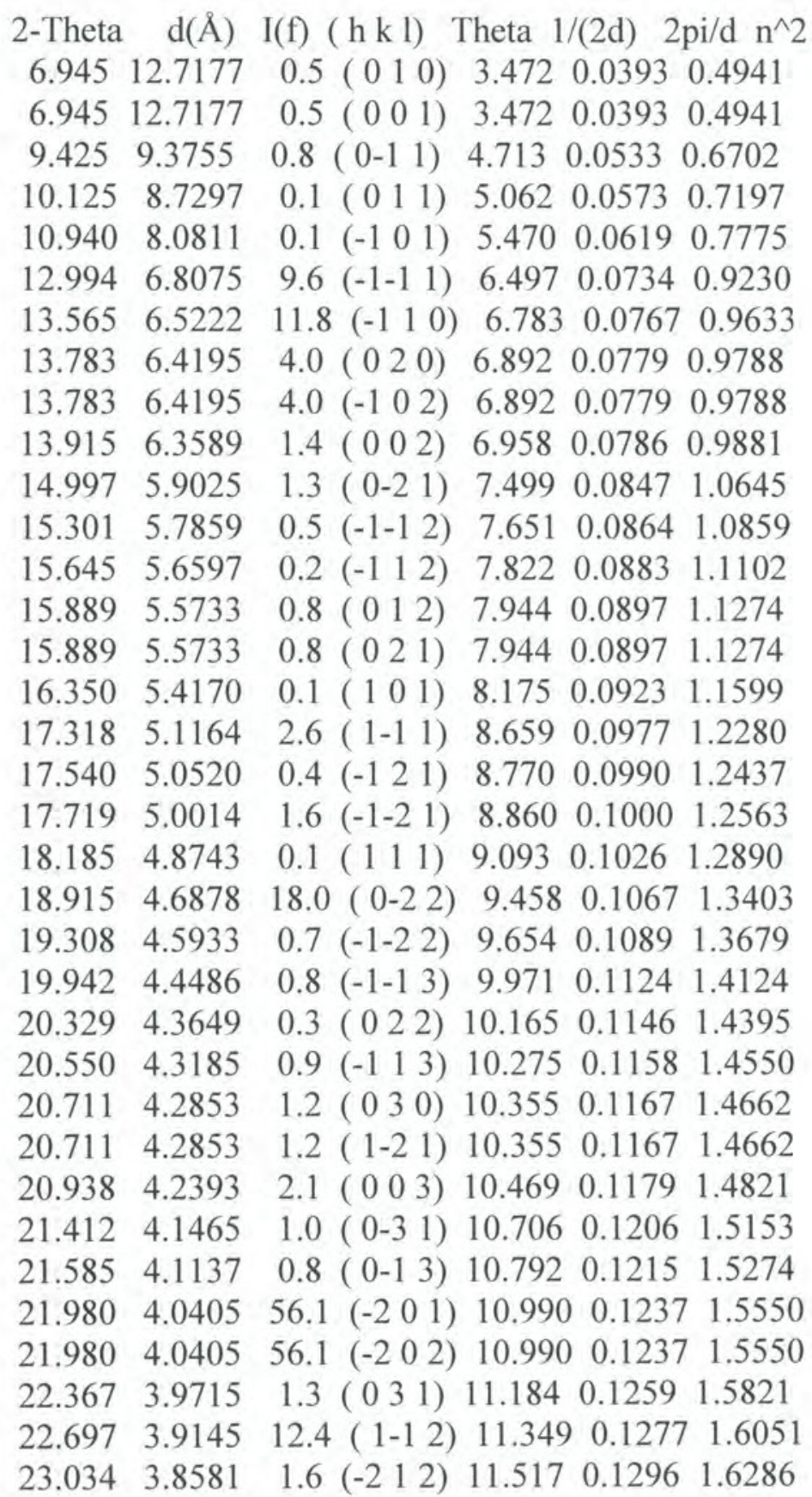


PNNL-20650, Rev. 2

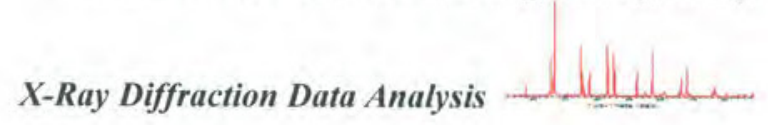

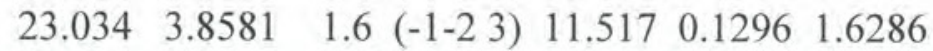

$$
\begin{aligned}
& \begin{array}{lllllllll}
23.501 & 3.7825 & 31.0 & (-1 & 3 & 0 & 11.750 & 0.1322 & 1.6611
\end{array} \\
& \begin{array}{llllllll}
23.670 & 3.7558 & 21.8 & \left(\begin{array}{lllll}
1 & 1 & 2
\end{array}\right) & 11.835 & 0.1331 & 1.6730
\end{array} \\
& \begin{array}{lllllllll}
24.091 & 3.6911 & 2.9 & \left(\begin{array}{lllll}
-2 & 0 & 3
\end{array}\right) & 12.046 & 0.1355 & 1.7023
\end{array} \\
& \begin{array}{lllllllll}
24.091 & 3.6911 & 2.9 & \left(\begin{array}{lllll}
-1 & 2 & 3
\end{array}\right) & 12.046 & 0.1355 & 1.7023
\end{array} \\
& \begin{array}{llllllll}
24.303 & 3.6594 & 2.4 & (0-23) & 12.151 & 0.1366 & 1.7170
\end{array}
\end{aligned}
$$

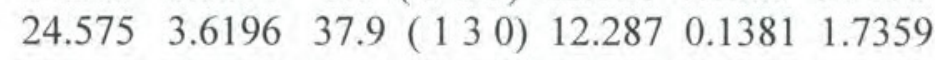

$$
\begin{aligned}
& \begin{array}{llllllll}
25.007 & 3.5580 & 1.0 & (-2-1 & 3 & 12.503 & 0.1405 & 1.7659
\end{array} \\
& \begin{array}{lllllllll}
25.159 & 3.5368 & 0.8 & \left(\begin{array}{lllll}
-2 & 1 & 3
\end{array}\right) & 12.579 & 0.1414 & 1.7765
\end{array} \\
& \begin{array}{llllllllll}
25.159 & 3.5368 & 0.8 & \left(\begin{array}{lllll}
-1 & 0 & 4
\end{array}\right) & 12.579 & 0.1414 & 1.7765
\end{array} \\
& \begin{array}{lllllllll}
25.343 & 3.5115 & 3.9 & \left(\begin{array}{llll}
-1 & 3 & 2
\end{array}\right) & 12.672 & 0.1424 & 1.7893
\end{array} \\
& \begin{array}{llllllll}
25.619 & 3.4743 & 5.3 & (1-31) & 12.809 & 0.1439 & 1.8085
\end{array} \\
& \begin{array}{llllllll}
25.730 & 3.4595 & 14.9 & (-1-14) & 12.865 & 0.1445 & 1.8162
\end{array} \\
& \begin{array}{lllllllll}
25.730 & 3.4595 & 14.9 & (-2 & 2 & 1 & 12.865 & 0.1445 & 1.8162
\end{array}
\end{aligned}
$$

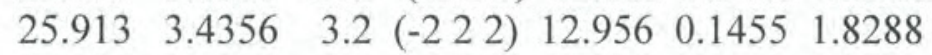

$$
\begin{aligned}
& \begin{array}{lllllllll}
25.979 & 3.4270 & 3.1 & \left(\begin{array}{llll}
0 & 2 & 3
\end{array}\right) & 12.989 & 0.1459 & 1.8334
\end{array} \\
& \begin{array}{llllllll}
26.160 & 3.4037 & 7.6 & (-2-2 & 2 & 13.080 & 0.1469 & 1.8460
\end{array} \\
& \begin{array}{lllllllll}
26.478 & 3.3635 & 29.7 & \left(\begin{array}{llll}
-1 & 1 & 4
\end{array}\right) & 13.239 & 0.1487 & 1.8680
\end{array} \\
& \begin{array}{llllllll}
26.478 & 3.3635 & 29.7 & (-2-2 & 1 & 13.239 & 0.1487 & 1.8680
\end{array} \\
& \begin{array}{lllllllll}
27.325 & 3.2611 & 57.2 & \left(\begin{array}{lllll}
1 & 3 & 1
\end{array}\right) & 13.663 & 0.1533 & 1.9267
\end{array}
\end{aligned}
$$

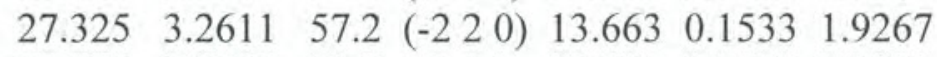

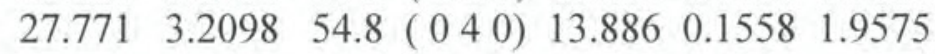

$$
\begin{aligned}
& \begin{array}{llllllll}
27.771 & 3.2098 & 54.8 & (-2-2 & 3 & 13.886 & 0.1558 & 1.9575
\end{array}
\end{aligned}
$$

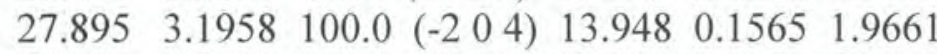

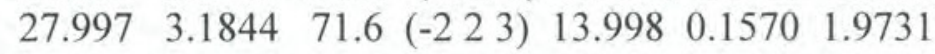

$$
\begin{aligned}
& \begin{array}{lllllllll}
28.042 & 3.1794 & 92.4 & \left(\begin{array}{llll}
2 & 0 & 1
\end{array}\right) & 14.021 & 0.1573 & 1.9762
\end{array} \\
& \begin{array}{llllllllll}
28.042 & 3.1794 & 92.4 & \left(\begin{array}{llll}
0 & 0 & 4
\end{array}\right) & 14.021 & 0.1573 & 1.9762
\end{array} \\
& \begin{array}{llllllll}
28.570 & 3.1219 & 44.4 & (-2-14) & 14.285 & 0.1602 & 2.0126
\end{array} \\
& \begin{array}{lllllllll}
28.570 & 3.1219 & 44.4 & (22 & 2 & 0 & 14.285 & 0.1602 & 2.0126
\end{array}
\end{aligned}
$$

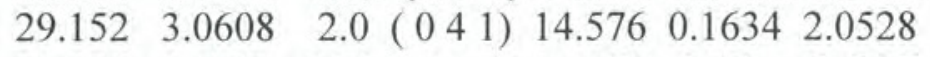

$$
\begin{aligned}
& \begin{array}{llllllllll}
29.349 & 3.0407 & 20.2 & \left(\begin{array}{lllll}
0 & 1 & 4
\end{array}\right) & 14.674 & 0.1644 & 2.0663
\end{array} \\
& 29.349 \quad 3.0407 \quad 20.2\left(\begin{array}{lllllll}
1-3 & 2 & 14.674 & 0.1644 & 2.0663
\end{array}\right.
\end{aligned}
$$

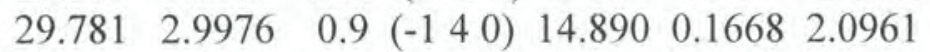

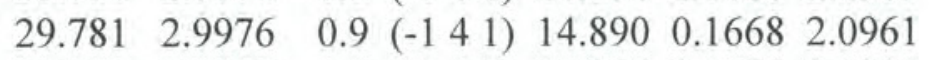

$$
\begin{aligned}
& \begin{array}{llllllll}
29.913 & 2.9847 & 1.0 & (-2314) & 14.956 & 0.1675 & 2.1051
\end{array} \\
& \begin{array}{lllllll}
29.913 & 2.9847 & 1.0 & \left(\begin{array}{llll}
1 & 1 & 3
\end{array}\right) & 14.956 & 0.1675 & 2.1051
\end{array}
\end{aligned}
$$

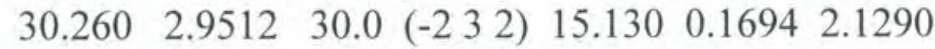

$$
\begin{aligned}
& \begin{array}{lllllll}
30.260 & 2.9512 & 30.0 & (0-42) & 15.130 & 0.1694 & 2.1290
\end{array} \\
& \begin{array}{lllllll}
30.447 & 2.9335 & 20.0 & (0-24) & 15.224 & 0.1704 & 2.1419
\end{array}
\end{aligned}
$$

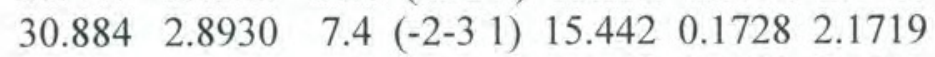

$$
\begin{aligned}
& \begin{array}{lllllll}
30.884 & 2.8930 & 7.4 & (-2-24) & 15.442 & 0.1728 & 2.1719
\end{array} \\
& \begin{array}{llllllll}
31.615 & 2.8277 & 24.6 & \left(\begin{array}{lllll}
1 & 3 & 2
\end{array}\right) & 15.808 & 0.1768 & 2.2220
\end{array}
\end{aligned}
$$

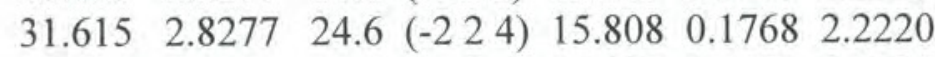

$$
\begin{aligned}
& \begin{array}{lllllllll}
31.758 & 2.8153 & 15.4 & \left(\begin{array}{llll}
-1 & 0 & 5
\end{array}\right) & 15.879 & 0.1776 & 2.2318
\end{array} \\
& \begin{array}{lllllll}
31.898 & 2.8033 & 10.0 & (-1-34) & 15.949 & 0.1784 & 2.2413
\end{array}
\end{aligned}
$$


PNNL-20650, Rev. 2

Battelle - Pacific Northwest National Laboratory

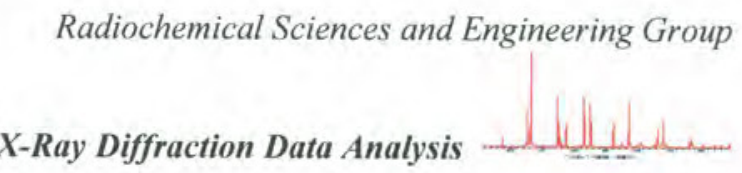

\begin{tabular}{|c|c|c|c|}
\hline 161 & 2.7810 & $2.1(-2.33) 16.080$ & $0.1798 \quad 2.2593$ \\
\hline 2.161 & 2.7810 & $2.1(221) 16.080$ & $0.1798 \quad 2.2593$ \\
\hline 2.856 & 2.7238 & $1.5\left(\begin{array}{lll}-3 & 0 & 2\end{array}\right) 16.428$ & 0.18362 .3068 \\
\hline 2.856 & 2.7238 & $1.5(-205) 16.428$ & 0.18362 .3068 \\
\hline 2.947 & 2.7164 & $1.1\left(\begin{array}{lll}-1 & 1 & 5\end{array}\right) 16.474$ & 0.18412 .3131 \\
\hline 3.046 & 2.7085 & $0.6\left(\begin{array}{lll}2 & 0 & 2\end{array}\right) 16.523$ & 0.18462 .3198 \\
\hline 3.046 & 2.7085 & $0.6(-1-43) 16.523$ & 0.18462 .3198 \\
\hline 335 & 2.6857 & $0.9\left(\begin{array}{l}14 \\
\end{array}\right) 16.667$ & 0.18622 .3395 \\
\hline 335 & 2.6857 & $0.9(-2-15) 16.667$ & 0.18622 .3395 \\
\hline 459 & 2.6760 & $1.9\left(\begin{array}{lll}-3 & 1 & 2\end{array}\right) 16.730$ & 0.18682 .3480 \\
\hline 719 & 2.6560 & $15.2(0-43) 16.859$ & 0.18832 .3657 \\
\hline 719 & 2.6560 & $15.2(-134) 16.859$ & 0.18832 .3657 \\
\hline 3.820 & 2.6482 & $10.3(0-34) 16.910$ & 0.18882 .3726 \\
\hline 820 & 2.6482 & $10.3(-3-12) 16.910$ & $0.1888 \quad 2.3726$ \\
\hline 4.215 & 2.6186 & 2) 17.107 & 0.19092 .3994 \\
\hline 215 & 2.6186 & 3) 17.107 & 0.19092 .3994 \\
\hline 1.532 & 2.5952 & $0.4(-2-34) 17.266$ & 0.19272 .4210 \\
\hline 532 & 2.5952 & $0.4(-311) \quad 17.266$ & 0.19272 .4210 \\
\hline 34.626 & 2.5884 & $0.4(-143) 17.313$ & 0.19322 .4274 \\
\hline .048 & 2.5582 & $5.7(2-22) 17.524$ & 0.19542 .4561 \\
\hline 35.048 & 2.5582 & $5.7(-3-11) 17.524$ & 0.19542 .4561 \\
\hline .263 & 2.5431 & $3.2(-2-25) 17.631$ & 0.19662 .4707 \\
\hline 35.263 & 2.5431 & $3.2\left(\begin{array}{ll}10 & 0\end{array}\right) 17.631$ & $0.1966 \quad 2.4707$ \\
\hline 510 & 2.5260 & $23.5(1$ & 0.19792 .4874 \\
\hline 35.510 & 2.5260 & $23.5(-242) 17.755$ & 0.19792 .4874 \\
\hline 35.745 & 2.5099 & $7.3(-3-14) 17.873$ & $0.1992 \quad 2.5034$ \\
\hline 35.881 & 2.5007 & $32.2(-2-42) 17.941$ & 0.19992 .5126 \\
\hline 35.881 & 2.5007 & $32.2\left(\begin{array}{lll}-3 & 1 & 4\end{array}\right) 17.941$ & 0.19992 .5126 \\
\hline 35.994 & 2.4931 & $18.4\left(\begin{array}{lll}-3 & 2 & 3\end{array}\right) 17.997$ & 0.20062 .5202 \\
\hline 36.185 & 2.4804 & $2.0(-240) 18.093$ & 0.20162 .5331 \\
\hline 36.185 & 2.4804 & $2.0(-3-2$ 2) 18.093 & 0.20162 .5331 \\
\hline 36.295 & 2.4731 & $2.3(-225) 18.148$ & 0.20222 .5406 \\
\hline 36.295 & 2.4731 & $2.3(-3-23) 18.148$ & $0.2022 \quad 2.5406$ \\
\hline 36.446 & 2.4632 & $1.4(-150) 18.223$ & $0.2030 \quad 2.5508$ \\
\hline 36.446 & 2.4632 & $015) 18.223$ & 0.20302 .5508 \\
\hline 36.536 & 2.4574 & $1.3\left(\begin{array}{lll}1 & 1 & 4\end{array}\right) 18.268$ & 0.20352 .5569 \\
\hline 36.536 & 2.4574 & $1.3(-151) 18.268$ & 0.20352 .5569 \\
\hline 36.850 & 2.4371 & $5.0(222) 18.425$ & 0.20522 .5781 \\
\hline 36.850 & 2.4371 & $5.0(-1-51) 18.425$ & $0.2052 \quad 2.5781$ \\
\hline 36.976 & 2.4292 & $5.4(0-2.5) 18.488$ & 0.20582 .5866 \\
\hline 36.976 & 2.4292 & $5.4\left(\begin{array}{lll}-3 & 1 & 0\end{array}\right) 18.488$ & $0.2058 \quad 2.5866$ \\
\hline 37.136 & 2.4190 & $2.7(142) 18.568$ & $0.2067 \quad 2.5974$ \\
\hline 37.136 & 2.4190 & $2.7(-1-35) 18.568$ & 0.20672 .5974 \\
\hline 37.348 & 2.4058 & $3.6(-1-52) 18.674$ & $0.2078 \quad 2.6117$ \\
\hline 37.348 & 2.4058 & $3.6(-243) 18.674$ & 0.20782 .6117 \\
\hline
\end{tabular}

F.98

Page 47 of 56 
PNNL-20650, Rev. 2

\begin{tabular}{|c|c|c|c|c|c|}
\hline & 00 & ( & & & \\
\hline 697 & 343 & $4.5(310)$ & 18.84 & 0 & \\
\hline 697 & 3843 & $4.5(1$ & 18.848 & & \\
\hline 782 & 3792 & $27 c_{-3}$ & 91 & & \\
\hline 096 & 3603 & $5.0(-152)$ & 19.048 & & \\
\hline & & 5.0 & & & \\
\hline 199 & .3541 & $3.0\left(\begin{array}{lll}-3 & 0 & 5\end{array}\right)$ & 0 & & \\
\hline & & $8.1(-3-15)$ & & & \\
\hline 3 & & 2) & 19 & & \\
\hline & & 4) & & & \\
\hline & & 16) & & & \\
\hline & 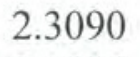 & ) & 19. & & (t: \\
\hline & & & & & \\
\hline 1 & 1 & & 19 & & 124 \\
\hline & & ) & & & \\
\hline 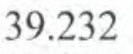 & 2.29 & 4) & 19. & 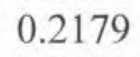 & \\
\hline & & & & & \\
\hline 55 & & ) & 19. & & \\
\hline & & & & & \\
\hline & 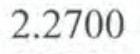 & 2) & 19 & & \\
\hline & & & & & \\
\hline 5 & a & & 19 & & \\
\hline & .26 & 6) & 19 & & 2.7 \\
\hline & 0 & & & & \\
\hline & 2 & $8(0$ & 19. & 0 & 2.7 \\
\hline & & & 20 & & \\
\hline & 2 & 6) & 20 & & 2.8 \\
\hline & & & & & \\
\hline & & 1) & 20 & 0 . & 2.8 \\
\hline & & & & & \\
\hline & 2.2 & 4) & 20 & 0.2 & 2.8537 \\
\hline & & & & 0.2 & 2.8537 \\
\hline & & 1.. & & 0.22 & 2.8 \\
\hline & 2.19 & $1.9(-3$ & 20. & 0.2282 & 2.8681 \\
\hline & 2.18 & 1.6 & 20. & 0.2291 & 2.8790 \\
\hline & 2.18 & $1.6(-1-45)$ & 20. & 0.2291 & 2.8790 \\
\hline & 2.17 & 2.2 & & 0.2300 & 2.8900 \\
\hline & 2.17 & 2.2 & 20. & 0.2300 & 2.8900 \\
\hline 17.001 & 2.15 & 3.0 & & & 2.9099 \\
\hline & 2.1592 & $3.0(-2-52)$ & 20.900 & 0.2316 & 2.9095 \\
\hline 72.17 & 2.1427 & 16.6 & & 0.2334 & 2.932 \\
\hline 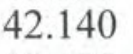 & 2.1427 & $16.6\left(\begin{array}{lll}0 & 6 & 0\end{array}\right)$ & 21.070 & 0.2334 & 2.932 \\
\hline . & & 3.0 & 21.2 & 0.2349 & 2.951 \\
\hline & 2.1290 & $3.0(053)$ & 21.2 & 0.2349 & 2.95 \\
\hline & 2.1177 & $3.6(134)$ & 21.330 & 0.2361 & 2.967 \\
\hline
\end{tabular}


PNNL-20650, Rev. 2

\begin{tabular}{|c|c|c|c|c|c|}
\hline 660 & 177 & 512 & & & \\
\hline 07 & .1112 & $2.1(-3-16)$ & & 2368 & 970 \\
\hline 120 & .0954 & $11.1(152)$ & 5 & 2386 & \\
\hline & 0954 & $11.1(-316)$ & & & \\
\hline & .0881 & $6.6(-2-36)$ & 1.648 & & \\
\hline & & & & & \\
\hline 44 & .0812 & $1.4(3$ & 23 & & \\
\hline & & 10,1 & & & \\
\hline & 2.07 & $1.2(-$ & & & \\
\hline & & & & & \\
\hline & 2 & & & & \\
\hline & & & & & \\
\hline 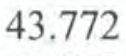 & 2.0 & 2. & & & \\
\hline & 2.00 & 2. & -0 & & \\
\hline & 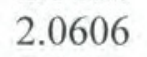 & & & & \\
\hline & 2.0. & $0.9(2$ & 22. & & \\
\hline & & & & & \\
\hline & 2.0 & $0.9(-$ & 22 & & \\
\hline & & $0.9(-1$ & & & \\
\hline & 2.0 & $0.7(-$ & 0 & & \\
\hline A. & 2.0 & $0=r$ & & & \\
\hline & 2.0 & $0.6(-2$ & 22. & & \\
\hline & 2.0 & ) & 2 & & \\
\hline (1.00 & 2.02 & $2.8(-1$ & 22.348 & & \\
\hline & 2.02 & $4.1\left(\begin{array}{lll}-4 & 0 & 4\end{array}\right)$ & 22.413 & 0.2 & 21 \\
\hline & 2.0 & $5.5(-2$ & 22.455 & 0.2479 & \\
\hline & 2.01 & $5.5\left(\begin{array}{lll}-4 & 0 & 2\end{array}\right)$ & 22.455 & 0.2479 & \\
\hline & & $3.1(-2$ & 22. & & \\
\hline 50 & 2.009 & $3.1(-4-13)$ & 22.545 & 0.2489 & 11 \\
\hline
\end{tabular}

6) PDF\#01-071-1403(RDB): Aegerine, calcian, aluminian (Sodium Calcium Iron Aluminum Silicate), Fe0.5185 Al0.4815 Ca0.466 Na0.534 Si2 O6

Radiation=CuKál Lambda $=1.5406 \quad$ Filter $=$

Ref: Curtis, L., Gittins, J., Kocman, V., Rucklidge, J.C., Hawthorne, F.C., Ferguson, R.B. Can. Mineral., v13 p62 (1975)

Monoclinic - Single Crystal, P2/n (13) $\quad \mathrm{Z}=4$

CELL: $9.622 \times 8.826 \times 5.279<90.0 \times 106.92 \times 90.0>\quad$ P.S $=\mathrm{mP} 40.00$

$\operatorname{Density}(\mathrm{c})=3.485$

Strong Lines: 2.98/X 2.53/4 2.53/4 2.90/4 2.48/4 2.48/4 2.55/3 2.55/3

2-Theta $\mathrm{d}(\AA) \quad \mathrm{I}(\mathrm{f}) \quad(\mathrm{h} \mathrm{k} \mathrm{l}) \quad$ Theta $1 /(2 \mathrm{~d}) \quad 2 \mathrm{pi} / \mathrm{d} \mathrm{n} \mathrm{n}^{\wedge} 2$

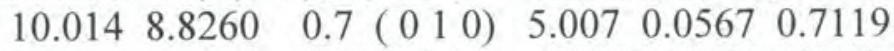

$\begin{array}{llllllll}13.889 & 6.3709 & 15.4 & \left(\begin{array}{lll}1 & 1 & 0\end{array}\right) & 6.945 & 0.0785 & 0.9862\end{array}$

$\begin{array}{llllllllll}17.383 & 5.0975 & 0.1 & \left(\begin{array}{lllll}-1 & 0 & 1\end{array}\right) & 8.691 & 0.0981 & 1.2326\end{array}$ 
PNNL-20650, Rev. 2

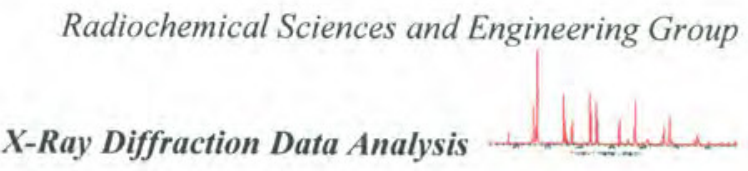

\begin{abstract}
$\begin{array}{llllllll}19.268 & 4.6027 & 1.7 & \left(\begin{array}{llll}2 & 0 & 0\end{array}\right) & 9.634 & 0.1086 & 1.3651\end{array}$
$\begin{array}{lllllllll}20.105 & 4.4130 & 18.5 & \text { ( } 020) & 10.053 & 0.1133 & 1.4238\end{array}$

$\begin{array}{lllllllll}20.105 & 4.4130 & 18.5 & \left(\begin{array}{lllll}-1 & 1 & 1\end{array}\right) & 10.053 & 0.1133 & 1.4238\end{array}$

$\begin{array}{lllllllll}21.759 & 4.0811 & 0.9 & (210) & 10.880 & 0.1225 & 1.5396\end{array}$

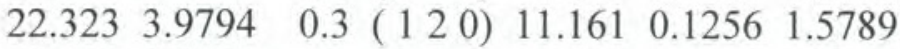

$\begin{array}{lllllllll}22.323 & 3.9794 & 0.3 & \left(\begin{array}{lllll}1 & 0 & 1\end{array}\right) & 11.161 & 0.1256 & 1.5789\end{array}$

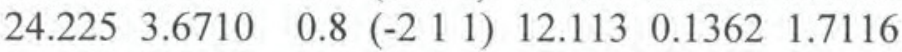

$\begin{array}{lllllllll}24.580 & 3.6188 & 1.1 & \left(\begin{array}{lllll}1 & 1 & 1\end{array}\right) & 12.290 & 0.1382 & 1.7363\end{array}$

$\begin{array}{lllllllll}26.806 & 3.3231 & 3.0 & \left(\begin{array}{llll}0 & 2 & 1\end{array}\right) & 13.403 & 0.1505 & 1.8907\end{array}$

$\begin{array}{llllllll}27.988 & 3.1854 & 11.3 & \text { (2 } 220) & 13.994 & 0.1570 & 1.9725\end{array}$

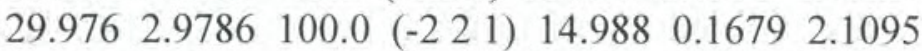

$\begin{array}{llllllll}30.826 & 2.8983 & 39.3 & \text { ( } 3110) & 15.413 & 0.1725 & 2.1679\end{array}$

$\begin{array}{lllllllll}31.043 & 2.8785 & 17.7 & \left(\begin{array}{lllll}-3 & 1 & 1\end{array}\right) & 15.522 & 0.1737 & 2.1828\end{array}$

$\begin{array}{lllllllll}31.514 & 2.8366 & 0.8 & \left(\begin{array}{llll}2 & 1 & 1\end{array}\right) & 15.757 & 0.1763 & 2.2151\end{array}$

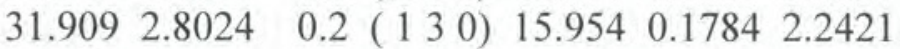

$\begin{array}{lllllllll}35.192 & 2.5481 & 25.1 & \left(\begin{array}{llll}-2 & 0 & 2\end{array}\right) & 17.596 & 0.1962 & 2.4659\end{array}$

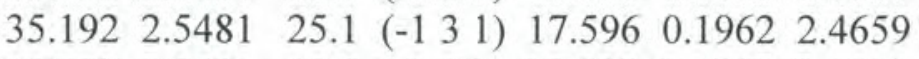

$\begin{array}{lllllllll}35.277 & 2.5421 & 15.9 & \left(\begin{array}{llll}0 & 3 & 1\end{array}\right) & 17.639 & 0.1967 & 2.4716\end{array}$

$\begin{array}{lllllllll}35.521 & 2.5252 & 40.4 & \left(\begin{array}{llll}-1 & 1 & 2\end{array}\right) & 17.760 & 0.1980 & 2.4882\end{array}$

$\begin{array}{lllllllll}35.521 & 2.5252 & 40.4 & \left(\begin{array}{lllll}0 & 0 & 2\end{array}\right) & 17.760 & 0.1980 & 2.4882\end{array}$

$\begin{array}{llllllll}35.607 & 2.5193 & 23.9 & \text { (3 } 220) & 17.804 & 0.1985 & 2.4940\end{array}$

$\begin{array}{lllllllll}36.215 & 2.4784 & 37.6 & \text { (2 2 1) } & 18.107 & 0.2017 & 2.5351\end{array}$

$\begin{array}{llllllll}36.215 & 2.4784 & 37.6 & (230) & 18.107 & 0.2017 & 2.5351\end{array}$

$\begin{array}{lllllllll}36.671 & 2.4487 & 0.8 & \left(\begin{array}{llll}-2 & 1 & 2\end{array}\right) & 18.335 & 0.2042 & 2.5660\end{array}$

$\begin{array}{llllllll}36.997 & 2.4278 & 0.6 & \left(\begin{array}{llll}0 & 1 & 2\end{array}\right) & 18.498 & 0.2059 & 2.5880\end{array}$

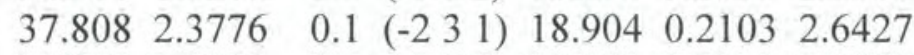

$\begin{array}{llllllll}38.047 & 2.3632 & 0.2 & \left(\begin{array}{llll}1 & 3 & 1\end{array}\right) & 19.023 & 0.2116 & 2.6588\end{array}$

$\begin{array}{lllllllll}38.476 & 2.3378 & 0.1 & \left(\begin{array}{llll}3 & 0 & 1\end{array}\right) & 19.238 & 0.2139 & 2.6876\end{array}$

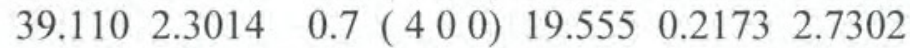

$\begin{array}{lllllllll}39.321 & 2.2895 & 0.5 & \left(\begin{array}{lllll}-4 & 1 & 1\end{array}\right) & 19.661 & 0.2184 & 2.7443\end{array}$

$\begin{array}{llllllll}39.858 & 2.2599 & 8.1 & \left(\begin{array}{llll}3 & 1 & 1\end{array}\right) & 19.929 & 0.2213 & 2.7803\end{array}$

$\begin{array}{lllllllll}40.379 & 2.2319 & 0.4 & \left(\begin{array}{llll}-3 & 1 & 2\end{array}\right) & 20.189 & 0.2240 & 2.8151\end{array}$

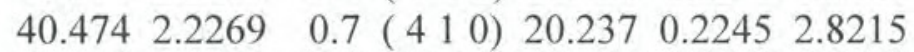

$\begin{array}{llllllll}40.979 & 2.2006 & 13.7 & \left(\begin{array}{llll}1 & 1 & 2\end{array}\right) & 20.489 & 0.2272 & 2.8552\end{array}$

$\begin{array}{llllllll}41.152 & 2.1918 & 8.6 & \left(\begin{array}{llll}0 & 2 & 2\end{array}\right) & 20.576 & 0.2281 & 2.8667\end{array}$

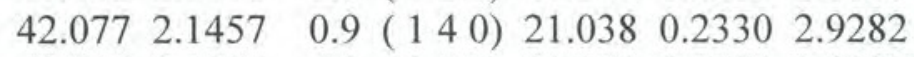

$\begin{array}{llllllll}42.536 & 2.1236 & 4.9 & \left(\begin{array}{l}3 \\ 3\end{array} 0\right) & 21.268 & 0.2354 & 2.9587\end{array}$

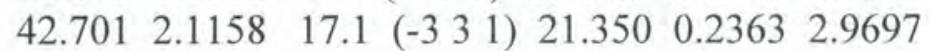

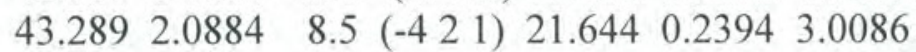

$\begin{array}{llllllll}43.786 & 2.0658 & 0.5 & \left(\begin{array}{lll}3 & 2 & 1\end{array}\right) & 21.893 & 0.2420 & 3.0415\end{array}$

$\begin{array}{llllllll}44.357 & 2.0406 & 0.5 & (420) & 22.178 & 0.2450 & 3.0791\end{array}$

$\begin{array}{llllllll}44.787 & 2.0220 & 12.4 & \left(\begin{array}{llll}0 & 4 & 1\end{array}\right) & 22.394 & 0.2473 & 3.1075\end{array}$

$\begin{array}{lllllll}44.787 & 2.0220 & 12.4 & \left(\begin{array}{llll}1 & 2 & 2\end{array}\right) & 22.394 & 0.2473 & 3.1075\end{array}$

$\begin{array}{llllllll}44.871 & 2.0184 & 14.0 & \left(\begin{array}{lll}-4 & 0 & 2\end{array}\right) & 22.435 & 0.2477 & 3.1130\end{array}$

$\begin{array}{lllllll}45.697 & 1.9838 & 5.2 & \left(\begin{array}{llll}2 & 0 & 2\end{array}\right) & 22.848 & 0.2520 & 3.1672\end{array}$
\end{abstract}

F.101 
PNNL-20650, Rev. 2

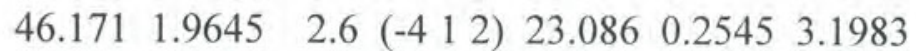

$\begin{array}{lllllllll}46.171 & 1.9645 & 2.6 & \left(\begin{array}{llll}-1 & 3 & 2\end{array}\right) & 23.086 & 0.2545 & 3.1983\end{array}$

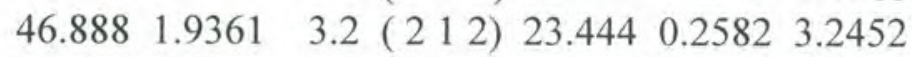

$\begin{array}{lllllllll}46.888 & 1.9361 & 3.2 & (-2 & 4 & 1 & 23.444 & 0.2582 & 3.2452\end{array}$

$\begin{array}{llllllll}47.088 & 1.9284 & 1.8 & \left(\begin{array}{llll}1 & 4 & 1\end{array}\right) & 23.544 & 0.2593 & 3.2583\end{array}$

$\begin{array}{llllllll}47.406 & 1.9162 & 0.4 & \left(\begin{array}{llll}-5 & 0 & 1\end{array}\right) & 23.703 & 0.2609 & 3.2790\end{array}$

$\begin{array}{llllllll}47.406 & 1.9162 & 0.4 & \left(\begin{array}{llll}0 & 3 & 2\end{array}\right) & 23.703 & 0.2609 & 3.2790\end{array}$

$\begin{array}{llllllll}48.515 & 1.8750 & 1.6 & \left(\begin{array}{llll}-5 & 1 & 1\end{array}\right) & 24.257 & 0.2667 & 3.3511\end{array}$

$\begin{array}{llllllll}49.099 & 1.8540 & 0.1 & \left(\begin{array}{llll}4 & 1 & 1\end{array}\right) & 24.550 & 0.2697 & 3.3890\end{array}$

$\begin{array}{llllllll}49.627 & 1.8355 & 1.4 & \left(\begin{array}{llll}-4 & 2 & 2\end{array}\right) & 24.813 & 0.2724 & 3.4231\end{array}$

$\begin{array}{lllllllll}49.777 & 1.8303 & 2.9 & \left(\begin{array}{llll}3 & 3 & 1 & 1\end{array}\right) & 24.889 & 0.2732 & 3.4329\end{array}$

$\begin{array}{lllllllll}50.215 & 1.8154 & 0.7 & \left(\begin{array}{lllll}-3 & 3 & 2\end{array}\right) & 25.108 & 0.2754 & 3.4611\end{array}$

$\begin{array}{llllllll}50.393 & 1.8094 & 1.5 & \text { (2 2 2 2) } & 25.196 & 0.2763 & 3.4725\end{array}$

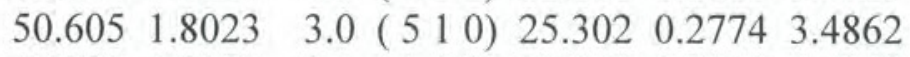

$\begin{array}{lllllllll}50.722 & 1.7984 & 3.1 & \left(\begin{array}{llll}1 & 3 & 2\end{array}\right) & 25.361 & 0.2780 & 3.4938\end{array}$

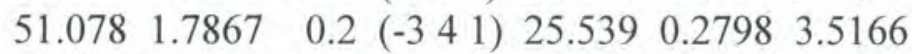

$\begin{array}{llllllll}51.391 & 1.7766 & 1.1 & (241) & 25.696 & 0.2814 & 3.5367\end{array}$

$\begin{array}{llllllll}51.746 & 1.7652 & 0.3 & (050) & 25.873 & 0.2833 & 3.5595\end{array}$

$\begin{array}{lllllllll}51.922 & 1.7596 & 0.3 & (-5 & -5 & 1 & 25.961 & 0.2842 & 3.5708\end{array}$

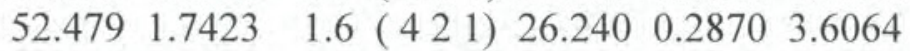

$\begin{array}{llllllll}52.761 & 1.7336 & 9.9 & (150) & 26.380 & 0.2884 & 3.6243\end{array}$

$\begin{array}{lllllllll}53.189 & 1.7207 & 0.5 & \left(\begin{array}{llll}-2 & 1 & 3\end{array}\right) & 26.595 & 0.2906 & 3.6516\end{array}$

$\begin{array}{lllllllll}53.334 & 1.7163 & 0.7 & \left(\begin{array}{lllll}-5 & 1 & 2\end{array}\right) & 26.667 & 0.2913 & 3.6608\end{array}$

$\begin{array}{lllllllll}53.334 & 1.7163 & 0.7 & \left(\begin{array}{llll}-1 & 1 & 3\end{array}\right) & 26.667 & 0.2913 & 3.6608\end{array}$

$\begin{array}{lllllllll}53.917 & 1.6992 & 0.3 & \left(\begin{array}{lll}5 & 2 & 0\end{array}\right) & 26.958 & 0.2943 & 3.6978\end{array}$

$\begin{array}{lllllllll}53.917 & 1.6992 & 0.3 & \left(\begin{array}{lllll}-3 & 0 & 3\end{array}\right) & 26.958 & 0.2943 & 3.6978\end{array}$

$\begin{array}{llllllll}54.136 & 1.6928 & 0.3 & (-14 & 4 & 27.068 & 0.2954 & 3.7117\end{array}$

$\begin{array}{lllllllll}54.304 & 1.6880 & 2.0 & \left(\begin{array}{llll}3 & 1 & 2\end{array}\right) & 27.152 & 0.2962 & 3.7224\end{array}$

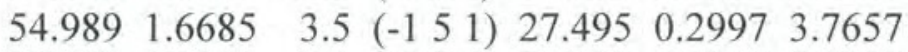

$\begin{array}{lllllllll}54.989 & 1.6685 & 3.5 & \left(\begin{array}{llll}-3 & 1 & 3\end{array}\right) & 27.495 & 0.2997 & 3.7657\end{array}$

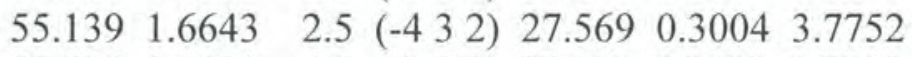

$\begin{array}{lllllll}55.239 & 1.6616 & 4.9 & (042) & 27.619 & 0.3009 & 3.7815\end{array}$

$\begin{array}{llllllll}55.851 & 1.6448 & 0.2 & \left(\begin{array}{llll}2 & 3 & 2\end{array}\right) & 27.925 & 0.3040 & 3.8200\end{array}$

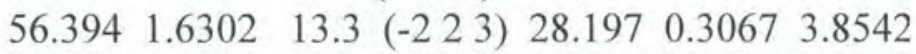

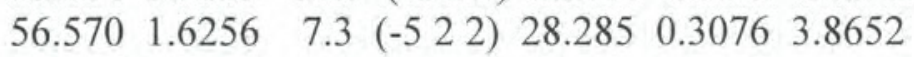

$\begin{array}{llllllll}56.570 & 1.6256 & 7.3 & \left(\begin{array}{lllll}-1 & 2 & 3\end{array}\right) & 28.285 & 0.3076 & 3.8652\end{array}$

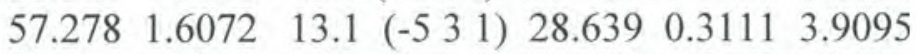

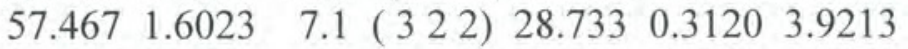

$\begin{array}{llllllll}57.847 & 1.5927 & 5.6(440) & 28.923 & 0.3139 & 3.9450\end{array}$

$\begin{array}{lllllll}57.847 & 1.5927 & 5.6(431) & 28.923 & 0.3139 & 3.9450\end{array}$

$\begin{array}{lllllll}58.056 & 1.5875 & 3.0 & \left(\begin{array}{llll}5 & 0 & 1\end{array}\right) & 29.028 & 0.3150 & 3.9580\end{array}$

$\begin{array}{llllllll}58.235 & 1.5830 & 0.4 & (14 & 2 & 29.117 & 0.3159 & 3.9691\end{array}$

$\begin{array}{llllllll}58.450 & 1.5777 & 0.2 & \left(\begin{array}{lllll}1 & 0 & 3\end{array}\right) & 29.225 & 0.3169 & 3.9825\end{array}$

$\begin{array}{lllllllll}58.450 & 1.5777 & 0.2 & \left(\begin{array}{lllll}-6 & 1 & 1\end{array}\right) & 29.225 & 0.3169 & 3.9825\end{array}$

$\begin{array}{llllllll}58.644 & 1.5729 & 0.4 & \left(\begin{array}{llll}-4 & 1 & 3\end{array}\right) & 29.322 & 0.3179 & 3.9946\end{array}$
\end{abstract}

\section{F.102}


PNNL-20650, Rev. 2

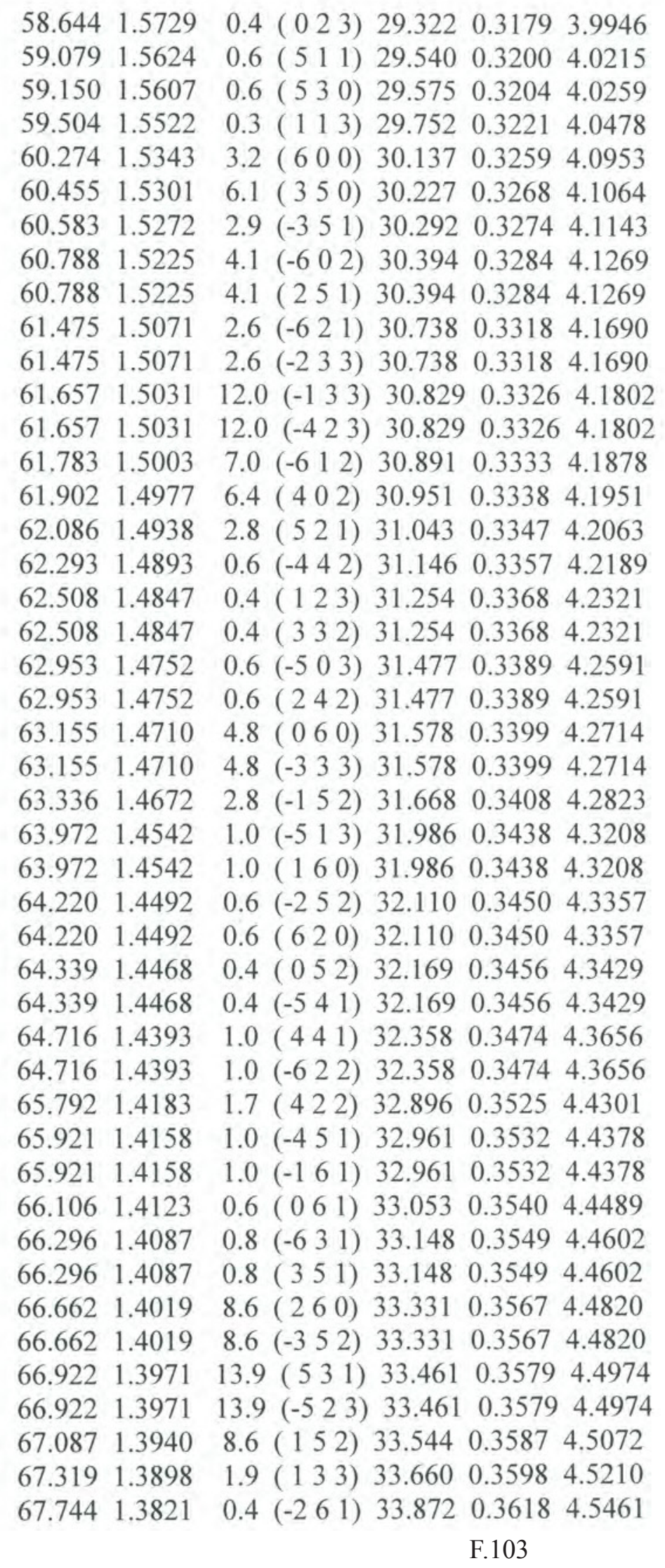


PNNL-20650, Rev. 2

\begin{abstract}
$\begin{array}{lllllll}67.964 & 1.3782 & 4.2 & \text { (2 } 223) & 33.982 & 0.3628 & 4.5591\end{array}$
$\begin{array}{llllllll}67.964 & 1.3782 & 4.2 & \left(\begin{array}{llll}1 & 6 & 1\end{array}\right) & 33.982 & 0.3628 & 4.5591\end{array}$

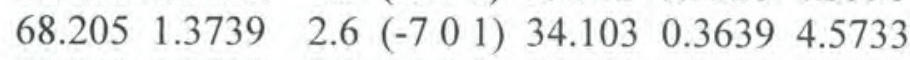

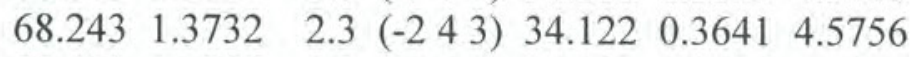

$\begin{array}{lllllllll}68.401 & 1.3704 & 1.0 & \left(\begin{array}{llll}-1 & 4 & 3\end{array}\right) & 34.201 & 0.3649 & 4.5849\end{array}$

$\begin{array}{llllllll}68.401 & 1.3704 & 1.0 & (-542) & 34.201 & 0.3649 & 4.5849\end{array}$

$\begin{array}{lllllllll}68.977 & 1.3604 & 0.1 & \left(\begin{array}{l}6 \\ 6\end{array} 0\right) & 34.488 & 0.3675 & 4.6187\end{array}$

$\begin{array}{lllllllll}69.142 & 1.3575 & 0.2 & \left(\begin{array}{lllll}-7 & 1 & 1\end{array}\right) & 34.571 & 0.3683 & 4.6284\end{array}$

$\begin{array}{llllllll}69.142 & 1.3575 & 0.2 & (342) & 34.571 & 0.3683 & 4.6284\end{array}$

$\begin{array}{lllllllll}69.821 & 1.3460 & 0.1 & \left(\begin{array}{llll}-3 & 4 & 3\end{array}\right) & 34.911 & 0.3715 & 4.6681\end{array}$

$\begin{array}{lllllllll}69.821 & 1.3460 & 0.1 & \left(\begin{array}{llll}6 & 1 & 1\end{array}\right) & 34.911 & 0.3715 & 4.6681\end{array}$

$\begin{array}{lllllll}70.273 & 1.3384 & 0.6 & (043) & 35.136 & 0.3736 & 4.6945\end{array}$

$\begin{array}{llllllll}70.496 & 1.3347 & 0.3 & \left(\begin{array}{l}4 \\ 4\end{array} 2\right) & 35.248 & 0.3746 & 4.7075\end{array}$

$\begin{array}{lllllllll}70.771 & 1.3302 & 0.1 & \left(\begin{array}{llll}-6 & 1 & 3\end{array}\right) & 35.386 & 0.3759 & 4.7235\end{array}$

$\begin{array}{llllllll}71.308 & 1.3215 & 6.0 & \text { (2 } 61 \text { 1) } & 35.654 & 0.3784 & 4.7546\end{array}$

$\begin{array}{llllllll}71.308 & 1.3215 & 6.0 & \left(\begin{array}{llll}-7 & 1 & 2\end{array}\right) & 35.654 & 0.3784 & 4.7546\end{array}$

$\begin{array}{llllllll}71.522 & 1.3181 & 6.0 & (252) & 35.761 & 0.3793 & 4.7669\end{array}$

$\begin{array}{lllllllll}71.522 & 1.3181 & 6.0 & (-533 & 3 & 35.761 & 0.3793 & 4.7669\end{array}$

$\begin{array}{llllllll}72.164 & 1.3079 & 0.4 & \left(\begin{array}{llll}3 & 1 & 3\end{array}\right) & 36.082 & 0.3823 & 4.8039\end{array}$

$\left.\begin{array}{lllllllll}72.349 & 1.3050 & 0.4 & (-2 & 1 & 4\end{array}\right) \begin{array}{llll}36.174 & 0.3831 & 4.8146\end{array}$

$\begin{array}{lllllllll}72.587 & 1.3013 & 3.7 & \text { (6 } 621 \text { ) } & 36.294 & 0.3842 & 4.8282\end{array}$

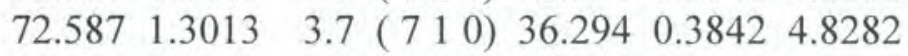

$\left.\begin{array}{lllllllll}72.734 & 1.2991 & 2.2 & (-5 & 5 & 1\end{array}\right) \begin{array}{llll}36.367 & 0.3849 & 4.8366\end{array}$

$\begin{array}{lllllllll}72.734 & 1.2991 & 2.2 & (-6 & 4 & 1 & 36.367 & 0.3849 & 4.8366\end{array}$

$\begin{array}{llllllll}72.984 & 1.2952 & 4.1 & (-443) & 36.492 & 0.3860 & 4.8510\end{array}$

$\begin{array}{lllllllll}72.984 & 1.2952 & 4.1 & \left(\begin{array}{llll}-3 & 1 & 4\end{array}\right) & 36.492 & 0.3860 & 4.8510\end{array}$

$\begin{array}{lllllllll}73.194 & 1.2920 & 2.1 & (451) & 36.597 & 0.3870 & 4.8630\end{array}$

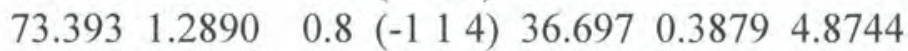

$\begin{array}{llllllll}73.393 & 1.2890 & 0.8 & (541) & 36.697 & 0.3879 & 4.8744\end{array}$

$\begin{array}{lllllllll}73.523 & 1.2871 & 0.4 & \left(\begin{array}{llll}-6 & 2 & 3\end{array}\right) & 36.761 & 0.3885 & 4.8817\end{array}$

$\begin{array}{lllllllll}73.669 & 1.2849 & 0.4 & \left(\begin{array}{lllll}-1 & 6 & 2\end{array}\right) & 36.835 & 0.3891 & 4.8901\end{array}$

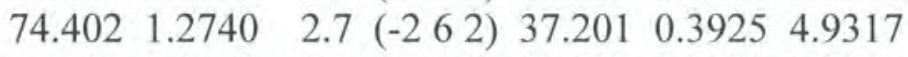

$\begin{array}{llllllll}74.402 & 1.2740 & 2.7 & \text { ( } 55 \text { 5 } 0) & 37.201 & 0.3925 & 4.9317\end{array}$

$\left.\begin{array}{llllllll}74.605 & 1.2711 & 6.8 & (0 & 6 & 2\end{array}\right) \begin{array}{llll}0 & 37.302 & 0.3934 & 4.9432\end{array}$

$\begin{array}{llllllll}74.894 & 1.2669 & 3.0 & (323) & 37.447 & 0.3947 & 4.9596\end{array}$

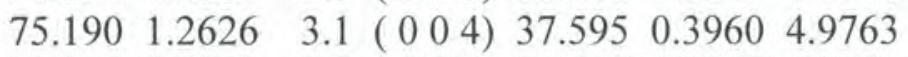

$\begin{array}{lllllllll}75.190 & 1.2626 & 3.1 & (-4 & 1 & 4 & 37.595 & 0.3960 & 4.9763\end{array}$

$\begin{array}{llllllll}75.398 & 1.2597 & 1.7 & (640) & 37.699 & 0.3969 & 4.9880\end{array}$

$\begin{array}{llllllll}75.398 & 1.2597 & 1.7 & (720) & 37.699 & 0.3969 & 4.9880\end{array}$

$\begin{array}{llllllll}75.859 & 1.2531 & 0.4 & (-642) & 37.930 & 0.3990 & 5.0140\end{array}$

$\begin{array}{llllllll}76.089 & 1.2499 & 0.4 & (170) & 38.045 & 0.4000 & 5.0269\end{array}$

$\begin{array}{llllllll}76.089 & 1.2499 & 0.4 & (-461) & 38.045 & 0.4000 & 5.0269\end{array}$

$\begin{array}{llllllll}76.457 & 1.2448 & 0.7 & (-73 & 3 & 38.229 & 0.4017 & 5.0475\end{array}$

$\begin{array}{lllllllll}76.457 & 1.2448 & 0.7 & (-253) & 38.229 & 0.4017 & 5.0475\end{array}$

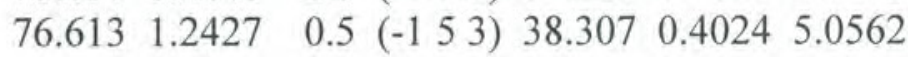

F.104 
PNNL-20650, Rev. 2

\begin{tabular}{|c|c|c|c|c|c|}
\hline 6.613 & 427 & $0.5(-552)$ & & & \\
\hline 6.866 & 1.2392 & $1.3(460)$ & 38.433 & 0.4035 & 5.0703 \\
\hline .866 & 1.2392 & $1.3(442)$ & 38.433 & 0.4035 & 5.0703 \\
\hline .110 & 1.2359 & $0.8(631)$ & 38.555 & 0.4046 & 08 \\
\hline 7.416 & 1.2318 & $5.3(352)$ & 38.708 & 0.4059 & 5.1009 \\
\hline 003 & 1.2240 & $1.6(-3.53)$ & 39.002 & 0.4085 & 5 \\
\hline 3.003 & 1.2240 & $1.6(-171)$ & 39.002 & 0.4085 & 5.1334 \\
\hline & 1.2169 & $0.2\left(\begin{array}{lll}-7 & 3 & 2\end{array}\right)$ & 39.272 & 0.4109 & 5. \\
\hline 78.545 & 1.2169 & $0.2(270)$ & 39.272 & 0.4109 & 5.1634 \\
\hline & 1.2 & 0.3 & 39.387 & 0.4119 & 5.17 \\
\hline 78.939 & 1.2118 & $0.3(2$ & 39.469 & 0.4126 & 5.1 \\
\hline 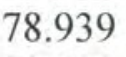 & 1.21 & $0.3(-7$ & 39.469 & 0.41 & 5.1 \\
\hline 79.198 & 1.208 & $0.2\left(\begin{array}{lll}-5 & 1 & 4\end{array}\right)$ & 39.599 & 0.4137 & 5.1992 \\
\hline & & 0.1 & 39.686 & 0.4145 & 5.208 \\
\hline & 1.20 & $0.4(-234)$ & 39.794 & 0.4154 & 5.2207 \\
\hline 9.589 & 1.203 & $0.4(-271)$ & 39.794 & 0.4154 & 5.22 \\
\hline
\end{tabular}

\section{7) PDF\#01-071-3770, Silicon, syn, Si}

Radiation $=$ CuKál $\quad$ Lambda $=1.5406$

Ref: Jette, E.R., Foote, F. J. Chem. Phys., v1 p753 (1933)

Cubic - Fd-3m (227) $\quad \mathrm{Z}=8$

CELL: $5.43077 \times 5.43077 \times 5.43077<90.0 \times 90.0 \times 90.0>P . S=c F 8.00$

$\operatorname{Density}(\mathrm{c})=2.329$

Strong Lines: 3.14/X 1.92/6 1.64/3 1.11/1 1.25/1 1.36/1 1.05/1 0.92/1

2-Theta $d(\AA) \quad I(f)\left(\right.$ h k l) Theta $1 /(2 \mathrm{~d}) \quad 2$ pi/d $\mathrm{n}^{\wedge} 2$

$\begin{array}{lllllllllll}28.443 & 3.1355 & 100.0 & \left(\begin{array}{lllllll}1 & 1 & 1\end{array}\right) & 14.222 & 0.1595 & 2.0039 & 3\end{array}$

$\begin{array}{llllllllll}47.304 & 1.9201 & 55.2 & (22 & 2 & 0 & 23.652 & 0.2604 & 3.2724 & 8\end{array}$

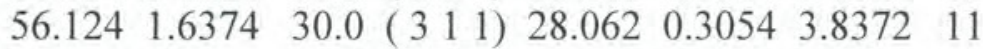

$\begin{array}{llllllllll}58.858 & 1.5677 & 0.1 & (22 & 2 & 2 & 29.429 & 0.3189 & 4.0078 & 12\end{array}$

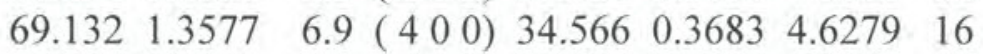

$\begin{array}{llllllllll}76.379 & 1.2459 & 9.6 & \left(\begin{array}{lllll}3 & 3 & 1\end{array}\right) & 38.189 & 0.4013 & 5.0431 & 19\end{array}$

$\begin{array}{lllllllll}88.033 & 1.1086 & 11.6 & (422) & 44.017 & 0.4510 & 5.6679 & 24\end{array}$

$\begin{array}{lllllllll}94.956 & 1.0452 & 6.1 & \left(\begin{array}{llll}5 & 1 & 1\end{array}\right) & 47.478 & 0.4784 & 6.0118 & 27\end{array}$

$\begin{array}{lllllllll}106.713 & 0.9600 & 3.4 & (440) & 53.356 & 0.5208 & 6.5448 & 32\end{array}$

$\begin{array}{llllllllll}114.097 & 0.9180 & 5.9 & \left(\begin{array}{lllll}5 & 3 & 1\end{array}\right) & 57.048 & 0.5447 & 6.8447 & 35\end{array}$

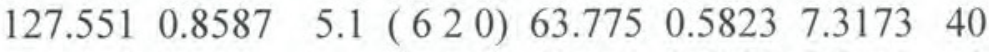

$\begin{array}{llllllll}136.901 & 0.8282 & 2.4 & \left(\begin{array}{lllll}5 & 3 & 3\end{array}\right) & 68.450 & 0.6037 & 7.5867 & 43\end{array}$ 
PNNL-20650, Rev. 2

Battelle - Pacific Northwest National Laboratory

Radiochemical Sciences and Engineering Group X-Ray Diffraction Data Analysis

Appendix 7

Supplemental XRD powder patterns

Figure 15 presents the XRD powder patterns for UDS core samples: TI040-419D-2X, TI040-420D-2X, TI040-421D-2X, and TI040-422D-2X along with the corresponding PDF cards. Figure 16 presents the XRD powder patterns for UDS container composites: TI040-792D-2X and TI040-793D-2X.

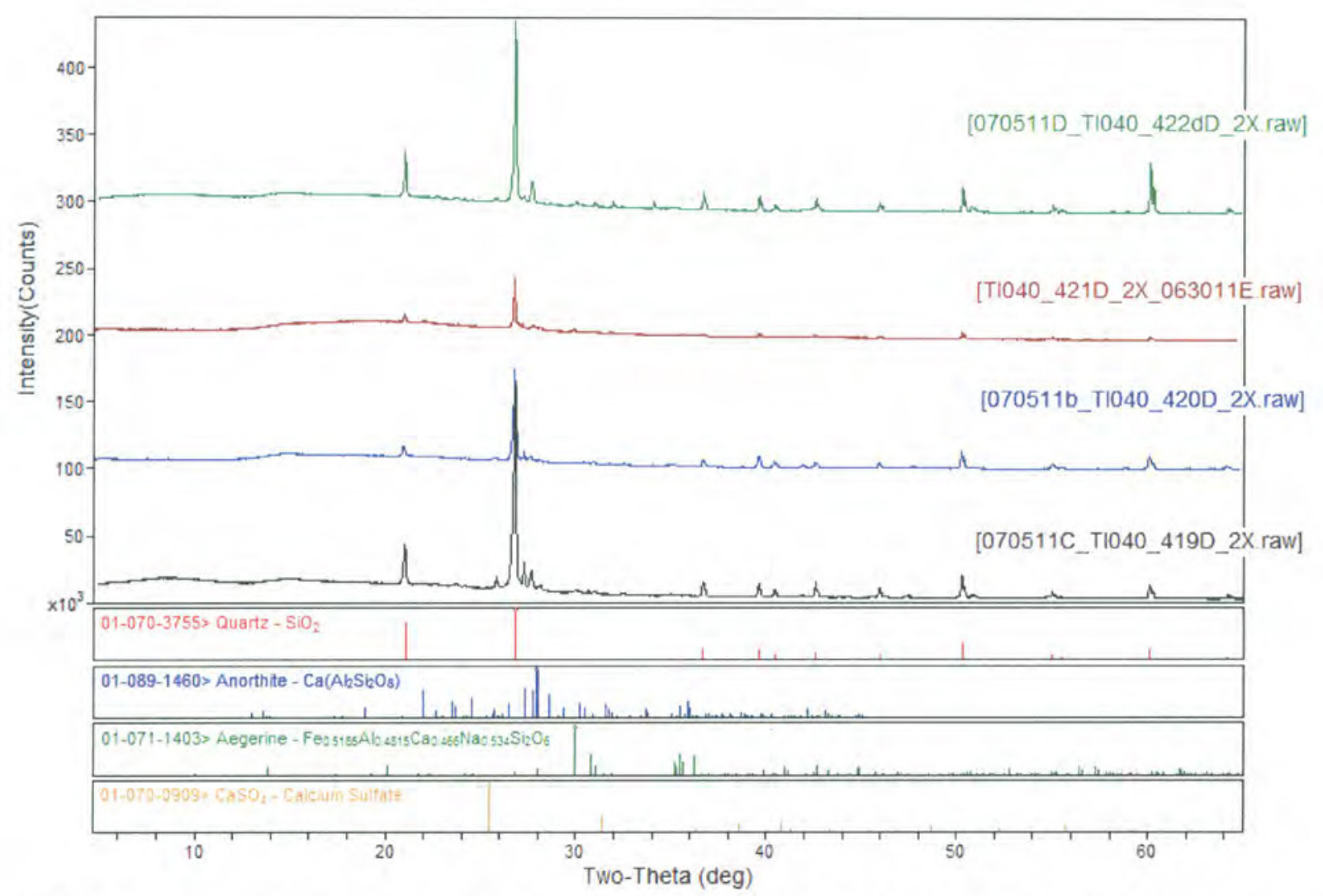

Figure 15. XRD powder patterns for TI040-419D-2X, TI040-420D-2X, TI040-421D-2X, and TI040-422D-2X along with the corresponding PDF cards. 
PNNL-20650, Rev. 2

Battelle - Pacific Northwest National Laboratory
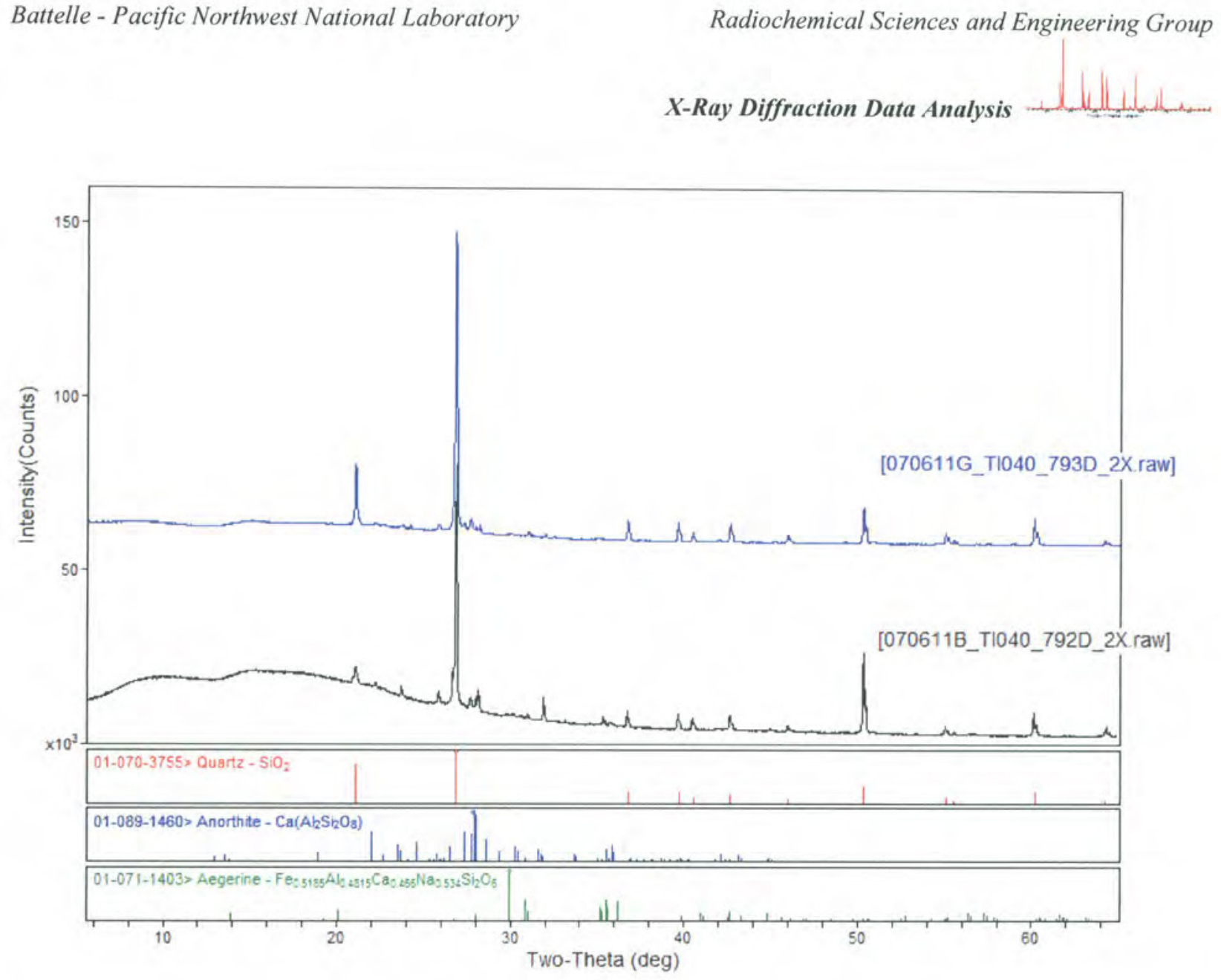

Figure 16. XRD powder patterns for TI040-792D-2X and TI040-793D-2X along with the corresponding PDF cards. 


\begin{tabular}{|c|c|c|c|}
\hline & $\begin{array}{c}\text { PL K Basin Proj } \\
52578\end{array}$ & \multicolumn{2}{|c|}{ Document Review Record } \\
\hline Title: & \multicolumn{3}{|c|}{ XRD Report for KW 210 Undissolved Solids } \\
\hline \multicolumn{3}{|c|}{ Please return the completed form to: Doinita Neiner } & Comments Due: \\
\hline \multicolumn{2}{|c|}{ Organization/Department: } & Designated Reviewer: & $\begin{array}{l}\text { Reviewer Signature/Date: } \\
\text { (Upon completion of review) }\end{array}$ \\
\hline & D9H63 & Mike Urie & \\
\hline
\end{tabular}

It is optional, but helpful when comments are identified as to type or if the reviewer wishes to specify mandatory comments requiring resolution. In this case, the following conventions apply:

*Type: $\mathbf{E}$-Editorial, addresses word processing errors that do not adversely impact the integrity of the calculation.

O-Optional, comment resolution would provide clarification, but does not impact the integrity of the work or calculations

M - Mandatory, comment shall be resolved; reviewer identifies impact on the integrity of the work or calculations

\begin{tabular}{|c|c|c|c|c|}
\hline $\begin{array}{c}\text { Comment } \\
\text { Number }\end{array}$ & $\begin{array}{c}\text { Section/ } \\
\text { Page }\end{array}$ & Type & Comment & Comment Resolution \\
\hline 1 & Figure 5 & E & Change "show" to "shown" or "is shown" & SNNE \\
\hline 2 & $\begin{array}{l}\text { Figures } \\
3,6,7,8,9\end{array}$ & E & Change "spectrum" to "spectra". & DONE \\
\hline & & & & \\
\hline & & & & \\
\hline & & & & \\
\hline & & & & \\
\hline & & & & \\
\hline & & & & \\
\hline & & & & \\
\hline & & & & \\
\hline & & & & \\
\hline & & & & \\
\hline & & & & \\
\hline & & & & \\
\hline
\end{tabular}

Concur with comment pesolution, review complete.




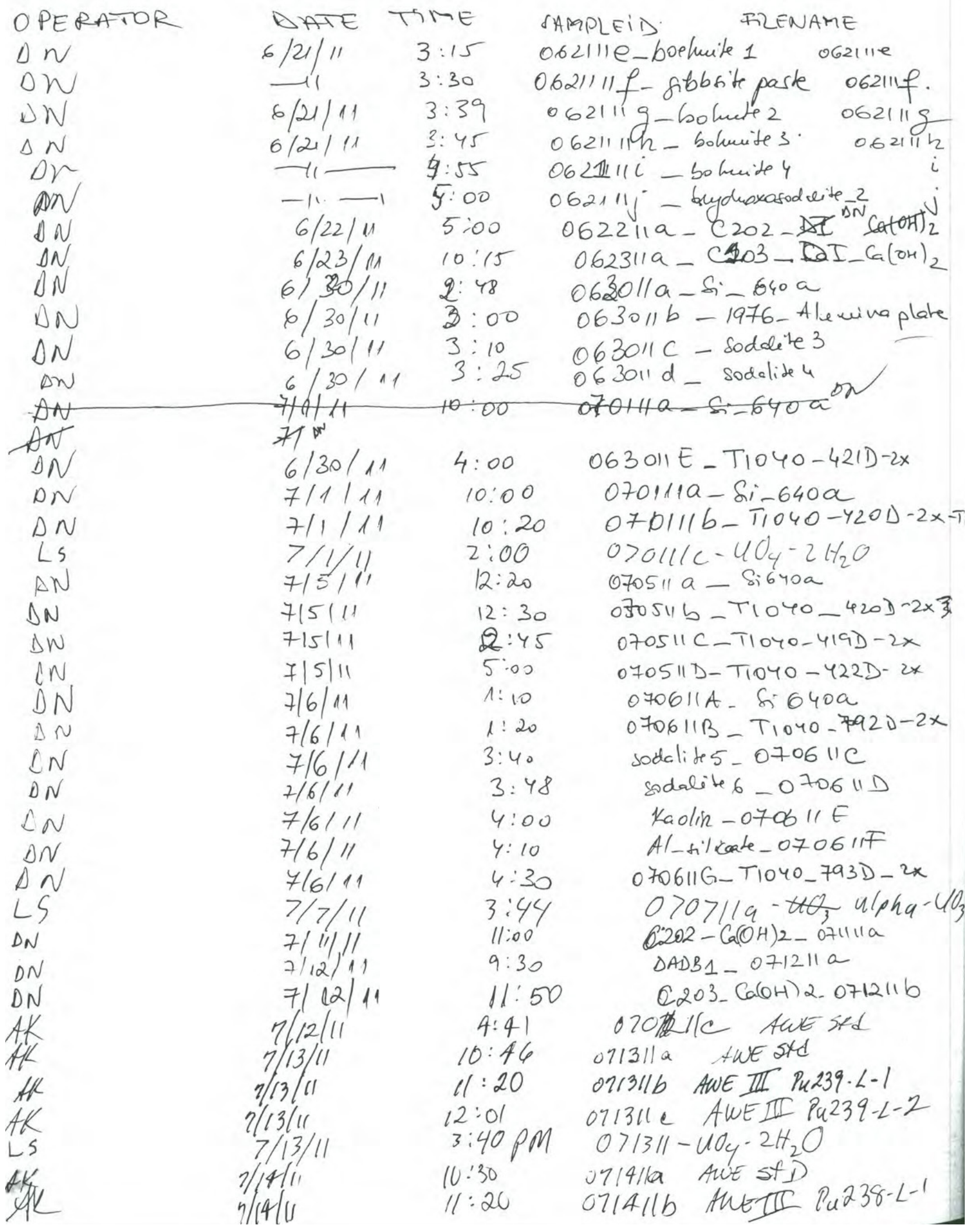


PNNL-20650, Rev. 2

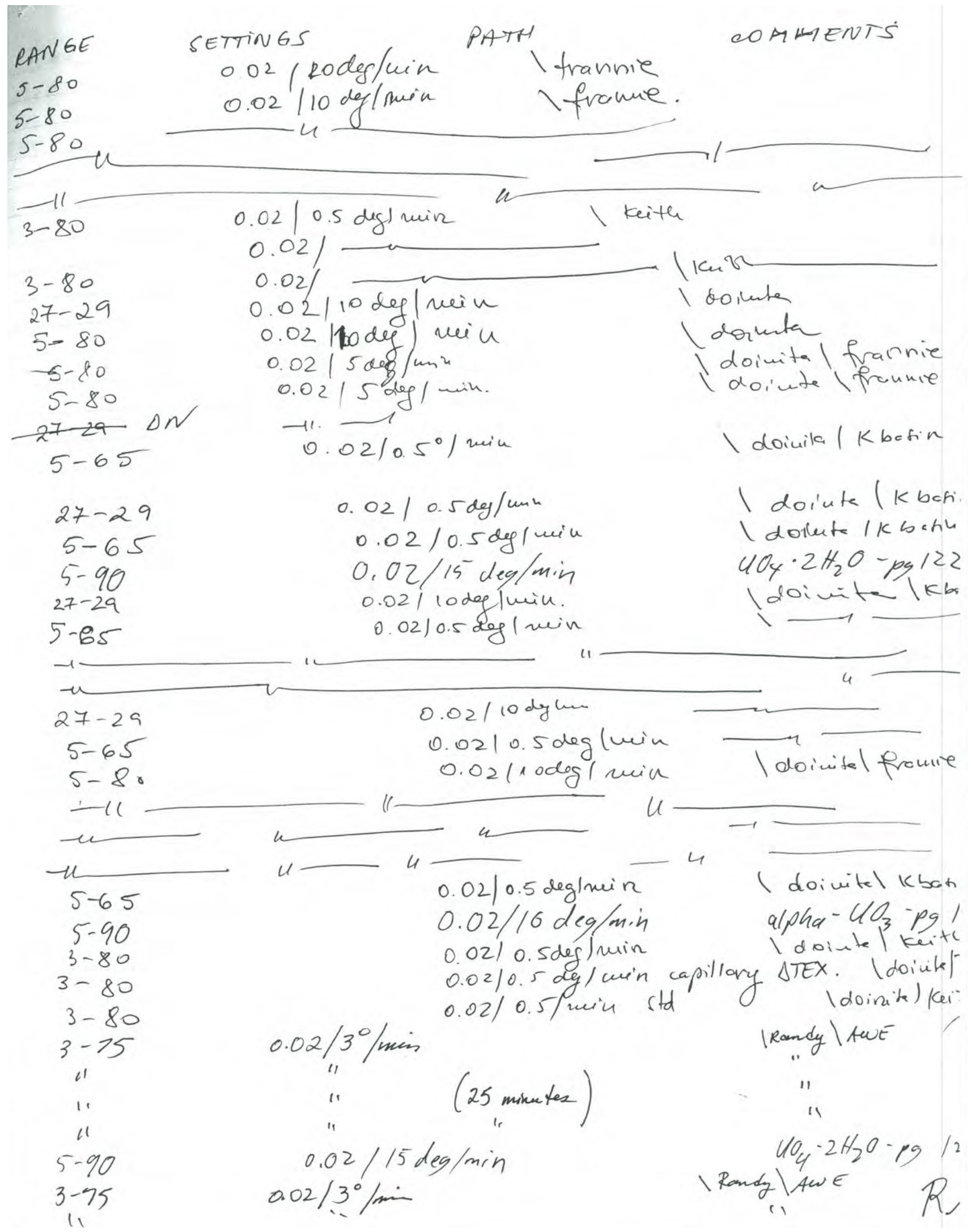




\section{PLANNING}

\section{Topic/Issue to be Evaluated: Surveillance of K Basin X-Ray Diffraction Analysis Report; KW 210 Undissolved Solids}

This surveillance was undertaken to evaluate the draft X-Ray Diffraction (XRD) Analysis Report, KW 210 Undissolved Solids for the samples listed in Table 1 collected during the implementation of test instruction, T1040, Processing of the Undissolved Solids Samples from the K West Basin Core Samples Collected from SCS-CON-210 for Additional Analysis.

\section{PERFORMANCE}

\begin{tabular}{|l|l|}
\hline Date Initiated: 16 June 2011 & Location: RPL \\
\hline Date Received: 25 July 2011 & Date Reviewed: 25 July 201 I \\
\hline Date Resubmitted: 27 July 2011 & Date Closed: 27 July 2011 \\
\hline Date Completed: 27 July 2011 & $\begin{array}{l}\text { DRAFT Report Issued: } 25 \text { July 2011 } \\
\text { FINAL Report Issued: } 27 \text { July } 2011\end{array}$ \\
\hline $\begin{array}{l}\text { Contacts:, Clark Carlson, Sandy Fiskum, } \\
\text { Doinita Neiner, Mike Urie }\end{array}$ & Org Code: PE137, D9H63 \\
\hline Surveillance Team: Lisa Middleton & \\
\hline
\end{tabular}

\section{PRELIMINARY INFORMATION}

\section{Source of Requirement(s):}

- KBC-33786, Rev. 2, December 2009; Quality Assurance Project Plan/Sampling and Analysis Plan (QAPjP/SAP): Quality Assurance Project Plan/Sampling and Analysis Plan for Containerized KW Settler Sludge

\section{Other Applicable Documents:}

- RPL-OP-001, Rev. 9, Routine Research Operations

- PNNL-RPG-268, Rev. 4, Solids Analysis by X-Ray Diffraction

- 52578-ADM-001, Rev. 0, K Basin Requests for Analysis and Guidelines for Data Reporting and Technical, QE, and Spreadsheet Reviews. 
Number: SR-52578-2011-034

\section{Purpose/Scope:}

The 52578 Project QE staff are required via the QA Program to perform a review of K Basin reports generated within the Radiochemical Processing Laboratory (RPL) to determine traceability and accuracy of data contributing to the completed report.

The draft report was reviewed to evaluate that the data are what they purport to be, that is, that the reported results reflect what was actually done and the results meet applicable requirements. When deficiencies in the data are identified, then those deficiencies need to be documented as a K Basin Occurrence Report for the data user's review and, when applicable, resolved by corrective action. The XRD analysis under this Analysis Request was performed to identify crystalline structures in roomtemperature-dried sludge, to identify hydrate compounds plus any other identifiable compounds. This includes noting the presence (or absence) of aluminum hydrates, uranium hydrates, uranium oxides as well as zirconium metal.

When issues were found, the cognizant staff were contacted to resolve issues prior to completion of the review.

\section{Approach:}

The draft report initiated by a $\mathrm{K}$ Basin Analysis Request form dated 6/16/1 I was provided as the basis for the data quality review. Six (6) samples were submitted on $6 / 16 / 11$ for analysis as noted in Table 1 :

Table 1: XRD Samples IDs \& Descriptions

\begin{tabular}{|c|c|c|c|c|c|}
\hline $\begin{array}{c}\text { Client Sample } \\
\text { ID }\end{array}$ & Sample Description & XRD Sample ID & $\begin{array}{c}\text { Internal } \\
\text { Standard }\end{array}$ & $\begin{array}{c}\text { Date } \\
\text { Analyzed }\end{array}$ & $\begin{array}{l}\text { Daily Performance } \\
\text { Check w/ NIST } \\
\text { SRM 640a? }\end{array}$ \\
\hline TI040-419D-2X & $\begin{array}{c}\text { KW210-A1 } \\
\text { undissolved solids } \\
\text { (UDS) }\end{array}$ & TI040-419D-2X & None & $7 / 5 / 11$ & $\begin{array}{c}\mathrm{Y} \\
0705011 \mathrm{a} \_S i 640 \mathrm{a}\end{array}$ \\
\hline \multirow{2}{*}{ TI040-420D-2X } & \multirow{2}{*}{ KW210-A4 UDS } & TI040-420D-2X & None & $7 / 5 / 11$ & $\begin{array}{c}\mathrm{Y} \\
0705011 \mathrm{a} \_ \text {Si640a }\end{array}$ \\
\hline & & $\begin{array}{c}\text { TI040-420D-2X- } \\
\text { TiO2 } \\
\end{array}$ & Rutile & $7 / 1 / 11$ & $\begin{array}{c}\text { Y } \\
0701011 \mathrm{a} \text { Si } 640 \mathrm{a} \\
\end{array}$ \\
\hline TI040-421D-2X & KW210-B1 UDS & T1040-421D-2X & None & $6 / 30 / 11$ & $\begin{array}{c}\text { Y } \\
063011 \mathrm{a} \text { Si_640a } \\
\end{array}$ \\
\hline TI040-422D-2X & KW210-B3 UDS & T1040-422D-2X & None & $7 / 5 / 11$ & $\begin{array}{c}\text { Y } \\
0705011 \mathrm{a} \text { Si640a } \\
\end{array}$ \\
\hline TI040-792D-2X & $\begin{array}{c}\text { KW210-CC, - } \\
2000 /+500 \mu \mathrm{m} \text { UDS }\end{array}$ & TI040-792D-2X & None & $7 / 6 / 11$ & $\begin{array}{c}\mathrm{Y} \\
0706011 \mathrm{a} \_ \text {Si640a }\end{array}$ \\
\hline TI040-793D-2X & $\begin{array}{c}\text { KW210-CC, }-500 \mu \mathrm{m} \\
\text { UDS }\end{array}$ & TI040-793D-2X & None & $7 / 6 / 11$ & $\begin{array}{c}\mathrm{Y} \\
0706011 \mathrm{a} \_ \text {Si640a } \\
\end{array}$ \\
\hline
\end{tabular}


PNNL-20650, Rev. 2

PROJECT 52578 - SURVEILLANCE REPORT

Number: SR-52578-2011-034

Page 3 of 5

\section{Requirements:}

$50-100 \%$ completeness is specified in KBC-33786, Rev. 2 with the specification that if significant gamma radioactivity is detected in the residue, then $100 \%$ completeness is required; otherwise $50 \%$ completeness is required. Data quality objectives for MDL, precision and accuracy were not specified in KBC-33786, Rev. 2 for XRD analysis.

Method requirements were that a semi-annual calibration using NIST SRM 1976 and a daily calibration using NIST SRM 640a were performed.

Semi-annual calibration using NIST SRM 1976, Corundum, was performed on 6/30/11; file name 063011b_1976_Aluminaplate. Daily calibrations were performed as noted above in Table 1. All samples were to be analyzed without internal standard, one sample (TI040-420D-2X) was to also be run with rutile as an internal standard, creating 7 samples total.

The report contained the following information:

1. Cover page

2. XRD Analysis Request Form/ XRD Mount Preparation Signed by Sandy Fiskum 6/16/11

3. XRD Analysis Bench Sheet - Sample Preparation (pp.2)

4. XRD Analysis Bench Sheet - Diffraction Measurements (pp.2)

5. Narrative Report and data tables

a. Table 1-Summary of Phases Identified in KW210 Undissolved Solids

b. Sample preparation

c. X-Ray powder diffraction data collection

d. X-Ray powder diffraction data analysis

e. Summary of findings

f. Sample Analyses

g. Appendix 1 - SRM 674 NIST Certificate

h. Appendix 2-Daily Performance Checks - 5/25/11

i. Appendix 3-SRM 640a NIST Certificate

j. Appendix 4-Quality Control/Calibration - 1/6/11

k. Appendix 5--SRM 1976 NIST Certificate

I. Appendix 6-PDF cards for the identified crystalline phases presented in report

m. Appendix 7 -Supplemental XRD Powder Patterns

n. DRR form signed by the Technical Reviewer, Mike Urie $7 / 21 / 11$

o. Copy of XRD run $\log (6 / 21 / 11-7 / 14 / 11), 2$ pages

\section{Procedure:}

PNNL-RPG-268, Rev. 4, Solids Analysis by X-Ray Diffraction 
Number: SR-52578-2011-034

M\&TE used:

\begin{tabular}{|c|c|c|c|c|}
\hline Location & Description & Manufacturer & Model & Unique ID \\
\hline RPL/409 & $\begin{array}{c}\text { X-Ray } \\
\text { Diffractometer }\end{array}$ & Rigaku & Ultima IV & $\begin{array}{c}\text { GD 3827N } \\
\text { /WD59716 }\end{array}$ \\
\hline & RGD & & & $\# 105$ \\
\hline & SRM & NIST & $640 \mathrm{a}$ & $640 \mathrm{a}$ \\
\hline & SRM & NIST & 674 & 674 \\
\hline & SRM & NIST & 1976 & 1976 \\
\hline
\end{tabular}

The completed surveillance report will be posted to the SharePoint site and submitted as a project record. Any documentation associated with this surveillance will be available in the Lead Auditor's office, Building 325, 5-G.

\section{RESULTS}

Findings are defined as a statement of fact relating to noncompliance with previously agreed-upon procedures, plans, codes, standards, specifications, or other forms of contractual or legal obligation. It should be understood that any lack of a finding in a specific area is not considered an indication that deficiencies do not exist.

Observations are defined as a weakness that if not corrected, could yield a departure from a requirement although the weakness is not necessarily a departure from requirements.

\section{DQO Compliance:}

$100 \%$ completeness was obtained. Semi-annual and monthly calibrations were performed as required. Sample preparation and analysis was documented using a checklist and therefore not resulting in a recurrence of the deficiency noted in D59205-2-211.

\section{Summary of Identified Concerns:}

Note: All concerns identified in the review were addressed in a comment resolution meeting on 26 July 2011, Deborah Coffey, Lisa Middleton and Doinita Neiner attended to disposition review comments. There were no outstanding identified findings or observations.

1. Page $9,2^{\text {nd }}$ paragraph, $2^{\text {nd }}$ sentence: The sentence should begin "Each sample was mixed..." instead of "A sample was mixed..." This clarifies that identical sample prep occurred for each sample. Sentence changed for clarification. No further action required, closed.

2. Page 10, Section 3.1: The acronym PDF needs to be defined in its first occurrence. The definition can then be removed from the second occurrence later in the paragraph. As a rulewhen defining an acronym, the full name is given first, followed by the acronym in parenthesis, such as: Powder Diffraction File (PDF). Acronym correctly defined. No further action required, closed.

3. Page 10, Section 3.1: The acronym RDB-ICSD needs to be defined. Acronym removed, not pertinent to the report. No further action required, closed. 
4. Page 15: The last sentence should read "The two duplicate samples confirm the diffraction patterns given the overall analysis method uncertainty." Not "The two duplicate samples reasonably reproduce the diffraction patterns given the overall experimental uncertainty." Sentence changed for clarification. No further action required, closed.

5. Page 16, Figure 5: The figure is too large for the page, exceeding the margin, so the right side of the figure is not visible. Figure resized to fit page. No further action required, closed.

6. Page $17,1^{\text {st }}$ sentence "samples" should be singular, "sample" since you are only talking about TI040-421D-2X. Plurality of "sample" changed. No further action required, closed.

7. Appendix 2, page 24: Where do the reference values in tables 4-7 come from? The value doesn't match values from either the standard certificate or the given PDF cards? The reference value now reflects the value given by the SRM. The PDF card for the SRM (01-071-3770) is now included in Appendix 6 as well. No further action required, closed.

\section{CORRECTIVE ACTION}

[X] None Required. [ ] Complete [ ] Follow up Corrective Action

\section{Surveillance Performed By:}

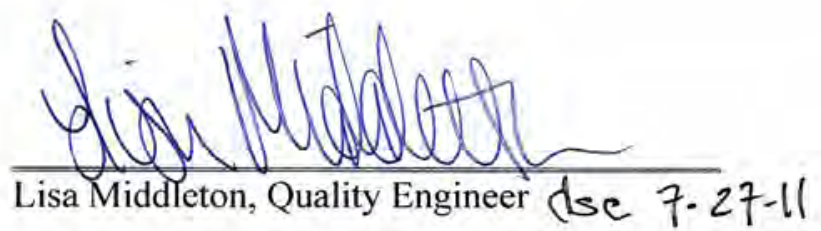

Date:

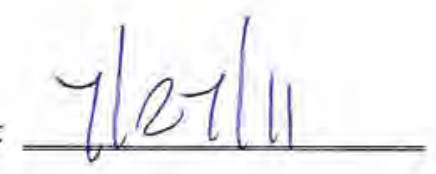

DISTRIBUTION: Clark Carlson, Sandy Fiskum, Matt Fountain, Doinita Neiner, Andy Schmidt, Rick Shimskey, Mike Urie, Deborah Coffey, Lisa Middleton 

PNNL-20650, Rev. 2

Appendix G

Particle Density 



\section{Appendix G}

\section{Particle Density}

Appendix G contains the particle density characterization report. Particle density was conducted on the size-fractionated samples collected from the Engineered Container SCS-CON-210 container composite. Any reference to an appendix ID within the particle density report refers to the self-contained appendices.

The following table of contents shows that pagination specific to Appendix G was applied to the analysis request form, particle density report (inclusive of self-contained appendices), pycnometer analysis data sheets document review record, and surveillance report. This pagination is sequential and consistent (e.g., page G.\#).

\section{Appendix G Table of Contents}

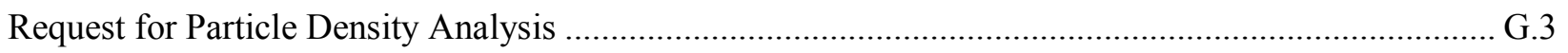

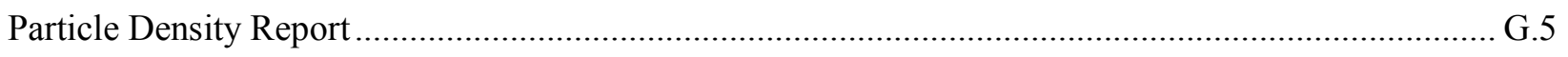

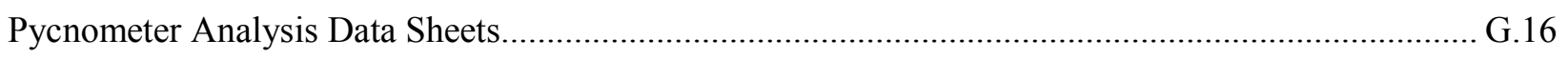

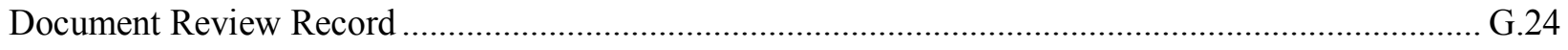

Surveillance of K Basin Particle Density Report SR-52578-2011-032 ….......................................... G.25 
G.2 


\section{Request for Particle Density Analysis by Gas Pycnometry}

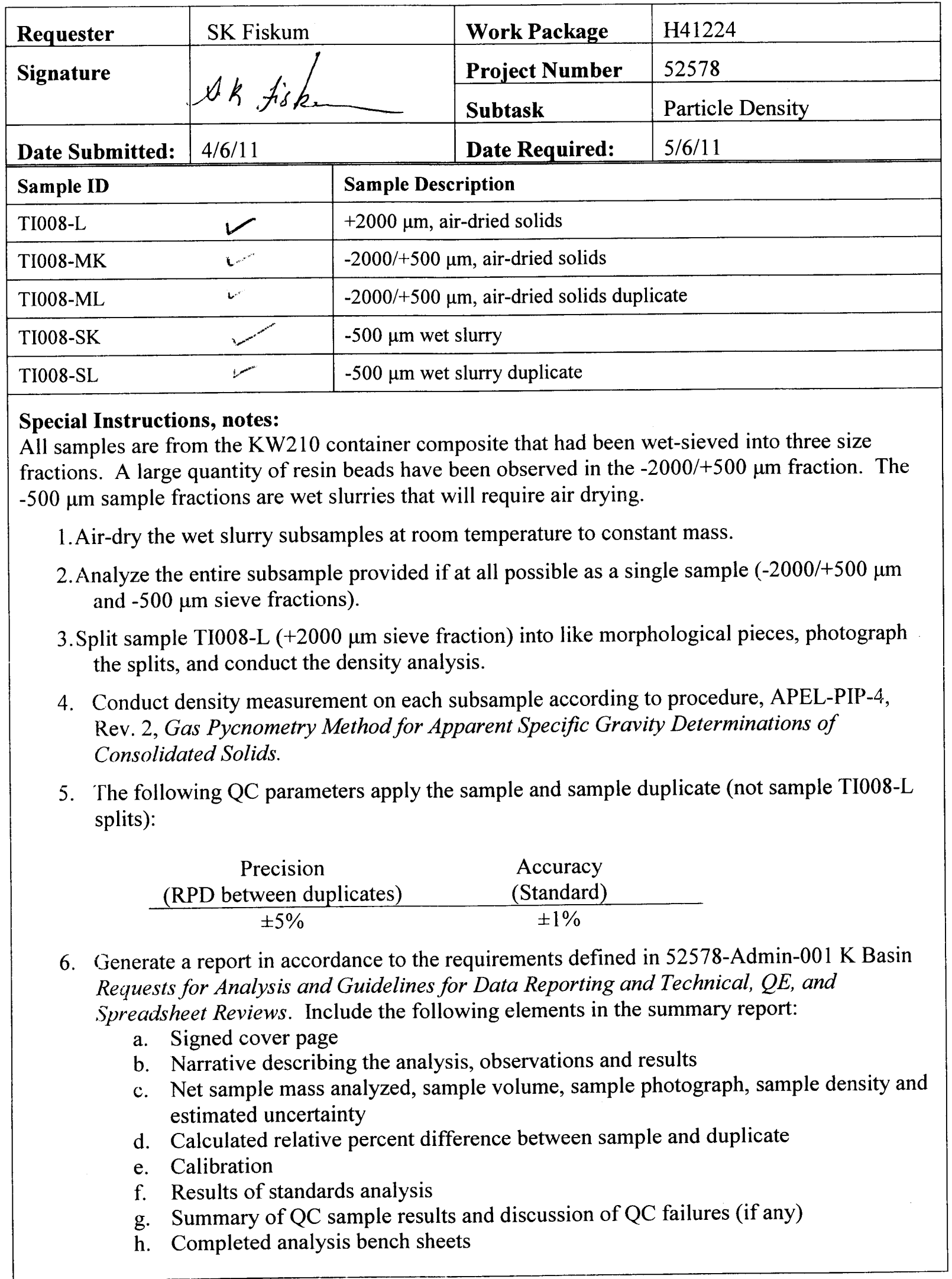

Page 1 of 2 
Special Instructions, notes, continued:

7. Submit report and data for technical review and obtain a Document Review Record (DRR) form; resolve comments, complete form showing resolution of comments and acceptance by Technical Reviewer. Have Technical Reviewer sign cover page

8. Submit report and data for QE review. Resolve draft surveillance report comments. Have QE Reviewer sign cover page 


\section{Particle Density Report}

\section{Battelle-Pacific Northwest National Laboratory Radiochemical Sciences and Engineering Group PO Box 999, Richland, Washington 99352}

\section{Project: \#52578}

Sample ID's: TI008-L, TI008-MK, TI008-ML, TI008-SK, TI008-SL

Procedure: APEL-PIP-4, Rev 2, Gas Pycnometry Method for Apparent Specific Gravity Determinations of Consolidated Solids

Analyst: Matthew Edwards Analysis Dates: 06/14/2011, 06/15/2011, 6/23/11

Instrument: AccuPyc 1330, Serial \#3955

Balance: Mettler AB104-S, Serial \#1129263167

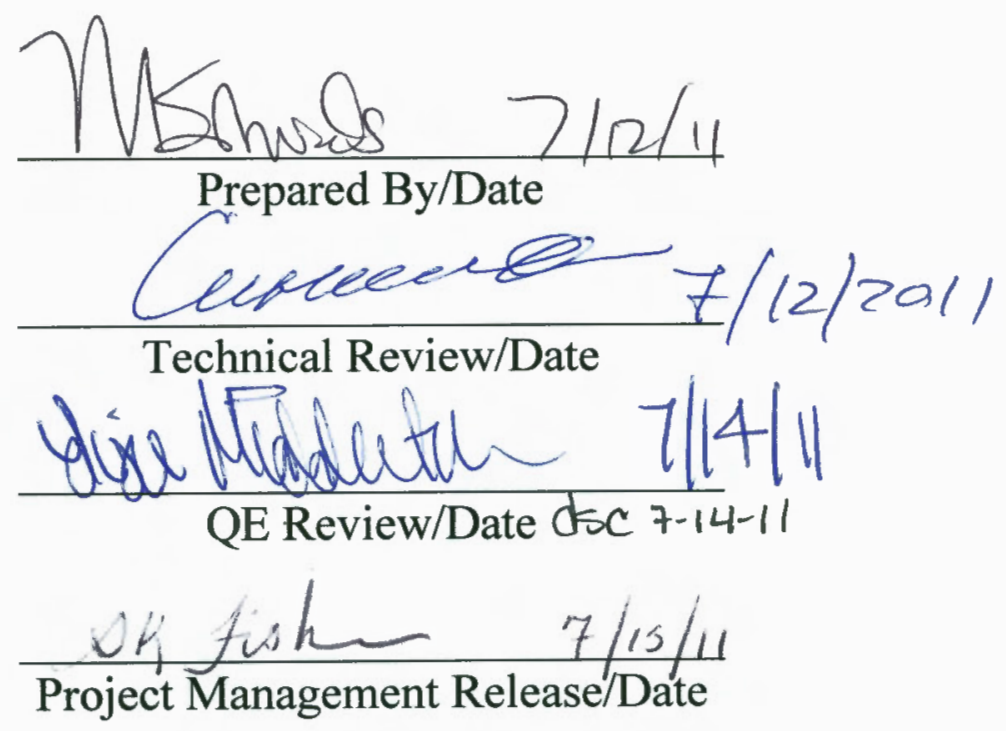




\subsection{Introduction}

This report addresses the particle density measurements of sieved fractions of the KW210 container material $(+2000,+500 /-2000$, and -500 micron sieve fractions).

\subsection{Instrumentation}

\section{Gas expansion pycnometer}

The AccuPyc 1300 gas expansion pycnometer measures the volume of displaced gas (ultra-high purity [UHP] helium) in a volume pre-calibrated sample chamber. The instrument consists of a sample chamber and an expansion chamber separated by an expansion valve. After inserting a sample in the sample chamber, the sample chamber is filled with helium and gas molecules that fill the pores of the sample. The helium is then discharged into the expansion chamber, which has a known internal volume. The pressure difference between the isolated sample chamber and that of the sample chamber and expansion chamber combined is used to calculate volume displacement using Boyle's Law (1)

$$
p V=k
$$

Where: $p$ denotes the pressure of the system, $V$ is the volume of the gas, and $k$ is a constant value representative of the pressure and volume of the system. Rearranging to fit our system gives (2)(Lowell, 2004).

$$
V_{s}=V_{c}+\frac{V_{r}}{1-\frac{P_{1}}{P_{2}}}
$$

Where $V_{s}$ is the sample volume, $V_{c}$ is the volume of the empty sample chamber, $V_{r}$ is the reference volume, $P_{1}$ is the first pressure (in the sample chamber only) and $P_{2}$ is the second pressure after expansion of the gas into the combined volumes of sample chamber and reference chamber. $V_{s}$ is then used with the pre-determined mass of the sample (m) to obtain particle density $(\rho)(3)$.

$$
\rho=m / V_{s}
$$




\subsection{Samples}

Five samples were submitted for analysis. Using the tare weight of the vial that was recorded in 52578-TI008, the dry mass provided was obtained and recorded in Table 1. The $-\mathrm{L}$ and $-\mathrm{M}$ samples had particles of varying shapes, sizes, and colors and the $-\mathrm{M}$ samples also had visible resin beads. Also of note, the $-\mathbf{M}$ samples appeared to have a significant charge as can be seen in Figure 1. The -S samples were a dark brown powder. Pictures of the sample materials were taken and can be seen in Figure 2 through Figure 8.

Table 1 Sample Masses

\begin{tabular}{|c|c|c|}
\hline $\begin{array}{c}\text { Sub divided fraction } \\
\text { identification }\end{array}$ & Physical description & Mass of subdivision (g) \\
\hline TI008-L & $\begin{array}{c}\text { Particles of varying shapes, sizes } \\
\text { and colors. }\end{array}$ & 0.7588 \\
\hline TI008-MK & $\begin{array}{c}\text { Particles of varying shapes, sizes } \\
\text { and colors. Resin beads evident. }\end{array}$ & 0.5231 \\
\hline TI008-ML & $\begin{array}{c}\text { Particles of varying shapes, sizes } \\
\text { and colors. Resin beads evident. }\end{array}$ & 0.5074 \\
\hline TI008-SK & Dark Brown powder & 0.6755 \\
\hline TI008-SL & Dark Brown powder & 0.3462 \\
\hline $\begin{array}{l}{ }^{1} \text { The tare mass of the vial was recorded in Test Instructions 52578-TI008. A gross weight of the sample was obtained. Mass of } \\
\text { subdivision is the difference between the gross and tare masses. }\end{array}$
\end{tabular}

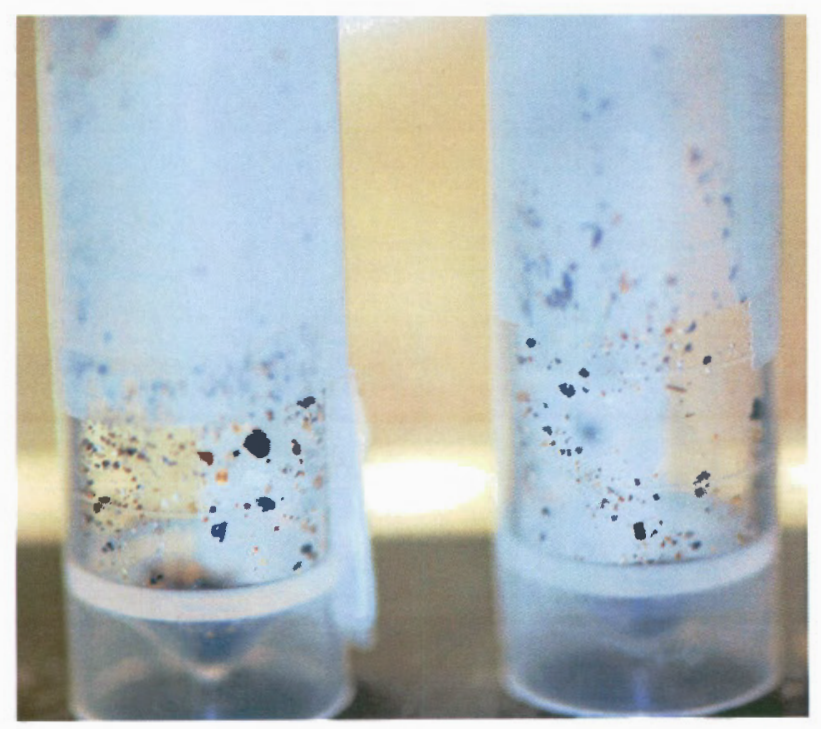

Figure 1 Sample Vials of TI008-MK \& ML 
PNNL-20650, Rev. 2

Particle Density Report

Page 4 of 6

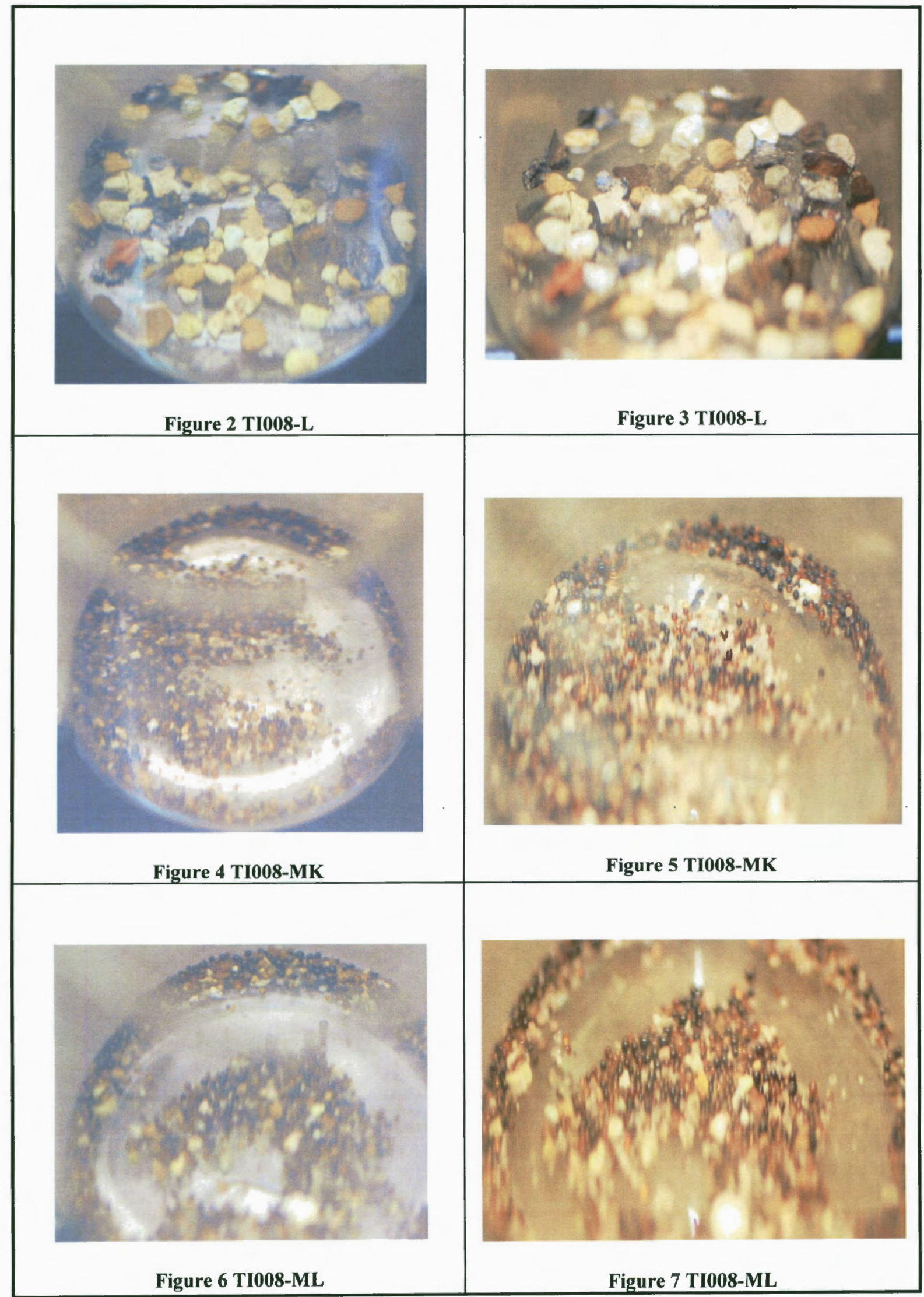




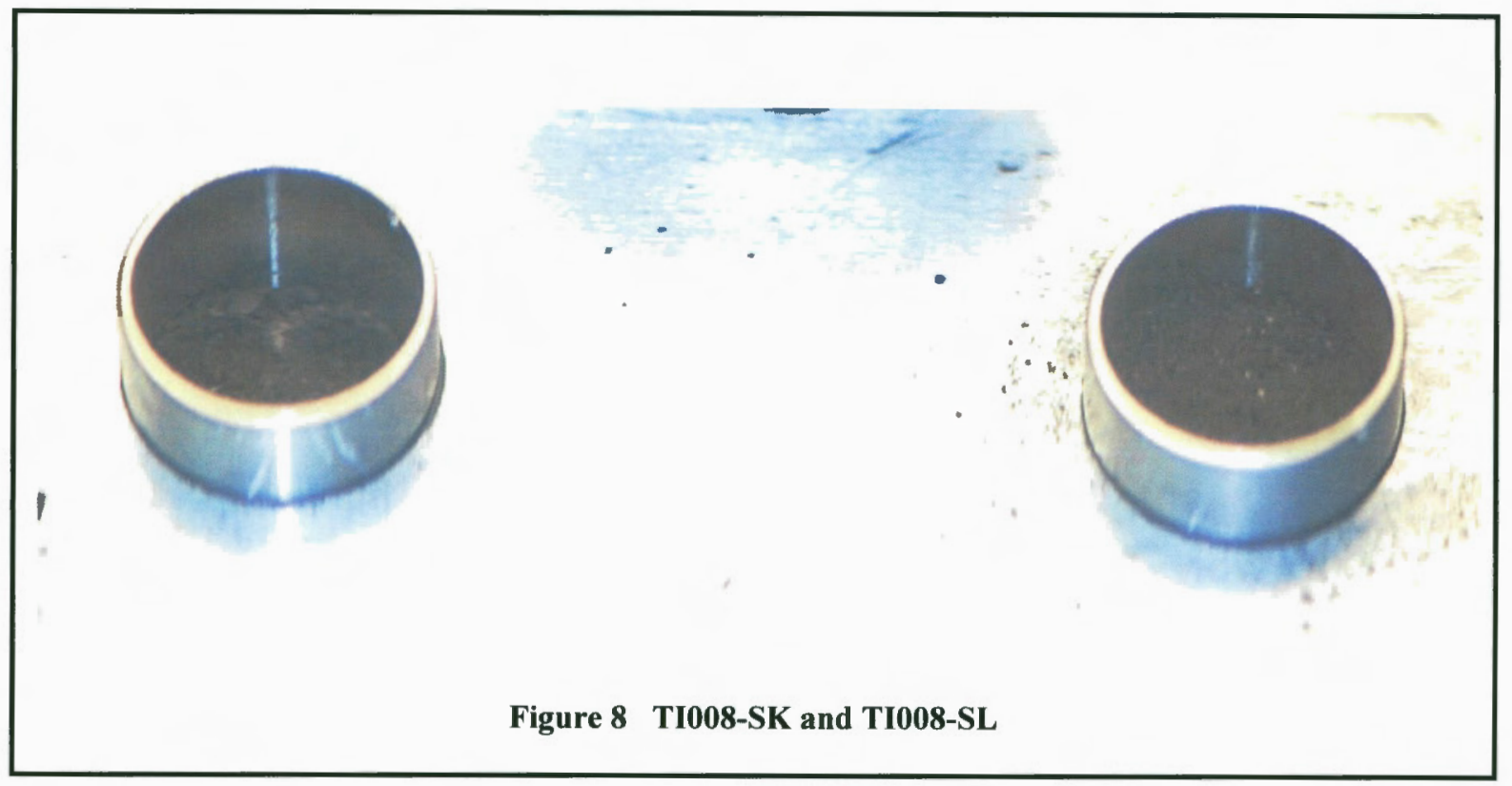

\subsection{Experimental}

As required by the analysis procedure APEL-PIP-4, Gas Pycnometry Method for Apparent Specific Gravity Determinations of Consolidated Solids, the AccuPyc 1330 Pycnometer was calibrated per the manufacturer's instructions using a verified calibration standard ( $\# 3955$, verified volume $0.718566 \mathrm{~cm}^{3}$ ) and confirmed with a second verified standard (\#1500, verified volume $0.7185465 \mathrm{~cm}^{3}$, expected density $14.9599 \mathrm{~g} / \mathrm{cm}^{3}$ ) at the beginning of each series of analyses, or at a minimum, daily. The calibration reports, provided from the instrument manufacture, are located in Appendix A.

During the course of K-Basin supported work, the calibration standard has been run multiple times. A summary of calibration data and a record of the pycnometer accuracy are provided in Appendix B. The calibration was considered acceptable if the measured volume of standard \#1500 was within $\pm 1.0 \%$ of the certified value. The results of the standard measurements are shown in Table 2 and were within tolerance.

Table 2 Standard \#1500 Measurements

\begin{tabular}{|c|c|c|}
\hline $\begin{array}{c}\text { Standard } \\
\text { Measurement, } \\
{\mathrm{g} / \mathrm{cm}^{3}}^{\mathbf{3}}\end{array}$ & $\begin{array}{c}\text { \% deviation from standard } \\
\text { density value of } 14.9599 \\
\mathrm{~g} / \mathrm{cm}^{3(1)}\end{array}$ & $\begin{array}{c}\text { Within } \pm 1 \% \text { DQO?, Y/N } \\
15.1094 \mathrm{~g} / \mathrm{cm}^{3}>X>14.8102 \\
\mathrm{~g} / \mathrm{cm}^{3}\end{array}$ \\
\hline 14.9815 & 0.15 & $\mathrm{Y}$ \\
\hline 14.9760 & 0.11 & $\mathrm{Y}$ \\
\hline 15.0014 & 0.28 & $\mathrm{Y}$ \\
\hline 14.9585 & 0.01 & $\bar{Y}$ \\
\hline
\end{tabular}


The balance (ID \#1129263167) calibration was verified by weighing 1, 10, and $20 \mathrm{~g}$ standard weights and the checks were considered acceptable when the measured values were within $\pm 1.0 \%$ of the standard values.

The samples were placed in a tared $1-\mathrm{cm}^{3}$ pycnometer sample cup and weighed. The pycnometer cup and sample were placed in the AccuPyc 1330 sample chamber and the chamber cap was fastened securely. The sample was analyzed five times using UHP helium, the results were averaged and the density with standard deviation was recorded. To the extent possible, the whole sample aliquot provided was used for measurements.

\subsection{Sample Results}

The samples were transferred to the analysis cup with the intent of using the entire sample provided. Table 3 shows the success of this effort as well as the density result in $\mathrm{g} / \mathrm{cm}^{3}$ along with \%RSD and \%RPD where applicable.

Table 3 Mass Provided and Analyzed and Results

\begin{tabular}{|c|c|c|c|c|c|c|}
\hline Sample & $\begin{array}{l}\text { Mass of } \\
\text { subdivision } \\
(\mathrm{g})^{(1)}\end{array}$ & $\begin{array}{c}\text { Mass } \\
\text { analyzed } \\
(\mathrm{g})^{(2)}\end{array}$ & $\begin{array}{c}\text { Difference } \\
\text { (g) }\end{array}$ & Density, $\mathrm{g} / \mathrm{cm}^{3}$ & $\begin{array}{c}\% \\
\text { RSD }\end{array}$ & $\begin{array}{c}\% \\
\text { RPD }\end{array}$ \\
\hline TI008-L & 0.7588 & 0.6780 & 0.0808 & 2.60 & 0.15 & $\overline{\mathrm{NA}}$ \\
\hline TI008-MK & 0.5231 & 0.4142 & 0.1089 & 1.62 & 0.04 & \multirow{2}{*}{0} \\
\hline TI008-ML & 0.5074 & 0.3420 & 0.1654 & 1.62 & 0.03 & \\
\hline TI008-SK & 0.6755 & 0.6355 & 0.0400 & 2.57 & 0.05 & \multirow{2}{*}{3} \\
\hline TI008-SL & 0.3462 & 0.2962 & 0.0500 & 2.49 & 0.07 & \\
\hline
\end{tabular}

\subsection{Data Quality Objectives}

The accuracy DQO of $\pm 1 \%$ was attained for balance and volume measures (refer to summary of calibration checks in Attachment B). The precision DQO of $\pm 5 \%$ RPD was met for all duplicate samples.

\subsection{Works Cited}

Lowell, J. (2004). Characterization of Porous Solids and Powders:Surface Area, Pore Size, and Density. Springer. 
PNNL-20650, Rev. 2

\section{Appendix A}

\section{Calibration Reports}




\section{[micromeritics ${ }^{\circ}$}

\section{Calibration Data Report AccuPyc 1330}

Serial Number: 3955

Volume of Calibration Standard:

\begin{tabular}{cc}
$\frac{0.718566}{1.2796}$ cubic centimeters \\
\hline 0.7286 & cubic centimeters \\
1.45 & cubic centimeters
\end{tabular}

Temperature Offset:

Average Cell Volume:

Average Expansion Volume:

1.45

Technician;

Date:
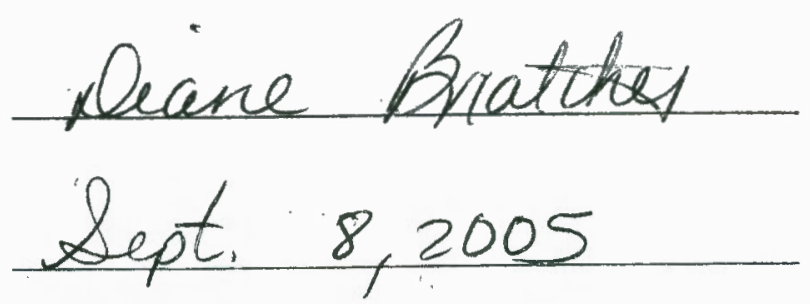
PNNL-20650, Rev. 2

$170-662-3666$

\section{[. $\mathbf{1}^{\circ}$ micromeritics}

\section{Calibration Data Report AccuPyc 1330}

Serial Number:

Volume of Calibration Standard:

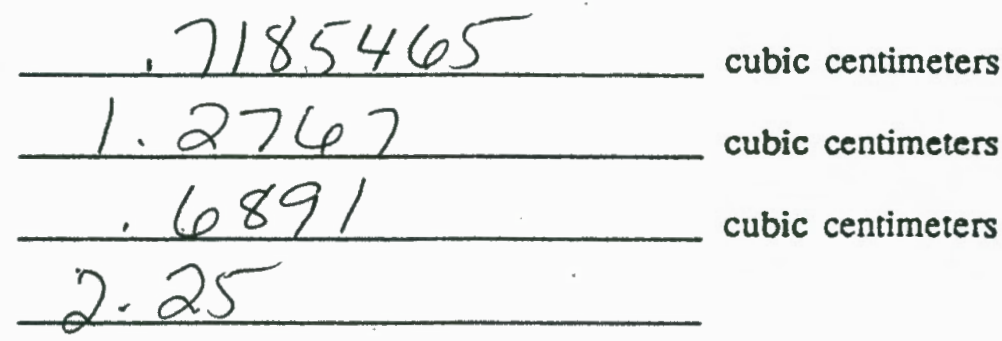

Timperature Offset:

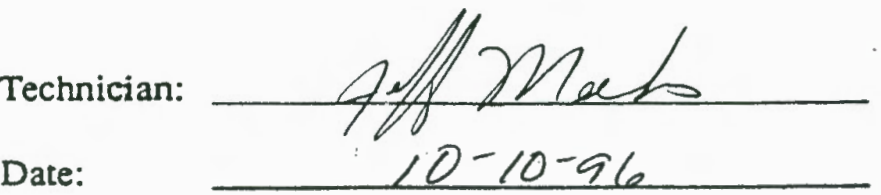


PNNL-20650, Rev. 2

\section{Appendix B}

\section{Summary of Calibration Data}


PNNL-20650, Rev. 2

\section{Appendix B}

Pycnometer

\begin{tabular}{|c|c|c|}
\hline $\begin{array}{c}\text { Calibration Standard } \\
(\# 3955)\end{array}$ & Verification Sta & ndard (\#1500) \\
\hline date & Density $\left(\mathrm{g} / \mathrm{cm}^{3}\right)$ & Volume $\left(\mathrm{cm}^{3}\right)$ \\
\hline $5 / 28 / 2009$ & 14.9802 & 0.7176 \\
\hline $6 / 2 / 2009$ & 14.9502 & 0.7190 \\
\hline $9 / 4 / 2009$ & 14.9596 & 0.7186 \\
\hline $9 / 4 / 2009$ & 14.9585 & 0.7186 \\
\hline $9 / 16 / 2009$ & 14.9733 & 0.7179 \\
\hline $9 / 17 / 2009$ & 14.9585 & 0.7186 \\
\hline $9 / 30 / 2009$ & 15.0215 & 0.7156 \\
\hline $5 / 25 / 2010$ & 14.9607 & 0.7185 \\
\hline $6 / 10 / 2010$ & 14.9607 & 0.7185 \\
\hline $2 / 7 / 2011$ & 14.9997 & 0.7166 \\
\hline $2 / 8 / 2011$ & 14.9686 & 0.7181 \\
\hline $2 / 9 / 2011$ & 14.9428 & 0.7194 \\
\hline $2 / 10 / 2011$ & 14.9603 & 0.7185 \\
\hline $2 / 10 / 2011$ & 14.9561 & 0.7187 \\
\hline $2 / 10 / 2011$ & 14.9555 & 0.7188 \\
\hline $2 / 10 / 2011$ & 14.9580 & 0.7186 \\
\hline $2 / 11 / 2011$ & 14.9602 & 0.7185 \\
\hline $2 / 14 / 2011$ & 14.9598 & 0.7185 \\
\hline $2 / 14 / 2011$ & 14.9566 & 0.7187 \\
\hline $3 / 15 / 2011$ & 14.9663 & 0.7182 \\
\hline $6 / 13 / 2011$ & 14.9818 & 0.7175 \\
\hline $6 / 14 / 2011$ & 14.9815 & 0.7175 \\
\hline $6 / 15 / 2011$ & 14.9760 & 0.7178 \\
\hline $6 / 20 / 2011$ & 15.0014 & 0.7166 \\
\hline $6 / 23 / 2011$ & 14.9585 & 0.7186 \\
\hline Average & 14.9682 & 0.7181 \\
\hline Standard Deviation & 0.0179 & 0.0009 \\
\hline *Accuracy & $100.06 \%$ & $99.94 \%$ \\
\hline
\end{tabular}

*Accuracy calulation for Volume (0.7186 cc certified):

$1-((0.7186-(0.7181)) / 0.7186)$

*Accuracy calulation for Density $\left(14.9599 \mathrm{~g} / \mathrm{cc}^{\otimes 0}\right)$ :

$1-((14.9599-(14.9682)) / 14.9599)$

${ }^{\circ}$ based on measured weight of $10.7494 \mathrm{~g}$ divided by certified volume of $0.7186 \mathrm{cc}$ 
PNNL-20650, Rev. 2

Pycnometer Analysis Sheet

APEL-PIP-4, Rev. 2

Pycnometer Information:

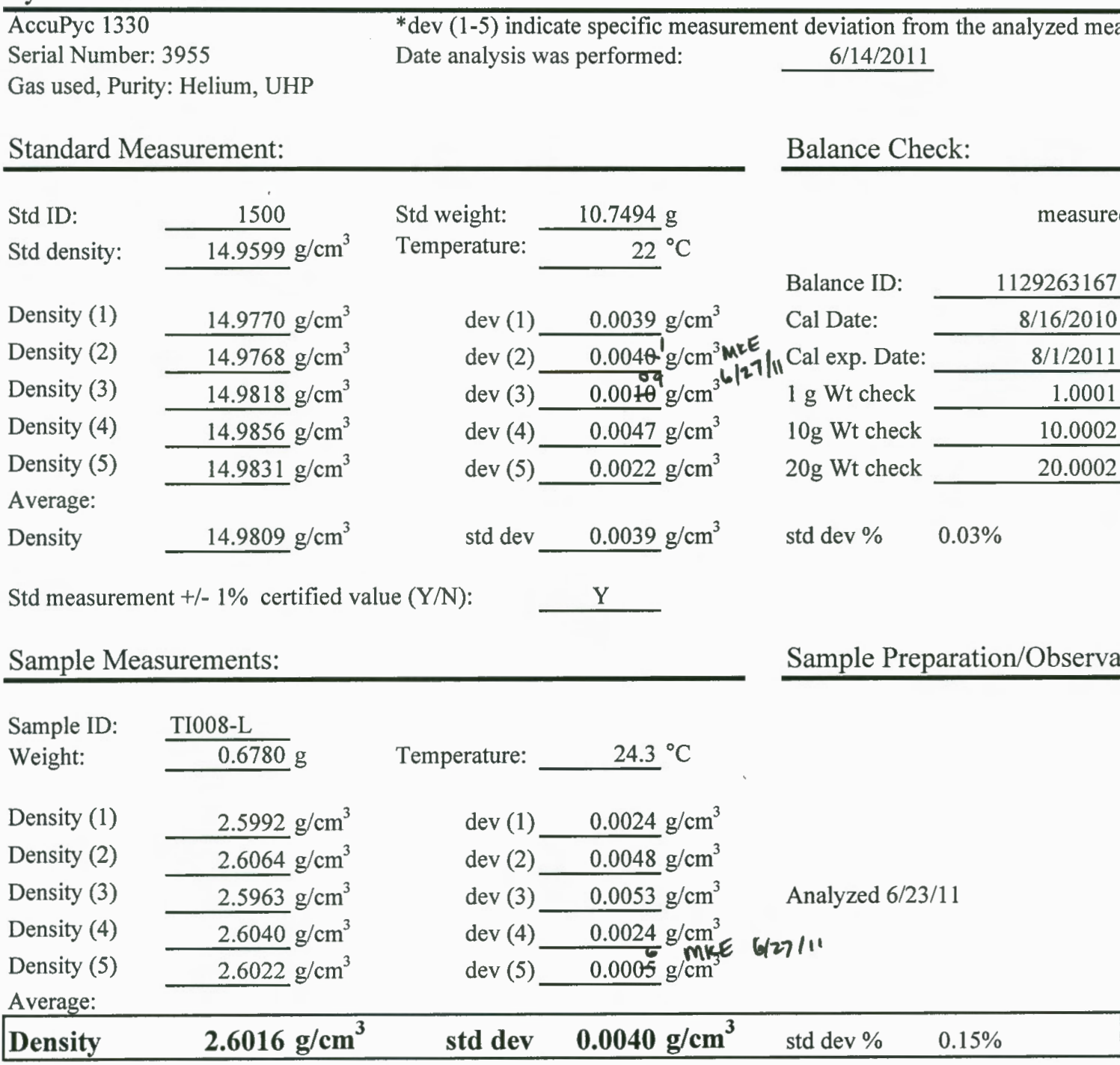

\begin{tabular}{|c|c|c|c|c|}
\hline $\begin{array}{l}\text { Sample ID: } \\
\text { Weight: }\end{array}$ & $\frac{\mathrm{TI} 008-\mathrm{MK}}{0.4142} \mathrm{~g}$ & Temperature: & $22.8^{\circ} \mathrm{C}$ & \\
\hline Density (1) & $1.6208 \mathrm{~g} / \mathrm{cm}^{3}$ & $\operatorname{dev}(1)$ & $0.0010 \mathrm{~g} / \mathrm{cm}^{3}$ & \\
\hline Density (2) & $1.6215 \mathrm{~g} / \mathrm{cm}^{3}$ & $\operatorname{dev}(2)$ & $0.0003 \mathrm{~g} / \mathrm{cm}^{3}$ & \\
\hline Density (3) & $1.6223 \mathrm{~g} / \mathrm{cm}^{3}$ & $\operatorname{dev}(3)$ & $0.0005 \mathrm{~g} / \mathrm{cm}^{3}$ & very statically charged \\
\hline Density (4) & $1.6220 \mathrm{~g} / \mathrm{cm}^{3}$ & $\operatorname{dev}(4)$ & $0.0002 \mathrm{~g} / \mathrm{cm}^{3}$ & Analyzed 6/14/11 \\
\hline Density (5) & $1.6223 \mathrm{~g} / \mathrm{cm}^{3}$ & $\operatorname{dev}(5)$ & $0.0005 \mathrm{~g} / \mathrm{cm}^{3}$ & \\
\hline Average: & & & & \\
\hline Density & $1.6218 \mathrm{~g} / \mathrm{cm}^{3}$ & std dev & $0.0006 \mathrm{~g} / \mathrm{cm}^{3}$ & std dev $\%$ \\
\hline
\end{tabular}
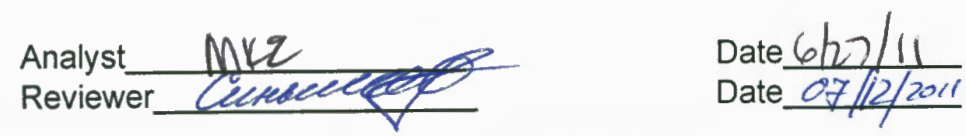
PNNL-20650, Rev. 2

Pycnometer Analysis Sheet

APEL-PIP-4, Rev. 2

(Page 2)

Sample Measurements:

Sample Preparation/Observations:

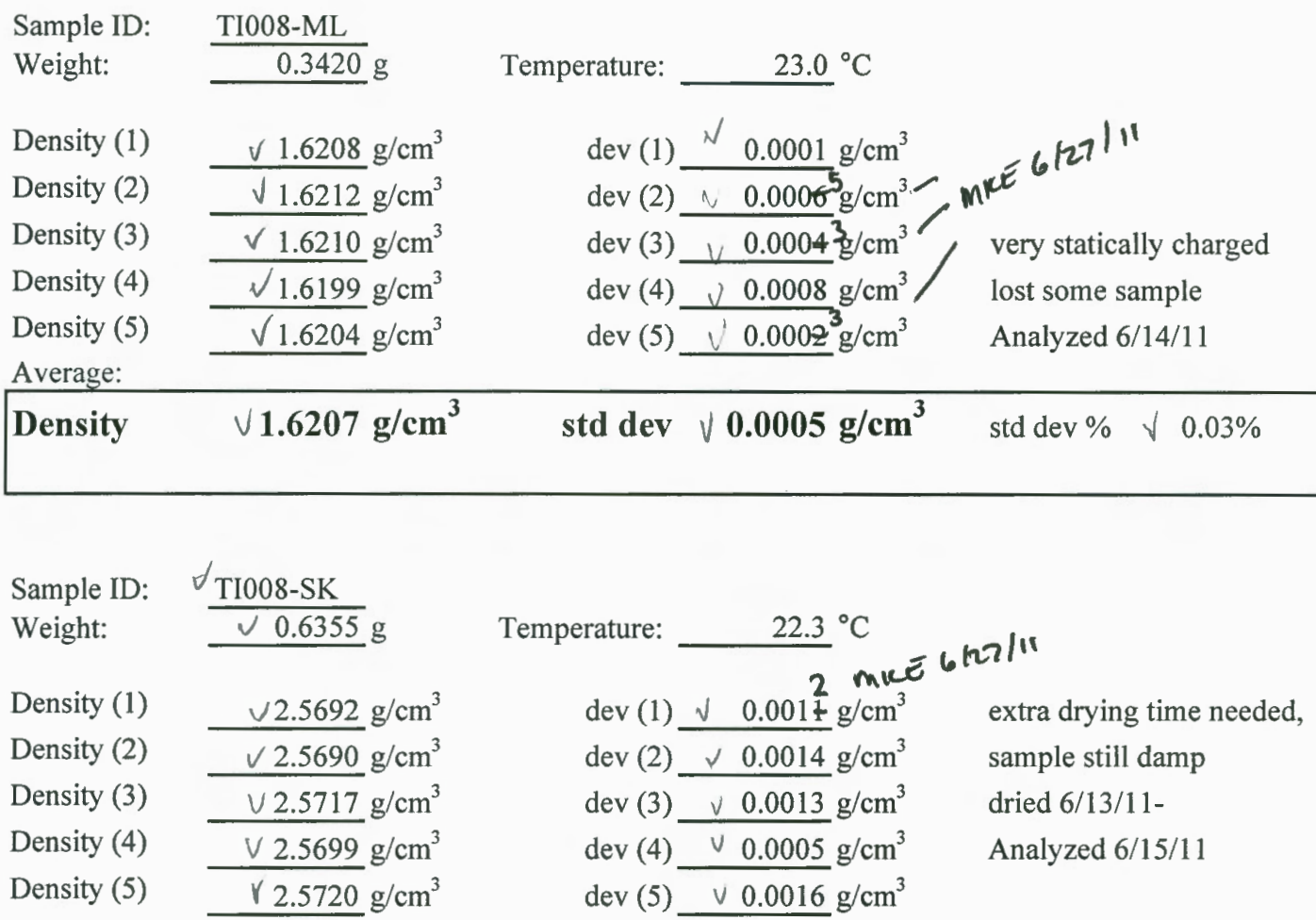

\begin{tabular}{|llllll|}
\hline Density & $\sqrt{2.5704} \mathrm{~g} / \mathrm{cm}^{3}$ & std dev & $\sqrt{0.0014} \mathbf{g} / \mathrm{cm}^{3}$ & std dev $\%$ & $V_{0.05 \%}$ \\
\hline
\end{tabular}

Sample ID: $\quad \checkmark$ TI008-SL

Weight: $\quad \bar{\downarrow} 0.2962 \mathrm{~g} \quad$ Temperature: $\quad 22.5^{\circ} \mathrm{C}$

\begin{tabular}{|c|c|c|c|c|}
\hline Density (1) & $\downarrow 2.4916 \mathrm{~g} / \mathrm{cm}^{3}$ & $\checkmark \operatorname{dev}(1)$ & $0.0020 \mathrm{~g} / \mathrm{cm}^{3}$ & \\
\hline Density (2) & $v 2.4939 \mathrm{~g} / \mathrm{cm}^{3}$ & $V_{\operatorname{dev}(2)}$ & $0.0003 \mathrm{~g} / \mathrm{cm}^{3}$ & extra drying time needed, \\
\hline Density (3) & $\sqrt{ } 2.4922 \mathrm{~g} / \mathrm{cm}^{3}$ & $\gamma \operatorname{dev}(3)$ & $0.0014 \mathrm{~g} / \mathrm{cm}^{3}$ & sample still damp \\
\hline Density (4) & $V 2.4950 \mathrm{~g} / \mathrm{cm}^{3}$ & $\checkmark \operatorname{dev}(4)$ & $0.0014 \mathrm{~g} / \mathrm{cm}^{3}$ & dried 6/13/11- \\
\hline Density (5) & V $2.4954 \mathrm{~g} / \mathrm{cm}^{3}$ & $\sqrt{\operatorname{dev}(5)}$ & $0.0018 \mathrm{~g} / \mathrm{cm}^{3}$ & Analyzed 6/15/11 \\
\hline
\end{tabular}

Average:

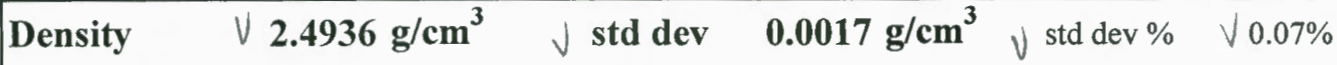

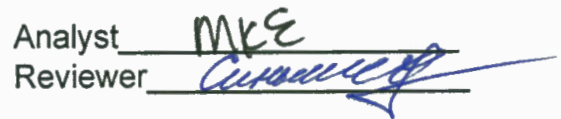

Date $\frac{6 / 27 / 11}{07 / 121 / 1}$ 
PNNL-20650, Rev. 2

\section{Pycnometer Analysis Sheet}

(Page 3)

**Tares from: TI008 and measured on balance N04143

**For Gross Weights:

Balance Check:

Date Performed: $6 / 13 / 2011$ measured $+/ \cdot 0.5 \%$

Balance ID: $\quad \frac{1.129 E+09}{8 / 16 / 2010}$

$(\mathrm{Y} / \mathrm{N})$

Cal Date: $\quad \frac{11298+09}{8 / 16 / 2010}$

Cal exp. Date: $-\frac{8 / 1 / 2011}{1.0000}$,

$1 \mathrm{~g} \mathrm{Wt}$ check $1.0000 \mathrm{~g}$,

$10 \mathrm{~g}$ Wt check $\frac{10.0002}{20.0003} \mathrm{~g}$

\begin{tabular}{c}
$\frac{Y}{Y}$ \\
\hline$Y$ \\
\hline$Y$
\end{tabular}

Masses Provided:

\begin{tabular}{|c|c|c|c|}
\hline Sample & Tare wt $(\mathrm{g})$ & $\begin{array}{l}\text { Gross } \\
\text { wt (g) }\end{array}$ & $\begin{array}{c}\text { Mass } \\
\text { Provided: }\end{array}$ \\
\hline TI008-L & $\vee 17.7301$ & 18.4889 & 0.7588 \\
\hline TI008-MK & $\sqrt{4.0141}$ & 4.5372 & 0.5231 \\
\hline TI008-ML & $\sqrt{3.9887}$ & 4.4961 & 0.5074 \\
\hline
\end{tabular}

Date Performed: $6 / 15 / 2011 \quad$ measured $+/=0.5 \%$

Standard Measurement:

\begin{tabular}{|c|c|c|c|c|c|c|c|}
\hline & \\
\hline Std ID: & \multicolumn{2}{|l|}{1500} & \multirow{2}{*}{$\begin{array}{l}\text { Std weight: } \\
\text { Temperature: }\end{array}$} & $10.7494 \mathrm{~g}$ & \multicolumn{3}{|c|}{ measured $+/-0.5 \%$} \\
\hline \multirow[t]{2}{*}{ Std density: } & \multicolumn{2}{|c|}{$14.9599 \mathrm{~g} / \mathrm{cm}^{3}$} & & $\sqrt{22.3}{ }^{\circ} \mathrm{C}$ & \multirow[b]{2}{*}{ Balance ID: } & \multirow[b]{2}{*}{1129263167} & \multirow[t]{2}{*}{$(\mathrm{Y} / \mathrm{N})$} \\
\hline & & & & & & & \\
\hline Density (1) & \multicolumn{2}{|c|}{$\forall 14.9777 \mathrm{~g} / \mathrm{cm}^{3}$} & $\operatorname{dev}(1)$ & $\checkmark \quad 0.0017 \mathrm{~g} / \mathrm{cm}^{3}$ & Cal Date: & $8 / 16 / 2010$ & \\
\hline Density (2) & \multicolumn{2}{|c|}{$\sqrt{ } 14.9741 \mathrm{~g} / \mathrm{cm}^{3}$} & $\operatorname{dev}(2)$ & $\mathrm{v} \quad 0.0019 \mathrm{~g} / \mathrm{cm}^{3}$ & Cal exp. Date: & $8 / 1 / 2011$ & $\mathrm{Y}$ \\
\hline Density (3) & \multicolumn{2}{|c|}{$v 14.9750 \mathrm{~g} / \mathrm{cm}^{3}$} & $\operatorname{dev}(3)$ & $0.0010 \mathrm{~g} / \mathrm{cm}^{3}$ & $1 \mathrm{~g} \mathrm{Wt}$ check & $\sqrt{1.0004} \mathrm{~g}$ & $\mathrm{Y}$ \\
\hline Density (4) & \multicolumn{2}{|c|}{$\sqrt{14.9759} \mathrm{~g} / \mathrm{cm}^{3}$} & $\operatorname{dev}(4)$ & $0.0001 \mathrm{~g} / \mathrm{cm}^{3}$ & $10 \mathrm{~g}$ Wt check & $\mathrm{V} \quad 10.0002 \mathrm{~g}$ & $\mathrm{Y}$ \\
\hline Density (5) & \multicolumn{2}{|c|}{$\sqrt{ } 14.9772 \mathrm{~g} / \mathrm{cm}^{3}$} & $\operatorname{dev}(5)$ & $\sqrt{ } 0.0012 \mathrm{~g} / \mathrm{cm}^{3}$ & $20 \mathrm{~g} \mathrm{Wt}$ check & \multirow[t]{2}{*}{$\sqrt{20.0006} \mathrm{~g}$} & $\mathrm{Y}$ \\
\hline Average: & & & & & & & \\
\hline Density & \multicolumn{2}{|c|}{$\mathrm{V} 14.9760 \mathrm{~g} / \mathrm{cm}^{3}$} & std dev & $\vee 0.0015 \mathrm{~g} / \mathrm{cm}^{3}$ & std $\operatorname{dev} \%$ & $0.01 \%$ & \\
\hline \multicolumn{4}{|c|}{ Std measurement $+/-1 \%$ certified value $(Y / N)$ : } & Y & & & \\
\hline \multicolumn{4}{|c|}{ Masses Provided: } & \multirow{3}{*}{\multicolumn{2}{|c|}{$\begin{array}{l}\text { extra drying time needed, } \\
\text { sample still damp }\end{array}$}} & & \\
\hline TI008-SK & $v_{17.4061}$ & 18.0816 & 0.6755 & & & & \\
\hline TI008-SL & $\sqrt{17.681}$ & 18.0272 & 0.3462 & & & & \\
\hline
\end{tabular}

Balance Check: 
PNNL-20650, Rev. 2

Pycnometer Analysis Sheet

APEL-PIP-4, Rev. 2

(Page 4)

Date Performed: $6 / 20 / 2011$ measured $+/-0.5 \%$

Standard Measurement:

Balance Check:

\begin{tabular}{|c|c|c|c|c|c|c|}
\hline \multirow{3}{*}{$\begin{array}{l}\text { Std ID: } \\
\text { Std density: }\end{array}$} & \multirow{3}{*}{$\frac{1500}{14.9599} \mathrm{~g} / \mathrm{cm}^{3}$} & \multirow{3}{*}{$\begin{array}{l}\text { Std weight: } \\
\text { Temperature: }\end{array}$} & \multirow{2}{*}{$\frac{10.7494}{23.5}{ }^{\circ} \mathrm{g}$} & \multicolumn{3}{|c|}{ measured $+/-0.5 \%$} \\
\hline & & & & \multirow[b]{2}{*}{ Balance ID: } & & \multirow[t]{2}{*}{$(\mathrm{Y} / \mathrm{N})$} \\
\hline & & & & & \multirow{2}{*}{$\frac{1129263167}{8 / 16 / 2010}$} & \\
\hline Density (1) & $15.0006 \mathrm{~g} / \mathrm{cm}^{3}$ & $\operatorname{dev}(1)$ & $0.0008 \mathrm{~g} / \mathrm{cm}^{3}$ & Cal Date: & & \multirow[b]{2}{*}{ Y } \\
\hline Density (2) & $15.0010 \mathrm{~g} / \mathrm{cm}^{3}$ & $\operatorname{dev}(2)$ & $0.0005 \mathrm{~g} / \mathrm{cm}^{3}$ & Cal exp. Date: & $8 / 1 / 2011$ & \\
\hline Density (3) & $15.0003 \mathrm{~g} / \mathrm{cm}^{3}$ & $\operatorname{dev}(3)$ & $0.0011 \mathrm{~g} / \mathrm{cm}^{3}$ & $1 \mathrm{~g} \mathrm{Wt}$ check & $\overline{1.0006} \mathrm{~g}$ & $\bar{Y}$ \\
\hline Density (4) & $15.0015 \mathrm{~g} / \mathrm{cm}^{3}$ & $\operatorname{dev}(4)$ & $0.0001 \mathrm{~g} / \mathrm{cm}^{3}$ & $10 \mathrm{~g} \mathrm{Wt}$ check & $10.0002 \mathrm{~g}$ & Y \\
\hline Density (5) & $15.0037 \mathrm{~g} / \mathrm{cm}^{3}$ & $\operatorname{dev}(5)$ & $0.0023 \mathrm{~g} / \mathrm{cm}^{3}$ & $20 \mathrm{~g}$ Wt check & $20.0006 \mathrm{~g}$ & $\bar{Y}$ \\
\hline Average: & \multirow[b]{2}{*}{$15.0014 \mathrm{~g} / \mathrm{cm}^{3}$} & & & & & \\
\hline Density & & std dev & $0.0014 \mathrm{~g} / \mathrm{cm}^{3}$ & std dev \% & $0.01 \%$ & \\
\hline \multicolumn{3}{|c|}{ Std measurement $+/-1 \%$ certified value $(\mathrm{Y} / \mathrm{N})$} & $\mathrm{Y}$ & & & \\
\hline Date Performed: & : 6/23/2011 & \multicolumn{2}{|l|}{ leasured $+/-0.5 \%$} & & & \\
\hline \multicolumn{4}{|c|}{ Standard Measurement: } & \multicolumn{2}{|c|}{ Balance Check: } & \\
\hline \multirow{3}{*}{$\begin{array}{l}\text { Std ID: } \\
\text { Std density: }\end{array}$} & \multirow{3}{*}{$\frac{1500}{14.9599} \mathrm{~g} / \mathrm{cm}^{3}$} & \multirow{3}{*}{$\begin{array}{l}\text { Std weight: } \\
\text { Temperature: }\end{array}$} & $10.7494 \mathrm{~g}$ & \multicolumn{3}{|c|}{ measured $+/-0.5 \%$} \\
\hline & & & $\sqrt{24.3^{\circ}}{ }^{\circ} \mathrm{C}$ & & & \multirow[t]{2}{*}{$(\mathrm{Y} / \mathrm{N})$} \\
\hline & & & & Balance ID: & 1129263167 & \\
\hline Density (1) & $14.9586 \mathrm{~g} / \mathrm{cm}^{3}$ & $\operatorname{dev}(1)$ & $0.0001 \mathrm{~g} / \mathrm{cm}^{3}$ & Cal Date: & $8 / 16 / 2010$ & \\
\hline Density (2) & $14.9579 \mathrm{~g} / \mathrm{cm}^{3}$ & $\operatorname{dev}(2)$ & $0.0006 \mathrm{~g} / \mathrm{cm}^{3}$ & Cal exp. Date: & $8 / 1 / 2011$ & Y \\
\hline Density (3) & $14.9588 \mathrm{~g} / \mathrm{cm}^{3}$ & $\operatorname{dev}(3)$ & $0.0003 \mathrm{~g} / \mathrm{cm}^{3}$ & $1 \mathrm{~g} \mathrm{Wt}$ check & $\forall 1.0003 \mathrm{~g}$ & $Y$ \\
\hline Density (4) & $14.9591 \mathrm{~g} / \mathrm{cm}^{3}$ & $\operatorname{dev}(4)$ & $0.0006 \mathrm{~g} / \mathrm{cm}^{3}$ & $10 \mathrm{~g}$ Wt check & $\sqrt{N 10.0002} \mathrm{~g}$ & $\mathrm{Y}$ \\
\hline Density (5) & $14.9581 \mathrm{~g} / \mathrm{cm}^{3}$ & $\operatorname{dev}(5)$ & $0.0004 \mathrm{~g} / \mathrm{cm}^{3}$ & $20 \mathrm{~g}$ Wt check & $20.0000 \mathrm{~g}$ & $\bar{Y}$ \\
\hline \multicolumn{3}{|l|}{ Average: } & & & & \\
\hline Density & V $14.9585 \mathrm{~g} / \mathrm{cm}^{3}$ & y std dev & $0.0005 \mathrm{~g} / \mathrm{cm}^{3}$ & $\checkmark$ std dev \% & $0.00 \%$ & \\
\hline \multicolumn{3}{|c|}{ Std measurement $+/-1 \%$} & $\mathrm{Y}$ & & & \\
\hline
\end{tabular}

Analyst $1 M \times \varepsilon$

Reviewer
Date $6 / 22 / 11$

Date $07 /(2) / 1$
4 
PNNL-20650, Rev. 2

Pycnometer Analysis Sheet

APEL-PIP-4, Rev. 2

Pycnometer Information:

AccuPyc $1330 \quad$ *dev (1-5) indicate specific measurement deviation from the analyzed mean

Serial Number: 3955

Gas used, Purity: Helium, UHP

Date analysis was performed:

6) $14 / 11$

Standard Measurement:

Balance Check:

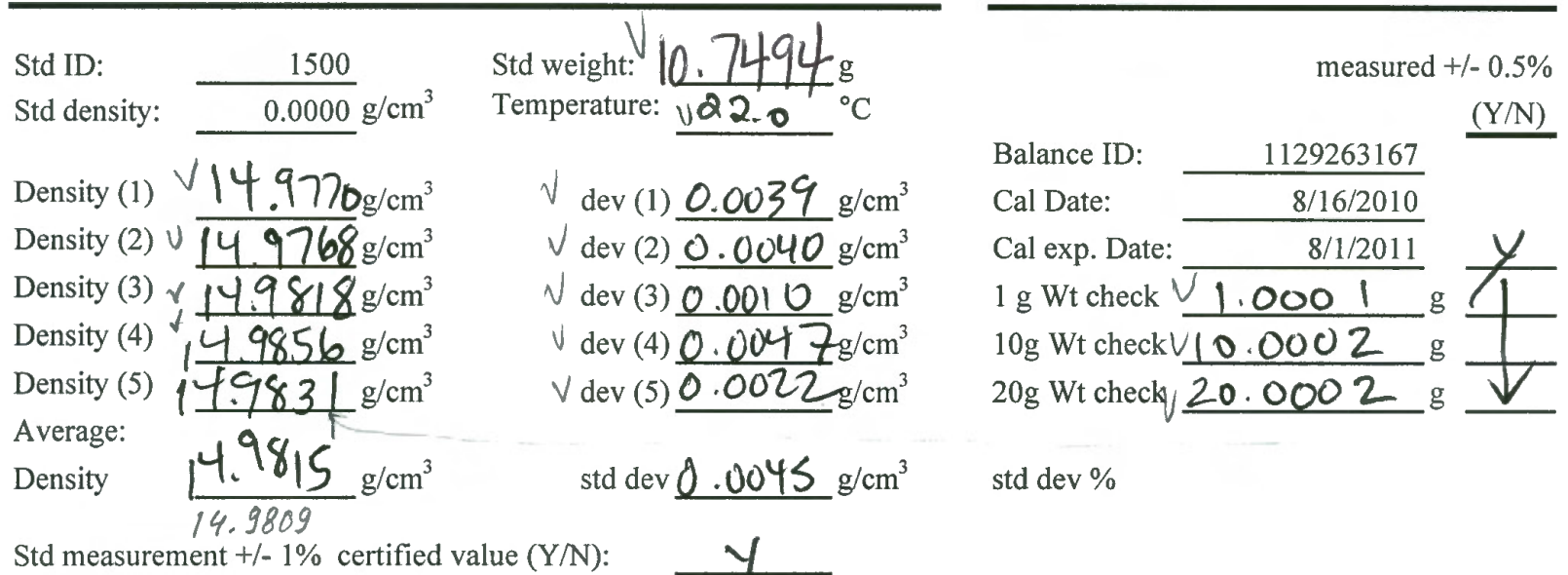

Sample Measurements:

Sample Preparation/Observations:

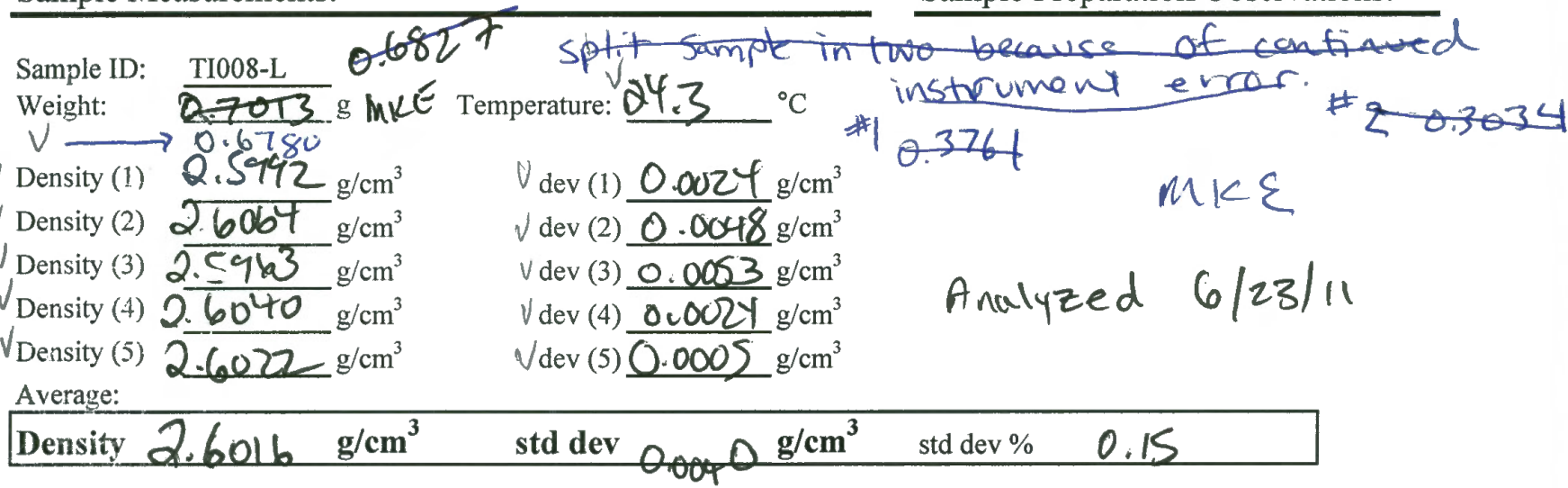

Sample iD: Tl008-MK

Weight: $\sqrt{0.4142^{\circ}} \quad$ Temperature: $22.8^{\circ} \mathrm{C}$

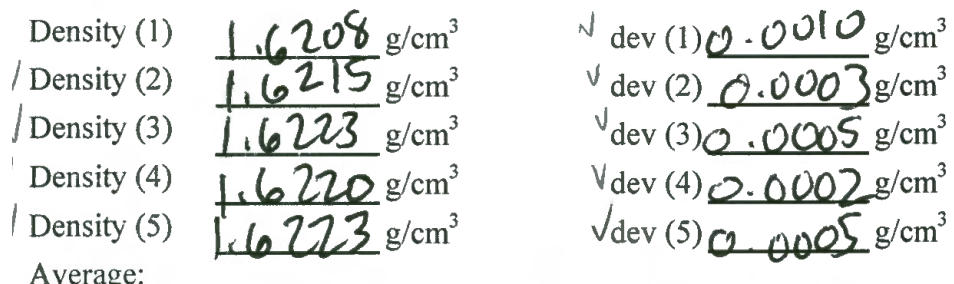

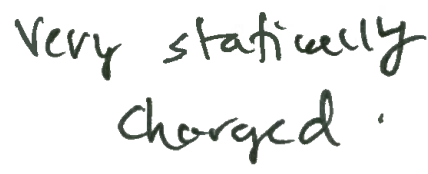

Density $1.6218^{\mathrm{g} / \mathrm{cm}^{3}} \quad$ std dev $0.0006 \mathrm{~g} / \mathrm{cm}^{3} \quad$ std dev \% 0.04

Analyst WKE

Reviewer
Date $6 / 27 / 11$

Date $07 / 12 / 1$ 
PNNL-20650, Rev. 2

Pycnometer Analysis Sheet

APEL-PIP-4, Rt

(Page 2)

Date analysis was performed:

Sample Measurements:

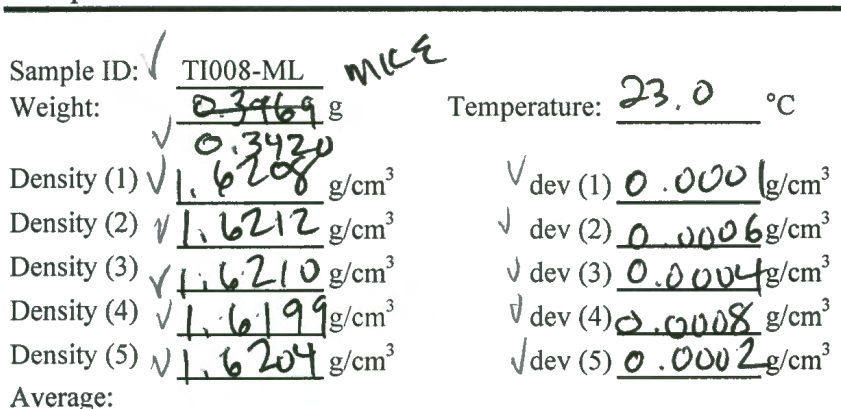

$6 / 14 / 11$

$6 / 15 / 11$

Sample Preparation/Observations:

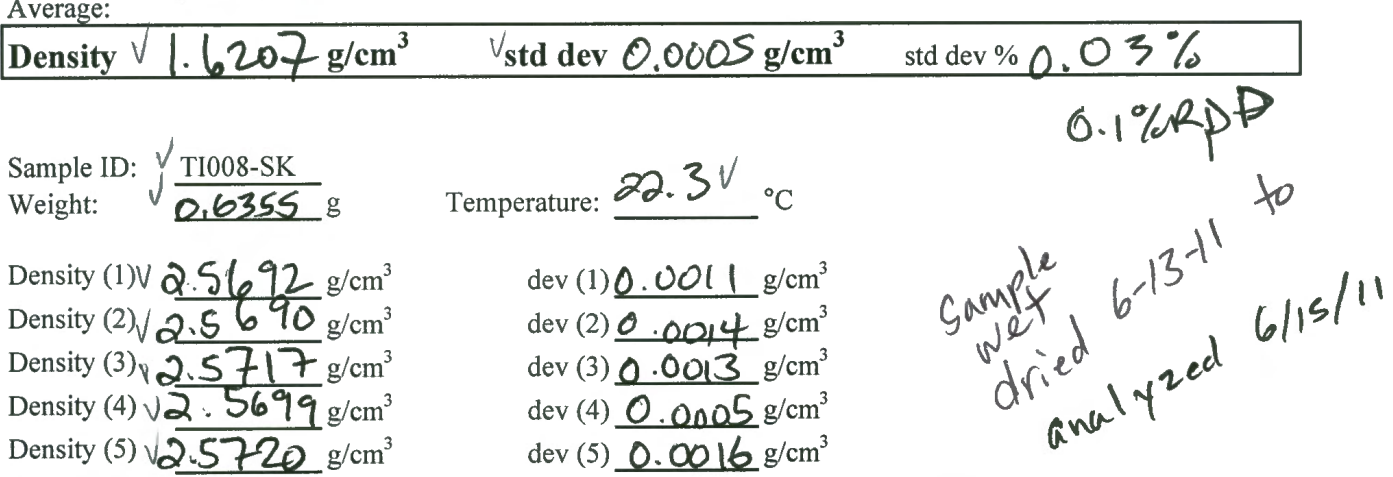

Average:

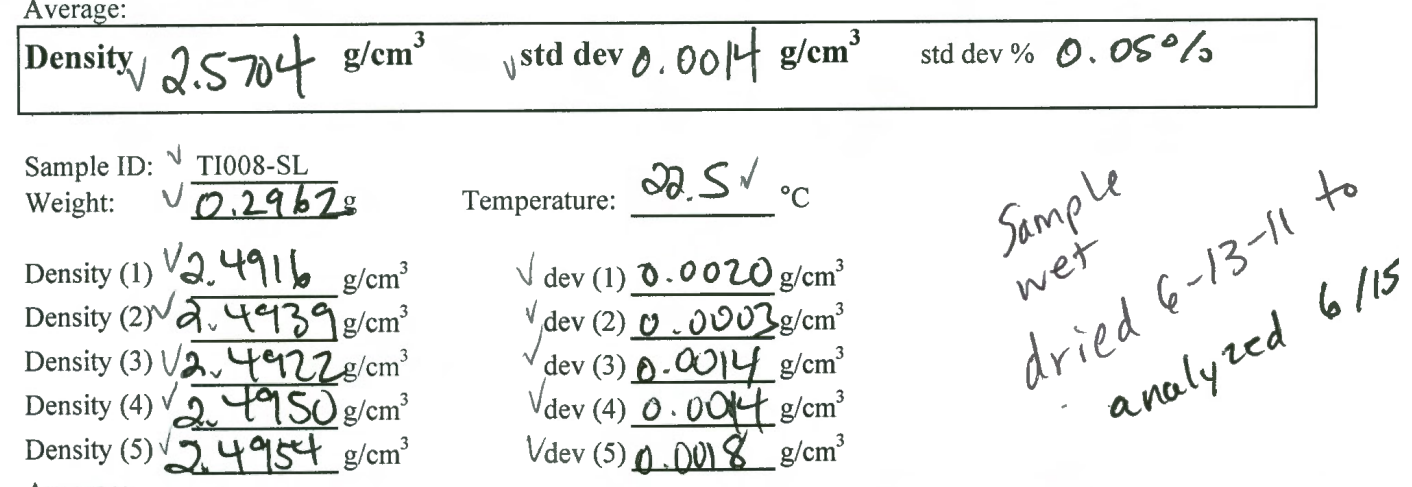

Density $2.4936 \mathrm{~g}^{\mathrm{g} \mathrm{cm}^{3}} \quad$ std dev $0.0017^{\mathrm{g} / \mathrm{cm}^{3}}$ std dev $\% 0.07$

3.0 RPD

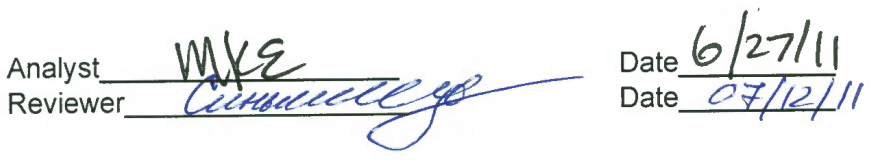


PNNL-20650, Rev. 2

'age 3)

Date analysis was performed: $\quad \frac{6 / 14 / 11}{6 / 15 / 11}$

"Tares from: TI008 measured on balance No4143

*For Gross Weights:

Balance Check:

ate Performed: $6 / 3 / 11$ measured $+1-0.5 \%$

$\begin{array}{ll}\text { alance ID: } & \frac{1.129 \mathrm{E}+09}{8 / 16 / 2010} \\ \text { al Date: } & \frac{8 / 1 / 2011}{2}\end{array}$

al exp. Date: $\frac{8 / 1 / 2011}{1 / 0000}$

g Wt check 1.0000 g

Jg Wt check $10.0002 \mathrm{~g} V$

$\mathrm{g}$ Wt check $20.0003 \mathrm{~g}$

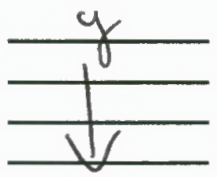

lasses Provided:

\begin{tabular}{|c|c|c|c|}
\hline Sample & Tare wt $(\mathrm{g})$ & $\begin{array}{c}\text { Gross } \\
\text { wt }(\mathrm{g})\end{array}$ & $\begin{array}{c}\text { Mass } \\
\text { Provided: }\end{array}$ \\
\hline I008-L & 17.7301 & 18.4889 & \\
\hline I008-MK & 4.0141 & 4.5372 & \\
\hline I008-ML & 3.9887 & 4.496 & mL \\
\hline $1008-\mathrm{SK}$ & 17.4061 & 174907 & $18.081 \mathrm{C}$ \\
\hline $1008-\mathrm{SL}$ & 17.6810 & 18.0272 & \\
\hline
\end{tabular}

$6 / 15 / 11$

lg wt. CK. $1.0004^{V}$ $\log$ wt. CK $10.0002 \mathrm{~V}$

$20 \mathrm{~g} w+\mathrm{CK} 20.0006 \mathrm{~V}$

$\lg 1.0006$

$\log 10.0002$

20 y 20.0006

ralyst a ak e

viewer
Std ck 6/15/11 Temp $22.3 \mathrm{C}$ 14.97770 .0017 $v 14.97410 .0019$

$v 14.9750 \quad 0.0010$

$v 14.9759 \quad 0.0001$

$\begin{array}{lll}v 14.9772 & 0.0012\end{array}$

$\begin{array}{lll}\frac{1+.9760}{15.0006} & 0.0000015 & \frac{0.01 \%}{23.5} \\ 15.0010 & 0.0005 & \\ 15.0003 & 0.0011 & \\ 15.0015 & 0.0001 \\ 15.0037 & 0.0023 \\ 15.0014 & 0.0014 & 0.01 \%\end{array}$

Date $\frac{6 / 27 / 11}{07 / 12 / 11}$

3

G.22 
PNNL-20650, Rev. 2

$$
\begin{aligned}
& \frac{6 / 23 / 11}{\lg V 1.0003} \\
& \log / 10.0002 \\
& \log 20.0000
\end{aligned}
$$

$$
\begin{aligned}
& \frac{\text { Std ck 6/23/11 }}{14.9586} 0.0001 \quad 24.3 c \\
& v 14.95790 .0006 \\
& \text {. } 14.95880 .0003 \\
& .14 .95910 .0006 \\
& .14 .95810 .0004 \\
& 14.9585 \quad 0.0005 \quad 0.00 \%
\end{aligned}
$$

Analyst mike 6/27/11

Reviewer Cimacleege of/12/11

G.23 


\begin{tabular}{|c|c|c|c|}
\hline \multicolumn{2}{|c|}{ RPL K Basin Project } & \multicolumn{2}{|c|}{$\begin{array}{l}\text { Document Review Record } \\
\text { MKE } 7 / 12 / 11\end{array}$} \\
\hline Title: & \multicolumn{3}{|c|}{ Particle Density Report bong Termive TI008 } \\
\hline \multicolumn{3}{|c|}{ Please return the completed form to: Matthew Edwards } & Comments Due: \\
\hline Org & nization/Department: & Designated Reviewer: & $\begin{array}{l}\text { Reviewer Signature/Date: } \\
\text { (Upon completion of review) }\end{array}$ \\
\hline & D9H63 & Sergey Sinkov & $07 / 12$ \\
\hline
\end{tabular}

It is optional, but helpful when comments are identified as to type or if the reviewer wishes to specify mandatory comments requiring resolution. In this case, the following conventions apply:

*Type: E-Editorial, addresses word processing errors that do not adversely impact the integrity of the calculation.

O-Optional, comment resolution would provide clarification, but does not impact the integrity of the work or calculations

M-Mandatory, comment shall be resolved; reviewer identifies impact on the integrity of the work or calculations

\begin{tabular}{|c|c|c|c|c|}
\hline $\begin{array}{l}\text { Comment } \\
\text { Number }\end{array}$ & $\begin{array}{l}\text { Section/ } \\
\text { Page }\end{array}$ & Type & Comment & Comment Resolution \\
\hline 1 & 2 of 7 & M & $\begin{array}{l}V_{\mathrm{T}} \text { in formula } 2 \text { does not match text in } \\
\text { paragraph below }\end{array}$ & Changed $V_{T}$ to $V_{r}$ in formula 2 to match text below \\
\hline 2 & $\begin{array}{l}\text { Analysis } \\
\text { Sheet }\end{array}$ & M & $\begin{array}{l}\text { Density (5) FOR THE Std measurement on } \\
\text { the completed analysis sheet does not } \\
\text { match the hard copy ( } 14.9831 \text { and } \\
14.9861) \text { This also changes the Average } \\
\text { density and std dev. }\end{array}$ & Transcription error. Changed value to 14.9831 \\
\hline 3 & $\begin{array}{l}\text { Analysis } \\
\text { Sheet }\end{array}$ & M & $\begin{array}{l}\text { Standard dev for the TI008-MK sample is } \\
\text { not filled in on the hard copy. }\end{array}$ & Added the std. dev. Value to the hard copy \\
\hline & & & & \\
\hline & & & & \\
\hline & & & & \\
\hline & & & & \\
\hline & & & & \\
\hline & & & & \\
\hline & & & & \\
\hline & & & & \\
\hline & & & & \\
\hline & & & 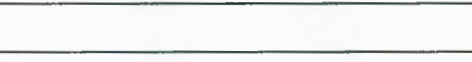 & 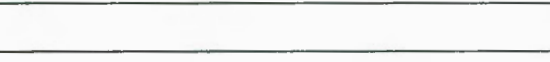 \\
\hline
\end{tabular}

Concur with comment resolution, review complete.
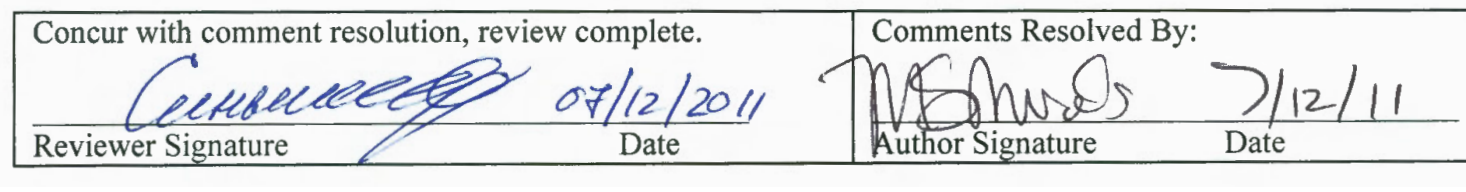


\section{PLANNING}

Topic/Issue to be evaluated: QE Review of Particle Density Report for TI008-L, TI008-MK, TI008-ML, TI008-SK and TI008-SL

This surveillance was undertaken to evaluate the draft Particle Density Report for five KW210 container material samples $(+2000,+500 /-2000$ and -500 micron sieve fractions and supporting data for the samples listed in Table 1.

\section{PERFORMANCE}

\begin{tabular}{|l|l|}
\hline Date Initiated: 6 April 2011 & Location: RPL \\
\hline $\begin{array}{l}\text { Date Received: 12 July 2011 } \\
\text { Date Reviewed: 13July 2011 } \\
\text { Date Completed: 14 July 2011 }\end{array}$ & $\begin{array}{l}\text { DRAFT Report Issued: 13 July 2011 } \\
\text { FINAL Report Issued: 14 July 2011 }\end{array}$ \\
\hline Contacts: Matt Edwards and Sergey Sinkov & Org Code: PE 137, D9H63 \\
\hline Surveillance Team: Lisa Middleton & \\
\hline
\end{tabular}

\section{PRELIMINARY INFORMATION}

\section{Source of Requirement(s):}

- KBC-33786, Rev. 2, December 2009; Quality Assurance Project Plan/Sampling and Analysis Plan (QAPjP/SAP): Quality Assurance Project Plan/Sampling and Analysis Plan for Containerized KW Settler Sludge

Other Applicable Documents:

- RPL-OP-001, Rev. 9, Routine Research Operations

- APEL-PIP-4, Rev. 2, Gas Pycnometry Method for Apparent Specific Gravity Determinations of Consolidated Solids

- 52578-ADM-001, Rev. 0, K Basin Requests for Analysis and Guidelines for Data Reporting and Technical, QE, and Spreadsheet Reviews.

\section{Purpose/Scope:}

The 52578 Project QE staff are required via the QA Program to perform a review of K Basin reports generated within the Radiochemical Processing Laboratory (RPL) to determine traceability and accuracy of data contributing to the completed report. 
Number: SR-52578-2011-032

Page 2 of 5

The draft report was reviewed to evaluate that the data are what they purport to be, that the reported results reflect what was actually done and the results meet applicable requirements. When deficiencies in the data are identified, then those deficiencies need to be documented as a K Basin Occurrence Report for the data user's review and, when applicable, resolved by corrective action.

\section{Requirements:}

The requirements for this work are defined in Table 3-10, KBC-33786 Rev 2; as well as the Analysis Request, applicable DQOs are:

- Precision $\pm 5 \%$

- Accuracy $\pm 1 \%$

The draft report itself was the basis for the data quality review.

When issues were found, the cognizant staff were contacted to resolve issues prior to completion of the review.

\section{APPROACH}

The Particle Density Report submitted for review consisted of the following for the five samples listed below in Table 1:

- Particle Density Report for five (5) KW210 samples (pp.7) including Figures 1-8, photographs of submitted samples

- Appendix A - Calibration Reports; Serial Numbers 1550 on $10 / 10 / 96$ and 3955 on $9 / 8 / 05$

- Appendix B - Summary of Calibration Data from 5/28/09 to 6/15/11

- Request for Particle Density Analysis by Gas Pycnometry (submitted 4/6/11)

- Pycnometer Analysis Sheet (pp.4) including M\&TE, standard 1550 density results $(\eta=5)$ on 6/14/11; Pycnometer Analysis Sheet with hand-written entries included (pp.4) - (bench sheets)

- Document Review Record, Sergey Sinkov, for Particle Density Report Long Term Archive, signed $7 / 12 / 11$

\begin{tabular}{|c|c|}
\hline Sample ID & Sample Description \\
\hline TI008-L & Particles of varying shapes, sizes \& colors. \\
\hline TI008-MK & $\begin{array}{c}\text { Particles of varying shapes, sizes and } \\
\text { colors. Resin beads evident. } \\
\text { Particles of varying shapes, sizes and } \\
\text { colors. Resin beads evident. }\end{array}$ \\
\hline TI008-ML & Dark Brown powder \\
\hline TI008-SK & Dark Brown powder \\
\hline TI008-SL & \\
\hline
\end{tabular}

Table 1. Subsample sieve fractions of KW210. 


\section{Approach:}

The issues identified in the QE review of the report are noted below and a copy of the draft report with comments was provided to the author.

Before the completed report was signed off for QE review, it was determined that all comments/concerns from the technical review were addressed. There were no outstanding identified findings or observations.

\section{Procedure Used:}

\section{APEL-PIP-4, Rev 2, Gas Pycnometry Method for Apparent Specific Gravity Determinations of Consolidated Solids}

\section{Sample Preparation:}

Five (5) samples were submitted for analysis; one sample (TI008-L) was to be split for duplicate analysis. Tare weights are recorded in completed K Basin test Instruction (TI), TI008.

\section{Samples Analyzed:}

Five samples were submitted for analysis as noted above. The samples were analyzed five times and the results averaged and mass and density reported.

\section{M\&TE Used:}

\begin{tabular}{|c|c|c|c|c|}
\hline Location & Manufacturer & Model & Unique ID & $\begin{array}{c}\text { RPL Calibration } \\
\text { Control } \\
\text { Tracking } \\
\text { Number }\end{array}$ \\
\hline RPL/506 & Mettler & AB104-S & $\begin{array}{c}1129263167 \\
\text { Used on } 6 / 14 / 11, \\
6 / 15 / 11,6 / 20 / 11 \& \\
6 / 23 / 11\end{array}$ & 1129263167 \\
& & & 3955 & NA \\
\hline
\end{tabular}

\section{RESULTS}

Findings are defined as a statement of fact relating to noncompliance with previously agreed-upon procedures, plans, codes, standards, specifications, or other forms of contractual or legal obligation. It should be understood that any lack of a finding in a specific area is not considered an indication that deficiencies do not exist.

Observations are defined as a weakness that if not corrected, could yield a departure from a requirement although the weakness is not necessarily a departure from requirements. 
PNNL-20650, Rev. 2

PROJECT 52578 - SURVEILLANCE REPORT

Number: SR-52578-2011-032

Page 4 of 5

\section{Summary of Identified Concerns:}

DQO Compliance:

- Required duplicates; precision $\pm 5 \%$; Yes

- Accuracy $\pm 1 \%$; Yes

Note: Concerns identified in the review are expected to be addressed in a comment resolution meeting to be scheduled by the report author when the comments have been addressed. All concerns identified in the review were addressed in a comment resolution meeting on 14 July 2011. Deborah Coffey, Lisa Middleton and Matt Edwards attended. There were no outstanding identified findings or observations.

Identified issues of concern were the following:

1. Page 4 of the bench sheets (hand-written) is dated $6 / 23 / 11$. The typed version is dated $6 / 20 / 11$. If the analysis was indeed done on $6 / 20 / 11$, the cover page of the report needs to be changed to reflect this. There is a second standard check below the $6 / 20$ on the typed version that is dated 6/23. Standard checks were done on both days, but the analysis of the sample was done on 6/23. Discussed, no further action required. Closed

2. Page 5 of report: Is the expected density of the secondary standard supposed to be 14.9598 instead of 14.9599 ? Should be 14.9599, changed. Steel ball used for standard weighed 10.7494 instead of previous weight used in other analyses (10.7483), giving a different density (14.9599). Values for weight and density given in Table 2 of the report were corrected to reflect values attained in this analysis. No further action required. Closed

3. Can the content of page 7 fit onto page 6 so there isn't a large gap in the report? Yes. No further action required. Closed

4. The first formula in Appendix B should say density instead of volume. Please fix the template you use as well, so this won't come up again. Template was already fixed from last time $\odot$ I had just printed this out before I made the template change. Fixed, no further action required. Closed

5. The values listed in the volume \& density formulas of Appendix B are incorrect. They should be $0.7181 \& 14.9682$ instead of $0.7183 \& 14.9654$ changed. Fixed, no further action required. Closed

6. Pycnometer Analysis Sheet: There are 8 deviations that the machine calculated wrong. Standard on page 1 \#s 2 \&3. TI008-L \#5. TI008-ML \#s 2, 3 \& 5. TI008-SK \# 1. $1^{\text {st }}$ standard on page 4 \# 2. See comment copy. Changed on sheets with pen and initialed and dated. Also changed future spreadsheets to calculate the number instead of an input from the instrument (since it has this issue occasionally). Fixed, no further action required. Closed

7. Pycnometer Analysis Sheets: It appears that the incorrect standard density was entered into the bench sheets. Shouldn't it be 14.9598 instead of 14.9599 ? No. 14.9599 is right. It is a calculation based on the standard weight and the calibrated volume. No further action required. Closed 


\section{CORRECTIVE ACTION}

[X] None Required. [ ] Complete [ ] Follow up Corrective Action

\section{Surveillance Performed By:}
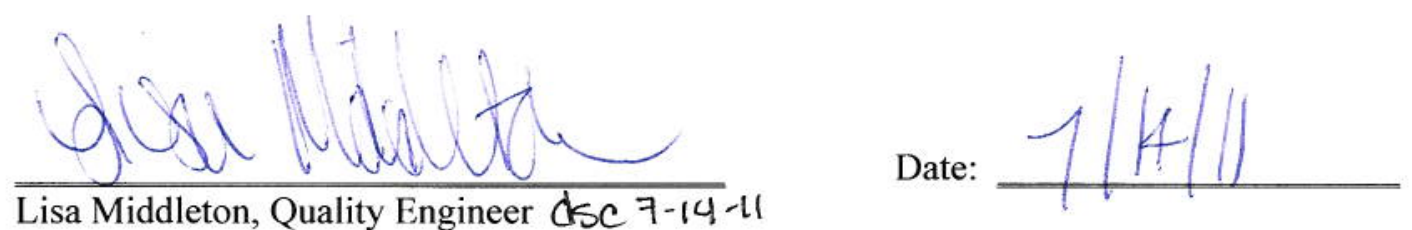

DISTRIBUTION: Clark Carlson, Matt Edwards, Sandy Fiskum, Andy Schmidt, Rick Shimskey, Sergey Sinkov, Deborah Coffey, Lisa Middleton 
PNNL-20650, Rev. 2

\section{Appendix H}

\section{Rheology}





\section{Appendix $\mathrm{H}$}

\section{Rheology}

Appendix H contains the rheology characterization report for the KW210 container composite samples and supporting documentation and calculations. The supporting documentation includes data reduction, calculation and graphing sheets, performance verification standard certificates, the referenced Nonconformance Report and the Surveillance Report .

The following table of contents shows that pagination specific to Appendix $\mathrm{H}$ was applied to the rheology report and supporting attachments. This pagination is sequential and consistent (e.g., page H.\#).

\section{Appendix H Table of Contents}

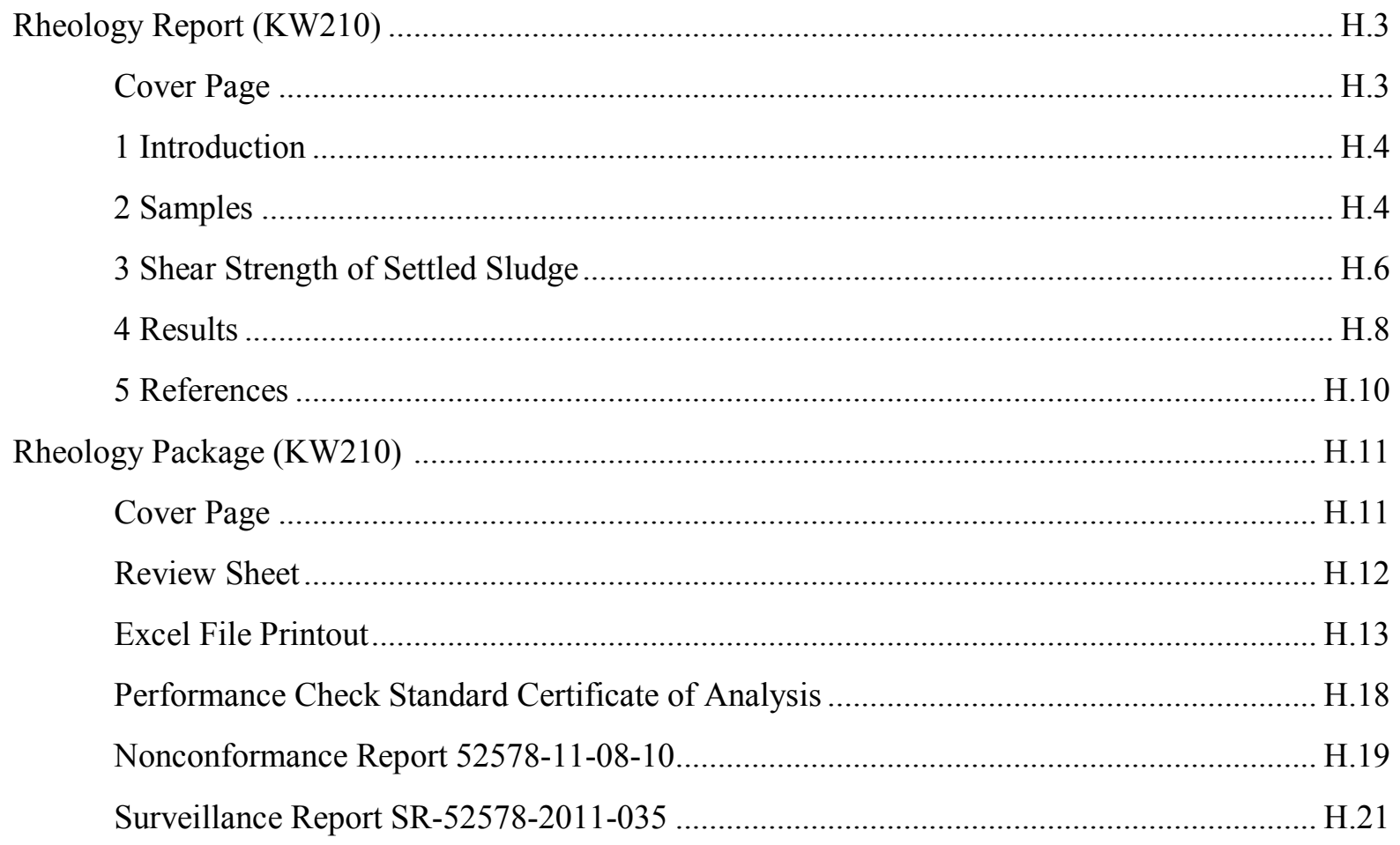





\section{Rheological Measurements for K West Basin Consolidated Container Composites SCS-CON-210}

\section{Battelle-Pacific Northwest National Laboratory Radiochemical Sciences and Engineering Group PO Box 999, Richland, Washington 99352}

Project: \#52578

Sample ID: KW210-CC-A

Supporting Test Instruction: 52578-TI039

Procedure: RPL-COLLOID-02, Rev. 2, Measurement of Physical and Rheological Properties of Solutions, Slurries and Sludges

Analyst: Carolyn Burns

Analysis Dates: 05/26/2011 through 06/21/2011

Instrument: Haake RV20-M5, SN\# 902398, with MV, MV2P and Vane Geometries

Supporting Electronic Report File Name: "TI-039 - 001 (Reviewed).xlsm"

File location: SharePoint site under TI-039 folder

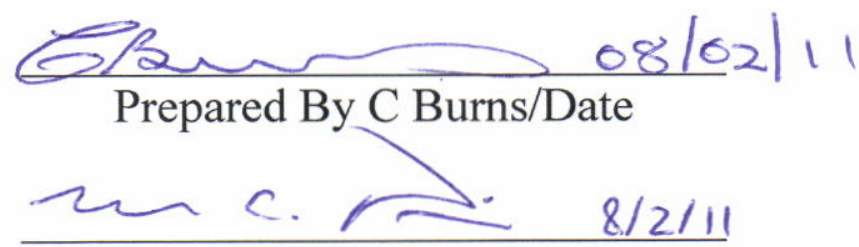

Reviewed By RC Daniel/Date

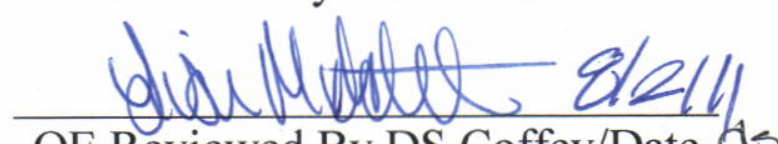

QE Reviewed By DS Coffey/Date Clsc 8-2-11

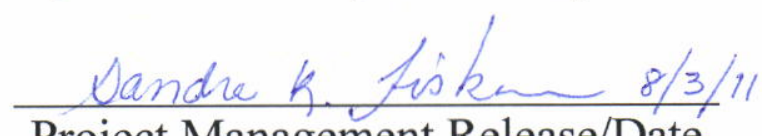

Project Management Release/Date 
PNNL-20650, Rev. 2

Carolyn Burns

Page 2 of 8

July 20, 2011

\section{Introduction}

The sludge from the K Basins Pits and Floors (referred to as Container Sludge) has been consolidated into five engineered containers located under water in the K-West (KW) Basin. The container sludge originating from the K-East (KE) Basin resides in containers SCS-CON-240, -CON-250, and -CON260. The container sludge originating from the KW Basin resides in SCS-CON-210 and -CON-220.

Two to four top-to-bottom core samples have been collected from each container and shipped to RPL. Previous test instructions have been implemented to reconstitute the sludge in a given core into a core composite sample. Sub-samples from each core composite within one Engineered Container have been consolidated into a single sample in an effort to represent the entire container (called a Container Composite). The Container Composite has been wet-sieved into +2000 micron, $-2000 /+500$ micron, and -500 micron particle size fractions. Subsamples of the -500 micron fraction have been collected to support rheological characterization of this material. In 2009, rheological measurements were performed on all of the container composites with the exception of SCS-CON-210. This report addresses the rheological characterization for subsamples of the container composites for SCS-CON210.

Figure 1 summarizes the processing steps for the preparation of the rheology composite sample. Samples were collected in equal volumes of dewatered sludge according to 52578-TI001 and-TI002. The volumes were not re-equalized after settling or further compaction upon standing. The four reserved "-A" subsamples were combined into one bottle, the settled solids volume was measured and the settled solids density calculated. The "A" bottle composite was then submitted for rheology testing. ${ }^{\text {(a) }}$ Thus rheology (shear strength only) was tested on the full container composite (unseived) as opposed to the -500 micron fraction. Viscosity could not be measured on the unsieved material because of issues associated with the large particles. This approach, which differs from that defined in the QAPjP/SAP, was directed by the Buyer's Technical Representative (BTR) based on sample volume limitations and evolving Sludge Treatment Project priorities. ${ }^{(b)}$

This data package summarizes the test approach and results of rheological measurements performed under Test Instruction 52578-TI039, Rheological Measurements for K West Basin Consolidated Container Composite SCS-CON-210. Shear strength measurements were performed on one KW210 container composite sample: KW210-CC-A. Flow curve measurements were not performed as previously discussed.

\section{Sample}

Table 1 identifies the sample to be subjected to shear strength measurements. The shear strength measurements were conducted in the Shielded Analytical Laboratory (SAL).

(a) This material is expected to be used for Treat/Transfer testing according to scope defined in PRC-STP-TP00261, Rev. 1 (Test Plan for Polymer Testing at the Pacific Nortbwest National Laboratory on Actual K Basin Sludge Incorporating Treat and Transfer While Performing Solids Recirculation Assessment, B Manning, 2/3/11) following testing for rheology.

(b) E-mail from WW Rutherford to SK Fiskum 2/14/11, "Concurrence requested for alternative subsampling and analysis of KW210 core samples." 
PNNL-20650, Rev. 2

Carolyn Burns

Page 3 of 8

July 20, 2011

Table 1. Container Composite KW210 Sample for Shear Strength Testing

\begin{tabular}{|l|l|l|l|}
\hline Sample ID & Sample Purpose. & Vessel Description & $\begin{array}{l}\text { Approximate Sample Size, } \\
\text { wet sludge basis }\end{array}$ \\
\hline \multicolumn{3}{|l|}{ Container Composite KW210, Subsample Collected under Test Instruction $52578-$ TI 008} \\
\hline KW210-CC-A & Shear Strength & $240 \mathrm{~mL}$ glass Qorpak & $\sim 180 \mathrm{~mL}$ \\
\hline
\end{tabular}

The general rheological characterization approach presented in this report was followed/cold tested for the characterization of K Basin sludge simulants in May 2009 (PNNL-18408). Key findings and lessons learned from this simulant characterization effort have been incorporated into this test instruction.

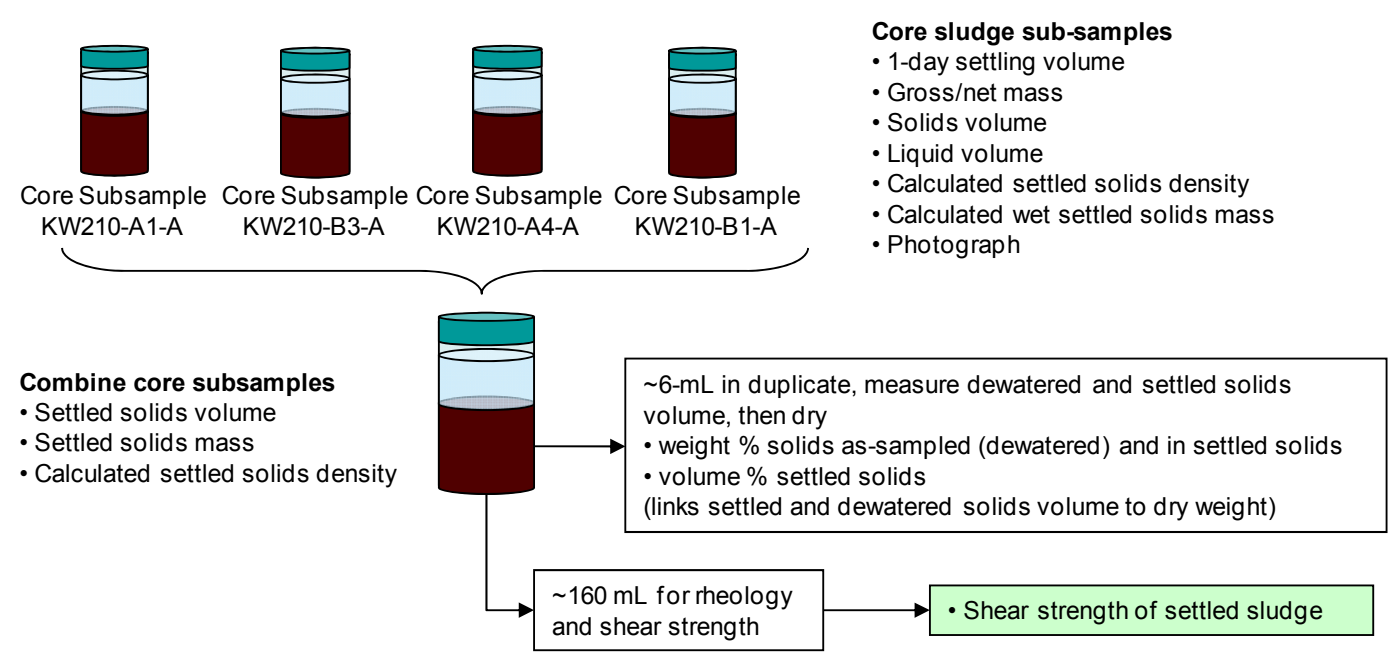

Figure 1. Overall Processing Schematic for the "A" Container Composite (shear strength sample preparation) 
PNNL-20650, Rev. 2

Carolyn Burns

Page 4 of 8

July 20, 2011

\section{Shear Strength of Settled Sludge}

\section{Test Equipment Description}

Shear strength was measured on the settled sludge sample in accordance with PNNL technical procedure, RPL-COLLOID-02, Rev. 2, "Measurement of Physical and Rheological Properties of Solutions Slurries and Sludges". The measurements were performed in SAL with a Haake RV20-M5 Viscometer. The RV20-M5 viscometer is capable of evaluating fluid rheological properties using Couette flow devices or shear strength using shear vanes. A shear vane tool was used for shear strength measurements. Table 2 summarizes the M5 measuring system information.

Table 2. Summary of Haake RV20 system with M5 measuring head

\begin{tabular}{||l|l||}
\hline Analyzer: & $\begin{array}{l}\text { Rotovisco } \mathbb{R} \text { RV20 Measuring System M with M5 } \\
\text { Measuring Head }\end{array}$ \\
\hline Measurement principle: & Controlled Rate \\
\hline Serial Number: & 902398 \\
\hline Torque Sensor Range & 0.49 to $49 \mathrm{mN} \cdot \mathrm{m}$ \\
\hline Rotational Rate Range & 0.05 to $500 \mathrm{RPM}$ \\
\hline Software & RheoWin Pro, Version 2.96, 1996 \\
\hline
\end{tabular}

\section{Background}

Shear strength is a semi-quantitative measurement of the stress required to yield a sample in shear such that it ceases to deform like a solid but instead flows like a viscous material with a finite viscosity. The shear strength of a sample may be dependent on sample history. The shear strength can be obtained by directly measuring resulting torque as a function of time when an immersed vane is slowly rotated in the sample material. The measured torque is converted to a shear stress by equations 1 and 2.

$$
\tau=\frac{T}{K}
$$

where

$$
K=\frac{\pi D^{3}}{2}\left(\frac{H}{D}+\frac{1}{3}\right)
$$

where $\quad \tau=$ calculated shear stress in Pascal

$\mathrm{T}=$ measured torque in Newton-meters

$\mathrm{K}=$ shear vane constant in cubic meters

$\mathrm{D}=$ shear vane diameter in meters

$\mathrm{H}=$ shear vane height in meters.

This equation is based on the premise that the diameter of the vane shaft is much smaller than the diameter of the vane itself. A typical stress/time profile is shown in Figure 2. The profile shows an initial linear region $\left(\tau_{\mathrm{y}}\right)$ followed by a nonlinear region, a stress maximum $\left(\tau_{\mathrm{s}}\right)$, and a stress decay region. The stress maximum is typically associated with the transition between the visco-elastic and fully viscous flow. The shear strength is defined as the transition between these two flows and is associated with the stress maximum. The shear strength of the material is denoted as $\tau_{\mathrm{S}}$. 
PNNL-20650, Rev. 2

Carolyn Burns

Page 5 of 8

July 20, 2011

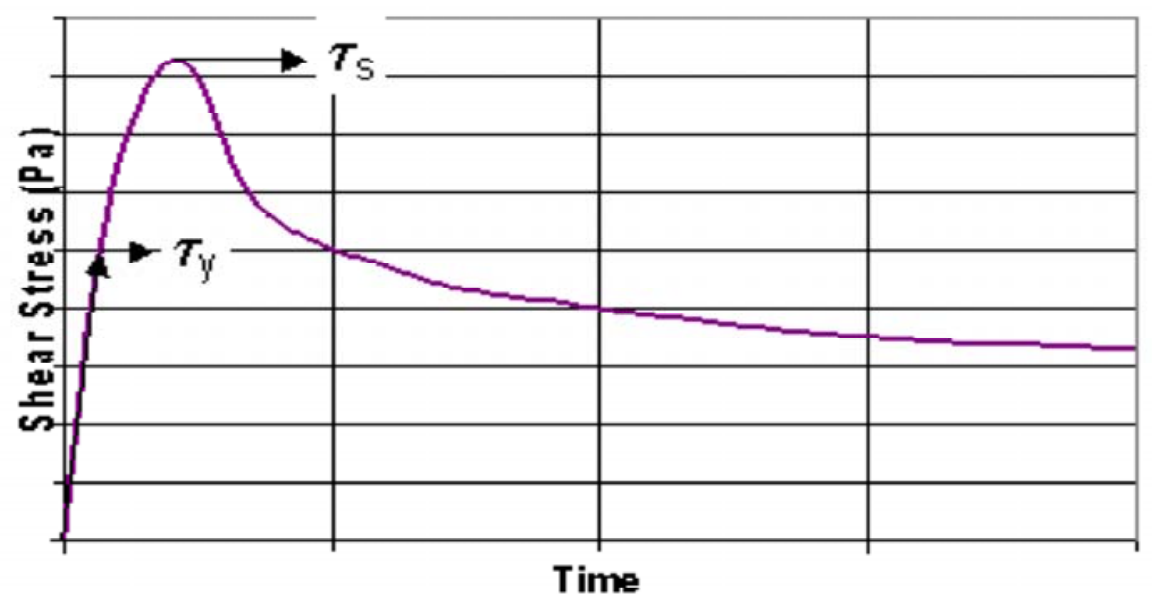

Figure 2. Typical Stress-Versus-Time Profile for a Shear Vane at Constant Shear Rate

\section{Sample Test Approach}

As shown in Table 1, the identified sample for shear strength was initially contained in a $240 \mathrm{~mL}$ Qorpak jar. The dimensions of this jar has been evaluated against PNNL technical procedure RPLCOLLOID-02, Rev. 2, to confirm that it meets the geometry requirements for a valid shear strength measurement; therefore shear strength measurements were performed in situ. Table 3 summarizes the matrix for the shear strength measurements. For the sample, up to 4 shear strength measurements were to be performed (depending on result reproducibility):

- One measurement on the "as received" sample where settling time is dependent on when the subsample was prepared and delivered to SAL for rheological measurements

- Two measurements at a consistent settling time ranging from 48 to $72 \mathrm{hrs}$

- A single replicate measurement of the two above was to be made should the second measurement of shear strength at $48-72 \mathrm{hrs}$ differ from the first measurement by $25 \%$ or greater.

The first measurement was performed on the as-received sample/as sampled material. A $6 \times 6 \mathrm{~mm}$ vane tool was implemented for the initial measurement, if the material strength was sufficiently low a second measurement may be possible. A larger, and hence more sensitive vane, would be implemented if the sample was undisturbed. Depending on the vane tool used for the remainder of the shear strength measurements, (determined after the initial measurement is performed) the sample size may only permit one sample measurement of the material at a point in time. After the initial measurement, the sample had been disturbed; therefore, following each measurement, the sample was remixed, and allowed to settle for a specified time, before the next measurement was taken.

As shown in Table 3, following the initial measurement, two measurements were performed on the sample after 48 to $72 \mathrm{hrs}$ of settling and under near identical conditions. These measurements were to be performed over a period of 6 to 9 days (to allow mixing/setting between each measurement). 
PNNL-20650, Rev. 2

Carolyn Burns

Page 6 of 8

July 20, 2011

Table 3. Test Matrix for Shear Strength Measurements

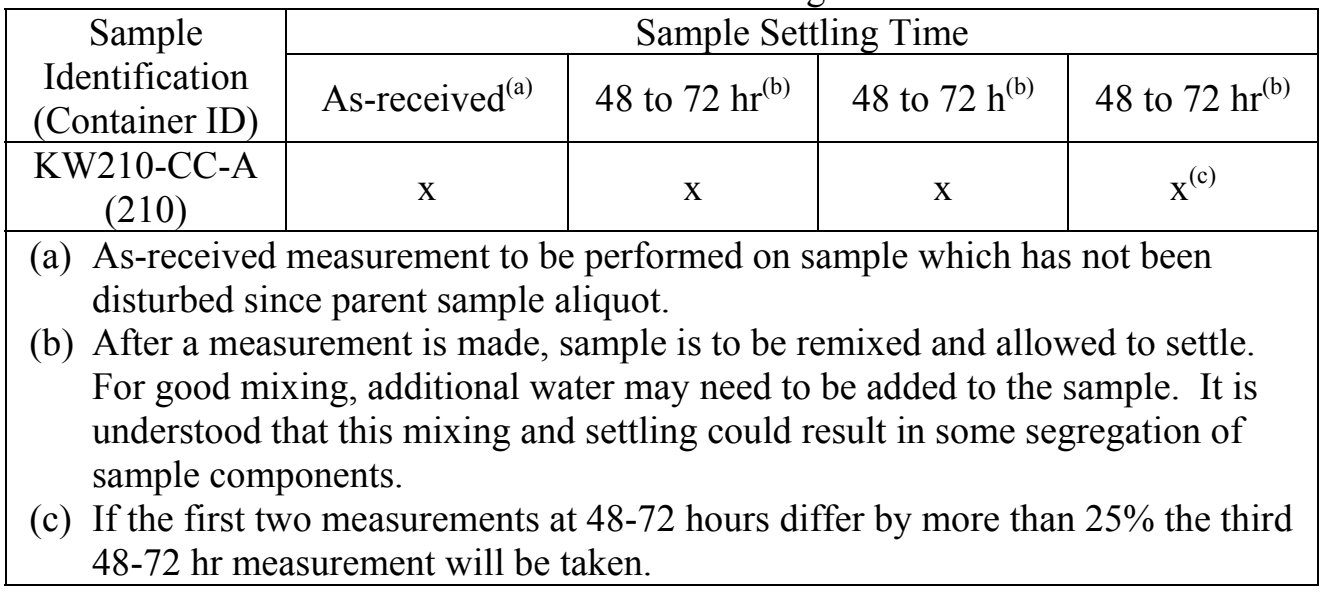

\section{Results}

Three shear strength measurements were performed on KW210-CC-A: one measurement of the "asreceived" settled solids strength and two measurements of the settled solids strength $\sim 48$-hrs after solids re-suspension. Shear strength measurements employed either a $6 \times 6 \mathrm{~mm}$ or a $16 \times 16 \mathrm{~mm}$ (diameter by height) vane tool. The sequence for the three shear strength measurements and mixing/resuspension techniques employed for sample KW210-CC-A are described in Table 4.

Table 4. Test sequence for shear strength measurements reported for sample KW210-CC-A.

\begin{tabular}{|c|c|c|c|}
\hline Sequence & Test & Description & Post-Test Mixing \\
\hline 1 & "As-Received" & $\begin{array}{l}\text { Shear strength measurement of } \\
\text { the "as-received" settled } \\
\text { solids. Approximately 2- } \\
\text { weeks of time between current } \\
\text { test and time of last solids } \\
\text { disruption. }\end{array}$ & $\begin{array}{l}\text { Sample homogenized and } \\
\text { transferred to a } 120 \mathrm{~mL} \\
\text { jar for subsequent } \\
\text { measurements. }\end{array}$ \\
\hline 2 & $\begin{array}{l}\text { First Mix, } \sim 48-\mathrm{hr} \\
\text { Settled }\end{array}$ & $\begin{array}{l}\text { Shear strength measurement } \\
\sim 48 \text {-hrs following post-test } \\
\text { mixing operations associated } \\
\text { with the "as-received" } \\
\text { measurements }\end{array}$ & $\begin{array}{l}\text { Mixed with a } 16 \times 16 \mathrm{~mm} \\
\text { vane impeller for } 2 \\
\text { minutes. Sample inverted } \\
\text { to inspect jar bottom for } \\
\text { complete mixing. }\end{array}$ \\
\hline 3 & $\begin{array}{l}\text { Second Mix, } \sim 48 \mathrm{hr} \\
\text { Settled }\end{array}$ & $\begin{array}{l}\text { Shear strength measurement } \\
48 \text {-hrs following post-test } \\
\text { mixing operations associated } \\
\text { with the first } 48 \text {-hr settled test. }\end{array}$ & $\begin{array}{l}\text { Mixed with a } 16 \times 16 \mathrm{~mm} \\
\text { vane impeller for } 2 \\
\text { minutes. Sample inverted } \\
\text { to inspect jar bottom for } \\
\text { complete mixing. }\end{array}$ \\
\hline
\end{tabular}


PNNL-20650, Rev. 2

Carolyn Burns

Page 7 of 8

July 20, 2011

Table 5. Settled solids and total slurry height inventories taken during testing associated with this data package.

\begin{tabular}{|c|c|c|c|c||}
\hline Sample ID & Test & $\begin{array}{c}\text { Settled Solids } \\
\text { Height, } \mathbf{c m}\end{array}$ & $\begin{array}{c}\text { Total Slurry } \\
\text { Height, } \mathbf{c m}\end{array}$ & $\begin{array}{c}\text { Temperature } \\
{ }^{\circ} \mathbf{C}\end{array}$ \\
\hline \multirow{3}{*}{ KW210-CC-A } & "As-received" & 3.2 & 7.5 & 27.3 \\
\cline { 2 - 5 } & First Mix & 4.7 & 5.8 & 29.5 \\
\cline { 2 - 5 } & Second Mix & 5.2 & 6 & 29.8 \\
\hline
\end{tabular}

Both before and during the course of the series of measurements outlined in Table 4, samples were monitored for loss of water. The total level of slurry (settled solids plus water) was adjusted as deemed necessary by the lead rheologist (Carolyn Burns) or cognizant scientist (Rick Shimskey). Water loss due to evaporation was offset in most cases by the DI water used to rinse down the vane tool after each measurement. Efforts were made to keep the total slurry volume of the sample constant, small volumes of water were used to wash down the vane tool after each measurement to minimize sample loss. Table 5 provides a summary of the sample volumes.

The results of shear strength testing are presented in Table 6. Sample test and mixing times are given in Table 7. All measurements were made at ambient cell temperature. "As-received" measurements corresponded to an overall settling time of $\sim 1824 \mathrm{hrs}(\sim 11$ weeks $)$; all other measurements corresponded to a settling period $\sim 48 \mathrm{hrs}$ following sample mixing.

The "as-received" sample, settling time of approximately 11 weeks, was measured using a $6 \times 6 \mathrm{~mm}$ vane tool to avoid exceeding the maximum operating torque of the RV20-M5 measuring system. This is consistent with guidance from CHPRC directing PNNL to use a $6 \times 6 \mathrm{~mm}$ vane tool (on October 11, 2010) for initial shear strength measurements. The initial strength of the sample was established to be within the working stress range of the $16 \times 16 \mathrm{~mm}$ vane and all subsequent measurements were made with this vane tool. The sample was transferred to a smaller container so that the $16 \times 16 \mathrm{~mm}$ vane tool could be used, as there was insufficient settled solids height to use this vane in the original container. Table 8 provides a summary of shear strength measurements. An average shear strength for sample KW210-CC-A is given; the average was determined using the first measurement from the first and second settle-and-mix operations. For each test, the vane was immersed into the center of jar at the indicated depth.

Table 6. Summary of shear strength measurement results.

\begin{tabular}{|c|c|c|c|c|c||}
\hline Sample ID & Test & $\begin{array}{c}\text { Vane Tool } \\
{[\mathbf{D} \times \mathbf{H}]} \\
\mathbf{m m}\end{array}$ & $\begin{array}{c}\text { Settling } \\
\text { Time, hrs }\end{array}$ & $\begin{array}{c}\text { Immersion } \\
\text { Depth, } \\
\mathbf{m m}\end{array}$ & $\begin{array}{c}\text { Shear } \\
\text { Strength, Pa }\end{array}$ \\
\hline \multirow{3}{*}{ KW210-CC-A } & "As-received" & $6 \times 6$ & 1824 & 6 & 281 \\
\cline { 2 - 6 } & First Mix & $16 \times 16$ & 50 & 16 & 63 \\
\cline { 2 - 5 } & Second Mix & $16 \times 16$ & \multirow{2}{*}{47} & 16 & 58 \\
\cline { 2 - 3 } & Second Mix & $16 \times 16$ & 26 & 113 \\
\hline
\end{tabular}

Table 7. Summary of Test and Mixing Times

\begin{tabular}{||c|c|c|c|c||}
\hline Sample ID & Test & Last Mixed & Sample Test & $\begin{array}{c}\text { Estimated Settling } \\
\text { Time, hrs }\end{array}$ \\
\hline \multirow{3}{*}{ KW210-CC-A } & "As-received" & $2011 / 03 / 1715: 19$ & $2011 / 06 / 0115: 00$ & 1824 \\
\cline { 2 - 5 } & First Mix & $2011 / 06 / 1015: 00$ & $2011 / 06 / 1216: 48$ & 52 \\
\cline { 2 - 5 } & Second Mix & $2011 / 06 / 1216: 55$ & $2011 / 06 / 1415: 38$ & 47 \\
\hline
\end{tabular}


Table 8. Shear Strength Summary Table for KW210-CC-A All measurements were taken at ambient cell temperature $\left(\sim 29^{\circ} \mathrm{C}\right)$

\begin{tabular}{|c|c|c|c|c|}
\hline Sample ID & Measurement & $\begin{array}{l}\text { Settling } \\
\text { Time (hr) }\end{array}$ & $\begin{array}{c}\text { Immersion } \\
\text { Depth (mm) }\end{array}$ & $\begin{array}{c}\text { Shear Strength } \\
(\mathrm{Pa})\end{array}$ \\
\hline \multirow{5}{*}{$\begin{array}{l}\text { KW210-CC-A } \\
\text { (KW210-SCC) }\end{array}$} & As-Received ${ }^{(\mathrm{c})}$ & 1824 & 6 & \multirow{5}{*}{$\begin{array}{c}\sim 281 \\
63^{(\mathrm{a})} \\
58^{(\mathrm{a})} \\
113 \\
\mathbf{6 0} \pm \mathbf{5}^{(\mathbf{b})}\end{array}$} \\
\hline & $1^{\text {st }} \mathrm{Mix}$ & 50 & 16 & \\
\hline & $2^{\text {nd }}$ Mix & 47 & 16 & \\
\hline & \multirow{2}{*}{$\begin{array}{c}2^{\text {nd }} \text { Mix } \\
\text { Average }\end{array}$} & 47 & 26 & \\
\hline & & & & \\
\hline
\end{tabular}

(a) Used in average calculation.

(b) Reported uncertainty is twice the standard error of the mean (which approximated the lower bound of the $95 \%$ confidence limits).

(c) Initial "as-received" strength measurement using a $6 \times 6 \mathrm{~mm}$ vane. Subsequent measurements taken with the $16 \times 16 \mathrm{~mm}$ vane.

\section{References}

HNF-SP-1201: Makenas, BJ, TL Welsh, RB Baker, GR Golcar, PR Bredt, AJ Schmidt and JM Tingey. 1997. Analysis of Sludge From Hanford K East Basin Canisters, HNF-SP-1201, Westinghouse Hanford Company, Richland, Washington.

PNNL-18408, Rev. 1: Burns, CA; M Luna, AJ Schmidt, 2011, Characterization of Settler Tank, KW Container and KE Container Sludge Simulants, PNNL-18408, Rev. 1., Pacific Northwest National Laboratory, Richland, Washington.

PNNL-19035: Fiskum SK, JM Billing, SJ Bos, CA Burns, CD Carlson, DS Coffey, JV Crum, RC Daniel, CH Delegard, MK Edwards, OT Farmer, LR Greenwood, SA Jones, D Neiner, BM Oliver, KN Pool, AJ Schmidt, RW Shimskey, CZ Soderquist, CJ Thompson, ML Thomas, T Trang-Le, and MW Urie. 2009. Characterization Data Package for Containerized Sludge Samples collected from Engineered Containers SCS-CON-240, 250, 260, and 220. PNNL-19035, Rev. 1, Pacific Northwest National Laboratory, Richland, Washington. 


\section{8-TI039-Rheology Data Package - Coverpage}

Description:

This Excel file data package documents measurement results (using Microsoft ${ }^{*}$ Office Excel) for rheology data collected under Test Instruction (Tl) 52578-TI039, Rheological Measurements for $\mathrm{K}$ West Basin Consolidated Container Composites SCS-CON-210. Results for sample KW210-CC-A are given.

Computer Employed:

MHP Dell Latitude D830 Laptop Computer (Property \# WE14110)

Operating Environment:

Microsoft Windows 7 Enterprise

Base Software Program:

Microsoft Office Professiona! Plus 2010 Excel Version 14.0.5128.5000 (32-bit)

Package Contents:

Package consists of this coverpage, a calculation review page, print-outs for one Microsoft ${ }^{\circ}$ office Excel workbook, and all other relevant documentation.

\begin{tabular}{|c|c|c|c|}
\hline Description & File Date & File Size & Number of Pages \\
\hline Data package coverpage & $\mathrm{n} / \mathrm{a}$ & $\mathrm{n} / \mathrm{a}$ & 1 \\
\hline Calculation review worksheet & $\mathrm{n} / \mathrm{a}$ & $\mathrm{n} / \mathrm{a}$ & 1 \\
\hline Excel printouts: "Tl-039 - 001 (Reviewed).xlsm" & $8 / 3 / 201111: 45$ & $45 \mathrm{~KB}$ & 5 \\
\hline Standard certificate: "Certificate 092710 (332587).pdf" & $5 / 13 / 201113: 20$ & $578 \mathrm{~KB}$ & 1 \\
\hline K-Basin Non-Conformance Report - NCR S2578-11-08-10 & $5 / 13 / 201113: 22$ & $853 \mathrm{~KB}$ & 2 \\
\hline K-Basin Surveillance Report - SR-52578-2011-035 & $n / a$ & $n / a$ & 5 \\
\hline
\end{tabular}

Package Generator Signature:

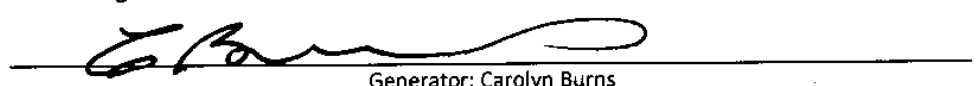




\section{Calculation Review Sheet}

\begin{tabular}{|c|c|}
\hline Reviewer Name: & Richard C. Daniel \\
\hline Reviewer Title: & S/E \\
\hline Review Date: & $7 / 26 / 2011$ \\
\hline \multirow{3}{*}{$\begin{array}{l}\text { Title of Spreadsheet } \\
\text { Calculation Reviewed: }\end{array}$} & One spreadsheet and a calibration certificate are included in this review package: \\
\hline & 1) "T1-039-001 (Reviewed).x1sm" \\
\hline & 2) "Certificate 092710 (332587).pdf" \\
\hline Revision Number: & $\mathrm{n} / \mathrm{a}$ \\
\hline Date Prepared: & $7 / 26 / 2011$ \\
\hline
\end{tabular}

Scope of Spreadsheet Review: (Check one or more of the following)

X General Validation Review: (General review \& spot checks)

Review of updated spreadsheet/calc (Revised portion only)

$100 \%$ Verification Review (Verification of all cells/calculations)

Independent calculation check (With hand calcs or independent spreadsheet)

Other:

CHECK UST

Spreadsheet/Caiculation Identification

Spreadsheet/Calculation Title:

Revision Number:

Date Prepared

Prepared by:

General Statement of Purpose:

General Description of Approach (note: specific

approaches may be described for each

section/subsection of spreadsheet/calculation)

Comments: $\quad$ Standard rheology spreadsheet, approach per RPL-COLLOID-02, Rev. 2

Assumptions

Are assumptions clearly stated?

Are assumptions supported/justified?

Are assumptions reasonable?

\begin{tabular}{|c|c|c|}
\multicolumn{1}{c}{ Yes } & No & NA \\
\hline & & $X$ \\
\hline$X$ & & \\
\hline$X$ & & \\
\hline$X$ & & \\
\hline & & \\
& & \\
& & \\
\hline
\end{tabular}

Comments: No comments

Input Values

Are input parameters correct (verified with source?)

Are parameter units consistent?

Are input values properly referenced?

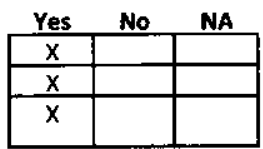

Comments: No comments

Equations/Approach

Are equations adequately defined?

Are equations properly referenced?

Are limitations of approach/equations identified?

Are equations appropriate?

Are units consistent?

\begin{tabular}{|c|l|l|} 
Yes & No & NA \\
\hline$X$ & & \\
\hline$X$ & & \\
\hline$X$ & & \\
\hline
\end{tabular}

Comments:

No comments

Results/Conclusions

Are formulas consistent in spreadsheet cells?

Are calculations correct?

Are conclusions consistent with results?

Are conclusions consistent with applicable limits?

Comments:

Page 1 of 1 
PNNL-20650, Rev. 2

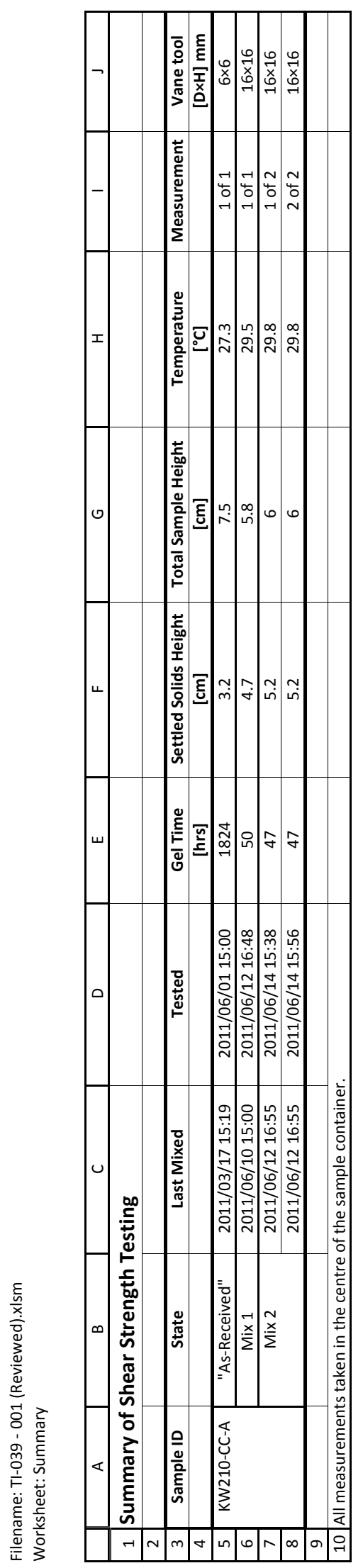


PNNL-20650, Rev. 2

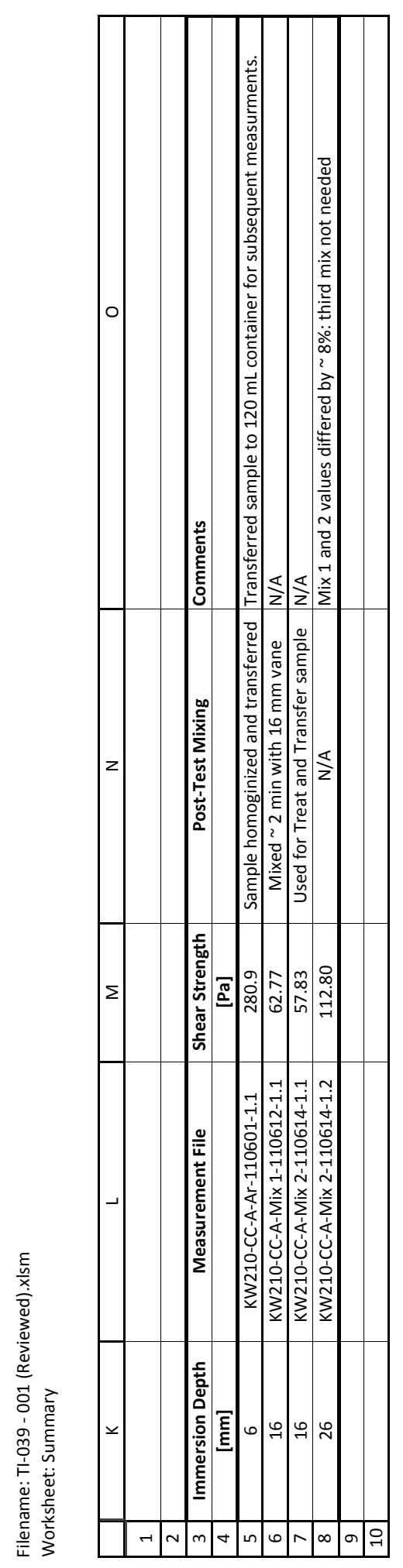

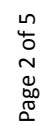

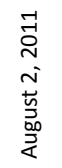


PNNL-20650, Rev. 2

Filename: TI-039 - 001 (Reviewed).xlsm

Worksheet: Shear Strength Table

\begin{tabular}{|c|c|c|c|c|c|c|c|}
\hline & A & $\mathrm{B}$ & $\mathrm{C}$ & $\mathrm{D}$ & $\mathrm{E}$ & $\mathrm{F}$ & G \\
\hline 1 & \multicolumn{4}{|c|}{ Shear Strength Summary Table (For Report) } & & & \\
\hline 2 & & & & & & & \\
\hline 3 & Sample ID & Test & Temperature & $\begin{array}{c}\text { Settling } \\
\text { Time }\end{array}$ & Location & $\begin{array}{c}\text { Immersion } \\
\text { Depth }\end{array}$ & Shear Strength \\
\hline 4 & & & $\left({ }^{\circ} \mathrm{C}\right)$ & (hr) & & $(\mathrm{mm})$ & (Pa) \\
\hline 5 & \multirow{4}{*}{ KW210-CC-A } & As received & 27.3 & 1824 & center & 6 & 280.9 \\
\hline 6 & & Mix 1 & 29.5 & 50 & center & 16 & 63 \\
\hline 7 & & Mix 2 & 29.8 & 47 & center & 16 & 58 \\
\hline 8 & & Mix 2 & 29.8 & 47 & center & 26 & 113 \\
\hline 9 & & & & & & & \\
\hline 10 & \multicolumn{4}{|c|}{ Average Shear Strength for KW210-CC-A at $48 \mathrm{hrs}$ Settling Time } & & & \\
\hline 11 & \multicolumn{3}{|c|}{ Based on $16 \mathrm{~mm}$ measurements (Mix 1 and 2) } & & & & \\
\hline 12 & Average & 60.3 & $\mathrm{~Pa}$ & & & & \\
\hline 13 & Standard Deviation & 3.5 & $\mathrm{~Pa}$ & & & & \\
\hline 14 & Standard Error & 2.5 & $\mathrm{~Pa}$ & & & & \\
\hline 15 & Formatted Result & $60 \pm 5$ & $\mathrm{~Pa}$ & & & & \\
\hline
\end{tabular}


PNNL-20650, Rev. 2

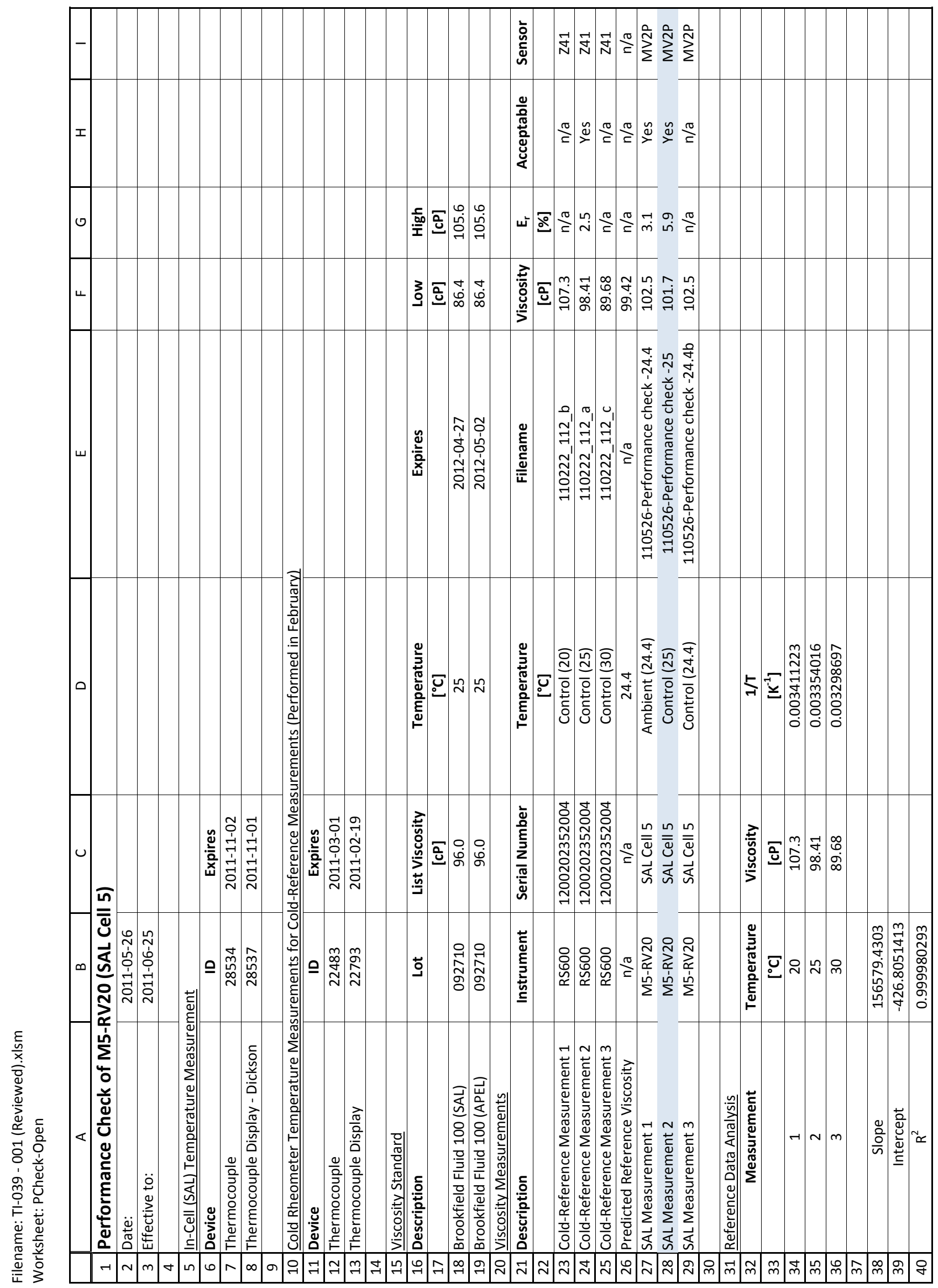

$n$
4
0
0
0
0
0
0
0 
PNNL-20650, Rev. 2

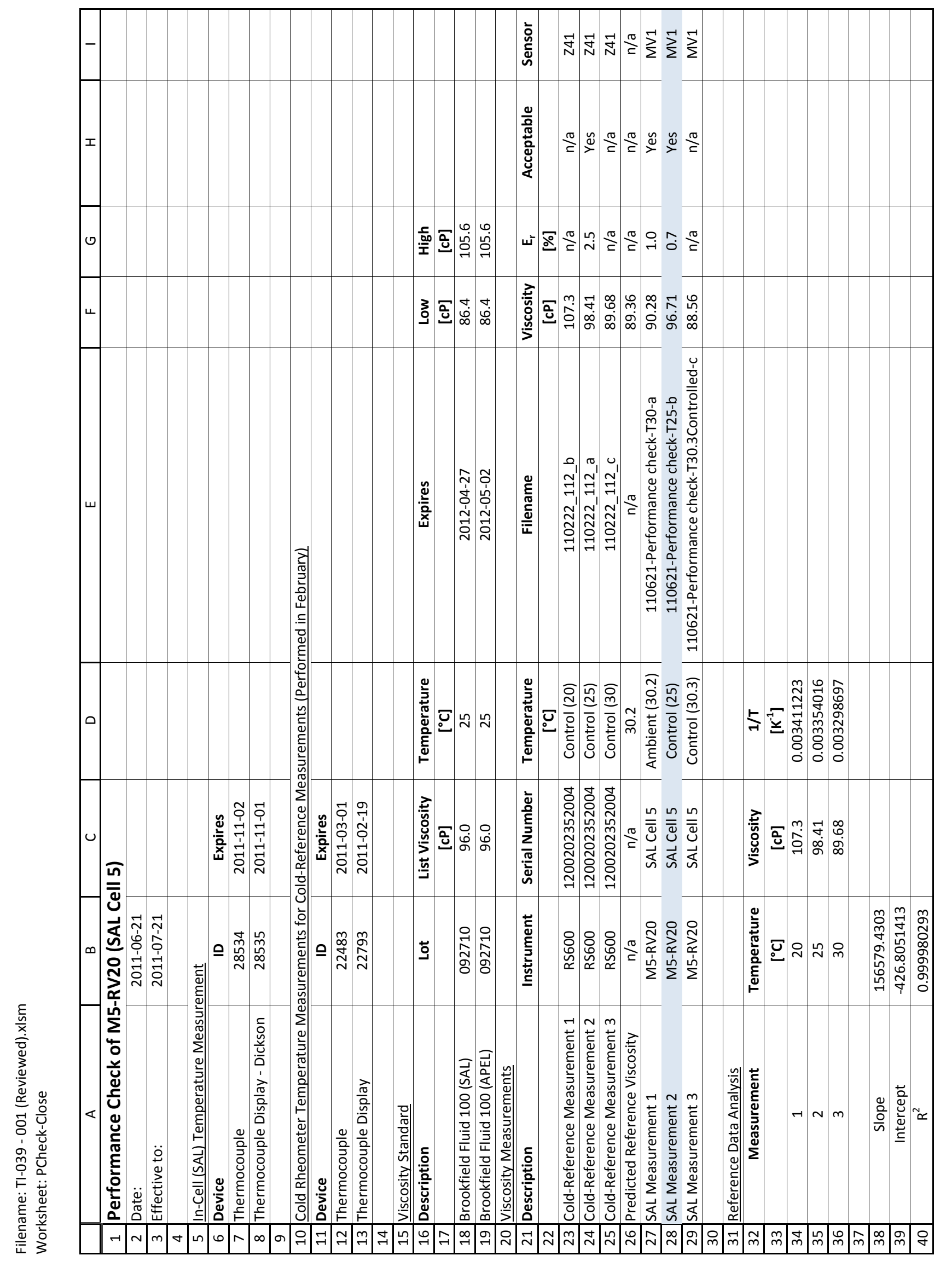


PNNL-20650, Rev. 2

ISO 9001 CERTIFIED Po \pm 136236 BC\#332587

CERTIFICATE OF VISCOSITY STANDARD CALIBRATION

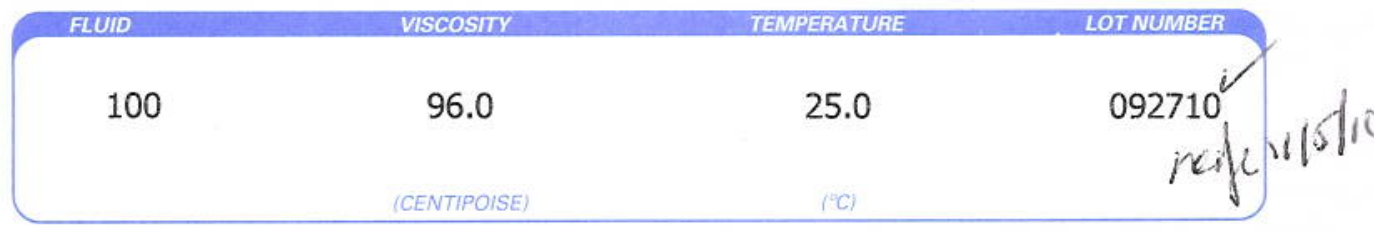

TEST INSTRUMENT

MODEL

LVDV-II+

MODEL

1529

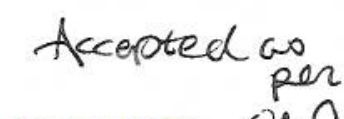

SERIAL NUMBER 6529213

188 dsceftery

SERIAL NUMBER $12-2-10$ A33422 NCR 52578 CALIBRATION OF THE ABOVE-MENTIONED BROOKFIELD VISCOSITY STANDARD IS ACCURATE TO WITHIN $1 \%$ OFITS STATED VALUA

THE VISCOSITY STANDARD OF THIS LOT NUMBER WAS MEASURED WITH A BROOKFIELD VISCOMETER THAT HAS BEEN CALIBRATED AGAINST A PRIMARY STANDARD, TRACEABLE TO THE NATIONAL INSTITJTE OF STANDARDS \& TECHNOLOGY.

use-as-is

BROOKFIELD ENGINEERING LABORATORIES, INC.

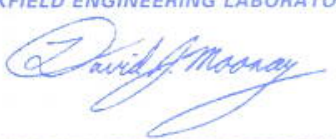

BROOKFIELD ENGINEERING LABORATORIES, INC.

11 Commerce Blvd., Middleboro, MA 02346-1031 U.S.A.

TEL. 800-628-8139 or 508-946-6200 FAX 508-946-6262

www.brookfieldengineering.com 


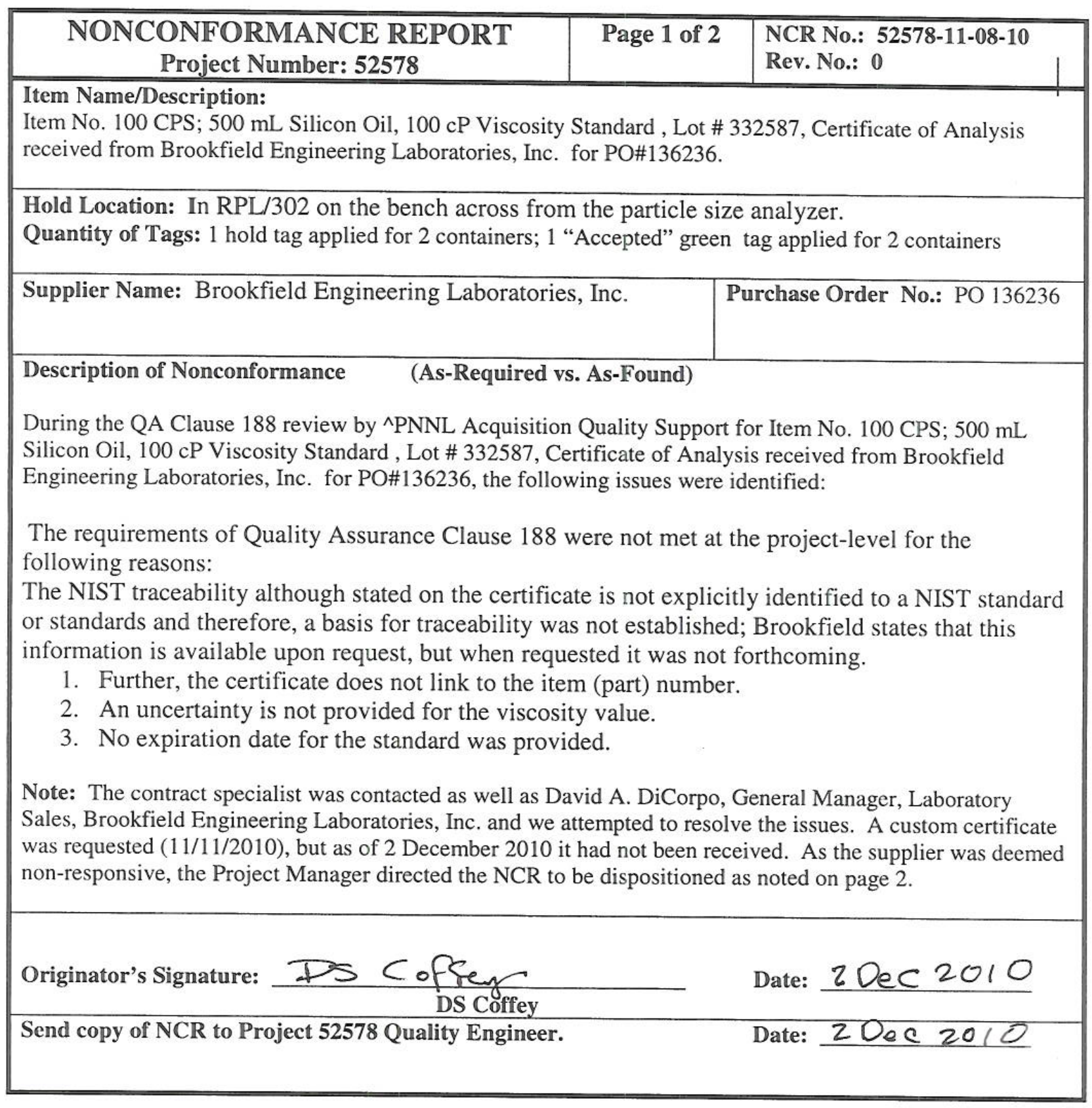




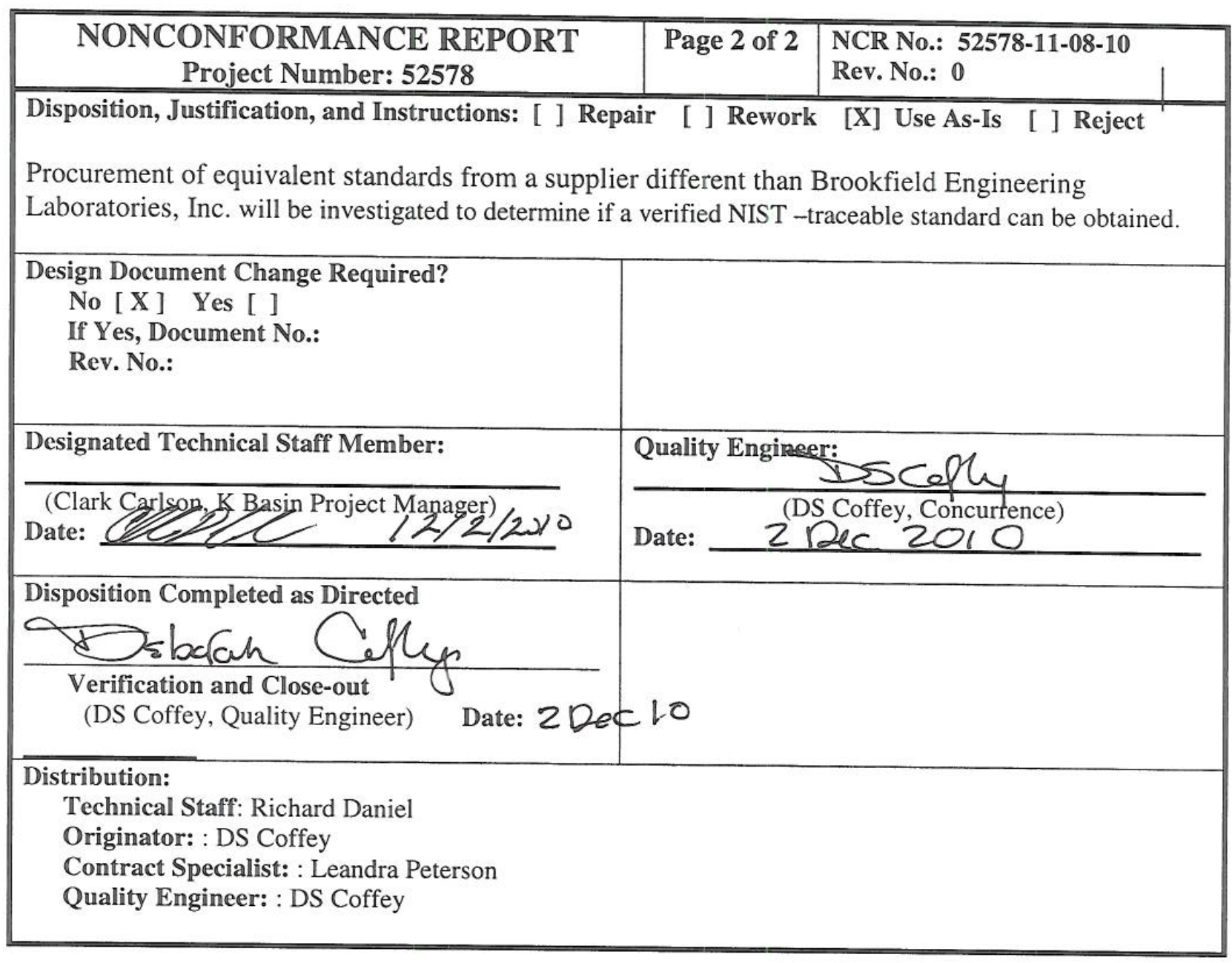




\section{PLANNING}

Topic/Issue to be evaluated: Surveillance of Completed K Basin TI, 52578-TI039, Rev. 0, Test Instruction for Rheological Measurements for $K$ West Basin Consolidated Container Composite SCS-CON-210.

This surveillance was undertaken to evaluate completed test instruction (TI), 52578-TI039, Test Instruction for Rheological Measurements for $K$ West Basin Consolidated Container Composite $S C S-C O N-210$, associated supporting information and spreadsheets, and the completed report, Rheological Measurements for $K$ West Basin Consolidated Container Composites SCS-CON210. The report was reviewed concurrently with TI039, but the report review will be documented via a Document Review Record (DRR) form. TI039 was used between 5/26/2011 and 7/26/2011. TI039 directs the performance of rheological measures for one sample using procedure, RPL-COLLOID-02, Rev. 2, Measurement of Physical and Rheological Properties of Solutions Slurries and Sludges. Carolyn Burns signed off TI steps. Richard Daniel performed a Technical Review of the TI.

\section{PERFORMANCE}

\begin{tabular}{|l|l|}
\hline $\begin{array}{l}\text { Date Initiated: 2 February 2011 } \\
\text { Date Completed: 26July 2011 }\end{array}$ & Location: RPL \\
\hline $\begin{array}{l}\text { Date Received: 27 July 2011 } \\
\text { Date Reviewed: 28 July 2011 }\end{array}$ & $\begin{array}{l}\text { DRAFT Report Issued: 29 July 2011 } \\
\text { FINAL Report Issued: 2 August 2011 }\end{array}$ \\
\hline $\begin{array}{l}\text { Contacts: Carolyn Burns, Clark Carlson, } \\
\text { Richard Daniel and Sandy Fiskum }\end{array}$ & Org Code: PE 137, D9H63 \\
\hline Surveillance Team: Lisa Middleton & \\
\hline
\end{tabular}

\section{Source of Requirement(s):}

- KBC-33786, Rev. 2, Quality Assurance Project Plan/Sampling and Analysis Plan (QAPjP/SAP): Quality Assurance Project Plan/Sampling and Analysis Plan for Containerized KW Settler Sludge; December 2009.

- CH2M Hill Plateau Remediation, Contract 41108, Statement of Work, Technical Support for Planning, Preparing, and Analyzing KW Settler Sludge (February 11, 2010).

- HNF-36985, Rev. 3, Data Quality Objectives for Sampling and Analysis of K Basin Sludge; July 2009

Other Applicable Documents:

- RPL-OP-001, Rev. 9, Routine Research Operations

- ADM-RSEG-BALANCES, Rev. 0, Balance Calibration Verification 
Number: SR-52578-2011-035

Page 2 of 5

\section{Purpose/Scope:}

The 52578 Project QE staff are required via the TI to perform a review of completed TIs. The document was reviewed to evaluate completeness, that all entries were recorded properly, the errors were corrected appropriately and when needed, that justification was present to describe data corrections, that any issues affecting data quality are documented as Occurrence Reports and that the attachments to the TI were at the stage to become a project record. Additionally, TI's are reviewed to assure that the technical reviews were performed and issues were dispositioned and that data are traceable to point of use, generally Excel spreadsheets, which also require technical review and comment/issue resolution. When issues were found, the cognizant staff were contacted to resolve issues prior to completion of the review.

\section{Approach:}

TI, 52578-TI039, required the rheology analysis of one sample as noted below:

\begin{tabular}{|c|c|c|c|}
\hline Sample ID & Sample Purpose. & Vessel Description & $\begin{array}{c}\text { Approximate Sample Size, } \\
\text { wet sludge basis }\end{array}$ \\
\hline \multicolumn{3}{|c|}{ Container Composite KW210, Subsample Collected under Test Instruction $52578-$ TI 008 } \\
\hline KW210-CC-A & Shear Strength & $240 \mathrm{~mL}$ glass Qorpak & $\sim 180 \mathrm{~mL}$ \\
\hline
\end{tabular}

The TI package submitted for review consisted of the following:

1. Completed TI, 52578-TI039, Rev. 0, Test Instruction for Rheological Measurements for K West Basin Consolidation Container Composite SCS-CON-210 (pp. 1-21) With the following inserted: Performance checks dated 5/26/11 \& 6/21/11 \& Flow curves/ rheograms dated 6/1/11, 6/12/11 \& $6 / 14 / 11$

2. Brookfield certificate of viscosity standard calibration (lot \# 092710)

3. Nonconformance Report - NCR 52578-11-08-10, related to QA Clause 188 review by $\wedge$ PNNL Acquisition Quality Support for Item No. 100 CPS; $500 \mathrm{~mL}$ Silicon Oil, $100 \mathrm{cP}$ Viscosity Standard, Lot \# 332587, Certificate of Analysis received from Brookfield Engineering Laboratories, Inc. for PO\#136236, the following issues were identified:

- Basis for NIST traceability was not demonstrated.

- Further, the certificate does not link to the item (part) number.

- An uncertainty was not provided for the viscosity value.

- No expiration date for the standard was provided.

Disposition was to use as-is, and identify for a different vendor for this standard

4. M\&TE including BPCL forms for Ohaus balance AV4101C (8031221019): 6/14/11 through 7/8/11\& Sartorius balance MSG234 (40023610/WD68444): 7/6/11 \&7/7/11 IS\&T Records of Calibration: duel element temperature display (28535) \& thermocouple (28534))

5. Calculation Review Sheet signed by Technical Reviewer Richard Daniel,7/26/11 for TI039-001 (Reviewed).xlsm

6. Spreadsheet named TI-039-001 (Reviewed).xlsm with the following worksheets:

- Summary

- Shear Strength Table

- PCheck-Open 
Number: SR-52578-2011-035

Page 3 of 5

- PCheck-Close

7. DRR for completed TI039 \& report Rheological Measurements for K West Basin Consolidated Container Composites SCS-CON-210, signed by Richard Daniel 7/27/11

\section{M\&E Used:}

\begin{tabular}{|l|c|c|c|c|}
\hline \multicolumn{1}{|c|}{ M\&TE } & Description & ID \#/Serial Number & Cal. Exp. & Dates Used \\
\hline Balance & Ohaus AV4101C & 8031221019 & $2 / 12$ & $6 / 10 / 11$ \\
\hline Balance & Sartorius MSG234 & $40023610 /$ WD68444 & $8 / 11$ & $6 / 10 / 11$ \\
\hline Thermocouple & $\begin{array}{c}\text { KMQSS-062U-6/Omega } \\
\text { type K }\end{array}$ & 28534 & $11 / 2 / 11$ & $6 / 1 / 11,6 / 12 / 11 \& 6 / 14 / 11$ \\
\hline $\begin{array}{l}\text { Dual Element } \\
\text { Temperature } \\
\text { Display }\end{array}$ & FT350/Dickson & $10217201 / 28535$ & $11 / 1 / 11$ & $6 / 1 / 11,6 / 12 / 11 \& 6 / 14 / 11$ \\
\hline
\end{tabular}

\section{Procedure Used:}

RPL-COLLOID-02, Rev. 2, Measurement of Physical and Rheological Properties of Solutions Slurries and Sludges

\section{RESULTS}

Findings are defined as a statement of fact relating to noncompliance with previously agreed-upon procedures, plans, codes, standards, specifications, or other forms of contractual or legal obligation. It should be understood that any lack of a finding in a specific area is not considered an indication that deficiencies do not exist.

Observations are defined as a weakness that if not corrected, could yield a departure from a requirement although the weakness is not necessarily a departure from requirements.

Note: All concerns identified in the review were addressed in a comment resolution meeting on 2 August 2011. Lisa Middleton, Deborah Coffey, Richard Daniel and Carolyn Burns attended.

Before the completed TI was signed off for QE review, it was determined that all comments/concerns were addressed. There were no outstanding identified findings or observations.

It was noted that there is an entry in the TI, step 3.1, dated before the TI was signed off for approval. The step, a performance check, was performed early due to the urgency of completing the work.

Issues of concern are summarized below:

1. Page 10, Table 6: Need to get initials \& signature from Richard Daniel. Completed, no further requirements. Closed.

2. Page 11, ID 1: Add balance model \# for Balance 1 (AV4101C). Added, no further action required. Closed 
3. Add page \#s to the attachments following page 17 (17A, 17B, 17C \&17 D). Completed, no further requirements. Closed

4. Page 19: Closing date is listed as $02 / 22 / 11$; however, analysis was done on $6 / 1 / 11,6 / 12 / 11 \&$ $6 / 14 / 11$. This doesn't make sense. The date of $2 / 22 / 11$ refers to when performance testing was done at APEL using the cold system, not in SAL. Opening was performed in SAL on 6/1/11; closing on 6/22/11. No further action required. Closed.

5. Add page \#s to the attachments following page 19 (19A, 19B, 19C 19D \& 19E). Completed, no further requirements. Closed

6. Page 21: A BPCL was not provided for balance \#2 (Sartorius MSG234 40023610/WD68444) used on 6/10/11. A completed BPCL was obtained for that time frame; however, a performance check was not performed on 6/10/11. This balance was actually used on 6/7/11. An acceptable performance check was performed. No further action required. Closed.

7. Page 21: A BPCL was not provided for balance \#1 (Ohaus AV4101C 8031221019) used on $6 / 10 / 11$. A completed BPCL was obtained for that time frame; however, a performance check was not performed on 6/10/11. This balance was actually used on 6/1/11. An acceptable performance check was performed. No further action required. Closed.

8. Page 21: The entry for "Balance ID" doesn't make sense. It looks to me like "12\#2". Should it be "\#1 \& \#2"? It should be \#1 \&\#2. Corrected, no further action required. Closed.

9. Excel file TI039-001 (Reviewed), Summary Tab, cell C6: Incorrect time listed. Should be 15:00 not 13:00. See page 17 of TI. Corrected, no further action required. Closed.

10. Excel file TI039-001 (Reviewed), PCheck-Open Tab: Thermocouple Display ID \& expiration date listed differs from the M\&TE listed in TI. Shouldn't it be 28535 exp. 11/1/11 instead of 28537 exp. 11/2/11? Corrected, no further action required. Closed.

11. Excel file TI039-001 (Reviewed), PCheck-Open Tab: Expiration date of viscosity standard listed differs from TI. TI says 4/27/12; spreadsheet says 2/5/12? There are 2 different standards, one for SAL \& one for APEL. Spreadsheet was modified to show the dates of the 2 different standards (4/27/12 \& 5/2/12). No further action required. Closed.

12. Excel file TI039-001 (Reviewed), PCheck-Close Tab: Thermocouple Display expiration date listed differs from the M\&TE listed in TI. Shouldn't it be 11/1/11 instead of 11/2/11? Corrected, no further action required. Closed.

13. Excel file TI039-001 (Reviewed), PCheck-Close Tab: Expiration date of viscosity standard listed differs from TI. TI says 4/27/12; spreadsheet says $2 / 5 / 12$ ? There are 2 different standards, one for SAL \& one for APEL. Spreadsheet was modified to show the dates of the 2 different standards $(4 / 27 / 12 \& 5 / 2 / 12)$. No further action required. Closed. 
Number: SR-52578-2011-035

Page 5 of 5

\section{CORRECTIVE ACTION}

[X] None Required. [ ] Complete [ ] Follow-up Corrective Action

Surveillance Performed By:
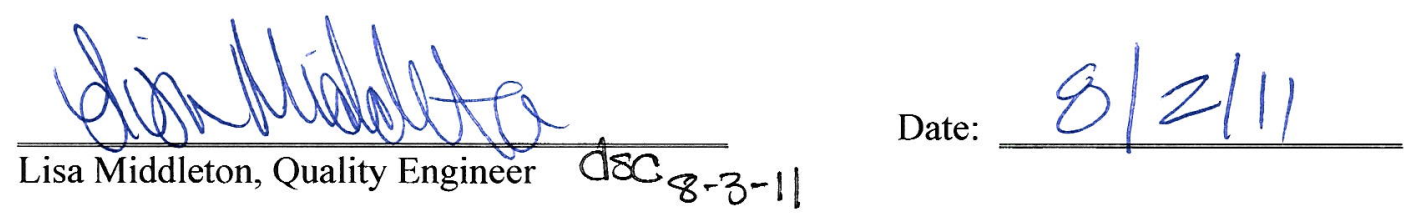

DISTRIBUTION: Carolyn Burns, Clark Carlson, Deborah Coffey, Richard Daniel, Sandy Fiskum, Matt Fountain, Rick Shimskey, and Andy Schmidt 

PNNL-20650, Rev. 2

\section{Appendix I}

\section{Particle Size Distribution}





\section{Appendix I}

\section{Particle Size Distribution}

Appendix I contains the particle size distribution characterization report for the KW210 container composite $-500 \mu \mathrm{m}$ size fraction samples and supporting documentation and calculations. The supporting documentation includes data manipulation, calculations, graphing sheets, performance verification standard certificates, and Surveillance Reports.

The following table of contents shows that pagination specific to Appendix I was applied to the particle size distribution characterization report and supporting attachments. This pagination is sequential and consistent (e.g., page I.\#).

\section{Appendix I Table of Contents}

Particle Size Distribution Report (KW210 -500 $\mu \mathrm{m}$ Sieve Fraction) ...................................................

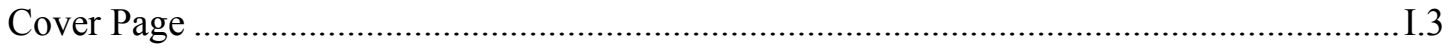

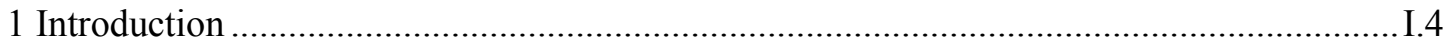

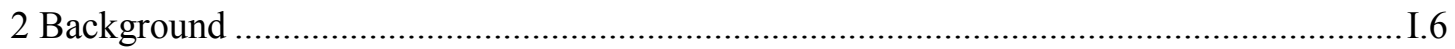

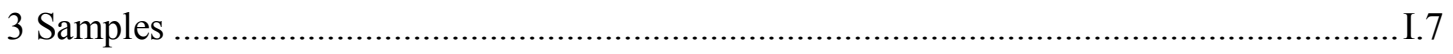

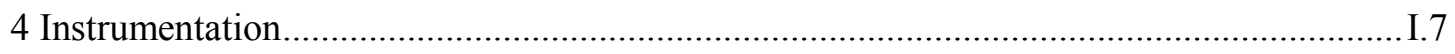

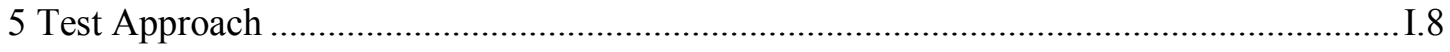

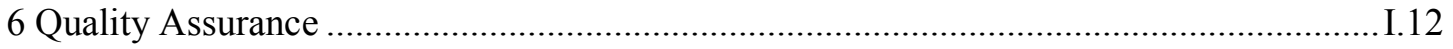

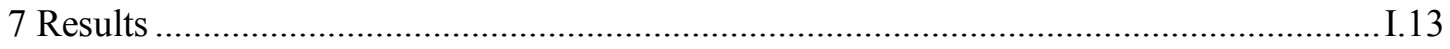

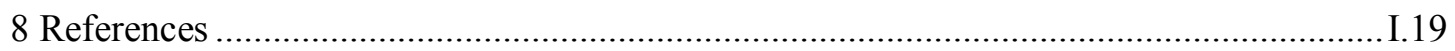

Particle Size Distribution Data Package (KW210 -500 $\mu \mathrm{m}$ Sieve Fraction) ......................................20

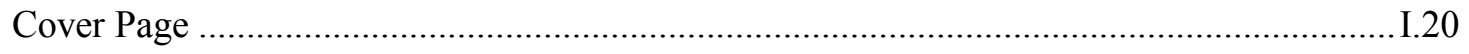

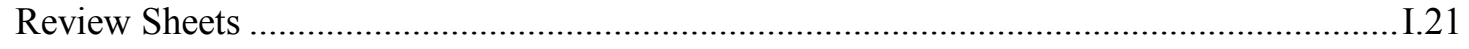

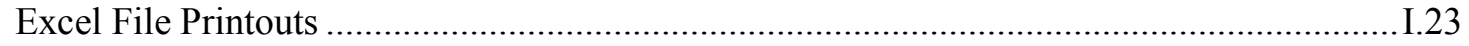

Performance Check Standard Certificate of Analysis ......................................................... 73

Surveillance Report SR-52578-2011-030 Rev. 0 ................................................................ 75

Surveillance Report SR-52578-2011-030 Rev. 1 ..............................................................78 



\section{Battelle-Pacific Northwest National Laboratory Radiochemical Sciences and Engineering Group \\ PO Box 999, Richland, Washington 99352}

Project: \#52578

Particle Size Analysis Performed Under Test Instruction 52578-TI-037, Particle Size Distribution Measurements for $K$ West Basin Consolidated Container Composite SCS-CON-210, Using Minus 500 Micron Particle Size Fraction Samples

Sample ID's: TI008-SP and TI008-SQ

Procedure: RPL-COLLOID-01, Rev. 1, Particle Size Analysis Using Malvern MS2000

Analyst: Carolyn Burns

Analysis Date: 06/15/2011

Instrument: Malvern Mastersizer 2000, SN\# MAL 100406, Hydro $\mu$ P Dispersion Unit, RPL Room 302

Supporting Electronic Report File Name: “52578-TI037 (Reviewed).xlsm"

File location: SharePoint site under TI-037 folder
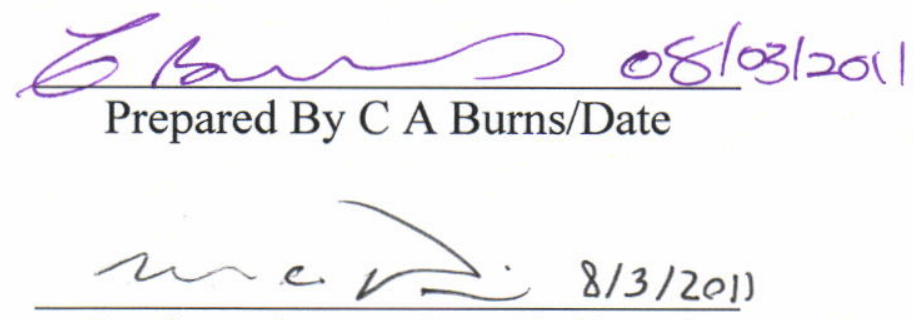

Reviewed By R C Daniel/Date

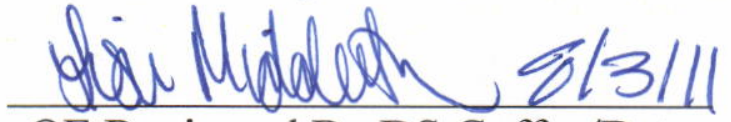

QE Reviewed By DS Coffee/Date dsc $8-3-11$

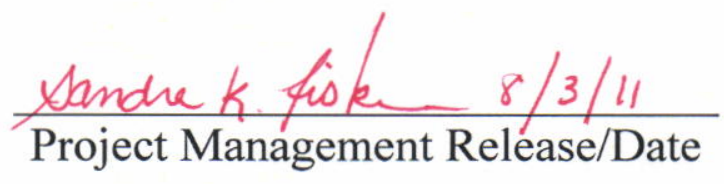


PNNL-20650, Rev. 2

\section{Introduction}

The sludge from the K Basin Pits and Floors (referred to as Container Sludge) has been consolidated into five engineered containers located under water in the K-West (KW) Basin. The container sludge originating from the K-East (KE) Basin resides in containers SCS-CON-240, -CON-250, and -CON260. The container sludge originating from the KW Basin resides in SCS-CON-210 and -CON-220.

Two to four top-to-bottom core samples have been collected from each container and shipped to RPL. Previous TIs have been implemented to reconstitute the sludge in a given core into a core composite sample. Sub-samples from each core composite within one Engineered Container have been consolidated into a single sample in an effort to represent the entire container (called a Container Composite). The Container Composite has been wet-sieved into +2000 micron, $-2000 /+500$ micron, and -500 micron particle size fractions. Subsamples of the -500 micron fraction have been collected to support particle size distribution (PSD) characterization of this material. The subsamples submitted for particle size analysis were suspended in DI water and removed from the hot cell. In 2009, all of the samples were analyzed with the exception of SCS-CON-210.

This data package summarizes the test approach and results of particle size analysis performed under Test Instruction 52578-TI-037, Particle Size Distribution Measurements for K West Basin Consolidated Container Composite SCS-CON-210, Using Minus 500 Micron Particle Size Fraction Samples. Particle size analyses were performed on a primary sample (Sample ID TI008-SP) and a duplicate sample (Sample ID TI008-SQ) from the KW210 container composite generated under 52578-TI008. Figure 1 shows the complete scope of characterization work being conducted on the Container Composite samples. The extent of this data package encompasses the analysis of PSD for the -500 micron sieve fraction of K-Basin sludge from Container Composite sample KW210. The work outlined in this data package is shaded in green in Figure 1. 

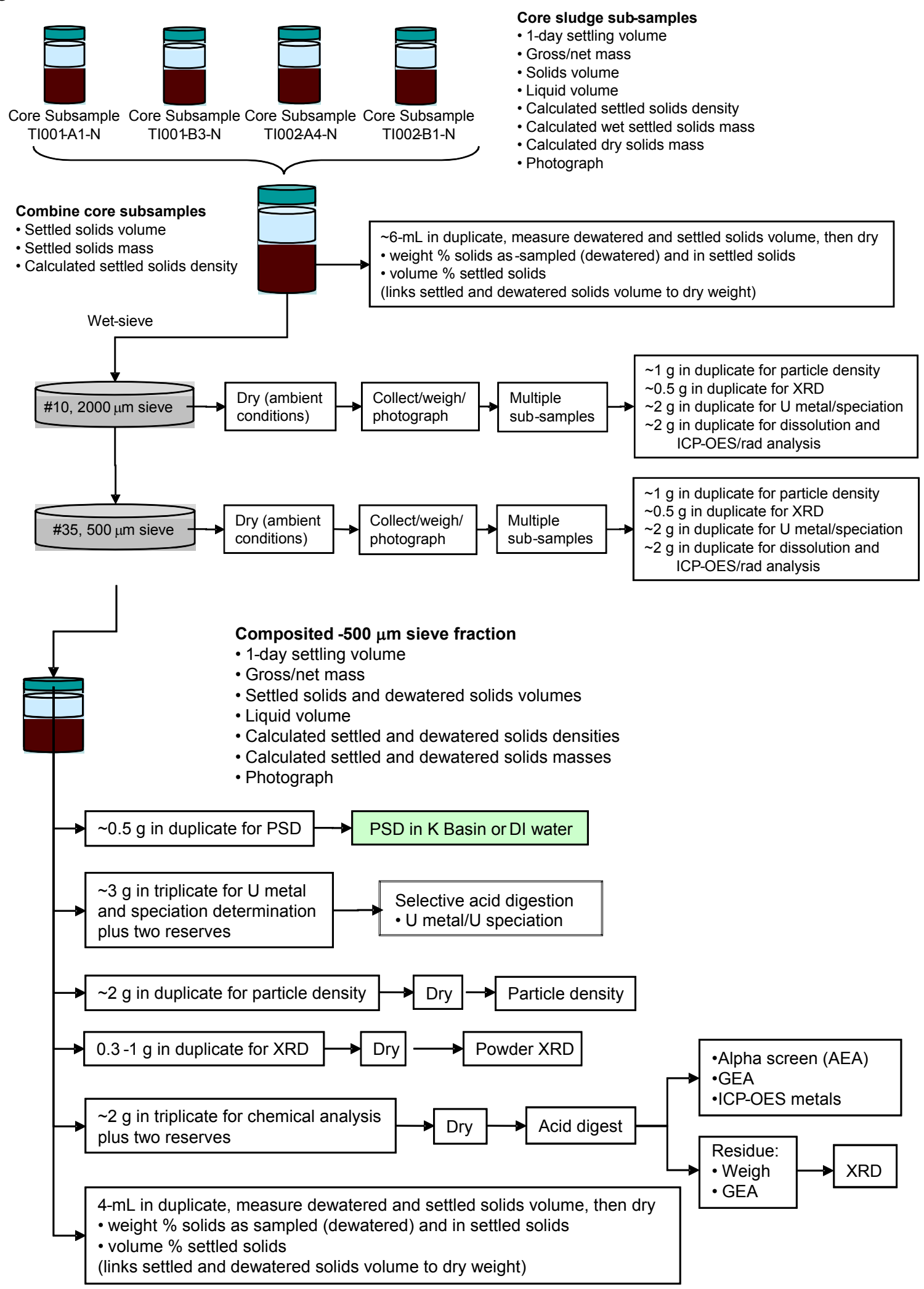

Figure 1. Overall Processing Schematic of Container Composite. PSD Characterization Scope is Highlighted in Green. 
August 3, 2011

\section{Background}

PSD describes the size fractionation of solid species in a given powder, dispersion, or slurry sample. PSD is typically described by either cumulative or differential population fraction versus a given particle size indicator. For example, the size distribution of particles in a slurry are often described using a histogram expressing the differential volume of particles falling between two equivalent sphere diameters over a large array of equivalent sphere diameters. PSD measurements can be accomplished using a number of approaches, such as settling experiments, microscopic imaging, and light obscuration and scattering.

The particle size measurements discussed herein are carried out on a Malvern Mastersizer 2000 particle size analyzer (Malvern Instruments, Inc., Southborough, MA). This instrument operates using the principle of laser diffraction (see Figure 2). Here, a monochromatic laser (red and/or blue) is directed through a transparent cell containing a dilute dispersion of the solid particles being analyzed. On the opposite side of the flow cell is a series of ring detectors capable of detecting the intensity of laser light at various scattering angles. If the laser does not strike a particle in the flow cell, it simply passes through the cell undisturbed and strikes the central detector. When the laser interacts with a particle, it is scattered at various angles. The scattered light is picked up across a number of rings of the detector, creating a unique "diffraction pattern" that can be mapped as a function of diffracted light intensity versus ring detector position. Prolonged observation of the light scattered from the dispersion allows a near complete sampling of the particle species contained therein (assuming all particles are uniformly dispersed). Comparison of the time-averaged diffraction signal against a "clean" reference cell signal generates a diffraction pattern unique to the test dispersion. Given the optical properties of the particulate and dispersing phases, mathematical analysis of the averaged "diffraction pattern" allows determination of size fractionation species contained in the dispersion.

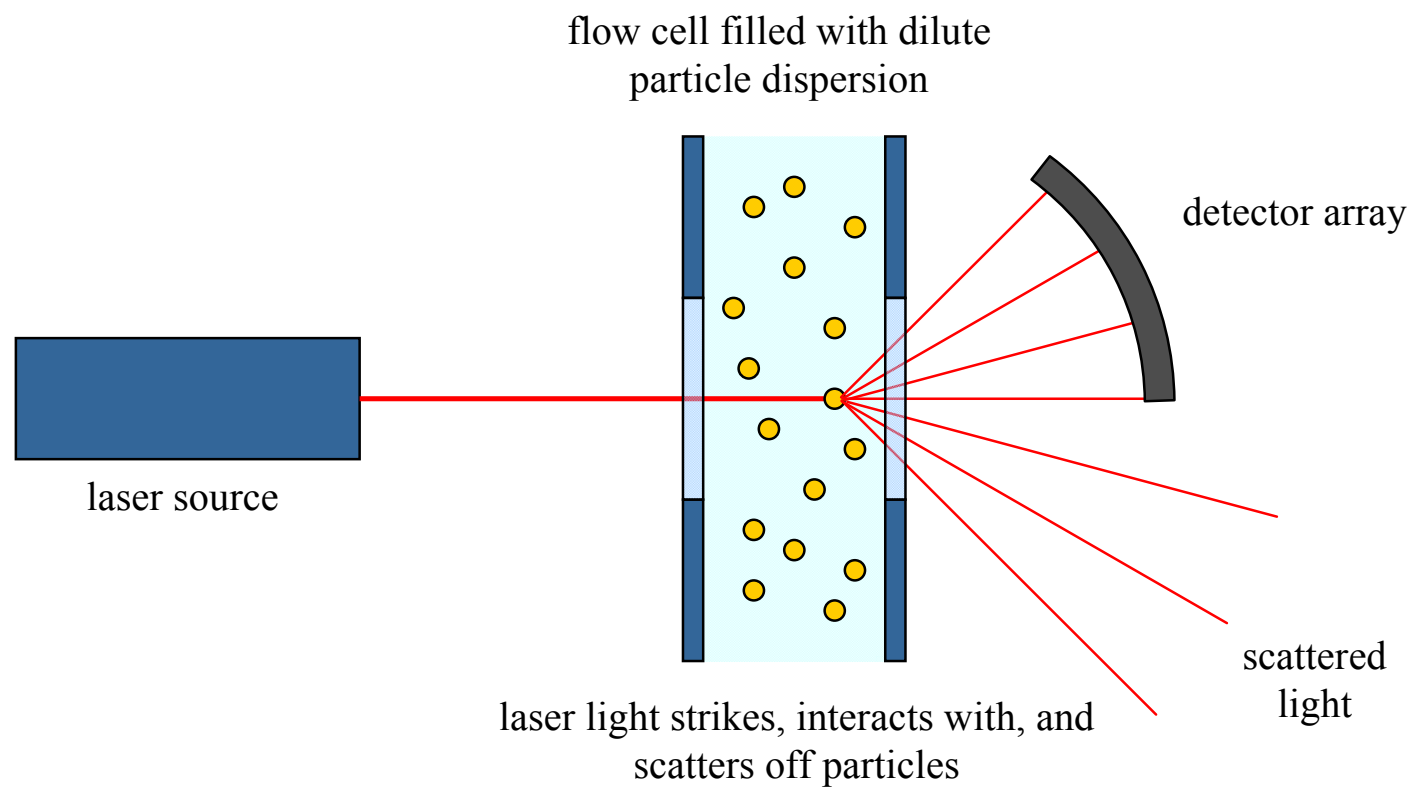

Figure 2. Schematic of a typical laser diffraction particle size analyzer.

It is important to recognize that particle size measurements by laser diffraction are intended to capture the size of a single, well-dispersed particle species. This "true" PSD captures all particles in the solid 
dispersion in a fully suspended and un-agglomerated primary particle state. Full dispersion at the primary particle level requires the correct selection of suspending phase chemistry, which is often modified through the use of dispersing agents or surfactants, and sufficient flow to suspend all particles during analysis. Complete suspension of all particle species requires sufficient mixing energy.

When dealing with complex dispersions, which contain multiple particle species and a broad distribution of sizes, finding the correct dispersing medium and measurement conditions may be difficult (if not impossible), as individual particle species in the solids mixture may have contradictory suspending phase chemistry requirements. As such, particle size analysis of complex solids dispersions is generally performed to determine the "apparent" PSD as a function of processing conditions such as flow rate and sonication and suspending phase chemistry such as $\mathrm{pH}$. The apparent PSD differs from the true PSD in two ways: 1) particle agglomerates exist and are treated as single particle species and 2) not all particles may be suspended at the flow conditions selected. Despite these short comings, apparent PSDs provide useful information about how the PSD of the test dispersion exists in the process from which it is derived and can highlight potential difficulties in suspending large/dense particles.

\section{Samples}

Samples for particle size analysis were collected under Test Instructions 52578-TI-008.

Table 1. Summary of Samples Provided for Particle Size Analysis.

\begin{tabular}{|l|l|l|c|}
\hline \multicolumn{1}{|c|}{ Sample ID } & \multicolumn{1}{|c|}{ Sample Purpose } & \multicolumn{1}{c|}{ Vessel Description } & $\begin{array}{c}\text { Sample Size } \\
\text { (wet sludge basis) }\end{array}$ \\
\hline TI008-SP & PSD & $\sim 20$-mL glass vial & $0.5 \mathrm{~g}$ \\
\hline TI008-SQ & PSD duplicate & $\sim 20$-mL glass vial & $0.5 \mathrm{~g}$ \\
\hline
\end{tabular}

\section{Instrumentation}

Particle size characterization was accomplished using a Mastersizer 2000 (Malvern Instruments, Inc., Southborough, MA 01772 USA) with a Hydro $\mu \mathrm{P}$ wet dispersion accessory. The Mastersizer has a nominal size measurement range of $0.02-2000 \mu \mathrm{m}$. The actual range is dependent on the accessory used as well as the properties of the solids being analyzed. When coupled with the Hydro $\mu \mathrm{P}$ wet dispersion accessory, the nominal measuring range is reduced to $0.02-150 \mu \mathrm{m}$ (also dependent on material density). Although particle sizes above $150 \mu \mathrm{m}$ can be observed with the Hydro $\mu \mathrm{P}$, reliable determination of their volume contribution is a challenge for the instrument and is highly dependent on the material density and size. Previous PSD determinations indicate that articles much larger than $\sim 600$ $\mu \mathrm{m}$ may "jam" or become stuck in the Hydro $\mu \mathrm{P}$ dispersion unit impeller, preventing their circulation to the flow cell observation window. Given that the container composite samples have been passed through a $500 \mu \mathrm{m}$ sieve, it was anticipated that there could be a remainder of large $(\sim 500 \mu \mathrm{m})$ particles that would not be able to pass through the dispersion unit and hence would not be accounted for in the PSD measurements obtained.

The Hydro $\mu \mathrm{P}$ wet dispersion accessory consists of a $20 \mathrm{~mL}$ sample flow cell with a continuously variable and independent pump and ultrasound. Both flow and sonication can be controlled and changed during measurement. As such, PSD measurements can be made before, during, and after sonication, allowing determination of the influence of each on the sample's PSD. The primary measurement functions of the Malvern analyzer are controlled through computer software. For the current measurements, Mastersizer 2000 software, Version 5.60 [Malvern Instruments, Ltd. Copyright 
(C) 1998-2009] was employed. Table 2 provides a summary of basic information regarding the analyzer and accessory located in Room 302 in the Radiological Processing Laboratory (RPL).

Table 2. Summary of Malvern Mastersizer 2000 Instrument Information.

\begin{tabular}{||l|l||}
\hline Analyzer: & Mastersizer 2000 \\
\hline Measurement principle: & Laser Diffraction (Mie Scattering) \\
\hline Analyzer Accessory: & Hydro $\mu \mathrm{P}$ \\
\hline Serial Number: & MAL 100406 \\
\hline Measurement Range: & $0.02-2000 \mu \mathrm{m}$ nominal $(0.02-150 \mu \mathrm{m}$ with accessory) \\
\hline Type: & $\begin{array}{l}\text { Flow cell system with continuously variable and } \\
\text { independent pump and ultrasound. }\end{array}$ \\
\hline Capacity: & $20 \mathrm{~mL}$ \\
\hline Pump Speed Range: & $0-5000 \mathrm{RPM}$ (variable) \\
\hline Ultrasound Power & $0-20 \mathrm{~W}$ (variable) \\
\hline Software Version/date & $5.60 / 1998-2009$ \\
\hline
\end{tabular}

\section{Test Approach}

Particle size distribution was determined according to the procedure RPL-COLLOID-01, Rev. 1, Particle Size Analysis Using Malvern MS2000. Before conducting particle-size measurements with KW210 samples, the PSD of a particle size standard traceable to the National Institute of Standards and Technology (NIST) was measured to verify instrument performance. The standard consisted of polydispersed silica particles with diameters falling primarily between 15-150 $\mu \mathrm{m}$. The results from this measurement were compared to the standard's certificate of analysis provided by the supplier to determine if the performance check indicated acceptable instrument performance (as dictated by RPLCOLLOID-01, Rev. 1). After completion of all PSD analyses associated with this test report, a closing performance check of the particle size analyzer was conducted.

For the PSD work described in this report, it was expected that the PSD measurements performed at initial recirculation times, without sonication, would be the most relevant PSD measurements for the sludge treatment project with respect to the current plans to load the sludge in the sludge transport and storage containers. These initial measurements (which correspond to a relatively unsheared slurry) would likely best reflect the expected PSD of the sludge particles in a settle/decant/filtration processing operation.

With this in mind, the following test approach was implemented to study and mitigate potential issues as described below:

- $\quad$ Subsampling - Samples were concentrated and required dilution to obtain a suitable sample for measurement. Efforts were made to withdraw sub-samples that best represent the submitted sample fraction.

- Quantity of Sample Material - when measuring the PSD of test material, the typical approach is to disperse (dilute) small aliquots of the concentrated (i.e., as-received) material in a suitable solvent (such as DI water or the particle suspending phase) contained within a variable-speed recirculator (Hydro $\mu \mathrm{P}$ dispersion unit). The sample quantity requirements are determined by monitoring the amount of light scattered by the diluted particle dispersion-referred to as obscuration. Sufficient sample material to yield obscuration values of 5 to $20 \%$ are generally considered acceptable for size measurements, which corresponds to sample sizes in the range of $0.1 \mathrm{~g}$ (for fine/less-dense particles) to $2 \mathrm{~g}$ for larger/more-dense particles. 
- The test approach employed for the concentrated K-Basin PSD samples was as follows. KBasin water was provided to use as the suspending phase for the analysis of these sample. The water was filtered through a $0.45 \mu \mathrm{m}$ syringe filter to remove particulate matter. First, the flow cell was filled with the suspending phase (filtered K-Basin water) and "as-received" test material was added drop wise until an acceptable obscuration was reached. For sample TI008-SP one drop of the as received material resulted in an obscuration of 16-20\%. For materials containing fine particles, obscuration values of the range $5-10 \%$ are more desirable to minimize the possibility of multiple scattering, which can bias the PSD if present. In contrast, one drop of the duplicate sample, TI008-SQ, resulted in an obscuration in the 5-10\% range. Side-by-side comparison of the two PSD sample vials found that the sample volume of TI008-SP was approximately half that of sample TI008-SQ. It was suspected that TI008-SP and may have lost some water due to evaporation resulting in the higher obscuration. Approximately $0.5 \mathrm{~mL}$ of filtered $\mathrm{KW}$ water was added to sample TI008-SP to obtain an obscuration value similar to that for sample TI008-SQ from one drop.

- Number of PSD Measurements at Each Condition - PSD characterizations at a given set of test conditions consisted of an average of three individual PSD measurements (with each measurement consisting of a 10 second red light observation and a 10 second blue light observation) for two sub-aliquots per sample. Data obtained for each of the sub-aliquots was averaged to obtain one PSD for each measuring condition per sample, i.e. at 1 minute recirculation (unsonicated), $100 \%$ sonication (20 watts) and post sonication.

Table 3 provides the matrix of conditions for each of the PSD samples listed in Table 1. The initial measurement conditions at 1 and 3 minute recirculation times are based on results obtained for the parameter evaluation study performed under Test Instruction, 52578-TI017. An additional data measurement set was collected (a post-sonicated measurement after 5 minutes of total recirculation) to further evaluate the effect of sonication on the measured PSD for the diluted samples.

Table 3. Modified Condition Matrix for PSD Determinations

\begin{tabular}{|c|c|c|c|}
\hline & $\begin{array}{l}\text { Recirculation } \\
\text { Rate, }^{\text {RPM }}{ }^{(a)}\end{array}$ & $\begin{array}{l}\text { Recirculation } \\
\text { Time, min }\end{array}$ & $\begin{array}{c}\text { Sonication }^{(\mathrm{a}, \mathrm{b})} \\
\text { power, time }\end{array}$ \\
\hline & $\begin{array}{l}2000 \\
2000 \\
2000\end{array}$ & $\begin{array}{l}1 \\
3 \\
5\end{array}$ & $\begin{array}{l}\text { Pre-sonication } \\
120 \mathrm{sec} / 100 \% \\
\text { Post-sonication }\end{array}$ \\
\hline \multicolumn{4}{|c|}{ Other Measures/Conditions } \\
\hline \multicolumn{4}{|c|}{$\begin{array}{l}\text { Number of PSD measurements/conditions: Each measurement consists of three } \\
\text { separate observations (with each observation consisting of a } 10 \text { second red light } \\
\text { measurement and a } 10 \text { second blue light measurement). }\end{array}$} \\
\hline \multicolumn{4}{|c|}{$\begin{array}{l}\text { The two initial measurement conditions for Recirculation RPM, recirculation time } \\
\text { and sonication power and time are based on results of the parameter evaluation in } \\
\text { Test Instruction 52578-TI017. A third measurement condition (at } 5 \text { minutes } \\
\text { recirculation) was added to further evaluate the impacts of sonication on the } \\
\text { measured PSD. } \\
\text { (b) The Hydro } \mu \text { P dispersion cell employed for particle size analysis is equipped with } \\
\text { variable power sonicator with an ultrasound power of up to } 20 \mathrm{~W} \text { (at an instrument } \\
\text { sonicator setting of } 100 \% \text { ). }\end{array}$} \\
\hline
\end{tabular}

Instrument Performance Check

As required by the procedure RPL-COLLOID-01, Rev. 1, the performance of the Malvern analyzer must be verified at the beginning of each series of analyses (with the period between performance 
checks not to exceed 90 days during use). Checks are performed using particle size standards traceable to the National Institute of Standards and Technology (NIST). Checks verify that the particle size analyzer can measure a PSD standard's d(50), the 50\% volume/weight fractile and mean particle size, to within $10 \%$ of the value specified on the manufacturer's certificate of analysis.

For the Mastersizer 2000 system employed for measurements (MAL100406 at RPL), two performance checks were conducted: 1) an opening performance check before the start of any PSD measurements on 06/15/11 and 2) a closing performance check at the end of measurements on the same day, 06/15/11. Both performance checks employed a Malvern Quality Audit Standard (QAS) QAS3004 supplied by Malvern Instruments, Inc. (Southborough, MA 01772 USA). The standard is traceable back to its certificate of analysis through a unique bottle number identifier.

The particle size standard was supplied as $0.10 \mathrm{~g}$ single shot of dry powder. Before measuring the performance check standard, the Hydro $\mu \mathrm{P}$ dispersion unit was filled with DI water. Next, the dry powder shot was added directly to the Hydro $\mu \mathrm{P}$ dispersion unit. A continuous pump speed of 2000 RPM was set to mix the flow cell contents and, after adequate mixing time, the PSD of the resulting dispersion was measured. The performance check size analysis employed a particle refractive index and absorption of 1.52 and 0 , respectively, and a suspending phase particle refractive index of 1.33 (for water).

The results of both the opening and closing performance checks are provided in Table 4. An average of three 2000 RPM measurements of the PSD indicated a d(50) of $63.112 \mu \mathrm{m}$ and $61.997 \mu \mathrm{m}$ for opening and closing performance checks, respectively, for the MS2000 particle size analyzer situated in RPL. These results deviate less than $2 \%$ from the $\mathrm{d}(50)$ listed on the standard's certificate of analysis from Malvern Instruments, Inc. In accordance with RPL-COLLOID-01, Rev. 1, an acceptable performance check is defined as: the $d(50)$ value for the standard is within $10 \%$ of the certified reference value.

Table 4. Performance check results for opening, and closing checks covering the period of measurement for PSD measurements for both instruments used for sample analysis under this test instruction.

\begin{tabular}{|c|c|c|c|}
\hline Size Range: & \multicolumn{3}{|l|}{15 to $150 \mu \mathrm{m}$} \\
\hline Catalogue \#: & \multicolumn{3}{|l|}{ QAS3004 } \\
\hline Bottle \# & \multicolumn{3}{|c|}{ 02/03858 (opening check), 02/03980 (closing check) } \\
\hline Weight: & \multicolumn{3}{|l|}{0.1 grams } \\
\hline \multicolumn{4}{|c|}{ Opening Check 06/15/11 } \\
\hline PSD Percentile & List & $\begin{array}{l}\text { Measured } \\
(\mu \mathrm{m})^{(\mathrm{a}),(\mathrm{c})}\end{array}$ & $E_{r}(\%)^{(b)}$ \\
\hline $\mathrm{d}(50)$ : & 62.362 & 63.112 & $1.20 \%$ \\
\hline \multicolumn{4}{|c|}{ Closing Check 06/15/11 } \\
\hline PSD Percentile & List & $\begin{array}{l}\text { Measured } \\
(\mu \mathrm{m})^{(\mathrm{a}),(\mathrm{c})}\end{array}$ & $\mathrm{E}_{\mathrm{r}}(\%)^{(\mathrm{b})}$ \\
\hline $\mathrm{d}(50):$ & 62.362 & 61.997 & $-0.59 \%$ \\
\hline \multicolumn{4}{|c|}{$\begin{array}{l}\text { (a) As measured for the period of performance applicable for this report. } \\
\text { (b) Calculated before rounding of significant figures in list and measured percentiles. } \\
\text { Defined as: } \mathrm{E}_{\mathrm{r}}(\%)=\left(\mathrm{X}_{\text {measured }}-\mathrm{X}_{\text {certified }}\right) / \mathrm{X}_{\text {certified }} * 100 \\
\text { (c) Average of triplicate measurements reported here as per the standard protocol of the } \\
\text { QAS supplier. }\end{array}$} \\
\hline
\end{tabular}


PNNL-20650, Rev. 2

Carolyn Burns

Page 9 of 17

August 3, 2011

Particle Size Characterization

All PSD measurements were performed with diluted "as-received" material with 1 drop of original sample diluted in a filled Hydro $\mu \mathrm{P}$ flow cell to achieve target test concentrations. Before each sampling event for PSD, the analyzer was drained, flushed with $20 \mathrm{~mL}$ of deionized (DI) water at least three times, filled with filtered K-Basin water, and brought into a measurement ready state. The analyst then examined the test sample to determine the best sampling approach. The dispersion unit flow cell was fully filled with filtered K-Basin water in preparation of sample introduction. Next, the sample was introduced to the Malvern dispersion unit and analyzed as follows:

1. The analyzer flow cell pump was set to the initial circulation rate of 2000 RPM (see Table 3).

2. The sample was prepared for analysis by re-suspending the settled solids. This was accomplished by initially agitating the sample vial to re-suspend the settled solids. The sample was then repeatedly pulsed with a $1 \mathrm{~mL}$ disposable plastic pipette until the contents were uniformly dispersed and representatively sub-sampled in the pipette. Each pulse involved drawing off a fraction of the sample into the pipette and immediately jetting the drawn liquid back into the sample vial.

3. Immediately after re-suspension, one drop of sample was introduced into the instrument (while the pump was active). For the current analyses, an obscuration of 5-20\% was targeted. The time at which the sample introduction was complete was noted and was associated with the start of sample recirculation.

4. The material (sample) and suspending phase optical properties were set in the analyzer software. The standard optical properties employed were an RI of 2.42 and an absorption index of 1 .

5. The sample PSD was measured under the conditions outlined in the sample test matrix (see Table 3).

For each test condition outlined in Table 3, the dispersion PSD was measured three times, with each measurement consisting of a 10 second red light observation and a 10 second blue light observation. An average of the 3 measurements was calculated, yielding one average PSD for each test condition per sample aliquot measured. Condition set points (i.e., pump speed and sonication) were changed at the specified recirculation times. Recirculation times were calculated as the difference between the current measurement time and the time sample dispersion was completed. PSD measurements were started at recirculation times of 1,3 , and 5 minutes

Analysis of the raw particle size data is performed automatically by the Mastersizer software immediately after each measurement. Analysis calculates the particle size distribution based on the scattered light intensity as a function of detection angle and the particle size model selected (e.g., single narrow, multiple narrow, or broad distributions). As indicated in the test steps outlined in the preceding paragraphs, the optical properties such as the refractive index (RI) of the sample and suspending phase must be entered into analyzer at the time of measurement. Table 5 outlines the optical properties employed in the current analysis. Selection of these optical properties is based on the results of the parameter evaluation study performed under Test Instruction, 52578-TI017. 
PNNL-20650, Rev. 2

Carolyn Burns

Page 10 of 17

August 3, 2011

Table 5. Default optical properties employed for PSD analysis of KW210 container composites. These properties were assessed through post-measurement reanalysis of KW240 (TI010-SP) data collected during the initial parameter evaluation study.

\begin{tabular}{||l|l||}
\hline Property & Values Employed \\
\hline Refractive Index (RI) & 2.42 \\
\hline Absorption Index & 1 \\
\hline Particle Shape & Irregular \\
\hline Model & General Purpose (Broad Distribution) \\
\hline
\end{tabular}

\section{Quality Assurance}

The quality assurance requirements defined in the QAPjP/SAP invoked Hanford Analytical Services Quality Assurance Requirements Document (HASQARD), Rev. 3 Volume 1 and 4 and balance calibration verification controls. These requirements have been implemented for this work as defined in this test instruction and are summarized in Table 6.

Table 6. QA requirements and implementation.

\begin{tabular}{|l|l||}
\hline QA Requirement & Implementation \\
\hline $\begin{array}{l}\text { Controlling sample } \\
\text { identification }\end{array}$ & $\begin{array}{l}\text { Samples generated during the execution of this TI will be uniquely identified } \\
\text { according to instruction in this TI. }\end{array}$ \\
\hline Container selection & $\begin{array}{l}\text { Containers were made of glass, consistent with the intended storage and } \\
\text { follow-on processing. }\end{array}$ \\
\hline Preservation & Sample preservation is not applicable for current scope. \\
\hline $\begin{array}{l}\text { Sample chain of custody } \\
\text { (COC) }\end{array}$ & $\begin{array}{l}\text { Internal COC is not required. Sample custody is maintained during the } \\
\text { sample preparation phase and any extra material is archived in case the } \\
\text { remainder is needed for reanalysis. Sample security is maintained by limited } \\
\text { access to the building and to laboratory areas. When the ASO laboratory } \\
\text { receives samples, an Analytical Service Request (ASR) is generated. Sample } \\
\text { processing steps are documented in TIs and on bench sheets. Processed } \\
\text { sample fractions are also uniquely identified. }\end{array}$ \\
\hline Sample storage & $\begin{array}{l}\text { There are no sample storage requirements for PSD samples; they are stored at } \\
\text { room temperature. }\end{array}$ \\
\hline $\begin{array}{l}\text { Sample handling and } \\
\text { transfer }\end{array}$ & $\begin{array}{l}\text { Handling and transfers was conducted in a manner to minimize potential for } \\
\text { contamination (clean containers and sampling devices), minimize loss (use } \\
\text { secondary containment during process operations-where practical), and } \\
\text { minimize tendency for sample to dry out (except where drying is required) } \\
\text { where the wetting agent is deionized (DI) water. }\end{array}$ \\
\hline Holding time limits & Holding time limits are not applicable to PSD measurement. \\
\hline Waste disposition & $\begin{array}{l}\text { Waste was processed through the normal waste operations protocols and } \\
\text { procedures. }\end{array}$ \\
\hline $\begin{array}{l}\text { Preparing required } \\
\text { quality control (QC) } \\
\text { samples }\end{array}$ & $\begin{array}{l}\text { The primary sample and duplicate were generated from independent replicate } \\
\text { tests, and are not considered true analytical duplicates. Therefore duplicate } \\
\text { precision criteria, (i.e., } \pm 20 \% \text { ) for the PSD was not applied to these samples. }\end{array}$ \\
\hline Balance checks & NA; balances are not typically used in PSD measurements. \\
\hline \hline
\end{tabular}


PNNL-20650, Rev. 2

Carolyn Burns

Page 11 of 17

August 3, 2011

\section{Results}

Particle size distribution measurements for samples derived from KW210 container composite sample are presented below. Table 7 summarizes the cumulative percent at 1 minute of recirculation

(unsonicated) and with sonication applied for the primary and duplicate samples, TI008-SP and TI008SQ, respectively, investigated in this study. The cumulative \% undersize data given in Table 7 has been plotted along with the volume contribution \% PSD and are shown in Figures 3 through 6 . The post sonicated data collected have been included on Figures 3 through 6 . Only the 1 minute recirculation time and the sonicated data have been included in Table 7; this is consistent with the work scope of this TI and previous reports. Post sonication data were collected as a precautionary measure, given the sample dilutions required to obtain a suitable sample obscuration. Dilute samples sometimes form bubbles and or agglomerate as a result of sonication. Neither was observed for either sample analyzed. The data obtained at 1 minute recirculation time are more representative of the suspended particles present during settling of actual sludge material while the sonicated data better represents the "true" PSD of the fully suspended sample particles. The average particle size distribution of the container composite KW210 - 500 $\mu \mathrm{m}$ size fraction is given in Table 8 and Figures 7 and 8 , again only the unsonicated and sonicated data is given in Tables 7 and 8 .

For all sample aliquots analyzed, grinding/knocking sounds could be heard inside the dispersion unit; this noise is indicative of large particles getting caught-up in and knocking on the internal parts of the dispersion. This would suggest that there were particles present in both samples provided for analysis with at least one linear dimension greater than $500 \mu \mathrm{m}$. Sieving through a $500 \mu \mathrm{m}$ sieve does not always eliminate elongated particles with one dimension greater than $500 \mu \mathrm{m}$. If large particles are held up in the dispersion components, they may not flow into the observation cell and be measured by the instrument. Additionally, hold-up of large particles may block flow of smaller particles, preventing their observation. The grinding/knocking sound was not heard once the instrument was flushed, cleaned and filled with K-Basin water for the background measurement, which suggests that the large particles did not accumulate in the system and effect subsequent measurements.

A summary of a select number of percentiles is given in Table 9; these data provide a brief overview of the particle sizes present, in particular the tails of the PSD (upper and lower limits). 
PNNL-20650, Rev. 2

Carolyn Burns

Page 12 of 17

August 3, 2011

Table 7. Cumulative Percent Undersize measured at 1 minute recirculation time (unsonicated) and during sonication at 3 minutes recirculation time.

\begin{tabular}{|c|c|c|c|c|}
\hline \multirow[b]{2}{*}{ Size, $\mu \mathrm{m}$} & \multicolumn{2}{|c|}{ (a)TI-008-SP } & \multicolumn{2}{|c|}{ (a) TI-008-SQ } \\
\hline & Unsonicated & $\begin{array}{c}100 \% \\
\text { Sonication }\end{array}$ & Unsonicated & $\begin{array}{c}100 \% \\
\text { Sonication }\end{array}$ \\
\hline 0.05 & 0 & 0 & 0 & 0 \\
\hline 0.5 & 0.28 & 0.64 & 0.00 & 0.32 \\
\hline 1 & 1.01 & 2.45 & 0.34 & 1.35 \\
\hline 2.5 & 4.51 & 7.53 & 2.06 & 4.12 \\
\hline 5 & 10.2 & 13.0 & 4.66 & 7.07 \\
\hline 10 & 16.1 & 19.1 & 7.31 & 10.5 \\
\hline 20 & 20.8 & 25.3 & 9.56 & 14.3 \\
\hline 40 & 24.9 & 31.8 & 11.7 & 18.8 \\
\hline 60 & 27.8 & 36.1 & 13.8 & 22.4 \\
\hline 80 & 30.1 & 38.9 & 15.5 & 24.9 \\
\hline 100 & 32.0 & 40.6 & 16.7 & 26.4 \\
\hline 125 & 34.0 & 42.1 & 18.0 & 27.5 \\
\hline 150 & 36.1 & 43.4 & 19.3 & 28.5 \\
\hline 175 & 38.5 & 45.0 & 21.3 & 30.1 \\
\hline 200 & 41.4 & 47.1 & 24.1 & 32.3 \\
\hline 300 & 56.7 & 60.2 & 41.5 & 47.8 \\
\hline 400 & 72.7 & 75.1 & 61.4 & 66.2 \\
\hline 500 & 85.2 & 87.0 & 77.6 & 81.1 \\
\hline 600 & 93.4 & 94.7 & 88.5 & 91.0 \\
\hline 700 & 98.0 & 98.7 & 95.1 & 96.7 \\
\hline 800 & 99.9 & 100 & 98.5 & 99.4 \\
\hline 1000 & 100 & 100 & 100 & 100 \\
\hline
\end{tabular}


PNNL-20650, Rev. 2

Carolyn Burns

Page 13 of 17

August 3, 2011

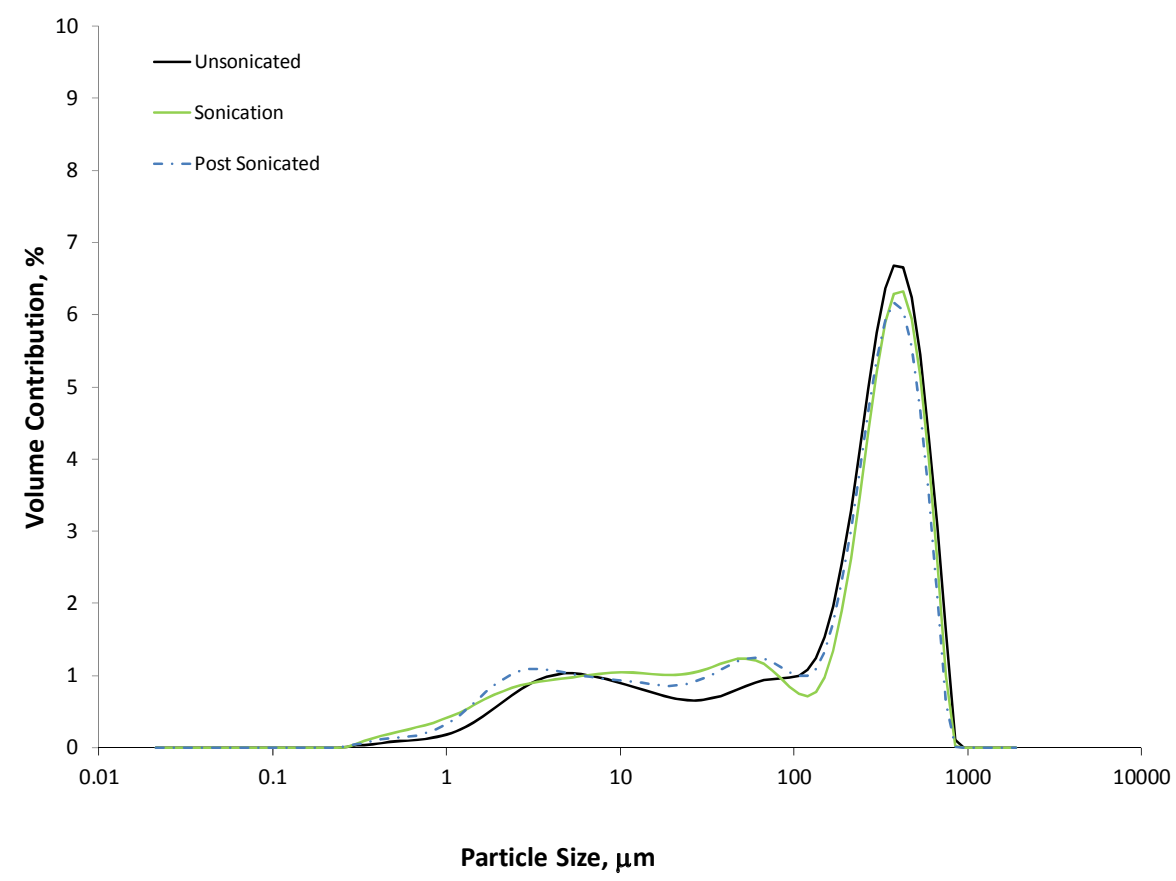

Figure 3. Average volume particle size distribution measured at 1 minute recirculation, $100 \%$ sonication and post sonication for sample TI008-SP. Based on the average of 6 PSD measurements generated from 2 aliquots.

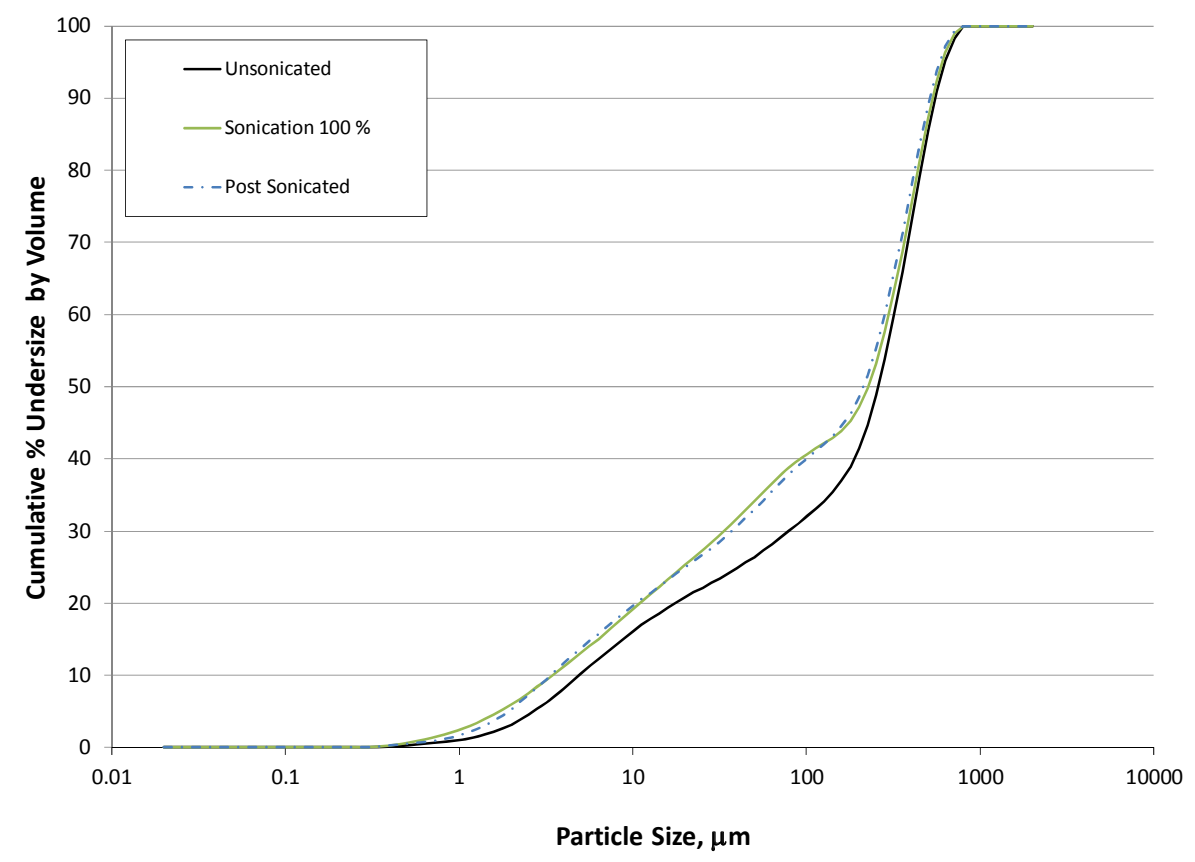

Figure 4. Average Cumulative \% undersize plot measured at 1 minute recirculation, $100 \%$ sonication and post sonication for sample TI008-SP. Based on the average of 6 PSD measurements generated from 2 aliquots. 
PNNL-20650, Rev. 2

Carolyn Burns

Page 14 of 17

August 3, 2011

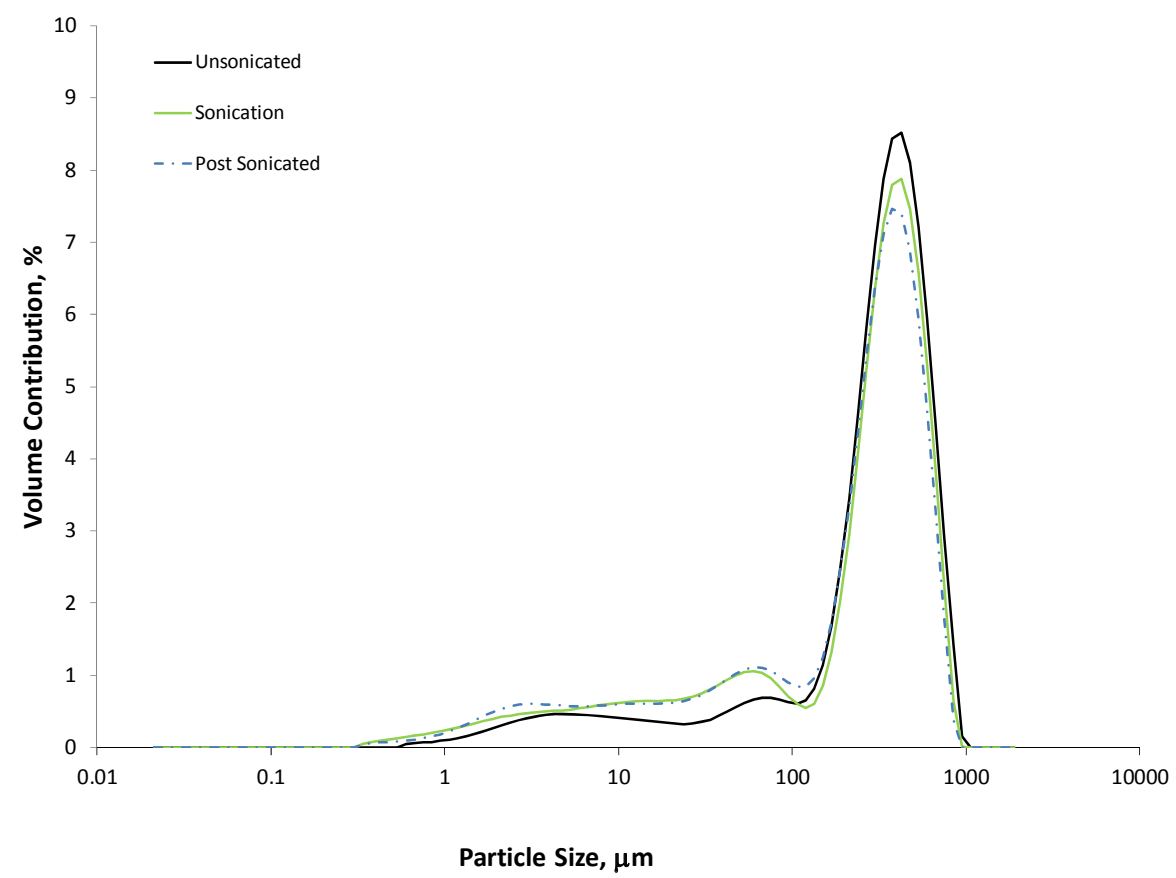

Figure 5. Average volume particle size distribution measured at 1 minute recirculation, $100 \%$ sonication, and post sonication for sample TI008-SQ. Based on the average of 6 PSD measurements generated from 2 aliquots.

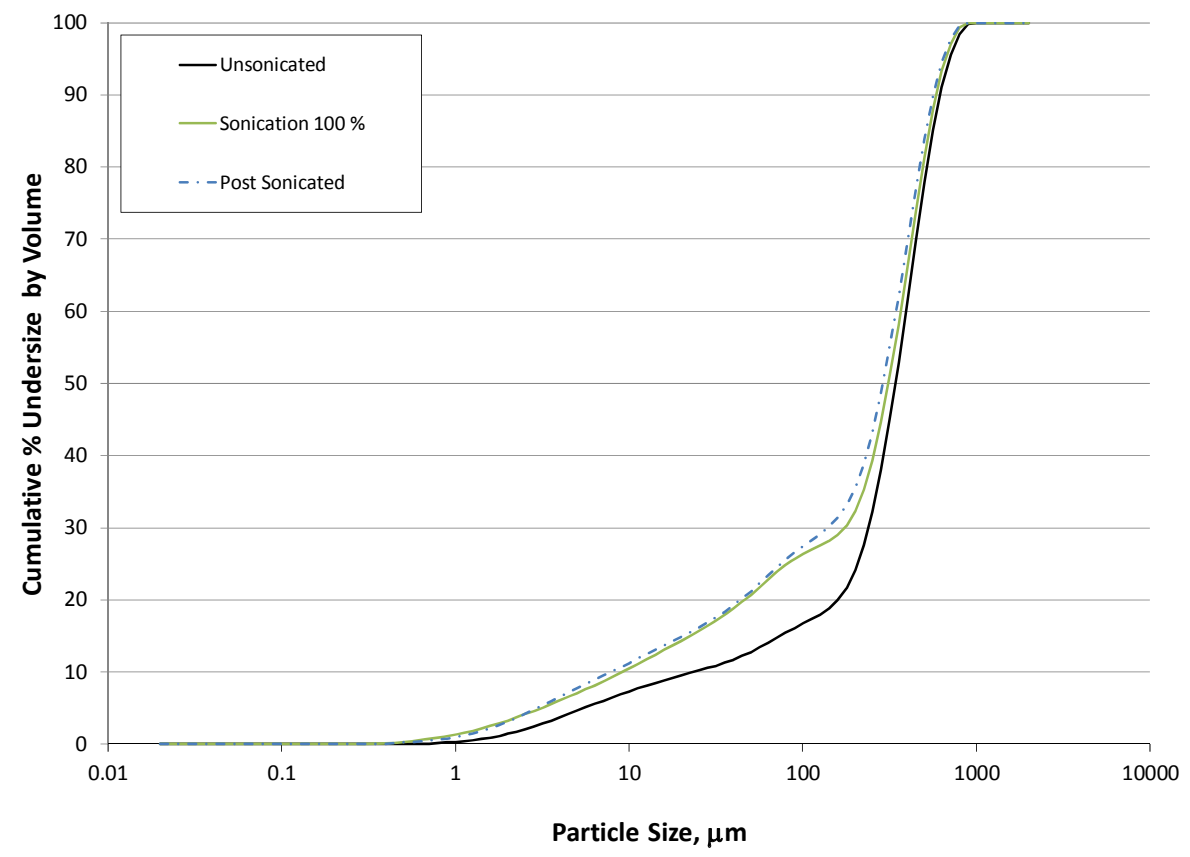

Figure 6. Average Cumulative \% undersize plot measured at 1 minute recirculation and $100 \%$ sonication for sample TI008-SQ. Based on the average of 6 PSD measurements generated from 2 aliquots. 
PNNL-20650, Rev. 2

Carolyn Burns

Page 15 of 17

August 3, 2011

Table 8. KW210 SCC Average Cumulative Percent Undersize measured at 1 minute recirculation time (unsonicated) and during sonication at 3 minutes recirculation time.

\begin{tabular}{|c|c|c|}
\hline \multirow[b]{2}{*}{ Size, $\mu \mathrm{m}$} & \multicolumn{2}{|c|}{ (a) KW210 SCC } \\
\hline & Unsonicated & $\begin{array}{c}100 \% \\
\text { Sonication }\end{array}$ \\
\hline 0.05 & 0 & 0 \\
\hline 0.5 & 0.14 & 0.48 \\
\hline 1 & 0.68 & 1.90 \\
\hline 2.5 & 3.28 & 5.83 \\
\hline 5 & 7.41 & 10.1 \\
\hline 10 & 11.7 & 14.8 \\
\hline 20 & 15.2 & 19.8 \\
\hline 40 & 18.3 & 25.3 \\
\hline 60 & 20.8 & 29.2 \\
\hline 80 & 22.8 & 31.9 \\
\hline 100 & 24.4 & 33.5 \\
\hline 125 & 26.0 & 34.8 \\
\hline 150 & 27.7 & 35.9 \\
\hline 175 & 29.9 & 37.5 \\
\hline 200 & 32.8 & 39.7 \\
\hline 300 & 49.1 & 54.0 \\
\hline 400 & 67.1 & 70.6 \\
\hline 500 & 81.4 & 84.0 \\
\hline 600 & 90.9 & 92.9 \\
\hline 700 & 96.5 & 97.7 \\
\hline 800 & 99.2 & 99.7 \\
\hline 1000 & 100 & 100 \\
\hline \multicolumn{3}{|c|}{$\begin{array}{l}\text { Based on the average of } 12 \text { PSD measurements } \\
\text { generated from } 4 \text { aliquots. }\end{array}$} \\
\hline
\end{tabular}


PNNL-20650, Rev. 2

Carolyn Burns

Page 16 of 17

August 3, 2011

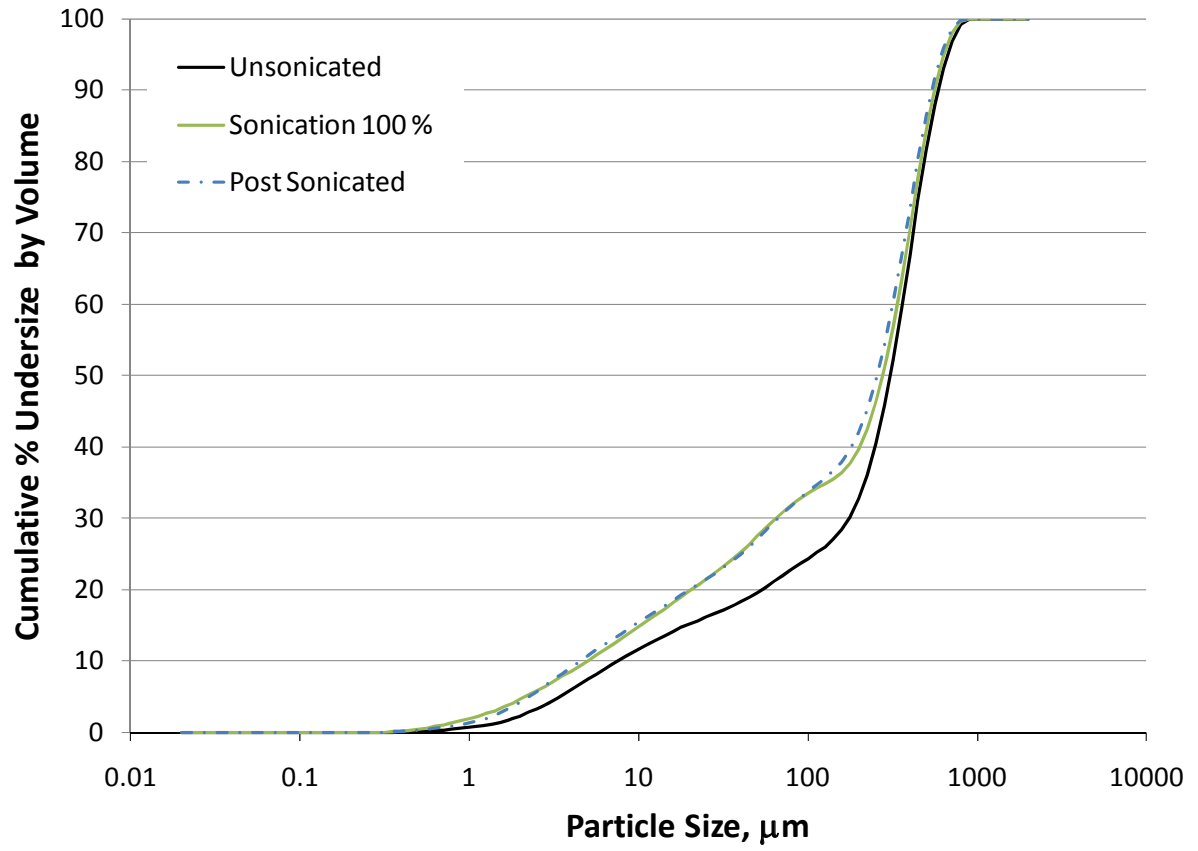

Figure 7. Average Cumulative Volume \% Undersize (Less Than $500 \mu \mathrm{m}$ Size Fraction) Plot of KW210-SCC, based on the average of 12 measurements generated from four aliquots, two aliquots from sample TI008-SP (primary) and two aliquots from TI008-SQ (duplicate)

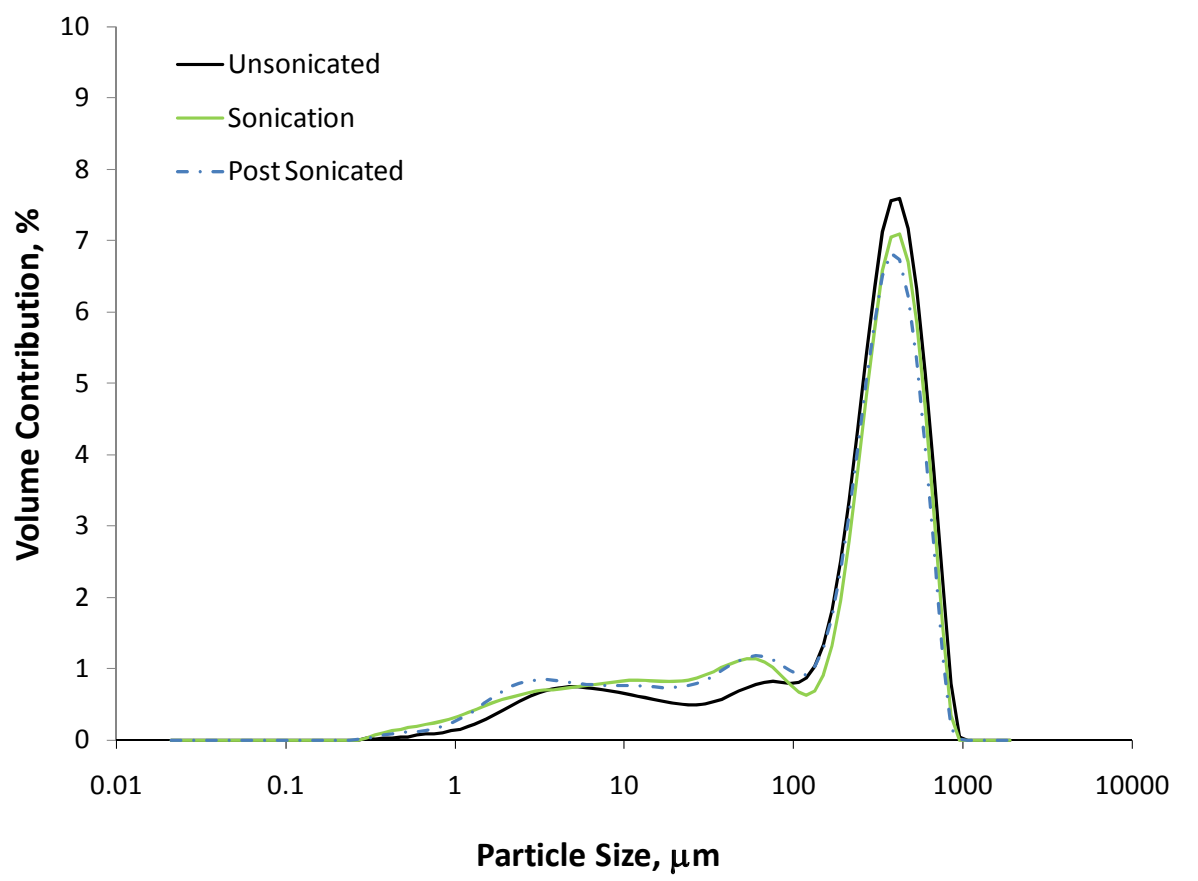

Figure 8. Average Volume \% PSD of Sample KW210-SCC (Less Than $500 \mu \mathrm{m}$ Size Fraction) Plot of KW210-SCC, based on the average of 12 measurements generated from four aliquots, two aliquots from sample TI008-SP (primary) and two aliquots from TI008-

SQ (duplicate) 
PNNL-20650, Rev. 2

Carolyn Burns

Page 17 of 17

August 3, 2011

Table 9. Summary table of selected percentile values for measurements taken at 1 minute recirculation time (unsonicated) and during sonication at 3 minutes recirculation time.

\begin{tabular}{|c|c|c|c|c|c|c|}
\hline & \multicolumn{2}{|c|}{${ }^{(\mathbf{a})}$ TI008-SP } & \multicolumn{2}{c|}{${ }^{(\mathbf{a})}$ TI008-SQ } & \multicolumn{2}{c|}{ KW210 SCC Average } \\
\hline Percentiles & $\begin{array}{c}\text { Unsonicate } \\
\mathbf{d}\end{array}$ & $\begin{array}{c}\mathbf{1 0 0} \\
\text { Sonicatio } \\
\mathbf{n}\end{array}$ & $\begin{array}{c}\text { Unsonicate } \\
\mathbf{d}\end{array}$ & $\begin{array}{c}\mathbf{1 0 0} \% \\
\text { Sonication }\end{array}$ & Unsonicated & $\begin{array}{c}\mathbf{1 0 0} \% \\
\text { Sonication }\end{array}$ \\
\hline $\mathrm{d}(0.01)$ & 0.99 & 0.60 & 1.65 & 0.83 & 1.24 & 0.68 \\
\hline $\mathrm{d}(0.05)$ & 2.69 & 1.72 & 5.45 & 3.10 & 3.42 & 2.15 \\
\hline $\mathrm{d}(0.10)$ & 4.92 & 3.45 & 23.3 & 9.12 & 7.52 & 4.96 \\
\hline $\mathrm{d}(0.50)$ & 259 & 227 & 342 & 312 & 305 & 276 \\
\hline $\mathrm{d}(0.90)$ & 552 & 533 & 619 & 587 & 588 & 562 \\
\hline $\mathrm{d}(0.95)$ & 628 & 605 & 699 & 663 & 666 & 636 \\
\hline $\mathrm{d}(0.99)$ & 737 & 711 & 823 & 776 & 786 & 750 \\
\hline
\end{tabular}

a) Based on the average of 6 PSD measurements generated from 2 aliquots.

b) Based on the averaged of 12 PSD measurements generated from 4 aliquots, two aliquots from TI008-SP (primary) and two aliquots from TI008-SQ (duplicate).

\section{References}

RPL-COLLOID-01, Rev. 1, Particle Size Analysis Using Malvern MS2000.

KBC-33786, Rev. 2, December 2009; Quality Assurance Project Plan/Sampling and Analysis Plan (QAPjP/SAP): Quality Assurance Project Plan/Sampling and Analysis Plan for Containerized KW Settler Sludge

CH2M Hill Plateau Remediation, Contract 41108, Statement of Work, Technical Support for Planning, Preparing, and Analyzing KW Settler Sludge (February 11, 2010).

Data Quality Objectives for Sampling and Analysis of K Basin Sludge HNF-36985, Rev. 3, July 2009 
PNNL-20650, Rev. 2

\section{8-TI037-PSD Data Package - Coverpage}

Description:

This Excel file data package documents measurement results (using Microsoft ${ }^{*}$ Office Excel) for particle size distribution (PSD) data collected under Test Instruction (TI) 52578-TI-037, Particle Size Distribution Measurements for K West Basin Consolidated Container Composite SCS-CON-210, Using Minus 500 Micron Particle Size Fraction Samples. Results for samples TI008-SP and TI008-SQ are given.

Computer Employed:

MHP Dell Latitude D830 Laptop Computer (Property \# WE14110)

Operating Environment:

Microsoft Windows 7 Enterprise

Base Software Program:

Microsoft Office Professional Plus 2010 Excel Version 14.0.5128.5000 (32-bit)

Package Contents:

Package consists of this coverpage, two calculation review pages, print-outs for two Microsoftº Office Excel workbooks, and all other relevant documentation.

\begin{tabular}{|c|c|c|c|}
\hline Description & File Date & File Size & Number of Pages \\
\hline Data package coverpage & $\mathrm{n} / \mathrm{a}$ & $\mathrm{n} / \mathrm{a}$ & 1 \\
\hline Calculation review worksheet & $\mathrm{n} / \mathrm{a}$ & $n / a$ & 1 \\
\hline Supplemental calculation review worksheet & $\mathrm{n} / \mathrm{a}$ & $\mathrm{n} / \mathrm{a}$ & 1 \\
\hline Excel printouts: "52578-T1037 (Reviewed).xlsm" & $7 / 7 / 201119: 55$ & $100 \mathrm{~KB}$ & 29 \\
\hline Excel printouts: "TI008 averaged data.xIsm" & $8 / 4 / 201115: 48$ & $73 \mathrm{~KB}$ & 21 \\
\hline Standard certificate: "Malvern Standard - QAS3004.pdf" & $5 / 3 / 201115: 04$ & $480 \mathrm{~KB}$ & 2 \\
\hline K-Basin Surveillance Report - SR-52578-2011-030 (Rev. 0) & $\mathrm{n} / \mathrm{a}$ & $\mathrm{n} / \mathrm{a}$ & 3 \\
\hline K-Basin Surveillance Report - SR-52578-2011-030 Rev. 1 & $\mathrm{n} / \mathrm{a}$ & $\mathrm{n} / \mathrm{a}$ & 4 \\
\hline
\end{tabular}

Package Generator Signature:

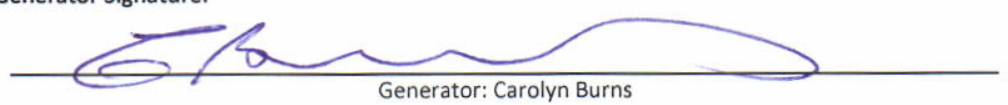


PNNL-20650, Rev. 2

\section{Calculation Review Sheet}

\begin{tabular}{|c|c|}
\hline Reviewer Name: & Richard C. Daniel \\
\hline Reviewer Title: & S/E \\
\hline Review Date: & $7 / 7 / 2011$ \\
\hline \multirow{3}{*}{$\begin{array}{l}\text { Title of Spreadsheet } \\
\text { Calculation Reviewed: }\end{array}$} & One spreadsheet and a calibration certificate are included in this review package: \\
\hline & 1) "52578-TI037 (Reviewed).xIsm" \\
\hline & 2) "Malvern Standard - QAS3004.pdf" \\
\hline Revision Number: & $\mathrm{n} / \mathrm{a}$ \\
\hline Date Prepared: & $7 / 7 / 2011$ \\
\hline
\end{tabular}

Scope of Spreadsheet Review: (Check one or more of the following)

\begin{tabular}{|c|l}
\hline$X$ & General Validation Review: (General review \& spot checks) \\
Review of updated spreadsheet/calc (Revised portion only) \\
$100 \%$ Verification Review (Verification of all cells/calculations) \\
Independent calculation check (With hand calcs or independent spreadsheet) \\
Other:
\end{tabular}

CHECK LIST

Spreadsheet/Calculation Identification

Spreadsheet/Calculation Title:

Revision Number:

Date Prepared

Prepared by:

General Statement of Purpose:

General Description of Approach (note: specific

approaches may be described for each

section/subsection of spreadsheet/calculation)

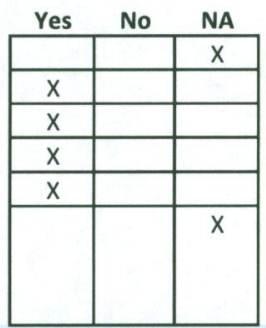

Comments: $\quad$ Standard PSD spreadsheet, approach per RPL-COLLOID-01, Rev. 1

Assumptions

Are assumptions clearly stated?

Are assumptions supported/justified?

Are assumptions reasonable?

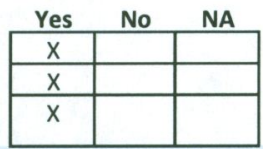

Comments: $\quad$ No comments

Input Values

Are input parameters correct (verified with source?)

Are parameter units consistent?

Are input values properly referenced?

\begin{tabular}{l} 
Yes \\
\begin{tabular}{|c|l|l|}
\hline$X$ & No & NA \\
\hline$X$ & & \\
\hline$X$ & & \\
\hline
\end{tabular} \\
\hline
\end{tabular}

Comments: No comments

Equations/Approach

Are equations adequately defined?

Are equations properly referenced?

Are limitations of approach/equations identified?

Are equations appropriate?

Are units consistent?

\begin{tabular}{|c|c|c|}
\multicolumn{1}{c}{ Yes } & No & \multicolumn{1}{c|}{ NA } \\
\hline$X$ & & \\
\hline & & $X$ \\
\hline & & $X$ \\
\hline$X$ & & \\
\hline$X$ & & \\
\hline
\end{tabular}

Comments: $\quad$ No comments

Results/Conclusions

Are formulas consistent in spreadsheet cells?

Are calculations correct?

Are conclusions consistent with results?

Are conclusions consistent with applicable limits?

Comments: No comments

\begin{tabular}{|c|c|c|}
\hline Yes & No & NA \\
\hline$x$ & & \\
\hline \multicolumn{3}{|l|}{$x$} \\
\hline & & $x$ \\
\hline & & $x$ \\
\hline
\end{tabular}

Reviewer Sign/Date: $\frac{\text { No comments }}{\text { Richard C. Daniel }}$

Page 1 of 1 
PNNL-20650, Rev. 2

\section{Calculation Review Sheet}

\begin{tabular}{|c|c|}
\hline Reviewer Name: & Richard C. Daniel \\
\hline Reviewer Title: & S/E \\
\hline Review Date: & $8 / 1 / 2011$ \\
\hline \multirow{4}{*}{$\begin{array}{l}\text { Title of Spreadsheet } \\
\text { Calculation Reviewed: } \\
\text { Revision Number: } \\
\text { Date Prepared: }\end{array}$} & This review covers the following supplemental files for the 52578-TI037-PSD data package \\
\hline & "TI008 averaged data.xlsm" \\
\hline & $\mathrm{n} / \mathrm{a}$ \\
\hline & $8 / 1 / 2011$ \\
\hline
\end{tabular}

Scope of Spreadsheet Review: (Check one or more of the following)

$\mathrm{X}$ General Validation Review: (General review \& spot checks)

Review of updated spreadsheet/calc (Revised portion only)

$100 \%$ Verification Review (Verification of all cells/calculations)

Independent calculation check (With hand calcs or independent spreadsheet)

CHECK LIST Other:

Spreadsheet/Calculation Identification

Spreadsheet/Calculation Title:

Revision Number:

Date Prepared

Prepared by:

General Statement of Purpose:

General Description of Approach (note: specific approaches may be described for each section/subsection of spreadsheet/calculation)

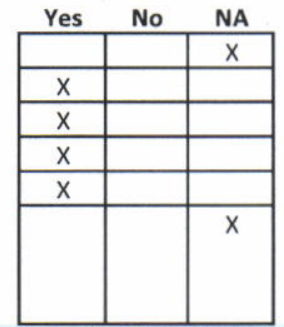

Comments: $\quad$ Standard PSD spreadsheet, approach per RPL-COLLOID-01, Rev. 1

\section{Assumptions}

Are assumptions clearly stated?

Are assumptions supported/justified?

Are assumptions reasonable?

\begin{tabular}{|c|c|c|}
\multicolumn{1}{c}{ Yes } & No & \multicolumn{1}{c|}{ NA } \\
\hline$X$ & & \\
\hline$X$ & & \\
\hline$X$ & & \\
\hline
\end{tabular}

Comments:

No comments

Input Values

Are input parameters correct (verified with source?)

Are parameter units consistent?

Are input values properly referenced?

\begin{tabular}{|c|c|c|}
\multicolumn{1}{c}{ Yes } & No & \multicolumn{1}{c|}{ NA } \\
\hline$X$ & & \\
\hline$X$ & & \\
\hline$X$ & & \\
\hline
\end{tabular}

Comments: No comments

Equations/Approach

Are equations adequately defined?

Are equations properly referenced?

Are limitations of approach/equations identified?

Are equations appropriate?

Are units consistent?

\begin{tabular}{|c|c|c|}
\multicolumn{1}{|c|}{ Yes } & \multicolumn{1}{c}{ No } & \multicolumn{1}{c}{ NA } \\
\hline$X$ & & \\
\hline & & $X$ \\
\hline & & $X$ \\
\hline$X$ & & \\
\hline$X$ & & \\
\hline
\end{tabular}

Comments: No comments

\section{Results/Conclusions}

Are formulas consistent in spreadsheet cells?

Are calculations correct?

Are conclusions consistent with results?

Are conclusions consistent with applicable limits?

Comments: No comments

Reviewer Sign/Date:

Richard C. Daniel $\mathrm{C}$

\begin{tabular}{|c|c|c|} 
Yes & \multicolumn{1}{c}{ No } & \multicolumn{1}{c}{ NA } \\
\hline$X$ & & \\
\hline$X$ & & \\
\hline & & $X$ \\
\hline & & $X$ \\
\hline
\end{tabular}

Page 1 of 1 
PNNL-20650, Rev. 2

Filename: 52578-TI037 (Reviewed).xIsm Worksheet: Summary Page

\begin{tabular}{|c|c|l|c|c|c|c|}
\hline & A & \multicolumn{1}{|c|}{ B } & C & D & E & F \\
\hline 1 & Summary of PSD Records & & & & \\
\hline 2 & Source File: & "52578-TI-037.mea" & & & & \\
\hline 3 & & & & & & \\
\hline & $\begin{array}{c}\text { Record } \\
\text { number }\end{array}$ & Sample Name & Average result records & $\begin{array}{c}\text { Pump } \\
\text { speed }\end{array}$ & $\begin{array}{c}\text { Stirrer } \\
\text { speed }\end{array}$ & $\begin{array}{c}\text { Ultrasonic } \\
\text { level }\end{array}$ \\
\hline 5 & 61 & TI008-SP-Unsonicated & $13,14,15,49,50,51^{\prime \prime \prime}$ & 2000 & 0 & 0 \\
\hline 6 & 62 & TI008-SP-Sonicated & $17,18,19,53,54,55^{\prime \prime \prime}$ & 2000 & 0 & 100 \\
\hline 7 & 65 & TI008-SQ-Sonicated & $29,30,31,41,42,43^{\prime \prime \prime \prime}$ & 2000 & 0 & 100 \\
\hline 8 & 66 & TI008-SQ-Post Sonicated & $33,34,35,45,46,47^{\prime \prime \prime}$ & 2000 & 0 & 0 \\
\hline 9 & 67 & TI008-SQ-Unsonicated & $25,26,27,37,38,39^{\prime \prime \prime}$ & 2000 & 0 & 0 \\
\hline 10 & 68 & TI008-SP-Post Sonicated & $21,22,23,57,58,59 " \prime \prime$ & 2000 & 0 & 0 \\
\hline
\end{tabular}


PNNL-20650, Rev. 2

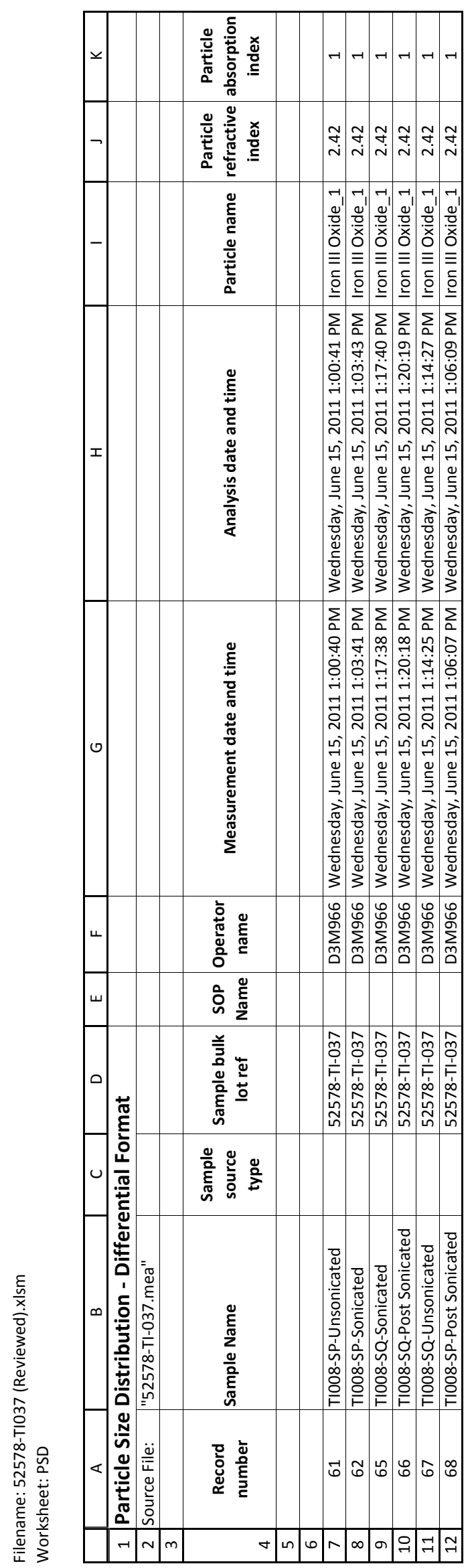


PNNL-20650, Rev. 2

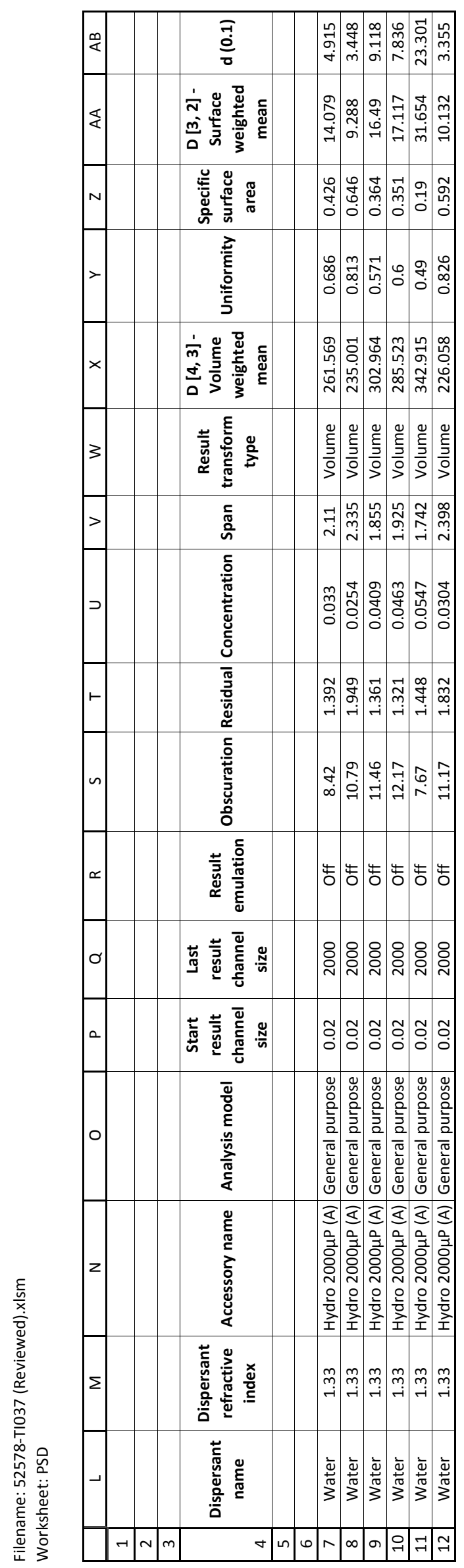


PNNL-20650, Rev. 2

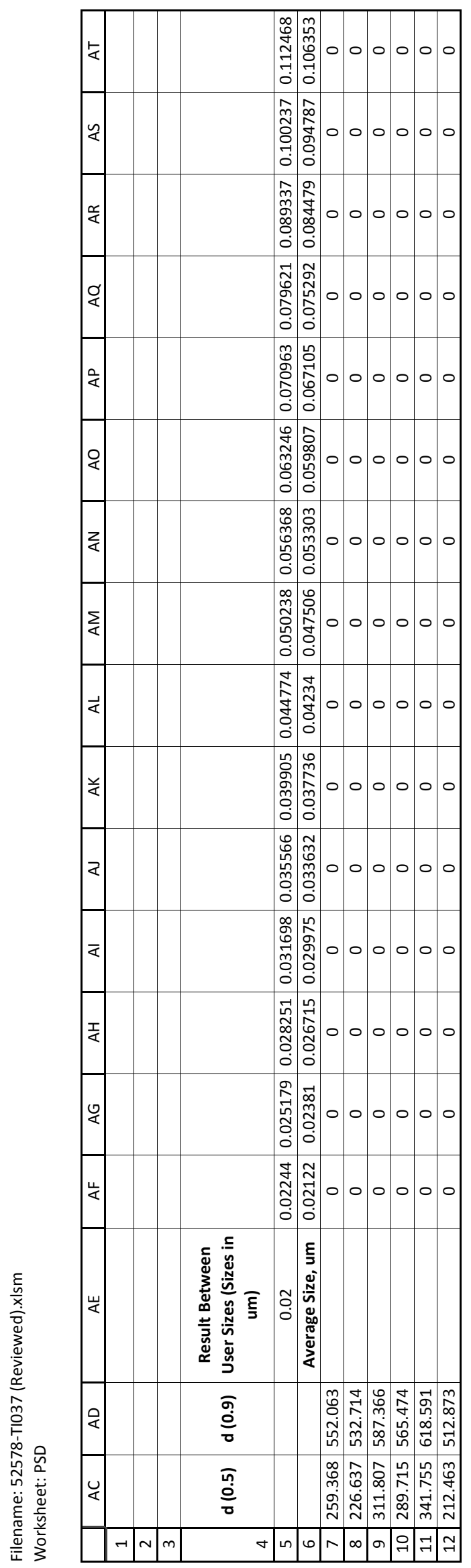


PNNL-20650, Rev. 2

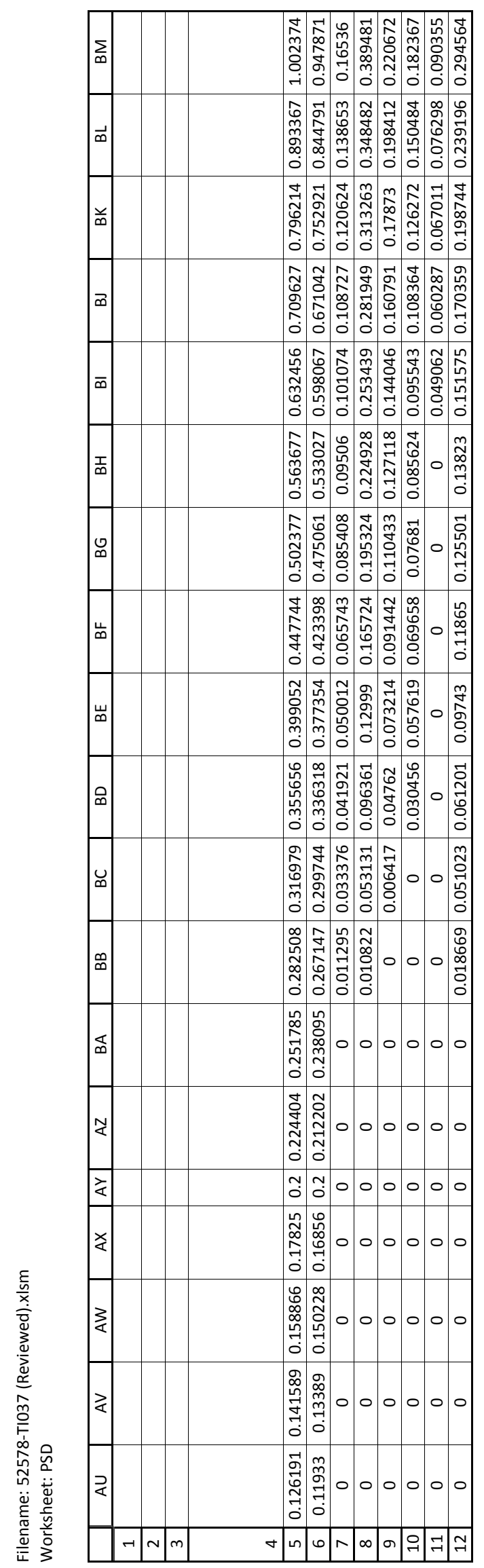

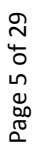


PNNL-20650, Rev. 2

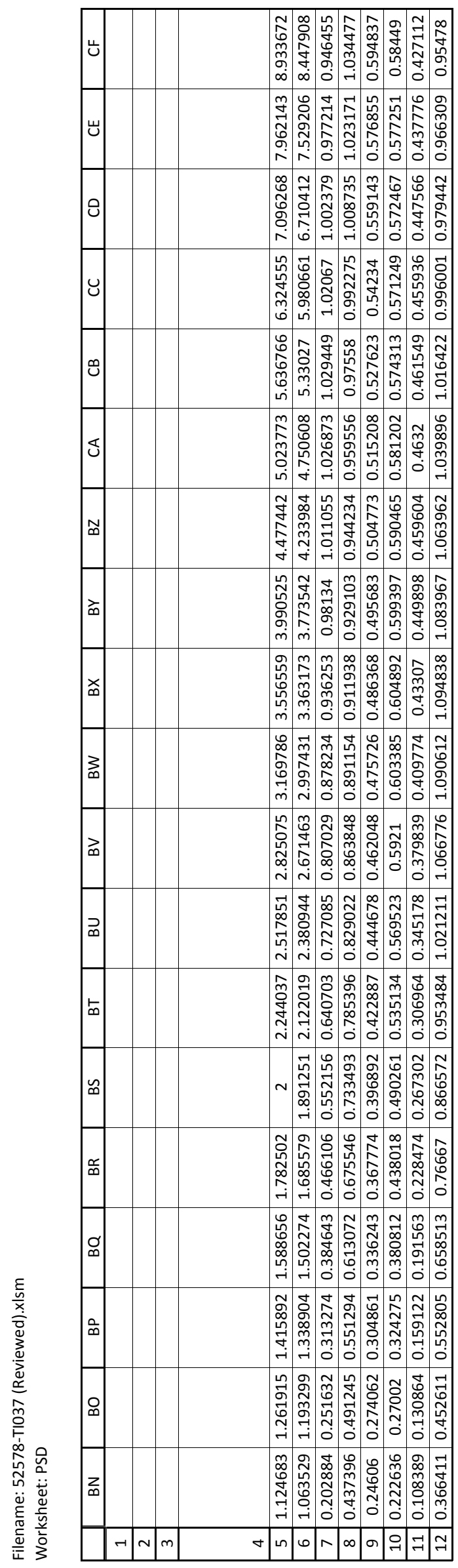

จิ
4
0
6
0
0
0
0 
PNNL-20650, Rev. 2

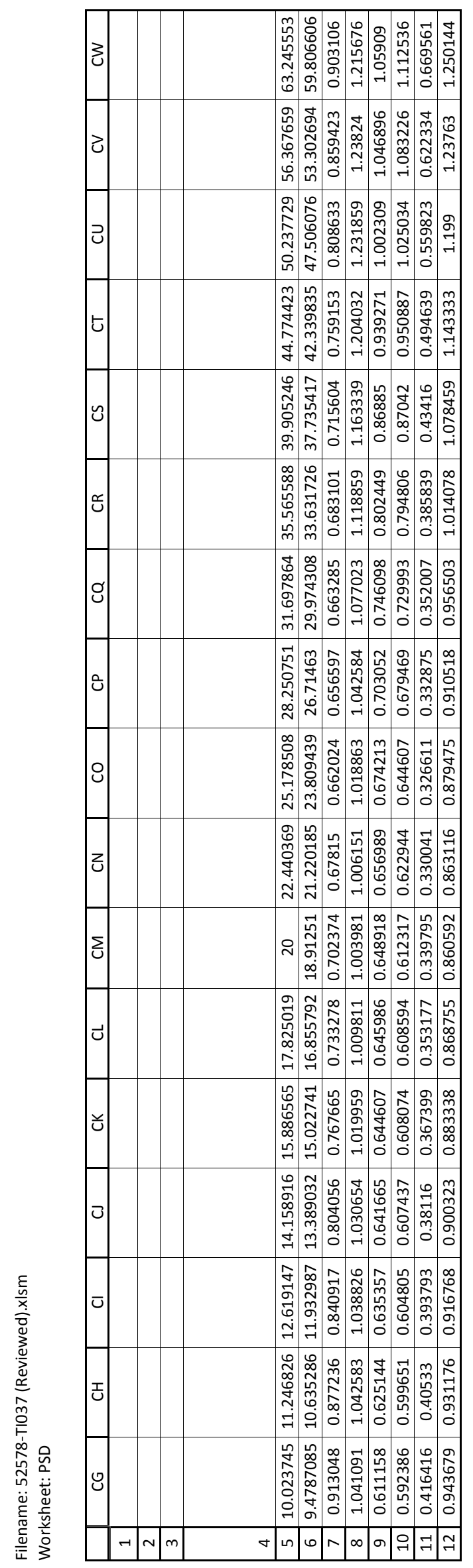

จิ
4
0
1
0
0
0
0 
PNNL-20650, Rev. 2

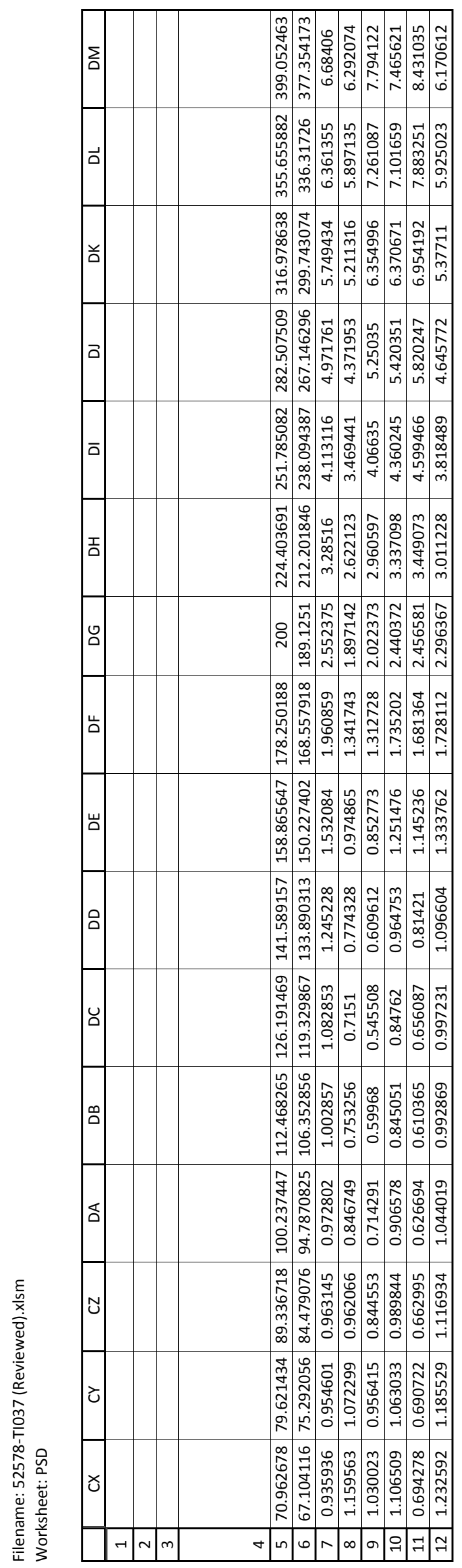

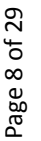


PNNL-20650, Rev. 2

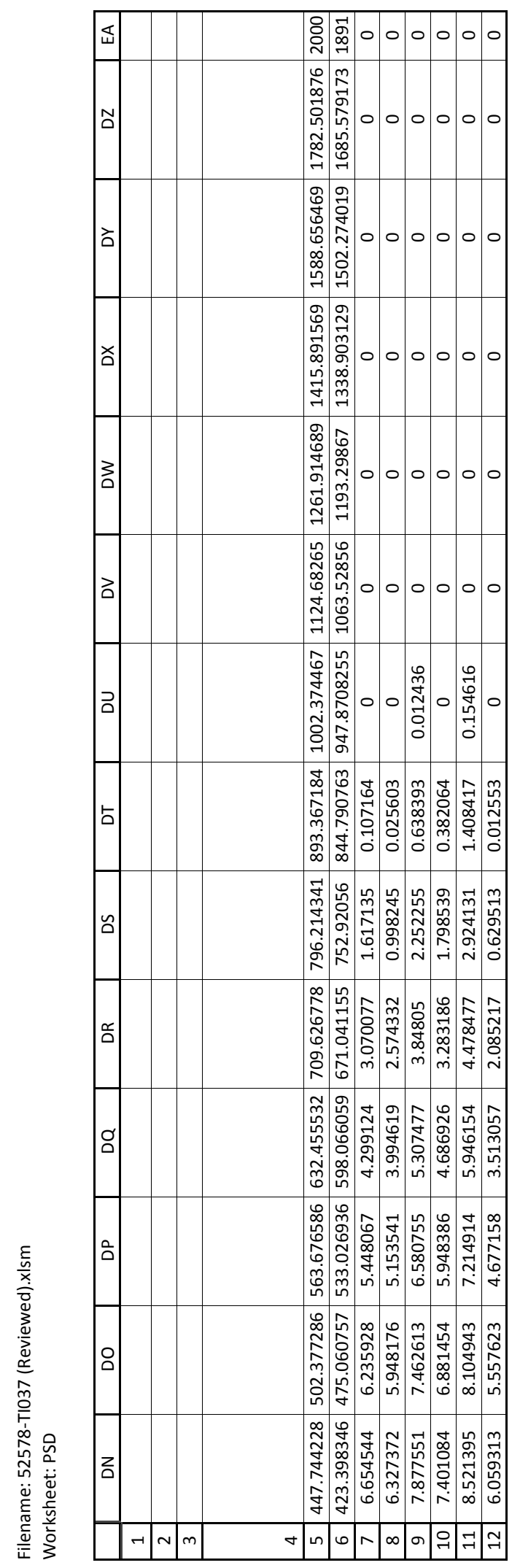

నิ
$\overleftarrow{4}$
0
0
0
0
0
0 
PNNL-20650, Rev. 2

Filename: 52578-TI037 (Reviewed).xlsm

Worksheet: PSD

\begin{tabular}{|r|l|}
\hline & \multicolumn{1}{|c|}{ EB } \\
\hline 1 & \\
\hline 2 & \\
\hline 3 & \\
\hline & Operator Notes \\
4 & \\
\hline 5 & \\
\hline 6 & \\
\hline 7 & Average of 6 measurements from 52578-TI-037.mea \\
\hline 8 & Average of 6 measurements from 52578-TI-037.mea \\
\hline 9 & Average of 6 measurements from 52578-TI-037.mea \\
\hline 10 & Average of 6 measurements from 52578-TI-037.mea \\
\hline 11 & Average of 6 measurements from 52578-TI-037.mea \\
\hline 12 & Average of 6 measurements from 52578-TI-037.mea \\
\hline
\end{tabular}

July 7, 2011

Page 10 of 29 
PNNL-20650, Rev. 2

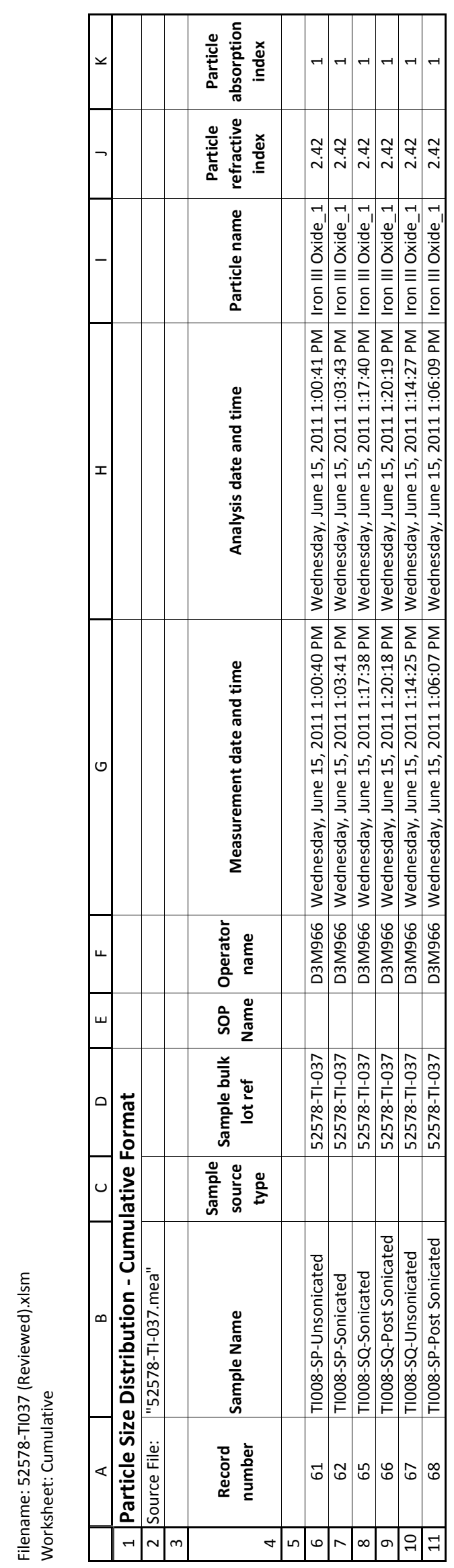

\begin{tabular}{l}
2 \\
\multirow{T}{0}{} \\
7 \\
7 \\
0 \\
0 \\
0 \\
0
\end{tabular} 
PNNL-20650, Rev. 2

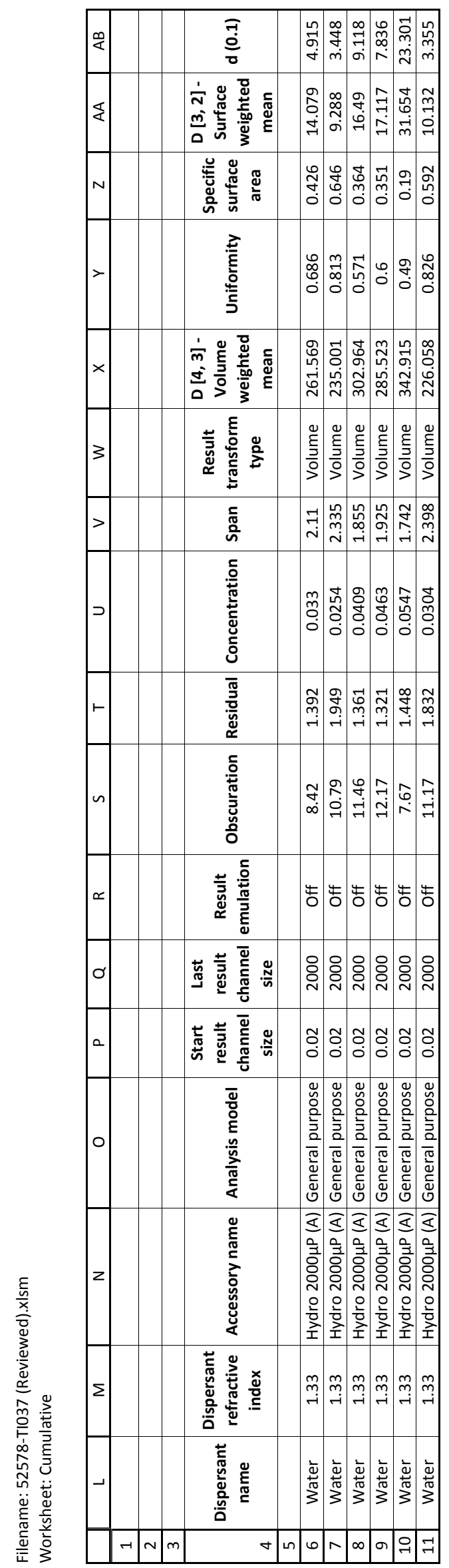

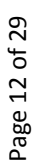


PNNL-20650, Rev. 2

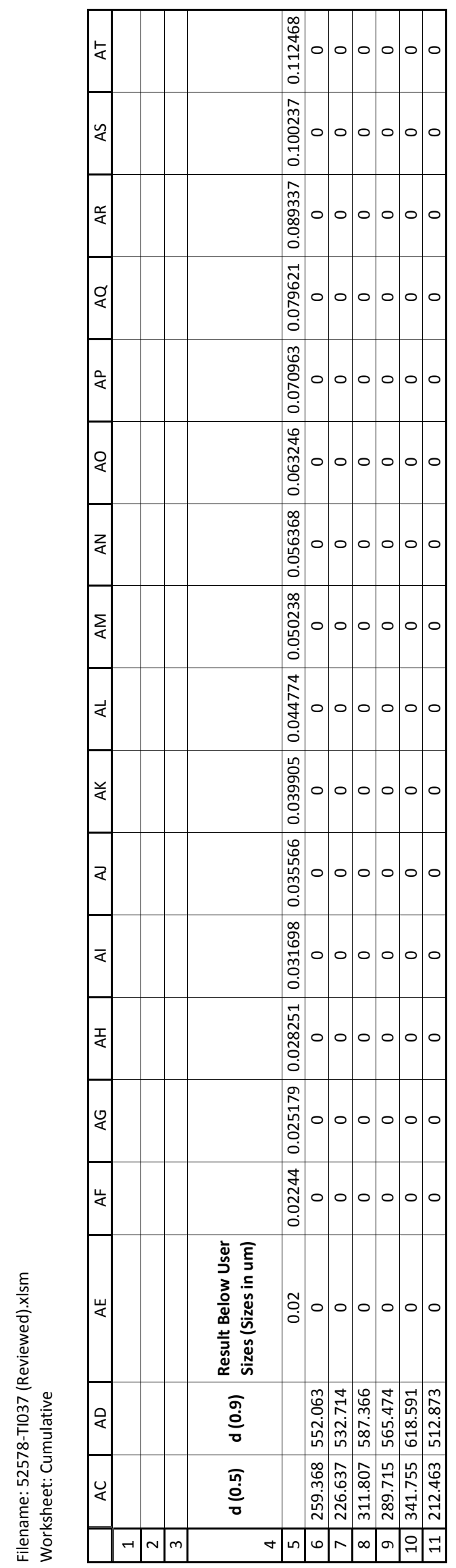


PNNL-20650, Rev. 2

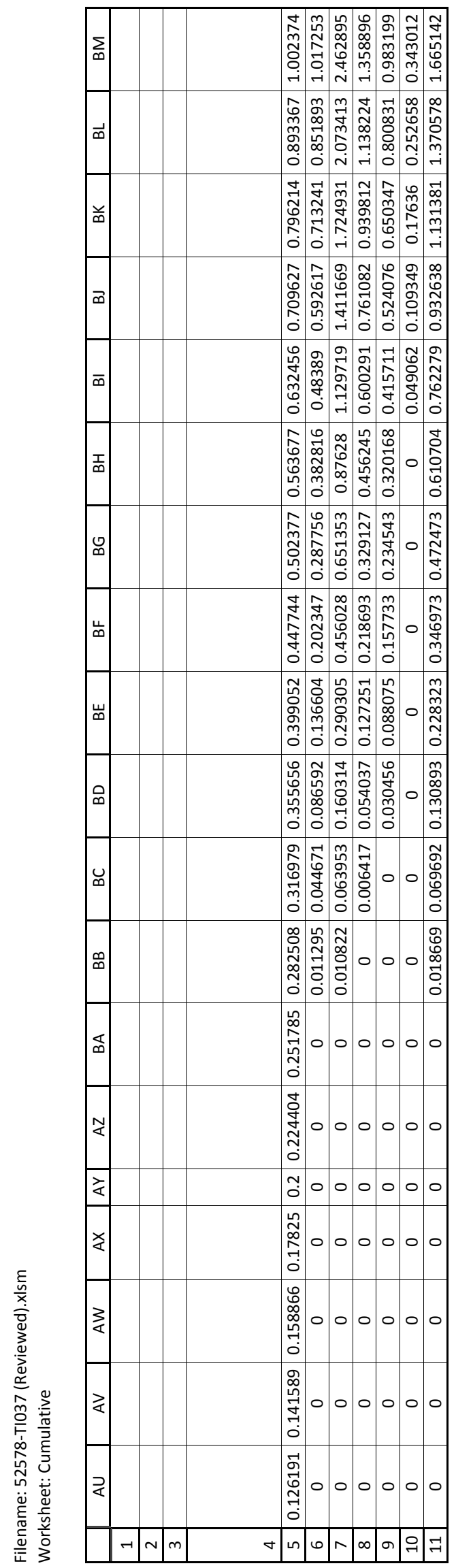

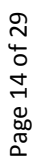


PNNL-20650, Rev. 2

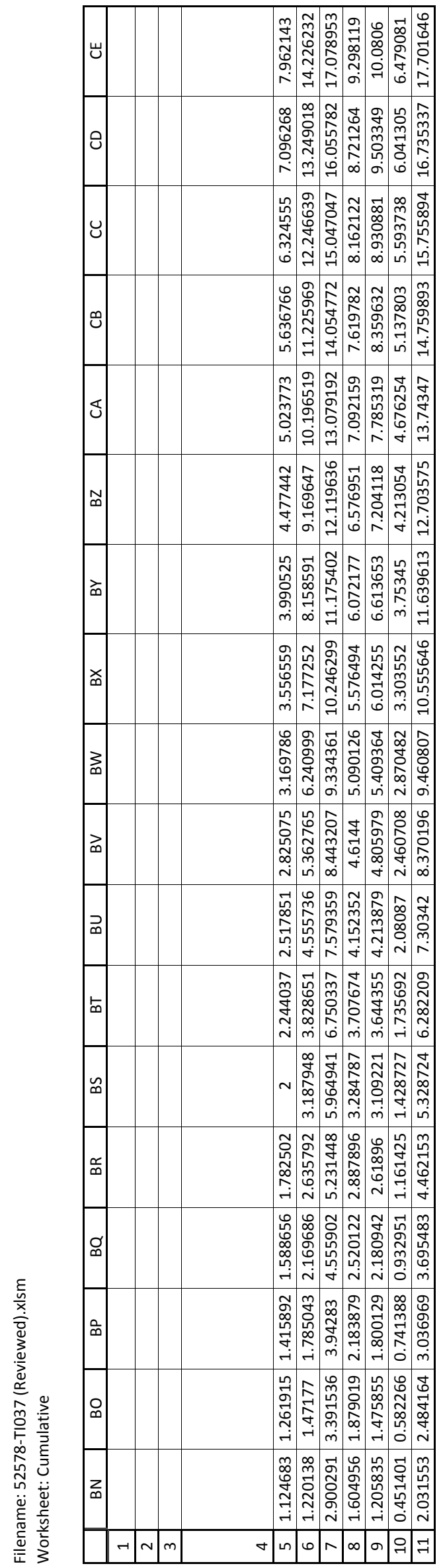

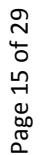


PNNL-20650, Rev. 2

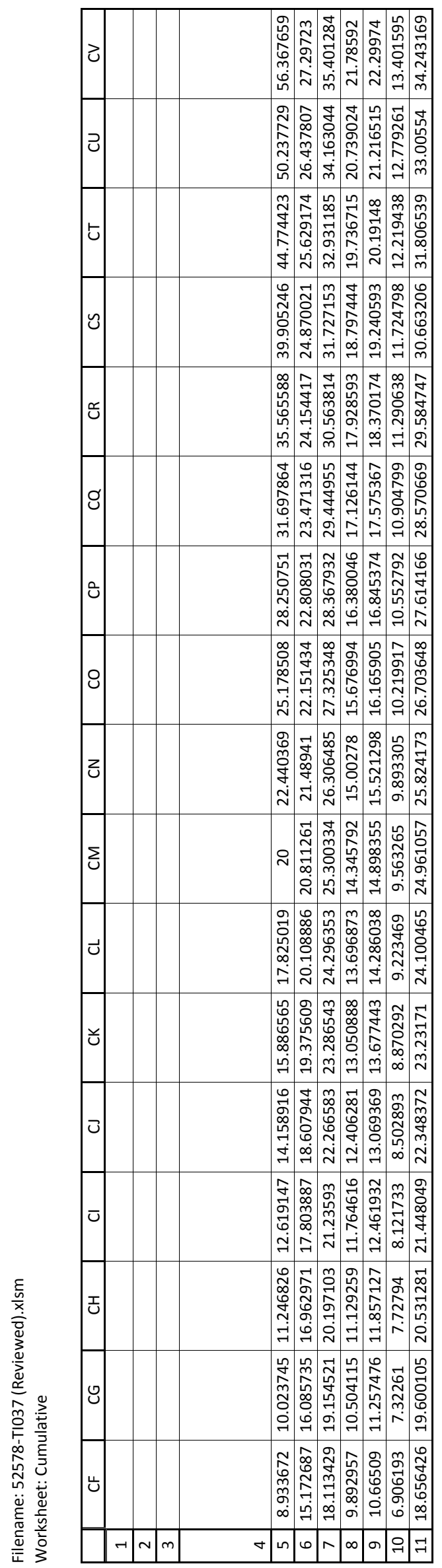

Tิ
4
0
0
0
0
0
0
0 
PNNL-20650, Rev. 2

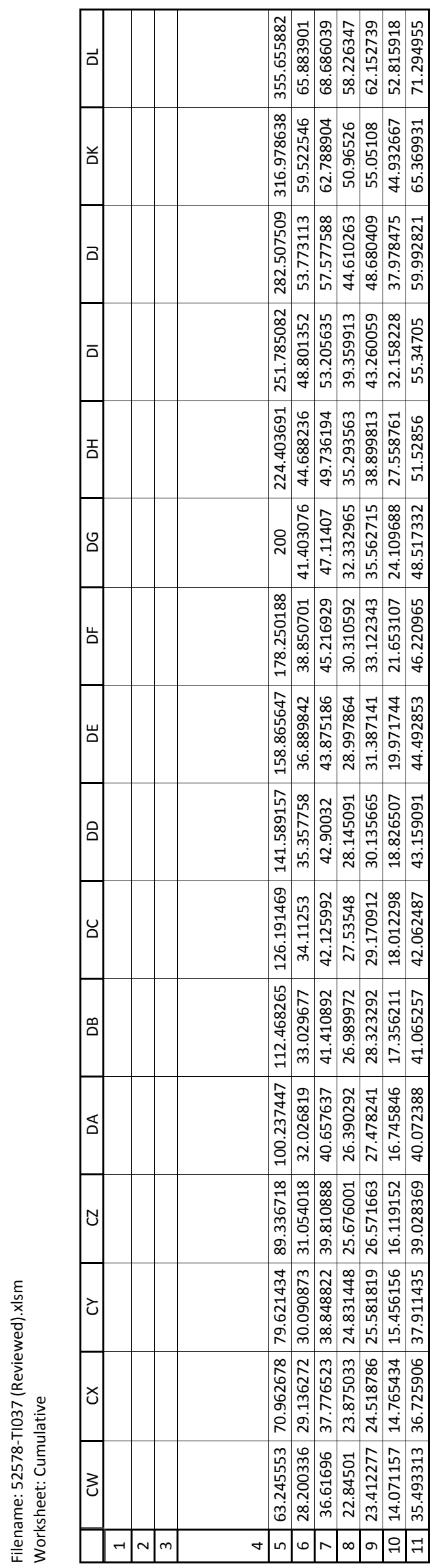

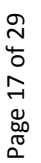


PNNL-20650, Rev. 2

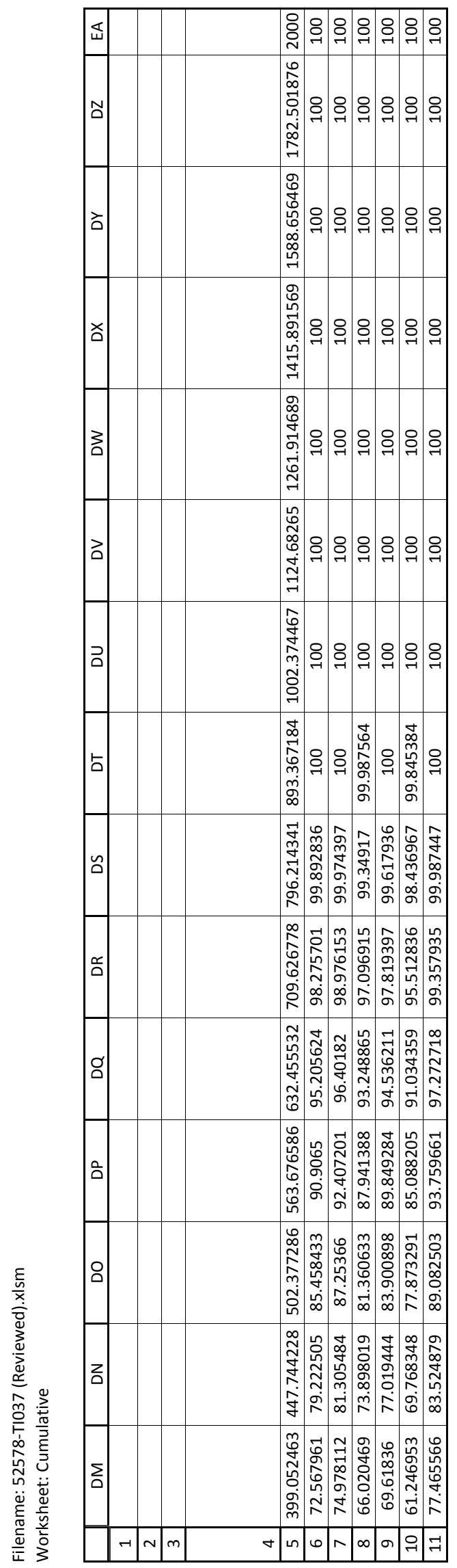

\begin{tabular}{l}
2 \\
\multirow{2}{0}{} \\
$\infty$ \\
01 \\
0 \\
0 \\
0 \\
0
\end{tabular} 
PNNL-20650, Rev. 2

Filename: 52578-TI037 (Reviewed).xlsm

Worksheet: Cumulative

\begin{tabular}{|r|l|}
\hline & \multicolumn{1}{|c|}{ EB } \\
\hline 1 & \\
\hline 2 & \\
\hline 3 & \\
\hline & Operator Notes \\
4 & \\
\hline 5 & \\
\hline 6 & Average of 6 measurements from 52578-TI-037.mea \\
\hline 7 & Average of 6 measurements from 52578-TI-037.mea \\
\hline 8 & Average of 6 measurements from 52578-TI-037.mea \\
\hline 9 & Average of 6 measurements from 52578-TI-037.mea \\
\hline 10 & Average of 6 measurements from 52578-TI-037.mea \\
\hline 11 & Average of 6 measurements from 52578-TI-037.mea \\
\hline
\end{tabular}


PNNL-20650, Rev. 2

Filename: 52578-TI037 (Reviewed).xlsm

Worksheet: Cumulative Tables

\begin{tabular}{|c|c|c|c|c|c|}
\hline & $\mathrm{A}$ & $\mathrm{B}$ & $\mathrm{C}$ & $\mathrm{D}$ & $\mathrm{E}$ \\
\hline 1 & \multicolumn{3}{|c|}{ Particle Size Distribution - Cumulative Table Format } & & \\
\hline 2 & Source File: & "52578-TI-037.mea" & & & \\
\hline \multicolumn{6}{|l|}{3} \\
\hline 4 & Record number & 61 & 62 & 67 & 65 \\
\hline 5 & Sample Name & TI008-SP-Unsonicated & TI008-SP-Sonicated & TI008-SQ-Unsonicated & TI008-SQ-Sonicated \\
\hline 6 & Analysis model & General purpose & General purpose & General purpose & General purpose \\
\hline 7 & Particle refractive index & 2.42 & 2.42 & 2.42 & 2.42 \\
\hline 8 & Pump speed & 2000 & 2000 & 2000 & 2000 \\
\hline 9 & & & & & \\
\hline 10 & Size, $\mu \mathrm{m}$ & \multicolumn{4}{|c|}{ Percent Cumulative Under Size by Volume, \% } \\
\hline 11 & 0.05 & 0 & 0 & 0 & 0 \\
\hline 12 & 0.5 & 0.28 & 0.64 & 0.00 & 0.32 \\
\hline 13 & 1 & 1.01 & 2.45 & 0.34 & 1.35 \\
\hline 14 & 2.5 & 4.51 & 7.53 & 2.06 & 4.12 \\
\hline 15 & 5 & 10.2 & 13.0 & 4.66 & 7.07 \\
\hline 16 & 10 & 16.1 & 19.1 & 7.31 & 10.5 \\
\hline 17 & 20 & 20.8 & 25.3 & 9.56 & 14.3 \\
\hline 18 & 40 & 24.9 & 31.8 & 11.7 & 18.8 \\
\hline 19 & 60 & 27.8 & 36.1 & 13.8 & 22.4 \\
\hline 20 & 80 & 30.1 & 38.9 & 15.5 & 24.9 \\
\hline 21 & 100 & 32.0 & 40.6 & 16.7 & 26.4 \\
\hline 22 & 125 & 34.0 & 42.1 & 18.0 & 27.5 \\
\hline 23 & 150 & 36.1 & 43.4 & 19.3 & 28.5 \\
\hline 24 & 175 & 38.5 & 45.0 & 21.3 & 30.1 \\
\hline 25 & 200 & 41.4 & 47.1 & 24.1 & 32.3 \\
\hline 26 & 300 & 56.7 & 60.2 & 41.5 & 47.8 \\
\hline 27 & 400 & 72.7 & 75.1 & 61.4 & 66.2 \\
\hline 28 & 500 & 85.2 & 87.0 & 77.6 & 81.1 \\
\hline 29 & 600 & 93.4 & 94.7 & 88.5 & 91.0 \\
\hline 30 & 700 & 98.0 & 98.7 & 95.1 & 96.7 \\
\hline 31 & 800 & 99.9 & 100 & 98.5 & 99.4 \\
\hline 32 & 1000 & 100 & 100 & 100 & 100 \\
\hline
\end{tabular}


PNNL-20650, Rev. 2

Filename: 52578-TI037 (Reviewed).xIsm

Worksheet: Percentiles

\begin{tabular}{|c|c|c|c|c|c|}
\hline & A & $B$ & C & $\mathrm{D}$ & $E$ \\
\hline 1 & \multicolumn{3}{|c|}{ Particle Size Distribution - Percentiles } & & \\
\hline 2 & Source File: & "52578-TI-037.mea" & & & \\
\hline \multicolumn{6}{|l|}{3} \\
\hline 4 & Record number & 61 & 62 & 67 & 65 \\
\hline 5 & Sample Name & TI008-SP-Unsonicated & TI008-SP-Sonicated & TI008-SQ-Unsonicated & TI008-SQ-Sonicated \\
\hline 6 & Pump speed & 2000 & 2000 & 2000 & 2000 \\
\hline 7 & Stirrer speed & 0 & 0 & 0 & 0 \\
\hline 8 & Ultrasonic level & 0 & 100 & 0 & 100 \\
\hline 9 & $d(0.010), \mu m$ & 0.99 & 0.60 & 1.65 & 0.83 \\
\hline 10 & $d(0.050), \mu m$ & 2.69 & 1.72 & 5.45 & 3.10 \\
\hline 11 & $d(0.100), \mu m$ & 4.92 & 3.45 & 23.3 & 9.12 \\
\hline 12 & $d(0.500), \mu m$ & 259 & 227 & 342 & 312 \\
\hline 13 & $d(0.900), \mu m$ & 552 & 533 & 619 & 587 \\
\hline 14 & $d(0.950), \mu m$ & 628 & 605 & 699 & 663 \\
\hline 15 & $\mathrm{~d}(0.990), \mu \mathrm{m}$ & 737 & 711 & 823 & 776 \\
\hline
\end{tabular}


PNNL-20650, Rev. 2

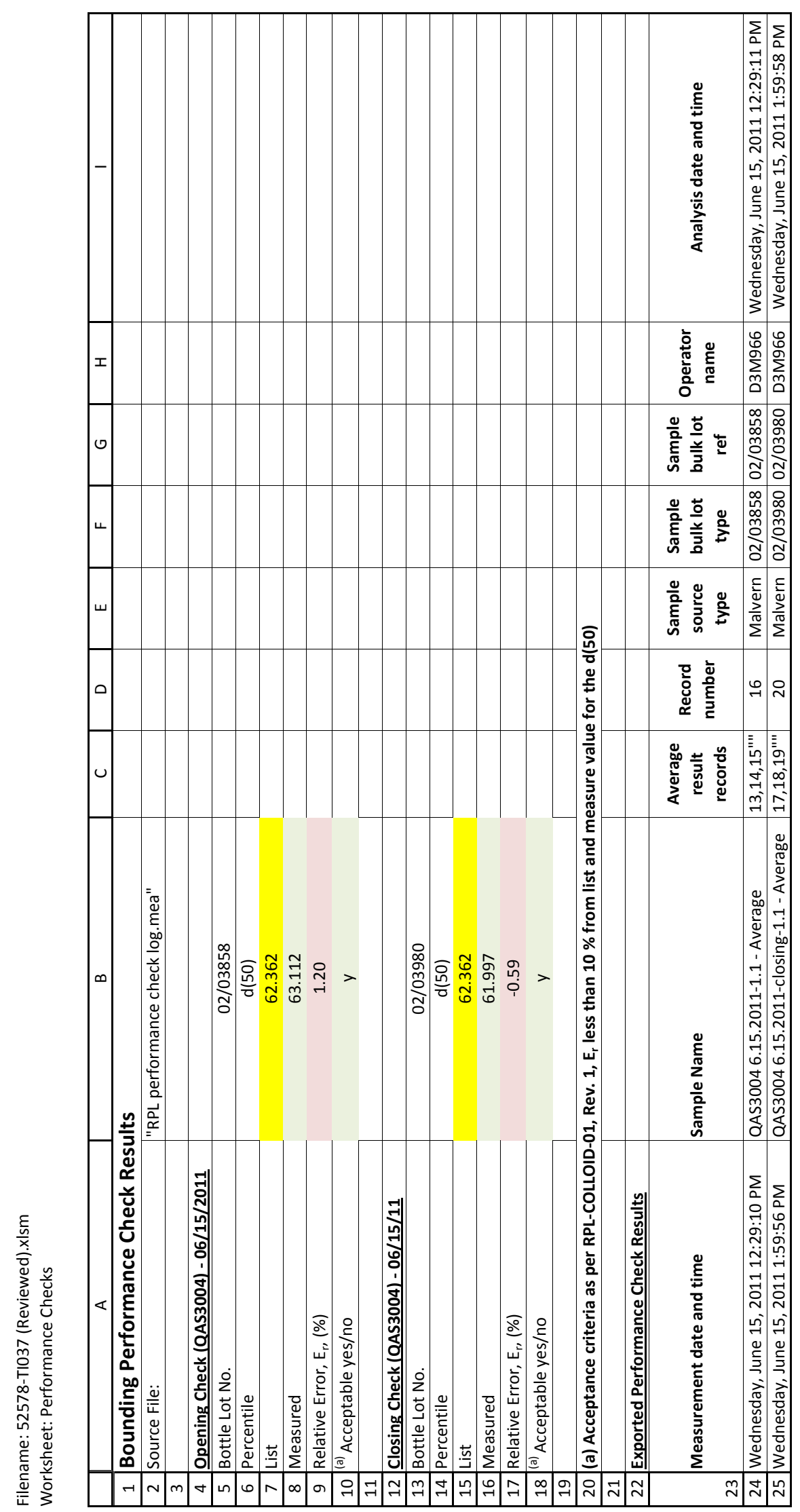

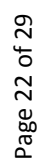


PNNL-20650, Rev. 2

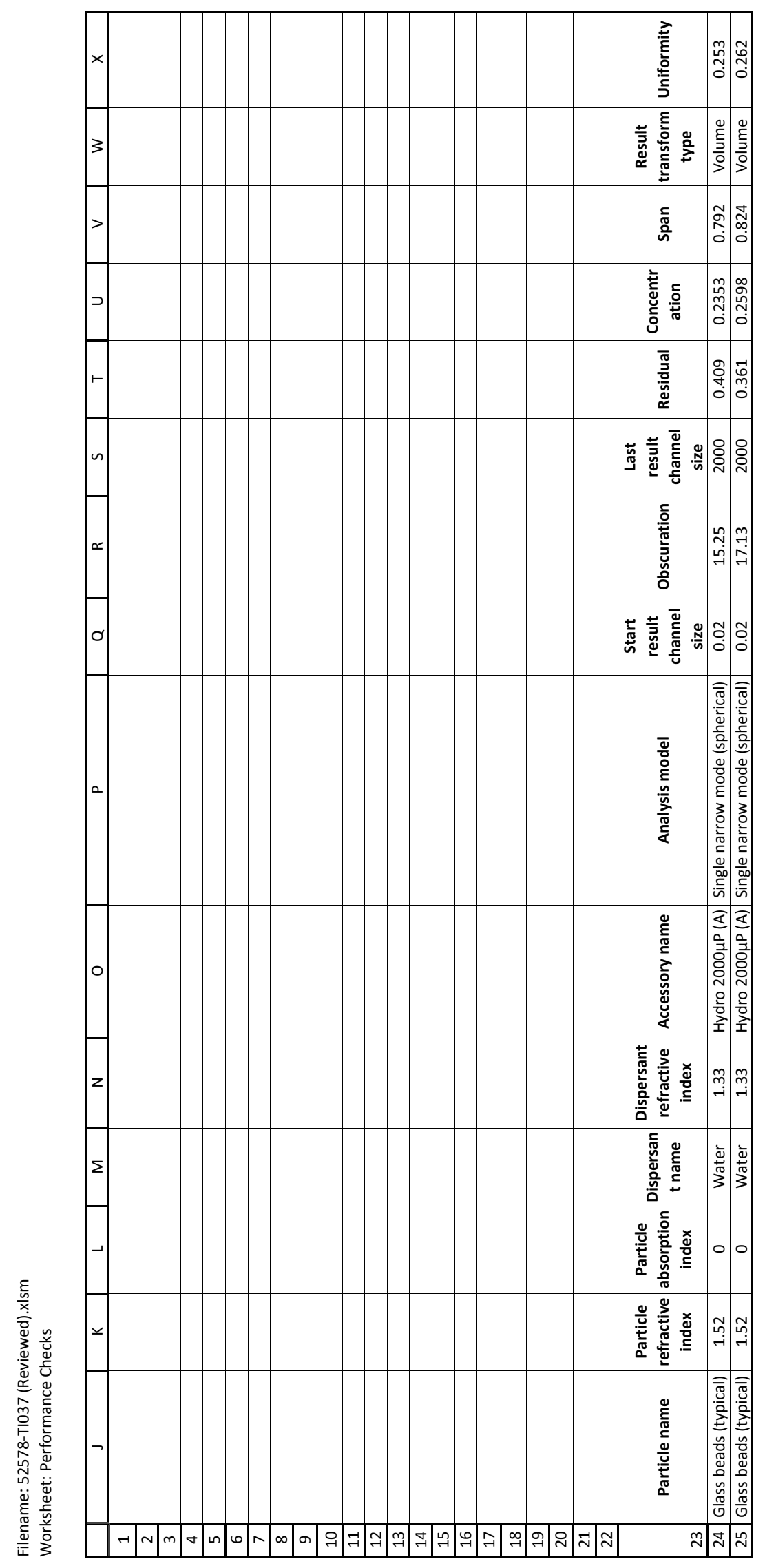

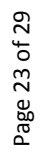


PNNL-20650, Rev. 2

Filename: 52578-TI037 (Reviewed).xlsm

Worksheet: Performance Checks

\begin{tabular}{|r|l|l|l|}
\hline & $Y$ & Z & AA \\
\hline 1 & & & \\
\hline 2 & & & \\
\hline 3 & & & \\
\hline 4 & & & \\
\hline 5 & & & \\
\hline 6 & & & \\
\hline 7 & & & \\
\hline 8 & & & \\
\hline 9 & & & \\
\hline 10 & & & \\
\hline 11 & & & \\
\hline 12 & & & \\
\hline 13 & & & \\
\hline 14 & & & \\
\hline 15 & & & \\
\hline 16 & & & \\
\hline 17 & & & \\
\hline 18 & & & \\
\hline 19 & & & \\
\hline 20 & & & \\
\hline 21 & & & \\
\hline 22 & & & \\
\hline & & & \\
& d (0.1) & d (0.5) & d (0.9) \\
\hline 23 & & & \\
\hline 24 & 41.303 & 63.112 & 91.269 \\
\hline 25 & 39.273 & 61.997 & 90.367 \\
\hline
\end{tabular}

July 7, 2011

Page 24 of 29 
PNNL-20650, Rev. 2

Filename: 52578-TI037 (Reviewed).xlsm

Worksheet: Graph Information

\begin{tabular}{|c|c|c|}
\hline & A & $\mathrm{B}$ \\
\hline 1 & \multicolumn{2}{|c|}{ Summary of Graphs and Graph Reference Cells } \\
\hline \multicolumn{3}{|l|}{2} \\
\hline 3 & Stand Alone Charts & \\
\hline \multicolumn{3}{|l|}{4} \\
\hline 5 & Graph \#: & 1 \\
\hline 6 & Worksheet Label: & TI-008-SP PSD chart \\
\hline 7 & Number of Data Series: & 3 \\
\hline 8 & Series 1 Formula: & =SERIES("Unsonicated",PSD!\$AF\$6:\$EA\$6,PSD!\$AF\$7:\$EA\$7,1) \\
\hline 9 & Series 2 Formula: & =SERIES("Sonication",PSD!\$AF\$6:\$EA\$6,PSD!\$AF\$8:\$EA\$8,2) \\
\hline 10 & Series 3 Formula: & =SERIES("Post Sonicated",PSD!\$AF\$6:\$EA\$6,PSD!\$AF\$12:\$EA 12,3$)$ \\
\hline \multicolumn{3}{|l|}{11} \\
\hline \multicolumn{3}{|l|}{12} \\
\hline 13 & Graph \#: & 2 \\
\hline 14 & Worksheet Label: & TI008-SQ PSD chart \\
\hline 15 & Number of Data Series: & 3 \\
\hline 16 & Series 1 Formula: & =SERIES("Unsonicated",PSD!\$AF\$6:\$EA\$6,PSD!\$AF\$11:\$EA\$11,1) \\
\hline 17 & Series 2 Formula: & =SERIES("Sonication",PSD!\$AF\$6:\$EA\$6,PSD!\$AF\$9:\$EA\$9,2) \\
\hline 18 & Series 3 Formula: & =SERIES("Post Sonicated",PSD!\$AF\$6:\$EA\$6,PSD!\$AF\$10:\$EA $\$ 10,3)$ \\
\hline \multicolumn{3}{|l|}{19} \\
\hline \multicolumn{3}{|l|}{20} \\
\hline 21 & Graph \#: & 3 \\
\hline 22 & Worksheet Label: & TI-008-SP CUM chart \\
\hline 23 & Number of Data Series: & 3 \\
\hline 24 & Series 1 Formula: & =SERIES("Unsonicated",Cumulative!\$AE\$5:\$EA\$5,Cumulative!\$AE\$6:\$EA\$6,1) \\
\hline 25 & Series 2 Formula: & =SERIES("Sonication $100 \% "$ "Cumulative!\$AE\$5:\$EA\$5,Cumulative!\$AE\$7:\$EA\$7,2) \\
\hline 26 & Series 3 Formula: & =SERIES("Post Sonicated",Cumulative!\$AE\$5:\$EA\$5,Cumulative!\$AE\$11:\$EA\$11,3) \\
\hline \multicolumn{3}{|l|}{27} \\
\hline \multicolumn{3}{|l|}{28} \\
\hline 29 & Graph \#: & 4 \\
\hline 30 & Worksheet Label: & TI-008-SQ CUM chart \\
\hline 31 & Number of Data Series: & 3 \\
\hline 32 & Series 1 Formula: & =SERIES("Unsonicated",Cumulative!\$AE\$5:\$EA\$5,Cumulative!\$AE\$10:\$EA\$10,1) \\
\hline 33 & Series 2 Formula: & 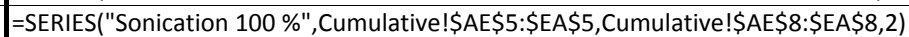 \\
\hline 34 & Series 3 Formula: & =SERIES("Post Sonicated",Cumulative!\$AE\$5:\$EA\$5,Cumulative!\$AE\$9:\$EA\$9,3) \\
\hline 35 & & \\
\hline
\end{tabular}


PNNL-20650, Rev. 2

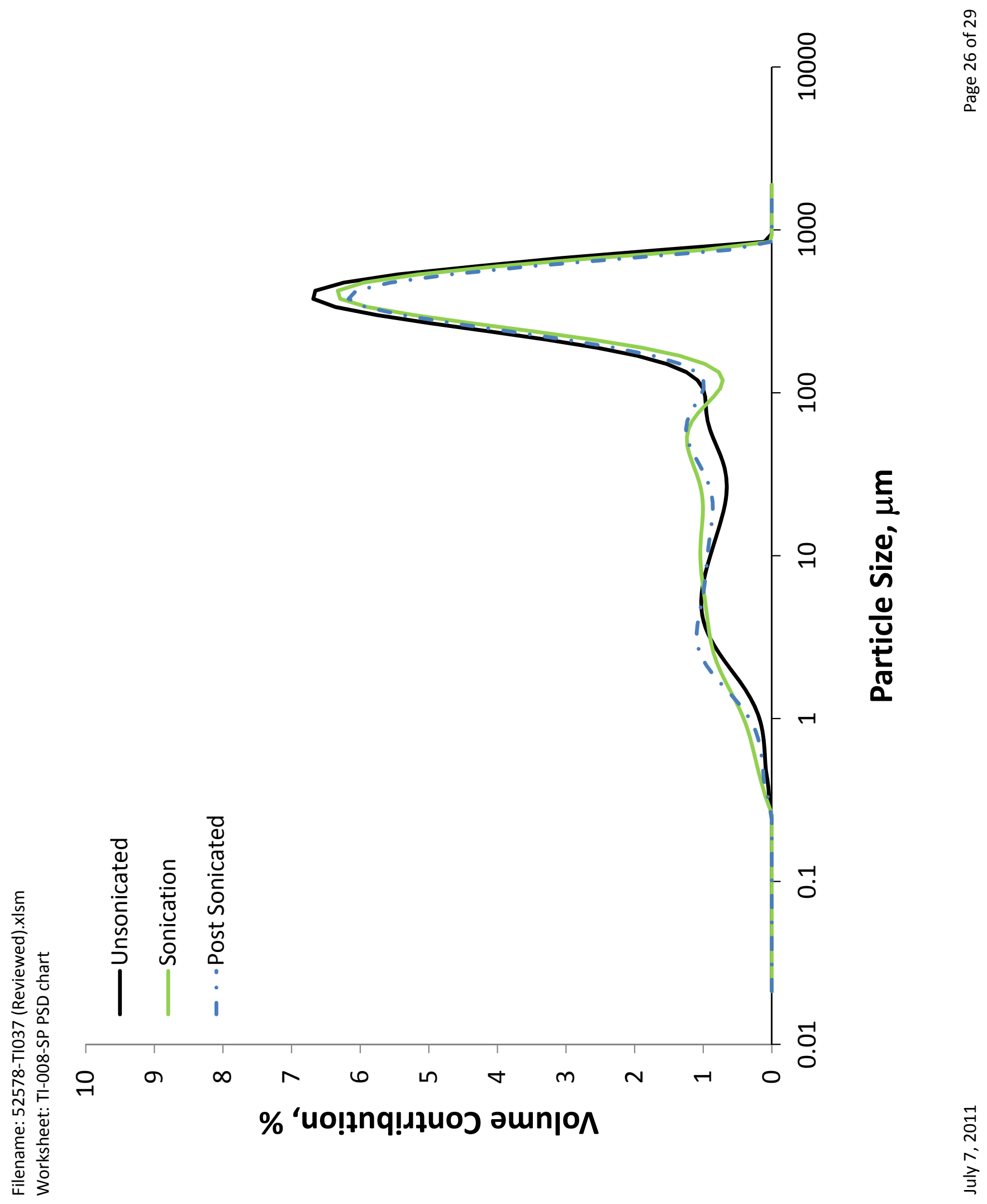


PNNL-20650, Rev. 2

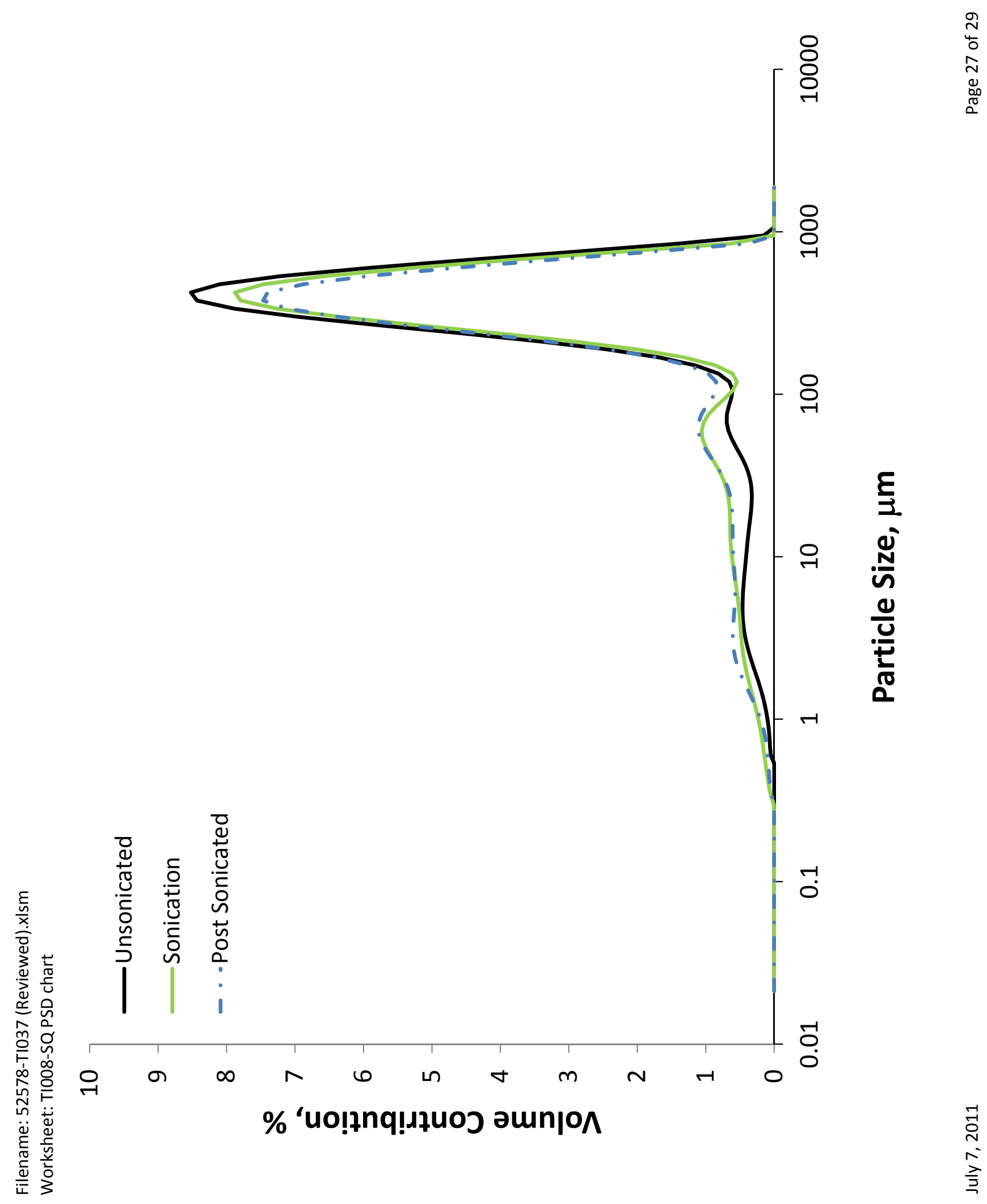


PNNL-20650, Rev. 2

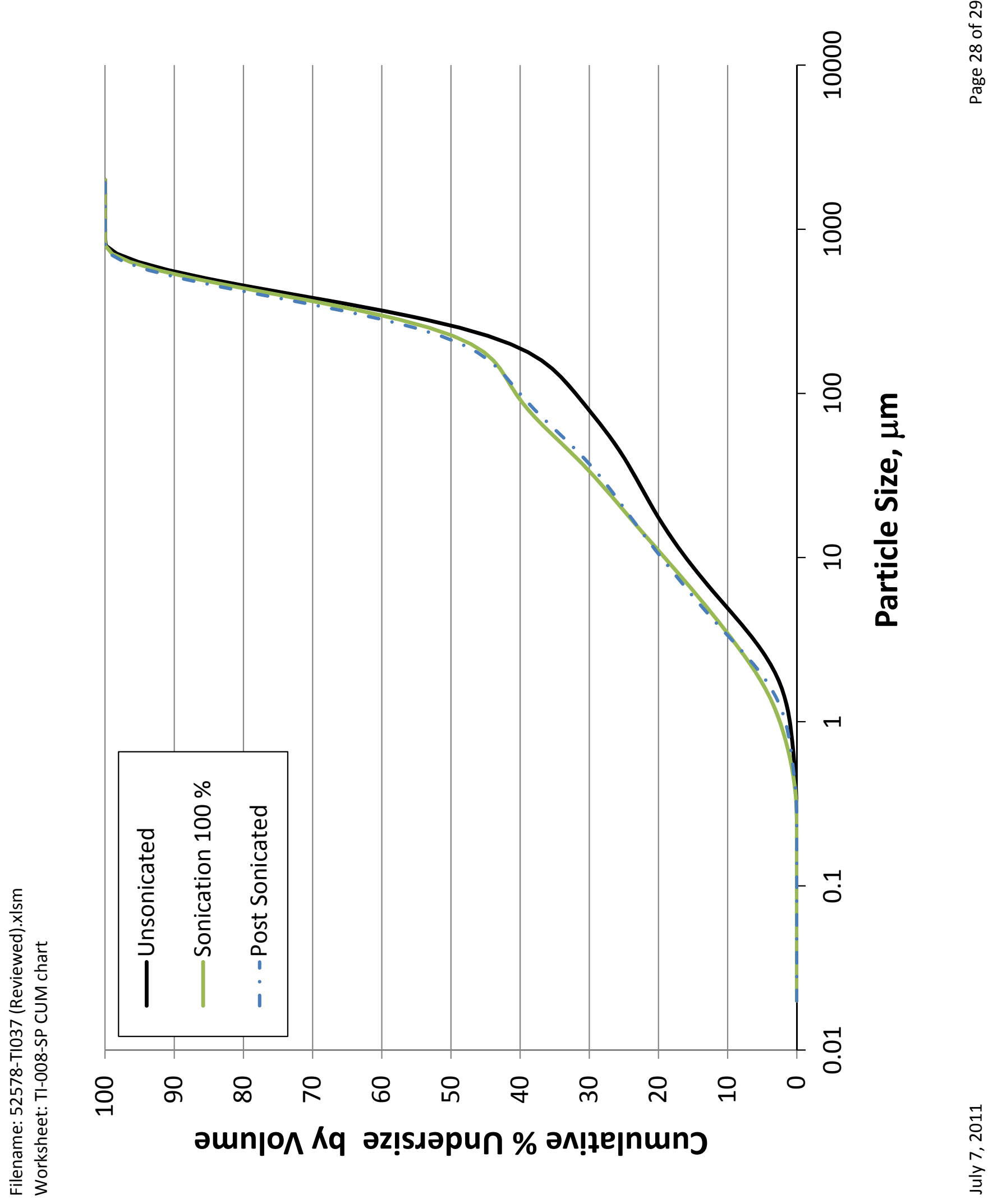


PNNL-20650, Rev. 2

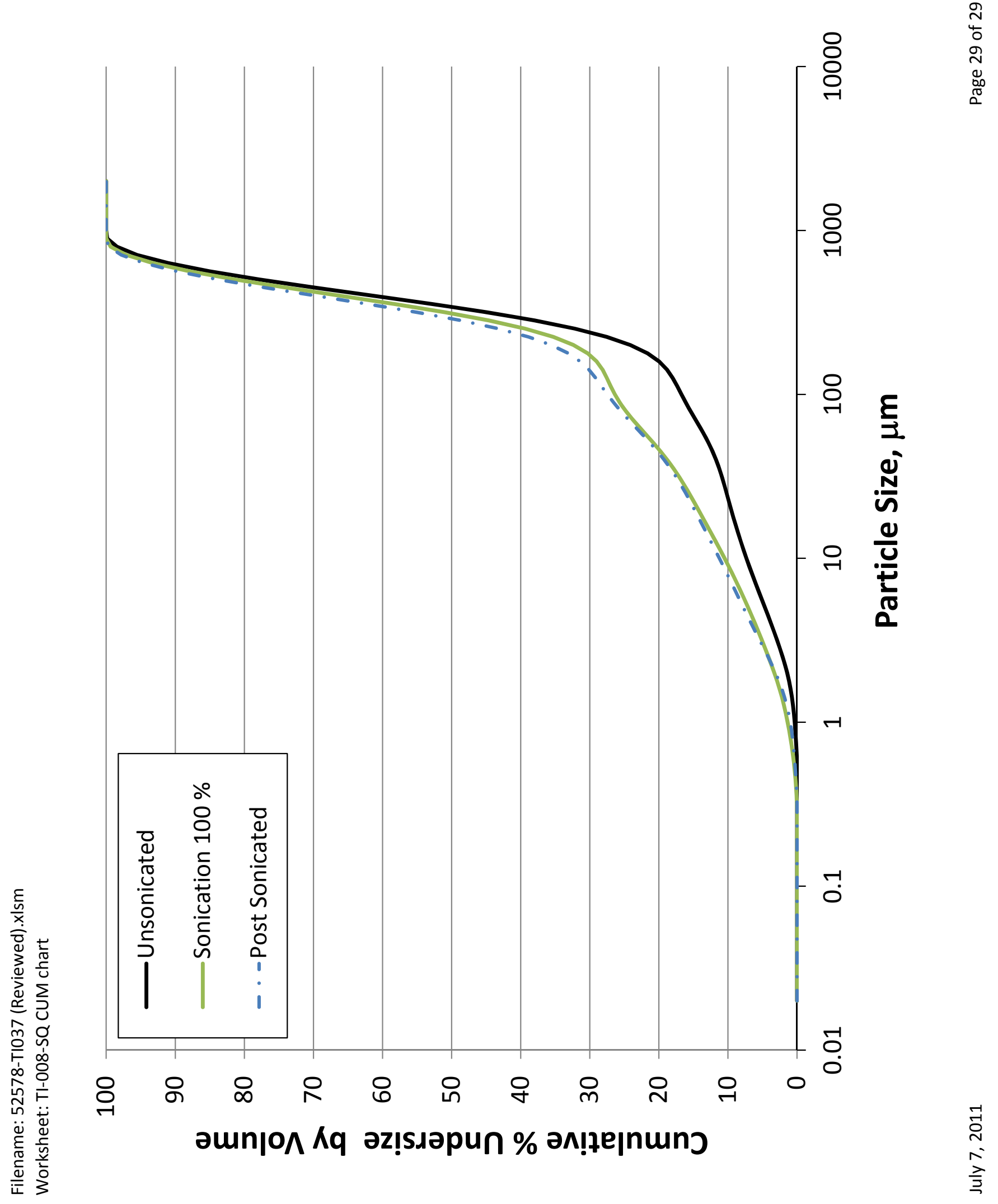


PNNL-20650, Rev. 2

Filename: TI008 averaged data.xlsm

Worksheet: summary

\begin{tabular}{|c|c|c|c|c|c|c|}
\hline & A & $\mathrm{B}$ & $\mathrm{C}$ & $\mathrm{D}$ & $\mathrm{E}$ & $\mathrm{F}$ \\
\hline 1 & \multicolumn{3}{|c|}{ Summary of Records - Supplemental Data Package } & & & \\
\hline 2 & \multicolumn{3}{|c|}{ This data package supplements KW210 PSD data in "52578-TI037 (Reviewed).xlsm" } & & & \\
\hline 3 & \multicolumn{4}{|c|}{ The source Malvern measurement file for the current data package is "52578-TI-037.mea" } & & \\
\hline \multicolumn{7}{|l|}{4} \\
\hline 5 & $\begin{array}{l}\text { Record } \\
\text { number }\end{array}$ & Sample Name & Average result records & $\begin{array}{l}\text { Pump } \\
\text { speed }\end{array}$ & $\begin{array}{l}\text { Stirrer } \\
\text { speed }\end{array}$ & $\begin{array}{c}\text { Ultrasonic } \\
\text { level }\end{array}$ \\
\hline 6 & 69 & TI008-Unsonicated Average & $13,14,15,25,26,27,37,38,39,49,50,51 " '$ & 2000 & 0 & 0 \\
\hline 7 & 70 & TI008-Sonicated Average & 17,18,19,29,30,31,41,42,43,53,54,55"' & 2000 & 0 & 100 \\
\hline 8 & 71 & TI008-Post Sonication Average & $21,22,23,33,34,35,45,46,47,57,58,59 " ' "$ & 2000 & 0 & 0 \\
\hline
\end{tabular}

August 3, 2011

Page 1 of 21 
PNNL-20650, Rev. 2

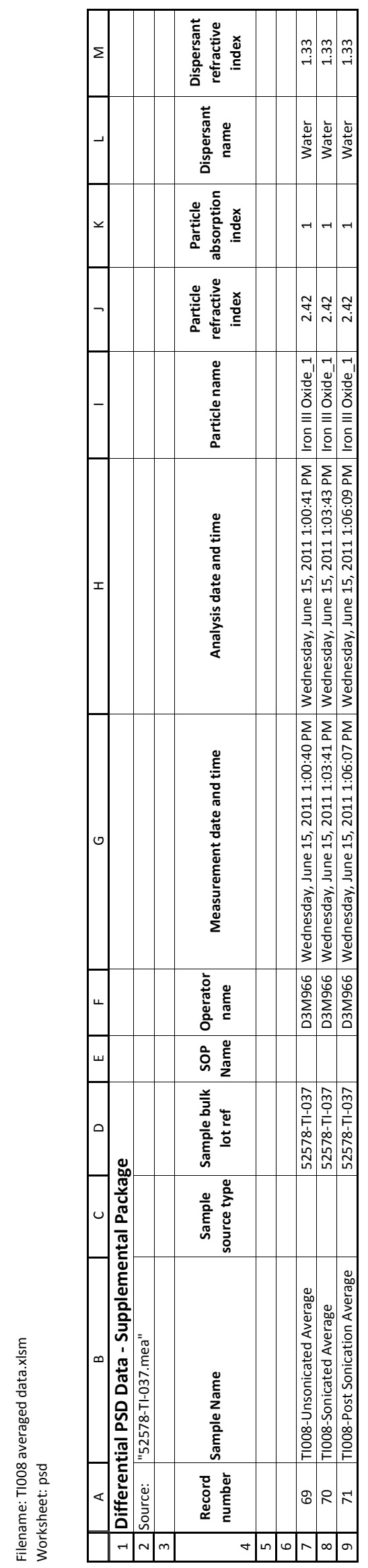

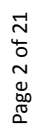


PNNL-20650, Rev. 2

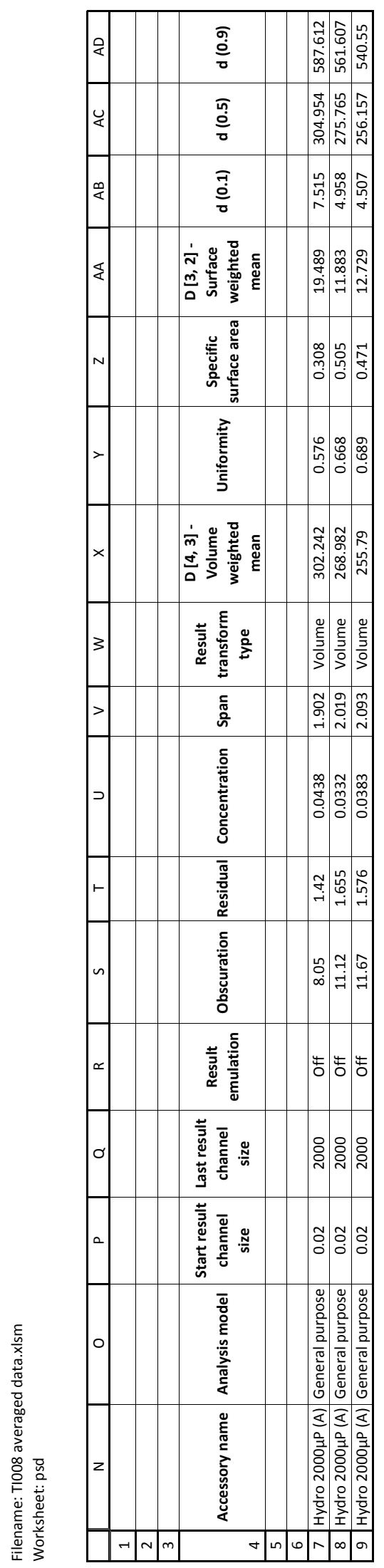


PNNL-20650, Rev. 2

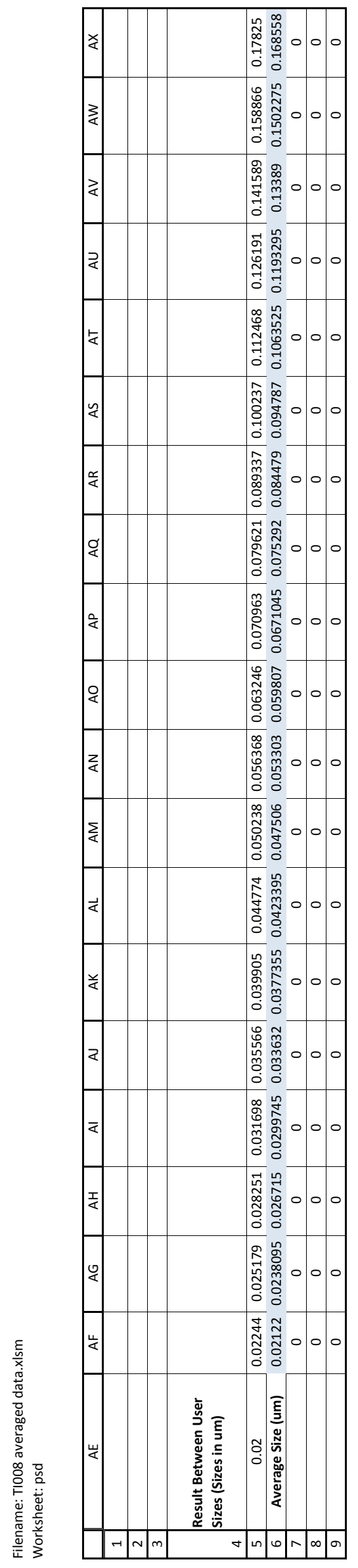


PNNL-20650, Rev. 2

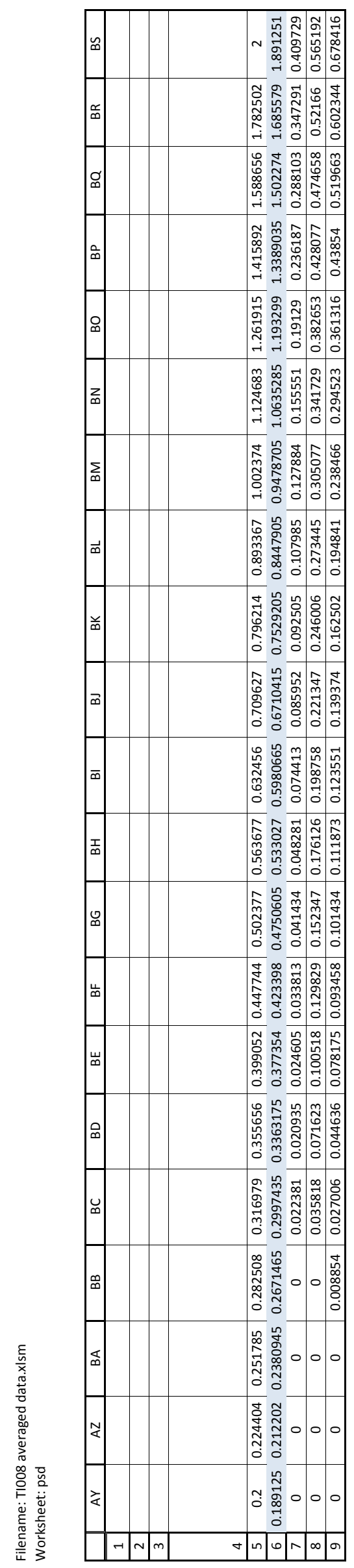


PNNL-20650, Rev. 2

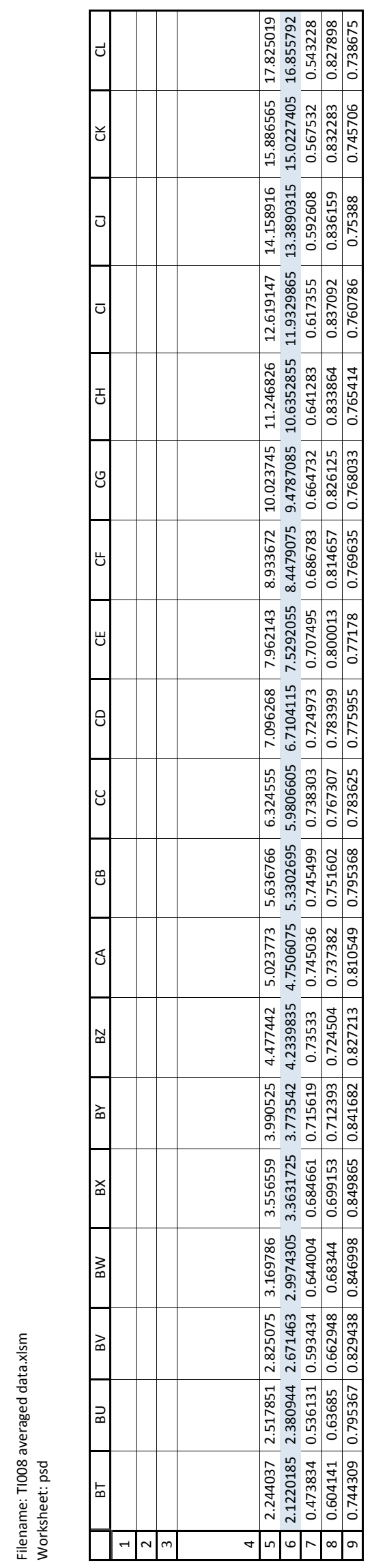


PNNL-20650, Rev. 2

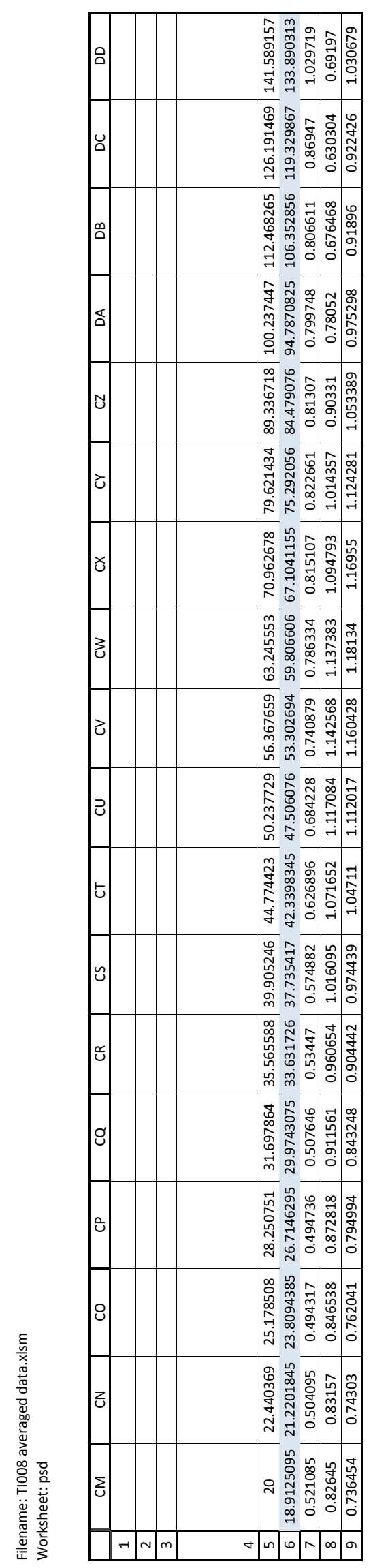


PNNL-20650, Rev. 2

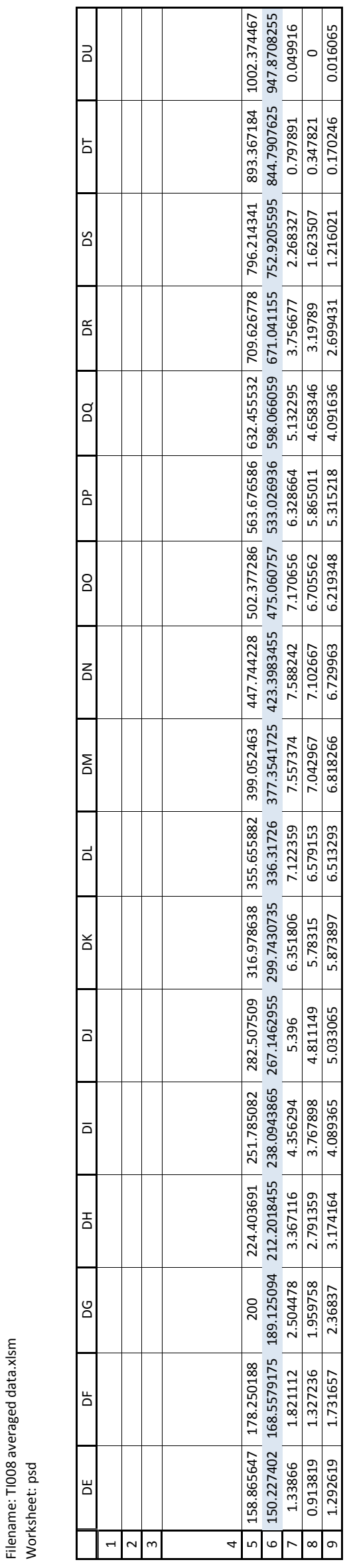


PNNL-20650, Rev. 2

Filename: TI008 averaged data.xIsm Worksheet: psd

\begin{tabular}{|c|c|c|c|c|c|c|c|}
\hline & DV & DW & DX & DY & DZ & EA & EB \\
\hline \multicolumn{8}{|l|}{1} \\
\hline \multirow{2}{*}{\multicolumn{8}{|c|}{$\frac{2}{3}$}} \\
\hline & & & & & & & \\
\hline 4 & & & & & & & Operator Notes \\
\hline 5 & 1124.68265 & 1261.914689 & 1415.891569 & 1588.656469 & 1782.501876 & 2000 & \\
\hline 6 & 1063.528559 & 1193.29867 & 1338.903129 & 1502.274019 & 1685.579173 & 1891.250938 & \\
\hline 7 & 0 & 0 & 0 & 0 & 0 & 0 & Average of 12 measurements from 52578-TI-037.mea \\
\hline 8 & 0 & 0 & 0 & 0 & 0 & 0 & Average of 12 measurements from 52578-TI-037.mea \\
\hline 9 & 0 & 0 & 0 & 0 & 0 & 0 & Average of 12 measurements from 52578-TI-037.mea \\
\hline
\end{tabular}


PNNL-20650, Rev. 2

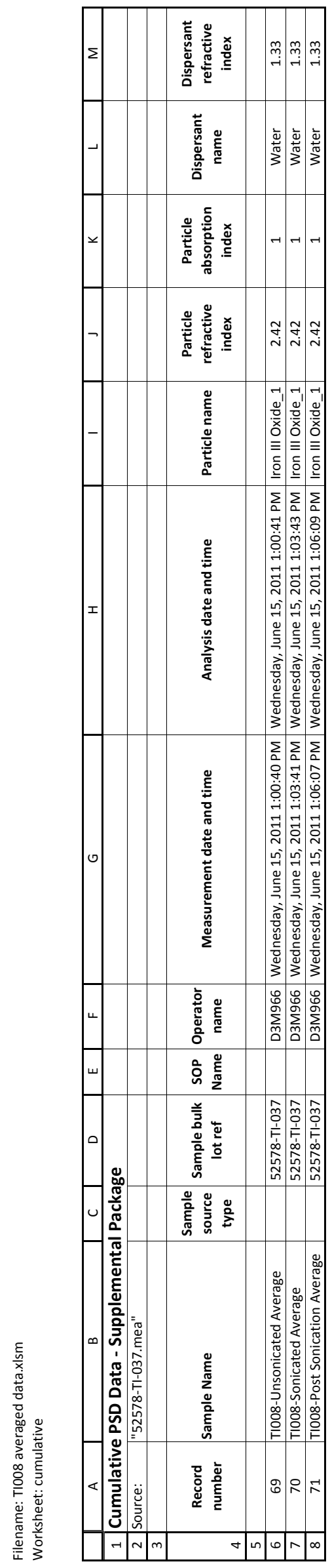


PNNL-20650, Rev. 2

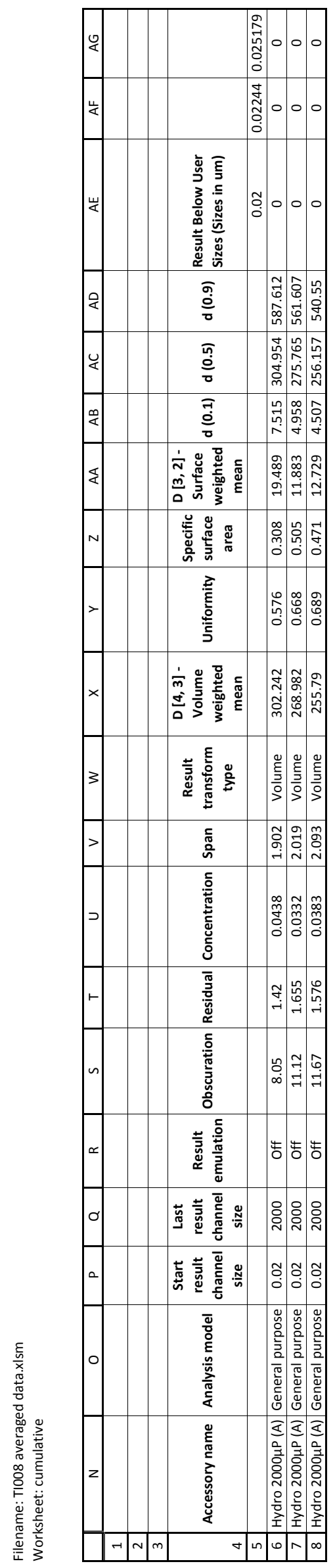


PNNL-20650, Rev. 2

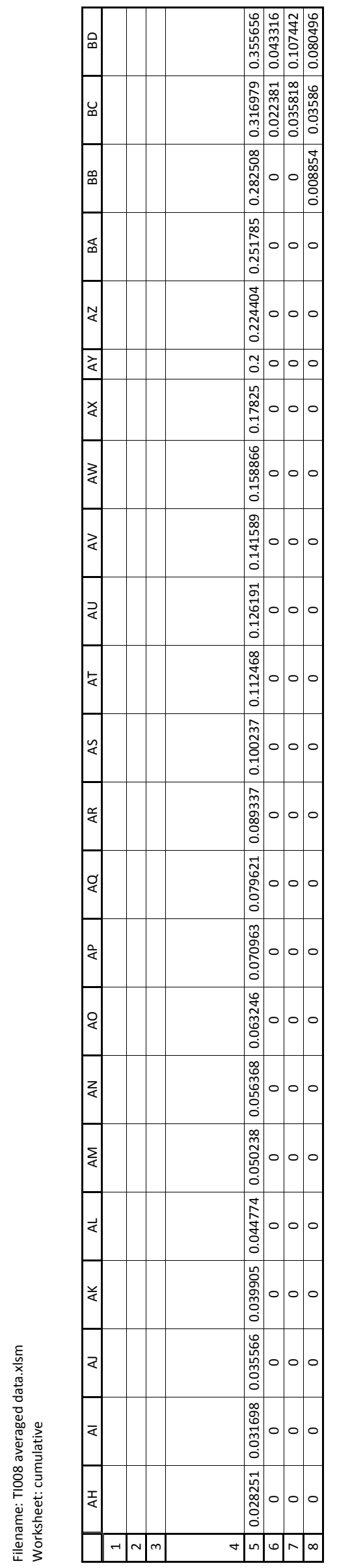

I
4
$\stackrel{0}{0}$
7
0
0
0
0 
PNNL-20650, Rev. 2

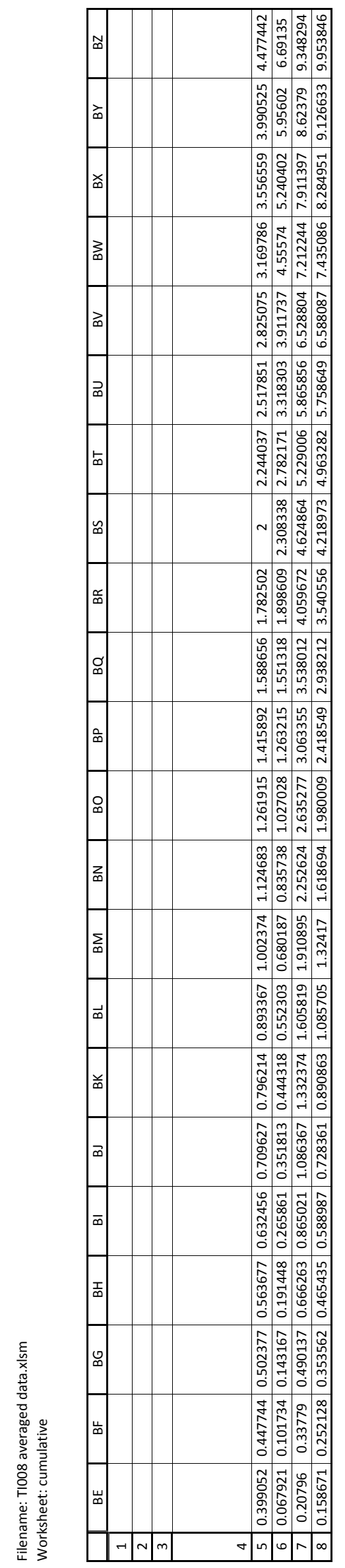


PNNL-20650, Rev. 2

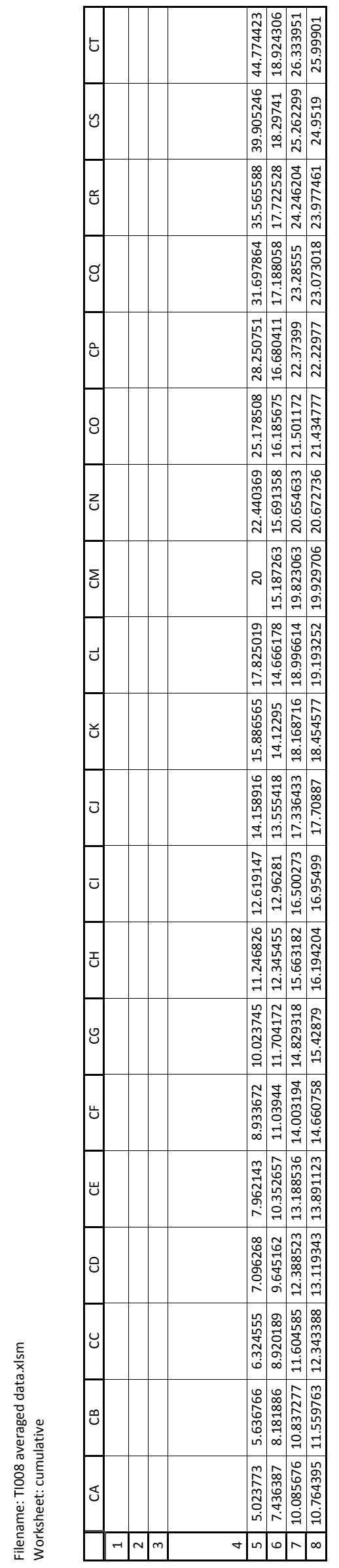


PNNL-20650, Rev. 2

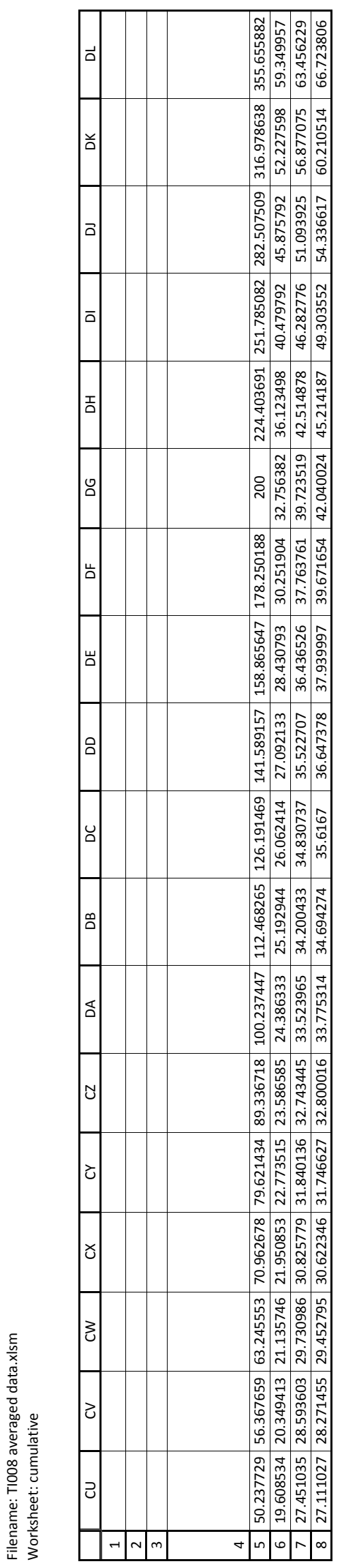

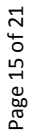


PNNL-20650, Rev. 2

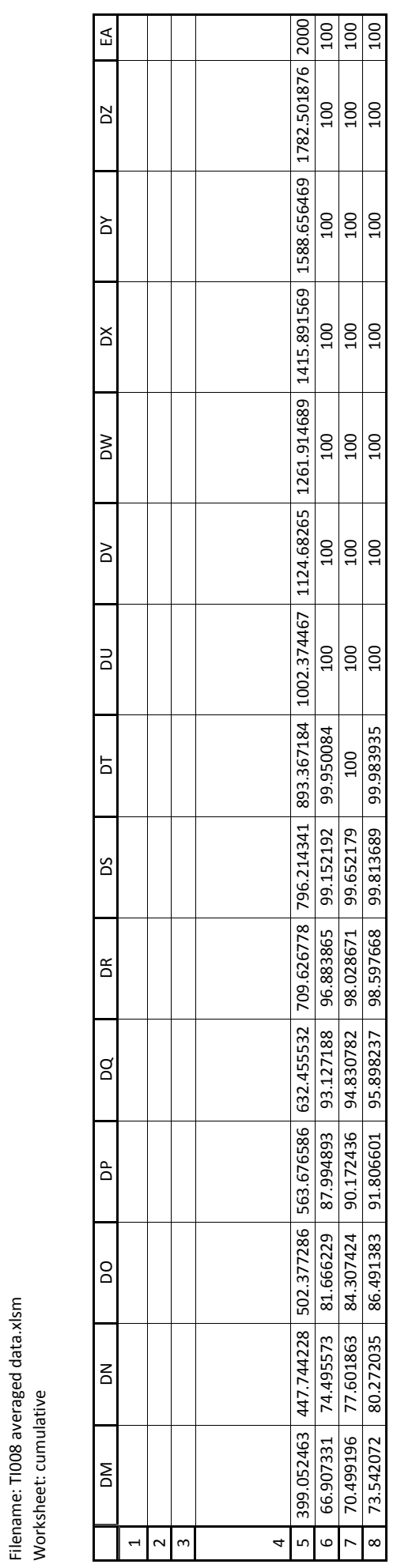

$\stackrel{1}{4}$
4
0
0
0
0
0
0
0

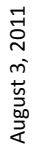


PNNL-20650, Rev. 2

Filename: TI008 averaged data.xlsm

Worksheet: cumulative

\begin{tabular}{|l|l|}
\hline & \multicolumn{1}{|c|}{ EB } \\
\hline 1 & \\
\hline 2 & \\
\hline 3 & \\
\hline & \\
& \\
4 & \\
\hline 5 & \\
\hline 6 & Average of 12 measurements from 52578-TI-037.mea \\
\hline 7 & Average of 12 measurements from 52578-TI-037.mea \\
\hline 8 & Average of 12 measurements from 52578-TI-037.mea \\
\hline
\end{tabular}

August 3, 2011

Page 17 of 21 
PNNL-20650, Rev. 2

Filename: TI008 averaged data.xIsm

Worksheet: cumulative table

\begin{tabular}{|c|c|c|c|c|}
\hline & A & $\mathrm{B}$ & $\mathrm{C}$ & $\mathrm{D}$ \\
\hline 1 & \multicolumn{3}{|c|}{ Cumulative PSD Data in Tabular Form - Supplemental Package } & \\
\hline 2 & Source: & "52578-TI-037.mea" & & \\
\hline 3 & & & & \\
\hline 4 & Record number: & 69 & 70 & 71 \\
\hline 5 & Sample name: & TI008-Unsonicated Average & TI008-Sonicated Average & TI008-Post Sonication Average \\
\hline 6 & Analysis model: & General purpose & General purpose & General purpose \\
\hline 7 & Particle refractive index: & 2.42 & 2.42 & 2.42 \\
\hline 8 & Pump speed, RPM: & 2000 & 2000 & 2000 \\
\hline 9 & Result Below Size, $\mu \mathrm{m}$ : & Cums & tive Percent Undersize, \% & Volume \\
\hline 10 & 0.05 & 0 & 0 & 0 \\
\hline 11 & 0.5 & 0.141 & 0.483 & 0.349 \\
\hline 12 & 1 & 0.677 & 1.904 & 1.319 \\
\hline 13 & 2.5 & 3.283 & 5.826 & 5.708 \\
\hline 14 & 5 & 7.406 & 10.055 & 10.731 \\
\hline 15 & 10 & 11.691 & 14.812 & 15.413 \\
\hline 16 & 20 & 15.187 & 19.823 & 19.93 \\
\hline 17 & 40 & 18.31 & 25.284 & 24.973 \\
\hline 18 & 60 & 20.771 & 29.214 & 28.912 \\
\hline 19 & 80 & 22.807 & 31.88 & 31.791 \\
\hline 20 & 100 & 24.37 & 33.509 & 33.756 \\
\hline 21 & 125 & 25.986 & 34.778 & 35.538 \\
\hline 22 & 150 & 27.715 & 35.942 & 37.253 \\
\hline 23 & 175 & 29.922 & 37.516 & 39.358 \\
\hline 24 & 200 & 32.756 & 39.724 & 42.04 \\
\hline 25 & 300 & 49.081 & 54 & 57.308 \\
\hline 26 & 400 & 67.065 & 70.647 & 73.683 \\
\hline 27 & 500 & 81.387 & 84.048 & 86.253 \\
\hline 28 & 600 & 90.945 & 92.868 & 94.194 \\
\hline 29 & 700 & 96.507 & 97.726 & 98.348 \\
\hline 30 & 800 & 99.214 & 99.689 & 99.833 \\
\hline 31 & 1000 & 100 & 100 & 100 \\
\hline
\end{tabular}


PNNL-20650, Rev. 2

Filename: TI008 averaged data.xIsm

Worksheet: graph information

\begin{tabular}{|c|c|c|}
\hline & A & B \\
\hline 1 & \multicolumn{2}{|c|}{ Summary of Graphs and Graph Reference Cells } \\
\hline 2 & & \\
\hline 3 & Stand Alone Charts & \\
\hline 4 & & \\
\hline 5 & Graph \#: & 1 \\
\hline 6 & Worksheet Label: & PSD chart \\
\hline 7 & Number of Data Series: & 3 \\
\hline 8 & Series 1 Formula: & $=$ =SERIES("Unsonicated",psd!\$AF\$6:\$EA\$6,psd!\$AF\$7:\$EA\$7,1) \\
\hline 9 & Series 2 Formula: & =SERIES("Sonication",psd!\$AF\$6:\$EA\$6,psd!\$AF\$8:\$EA\$8,2) \\
\hline 10 & Series 3 Formula: & =SERIES("Post Sonicated",psd!\$AF\$6:\$EA\$6,psd!\$AF\$9:\$EA\$9,3) \\
\hline 11 & & \\
\hline 12 & & \\
\hline 13 & Graph \#: & 2 \\
\hline 14 & Worksheet Label: & cumulative chart \\
\hline 15 & Number of Data Series: & 3 \\
\hline 16 & Series 1 Formula: & =SERIES("Unsonicated",cumulative!\$AE\$5:\$EA\$5,cumulative!\$AE\$6:\$EA 6,1$)$ \\
\hline 17 & Series 2 Formula: & $=$ =SERIES("Sonication $100 \% "$ "cumulative!\$AE\$5:\$EA\$5,cumulative!\$AE\$7:\$EA\$7,2) \\
\hline 18 & Series 3 Formula: & =SERIES("Post Sonicated",cumulative!\$AE\$5:\$EA\$5,cumulative!\$AE\$8:\$EA\$8,3) \\
\hline 19 & & \\
\hline
\end{tabular}


PNNL-20650, Rev. 2

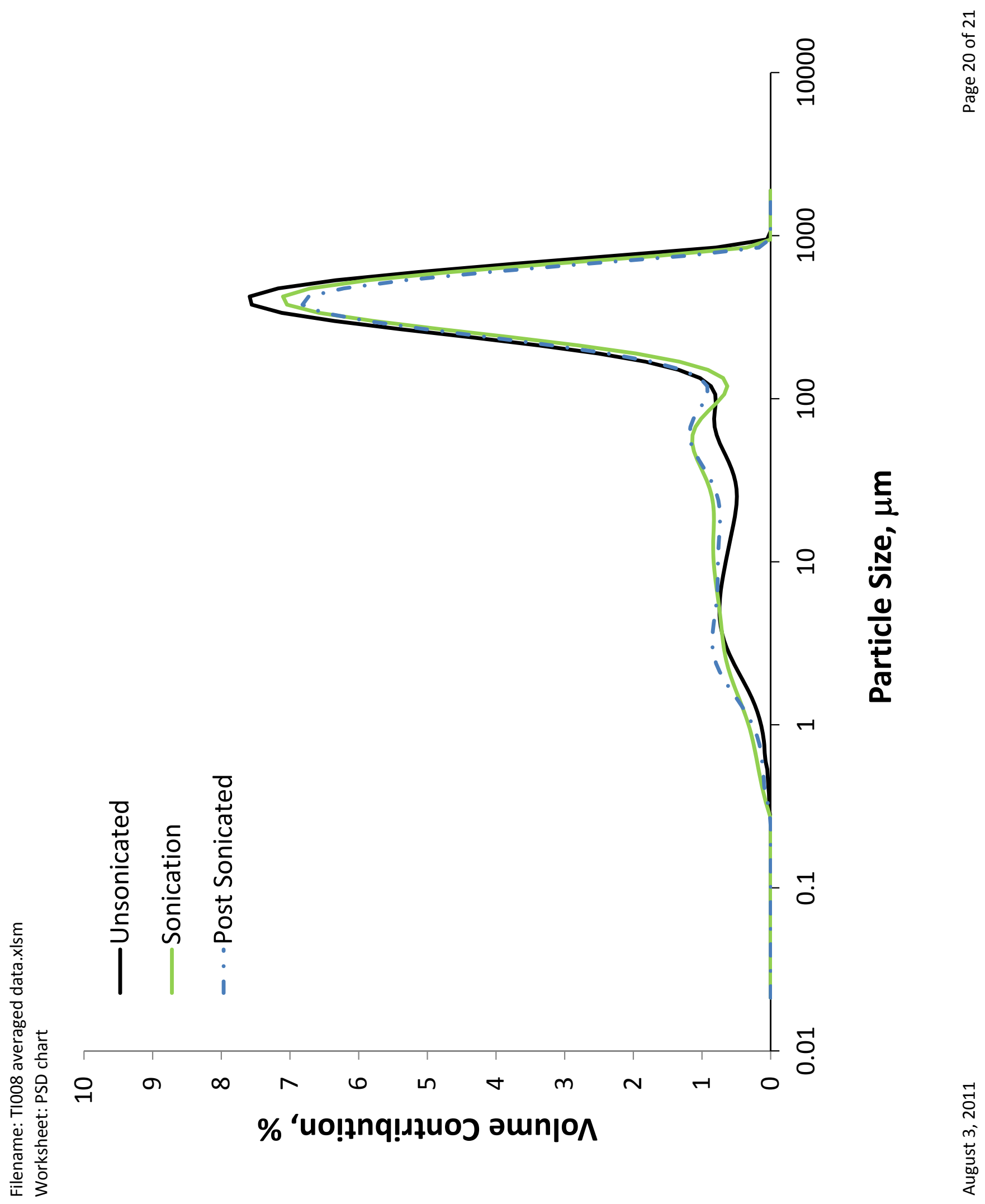


PNNL-20650, Rev. 2

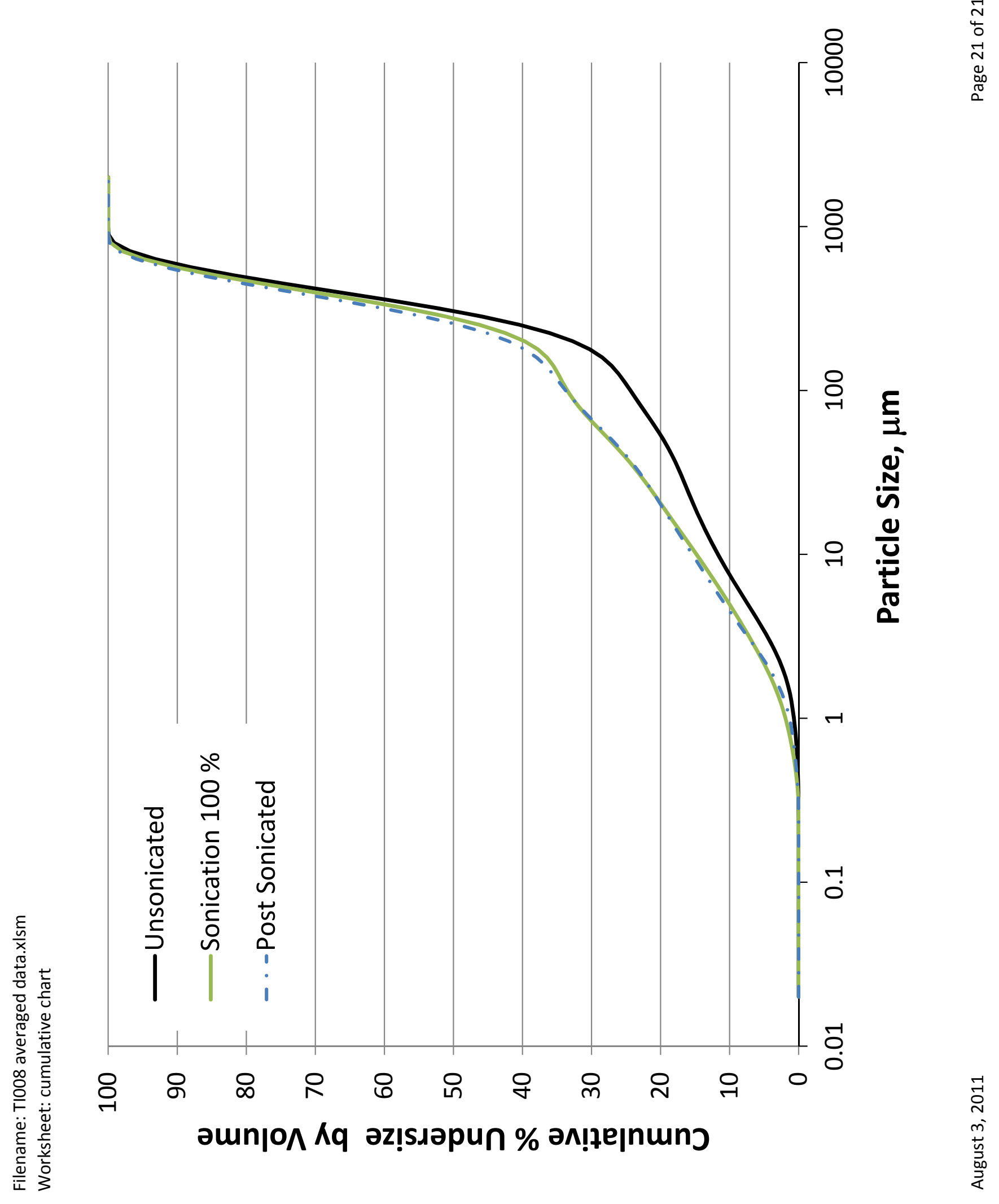


PNNL-20650, Rev. 2
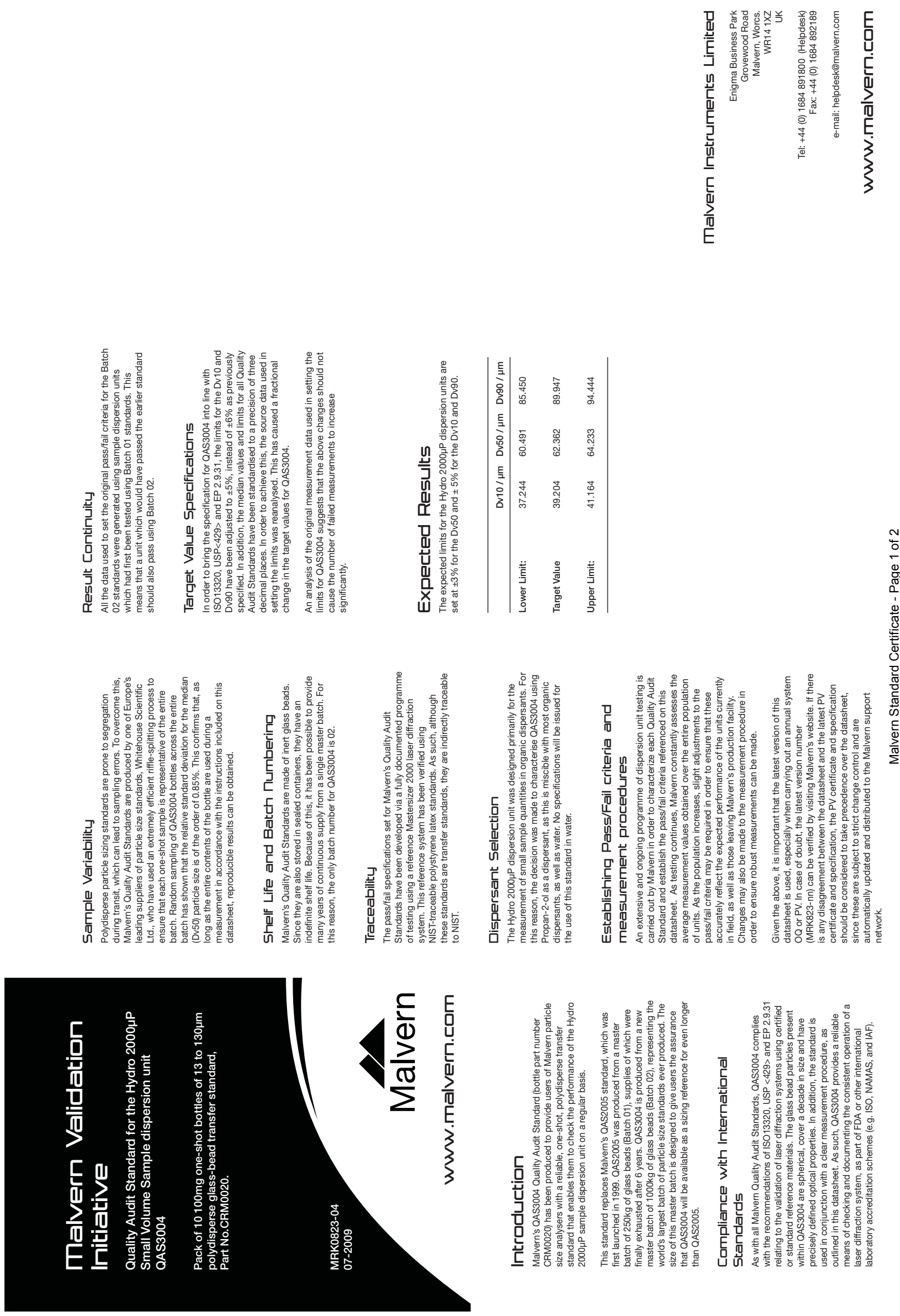

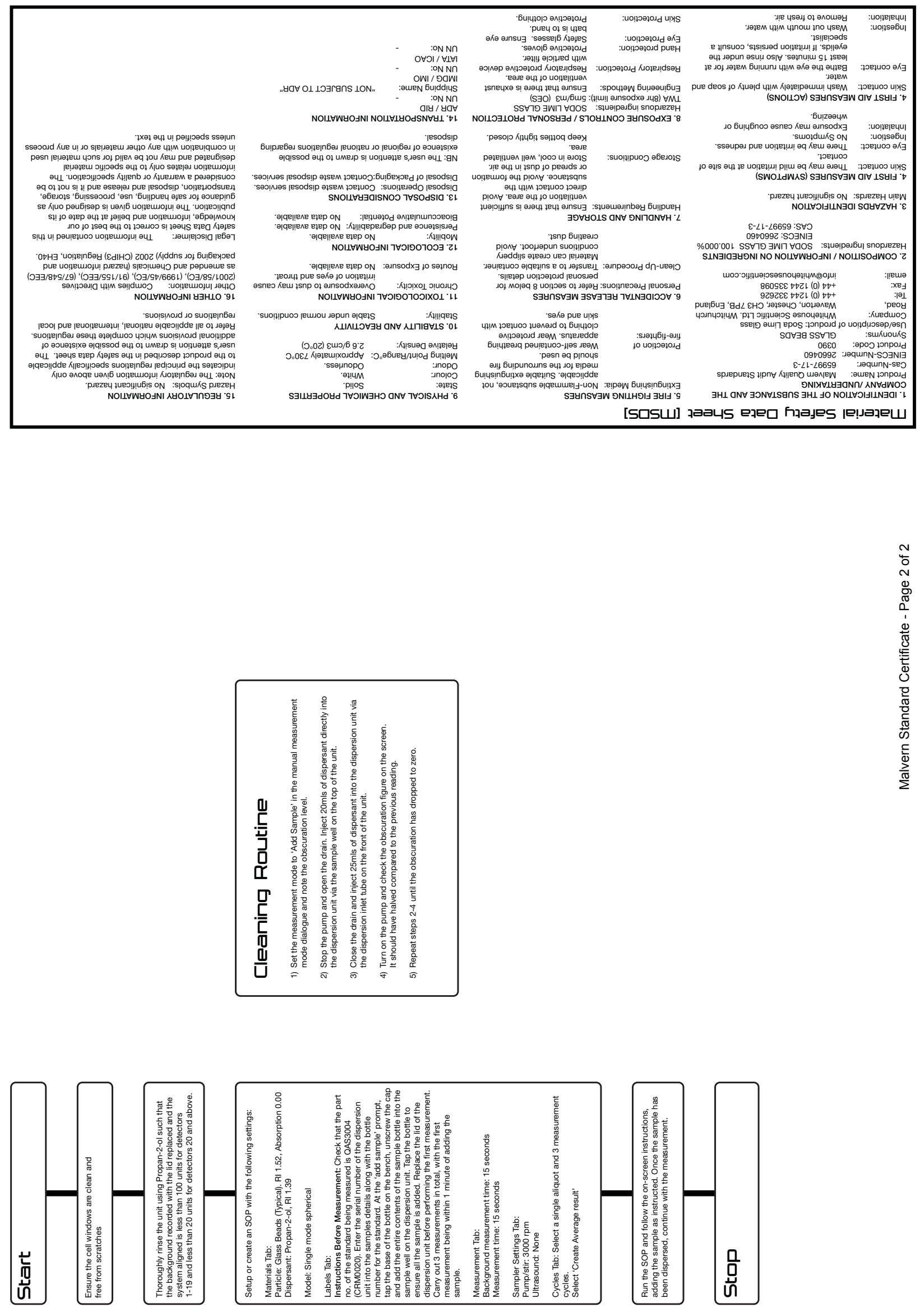


\section{PLANNING}

Topic/Issue to be evaluated: Surveillance of Completed K Basin TI, 52578-TI037, Rev. 0, Test Instruction for Particle Size Distribution Measurements for K West Basin Consolidated Container Composite SCS-CON-210, Minus 500 micron Particle Size Fraction Samples.

This surveillance was undertaken to evaluate completed test instruction (TI), 52578-TI037, Test Instruction for Particle Size Distribution Measurements for $K$ West Basin Consolidated Container Composite SCS-CON-210, Minus 500 micron Particle Size Fraction Samples, associated supporting information and spreadsheets, and the completed report, Particle Size Analysis Performed Under Test Instruction 52578-TI-037, Particle Size Distribution Measurements for K West Basin Consolidated Container Composite SCS-CON-210, Using Minus 500 Micron Particle Size Fraction Samples. The report was reviewed concurrently with TI037, but the report review will be documented via a Document Review Record (DRR) form. TI037 was used between 6/15/2011 and 7/7/2011. TI037 directs the performance of particle size distribution measurements for 2 samples using procedure, RPL-COLLOID01, Rev. 1, Particle Size Analysis Using Malvern MS2000. Carolyn Burns signed off TI steps. Richard Daniel performed a Technical Review of the TI.

\section{PERFORMANCE}

\begin{tabular}{|l|l|}
\hline $\begin{array}{l}\text { Date Initiated: 15 June 2011 } \\
\text { Date Completed: } 7 \text { July } 2011\end{array}$ & Location: RPL \\
\hline $\begin{array}{l}\text { Date Received: 8 July 2011 } \\
\text { Date Reviewed: 11 July 2011 }\end{array}$ & DRAFT Report Issued: 11 July 2011 \\
\hline $\begin{array}{l}\text { Contacts: Carolyn Burns, Clark Carlson, } \\
\text { Richard Daniel and Sandy Fiskum }\end{array}$ & Org Code: PE 137, D9H63 \\
\hline Surveillance Team: Lisa Middleton & \\
\hline
\end{tabular}

\section{PRELIMINARY INFORMATION}

\section{Source of Requirement(s):}

- $\quad$ KBC-33786, Rev. 2, December 2009; Quality Assurance Project Plan/Sampling and Analysis Plan (QAPjP/SAP): Quality Assurance Project Plan/Sampling and Analysis Plan for Containerized KW Settler Sludge

- Data Quality Objectives for Sampling and Analysis of K Basin Sludge HNF-36985, Rev. 3, July 2009

Other Applicable Documents:

- RPL-OP-001, Rev. 9, Routine Research Operations

- ADM-RSEG-BALANCES, Rev. 0, Balance Calibration Verification 
Number: SR-52578-2011-030

Page 2 of 3

The 52578 Project QE staff are required via the TI to perform a completeness review. The document was reviewed to evaluate that all entries were recorded properly, the errors were corrected appropriately and when needed, that justification was present to describe data corrections, and that the attachments to the TI were at the stage to become a project record. When issues were found, the cognizant staff were contacted to resolve issues prior to completion of the review.

\section{Approach:}

The TI package submitted for review consisted of the following:

1. Completed TI, 52578-T1037, Rev. 0, Test Instruction for Particle Size Distribution Measurements for K West Basin Consolidated Container Composite SCS-CON-210, Minus 500 micron Particle Size Fraction Samples (pp. 1-19)

2. DRR \& Calculation Review Sheet, both signed by Richard Daniel on 7/7/11

3. Excel File: 52578-TI037 (Reviewed)

4. PDF version of Excel file with cover page \& review form

5. Word File: 52578-T1037 Writeup (ITR) Rev B (Report: Particle Size Analysis Performed Under Test Instruction 52578-TI-037, Particle Size Distribution Measurements for K West Basin Consolidated Container Composite SCS-CON-210, Using Minus 500 Micron Particle Size Fraction Samples)

6. PDF of Word file listed above with reviewer mark-up.

\section{M\&E Used:}

\begin{tabular}{|c|c|c|}
\hline Description & ID \#/Serial Number & Dates Used \\
\hline $\begin{array}{c}\text { Malvern Mastersizer } \\
2000\end{array}$ & MAL100406 & $6 / 15 / 11$ \\
\hline
\end{tabular}

Procedure Used:

RPL-COLLOID-01, Rev. 1, Particle Size Analysis Using Malvern MS2000

\section{RESULTS}

Findings are defined as a statement of fact relating to noncompliance with previously agreed-upon procedures, plans, codes, standards, specifications, or other forms of contractual or legal obligation. It should be understood that any lack of a finding in a specific area is not considered an indication that deficiencies do not exist.

Observations are defined as a weakness that if not corrected, could yield a departure from a requirement although the weakness is not necessarily a departure from requirements.

Note: All concerns identified in the review were addressed; a comment resolution meeting was not necessary. 
Before the completed TI was signed off for QE review, it was determined that all comments/concerns were addressed. There were no outstanding identified findings or observations.

Issues of concern are summarized below:

1. Line-outs need to be initialed \& dated on pages $8,16 \& 17$. Completed, no further action required; closed.

2. The Result Analysis Reports inserted into the TI need to have page \#'s added to them (e.g. 16A, 17A, 17B ...). Completed, no further action required; closed.

\section{CORRECTIVE ACTION}

[X] None Required. [ ] Complete [ ] Follow-up Corrective Action

\section{Surveillance Performed By:}

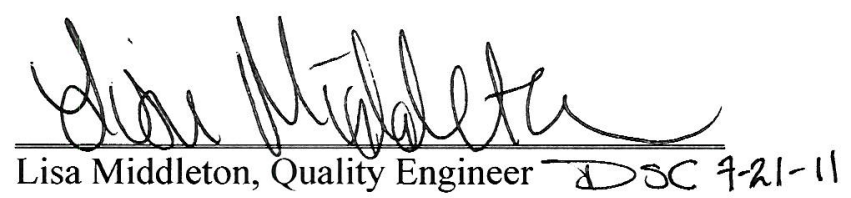

Date:

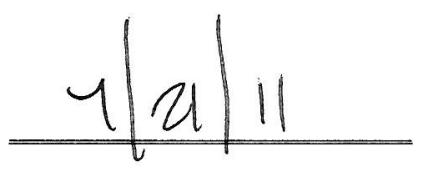

DISTRIBUTION: Carolyn Burns, Clark Carlson, Deborah Coffey, Richard Daniel, Sandy Fiskum, Matt Fountain, Rick Shimskey, and Andy Schmidt 


\section{PLANNING}

Topic/Issue to be evaluated: Surveillance of Completed K Basin TI, 52578-TI037, Rev. 0, Test Instruction for Particle Size Distribution Measurements for $K$ West Basin Consolidated Container Composite SCS-CON-210, Minus 500 micron Particle Size Fraction Samples.

This report has been revised to include the addition of a data reduction spreadsheet that was added to the report and was not included in the report when submitted for review.

This surveillance was undertaken to evaluate completed test instruction (TI), 52578-TI037, Test Instruction for Particle Size Distribution Measurements for $K$ West Basin Consolidated Container Composite SCS-CON-210, Minus 500 micron Particle Size Fraction Samples, associated supporting information and spreadsheets, and the completed report, Particle Size Analysis Performed Under Test Instruction 52578-T7-037, Particle Size Distribution Measurements for K West Basin Consolidated Container Composite SCS-CON-210, Using Minus 500 Micron Particle Size Fraction Samples. The report was reviewed concurrently with $\mathrm{T} 1037$, but the report review will be documented via a Document Review Record (DRR) form. TI037 was used between 6/15/2011 and 7/7/2011. TI037 directs the performance of particle size distribution measurements for 2 samples using procedure, RPL-COLLOID01, Rev. 1, Particle Size Analysis Using Malvern MS2000. Carolyn Burns signed off TI steps. Richard Daniel performed a Technical Review of the TI.

\section{PERFORMANCE}

\begin{tabular}{|l|l|}
\hline $\begin{array}{l}\text { Date Initiated: 15 June 2011 } \\
\text { Date Completed: 7 July 2011 }\end{array}$ & Location: RPL \\
\hline $\begin{array}{l}\text { Date Received: 8 July 2011 } \\
\text { Date Reviewed: } 11 \text { July 2011 }\end{array}$ & $\begin{array}{l}\text { DRAFT Report Issued: 11 July 2011 } \\
\text { FINAL Report Issued: 21 July 2011 } \\
\text { REVISED Report Issued: 3 August 2011 }\end{array}$ \\
\hline $\begin{array}{l}\text { Contacts: Carolyn Burns, Clark Carlson, } \\
\text { Richard Daniel and Sandy Fiskum }\end{array}$ & Org Code: PE 137, D9H63 \\
\hline Surveillance Team: Lisa Middleton & \\
\hline
\end{tabular}

\section{PRELIMINARY INFORMATION}

\section{Source of Requirement(s):}

- KBC-33786, Rev. 2, December 2009; Quality Assurance Project Plan/Sampling and Analysis Plan (QAPjP/SAP): Quality Assurance Project Plan/Sampling and Analysis Plan for Containerized KW Settler Sludge

- Data Quality Objectives for Sampling and Analysis of K Basin Sludge HNF-36985, Rev. 3, July 2009

Other Applicable Documents:

- RPL-OP-001, Rev. 9, Routine Research Operations 
Number: SR-52578-2011-030 Rev. 1

- ADM-RSEG-BALANCES, Rev. 0, Balance Calibration Verification

\section{Purpose/Scope:}

The 52578 Project QE staff are required via the TI to perform a completeness review. The document was reviewed to evaluate that all entries were recorded properly, the errors were corrected appropriately and when needed, that justification was present to describe data corrections, and that the attachments to the TI were at the stage to become a project record. When issues were found, the cognizant staff were contacted to resolve issues prior to completion of the review.

\section{Approach:}

The TI package submitted for review consisted of the following:

1. Completed TI, 52578-T1037, Rev. 0, Test Instruction for Particle Size Distribution Measurements for $K$ West Basin Consolidated Container Composite SCS-CON-210, Minus 500 micron Particle Size Fraction Samples (pp. 1-19)

2. DRR \& Calculation Review Sheet, both signed by Richard Daniel on 7/7/11

3. Excel File: 52578-T1037 (Reviewed).xlsm with the following tabs:

- Summary Page

- Performance Checks

- PSD

- Cumulative

- Cumulative Tables

- Percentiles

- TI-008-SP PSD Chart

- TI-008-SQ PSD Chart

- TI-008-SP CUM Chart

- TI-008-SQ CUM Chart

4. PDF version of Excel file with cover page \& review form

5. Word File: 52578-TI037 Writeup (ITR) Rev B.doc (Report: Particle Size Analysis Performed Under Test Instruction 52578-TI-037, Particle Size Distribution Measurements for K West Basin Consolidated Container Composite SCS-CON-210, Using Minus 500 Micron Particle Size Fraction Samples)

6. PDF of Word file listed above with reviewer mark-up.

7. A new Excel spreadsheet was submitted for review on 2 August 2011 (TI008 average data.xls), containing the following tabs:
- Summary
- PSD
- Cumulative
- Percentiles
- Cumulative Chart
- Cumulative Table
- PSD Chart 
Number: SR-52578-2011-030 Rev. 1

Page 3 of 4

M\&E Used:

\begin{tabular}{|c|c|c|}
\hline Description & ID \#/Serial Number & Dates Used \\
\hline $\begin{array}{c}\text { Malvern Mastersizer } \\
2000\end{array}$ & MAL100406 & $6 / 15 / 11$ \\
\hline
\end{tabular}

Procedure Used:

RPL-COLLOID-01, Rev. 1, Particle Size Analysis Using Malvern MS2000

\section{RESULTS}

Findings are defined as a statement of fact relating to noncompliance with previously agreed-upon procedures, plans, codes, standards, specifications, or other forms of contractual or legal obligation. It should be understood that any lack of a finding in a specific area is not considered an indication that deficiencies do not exist.

Observations are defined as a weakness that if not corrected, could yield a departure from a requirement although the weakness is not necessarily a departure from requirements.

Note: All concerns identified in the review were addressed; a comment resolution meeting was not necessary.

Before the completed TI was signed off for QE review, it was determined that all comments/concerns were addressed. There were no outstanding identified findings or observations.

Issues of concern are summarized below:

1. Line-outs need to be initialed \& dated on pages $8,16 \& 17$. Completed, no further action required; closed.

2. The Result Analysis Reports inserted into the TI need to have page \#'s added to them (e.g. 16A, 17A, 17B...). Completed, no further action required; closed.

3. The Excel file: TI008 averaged data.xls was reviewed on 8/3/11 along with a revised copy of the PSD Report. There were no changes identified for the Excel file. Editorial comments in the report were recorded using the "track changes" function. All comments in report were addressed. No further action required, closed. 


\section{CORRECTIVE ACTION}

[X] None Required. [ ] Complete [ ] Follow-up Corrective Action

\section{Surveillance Performed By:}

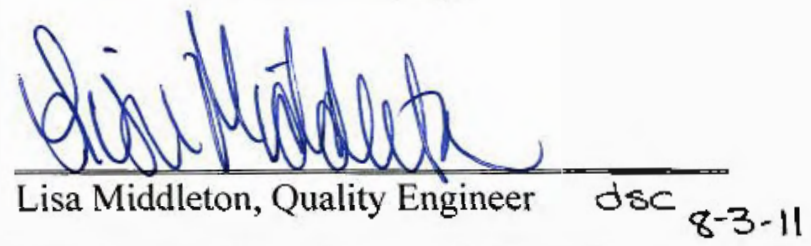

Date:

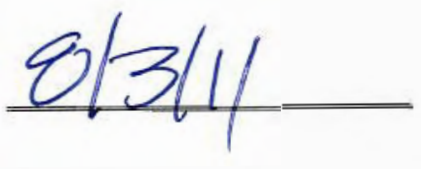

DISTRIBUTION: Carolyn Burns, Clark Carlson, Deborah Coffey, Richard Daniel, Sandy Fiskum, Matt Fountain, Rick Shimskey, and Andy Schmidt 



\section{Appendix J}

\section{Verification Processes and Revision 2 Correction Summary}


PNNL-20650, Rev. 2

\section{Appendix $\mathbf{J}$}

\section{Verification Processes and Revision 2 Correction Summary}

In accordance with the K Basin Quality Assurance Project Plan/Sampling and Analysis Plan (QAPjP/SAP), KBC-33786, Rev. 2 (Baker et al. 2009), the Analytical Support Operations (ASO) procedures, and the quality management system, data verification activities were performed to confirm and document that the reported results are accurate and reflect the characterization analyses that were actually performed and that the quality control (QC) sample results and data met the applicable requirements. Activities associated with sample receipt, consolidation, subsampling, and characterization were complex. The QAPjP/SAP requirements were implemented and controlled by adherence to the ASO Quality Assurance (QA) Plan, sample preparation and analysis procedures, and controlling work documents (e.g., test instructions [TIs]). The ASO QA plan, procedures, and work documents are consistent and compliant with DOE/RL-96-68, HASQARD, Revision 3, Volume 1, Administrative Requirements and Volume 4, Laboratory Technical Requirements. A number of approaches were employed to verify the data from origination through final reporting and these approaches are discussed in this appendix.

A summary of the changes made in developing Revision 2 of this data package is found in Section J.7. These changes are based on responding to general corrections identified during the Sludge Treatment Project (STP) validation and assessment activities (performed after issuance of Revision 1 of this data package) and minor changes directed from PNNL project management.

\section{J.1 Sample Receipt and Preparation}

Table J.1 summarizes the verification review requirements corresponding to the documentation of sample receipt with the corresponding record location.

Table J.1. Summary of Verification Records for Sample Receipt

\begin{tabular}{lll}
\hline \multicolumn{1}{c}{ Requirement } & \multicolumn{1}{c}{ Verification Record } & \multicolumn{1}{c}{ Record Location } \\
\hline $\begin{array}{l}\text { Completed Chain of } \\
\text { Custody (COC) forms }\end{array}$ & Signed and dated COC forms & Appendix A of this report \\
$\begin{array}{l}\text { Observations of } \\
\text { samples/sludge polybottles }\end{array}$ & $\begin{array}{l}\text { Reviewer signature and date } \\
\text { in laboratory record book } \\
\text { (LRB) and on front cover of } \\
\text { completed Tis }\end{array}$ & $\begin{array}{l}\text { DVD-52578-PNNL-20650 } \\
\text { Rev. 2, Section III, } \\
\text { September 2013 }\end{array}$ \\
$\begin{array}{lll}\text { Provided sample } \\
\begin{array}{l}\text { Complete summary provided } \\
\text { nomenclation (ID) }\end{array}\end{array}$ & Section 2.0 of report \\
\hline
\end{tabular}

Images of the $\mathrm{COC}$ forms submitted with each polybottle pair containing $\mathrm{K}$ Basin sludge are provided in Appendix A. The COCs have been reviewed for completeness by the project technical staff and the Quality Engineer (QE). 
Sample ID nomenclature used within this characterization effort included client-provided identification (i.e., engineered container IDs [e.g., SCS-CON-210] and core IDs [e.g., A1]), and Pacific Northwest National Laboratory (PNNL) identification (i.e., TI subsample IDs [e.g., TI001-A1-B], ASO laboratory analytical services request (ASR) numbers [e.g., 8798], and Radiochemical Processing Laboratory (RPL) analytical laboratory IDs [e.g., 11-0419]). Section 2.0 of this report includes sample ID cross reference tables to assist the reader in the navigation of both the document and the appendices.

Table J.2 summarizes the approach for verification reviews to evaluate work against requirements corresponding to sample consolidation, subsampling, and other characterization testing controlled via test instructions (e.g., rheology, particle size analyses) along with the corresponding location of the objective review.

Table J.2. Summary of Verification Records for Test Instructions

\begin{tabular}{|c|c|c|}
\hline Requirement & Verification Record & Record Location \\
\hline \multicolumn{3}{|l|}{ Planning } \\
\hline $\begin{array}{l}\text { Work document (TI) includes steps to obtain } \\
\text { required samples and data meeting technical } \\
\text { compliance. }\end{array}$ & $\begin{array}{l}\text { Technical reviewer signature and } \\
\text { date }\end{array}$ & $\begin{array}{l}\text { Front cover of completed TI, } \\
\text { DVD-52578-PNNL-20650 } \\
\text { Rev. 2, Section III, }\end{array}$ \\
\hline $\begin{array}{l}\text { Work document collects data and samples } \\
\text { consistent with the QAPjP/SAP. }\end{array}$ & $\begin{array}{l}\text { Project manager signature and } \\
\text { date }\end{array}$ & September 2013 \\
\hline $\begin{array}{l}\text { Work document meets CH2MHill Plateau } \\
\text { Remediation Company (CHRPC) Sludge } \\
\text { Treatment Project (STP) requirements, } \\
\text { consistent with the QAPjP/SAP. }\end{array}$ & $\begin{array}{l}\text { Buyer's Technical Representative } \\
\text { (BTR) signature and date }\end{array}$ & \\
\hline $\begin{array}{l}\text { Work document appropriately captures } \\
\text { measurement and test equipment requirements } \\
\text { and documentation. }\end{array}$ & QE signature and date & \\
\hline \multicolumn{3}{|l|}{ Execution/Completion } \\
\hline $\begin{array}{l}\text { Steps in work document have been completed, } \\
\text { changes have been documented, records are } \\
\text { logical and understood, collected data are in } \\
\text { technical compliance with project } \\
\text { requirements. }\end{array}$ & $\begin{array}{l}\text { Technical reviewer signature and } \\
\text { date }\end{array}$ & $\begin{array}{l}\text { Front cover of completed TI, } \\
\text { DVD-52578-PNNL-20650 } \\
\text { Rev. 2, Section III, } \\
\text { September } 2013\end{array}$ \\
\hline $\begin{array}{l}\text { Steps in work document have been conducted } \\
\text { in accordance with QA requirements. }\end{array}$ & QE signature on completed TI & \\
\hline $\begin{array}{l}\text { Transcriptions into data-calculation } \\
\text { spreadsheets and data-reduction calculations } \\
\text { and measurement uncertainties from } \\
\text { completed TI are properly conducted. }\end{array}$ & $\begin{array}{l}\text { Technical reviewer signature and } \\
\text { date }\end{array}$ & $\begin{array}{l}\text { Calculation spreadsheet } \\
\text { review form, DVD-52578- } \\
\text { PNNL-20650 Rev. 2, Section } \\
\text { III, September } 2013\end{array}$ \\
\hline $\begin{array}{l}\text { Overall check for completeness of each TI, } \\
\text { data traceability, associated attachments, and } \\
\text { spreadsheets including identification and } \\
\text { disposition of all issues. }\end{array}$ & $\begin{array}{l}\text { TI surveillance report signed by } \\
\text { QE }\end{array}$ & $\begin{array}{l}\text { DVD-52578-PNNL-20650 } \\
\text { Rev. 2, Section III, } \\
\text { September } 2013\end{array}$ \\
\hline
\end{tabular}

The activities associated with sample receipt, consolidation, subsampling, and physical characterization were controlled and documented in detailed TIs. ${ }^{\text {(a) }}$ The TIs were reviewed by at least one

(a) Completed TIs are provided in digital format; see DVD-52578-PNNL-20650 Rev. 2, Section III, September 2013. A listing of the applicable TIs is also provided within the summary report, just after the table of contents. 
technical reviewer, the $\mathrm{QE}$, and the client before issuance. These reviews were performed to verify that all client data needs were addressed during planning for processing activities. After the TIs were completed, the primary user performed a completeness review and the technical reviewer performed a technical review. Calculation spreadsheets were developed for each processing TI to determine various physical parameters (e.g., sample mass, settled sludge density). Spreadsheet reviews included a technical review of data entries from TIs into the calculation spreadsheet as well as calculation algorithms, crossreferences to other cells, assignments of uncertainties, error propagation (where applicable), and overall reasonableness and consistency of the data. Spreadsheet reviews were performed by annotating issues and capturing responses to questions raised. The calculation spreadsheets, with documentation of the reviews, are included as part of the completed TIs.

Formal QE reviews of TIs, attachments, and supporting spreadsheets were performed to verify all entries were recorded properly, errors were corrected appropriately, justification was present (when needed) to describe data corrections, data traceability was evident, and TIs and attachments were acceptable to become a project record. Identified issues and concerns were discussed and resolved before the TI review was signed by the QE. QE reviews were documented as formal surveillance reports and are included as part of the completed TI. When necessary, Occurrence Reports were initiated to track discussions with the client on issues of concern and to determine if the occurrence needed to be elevated to a Deficiency Report or Nonconformance Report.

\section{J.2 Sample Analysis and Characterization}

Table J.3 summarizes the records documenting the planning for sample preparation and analysis, completed TIs, data forms, summary reports, and supporting information, along with the corresponding location of records indicating technical and QE review.

The data quality objectives (DQOs) defined in the QAPjP/SAP documents identify processes/analytical techniques for each characterization parameter (e.g., ${ }^{137} \mathrm{Cs}$ concentration using gamma energy analyses [GEA] and detection limit, and precision and accuracy acceptance criteria). Applicable procedure DQOs were also considered and evaluated relative to the QC sample results. Whenever there was a discrepancy between the DQOs in the ASR or the ASO procedure/ASO QA Plan, the DQOs in the ASRs (project Special Instructions) took precedence as the Special Instructions conveyed the specific K Basin project requirements. The specific PNNL/ASO procedures used for the various analyses are documented in DVD-52578-PNNL-20650 Rev. 2, Section II, September 2013. Procedures used to measure nuclear material in core samples for nuclear material accountability purposes were provided to the safeguards representative for review before use. The approach and control limits used by the laboratory for balance accuracy and linearity checks were provided to the safeguards representative for review and acceptance before use.

As previously noted, elemental and isotopic analyses and associated QC requirements in the QAPjP/SAP were provided to the ASO in the form of Special Instructions appended to the ASRs. Because the analytical requirements were complex in nature, these Special Instructions were reviewed and approved both internally by PNNL staff (author, technical reviewer, ASO Manager, and QE) and by the contract BTR to help make sure that all requirements were properly rolled out to the ASO. As with the TIs, approval/concurrence was documented by signature on the Special Instructions. The ASRs and the Special Instructions are included in the appendices of this report. 
DQOs for non-ASO analyses (X-ray diffraction [XRD], isotopic Pu and $\mathrm{U}$, and particle density) were conveyed to the analysts via Analysis Request forms. Procedures used for non-ASO measures were provided to the project for review before use. These analyses were conducted according to the Analysis Requests and PNNL test procedures. Particle size distribution (PSD) and rheology were conducted according to PNNL procedures with specific processing parameters defined in TIs. These TIs were reviewed and signed before implementation using the same protocol applied to the sample receipt and processing TIs. These completed TIs are also provided in the digital format (DVD-52578-PNNL-20650 Rev. 2, Section III, September 2013).

Table J.3. Summary of Verification Records for Analytical Sample Preparation and Analysis

\begin{tabular}{lll}
\hline \multicolumn{1}{c}{ Requirement } & \multicolumn{1}{c}{ Verification Record } & \multicolumn{1}{c}{ Record Location } \\
\hline Planning & & \\
Work documents (ASR Special Instructions) identify & ASR Special Instructions & Appendices B, C, and D \\
all subsamples and specific analyses to be performed. & $\begin{array}{l}\text { signatures on ASR Special } \\
\text { All method and QAPjP/SAP QC requirements have }\end{array}$ & \\
$\begin{array}{l}\text { Instructions as follows: } \\
\text { been identified and properly communicated to the }\end{array}$ & $\begin{array}{l}\text { - cognizant scientist } \\
\text { analytical laboratory. }\end{array}$ & \\
& - project manager & \\
\hline
\end{tabular}

Execution/Completion

For each ASO analysis, a technical review has been performed, consisting of the following elements:

- transcription accuracy

- correctness of calculations

- evaluation of overall consistency and reasonableness of data.

For each ASO analysis, a QE review has been performed, consisting of the following elements:

- evaluation of project, ASR, and method DQOs against QC sample results

- transcription accuracy

- correctness of calculations

- evaluation of overall consistency and reasonableness of data

- implementation of appropriate corrective action, when necessary.

- data traceability

- documentation of QC sample results not meeting DQOs and other data quality issues.

Non-ASO analysis (such as particle density, isotopic distribution by mass spectrometry, XRD, rheology, PSD, etc.) reports are subjected to a technical review with the following general elements.

- evaluation of method and QC performance

- evaluation of compliance with technical and QC requirements, as defined in the ASR and Special Instruction

- transcription accuracy

- correctness of calculations

- evaluation of overall consistency and reasonableness of data.
Technical reviewer signature on the completed ASO analytical report

QE review surveillance reports

ASO reports in Appendices B, C, D

Technical reviewer signature on completed technical report
Appendices E, F, G, H, and I 
Table J.3. (contd)

\begin{tabular}{|c|c|c|}
\hline Requirement & Verification Record & Record Location \\
\hline $\begin{array}{l}\text { Non-ASO analysis (particle density, isotopic } \\
\text { distribution by thermal ionization mass spectrometry, } \\
\text { XRD, rheology, and PSD) reports are subjected to a } \\
\text { QE review with the following general elements. } \\
\text { - evaluation of project, Analysis Request, and } \\
\text { method DQOs against QC sample results } \\
\text { - transcription accuracy } \\
\text { - correctness of calculations } \\
\text { - evaluation of overall consistency and } \\
\text { reasonableness of data } \\
\text { - data traceability } \\
\text { - implementation of appropriate corrective action, } \\
\text { - when necessary } \\
\text { documentation of QC sample results not meeting } \\
\text { DQOs and other data quality issues. }\end{array}$ & $\begin{array}{l}\text { QE reviewer signature on } \\
\text { completed technical report }\end{array}$ & $\begin{array}{l}\text { Appendices E, F, G, H, } \\
\text { and I }\end{array}$ \\
\hline $\begin{array}{l}\text { Documentation of occurrences, deficiencies, and } \\
\text { nonconformance }\end{array}$ & $\begin{array}{l}\text { Occurrence Reports signed by } \\
\text { the project manager and the } \\
\text { QE }\end{array}$ & $\begin{array}{l}\text { Appendices B, C, D, E, F, } \\
\text { and G and summarized in } \\
\text { Table J.4 }\end{array}$ \\
\hline
\end{tabular}

\section{J.3 Data Reporting}

Preliminary ASO data reports received internal technical reviews (by ASO staff) and provided to PNNL project staff and the BTR. This early project review facilitated preliminary data reporting and timely identification of issues and concerns. Technical staff evaluated results received from the ASO for overall consistency between duplicates and processes. Where possible, duplicative analysis results were compared (e.g. ${ }^{241} \mathrm{Am}$ concentrations determined by GEA and alpha energy analysis [AEA] and total U concentrations determined by kinetic phosphorescence analysis and inductively coupled plasma optical emission spectrometry [ICP-OES] ) and isotopic ratios (e.g., ${ }^{241} \mathrm{Am}:{ }^{154} \mathrm{Eu}$ and ${ }^{238} \mathrm{Pu}:{ }^{239+240} \mathrm{Pu}$ ) were reviewed for consistency.

Each ASO data report includes sections that address each of the analytical QC samples and DQO compliance to the procedure requirements and to requirements identified in the ASR/Special Instructions and the QAPjP/SAP. Each ASO analysis report was reviewed and signed by the preparer and the technical reviewer. The report and supporting data were then forwarded to the ASO QE for review. The QE reviews were documented as surveillance reports and were performed to verify that data were what they purported to be (i.e., the reported results reflected what was actually done and the results meet applicable requirements, particularly procedure and project data quality objectives). Identified issues and concerns were discussed and resolved before the QE signed and issued the surveillance report. All QE review comments were resolved before the analytical report was finalized. The QE review and comment resolution are documented in surveillance reports that are available in the ASO records. The ASO Lead also performed a final review of each ASO report for completeness and provided authorization to release the data.

Individual, non-ASO reports (e.g., XRD, PSD, particle density, rheology, and isotopic analysis by thermal ionization mass spectrometry [TIMS]) contributing to the final $\mathrm{K}$ Basin reports are provided in appendices. These reports were subject to a minimum of technical and QE reviews. The QE reviews 
were documented as formal surveillance reports and were performed to verify that data were what they purported to be, that is, that the reported results reflected what was actually done and the results meet applicable requirements, particularly procedure and project data quality objectives. Identified issues and concerns were discussed and resolved before the QE signed the surveillance report. Surveillance reports are included in the relevant appendices. When necessary, Occurrence Reports were initiated to track discussions with the client on issues of concern and to determine if the occurrence needed to be elevated to a Deficiency Report or Nonconformance Report.

Tiered reviews of the completed sections of the summary K Basin report were conducted. Reviews were conducted by data contributors (e.g., ASO staff, X-ray spectroscopist), staff tasked with verifying numerical data entries (transcriptions from calculation spreadsheets to report tables) and result discussion text, an independent technical reviewer (for overall reasonableness and accuracy of presentation and conclusions), the RPL project management staff, and the assigned QE.

\section{J.4 Occurrences, Deficiencies, and Nonconformance}

The process for documenting issues of concern and determining if significant conditions adverse to quality were indicated, Occurrence Report forms, Deficiency Report forms, and Nonconformance Report forms were generated, as applicable. The project manager and QE evaluated occurrences for conditions adverse to quality to determine if the occurrence was a significant condition adverse to quality that required elevation to a deficiency or nonconformance. These terms are defined as follows:

- Occurrences are defined to be any issue affecting sample integrity or data quality. Occurrences were tracked to document any issue of concern to bring the issue to the attention of project management and the client and when applicable, to define appropriate corrective actions or paths forward.

- Deficiencies are defined as failures to follow work-controlling documents such as test plans, TIs, or procedures. Deficiency Reports may result from Occurrence Reports that are determined by the responsible manager to be a significant condition adverse to quality requiring corrective action and tracking to closure or may be issued without an Occurrence Report when it is clear that a failure to follow work-controlling documents occurred.

- Nonconformances are defined as failures of items to meet specifications or to operate as expected; this includes using out-of-service equipment and measuring and test equipment used outside of a valid calibration interval and other types of calibration-related issues. Nonconformance Reports result from occurrences that are determined by the responsible manager to be a significant condition adverse to quality requiring disposition of the item (e.g., use as-is, reject, repair, re-work), corrective action, and tracking to closure.

Occurrence Reports were uniquely identified and include a description of the issue, affected samples, investigative findings, recommended actions, and an assessment of significant impact to data quality. Occurrence Reports were used to inform the client and establish concurrence with respect to recommended actions. Project management made the determination as to whether the issues needed to be elevated to formal Nonconformance Reports or Deficiency Reports. Table J.4 provides summaries of the issued Occurrence Reports and identifies the location where these records can be found. No Deficiency Reports were generated for this scope of the K Basin work. One Nonconformance Report was generated. 
PNNL-20650, Rev. 2

Table J.4. Occurrence Report Summary

\begin{tabular}{|c|c|c|}
\hline ID & Summary & Record Location \\
\hline OR-52578-04-18-11 & $\begin{array}{l}\text { ASR } 8798 \text {, two duplicate pair results for GEA exceed the } \pm 20 \% \\
\text { relative percent difference (RPD) requirement }\left({ }^{60} \mathrm{Co} \text { RPD for TI001- }\right. \\
\left.\text { A1 pair was } 48 \% \text { and }{ }^{155} \mathrm{Eu} \text { RPD for TI002-B1 pair was } 32 \%\right) \text {. }\end{array}$ & Appendix B \\
\hline OR-52578-04-20-11 & $\begin{array}{l}\text { ASR } 8798 \text {, three duplicate pair core sample results on several analytes } \\
\text { exceed } \pm 20 \% \text { RPD requirement; the matrix spike sample results for } \\
\text { Sb, Tl, Zn, and Zr exceed } \pm 20 \% \text { accuracy requirement. }\end{array}$ & Appendix B \\
\hline OR-52578-04-29-11 & $\begin{array}{l}\text { ASR } 8824 \text {, the MCC sample duplicate pair results exceed } \pm 20 \% \text { RPD } \\
\text { requirement for ICP-OES results from PNL-ALO- } 129 \text { acid digest and } \\
{[\mathrm{Na}] \text { in preparation blank was greater than } 5 \% \text { present in samples. }}\end{array}$ & Appendix C \\
\hline OR-52578-05-09-11 & $\begin{array}{l}\text { Minor isotopes in the U TIMS standard did not meet the } \pm 2 \% \\
\text { acceptance criterion; all }{ }^{238} \mathrm{Pu} \text { sample and duplicate pair precision } \\
\text { results exceed } \pm 20 \% \text { RPD requirement; }{ }^{241} \mathrm{Pu} \text { sample TI001-A1 } \\
\text { duplicate pair precision result exceeds } \pm 20 \% \text { RPD requirement. }\end{array}$ & Appendix E \\
\hline OR-52578-05-19-11 & $\begin{array}{l}\text { ASR 8824, U metal duplicate pair results exceed } \pm 20 \% \text { RPD } \\
\text { requirement (RPD for samples TI008-ME and TI008-MF was } 86 \% \text { ). }\end{array}$ & Appendix C \\
\hline OR-52578-05-26-11 & $\begin{array}{l}\text { ASR } 8798 \text {, U metal duplicate pair exceeds } \pm 20 \% \text { RPD requirement } \\
\text { (RPD for TI002-A4 pair was } 54 \% \text { ). }\end{array}$ & Appendix B \\
\hline OR-52578-05-27-11 & $\begin{array}{l}\text { ASR } 8798 \text {, two duplicate pair results for }{ }^{237} \mathrm{~Np} \text { exceed } \pm 20 \% \text { RPD } \\
\text { requirement (RPD for TI001-A1 pair was } 22 \% \text { and RPD for TI002-B1 } \\
\text { pair was } 82 \% \text { RPD) }\end{array}$ & Appendix B \\
\hline OR-52578-06-27-11 & $\begin{array}{l}\text { ASR } 8824 \text {, GEA and AEA MCC duplicate pair results exceed } \pm 20 \% \\
\text { RPD requirement. }\end{array}$ & Appendix C \\
\hline OR-52578-06-29-11 & $\begin{array}{l}\text { Four PNNL subsample IDs were the same as the client sample IDs: } \\
\text { KW210-A1-A, KW210-A4-A, KW210-B1-A, and KW210-B3-A and } \\
\text { did not contain the TI ID. These samples were combined to create } \\
\text { one of the container composites. }\end{array}$ & $\begin{array}{l}\text { Section III, DVD- } \\
\text { 52578-PNNL- } \\
\text { 20650 Rev. 2, } \\
\text { September } 2013\end{array}$ \\
\hline OR-52578-06-30-11 & $\begin{array}{l}\text { ASR } 8798, \text { total organic carbon (TOC) samples were dried to constant } \\
\text { mass at } 105^{\circ} \mathrm{C} \text { before analysis as per the analysis procedure, but there } \\
\text { was a QAPjP/SAP statement that this analysis was to be performed on } \\
\text { a wet sample. }\end{array}$ & Appendix B \\
\hline OR-52578-07-19-11 & $\begin{array}{l}\text { ASR } 8798, \text { TOC/TIC Method QC, duplicate/replicate samples and } \\
\text { analytical spike sample did not meet method and project acceptance } \\
\text { criteria. }\end{array}$ & Appendix E \\
\hline OR-52578-08-19-11 & $\begin{array}{l}\text { Gelatinous material impact on settled sludge density for sample } \\
\text { TI002-A4-N. }\end{array}$ & $\begin{array}{l}\text { 52578-TI002, } \\
\text { Section III, DVD- } \\
\text { 52578-PNNL- } \\
\text { 20650 Rev. 2, } \\
\text { September } 2013\end{array}$ \\
\hline OR-52578-08-31-11 & $\begin{array}{l}\text { ASR } 8878 \text {, detection limit for gross alpha analysis samples was } \\
\text { greater than the requested detection limit of } 1.0 \mathrm{E}-03 \mu \mathrm{Ci} / \mathrm{g} \text {. }\end{array}$ & Appendix D \\
\hline
\end{tabular}

Table J.5. Nonconformance Report Summary

\begin{tabular}{clc}
\hline ID & \multicolumn{1}{c}{ Summary } & Record Location \\
\hline NCR-52578-11-08-10 & $\begin{array}{l}\text { The viscosity standard certificate was not supported for NIST } \\
\text { traceability by the vendor and therefore did not meet PNNL } \\
\text { Quality Assurance Clause 188 requirements. }\end{array}$ & Appendix H \\
\hline
\end{tabular}




\section{J.5 Surveillances}

There were six QE surveillances of completed TIs, three surveillances of supporting LRB entries, one surveillance of two data analysis Excel spreadsheets, and four data report surveillances performed. In two cases (rheology and PSD), reviews of completed TIs and draft reports were conducted concurrently and summarized under one surveillance report for each analysis activity. Surveillances for ASRs 8798, 8824, 8878 and 8919 are available in the ASO records system. Table J.6 provides a summary and location of surveillance reports.

Table J.6. Surveillance Report Summary

\begin{tabular}{|c|c|c|c|}
\hline Number & Subject & $\begin{array}{l}\text { Report Issued/ } \\
\text { Summary of Concerns }\end{array}$ & Associated Record \\
\hline $\begin{array}{l}\text { SR-52578- } \\
2011-009\end{array}$ & $\begin{array}{l}\text { QE Surveillance of RPL K Basin LRB } \\
60605 \text {, pp. } 29 \text { to 60, March 2011, which } \\
\text { covered LRB entries from 8/9/10 to } 2 / 2 / 11\end{array}$ & $\begin{array}{l}3 / 18 / 11 \\
\text { All comments } \\
\text { addressed. }\end{array}$ & $\begin{array}{l}\text { LRBs, Section III, } \\
\text { DVD-52578-PNNL- } \\
20650 \text { Rev. 2, } \\
\text { September } 2013\end{array}$ \\
\hline $\begin{array}{l}\text { SR-52578- } \\
2011-021\end{array}$ & $\begin{array}{l}\text { QE Surveillance of RPL K Basin LRB } \\
\text { 60605, pp. } 65 \text { to } 77 \text {, May 2011, which } \\
\text { covered LRB entries from 2/25/11 to 5/5/11 }\end{array}$ & $\begin{array}{l}\text { 6/3/11 } \\
\text { All comments } \\
\text { addressed. }\end{array}$ & $\begin{array}{l}\text { LRBs, Section III, } \\
\text { DVD-52578-PNNL- } \\
\text { 20650 Rev. 2, } \\
\text { September } 2013\end{array}$ \\
\hline $\begin{array}{l}\text { SR-52578- } \\
2011-023\end{array}$ & $\begin{array}{l}\text { QE Surveillance of Completed } 52578- \\
\text { TI001, K West Basin Sample Receipt and } \\
\text { Preparation of Consolidated Core } \\
\text { Composites: SCS-CON-210-A1 and } \\
\text { SCS-CON-210-B3 }\end{array}$ & $\begin{array}{l}\text { 6/14/11 } \\
\text { All comments } \\
\text { addressed. }\end{array}$ & $\begin{array}{l}\text { TIs, 52578-TI001 } \\
\text { Section III, DVD- } \\
\text { 52578-PNNL-20650 } \\
\text { Rev. 2, September } \\
\text { 2013 }\end{array}$ \\
\hline $\begin{array}{l}\text { SR-52578- } \\
2011-024\end{array}$ & $\begin{array}{l}\text { QE Surveillance of Completed } 52578- \\
\text { TI002, K West Basin Sample Receipt and } \\
\text { Preparation of Consolidated Core } \\
\text { Composites: SCS-CON-210-A4 and } \\
\text { SCS-CON-210-B1 }\end{array}$ & $\begin{array}{l}\text { 6/14/11 } \\
\text { All comments } \\
\text { addressed. }\end{array}$ & $\begin{array}{l}\text { TIs, 52578-TI002 } \\
\text { Section III, DVD- } \\
\text { 52578-PNNL-20650 } \\
\text { Rev. 2, September } \\
\text { 2013 }\end{array}$ \\
\hline $\begin{array}{l}\text { SR-52578- } \\
2011-025\end{array}$ & $\begin{array}{l}\text { QE Surveillance of completed } 52578 \text { - } \\
\text { TI008, Preparation of K West Basin } \\
\text { Consolidated Container Composite for } \\
\text { SCS-CON-210 }\end{array}$ & $\begin{array}{l}6 / 23 / 11 \\
\text { All comments } \\
\text { addressed. }\end{array}$ & $\begin{array}{l}\text { TIs, 52578-TI008 } \\
\text { Section III, DVD- } \\
\text { 52578-PNNL-20650 } \\
\text { Rev. 2, September } \\
\text { 2013 }\end{array}$ \\
\hline $\begin{array}{l}\text { SR-52578- } \\
\text { 2011-027, } \\
\text { Rev. } 1\end{array}$ & $\begin{array}{l}\text { QE Review of TIMS Report for Isotopic } \\
\text { Analysis of KW } 210 \text { Core Samples }\end{array}$ & $\begin{array}{l}8 / 3 / 11 \\
\text { All comments addressed } \\
\text { and OR 52578-05-09-11 } \\
\text { issued. }\end{array}$ & Appendix E \\
\hline $\begin{array}{l}\text { SR-52578- } \\
2011-029\end{array}$ & $\begin{array}{l}\text { QE Surveillance of } X \text {-Ray Diffraction } \\
\text { Analysis Report } K W 210 \text { Container } \\
\text { Composites }\end{array}$ & $\begin{array}{l}\text { 7/20/11 } \\
\text { All comments } \\
\text { addressed. }\end{array}$ & Appendix F \\
\hline $\begin{array}{l}\text { SR-52578- } \\
\text { 2011-030, } \\
\text { Rev. } 1\end{array}$ & $\begin{array}{l}\text { QE Surveillance of Completed 52578- } \\
\text { TI037, Particle Size Distribution } \\
\text { Measurements for K West Basin } \\
\text { Consolidated Container Composite SCS- } \\
\text { CON-210, Using Minus } 500 \text { micron } \\
\text { Particle Size Fraction Samples }\end{array}$ & $\begin{array}{l}\text { 8/3/11 } \\
\text { All comments } \\
\text { addressed. }\end{array}$ & $\begin{array}{l}\text { TIs, 52578-TI037 } \\
\text { Section III, DVD- } \\
\text { 52578-PNNL-20650 } \\
\text { Rev. 2, September } \\
\text { 2013 }\end{array}$ \\
\hline
\end{tabular}


PNNL-20650, Rev. 2

Table J.6. (contd)

\begin{tabular}{|c|c|c|c|}
\hline Number & Subject & $\begin{array}{c}\text { Report Issued/ } \\
\text { Summary of Concerns }\end{array}$ & Associated Record \\
\hline $\begin{array}{l}\text { SR-52578- } \\
2011-032\end{array}$ & $\begin{array}{l}\text { QE Surveillance of Particle Density Report } \\
\text { for TI008-L, TI008-MK, TI008-ML, TI008- } \\
\text { SK \& TI008-SL }\end{array}$ & $\begin{array}{l}\text { 7/14/11 } \\
\text { All comments } \\
\text { addressed. }\end{array}$ & Appendix G \\
\hline $\begin{array}{l}\text { SR-52578- } \\
2011-33\end{array}$ & $\begin{array}{l}\text { QE Surveillance of Completed 52578-TI- } \\
040, \text { Processing of the Undissolved Solids } \\
\text { Samples from the K West Basin Core } \\
\text { Samples Collected from SCS-CON-210 for } \\
\text { Additional Analysis }\end{array}$ & $\begin{array}{l}\text { 7/28/11 } \\
\text { All comments } \\
\text { addressed. }\end{array}$ & $\begin{array}{l}\text { TIs, 52578-TI040 } \\
\text { Section III, DVD- } \\
\text { 52578-PNNL-20650 } \\
\text { Rev. 2, September } \\
\text { 2013 }\end{array}$ \\
\hline $\begin{array}{l}\text { SR-52578- } \\
2011-034\end{array}$ & $\begin{array}{l}\text { QE Surveillance of XRD Analysis Report } \\
\text { for KW210 Undissolved Solids }\end{array}$ & $\begin{array}{l}7 / 27 / 11 \\
\text { All comments } \\
\text { addressed. }\end{array}$ & Appendix F \\
\hline $\begin{array}{l}\text { SR-52578- } \\
2011-035\end{array}$ & $\begin{array}{l}\text { QE Surveillance of completed K Basin TI, } \\
52578 \text {-TI } 039 \text {, Rheological Measurements } \\
\text { for K West Basin Consolidated Container } \\
\text { Composites SCS-CON-210 }\end{array}$ & $\begin{array}{l}8 / 2 / 11 \\
\text { All comments } \\
\text { addressed. }\end{array}$ & Appendix $\mathrm{H}$ \\
\hline $\begin{array}{l}\text { SR-52578- } \\
2011-041\end{array}$ & $\begin{array}{l}\text { QE Surveillance of: Surveillance of RPL } \\
\text { K Basin LRB 60605, pp. } 82 \text { to } 92 \text {, } \\
\text { September 2011, which covered LRB } \\
\text { entries from 5/20/11 to } 8 / 5 / 11\end{array}$ & $\begin{array}{l}\text { 9/13/11 } \\
\text { All comments } \\
\text { addressed. }\end{array}$ & $\begin{array}{l}\text { LRBs, Section III, } \\
\text { DVD-52578-PNNL- } \\
\text { 20650 Rev. 2, } \\
\text { September } 2013\end{array}$ \\
\hline $\begin{array}{l}\text { SR-52578- } \\
2011-042\end{array}$ & $\begin{array}{l}\text { QE Surveillance of Two Spreadsheets: } \\
\text { ASR } 8824 \text { Data Reduction Rev1.xlsx and } \\
\text { ASR } 8798 \text { Data Reduction Rev2.xlsx }\end{array}$ & $\begin{array}{l}\text { 9/26/11 } \\
\text { All comments } \\
\text { addressed. }\end{array}$ & $\begin{array}{l}\text { ASR Data } \\
\text { Reductions, Section } \\
\text { III, DVD-52578- } \\
\text { PNNL-20650 Rev. 2, } \\
\text { September 2013 }\end{array}$ \\
\hline
\end{tabular}

\section{J.6 Technical Reviewers}

Table J.7 provides technical reviewers' names, qualifications, and areas of review for this project.

Table J.7. Technical Reviewers Supporting the K Basin Sludge Characterization Activities

\begin{tabular}{|c|c|c|}
\hline General Review Area & Reviewer & Qualifications \\
\hline $\begin{array}{l}\text { TI review before } \\
\text { implementation }\end{array}$ & AJ Schmidt & $\begin{array}{l}\text { MS Chemical Engineering; } 22 \text { years of experience, including } \\
14 \text { years with K Basin Sludge characterization, testing and } \\
\text { evaluation. }\end{array}$ \\
\hline \multirow[t]{2}{*}{$\begin{array}{l}\text { TIs, supporting data calculation } \\
\text { spreadsheet, and LRB }\end{array}$} & SK Fiskum & $\begin{array}{l}\text { BS Chemistry, } 26 \text { years of chemical and radiochemical } \\
\text { characterization and reporting experience. }\end{array}$ \\
\hline & $\begin{array}{l}\text { RW } \\
\text { Shimskey }\end{array}$ & $\begin{array}{l}\text { BS Chemical Engineering, PE License; } 7 \text { years of radiochemical } \\
\text { processing, characterization, and reporting experience at PNNL; } \\
11 \text { years of zirconium/titanium processing experience geared to } \\
\text { the nuclear cladding and aerospace industry. }\end{array}$ \\
\hline $\begin{array}{l}\text { TIs, supporting data calculation } \\
\text { spreadsheet, LRB, ASRs, } \\
\text { particle density, PSD, rheology, } \\
\text { XRD, TIMS, and } \\
\text { characterization analyses }\end{array}$ & DS Coffey & $\begin{array}{l}\text { BS Biology/Chemistry, MS Resource Ecology, } 2 \text { years as } \\
\text { Laboratory Director and } 11 \text { years as deputy and Quality Manager } \\
\text { EPA ERL laboratory, } 5 \text { years as Deputy QM WIPP Sandia } \\
\text { National Laboratory, } 11 \text { years as QE Battelle, } 30 \text { years of } \\
\text { analytical data reporting with focus on regulatory work. }\end{array}$ \\
\hline
\end{tabular}


Table J.7. (contd)

\begin{tabular}{|c|c|c|}
\hline General Review Area & Reviewer & Qualifications \\
\hline $\begin{array}{l}\text { TIs, supporting data calculation } \\
\text { spreadsheet, LRB, PSD, } \\
\text { rheology, XRD and } \\
\text { characterization analyses }\end{array}$ & $\begin{array}{l}\text { LA } \\
\text { Middleton }\end{array}$ & $\begin{array}{l}\text { BS Environmental Science (with a minor in Chemistry), } 2 \text { years } \\
\text { of experience as QE at PNNL. }\end{array}$ \\
\hline TOC reports & KA Pool & $\begin{array}{l}\text { BS Chemistry, } 24 \text { years of experience in chemical and } \\
\text { radiochemical characterization and reporting. }\end{array}$ \\
\hline ICP-OES reports & $\begin{array}{l}\text { CJ } \\
\text { Thompson }\end{array}$ & $\begin{array}{l}\text { BS Chemistry, MS Analytical Chemistry, } \mathrm{PhD} \text { Analytical } \\
\text { Chemistry; } 16 \text { years of experience in chemical characterization } \\
\text { and reporting. }\end{array}$ \\
\hline Radiochemistry reports & $\begin{array}{l}\mathrm{CZ} \\
\text { Soderquist }\end{array}$ & $\begin{array}{l}\text { MS, Chemistry, } 27 \text { years of experience in radioanalytical } \\
\text { chemistry. }\end{array}$ \\
\hline Mass spectrometry reports & JC Braley & $\begin{array}{l}\text { PhD Chemistry, } 1 \text { year of post-degree experience with an } \\
\text { emphasis on radioanalytical and separations chemistry. }\end{array}$ \\
\hline Radiochemistry reports & $\begin{array}{l}\text { LR } \\
\text { Greenwood }\end{array}$ & $\begin{array}{l}\text { PhD Nuclear Physics, Laboratory Fellow, } 40 \text { years of experience } \\
\text { with reactor dosimetry, nuclear data measurements, and radiation } \\
\text { detector development; team lead for Radiochemistry; project } \\
\text { manager on numerous projects }\end{array}$ \\
\hline Radiochemistry reports & TT Trang-Le & $\begin{array}{l}\text { BS Mathematics-Actuarial Science, } 16 \text { years of experience in } \\
\text { radioanalytical reporting. }\end{array}$ \\
\hline XRD reports & MW Urie & $\begin{array}{l}\text { BS Chemistry, BS Electrical Engineering, } 43 \text { years of chemical } \\
\text { characterization; } 20 \text { years of XRF, XRD, Auger, and physical } \\
\text { measurements experience. }\end{array}$ \\
\hline $\begin{array}{l}\text { Particle density and } \\
\text { U speciation reports }\end{array}$ & SI Sinkov & $\begin{array}{l}\text { PhD Chemistry (with an emphasis on chemistry of transuranium } \\
\text { elements), } 26 \text { years of experience, including } 6 \text { years with } \\
\text { K Basin sludge characterization, testing, and reporting. }\end{array}$ \\
\hline Rheology report & CA Burns & $\begin{array}{l}\text { PhD Chemistry (with an emphasis on surface and colloid } \\
\text { science), } 5 \text { years of post-degree experience in the } \\
\text { characterization of physical properties of both radiological and } \\
\text { non radiological materials. }\end{array}$ \\
\hline PSD report & RC Daniel & $\begin{array}{l}\text { PhD Chemical Engineering (with an emphasis on surface and } \\
\text { colloid science), } 5 \text { years of post-degree experience in the size } \\
\text { characterization of radiological dispersions. }\end{array}$ \\
\hline $\begin{array}{l}\text { Mass spectrometry reports and } \\
\text { mass measurement reports }\end{array}$ & CH Delegard & $\begin{array}{l}\text { BS Chemistry, } 38 \text { years of chemical, radiochemical, and } \\
\text { safeguards characterization and reporting experience. }\end{array}$ \\
\hline $\begin{array}{l}\text { Transcription review of } \\
\text { analytical data calculation } \\
\text { spreadsheets and ASO reports; } \\
\text { data incorporation into } \\
\text { summary report; transcription } \\
\text { accuracy }\end{array}$ & M Luna & $\begin{array}{l}\text { BS Chemistry/Biology, } 4 \text { years of experience in the } \\
\text { characterization of physical properties of both radiological and } \\
\text { non radiological materials, testing, and reporting, } 8 \text { years of } \\
\text { experience in Chemical/Biological warfare agents and clinical } \\
\text { pathogens decontamination, and } 7 \text { years studying molecular } \\
\text { process and bioengineering autoimmune and infectious diseases. }\end{array}$ \\
\hline $\begin{array}{l}\text { Technical review of completed } \\
\text { report }\end{array}$ & JM Tingey & $\begin{array}{l}\text { PhD Physical Chemistry, } 27 \text { years of experience in the } \\
\text { characterization and treatment of nuclear wastes and spent } \\
\text { nuclear fuel, basic and applied actinide chemistry, plutonium and } \\
\text { spent fuel processing, and colloid chemistry. Several years } \\
\text { experience in characterization of K Basin sludges. }\end{array}$ \\
\hline
\end{tabular}




\section{J.7 STP Validation and Revision 2 Corrections}

The initial report (Revision 1) was delivered to CHPRC for data validation by the STP. The validation activity resulted in identification of errata and provided the basis for Revision 2 to this report. Table J.8 summarizes the errata identified during the STP validation process and the corrections made to the report. Additional editorial changes directed by the project are summarized in Table J.9.

Table J.8. Summary of STP Validation Errata and Changes Made in Revision 2

\begin{tabular}{|c|c|c|}
\hline $\begin{array}{l}\text { PNNL-20650, Rev. } 1 \\
\text { Report Location }\end{array}$ & Errata Summary & Correction Made \\
\hline Page xiii, Definitions section & $\begin{array}{l}\text { The given definition of "Dry Sludge: } \\
\text { Sludge that has been dried to a constant } \\
\text { mass at } 105^{\circ} \text { C." A clarifying definition } \\
\text { should be added for the term "As-Sampled" } \\
\text { since there is a tendency to equate "as- } \\
\text { sampled" and "dewatered" (in-part because } \\
\text { Test Instructions have equated these terms } \\
\text { in their "Relevant Terms" definition section } \\
\text { [e.g., page } 11 \text { of } 52578-T I 008 \text { where } \\
\text { sieving was done includes a definition for } \\
\text { "Dewatered or As-Sampled"]). }\end{array}$ & $\begin{array}{l}\text { Definition of "as-sampled" added, } \\
\text { "The actual state of the sludge at } \\
\text { the point of sampling; in most } \\
\text { instances this is the "dewatered" } \\
\text { sludge state, but in the case of } \\
\text { sieved sample material retained on } \\
\text { a sieve it is taken as "air-dried" } \\
\text { (dried at ambient conditions in the } \\
\text { hot cell)." } \\
\text { The Definition now appears on } \\
\text { page xv of revision } 2 \text {. }\end{array}$ \\
\hline $\begin{array}{l}\text { Page } 2.3 \\
\text { Figure } 2.1\end{array}$ & $\begin{array}{l}\text { Under icon for "KW \& KE } 230 \text { ", in gray } \\
\text { square, under "59205-TI023" it is indicated } \\
\text { this TI addresses "Cores A4 and B2" the } \\
\text { correct cores are "Cores A2 and B4". }\end{array}$ & $\begin{array}{l}\text { Figure } 2.1 \text { changed to reflect } \\
\text { correct core identifications. }\end{array}$ \\
\hline $\begin{array}{l}\text { Page } 2.10 \\
\text { Table } 2.3 \\
\text { Column "Subsample ID" } \\
\text { Archive samples; } \\
\text { Page } 2.11 \\
\text { Table } 2.4 \\
\text { Column "Subsample ID" } \\
\text { Archive samples; } \\
\text { Page } 2.12 \\
\text { Table } 2.5 \\
\text { Column "Subsample ID" } \\
\text { Archive samples; } \\
\text { Page } 2.13 \\
\text { Table } 2.6 \\
\text { Column "Subsample ID" } \\
\text { Archive samples }\end{array}$ & $\begin{array}{l}\text { Page } 2.10 \text { Table } 2.3 \text {, page } 2.11 \text { Table } 2.4 \text {, } \\
\text { page } 2.12 \text { Table } 2.5 \text {, and page } 2.13 \text { Table } \\
\text { 2.6: in the column under "Subsample ID" } \\
\text { for the archive samples that are the last } \\
\text { group of samples shown in this column, the } \\
\text { sample prefixes have incorrectly been } \\
\text { shown as "TI001" or "TI002"; the actual } \\
\text { sample ID prefix for all these archive } \\
\text { samples is "KW210". }\end{array}$ & $\begin{array}{l}\text { Sample identifications corrected. } \\
\text { Table } 2.4 \text { footnote }(d) \text { was } \\
\text { similarly amended to reflect } \\
\text { nomenclature. }\end{array}$ \\
\hline $\begin{array}{l}\text { Page } 2.20 \\
\text { Table } 2.9 \text {, } \\
\text { Column "As-sampled Mass" }\end{array}$ & $\begin{array}{l}\text { Added footnote (a) to table to clarify the as- } \\
\text { sampled mass basis for middle and large } \\
\text { sieve fraction samples is based on air-dried } \\
\text { sample masses. }\end{array}$ & $\begin{array}{l}\text { Added footnote (a) which states, } \\
\text { "The as-sampled mass basis for all } \\
\text { large and medium sieve fraction } \\
\text { samples (i.e. TI008-LA and TI008- } \\
\mathrm{M}^{*} \text {, where * is A-L) is "air dried" } \\
\text { and thus the sample may contain } \\
\text { some residual water." All other } \\
\text { existing footnote identities were } \\
\text { incremented up by one letter. }\end{array}$ \\
\hline
\end{tabular}


Table J.8. (contd)

\begin{tabular}{|c|c|c|}
\hline $\begin{array}{l}\text { PNNL-20650, Rev. } 1 \\
\text { Report Location }\end{array}$ & Errata Summary & Correction Made \\
\hline $\begin{array}{l}\text { Page } 5.5 \text {, Section 5.2, second } \\
\text { sentence in first paragraph }\end{array}$ & $\begin{array}{l}\text { "Appendix J contains the detailed rheology } \\
\text { including shear strength rheograms." The } \\
\text { actual Appendix is "H" instead of indicated } \\
\text { "J". Also the ending phrase "... including } \\
\text { strength rheograms" should be deleted. The } \\
\text { rheograms were not collected; only shear } \\
\text { strength measures were collected. }\end{array}$ & $\begin{array}{l}\text { Sentence now reads, "Appendix H } \\
\text { contains the detailed rheology } \\
\text { report." and the ending phrase was } \\
\text { deleted. }\end{array}$ \\
\hline Page 5.10, Table 5.5 title & $\begin{array}{l}\text { In the table title, sample number should be } \\
\text { KW210-CC-N rather than indicated } \\
\text { KW210-SS-N. }\end{array}$ & $\begin{array}{l}\text { Sample identification corrected to } \\
\text { KW210-CC-N. }\end{array}$ \\
\hline $\begin{array}{l}\text { Page 5.21, Table 5.15, MCC } \\
\text { U Metal, As-sampled basis }\end{array}$ & $\begin{array}{l}\text { For MCC samples, table should include } \\
\text { clarification that "as-sampled" basis is "air } \\
\text { dried". }\end{array}$ & $\begin{array}{l}\text { Inserted new footnote (b) which } \\
\text { states, "The as-sampled mass basis } \\
\text { for medium sieve fraction samples } \\
\text { (i.e. TI008-ME and TI008-MF) is } \\
\text { air dried and thus the sample may } \\
\text { contain some residual water. } \\
\text { Existing footnotes (b) through (d) } \\
\text { were incremented up by one letter. }\end{array}$ \\
\hline $\begin{array}{l}\text { Page 5.21, Table 5.15, } \\
\text { footnote (c) }\end{array}$ & $\begin{array}{l}\text { In the footnote (c), “ } 0.474 \mathbf{g}_{\text {sampled }} / \mathbf{g}_{\text {dry }} \\
\text { should be: } 0.474 \mathbf{g}_{\text {dry }} / \mathbf{g}_{\text {sampled. }}\end{array}$ & $\begin{array}{l}\text { Units corrected to } \mathbf{g}_{\text {dry }} / \mathbf{g}_{\text {sampled }} \text { in } \\
\text { footnote (d) of revision } 2 \text {. }\end{array}$ \\
\hline $\begin{array}{l}\text { Page } 5.21 \text {, Table } 5.15 \text {, } \\
\text { footnote }(\mathrm{d})\end{array}$ & $\begin{array}{l}\text { In the footnote (d) reference, "(Fiskum et al. } \\
2011 \text { a, Appendix J)," the "2011a" should be } \\
\text { "2011b". }\end{array}$ & $\begin{array}{l}\text { Reference correction to Fiskum et } \\
\text { al. } 2011 \text { b on footnote (e) of } \\
\text { revision } 2 \text {. }\end{array}$ \\
\hline $\begin{array}{l}\text { Page 5.22, first paragraph, } \\
\text { fourth sentence }\end{array}$ & $\begin{array}{l}\text { The reference for "(Fiskum et al. 2011, } \\
\text { Appendix J)," the " } 2011 \text { " should be } \\
\text { "2011b". }\end{array}$ & $\begin{array}{l}\text { Reference corrected in text to read } \\
\text { Fiskum et al. } 2011 \mathrm{~b} \text {. }\end{array}$ \\
\hline $\begin{array}{l}\text { Page } 5.23 \text {, Table } 5.17 \text {, } \\
\text { Sample ID column }\end{array}$ & $\begin{array}{l}\text { The three samples identified with "TI009" } \\
\text { prefixes should have prefixes of "TI008". }\end{array}$ & $\begin{array}{l}\text { Corrected all TI009 prefixes to } \\
\text { TI008. }\end{array}$ \\
\hline $\begin{array}{l}\text { Page } 5.31 \text {, Table } 5.24 \text {, As- } \\
\text { measured }{ }^{60} \text { Co concentration } \\
\text { for sample TI008-SA }\end{array}$ & $\begin{array}{l}\text { Value of Co- } 60 \text { concentration should be } \\
\mathbf{1 . 1 2 E - 3} \text { instead of } \mathbf{1 . 2 E}-\mathbf{3} \text {. Also under the } \\
\text { "Relative Amount" Co- } 60 \text { should be } \mathbf{0 . 2 5} \% \\
\text { instead of to } \mathbf{0 . 2 7} \% \text {. \{Related to this: On } \\
\text { the PNNL-20650 Rev } 1 \text { DVD, under } \\
\text { Section III, that includes data analysis MS } \\
\text { Excel files, under "ASR } 8824 \text { Data } \\
\text { Reduction Rev1", tab "Radchem" the } \\
\text { spreadsheet cell O43 (the concentration of } \\
\text { Co-60 in sample TI008-SA) should be } \\
\mathbf{1 . 1 2 E - 3 ~} \mu \mathrm{Ci} / \mathrm{g} \text { instead of the indicated } \\
\mathbf{1 . 2 E - 3} \mu \mathrm{Ci} / \mathrm{g} \text { to match the ASO reported } \\
\text { value (page C.36). This in-turn changes the } \\
\text { relative Co-60 value in cell Q60 to } \mathbf{0 . 2 5 \%} \\
\text { instead of the indicated } \mathbf{0 . 2 7 \%} \text {.\}. }\end{array}$ & $\begin{array}{l}\text { As-measured }{ }^{60} \text { Co concentration } \\
\text { changed from } 1.2 \mathrm{E}-3 \text { to } 1.12 \mathrm{E}-3 \\
\text { and relative amount changed from } \\
0.27 \% \text { to } 0.25 \% \text {. } \\
\text { Spreadsheet "ASR8824 Data } \\
\text { Reduction Rev.1.xlsx, worksheet } \\
\text { "radchem", cell O43, updated to } \\
1.12 \mathrm{E}-3 \text {. Spreadsheet worksheet } \\
\text { "overview" updated to reflect } \\
\text { change and the spreadsheet } \\
\text { filename revised to "ASR } 8824 \\
\text { Data Reduction Rev.2.xlsx. The } \\
\text { spreadsheet package was reviewed } \\
\text { and added to the DVD data } \\
\text { package for this report. }\end{array}$ \\
\hline $\begin{array}{l}\text { Page 5.33, Table 5.26, } \\
\text { "(RPL/ASO Parent ID) Sieve } \\
\text { Fraction" column }\end{array}$ & $\begin{array}{l}\text { The sample identifications indicated as } \\
\text { "(11-0792-D)" and "(11-0793-D) should be } \\
\text { "(11-0792-129-D)" and "(11-0793-129-D)." }\end{array}$ & $\begin{array}{l}\text { Sample identifications changed to } \\
11-0792-129-D \text { and } \\
11-0793-129-D \text {. }\end{array}$ \\
\hline
\end{tabular}


PNNL-20650, Rev. 2

Table J.8. (contd)

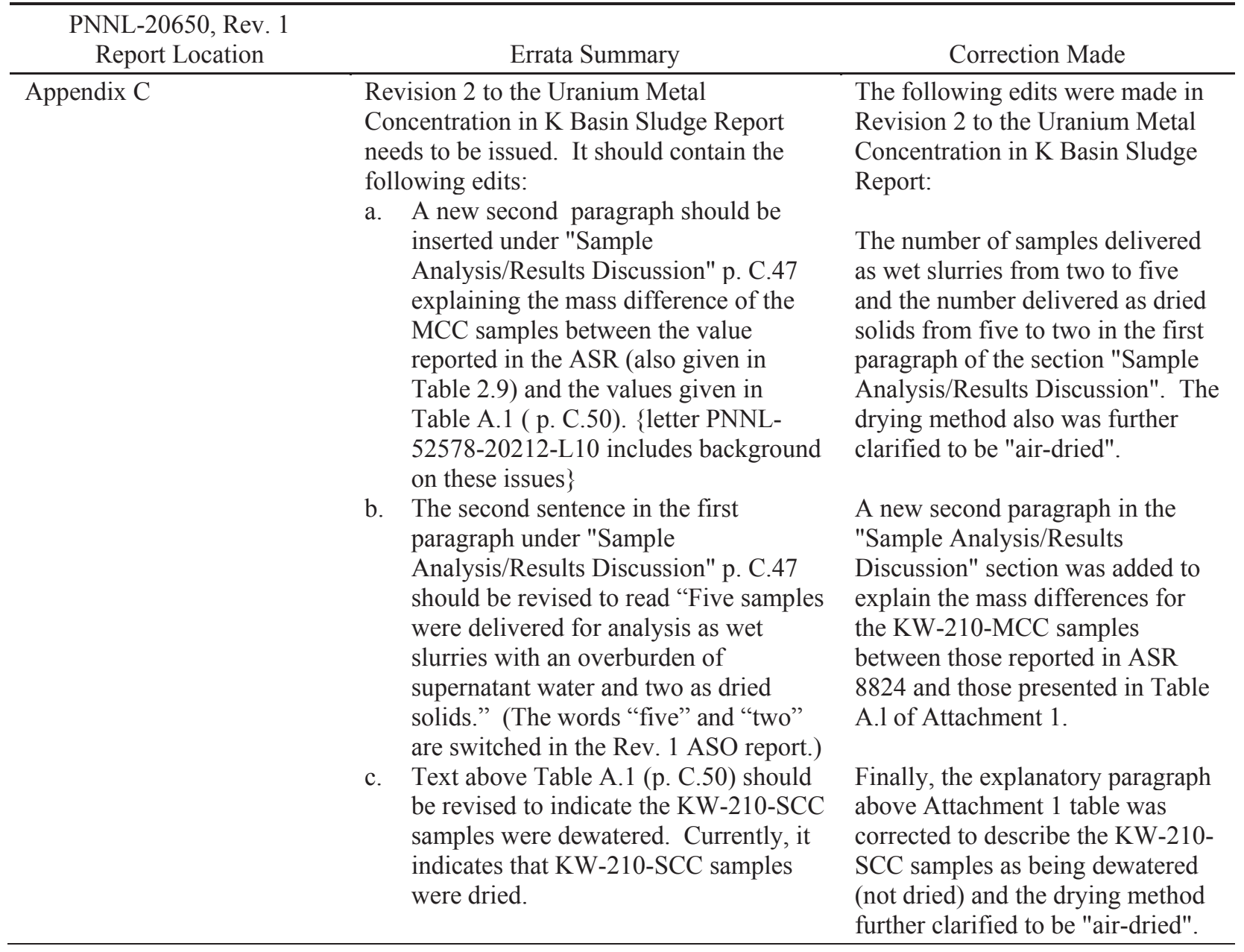

Table J.9. Summary of PNNL Directed Changes Made in Revision 2

\begin{tabular}{lll}
\hline \multicolumn{1}{c}{$\begin{array}{c}\text { PNNL-20650, Rev. 1 } \\
\text { Report Location }\end{array}$} & \multicolumn{1}{c}{ Editorial Change Summary } & \multicolumn{1}{c}{ Correction Made } \\
\hline Disclaimer page & $\begin{array}{l}\text { Provide name, address, phone number, and } \\
\text { email address of two agencies at which } \\
\text { this report will be archived and available at } \\
\text { request. }\end{array}$ & $\begin{array}{l}\text { Provided contact information to } \\
\text { request a copy of this report. }\end{array}$ \\
$\begin{array}{l}\text { Page ii, Revision history } \\
\text { table }\end{array}$ & $\begin{array}{l}\text { Provide new entry to revision table for } \\
\text { report revision 2. The description column } \\
\text { explains the corrections are a direct result } \\
\text { of the STP validation process. }\end{array}$ & $\begin{array}{l}\text { Inserted new entry in revision } \\
\text { history table reflecting the changes } \\
\text { made to this report. }\end{array}$ \\
$\begin{array}{l}\text { Page iii, Certification } \\
\text { statement }\end{array}$ & $\begin{array}{l}\text { Include a new certification page for } \\
\text { revision 2 and keep the original } \\
\text { certification page generated for Revision } \\
\text { 1. }\end{array}$ & $\begin{array}{l}\text { Inserted new page for certification } \\
\text { for revision 2 prior to the original } \\
\text { certification page. }\end{array}$ \\
Page ix, x, and xi, Acronyms & $\begin{array}{l}\text { Acronym definitions needed for DVD, } \\
\text { NARA, and TRIM. }\end{array}$ & $\begin{array}{l}\text { The DVD, NARA, and TRIM } \\
\text { acronyms were defined in Rev. 2 of } \\
\text { the report. }\end{array}$ \\
\hline
\end{tabular}


Table J.9. (contd)

\begin{tabular}{|c|c|c|}
\hline $\begin{array}{l}\text { PNNL-20650, Rev. } 1 \\
\text { Report Location }\end{array}$ & Editorial Change Summary & Correction Made \\
\hline $\begin{array}{l}\text { Page xxv, DVD TOC, } \\
\text { Section III, ASR Data } \\
\text { Reductions }\end{array}$ & $\begin{array}{l}\text { Provide revision } 2 \text { spreadsheet name to list } \\
\text { of files. }\end{array}$ & $\begin{array}{l}\text { Spreadsheet "ASR } 8824 \text { Data } \\
\text { Reduction Rev2.xlsx" included in } \\
\text { DVD folder file list on page xxvii } \\
\text { of revision } 2 \text {. }\end{array}$ \\
\hline $\begin{array}{l}\text { Page xxiii, DVD TOC } \\
\text { introduction }\end{array}$ & $\begin{array}{l}\text { New report revision and publish date } \\
\text { requires DVD title and electronic report } \\
\text { name updates. }\end{array}$ & $\begin{array}{l}\text { Revision } 1 \text { replaced with Revision } 2 \\
\text { and October } 2011 \text { replaced with } \\
\text { September } 2013 \text { on page xxv of } \\
\text { revision } 2 \text {. }\end{array}$ \\
\hline Page 2.1 , footnote (b) & $\begin{array}{l}\text { Reflect change in the name of the DVD in } \\
\text { footnote (b) on this page. }\end{array}$ & $\begin{array}{l}\text { Footnote (b) on page } 2.1 \text { now shows } \\
\text { DVD-52578-PNNL-20650 Rev. } 2 \text {, } \\
\text { Section III, September } 2013 \text {. }\end{array}$ \\
\hline $\begin{array}{l}\text { Page } 2.4 \text {, first paragraph, } \\
\text { second sentence }\end{array}$ & $\begin{array}{l}\text { Reflect change in the name of the DVD on } \\
\text { this page. }\end{array}$ & $\begin{array}{l}\text { Sentence now references DVD- } \\
\text { 52578-PNNL-20650 Rev. } 2 \text {, } \\
\text { Section III, September } 2013 \text {. }\end{array}$ \\
\hline $\begin{array}{l}\text { Page } 2.27 \text {, Section } 2.4 \text {, fourth } \\
\text { paragraph }\end{array}$ & $\begin{array}{l}\text { Reflect change in the name of the DVD on } \\
\text { this page. }\end{array}$ & $\begin{array}{l}\text { Sentence now references DVD- } \\
\text { 52578-PNNL-20650 Rev. } 2 \text {, } \\
\text { Section II, September } 2013 .\end{array}$ \\
\hline $\begin{array}{l}\text { Page } 3.5 \text {, Table } 3.1 \text {, second } \\
\text { table note }\end{array}$ & $\begin{array}{l}\text { Reflect change in the name of the DVD on } \\
\text { this page. }\end{array}$ & $\begin{array}{l}\text { Sentence now references DVD- } \\
\text { 52578-PNNL-20650 Rev. } 2 \\
\text { Section III, September } 2013 \text {. }\end{array}$ \\
\hline Page 3.6, footnote (b) & $\begin{array}{l}\text { Reflect change in the name of the DVD on } \\
\text { this page. }\end{array}$ & $\begin{array}{l}\text { Sentence now references DVD- } \\
\text { 52578-PNNL-20650 Rev. 2, } \\
\text { Section III, September } 2013\end{array}$ \\
\hline $\begin{array}{l}\text { Page } 3.7 \text {, second paragraph, } \\
\text { third sentence }\end{array}$ & $\begin{array}{l}\text { Reflect change in the name of the DVD on } \\
\text { this page. }\end{array}$ & $\begin{array}{l}\text { Sentence now references DVD- } \\
\text { 52578-PNNL-20650 Rev. } 2 \text {, } \\
\text { Section III, September } 2013 \text {. }\end{array}$ \\
\hline $\begin{array}{l}\text { Page } 4.2 \text {, first paragraph, } \\
\text { third sentence }\end{array}$ & $\begin{array}{l}\text { Reflect change in the name of the DVD on } \\
\text { this page. }\end{array}$ & $\begin{array}{l}\text { Sentence now references DVD- } \\
\text { 52578-PNNL-20650 Rev. } 2 \text {, } \\
\text { Section IV, September } 2013 \text {. }\end{array}$ \\
\hline $\begin{array}{l}\text { Page } 4.9 \text {, Figure } 4.10 \text {, } \\
\text { bottom-right image }\end{array}$ & $\begin{array}{l}\text { The sample ID prefix has been incorrectly } \\
\text { shown as "TI001," the actual sample ID } \\
\text { prefix for all archive samples is "KW210." }\end{array}$ & $\begin{array}{l}\text { The sample ID for the image now } \\
\text { reads KW210-B3-Archive-1. }\end{array}$ \\
\hline $\begin{array}{l}\text { Page } 4.12 \text {, Table } 4.2 \text {, table } \\
\text { note }\end{array}$ & $\begin{array}{l}\text { Reflect change in the name of the DVD on } \\
\text { this page. }\end{array}$ & $\begin{array}{l}\text { Sentence now references DVD- } \\
\text { 52578-PNNL-20650 Rev. } 2 \text {, } \\
\text { Section III, September } 2013 \text {. }\end{array}$ \\
\hline $\begin{array}{l}\text { Page } 4.17 \text {, Table } 4.6 \text {, third } \\
\text { table note }\end{array}$ & $\begin{array}{l}\text { Reflect change in the name of the DVD on } \\
\text { this page. }\end{array}$ & $\begin{array}{l}\text { Sentence now references DVD- } \\
\text { 52578-PNNL-20650 Rev. } 2 \text {, } \\
\text { Section III, September } 2013 \text {. }\end{array}$ \\
\hline $\begin{array}{l}\text { Page } 4.18 \text {, Table } 4.7 \text {, } \\
\text { footnote (a) }\end{array}$ & $\begin{array}{l}\text { Reflect change in the name of the DVD on } \\
\text { this page. }\end{array}$ & $\begin{array}{l}\text { Sentence now references DVD- } \\
\text { 52578-PNNL-20650 Rev. } 2 \text {, } \\
\text { Section III, September } 2013 \text {. }\end{array}$ \\
\hline $\begin{array}{l}\text { Page } 4.19 \text {, Table } 4.8 \text {, second } \\
\text { table note }\end{array}$ & $\begin{array}{l}\text { Reflect change in the name of the DVD on } \\
\text { this page. }\end{array}$ & $\begin{array}{l}\text { Sentence now references DVD- } \\
\text { 52578-PNNL-20650 Rev. } 2 \text {, } \\
\text { Section III, September 2013. }\end{array}$ \\
\hline $\begin{array}{l}\text { Page } 4.20 \text {, Table } 4.9 \text {, second } \\
\text { table note }\end{array}$ & $\begin{array}{l}\text { Reflect change in the name of the DVD on } \\
\text { this page. }\end{array}$ & $\begin{array}{l}\text { Sentence now references DVD- } \\
\text { 52578-PNNL-20650 Rev. } 2 \text {, } \\
\text { Section III, September } 2013 \text {. }\end{array}$ \\
\hline
\end{tabular}


Table J.9. (contd)

\begin{tabular}{|c|c|c|}
\hline $\begin{array}{l}\text { PNNL-20650, Rev. } 1 \\
\text { Report Location }\end{array}$ & Editorial Change Summary & Correction Made \\
\hline $\begin{array}{l}\text { Page } 4.34 \text {, Table } 4.17 \text {, second } \\
\text { table note }\end{array}$ & $\begin{array}{l}\text { Reflect change in the name of the DVD on } \\
\text { this page. }\end{array}$ & $\begin{array}{l}\text { Sentence now references DVD- } \\
\text { 52578-PNNL-20650 Rev. 2, } \\
\text { Section III, September 2013. }\end{array}$ \\
\hline $\begin{array}{l}\text { Page } 4.40 \text {, Table } 4.21 \text {, table } \\
\text { note }\end{array}$ & $\begin{array}{l}\text { Reflect change in the name of the DVD on } \\
\text { this page. }\end{array}$ & $\begin{array}{l}\text { Sentence now references DVD- } \\
\text { 52578-PNNL-20650 Rev. } 2 \text {, } \\
\text { Section III, September } 2013 \text {. }\end{array}$ \\
\hline $\begin{array}{l}\text { Page } 4.42 \text {, Table } 4.22 \text {, Core } \\
\text { Sample column }\end{array}$ & Correct core sample ID from B4 to B3 & Core sample ID corrected to B3. \\
\hline Page 5.2, footnote (a) & $\begin{array}{l}\text { Reflect change in the name of the DVD on } \\
\text { this page. }\end{array}$ & $\begin{array}{l}\text { Sentence now references DVD- } \\
\text { 52578-PNNL-20650 Rev. 2, } \\
\text { Section III, September 2013. }\end{array}$ \\
\hline $\begin{array}{l}\text { Page } 5.39 \text {, Table } 5.29, \\
\text { footnote (a) }\end{array}$ & $\begin{array}{l}\text { Reflect change in the name of the DVD on } \\
\text { this page. }\end{array}$ & $\begin{array}{l}\text { Sentence now references DVD- } \\
\text { 52578-PNNL-20650 Rev. } 2 \\
\text { Section III, September 2013. }\end{array}$ \\
\hline $\begin{array}{l}\text { Page 6.1, first paragraph, } \\
\text { second sentence }\end{array}$ & $\begin{array}{l}\text { Reflect change in the name of the DVD on } \\
\text { this page. }\end{array}$ & $\begin{array}{l}\text { Sentence now references DVD- } \\
\text { 52578-PNNL-20650 Rev. 2, } \\
\text { Section I-IV, September } 2013 .\end{array}$ \\
\hline Page 6.1, Introduction & $\begin{array}{l}\text { Add statement that references where DVD } \\
\text { is stored for potential public inquiry. Add } \\
\text { statement how this records storage differs } \\
\text { from ASO records storage. }\end{array}$ & $\begin{array}{l}\text { Text now reads: } \\
\text { The contents of the DVD are stored } \\
\text { in the PNNL records electronic } \\
\text { database Total Records Information } \\
\text { Management (TRIM) records } \\
\text { system. The long-term storage of } \\
\text { ASO-specific records are maintained } \\
\text { in the National Archives and } \\
\text { Records Administration (NARA) } \\
\text { Federal Records Center (Seattle, } \\
\text { Washington) (hardcopy), which in } \\
\text { turn is based on DOE and other } \\
\text { Federal records management } \\
\text { requirements. }\end{array}$ \\
\hline $\begin{array}{l}\text { Page } 6.1 \text {, Section } 6.1 \text {, end of } \\
\text { first paragraph }\end{array}$ & Reflect the change and addition of NARA. & $\begin{array}{l}\text { Text addition in bold: (Note: All } \\
\text { records submitted for storage } \\
\text { created by PNNL under the } \\
\text { Battelle/DOE Operating Contract } \\
\text { [DE-ACO5-76RL01830] are } \\
\text { transferred to the NARA Federal } \\
\text { Records Center in Seattle, } \\
\text { Washington.) }\end{array}$ \\
\hline $\begin{array}{l}\text { Page } 6.2 \text {, Table } 6.1 \text {, position } \\
\text { "PNNL Records } \\
\text { Management; Records } \\
\text { Storage and Retrieval" }\end{array}$ & $\begin{array}{l}\text { Update PNNL Records Management } \\
\text { Contact. }\end{array}$ & $\begin{array}{l}\text { Patricia Eick has been replaced by } \\
\text { Terri Mooney; her contact } \\
\text { information was added. }\end{array}$ \\
\hline Appendix J, page J.1 & $\begin{array}{l}\text { Add new paragraph describing the genesis } \\
\text { of the data package Revision } 2 \text { relative to } \\
\text { the STP validation. }\end{array}$ & $\begin{array}{l}\text { New paragraph } 2 \text { was added to page } \\
\text { J.1 describing the reason for issuing } \\
\text { Revision } 2 \text {. }\end{array}$ \\
\hline
\end{tabular}


PNNL-20650, Rev. 2

Table J.9. (contd)

\begin{tabular}{lll}
\hline \multicolumn{1}{c}{$\begin{array}{c}\text { PNNL-20650, Rev. 1 } \\
\text { Report Location }\end{array}$} & \multicolumn{1}{c}{ Editorial Change Summary } & \multicolumn{1}{c}{ Correction Made } \\
\hline $\begin{array}{l}\text { Appendix J } \\
\text { Throughout }\end{array}$ & Reflect change in the name of the DVD. & $\begin{array}{l}\text { Rev.1 was changed to Rev. 2 and } \\
\text { October 2011 was changed to } \\
\text { September 2013. }\end{array}$ \\
Appendix J & $\begin{array}{l}\text { Add new subsection to discuss the STP } \\
\text { validation and changes made for } \\
\text { Revision 1. }\end{array}$ & $\begin{array}{l}\text { Added subsection J.7 "STP } \\
\text { Validation and Revision 2 } \\
\text { corrections." }\end{array}$ \\
\hline
\end{tabular}

